Graduate Texts in Physics

Helmut Wiedemann

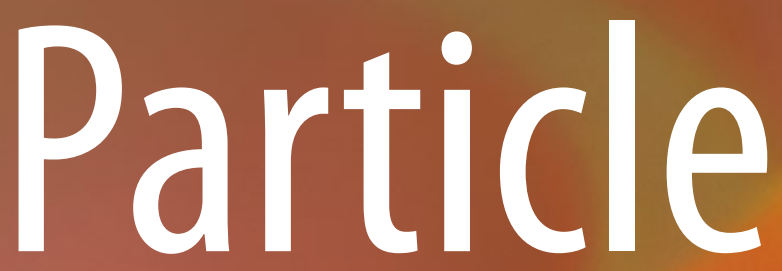

Accelerator

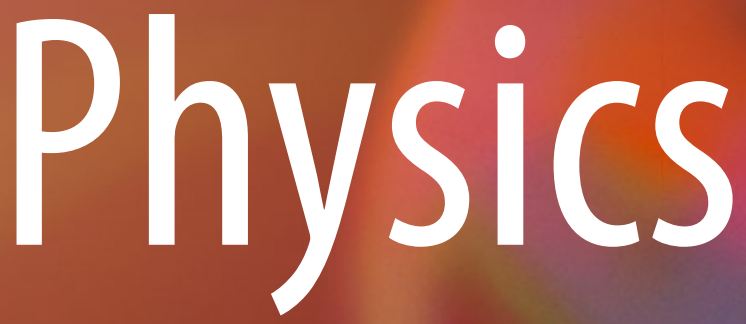

Fourth Edition

Springer 


\section{Graduate Texts in Physics}

\section{Series Editors}

Sadri Hassani

Illinois, USA

W.J. Munro

Kanagawa, Japan

Richard Needs

Cambridge, UK

William T. Rhodes

Florida, USA

Martin Stutzmann

Garching, Germany

Andreas Wipf

Jena, Germany 


\section{Graduate Texts in Physics}

Graduate Texts in Physics publishes core learning/teaching material for graduateand advanced-level undergraduate courses on topics of current and emerging fields within physics, both pure and applied. These textbooks serve students at the MS- or PhD-level and their instructors as comprehensive sources of principles, definitions, derivations, experiments and applications (as relevant) for their mastery and teaching, respectively. International in scope and relevance, the textbooks correspond to course syllabi sufficiently to serve as required reading. Their didactic style, comprehensiveness and coverage of fundamental material also make them suitable as introductions or references for scientists entering, or requiring timely knowledge of, a research field.

More information about this series at www.springer.com/series/8431 
Helmut Wiedemann

\section{Particle Accelerator Physics}

Fourth Edition

算 Springer 


\author{
Helmut Wiedemann \\ Emeritus Professor of Applied Physics \\ and of the Stanford Synchrotron \\ Radiation Laboratory \\ Stanford University \\ Stanford \\ California, USA
}

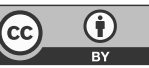

ISSN 1868-4513

Graduate Texts in Physics

ISBN 978-3-319-18316-9

DOI 10.1007/978-3-319-18317-6
ISSN 1868-4521 (electronic)

ISBN 978-3-319-18317-6 (eBook)

Library of Congress Control Number: 2015945573

Open Access This book was originally published with exclusive rights reserved by the Publisher in 2015 and was licensed as an open access publication in November 2019 under the terms of the Creative Commons Attribution 4.0 International License (http://creativecommons.org/licenses/by/4.0/), which permits use, sharing, adaptation, distribution and reproduction in any medium or format, as long as you give appropriate credit to the original author(s) and the source, provide a link to the Creative Commons license if changes were made.

The images or other third party material in this book may be included in the book's Creative Commons license, unless indicated otherwise in a credit line to the material or in the Correction Note appended to the book. For details on rights and licenses please read the Correction https://doi.org/10.1007/978-3-319-18317-6_28. If material is not included in the book's Creative Commons license and your intended use is not permitted by statutory regulation or exceeds the permitted use, you will need to obtain permission directly from the copyright holder.

(C) The Editor(s) (if applicable) and The Author(s) 2015, corrected publication 2019

The use of general descriptive names, registered names, trademarks, service marks, etc. in this publication does not imply, even in the absence of a specific statement, that such names are exempt from the relevant protective laws and regulations and therefore free for general use.

The publisher, the authors and the editors are safe to assume that the advice and information in this book are believed to be true and accurate at the date of publication. Neither the publisher nor the authors or the editors give a warranty, expressed or implied, with respect to the material contained herein or for any errors or omissions that may have been made. The publisher remains neutral with regard to jurisdictional claims in published maps and institutional affiliations.

The original version of this book was revised.

The correction to this book can be found at https://doi.org/10.1007/978-3-319-18317-6_28

Printed on acid-free paper

Springer International Publishing AG Switzerland is part of Springer Science+Business Media (www.springer.com) 
To my sons and students 



\section{Preface to Fourth Edition}

Just 20 years have passed since the first edition. During those years, the book has gone through several phases starting with the two volume edition 1 and 2 . Finally in 2007, both volumes and the book on Synchrotron Radiation have been combined into the Third-Edition as one volume to serve as a textbook for students and beginners as well as a reference book for the practitioners. Now it has become necessary to review the text and upgrade to include new developments. It also has become apparent that the decision for the Third-Edition to eliminate introductory accelerator physics was not correct. Use of this text for beginners is quite broad, and the introduction to accelerator physics is desired. Therefore, three chapters have been added at the beginning to introduce a variety of accelerators and their functioning. In support to teaching, many problems with solutions have been added for those chapters. The author also tried to distinguish between introductory chapters and chapters which lead to more detailed subjects and show proofs. Chapters which can be skipped on a first reading have been labeled with a star *

As mentioned, the text includes many problems with and without solutions. The idea was to give solutions for the beginners while more advanced problems are not suitable for solutions in a textbook. Accelerator physics is not a collection of homework problems. Many questions and problems are rather complex and need to be treated in context with their impact on other systems. In most cases, there is no one optimum solution. Individual parameter choices must be made and modified according to their impact on other systems. Choices in beam dynamics, for example, have an impact on magnet design or RF-system parameters, etc. affecting the design of power supplies or financial budget. Straightforward design choices permeate through almost all other components requiring careful evaluation. Often the consequence of one parameter choice on other systems will become apparent only after considerable further design optimization. Unfortunately, often compromises must be made because of financial considerations. Work in accelerator physics includes often several approximations, and the designer should not hesitate to start over again with new insight. All this cannot be included in problem solutions in a textbook. However, it seemed to the author interesting to throw up such design problems which the interested reader can use to make his/her choices. 
Finally, in the last chapter on Free Electron Lasers, a short introduction into the components of a SASE-FEL facility is given. This introduction must be short and limited to the discussion of issues and function of main components in this text. Much more detail is required to design such a facility and a dedicated textbook is desirable.

I would like to thank all staff at Springer Publishing, especially the Editor Dr. Christian Caron, Production Coordinator Mrs. Birgit Muench, the Production Editor and Manager Ms. Shanthi Ramamoorthy, and Ms. Fathima Rizwana for their careful editing, support, and help before and during the production process.

Chiang Mai, Thailand

Helmut Wiedemann

February, 2015 


\section{Preface to Third Edition}

This issue of Particle Accelerator Physics is intended to combine the content of two earlier volumes and the volume on synchrotron radiation into one reference book. This book is designed for the serious scientist and student to acquire the underlying physics of electron accelerator physics. Introductory discussions on various types of accelerators have been eliminated, being well documented in the literature. Beam optics has been formulated in a general way as to be applicable also to proton and ion beams. Following the requests of many readers many solutions to exercises are given in the Appendix. Breaking with the author's preference, Standard International units are used in this edition. In Appendix B, transformation rules are given to convert formulae between SI and cgs systems. In the process of rewriting the texts, known typographical and real errors have been corrected. The author wishes to express his sincere appreciation to all readers pointing out such errors.

I would like to thank all staff at Springer who have contributed to the publication of this text. Foremost, I thank Dr. Christian Caron for his suggestion and encouragement to combine several textbooks into one reference volume. For the expert editing and cover design I thank Mrs. Birgit Muench and her staff. Finally, it is a pleasure to thank Ms. Bhawna Narang from Techbooks for her patient and thorough preparation of the proofs and final printing.

Nakhon Ratchasima, Thailand

Helmut Wiedemann

March 2007 



\section{Preface to First Edition, Volume I}

The purpose of this book is to provide a comprehensive introduction into the physics of particle accelerators and particle beam dynamics.Particle accelerators have become important research tools in high energy physics as well as sources of incoherent and coherent radiation from the far infra red to hard X-rays for basic and applied research. During years of teaching accelerator physics, it became clear that the single most annoying obstacle to get introduced into the field is the absence of a suitable textbook. Indeed most information about modern accelerator physics is contained in numerous internal notes from authors working mostly in high energy physics laboratories all over the world.

This text intends to provide a broad introduction and reference book into the field of accelerators for graduate students, engineers, and scientists summarizing many ideas and findings expressed in such internal notes and elsewhere. In doing so, theories are formulated in a general way to become applicable for any kind of charged particles. Writing such a text, however, poses the problem of correct referencing of original ideas. I have tried to find the earliest references among more or less accessible notes and publications and have listed those although the reader may have difficulty to obtain the original paper. In spite of great effort to be historically correct, I apologize for possible omissions and misquotes. This situation made it necessary to rederive again some of such ideas rather than quote the results and refer the interested reader to the original publication. I hope this approach will not offend the original authors, but rather provides a broader distribution of their original ideas, which have become important to the field of accelerator physics.

This text is split into two volumes. The first volume is designed to be selfcontained and is aimed at newcomers into the field of accelerator physics, but also to those who work in related fields and desire some background on basic principles of raccelerator physics. The first volume therefore gives an introductory survey of fundamental principles of particle acceleration followed by the theory of linear beam dynamics in the transverse as well as longitudinal phase space including a detailed discussion of basic magnetic focusing units. Concepts of single and multi-particle beam dynamics are introduced. 
Synchrotron radiation, its properties and effect on beam dynamics and electron beam parameters, is described in considerable detail followed by a discussion of beam instabilities on an introductory level, beam lifetime and basic lattice design concepts. The second volume is aimed specifically to those students, engineers, and scientists who desire to immerse themselves deeper into the physics of particle accelerators. It introduces the reader to higher order beam dynamics, Hamiltonian particle dynamics, general perturbation theory, nonlinear beam optics, chromatic and geometric aberrations, and resonance theory. The interaction of particle beams with rf fields of the accelerating system and beam loading effects are described in some detail relevant to accelerator physics. Following a detailed derivation of the theory of synchrotron radiation particle beam phenomena are discussed while utilizing the Vlasov and Fokker Planck equations leading to the discussion of beam parameters and their manipulation and collective beam instabilities. Finally, design concepts and new developments of particle accelerators as synchrotron radiation sources or research tools in high energy physics are discussed in some detail.

This text grew out of a number of lecture notes for accelerator physics courses at Stanford University, the Synchrotron Radiation Research Laboratory in Taiwan, the University of Sao Paulo in Brazil, the International Center for Theoretical Physics in Trieste and the US Particle Accelerator School as well as from interaction with students attending those classes and my own graduate students.

During almost 30 years in this field, I had the opportunity to work with numerous individuals and accelerators in laboratories around the world. Having learned greatly from these interactions, I would like to take this opportunity to thank all those who interacted with me and have had the patience to explain their ideas, share their results, or collaborate with me. The design and construction of new particle accelerators provides a specifically interesting period to develop and test theoretically new ideas, to work with engineers and designers, to see theoretical concepts become hardware and to participate in the excitement of commissioning and optimization. I have had a number of opportunities for such participation at the Deutsches Elektronen Synchrotron, DESY, in Hamburg, Germany and at the Stanford University at Stanford, California and am grateful to all colleagues who hosted and collaborated with me. I wished I could mention them individually and apologize for not doing so.

A special thanks goes to the operators of the electron storage rings SPEAR and PEP at the Stanford Linear Accelerator Center, specifically to T. Taylor, W. Graham, E. Guerra, and M. Maddox, for their dedicated and able efforts to provide me during numerous shifts over many years with a working storage ring ready for machine physics experimentation. 
I thank Mrs. Joanne Kwong, who typed the initial draft of this text and introduced me into the intricacies of TEX typesetting. The partial support by the Department of Energy through the Stanford Synchrotron Radiation Laboratory in preparing this text is gratefully acknowledged. Special thanks to Dr. C. Maldonado for painstakingly reading the manuscript. Last but not least, I would like to thank my family for their patience in dealing with an "absent" husband and father.

Palo Alto, CA, USA

Helmut Wiedemann

December 1992 



\section{Preface to First Edition, Volume II}

This text is a continuation of the first volume on "Basic Principles and Linear Beam Dynamics". While the first volume has been written as an introductory overview into beam dynamics it does not include more detailed discussion of nonlinear and higher order beam dynamics or the full theory of synchrotron radiation from relativistic electron beams. Both issues are, however, of fundamental importance for the design of modern particle accelerators. In this volume beam dynamics is formulated within the realm of Hamiltonian dynamics leading to the description of multiparticle beam dynamics with the Vlasov equation and including statistical processes with the Fokker Planck equation. Higher order perturbations and aberrations are discussed in detail including Hamiltonian resonance theory and higher order beam dynamics. The discussion of linear beam dynamics in Vol. I is completed here with the derivation of the general equation of motion including kinematic terms and coupled motion. Building on the theory of longitudinal motion in Vol. I the interaction of a particle beam with the rf system including beam loading, higher order phase focusing and combination of acceleration and transverse focusing is discussed. The emission of synchrotron radiation greatly affects the beam quality of electron or positron beams and we therefore derive the detailed theory of synchrotron radiation including spatial and spectral distribution as well as properties of polarization. The results of this derivation are then applied to insertion devices like undulator and wiggler magnets. Beam stability in linear and circular accelerators is compromised by the interaction of the electrical charge in the beam with its environment leading to instabilities. Theoretical models of such instabilities are discussed and scaling laws for the onset and rise time of instabilities derived. Although this text builds up on Vol. I, it relates to it only as a reference for basic issues of accelerator physics which could be obtained as well elsewhere. This volume is aimed specifically to those students, engineers and scientists who desire to acquire a deeper knowledge of particle beam dynamics in accelerators. To facilitate the use of this text as a reference many of the more important results are emphasized by a frame for quick detection. Consistent with Vol. I we use the cgs system of units. However, for the convenience of the reader who is used to the system of international units conversion factors have been added whenever such conversion is necessary, e.g. whenever 
electrical or magnetic units are used. These conversion factors are enclosed in square brackets like $\sqrt{4 \pi \epsilon_{0}}$ and should be ignored by those who use formulas in the cgs system. The conversion factors are easy to identify since they include only the constants $c, \pi, \epsilon_{0}, \mu_{0}$ and should therefore not mixed up with other factors in square brackets. For the convenience of the reader the source of these conversion factors is compiled in the appendix together with other useful tools.

I would like to thank Joanne Kwong, who typed the initial draft of this text and introduced me into the intricacies of TEX typesetting and to my students who guided me by numerous inquisitive questions. Partial support by the Division of Basic Energy Sciences in the Department of Energy through the Stanford Synchrotron Radiation Laboratory in preparing this text is gratefully acknowledged. Special thanks to Dr. C. Maldonado for painstakingly reading the manuscript and to the editorial staff of Springer Verlag for the support during the preparation of this text.

Palo Alto, CA, USA

Helmut Wiedemann

March 1994 


\section{Contents}

\section{Part I Introduction}

1 Introduction to Accelerator Physics ........................... 3

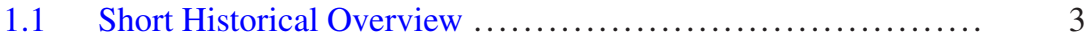

1.2 Particle Accelerator Systems ........................... 7

1.2.1 Main Components of Accelerator Facilities .......... 7

1.2.2 Applications of Particle Accelerators ................ 10

1.3 Definitions and Formulas .............................. 11

1.3.1 Units and Dimensions ......................... 11

1.3.2 Maxwell's Equations............................ 13

1.4 Primer in Special Relativity.............................. 14

1.4.1 Lorentz Transformation......................... 15

1.4.2 Lorentz Invariance .............................. 18

1.4.3 Spatial and Spectral Distribution of Radiation ........ 22

1.4.4 Particle Collisions at High Energies .................. 24

1.5 Principles of Particle-Beam Dynamics ...................... 26

1.5.1 Electromagnetic Fields of Charged Particles .......... 26

1.5.2 Vector and Scalar Potential ........................ 27

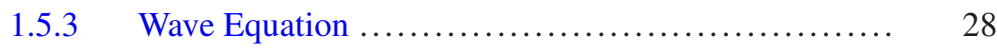

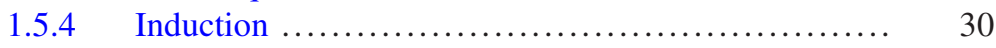

1.5.5 Lorentz Force ............................... 30

1.5.6 Equation of Motion ........................... 31

1.5.7 Charged Particles in an Electromagnetic Field ........ 33

1.5.8 Linear Equation of Motion ........................ 34

1.5.9 Energy Conservation............................. 35

1.5.10 Stability of a Charged-Particle Beam ............... 37

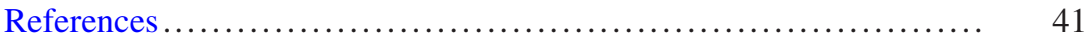

2 Linear Accelerators ......................................... 43

$2.1 \quad$ Principles of Linear Accelerators ........................... 43

2.1.1 Charged Particles in Electric Fields ................. 44

2.1.2 Electrostatic Accelerators........................ 45 
2.2 Electric Field Components ........................... 48

2.2.1 Electrostatic Deflectors ....................... 48

2.2.2 Electrostatic Focusing Devices .................. 49

2.2.3 Iris Doublet ................................. 51

2.2.4 Einzellens.................................... 52

$2.3 \quad$ Acceleration by rf Fields ................................. 54

2.3.1 Basic Principle of Microwave Linear Accelerators .... 54

References........................................... 57

3 Circular Accelerators .................................... 59

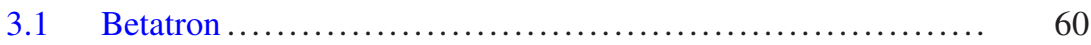

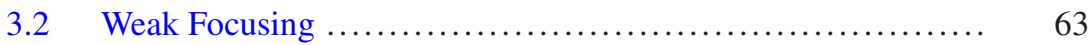

3.3 Adiabatic Damping.................................. 66

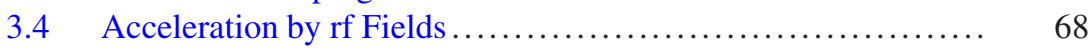

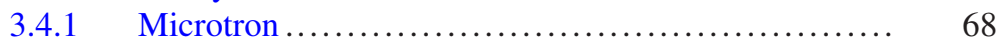

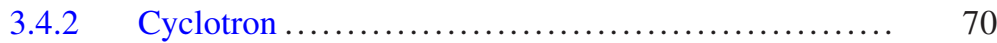

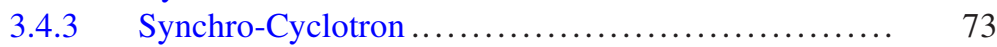

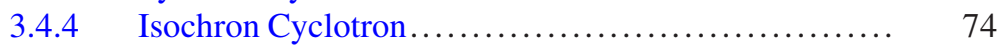

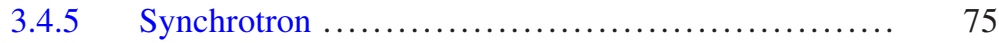

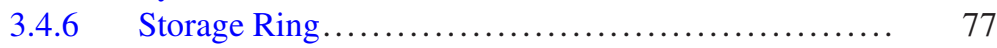

3.4.7 Summary of Characteristic Parameters .............. 77

References............................................. 79

\section{Part II Tools We Need}

4 Elements of Classical Mechanics............................ 83

4.1 How to Formulate a Lagrangian? ...................... 85

4.1.1 The Lagrangian for a Charged Particle in an EM-Field ................................. 85

4.2 Lorentz Force ......................................... 86

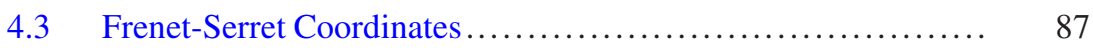

4.4 Hamiltonian Formulation ............................. 88

4.4.1 Cyclic Variables ................................ 90

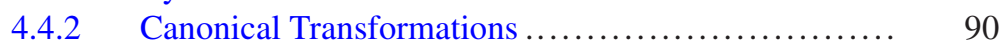

4.4.3 Curvilinear Coordinates ....................... 93

4.4.4 Extended Hamiltonian .......................... 95

4.4.5 Change of Independent Variable .................. 96

References........................................... 98

5 Particle Dynamics in Electro-Magnetic Fields ................. 99

$5.1 \quad$ The Lorentz Force .................................... 99

5.2 Fundamentals of Charged Particle Beam Optics.............. 100

5.2.1 Particle Beam Guidance ....................... 100

5.2.2 Particle Beam Focusing ....................... 102

5.3 Equation of Motion ............................... 106 
5.4 Equations of Motion from the Lagrangian and Hamiltonian ..... 109

5.4.1 Equations of Motion from Lagrangian ............... 110

5.4.2 Canonical Momenta.............................. 112

5.4.3 Equation of Motion from Hamiltonian............... 112

5.4.4 Harmonic Oscillator ............................ 114

5.4.5 Action-Angle Variables ............................ 115

5.5 Solutions of the Linear Equations of Motion ............... 116

5.5.1 Linear Unperturbed Equation of Motion............. 117

5.5.2 Matrix Formulation ........................... 118

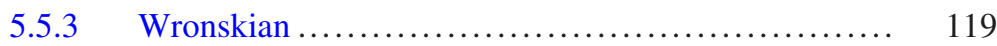

5.5.4 Perturbation Terms ............................ 120

References............................................. 123

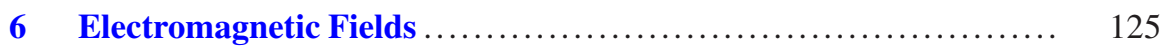

6.1 Pure Multipole Field Expansion ....................... 125

6.1.1 Electromagnetic Potentials and Fields for Beam Dynamics ........................... 126

6.1.2 Fields, Gradients and Multipole Strength Parameter... 128

6.1.3 Main Magnets for Beam Dynamics ................. 131

6.1.4 Multipole Misalignment and "Spill-down" .......... 137

6.2 Main Magnet Design Criteria ............................ 138

6.2.1 Design Characteristics of Dipole Magnets........... 138

6.2.2 Quadrupole Design Concepts ..................... 140

6.3 Magnetic Field Measurement........................... 145

6.3.1 Hall Probe .................................. 147

6.3.2 Rotating Coil ...................................... 148

6.4 General Transverse Magnetic-Field Expansion ................ 152

6.4.1 Pure Multipole Magnets ........................ 153

6.4.2 Kinematic Terms .................................. 155

6.5 Third-Order Differential Equation of Motion ................ 160

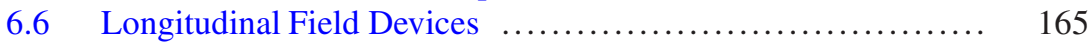

6.7 Periodic Wiggler Magnets .............................. 167

6.7.1 Wiggler Field Configuration..................... 168

6.8 Electrostatic Quadrupole ............................. 172

References................................................ 174

\section{Part III Beam Dynamics}

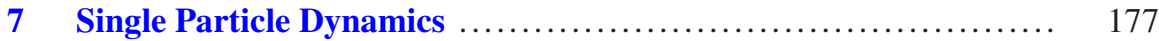

7.1 Linear Beam Transport Systems ......................... 178

7.1.1 Nomenclature ............................... 179

7.2 Matrix Formalism in Linear Beam Dynamics ............... 180

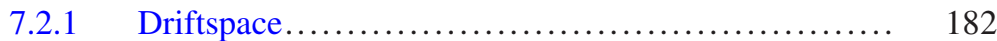

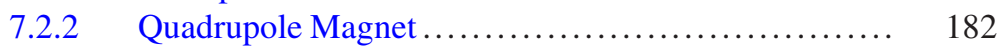

7.2.3 Thin Lens Approximation ....................... 184

7.2.4 Quadrupole End Field Effects ................... 187 


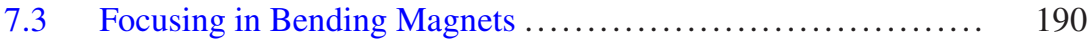

7.3.1 Sector Magnets .................................... 191

7.3.2 Fringe Field Effects .......................... 193

7.3.3 Finite Pole Gap ................................ 195

7.3.4 Wedge Magnets .............................. 196

7.3.5 Rectangular Magnet................................. 198

7.3.6 Focusing in a Wiggler Magnet .................. 200

7.3.7 Hard-Edge Model of Wiggler Magnets .............. 203

7.4 Elements of Beam Dynamics ......................... 205

7.4.1 Building Blocks for Beam Transport Lines........... 205

7.4.2 Isochronous Systems......................... 208

References ............................................... 211

8 Particle Beams and Phase Space ......................... 213

$8.1 \quad$ Beam Emittance ....................................... 214

8.1.1 Liouville's Theorem ........................... 215

8.1.2 Transformation in Phase Space.................... 218

8.1.3 Beam Matrix .............................. 222

$8.2 \quad$ Betatron Functions .................................. 227

8.2.1 Beam Envelope............................. 230

8.3 Beam Dynamics in Terms of Betatron Functions............. 231

8.3.1 Beam Dynamics in Normalized Coordinates .......... 233

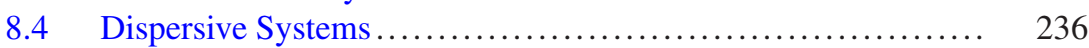

8.4.1 Analytical Solution ........................... 237

8.4.2 $3 \times 3$-Transformation Matrices ................... 238

8.4.3 Linear Achromat ................................ 240

8.4.4 Spectrometer ................................ 244

8.4.5 Measurement of Beam Energy Spectrum ........... 245

8.4.6 Path Length and Momentum Compaction ............. 248

References.......................................... 251

9 Longitudinal Beam Dynamics ............................. 253

9.1 Longitudinal Particle Motion ........................... 254

9.1.1 Longitudinal Phase Space Dynamics ............... 256

9.2 Equation of Motion in Phase Space .................... 259

9.2.1 Small Oscillation Amplitudes ...................... 262

9.2.2 Phase Stability ............................. 266

9.2.3 Acceleration of Charged Particles................ 270

9.3 Longitudinal Phase Space Parameters...................... 274

9.3.1 Separatrix Parameters ........................... 274

9.3.2 Momentum Acceptance........................ 275

9.3.3 Bunch Length ................................ 278

9.3.4 Longitudinal Beam Emittance..................... 280

9.3.5 Phase Space Matching ....................... 282 


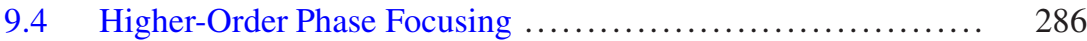

9.4.1 Dispersion Function in Higher Order .............. 287

9.4.2 Path Length in Higher Order ...................... 289

9.4.3 Higher Order Momentum Compaction Factor......... 291

9.4.4 Higher-Order Phase Space Motion................. 292

9.4.5 Stability Criteria .................................. 296

References............................................ 302

10 Periodic Focusing Systems ................................ 303

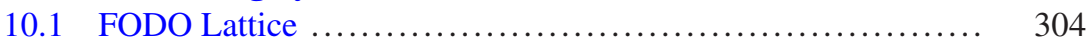

10.1.1 Scaling of FODO Parameters................... 305

10.1.2 Betatron Motion in Periodic Structures ................ 309

10.1.3 General FODO Lattice......................... 311

10.2 Beam Dynamics in Periodic Closed Lattices ................ 315

10.2.1 Hill's Equation ............................. 315

10.2.2 Periodic Betatron Functions ..................... 318

10.2.3 Periodic Dispersion Function...................... 321

10.2.4 Periodic Lattices in Circular Accelerators ............ 329

10.3 FODO Lattice and Acceleration ........................ 339

10.3.1 Lattice Structure................................. 339

10.3.2 Transverse Beam Dynamics and Acceleration ........ 341

References......................................... 349

\section{Part IV Beam Parameters}

11 Particle Beam Parameters ................................. 353

11.1 Definition of Beam Parameters .......................... 353

11.1.1 Beam Energy ................................. 353

11.1.2 Time Structure ............................... 354

11.1.3 Beam Current ..................................... 354

11.1.4 Beam Dimensions .............................. 356

11.2 Damping ................................................ 358

11.2.1 Robinson Criterion ............................ 358

11.3 Particle Distribution in Longitudinal Phase Space............. 365

11.3.1 Energy Spread .............................. 366

11.3.2 Bunch Length .................................. 368

11.4 Transverse Beam Emittance ................................ 368

11.4.1 Equilibrium Beam Emittance................... 369

11.4.2 Emittance Increase in a Beam Transport Line ......... 371

11.4.3 Vertical Beam Emittance ........................ 371

11.4.4 Beam Sizes ...................................... 373

11.4.5 Beam Divergence ............................. 375

11.5 Variation of the Damping Distribution ..................... 375

11.5.1 Damping Partition and Rf-Frequency ............. 375 
11.6 Variation of the Equilibrium Beam Emittance ............... 377

11.6.1 Beam Emittance and Wiggler Magnets .............. 377

11.6.2 Damping Wigglers ........................... 380

11.7 Robinson Wiggler ................................. 382

11.7.1 Damping Partition and Synchrotron Oscillation....... 382

11.7.2 Can We Eliminate the Beam Energy Spread? ......... 384

11.8 Beam Life Time .................................... 385

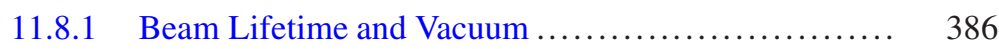

11.8.2 Ultra High Vacuum System....................... 395

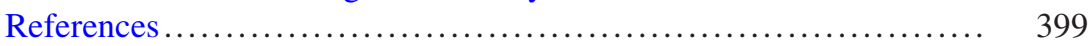

12 Vlasov and Fokker-Planck Equations ...................... 401

12.1 The Vlasov Equation .................................... 402

12.1.1 Betatron Oscillations and Perturbations .............. 408

12.1.2 Damping ..................................... 410

12.2 Damping of Oscillations in Electron Accelerators............. 411

12.2.1 Damping of Synchrotron Oscillations .............. 412

12.2.2 Damping of Vertical Betatron Oscillations ........... 416

12.2.3 Robinson's Damping Criterion ..................... 419

12.2.4 Damping of Horizontal Betatron Oscillations ......... 422

12.3 The Fokker-Planck Equation ....................... 422

12.3.1 Stationary Solution of the Fokker-Planck Equation ... 425

12.3.2 Particle Distribution within a Finite Aperture ........ 430

12.3.3 Particle Distribution in the Absence of Damping ..... 432

References.......................................... 435

13 Equilibrium Particle Distribution ............................ 437

13.1 Particle Distribution in Phase Space..................... 437

13.1.1 Diffusion Coefficient and Synchrotron Radiation ...... 438

13.1.2 Quantum Excitation of Beam Emittance............... 440

13.2 Equilibrium Beam Emittance .......................... 441

13.2.1 Horizontal Equilibrium Beam Emittance .............. 441

13.2.2 Vertical Equilibrium Beam Emittance ............... 442

13.3 Equilibrium Energy Spread and Bunch Length ................ 444

13.3.1 Equilibrium Beam Energy Spread ................. 444

13.3.2 Equilibrium Bunch Length ......................... 444

13.4 Phase-Space Manipulation ............................ 446

13.4.1 Exchange of Transverse Phase-Space Parameters ..... 446

13.4.2 Bunch Compression ............................. 446

13.4.3 Alpha Magnet .................................. 449

13.5 Polarization of a Particle Beam ........................... 453

References........................................... 457 
14 Beam Emittance and Lattice Design...................... 459

14.1 Equilibrium Beam Emittance in Storage Rings ................ 461

14.1.1 FODO Lattice .................................. 461

14.1.2 Minimum Beam Emittance ...................... 462

14.2 Absolute Minimum Emittance........................... 465

14.3 Beam Emittance in Periodic Lattices ......................... 468

14.3.1 The Double Bend Achromat Lattice (DBA) .......... 469

14.3.2 The FODO Lattice .............................. 470

14.3.3 Optimum Emittance for Colliding Beam

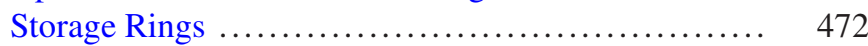

References............................................ 472

\section{Part V Perturbations}

15 Perturbations in Beam Dynamics......................... 477

15.1 Magnet Field and Alignment Errors ...................... 478

15.1.1 Self Compensation of Perturbations ............... 479

15.2 Dipole Field Perturbations .......................... 480

15.2.1 Dipole Field Errors and Dispersion Function.......... 482

15.2.2 Perturbations in Open Transport Lines................ 482

15.2.3 Existence of Equilibrium Orbits.................. 484

15.2.4 Closed Orbit Distortion .......................... 486

15.2.5 Statistical Distribution of Dipole Field

15.2.6 Dipole Field Errors in Insertion Devices.............. 492

15.2.7 Closed Orbit Correction ................................ 494

15.2.8 Response Matrix ............................ 496

15.2.9 Orbit Correction with Single Value
Decomposition (SVD) ....................... 497

15.3 Quadrupole Field Perturbations ............................ 499

15.3.1 Betatron Tune Shift ......................... 500

15.3.2 Optics Perturbation Due to Insertion Devices ......... 502

15.3.3 Resonances and Stop Band Width .................. 503

15.3.4 Perturbation of Betatron Function.................... 506

15.4 Chromatic Effects in a Circular Accelerator .................. 509

15.4.1 Chromaticity .................................. 509

15.4.2 Chromaticity Correction ........................ 513

15.4.3 Chromaticity in Higher Approximation............... 514

15.4.4 Non-linear Chromaticity ............................ 517

15.5 Kinematic Perturbation Terms ............................ 522

15.6 Perturbation Methods in Beam Dynamics .................. 524

15.6.1 Periodic Distribution of Statistical Perturbations ...... 525

15.6.2 Periodic Perturbations in Circular Accelerators ........ 528

15.6.3 Statistical Methods to Evaluate Perturbations ......... 530 
15.7 Control of Beam Size in Transport Lines ................ 531

References ............................................ 538

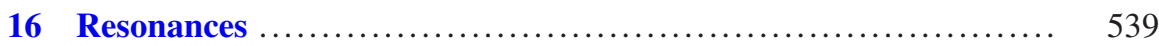

$16.1 \quad$ Lattice Resonances ..................................... 539

16.1.1 Resonance Conditions ............................. 540

16.1.2 Coupling Resonances .......................... 544

16.1.3 Resonance Diagram............................... 545

16.2 Hamiltonian Resonance Theory ........................... 547

16.2.1 Non-linear Hamiltonian............................. 547

16.2.2 Resonant Terms ............................... 550

16.2.3 Resonance Patterns and Stop-Band Width............. 553

16.2.4 Half-Integer Stop-Band ........................... 555

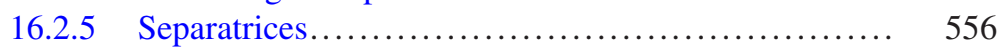

16.2.6 General Stop-Band Width ...................... 558

16.3 Third-Order Resonance ................................. 560

16.3.1 Particle Motion in Phase Space .................... 563

References......................................... 564

17 Hamiltonian Nonlinear Beam Dynamics....................... 565

17.1 Higher-Order Beam Dynamics ............................ 565

17.1.1 Multipole Errors............................... 565

17.1.2 Non-linear Matrix Formalism ....................... 569

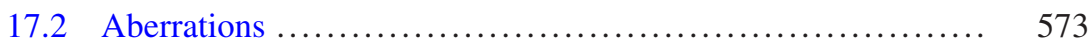

17.2.1 Geometric Aberrations........................ 575

17.2.2 Filamentation of Phase Space ................... 581

17.2.3 Chromatic Aberrations.............................. 584

17.2.4 Particle Tracking .................................. 587

17.3 Hamiltonian Perturbation Theory ........................ 588

17.3.1 Tune Shift in Higher Order ...................... 595

References............................................ 599

\section{Part VI Acceleration}

18 Charged Particle Acceleration ............................. 603

18.1 Rf-Waveguides and Cavities ............................ 603

18.1.1 Wave Equation ................................... 604

18.1.2 Rectangular Waveguide Modes ...................... 605

18.1.3 Cylindrical Waveguide Modes ................... 610

18.2 Rf-Cavities.......................................... 614

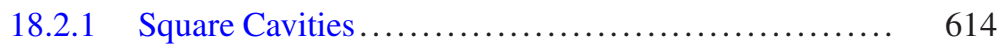

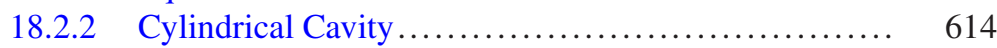

18.2.3 Energy Gain ..................................... 616

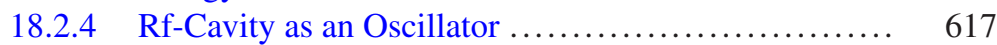

18.2.5 Cavity Losses and Shunt Impedance................. 619 


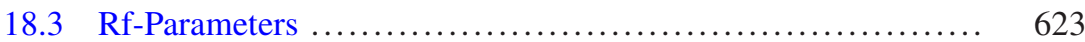

18.3.1 Synchronous Phase and Rf-voltage ................ 625

18.4 Linear Accelerator..................................... 625

18.4.1 Basic Waveguide Parameters .................... 626

18.4.2 Particle Capture in a Linear Accelerator Field ........ 632

18.5 Preinjector and Beam Preparation ........................... 634

18.5.1 Prebuncher ...................................... 634

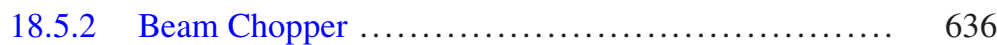

18.5.3 Buncher Section.................................. 638

References .............................................. 640

19 Beam-Cavity Interaction ................................ 641

19.1 Coupling Between rf-Field and Particles.................... 641

19.1.1 Network Modelling of an Accelerating Cavity ........ 642

19.2 Beam Loading and Rf-System ......................... 645

19.3 Higher-Order Mode Losses in an Rf-Cavity ................. 650

19.3.1 Efficiency of Energy Transfer from Cavity to Beam ... 653

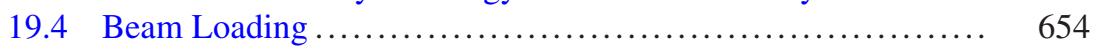

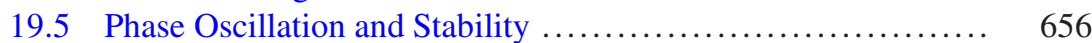

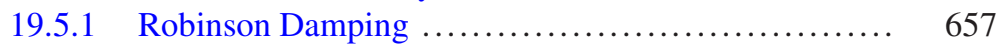

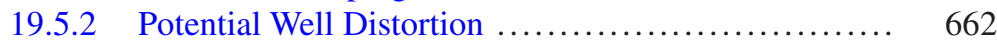

References........................................... 665

\section{Part VII Coupled Motion}

20 Dynamics of Coupled Motion ................................. 669

20.1 Equations of Motion in Coupled Systems.................... 669

20.1.1 Coupled Beam Dynamics in Skew Quadrupoles ...... 670

20.1.2 Particle Motion in a Solenoidal Field ................ 672

20.1.3 Transformation Matrix for a Solenoid Magnet ........ 675

20.2 Betatron Functions for Coupled Motion .................... 678

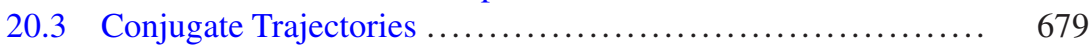

20.4 Hamiltonian and Coupling ............................ 685

20.4.1 Linearly Coupled Motion ............................ 686

20.4.2 Higher-Order Coupling Resonances ............... 695

20.4.3 Multiple Resonances.............................. 695

References................................................ 697

\section{Part VIII Intense Beams}

21 Statistical and Collective Effects .......................... 701

21.1 Statistical Effects ................................. 702

21.1.1 Schottky Noise ............................. 702

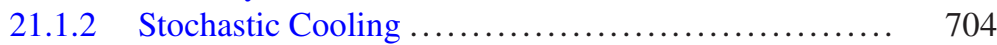

21.1.3 Touschek Effect .............................. 705

21.1.4 Intra-Beam Scattering......................... 706 


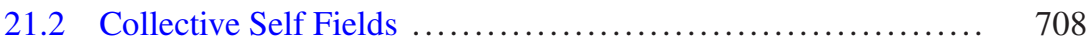

21.2.1 Self Field for Elliptical Particle Beams .............. 709

21.2.2 Beam-Beam Effect ............................ 712

21.2.3 Transverse Self Fields ......................... 715

21.2.4 Fields from Image Charges ...................... 715

21.2.5 Space-Charge Effects ............................. 720

21.2.6 Longitudinal Space-Charge Field ................ 725

21.3 Beam-Current Spectrum ............................ 727

21.3.1 Longitudinal Beam Spectrum .................... 727

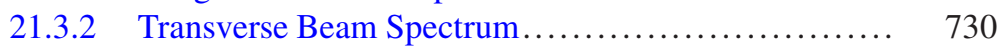

References.......................................... 734

22 Wake Fields and Instabilities ............................... 737

22.1 Definitions of Wake Field and Impedance .................. 738

22.1.1 Parasitic Mode Losses and Impedances............. 739

22.1.2 Longitudinal Wake Fields ........................ 743

22.1.3 Transverse Wake Fields ............................ 749

22.1.4 Panofsky-Wenzel Theorem ..................... 750

22.2 Impedances in an Accelerator Environment ................ 751

22.2.1 Space-Charge Impedance...................... 751

22.2.2 Resistive-Wall Impedance ..................... 752

22.2.3 Cavity-Like Structure Impedance ................. 753

22.2.4 Overall Accelerator Impedance ..................... 754

22.2.5 Broad-Band Wake Fields in a Linear Accelerator ...... 756

22.3 Coasting-Beam Instabilities ......................... 756

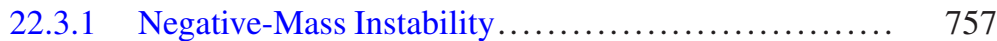

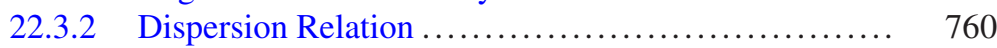

22.3.3 Landau Damping ............................. 767

22.3.4 Transverse Coasting-Beam Instability .............. 769

22.4 Longitudinal Single-Bunch Effects ...................... 771

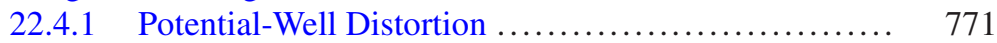

22.5 Transverse Single-Bunch Instabilities ...................... 779

22.5.1 Beam Break-Up in Linear Accelerators............... 779

22.5.2 Fast Head-Tail Effect .............................. 781

22.5.3 Head-Tail Instability ............................ 786

22.6 Multi-Bunch Instabilities .............................. 789

References................................................ 795

\section{Part IX Synchrotron Radiation}

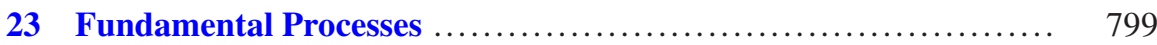

23.1 Radiation from Moving Charges ........................ 799

23.1.1 Why Do Charged Particles Radiate? ................. 800

23.1.2 Spontaneous Synchrotron Radiation .................. 801

23.1.3 Stimulated Radiation........................... 802

23.1.4 Electron Beam................................ 803 
23.2 Conservation Laws and Radiation .......................... 804

23.2.1 Cherenkov Radiation ........................... 805

23.2.2 Compton Radiation ............................... 806

23.3 Electromagnetic Radiation .............................. 807

23.3.1 Coulomb Regime .............................. 808

23.3.2 Radiation Regime ........................... 809

References............................................. 813

24 Overview of Synchrotron Radiation ........................... 815

24.1 Radiation Sources .................................. 816

24.1.1 Bending Magnet Radiation ...................... 816

24.1.2 Superbends ................................ 817

24.1.3 Wavelength Shifter.............................. 818

24.1.4 Wiggler Magnet Radiation ........................ 819

24.1.5 Undulator Radiation ............................ 822

24.2 Radiation Power ...................................... 830

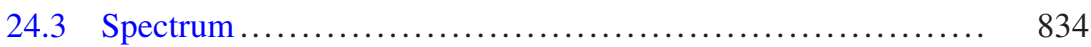

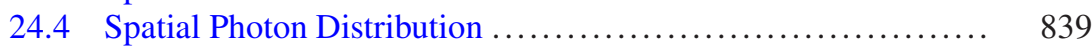

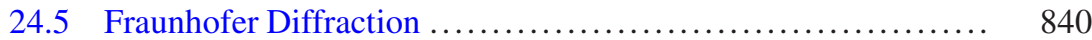

24.6 Spatial Coherence .................................... 843

24.7 Temporal Coherence .................................. 846

24.8 Spectral Brightness .................................... 848

24.8.1 Matching .................................... 849

24.9 Photon Source Parameters .......................... 851

References.......................................... 854

25 Theory of Synchrotron Radiation ............................. 857

25.1 Radiation Field .................................... 857

25.2 Total Radiation Power and Energy Loss .................... 864

25.2.1 Transition Radiation ........................... 865

25.3 Spatial Radiation Distribution ......................... 868

25.3.1 Radiation Lobes .................................... 868

25.4 Radiation Field in the Frequency Domain .................. 873

25.4.1 Spectral Distribution in Space and Polarization ........ 877

25.4.2 Spectral and Spatial Photon Flux................... 879

25.4.3 Harmonic Representation........................... 880

25.4.4 Spatial Radiation Power Distribution ............... 881

25.5 Asymptotic Solutions ............................. 883

25.5.1 Low Frequencies and Small Observation Angles ..... 884

25.5.2 High Frequencies or Large Observation Angles....... 884

25.6 Angle-Integrated Spectrum ........................... 885

25.7 Statistical Radiation Parameters ........................... 891

References............................................. 893 
26 Insertion Device Radiation $\ldots \ldots \ldots \ldots \ldots \ldots \ldots \ldots \ldots \ldots \ldots \ldots \ldots . \ldots \ldots \ldots$

26.1 Particle Dynamics in a Periodic Field Magnet................ 896

26.2 Undulator Radiation ....................................... 899

26.2.1 Fundamental Wavelength ....................... 899

26.2.2 Radiation Power................................ 900

26.2.3 Spatial and Spectral Distribution .................... 901

26.2.4 Line Spectrum................................... 914

26.2.5 Spectral Undulator Brightness....................... 917

26.3 Elliptical Polarization ................................ 918

26.3.1 Elliptical Polarization from Bending Magnet

Radiation ................................. 918

26.3.2 Elliptical Polarization from Periodic Insertion

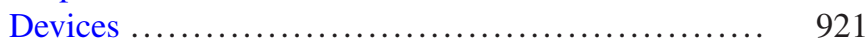

References ............................................ 927

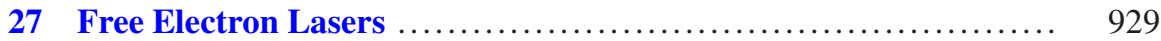

27.1 Small Gain Regime............................... 930

27.1.1 Energy Transfer ............................. 932

27.1.2 Equation of Motion ........................... 934

27.1.3 FEL-Gain ...................................... 937

27.2 High Gain Free Electron Laser .......................... 942

27.2.1 Electron Dynamics in a SASE FEL ............... 942

27.2.2 Electron Source ................................. 945

27.2.3 Beam Dynamics.............................. 945

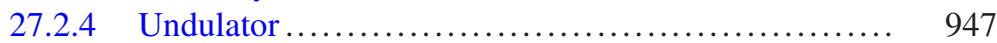

References............................................ 947

Correction to: Particle Accelerator Physics ............... C1

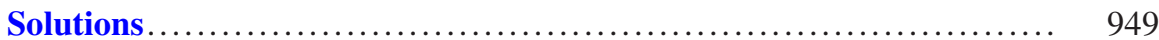

A Useful Mathematical Formulae ............................ 983

A.1 Vector Algebra.......................................... 983

A.1.1 Differential Vector Expressions .................... 984

A.1.2 Algebraic Relations ........................... 984

A.1.3 Differential Relations .......................... 985

A.1.4 Partial Integration ................................ 985

A.1.5 Trigonometric and Exponential Functions ............ 985

A.1.6 Integral Relations ............................ 986

A.1.7 Dirac's Delta Function ............................. 986

A.1.8 Bessel's Functions ............................. 986

A.1.9 Series Expansions ............................... 987

A.1.10 Fourier Series ..................................... 987

A.1.11 Coordinate Transformations ....................... 988 
B Physical Formulae and Parameters .......................... 993

B.1 Physical Constants ................................ 993

B.2 Relations of Fundamental Parameters ....................... 994

B.3 Unit Conversions .................................... 994

B.4 Maxwell's Equations .................................. 995

B.5 Wave and Field Equations ........................... 995

B.6 Relativistic Relations .................................... 996

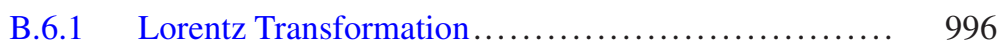

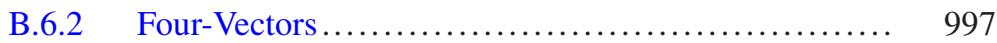

B.6.3 Square of the 4-Acceleration ....................... 998

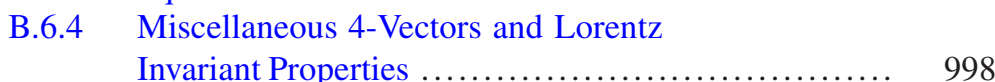

B.7 Transformation Matrices in Beam Dynamics ................... 998

B.8 General Transformation Matrix........................... 999

B.8.1 Symmetric Magnet Arrangement ................. 999

B.8.2 Inverse Transformation Matrix ................... 1000

B.9 Specific Transformation Matrices ...................... 1000

B.9.1 Drift Space ................................. 1000

B.9.2 Bending Magnets ............................ 1000

B.9.3 Quadrupole .............................. 1003

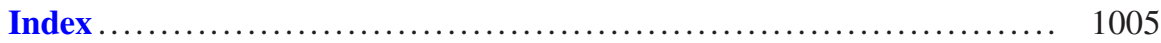


Part I

Introduction 


\section{Chapter 1 \\ Introduction to Accelerator Physics}

The development of charged particle accelerators and it's underlying principles has its basis on the theoretical and experimental progress in fundamental physical phenomena. While active particle accelerator experimentation started seriously only in the twentieth century, it depended on the basic physical understanding of electromagnetic phenomena as investigated both theoretically and experimentally mainly during the nineteenth and beginning twentieth century. In this introduction we will recall briefly the history leading to particle accelerator development, applications and introduce basic definitions and formulas governing particle beam dynamics.

\subsection{Short Historical Overview}

The history and development of particle accelerators is intimately connected to the discoveries and understanding of electrical phenomena and the realization that the electrical charge comes in lumps carried as a specific property by individual particles. It is reported that the Greek philosopher and mathematician Thales of Milet, who was born in $625 \mathrm{BC}$ first observed electrostatic forces on amber. The Greek word for amber is electron or $\eta \lambda \epsilon \kappa \tau \rho o v$ and has become the origin for all designations of electrical phenomena and related sciences. For more than 2000 years this observation was hardly more than a curiosity. In the eighteenth century, however, electrostatic phenomena became quite popular in scientific circles and since have been developed into a technology which by now completely embraces and dominates modern civilization as we know it.

It took another 100 years before the carriers of electric charges could be isolated. Many systematic experiments were conducted and theories developed to formulate the observed electrical phenomena mathematically. It was Coulomb, who in 1785 first succeeded to quantify the forces between electrical charges which we now call

This chapter has been made Open Access under a CC BY 4.0 license. For details on rights and licenses please read the Correction https://doi.org/10.1007/978-3-319-18317-6_28 
Coulomb forces. As more powerful sources for electrical charges became available, glow discharge phenomena were observed and initiated an intensive effort on experimental observations during most of the second half of the nineteenth century. It was the observations of these electrical glow discharge phenomena that led the scientific community to the discovery of elementary particles and electromagnetic radiation which are both basic ingredients for particle acceleration.

Research leading to the discovery of elementary particles and to ideas for the acceleration of such particles is dotted with particularly important milestones which from time to time set the directions for further experimental and theoretical research. It is obviously somewhat subjective to choose which discoveries might have been the most influential. Major historical discoveries leading to present day particle accelerator physics started to happen more than a 150 years ago:

1815 The physician and chemist W. Proust postulates, initially anonymous, that all atoms are composed of hydrogen atoms and that therefore all atomic weights come in multiples of the weight of a hydrogen atom.

1839 M. Faraday [1] publishes his experimental investigations of electricity and described various phenomena of glow discharge.

1858 J. Plücker [2] reports on the observation of cathode rays and their deflection by magnetic fields. He found the light to become deflected in the same spiraling direction as Ampere's current flows in the electromagnet and therefore postulated that the electric light, as he calls it, under the circumstances of the experiment must be magnetic.

1867 L. Lorenz working in parallel with J.C. Maxwell on the theory of electromagnetic fields formulates the concept of retarded potentials although not yet for moving point charges.

1869 J.W. Hittorf [3], a student of Plücker, started his thesis paper with the statement (translated from german): "The undisputed darkest part of recent theory of electricity is the process by which in gaseous volumes the propagation of electrical current is effected". Obviously observations with glow discharge tubes displaying an abundance of beautiful colors and complicated reactions to magnetic fields kept a number of researchers fascinated. Hittorf conducted systematic experiments on the deflection of the light in glow discharges by magnetic fields and corrected some erroneous interpretations by Plücker.

1871 C.F. Varley postulates that cathode rays are particle rays.

1874 H. von Helmholtz postulates atomistic structure of electricity.

1883 J.C. Maxwell publishes his Treatise on Electricity and Magnetism.

1883 T.A. Edison discovers thermionic emission.

1886 E. Goldstein [4] observed positively charged rays which he was able to isolate from a glow discharge tube through channels in the cathode. He therefore calls these rays Kanalstrahlen.

1887 H. Hertz discoveries transmission of electromagnetic waves and photoelectric effect.

1891 G.J. Stoney introduces the name electron. 
1895 H.A. Lorentz formulates electron theory, the Lorentz force equation and Lorentz contraction.

1894 P. Lenard builts a discharge tube that allows cathode rays to exit to atmospheric air.

1895 W. Röntgen discovers X-rays.

1895 E. Wiedemann [5] reports on a new kind of radiation studying electrical sparks.

1897 J.J. Thomson measures the e/m-ratio for kanal and cathode rays with electromagnetic spectrometer and found the $\mathrm{e} / \mathrm{m}$ ratio for cathode rays to be larger by a factor of 1,700 compared to the e/m ratio for kanal rays. He concluded that cathode rays consist of free electricity giving evidence to free electrons.

1897 J. Larmor formulates concept of Larmor precession.

1898 A. Liènard calculates the electric and magnetic field in the vicinity of a moving point charge and evaluated the energy loss due to electromagnetic radiation from a charged particle travelling on a circular orbit.

1900 E. Wiechert derives expression for retarded potentials of moving point charges.

1901 W. Kaufmann, first alone, and in 1907 together with A.H. Bucherer measure increase of electron mass with energy. First experiment in support of theory of special relativity.

1905 A. Einstein publishes theory of special relativity.

1906 J.J. Thomson [6] explains the emission of this radiation as being caused by acceleration occurring during the collision of charged particles with other atoms and calculated the energy emitted per unit time to be $\left(2 e^{2} f^{2}\right) /(3 V)$, where $e$ is the charge of the emitting particle, $f$ the acceleration and $V$ the velocity of light.

1907 G.A. Schott [7, 8] formulated the first theory of synchrotron radiation in an attempt to explain atomic spectra.

1909 R.A. Millikan starts measuring electric charge of electron.

1913 First experiment by J. Franck and G. Hertz to excite atoms by accelerated electrons.

1914 E. Marsden produces first proton beam irradiating paraffin with alpha particles.

1920 H. Greinacher [9] builts first cascade generator.

1922 R. Wideroe as a graduate student sketches ray transformer (betatron).

1924 G. Ising [10] invents as a student the electron linac with drift tubes and spark gap excitation.

1928 R. Wideroe [11] reports first operation of linear accelerator with potassium and sodium ions. Discusses operation of betatron and failure to get beam for lack of focusing.

1928 P.A.M. Dirac predicts existence of positrons.

1931 R.J. Van de Graaff [12] builts first high voltage generator.

1932 Lawrence and Livingston [13] accelerate first proton beam from 1.2 MeV cyclotron employing weak focusing. 
1932 J.D. Cockcroft and E.T.S. Walton [14] use technically improved cascade generator to accelerate protons and initiate first artificial atomic reaction: $\mathrm{Li}+$ $p \rightarrow 2 \mathrm{He}$.

1932 in the same year, C.D. Andersen discovers positrons, neutrons were discovered by J. Chadwick, and H.C. Urey discoveries deuterons.

1939 W.W. Hansen, R. Varian and his brother S. Varian invent klystron microwave tube at Stanford.

1941 D.W. Kerst and R. Serber [15] complete first functioning betatron.

1941 B. Touschek and R. Wideroe formulate storage ring principle.

1944 D. Ivanenko and I. Ya. Pomeranchuk [16] and J. Schwinger [17] point out independently an energy limit in circular electron accelerators due to synchrotron radiation losses.

1945 V.I. Veksler [18] and E.M. McMillan [19] independently discover the principle of phase focusing.

1945 J.P. Blewett [20] experimentally discovers synchrotron radiation by measuring the energy loss of electrons.

1947 L.W. Alvarez [21] designs first proton linear accelerator at Berkeley.

1948 E.L. Ginzton et al. [22] accelerate electrons to $6 \mathrm{MeV}$ with Mark I at Stanford.

1949 McMillan et al. commissioned $320 \mathrm{MeV}$ electron synchrotron.

1950 N. Christofilos [23] formulates concept of strong focusing.

1952 M.S. Livingston et al. [24] describe design for $2.2 \mathrm{GeV}$ Cosmotron in Brookhaven.

1951 H. Motz [25] builds first wiggler magnet to produce quasi monochromatic synchrotron radiation.

1952 E. Courant et al. [26] publish first paper on strong focusing.

1954 R.R. Wilson et al. operate first AG electron synchrotron in Cornell at $1.1 \mathrm{GeV}$.

1954 Lofgren et al. accelerate protons to $5.7 \mathrm{GeV}$ in Bevatron.

1955 M. Chodorow et al. [27] complete $600 \mathrm{MeV}$ MARK III electron linac.

1955 M. Sands [28] define limits of phase focusing due to quantum excitation.

1959 E. Courant and Snyder [29] publish their paper on the Theory of the Alternating-Gradient Synchrotron.

Research and development in accelerator physics blossomed significantly during the 1950s supported by the development of high power radio frequency sources and the increased availability of government funding for accelerator projects. Parallel with the progress in accelerator technology, we also observe advances in theoretical understanding, documented in an increasing number of publications. It is beyond the scope of this text to only try to give proper credit to all major advances in the past 60 years and refer the interested reader to more detailed references. 


\subsection{Particle Accelerator Systems}

Particle accelerators come in many forms applying a variety of technical principles. All are based on the interaction of the electric charge with static and dynamic electromagnetic fields and it is the technical realization of these interactions that leads to the different types of particle accelerators. Electromagnetic fields are used over most of the available frequency range from static electric fieldsto ac magnetic fields in betatrons oscillating at 50 or $60 \mathrm{~Hz}$, to radio frequency fields in the $\mathrm{MHz}$ to $\mathrm{GHz}$ range and ideas are being explored to use laser beams to generate high field particle acceleration.

In this text, we will not discuss the different technical realization of particle acceleration but rather concentrate on basic principles which are designed to help the reader to develop technical solutions for specific applications meeting basic beam stability requirements. For particular technical solutions we refer to the literature. Further down we will discuss briefly basic accelerator types and their theoretical back ground. Furthermore, to discuss basic principles of particle acceleration and beam dynamics it is desirable to stay in contact with technical reality and reference practical and working solutions. We will therefore repeatedly refer to certain types of accelerators and apply theoretical beam dynamics solutions to exhibit the salient features and importance of the theoretical ideas under discussion. In these references we use mostly such types of accelerators which are commonly used and are extensively publicized.

\subsubsection{Main Components of Accelerator Facilities}

In the following paragraphs we describe components of particle accelerators in a rather cursory way to introduce the terminology and overall features. Particle accelerators consist of two basic units, the particle source or injector and the main accelerator. The particle source comprises all components to generate a beam of desired particles.

Generally glow discharge columns are used to produce proton or ion beams, which then are first accelerated in electrostatic accelerators like a Van de Graaff or Cockcroft-Walton accelerator and then in an Alvarez-type linear accelerator. To increase the energy of heavy ion beams the initially singly charged ions are, after some acceleration, guided through a thin metal foil to strip more electrons off the ions. More than one stripping stage may be used at different energies to reach the maximum ionization for most efficient acceleration.

Much more elaborate measures must be used to produce antiprotons. Generally a high energy proton beam is aimed at a heavy metal target, where, through hadronic interactions with the target material, among other particles antiprotons are generated. Emerging from the target, these antiprotons are collected by strong focusing devices and further accelerated. 
Electrons are commonly generated from a heated cathode, also called a thermionic gun, which is covered on the surface by specific alkali oxides or any other substance with a low work function to emit electrons at technically practical temperatures. Another method to create a large number of electrons within a short pulse uses a strong laser pulse directed at the surface of a photo cathode. Systems where the cathode is inserted directly into an accelerating rf field are called $\mathrm{rf}$ guns. Positrons are created the same way as antiprotons by aiming high energy electrons on a heavy metal target where, through an electromagnetic shower and pair production, positrons are generated. These positrons are again collected by strong magnetic fields and further accelerated.

Whatever the method of generating particles may be, in general they do not have the time structure desired for further acceleration or special application. Efficient acceleration by rf fields occurs only during a very short period per oscillation cycle and most particles would be lost without proper preparation. For high beam densities it is desirable to compress the continuous stream of particles from a thermionic gun or a glow discharge column into a shorter pulse with the help of a chopper device and/or a prebuncher. The chopper may be a mechanical device or a deflecting magnetic or rf field moving the continuous beam across the opening of a slit. At the exit of the chopper we observe a series of beam pulses, called bunches, to be further processed by the prebuncher. Here early particles within a bunch are decelerated and late particles accelerated. After a well defined drift space, the bunch length becomes reduced due to the energy dependence of the particle velocity. Obviously this compression works only as long as the particles are not relativistic while the particle velocity can be modulated by acceleration or deceleration.

No such compression is required for antiparticles, since they are produced by high energetic particles having the appropriate time structure. Antiparticle beams emerging from a target have, however, a large beam size and beam divergence. To make them suitable for further acceleration they are generally stored for some time in a cooling or damping ring. Such cooling rings are circular "accelerators" where particles are not accelerated but spend just some time circulating. Positrons circulating in such storage rings quickly lose their transverse momenta and large beam divergence through the emission of synchrotron radiation. In the case of antiprotons, external fields are applied to damp the transverse beam size or they circulate against a strong counterrotating electron beam loosing transverse momentum through scattering.

Antiparticles are not always generated in large quantities. On the other hand, the accelerator ahead of the conversion target can often be pulsed at a much higher rate than the main accelerator can accept injection. In such cases, the antiparticles are collected from the rapid cycling injector in an accumulator ring and then transferred to the main accelerator when required.

Particle beams prepared in such a manner may now be further accelerated in linear or circular accelerators. A linear accelerator consists of a linear sequence of many accelerating units where accelerating fields are generated and timed such that particles absorb and accumulate energy from each acceleration unit. Most commonly used linear accelerators consist of a series of cavities excited by 
radio frequency sources to high accelerating fields. In the induction accelerator, each accelerating unit consists of a transformer which generates from an external electrical pulse a field on the transformer secondary which is formed such as to allow the particle beam to be accelerated. Such induction accelerators can be optimized to accelerate very high beam currents to medium beam energies.

For very high beam energies linear accelerators become very long and costly. Such practical problems can be avoided in circular accelerators where the beam is held on a circular path by magnetic fields in bending magnets and passing repeatedly every turn through accelerating sections, similar to those in a linear accelerator. This way, the particles gain energy from the accelerating cavities at each turn and reach higher energies while the fields in the bending magnets are raised.

The basic principles to accelerate particles of different kind are similar and we do not need to distinguish between protons, ions, and electrons. Technically, individual accelerator components differ more or less to adjust to the particular beam parameters which have mostly to do with the particle velocities. For highly relativistic particles the differences in beam dynamics vanish. Protons and ions are more likely to be nonrelativistic and therefore vary the velocity as the kinetic energy is increased, thus generating problems of synchronism with the oscillating accelerating fields which must be solved by technical means.

After acceleration in a linear or circular accelerator the beam can be directed onto a target, mostly a target of liquid hydrogen, to study high energy interactions with the target protons. Such fixed target experimentation dominated nuclear and high energy particle physics from the first applications of artificially accelerated particle beams far into the 1970s and is still a valuable means of basic research. Obviously, it is also the method in conjunction with a heavy metal target to produce secondary particles like antiparticles for use in colliding beam facilities and mesons for basic research.

To increase the center-of-mass energy for basic research, particle beams are aimed not at fixed targets but to collide head on with another beam. This is one main goal for the construction of colliding beam facilities or storage rings. In such a ring, particle and antiparticle beams are injected in opposing directions and made to collide in specifically designed interaction regions. Because the interactions between counter orbiting particles is very rare, storage rings are designed to allow the beams to circulate for many turns with beam life times of several hours to give the particles ample opportunity to collide with other counter rotating particles. Of course, beams can counter rotate in the same magnetic fields only if one beam is made of the antiparticles of the other beam while two intersecting storage rings must be employed to allow the collision of unequal particles.

The circulating beam in an electron storage ring emits synchrotron radiation due to the transverse acceleration during deflection in the bending magnets. This radiation is highly collimated in the forward direction, of high brightness and therefore of great interest for basic and applied research, technology, and medicine.

Basically the design of a storage ring is the same as that for a synchrotron allowing some adjustment in the technical realization to optimize the desired features of acceleration and long beam lifetime, respectively. Beam intensities are 
generally very different in a synchrotron from that in a storage ring. In a synchrotron, the particle intensity is determined by the injector and this intensity is much smaller than desired in a storage ring. The injection system into a storage ring is therefore designed such that many beam pulses from a linear accelerator, an accumulator ring or a synchrotron can be accumulated. A synchrotron serving to accelerate beam from a low energy preinjector to the injection energy of the main facility, which may be a larger synchrotron or a storage ring, is also called a booster synchrotron or short a booster.

Although a storage ring is not used for particle acceleration it often occurs that a storage ring is constructed long after and for a higher beam energy than the injector system. In this case, the beam is accumulated at the maximum available injection energy. After accumulation the beam energy is slowly raised in the storage ring to the design energy by merely increasing the strength of the bending and focusing magnets.

Electron positron storage rings have played a great role in basic high-energy research. For still higher collision energies, however, the energy loss due to synchrotron radiation has become a practical and economic limitation. To avoid this limit, beams from two opposing linear accelerators are brought into head on collision at energies much higher than is possible to produce in circular accelerators. To match the research capabilities in colliding beam storage rings, such linear colliders must employ sophisticated beam dynamics controls, focusing arrangements and technologies similar to X-ray laser systems now operating.

\subsubsection{Applications of Particle Accelerators}

Particle accelerators are mainly known for their application as research tools in nuclear and high energy particle physics requiring the biggest and most energetic facilities. Smaller accelerators, however, have found broad applications in a wide variety of basic research and technology, as well as medicine. In this text, we will not discuss the details of all these applications but try to concentrate only on the basic principles of particle accelerators and the theoretical treatment of particle beam dynamics and instabilities. An arbitrary and incomplete listing of applications for charged particle beams and their accelerators is given for reference to the interested reader:

Nuclear physics

Electron/proton accelerators

Ion accelerators/colliders

Continuous beam facility

High-energy physics

Fixed target accelerator

Colliding beam storage rings

Linear colliders
Power generation

Inertial fusion

Reactor fuel breeding

Industry

Radiography by x-rays

Ion implantation

Isotope production/separation

Materials testing/modification 


\author{
Food sterilization \\ X-ray lithography \\ Synchrotron radiation \\ Basic atomic and molecular physics \\ Condensed matter physics \\ Earth sciences \\ Material sciences \\ Chemistry \\ Molecular and cell biology \\ Surface/interface physics
}

\author{
Coherent radiation \\ Free electron lasers, X-FEL \\ Microprobe \\ Holography \\ Medicine \\ Radiotherapy \\ Health physics \\ Microsurgery with tunable FEL \\ Sterilization
}

This list is by no means exhaustive and additions must be made at an impressive pace as the quality and characteristics of particle beams become more and more sophisticated, predictable and controllable. Improvements in any parameter of particle beams create opportunities for new experiments and applications which were not possible before. More detailed information on specific uses of particle accelerators as well as an extensive catalogue of references has been compiled by Scharf [30].

\subsection{Definitions and Formulas}

Particle beam dynamics can be formulated in a variety units and it is therefore prudent to define the units used in this text to avoid confusion. In addition, we recall fundamental relations of electromagnetic fields and forces as well as some laws of special relativity to the extend that will be required in the course of discussions.

\subsubsection{Units and Dimensions}

A set of special physical units, selected primarily for convenience, are most commonly used to quantify physical constants in accelerator physics. The use of many such units is often determined more by historical developments than based on the choice of a consistent set of quantities useful for accelerator physics.

Generally, accelerator physics theory is formulated in the metric mks-system of units or SI-units which we follow also in this text. For readers used to cgs units, we include here conversion tables for convenience. To measure the energy of charged particles the unit Joule is actually used very rarely. The basic unit of energy in particle accelerator physics is the electron Volt $(\mathrm{eV})$, which is the kinetic energy a particle with one basic unit of electrical charge $e$ would gain while being accelerated between two conducting plates at a potential difference of $1 \mathrm{~V}$. Therefore, $1 \mathrm{eV}$ is equivalent to $1.60217733 \times 10^{-19} \mathrm{~J}$. Specifically, we will often 
use derivatives of the basic units to express actual particle energies in a convenient form:

$$
1 \mathrm{keV}=1000 \mathrm{eV} ; 1 \mathrm{MeV}=10^{6} \mathrm{eV} ; 1 \mathrm{GeV}=10^{9} \mathrm{eV} ; 1 \mathrm{TeV}=10^{12} \mathrm{eV}
$$

To describe particle dynamics we find it necessary to sometimes use the particle's momentum and sometimes the particle's energy. The effect of the Lorentz force from electric or magnetic fields is inversely proportional to the momentum of the particle. Acceleration in rf fields, on the other hand, is most conveniently measured by the increase in kinetic or total energy.

In an effort to simplify the technical jargon used in accelerator physics the term energy is used for all three quantities although mathematically the momentum is then multiplied by the velocity of light for dimensional consistency. There are still numerical differences which must be considered for all but very highly relativistic particles. Where we need to mention the pure particle momentum and quote a numerical value, we generally use the total energy divided by the velocity of light with the unit $\mathrm{eV} / \mathrm{c}$. With this definition a particle of energy $c p=1 \mathrm{eV}$ would have a momentum of $p=1 \mathrm{eV} / \mathrm{c}$.

An additional complication arises in the case of composite particles like heavy ions, consisting of protons and neutrons. In this case, the particle energy is not quoted for the whole ion but in terms of the energy per nucleon.

The particle beam current is measured generally in Amperes, no matter what general system of units is used but also occasionally in terms of the total charge or number of particles. The current is then the total charge $Q$ passing a point during the time $t$. Depending on the time duration one gets an instantaneous current or some average current. Therefore a quotation of the particle current requires also the definition of the time structure of the beam. In circular accelerators, for example, the average beam current $I$ relates directly to the beam intensity or the number of circulating particles $N$. If $\beta c$ is the velocity of the particle and $Z$ the charge multiplicity, we get for the relation of beam current and beam intensity

$$
I=e Z f_{\mathrm{rev}} N
$$

where the revolution frequency $f_{\mathrm{rev}}=\beta c / C$ and $C$ is the circumference of the circular accelerator. This is the average circulating current to be distinguished from the bunch current or peak bunch current, which is the charge per bunch $q$ divided by the duration of the bunch.

For a linear accelerator or beam transport line where particles come by only once, the definition of the beam current is more subtle. We still have a simple case if the particles come by in a continuous stream in which case the beam current is proportional to the particle flux $\dot{N}$ or $I=e Z \dot{N}$. This case, however, occurs very rarely since particle beams are generally accelerated by rf fields. As a consequence there is no continuous flux of particles reflecting the time varying acceleration of the rf field. The particle flux therefore is better described by a series of equidistant particle bunches separated by an integral number of wavelengths of the accelerating 
Table 1.1 Numerical conversion factors

\begin{tabular}{l|l|l}
\hline Quantity & \multicolumn{2}{|l}{ Replace cgs parameter by practical units } \\
\hline Potential & 1 esu & $300 \mathrm{~V}$ \\
\hline Electrical field & 1 esu & $310^{4} \mathrm{~V} / \mathrm{m}$ \\
\hline Current & $1 \mathrm{esu}$ & $0.1 \cdot c \mathrm{~A}$ \\
\hline Charge & $1 \mathrm{esu}$ & $0.333310^{-9} \mathrm{C}$ \\
\hline Force & $1 \mathrm{dyn}$ & $10^{-5} \mathrm{~N}$ \\
\hline Energy & $1 \mathrm{eV}$ & $1.60210^{-19} \mathrm{~J}$ \\
\cline { 2 - 3 } & $1 \mathrm{eV}$ & $1.60210^{-12} \mathrm{erg}$ \\
\hline
\end{tabular}

rf field. Furthermore, the acceleration often occurs only in bursts or pulses producing either a single bunch of particles or a string of many bunches. In these cases we distinguish between different current definitions. The peak current is the peak instantaneous beam current for a single bunch, while the average current is defined as the particle flux averaged over the duration of the beam pulse or any other given time period, e.g. $1 \mathrm{~s}$.

Magnetic fields are quoted either in Tesla or Gauss. ${ }^{1}$ Similarly, field gradients and higher derivatives are expressed in Tesla per meter or Gauss per centimeter. Frequently we find the need to perform numerical calculations with parameters given in different units. Some helpful numerical conversions from cgs to mks-units are compiled in Table 1.1.

Similar conversion factors can be derived for electromagnetic quantities in formulas by comparisons of similar equations in the MKS and cgs-system. Table 1.2 includes some of the most frequently used conversions. The absolute dielectric constant is

$$
\varepsilon_{0}=\frac{10^{7}}{4 \pi c^{2}} \frac{\mathrm{C}}{\mathrm{Vm}}=8.854 \times 10^{-12} \frac{\mathrm{C}}{\mathrm{Vm}}
$$

and the absolute is

$$
\mu_{0}=4 \pi 10^{-7} \frac{\mathrm{Vs}}{\mathrm{Am}}=1.2566 \times 10^{-6} \frac{\mathrm{Vs}}{\mathrm{Am}}
$$

Both constants are related by $c^{2} \varepsilon_{0} \mu_{0}=1$. Using these conversion factors it is possible to convert formulas in cgs units into the equivalent form for mks-units.

\subsubsection{Maxwell's Equations}

Predictable control of charged particles is effected only by electric and magnetic fields and beam dynamics is the result of such interaction. We try to design and

\footnotetext{
${ }^{1}$ Because of its wide use, we use in rare cases the unit Gauss even though it is not a SI unit (1 Gauss $=0.0001$ Tesla $=0.1 \mathrm{mT}$ ).
} 
Table 1.2 Conversion factors for equations

\begin{tabular}{l|l|l}
\hline Quantity & $\begin{array}{l}\text { Replace cgs-parameter } \\
\text { by mks-parameter }\end{array}$ \\
\hline Potential & $V_{\mathrm{cgs}}$ & $\sqrt{4 \pi \varepsilon_{0}} V_{\mathrm{mks}}$ \\
\hline Electric field & $E_{\mathrm{cgs}}$ & $\sqrt{4 \pi \varepsilon_{0}} E_{\mathrm{mks}}$ \\
\hline Current & $I_{\mathrm{cgs}}$ & $\frac{1}{\sqrt{4 \pi \varepsilon_{0}}} I_{\mathrm{mks}}$ \\
\hline Current density & $j_{\mathrm{cgs}}$ & $\frac{1}{\sqrt{4 \pi \varepsilon_{0}}} j_{\mathrm{mks}}$ \\
\hline Charge & $q_{\mathrm{cgs}}$ & $\frac{1}{\sqrt{4 \pi \varepsilon_{0}}} q_{\mathrm{mks}}$ \\
\hline Charge density & $\rho_{\mathrm{cgs}}$ & $\frac{1}{\sqrt{4 \pi \varepsilon_{0}}} \rho_{\mathrm{mks}}$ \\
\hline Conductivity & $\sigma_{\mathrm{cgs}}$ & $\frac{1}{\sqrt{4 \pi \varepsilon_{0}}} \sigma_{\mathrm{mks}}$ \\
\hline Inductance & $L_{\mathrm{cgs}}$ & $4 \pi \varepsilon_{0} L_{\mathrm{mks}}$ \\
\hline Capacitance & $C_{\mathrm{cgs}}$ & $\frac{1}{4 \pi \varepsilon_{0}} C_{\mathrm{mks}}$ \\
\hline Magnetic field & $H_{\mathrm{cgs}}$ & $\sqrt{4 \pi \mu_{0}} H_{\mathrm{mks}}$ \\
\hline Magnetic induction & $B_{\mathrm{cgs}}$ & $\frac{4 \pi}{\mu_{0}} B_{\mathrm{mks}}$ \\
\hline
\end{tabular}

formulate electromagnetic fields in a way that can be used to accurately predict the behavior of charged particles. To describe the general interaction of fields based on electric currents in specific devices and free charged particles which we want to preserve, guide and focus, we use as a starting point Maxwell's equations:

$$
\begin{array}{ll}
\nabla(\epsilon \boldsymbol{E})=\frac{\rho}{\epsilon_{0}}, & \text { Coulomb's law, } \\
\nabla \boldsymbol{B}=0, & \\
\nabla \times \boldsymbol{E}=-\frac{\partial}{\partial t} \boldsymbol{B}, & \text { Faraday's law, } \\
\nabla \times\left(\frac{1}{\mu} \boldsymbol{B}\right)=\mu_{0} \boldsymbol{j}+\frac{1}{c^{2}} \frac{\partial}{\partial t}(\epsilon \boldsymbol{E}) . & \text { Ampère's law, }
\end{array}
$$

consistent with the SI-system of units by inclusion of the unit scale factors $\epsilon_{0}$ and $\mu_{0}$. The quantities $\epsilon$ and $\mu$ are the relative dielectric constant and magnetic permeability of the surrounding materials, respectively. Integration of one or the other of Maxwell's equations results, for example, in the fields from singly charged particles or those of an assembly of particles travelling along a common path and forming a beam. Applying Maxwell's equations, we will make generous use of algebraic relations which have been collected in Appendix A.

\subsection{Primer in Special Relativity}

In accelerator physics the dynamics of particle motion is formulated for a large variety of energies from nonrelativistic to highly relativistic values and the equations of motion obviously must reflect this. Relativistic mechanics is therefore a fundamental ingredient of accelerator physics and we will recall a few basic relations of relativistic particle mechanics from a variety of more detailed derivations in 
generally available textbooks. Beam dynamics is expressed in a laboratory by a fixed system of coordinates but some specific problems are better discussed in the moving coordinate system of particles. Transformation between the two systems is effected through a Lorentz transformation.

\subsubsection{Lorentz Transformation}

Physical phenomena can appear different for observers in different systems of reference. Yet, the laws of nature must be independent of the reference system. In classical mechanics, we transform physical laws from one to another system of reference by way of the Galileo transformation $z^{*}=z-v t$ assuming that one system moves with velocity $v$ along the $z$-axis of the other system.

As the velocities of bodies under study became faster, it became necessary to reconsider this simple transformation leading to Einstein's special theory of relativity. Maxwell's equations result in electromagnetic waves expanding at a finite velocity and do not contain any reference to a specific system of reference. Any attempt to find a variation of the "velocity of light" with respect to moving reference systems failed, most notably in Michelson's experiments. The expansion velocity of electromagnetic waves is therefore independent of the reference system and is finite.

Any new transformation laws must include the observation that no element of energy can travel faster than the speed of light. The new transformation formulae combine space and time and are for a reference system $\mathcal{L}^{*}$ moving with velocity $v_{z}=c \beta_{z}$ along the $z$-axis with respect to the stationary system $\mathcal{L}$.

$$
\begin{aligned}
& x=x^{*}, \\
& y=y^{*}, \\
& z=\gamma\left(z^{*}+\beta_{z} c t^{*}\right), \\
& c t=\gamma\left(\beta_{z} z^{*}+c t^{*}\right),
\end{aligned}
$$

where the relativistic factor is

$$
\gamma=\frac{1}{\sqrt{1-\beta_{z}^{2}}}
$$

with

$$
\beta_{z}=v_{z} / c
$$

and where all quantities designated with ${ }^{*}$ are defined in the moving system $\mathcal{L}^{*}$. Of course, either system is moving relative to the other and we will use this 
relativity in various circumstances depending on whether quantities are known in the laboratory or moving system. The Lorentz transformations can be expressed in matrix formulation by

$$
\left(\begin{array}{c}
x \\
y \\
z \\
c t
\end{array}\right)=\left(\begin{array}{cccc}
1 & 0 & 0 & 0 \\
0 & 1 & 0 & 0 \\
0 & 0 & \gamma & +\beta \gamma \\
0 & 0 & +\beta \gamma & \gamma
\end{array}\right)\left(\begin{array}{c}
x^{*} \\
y^{*} \\
z^{*} \\
c t^{*}
\end{array}\right)=\mathcal{M}_{\mathrm{L}}\left(\begin{array}{c}
x^{*} \\
y^{*} \\
z^{*} \\
c t^{*}
\end{array}\right)
$$

and the inverse transformation is the same except that the velocity or $\beta$ changes sign $(v \rightarrow-v)$.

\section{Lorentz Transformation of Fields}

Without proof, electromagnetic fields transform between reference systems in relative motion like

$$
\left(\begin{array}{c}
E_{x} \\
E_{y} \\
E_{z} \\
c B_{x} \\
c B_{y} \\
c B_{z}
\end{array}\right)=\left(\begin{array}{cccccc}
\gamma & 0 & 0 & 0 & +\gamma \beta_{z} & 0 \\
0 & \gamma & 0 & -\gamma \beta_{z} & 0 & 0 \\
0 & 0 & 1 & 0 & 0 & 0 \\
0 & -\gamma \beta_{z} & 0 & \gamma & 0 & 0 \\
+\gamma \beta_{z} & 0 & 0 & 0 & \gamma & 0 \\
0 & 0 & 0 & 0 & 0 & 1
\end{array}\right)\left(\begin{array}{c}
E_{x}^{*} \\
E_{y}^{*} \\
E_{z}^{*} \\
c B_{x}^{*} \\
c B_{y}^{*} \\
c B_{z}^{*}
\end{array}\right) .
$$

Again, for the inverse transformation only the sign of the relative velocity must be changed, $\beta_{z} \rightarrow-\beta_{z}$. According to this transformation of fields, a pure static magnetic field in the laboratory system $\mathcal{L}$, for example, becomes an electromagnetic field in the moving system $\mathcal{L}^{*}$. An undulator field, therefore, looks to an electron like a virtual photon with an electromagnetic field like a laser field and both interactions can be described by Compton scattering.

\section{Lorentz Contraction}

Characteristic for relativistic mechanics is the Lorentz contraction and time dilatation, both of which become significant in the description of particle dynamics. To describe the Lorentz contraction, we consider a rod at rest in the stationary system $\mathcal{L}$ along the $z$-coordinate with a length $\ell=z_{2}-z_{1}$. In the system $\mathcal{L}^{*}$, which is moving with the velocity $v_{z}$ in the positive $z$-direction with respect to $\mathcal{L}$, the rod appears to have the length $\ell^{*}=z_{2}^{*}-z_{1}^{*}$. By a Lorentz transformation we can relate that to the length in the $\mathcal{L}$-system. Observing both ends of the rod at the same time the lengths of the rod as observed from both systems relate like $\ell=z_{2}-z_{1}=\gamma\left(z_{2}^{*}+v_{z} t_{2}^{*}\right)-\gamma\left(z_{1}^{*}+v_{z} t_{1}^{*}\right)=\gamma \ell^{*}$ or

$$
\ell=\gamma \ell^{*} .
$$


A rod at rest in system $\mathcal{L}$ appears shorter in the moving particle system $\mathcal{L}^{*}$ by a factor $\gamma$ and is always longest in it's own rest system. For example, the periodicity of an undulator $\lambda_{\mathrm{p}}$ becomes Lorentz contracted to $\lambda_{\mathrm{p}} / \gamma$ as seen by relativistic electrons. Because of the Lorentz contraction, the volume of a body at rest in the system $\mathcal{L}$ appears also reduced in the moving system $\mathcal{L}^{*}$ and we have for the volume of a body in three dimensional space

$$
V=\gamma V^{*}
$$

Only one dimension of this body is Lorentz contracted and therefore the volume scales only linearly with $\gamma$. As a consequence, the charge density $\rho$ of a particle bunch with the volume $V$ is lower in the laboratory system $\mathcal{L}$ compared to the density in the system moving with this bunch and becomes

$$
\rho=\frac{\rho *}{\gamma}
$$

\section{Time Dilatation}

Similarly, we may derive the time dilatation or the elapsed time between two events occurring at the same point in both coordinate systems. Applying the Lorentz transformations we get from (1.5) with $z_{2}^{*}=z_{1}^{*}$

$$
\Delta t=t_{2}-t_{1}=\gamma\left(t_{2}^{*}+\frac{\beta_{z} z_{2}^{*}}{c}\right)-\gamma\left(t_{1}^{*}+\frac{\beta_{z} z_{1}^{*}}{c}\right)
$$

or

$$
\Delta t=\gamma \Delta t^{*}
$$

For a particle at rest in the moving system $\mathcal{L}^{*}$ the time $t^{*}$ varies slower than the time in the laboratory system. This is the mathematical expression for the famous twin paradox where one of the brothers moving in a space capsule at relativistic speed would age slower than his twin brother staying back. This phenomenon gains practical importance for unstable particles. For example, high-energy pions, observed in the laboratory system, have a longer lifetime by the factor $\gamma$ compared to low-energy pions with $\gamma=1$. As a consequence, high energy unstable particles, like pions and muons, live longer and can travel farther as measured in the laboratory system, because the particle decay time is a particle property and is therefore measured in its own moving system. This is important. For example, in medical applications when a beam of pions has to be transported from the highly radioactive target area to a radiation free environment for the patient for cancer treatment. 


\subsubsection{Lorentz Invariance}

Briefly, we have to introduce 4-vectors, because they will make later discussions much easier and illuminate fundamental properties of synchrotron radiation which is emitted in the particle system, but observed in the laboratory system as we will see later in this section. Four-vectors have a special significance in physics. As their name implies, four physical quantities can form a 4-vector which has convenient properties when viewed in different reference systems. The components of spacetime, for example, form a 4-vector $\tilde{\boldsymbol{s}}=(x, y, z, \mathrm{i} c t)$. To identify 4-vectors, we add a tilde $\tilde{\boldsymbol{s}}$ to the symbols. All true 4 -vectors transform like the space-time coordinates through Lorentz transformations.

$$
\tilde{\boldsymbol{a}}=\mathcal{M}_{\mathrm{L}} \tilde{\boldsymbol{a}}^{*}
$$

\section{Invariance to Lorentz Transformations}

The length of 4-vectors is the same in all reference systems and is therefore open to measurements and comparisons independent of the location of the experimenter. In fact, it can be shown (exercise) that even the product of two arbitrary 4vectors is Lorentz invariant. Take two 4-vectors in an arbitrary frame of reference $\tilde{\boldsymbol{a}}^{*}=\left(a_{1}^{*}, a_{2}^{*}, a_{3}^{*}, \mathrm{i} a_{4}^{*}\right)$ and $\tilde{\boldsymbol{b}}^{*}=\left(b_{1}^{*}, b_{2}^{*}, b_{3}^{*}, \mathrm{i} b_{4}^{*}\right)$ and form the product $\tilde{\boldsymbol{a}}^{*} \tilde{\boldsymbol{b}}^{*}$ in component form. A Lorentz transformation on both 4 -vectors gives $\tilde{\boldsymbol{a}}^{*} \tilde{\boldsymbol{b}}^{*}=\tilde{\boldsymbol{a}} \tilde{\boldsymbol{b}}$, which is the same in any reference system and is therefore Lorentz invariant. Specifically, the length of any 4-vector is Lorentz invariant.

\section{Space-Time}

Imagine a light flash to originate at the origin of the coordinate system $\mathcal{L}(x, y, z)$. At the time $t$, the edge of this expanding light flash has expanded with the velocity of light to

$$
x^{2}+y^{2}+z^{2}=c^{2} t^{2} .
$$

Observing the same light flash from a moving system, we apply a Lorentz transformation from the laboratory system $\mathcal{L}$ to the moving system $\mathcal{L}^{*}$ and get

$$
x^{* 2}+y^{* 2}+z^{* 2}=c^{2} t^{* 2}
$$

demonstrating the invariance of the velocity of light $c$ as has been experimentally verified by Michelson and Morley in 1887. The velocity of light is the same in all reference systems and its value is

$$
c=299,792,458 \mathrm{~m} / \mathrm{s} \text {. }
$$


The components of the space-time 4-vector are

$$
\tilde{\boldsymbol{s}}=\left(x_{1}, x_{2}, x_{3}, x_{4}\right)=(x, y, z, \mathrm{i} c t),
$$

where the time component has been multiplied by $c$ to give all components the same dimension. From the Lorentz invariant world time $\tau$, defined as

$$
c \tau=\sqrt{-\tilde{\boldsymbol{s}}^{2}}
$$

we get

$$
\begin{aligned}
c \mathrm{~d} \tau & =\sqrt{c^{2}(\mathrm{~d} t)^{2}-(\mathrm{d} x)^{2}-(\mathrm{d} y)^{2}-(\mathrm{d} z)^{2}}=\sqrt{c^{2}-\left(v_{x}^{2}+v_{y}^{2}+v_{z}^{2}\right)} \mathrm{d} t \\
& =\sqrt{c^{2}-v^{2}} \mathrm{~d} t=\sqrt{1-\beta^{2}} c \mathrm{~d} t
\end{aligned}
$$

a relation, we know from the Lorentz transformation as time dilatation $\mathrm{d} \tau=\frac{1}{\gamma} \mathrm{d} t$.

Other 4-vectors can be formulated and often become relevant in accelerator physics as, for example, those listed below. More 4-vectors are listed in Appendix B.

\section{Four-Velocity}

A velocity 4-vector can be derived from the space-time 4-vector by simple differentiation

$$
\tilde{\boldsymbol{v}}=\frac{\mathrm{d} \tilde{\boldsymbol{s}}}{\mathrm{d} \tau}=\gamma \frac{\mathrm{d} \tilde{\boldsymbol{s}}}{\mathrm{d} t}=\gamma(\dot{x}, \dot{y}, \dot{z}, \mathrm{i} c)
$$

Evaluating the square of the velocity 4-vector we find $\tilde{\boldsymbol{v}}^{2}=\gamma \boldsymbol{v}^{2}-\gamma c^{2}=-c^{2}$ in the rest frame and in any other reference frame. The velocity of light is the same in any reference system as experimentally verified by Michelson and Morley.

\section{Four-Acceleration}

From the velocity 4 -vector, we derive the 4-acceleration

$$
\tilde{\boldsymbol{a}}=\frac{\mathrm{d} \tilde{\boldsymbol{v}}}{\mathrm{d} \tau}=\gamma \frac{\mathrm{d}}{\mathrm{d} t}\left(\gamma \frac{\mathrm{d} \tilde{\boldsymbol{s}}}{\mathrm{d} t}\right)=\gamma^{2} \frac{\mathrm{d}^{2} \tilde{\boldsymbol{s}}}{\mathrm{d} t^{2}}+\gamma \tilde{\boldsymbol{v}} \frac{\mathrm{d} \gamma}{\mathrm{d} t}=\gamma^{2} \frac{\mathrm{d}^{2} \tilde{\boldsymbol{s}}}{\mathrm{d} t^{2}}+\tilde{\boldsymbol{v}} \frac{\gamma^{3}}{c^{2}}(\boldsymbol{v} \boldsymbol{a})
$$

or in component form $\tilde{\boldsymbol{a}}=\left(\tilde{a}_{x}, \tilde{a}_{y}, \tilde{a}_{z}, \mathrm{i} \tilde{a}_{t}\right)$, we get $\tilde{a}_{x}=\gamma^{2} a_{x}+\gamma^{4} \beta_{x}(\boldsymbol{\beta} \boldsymbol{a}), . ., \tilde{a}_{t}=$ $\gamma^{4}(\boldsymbol{\beta} \boldsymbol{a})$ where $\boldsymbol{a}=(\ddot{x}, \ddot{y}, \ddot{z})$ is the ordinary acceleration. The Lorentz invariance of $\tilde{\boldsymbol{a}}^{2}$ becomes important to describe the emission of synchrotron radiation from a 
relativistic charged particle and observation in a laboratory reference frame. Conversely, experimental verification of the theory of synchrotron radiation validates the invariance of $\tilde{\boldsymbol{a}}^{2}$.

\section{Momentum-Energy 4-Vector}

An important 4-vector is the 4-momentum or momentum-energy 4-vector defined by the canonical momentum $c \boldsymbol{p}$ and total energy $E$

$$
c \tilde{\boldsymbol{p}}=\left(c p_{x}, c p_{y}, c p_{z}, \mathrm{i} E\right) .
$$

The length of the energy-momentum 4-vector $c \tilde{\boldsymbol{p}}=\left(c p_{x}, c p_{y}, c p_{z}, \mathrm{i} E\right)$ can be determined by going into the rest frame where the momentum is zero and we get

$$
c^{2} \tilde{\boldsymbol{p}}^{2}=c^{2} p_{x}^{2}+c^{2} p_{y}^{2}+c^{2} p_{z}^{2}-E^{2}=-A^{2} m^{2} c^{4},
$$

where we have set $E_{0}=A m c^{2}$ for a particle with atomic mass $A$. From this the total energy is

$$
E^{2}=c^{2} \boldsymbol{p}^{2}+A^{2} m^{2} c^{4}
$$

demonstrating the experimentally verifiable fact that the particle mass is Lorentz invariant.

We look now for an expression of (1.26) without the use of velocities and derive from the product of the velocity and momentum-energy 4-vectors

$$
(\gamma \boldsymbol{v}, \mathrm{i} \gamma c)(c \boldsymbol{p}, \mathrm{i} E)=\gamma \boldsymbol{v} \boldsymbol{c p}-c \gamma E=-c A m c^{2}
$$

an expression for the momentum $c p=\frac{\gamma E-A m c^{2}}{\gamma \beta}$ since $\boldsymbol{p} \| \boldsymbol{\beta}$. Inserting this into (1.26), we get $E^{2}=\left(\frac{\gamma E-A m c^{2}}{\gamma \beta}\right)^{2}+A^{2} m^{2} c^{4}$, and with $\beta^{2} \gamma^{2}=\gamma^{2}-1$

$$
\gamma=\frac{E}{A m c^{2}}
$$

defining the relativistic factor $\gamma$ in terms of energies. Sometimes, authors attach this relativistic factor to the mass and assume thereby an increasing moving mass. Einstein's point of view is expressed in the following quote: "It is not good to introduce the concept of the mass of a moving body $M=\gamma m_{0}$ for which no clear definition can be given. It is better to introduce no mass concept other than the 'rest mass' $m_{0}$. Instead of introducing $M$ it is better to mention the expression for the momentum and energy of a body in motion." In this book, we take the rest mass $m_{0}$ as an invariant. 
The total energy of a particle is given by

$$
E=\gamma E_{0}=\gamma A m c^{2}
$$

where $E_{0}=A m c^{2}$ is the rest energy of the particle and $A$ the atomic mass. For electrons we assume that $A=1$ and $m=m_{\mathrm{e}}$. Since in this text we concentrate mainly on electrons and protons, we assume $A=1$. The kinetic energy is defined as the total energy minus the rest energy

$$
E_{\text {kin }}=E-E_{0}=(\gamma-1) m c^{2} .
$$

The change in kinetic energy during acceleration is equal to the product of the accelerating force and the path length over which the force acts on the particle. Since the force may vary along the path we use the integral

$$
\Delta E_{\mathrm{kin}}=\int_{L_{\mathrm{acc}}} \mathbf{F} \mathrm{d} \mathbf{s}
$$

to define the energy increase. The length $L_{\mathrm{acc}}$ is the path length through the accelerating field. In discussions of energy gain through acceleration, we consider only energy differences and need therefore not to distinguish between total and kinetic energy. The particle momentum finally is defined by

$$
c^{2} p^{2}=E^{2}-E_{0}^{2}
$$

or

$$
c p=\sqrt{E^{2}-E_{0}^{2}}=m c^{2} \sqrt{\gamma^{2}-1}=\gamma \beta m c^{2}=\beta E,
$$

where $\beta=v / c$. The simultaneous use of the terms energy and momentum might seem sometimes to be misleading as we discussed earlier. In this text, however, we will always use physically correct quantities in mathematical formulations even though we sometimes use the term energy for the quantity $c p$. In electron accelerators the numerical distinction between energy and momentum is insignificant since we consider in most cases highly relativistic particles. For proton accelerators and even more so for heavy ion accelerators the difference in both quantities becomes, however, significant.

Often we need differential expressions or expressions for relative variations of a quantity in terms of variations of another quantity. Such relations can be derived from the definitions in this section. By variation of (1.33), for example, we get

$$
\mathrm{d} c p=\frac{m c^{2}}{\beta} \mathrm{d} \gamma=\frac{\mathrm{d} E}{\beta}=\frac{\mathrm{d} E_{\text {kin }}}{\beta}
$$


and

$$
\frac{\mathrm{d} c p}{c p}=\frac{1}{\beta^{2}} \frac{\mathrm{d} \gamma}{\gamma} .
$$

Varying (1.32) and replacing $\mathrm{d} \gamma$ from (1.33) we get

$$
\mathrm{d} c p=\gamma^{3} m c^{2} \mathrm{~d} \beta
$$

and

$$
\frac{\mathrm{d} c p}{c p}=\gamma^{2} \frac{\mathrm{d} \beta}{\beta}
$$

\section{Photon 4-Vector}

An analogous 4-vector can be formulated for photons using deBroglie's relations $\boldsymbol{p}=\hbar \boldsymbol{k}$ and $E=\hbar \omega$ for $c \tilde{\boldsymbol{k}}=\left(c k_{x}, c k_{y}, c k_{z}, \mathrm{i} \omega\right)$. Since the energy-momentum 4-vector is derived from the canonical momentum, we will have to modify this 4vector when electromagnetic fields are present.

\section{Force 4-Vector}

The force 4-vector is the time derivative of the energy-momentum 4-vector $(c \dot{\boldsymbol{p}}, \mathrm{i} \dot{E})$, which is consistent with the observation (so far) that the rest mass does not change with time.

\section{Electro-magnetic 4-Vector}

The electromagnetic-potential 4-vector is $(c \boldsymbol{A}, \mathrm{i} \phi)$.

\subsubsection{Spatial and Spectral Distribution of Radiation}

Of great importance in accelerator and synchrotron radiation physics is the Lorentz invariance of the product of two 4-vectors. Electromagnetic fields emanating from relativistic charges can be described by plane waves $E^{*}=E_{0}^{*} \mathrm{e}^{\mathrm{i} \Phi^{*}}$, where $\Phi^{*}=$ $\omega^{*} t^{*}-k^{*} n^{*} \boldsymbol{r}^{*}$ is the phase of the wave in the particle system and is Lorentz invariant. This invariance stems from the fact that the phase can be formulated as the product of the photon and space-time 4-vectors

$$
c \tilde{\boldsymbol{p}} \cdot \tilde{\boldsymbol{s}}=[c k n, \mathrm{i} \omega][z, \mathrm{i} c t],
$$


where we have set $\boldsymbol{k}=\boldsymbol{n} \boldsymbol{k}$ with $\boldsymbol{n}$ being the unit vector in the direction of wave propagation. Using $k=\omega / c$ the phase as measured in the laboratory $\mathcal{L}$ is the same as that in the particle frame of reference $\mathcal{L}^{*}$

$$
\omega^{*}\left[\left(n_{x}^{*} x^{*}+n_{y}^{*} y^{*}+n_{z}^{*} z^{*}\right)-c t^{*}\right]=\omega\left[\left(n_{x} x+n_{y} y+n_{z} z\right)-c t\right]=\text { invariant. }
$$

To derive the relationships between similar quantities in both systems, we use the Lorentz transformation (1.8), noting that the particle reference frame is the frame, where the particle or radiation source is at rest, and replace the coordinates $\left(x^{*}, y^{*}, z^{*}, c t^{*}\right)$ by those in the laboratory system for

$$
\begin{aligned}
& \omega^{*}\left[\left(n_{x}^{*} x^{*}+n_{y}^{*} y^{*}+n_{z}^{*} z^{*}\right)-c t^{*}\right] \\
& =\omega^{*}\left[n_{x}^{*} x+n_{y}^{*} y+n_{z}^{*}(\gamma z-\beta \gamma c t)-(-\beta \gamma z+\gamma c t)\right] \\
& =\omega\left[\left(n_{x} x+n_{y} y+n_{z} z\right)-c t\right],
\end{aligned}
$$

from which one can isolate, for example, a relation between $\omega^{*}$ and $\omega$. Since the space-time coordinates are independent from each other, we may equate their coefficients on either side of the equation separately.

\section{Spectral Distribution}

In so doing, the $c t$-coefficients define the transformation of the oscillation frequency

$$
\omega^{*} \gamma\left(1+\beta_{z} n_{z}^{*}\right)=\omega
$$

which expresses the relativistic Doppler effect. Looking parallel and opposite to the direction of particle motion $n_{z}^{*}=1$, the observed oscillation frequency is increased by the factor $\left(1+\beta_{z}\right) \gamma \approx 2 \gamma$ for highly relativistic particles. The Doppler effect is reduced (red shifted) if the radiation is viewed at some finite angle $\Theta$ with respect to the direction of motion of the source. In these cases $n_{z}^{*}=\cos \Theta^{*}$ and the frequency shift can be very large for highly relativistic particles with $\gamma \gg 1$.

\section{Spatial Distribution}

Similarly, we obtain the transformation of spatial directions from

$$
n_{x}=\frac{n_{x}^{*}}{\gamma\left(1+\beta_{z} n_{z}^{*}\right)}, \quad n_{y}=\frac{n_{y}^{*}}{\gamma\left(1+\beta_{z} n_{z}^{*}\right)}, \quad n_{z}=\frac{\beta_{z}+n_{z}^{*}}{\left(1+\beta_{z} n_{z}^{*}\right)} .
$$


These transformations define the spatial distribution of radiation in the laboratory system. In case of transverse acceleration the radiation in the particle rest frame is distributed like $\cos ^{2} \Theta^{*}$ about the direction of motion. This distribution becomes greatly collimated into the forward direction in the laboratory system. With $n_{x}^{* 2}+$ $n_{y}^{* 2}=\sin ^{2} \Theta^{*}$ and $n_{x}^{2}+n_{y}^{2}=\sin ^{2} \Theta \approx \Theta^{2}$ and $n_{z}^{*}=\cos \Theta^{*}$, we find

$$
\Theta \approx \frac{\sin \Theta^{*}}{\gamma\left(1+\beta \cos \Theta^{*}\right)}
$$

In other words, radiation from relativistic particles, emitted in the particle system into an angle $-\pi / 2<\Theta^{*}<\pi / 2$ appears in the laboratory system highly collimated in the forward direction within an angle of

$$
\Delta \Theta \approx \pm \frac{1}{\gamma} .
$$

This angle is very small for highly relativistic electrons like those in a storage ring, where $\gamma$ is of the order of $10^{3}-10^{4}$.

\subsubsection{Particle Collisions at High Energies}

The most common use of high-energy particle accelerators has been for basic research in elementary particle physics. Here, accelerated particles are aimed at a target, which incidentally may be just another particle beam, and the researchers try to analyze the reaction of high-energy particles colliding with target particles. The available energy from the collision depends on the kinematic parameters of the colliding particles. We define a center of mass coordinate system which is the system that moves with the center of mass of the colliding particles. In this system the vector sum of all momenta is zero and is preserved through the collision. Similarly, the total energy is conserved and we may define a center of mass energy the same way the rest energy of a single particle is defined by

$$
E_{\mathrm{cm}}^{2}=\left(\sum_{i} E_{i}\right)^{2}-\left(\sum_{i} c p_{i}\right)^{2},
$$

where the summation is taken over all particles forming the center of mass system. The center of mass energy includes all old particle masses but also new masses of new particles which have not been there before. We apply this to two colliding particles with masses $m_{1}$ and $m_{2}$ and velocities $\mathbf{v}_{\mathbf{1}}$ and $\mathbf{v}_{\mathbf{2}}$, respectively,

$$
\left(m_{1}, \mathbf{v}_{1}\right) \longrightarrow \longleftarrow\left(m_{2}, \mathbf{v}_{2}\right)
$$


The center of mass energy for this system of two colliding particles is then

$$
\begin{aligned}
E_{\mathrm{cm}}^{2} & =\left[\sum_{i=1}^{2}\left(E_{\mathrm{kin}}+m c^{2}\right)_{i}\right]^{2}-\left[\sum_{i=1}^{2} c p_{i}\right]^{2} \\
& =\left(\gamma_{1} m_{1}+\gamma_{2} m_{2}\right)^{2} c^{4}-\left(\gamma_{1} \beta_{1} m_{1}+\gamma_{2} \beta_{2} m_{2}\right)^{2} c^{4}
\end{aligned}
$$

We apply these kinematic relations to a proton $\left(m_{1}=m_{\mathrm{p}}\right)$ of energy $\gamma$ colliding with a proton at rest in a target. For a target proton at rest with $\gamma_{2}=1, m_{2}=m_{\mathrm{p}}, \beta_{2}=0$ and $\beta \gamma=\sqrt{\gamma^{2}-1}$, the center of mass energy is

$$
E_{\mathrm{cm}}^{2}=(\gamma+1)^{2} m_{\mathrm{p}}^{2} c^{4}-\left(\gamma^{2}-1\right) m_{\mathrm{p}}^{2} c^{4}
$$

or after some manipulations

$$
E_{\mathrm{cm}}=\sqrt{2(\gamma+1)} m_{\mathrm{p}} c^{2}
$$

The available energy for high-energy reactions after conservation of energy and momentum for the whole particle system is the center of mass energy minus the rest energy of the particles that need to be conserved. If, for example, two protons collide, high-energy physics conservation laws tell us that the hadron number must be conserved and therefore the reaction products must include two units of the hadron number. In the most simple case the reaction will produce just two protons and some other particles with a total energy equal to the available energy

$$
E_{\text {avail }}=E_{\mathrm{cm}}-2 m_{\mathrm{p}} c^{2}=[\sqrt{2(\gamma+1)}-2] m_{\mathrm{p}} c^{2}
$$

The energy available from such reactions increases only like the square root of the energy of the accelerated particle which makes such stationary target physics an increasingly inefficient use of high-energy particles. A significantly more efficient way of using the energy of colliding particles can be obtained by head on collision of two equal particles of equal energy. In this case $\gamma_{1}=\gamma_{2}=\gamma$, the mass of the colliding particles is $m_{1}=m_{2}=m_{\mathrm{p}}$, and $\beta_{1}=-\beta_{2}=\beta$. In this case, the center of mass energy is simply twice the energy of each of the particles

$$
E_{\mathrm{cm}}=2 \gamma m c^{2}=2 E .
$$

In colliding beam facilities, where particles collide with their antiparticles no particle type conservation laws must be obeyed and therefore the total energy of both particles becomes available for the production of new particles at the collision point. In a similar way we may calculate the available energy for a variety of collision scenar- 
ios like the collision of an accelerated electron with a stationary proton, the head on collision of electrons with protons or collisions involving high-energy heavy ions.

\subsection{Principles of Particle-Beam Dynamics}

Accelerator physics relates primarily to the interaction of charged particles with electromagnetic fields. Detailed knowledge of the functionality of this interaction allows the design of accelerators meeting specific goals and the prediction of charged particle beam behavior in those accelerators. The interplay between particles and fields is called beam dynamics. In this section, we recall briefly some features of electromagnetic fields and fundamental processes of classical and relativistic mechanics as they relate to particle beam dynamics.

\subsubsection{Electromagnetic Fields of Charged Particles}

Predictable control of charged particles is effected only by electric and magnetic fields and beam dynamics is the result of such interaction. We try to design and formulate electromagnetic fields in a way that can be used to accurately predict the behavior of charged particles. To describe the general interaction of fields based on electric currents in specific devices and free charged particles which we want to preserve, guide and focus, we use as a starting point Maxwell's equations (1.4).

\section{Electric Field of a Point Charge}

First, we apply Gauss' theorem to a point charge $q$ at rest. The natural coordinate system is the polar system because the fields of a point charge depend only on the radial distance from the charge. We integrate Coulomb's law (1.4) over a spherical volume containing the charge $q$ at its center. With $\mathrm{d} V=4 \pi r^{2} \mathrm{~d} r$ the integral becomes $\int \nabla \boldsymbol{E} \mathrm{d} V=\int_{0}^{R} \frac{1}{r^{2}} \frac{\partial}{\partial r}\left(r^{2} E_{r}\right) \mathrm{d} V=4 \pi R^{2} E_{r}(R)$, where $R$ is the radial distance from the charge. On the r.h.s. of Coulomb's law (1.4), an integration over all the charge $q$ gives $\int \frac{\rho}{\epsilon_{0} \epsilon} \mathrm{d} V=\frac{q}{\epsilon_{0} \epsilon}$ and the electric field of a point charge at distance $R$ is

$$
E_{r}(R)=\frac{1}{4 \pi \epsilon_{0} \epsilon} \frac{q}{R^{2}} .
$$

The electric field is proportional to the charge and decays quadratically with distance $R$. 


\section{Fields of a Charged Particle Beam}

Many charged particles, travelling along the same path form a beam. This particle beam generates an electric as well as a magnetic field. The proper coordinates are now cylindrical and Coulomb's law is

$$
\nabla \boldsymbol{E}=\frac{1}{r} \frac{\partial}{\partial r}\left(r E_{r}\right)+\frac{1}{r} \underbrace{\frac{\partial E_{\varphi}}{\partial \varphi}}_{=0}+\underbrace{\frac{\partial E_{z}}{\partial z}}_{=0}=\frac{1}{r} \frac{\partial}{\partial r}\left(r E_{r}\right)=\frac{\rho_{0}}{\epsilon_{0} \epsilon},
$$

where $\rho_{0}$ is the charge density in the particle beam. We assume a uniform continuous beam and expect therefore no azimuthal or longitudinal dependence, leaving only the radial dependence. Radial integration over a cylindrical volume of unit length collinear with the beam gives with the volume element $\mathrm{d} V=2 \pi r \mathrm{~d} r$, on the 1.h.s. $\left|r E_{r}\right|_{0}^{r} 2 \pi$. The r.h.s. is $\frac{\rho_{0}}{\epsilon_{0} \epsilon} \pi r^{2}$ and the electric field for a uniformly charged particle beam with radius $R$ is

$$
E_{r}(r)=\left\{\begin{array}{ll}
\frac{\rho_{0}}{2 \epsilon_{0} \epsilon} r & \text { for } r<R \\
\frac{\rho_{0}}{2 \epsilon_{0} \epsilon} \frac{R^{2}}{r} & \text { for } r>R
\end{array} .\right.
$$

The magnetic field for the same beam can be derived by applying Stoke's theorem on Ampere's law to give after integration

$$
B_{\varphi}(r)=\left\{\begin{array}{ll}
\frac{1}{2} \mu_{0} \mu j_{0} r & \text { for } r<R \\
\frac{1}{2} \mu_{0} \mu j_{0} \frac{R^{2}}{r} & \text { for } r>R
\end{array} .\right.
$$

The fields increase linearly within the beam and decay again like $1 / r$ outside the beam. Real particle beams do not have a uniform distribution and therefore a form function must be included in the integration. In most cases, the radial particle distribution is bell shaped or Gaussian. Both distributions differ little in the core of the beam and therefore a convenient assumption is that of a Gaussian distribution for which the fields will be derived in Problem 1.3.

\subsubsection{Vector and Scalar Potential}

By virtue of Maxwell's equation $\nabla \boldsymbol{B}=0$ one can derive the magnetic field from a vector potential $\boldsymbol{A}$ defined by $\boldsymbol{B}=\nabla \times \boldsymbol{A}$.Faraday's law can be used to derive also the electric field from potentials. The equation $\boldsymbol{\nabla} \times \boldsymbol{E}=-\frac{\partial}{\partial t} \boldsymbol{B}=-\frac{\partial}{\partial t}(\boldsymbol{\nabla} \times \boldsymbol{A})$ can be written like $\nabla \times(\boldsymbol{E}+\dot{\boldsymbol{A}})=0$, and solved by $\boldsymbol{E}=-\frac{\partial \boldsymbol{A}}{\partial t}-\nabla V$, where we added the gradient of a scalar potential function $V$ which does not alter the validity 
of Maxwell's equations for all fields so defined. To summarize, both, electric and magnetic fields can be derived from a scalar $V$ and vector $\boldsymbol{A}$ potential

$$
\begin{aligned}
& \boldsymbol{B}=\nabla \times \boldsymbol{A}-\nabla V, \\
& \boldsymbol{E}=-\frac{\partial \boldsymbol{A}}{\partial t}-\nabla V .
\end{aligned}
$$

These definitions of the magnetic and electric fields from potentials will not alter the validity of Maxwell's equations as can be verified by backinsertion.

\subsubsection{Wave Equation}

From Ampere's law both the vector and scalar potentials can be derived. Replacing in Ampère's law $\nabla \times \boldsymbol{B}=\mu_{0} \mu \boldsymbol{j}+\frac{\epsilon \mu}{c^{2}} \dot{\boldsymbol{E}}$ the fields with their expressions in terms of potentials, we get $\nabla \times(\nabla \times \boldsymbol{A})=\mu_{0} \mu \boldsymbol{j}+\frac{\epsilon \mu}{c^{2}}(-\ddot{\boldsymbol{A}}-\nabla \dot{V})$ and with $\nabla \times(\nabla \times \boldsymbol{A})=$ $\nabla(\nabla \boldsymbol{A})-\nabla^{2} \boldsymbol{A}$

$$
\nabla^{2} \boldsymbol{A}-\frac{\epsilon \mu}{c^{2}} \frac{\partial^{2} \boldsymbol{A}}{\partial t^{2}}=-\mu_{0} \mu \boldsymbol{j}+\nabla \underbrace{\left(\nabla \boldsymbol{A}+\frac{\epsilon \mu}{c^{2}} \dot{V}\right)}_{=0} .
$$

At this point, we specify the potential function $V$ such that it meets the condition

$$
\nabla \boldsymbol{A}+\frac{\epsilon \mu}{c^{2}} \dot{V}=0
$$

thereby simplifying greatly (1.53) and separating both potentials. This condition is called the Lorenz gauge and the resulting wave equation is

$$
\nabla^{2} \boldsymbol{A}-\frac{\epsilon \mu}{c^{2}} \frac{\partial^{2} \boldsymbol{A}}{\partial t^{2}}=-\mu_{0} \mu \boldsymbol{j} .
$$

The vector potential is clearly defined by the placement of electrical currents $\boldsymbol{j}$. We will use this property later in the design of, for example, magnets for particle beam guidance. Similarly, the wave equation for the scalar potential is

$$
\nabla^{2} V-\frac{1}{c^{2}} \frac{\partial^{2} V}{\partial t^{2}}=-\frac{\rho}{\epsilon_{0} \epsilon}
$$

Knowledge of the placement of electrical charges defines uniquely the scalar potential function. However, because the velocity of electro-magnetic waves is finite, the potentials at the observation point depend on the charges and currents 
at the retarded time, e.g. the location when the electro-magnetic waves have been emitted. The second order differential equations (1.55), (1.56) can be integrated readily and the potentials are

$$
\boldsymbol{A}(P, t)=\left.\frac{\mu_{0} \mu}{4 \pi} \int \frac{\boldsymbol{j}(x, y, z)}{R(x, y, z)}\right|_{t_{\mathrm{r}}} \mathrm{d} x \mathrm{~d} y \mathrm{~d} z
$$

and

$$
V(P, t)=\left.\frac{1}{4 \pi \epsilon_{0} \epsilon} \int \frac{\rho(x, y, z)}{R(x, y, z)}\right|_{t_{\mathrm{r}}} \mathrm{d} x \mathrm{~d} y \mathrm{~d} z .
$$

Integration over all currents and charges at the retarded distance $R_{t_{\mathrm{r}}}$ from the observation point $P$ results in the definition of the vector and scalar potential at the point $P$. Both electric and magnetic fields may be derived as discussed in the last section.

The wave equation just derived has special relevance for static fields where the Lorenz gauge reduces to the Coulomb gauge

$$
\nabla \boldsymbol{A}=0
$$

and (1.55) and (1.56) reduce in a charge and current free environment to the Laplace equation being equal to zero

$$
\begin{aligned}
& \Delta A=0, \\
& \Delta V=0 .
\end{aligned}
$$

Static magnetic and electric fields used in beam dynamics will be derived from theses potentials being solutions of the Laplace equation.

\section{Lienard-Wiechert Potentials}

For a point charge $e$ at rest, we can integrate (1.57) readily to get $A(R, t)=0$ and $V(R, t)=\frac{e}{4 \pi \epsilon_{0} \epsilon R}$. On the other hand, in case of a moving point charge the potentials cannot be obtained by simply integrating over the "volume" of the point charge. The motion of the charge must be taken into account and the result of a proper integration (see Chap. 25) are the Liénard-Wiechert potentials for moving charges [31, 32]

$$
\boldsymbol{A}(R, t)=\left.\frac{\mu_{0} \mu c}{4 \pi} \frac{q}{R} \frac{\boldsymbol{\beta}}{1+\boldsymbol{n} \boldsymbol{\beta}}\right|_{t_{\mathrm{r}}}
$$

and

$$
V(R, t)=\left.\frac{1}{4 \pi \epsilon_{0}} \frac{q}{R} \frac{1}{1+\boldsymbol{n} \boldsymbol{\beta}}\right|_{t_{\mathrm{r}}} .
$$


These potentials describe the radiation fields of synchrotron radiation being emitted from relativistic electrons.

\subsubsection{Induction}

Applying Stokes' theorem to Faraday's law (1.4), we get on the 1.h.s. a line integral along the boundaries of the surface area $S$, which is equivalent to a voltage. On the r.h.s. the magnetic flux passing through the surface $S$ is integrated and

$$
\int_{S}[\nabla \times \boldsymbol{E}] \mathrm{d} \boldsymbol{a}=\oint \boldsymbol{E} \mathrm{d} s=-\int_{S} \frac{\partial \boldsymbol{B}}{\partial t} \mathrm{~d} \boldsymbol{a}=-\frac{\partial \Phi}{\partial t} .
$$

By virtue of Faraday's law, the time varying magnetic flux $\Phi$ through the area $S$ generates an electromotive force along the boundaries of $S$. In accelerator physics this principle is applied in the design of a betatron. Similarly, from the second term on the right hand side in Ampère's law (1.4), we get a magnetic induction from a time varying electric field. Both phenomena play together to form the principle of induction or, in a particular example, that of a transformer.

\subsubsection{Lorentz, Force}

The trajectories of charged particles can be influenced only by electric and magnetic fields through the Lorentz force

$$
\boldsymbol{F}_{\mathrm{L}}=q \boldsymbol{E}+q(\boldsymbol{v} \times \boldsymbol{B}) .
$$

Guiding particles through appropriate electric or magnetic fields is called particle beam optics or beam dynamics. Knowledge of the location and amplitudes of electric and magnetic fields allows us to predict the path of charged particles. Closer inspection of (1.64) shows that the same force from electric or magnetic fields can be obtained if $\boldsymbol{E}=v \boldsymbol{B}$, where we have assumed that the particle velocity is orthogonal to the magnetic field, $\boldsymbol{v} \perp \boldsymbol{B}$. For relativistic particles $v \approx c$ and to get the same force from an electric field as from, say a 1 Tesla magnetic field, one would have to have an unrealistic high field strength of $\boldsymbol{E} \approx 300 \mathrm{MV} / \mathrm{m}$. For this reason, magnetic fields are used to deflect or focus relativistic charged particles. For sub-relativistic particles like ion beams with velocities $v \ll c$, on the other hand, electric fields may be more efficient. 


\subsubsection{Equation of Motion}

Accelerator physics is to a large extend the description of charged particle dynamics in the presence of external electromagnetic fields or of fields generated by other charged particles. We use the Lorentz force to formulate particle dynamics under the influence of electromagnetic fields. Whatever the interaction of charged particles with electromagnetic fields and whatever the reference system may be, we depend in accelerator physics on the invariance of the Lorentz force equation under coordinate transformations. All acceleration and beam guidance in accelerator physics will be derived from the Lorentz force. For simplicity, we use throughout this text particles with one unit of electrical charge $e$ like electrons and protons. In case of multiply charged ions the single charge $e$ must be replaced by $e Z$ where $Z$ is the charge multiplicity of the ion. Both components of the Lorentz force are used in accelerator physics where the force due to the electrical field is mostly used to actually increase the particle energy while magnetic fields are used to guide particle beams along desired beam transport lines. This separation of functions, however, is not exclusive as the example of the betatron accelerator shows where particles are accelerated by time dependent magnetic fields. Similarly electrical fields are used in specific cases to guide or separate particle beams.

Relating the Lorentz force to particle momentum or kinetic energy, we know from definitions in classical mechanics that

$$
\left.\begin{array}{c}
\Delta \boldsymbol{p}=\int \boldsymbol{F}_{\mathrm{L}} \mathrm{d} t \\
\Delta E_{\mathrm{kin}}=\int \boldsymbol{F}_{\mathrm{L}} \mathrm{d} \mathbf{s}
\end{array}\right\} \quad \underset{\mathrm{d} s=\boldsymbol{v} \mathrm{d} t}{\longrightarrow} \quad \boldsymbol{\beta} \Delta c \boldsymbol{p}=\Delta E_{\mathrm{kin}},
$$

where $\boldsymbol{\beta}=\boldsymbol{v} / c$. The Lorentz force can be expressed in terms of fields and the change of kinetic energy becomes

$$
\begin{aligned}
\Delta E_{\mathrm{kin}} & =\int \boldsymbol{F}_{\mathrm{L}} \mathrm{d} \boldsymbol{s}=q \int[\boldsymbol{E}+(\boldsymbol{v} \times \boldsymbol{B})] \mathrm{d} \boldsymbol{s} \\
& =q \int \boldsymbol{E} \mathrm{d} \boldsymbol{s}+q \int \underbrace{(\boldsymbol{v} \times \boldsymbol{B}) \boldsymbol{v}}_{=0} \mathrm{~d} t,
\end{aligned}
$$

which indicates that an electric field component in the direction of particle motion does increase the particle's kinetic energy, while the magnetic field does not contribute any acceleration. Magnetic fields are used only to deflect a particle's path by changing the direction of its momentum vector.

It becomes obvious that the kinetic energy of a particle changes whenever it travels in an accelerating electric field $\boldsymbol{E}$ and the acceleration occurs in the direction of the electric field. This acceleration is independent of the particle velocity and acts even on a particle at rest $\boldsymbol{v}=0$. The second component of the Lorentz force in contrast depends on the particle velocity and is directed normal to the direction of propagation and normal to the magnetic field direction. We find therefore from 
(1.66) the result that the kinetic energy is not changed by the presence of magnetic fields since the scalar product $(\boldsymbol{v} \times \boldsymbol{B}) \boldsymbol{v}=0$ vanishes. The magnetic field causes only a deflection of the particle trajectory.

The Lorentz force (1.64) in conjunction with (1.65) is used to derive the equation of motion for charged particles in the presence of electromagnetic fields

$$
\frac{\mathrm{d} \boldsymbol{p}}{\mathrm{d} t}=\frac{\mathrm{d}}{\mathrm{d} t}(A m \gamma \boldsymbol{v})=e Z \boldsymbol{E}+e Z(\boldsymbol{v} \times \boldsymbol{B}),
$$

where $Z$ is the charge multiplicity of the charged particle and $A$ the atomic mass. For simplicity we drop from here on the factors $A$ and $Z$ since they are different from unity only for ion beams. For ion accelerators we note therefore that the particle charge $e$ must be replaced by $e Z$ and the mass by $A m$.

Both relations in (1.65) can be used to describe the effect of the Lorentz force on particles. However, ease of mathematics makes us use one or the other. We use the first equation for dynamics in magnetic fields and the second for that in accelerating fields. Since the energy or the particle velocity does not change in a magnetic field it is straightforward to calculate $\Delta \boldsymbol{p}$. On the other hand, accelerating fields do change the particle's velocity which must be included in the time integration to get $\Delta p$. Calculating $\Delta E_{\text {kin }}$, we need to know only the spatial extend and magnitude of the accelerating fields to perform the integration.

The particle momentum $\boldsymbol{p}=\gamma m \boldsymbol{v}$ and it's time derivative

$$
\frac{\mathrm{d} \boldsymbol{p}}{\mathrm{d} t}=m \gamma \frac{\mathrm{d} \boldsymbol{v}}{\mathrm{d} t}+m \boldsymbol{v} \frac{\mathrm{d} \gamma}{\mathrm{d} t} .
$$

With

$$
\frac{\mathrm{d} \gamma}{\mathrm{d} t}=\frac{\mathrm{d},}{\mathrm{d} \beta} \frac{\mathrm{d} \beta}{\mathrm{d} t}=\gamma^{3} \frac{\beta}{c} \frac{\mathrm{d} v}{\mathrm{~d} t}
$$

we get from (1.68) the equation of motion

$$
\boldsymbol{F}=\frac{\mathrm{d} \boldsymbol{p}}{\mathrm{d} t}=m\left(\gamma \frac{\mathrm{d} \boldsymbol{v}}{\mathrm{d} t}+\gamma^{3} \frac{\beta}{c} \frac{\mathrm{d} v}{\mathrm{~d} t} \boldsymbol{v}\right) .
$$

For a force parallel to the particle propagation $\boldsymbol{v}$, we have $\dot{v} \boldsymbol{v}=\dot{\boldsymbol{v}} v$ and (1.70) becomes

$$
\frac{\mathrm{d} \boldsymbol{p}_{\|}}{\mathrm{d} t}=m \gamma\left(1+\gamma^{2} \beta \frac{v}{c}\right) \frac{\mathrm{d} \boldsymbol{v}_{\|}}{\mathrm{d} t}=m \gamma^{3} \frac{\mathrm{d} \boldsymbol{v}_{\|}}{\mathrm{d} t} .
$$

On the other hand, if the force is directed normal to the particle propagation, we have $\mathrm{d} v / \mathrm{d} t=0$ and (1.70) reduces to

$$
\frac{\mathrm{d} \boldsymbol{p}_{\perp}}{\mathrm{d} t}=m \gamma \frac{\mathrm{d} \boldsymbol{v}_{\perp}}{\mathrm{d} t} .
$$


It is obvious from (1.71) and (1.72) how differently the dynamics of particle motion is affected by the direction of the Lorentz force. Specifically the dynamics of highly relativistic particles under the influence of electromagnetic fields depends greatly on the direction of the force with respect to the direction of particle propagation. The difference between parallel and perpendicular acceleration will have a great impact on the design of electron accelerators. As we will see later, the acceleration of electrons is limited due to the emission of synchrotron radiation. This limitation, however, is much more severe for electrons in circular accelerators where the magnetic forces act perpendicularly to the propagation compared to the acceleration in linear accelerators where the accelerating fields are parallel to the particle propagation. This argument is also true for protons or for that matter, any charged particle, but because of the much larger particle mass the amount of synchrotron radiation is generally negligibly small.

\subsubsection{Charged Particles in an Electromagnetic Field}

An electromagnetic field exerts a force on a charged particle. A magnetic field or transverse electric field can deflect the beam and we use magnets as guiding and focusing elements for particle beam dynamics. This dynamics guides the particles on a path which is in equilibrium between the Lorentz force and the centrifugal force. A charged particle in a magnetic field follows a path defined by the equilibrium between centrifugal and Lorentz force

$$
\frac{\gamma m v^{2}}{\rho} \boldsymbol{n}+e[\boldsymbol{v} \times \boldsymbol{B}]=0,
$$

where $\boldsymbol{n}$ is the unit vector in the direction of the centrifugal force, $1 / \rho$ the local curvature and $m$ the mass of the particle with charge $e$. For a magnetic field orthogonal to the velocity vector of the particle the vector product is always parallel and opposite to $\boldsymbol{n}$ and (1.73) reduces to

$$
\frac{\gamma m v^{2}}{\rho}=-e v B_{\perp}
$$

with the local bending radius

$$
\frac{1}{\rho}=\frac{e c B}{\beta E_{\mathrm{tot}}}=\frac{e c B_{\perp}}{c p} .
$$

The plane of the particle path is orthogonal to the transverse magnetic field. In a uniform magnetic field the particle follows the path of an arc with radius

$$
\frac{1}{\rho}\left[\mathrm{m}^{-1}\right]=0.2995 \frac{B_{\perp}[\mathrm{T}]}{c p[\mathrm{GV}]} \approx 0.3 \frac{B_{\perp}[\mathrm{T}]}{c p[\mathrm{GV}]}
$$


in more practical units. We have a similar situation with respect to a transverse electrical field. Here, the centrifugal force is now

$$
\frac{\gamma m v^{2}}{\rho}+e \boldsymbol{E}_{\perp}=0
$$

or

$$
\frac{1}{\rho}=-\frac{e \boldsymbol{E}_{\perp}}{\gamma m c^{2} \beta^{2}}=-\frac{e \boldsymbol{E}_{\perp}}{\beta^{2} E_{\mathrm{tot}}},
$$

or in more practical units

$$
\frac{1}{\rho}\left[\mathrm{m}^{-1}\right]=-\frac{\boldsymbol{E}_{\perp}[\mathrm{V} / \mathrm{m}]}{\beta c p[\mathrm{GV}]}
$$

Here, some caution is appropriate, because during the deflection the unit vector $\boldsymbol{n}$ is changing direction while the electric field may not change direction as in the case of a field between parallel straight plates. However, if the electrodes are bend along the expected particle path, the direction of the electric field is changing with $\boldsymbol{n}$ or the deflection of the beam.

\subsubsection{Linear Equation of Motion}

We have now all ingrediences to formulate an equation of motion in linear approximation. Analytical geometry tells us that the curvature is given in cartesian coordinates by

$$
\kappa=\frac{-x^{\prime \prime}}{{\sqrt{1+x^{\prime 2}}}^{3}} .
$$

This equation can be simplified if we assume that $x^{\prime} \approx 0$. We recognize this from light optics as the paraxial approximation where all trajectories or rays are assumed to be close to the optical axis. This approximation suits beam dynamics very well since we try hard to keep all particles within a rather narrow vacuum chamber. Therefore (1.80) reduces with (1.75) to

$$
\kappa \approx-x^{\prime \prime}=\frac{e c B_{y}}{c p} .
$$

The magnetic fields will have two main components, the guiding field for bending and a focusing field. Both fields together can be expressed by $B_{y}=B_{0 y}+g x$, where $B_{0 y}$ is the bending field and $g$ the field gradient $g=\partial B_{y} / \partial x$. The particle beam is not perfectly monochromatic and we account for this by expanding the particle energy 
to first order $\frac{1}{c p} \approx \frac{1}{c p_{0}}(1-\delta)$, where $\delta=\Delta p / p_{0}$. With this we get the equation of motion

$$
x^{\prime \prime}=\frac{e c B_{y}}{c p}=\frac{e c}{c p_{0}}(1-\delta)\left(B_{0 y}+g x\right)
$$

or keeping only linear terms in $x$ and $\delta$

$$
x^{\prime \prime}+k x=-\frac{1}{\rho_{0}}+\frac{1}{\rho_{0}} \delta .
$$

Here we have introduced the quadrupole focusing strength $k=\frac{e c}{c p_{0}} g x$ and the bending radius is taken to be in the horizontal plane. The solution of this equation of motion will be very complicated due to the arbitrary layout of the beam transport line or $\rho_{0}(z)$. we are not interested in a mathematical formulation of this layout, but are interested only on the deviation of a particle from the desired transport line layout as defined by the location of magnets. We may transform away the beam line layout by merely dropping the $\frac{1}{\rho_{0}}$-term from (1.82) to get finally the linear equation of motion for particle dynamics

$$
x^{\prime \prime}+k(z) x=+\frac{1}{\rho_{0}(z)} \delta .
$$

Later we will introduce this coordinate system rigorously. This looks basically like the differential equation of a harmonic oscillator if it were not for the fact that the magnet strengths are functions of $z$.However, the solutions will be of oscillatory nature describing the particle motion in the restoring fields of the focusing devices. Actual analytical solutions will be discussed in great detail later in this text.

\subsubsection{Energy Conservation}

The rate of work done in a charged particle-field environment is defined by the Lorentz force and the particle velocity $\boldsymbol{F}_{\mathrm{L}} \boldsymbol{v}=e \boldsymbol{E} \boldsymbol{v}+e(\boldsymbol{v} \times \boldsymbol{B}) \boldsymbol{v}$. Noting that $(\boldsymbol{v} \times \boldsymbol{B}) \boldsymbol{v}=0$, we set $\boldsymbol{e} \boldsymbol{E}=\boldsymbol{j} \boldsymbol{E}$, and the total rate of work done by all particles and fields can be obtained by integrating Ampère's law(1.4) over all currents and fields

$$
\int \boldsymbol{j} \boldsymbol{E} \mathrm{d} V=\epsilon_{0} \epsilon \int\left(c^{2}(\nabla \times \boldsymbol{B})-\dot{\boldsymbol{E}}\right) \boldsymbol{E} \mathrm{d} V
$$


With the vector relation $\nabla(a \times b)=b(\nabla \times a)-a(\nabla \times b)$

$$
\begin{aligned}
\int \boldsymbol{j} \boldsymbol{E} \mathrm{d} V & =\epsilon_{0} \epsilon \int[c^{2} \boldsymbol{B} \underbrace{\nabla \times \boldsymbol{E}}_{=-\dot{\boldsymbol{B}}}-c^{2} \nabla(\boldsymbol{E} \times \boldsymbol{B})-\dot{\boldsymbol{E}} \boldsymbol{E}] \mathrm{d} V \\
& =-\int\left[\frac{\mathrm{d} u}{\mathrm{~d} t}+\epsilon_{0} \epsilon c^{2} \nabla(\boldsymbol{E} \times \boldsymbol{B})\right] \mathrm{d} V,
\end{aligned}
$$

where an energy density has been defined by

$$
u=\frac{1}{2} \epsilon_{0}\left(E^{2}+c^{2} B^{2}\right)
$$

Applying Gauss's theorem to the vector product in (1.85), we get an expression for the energy conservation of the complete particle-field system

$$
\underbrace{\frac{\mathrm{d}}{\mathrm{d} t} u \mathrm{~d} V}_{\begin{array}{c}
\text { change of } \\
\text { field energy }
\end{array}}+\underbrace{\int \boldsymbol{j E} \mathrm{d} V}_{\begin{array}{c}
\text { particle energy } \\
\text { loss or gain }
\end{array}}+\underbrace{\oint \boldsymbol{S n} \mathrm{d} a}_{\begin{array}{c}
\text { radiation loss through } \\
\text { closed surface } a
\end{array}}=0 .
$$

This equation expresses the conservation of energy relating the change in field energy and particle acceleration with a new quantity describing energy loss or gain through radiation.

\section{Poynting Vector}

The third integral in (1.87) is performed over a surface enclosing all charges and currents considered. The Poynting vector $S$ is the energy loss/gain through a unit surface element in the direction of the unit vector $\boldsymbol{n}$ normal to the surface defined by

$$
\boldsymbol{S}=\frac{1}{\mu_{0} \mu}[\boldsymbol{E} \times \boldsymbol{B}] .
$$

Equation (1.88) exhibits characteristic features of electromagnetic radiation. Both, electric and magnetic radiation fields are orthogonal to each other $(\boldsymbol{E} \perp \boldsymbol{B})$, orthogonal to the direction of propagation $(\boldsymbol{E} \perp \boldsymbol{n}, \boldsymbol{B} \perp \boldsymbol{n})$, and the vectors $\boldsymbol{E}, \boldsymbol{B}, \boldsymbol{S}$ form a right handed orthogonal system. For plane waves $\boldsymbol{n} \times \boldsymbol{E}=c \boldsymbol{B}$ and

$$
\boldsymbol{S}=\frac{1}{c \mu_{0} \mu} \boldsymbol{E}^{2} \boldsymbol{n} .
$$

Knowing the electric fields we may determine the Poynting vector describing electro-magnetic waves or synchrotron radiation. 


\subsubsection{Stability of a Charged-Particle Beam}

Individual particles in an intense beam are under the influence of strong repelling electrostatic forces creating the possibility of severe stability problems. Particle beam transport over long distances could be greatly restricted unless these spacecharge forces can be kept under control. First, it is interesting to calculate the magnitude of the problem.

If all particles would be at rest within a small volume, we would clearly expect the particles to quickly diverge from the center of charge under the influence of the repelling space charge forces from the other particles. This situation may be significantly different in a particle beam where all particles propagate in the same direction. We will therefore calculate the fields generated by charged particles in a beam and derive the corresponding Lorentz force due to these fields. Since the Lorentz force equation is invariant with respect to coordinate transformations, we may derive this force either in the laboratory system or in the moving system of the particle bunch.

From (1.49) and (1.50) we determine the Lorentz force due to electro-magnetic fields generated by the beam itself and acting on a particle within that beam. From (1.49) and (1.50) we get

$$
F_{r}=e\left(E_{r}-v B_{\varphi}\right)=\frac{e}{2 \epsilon_{0} \epsilon} \frac{\rho_{0}}{\gamma^{2}} r
$$

Only the radial component of the Lorentz force is finite. The Lorentz force remains repelling but due to a relativistic effect we find that the repelling electrostatic force at higher energies is increasingly compensated by the magnetic field. The total Lorentz force due to space charges therefore vanishes like $\gamma^{-2}$ for higher energies. Obviously this repelling space charge force is much stronger for proton and especially for ion beams because of the smaller value for $\gamma$ and, in the case of ions, because of the larger charge multiplicity which increases the space-charge force by a factor of $Z$.

We find the same result if we derive the Lorentz force in the moving system $\mathcal{L}^{*}$ of the particle beam and then transform to the laboratory system. In this moving system we have obviously only the repelling electrostatic force since the particles are at rest and the only field component is the radial electrical field which is from (1.49)

$$
F_{r}^{*}=e E_{r}^{*}=\frac{e}{2 \epsilon_{0} \epsilon} \rho_{0}^{*} r^{*}
$$

Transforming this equation back into the laboratory system we note that the force is purely radial and therefore acts only on the radial momentum. With $F_{r}=\mathrm{d} p_{r} / \mathrm{d} t$ and $p_{r}=p_{r}^{*}$ we find $F^{*}=\gamma F_{r}$ since $\mathrm{d} t=\gamma \mathrm{d} t^{*}$. The charge densities in both 
systems are related by $\rho^{*}=\rho / \gamma$, the radii by $r^{*}=r$, and the Lorentz force in the laboratory system becomes thereby

$$
F_{r}=\frac{e}{2 \epsilon_{0} \epsilon} \frac{\rho_{0}}{\gamma^{2}} r
$$

in agreement with (1.90).

We obtained the encouraging result that at least relativistic particle beams become stable under the influence of their own fields. For lower particle energies, however, significant diverging forces must be expected and adequate focusing measures must be applied. The physics of such space charge dominated beams is beyond the scope of this book and is treated elsewhere, for example in considerable detail in [33].

\section{Problems}

1.1 (S). Use the definition for $\beta$, the momentum, the total and kinetic energy and derive expressions $p\left(\beta, E_{\mathrm{kin}}\right), p\left(E_{\mathrm{kin}}\right)$ and $E_{\mathrm{kin}}(\gamma)$. Simplify the expressions for very large energies, $\gamma \gg 1$. Derive from these relativistic expressions the classical nonrelativistic formulas.

1.2 (S). Prove the validity of the field equations $E_{r}=\frac{1}{2 \epsilon_{0}} \rho_{0} r$ and $B_{\varphi}=\frac{1}{2} \mu_{0} \beta \rho_{0} r$ for a uniform cylindrical particle beam with constant charge density $\rho_{0}$ within a radius $r<R$. Derive the field expressions for $r>R$.

1.3 (S). Derive the electric and magnetic fields of a beam with a radial charge distribution $\rho(r, \varphi, z)=\rho(r)$. Derive the field equations for a Gaussian charge distribution with standard deviation $\sigma$ given by $\rho(r)=\rho_{0} \exp \left[-r^{2} /\left(2 \sigma^{2}\right)\right]$. What are the fields for $r=0$ and $r=\sigma$ ?

1.4 (S). A circular accelerator with a circumference of $300 \mathrm{~m}$ contains a uniform distribution of singly charged particles orbiting with the speed of light. If the circulating current is $1 \mathrm{amp}$, how many particles are orbiting? We instantly turn on an ejection magnet so that all particles leave the accelerator during the time of one revolution. What is the peak current at the ejection point? How long is the current pulse duration? If the accelerator is a synchrotron accelerating particles at a rate of 10 acceleration cycles per second, what is the average ejected particle current?

1.5 (S). A proton with a kinetic energy of $1 \mathrm{eV}$ is emitted parallel to the surface of the earth. What is the bending radius due to gravitational forces? What are the required transverse electrical and magnetic fields to obtain the same bending radius? What is the ratio of electrical to magnetic field? Is this ratio different for a proton energy of say $10 \mathrm{TeV}$ ? Why? (gravitational constant $6.67259 \times 10^{-11} \mathrm{~m}^{3} \mathrm{~kg}^{-1} \mathrm{~s}^{-2}$ ). 
1.6 (S). Consider a highly relativistic electron bunch of $n=10^{10}$ uniformly distributed electrons. The bunch has the form of a cylindrical slug, $\ell=1 \mathrm{~mm}$ long and a radius of $R=0.1 \mu \mathrm{m}$. What is the electrical and magnetic field strength at the surface of the beam. Calculate the peak electrical current of the bunch. If two such beams in a linear collider with an energy of $500 \mathrm{GeV}$ pass by each other at a distance of $10 \mu \mathrm{m}$ (center to center), what is the deflection angle of each beam due to the field of the other beam?

1.7 (S). Show that for plane waves $\boldsymbol{n} \times \boldsymbol{E}=c \boldsymbol{B}$.

1.8 (S). Show that the product of two 4 -vectors is Lorentz invariant.

1.9 (S). Prove that the 4-acceleration is indeed given by (1.23).

1.10 (S). Using 4-vectors, derive the frequency of an outgoing photon from a headon Compton scattering process of an electron with a photon of frequency $\omega_{\mathrm{L}}$.

1.11 (S). Using 4-vectors, derive the frequency of an outgoing photon from a headon Compton scattering process of an electron with the field of an undulator with period $\lambda_{\mathrm{u}}$.

1.12. Protons are accelerated to a kinetic energy of $200 \mathrm{MeV}$ at the end of the Fermilab Alvarez linear accelerator. Calculate their total energy, their momentum and their velocity in units of the velocity of light $\left(m_{\mathrm{p}} c^{2}=938.27 \mathrm{MeV}\right)$.

1.13. Consider electrons to be accelerated in the $L=3 \mathrm{~km}$ long SLAC linear accelerator with a uniform gradient of $20 \mathrm{MeV} / \mathrm{m}$. The electrons have a velocity $v=\frac{1}{2} c$ at the beginning of the linac. What is the length of the linac in the rest frame of the electron? Assume the particles at the end of the $3 \mathrm{~km}$ long linac would enter another $3 \mathrm{~km}$ long tube and coast through it. How long would this tube appear to be to the electron?

1.14 (S). A positron beam of energy $E$ accelerated in a linac hits a fixed hydrogen target. What is the available energy from a collision with a target electron assumed to be at rest? Compare this available energy with that obtained in a linear collider where electrons and positrons from two similar linacs collide head on at the same energy.

1.15 (S). The SPEAR colliding beam storage ring has been constructed originally for electron and positron beams to collide head on with an energy of up to $3.5 \mathrm{GeV}$. At $1.55 \mathrm{GeV}$ per beam a new particle, the $\psi / J$-particle, was created. In a concurrent fixed target experiment at BNL, such $\psi / J$-particle have been produced by protons hitting a hydrogen target. What proton energy was required to produce the new particle? Determine the positron energy needed to create $\psi / J$-particles by collisions with electrons in a fixed target.

1.16. A charged pion meson has a rest energy of $139.568 \mathrm{MeV}$ and a mean life time of $\tau_{0 \pi}=26.029 \mathrm{~ns}$ in its rest frame. What are the life times $\tau_{\pi}$, if accelerated to a kinetic energy of $20 \mathrm{MeV}$ ? and $100 \mathrm{MeV}$ ? A pion beam decays exponentially like 
$\mathrm{e}^{-t / \tau_{\pi}}$. At what distance from the source will the pion beam intensity have fallen to $50 \%$, if the kinetic energy is $20 \mathrm{MeV}$ ? or $100 \mathrm{MeV}$ ?

1.17 (S). Assume you want to produce antiprotons by accelerating protons and letting them collide with other protons in a stationary hydrogen target. What is the minimum kinetic energy the accelerated protons must have to produce antiprotons? Use the reaction $p+p \rightarrow p+p+p+\bar{p}$.

1.18. Use the results of Problem 1.3 and consider a parallel beam at the beginning of a long magnet free drift space. Follow a particle under the influence of the beam self fields starting at a distance $r_{0}=\sigma$ from the axis. Derive the radial particle distance from the axis as a function of $z$.

1.19. Show that (1.57) is indeed a solution of (1.55).

1.20. Express the equation of motion (1.67) for $Z=1$ in terms of particle acceleration, velocity and fields only. Verify from this result the validity of (1.71) and (1.72).

1.21. Plot on log-log scale the velocity $\beta$, total energy as a function of the kinetic energy for electrons, protons, and gold ions $\mathrm{Au}^{+14}$. Vary the total energy from $0.01 m c^{2}$ to $10^{4} m c^{2}$. Why does the total energy barely change at low kinetic energies.

1.22. The design for a Relativistic Heavy Ion Collider calls for the acceleration of completely ionized gold atoms in a circular accelerator with a bending radius of $\rho=242.78 \mathrm{~m}$ and superconducting magnets reaching a maximum field of $34.5 \mathrm{~kg}$. What is the maximum achievable kinetic energy per nucleon for gold ions $\mathrm{Au}^{+77}$ compared to protons? Calculate the total energy, momentum, and velocity of the gold atoms $\left(\mathrm{A}_{\mathrm{Au}}=197\right)$.

1.23. Gold ions $\mathrm{Au}^{+14}$ are injected into the Brookhaven Alternating Gradient Synchrotron AGS at a kinetic energy per nucleon of $72 \mathrm{MeV} / \mathrm{u}$. What is the velocity of the gold ions? The AGS was designed to accelerate protons to a kinetic energy of $28.1 \mathrm{GeV}$. What is the corresponding maximum kinetic energy per nucleon for these gold ions that can be achieved in the AGS? The circulating beam is expected to contain $6 \cdot 10^{9}$ gold ions. Calculate the beam current at injection and at maximum energy assuming there are no losses during acceleration. The circumference of the AGS is $C_{\mathrm{AGS}}=807.1 \mathrm{~m}$. Why does the beam current increase although the circulating charge stays constant during acceleration?

1.24. Particles undergo elastic collisions with gas atoms. The rms multiple scattering angle is given by $\sigma_{\theta} \approx Z \frac{20(\mathrm{MeV} / \mathrm{c})}{\beta p} \sqrt{\frac{s}{\ell_{\mathrm{r}}}}$, where $Z$ is the charge multiplicity of the beam particles, $s$ the distance travelled and $\ell_{\mathrm{r}}$ the radiation length of the scattering material (for air the radiation length at atmospheric pressure is $\ell_{\mathrm{r}}=500 \mathrm{~m}$ or $60.2 \mathrm{~g} / \mathrm{cm}^{2}$ ). Derive an approximate expression for the beam radius as a function of $s$ due to scattering. What is the approximate tolerable gas pressure in a proton storage ring if a particle beam is supposed to orbit for $20 \mathrm{~h}$ and the elastic gas scattering shall not increase the beam size by more than a factor of two during that time? 


\section{References}

1. M. Faraday, Poggendorf Ann. 48, 430 (1839)

2. J. Pluecker, Ann. Phys. Chem. 13, 88 (1858)

3. J.W. Hittorf, Ann. Phys. Chem. 5 Reihe 16, 1 (1869)

4. E. Goldstein, Monatsberichte der Königlich Preussischen Akademie der Wissenschaften, p. 284 (1876)

5. E. Wiedemann, Z. Elektrochem. 8, 155 (1895)

6. J.J. Thomson, Conduction of Electricity Through Gases, vol. 161 (Cambridge University Press, Cambridge, 1906), p. 602

7. G.A. Schott, Ann. Phys. 24, 635 (1907)

8. G.A. Schott, Phil. Mag. [6] 13, 194 (1907)

9. H. Greinacher, Z. Phys. 4, 195 (1921)

10. G. Ising, Ark. Mat. Astron. Fys. 18, 1 (1924)

11. R. Wideroe, Arch. Elektrotech. 21, 387 (1928)

12. R.J. Van de Graaff, pr 38, 1919 (1931)

13. E.O. Lawrence, M.S. Livingston, pr 40, 19 (1932)

14. J.D. Cockcroft, E.T.S. Walton, Proc. Roy. Soc A 136, 619 (1932)

15. D.W. Kerst, R. Serber, Phys. Rev. 60, 47 (1941)

16. D. Ivanenko, A.A. Sokolov, DAN(USSR) 59, 1551 (1972)

17. J.S. Schwinger, On the classical radiation of accelerated electrons. Phys. Rev. 75, 1912 (1949)

18. V.I. Veksler, DAN(USSR) 44, 393 (1944)

19. E.M. McMillan, Phys. Rev. 68, 143 (1945)

20. J.P. Blewett, Phys. Rev. 69, 87 (1946)

21. L.W. Alvarez, Phys. Rev. 70, 799 (1946)

22. E.L. Ginzton, W.W. Hansen, W.R. Kennedy, Rev. Sci. Inst. 19, 89 (1948)

23. N. Christofilos, US Patent No 2,736,766 (1950)

24. M.S. Livingston, J.P. Blewett, G.K. Green, L.J. Haworth, Rev. Sci. Instrum. 21,7 (1950)

25. H. Motz, J. Appl. Phys. 22, 527 (1951)

26. E.D. Courant, M.S. Livingston, H.S. Snyder, Phys. Rev. 88, 1190 (1952)

27. M. Chodorow, E.L. Ginzton, W.W. Hansen, R.L. Kyhl, R. Neal, W.H.K. Panofsky, Rev. Sci. Instrum. 26, 134 (1955)

28. M. Sands, Phys. Rev. 97, 470 (1955)

29. E.D. Courant, H.S. Snyder, Appl. Phys. 3, 1 (1959)

30. W. Scharf, Particle Accelerators and Their Uses (Harwood Academic, New York, 1985)

31. A. Liènard, L'Eclaire Electrique 16, 5 (1898)

32. E. Wiechert, Arch. Neerl. 5, 546 (1900)

33. M. Reiser, Theory and Design of Charged Particle Beams (Wiley, New York, 1994) 


\section{Chapter 2 \\ Linear Accelerators}

Before we address the physics of beam dynamics in accelerators it seems appropriate to discuss briefly various methods of particle acceleration as they have been developed over the years. It would, however, exceed the purpose of this text to discuss all variations in detail. Fortunately, extensive literature is available on a large variety of different accelerators and therefore only fundamental principles of particle acceleration shall be discussed here. A valuable source of information for more detailed information on the historical development of particle accelerators is Livingston's collection of early publications on accelerator developments [8].

The development of charged-particle accelerators has progressed along double paths which by the appearance of particle trajectories are distinguished as linear accelerators and circular accelerators. Particles in linear accelerators travel on a straight line and pass only once through the accelerator structure while in a circular accelerator they follow a closed orbit periodically for many revolutions accumulating energy at every passage of the accelerating structure.

\subsection{Principles of Linear Accelerators}

No fundamental advantage or disadvantage can be claimed for one or the other class of accelerators. It is mostly the particular application and sometimes the available technology that determines the choice between both classes. Both types have been invented and developed throughout the twentieth century, and continue to be improved and optimized as associated technologies advance. In this chapter we will concentrate on linear accelerators and postpone the discussion on circular accelerators to the next chapter. In linear accelerators the particles are accelerated by definition along a straight path by either electrostatic fields or microwave fields.

This chapter has been made Open Access under a CC BY 4.0 license. For details on rights and licenses please read the Correction https://doi.org/10.1007/978-3-319-18317-6_28

(C) The Author(s) 2015

H. Wiedemann, Particle Accelerator Physics, Graduate Text in Physics, https://doi.org/10.1007/978-3-319-18317-6_2 


\subsubsection{Charged Particles in Electric Fields}

In accelerator physics all forces on charged particles originate from electromagnetic fields. For particle acceleration we consider only the electric-field term of the Lorentz force. The nature of the electric field can be static, pulsed, generated by a time varying magnetic field or a microwave field. Both the electric and magnetic fields are connected by Maxwell's equations. Such fields are generated by appropriate sources hooked up to an accelerating section which, in the case of electro-static fields, consists of just two electrodes with the particle source at the potential of one electrode and a hole in the center of the other electrode to let the accelerated particles pass through. Special resonant cavities are used as accelerating sections with two holes on the axis of the cavity to let the beam pass through. Either field can be represented by the plane wave equation

$$
\mathbf{E}(\psi)=\mathbf{E}_{0} \mathrm{e}^{\mathrm{i}(\omega t-k s)}=\mathbf{E}_{0} \mathrm{e}^{\mathrm{i} \psi},
$$

where $\omega$ is the frequency and $k$ the wave number including the case of static fields with $\omega=0$ and $k=0$. The Lorentz force acting on an electric charge is

$$
\mathbf{F}_{\mathrm{L}}=\frac{\mathrm{d}}{\mathrm{d} t} m c \gamma \boldsymbol{\beta}=e \mathbf{E}(\psi)
$$

and the equation of motion for particles under this force is

$$
\frac{\mathrm{d}}{\mathrm{d} t} m c \gamma \boldsymbol{\beta}=e \mathbf{E}(\psi) .
$$

Integration of (2.3) results in an expression for the momentum gain of the particle

$$
\Delta p=m c\left(\gamma \beta-\gamma_{0} \beta_{0}\right)=e \int \mathbf{E}(\psi) \mathrm{d} t,
$$

where $m c \gamma_{0} \beta_{0}$ is the initial momentum of the particle. Generally, it is somewhat complicated to perform a time integration which requires the tracking of particles though the accelerating cavity. To simplify the calculation, we look for the gain in kinetic energy which reduces (2.4) to a spatial integration of the electric field in the accelerating cavity. This integral is a property of the cavity and is independent of particle motion. With $\beta \Delta c p=\Delta E_{\text {kin }}$ the energy gain for particles passing through the accelerating section is

$$
\Delta E_{\mathrm{kin}}=e \int_{L_{\mathrm{cy}}} \mathbf{E}(\psi) \mathrm{d} \mathbf{s}
$$

where $L_{\mathrm{cy}}$ is the length of the accelerating section. 
The effectiveness of acceleration in a microwave field depends greatly on the phase relationship of the field with the particle motion. For successful particle acceleration we expect therefore the need to meet specific synchronicity conditions to ensure acceleration.

\subsubsection{Electrostatic Accelerators}

In electrostatic accelerators the potential difference between two electrodes is used for particle acceleration as shown in Fig. 2.1. The most simple such arrangement has been used now for almost two centuries in glow discharge tubes for fundamental research on the nature of plasmas, as light sources or as objects of aesthetic interest due to colorful phenomena in such tubes. In another, more modern application electrons are accelerated in an x-ray tube by high electrostatic fields and produce after striking a metal target intense $\mathrm{x}$-rays used in medicine and industry.

The voltages that can be achieved by straight voltage transformation and rectification are quite limited by electrical breakdown effects to a few 10,000 V/cm. More sophisticated methods of producing high voltages therefore have been developed to reach potential differences of up to several million volts.

To distribute evenly the electric fields of high potential differences a series of irises are distributed along the acceleration column and separated by appropriate resistors to break down the high voltage into smaller steps between the irises. As an added benefit we also gain focusing of the particle beam as will be discussed later in this chapter.

A variety of techniques to obtain high voltages have been developed and applied to particle acceleration with more or less success. We will discus briefly a few of theses techniques because they are still used.

Fig. 2.1 Principle of electrostatic accelerators

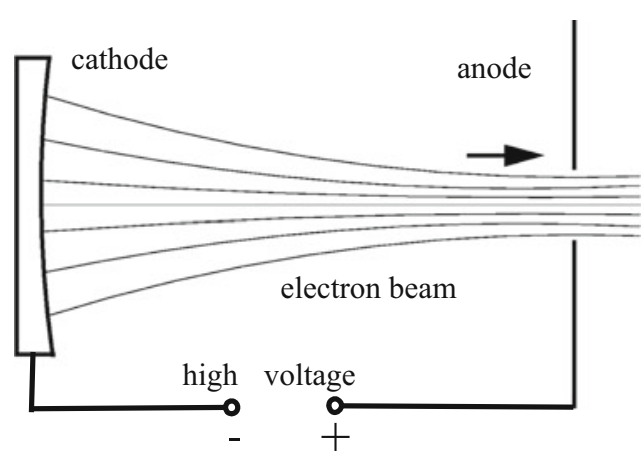




\section{Cascade Generators}

The basic method implemented in the cascade generator is that of a voltage multiplier circuit which has been proposed by Greinacher [5] in 1914 and Schenkel [10] in 1919 which allows to achieve a multiplication of the voltage across the plates of a capacitor. A set of capacitors are charged through appropriately placed diodes from an alternating current source (Fig. 2.2) in such a way that during the positive half wave, half the capacitors are charged to a positive voltage and during the negative half wave, the other half of the capacitors are charged to a negative voltage thus providing twice the maximum ac voltage. By arranging $2 N$ capacitors in this way the charging voltage can be multiplied by the factor $N$.

While there is no fundamental limit to the total voltage, high voltage break down will impose a technical limit on the maximum achievable voltage. Based on this method Cockcroft and Walton [3] developed appropriate high-voltage techniques and built the first high energy particle accelerator reaching voltages as high as several million Volt. Applying the high voltage to a beam of protons they were able for the first time to initiate through artificially accelerated protons a nuclear reaction. In this case it was the conversion of a Lithium nucleus into two helium nuclei, in the reaction

$$
\mathrm{p}+\mathrm{Li} \rightarrow 2 \mathrm{He}
$$

Such Cockcroft-Walton accelerators turned out to be very efficient and are still used as the first step in modern proton accelerator systems. Obviously with this kind of voltage generation it is not possible anymore to produce a continuous stream of particles. Because of the switching process, there is a time to charge the capacitors followed by a time to apply the multiplied voltage to particle acceleration. As a consequence, we observe a pulsed particle beam from a Cockroft-Walton accelerator.

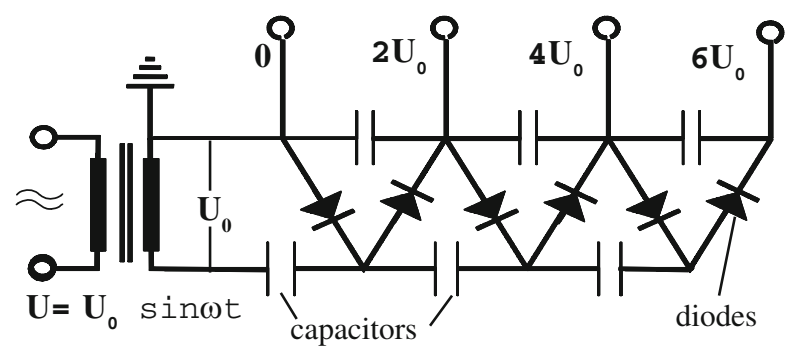

Fig. 2.2 Cascade generator (schematic) 


\section{Van de Graaff Accelerator}

Much higher voltages can be reached with a Van de Graaff accelerator [12]. Here, electric charge is extracted by field emission from a pointed metal electrode and sprayed onto an isolated endless belt. This belt is moved by motor action to carry the charge to the inside of a hollow sphere, where the charge is stripped off again by reverse field emission onto a pointed metal electrode which is connected to the inside of the sphere. The principle of this electrostatic generator is shown in Fig. 2.3. Electrical charges in a metallic conductor collect on the outside and it is therefore possible to continuously accumulate electrical charge by deposition to the inside surface of a hollow metallic sphere. If the whole system is placed into a high pressure vessel filled with an electrically inert gas like Freon or $\mathrm{SF}_{6}$, voltages as high as 20 million volts can be reached.

The high voltage can be used to accelerate electrons as well as protons or ions. In the latter two cases more than double the accelerating voltage can be achieved in a Tandem Van de Graaff accelerator. If a proton beam must be accelerated, the accelerating process would start with negatively charged hydrogen ions $\mathrm{H}^{-}$from a plasma discharge tube which are then accelerated say from ground potential to the full Van de Graaff voltage $+V$. At that point the two electrons of the negative hydrogen ion are stripped away by a thin foil or gas curtain resulting by charge exchange in positively charged protons which can be further accelerated between the potential $+V$ and ground potential to a total kinetic energy $E_{\text {kin }}=e 2 V$.

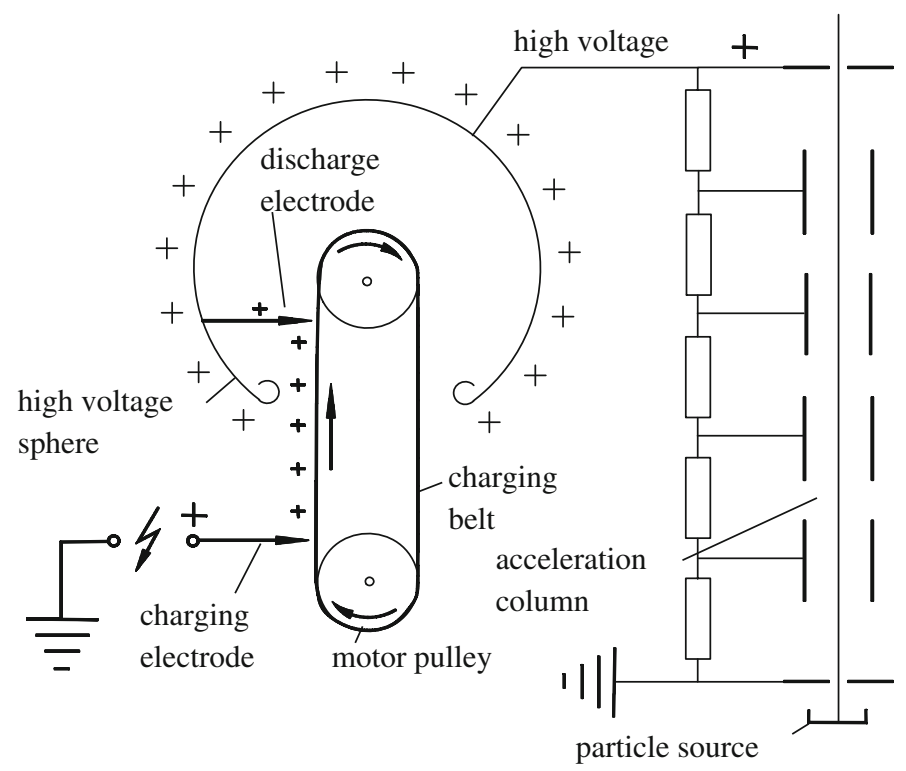

Fig. 2.3 Van de Graaff accelerator (schematic) 
High electrostatic voltages from a Van de Graaff generator cannot be applied directly to just two electrodes as shown in Fig. 2.1. Because of the great distance between the electrodes necessary to avoid voltage break down the fields would not be distributed uniformly along the axis of the acceleration column. Therefore, the voltage is applied to a series of resistors connected to iris electrodes which allow a uniform distribution of the electrical field along the acceleration column as shown in Fig. 2.3. A more detailed review of the development of electro-static high voltage generators can be found in [13].

\subsection{Electric Field Components}

In our discussion we concentrate first on the interaction of particles with electrostatic fields. Such field components function both as focusing and acceleration devices. Electro-static fields are commonly employed for low energy, nonrelativistic particles. As was discussed earlier, magnetic devices are most effective at high energies when particle velocities are close to the speed of light. At lower velocities, magnetic fields loose their efficiency and are often replaced by more economic electric field devices and at very low energies electric fields are used almost exclusively.

\subsubsection{Electrostatic Deflectors}

The electric field $\boldsymbol{E}$ between two parallel metallic electrodes is uniform and can be used to deflect a particle beam. To get a uniform field, we generate equipotential surfaces by placing metallic electrodes at, for example, $x= \pm G=$ const. and applying a voltage difference $V$ between the electrodes. The Lorentz force of the electric field on a charged particle is by virtue of d'Alembert's principle equal to the centrifugal force and is for a horizontal deflection

$$
e Z \boldsymbol{E}_{x}=\frac{\gamma A m c^{2} \beta^{2}}{\rho} .
$$

Here we have assumed that the electric field is parallel to the vector from the particle to the center of curvature. That is true for parallel plates which are curved to follow the curvature or almost true for straight parallel plates if the deflection angle is very small. Solving for the curvature, we get

$$
\frac{1}{\rho}=\frac{e Z E_{x}}{\gamma A m c^{2} \beta^{2}}=\frac{e Z V}{2 G} \frac{1}{E_{\text {kin }}} \frac{\gamma}{\gamma+1},
$$


where $2 G$ is the distance and $V$ the voltage between the electrodes. We kept here the relativistic notation to cover the rare use of electrostatic fields on high energy beams for small deflections which cannot be done by magnetic fields. For nonrelativistic particles, (2.8) reduces to $\frac{1}{\rho}=\frac{e Z E_{x}}{A m v^{2}}$ or in case of an ion beam with charge multiplicity $Z$ and kinetic energy per nucleon $E_{\mathrm{kin}}=\frac{1}{2} m v^{2}$

$$
\frac{1}{\rho}\left(\mathrm{m}^{-1}\right)=\frac{e \mathrm{Z} \boldsymbol{E}_{x}(\mathrm{~V} / \mathrm{m})}{2 A E_{\mathrm{kin}}(\mathrm{eV})}
$$

where $\boldsymbol{E}_{x}=V /(2 G)$ is the electric field between the electrodes.

\subsubsection{Electrostatic Focusing Devices}

The most simple electro-static device with focusing properties is an iris electrode on some potential and coaxial with the path of a charged particle beam as shown in Fig. 2.4.

To determine the field configuration and focusing properties, we note that the electric potential distribution $V(r, z)$ in the vicinity of the iris is rotationally symmetric and expanding into a Taylor series about $r=0$ this symmetry requires all odd terms of the expansion to vanish.

$$
V(r, z)=V_{0}(z)+\frac{1}{2} \ddot{V}_{0}(z) r^{2}+\frac{1}{24} \frac{\partial^{4} V_{0}(z)}{\partial r^{4}} r^{4}+\cdots
$$

Derivatives with respect to $r$ are indicated with a dot and derivatives with respect to $z$ with a prime. To be a real potential solution (2.10) must also be a solution of the Laplace equation

$$
\Delta V=\frac{\partial^{2} V}{\partial r^{2}}+\frac{1}{r} \frac{\partial V}{\partial r}+\frac{\partial^{2} V}{\partial z^{2}}=0 .
$$

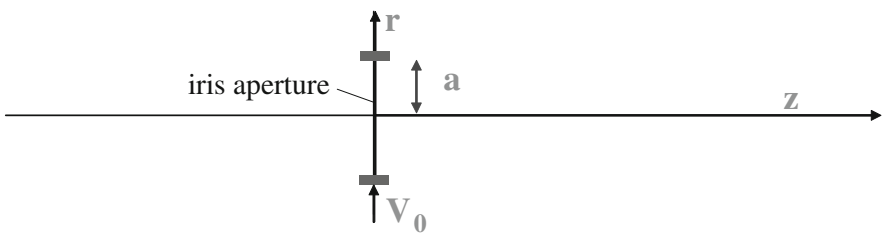

Fig. 2.4 Iris electrode 
Inserting (2.10) into (2.11) results in

$$
0=\ddot{V}_{0}+\frac{1}{2} \frac{\partial^{4} V_{0}}{\partial r^{4}} r^{2}+\frac{1}{2} \dddot{V}_{0} r+\ddot{V}_{0}+\frac{1}{6} \frac{\partial^{4} V_{0}}{\partial r^{4}} r^{2}+V_{0}^{\prime \prime}+\frac{1}{2} \ddot{V}_{0}^{\prime \prime} r^{2}+\cdots,
$$

where the coefficients of each term $r^{n}$ must be equal to zero separately to give $2 \ddot{V}_{0}+$ $V_{0}^{\prime \prime}=0, \dddot{V}_{0}=0$ and $\frac{2}{3} \frac{\partial^{4} V_{0}}{\partial r^{4}}+\frac{1}{2} \ddot{V}_{0}^{\prime \prime}=0$. Using these relations, we set $\frac{\partial^{4} V_{0}}{\partial r^{4}}=\frac{3}{8} \frac{\partial^{4} V_{0}}{\partial z^{4}}$ and the potential function is

$$
V(r, z)=V_{0}(z)-\frac{1}{4} V_{0}^{\prime \prime}(z) r^{2}+\frac{1}{64} \frac{\partial^{4} V_{0}(z)}{\partial z^{4}} r^{4}+\cdots
$$

The on-axis $(r=0)$ field component is

$$
E_{z}=-V_{0}^{\prime}(z)
$$

and from $\nabla \boldsymbol{E}=0$ or $\frac{\partial E_{z}}{\partial z}=-\frac{1}{r} \frac{\partial}{\partial r}\left(r E_{r}\right)$, we get by integration

$$
E_{r}=-\frac{r}{2} \frac{\partial E_{z}}{\partial z}=\frac{1}{2} V_{0}^{\prime \prime}(z) r
$$

Knowing the field components, we can derive the focusing properties by integrating the radial equation of motion $m \ddot{r}=m v^{2} r^{\prime \prime}=q E_{r}$, where $v$ and $q$ are the particle velocity and charge, respectively. We use Fig. 2.5 to define the integration

$$
r_{2}^{\prime}-r_{1}^{\prime}=\frac{q}{m v^{2}} \int_{z_{1}}^{z_{2}} E_{r} \mathrm{~d} z=-\frac{q}{2 m v^{2}} \int_{z_{1}}^{z_{2}} r \frac{\partial E_{r}}{\partial z} \mathrm{~d} z
$$

and solve in thin lens approximation $(r=\operatorname{cost}, v=\operatorname{cost})$

$$
r_{2}^{\prime}-r_{1}^{\prime}=-\frac{q r_{1}}{2 m v^{2}}\left(E_{2}-E_{1}\right)
$$

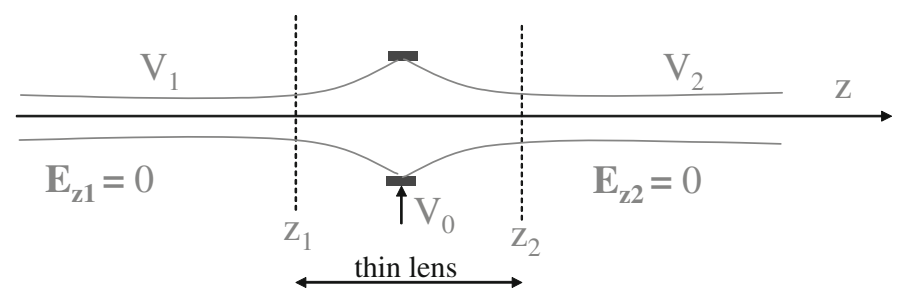

Fig. 2.5 Focusing by an iris electrode 
With $\frac{1}{2} m v^{2}=q V_{0}$ and setting $E=-V^{\prime}$, (2.16) becomes $r_{2}^{\prime}-r_{1}^{\prime}=\frac{r_{1}}{4} \frac{V_{2}^{\prime}-V_{1}^{\prime}}{V_{0}}$ and the focal length of the iris electrode is

$$
\frac{1}{f}=\frac{V_{2}^{\prime}-V_{1}^{\prime}}{4 V_{0}}
$$

and the transformation matrix is finally

$$
\mathcal{M}_{\text {iris }}=\left(\begin{array}{cc}
1 & 0 \\
\frac{V_{2}^{\prime}-V_{1}^{\prime}}{V_{0}} & 1
\end{array}\right) \text {. }
$$

From the transformation matrix or focal length it is obvious that there is no focusing for a symmetric iris electrode where $V_{2}^{\prime}=V_{1}^{\prime}$. On the other hand, an asymmetric potential is not possible without additional electrodes. We investigate therefore the properties of an iris doublet.

\subsubsection{Iris Doublet}

We now investigate the particle dynamics for an iris doublet as shown in Fig. 2.6. Between both electrodes a distance $d$ apart, the potential varies linearly from $V_{1}$ to $V_{2}$. The doublet has three active parts, two iris electrodes and the drift space between them. The transformation matrices for both iris electrodes are

$$
\mathcal{M}_{1}=\left(\begin{array}{cc}
1 & 0 \\
\frac{V_{2}-V_{1}}{4 d V_{1}} & 1
\end{array}\right) \quad \text { and } \quad \mathcal{M}_{2}=\left(\begin{array}{cc}
1 & 0 \\
\frac{V_{2}-V_{1}}{4 d V_{2}} & 1
\end{array}\right) .
$$

The transformation matrix for the drift space between the electrodes can be derived from the particle trajectory

$$
r(z)=r_{1}+\int_{0}^{z} r^{\prime}(\bar{z}) \mathrm{d} \bar{z}=r_{1}+\int_{0}^{z} \frac{r^{\prime} p_{1}}{p_{1}+\Delta p(\bar{z})} \mathrm{d} \bar{z} .
$$

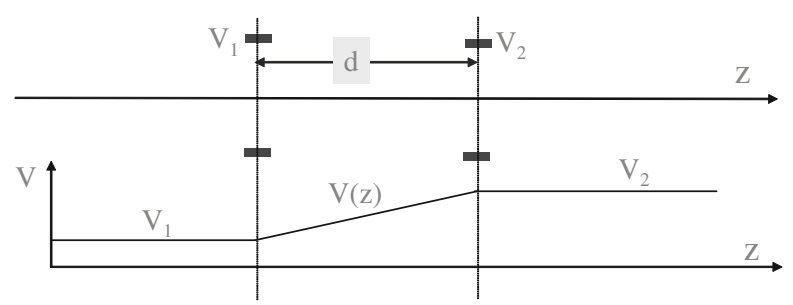

Fig. 2.6 Focusing by an iris doublet 
The particle momentum varies between the electrodes from $p_{1}=\sqrt{2 m E_{\mathrm{kin}}}$ to $p_{1}+$ $\Delta p(\bar{z})=\sqrt{2 m\left(E_{\text {kin }}+q \frac{V_{2}-V_{1}}{d} z\right)}$ and the integral in (2.20) becomes

$$
\int_{0}^{d} \frac{\mathrm{d} \bar{z}}{\sqrt{1+\frac{V_{2}-V_{1}}{E_{\text {kin }}} \bar{z}}}=\left.\frac{2 E_{\mathrm{kin}} d}{q\left(V_{2}-V_{1}\right)} \sqrt{1+q \frac{V_{2}-V_{1}}{E_{\mathrm{kin}} d}} \bar{z}\right|_{0} ^{d}=\frac{2 d \sqrt{V_{1}}}{\sqrt{V_{2}}+\sqrt{V_{1}}} .
$$

The particle trajectory at the location of the second electrode is $r(d)=r_{2}=r_{1}+$ $\frac{2 d \sqrt{V_{1}}}{\sqrt{V_{2}}+\sqrt{V_{1}}} r_{1}^{\prime}$ and its derivative $r_{2}^{\prime}=r_{1}^{\prime} \sqrt{V_{1}} / \sqrt{V_{2}}$ from which we can deduce the transformation matrix

$$
\mathcal{M}_{\mathrm{d}}=\left(\begin{array}{cc}
1 & \frac{2 d \sqrt{V_{1}}}{\sqrt{V_{2}}+\sqrt{V_{1}}} \\
0 & \frac{\sqrt{V_{1}}}{\sqrt{V_{2}}}
\end{array}\right)
$$

We may now collect all parts and get the transformation matrix for the iris doublet

$$
\mathcal{M}_{\mathrm{db}}=\mathcal{M}_{2} \mathcal{M}_{\mathrm{d}} \mathcal{M}_{1}=\left(\begin{array}{cc}
\frac{1}{2}(R+1) & \frac{2 d}{1+R} \\
\frac{\left(R^{2}-1\right)(3 R+1)}{8 d R^{2}} & \frac{3 R-1}{2 R^{2}}
\end{array}\right),
$$

where $R=\sqrt{V_{2}} / \sqrt{V_{1}}$. Unfortunately, this doublet is still not very convenient since it still changes the energy of the particle as indicated by the fact that the determinant $\operatorname{det}\left(\mathcal{M}_{\mathrm{db}}\right)=1 / R$. As indicated earlier this focusing device is also an accelerating structure. Any two adjacent irises along a high voltage accelerating structure act like a focusing device while accelerating particles.

\subsubsection{Einzellens}

To obtain a focusing device that does not change the particle energy, we combine two doublets to form a symmetric triplet as shown in Fig. 2.7. The transformation

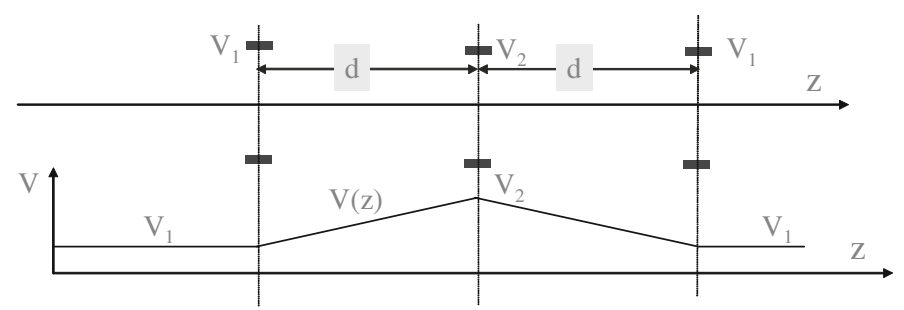

Fig. 2.7 Structure of an Einzellens 
matrix for such an Einzellens is then the product of two symmetric doublets

$$
\mathcal{M}_{\mathrm{el}}=\mathcal{M}_{2 \mathrm{~d}}\left(V_{2}, V_{1}\right) \mathcal{M}_{1 \mathrm{~d}}\left(V_{1}, V_{2}\right)=\left(\begin{array}{ll}
m_{11} & m_{12} \\
m_{21} & m_{22}
\end{array}\right),
$$

where

$$
\begin{array}{ll}
m_{11}=4-\frac{3 R}{2}-\frac{3}{2 R}, & m_{12}=\frac{2 d}{R} \frac{3 R-1}{1+R}, \\
m_{21}=\frac{3\left(R^{2}-1\right)(1-R)(3-R)}{8 d R}, & m_{22}=4-\frac{3}{2 R}-\frac{3 R}{2},
\end{array}
$$

and $R=\frac{\sqrt{V_{2}}}{\sqrt{V_{1}}}=\sqrt{1+\frac{V}{V_{1}}}=\sqrt{1+\frac{q V}{E_{\text {kin }}}}$. The Einzellens displays some peculiar focusing properties depending on the potentials involved compared with the particle's kinetic energy. The focal length of the Einzellens is

$$
\frac{1}{f}=\frac{3}{8 d R}\left(1-R^{2}\right)(R-1)(3-R) .
$$

Varying the potential $V$, we obtain varying focusing conditions as summarized in the following table and plotted as a function of $R$ in Fig. 2.8.

The results of focusing properties in an Einzellens are compiled in the following table. Depending on the chosen voltage the Einzel-lens can be focusing or defocusing.

$$
\begin{array}{ccccc}
V & V<-V_{1} & -V_{1}<V<0 & 0<V<8 V_{1} & V>8 V_{1} \\
R \text { imaginary } & 0<R<1 & 1<R<3 & R>3 \\
1 / f \text { no solution } & >0 & >0 & <0 \\
\text { n/a } & \text { focusing } & \text { focusing } & \text { de-focusing }
\end{array}
$$

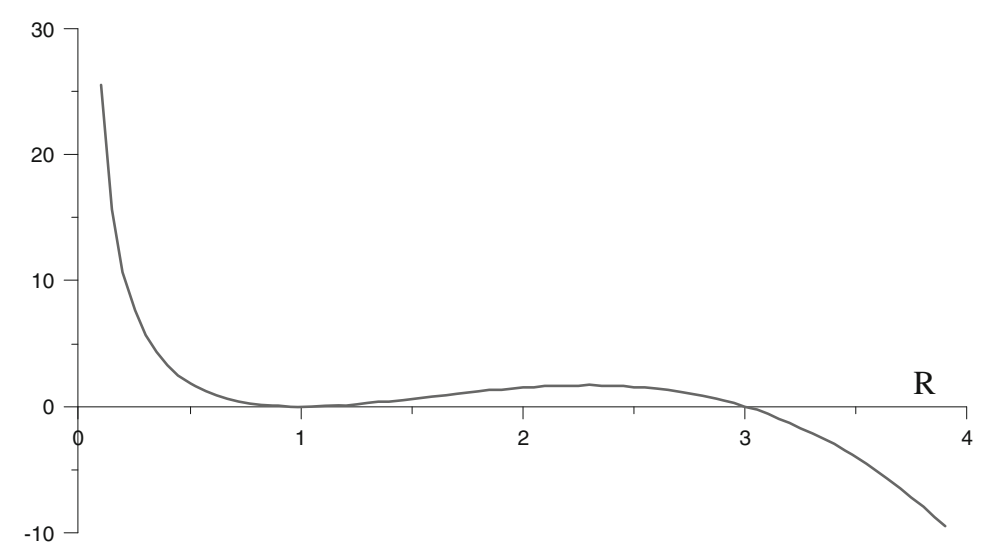

Fig. 2.8 Focusing $8 d / 3 f$ from (2.26) in an Einzellens as a function of $R$ 
The practical focusing regime is limited to $0<R<1$. For $1<R<3$ the focusing is very weak and for $R>3$ the Einzellens is defocusing.

\subsection{Acceleration by rf Fields}

The most successful acceleration of particles is based on the use of rf fields for which by now powerful sources exist. Very high accelerating voltages can be achieved in resonant rf cavities far exceeding those obtainable in electrostatic accelerators of similar dimensions. Particle acceleration in linear accelerators as well as in circular accelerators are based on the use of rf fields and we will in the following sections and in the next chapter discuss the principles of the more important types of particle accelerators.

\subsubsection{Basic Principle of Microwave Linear Accelerators}

The principle of the linear accelerator based on microwave fields and drift tubes was proposed by Ising [6] and Wideroe. [14] The accelerator consists of a series of coaxial metallic tubes where the accelerating field is generated in gaps between adjacent tubes. In this method particles are accelerated by repeated application of $\mathrm{rf}$ fields. Wideroe constructed such an accelerator and was able to accelerate potassium ions up to $50 \mathrm{keV}$.

While the principle is simple, the realization requires specific conditions to ensure that the particles are exposed to only accelerating rf fields. The particles travel through the metallic tubes while the field is not suitable for acceleration as shown in Fig. 2.9. The tubes shield the particles from external rf fields and the length of the tube segments are chosen such that the particles reach the gap between two successive tubes only when the rf field is accelerating.

\section{Synchronicity Condition}

For efficient acceleration the motion of the particles must be synchronized with the rf fields in the accelerating sections. The distance between the center of two adjacent gaps must be equal to the travel time of the particles from one gap to the next. The length of the drift tubes are chosen such that the particles travel for most of the rf period in the field free interior and emerge in a gap to the next drift tube at a moment the field is accelerating. The length of the shielding tubes is therefore almost as long as it take the particles to travel in a full $\mathrm{rf}$ period. In this case, we have synchronism between particle motion and rf field and the length of the $i$ th drift tube/section is

$$
L_{i} \approx v_{i} T_{\mathrm{rf}}
$$




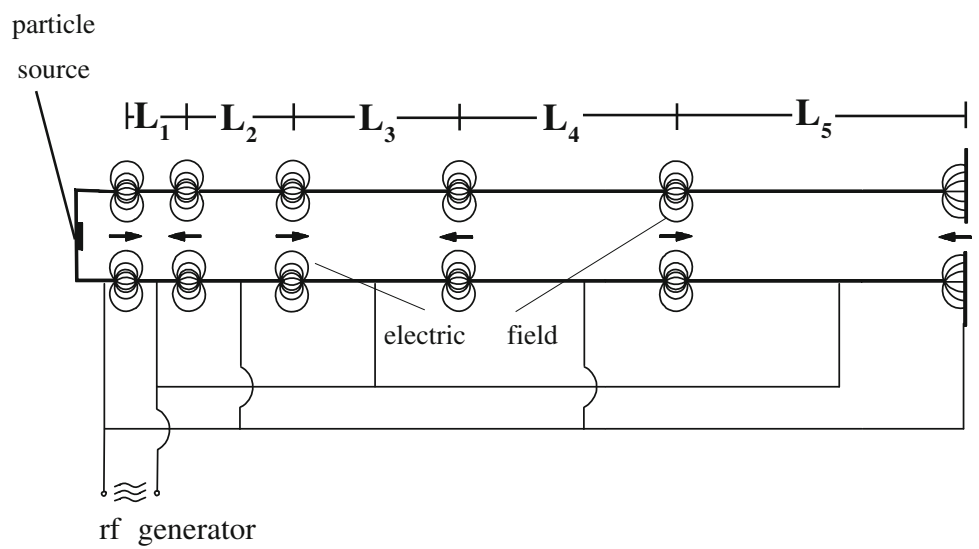

Fig. 2.9 Wideroe linac structure (schematic)

where $v_{i}$ is the velocity of the particles in the $i$ th section and $T_{\mathrm{rf}}$ the $\mathrm{rf}$ period.

Stimulated by the successful acceleration of potassium ions by Wideroe, a group led by Sloan and Lawrence at Berkeley were able to build a $50 \mathrm{~kW}$ rf generator oscillating at $10 \mathrm{MHz}$ and delivering a gap voltage of $42 \mathrm{kV}$. Applying this to 30 acceleration tubes they were able to accelerate mercury ions to a total kinetic energy of $1.26 \mathrm{MeV}$ [11].

In the 1920s when this principle was developed it was difficult to build high frequency generators at significant power. In $1928 \mathrm{rf}$ generators were available only up to about $7 \mathrm{MHz}$ and numerical evaluation of (2.27) shows that this principle was useful only for rather slow particles like low energy protons and ions. The drift tubes can become very long for low rf frequencies and particles traveling with, for example, half the speed of light would require a drift tube length of $10.7 \mathrm{~m}$ at $7 \mathrm{MHz}$. Such long drift tubes add up quickly to a very long accelerators before the particles approach the speed of light. To reduce the length of the tubes, higher frequencies are required.

Further progress in the development of rf linear accelerators therefore depended greatly on the development of rf equipment at high frequency which happened during World War II in connection with the development of radar systems. In 1937, Hansen and the Varian brothers invented the klystron at Stanford. Soon the feasibility of high power klystrons had been established [2] which to this date is one of the most efficient rf amplifiers available. The first klystron was developed for $3,000 \mathrm{MHz}$ which is still the preferred frequency for high energy electron linear accelerators. The klystron principle is economically feasible from about $100 \mathrm{MHz}$ to more than $10 \mathrm{GHz}$. With such a wide range of high frequencies available, the principle of rf acceleration in linear accelerators has gained quick and continued prominence for the acceleration of protons as well as electrons.

Going to higher frequencies, however, the capacitive nature of the Wideroe structure becomes very lossy due to electromagnetic radiation. To overcome this 


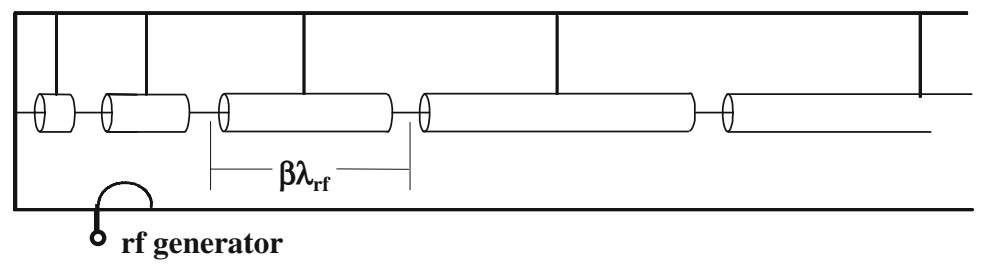

Fig. 2.10 Alvarez linac structure (schematic)

difficulty, Alvarez [1] proposed to enclose the gaps between the tubes by metallic cavities (Fig. 2.10). The acceleration section would now be composed of a series of tubes forming, together with the outer enclosure, a resonant cavity.

This Alvarez structure is still the preferred preaccelerator to accelerate protons and ions from a few hundred $\mathrm{keV}$ out of a Cockroft-Walton electrostatic generator to a few hundred $\mathrm{MeV}$ for injection into a booster synchrotron. Because of the lower velocity of protons and ions at up to a few hundred $\mathrm{MeV}$ the operating frequency for proton linacs is generally around $200 \mathrm{MHz}$.

Radio frequencies of 3,000 MHz and higher are desired for electron acceleration. In Chap. 18.4 we will discuss in more detail the basic features and scaling of high frequency accelerating structures to give the interested reader the tools to understand the scaling and limitations of basic linear accelerator physics. For more detailed discussion of rf aspects in linear electron accelerators, the reader is referred to the literature $[4,7,9]$.

\section{Problems}

2.1 (S). Derive the geometry of electrodes for a horizontally deflecting electric dipole with an aperture radius of $2 \mathrm{~cm}$ which is able to deflect an electron beam with a kinetic energy of $10 \mathrm{MeV}$ by $10 \mathrm{mrad}$. The dipole be $0.1 \mathrm{~m}$ long. What is the electric field required between the electrodes?

2.2 (S). Calculate the minimum power rating for the motor driving the charging belt of a Van de Graaff accelerator while producing a charge current of $100 \mathrm{~mA}$ at $5 \mathrm{MV}$.

2.3 (S). Calculate the length for the first four drift tubes of a Wideroe linac for the following parameters: starting kinetic energy is $100 \mathrm{keV}$, the energy gain per gap is $1 \mathrm{MeV}$, and the microwave frequency $7 \mathrm{MHz}$. Assume the gaps to be of zero length for simplicity. Perform the calculations for both electrons and singly charged potassium ions $\left(\mathrm{A}_{\mathrm{K}}=39.0983 \mathrm{amu} \approx 39 ; 1 \mathrm{amu} \approx 1 \mathrm{GeV}\right)$ and compare the results. 


\section{References}

1. L.W. Alvarez, Phys. Rev. 70, 799 (1946)

2. M. Chodorow, E.L. Ginzton, I.R. Neilsen, S. Sonkin, Proc. IRE 41, 1584 (1953)

3. J.D. Cockcroft, E.T.S. Walton, Proc. Roy. Soc A 136, 619 (1932)

4. E.L. Ginzton, W.W. Hansen, W.R. Kennedy, Rev. Sci. Inst. 19, 89 (1948)

5. H. Greinacher, Z. Physik 4, 195 (1921)

6. G. Ising, Arkiv för Matematik, Astronomi och Fysik 18, 1 (1924)

7. P. Lapostolle, A. Septier (eds.), Linear Accelerators (North-Holland, Amsterdam, 1970)

8. M.S. Livingston (ed.), The Development of High-Energy Accelerators (Dover, New York, 1966)

9. R. Neal (ed.), The 2 Mile Linear Accelerator (Benjamin, New York, 1968)

10. M. Schenkel, Elektrotech. Z. 40, 333 (1919)

11. D.H. Sloan, E.O. Lawrence, Phys. Rev. 38, 2021 (1931)

12. R.J. Van de Graaff, Phys. Rev. 38, 1919 (1931)

13. R.J. Van de Graaff, J.G. Trump, W.W. Buechner, Rep. Prog. Phys. 11, 1 (1948)

14. R. Wideroe, Archiev für Elektrotechnik 21, 387 (1928) 


\section{Chapter 3 \\ Circular Accelerators}

Parallel with the development of electrostatic and linear rf accelerators the potential of circular accelerators was recognized and a number of ideas for such accelerators have been developed over the years. Technical limitations for linear accelerators encountered in the early 1920 s to produce high-power rf waves stimulated the search for alternative accelerating methods or ideas for accelerators that would use whatever little rf fields could be produced as efficiently as possible.

Interest in circular accelerators quickly moved up to the forefront of accelerator design and during the 1930s made it possible to accelerate charged particles to many million electron volts. Only the invention of the rf klystron by the Varian brothers at Stanford in 1937 gave the development of linear accelerators the necessary boost to reach par with circular accelerators again. Since then both types of accelerators have been developed further and neither type has yet outperformed the other. In fact, both types have very specific advantages and disadvantages and it is mainly the application that dictates the use of one or the other.

Circular accelerators are based on the use of magnetic fields to guide the charged particles along a closed orbit. The acceleration in all circular accelerators but the betatron is effected in one or few accelerating cavities which are traversed by the particle beam many times during their orbiting motion. This greatly simplifies the rf system compared to the large number of energy sources and accelerating sections required in a linear accelerator. While this approach seemed at first like the perfect solution to produce high energy particle beams, its progress soon became limited for the acceleration of electrons by copious production of synchrotron radiation.

The simplicity of circular accelerators and the absence of significant synchrotron radiation for protons and heavier particles like ions has made circular accelerators the most successful and affordable principle to reach the highest possible proton energies for fundamental research in high energy physics. Protons are being accelerated into the $\mathrm{TeV}$ range in the Large Hadron Collider (LHC) at CERN in Geneva, Switzerland [1].

This chapter has been made Open Access under a CC BY 4.0 license. For details on rights and licenses please read the Correction https://doi.org/10.1007/978-3-319-18317-6_28 
For electrons the principle of circular accelerators has reached a technical and economic limit at about $28 \mathrm{GeV}$ [2] due to synchrotron radiation losses, which make it increasingly harder to accelerate electrons to higher energies [3]. Further progress in the attempt to reach higher electron energies is being pursued through the principle of linear colliders $[4,5]$, where synchrotron radiation is avoided.

Many applications for accelerated particle beams, however, exist at significantly lower energies and a multitude of well developed principles of particle acceleration are available to satisfy those needs. We will discuss only the basic principles behind most of these low- and medium-energy accelerators in this text and concentrate in more detail on the beam physics in synchrotrons and storage rings. Well documented literature exists for smaller accelerators and the interested reader is referred to the bibliography at the end of this text.

\subsection{Betatron}

The first "circular electron accelerator" has been invented and developed a hundred years ago in the form of an electrical current transformer. Here we find the electrons in the wire of a secondary coil accelerated by an electro motive force generated by a time varying magnetic flux through the area enclosed by the secondary coil. This idea was picked up independently by several researchers [6, 7]. Wideroe finally recognized the importance of a fixed orbit radius and formulated the Wideroe $\frac{1}{2}$ condition, which is a necessary although not sufficient condition for the successful operation of a beam transformer or betatron as it was later called, because it functions optimally only for the acceleration of beta rays or electrons [8].

The betatron makes use of the transformer principle, where the secondary coil is replaced by an electron beam circulating in a closed doughnut shaped vacuum chamber. A time-varying magnetic field is enclosed by the electron orbit and the electrons gain an energy in each turn which is equal to the electro-motive force generated by the varying magnetic field. The principle arrangement of the basic components of a betatron are shown in Fig. 3.1.

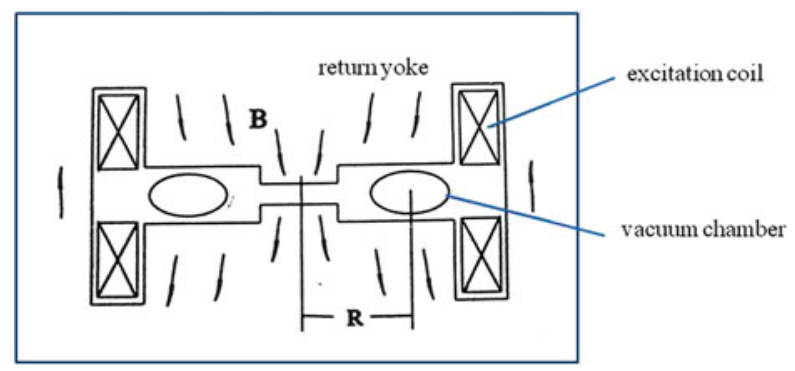

Fig. 3.1 The principle of acceleration in a betatron (schematic) 
The accelerating field is determined by integrating Maxwell's equation

$$
\nabla \times \mathbf{E}=-\frac{\partial}{\partial t} \mathbf{B}
$$

and utilizing Stokes's theorem, we obtain the energy gain per turn

$$
\oint \mathbf{E d} \mathbf{s}=-\frac{\partial \Phi}{\partial t}
$$

where $\Phi$ is the magnetic flux enclosed by the integration path, which is identical to the design orbit of the beam. The particles follow a circular path under the influence of the Lorentz force in a uniform magnetic field. We use a cylindrical coordinate system $(r, \varphi, y)$, where the particles move with the coordinate $\varphi$ clockwise along the orbit. From (1.74) we get for the particle momentum

$$
c p=\gamma c m v=e c r B_{\perp} .
$$

The accelerating force is equal to the rate of change of the particle momentum and can be obtained from the time derivative of (3.3). This force must be proportional to the azimuthal electric field component $E_{\varphi}$ on the orbit

$$
\frac{\mathrm{d} p}{\mathrm{~d} t}=-e\left(\frac{\mathrm{d} r}{\mathrm{~d} t} B_{\perp}+r \frac{\mathrm{d} B_{\perp}}{\mathrm{d} t}\right)=e E_{\varphi} .
$$

Following Wideroe's requirement for a constant orbit $\mathrm{d} r / \mathrm{d} t=0$ allows the containment of the particle beam in a doughnut shaped vacuum chamber surrounding the magnetic field. The induced electric field has only an angular component $E_{\varphi}$ since we have assumed that the magnetic field enclosed by the circular beam is uniform or at least rotationally symmetric. While noting that for a positive rate of change for the magnetic field the induced azimuthal electric field is negative, the left hand side of (3.2) then becomes simply

$$
\oint \mathbf{E d} \mathbf{s}=-\int E_{\varphi} R \mathrm{~d} \varphi=-2 \pi R E_{\varphi}
$$

On the other hand, we have from (3.4)

$$
e E_{\varphi}=-e R \frac{\mathrm{d} B_{\perp}(R)}{\mathrm{d} t}
$$

and using (3.5), (3.6) in (3.2) we get

$$
\frac{\mathrm{d} \Phi}{\mathrm{d} t}=2 \pi R^{2} \frac{\mathrm{d} B_{\perp}(R)}{\mathrm{d} t} .
$$


Noting that the complete magnetic flux enclosed by the particle orbit can also be expressed by an average field enclosed by the particle orbit, we have $\Phi=\pi R^{2} \bar{B}(R)$, where $\bar{B}(R)$ is the average magnetic induction within the orbit of radius $R$. The rate of change of the magnetic flux becomes

$$
\frac{\mathrm{d} \Phi}{\mathrm{d} t}=\pi R^{2} \frac{\mathrm{d} \bar{B}_{\perp}(R)}{\mathrm{d} t}
$$

and comparing this with (3.7) we obtain the Wideroe $\frac{1}{2}$-condition

$$
B_{\perp}(R)=\frac{1}{2} \bar{B}_{\perp}(R)
$$

which requires for orbit stability that the field at the orbit be half the average flux density through the orbit. This condition must be met in order to obtain orbital-beam stability in a betatron accelerator. By adjusting the total magnetic flux through the particle orbit such that the average magnetic field within the orbit circle is twice the field strength at the orbit, we are in a position to accelerate particles on a circle with a constant radius $R$ within a doughnut shaped vacuum chamber.

The basic components of a betatron, shown in Fig. 3.1, have rotational symmetry. In the center of the magnet, we recognize two magnetic gaps of different aperture. One gap at $R$ provides the bending field for the particles along the orbit. The other gap in the midplane of the central return yoke is adjustable and is being used to tune the magnet such as to meet the Wideroe $\frac{1}{2}$-condition. The magnetic field is generally excited by a resonance circuit cycling at the ac frequency of the main electricity supply. In this configuration the magnet coils serve as the inductance and are connected in parallel with a capacitor bank tuned to the ac frequency of 50 or $60 \mathrm{~Hz}$.

The rate of momentum gain is derived by integration of (3.4) with respect to time and we find that the change in momentum is proportional to the change in the magnetic field

$$
\triangle p=R \int \frac{\mathrm{d} B_{\perp}}{\mathrm{d} t} \mathrm{~d} t=e R \Delta B_{\perp}
$$

The particle momentum depends only on the momentary magnetic field and not on the rate of change of the field. For slowly varying magnetic fields the electric field is smaller but the particles will make up the reduced acceleration by travelling around the orbit more often. While the magnet cycling rate does not affect the particle energy it certainly determines the available flux of accelerated particles per unit time. The maximum particle momentum is determined only by the orbit radius and the maximum magnetic field at the orbit during the acceleration cycle

$$
c p_{\max }=e c R B_{\max }(R) .
$$


The betatron principle works for any charged particle and for all energies since the stability condition (3.9) does not depend on particle parameters. In praxis, however, we find that the betatron principle is unsuitable to the acceleration of heavy particles like protons. The magnetic fields in a betatron as well as the size of the betatron magnet set practical limits to the maximum momentum achievable. Donald Kerst built the largest betatron ever constructed with an orbit radius of $R=1.23 \mathrm{~m}$, a maximum magnetic field at the orbit of $8.1 \mathrm{kG}$ and a total magnet weight of 350 tons reaching the maximum expected particle momentum of $300 \mathrm{MeV} / \mathrm{c}$ at $60 \mathrm{~Hz}$.

For experimental applications we are interested in the kinetic energy of the accelerated particles. In case of electrons the rest mass is small compared to the maximum momentum of $c p=300 \mathrm{MeV}$ and therefore the kinetic electron energy from this betatron is

$$
E_{\mathrm{kin}} \approx c p=300 \mathrm{MeV} \text {. }
$$

In contrast to this result, we find the achievable kinetic energy for a proton to be much smaller

$$
E_{\mathrm{kin}} \approx \frac{1}{2} \frac{(c p)^{2}}{m_{\mathrm{p}} c^{2}}=48 \mathrm{MeV}
$$

because of the large mass of protons.

The betatron produces a pulse of accelerated particles once per ac cycle. To gain the maximum energy, the ac field is biased by a dc current and acceleration occurs from the minimum ac field to the maximum ac field. At the maximum field the beam can be ejected for applications.

Different, more efficient accelerating methods have been developed for protons, and betatrons are therefore used exclusively for the acceleration of electrons as indicated by it's name. Most betatrons are designed for modest energies of up to $45 \mathrm{MeV}$ and are used to produce electron and hard x-ray beams for medical applications or in technical applications to, for example, examine the integrity of full penetration welding seams in heavy steel containers.

\subsection{Weak Focusing}

The Wideroe $\frac{1}{2}$-condition is a necessary condition to obtain a stable particle orbit at a fixed radius $R$. This stability condition, however, is not sufficient for particles to survive the accelerating process. Any particle starting out with, for example, a slight vertical slope would, during the acceleration process, follow a continuously spiraling path until it hits the top or bottom wall of the vacuum chamber and gets lost. Constructing and testing the first, although unsuccessful, beam transformer, Wideroe recognized [8] the need for beam focusing, a need which has become a fundamental part of all future particle accelerator designs. First theories on beam 
stability and focusing have been pursued by Walton [9] and later by Steenbeck, who formulated a stability condition for weak focusing and applied it to the design of the first successful construction and operation of a betatron in 1935 at the SiemensSchuckert Company in Berlin reaching an energy of $1.9 \mathrm{MeV}$ [10] although at a very low intensity measurable only with a Geiger counter. The focusing problems in a betatron were finally solved in a detailed orbit analysis by Kerst and Serber [11].

To derive the beam stability condition we note that (1.74) is true only at the ideal orbit $r=R$. For any other orbit radius $r$ the restoring force is

$$
F_{x}=\frac{\gamma m v^{2}}{r}-e v B_{y}
$$

Here we use a cartesian coordinate system which moves with the particle along the orbit with $\mathbf{x}$ pointing in the radial and $\mathbf{y}$ in the axial direction.

In a uniform magnetic field the restoring force would be zero for any orbit. To include focusing we assume that the magnetic field at the orbit includes a gradient such that for a small deviation $x$ from the ideal orbit, $r=R+x=R(1+x / R)$, the magnetic guide field becomes

$$
B_{y}=B_{0 y}+\frac{\partial B_{y}}{\partial x} x=B_{0 y}\left(1+\frac{R}{B_{0 y}} \frac{\partial B_{y}}{\partial x} \frac{x}{R}\right) .
$$

After insertion of (3.15) into (3.14) the restoring force is

$$
F_{x} \approx \frac{\gamma m v^{2}}{R}\left(1-\frac{x}{R}\right)-e v B_{0 y}\left(1-n \frac{x}{R}\right)
$$

where we assumed $x \ll R$ and defined the field index

$$
n=-\frac{R}{B_{0 y}} \frac{\partial B_{y}}{\partial x} .
$$

With (1.74) we get for the horizontal restoring force

$$
F_{x}=-\frac{\gamma m v^{2}}{R} \frac{x}{R}(1-n) .
$$

The equation of motion under the influence of the restoring force in the deflecting or horizontal plane is with $F_{x}=\gamma m \ddot{x}$

$$
\ddot{x}+\omega_{x}^{2} x=0,
$$

which has the exact form of a harmonic oscillator with the frequency

$$
\omega_{x}=\frac{v}{R} \sqrt{1-n}=\omega_{0} \sqrt{1-n}
$$


where $\omega_{0}$ is the orbital revolution frequency. The particle performs oscillations about the ideal or reference orbit with the amplitude $x(z)$ and the frequency $\omega_{x}$. Because this focusing feature was discovered in connection with the development of the betatron we refer to this particle motion as betatron oscillations with the betatron frequency $\omega_{x}$. From (3.20) we note a stability criterion, which requires that the field index not exceed unity to prevent the betatron oscillation amplitude to grow exponentially,

$$
n<1 \text {. }
$$

The particle beam stability discussion is complete only if we also can show that there is stability in the vertical plane. A vertical restoring force requires a finite horizontal field component $B_{x}$ and the equation of motion becomes

$$
\gamma m \ddot{y}=e v B_{x} .
$$

Maxwell's curl equation $\frac{\partial B_{x}}{\partial y}-\frac{\partial B_{y}}{\partial x}=0$ can be integrated and the horizontal field component is with (3.15), (3.17)

$$
B_{x}=\int \frac{\partial B_{y}}{\partial x} \mathrm{~d} y=-\int n \frac{B_{0 y}}{R} \mathrm{~d} y=-n \frac{B_{0 y}}{R} y .
$$

Insertion of (3.23) and (1.74) into (3.22) results in the equation of motion for the vertical plane in the form of

$$
\ddot{y}+\omega_{y}^{2} y=0,
$$

where the vertical betatron oscillation frequency is

$$
\omega_{y}=\omega_{0} \sqrt{n}
$$

Particles perform stable betatron oscillations about the horizontal mid plane with the vertical betatron frequency $\omega_{y}$ as long as the field index is positive

$$
n>0 \text {. }
$$

In summary, we have found that a field gradient in the magnetic guide field can provide beam stability in both the horizontal and vertical plane provided that the field index meets the criterion

$$
0<n<1
$$

which has been first formulated and applied by Steenbeck [10] and is therefore also called Steenbeck's stability criterion. 
A closer look at (3.20) shows that the field index actually provides defocusing in the horizontal plane. The reason why we get focusing in both planes is that there is a strong natural focusing from the sector type magnet which is larger than the defocusing from the field gradient. This focusing is of geometric nature and relates to the length of the orbit. A particle travelling, for example, parallel to and outside the ideal orbit is deflected more because it follows a longer path in the uniform magnetic field than a particle following the ideal orbit leading to effective focusing toward the ideal orbit. Conversely, a particle traveling parallel to and inside the ideal orbit is deflected less and therefore again is deflected toward the ideal orbit.

The stability condition (3.21) actually stipulates that the defocusing from the field index in the horizontal plane be less than the focusing of the sector magnet allowing to choose the sign of the field index such that it provides focusing in the vertical plane. Basically the field gradient provides a means to distribute the strong sector magnet focusing. This method of beam focusing is known as weak focusing in contrast to the principle of strong focusing, which will be discussed extensively in the remainder of this text.

\subsection{Adiabatic Damping}

During the discussion of transverse focusing we have neglected the effect of acceleration. To include the effect of acceleration into our discussion on beam dynamics, we use as an example the Lorentz force equation for the vertical motion. The equation of motion is

$$
\frac{\mathrm{d}}{\mathrm{d} t}(\gamma m \dot{y})=e v_{z} B_{x}
$$

where we used the fields $\mathbf{B}=\left(B_{x}, B_{y}, 0\right)$ in a cartesian coordinate system $(x, y, z)$. Evaluating the differentiation, we get the equation of motion at the equilibrium orbit

$$
\gamma m \ddot{y}+\dot{\gamma} m \dot{y}=e \omega_{0} R B_{x} .
$$

Inserting (3.23) into (3.29) results in the equation of motion in the vertical plane under the influence of accelerating electrical and focusing magnetic fields

$$
\ddot{y}+\frac{\dot{E}}{E} \dot{y}+n \omega_{0}^{2} y=0,
$$

where $\dot{E}$ is the energy gain per unit time. This is the differential equation of a damped harmonic oscillator with the solution

$$
y=y_{0} \mathrm{e}^{-\alpha_{y} t} \cos \omega_{y} t
$$


where $\omega_{y} \approx \omega_{0} \sqrt{n}$ and the damping decrement

$$
\alpha_{y}=\frac{1}{2} \frac{\dot{E}}{E} .
$$

For technically feasible acceleration $\dot{E}$ the damping time $\tau_{y}=\alpha_{y}^{-1}$ is very long compared to the oscillation period and we therefore may consider for the moment the damping as a constant. The envelope $y_{\max }=y_{0} e^{-\alpha_{y} t}$ of the oscillation (3.31) decays like

$$
\mathrm{d} y_{\max }=-\frac{1}{2} \frac{\dot{E}}{E} y_{\max } \mathrm{d} t,
$$

which after integration becomes

$$
\frac{y_{\max }}{y_{0, \max }}=\sqrt{\frac{E_{0}}{E}} .
$$

The betatron oscillation amplitude is reduced as the particle energy increases. This type of damping is called adiabatic damping. Similarly, the slope $y^{\prime}$ as well as the horizontal oscillation parameters experience the same effect of adiabatic damping during acceleration. This damping is due to the fact that the longitudinal particle momentum is increasing during acceleration while the transverse momentum is not. For a particle beam we define a beam emittance in both planes by the product $\epsilon_{u}=$ $u_{\max } u_{\max }^{\prime}$, where $u$ stands for $x$ or $y$. Due to adiabatic damping this beam emittance is reduced inversely proportional to the energy like

$$
\epsilon \sim \frac{1}{E}
$$

No specific use has been made of the principle of betatron acceleration to derive the effect of adiabatic damping. We therefore expect this effect to be generally valid for any kind of particle acceleration.

The development of the betatron was important for accelerator physics for several reasons. It demonstrated the need for particle focusing, the phenomenon of adiabatic damping and stimulated Schwinger to formulate the theory of synchrotron radiation [3]. He realized that the maximum achievable electron energy in a betatron must be limited by the energy loss due to synchrotron radiation. Postponing a more detailed discussion of synchrotron radiation to Chap. 24, we note that the instantaneous synchrotron radiation power is given by $P_{\gamma} \propto E^{2} F_{\perp}^{2}$, where $F_{\perp}$ is the transverse force on the particle and the energy loss per turn to synchrotron radiation in a circular electron accelerator is given by (24.41). The energy loss increases rapidly with the fourth power of energy and can lead quickly to an energy limitation of the accelerator when the energy loss per turn becomes equal to the energy gain. 


\subsection{Acceleration by rf Fields}

Most types of circular particle accelerators utilize compact accelerating cavities, which are excited by rf sources. Particles traverse this cavity periodically and gain energy from the electromagnetic fields in each passage. The bending magnet field serves only as a beam guidance system to allow the repeated passage of the particle beam through the cavity. Technically, this type of accelerator seem to be very different from the principle of the betatron. Fundamentally, however, there is no difference. We still rely on the transformer principle, which in the case of the betatron looks very much like the familiar transformers at low frequencies, while accelerating cavities are transformer realizations for very high frequencies. Electric fields are generated in both cases by time varying magnetic fields.

Since the cavity fields are oscillating, acceleration is not possible at all times and for multiple accelerations we must meet specific synchronicity condition between the motion of particles and the field oscillation. The time it takes the particles to travel along a full orbit must be an integer multiple of the oscillation period for the radio frequency field. This synchronization depends on the particle velocity, path length, magnetic fields employed, and on the rf frequency. Specific control of one or more of these parameters defines the different types of particle accelerators to be discussed in the following subsections.

\subsubsection{Microtron}

The schematic configuration of a microtron [12] is shown in Fig. 3.2. Particles emerging from a source pass through the accelerating cavity and follow then a circular orbit in a uniform magnetic field $B_{y}$ leading back to the accelerating cavity. After each acceleration the particles follow a circle with a bigger radius till they reach the boundary of the magnet.

The bending radius of the orbit can be derived from the Lorentz force equation (1.74)

$$
\frac{1}{\rho}=\frac{e c B}{c p}=\frac{e c B_{y}}{\gamma \beta m c^{2}},
$$

and the revolution time for a particle traveling with velocity $v$ is

$$
\tau=\frac{2 \pi \rho}{v}=\frac{2 \pi m c}{e} \frac{\gamma}{B_{y}} .
$$

The revolution time is therefore proportional to the particle energy and inversely proportional to the magnetic field. It is interesting to note that for subrelativistic particles, where $\gamma \approx 1$, the revolution time is constant even though the particle 


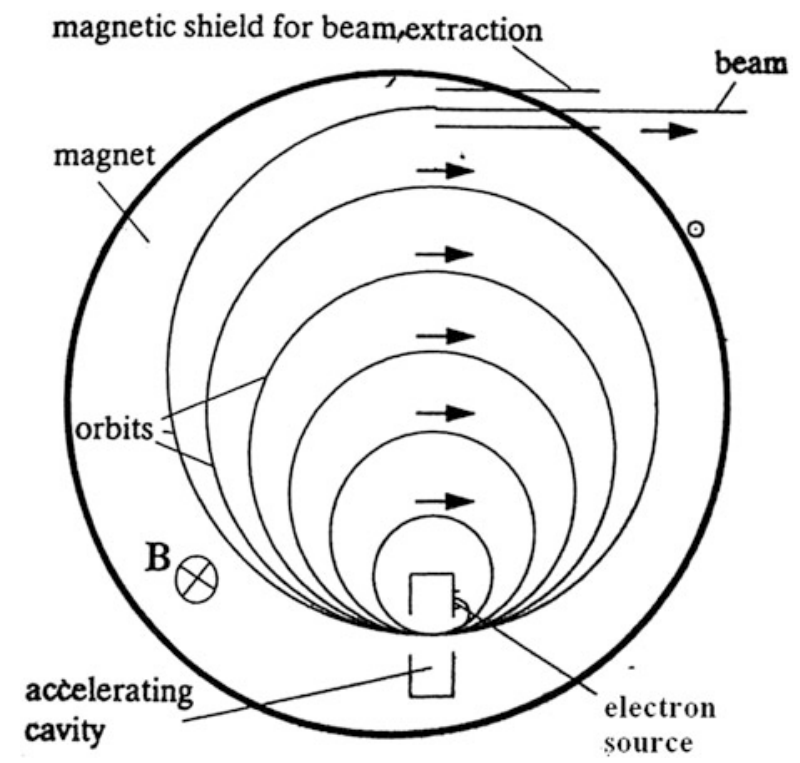

Fig. 3.2 The principle of a microtron accelerator (schematic)

momentum increases. The longer path length for the higher particle momentum is compensated by the higher velocity. As particles reach relativistic energies, however, this synchronism starts to fail. To still achieve continued acceleration, specific conditions must be met.

A particle having completed the $n$th turn passes through the cavity and it's energy is increased because of acceleration. The change in the revolution time during the $(n+1)$ st turn compared to the $n$th turn is proportional to the energy increase $\Delta \gamma$. The increase in the revolution time must be an integer multiple of the radio frequency period. Assuming that the revolution time along the first innermost circle, when the particle energy is still $\gamma \approx 1$, is equal to one rf period we conclude that synchronism is preserved for all turns if

$$
\Delta \gamma=1
$$

or integer multiples. In order to make a microtron functional the energy gain from the accelerating cavity in each passage must be

$$
\begin{array}{cl}
\Delta E_{\mathrm{e}}=511 \mathrm{keV} & \text { for electrons and } \\
\Delta E_{\mathrm{p}}=938 \mathrm{MeV} & \text { for protons. }
\end{array}
$$

While it is possible to meet the condition for electrons it is technically impossible at this time to achieve accelerating voltages of almost $1 \mathrm{GV}$ in an accelerating cavity 


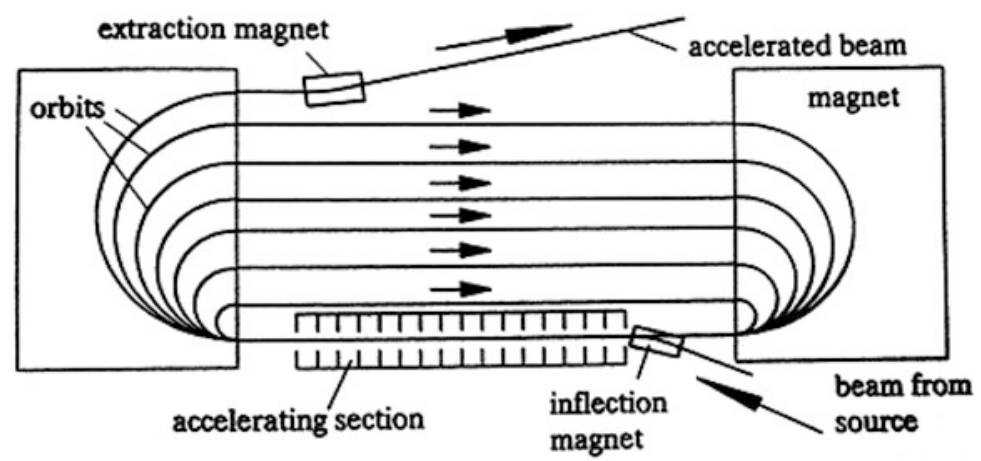

Fig. 3.3 Race track microtron [13] (schematic)

of reasonable length. The principle of microtrons is therefore specifically suited for the acceleration of electrons.

The size of the magnet imposes a practical limit to the maximum particle energy. A single magnet scales like the third power of the bending radius and therefore the weight of the magnet also scales like the third power of the maximum particle energy. Single magnet microtrons are generally used only to accelerate electrons to energies up to about $25-30 \mathrm{MeV}$.

To alleviate the technical and economic limitations as well as to improve control of the synchronicity condition, the concept of a race track microtron has been developed $[13,14]$. In this type of microtron the magnet is split in the middle and normal to the orbit plane and pulled apart as shown in Fig. 3.3. The space opened up provides space for a short linear accelerator which allows the acceleration of electrons by several units in $\gamma$ thus reducing the number of orbits necessary to reach the desired energy. The magnets are flat and scale primarily only like the square of the bending radius.

\subsubsection{Cyclotron}

The synchronicity condition of a microtron proved to be too severe for the successful acceleration of heavier particles like protons. In drawing this conclusion, however, we have ignored the trivial synchronicity condition $\Delta \gamma=0$. This condition demands that the particle energy be nonrelativistic which limits the maximum achievable energy to values much less than the rest energy. This limitation is of no interest for electrons since electro-static accelerators would provide much higher energies than that. For protons, however, energies much less than the rest energy of $938 \mathrm{MeV}$ are of great interest. This was recognized in 1930 by Lawrence and Edlefsen [15] in the process of inventing the principle of the cyclotron and the first such device was built by Lawrence and Livingston in 1932 [16]. 
Fig. 3.4 Principle of a cyclotron [16] (schematic). Vertical (top) and horizontal (bottom) mid plane cross section
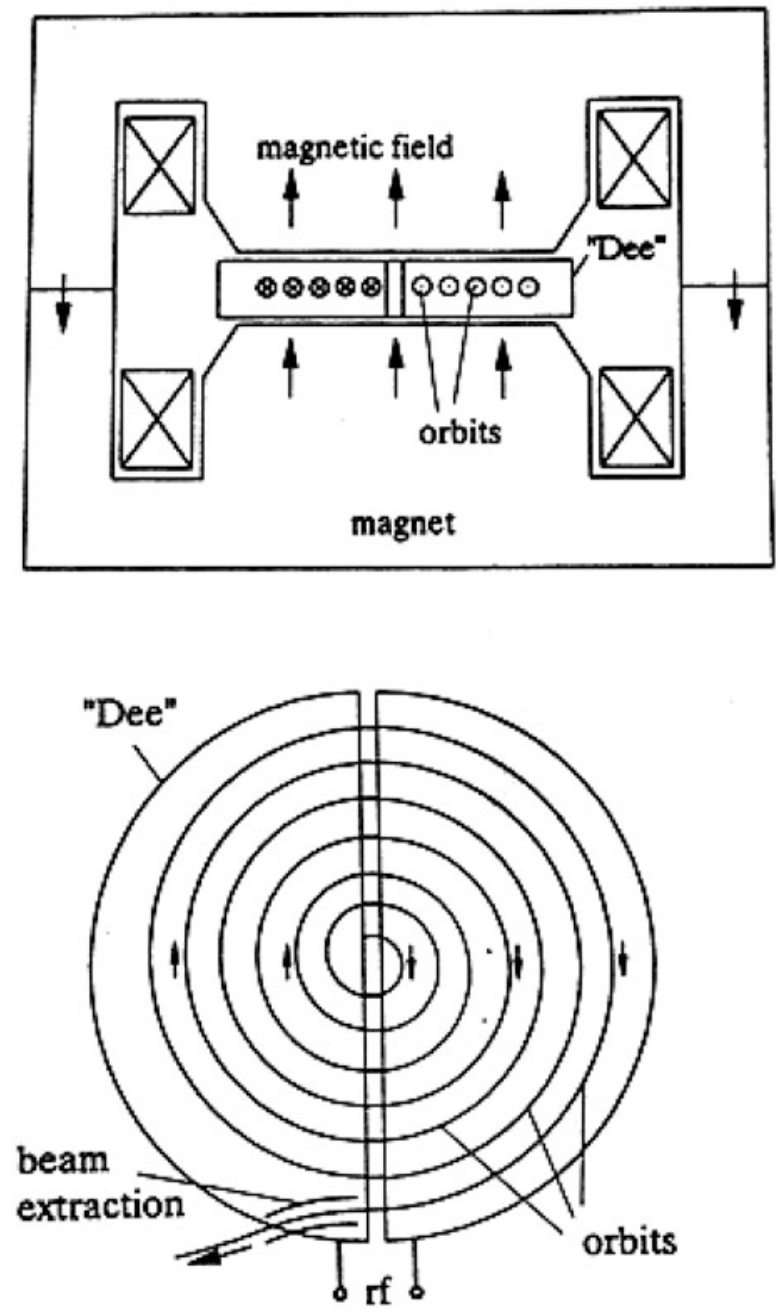

The cyclotron principle employs a uniform magnetic field and an rf cavity that extends over the whole aperture of the magnet as shown in Fig. 3.4. The accelerating cavity has basically the form of a pill box cut in two halves, where the accelerating fields are generated between those halves and are placed between the poles of the magnet. Because of the form of the half pill boxes, these cavities are often called the D's of a cyclotron. The particle orbits occur mostly in the field free interior of the $D$ 's and traverse the accelerating gaps between the two $D$ 's twice per revolution. Due to the increasing energy, the particle trajectories spiral to larger and larger radii. The travel time within the D's is adjusted by the choice of the magnetic field such 
that it is equal to half the radio frequency period. The principle of the cyclotron is basically the application of the Wideroe linac in a coiled up version to save space and rf equipment. Fundamentally, however, the use of field free tubes or $D$ 's with increasing path length between accelerating gaps is the same.The revolution time in a cyclotron is given by (3.37) where now $\gamma=1$ and the acceleration of ions with a charge multiplicity $Z$ is allowed. Keeping the magnetic field constant, we have a constant revolution frequency and may therefore apply a constant radio frequency

$$
f_{\mathrm{rf}}=\frac{Z e B_{y}}{2 \pi m c \gamma} h=\text { const. }
$$

The principle of the cyclotron is limited to nonrelativistic particles. Protons are sufficiently nonrelativistic up to kinetic energies of about $20-25 \mathrm{MeV}$ or about $2.5 \%$ of the rest energy. As the particles become relativistic the revolution frequency becomes smaller and the particles get out of synchronism with the radio frequency $f_{\mathrm{rf}}$.

The radio frequency depends on the charge multiplicity $Z$ of the particles to be accelerated and on the magnetic field $B_{y}$. Evaluating (3.40), the following frequencies are required for different types of particles

$$
\begin{aligned}
f_{\mathrm{rf}}[\mathrm{MHz}] & =1.53 \cdot B_{y}[\mathrm{kG}] & & \text { for protons, } \\
& =0.76 \cdot B_{y}[\mathrm{kG}] & & \text { for deuterons, } \\
& =0.76 \cdot B_{y}[\mathrm{kG}] & & \text { for } \mathrm{He}^{++} .
\end{aligned}
$$

A closer inspection of the synchronicity condition, however, reveals that these are only the lowest permissible rf frequencies. Any odd integer multiple of the frequencies (3.41) would be acceptable too.

As long as particles do not reach relativistic energies, the maximum achievable kinetic energy $E_{\text {kin }}$ depends on the type of the particle, the magnetic field $B$, and the maximum orbit radius $R$ possible in the cyclotron and is given by

$$
E_{\text {kin }}=\frac{1}{2} m v^{2}=\frac{(c p)^{2}}{2 m c^{2}}=\frac{Z^{2} e^{2} B_{y}^{2} R^{2}}{2 m c^{2}} .
$$

Examples of numerical relations are

$$
\begin{aligned}
E_{\mathrm{kin}}[\mathrm{MeV}] & =0.48 B_{y}^{2}\left[\mathrm{kG}^{2}\right] R^{2}\left[\mathrm{~m}^{2}\right] & & \text { for protons, } \\
& =0.24 B_{y}^{2}\left[\mathrm{kG}^{2}\right] R^{2}\left[\mathrm{~m}^{2}\right] & & \text { for deuterons, } \\
& =0.48 B_{y}^{2}\left[\mathrm{kG}^{2}\right] R^{2}\left[\mathrm{~m}^{2}\right] & & \text { for } \mathrm{He}^{++} .
\end{aligned}
$$


The particle flux reflects the time structure of the radio frequency field. For a continuous radio frequency field the particle flux is also "continuous" with micro bunches at distances equal to the oscillation period of the accelerating field. For a pulsed rf system obviously the particle flux reflects this macropulse structure on top of the micropulses.

\subsubsection{Synchro-Cyclotron}

The limitation to nonrelativistic energies of the cyclotron principle is due only to the assumption that the radio frequency be constant. This mode of operation for $\mathrm{rf}$ systems is desirable and most efficient but is not a fundamental limitation. Technical means are available to vary the radio frequency in an accelerating cavity.

As the technology for acceleration to higher and higher energies advances, the need for particle beam focusing becomes increasingly important. In the transverse plane this is achieved by the weak focusing discussed earlier. In the longitudinal phase space stability criteria have not been discussed yet. Veksler [17] and McMillan [18] discovered and formulated independently the principle of phase focusing, which is a fundamental focusing property for high energy particle accelerators based on accelerating microwave frequency fields and was successfully tested only one year later [19]. We will discuss this principle of phase focusing in great detail in Chap. 9.

Both the capability of varying the rf frequency and the principle of phase focusing is employed in the synchro-cyclotron. In this version of the cyclotron, the microwave frequency varies as the relativistic factor $\gamma$ deviates from unity. Instead of (3.40) we have for the revolution frequency or microwave frequency

$$
f_{\mathrm{rf}}=\frac{Z e B_{y}}{2 \pi \gamma m c} h,
$$

where $h$ is an integer called the harmonic number. Since $B=$ const the radio frequency must be adjusted like

$$
f_{\mathrm{rf}} \sim 1 / \gamma(t)
$$

to keep synchronism. The momentary particle energy $\gamma(t)$ can be derived from the equation of motion $1 / \rho=Z e B /(c p)$ which we solve for the kinetic energy

$$
\sqrt{E_{\mathrm{kin}}\left(E_{\mathrm{kin}}+2 m c^{2}\right)}=e Z B_{y} \rho \text {. }
$$

The largest accelerator ever built based on this principle is the 184 inch synchro cyclotron at the Lawrence Berkley Laboratory (LBL) in 1946 [20]. The magnet weight was 4,300 tons, produces a maximum magnetic field of $15 \mathrm{kG}$ and has a maximum orbit radius of $2.337 \mathrm{~m}$. From (3.46) we conclude that the maximum 
kinetic proton energy should be $E_{\text {kin,max }}=471 \mathrm{MeV}$ while $350 \mathrm{MeV}$ have been achieved. The discrepancy is mostly due to the fact that the maximum field of $15 \mathrm{kG}$ does not extend out to the maximum orbit due to focusing requirements. The principle of the synchro cyclotron allows the acceleration of particles to rather large energies during many turns within the cyclotron magnet. This long path requires the addition of weak focusing as discussed in connection with the betatron principle to obtain a significant particle flux at the end of the acceleration period. From the discussion in Sect. 3.2 we know that efficient focusing in both planes requires the vertical magnetic field component to drop off with increasing radius. For equal focusing in both planes the field index should be $n=\frac{1}{2}$ and the magnetic field scales therefore like

$$
B_{y}(\rho) \sim \frac{1}{\sqrt{\rho}} .
$$

The magnetic field is significantly lower at large radii compared to the center of the magnet.

This magnetic field dependence on the radial position leads also to a modification of the frequency tracking condition (3.45). Since both the magnetic field and the particle energy change, synchronism is preserved only if the rf frequency is modulated like

$$
f_{\mathrm{rf}} \sim \frac{B_{y}[\rho(t)]}{\gamma(t)} .
$$

Because of the need for frequency modulation, the particle flux has a pulsed macro structure equal to the cycling time of the rf modulation. A detailed analysis of the accelerator physics issues of a synchro-cyclotron can be found in [21].

\subsubsection{Isochron Cyclotron}

The frequency modulation in a synchro cyclotron is technically complicated and must be different for different particle species. A significant breakthrough occurred in this respect when Thomas [22] realized that the radial dependence of the magnetic field could be modified in such a way as to match the particle energy $\gamma$. The condition (3.48) becomes in this case

$$
f_{\mathrm{rf}} \sim \frac{B_{y}[\rho(t)]}{\gamma(t)}=\text { const. }
$$

To reconcile (3.49) with the focusing requirement, strong azimuthal variations of the magnetic fields are introduced

$$
\frac{\partial B_{y}(\rho, \varphi)}{\partial \varphi} \neq 0 .
$$


In essence, the principle of weak focusing is replaced by strong focusing, to be discussed later, with focusing forces established along the particle trajectory while meeting the synchronicity condition only on average in each turn such that

$$
\frac{1}{2 \pi} \oint B_{y}[\rho(t), \varphi] \mathrm{d} \varphi \sim \gamma(t) .
$$

Isochron cyclotrons produce a continuous beam of micro bunches at the rf frequency and are used frequently for acceleration of protons and ions for cancer therapy.

The development of circular accelerators has finally made a full circle. Starting from the use of a constant radio frequency field to accelerate particles we found the need for frequency modulation to meet the synchronicity condition for particles through the relativistic transition regime. Application of sophisticated magnetic focusing schemes, which are now known as strong focusing, finally allowed to revert back to the most efficient way of particle acceleration with constant fixed radio frequency fields.

\subsubsection{Synchrotron}

The maximum particle energy is limited to a few hundred $\mathrm{MeV}$ as long as one stays with the basic cyclotron principle because the volume and therefore cost for the magnet becomes prohibitively large. Higher energies can be achieved and afforded if the orbit radius $R$ is kept constant. In this case the center of the magnet is not needed anymore and much smaller magnets can be employed along the constant particle orbit. Equation (3.36) is still applicable but we keep now the orbit radius constant and have the design condition

$$
\frac{1}{R}=\frac{e c B_{y}}{c p}=\text { const . }
$$

This condition can be met for all particle energies by ramping the magnet fields proportional to the particle momentum. Particles are injected at low momentum and are then accelerated while the bending magnet fields are increased to keep the particles on a constant radius while they gain energy. The particle beam from such a synchrotron is pulsed with a repetition rate determined by the magnetic field cycling. The synchronicity condition

$$
f_{\mathrm{rf}}=\frac{Z e c B_{y}}{2 \pi \gamma m c^{2}} h
$$

is still valid, but because the magnetic field is varied proportional to the particle momentum we expect the frequency to require adjustment as long as there is sufficient difference between particle energy and momentum. 


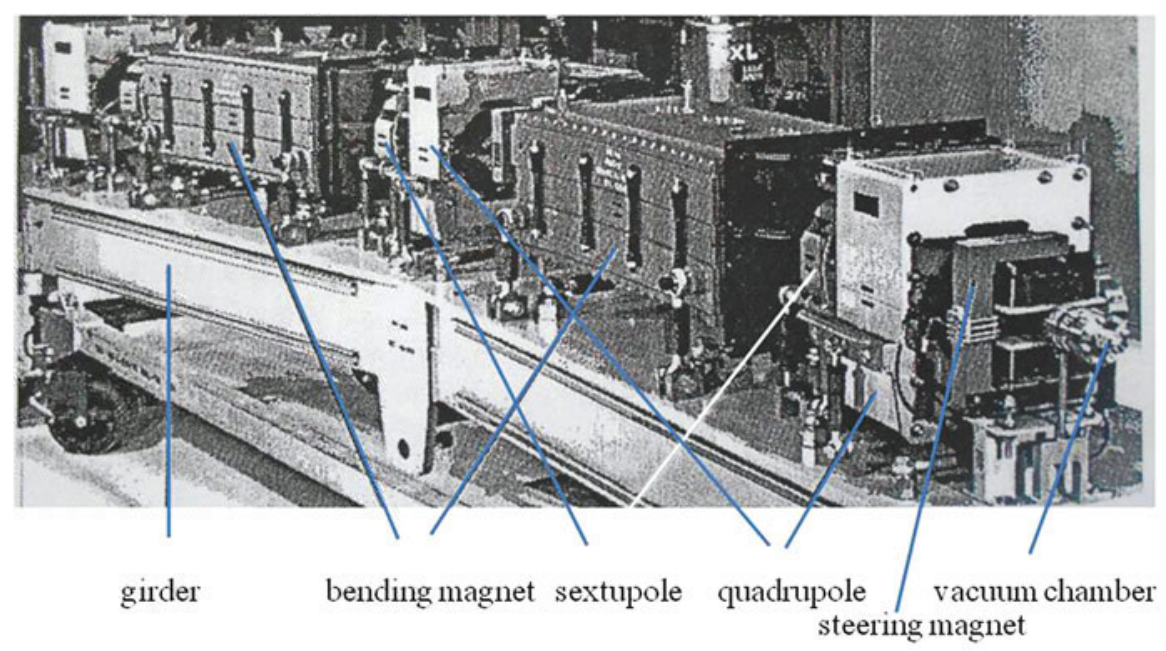

Fig. 3.5 Magnet arrangement in a synchrotron [23]

For highly relativistic particles a solution for particle acceleration has been found which does not require a prohibitively large magnet and where the radio frequency fields can be of constant frequency for optimal efficiency. This is the case for electron synchrotrons with an initial energy of at least a few tens of MeV. For this reason electrons are generally injected into a synchrotron at energies of more than about $10-20 \mathrm{MeV}$ from a linear accelerator or a microtron. Figure 3.5 shows an example of a synchrotron used for the injection of electrons into a storage ring [23].

For heavier particles, however, we are back to the need for some modest frequency modulation during the early phases of acceleration. From (3.53) we expect the revolution frequency to vary like

$$
f_{\text {rev }}(t)=\frac{Z e c B_{y}}{2 \pi c p} \beta(t) \propto \beta(t)
$$

To preserve the synchronicity condition, the radio frequency must be an integer multiple of the revolution frequency and must be modulated in proportion of the varying revolution frequency. The ratio of the $\mathrm{rf}$ frequency to revolution frequency is called the harmonic numberdefined by

$$
f_{\mathrm{rf}}=h f_{\text {rev }}
$$

The maximum energy in a synchrotron is determined by the ring radius $R$, and the maximum magnetic field $B_{y}$, and is

$$
c p_{\max }(\mathrm{GeV})=\sqrt{E_{\text {kin }}\left(E_{\text {kin }}+2 m c^{2}\right)}=C_{p} B_{y}[\mathrm{~T}] R[\mathrm{~m}],
$$


where

$$
C_{p}=0.2997926 \frac{\mathrm{GeV}}{\mathrm{Tm}} \text {. }
$$

Early synchrotrons have been constructed with weak focusing bending magnets which in addition to the dipole field component also included a field gradient consistent with a field index meeting the focusing condition (3.27). More detailed information about early weak focusing synchrotrons can be obtained from [21, 24].

With the discovery of the principle of strong focusing by Christofilos [25] and independently by Courant et. al. [26] in 1952 much more efficient synchrotrons could be designed. The apertures in the magnets could be reduced by up to an order of magnitude thus allowing the design of high-field magnets at greatly reduced overall magnet size and cost. The physics of strong focusing will be discussed in great detail in subsequent chapters.

Synchrotrons are the workhorse in particle acceleration and are applied for electron acceleration as well as proton and ion acceleration to the highest energies. In more modern proton accelerators superconducting magnets are employed to reach energies in excess of $1 \mathrm{TeV}$.

\subsubsection{Storage Ring}

Although not an accelerator in the conventional sense, a particle storage ring can be considered as a synchrotron frozen in time. While the basic functioning of a storage ring is that of a synchrotron, particle beams are generally not accelerated but only stored to orbit for long times of several hours. Bruno Touschek and R. Wideroe invented this principle in 1941 while working (not completely by their own free will) at the Hamburg University for application in high energy physics to bring two counter rotating beams of particles and antiparticles into collision and study high energy elementary particle processes. A newer and more copious application of the storage ring principle arose from the dedicated production of synchrotron radiation for basic and applied research and technology. The principles, details and functioning of synchrotrons and storage rings will occupy most of our discussions in this text.

\subsubsection{Summary of Characteristic Parameters}

It is interesting to summarize the basic principles for the different particle accelerators discussed. All are based on two relations, the equation of motion (3.14) and the synchronicity condition (3.37). Depending on which parameter in these two relations we want to keep constant or let vary, different acceleration principles apply 
Table 3.1 Parameter properties for different acceleration principles

\begin{tabular}{|c|c|c|c|c|c|c|}
\hline Principle & Energy & Velocity & Orbit & Field & Frequency & Particle flux \\
\hline & $\gamma$ & $v$ & $\rho$ & $B$ & $f_{\mathrm{rf}}$ & \\
\hline Microtron & var. & $c$ & $\sim p$ & const. & const. & const. $^{\mathrm{a}}$ \\
\hline Cyclotron & 1 & var. & $\sim v$ & const. & const. & const. $^{\mathrm{a}}$ \\
\hline Synchro-cyclotron & var. & var. & $\sim p$ & $B(\rho)$ & $\sim \frac{B(\rho)}{\gamma(t)}$ & pulsed \\
\hline Isochron cyclotron & var. & var. & $\rho(p)$ & $B(\rho, \varphi)$ & const. & const. $^{\mathrm{a}}$ \\
\hline Proton synchrotron & var. & var. & $R$ & $\sim p(t)$ & $\sim v(t)$ & pulsed \\
\hline Electron synchrotron & var. & $c$ & $R$ & $\sim p(t)$ & const. & pulsed \\
\hline
\end{tabular}

a continuous beam, although rf-modulated

with varying advantages and disadvantages. In Table 3.1 these parameters and their disposition are compiled for the acceleration principles discussed above.

There are two particle parameters and three technical device parameters which define the mode of accelerator operation. Interestingly enough, most of the discussed acceleration methods have their proper application and are used either as stand alone accelerators for research and technology or are part of an acceleration chain for high energy particle accelerators.

For example, it makes no sense to construct a proton synchrotron, where the protons must be injected directly from the source at very low energies. The proper way is to first accelerate the protons with electro-static fields, for example in a Cockcroft-Walton accelerator followed by a medium energy linear accelerator (e.g. an Alvarez structure) to reach a high enough energy of a few hundred $\mathrm{MeV}$ for efficient injection into a synchrotron. Similarly, we accelerate an electron beam first in a linac or microtron before injection into a synchrotron or storage ring.

\section{Problems}

3.1 (S). Consider the Kerst betatron cycling at $60 \mathrm{~Hz}$. Electrons are injected at $50 \mathrm{keV}$ kinetic energy into this betatron. Calculate the magnetic field on the orbit at injection and the energy gain per turn for the first turn and at a time when the electron has gained $20 \mathrm{MeV}$. Discuss the reason for the difference in the energy gain per turn (use linear dependence of field, $B \approx B_{0} \omega t$ ).

3.2 (S). What is the total excitation current in each of the two coils for a betatron with an orbit radius of $R=0.4 \mathrm{~m}$, a maximum electron momentum of $c p=42 \mathrm{MeV}$ and a gap of $g=10 \mathrm{~cm}$ between the poles (hint: apply Ampère's law).

3.3 (S). Calculate the frequency variation required to accelerate protons or deuterons in a synchro cyclotron from a kinetic energy of $E_{\text {kin } 0}=100 \mathrm{keV}$ to an end energy of $E_{\mathrm{kin}}=600 \mathrm{MeV}$. Keep the magnetic field constant and ignore weak focussing. Derive formula for the rf frequency as a function of the kinetic 
energy and generate a graph of $f_{\mathrm{rf}} / f_{\mathrm{rf}, 0}$ vs. $E_{\text {kin }}$ from just a few points. How big would the frequency swing be for electrons?

3.4 (S). Calculate the electron beam current in the Kerst betatron that would produce a total synchrotron radiation power of 1 Watt at $c p=300 \mathrm{MeV}$.

3.5 (S). Try to "design" a microtron for a maximum electron energy of $E=$ $25 \mathrm{MeV}$ at a magnetic field of $B=2140 \mathrm{G}$. (a) What is the diameter of the last circular trajectory at $25 \mathrm{MeV}$ ? (b) Sketch a cross section of the magnet with excitation coils. Magnet poles must extend radially at least by 1.5 gap heights beyond the maximum orbit to obtain good field quality. Use a total coil cross section of $5 \mathrm{~cm}^{2}$. Choose your own gap height. (c) What is the electrical power required to operate each coil assuming copper and a copper fill factor of $75 \%$. That means $75 \%$ of the coil cross section is copper and the rest is for insulation and cooling. Do you think your coil needs water cooling? (for simplicity assume the coil length to be equal to the length of the last trajectory plus $10 \%$.) (d) How does the electrical power requirement change if you change the number of turns in the coil thereby changing the electrical current. Keep in either case $75 \%$ fill factor.

3.6 (S). Consider the Fermilab $400 \mathrm{GeV} / \mathrm{c}$ synchrotron which has a circumference of $6,000 \mathrm{~m}$. Protons are injected at $10 \mathrm{GeV} / \mathrm{c}$ momentum. Calculate the frequency swing necessary for synchronicity during the acceleration cycle.

\section{References}

1. J.M. Peterson, Physics of Particle Accelerators. AIP, vol. 184 (American Institute of Physics, New York, 1989), pp. 2240

2. Design study of a 15 to 100 gev positron-electron colliding beam machine(lep). Technical Report ISR-LEP 78-17, CERN, CERN, Geneva (1978)

3. J.S. Schwinger, On the classical radiation of accelerated electrons. Phys. Rev. 75, 1912 (1949)

4. Slac linear collider, conceptual design report. Technical Report SLAC-229, SLAC, Stanford (1981)

5. M. Tigner, Nuovo Cimento 37, 1228 (1965)

6. G. Breit, M.A. Tuve, Carnegie Institution Year Book, vol. 27 (Carnegie Institution for Science, Washington, 1927), pp. 209

7. J. Slepian, US Patent No 1,645,304 (1922)

8. R. Wideroe, Archiev für Elektrotechnik 21, 387 (1928)

9. E.T.S. Walton, Proc. Camb. Philos. Soc. 25, 569 (1929)

10. M. Steenbeck, US Patent No 2,103,303 (1935)

11. D.W. Kerst, R. Serber, Phys. Rev. 60, 47 (1941)

12. S.P. Kapitza, V.N. Melekhin, The Microtron (Harwood Academic, London, 1987)

13. M. Eriksson, Nucl. Instrum. Methods 203, 1 (1982)

14. B.H. Wiik, Nucl. Instrum. Methods 56, 197 (1967)

15. E.O. Lawrence, N.E. Edlefsen, Science 72, 378 (1930)

16. E.O. Lawrence, M.S. Livingston, Phys. Rev. 40, 19 (1932)

17. V.I. Veksler, DAN(USSR) 44, 393 (1944)

18. E.M. McMillan, Phys. Rev. 68, 143 (1945)

19. J.R. Richardson, K.R. MacKenzie, E.J. Lofgren, B.T. Wright, Phys. Rev. 69, 669 (1946) 
20. W.M. Brobeck, E.O. Lawrence, K.R. MacKenzie, E.M. McMillan, R. Serber, D.C. Sewell, K.M. Simpson, R.L. Thornton, Phys. Rev. 71, 449 (1947)

21. D. Bohm, L. Foldy, Phys. Rev. 72, 649 (1947)

22. L.H. Thomas, Phys. Rev. 54, 580 (1938)

23. 1-2 gev synchrotron radiation source, conceptual design report. Technical Report PUB-5172 Rev., Lawrence Berkeley Laboratory, UCB, Berkeley (1986)

24. M.S. Livingston, J.P. Blewett, G.K. Green, L.J. Haworth, Rev. Sci. Instrum. 21, 7 (1950)

25. N. Christofilos, US Patent No 2,736,766 (1950)

26. E.D. Courant, M.S. Livingston, H.S. Snyder, Phys. Rev. 88, 1190 (1952) 
Part II

Tools We Need 


\section{Chapter 4 \\ Elements of Classical Mechanics*}

Based on d'Alembert's principle, we formulate Hamilton's integral principle by defining a function $L=L\left(q_{i}, \dot{q}_{i}, t\right)$ such that for any mechanical system the variation of the integral $\int_{t_{0}}^{t_{1}} L \mathrm{~d} t$ vanishes along any real path (Fig. 4.1) so that

$$
\delta \int_{t_{0}}^{t_{1}} L(t) \mathrm{d} t=0
$$

Here, the variables $\left(q_{i}, \dot{q}_{i}, t\right)$ are the coordinates and velocities, respectively, and $t$ is the independent variable time. We may expand this function and get

$$
\delta \int_{t_{0}}^{t_{1}} L \mathrm{~d} t=\int \sum_{i} \frac{\partial L}{\partial q_{i}} \delta q_{i} \mathrm{~d} t+\int \sum_{i} \frac{\partial L}{\partial \dot{q}_{i}} \delta \dot{q}_{i} \mathrm{~d} t
$$

The second term can be modified using the assumption of the variational theorem which requires that $\delta q_{i}=0$ at the beginning and end of the path. The second term can be integrated by parts and is

$$
\int \frac{\partial L}{\partial \dot{q}_{i}} \delta \dot{q}_{i} \mathrm{~d} t=\int \frac{\partial L}{\partial \dot{q}_{i}} \frac{\mathrm{d}}{\mathrm{d} t} \delta q_{i} \mathrm{~d} t=\underbrace{\left.\frac{\partial L}{\partial \dot{q}_{i}} \frac{\mathrm{d}}{\mathrm{d} t} \delta q_{i}\right|_{t_{0}} ^{t_{1}}}_{=0}-\int \frac{\mathrm{d}}{\mathrm{d} t} \frac{\partial L}{\partial \dot{q}_{i}} \delta q_{i} \mathrm{~d} t .
$$

Both terms can now be combined for

$$
\delta \int_{t_{0}}^{t_{1}} L \mathrm{~d} t=\int \sum_{i}\left(\frac{\partial L}{\partial q_{i}}-\frac{\mathrm{d}}{\mathrm{d} t} \frac{\partial L}{\partial \dot{q}_{i}}\right) \delta q_{i} \mathrm{~d} t=0 .
$$

This chapter has been made Open Access under a CC BY 4.0 license. For details on rights and licenses please read the Correction https://doi.org/10.1007/978-3-319-18317-6_28 
Fig. 4.1 Variational principle

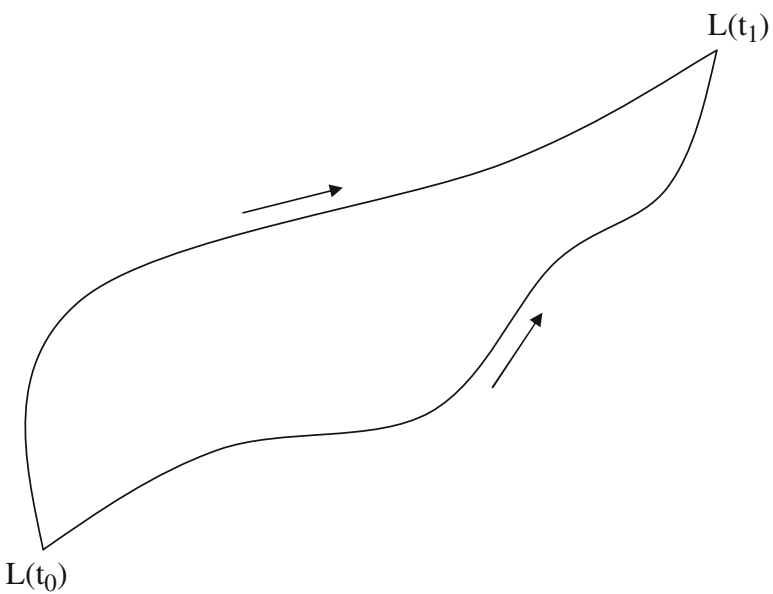

This integral is zero for any arbitrary path if and only if the integrand vanishes for each component ${ }_{i}$ independently. The resulting equations are called the EulerLagrange equations

$$
\frac{\mathrm{d}}{\mathrm{d} t} \frac{\partial L}{\partial \dot{q}_{i}}-\frac{\partial L}{\partial q_{i}}=0 .
$$

Bypassing a more accurate discussion [1], we guess at the nature of the EulerLagrange equations by considering a falling mass $m$. The kinetic energy is $T=$ $\frac{1}{2} m v^{2}$ and the potential energy $V=g x$, where $g$ is the gravitational force. If we set $L=T-V=\frac{1}{2} m v^{2}-g x$ and apply (4.5), we get $m \dot{v}=g$ which is the well known classical equation of motion for a falling mass in a gravitational field. The time independent Lagrangian can be defined by

$$
L=T-V
$$

and the Lagrange function therefore has the dimension of an energy. Furthermore, in analogy with basic mechanics like a falling mass, we can define the momenta of a system by

$$
P_{i}=\frac{\partial L}{\partial \dot{q}_{i}}
$$

and call them the generalized canonical momenta. We use a capital $P$ for the canonical momentum to distinguish it from the ordinary momentum $p$. Both are different only when electromagnetic fields are involved. 


\subsection{How to Formulate a Lagrangian?}

To formulate an expression for the Lagrangian is a creative process of physics. Whatever expression one might propose, it should be independent of a particular reference system and therefore Lorentz invariant. Earlier, we have learned that the product of two 4-vectors is Lorentz invariant and the product of two, not necessarily different, 4-vectors is therefore a good choice to form a Lagrangian. We investigate, for example, the product of the momentum-energy $\left(c p_{x}^{*}, c p_{y}^{*}, c p_{z}^{*}, \mathrm{i} E^{*}\right)=$ $\left(0,0,0, \mathrm{i} m c^{2}\right)$ and the differential space-time 4-vectors $\left(\mathrm{d} x^{*}, \mathrm{~d} y^{*}, \mathrm{~d} z^{*}, \mathrm{i} c \mathrm{~d} \tau\right)$ in the particle rest frame and get

$$
\frac{1}{c}\left(\mathrm{~d} x^{*}, \mathrm{~d} y^{*}, \mathrm{~d} z^{*}, \mathrm{i} c \mathrm{~d} \tau\right)\left(c p_{x}^{*}, c p_{y}^{*}, c p_{z}^{*}, \mathrm{i} E^{*}\right)=-m c^{2} \mathrm{~d} \tau=-m c^{2} \sqrt{1-\beta^{2}} \mathrm{~d} t .
$$

This expression has the dimension of an energy and is Lorentz invariant. We consider therefore this as the Lagrangian for a particle at rest being observed from a relatively moving laboratory system

$$
L=-m c^{2} \sqrt{1-\beta^{2}} .
$$

The conjugate momentum is from (4.7) for the $x$-component

$$
P_{x}=-m \frac{-v_{x}}{\sqrt{1-\beta^{2}}}=\gamma m v_{x}
$$

and the equation of motion $\frac{\mathrm{d}}{\mathrm{d} t} \frac{\partial L}{\partial v_{x}}-\frac{\partial L}{\partial x}$ becomes

$$
\frac{\mathrm{d} P_{x}}{\mathrm{~d} t}=0
$$

indicating that the particle is in uniform motion with velocity $\beta$.

The Lagrangian (4.9) is consistent with classical experience if we set $\beta \ll 1$ and $L=-m c^{2} \sqrt{1-\beta^{2}} \approx-m c^{2}+\frac{1}{2} m v^{2}$. Since we use only derivatives of the Lagrangian, we may ignore the constant $-m c^{2}$ and end up with the kinetic energy of the free particle.

\subsubsection{The Lagrangian for a Charged Particle in an EM-Field}

The interaction between charged particle and electromagnetic field depends only on the particle charge and velocity and on the field. We try therefore the product of field and velocity 4 -vector. Formulating this product in the laboratory system, where the 
fields have been generated, we get.

$$
e\left(A_{x}, A_{y}, A_{z}, \mathrm{i} \phi\right) \gamma\left(v_{x}, v_{y}, v_{z}, \mathrm{i}\right)=e \gamma(\boldsymbol{A} \boldsymbol{v}-\phi) .
$$

Noting that $\gamma \mathrm{d} \tau=\mathrm{d} t$, the extension to the Lagrange function in the presence of electromagnetic fields is

$$
L=-m c^{2} \sqrt{1-\beta^{2}}+e A v-e \phi .
$$

The canonical momentum is from (4.7)

$$
\boldsymbol{P}=\frac{m \boldsymbol{v}}{\sqrt{1-\beta^{2}}}+e \boldsymbol{A}=\gamma m \boldsymbol{v}+e \boldsymbol{A}=\boldsymbol{p}+e \boldsymbol{A},
$$

where $\boldsymbol{p}$ is the ordinary momentum. Equation (4.13) is consistent with $L=T-V$, where the potential $V=e \phi-e A v$.

\subsection{Lorentz Force}

The conjugate momenta in Cartesian coordinates $\boldsymbol{r}=(x, y, z)$ can be derived from (4.5) with (4.13)

$$
\dot{\boldsymbol{P}}=\frac{\partial L}{\partial \boldsymbol{r}}=e \boldsymbol{\nabla}(\boldsymbol{A v})-e \boldsymbol{\nabla} \phi=e(\boldsymbol{v} \nabla) \boldsymbol{A}+e[\boldsymbol{v} \times(\nabla \times \boldsymbol{A})]-e \boldsymbol{\nabla} \phi,
$$

where we used the algebraic relation (A.18). Insertion into

$$
\frac{\mathrm{d}}{\mathrm{d} t} \frac{\partial L}{\partial \dot{\boldsymbol{r}}}=\frac{\mathrm{d} \boldsymbol{P}}{\mathrm{d} t}=\frac{\mathrm{d}}{\mathrm{d} t}(\boldsymbol{p}+e \boldsymbol{A})=e(\boldsymbol{v} \nabla) \boldsymbol{A}+e[\boldsymbol{v} \times(\nabla \times \boldsymbol{A})]-e \boldsymbol{\nabla} \phi
$$

results with $\dot{\boldsymbol{r}}=\boldsymbol{v}$ and $\frac{\mathrm{d} \boldsymbol{A}}{\mathrm{d} t}=\frac{\partial \boldsymbol{A}}{\partial t}+(\boldsymbol{v} \boldsymbol{\nabla}) \boldsymbol{A}$ in an expression for the ordinary momentum $\boldsymbol{p}$

$$
\frac{\mathrm{d} \boldsymbol{p}}{\mathrm{d} t}=-e \frac{\partial \boldsymbol{A}}{\partial t}+e[\boldsymbol{v} \times(\nabla \times \boldsymbol{A})]-e \nabla \phi
$$

Converting potentials to fields, we recover the Lorentz force $\boldsymbol{F}_{\mathrm{L}}=\frac{\mathrm{d} \boldsymbol{p}}{\mathrm{d} t}$ or

$$
\boldsymbol{F}_{\mathrm{L}}=e \boldsymbol{E}+e(\boldsymbol{v} \times \boldsymbol{B}) .
$$




\subsection{Frenet-Serret Coordinates}

A particle trajectory follows a path described by

$$
\boldsymbol{r}(z)=\boldsymbol{r}_{0}(z)+\delta \boldsymbol{r}(z)
$$

Here $\boldsymbol{r}_{0}(z)$ is the ideal path for beam dynamics and an orthogonal coordinate system moves along this path with its origin at $\boldsymbol{r}_{0}(z)$ as shown in Fig. 4.2. For this FrenetSerret coordinate system we define three vectors

$$
\begin{array}{ll}
\boldsymbol{u}_{x}(z) & \text { unit vector } \perp \text { to trajectory } \\
\boldsymbol{u}_{z}(z)=\frac{\mathrm{d} \boldsymbol{r}_{0}(z)}{\mathrm{d} z} & \text { unit vector } \| \text { to trajectory } \\
\boldsymbol{u}_{y}(z)=\boldsymbol{u}_{z}(z) \times \boldsymbol{u}_{x}(z) & \text { unit binormal vector }
\end{array}
$$

to form an orthogonal coordinate system moving along the trajectory with a reference particle at $\boldsymbol{r}_{0}(z)$. In beam dynamics, we identify the plane defined by vectors $\boldsymbol{u}_{x}$ and $\boldsymbol{u}_{z}(z)$ as the horizontal plane and the plane orthogonal to it as the vertical plane, parallel to $\boldsymbol{u}_{y}$. Change in vectors are determined by curvatures.

$$
\frac{\mathrm{d} \boldsymbol{u}_{x}(z)}{\mathrm{d} z}=\kappa_{x} \boldsymbol{u}_{z}(z), \quad \text { and } \quad \frac{\mathrm{d} \boldsymbol{u}_{y}(z)}{\mathrm{d} z}=\kappa_{y} \boldsymbol{u}_{z}(z),
$$

where $\left(\kappa_{x}, \kappa_{y}\right)$ are the curvatures in the horizontal and vertical plane respectively. The particle trajectory can now be described by

$$
\boldsymbol{r}(x, y, z)=\boldsymbol{r}_{0}(z)+x(z) \boldsymbol{u}_{x}(z)+y(z) \boldsymbol{u}_{y}(z),
$$

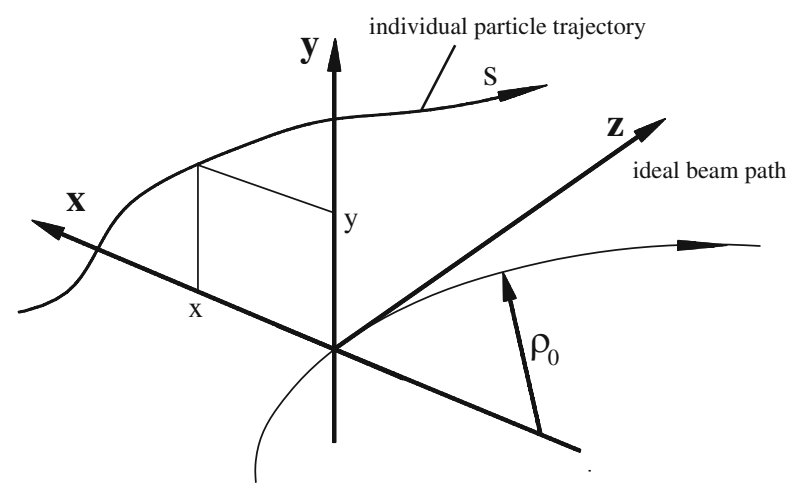

Fig. 4.2 Frenet-Serret coordinate system 
where $\boldsymbol{r}_{0}(z)$ is the location of the coordinate system's origin (reference particle) and $(x, y)$ are the deviations of a particular particle from $\boldsymbol{r}_{0}(z)$. The derivative with respect to $z$ is then

$$
\frac{\mathrm{d}}{\mathrm{d} z} \boldsymbol{r}(x, y, z)=\frac{\mathrm{d} \boldsymbol{r}_{0}}{\mathrm{~d} z}+x(z) \frac{\mathrm{d} \boldsymbol{u}_{x}(z)}{\mathrm{d} z}+y(z) \frac{\mathrm{d} \boldsymbol{u}_{y}(z)}{\mathrm{d} z}+x^{\prime}(z) \boldsymbol{u}_{x}(z)+y^{\prime}(z) \boldsymbol{u}_{y}(z)
$$

or with (4.19) and (4.20)

$$
\mathrm{d} \boldsymbol{r}=\boldsymbol{u}_{x} \mathrm{~d} x+\boldsymbol{u}_{y} \mathrm{~d} y+\boldsymbol{u}_{z} h \mathrm{~d} z
$$

where

$$
h=1+\kappa_{0 x} x+\kappa_{0 y} y .
$$

Using these Frenet-Serret coordinates, we are able to describe particle trajectories much more efficient than we could do in Cartesian coordinates. Essentially, we have transformed away the ideal path or the geometry of the design beam transport line which is already well known to us from the placement of beam guidance elements. The new coordinates measure directly the deviation of any particles from the reference particle.

We may use these relations to introduce a transformation, from the Cartesian coordinate system to curvilinear Frenet-Serret coordinates, in the Lagrangian $L=-m c^{2} \sqrt{1-\beta^{2}}+e \dot{\boldsymbol{r}} \boldsymbol{A}-e \phi$. In the new coordinates, $\sqrt{1-\beta^{2}}=$ $\sqrt{1-\frac{1}{c^{2}}\left(\dot{x}^{2}+\dot{y}^{2}+h^{2} \dot{z}^{2}\right)}, \dot{\boldsymbol{r}} A=\dot{x} A_{x}+\dot{y} A_{y}+h \dot{z} A_{z}$ and the Lagrangian becomes in curvilinear coordinates of beam dynamics

$$
L=-m c^{2} \sqrt{1-\frac{1}{c^{2}}\left(\dot{x}^{2}+\dot{y}^{2}+h^{2} \dot{z}^{2}\right)}+e\left(\dot{x} A_{x}+\dot{y} A_{y}+h \dot{z} A_{z}\right)-e \phi .
$$

\subsection{Hamiltonian Formulation}

Like any other mechanical system, particle beam dynamics in the presence of external electromagnetic fields can be described and studied very generally through the Hamiltonian formalism. The motion of particles in beam transport systems, expressed in normalized coordinates, is that of a harmonic oscillator and deviations caused by nonlinear restoring forces appear as perturbations of the harmonic oscillation. Such systems have been studied extensively in the past and powerful mathematical tools have been developed to describe the dynamics of harmonic oscillators under the influence of perturbations. Of special importance is the Hamiltonian formalism which we will apply to the dynamics of charged particles. Although this theory is well documented in many text books, for example in [1, 2], we will shortly recall the Hamiltonian theory with special attention to the application in charged particle dynamics. 
The canonical variables in the Hamiltonian theory are the coordinates and momenta rather than coordinates and velocities used in the Lagrangian. We use a coordinate transformation $\left(q_{i}, \dot{q}_{i}, t\right) \Longrightarrow\left(q_{i}, P_{i}, t\right)$ through the definition of the momenta $P_{i}=\partial L / \partial \dot{q}_{i}$ and define the Hamiltonian function by

$$
H\left(q_{i}, p_{i}\right)=\sum \dot{q}_{i} P_{i}-L\left(q_{i}, \dot{q}_{i}\right)
$$

In analogy to the Lagrangian, we find that $\dot{q}_{i} P_{i}=2 T$ and the Hamiltonian which does not depend on the time explicitly is therefore the sum of kinetic and potential energy

$$
H=T+V
$$

This will become useful later since we often know forces acting on particles which can be derived from a potential. Similar to the Euler-Lagrange equations, we define Hamiltonian equations by

$$
\frac{\partial H}{\partial q_{i}}=-\dot{P}_{i}, \quad \text { and } \quad \frac{\partial H}{\partial P_{i}}=+\dot{q}_{i} .
$$

With $L=-m c^{2} \sqrt{1-\beta^{2}}+e \boldsymbol{A} \boldsymbol{v}-e \phi$ and replacing velocities with momenta the Hamiltonian becomes

$$
H\left(q_{i}, P_{i}\right)=\sum \dot{q}_{i} P_{i}+m c^{2} \sqrt{1-\beta^{2}}-e \boldsymbol{A} \dot{\boldsymbol{q}}+e \phi,
$$

where $\boldsymbol{q}=\left(q_{1}, q_{2}, . ., q_{i}, ..\right)$ and $\boldsymbol{A}=\left(A_{1}, A_{2}, . ., A_{i}, ..\right)$, etc. and the canonical momentum is defined in (4.14). The canonical momentum $\boldsymbol{P}$ is from (4.14) the combination of the ordinary particle momentum $\boldsymbol{p}=\gamma m \dot{\boldsymbol{q}}$ and field momentum $e \boldsymbol{A}$. Insertion into the Hamiltonian and reordering gives $(H-e \phi)^{2}=m^{2} c^{4}+$ $c^{2}(\boldsymbol{P}-e \boldsymbol{A})^{2}$, or

$$
c^{2}(\boldsymbol{P}-e \boldsymbol{A})^{2}-(H-e \phi)^{2}=-m^{2} c^{4},
$$

The Hamiltonian (4.30) is equal to the square of the length of the energy momentum 4-vector $[c \boldsymbol{P}, \mathrm{i} E]$, where $E=H-e \phi$, and is therefore Lorentz invariant. A more familiar form is

$$
H=e \phi+\sqrt{c^{2}(\boldsymbol{P}-e \boldsymbol{A})^{2}+m^{2} c^{4}} .
$$

In nonrelativistic mechanics, the Hamiltonian becomes with $\beta \ll 1$ and ignoring the constant $m c^{2}$

$$
H_{\text {class }} \approx \frac{1}{2} m v^{2}+e \phi,
$$

which is the sum of kinetic and potential energy. 


\subsubsection{Cyclic Variables}

The solution of the equations of motion become greatly simplified in cases, where the Hamiltonian does not depend on one or more of the coordinates or momenta. In this case one or more of the Hamiltonian equations (4.28) are zero and the corresponding conjugate variables are constants of motion. Of particular interest for particle dynamics or harmonic oscillators are the cases where the Hamiltonian does not depend on say the coordinate $q_{i}$ but only on the momenta $P_{i}$. In this case we have

$$
H=H\left(q_{1}, \ldots q_{i-1}, q_{i+1} \ldots, P_{1}, P_{2} \ldots, P_{i}, \ldots\right)
$$

and the first Hamiltonian equation becomes

$$
\frac{\partial H}{\partial q_{i}}=-\dot{P}_{i}=0 \quad \text { or } \quad P_{i}=\text { const } .
$$

Coordinates $q_{i}$ which do not appear in the Hamiltonian are called cyclic coordinates and their conjugate momenta are constants of motion. From the second Hamiltonian equation we get with $P_{i}=$ const.

$$
\frac{\partial H}{\partial p_{i}}=\dot{q}_{i}=a_{i}=\text { const },
$$

which can be integrated immediately for

$$
q_{i}(t)=a_{i} t+c_{i},
$$

where $c_{i}$ is the integration constant. It is obvious that the complexity of a mechanical system can be greatly reduced if by a proper choice of canonical variables some or all dependence of the Hamiltonian on space coordinates can be eliminated. We will derive the formalism that allows the transformation of canonical coordinates into new ones, where some of them might be cyclic.

Example: Assume that the Hamiltonian does not depend explicitly on the time, then $\frac{\partial H}{\partial t}=0$ and the momentum conjugate to the time is a constant of motion.

From the second Hamilton equation, we have $\frac{\partial H}{\partial p_{i}}=\frac{\mathrm{d}}{\mathrm{d} t} t=1$ and the momentum conjugate to the time is therefore the total energy $p_{i}=H=$ const. The total energy of a system with a time independent Hamiltonian is constant and equal to the value of the Hamiltonian.

\subsubsection{Canonical Transformations}

For mechanical systems which allow in principle a formulation in terms of cyclic variables, we need to derive rules to transform one set of variables to another 
set, while preserving their property of being conjugate variables appropriate to formulate the Hamiltonian for the system. In other words, the coordinate transformation must preserve the variational principle (4.1). Such transformations are called canonical transformations $\bar{q}_{k}=f_{k}\left(q_{i}, P_{i}, t\right)$ and $\bar{P}_{k}=g_{k}\left(q_{i}, P_{i}, t\right)$, where $\left(q_{i}, P_{i}, t\right)$ are the old and $\left(\bar{q}_{k}, \bar{P}_{k}, t\right)$ the new coordinates. The variational principle reads now

$$
\delta \int\left(\sum_{k} \dot{q}_{k} P_{k}-H\right) \mathrm{d} t=0 \quad \text { and } \quad \delta \int\left(\sum_{k} \dot{\bar{q}}_{k} \bar{P}_{k}-\bar{H}\right) \mathrm{d} t=0 .
$$

The new Hamiltonian $\bar{H}$ need not be the same as the old Hamiltonian $H$ nor need both integrands be the same. Both integrands can differ, however, only by a total time derivative of an otherwise arbitrary function $G$

$$
\sum_{k} \dot{q}_{k} P_{k}-H=\sum_{k} \dot{\bar{q}}_{k} \bar{P}_{k}-\bar{H}+\frac{\mathrm{d} G}{\mathrm{~d} t} .
$$

After integration $\int \frac{\mathrm{d} G}{\mathrm{~d} t} \mathrm{~d} t$ becomes a constant and the variation of the integral obviously vanishes under the variational principle (Fig. 4.1). The arbitrary function $G$ is called the generating function and may depend on some or all of the old and new variables

$$
G=G\left(q_{k}, \bar{q}_{k}, P_{k}, \bar{P}_{k}, t\right) \quad \text { with } \quad 0 \leq k \leq N
$$

The generating functions are functions of only $2 N$ variables, coordinates and momenta. Of the $4 N$ variables only $2 N$ are independent because of another $2 N$ transformation equations (4.36). We may now choose any two of four variables to be independent keeping only in mind that one must be an old and one a new variable. Depending on our choice for the independent variables, the generating function may have one of four forms

$$
\begin{array}{ll}
G_{1}=G_{1}(q, \bar{q}, t), & G_{3}=G_{3}(P, \bar{q}, t), \\
G_{2}=G_{2}(q, \bar{P}, t), & G_{4}=G_{4}(P, \bar{P}, t),
\end{array}
$$

where we have set $q=\left(q_{1}, q_{2}, \ldots q_{N}\right)$ etc. We take, for example, the generating function $G_{1}$, insert the total time derivative

$$
\frac{\mathrm{d} G_{1}}{\mathrm{~d} t}=\sum_{k} \frac{\partial G_{1}}{\partial q_{k}} \frac{\partial q_{k}}{\partial t}+\sum_{k} \frac{\partial G_{1}}{\partial p_{k}} \frac{\partial P_{k}}{\partial t}+\frac{\partial G_{1}}{\partial t}
$$

in (4.37) and get after some sorting

$$
\sum_{k} \dot{q}_{k}\left(P_{k}-\frac{\partial G_{1}}{\partial q_{k}}\right)-\sum_{k} \dot{\bar{q}}_{k}\left(\bar{P}_{k}+\frac{\partial G_{1}}{\partial \bar{q}_{k}}\right)-\left(H-\bar{H}+\frac{\partial G_{1}}{\partial t}\right)=0 .
$$


Both, old and new variables are independent and the expressions in the brackets must therefore vanish separately leading to the defining equations

$$
P_{k}=\frac{\partial G_{1}}{\partial q_{k}}, \quad \bar{P}_{k}=-\frac{\partial G_{1}}{\partial \bar{q}_{k}}, \quad H=\bar{H}-\frac{\partial G_{1}}{\partial t} .
$$

Variables for which (4.42) hold are called canonical variables and the transformations (4.36) are called canonical.

Generating functions for other pairings of new and old canonical variables can be obtained from $G_{1}$ by Legendre transformations of the form

$$
G_{2}(q, \bar{P}, t)=G_{1}(q, \bar{q}, t)+q \bar{P} .
$$

Equations (4.42) can be expressed in a general form for all four different types of generating functions. We write the general generating equation as $G=$ $G\left(x_{k}, \bar{x}_{k}, t\right)$,where the variables $x_{k}$ and $\bar{x}_{k}$ can be either coordinates or momenta. Furthermore, $x_{k}$ and $\bar{x}_{k}$ are the old and new coordinates or momenta respectively and the $\left(y_{k}, \bar{y}_{k}\right)$ are the conjugate coordinates or momenta to $\left(x_{k}, \bar{x}_{k}\right)$. Then

$$
\begin{aligned}
y_{k} & = \pm \frac{\partial}{\partial x_{k}} G\left(x_{k}, \bar{x}_{k}, t\right), \\
\bar{y}_{k} & =\mp \frac{\partial}{\partial \bar{x}_{k}} G\left(x_{k}, \bar{x}_{k}, t\right), \\
H & =\bar{H}-\frac{\partial}{\partial t} G\left(x_{k}, \bar{x}_{k}, t\right) .
\end{aligned}
$$

The upper signs are to be used if the derivatives are taken with respect to coordinates and the lower signs if the derivatives are taken with respect to momenta. It is not obvious which type of generating function should be used for a particular problem. However, the objective of canonical transformations is to express the problem at hand in as many cyclic variables as possible. Any form of generating function that achieves this goal is therefore appropriate. To illustrate the use of generating functions for canonical transformations, we will discuss a few very examples. For an identity transformation we use a generating function of the form

$$
G=q_{1} \bar{P}_{1}+q_{2} \bar{P}_{2}+\ldots
$$

and get with (4.44) and $i=1,2, \ldots N$ the identities

$$
P_{i}=-\frac{\partial G}{\partial q_{i}}=\bar{P}_{i}, \quad \text { and } \quad \bar{q}_{i}=+\frac{\partial G}{\partial \bar{P}_{i}}=q_{i} .
$$


A transformation from rectangular $(x, y, z)$ to cylindrical $(r, \varphi, z)$ coordinates is defined by the generating function

$$
G(P, \bar{q})=-P_{x} r \cos \varphi-P_{y} r \sin \varphi-P_{z} z
$$

and the transformation relations are

$$
\begin{array}{ll}
x=-\frac{\partial G}{\partial p_{x}}=r \cos \varphi, & P_{r}=-\frac{\partial G}{\partial r}=+P_{x} \cos \varphi+P_{y} \sin \varphi \\
y=-\frac{\partial G}{\partial p_{y}}=r \sin \varphi, & P_{\varphi}=-\frac{\partial G}{r \partial \varphi}=-P_{x} \sin \varphi+P_{y} \cos \varphi \\
z=-\frac{\partial G}{\partial p_{z}}=z, & P_{z}=-\frac{\partial G}{\partial z}=P_{z} .
\end{array}
$$

Similarly, relations for the transformation from rectangular to polar coordinates can be derived from the generating function

$$
G=-P_{x} r \cos \varphi \sin \vartheta-P_{y} r \sin \varphi \sin \vartheta-P_{z} r \cos \vartheta
$$

It is not always obvious if a coordinate transformation is canonical. To identify a canonical transformation, we use Poisson brackets [1] defined by

$$
\left[f_{k}\left(q_{i}, P_{j}\right), g_{k}\left(q_{i}, P_{j}\right)\right]=\sum_{i}\left(\frac{\partial f_{k}}{\partial q_{i}} \frac{\partial g_{k}}{\partial P_{j}}-\frac{\partial f_{k}}{\partial P_{j}} \frac{\partial g_{k}}{\partial q_{i}}\right) \text {. }
$$

It can be shown [1] that the new variables $\bar{q}_{k}, \bar{P}_{k}$ or (4.36) are canonical if and only if the Poisson brackets

$$
\left[\bar{P}_{i}, \bar{P}_{j}\right]=0 \quad\left[\bar{q}_{i}, \bar{q}_{j}\right]=0 \quad\left[\bar{q}_{i}, \bar{P}_{j}\right]=\lambda \delta_{i j}
$$

where $\delta_{i j}$ is the Kronecker symbol and the factor $\lambda$ is a scale factor for the transformation. To preserve the scale in phase space, the scale factor must be equal to unity, $\lambda=1$. While the formalism for canonical transformation is straight-forward, we do not get a hint as to the optimum set of variables for a particular mechanical system. In the next sections we will see, however, that specific transformations have been identified and developed which prove especially useful for a whole class of mechanical systems.

\subsubsection{Curvilinear Coordinates}

The choice of a particular coordinate system, of course, must not alter the physical result and from this point of view any coordinate system could be used. However, it soon becomes clear that the pursuit of physics solutions can be mathematically much easier in one coordinate system that in another. For systems which are symmetric about a point we would use polar coordinates, for systems which are symmetric 
about a straight line we use cylindrical coordinates. In beam dynamics there is no such symmetry, but we have a series of magnets and other components aligned along some, not necessarily straight, line. The collection of these elements is what we call a beam line. The particular arrangement of elements is in most cases not determined by physics but other more practical considerations. The matter of fact is that we know about the "ideal" path and that all particle should travel along a path being defined by the physical centers of the beam line elements. In a Cartesian coordinate system fixed to the stars the result of "ideal" beam dynamics would be a complicated mathematical expression trying to describe the "ideal" path in which we have no interest, since we already know where it is. What we are interested in is the deviation a particular particle might have from the ideal path. The most appropriate coordinate system would therefore be one which moves along the ideal path. In Sect. 4.3 we have introduced such a curvilinear reference system also known as the Frenet-Serret reference system. The transformation from Cartesian to Frenet-Serret coordinates can be derived from the generating function formed from the old momenta and the new coordinates

$$
G\left(z, x, y, P_{\mathrm{c}, z}, P_{\mathrm{c}, x}, P_{\mathrm{c}, y}\right)=-\left(c \boldsymbol{P}_{\mathrm{c}}-e c \boldsymbol{A}_{\mathrm{c}}\right)\left[\boldsymbol{r}_{0}(z)+x \boldsymbol{u}_{x}(z)+y \boldsymbol{u}_{y}(z)\right] .
$$

The momenta and fields in the old Cartesian coordinate system are designated with the index ${ }_{\mathrm{c}}$ and the new canonical momenta $P$ in the Frenet-Serret system are then in both systems while noting that the transverse momenta are the same

$$
\begin{aligned}
& \left(c P_{z}-e c A_{z} h\right)=-\frac{\partial G}{\partial z}=\left(c P_{z}-e c A_{z}\right)_{c} h \\
& \left(c P_{x}-e c A_{x}\right)=-\frac{\partial G}{\partial x}=\left(c P_{x}-e c A_{x}\right)_{c} \\
& \left(c P_{y}-e c A_{y}\right)=-\frac{\partial G}{\partial y}=\left(c P_{y}-e c A_{y}\right)_{c}
\end{aligned}
$$

with $h$ as defined in (4.24). The Hamiltonian $H_{\mathrm{c}}=e \phi+c \sqrt{m^{2} c^{2}+(\boldsymbol{P}-e \boldsymbol{A})_{\mathrm{c}}^{2}}$ in Cartesian coordinates transforms to the one in curvilinear coordinates of beam dynamics

$$
H=e \phi+c \sqrt{m^{2} c^{2}+\frac{\left(P_{z}-e A_{z} h\right)^{2}}{h^{2}}+\left(P_{x}-e A_{x}\right)^{2}+\left(P_{y}-e A_{y}\right)^{2}} .
$$

For a particle travelling through a uniform field $B_{y}$, we have $\boldsymbol{A}=\left(0,0, A_{z}\right)=$ $\left(0,0,-B_{y} x\right), P_{x, y}=p_{x, y}$, and the Hamiltonian is with $A_{z}=A_{\mathrm{c}, z} h$

$$
H_{\mathrm{h}}=e \phi+c \sqrt{m^{2} c^{2}+p_{x}^{2}+p_{y}^{2}+\frac{1}{h^{2}}\left(P_{z}+e B_{y} h x\right)^{2}} .
$$


The distinction, we make here on fields in curvilinear and Cartesian coordinates stems from the practice to build magnets in a certain way. Dipole magnets are designed carefully to have a uniform field in the beam area along the curved path, which is not consistent with the transformation of a uniform dipole field in Cartesian coordinates.

\subsubsection{Extended Hamiltonian}

The Hamiltonian as derived so far depends on the canonical variables $\left(q_{i}, P_{i}\right)$ and the independent variable $t$ or $z$ defined for individual particles. This separate treatment of the independent variable can be eliminated by formulating an extended Hamiltonian in which all coordinates are treated the same.

Starting with $H\left(q_{1}, q_{2} \ldots q_{\mathrm{f}}, P_{1}, P_{2}, P_{3} \ldots P_{\mathrm{f}}, t\right)$, we introduce the independent variables $\left(q_{0}, P_{0}\right)$ by setting

$$
q_{0}=t \quad \text { and } \quad P_{0}=-H
$$

and obtain a new Hamiltonian

$$
\mathcal{H}\left(q_{0}, q_{1}, q_{2} \ldots q_{\mathrm{f}}, P_{0}, P_{1}, P_{2}, P_{3} \ldots P_{\mathrm{f}}\right)=H+P_{0}=0
$$

and Hamilton's equations are then

$$
\left.\begin{array}{c}
\frac{\mathrm{d} q_{i}}{\mathrm{~d} t}=\frac{\partial \mathcal{H}}{\partial P_{i}} \\
\frac{\mathrm{d} P_{i}}{\mathrm{~d} t}=-\frac{\partial \mathcal{H}}{\partial q_{i}}
\end{array}\right\} \quad \text { for } \quad i=0,1,2 \ldots
$$

In particular for $i=0$ the equations are

$$
\frac{\mathrm{d} q_{0}}{\mathrm{~d} t}=1 \rightarrow q_{0}=t+C_{1}
$$

and

$$
\frac{\mathrm{d} P_{0}}{\mathrm{~d} t}=-\frac{\partial \mathcal{H}}{\partial q_{0}}=-\frac{\mathrm{d} \mathcal{H}}{\mathrm{d} t} \quad \Longrightarrow \quad P_{0}=-\mathcal{H}+C_{2}
$$

The momentum conjugate to the time is equal to the Hamiltonian and since $\mathcal{H} \neq$ $\mathcal{H}(t)$ for static fields, it follows that

$$
\frac{\mathrm{d} P_{0}}{\mathrm{~d} t}=0 \quad \Longrightarrow \quad \mathcal{H}=\text { const. }
$$


Now, the independent variable is no more distinguishable from all other coordinates, the Hamiltonian is expressed as a function of coordinates and momenta only.

\subsubsection{Change of Independent Variable}

Since no particular coordinate is designated as the independent variable, we may use any of the coordinates as that. For example, we prefer often to use the longitudinal coordinate $z$ as the independent variable rather than the time $t$. More generally, consider to change the independent variable from $q_{i}$ to $q_{j}$. Defining, for example, $q_{3}$ as the new independent variable, we solve $\mathcal{H}$ for $P_{3}$

$$
P_{3}=-K\left(q_{0}, q_{1}, q_{2}, q_{3} \ldots q_{\mathrm{f},}, P_{0}, P_{1}, P_{2}, P_{4}, \ldots P_{\mathrm{f}}\right)
$$

and define a new extended Hamiltonian

$$
\mathcal{K}=P_{3}+K=0 .
$$

Then the equations

$$
\begin{gathered}
\frac{\partial \mathcal{K}}{\partial P_{3}}=\frac{\mathrm{d} q_{3}}{\mathrm{~d} q_{3}}=1, \\
-\frac{\partial \mathcal{K}}{\partial q_{3}}=\frac{\mathrm{d} P_{3}}{\mathrm{~d} q_{3}}=-\frac{\partial K}{\partial q_{3}}, \\
\frac{\partial \mathcal{K}}{\partial P_{i \neq 3}}=\frac{\mathrm{d} q_{i \neq 3}}{\mathrm{~d} q_{3}}=\frac{\partial K}{\partial P_{i \neq 3}}, \\
-\frac{\partial \mathcal{K}}{\partial q_{i \neq 3}}=\frac{\mathrm{d} P_{i \neq 3}}{\mathrm{~d} P_{3}}=-\frac{\partial K}{\partial q_{i \neq 3}}
\end{gathered}
$$

with the Hamiltonian

$$
K=-p_{3}
$$

As an example, to use the longitudinal coordinate $z$ rather than the time $t$ as the independent variable, we start with (4.54)

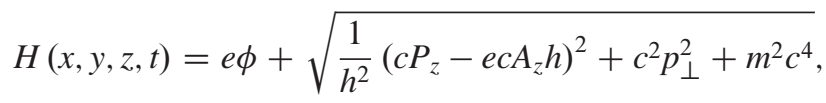


where $p_{\perp}^{2}=p_{x}^{2}+p_{y}^{2}$. The longitudinal momentum is

$$
c P_{z}=c e A_{z} h+h \sqrt{(H-e \phi)^{2}-\left(c p_{\perp}\right)^{2}-m^{2} c^{4}}=c e A_{z} h+h \sqrt{c^{2} p^{2}-c^{2} p_{\perp}^{2}},
$$

where $E^{2}=(H-e \phi)^{2}=(c p)^{2}+\left(m c^{2}\right)^{2}$ has been used. We further normalize to the momentum $p$ and use trajectory slopes, $x^{\prime}=\mathrm{d} x / \mathrm{d} z=p_{x} / p_{z}$ etc. rather than momenta. With this, the new Hamiltonian is $K\left(x, x^{\prime}, y, y^{\prime}, z\right)=-P_{z} / p$ or using $P_{z} / p=e A_{z} / p+h \sqrt{1-p_{\perp}^{2} / p^{2}}$ and $p_{\perp}^{2} / p^{2} \approx x^{\prime 2}+y^{\prime 2}$

$$
K\left(x, x^{\prime}, y, y^{\prime}, z\right)=-\frac{e A_{z} h}{p}-h \sqrt{1-x^{\prime 2}-y^{\prime 2}} .
$$

In beam dynamics, we restrict ourselves to paraxial beams, where $x^{\prime} \ll 1$ and $y^{\prime} \ll 1$, and the momentum $p \approx p_{z}$. Note, $\boldsymbol{p}$ may not be the canonical momentum if there is an electromagnetic field present, but $\boldsymbol{P}=\boldsymbol{p}+\boldsymbol{e A}$ is canonical. In this last step, we seem to have lost terms involving transverse vector potential components. This meets with the requirements of almost all beam transport lines, where we use predominantly transverse fields which can be derived from the $A_{z}$-component only. This is not true when we consider, for example, solenoid fields which occur rather seldom and will be treated separately as perturbations. Finally, we separate the ideal particle momentum $p_{0}$ from the momentum deviation $\delta=\Delta p / p_{0}$ and while ignoring higher order terms in $\delta$ replace $1 / p=1 /\left[p_{0}(1+\delta)\right] \approx \frac{1}{p_{0}}(1-\delta)$ in the Hamiltonian for

$$
K\left(x, x^{\prime}, y, y^{\prime}, z\right) \approx-\frac{e A_{z} h}{p_{0}}(1-\delta)-h \sqrt{1-x^{\prime 2}-y^{\prime 2}} .
$$

As discussed before, magnetic fields for particle beam dynamics can be derived from a single component $A_{z}$ of the vector potential and the task to determine equations of motion is now reduced to that of determining the vector potential for the magnets in use. The equations of motion are from (4.69)

$$
\begin{aligned}
& \frac{\partial K}{\partial x}=-x^{\prime \prime}=-\frac{e c}{c p_{0}} \frac{\partial A_{z} h}{\partial x}(1-\delta)-\kappa_{0 x} \sqrt{1-x^{\prime 2}-y^{\prime 2}}, \\
& \frac{\partial K}{\partial y}=-y^{\prime \prime}=-\frac{e c}{c p_{0}} \frac{\partial A_{z} h}{\partial y}(1-\delta)-\kappa_{0 y} \sqrt{1-x^{\prime 2}-y^{\prime 2}} .
\end{aligned}
$$

With $h B_{y}=-\frac{\partial A_{z} h}{\partial x}$ and $h B_{x}=\frac{\partial A_{z} h}{\partial y}$ the equations of motion become finally in paraxial approximation

$$
\begin{aligned}
& x^{\prime \prime}+\frac{e c}{c p_{0}} B_{y} h(1-\delta)-\kappa_{0 x}=0, \\
& y^{\prime \prime}-\frac{e c}{c p_{0}} B_{x} h(1-\delta)-\kappa_{0 y}=0 .
\end{aligned}
$$


These are the equations of motion in curvilinear coordinates under the influence of the magnetic field $\left(B_{x}, B_{y}\right)$.

\section{Problems}

4.1 (S). Show that the Hamiltonian transforms like $\mathcal{H}_{\varphi}=\frac{\mathrm{d} t}{\mathrm{~d} \varphi} \mathcal{H}_{t}$, if the independent variable is changed from $t$ to $\varphi$.

4.2 (S). Derive from the Lagrangian (4.25) the equation of motion.

4.3. Show that the transformations [a.), c.) for upper signs, d.) for $\epsilon=0$ ] are canonical and $[\mathrm{b}),. \mathrm{c}$.) for lower signs, d.) for $\epsilon \neq 0]$ are not:
a.) $\begin{aligned} & q_{1}=x_{1} p_{1}=\dot{x}_{1} \\ & q_{2}=x_{2} p_{2}=\dot{x}_{2}\end{aligned}$
b.) $q=r \cos \psi, \quad p=r \sin \psi$
c.) $\begin{aligned} & q_{1}=x_{1}, \quad p_{1}=\dot{x}_{1} \pm \dot{x}_{2}, \\ & q_{2}=x_{1} \pm x_{2}, p_{2}=\dot{x}_{2}\end{aligned}$
d.) $q=q_{0} e^{\epsilon}, \quad p=p_{0} e^{\epsilon}$

Show the formalism you use.

\section{References}

1. H. Goldstein, Classical Mechanics (Addison-Wesley, Reading, 1950)

2. L.D. Landau, E.M. Lifshitz, Mechanics (Pergamon, Oxford, 1976) 


\section{Chapter 5 \\ Particle Dynamics in Electro-Magnetic Fields}

The most obvious components of particle accelerators and beam transport systems are those that provide the beam guidance and focusing system. Whatever the application may be, a beam of charged particles is expected by design to follow closely a prescribed path along a desired beam transport line or along a closed orbit in case of circular accelerators. The forces required to bend and direct the charged particle beam or provide focusing to hold particles close to the ideal path are known as the Lorentz forces and are derived from electric and magnetic fields through the Lorentz equation.

\subsection{The Lorentz Force}

For a particle carrying a single basic unit of electrical charge the Lorentz force is

$$
\boldsymbol{F}=e \boldsymbol{E}+e[\boldsymbol{v} \times \boldsymbol{B}],
$$

where $e$ is the basic unit of electrical charge [1].

The vectors $\boldsymbol{E}$ and $\boldsymbol{B}$ are the electrical and magnetic field vectors, respectively, and $\boldsymbol{v}$ is the velocity vector of the particle. The evolution of particle trajectories under the influence of Lorentz forces is called beam dynamics or beam optics. The basic formulation of beam dynamics relies only on linear fields which are independent of or only linearly dependent on the distance of a particular particle from the ideal trajectory. The mathematical description of particle trajectories in the presence of only such linear fields is called linear beam dynamics.

The Lorentz force has two components originating from either an electrical field $\boldsymbol{E}$ or a magnetic field $\boldsymbol{B}$. For relativistic particles $(v \approx c)$ we find that the force from a magnetic field of $1 \mathrm{~T}$, for example, is equivalent to that for an electrical field of $300 \mathrm{MV} / \mathrm{m}$. Since it is technically straight forward to generate magnetic fields of the

This chapter has been made Open Access under a CC BY 4.0 license. For details on rights and licenses please read the Correction https://doi.org/10.1007/978-3-319-18317-6_28 
order of $1 \mathrm{~T}$, but rather difficult to establish the equivalent electric fields of $3 \mathrm{MV} / \mathrm{cm}$, it becomes apparent that most beam guidance and focusing elements for relativistic particle beams are based on magnetic fields. At low particle energies $(v \ll c)$ this preference is less clear and justified since the effectiveness of magnetic fields to bend particles is reduced proportional to the particle velocity $\beta=v / c$.

\subsection{Fundamentals of Charged Particle Beam Optics}

Magnetic as well as electric fields can be produced in many ways and appear in general in arbitrary directions and varying strength at different locations. It is impossible to derive a general mathematical formula for the complete path of charged particles in an arbitrary field distribution. To design particle beam transport systems, we therefore adopt some organizing and simplifying requirements on the characteristics of electro-magnetic fields used.

The general task in beam optics is to transport charged particles from point $A$ to point $B$ along a desired path. We call the collection of bending and focusing magnets installed along this ideal path the magnet lattice and the complete optical system including the bending and focusing parameters a beam transport system. Two general cases can be distinguished in beam transport systems. Systems that display neither symmetry nor periodicity and transport systems that include a symmetric or periodic array of magnets. Periodic or symmetric transport systems can be repeated an arbitrary number of times to produce longer transport lines. A specific periodic magnet lattice is obtained if the arrangement of bending magnets forms a closed loop. In our discussions of transverse beam dynamics, we will make no particular distinction between open beam transport lines and circular lattices except in such cases when we find the need to discuss special eigensolutions for closed periodic lattices. We will therefore use the terminology of beam transport systems when we discuss beam optics results applicable to both types of lattices and refer to circular accelerator lattices when we derive eigenfunctions characteristic only to periodic and closed magnet lattices.

\subsubsection{Particle Beam Guidance}

To guide a charged particle along a predefined path, magnetic fields are used which deflect particles as determined by the equilibrium of the centrifugal force and Lorentz force

$$
m \gamma v^{2} \boldsymbol{\kappa}+e[\boldsymbol{v} \times \boldsymbol{B}]=0
$$

where $\kappa=\left(\kappa_{x}, \kappa_{y}, 0\right)$ is the local curvature vector of the trajectory which is pointing in the direction of the centrifugalforce. 
We assume in general that the magnetic field vector $\boldsymbol{B}$ is oriented normal to the velocity vector $\boldsymbol{v}$. This means we restrict the treatment of linear beam dynamics to purely transverse fields. The restriction to purely transverse field components has no fundamental reason other than to simplify the formulation of particle beam dynamics. The dynamics of particle motion in longitudinal fields will be discussed in Chap. 9. As mentioned earlier, the transverse components of the particle velocities for relativistic beams are small compared to the particle velocity $v_{z},\left(v_{x} \ll v_{z}, v_{y} \ll\right.$ $\left.v_{z}, v_{z} \approx v_{s}\right)$. While we use a curvilinear coordinate system $(x, y, z)$ following the ideal path, we sometimes need to follow a particular particle trajectory for which we use the coordinate $s$. With these assumptions, the bending radius for the particle trajectory in a magnetic field is from (5.2) with $p=\gamma m v$

$$
\boldsymbol{\kappa}_{x, y}=\mp \frac{e c}{\beta E} \boldsymbol{B}_{y, x}
$$

and the angular frequency of revolution of a particle on a complete orbit normal to the field $B$ is

$$
\omega_{L}=\left|\frac{e c}{E} B\right|
$$

which is also called the cyclotron or Larmor frequency [2]. The sign in (5.3) has been chosen to meet the definition of curvature in analytical geometry where the curvature is negative if the tangent to the trajectory rotates counterclockwise. Often, the beam rigidity, defined as

$$
|B \rho|=\frac{p_{0}}{e}
$$

is used to normalize the magnet strength. Using more practical units the expressions for the beam rigidity and bending radius become

$$
B \rho(\mathrm{T} \mathrm{m})=\frac{10}{2.998} \beta E(\mathrm{GeV})
$$

and

$$
\frac{1}{\rho}\left(\mathrm{m}^{-1}\right)=\frac{B}{B \rho}=0.2998 \frac{|B(\mathrm{~T})|}{\beta E(\mathrm{GeV})},
$$

where we have dropped the sign for the bending radius. For relativistic particles this expression is further simplified since $\beta \approx 1$. The deflection angle in a magnetic field is

$$
\theta=\int \frac{\mathrm{d} z}{\rho}
$$


or for a uniform field like in a dipole magnet of arc length $\ell_{\mathrm{m}}$ the deflection angle is $\theta=\ell_{\mathrm{m}} / \rho$.

In this textbook, singly charged particles will be assumed unless otherwise noted. For multiply charged particles like ions, the electrical charge $e$ in all equations must be replaced by $e Z$ if, for example, ions of net charge $Z$ are to be considered. Since it is also customary not to quote the total ion energy, but the energy per nucleon, (5.7) becomes for ions

$$
\frac{1}{\rho}\left(\mathrm{m}^{-1}\right)=0.2998 \frac{Z}{A} \frac{|B(\mathrm{~T})|}{\beta E(\mathrm{GeV} / \mathrm{u})},
$$

where $E$ is the total energy per nucleon and $A$ the atomic mass.

Beam guiding or bending magnets and focusing devices are the most obvious elements of a beam transport system and we will shortly discus such magnets in more detail. Later, in Chap. 6, we will introduce all multipole magnets in a more formal way.

\subsubsection{Particle Beam Focusing}

Similar to the properties of light rays, particle beams also have a tendency to spread out due to an inherent beam divergence. To keep the particle beam together and to generate specifically desired beam properties at selected points along the beam transport line, focusing devices are required. In photon optics that focusing is provided by glass lenses. The characteristic property of such focusing lenses is that a light ray is deflected by an angle proportional to the distance of the ray from the center of the lens (Fig. 5.1). With such a lens a beam of parallel rays can be focused to a point and the distance of this focal point from the lens is called the focal length.

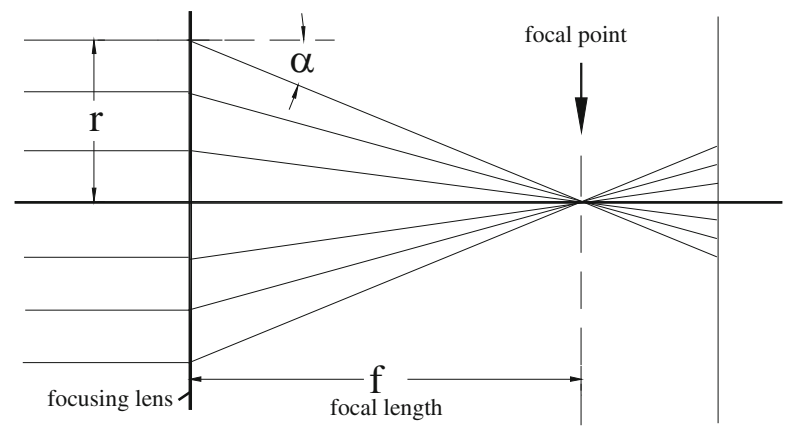

Fig. 5.1 Principle of focusing 
Any magnetic field that deflects a particle by an angle proportional to its distance $r$ from the axis of the focusing device will act in the same way as a glass lens does in the approximation of paraxial, geometric optics for visible light. If $f$ is the focal length, the deflection angle $\alpha$ is defined from Fig. 5.1 by

$$
\alpha=-\frac{r}{f}
$$

A similar focusing property can be provided for charged particle beams by the use of azimuthal magnetic fields $B_{\varphi}$ with the property

$$
\alpha=-\frac{\ell}{\rho}=-\frac{e c}{\beta E} B_{\varphi} \ell=-\frac{e c}{\beta E} g r \ell,
$$

where $\ell$ is the path length of the particle trajectory in the magnetic field $B_{\varphi}$ and $g$ is the field gradient defined by $B_{\varphi}=g r$ or by $g=\mathrm{d} B_{\varphi} / \mathrm{d} r$. Here we have assumed the length $\ell$ to be short compared to the focal length such that $r$ does not change significantly within the magnetic field. If this is not allowable, the product $B_{\varphi} \ell$ must be replaced by the integral $\int B_{\varphi} \mathrm{d} \sigma$.

To get the focusing property (5.10) we require a linear dependence on $r$ of either the magnetic field $B_{\varphi}$ or of the magnet length. We choose the magnetic field to increase linearly with the distance $r$ from the axis of the focusing device while the magnet length remains constant.

A magnetic field that provides the required focusing property of (5.11) can be found, for example, in a conductor carrying a uniform current density. Clearly, such a device does not seem very useful for particle beam focusing. To improve the "transparency" for particles, Panofsky and Baker [3] proposed to use a plasma lens "which contains a longitudinal arc of nearly uniform current density" and a similar device has been proposed in [4]. Still another variation of this concept is the idea to use an evenly distributed array of wires, called the wire lens [5], simulating a uniform longitudinal current distribution. The strength of such lenses, however, is not sufficient for focusing of high energy particles even if we ignore the obvious scattering problems. Both issues, however, become irrelevant, where focusing is required in combination with particle conversion targets. Here, for example, a Lithium cylinder, called a Lithium lens, carrying a large pulsed current can be used to focus positrons or antiprotons emerging from conversion targets $[6,7]$.

A different type of focusing device is the parabolic current sheet lens. In its simplest form, the current sheet lens is shown in Fig. 5.2. The rotational symmetric lens produces an azimuthal magnetic field which scales inversely proportional to $r$, $B_{\varphi} \sim 1 / r$. Since the length of the lens scales like $\ell \sim r^{2}$, the deflection of a particle trajectory increases linear with $r$ as desired for a focusing lens.

The field strength depends on the particular parameter of the paraboloid used for the current sheet and the electrical current. The magnetic field is from Maxwell's 


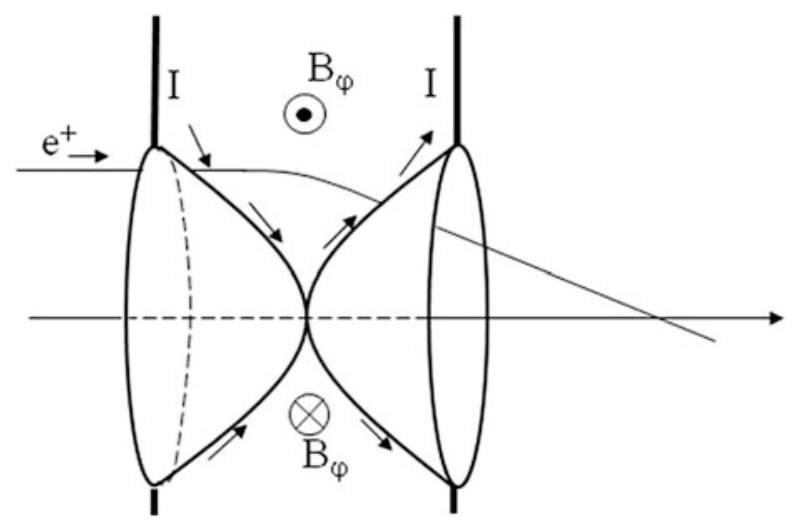

Fig. 5.2 Parabolic current sheet lens (schematic)

equation

$$
B_{\varphi}(\mathrm{T})=\frac{\mu_{0}}{2 \pi} \frac{I(\mathrm{~A})}{r(\mathrm{~m})}
$$

and with $\ell=a r^{2}$ the product of the field gradient $g=\partial B_{\varphi} / \partial r$ and the length $\ell$ is

$$
g \ell(\mathrm{T})=\frac{\mu_{0}}{2 \pi} a\left(\mathrm{~m}^{-1}\right) I(\mathrm{~A}) .
$$

The use of a parabolic shape for the current sheet is not fundamental. Any form with the property $\ell \sim r^{2}$ will provide the desired focusing properties. A geometric variation of such a system is used in high energy physics to focus a high energy $\mathrm{K}$-meson beam emerging from a target into the forward direction [8, 9]. Since the decaying kaon beam produces neutrinos among other particles this device is called a neutrino horn. On a much smaller scale compared to the neutrino horn a similar focusing devices can be used to focus positrons from a conversion target into the acceptance of a subsequent accelerator $[10,11]$.

This type of lens may be useful for specific applications but cannot be considered a general focusing device, where an aperture, free of absorbing material, is required to let particles pass without being scattered. The most suitable device that provides a material free aperture and the desired focusing field is called a quadrupole magnet. As will be discussed in Chap. 6 the magnetic field can be derived in Cartesian coordinates from the scalar potential $V=-g x y$

$$
\begin{aligned}
& -\frac{\partial V}{\partial x}=B_{x}=g y, \\
& -\frac{\partial V}{\partial y}=B_{y}=g x .
\end{aligned}
$$


Fig. 5.3 Magnetic field pattern for a quadrupole magnet

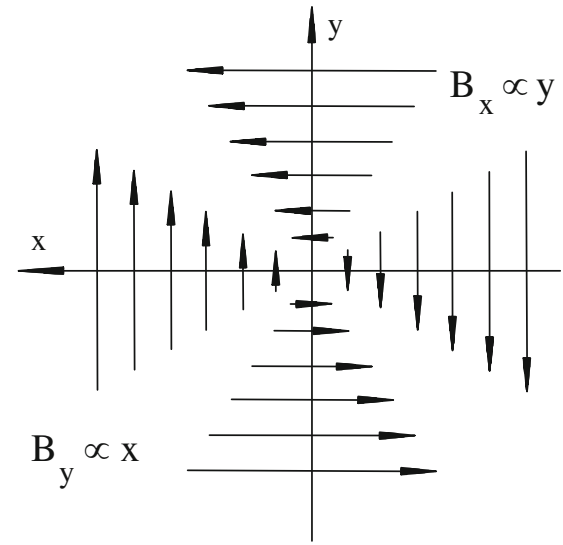

Such fields clearly deflect a particle trajectory proportional to its distance from the optical axis as we would expect for a focusing element. Magnetic equipotential surfaces with a hyperbolic profile will be suitable to create the desired fields. The field pattern of a quadrupole magnet is shown schematically in Fig. 5.3

In beam dynamics, it is customary to define an energy independent focusing strength. Similar to the definition of the bending curvature in (5.3) we define a focusing strength $k$ by

$$
k=\frac{e}{p} g=\frac{e c}{\beta E} g
$$

and the focal length of the magnetic device is from (5.11)

$$
f^{-1}=k \ell
$$

In more practical units, the focusing strength is given in analogy to (5.7) by

$$
k\left(\mathrm{~m}^{-2}\right)=0.2998 \frac{g(\mathrm{~T} / \mathrm{m})}{\beta E(\mathrm{GeV})} .
$$

Multiplication with $Z / A$ gives the focusing strength for ions of charge multiplicity $Z$ and atomic weight $A$. Consistent with the sign convention of the Frenet-Serret coordinate system, the field directions are chosen such that a positively charged particle like a proton or positron moving at a distance $x>0$ parallel to the $z$-axis is deflected toward the center (focusing), while the same particle with a vertical offset from the $z$-axis $(y>0)$ becomes deflected upward (defocusing).

Quadrupole magnets are focusing only in one plane and defocusing in the other. This property is a result of Maxwell's equations but does not diminish the usefulness of quadrupole magnets as focusing elements. A combination of quadrupoles can become a system that is focusing in both planes of a Cartesian coordinate system. 
From paraxial light optics it is known that the total focal length of a combination of two lenses with focal lengths $f_{1}$ and $f_{2}$ and separated by a distance $d$ is given by

$$
\frac{1}{f}=\frac{1}{f_{1}}+\frac{1}{f_{2}}-\frac{d}{f_{1} f_{2}} .
$$

A specific solution is $f_{1}=-f_{2}$ and a quadrupole doublet with this property is focusing in both the horizontal and vertical plane with equal focal length $1 / f=$ $d /\left|f_{1} f_{2}\right|$. Equation (5.19) allows many other solutions different from the simple assumption made here. The fundamental observation is here that there exist indeed combinations of focusing and defocusing quadrupoles which can be made focusing in both planes and are therefore useful for charged particle beam focusing.

\subsection{Equation of Motion}

We use magnetic fields to guide charged particles along a prescribed path or at least keep them close by. This path, or reference trajectory, is defined geometrically by straight sections and bending magnets only. In fact it is mostly other considerations, like the need to transport from an arbitrary point $\mathrm{A}$ to point $\mathrm{B}$ in the presence of building constraints, that determine a particular path geometry. We place dipole magnets wherever this path needs to be deflected and have straight sections in between. Quadrupole and higher order magnets do not influence this path but provide the focusing forces necessary to keep all particles close to the reference path.

The most convenient coordinate system to describe particle motion is the FrenetSerret system that follows with the particle along the reference path. In other words, we use a curvilinear coordinate system as defined mathematically by (4.19). The curvatures are functions of the coordinate $z$ and are nonzero only where there are bending magnets. In deriving the equations of motion, we limit ourselves to the horizontal plane only. The generalization to both horizontal and vertical plane is straightforward. We calculate the deflection angle of an arbitrary trajectory for an infinitesimal segment of a bending magnet with respect to the ideal trajectory. Using the notation of Fig. 5.4 the deflection angle of the ideal path is $\mathrm{d} \varphi_{0}=\mathrm{d} z / \rho_{0}$ or utilizing the curvature to preserve the directionality of the deflection

$$
\mathrm{d} \varphi_{0}=+\kappa_{0} \mathrm{~d} z
$$

where $\kappa_{0}$ is the curvature of the ideal path. The deflection angle for an arbitrary trajectory is then given by

$$
\mathrm{d} \varphi=+\kappa \mathrm{d} s .
$$


Fig. 5.4 Particle trajectories in deflecting systems. Reference path $z$ and individual particle trajectory $s$ have in general different bending radii

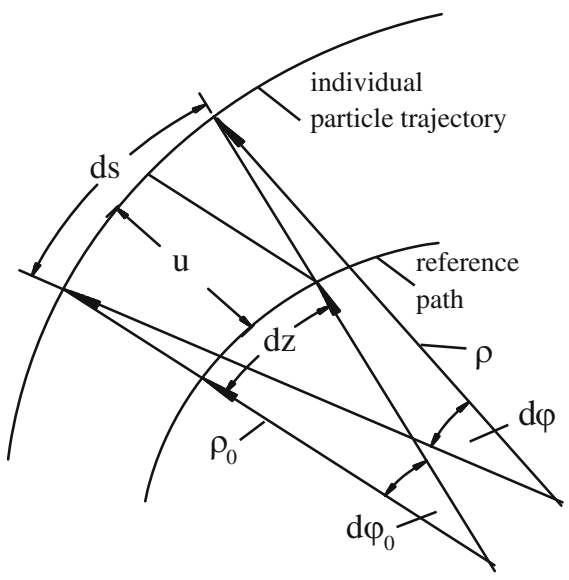

The ideal curvature $\kappa_{0}$ is evaluated along the reference trajectory $u=0$ for a particle with the ideal momentum. In linear approximation with respect to the coordinates the path length element for an arbitrary trajectory is

$$
\mathrm{d} s=\left(1+\kappa_{0} u\right) \mathrm{d} z+\mathcal{O}(2),
$$

where $u=x$ or $y$ is the distance of the particle trajectory from the reference trajectory in the deflecting plane.

The magnetic fields depend on $z$ in such a way that the fields are zero in magnet free sections and assume a constant value within the magnets. This assumption results in a step function distribution of the magnetic fields and is referred to as the hard edge model, generally used in beam dynamics. The path is therefore composed of a series of segments with constant curvatures. To obtain the equations of motion with respect to the ideal path we subtract from the curvature $\kappa$ for an individual particle the curvature $\kappa_{0}$ of the ideal path at the same location.

Since $u$ is the deviation of a particle from the ideal path, we get for the equation of motion in the deflecting plane with respect to the ideal path from Fig. 5.4 and (5.20), (5.21) with $u^{\prime \prime}=-\left(\mathrm{d} \varphi / \mathrm{d} z-\mathrm{d} \varphi_{0} / \mathrm{d} z\right)$,

$$
u^{\prime \prime}=-\left(1+\kappa_{0} u\right) \kappa+\kappa_{0},
$$

where the derivations are taken with respect to $z$. In particle beam dynamics, we generally assume paraxial beams, $u^{\prime 2} \ll 1$ since the divergence of the trajectories $u^{\prime}$ is typically of the order of $10^{-3} \mathrm{rad}$ or less and terms in $u^{\prime 2}$ can therefore be neglected. Where this assumption leads to intolerable inaccuracies the equation of motion must be modified accordingly.

The equation of motion for charged particles in electromagnetic fields can be derived from (5.23) and the Lorentz force. In case of horizontal deflection, the curvature is $\kappa=\kappa_{x}$ and expressing the general field by its components, we have 
from $(5.3)$

$$
\kappa_{x}=\frac{1}{1+\delta}\left(\kappa_{0 x}+k x+\frac{1}{2} m x^{2}+\ldots\right),
$$

where we expanded the field into components up to second order. Such magnetic field expansions will be discussed in much detail in Chap. 6. Here, we use just the three lowest order multipoles, a bending magnet, a quadrupole and a sextupole.

A real particle beam is never monochromatic and therefore effects due to small momentum errors must be considered. This can be done by expanding the particle momentum in the vicinity of the ideal momentum $p_{0}$

$$
\frac{1}{p}=\frac{1}{p_{0}(1+\delta)} \approx \frac{1}{p_{0}}(1-\delta+\ldots) .
$$

We are now ready to apply (5.23) to the horizontal plane, set $u=x$ and $\kappa=\kappa_{x}$ and get with (5.23), (5.24), while retaining only linear and quadratic terms in $\delta, x$ and $y$, the equation of motion

$$
\begin{aligned}
x^{\prime \prime}+\left(k+\kappa_{0 x}^{2}\right) x=\kappa_{0 x}(\delta- & \left.\delta^{2}\right)+\left(k+\kappa_{0 x}^{2}\right) x \delta \\
& -\frac{1}{2} m x^{2}-\kappa_{0} k x^{2}+\mathcal{O}(3) .
\end{aligned}
$$

Here, we have used energy independent field strength parameters as defined in (5.3) and (5.16).

It is interesting to identify each term with well known observations and terminology from geometric light optics. The $\left(k+\kappa_{0 x}^{2}\right) x$-term describes the focusing effects from quadrupoles and a pure geometrical focusing from bending in a sector magnet. Sector magnets are the natural bending magnets for a curvilinear coordinate system. However, in a uniform field sector magnet particles travel longer path for $x>0$ and a shorter path for $x<0$ leading directly to a focusing effect in the deflecting plane. In the nondeflecting plane there is no focusing. A dispersive effect arises from $\kappa_{0 x}\left(\delta-\delta^{2}\right)$ which reflects the varying deflection angle for particles which do not have the ideal design energy. Focusing is also energy dependent and the term $\left(k+\kappa_{0 x}^{2}\right) x \delta$ gives rise to chromatic aberrations describing imaging errors due to energy deviation. The term $-k \kappa_{0 x} x^{2}$ has no optical equivalent (it would be a focusing prism) and must be included only if there is focusing and bending present in the same magnet like in a synchrotron magnet. The last term we care about here is the sextupole term $-\frac{1}{2} m x^{2}$ which introduces both chromatic and geometric aberration. The chromatic aberration from sextupoles can be used to cancel some of the chromatic aberration (chromaticity) from quadrupoles, but in doing so we introduce a quadratic effect which leads to geometric aberrations. This is similar to the chromatic correction in optical systems by using different kinds of glasses. We will discuss these perturbatory effects in much more detail later. 
The equation of motion in the vertical plane can be derived in a similar way by setting $u=y$ in (5.23) and $\kappa=\kappa_{y}$. Consistent with the sign convention of the Frenet-Serret coordinate system (5.24) becomes for the vertical plane

$$
\kappa_{y}=\kappa_{0 y}+k y+m x y+\ldots \mathcal{O}(3)
$$

and the equation of motion in the vertical plane is

$$
y^{\prime \prime}-\left(k-\kappa_{0 y}^{2}\right) y=\kappa_{0 y} \delta-\left(k-\kappa_{0 y}^{2}\right) y \delta+m x y+\kappa_{0 y} k y^{2}+\mathcal{O}(3) .
$$

Of course, in most cases $\kappa_{0 y}=0$. In particular, we find for cases, where the deflection occurs only in one plane say the horizontal plane, that the equation of motion in the vertical plane becomes simply

$$
y^{\prime \prime}-k y=-k y \delta+m x y+\mathcal{O}(3)
$$

which to the order of approximation considered is independent of the strength of the horizontal bending field.

The magnet parameters $\kappa_{0}, k$, and $m$ are functions of the independent coordinate $z$. In real beam transport lines, these magnet strength parameters assume constant, non zero values within individual magnets and become zero in drift spaces between the magnets. The task of beam dynamics is to distribute magnets along the beam transport line in such a way that the solutions to the equations of motion result in the desired beam characteristics.

\subsection{Equations of Motion from the Lagrangian and Hamiltonian*}

In this section, we will formulate the Lagrangian and Hamiltonian suitable for the study of particle beam dynamics. Specifically, we will work in the curvilinear coordinate system and use the longitudinal coordinate $z$ as the independent variable rather than the time $t$. This is of particular importance because the time is measured along each particular trajectory and is therefore evolving differently for each particle in relation to the $z$-coordinate. The time is related to the particle position $s=v t$ along its trajectory and through its velocity while the $z$-coordinate can function as a general reference for all particles.

We will study both the Lagrangian and Hamiltonian formulation together to clearly define canonical momenta and facilitate the study of particle dynamics with the support of the full Hamiltonian theory. Depending on the problem at hand, it may be easier to start with one or the other formulation. 


\subsubsection{Equations of Motion from Lagrangian}

In Chap. 4 we have derived the Lagrangian (4.25) in the curvilinear coordinate system of beam dynamics

$$
L=-m c \sqrt{c^{2}-\dot{x}^{2}-\dot{y}^{2}-h^{2} \dot{z}^{2}}+e\left(\dot{x} A_{x}+\dot{y} A_{y}+h \dot{z} A_{\mathrm{c}, z}\right)-e \phi,
$$

which controls the movement of charged particles in an electromagnetic field. The magnetic fields can be derived from the potentials by

$$
\begin{aligned}
\boldsymbol{B} & =\boldsymbol{\nabla} \mathbf{A} \\
& =\frac{1}{h}\left[\frac{\partial\left(h A_{\mathrm{c}, z}\right)}{\partial y}-\frac{\partial A_{y}}{\partial z}\right] \mathbf{x}+\frac{1}{h}\left[\frac{\partial A_{x}}{\partial z}-\frac{\partial\left(h A_{\mathrm{c}, z}\right)}{\partial x}\right] \mathbf{y}+\left[\frac{\partial A_{y}}{\partial x}-\frac{\partial A_{x}}{\partial y}\right] \mathbf{z},
\end{aligned}
$$

where $h=1+\kappa_{x} x+\kappa_{y} y$, while the electric fields are $\boldsymbol{E}=-\nabla V$. The equations of motion are the Lagrangian equations and are in component form

$$
\begin{aligned}
\frac{\mathrm{d}}{\mathrm{d} t}(\gamma m \dot{x}) & =\gamma m h \kappa_{x} \dot{z}^{2}+e\left(\dot{y} B_{z}-h \dot{z} B_{y}\right)+e E_{x}, \\
\frac{\mathrm{d}}{\mathrm{d} t}(\gamma m \dot{y}) & =\gamma m h \kappa_{y} \dot{z}^{2}+e\left(-\dot{x} B_{z}+h \dot{z} B_{x}\right)+e E_{y}, \\
\frac{\mathrm{d}}{\mathrm{d} t}(\gamma m h \dot{z}) & =-\gamma m\left(\kappa_{x} \dot{x}+\kappa_{y} \dot{y}\right) \dot{z}+e\left(\dot{x} B_{y}-\dot{y} B_{x}\right)+e E_{z},
\end{aligned}
$$

where $\beta=\frac{1}{c} \sqrt{\dot{x}^{2}+\dot{y}^{2}+h^{2} \dot{z}^{2}}$ and $\gamma$ the relativistic factor. The first two equations describe the transverse particle motion which we will later call betatron motion or betatron oscillations. The third equation describes the longitudinal or synchrotron oscillation, where the main restoring force comes from the accelerating microwave field $e E_{z}$.

It is customary to replace the time variable by the position variable $z$ along the ideal path. Each particle travels along its own path $s$ at a velocity $v=\mathrm{d} s / \mathrm{d} t$ and we change the independent variable with the substitution

$$
\frac{\mathrm{d}}{\mathrm{d} t}=v \frac{\mathrm{d}}{\mathrm{d} s}=v \frac{\mathrm{d} z}{\mathrm{~d} s} \frac{\mathrm{d}}{\mathrm{d} z}=\frac{v}{s^{\prime}} \frac{\mathrm{d}}{\mathrm{d} z},
$$

where the quantity

$$
s^{\prime}=\sqrt{x^{\prime 2}+y^{\prime 2}+h^{2}} .
$$

The primes are used to indicate a derivation with respect to $z$ like $s^{\prime}=\mathrm{d} s / \mathrm{d} z$. The Lagrangian with $z$ as the independent variable rather than $t$ can be derived from (5.30) with (5.33) to give with the momentum deviation $\delta=\left(p-p_{0}\right) / p_{0}$ from the 
ideal momentum $p_{0}$

$$
\tilde{L}\left(x, x^{\prime}, y, y^{\prime}, z\right)=s^{\prime}+(1-\delta) \frac{e}{p_{0}}\left(x^{\prime} A_{x}+y^{\prime} A_{y}+h A_{\mathrm{c}, z}\right)-s^{\prime} \frac{e \phi}{\gamma m v^{2}} .
$$

Applying this to (5.32), the equations of motion are with $p=m \gamma v$

$$
\begin{aligned}
x^{\prime \prime}-\frac{s^{\prime \prime}}{s^{\prime}} x^{\prime} & =\kappa_{x} h-(1-\delta) \frac{e}{p_{0}} s^{\prime}\left(h B_{y}-y^{\prime} B_{z}\right)+s^{\prime 2} \frac{e E_{x}}{\gamma m v^{2}}, \\
y^{\prime \prime}-\frac{s^{\prime \prime}}{s^{\prime}} y^{\prime} & =\kappa_{y} h+(1-\delta) \frac{e}{p_{0}} s^{\prime}\left(h B_{x}-x^{\prime} B_{z}\right)+s^{\prime 2} \frac{e E_{y}}{\gamma m v^{2}}, \\
\frac{s^{\prime \prime}}{s^{\prime}} & =\frac{1}{h}\left[\kappa_{x}^{\prime} x+\kappa_{y}^{\prime} y+2\left(\kappa_{x} x^{\prime}+\kappa_{y} y^{\prime}\right)\right] \\
& -\frac{1-\delta}{h} \frac{e}{p_{0}} s^{\prime}\left(x^{\prime} B_{y}-y^{\prime} B_{x}\right)-s^{\prime 2} \frac{e E_{z}}{\gamma m v^{2}} .
\end{aligned}
$$

So far, no approximations were made and the equations of motion are fully Hamiltonian or symplectic. Equations (5.36), however, are not suited for analytical treatment and we use therefore often the paraxial approximation also known from geometric light optics where particle trajectories are assumed to stay in the vicinity of the optical path keeping all slopes small $\left(x^{\prime} \ll 1, y^{\prime} \ll 1, s^{\prime} \approx 1\right)$. Equation in (5.36c) describes again synchrotron motion and degenerates in the case where there are no electric fields to an equation that can be used to replace the factor $s^{\prime \prime} / s^{\prime}$ in the betatron equations. Since $s^{\prime 2} \approx 1$ for paraxial beams and terms like $\left(\kappa_{x}^{\prime}, \kappa_{y}^{\prime}\right)$ vanish in this approximation, we have $s^{\prime \prime} / s^{\prime} \approx 0$ and (5.36) becomes

$$
\begin{aligned}
& x^{\prime \prime} \approx \kappa_{x} h-(1-\delta) \frac{e}{p_{0}}\left(h B_{y}-y^{\prime} B_{z}\right)+\frac{e E_{x}}{\gamma m v^{2}}, \\
& y^{\prime \prime} \approx \kappa_{y} h+(1-\delta) \frac{e}{p_{0}}\left(h B_{x}-x^{\prime} B_{z}\right)+\frac{e E_{y}}{\gamma m v^{2}} .
\end{aligned}
$$

Of course, strictly speaking, these equations are not anymore symplectic, which is of no practical consequence as far as beam optics goes. Yet, in modern circular accelerators, particle beam stability can often be assured only by numerical tracking calculations. This process applies the equations of motion very often and even small approximations or deviations from symplecticity can introduce false dissipating forces leading to erroneous results. 


\subsubsection{Canonical Momenta}

The Lagrangian (5.35) defines the canonical momenta by derivation with respect to velocities

$$
\begin{aligned}
P_{x} & =\frac{\partial \tilde{L}}{\partial x^{\prime}}=\frac{\partial s^{\prime}}{\partial x^{\prime}}\left(1-\frac{e \phi}{\gamma m v^{2}}\right)+(1-\delta) \frac{e}{p_{0}} A_{x} \\
& =\frac{x^{\prime}}{s^{\prime}}\left(1-\frac{e \phi}{\gamma m v^{2}}\right)+(1-\delta) \frac{e}{p_{0}} A_{x}, \\
P_{y} & =\frac{\partial \tilde{L}}{\partial y^{\prime}}=\frac{\partial s^{\prime}}{\partial y^{\prime}}\left(1-\frac{e \phi}{\gamma m v^{2}}\right)+(1-\delta) \frac{e}{p_{0}} A_{y} \\
& =\frac{y^{\prime}}{s^{\prime}}\left(1-\frac{e \phi}{\gamma m v^{2}}\right)+(1-\delta) \frac{e}{p_{0}} A_{y} .
\end{aligned}
$$

Note, in this formulation, the canonical momenta are dimensionless because they are normalized to the total momentum $p$.

\subsubsection{Equation of Motion from Hamiltonian}

Knowledge of the Lagrangian and canonical momenta gives us the means to formulate the Hamiltonian of the system. In doing so, we use conjugate coordinates $\left(q_{i}, P_{i}\right)$ only, ignore the electric field and get from (5.38) $x^{\prime}=\left(P_{x}-\frac{e}{p} A_{x}\right) s^{\prime}$, etc. and the Hamiltonian $H=H\left(x, P_{x}, y, P_{y}, z\right)$ is by definition with (5.35)

$$
\begin{aligned}
H & =x^{\prime} P_{x}+y^{\prime} P_{y}-L\left(x, x^{\prime}, y, y^{\prime}, z\right) \\
& =-\frac{e}{p} A_{z} h-s^{\prime}\left[1-\left(P_{x}-\frac{e A_{x}}{p}\right)^{2}-\left(P_{y}-\frac{e A_{y}}{p}\right)^{2}\right]
\end{aligned}
$$

From (5.34) and (5.38), we have $s^{\prime 2}=s^{\prime 2}\left(P_{x}-\frac{e}{p} A_{x}\right)^{2}-s^{\prime 2}\left(P_{y}-\frac{e}{p} A_{y}\right)^{2}+h^{2}$ or $\left(h / s^{\prime}\right)^{2}=1-\left(P_{x}-\frac{e}{p} A_{x}\right)^{2}-\left(P_{y}-\frac{e}{p} A_{y}\right)^{2}$ and introducing this in the Hamiltonian, we get finally

$$
H\left(x, P_{x}, y, P_{y}, z\right)=-\frac{e}{p} A_{z} h-h \sqrt{1-\left(P_{x}-\frac{e A_{x}}{p}\right)^{2}-\left(P_{y}-\frac{e A_{y}}{p}\right)^{2}}
$$

where for practical applications, we set $e / p \approx(1-\delta) e / p_{0}$. We may restrict ourselves further to paraxial beams for which $\left(P_{x, y}-\frac{e}{p} A_{x, y}\right) \ll 1$ allowing to 
expand the square root and the Hamiltonian is in lowest order

$$
\begin{aligned}
H \approx- & (1-\delta) \frac{e}{p_{0}} A_{z} h-h \\
& +\frac{1}{2} h\left[P_{x}-(1-\delta) \frac{e A_{x}}{p_{0}}\right]^{2}+\frac{1}{2} h\left[P_{y}-(1-\delta) \frac{e A_{y}}{p_{0}}\right]^{2} .
\end{aligned}
$$

Replacing in (5.40) the normalized canonical momenta $\left(P_{x}, P_{y}\right)$ by normalized ordinary momenta $\left(p_{x}, p_{y}\right)$ and setting $p_{x}=x^{\prime}$ and $p_{y}=y^{\prime}$, the Hamiltonian assumes a more familiar form

$$
K\left(x, x^{\prime}, y, y^{\prime}, z\right) \approx-\frac{e}{p_{0}} A_{z} h(1-\delta)-h \sqrt{1-x^{\prime 2}-y^{\prime 2}},
$$

where the momenta $p_{x, y}$ or $\left(x^{\prime}, y^{\prime}\right)$ in the presence of fields are not canonical anymore and where second order terms in $\delta$ are dropped. As we will see, however, beam dynamics is based predominantly on fields which can be derived from a potential of the form $\boldsymbol{A}\left(0,0, A_{z}\right)$ and consequently, the ordinary momenta are indeed also canonical. We seem to have made a total circle coming from velocities $(\dot{x}, \dot{y})$ to slopes $\left(x^{\prime}, y^{\prime}\right)$ in the Lagrangian to normalized canonical momenta $\left(p_{x}, p_{y}\right)$ back to slopes $\left(x^{\prime}, y^{\prime}\right)$ which we know now to be canonical momenta for most of the fields used in beam dynamics.

The equations of motion can now be derived from the Hamiltonian (5.42) in curvilinear coordinates.

$$
\frac{\partial K}{\partial x}=-P_{x}^{\prime}
$$

where $P_{x}=x^{\prime}-\frac{e}{p} A_{x}$ and $P_{x}^{\prime}=x^{\prime \prime}$. The magnetic field $h B_{y}=\left(\frac{\partial A_{x}}{\partial z}-\frac{\partial h A_{z}}{\partial x}\right)$ does not depend on $z$, e.g. $\partial A_{x} / \partial z=0$. While ignoring any coupling into the vertical plane $(y \equiv 0)$, the equation of motion (5.43) is,

$$
-x^{\prime \prime}=-\frac{e}{p_{0}}(1-\delta) \frac{\partial h A_{z}}{\partial x}-\kappa_{x} \sqrt{1-x^{\prime 2}-y^{\prime 2}}
$$

or with $\kappa_{x} \neq 0, \kappa_{y}=0, h=1+\kappa_{x} x$ and expanding only to second order in $x, x^{\prime}, y, y^{\prime}, \delta$

$$
\begin{aligned}
x^{\prime \prime} & =-\frac{1}{\rho}(1-\delta) h+\kappa_{x} \sqrt{1-x^{\prime 2}-y^{\prime 2}} \\
& \approx-\frac{1}{\rho}(1-\delta) h+\kappa_{x}\left(1-\frac{1}{2} x^{\prime 2}-\frac{1}{2} y^{\prime 2}\right) \\
& \approx-\frac{1}{\rho}+\frac{1}{\rho} \delta-(1-\delta) \frac{1}{\rho} \kappa_{x} x+\kappa_{x}+\mathcal{O}(3) .
\end{aligned}
$$


The general curvature $\kappa$ can be expanded into, for example, a dipole $\kappa_{x}$, a quadrupole $k x$ and a sextupole field $\frac{1}{2} m x^{2}$ for $\frac{1}{\rho}=\kappa_{x}+k x+\frac{1}{2} m x^{2}+\mathcal{O}(3)$ resulting in the equation of motion

$$
\begin{gathered}
x^{\prime \prime}=-\kappa_{x}-k x-\frac{1}{2} m x^{2}+\kappa_{x} \delta+k x \delta-\kappa_{x}^{2} x+\kappa_{x}^{2} x \delta-k \kappa_{x} x^{2}+\kappa_{x}+\mathcal{O}(3), \text { or } \\
x^{\prime \prime}+\left(k+\kappa_{x}^{2}\right) x=\kappa_{x} \delta+\left(k+\kappa_{x}^{2}\right) x \delta-\frac{1}{2} m x^{2}-k \kappa_{x} x^{2}+\mathcal{O}(3)
\end{gathered}
$$

in agreement with (5.26). Similarly, we may derive the equation of motion for the vertical plane and get with $\frac{1}{\rho_{y}}=-\frac{e B_{x}}{p_{0}}=-\kappa_{y}+k y+m x y+\mathcal{O}(3)$

$$
y^{\prime \prime}-\left(k-\kappa_{y}^{2}\right) y=\kappa_{y} \delta-\left(k-\kappa_{y}^{2}\right) y \delta+m x y+k \kappa_{y} y^{2}+\mathcal{O}(3)
$$

in agreement with (5.28).

\subsubsection{Harmonic Oscillator}

Particle dynamics will be based greatly on the understanding of harmonic oscillators under the influence of perturbations. We therefore discuss here the Hamiltonian for a harmonic oscillator. To do that, we start from (5.42), eliminate the magnetic field $A_{z}=0$, ignore the curvature $(h=1)$ and remember that we have to reintroduce the potential by a function $V$. Furthermore, we use the time $t=z / c$ as the independent variable again. With this, we derive from (5.42) the Hamiltonian

$$
K\left(x, x^{\prime}, z\right) \approx-V-\sqrt{1-x^{\prime 2}} \approx-V-\left(1-\frac{1}{2} x^{\prime 2}\right) .
$$

The potential for a harmonic oscillator derives from a restoring force $-D x$ and is $-\frac{1}{2} D x^{2}$. A new Hamiltonian is then

$$
\mathcal{K}=\frac{1}{2} x^{\prime 2}+\frac{1}{2} D x^{2}
$$

and the equations of motion are

$$
\begin{aligned}
& \frac{\partial \mathcal{K}}{\partial x}=-x^{\prime \prime}=D x, \\
& \frac{\partial \mathcal{K}}{\partial x^{\prime}}=x^{\prime}=x^{\prime} .
\end{aligned}
$$

The Hamiltonian could have been formulated directly considering that it is equal to the sum of kinetic $T$ and potential $V$ energy $\mathcal{K}=T+V$. 


\subsubsection{Action-Angle Variables}

Particularly important for particle beam dynamics is the canonical transformation from Cartesian coordinates $(w, \dot{w}, \varphi)$ to action-angle variables $(J, \psi, \varphi)$. This class of transformations is best suited for harmonic oscillators like charged particles under the influence of focusing restoring forces. We assume the equations of motion to be expressed in normalized coordinates of particle beam dynamics with the independent variable $\varphi$ instead of the time. As we will discuss later, it is necessary in beam dynamics to transform ordinary Cartesian coordinates $\left(x, x^{\prime}, z\right)$ into normalized coordinates $(w, \dot{w}, \varphi)$. The generating function for the transformation to action-angle variables $(J, \psi, \varphi)$ is of the form $G_{1}$ in (4.39) which can be written with some convenient constant factors as

$$
G=-\frac{1}{2} \nu w^{2} \tan (\psi-\vartheta)
$$

where $\vartheta$ is an arbitrary phase. Applying (4.44) to the generating function (5.52) we get with $\dot{w}=\mathrm{d} w / \mathrm{d} \varphi$

$$
\begin{aligned}
& \frac{\partial G}{\partial w}=\dot{w}=-v w \tan (\psi-\vartheta), \\
& \frac{\partial G}{\partial \psi}=-J=-\frac{1}{2} \frac{v w^{2}}{\cos ^{2}(\psi-\vartheta)} .
\end{aligned}
$$

Solving for $w$ and $\dot{w}$ the equations take the form

$$
\begin{aligned}
& w=\sqrt{\frac{2 J}{v}} \cos (\psi-\vartheta), \\
& \dot{w}=-\sqrt{2 v J} \sin (\psi-\vartheta) .
\end{aligned}
$$

To determine whether the transformation to action-angle variables has led us to cyclic variables we will use the unperturbed Hamiltonian, while ignoring perturbations, and substitute the old variables by new ones through the transformations (5.54). The generating function (5.52) does not explicitly depend on the independent variable $\varphi$ and the new Hamiltonian is therefore given by

$$
H=v J
$$

The independent variable $\psi$ is obviously cyclic and from $\partial H / \partial \psi=0=\dot{J}$ we find the first invariant or constant of motion

$$
J=\text { const. }
$$


The second Hamiltonian equation

$$
\frac{\partial H}{\partial J}=\dot{\psi}=v
$$

defines the frequency of the oscillator which is a constant of motion since the action $J$ is invariant. The betatron frequency or tune

$$
v=v_{0}=\text { const }
$$

and the angle variable $\psi$ is the betatron phase. Eliminating the betatron phase $\psi$ from (5.54), we obtain an expression of the action in normalized coordinates

$$
J=\frac{1}{2} v_{0} w^{2}+\frac{1}{2} \frac{\dot{w}^{2}}{v_{0}} .
$$

Both terms on the r.h.s. can be associated with the potential and kinetic energy of the oscillator, respectively, and the constancy of the action $J$ is synonymous with the constancy of the total energy of the oscillator.

\subsection{Solutions of the Linear Equations of Motion}

Equations (5.26), (5.28) are the equations of motion for strong focusing beam transport systems $[12,13]$, where the magnitude of the focusing strength is a free parameter. No general analytical solutions are available for arbitrary distributions of magnets. We will, however, develop mathematical tools which make use of partial solutions to the differential equations, of perturbation methods and of particular design concepts for magnets to arrive at an accurate prediction of particle trajectories. One of the most important "tools" in the mathematical formulation of a solution to the equations of motion is the ability of magnet builders and alignment specialists to build magnets with almost ideal field properties and to place them precisely along a predefined ideal path. In addition, the capability to produce almost monochromatic particle beams is of great importance for the determination of the properties of particle beams. As a consequence, all terms on the right-hand side of (5.26), (5.28) can and will be treated as small perturbations and mathematical perturbation methods can be employed to describe the effects of these perturbations on particle motion.

We further notice that the left-hand side of the equations of motion resembles that of a harmonic oscillator although with a time dependent frequency. By a proper transformation of the variables we can, however, express (5.26), (5.28) exactly in the form of the equation for a harmonic oscillator with constant frequency. This transformation is very important because it allows us to describe the particle motion mostly as that of a harmonic oscillator under the influence of weak perturbation 
terms on the right-hand side. A large number of mathematical tools developed to describe the dynamics of harmonic oscillators become therefore available for charged particle beam dynamics.

\subsubsection{Linear Unperturbed Equation of Motion}

In our attempt to solve the equations of motion (5.26), (5.28), we first try to solve the homogeneous differential equation

$$
u^{\prime \prime}+K u=0
$$

where $u$ stands for $x$ or $y$ and where, for the moment, we assume $K$ to be constant with $K=k+\kappa_{x}^{2}$ or $K=-\left(k-\kappa_{y}^{2}\right)$, respectively. The principal solutions of this differential equation are for $K>0$

$$
C(z)=\cos (\sqrt{K} z) \quad \text { and } \quad S(z)=\frac{1}{\sqrt{K}} \sin (\sqrt{K} z),
$$

and for $K<0$

$$
C(z)=\cosh (\sqrt{|K|} z) \quad \text { and } \quad S(z)=\frac{1}{\sqrt{|K|}} \sinh (\sqrt{|K|} z) .
$$

These linearly independent solutions satisfy the following initial conditions

$$
\begin{aligned}
& C(0)=1, \quad C^{\prime}(0)=\mathrm{d} C / \mathrm{d} z=0 \\
& S(0)=0, \quad S^{\prime}(0)=\mathrm{d} S / \mathrm{d} z=1
\end{aligned}
$$

Any arbitrary solution $u(z)$ can be expressed as a linear combination of these two principal solutions

$$
\begin{gathered}
u(z)=C(z) u_{0}+S(z) u_{0}^{\prime}, \\
u^{\prime}(z)=C^{\prime}(z) u_{0}+S^{\prime}(z) u_{0}^{\prime},
\end{gathered}
$$

where $u_{0}, u_{0}^{\prime}$ are arbitrary initial parameters of the particle trajectory and derivatives are taken with respect to the independent variable $z$.

In a general beam transport system, however, we cannot assume that the magnet strength parameter $K$ remains constant and alternative methods of finding a solution for the particle trajectories must be developed. Nonetheless it has become customary to formulate the general solutions for $K=K(z)$ similar to the principal solutions found for a harmonic oscillator with a constant restoring force. Specifically, solutions can be found for any arbitrary beam transport line which satisfy the 
initial conditions (5.63). These principal solutions are the so-called sine like and cosine like solutions and we will derive the conditions for such solutions. For the differential equation

$$
u^{\prime \prime}+K(z) u=0
$$

with a time dependent restoring force $K(z)$, we make an ansatz for the general solutions in the form (5.64). Introducing the ansatz (5.64) into (5.65) we get after some sorting

$$
\left[S^{\prime \prime}(z)+K(z) S(z)\right] u_{0}+\left[C^{\prime \prime}(z)+K(z) C(z)\right] u_{0}^{\prime}=0 .
$$

This equation must be true for any pair of initial conditions $\left(u_{0}, u_{0}^{\prime}\right)$ and therefore the coefficients must vanish separately

$$
\begin{aligned}
& C^{\prime \prime}(z)+K(z) C(z)=0, \\
& S^{\prime \prime}(z)+K(z) S(z)=0 .
\end{aligned}
$$

The general solution of the equation of motion (5.65) can be expressed by a linear combination of a pair of solutions satisfying the differential equations (5.66) and the boundary conditions (5.63).

It is impossible to solve (5.66) analytically in a general way that would be correct for arbitrary distributions of quadrupoles $K(z)$. Purely numerical methods to solve the differential equations (5.66) maybe practical but are conceptually unsatisfactory since this method reveals little about characteristic properties of beam transport systems. It is therefore not surprising that other more revealing and practical methods have been developed to solve the beam dynamics of charged particle beam transport systems.

\subsubsection{Matrix Formulation}

The solution (5.64) of the equation of motion (5.65) may be expressed in matrix formulation

$$
\left[\begin{array}{c}
u(z) \\
u^{\prime}(z)
\end{array}\right]=\left[\begin{array}{cc}
C(z) & S(z) \\
C^{\prime}(z) & S^{\prime}(z)
\end{array}\right]\left[\begin{array}{c}
u_{0} \\
u_{0}^{\prime}
\end{array}\right] .
$$

If we calculate the principal solutions of (5.65) for individual magnets only, we obtain such a transformation matrix for each individual element of the beam transport system. Noting that within each of the beam line elements, whether it be a drift space or a magnet, the restoring forces are indeed constant, we may use within each single beam line element the simple solutions (5.61) or (5.62) for 
the equation of motion (5.65). With these solutions, we are immediately ready to form transformation matrices for each beam line element. In matrix formalism, we are able to follow a particle trajectory along a complicated beam line by repeated matrix multiplications from element to element. This procedure is widely used in accelerator physics and lends itself particularly effective for applications in computer programs. With this method we have completely eliminated the need to solve the differential equation (5.65), which we could not have succeeded in doing anyway without applying numerical methods. The simple solutions (5.61), (5.62) will suffice to treat most every beam transport problem.

\subsubsection{Wronskian}

The transformation matrix just derived has special properties well-known from the theory of linear homogeneous differential equation of second order [14]. Only a few properties relevant to beam dynamics shall be repeated here. We consider the linear homogeneous differential equation of second order

$$
u^{\prime \prime}+v(z) u^{\prime}+w(z) u=0 .
$$

For such an equation, the theory of linear differential equations provides us with a set of theorems describing the properties of the solutions

- there is only one solution that meets the initial conditions $u\left(z_{0}\right)=u_{0}$ and $u^{\prime}\left(z_{0}\right)=u_{0}^{\prime}$ at $z=z_{0}$

- because of the linearity of the differential equation, $c u(z)$ is also a solution if both $u(z)$ is a solution and if $c=$ const. ,

- if $u_{1}(z)$ and $u_{2}(z)$ are two solutions, any linear combination thereof is also a solution.

The two linearly independent solutions $u_{1}(z)$ and $u_{2}(z)$ can be used to form the Wronskian determinant or short the Wronskian

$$
W=\left|\begin{array}{ll}
u_{1}(z) & u_{2}(z) \\
u_{1}^{\prime}(z) & u_{2}^{\prime}(z)
\end{array}\right|=u_{1} u_{2}^{\prime}-u_{2} u_{1}^{\prime} .
$$

This Wronskian has remarkable properties which become of great fundamental importance in beam dynamics. Both $u_{1}$ and $u_{2}$ are solutions of (5.68). Multiplying and combining both equations like

$$
\begin{array}{l|l}
u_{1}^{\prime \prime}+v(z) u_{1}^{\prime}+w(z) u_{1}=0 & \mid \cdot-u_{2} \\
u_{2}^{\prime \prime}+v(z) u_{2}^{\prime}+w(z) u_{2}=0 & \mid \cdot u_{1}
\end{array}
$$


gives

$$
\left(u_{1} u_{2}^{\prime \prime}-u_{2} u_{1}^{\prime \prime}\right)+v(z)\left(u_{1} u_{2}^{\prime}-u_{2} u_{1}^{\prime}\right)=0,
$$

which will allow us to derive a single differential equation for the Wronskian. Making use of (5.69) and forming the derivative $\mathrm{d} W / \mathrm{d} z=u_{1} u_{2}^{\prime \prime}-u_{2} u_{1}^{\prime \prime}$, we obtain the differential equation

$$
\frac{\mathrm{d} W}{\mathrm{~d} z}+v(z) W(z)=0
$$

which can be integrated immediately to give

$$
W(z)=W_{0} \mathrm{e}^{-\int_{z_{0}}^{z} v(\bar{z}) \mathrm{d} \bar{z}}
$$

In the case of linear beam dynamics, we have $v(z) \equiv 0$ as long as we do not include dissipating forces like acceleration or energy losses into synchrotron radiation and therefore $W(z)=W_{0}=$ const. We use the sine and cosine like solutions as the two independent solutions and get from (5.69) with (5.63)

$$
W_{0}=C_{0} S_{0}^{\prime}-C_{0}^{\prime} S_{0}=1
$$

For the transformation matrix of an arbitrary beam transport line with negligible dissipating forces, we finally get the general result

$$
W(z)=\left|\begin{array}{cc}
C(z) & S(z) \\
C^{\prime}(z) & S^{\prime}(z)
\end{array}\right|=1 .
$$

This result will be used repeatedly to prove useful general characteristics of particle beam optics, in particular, this is another formulation of Liouville's theorem stating that the phase space density under these conditions is preserved. From the generality of the derivation, we conclude that the Wronskian is equal to unity, or phase space preserving, for any arbitrary beam line that is described by (5.68) if $v(z)=0$ and $w(z)=K(z)$.

\subsubsection{Perturbation Terms}

The principal solutions of the homogeneous differential equation give us the basic solutions in beam dynamics. We will, however, repeatedly have the need to evaluate the impact of perturbations on basic particle motion. These perturbations are effected by any number of terms on the r.h.s. of the equations of motion (5.26), (5.28). The principal solutions of the homogeneous equation of motion can be used 
to find particular solutions $P(z)$ for inhomogeneous differential equations including perturbations of the form

$$
P^{\prime \prime}(z)+K(z) P(z)=\tilde{p}(z)
$$

where $\tilde{p}(z)$ stands for any one or more perturbation terms in (5.26), (5.28). For simplicity, only the $z$-dependence is indicated in the perturbation term although in general they also depend on the transverse particle coordinates. A solution $P(z)$ of this equation can be found from

$$
P(z)=\int_{0}^{z} \tilde{p}(\tilde{z}) G(z, \tilde{z}) \mathrm{d} \tilde{z}
$$

where $G(z, \tilde{z})$ is a Green's function which can be constructed from the principal solutions of the homogeneous equation

$$
G(z, \tilde{z})=S(z) C(\tilde{z})-C(z) S(\tilde{z}) .
$$

After insertion into (5.75) a particular solution for the perturbation can be found from

$$
P(z)=S(z) \int_{0}^{z} \tilde{p}(\tilde{z}) C(\tilde{z}) \mathrm{d} \tilde{z}-C(z) \int_{0}^{z} \tilde{p}(\tilde{z}) S(\tilde{z}) \mathrm{d} \tilde{z} .
$$

The general solution of the equations of motion (5.26), (5.28) then is given by the combination of the two principal solutions of the homogenous part of the differential equation and a particular solution for the inhomogeneous differential equation

$$
u(z)=a C_{u}(z)+b S_{u}(z)+\delta P_{u}(z),
$$

where the coefficients $a$ and $b$ are arbitrary constants to be determined by the initial parameters of the trajectory. We have also used the index ${ }_{u}$ to indicate that these functions must be defined separately for $u=x$ and $y$.

Because of the linearity of the differential equation we find a simple superposition of the general solutions of the homogeneous equation and a particular solution for the inhomogeneous equations for any number of small perturbations. This is an important feature of particle beam dynamics since it allows us to solve the equation of motion up to the precision required by a particular application. While the basic solutions are very simple, corrections can be calculated for each perturbation term separately and applied as necessary. However, these statements, true in general, must be used carefully. In special circumstances even small perturbations may have a great effect on the particle trajectory if there is a resonance or if a particular instability occurs. With these caveats in mind one can assume that in a well defined particle beam line with reasonable beam sizes and well designed and constructed magnets the perturbations are generally small and that mathematical perturbations 
methods are applicable. Specifically, we will in most cases assume that the $(x, y)$ amplitudes appearing in some of the perturbation terms can be replaced by the principal solutions of the homogeneous differential equations.

\section{Dispersion Function}

One of the most important perturbations derives from the fact that the particle beams are not quite monochromatic but have a finite spread of energies about the nominal energy $c p_{0}$. The deflection of a particle with the wrong energy in any magnetic or electric field will deviate from that for an ideal particle. The variation in the deflection caused by such a chromatic error $\Delta p$ in bending magnets is the lowest order of perturbation given by the term $\delta / \rho_{0}$, where $\delta=\Delta p / p_{0} \ll 1$. We will ignore for now all terms quadratic or of higher order in $\delta$ and use the Green's function method to solve the perturbed equation

$$
u^{\prime \prime}+K(z) u=\kappa_{0 u}(z) \delta .
$$

In (5.78) we have derived a general solution for the equation of motion for any perturbation and applying this to (5.79), we get

$$
\begin{aligned}
& u(z)=a C_{u}(z)+b S_{u}(z)+\delta D_{u}(z), \\
& u^{\prime}(z)=a C_{u}^{\prime}(z)+b S_{u}^{\prime}(z)+\delta D_{u}^{\prime}(z),
\end{aligned}
$$

where we have set $P_{u}(z)=\delta D_{u}(z)$ and used (5.77) to obtain

$$
D_{u}(z)=\int_{0}^{z} \kappa_{0 u}(\tilde{z})\left[S_{u}(z) C_{u}(\tilde{z})-C_{u}(z) S_{u}(\tilde{z})\right] \mathrm{d} \tilde{z} .
$$

We have made use of the fact that like the perturbation the particular solution must be proportional to $\delta$ as well. The function $D_{u}(z)$ is called the dispersion function and the physical interpretation is simply that the function $\delta D_{u}(z)$ determines the offset of the reference trajectory from the ideal path for particles with a relative energy deviation $\delta$ from the ideal momentum $c p_{0}$.

This result shows that the dispersion function generated in a particular bending magnet does not depend on the dispersion at the entrance to the bending magnet which may have been generated by upstream bending magnets. The dispersion generated by a particular bending magnet reaches the value $D_{u}\left(L_{\mathrm{m}}\right)$ at the exit of the bending magnet of length $L_{\mathrm{m}}$ and propagates from there on through the rest of the beam line just like any other particle trajectory. This can be seen from (5.81), where we have for $z>L_{\mathrm{m}}$

$$
D_{u}(z)=S_{u}(z) \int_{0}^{L_{\mathrm{m}}} \kappa_{u}(\tilde{z}) C_{u}(\tilde{z}) \mathrm{d} \tilde{z}-C_{u}(z) \int_{0}^{L_{\mathrm{m}}} \kappa_{u}(\tilde{z}) S_{u}(\tilde{z}) \mathrm{d} \tilde{z},
$$


which has exactly the form of (5.64) describing the trajectory of a particle starting with initial parameters at the end of the bending magnet given by the integrals. With the solution (5.80) we can expand the $2 \times 2$-matrix in (5.67) into a $3 \times 3$-matrix, which includes the first order chromatic correction

$$
\left(\begin{array}{c}
u(z) \\
u^{\prime}(z) \\
\delta
\end{array}\right)=\left(\begin{array}{ccc}
C_{u}(z) & S_{u}(z) & D_{u}(z) \\
C_{u}^{\prime}(z) & S_{u}^{\prime}(z) & D_{u}^{\prime}(z) \\
0 & 0 & 1
\end{array}\right)\left(\begin{array}{c}
u\left(z_{0}\right) \\
u^{\prime}\left(z_{0}\right) \\
\delta
\end{array}\right)
$$

Here we have assumed that the particle energy and energy deviation remains constant along the beam line. This representation of the first order chromatic aberration will be used extensively in particle beam optics.

\section{Problems}

5.1 (S). Derive (5.32a) and (5.32 c) from the Lagrange equations. Show all steps.

5.2 (S). Derive the Lagrangian (5.35) from (5.30) (Hint: Its the variational principle $\delta \int L \mathrm{~d} t=0$ that needs to be transformed).

5.3 (S). Verify the numerical validity of (5.7).

5.4 (S). Show that (5.77) is indeed a solution of (5.74).

5.5 (S). Transform the Hamiltonian (5.49) of a harmonic oscillator into actionangle variables and show that the frequency is $v=\sqrt{D}$. Derive the equation of motion.

5.6. Show the validity of the transformation equations (5.54a) and (5.54b). Interpret the physical meaning of (5.56) and (5.57).

\section{References}

1. E.R. Cohen, B.N. Taylor, Rev. Mod. Phys. 59, 1121 (1987)

2. J. Larmor, Philos. Mag. 44, 503 (1897)

3. W.K.H. Panofsky, W.R. Baker, Rev. Sci. Instrum. 21, 445 (1950)

4. E.G. Forsyth, L.M. Lederman, J. Sunderland, IEEE Trans. NS 12, 872 (1965)

5. D. Luckey, Rev. Sci. Instrum. 31, 202 (1960)

6. B.F. Bayanov, G.I. Silvestrov, Zh. Tekh. Fiz. 49, 160 (1978)

7. B.F. Bayanov, J.N. Petrov, G.I. Silvestrov, J.A. Maclachlan, G.L. Nicholls, Nucl. Instrum. Methods 190, 9 (1981)

8. S. Van der Meer, Technical report, CERN (1961)

9. E. Regenstreif, Technical Report, CERN 64-41, CERN, Geneva (1964)

10. G.I. Budker, in International Conference on High Energy Accelerators (Dubna, 1973), p. 69

11. H. Wiedemann, Technical Report, H-14, DESY, Hamburg (1966) 
12. N. Christofilos, US Patent No 2,736,766 (1950)

13. E.D. Courant, M.S. Livingston, H.S. Snyder, Phys. Rev. 88, 1190 (1952)

14. E.D. Courant, H.S. Snyder, Appl. Phys. 3, 1 (1959) 


\section{Chapter 6 \\ Electromagnetic Fields}

Beam dynamics is effected by electromagnetic fields. Generally, magnetic fields are used for relativistic particle guidance and focusing while electric fields are mostly used in the form of electro-static fields or microwaves for acceleration of the particles. In this chapter, we will discuss in more detail the magnetic fields and their generation as they are used in beam dynamics. From (1.52), (1.51) we know how to derive static electric and magnetic fields from a vector or scalar potential by solving their Laplace equations.

\subsection{Pure Multipole Field Expansion}

Special desired effects on particle trajectories require specific magnetic fields. Dipole fields are used to bend particle beams and quadrupole magnets serve, for example, as beam focusing devices. To obtain an explicit formulation of the equations of motion of charged particles in an arbitrary magnetic field, we derive the general magnetic fields consistent with Maxwells equations.

Although we have identified a curvilinear coordinate system moving together with particles to best fit the needs of beam dynamics, we use in this section first, for simplicity, a fixed, right-handed Cartesian coordinate system $(x, y, z)$. By doing so, we assume straight magnets and neglect the effects of curvature. Later in this chapter, we will derive both the electromagnetic fields and equations of motion in full rigor.

This chapter has been made Open Access under a CC BY 4.0 license. For details on rights and licenses please read the Correction https://doi.org/10.1007/978-3-319-18317-6_28 


\subsubsection{Electromagnetic Potentials and Fields for Beam Dynamics}

Earlier we have derived the potentials from the wave equation in a charge and current free static environment. This is the beam environment and we want to formulate fields for beam dynamics there. In the same environment Maxwell's equations reduce to $\nabla \boldsymbol{B}=0$ and $\boldsymbol{\nabla} \times \boldsymbol{B}=0$ and can be used directly. Based on these equations, the magnetic fields can be derived from potentials by (1.51) as previously defined. Electrostatic fields are derived from a scalar potential alone according to (1.52).

In beam dynamics we use mostly purely transverse magnetic fields and from the definition of the magnetic field by the vector potential we find that only the component $A_{z} \neq 0$ collapsing practically to a scalar. To simplify math, we try to formulate a complex potential for transverse only fields and set

$$
P(z)=A_{z}(z)+\mathrm{i} V(z),
$$

where $z=x+\mathrm{i} y$. We define also a complex field which we hope to derive from the complex potential. The usual derivation of fields from potentials with $B=$ $B_{x}+\mathrm{i} B_{y}=-\frac{\partial P}{\partial z}$, however, does not work as can be shown by back-substitution. On the other hand, the conjugate complex form

$$
B^{*}=B_{x}-\mathrm{i} B_{y}=\mathrm{i} \frac{\partial P}{\partial z}
$$

is a valid, Maxwell compliant formulation. This is true because only the second formulation is an analytical function $f(z)=u(x, y)+\mathrm{i} v(x, y)$ meeting the CauchyRiemann conditions

$$
\frac{\partial u}{\partial x}=\frac{\partial v}{\partial y} \text { and } \frac{\partial u}{\partial y}=-\frac{\partial v}{\partial x}
$$

and are solutions of the Laplace equation. Evaluating (6.2) we get while dropping the index $z_{z}$ in the non-zero component of the vector potential $\left(A_{z}=A\right)$

$$
B^{*}=B_{x}-\mathrm{i} B_{y}=\mathrm{i} \frac{\partial P}{\partial z}=\mathrm{i} \frac{\partial A+\mathrm{i} \partial V}{\partial x+\mathrm{i} \partial y}=\mathrm{i} \frac{\frac{\partial A}{\partial x}+\mathrm{i} \frac{\partial V}{\partial x}}{1+\mathrm{i} \frac{\partial y}{\partial x}}=\mathrm{i} \frac{\partial A}{\partial x}-\frac{\partial V}{\partial x}
$$

because $x \perp y$. Similarly,

$$
B^{*}=B_{x}-\mathrm{i} B_{y}=\mathrm{i} \frac{\partial P}{\partial z}=\mathrm{i} \frac{\partial A+\mathrm{i} \partial V}{\partial x+\mathrm{i} \partial y}=\mathrm{i} \frac{\frac{\partial A}{\partial y}+\mathrm{i} \frac{\partial V}{\partial y}}{\frac{\partial x}{\partial y}+\mathrm{i}}=\frac{\partial A}{\partial y}+\mathrm{i} \frac{\partial V}{\partial y}
$$


Equating real and imaginary terms on both sides we may now express the field components like

$$
\begin{aligned}
& B_{x}=-\frac{\partial V}{\partial x} \text { and } B_{y}=-\frac{\partial V}{\partial y}, \text { or } \\
& B_{x}=+\frac{\partial A}{\partial y} \text { and } B_{y}=-\frac{\partial A}{\partial x},
\end{aligned}
$$

which are just the Cauchy-Riemann conditions for the complex magnetic field $B^{*}$. Both field definitions are valid definitions.

The potential of real magnets can be expanded into a power series defining all multipoles. Any function of an analytical function is also an analytical function. Especially, the power series

$$
P(z)=\sum_{n \geq 0} C_{n}(x+\mathrm{i} y)^{n}=\sum_{n \geq 0} C_{n} z^{n}=\sum_{n \geq 0} C_{n} r^{n} \mathrm{e}^{\mathrm{i} n \varphi}
$$

is an analytical function and therefore all components $P_{n}$ are complex solutions of the Laplace equation with complex amplitudes

$$
C_{n}=\lambda_{n}+\mathrm{i} \mu_{n}
$$

The coefficients $\lambda_{n}$ are for upright multipoles while the $\mu_{n}$ are those of skew multipoles. Upright multipoles are characterized by midplane symmetry which requires that for $y=0$ the horizontal fields vanish $B_{x}(y=0)=0$ and only vertical field components exist $B_{y}(y=0) \neq 0$. In beam dynamics we almost exclusively use upright magnets. This ansatz is not the most general solution of the Laplace equation, but includes all main multipole fields used in beam dynamics. Later, we will derive a solution that includes all terms allowed by the Laplace equation in a curvilinear coordinate system. Both, the real and imaginary part, are two independent solutions of the same Laplace equation. All coefficients $\lambda_{n}, \mu_{n}$ are still functions of $z$ although we do not indicate this explicitly.

We distinguish between the electrical potential $V_{\mathrm{e}}$ and the magnetic potential $V_{\mathrm{m}}$. Since the Laplace equation is valid for both the electric as well as the magnetic field in a material free region, no real distinction between both fields had to be made. In reality, we rarely design devices which include more than one term of the field expansion. It is therefore appropriate to decompose the general field potential in (6.7) into its independent multipole terms. To keep the discussion simple, we ignore here electric fields. 


\subsubsection{Fields, Gradients and Multipole Strength Parameter}

In (6.7) we used general coefficients which must be related to fields and field gradients. Furthermore, we are looking for energy independent magnet strength parameters which are almost exclusively used in beam dynamics. The particular field patterns for multipole magnets can be derived from the complex potential by differentiation to get the fields (6.6a). Although fields can be derived from both the vector and scalar potential, we will use only the latter to define the fields for beam dynamics.

The first term in (6.7) $C_{0}$ is a constant and will not contribute to transverse fields. However $C_{0}(z)$ and will therefore show up for longitudinal fields which we will discuss in Sect. 6.6. In Table 6.1 the scalar potentials are listed for the first five multipoles. In this list we have already introduced more practical quantities to be further defined. The coefficients $\left(\lambda_{n}, \mu_{n}\right)$ have been replaced by field gradients

$$
\begin{aligned}
& \lambda_{n}=-\frac{1}{n !} s_{n} \text { and } \\
& \mu_{n}=-\frac{1}{n !} \underline{s}_{n},
\end{aligned}
$$

which are defined for upright and skew magnets of order $n$ by

$$
\begin{aligned}
s_{n}\left(\mathrm{~T} / \mathrm{m}^{n-1}\right) & =+\left.\frac{\partial^{n-1} B_{y}}{\partial x^{n-1}}\right|_{\substack{x=0 \\
y=0}}, \quad n=1,2,3 \ldots \text { and } \\
\underline{s}_{n}\left(\mathrm{~T} / \mathrm{m}^{n-1}\right) & =-\left.\frac{\partial^{n-1} B_{x}}{\partial x^{n-1}}\right|_{\substack{x=0 \\
y=0}},
\end{aligned}
$$

respectively. Following common practice we use special letters for fields and gradients in low order multipoles (see Table 6.2, left column). In anticipation of formulating equations of motion we further introduce energy independent field gradients. Fields and gradients are not convenient for beam dynamics where we design energy independent beam transport systems. This we can do by a normalization that includes a general energy factor called the beam rigidity or just

Table 6.1 Magnetic multipole potentials

\begin{tabular}{l|l}
\hline Dipole & $-V_{1}=-B_{x} x-B_{y} y$ \\
\hline Quadrupole & $-V_{2}=-\frac{1}{2} \underline{g}\left(x^{2}-y^{2}\right)+g x y$, \\
\hline Sextupole & $-V_{3}=-\frac{1}{6} s_{3}\left(x^{3}-3 x y^{2}\right)+\frac{1}{6} s_{3}\left(3 x^{2} y-y^{3}\right)$, \\
\hline Octupole & $-V_{4}=-\frac{1}{24} s_{4}\left(x^{4}-6 x^{2} y^{2}+y^{4}\right)+\frac{1}{24} s_{4}\left(x^{3} y-x y^{3}\right)$, \\
\hline Decapole & $-V_{5}=-\frac{1}{120} s_{5}\left(x^{5}-10 x^{3} y^{2}+5 x y^{4}\right)+\frac{1}{120} s_{5}\left(5 x^{4} y-10 x^{2} y^{3}+y^{5}\right)$ \\
\hline
\end{tabular}


Table 6.2 Field gradient nomenclature for low order multipoles

\begin{tabular}{l|l|l}
\hline Dipole & $B_{y}$ & $\frac{e}{p_{0}} B_{y}=\frac{1}{\rho}$ \\
\hline Quadrupole & $\frac{\partial B_{y}}{\partial x}=g$ & $\frac{e}{p_{0}} \frac{\partial B_{y}}{\partial x}=k$ \\
\hline Sextupole & $\frac{\partial^{2} B_{y}}{\partial x^{2}}=s$ & $\frac{e}{p_{0}} \frac{\partial B_{y}}{\partial x}=m$ \\
\hline Octupole & $\frac{\partial^{3} B_{y}}{\partial x^{3}}=s_{4}$ & $\frac{e}{p_{0}} \frac{\partial B_{y}}{\partial x}=r$ \\
\hline Decapole & $\frac{\partial^{4} B_{y}}{\partial x^{4}}=s_{5}$ & $\frac{e}{p_{0}} \frac{\partial B_{y}}{\partial x}=S_{5}$ \\
\hline
\end{tabular}

the "Brho"from its mathematical form as

$$
R_{\mathrm{b}}=B \rho=\frac{p_{0}}{e}=\frac{\beta E}{c e}=\frac{1}{0.29979} \beta E(\mathrm{GV}) .
$$

This normalization factor is different for electrical and magnetic fields

$$
\begin{aligned}
& R_{\mathrm{b}, \mathrm{m}}=\frac{\beta E(\mathrm{GV})}{0.29979} \quad \text { for magnetic fields, and } \\
& R_{\mathrm{b}, \mathrm{e}}=\frac{\beta^{2} E(\mathrm{GV})}{0.29979} \quad \text { for electric fields. }
\end{aligned}
$$

This difference will obviously vanish for highly relativistic particles $(\beta \approx 1)$. In beam dynamics we use for relativistic beams mostly magnets and therefore we will use in this book the beam rigidity for magnetic fields $R_{\mathrm{b}, \mathrm{m}}$ unless otherwise noted. For low order magnet strength parameters we use $\kappa_{y}, k, m$ for bending magnets, quadrupoles and sextupoles, respectively as shown in the right column of Table 6.2. In Chap. 4 the particle path in a uniform field $B$ has been derived as an arc with radius $\rho$

$$
\frac{1}{\rho}=\frac{e c}{\beta E} B_{y} .
$$

This equation illustrates directly the normalization with a factor equal to the product of $B \rho$. Applied to a bending magnet, for example, we find that the curvature $\kappa_{x}=$ $1 / \rho$ is the normalized quantity for the uniform bending field $B_{y}$. Since we rarely deal with vertical bending magnets we drop the index $y$ in $B_{y}$ and the index $x$ in $\kappa_{x}$.

In (6.14) the curvature or the field can be treated very generally not just as the properties of a bending magnet. Equation (6.19) can be used as the general field and we obtain by multiplication with the beam rigidity

$$
\frac{1}{\rho}=\frac{1}{\rho_{0}}+k x+\frac{1}{2} m x^{2}+\frac{1}{6} r x^{3}+\ldots=\sum_{n=1}^{\infty} S_{n} x^{n-1},
$$


Table 6.3 Upright multipole fields

\begin{tabular}{l|l|l}
\hline Dipole & $\frac{e}{p_{0}} B_{x}=0$ & $\frac{e}{p_{0}} B_{y}=\frac{e}{p_{0}} B_{y 0}$ \\
\hline Quadrupole & $\frac{e}{p_{0}} B_{x}=k y$ & $\frac{e}{p_{0}} B_{y}=k x$ \\
\hline Sextupole & $\frac{e}{p_{0}} B_{x}=m x y$ & $\frac{e}{p_{0}} B_{y}=\frac{1}{2} m\left(x^{2}-y^{2}\right)$ \\
\hline Octupole & $\frac{e}{p_{0}} B_{x}=\frac{1}{6} r\left(3 x^{2} y-y^{3}\right)$ & $\frac{e}{p_{0}} B_{y}=\frac{1}{6} s_{4}\left(x^{3}-3 x y^{2}\right)$ \\
\hline Decapole & $\frac{e}{p_{0}} B_{x}=+\frac{1}{24} s_{5}\left(x^{3} y-x y^{3}\right)$ & $\frac{e}{p_{0}} B_{y}=+\frac{1}{24} s_{5}\left(x^{4}-6 x^{2} y^{2}+y^{4}\right)$ \\
\hline
\end{tabular}

Table 6.4 Rotated or skew multipole fields

\begin{tabular}{l|l|l}
\hline Dipole $\left(90^{\circ}\right)$ & $\frac{e}{p_{0}} B_{x}=\frac{e}{p_{0}} B_{x 0}$ & $\frac{e}{p_{0}} B_{y}=0$ \\
\hline Quadrupole $\left(45^{\circ}\right)$ & $\frac{e}{p_{0}} B_{x}=-\underline{k} x$ & $\frac{e}{p_{0}} B_{y}=+\underline{k} y$ \\
\hline Sextupole $\left(30^{\circ}\right)$ & $\frac{e}{p_{0}} B_{x}=-\frac{1}{2} \underline{m}\left(x^{2}-y^{2}\right)$ & $\frac{e}{p_{0}} B_{y}=+\underline{m} x y$ \\
\hline Octupole $\left(22.5^{\circ}\right)$ & $\frac{e}{p_{0}} B_{x}=-\frac{1}{6} \underline{r}\left(x^{3}-3 x y^{2}\right)$ & $\frac{e}{p_{0}} B_{y}=-\frac{1}{6} \underline{r}\left(3 x^{2} y-y^{3}\right)$ \\
\hline Decapole $\left(18^{\circ}\right)$ & $\frac{e}{p_{0}} B_{x}=-\frac{1}{24} \underline{\underline{s}}\left(x^{4}-6 x^{2} y^{2}+y^{4}\right)$ & $\frac{e}{p_{0}} B_{y}=+\frac{1}{24} \underline{s} \underline{s}\left(x^{3} y-x y^{3}\right)$ \\
\hline
\end{tabular}

The angles indicate the orientation of the central pole with respect to the $y$-axis (the $y$-axis is at $\left.90^{\circ}\right)$

where $\frac{1}{\rho_{0}}$ is the pure dipole field and the multipole magnet strengths

$$
S_{n}=\frac{e c}{\beta E} s_{n}
$$

or in more practical units

$$
S_{n}\left(\mathrm{~m}^{-n}\right)=0.29979 \cdot s_{n}\left(\mathrm{~T} / \mathrm{m}^{n-1}\right)
$$

This gives us immediately the normalization for quadrupoles, sextupoles and higher order multipoles. These parameters are used in beam dynamics as the energy independent magnet strengths while field gradients would scale with beam energy. From Table 6.1 we get by differentiation for upright multipoles the fields for low order upright multipole magnets which are compiled in Table 6.3.

The other class of magnets does not have mid-plane symmetry but the magnets have the same field patterns as the corresponding upright magnets, yet are rotated about the $z$-axis by an angle $\phi_{n}=\pi /(2 n)$, where $n$ is the order of the multipole. These magnets are rarely used in beam dynamics and if so mostly as corrections to field errors. For example, misaligned quadrupoles can create a skew field causing undesired coupling of particle motion between horizontal and vertical plane. Such coupling can be compensated by installing skew quadrupoles. From the expressions for the multipole potentials in Table 6.1 we obtain again the multipole field components which are compiled up to decapoles in Table 6.4.

The characteristic difference between the two sets of field solutions is that the fields of upright linear magnets in Table 6.3 do not cause coupling for particles 
traveling in the horizontal or vertical midplane, in contrast to the rotated magnet fields of Table 6.4 which would deflect particles out of the horizontal midplane. In linear beam dynamics, where we use only dipole and upright quadrupole magnets, the particle motion in the horizontal and vertical plane are completely independent. This is a highly desirable "convenience" without which particle beam dynamics would be much more complicated and less predictable. Since there is no particular fundamental reason for a specific orientation of magnets in a beam transport systems, we may as well use that orientation that leads to the simplest and most predictable results. We will therefore use exclusively upright magnet orientation for the main magnets and treat the occasional need for rotated magnets as a perturbation. In summary, the general magnetic field equation including only the most commonly used upright multipole elements are given by

$$
\begin{aligned}
& \frac{e}{p_{0}} B_{x}=\quad+k y+m x y+\frac{1}{6} r\left(3 x^{2} y-y^{3}\right)+\ldots \\
& \frac{e}{p_{0}} B_{y}=\frac{1}{\rho_{0}}+k x+\frac{1}{2} m\left(x^{2}-y^{2}\right)+\frac{1}{6} r\left(x^{3}-3 x y^{2}\right)+\ldots
\end{aligned}
$$

Sometimes it is interesting to investigate the particle motion only in the horizontal midplane, where $y=0$. In this case we expect the horizontal field components $B_{x}$ of all multipoles to vanish and any deflection or coupling is thereby eliminated. In such circumstances, the particle motion is completely contained in the horizontal plane and the general fields to be used are given by

$$
\begin{aligned}
& \frac{e}{p_{0}} B_{x}=0 \\
& \frac{e}{p_{0}} B_{y}=\frac{1}{\rho_{0}}+k x+\frac{1}{2} m x^{2}+\frac{1}{6} r x^{3}+\ldots+\frac{1}{(n-1) !} S_{n} x^{n-1}
\end{aligned}
$$

\subsubsection{Main Magnets for Beam Dynamics}

The feasibility of any accelerator or beam transport line design depends fundamentally on the parameters and diligent fabrication of technical components composing the system. Not only need the magnets be designed such as to minimize undesirable higher order multipole fields but they also must be designed such that the desired parameters are within technical limits. Most magnets constructed for beam transport lines are electromagnets rather than permanent magnets. The magnets are excited by electrical current carrying coils wound around magnet poles or in the case of superconducting magnets by specially shaped and positioned current carrying coils. In this section, we will discuss briefly some fundamental design concepts and limits for most commonly used iron dominated bending and quadrupole magnets as a guide for the accelerator designer towards a realistic design. For more detailed 
discussions on technical magnet designs we refer to related references, for example $[1,2]$.

Iron dominated magnets are the most commonly used magnets for particle beam transport systems. Only where very high particle energies and magnetic fields are required, superconducting magnets are used with maximum magnetic fields of 6 $10 \mathrm{~T}$ compared to the maximum field in an iron magnet of about $2 \mathrm{~T}$. Although saturation of ferromagnetic material imposes a definite limit on the strength of iron dominated magnets, most accelerator design needs can be accommodated within this limit.

We are now in a position to determine the fields for any multipole. This will be done in this section for magnetic fields most commonly used in particle transport systems, the bending field and the focusing quadrupole field. Only for very special applications are two or more multipole field components desired in the same magnet like in a gradient bending magnet or synchrotron magnet.

\section{Deflecting Magnets}

For the bending field $n=1$ and we get from (6.7) the magnetic potential

$$
P_{1}(x, y)=A_{1}+\mathrm{i} V_{1}=C_{1}(x+\mathrm{i} y)=\left(\lambda_{1} x-\mu_{1} y\right)+\mathrm{i}\left(\lambda_{1} y+\mu_{1} x\right) .
$$

in case of bending magnets, the skew type is a vertical bending magnet which is used in beam dynamics very rarely. The equipotential lines in the transverse $(x, y)$ plane along which the scalar potential is constant are determined for the first order potential by

$$
V_{1}=\lambda_{1} y+\mu_{1} x=\mathrm{const}
$$

and the corresponding electromagnetic field is given in component formulation by the vector

$$
\boldsymbol{B}=\left(-\mu_{1},-\lambda_{1}, 0\right)
$$

Equation (6.22) defines the lowest order transverse field in beam guidance or beam transport systems, is uniform in space and is called a dipole field. To simplify the design of beam transport systems it is customary to use dipole fields that are aligned with the coordinate system such as to exert a force on the particles only in the horizontal $x$ - or only in the vertical $y$-direction. With these definitions, we have for a horizontally deflecting magnet $\left(\lambda_{1} \neq 0, \mu_{1}=0\right)$ and for a vertically deflecting magnet $\left(\lambda_{1}=0, \mu_{1} \neq 0\right)$. To design a pure dipole magnet, we would place iron surfaces at equipotential lines. Specifically, for a horizontally deflecting magnet the equipotential lines are at

$$
y= \pm G
$$


to define a uniform vertical field within a vertical magnet aperture of $2 G$. Infinitely long magnets are assumed and the equipotential surface is defined by the same line anywhere along $z$.

As mentioned above, vertical bending magnets are rarely used in accelerator physics. Yet, there are special instances, especially in beam transport lines where vertical bending magnets are required. In those cases we would just introduce a vertical curvature $\kappa_{y}$ in $(6.18 \mathrm{a})$ or $(6.19 \mathrm{a})$ cover the vertical dispersion function. Outside the bending magnet the dispersion behaves just like a particle trajectory and therefore the quadrupoles do not have to be rotated or modified.

\section{Focusing Device}

The most suitable device that provides a material free aperture and a desired focusing field is a quadrupole magnet. The magnetic field can be derived from the term $n=2$ of the scalar potential (6.7)

$$
P_{2}(x, y)=C_{2}(x+\mathrm{i} y)^{2}=C_{2}\left(x^{2}-y^{2}+\mathrm{i} 2 x y\right) .
$$

Similar to the dipole case, both the real and imaginary parts are two independent solutions of the same Laplace equation and therefore the potential for both components can be written in the form

$$
P_{2}(x, y)=A_{2}+\mathrm{i} V_{2}=\lambda_{2}\left(x^{2}-y^{2}\right)-2 \mu_{2} x y+\mathrm{i}\left[2 \lambda_{2} x y+\mu_{2}\left(x^{2}-y^{2}\right)\right] .
$$

Both the real and imaginary solutions are independent solutions with independent coefficients. Coefficient $-2 \lambda_{2}=g$ is equal to the field gradient for an upright quadrupole and $-2 \mu_{2}=g$, which is the field gradient of a skew quadrupole. Separating both solutions, equipotential lines in the transverse $(x, y)$-plane for both second order potentials can be defined by

$$
\begin{aligned}
x^{2}-y^{2} & =\text { const }, \quad \text { for the skew quadrupole } \quad \text { and } \\
x y & =\text { const. } \quad \text { for the upright quadrupole. }
\end{aligned}
$$

Magnetic equipotential surfaces with a profile following the desired scalar potential (6.1.3) will be suitable to create the desired fields. The field pattern of an upright quadrupole magnet (6.26b) is shown schematically in Fig. 6.1 (left) together with the pole configuration for a rotated quadrupole Fig. 6.1 (right).

\section{Synchrotron Magnet}

Sometimes a combination of both, the dipole field of a bending magnet and the focusing field of a quadrupole, is desired for compact beam transport lines to form 


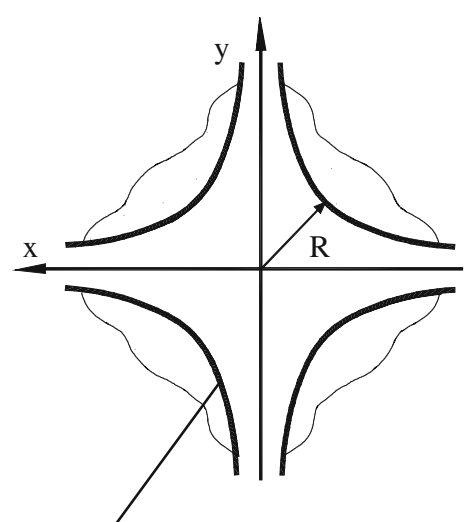

pole profile

$$
x y=1 / 2 R^{2}
$$

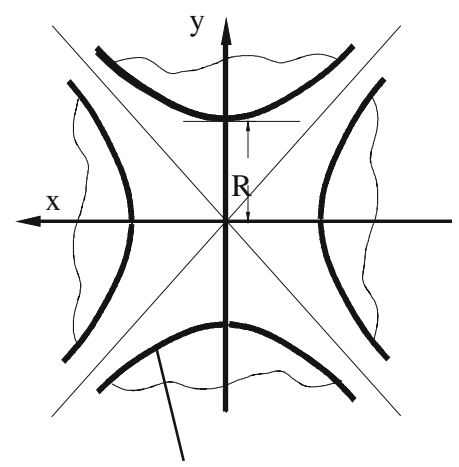

pole profile

$x^{2}-y^{2}=1 / 2 R^{2}$

Fig. 6.1 Pole shape of an upright quadrupole (left) and of a rotated quadrupole magnet (right)

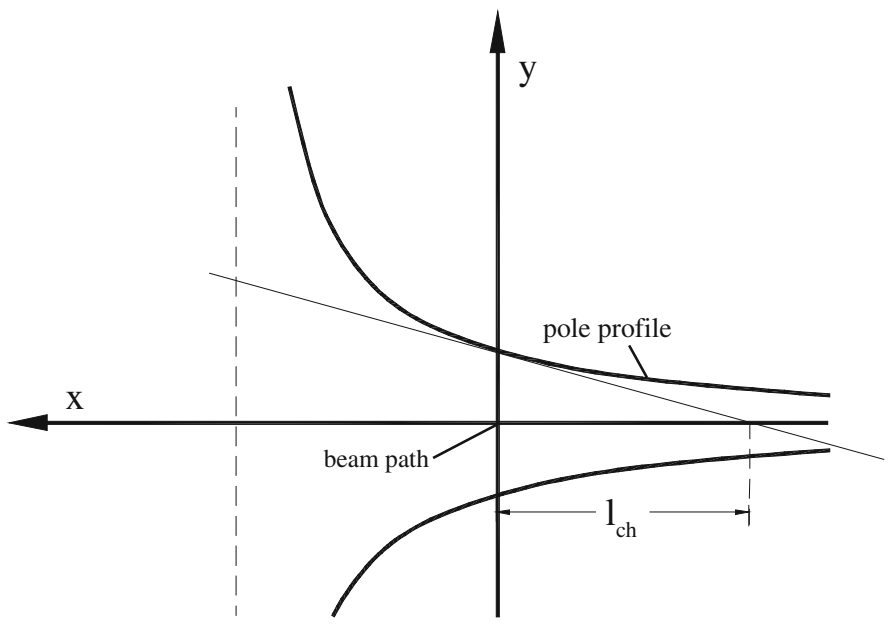

Fig. 6.2 Pole profile for a synchrotron magnet (schematic)

what is called a synchrotron magnet. The name comes from the use of such magnets for early synchrotron accelerators. The fields can be derived just like the dipole and quadrupole fields from the two-term potential (6.7) with $n=1$ and $n=2$.

Such a magnet actually is nothing but a transversely displaced quadrupole. The field in a quadrupole displaced by $x_{0}$ from the beam axis is $B_{y}=g\left(x-x_{0}\right)=g x-g x_{0}$ and a particle traversing this quadrupole at $x=0$ will be deflected by the field $B_{y}=$ $g x_{0}$. At the same time, we still observe focusing corresponding to the quadrupole field gradient $g$. The pole cross section of such a magnet is shown in Fig. 6.2. 
The deviation from parallelism of the magnet poles at the reference trajectory is often quantified by the characteristic length defined by

$$
\ell_{\mathrm{ch}}=\frac{B_{y}}{g}=\frac{1}{\rho_{0} k} \text {. }
$$

Geometrically this characteristic length is equal to the distance from the reference trajectory to that point at which the tangents from the two magnet poles at the vertical reference plane would touch (Fig. 6.2).

\section{Higher Order Multipole Magnets}

In a general beam transport line we use bending and quadrupole magnets to guide and focus a particle beam. For more sophisticated systems, however, we experience chromatic aberrations as is known from light optics. Particles with slightly different energies are focused differently and the image becomes blurred. In light optics such aberrations are partially corrected by the use of glasses with different refractive indices. In particle optics we use sextupoles. As the name indicates this magnet is composed of six poles. The complex potential is

$$
\begin{array}{r}
P_{3}(z)=A_{3}+\mathrm{i} V_{3}=C_{3}(x+\mathrm{i} y)^{3}=\lambda_{3}\left(x^{3}-3 x y^{2}\right)-\mu_{3}\left(3 x^{2} y-y^{3}\right) \\
+\mathrm{i}\left[\lambda_{3}\left(3 x^{2} y-y^{3}\right)+\mu_{3}\left(x^{3}-3 x y^{2}\right)\right] .
\end{array}
$$

Only upright sextupoles are used in beam dynamics for which $-6 \lambda_{3}=s_{3}$ the ideal fields are

$$
\frac{e}{p_{0}} B_{x}=-m x y \text { and } \frac{e}{p_{0}} B_{y}=-\frac{1}{2} m\left(x^{2}-y^{2}\right) .
$$

The pole profile is given by the scalar potential $V_{3}$

$$
V_{3}=3 x^{2} y-y^{3}=\mathrm{const}
$$

which describes the center poles along the vertical axis. To get the other poles one must rotate the center pole by $60^{\circ}$. The aperture radius $R$ must be chosen like in the case of the quadrupole from other consideration related to the application and beam requirement. The actual sextupole profile (6.30) is then given for the center pole by

$$
3 x^{2} y-y^{3}=-R^{3} .
$$

The magnet pole shapes for sextupole octupole or higher order magnets are shown in Fig. 6.3. Odd order multipoles like dipoles, sextupoles, decapoles etc. are characterized by central poles along the vertical axis (Fig. 6.3 left). Even order 


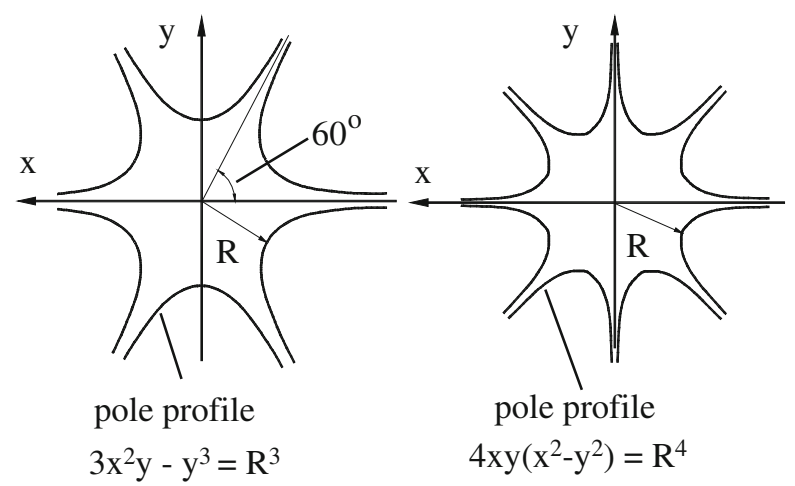

Fig. 6.3 Pole profile for an upright sextupole (left) and octupole (right) magnet

multipoles have no poles along the horizontal or vertical axis (Fig. 6.3 right). The profile can be derived directly from the respective potential (6.7). Only the profile of one pole must be determined since the other poles are generated by simple rotation of the first pole by multiples of the angle $90^{\circ} / n$, where $n$ is the order of the multipole. Multipoles of higher order than sextupoles are rarely used in accelerator physics but can be derived from the appropriate multipole potentials.

For an arbitrary single higher order multipole the field components can be derived from its potential (6.7)

$$
P_{n}(x, y)=A_{n}+\mathrm{i} V_{n}=C_{n}(x+\mathrm{i} y)^{n} .
$$

From this equation it is straight forward to extract an expression for the potential of any multipole field satisfying the Laplace equation. Since both electrical and magnetic fields may be derived from the Laplace equation, we need not make any distinction here and may use (6.32) as an expression for the electrical as well as the magnetic potential.

As mentioned before, it is useful to keep both sets of solutions $\left(\lambda_{n}, \mu_{n}\right)$ separate because they describe two distinct orientations of multipole fields. For a particular multipole both orientations can be realized by a mere rotation of the element about its axis. Only the solution $\lambda_{n}$ has what is called midplane symmetry with the property that $B_{n y}(x, y)=B_{n y}(x,-y)$. In this symmetry, there are no horizontal field components in the midplane, $B_{n x}(x, 0) \equiv 0$, and a particle travelling in the horizontal mid plane will remain in this plane. We call all magnets in this class upright magnets. The magnets defined by $\mu_{n} \neq 0$ we call rotated or skew magnets since they differ from the upright magnets only by a rotation about the magnet axis. In real beam transport systems, we use almost exclusively magnetic fields with midplane symmetry. 

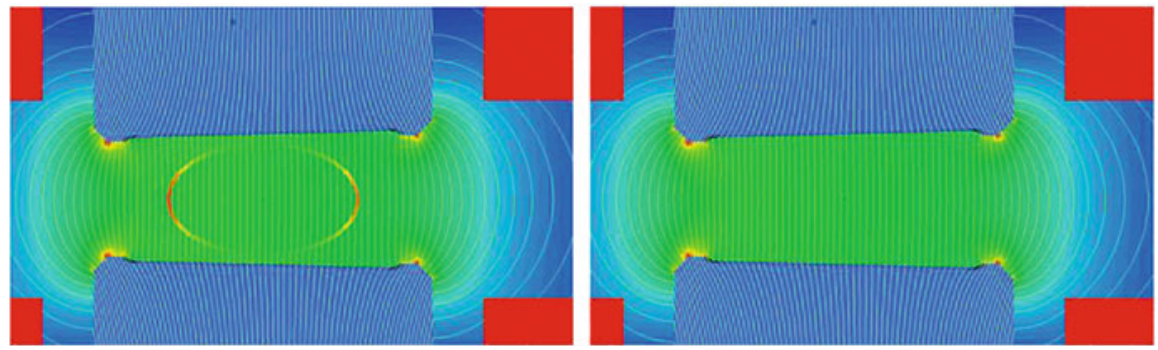

Fig. 6.4 Simulation of the dipole field through a vacuum chamber which is magnetic $(\mu=1.8)$ (left). The same situation is shown on the right side after annealing of the vacuum chamber

\section{Vacuum Chamber Material}

We have made great efforts to optimize the multipole field quality, but much of this can be destroyed again with the installation of a vacuum chamber. The vacuum chamber must be made of material which is non-magnetic. This is no problem with Aluminum or Copper but great care must be exercised with steel chambers. Nonmagnetic material with a permeability of some $\mu=1.01$ or $=1.02$ should be used. If the permeability is greater, the vacuum chamber walls concentrate magnetic flux which distorts the desired field. A field simulation with vacuum chamber is shown in Fig. $6.4^{1}$ where we note the field concentration in two parts of the vacuum chamber (left) which has a permeability of $\mu=1.8$. The simulation is for the NSRRC booster where beam could not be stored at injection energy of $150 \mathrm{MeV}$ because of the magnetic properties of the vacuum chamber. After annealing to about $1,050^{\circ} \mathrm{C}$ the permeability was reduced to $\mu \approx 1.01-1.02$ and the effect of the vacuum chamber has been clearly eliminated (right). Similar effects on the ideal magnetic field can occur in any other multipole. While the perturbation seems small and barely noticeable it is big enough to prevent storage of a beam in a circular accelerator.

\subsubsection{Multipole Misalignment and "Spill-down"}

In beam dynamics it is very important to align magnets very precise. However, there are limits and we need to know what happens if we misalign magnets. We consider first only a rotational misalignment by the angle $\delta$. The scalar potential is then

$$
P_{n}(r, \varphi)=C_{n} r^{n} \mathrm{e}^{\mathrm{i} n(\varphi-\delta)} .
$$

\footnotetext{
${ }^{1}$ The author thanks Jyh-Chyuan Jan, Cheng-Ying Kuo and Ping J. Chou from NSRRC, Taiwan for the pictures showing the effect of a magnetized vacuum chamber based on simulations.
} 
Expanding this, we get for small rotations such that $n \delta \ll 1$

$$
P_{n}(r, \varphi)=C_{n} r^{n} \mathrm{e}^{\mathrm{i} n \varphi} \mathrm{e}^{-\mathrm{i} n \delta} \approx C_{n} r^{n} \mathrm{e}^{\mathrm{i} n \varphi}(1-\mathrm{i} n \delta) .
$$

The rotational error $\delta$ has not altered the original magnetic field, but has added a small skew component of the same magnet. Much more dramatic are lateral misalignments. Here, we start from (6.32) and misplace the magnet by the amount $\delta z=\delta x+\mathrm{i} \delta y$.

$$
P_{n}(x, y)=C_{n}(z+\delta z)^{n} .
$$

This can be expanded for

$$
\begin{aligned}
P_{n}(x, y) & =C_{n}(z+\delta z)^{n} \\
& \approx C_{n} z^{n}+C_{n}\left[\left(\begin{array}{l}
n \\
1
\end{array}\right) z^{n-1} \delta z+\left(\begin{array}{l}
n \\
2
\end{array}\right) z^{n-2} \delta z^{2}+\ldots+\left(\begin{array}{l}
n \\
n
\end{array}\right) \delta z^{n}\right] .
\end{aligned}
$$

The original field is still preserved, but now many lower order terms appear. Actually, for a lateral misalignment all lower order magnetic field components appear, a phenomenon that is called "spill-down". These lower order fields cause orbit distortions, focusing errors and errors in the chromaticity, which all have to be compensated.

\subsection{Main Magnet Design Criteria}

In this section we will shortly discuss the design criteria for the main beam dynamics magnets like bending magnets and quadrupoles. For more detailed studies on magnets the reader is referred to relevant texts like [2].

\subsubsection{Design Characteristics of Dipole Magnets}

The expressions for the magnetic potentials give us a guide to design devices that generate the desired fields. Multipole fields are generated mostly in one of two ways: as iron dominated magnets, or by proper placement of electrical current carrying conductors. The latter way is mostly used in high field superconducting magnets, where fields beyond the general saturation level of about $2 \mathrm{~T}$ for iron are desired.

In iron dominated magnets, fields are determined by the shape of the iron surfaces. Just like metallic surfaces are equipotential surfaces for electrical fields, so are surfaces of ferromagnetic material, like iron in the limit of infinite magnetic permeability, equipotential surfaces for magnetic fields. Actually, for practical applications the permeability only has to be large just like the conductivity must be large to make a metallic surface an equipotential surface. This approximate property 
of iron surfaces can be exploited for the design of unsaturated or only weakly saturated magnets. For preliminary design calculations, we assume infinite permeability. Where effects of finite permeability or magnetic saturation become important, the fields are determined numerically by mathematical relaxation methods. In this text, we will not be able to discuss the details of magnet design and construction but will concentrate only on the main magnet features from a beam dynamics point of view. A wealth of practical experience in the design of iron dominated accelerator magnets, including an extensive list of references, is compiled in a review article by Fischer [1] and a monograph by Tanabe [2].

\section{Excitation Current and Saturation in a Bending Magnet}

A dipole field can be generated, for example, in an electromagnet as shown in Fig. 6.5 where the beam would travel normal to the cross section into the center of the magnet.

The magnetic field $\boldsymbol{B}$ is generated by an electrical current $I$ in current carrying coils surrounding magnet poles. A ferromagnetic return yoke surrounds the excitation coils providing an efficient return path for the magnetic flux. The magnetic field is determined by Ampere's law

$$
\nabla \times \frac{\boldsymbol{B}}{\mu_{\mathrm{r}}}=\mu_{0} \boldsymbol{j},
$$

where $\mu_{\mathrm{r}}$ is the relative permeability of the ferromagnetic material and $j$ is the current density in the coils. Integrating (6.37) along a closed path like the one shown in Fig. 6.5 and using Stokes' theorem gives

$$
2 G B_{0}+\int_{\text {iron }} \frac{\boldsymbol{B}}{\mu_{\mathrm{r}}} \mathrm{d} \boldsymbol{\sigma}=\mu_{0} I_{\mathrm{tot}},
$$

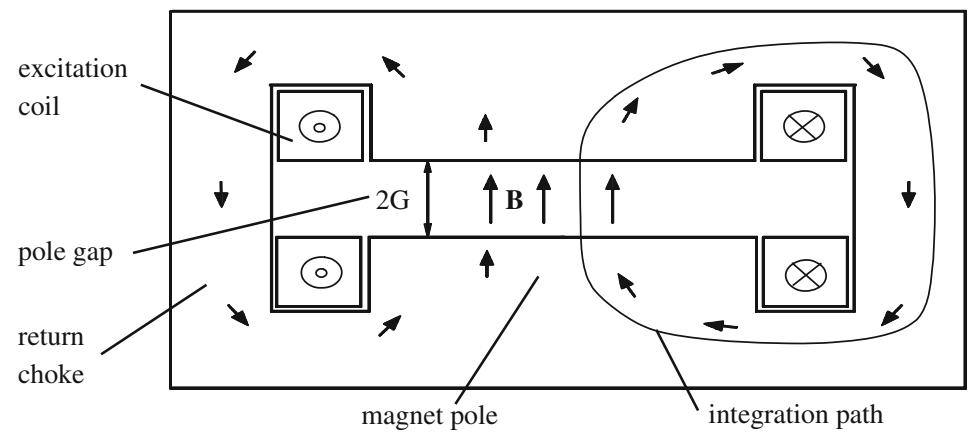

Fig. 6.5 Cross section of a dipole magnet (schematic) 
where $B_{0}$ is the magnetic field in the center of the magnet aperture between and normal to the parallel magnet poles with a gap distance of $2 G$. The integral term in (6.38) is zero or negligibly small in most cases assuming infinite or a very large permeability within the magnetic iron. $I_{\text {tot }}=2 I_{\text {coil }}$ is the total current flowing in the complete cross section of both coils. Solving (6.38) for the total current in each coil we get in more practical units

$$
I_{\text {coil }}(\mathrm{A})=\frac{1}{\mu_{0}} B_{0}(\mathrm{~T}) G(\mathrm{~m}),
$$

which is proportional to the magnetic field and the aperture between the magnet poles.

As a practical example, we consider a magnetic field of $1 \mathrm{~T}$ in a dipole magnet with an aperture of $2 G=10 \mathrm{~cm}$. From (6.39), a total electrical excitation current of about 40,000 A is required in each of two excitation coils to generate this field. Since the coil in general is composed of many turns, the actual electrical current is much smaller by a factor equal to the number of turns and the total coil current $I_{\text {coil }}$ is therefore often measured in units of Ampere-turns. For example, a coil composed of 40 windings with sufficient cross section to carry an electrical current of 1,000 A would provide the total required current of 40,000 A-turns.

As a rule of thumb to get a good field quality within an aperture width equal to the full gap height the pole width should be at least 3-times the full gap height. Narrower pole profiles require shimming of the pole profile. There are elaborate way to shape the pole profile for a bending magnet [2] but there are also more simple ways. The drop-off of the field towards the side of the poles can be to some extend extended further out by adding to the pole profile a straight line shim to slightly reduce the pole gap around the edges of the poles. This shim need not be more elaborate than a line segment to reduce the gap followed by a horizontal section to the edge of the pole. Such shims may start around half a full gap size from the center with a gentle slope and rarely a thickness of more than $0.5-1 \mathrm{~mm}$. We will discuss such shims in more detail in connection with quadrupole design.

Saturation effects are similar to those in a quadrupole magnet which will be discussed in the next section. Like in any magnet the first sign of saturation show up most likely at the pole root where the poles join the return yoke. That is so because much magnetic flux comes into the pole from the sides along the length of the pole thus increasing the magnetic flux density. One way out is to shape the pole pieces like wedges with increasing cross section towards the return yoke. Any saturation in the return yoke is easily avoided by increasing the thick ness of the iron in the return yoke.

\subsubsection{Quadrupole Design Concepts}

Quadrupoles together with bending magnets are the basic building blocks for charged particle beam transport systems and serve as focusing devices to keep 
the particle beam close to the desired beam path. The magnet pole profile for a quadrupole can be derived the same way as that for a dipole magnet. Placing an iron boundary in the shape of a hyperbola generates the equipotential surface required for an upright quadrupole, or mathematically

$$
x y=\text { const } .
$$

The inscribed radius of the iron free region is $R$ and the constant in (6.40) is therefore $(R / \sqrt{2})^{2}=\frac{1}{2} R^{2}$ as shown in Fig. 6.1. The pole shape or pole profile for a quadrupole with bore radius $R$ is then defined by the equation

$$
x y= \pm \frac{1}{2} R^{2} .
$$

Similarly, the pole profile of a rotated quadrupole is given by

$$
x^{2}-y^{2}= \pm R^{2} .
$$

This is the same hyperbola as (6.41) but rotated by $45^{\circ}$. Both (6.41) and (6.42) describe four symmetrically aligned hyperbolas which become the surfaces of the ferromagnetic poles producing an ideal quadrupole field. Magnetization at alternating polarity of each pole generates a sequence of equally strong north and south poles.

In a real quadrupole, we cannot use infinitely wide hyperbolas but must cutoff the poles at some width. In Fig. 6.6 some fundamental design features and parameters for a real quadrupole are shown and we note specifically the finite pole width to make space for the excitation coils. Since only infinitely wide hyperbolic poles create a pure quadrupole field, we expect the appearance of higher multipole field errors characteristic for a finite pole width.

\section{Pole Profile Shimming}

While in an ideal quadrupole the field gradient along, say, the $x$-axis would be constant, we find for a finite pole width a drop off of the field and gradient approaching the corners of poles. Different magnet designer apply a variety of pole shimming methods. In this text we use tangent shimming as described below. The field drop off at the pole edge can be reduced to some extend if the hyperbolic pole profile continues into its tangent close to the pole corner as indicated in Fig. 6.6.

This adds some iron to increase the field where the field would otherwise fall below the desired value. The starting point of the tangent determines greatly the final gradient homogeneity in the quadrupole aperture. In Fig. 6.7 the gradient along the $x$-axis is shown for different starting points of the tangent. There is obviously an optimum point for the tangent to minimize the gradient error over a wide aperture. 


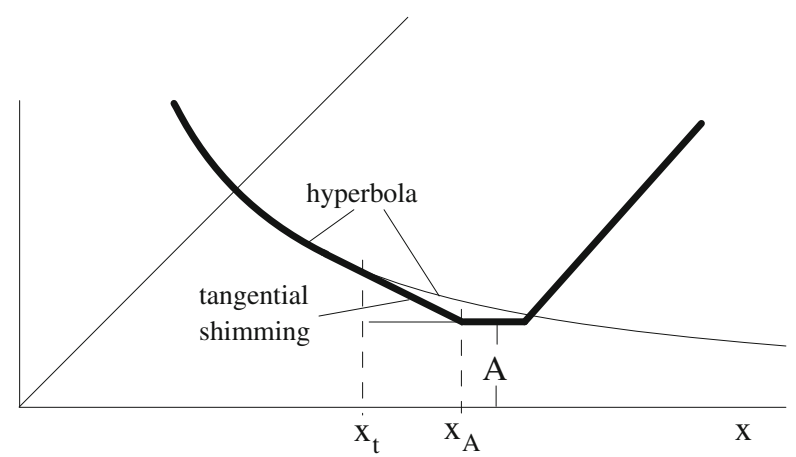

Fig. 6.6 Quadrupole design features

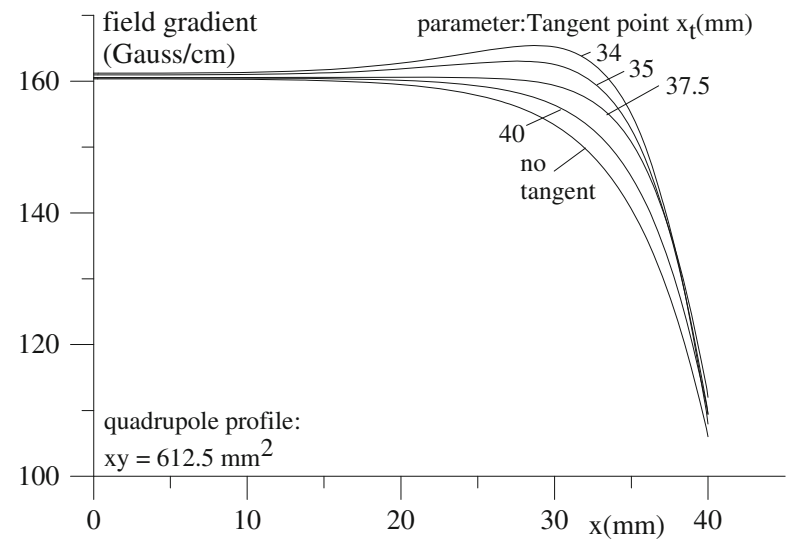

Fig. 6.7 Empirical field gradient and pole profile shimming for a particular quadrupole as determined by numerical simulations with the program MAGNET [3]

Application of tangent shimmingmust be considered as a fine adjustment of the field quality rather than a means to obtain a large good field aperture as becomes apparent from Fig. 6.7. The good field aperture is basically determined by the width of the pole. While optimizing the tangent point, we find an empirical correlation between gradient tolerance (Fig. 6.8) within an aperture region $x \leq X_{\mathrm{F}}$ and the pole width expressed by the minimum pole distance $A$. The good field region increases as the pole gets wider. For initial design purposes, we may use Fig. 6.8 to determine the pole width from $A$ based on the desired good field region $X_{\mathrm{F}}$ and gradient field quality.

The final design of a magnet pole profile is made with the help of computer codes which allow the calculation of magnet fields from a given pole profile with saturation characteristics determined from a magnetization curve. Widely used computer codes for magnet design are, for example, MAGNET [3] and POISSON [4]. 


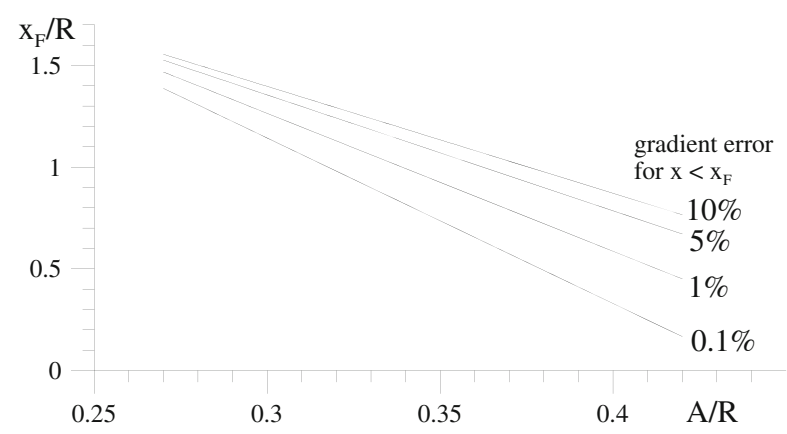

Fig. 6.8 Field gradient tolerances as a function of pole profile parameters calculated with MAGNET

Field errors in iron dominated magnets have two distinct sources, the finite pole width and mechanical manufacturing and assembly tolerances. From symmetry arguments, we can deduce that field errors due to the finite pole width produce only select multipole components. In a quadrupole, for example, only $(2 n+1) \cdot 4$-pole fields like 12-pole or 20-pole fields are generated. Similarly in a dipole of finite pole width only $(2 n+1) \cdot 2$-pole fields exist. We call these multipole field components often the allowed multipole errors. Manufacturing and assembly tolerances on the other hand do not exhibit any symmetry and can cause the appearance of any multipole field error.

The particular choice of some geometric design parameters must be checked against technical limitations during the design of a beam transport line. One basic design parameter for a quadrupole is the bore radius $R$ which depends on the aperture requirements of the beam. Addition of some allowance for the vacuum chamber and mechanical tolerance between chamber and magnet finally determines the quadrupole bore radius.

\section{Excitation Current and Saturation}

The field gradient is determined by the electrical excitation current in the quadrupole coils. Similar to the derivation for a bending magnet, we may derive a relation between field gradient and excitation current from Maxwell's curl equation. To minimize unnecessary mathematical complexity, we choose an integration path as indicated in Fig. 6.9 which contributes to the integral $\oint \mathbf{B}_{s} \mathrm{~d} \mathbf{s}$ only in the aperture of the quadrupole.

Starting from the quadrupole axis along a path at $45^{\circ}$ with respect to the horizontal or vertical plane toward the pole tip, we have

$$
\frac{1}{\mu_{\mathrm{r}}} \oint \mathbf{B}_{s} \mathrm{~d} \mathbf{s}=\int_{0}^{R} B_{r} \mathrm{~d} r=\mu_{0} I_{\mathrm{tot}}
$$


Fig. 6.9 Determination of the field gradient from the excitation current

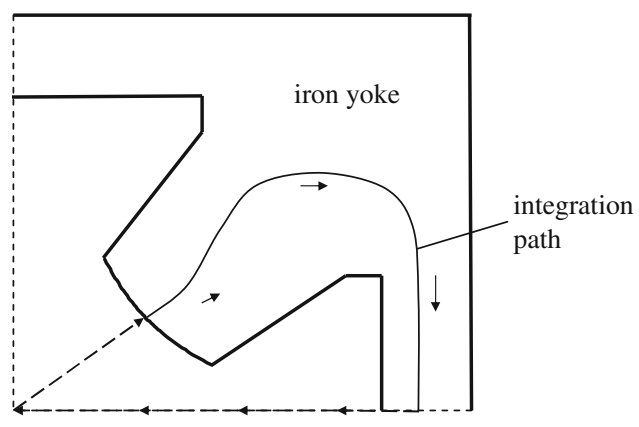

Since $B_{x}=g y$ and $B_{y}=g x$, the radial field component is $B_{r}=\sqrt{B_{x}^{2}+B_{y}^{2}}=g r$ and the excitation current from (6.43) is given by

$$
I_{\text {tot }}(\mathrm{A} \times \text { turns })=\frac{1}{2 \mu_{0}} g\left(\frac{\mathrm{T}}{\mathrm{m}}\right) R^{2}(\mathrm{~m}) .
$$

The space available for the excitation coils or coil slot in a real quadrupole design determines the maximum current carrying capability of the coil. Common materials for magnet coils are copper or aluminum. The electrical heating of the coils depends on the current density and a technically feasible balance between heating and cooling capability must be found. As a practical rule the current density in regular beam transport magnets should not exceed about $6-8 \mathrm{~A} / \mathrm{mm}^{2}$. This is more an economical than a technical limit and up to about a factor of two higher current densities could be used for special applications. The total required coil cross section, however, including an allowance for insulation material between coil windings and about 15-20\% for water cooling holes in the conductor depends on the electrical losses in the coil. The aperture of the water cooling holes is chosen such that sufficient water cooling can be provided with an allowable water temperature increase which should be kept below $40^{\circ} \mathrm{C}$ to avoid boiling of the cooling water at the surface and loss of cooling power. A low temperature rise is achieved if the water is rushed through the coil at high pressure in which case undesirable vibrations of the magnets may occur. The water cooling hole in the conductor must therefore be chosen with all these considerations in mind. Generally the current density averaged over the whole coil cross section is about $60-70 \%$ of that in the conductor.

In practical applications, we find the required coil cross section to be significant compared to the magnet aperture leading to a long pole length and potential saturation. To obtain high field limits due to magnetic saturation, iron with a low carbon content is used for most magnet applications in particle beam lines. Specifically, we require the carbon content for most high quality magnets to be no more than about $1 \%$. In Fig. 6.10 the magnetization curve and the permeability as a function of the excitation are shown for iron with $0.5 \%$ carbon content. We note a steep drop in the permeability above $1.6 \mathrm{~T}$ reaching full saturation at about $2 \mathrm{~T}$. 
Fig. 6.10 Magnetization and 10000 permeability of typical low carbon steel as a function of excitation

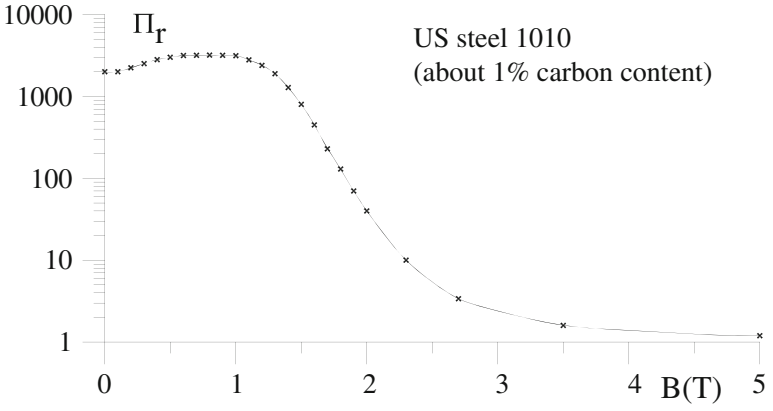

A magnet has an acceptable saturation level if the magnetic permeability anywhere over the cross section of the magnet remains large compared to unity, $\mu_{\mathrm{r}} \gg 1$.

Severe saturation effects at the corners of the magnet pole profile can be avoided if the maximum field gradient, as a rule of thumb, is chosen such that the pole tip field does not exceed a value of $B_{\mathrm{p}}=0.8-1 \mathrm{~T}$. This limits the maximum field gradient to $g_{\max }=B_{\mathrm{p}} / R$ and the quadrupole length must therefore be long enough to reach the focal length desired in the design of the beam transport line. Saturation of the pole corners introduces higher-order multipoles and must therefore be kept to a minimum.

Other saturation effects may occur at the pole root where all magnetic flux from a pole including fringe fields are concentrated. If the pole root is too narrow, the flux density is too high and saturation occurs. This does not immediately affect the field quality in the central aperture, but requires higher excitation currents. A similar effect may occur in the return yokes if the field density is too high because of too small an iron cross section. In Fig. 6.11 a permeability plot is shown for a magnet driven into severe saturation. Low values of the permeability indicate high saturation, which is evident in the pole root.

By increasing the width of the pole root the saturation is greatly reduced as shown in Fig. 6.12. To minimize pole root saturation the pole length should be as short as possible because less flux is drawn through the side of the pole. Unfortunately, this also reduces the space available for the excitation coils leading to excessively large current densities. To reduce this conflict, the pole width is usually increased at the pole root rather than shortening the pole length.

In addition to pole root saturation, we may also experience return yoke saturation, which is easily avoided by increasing its thickness.

\subsection{Magnetic Field Measurement}

The quality of the magnetic fields translates immediately into the quality and stability of the particle beam. The precision of the magnetic fields determines the predictability of the beam dynamics designs. While we make every effort to 


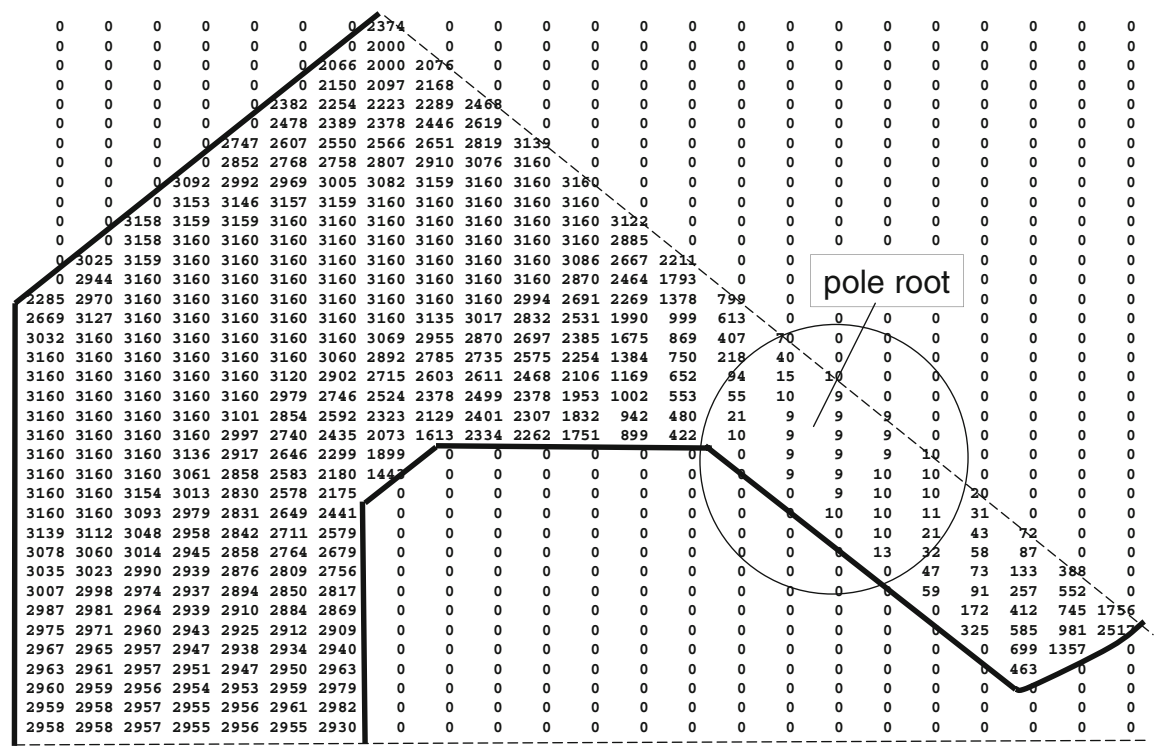

Fig. 6.11 Permeability values are plotted in a grid over the iron cross section of a highly excited quadrupole. We note the significantly reduced permeability $(\mu \ll 100)$ in the narrow pole root

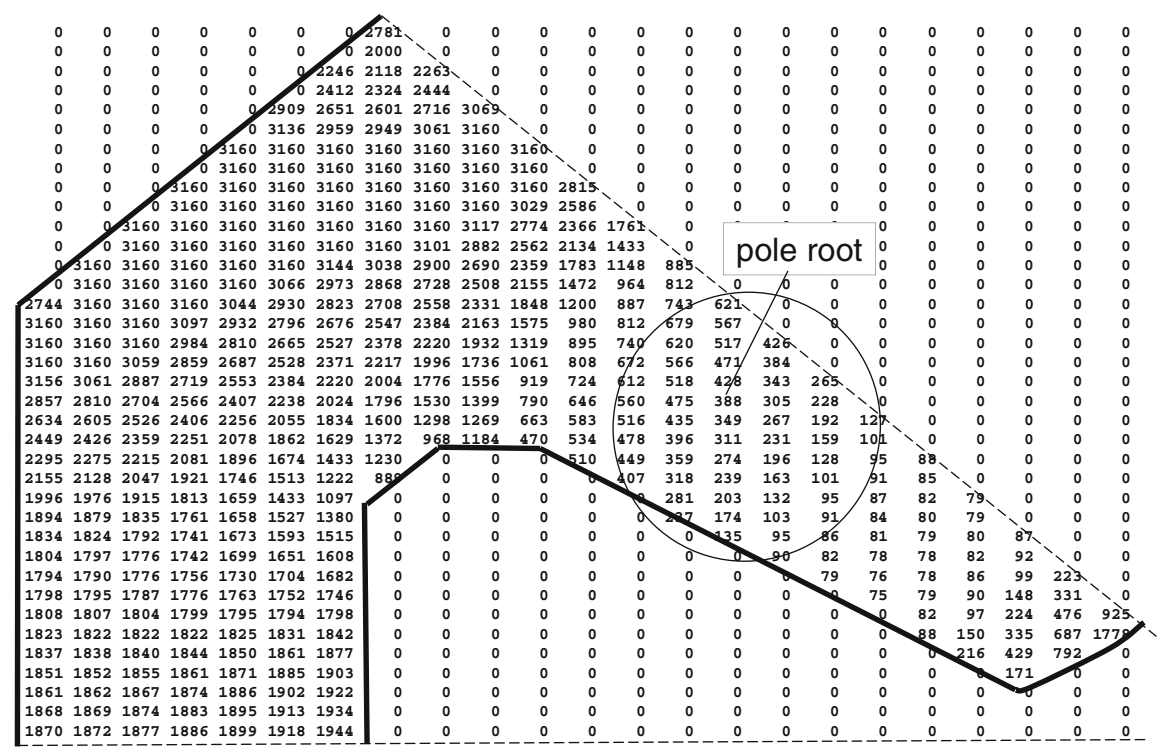

Fig. 6.12 Permeability values are plotted in a grid over the iron cross section of a highly excited quadrupole. We note the significantly reduced permeability $(\mu \ll 100)$ in the narrow pole root 
construct magnets as precise as possible, we cannot avoid the appearance of higher multipole fields due to finite pole widths or machining and assembly tolerances. Therefore, precise magnetic field measurements are required. While detailed discussions of magnetic field measurement technology exceeds the goals of this book, the issue is too important to ignore completely and we will discuss this topic in an introductory way. For more detailed information, please consult texts like [2].

\subsubsection{Hall Probe}

The Hall probe is the most commonly used device to measure the magnetic field. Its principle is based on the Lorentz force on moving charges. Use a small piece of metallic foil, say $1 \times 1 \mathrm{~mm}^{2}$, send an electrical current in one direction through the foil and place the foil into a magnetic field such that the field penetrates the plane of the foil. The moving electrons feel the Lorentz force due to the presence of the magnetic field and are pulled off a straight path, thus accumulating charge on one side of the foil. That charge accumulation causes with the other side of the foil a potential difference, the Hall voltage, which can be measured and which is proportional to the magnetic field component passing orthogonally through the foil. The material of commercial Hall probes is not a metallic foil but some material which contains many electrons with great mobility to maximize the sensitivity of the probe. The size of the probe is made very small for maximum resolution because the probe measures the average field across the area of the foil. Typical areas of a Hall probe may be in the $\mu m$ range which provides a high resolution as desired in magnetic field measurements for beam dynamics. Figure 6.13 shows the principle functioning of a Hall Probe.

By computer controlled precise movement of the Hall probe from point to point within the magnet aperture, the magnetic field can be mapped to high precision. The measurements can then be analysed as to field errors, multipole content and fringe field effects.

Fig. 6.13 Hall probe (schematic) ( $I$ activation current, $B$ magnetic field, $v_{\mathrm{e}}$ velocity of electrons, $F_{\mathrm{L}}$ Lorentz force, $\pm V$ signal voltage)

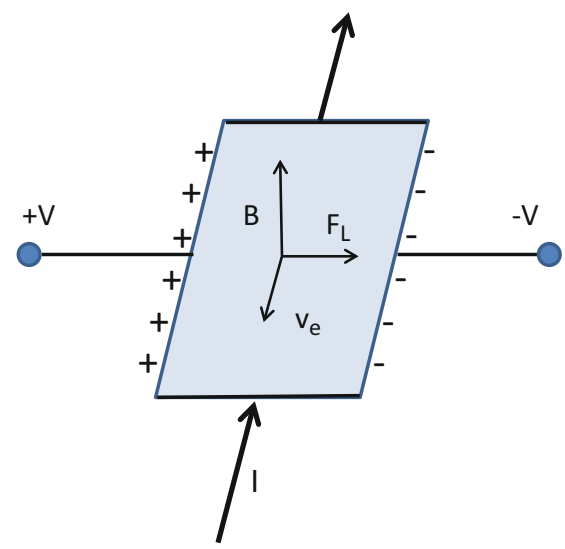


Fig. 6.14 Rotating coil in a magnet to determine higher order multipole components

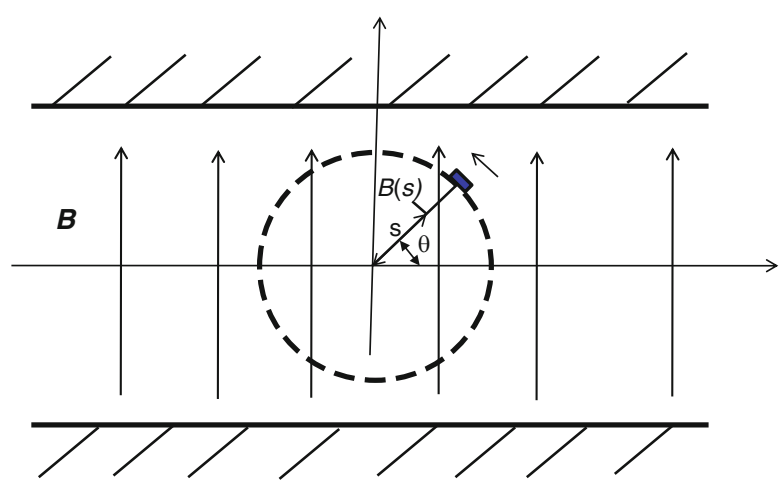

\subsubsection{Rotating Coil}

In practice, however, the particles in a beam integrate through a whole magnet and we are therefore bound to do the same with Hall probe measurements. A faster method, and actually more precise method for higher order multipole fields, is a rotating coil as shown in Fig. 6.14. Here, a coil wound of very thin electrical wire is installed coaxial within the magnet aperture. Rotating the coil produces a time dependent voltage which includes all fields within the cross section of the coil and integrated along the length of the coil. The length of most coils extends well beyond the ends of the magnet while very short coils may be used to specifically probe local fields like fringe fields in the ends of magnets. As the coil rotates the induced voltage is recorded measuring the integrated field along the length of the coil. The induced voltage is $V=-\frac{\mathrm{d} \Phi}{\mathrm{d} t}$ and the magnetic flux

$$
\Phi=L_{\mathrm{eff}} \int B(s) \mathrm{d} s
$$

where $L_{\text {eff }}$ is the effective length of the magnet. The integration is taken from the axis to the radial extent of the coil. With $\frac{\mathrm{d} \Phi}{\mathrm{d} t}=\frac{\mathrm{d} \Phi}{\mathrm{d} s} \frac{\mathrm{d} s}{\mathrm{~d} t}=L_{\mathrm{eff}} B(s) \frac{\mathrm{d} s}{\mathrm{~d} t}$ the induced voltage is $V=-L_{\text {eff }} B(s) \frac{\mathrm{d} s}{\mathrm{~d} t}$ and the integrated voltage is

$$
\int V \mathrm{~d} t=-L_{\mathrm{eff}} \int_{0}^{r} B(s) \mathrm{d} s .
$$

With $B_{x}=\frac{\partial A}{\partial y}$ and $B_{y}=-\frac{\partial A}{\partial x}$, we get $B(s)=-B_{x} \sin \theta+B_{y} \cos \theta=-\frac{\mathrm{d} A}{\mathrm{~d} s}$ and

$$
\int V \mathrm{~d} t=-L_{\mathrm{eff}} A(\theta)=\sum_{n}\left[p_{n} \cos \left(n \theta+\psi_{n}\right)+q_{n} \sin \left(n \theta+\psi_{n}\right)\right],
$$

where we have also introduced the Fourier transform of the signal. The vector potential is used to determine the fields because of simplicity of math. The Fourier 
transform will help us to determine the multipole strength and orientation. For the $n$-multipole

$$
\begin{aligned}
\left.\int V \mathrm{~d} t\right|_{n} & =-L_{\mathrm{eff}} A_{n} \\
& =-L_{\mathrm{eff}}\left|C_{n}\right| r_{0}^{n}\left(\cos n \theta \cos \psi_{n}-\sin n \theta \sin \psi_{n}\right) \\
& =p_{n} \cos \left(n \theta+\psi_{n}\right)+q_{n} \sin \left(n \theta+\psi_{n}\right)
\end{aligned}
$$

where $r_{0}$ is the radius of the coil and $A_{n}=\operatorname{Re} P_{n}=\left|C_{n}\right| r_{0}^{n} \cos \left(n \theta+\psi_{n}\right)$. To maximize the signal, the coil radius $r_{0}$ should be about $80 \%$ of the aperture radius. Larger coils would not fit the magnet aperture. The phase $\psi_{n}$ defines the orientation of the $n$-multipole. From (6.48) the multipole strength is

$$
L_{\text {eff }}\left|C_{n}\right|=\frac{\sqrt{p_{n}^{2}+q_{n}^{2}}}{r_{0}^{n}}
$$

and the orientation

$$
\psi_{n}=-\arctan \frac{q_{n}}{p_{n}}
$$

From the Fourier coefficients $\left(p_{n}, q_{n}\right)$ of the measured signal $\int V \mathrm{~d} t$ and knowledge of the coil size $r_{0}$ we can determine the strength $C_{n}$ and orientation $\psi_{n}$ of all multipole limited only by the sensitivity of the experimental setup. The magnetic fields are given by

$$
\begin{aligned}
B_{n x}-i B_{n y} & =i P^{\prime}=i n C_{n}|z|^{n-1} e^{\mathrm{i}(n-1) \theta} \\
& =n\left|C_{n}\right| r_{0}^{n-1}\left\{-\sin \left[(n-1) \theta+\psi_{n}\right]+\mathrm{i} \cos \left[(n-1) \theta+\psi_{n}\right]\right\}
\end{aligned}
$$

or

$$
\begin{aligned}
& B_{n x}=-n r_{0}^{n-1}\left[-\mu_{n} \cos (n-1) \theta+\lambda_{n} \sin (n-1) \theta\right], \quad \text { and } \\
& B_{n y}=-n r_{0}^{n-1}\left[+\lambda_{n} \cos (n-1) \theta-\mu_{n} \sin (n-1) \theta\right] .
\end{aligned}
$$

with

$$
\begin{aligned}
\left|C_{n}\right| \sin \psi_{n} & =-\mu_{n}=a_{n} \frac{B_{\text {main }}\left(r_{0}\right)}{n r_{0}^{n-1}} \text { and } \\
\left|C_{n}\right| \cos \psi_{n} & =+\lambda_{n}=-b_{n} \frac{B_{\text {main }}\left(r_{0}\right)}{n r_{0}^{n-1}} .
\end{aligned}
$$




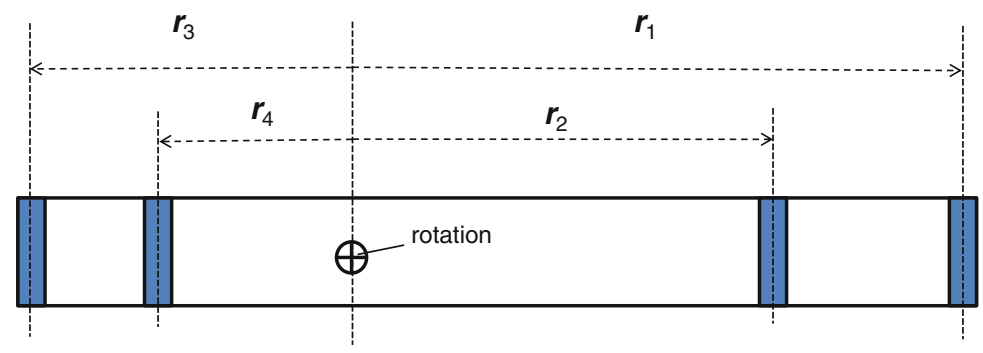

Fig. 6.15 Twin coil to determine higher order multipole components

The field components at an arbitrary radius $r$ are finally

$$
\begin{aligned}
\frac{B_{n x}}{B_{\text {main }}} & =\left(\frac{r}{r_{0}}\right)^{n-1}\left[b_{n} \sin (n-1) \theta+a_{n} \cos (n-1) \theta\right], \text { and } \\
\frac{B_{n y}}{B_{\text {main }}} & =\left(\frac{r}{r_{0}}\right)^{n-1}\left[b_{n} \cos (n-1) \theta+a_{n} \sin (n-1) \theta\right],
\end{aligned}
$$

where $B_{\text {main }}$ is the main magnet field at $r_{0}$. The signal obtained from a rotating coil can be used to determine the strength and orientation of higher multipoles.

\section{Practical Considerations}

The signals from higher multipoles are measured in the presence of the strong main field. The dynamic range of the equipment and integrator may not be wide enough to yield precise multipole information. It would be a great advantage if the signal from the main field could be compensated or at least be reduced to the level of the multipole signal. This is possible with multiple coils as shown in Fig. 6.15.

Here the signals from two coils are processed such that the main field is "bucked" out. There is an outer coil at $r_{1}, r_{3}$ and an inner coil $r_{2}, r_{4}$. The signal from the outer coil is

$$
\left.\int V \mathrm{~d} t\right|_{\text {outer coil }}=L_{\mathrm{eff}} m_{\mathrm{o}} \sum_{n}\left|C_{n}\right|\left(r_{1}^{n}-r_{3}^{n}\right) \cos \left(n \theta+\psi_{n}\right)
$$

and from the inner coil

$$
\left.\int V \mathrm{~d} t\right|_{\text {inner coil }}=L_{\text {eff }} m_{\mathrm{i}} \sum_{n}\left|C_{n}\right|\left(r_{2}^{n}-r_{4}^{n}\right) \cos \left(n \theta+\psi_{n}\right)
$$


forming a combined signal

$$
\left.\int V \mathrm{~d} t\right|_{\text {compensated }}=L_{\mathrm{eff}} \sum_{n \geq 0}\left|C_{n}\right|\left[m_{\mathrm{o}}\left(r_{1}^{n}-r_{3}^{n}\right)+m_{\mathrm{i}}\left(r_{2}^{n}-r_{4}^{n}\right)\right] \cos \left(n \theta+\psi_{n}\right) .
$$

Here, $m_{\mathrm{o}}$ and $m_{\mathrm{i}}$ are the turns in the outer and inner coil, respectively. Defining $\beta_{1}=\left|\frac{r_{3}}{r_{1}}\right|, \beta_{1}=\left|\frac{r_{4}}{r_{2}}\right|, \rho=\frac{r_{2}}{r_{1}}$ and $\mu=\frac{m_{\mathrm{i}}}{m_{\mathrm{o}}}$ the combined signal (6.57) is for the signal from the uncompensated outer coil

$$
\left.\int V \mathrm{~d} t\right|_{\text {uncompensated }}=L_{\text {eff }} m_{\mathrm{o}} \sum_{n \geq 0}\left|C_{n}\right| r_{1}^{n} S_{N} \cos \left(n \theta+\psi_{n}\right)
$$

and for the compensated coil signal

$$
\left.\int V \mathrm{~d} t\right|_{\text {compensated }}=L_{\mathrm{eff}} m_{\mathrm{o}} \sum_{n \geq 0}\left|C_{n}\right| r_{1}^{n} s_{n} \cos \left(n \theta+\psi_{n}\right)
$$

The signal sensitivity for the uncompensated coil is

$$
S_{N}=1-\left(-\beta_{1}\right)^{N}
$$

where $N$ represents the order of the main magnet field and the compensated coil has the sensitivity $s_{n}$ for the $n$ th-order multipole

$$
s_{n}=1-\left(-\beta_{1}\right)^{n}-\mu \rho^{n}\left[1-\left(-\beta_{2}\right)^{n}\right],
$$

where $n$ represents the $n$th order multipole. By choosing parameters such that $s_{n}$ becomes zero for the desired values of $n$, we may eliminated electronically the large signal from the main magnet field. For example, in case of a quadrupole, we would like to compensate the quadrupole field and the dipole field which may appear as a "spill-down" from a misaligned quadrupole. In this case, we would want to set $s_{1}=$ $1+\beta_{1}-\mu \rho\left[1+\beta_{2}\right] \approx 0$ and $s_{2} \approx 1-\beta_{1}^{2}-\mu \rho\left[1-\beta_{2}^{2}\right]=0$ and build a specific measurement coil for quadrupoles. Selecting arbitrarily $\mu=2$ and $\rho=0.625$ the desired sensitivity will be zero with $\beta_{1}=0.5$ and $\beta_{2}=0.2$. All other sensitivities are at least $60 \%$ and well known to be included in the analysis. It is not necessary that the main fields are bucked perfectly. It's sufficient if their signal is reduced to the level of the higher order multipole signals.

The whole magnetic measurement would record the signals from both coils separately and produce the strength and orientation of the main field for $n=N$ according to (6.49) and (6.50) while the same multipole parameters are derived from the same equations based on the compensated signal and including the calculated sensitivities. 
Magnetic field measurements have developed very far and have reached a level of accuracy and precision that fully meets the demands of beam dynamics. Especially, the determination of the multipole content is important to ensure the stability of a beam in, for example, a storage ring. While the effects of multipole fields cannot be analyzed analytically, we may track particles many times around the storage ring in the presence of these multipole fields and thus define beam stability and the dynamic aperture.

\subsection{General Transverse Magnetic-Field Expansion*}

In the previous section, we discussed solutions to the Laplace equation which included only pure transverse multipole components in a cartesian coordinate system thus neglecting all kinematic effects caused by the curvilinear coordinate system of beam dynamics. These approximations eliminate many higher-order terms which may become of significance in particular circumstances. In preparation for more sophisticated beam transport systems and accelerator designs aiming, for example, at ever smaller beam emittances it becomes imperative to consider higherorder perturbations to preserve desired beam characteristics. To obtain all field components allowed by the Laplace equation, a more general ansatz for the field expansion must be made. Here we restrict the discussion to scalar potentials only which are sufficient to determine all fields $[5,6]$.

Since we use a curvilinear coordinate system for beam dynamics, we use the same for the magnetic-field expansion and express the Laplace equation for the complex potential $P$ in these curvilinear coordinates

$$
\Delta V=\frac{1}{h}\left[\frac{\partial}{\partial x}\left(h \frac{\partial V}{\partial x}\right)+\frac{\partial}{\partial y}\left(h \frac{\partial V}{\partial y}\right)+\frac{\partial}{\partial z}\left(\frac{1}{h} \frac{\partial V}{\partial z}\right)\right]=0,
$$

where $h=1+\kappa_{x} x+\kappa_{y} y$ and $\kappa_{x}, \kappa_{y}$ the ideal curvatures in the horizontal and vertical plane, respectively. We also assume that the particle beam may be bend horizontally as well as vertically. For the general solution of the Laplace equation (6.62) we use an ansatz in the form of a power expansion

$$
\frac{e c}{\beta E} V(x, y, z)=-\sum_{p, q \geq 0} A_{p q}(z) \frac{x^{p}}{p !} \frac{y^{q}}{q !},
$$

where we have added the beam rigidity to facilitate the quantities for application in beam dynamics and where the coefficients $A_{p q}(z)$ are functions of $z$. Terms with negative indices $p$ and $q$ are excluded to avoid nonphysical divergences of the potential at $x=0$ or $y=0$. We insert this ansatz into (6.62), collect all terms of equal powers in $x$ and $y$ and get

$$
\sum_{p \geq 0} \sum_{q \geq 0}\left\{F_{p q}\right\} \frac{x^{p}}{(p-2) !} \frac{y^{q}}{(q-2) !} \equiv 0,
$$


where $\left\{F_{p q}\right\}$ represents the collection of all coefficients for the term $x^{p} y^{q}$. For (6.64) to be true for all values of the coordinates $x$ and $y$, we require that every coefficient $F_{p q}$ must vanish individually. Setting $F_{p q}=0$ leads to the recursion formula

$$
\begin{aligned}
A_{p, q+2}+A_{p+2, q} & =-\kappa_{x}(3 p+1) A_{p+1, q}-\kappa_{y}(3 q+1) A_{p, q+1} \\
& -3 \kappa_{y} q A_{p+2, q-1}-3 \kappa_{x} p A_{p-1, q+2} \\
& -2 \kappa_{x} \kappa_{0 y} q(3 p+1) A_{p+1, q-1}-2 \kappa_{x} \kappa_{y} p(3 q+1) A_{p-1, q+1} \\
& -3 \kappa_{y}^{2} q(q-1) A_{p+2, q-2}-3 \kappa_{x}^{2} p(p-1) A_{p-2, q+2} \\
& -\kappa_{x}^{3} p\left(p^{2}-3 p+2\right) A_{p-3, q+2}-\kappa_{y}^{3} q\left(q^{2}-3 q+2\right) A_{p+2, q-3} \\
& -\kappa_{x} \kappa_{y}^{2} q(q-1+3 p q-3 p) A_{p+1, q-2} \\
& -\kappa_{x}^{2} \kappa_{y} p(p-1+3 p q-3 q) A_{p-2, q+1} \\
& -\kappa_{y} q\left(3 \kappa_{x}^{2} p^{2}-\kappa_{x}^{2} p+\kappa_{y}^{2} q^{2}-2 \kappa_{y}^{2} q+\kappa_{y}^{2}\right) A_{p, q-1} \\
& -\kappa_{x} p\left(3 \kappa_{y}^{2} q^{2}-\kappa_{y}^{2} q+\kappa_{x}^{2} p^{2}-2 \kappa_{x}^{2} p+\kappa_{x}^{2}\right) A_{p-1, q} \\
& -(3 p-1) p \kappa_{x}^{2} A_{p, q}-(3 q-1) q \kappa_{y}^{2} A_{p, q} \\
& -A_{p, q}^{\prime \prime}-\kappa_{x} p A_{p-1, q}^{\prime \prime}-\kappa_{y} q A_{p, q-1}^{\prime \prime}-\kappa_{x}^{\prime} p A_{p-1, q}^{\prime}-\kappa_{y}^{\prime} q A_{p, q-1}^{\prime}
\end{aligned}
$$

which allows us to determine all coefficients $A_{p q}$. We note that all terms on the right hand side are kinematic terms originating from the curvilinear coordinate system. The derivatives, indicated by a prime, are understood to be taken with respect to the independent variable $z$, like $A^{\prime}=\mathrm{d} A / \mathrm{d} z$, etc. Equation (6.65) is a recursion formula for the field coefficients $A_{p q}$ and we have to develop a procedure to obtain all terms consistent with this expression.

\subsubsection{Pure Multipole Magnets}

The Laplace equation is of second order and therefore we cannot derive coefficients of quadratic or lower order from the recursion formula. The lowest-order coefficient $A_{00}$ represents a constant potential independent of the transverse coordinates $x$ and $y$ and since this term does not contribute to a transverse field component, we will ignore it in this section. However, since this term depends on $z$ we cannot neglect this term where longitudinal fields such as solenoid fields are important. Such fields will be discussed separately in Sect. 6.6 and therefore we set here for simplicity

$$
A_{00}=0
$$


The terms linear in $x$ or $y$ are the curvatures in the horizontal and vertical plane as defined previously

$$
A_{10}=-\kappa_{y} \quad \text { and } \quad A_{01}=\kappa_{x},
$$

and

$$
\begin{array}{rlrl}
\kappa_{x} & =-x^{\prime \prime}=+\frac{e}{p} B_{y} & \text { with } & \mid \frac{e}{p} B_{y} \\
\kappa_{y}=-y^{\prime \prime} & =-\frac{e}{p} B_{x} & \text { with } & \\
\frac{e}{p} B_{x} & =\frac{1}{\rho_{y}} .
\end{array}
$$

Finally, the quadratic terms proportional to $x$ and $y$ are identical to the quadrupole strength parameters

$$
A_{20}=-\underline{k}, \quad A_{11}=k, \quad A_{02}=\underline{k} .
$$

With these definitions of the linear coefficients, we may start exploiting the recursion formula. All terms on the right-hand side of (6.65) are of lower order than the two terms on the left-hand side which are of order $n=p+q+2$. The left-hand side is composed of two contributions, one resulting from pure multipole fields of order $n$ and the other from higher-order field terms of lower-order multipoles.

In (6.65) we identify and separate from all other terms the pure multipole terms of order $n$ which do not depend on lower-order multipole terms like kinematic terms by setting

$$
A_{p+2, q, n}+A_{p, q+2, n}=0 \text { for } p+q+2 \leq n
$$

and adding the index $n$ to indicate that these terms are the pure $n$ th-order multipoles. Only the sum of two terms can be determined which means both terms have the same value but opposite signs. For $n=3$ we have, for example, $A_{30}=-A_{12}$ or $A_{21}=-A_{03}$ and a comparison with the potentials of pure multipoles of Table 6.5 shows that $A_{30}=-\underline{m}$ and $A_{21}=m$. Similar correlations can be formulated for all higher order multipole. ${ }^{2}$ Analogous to dipoles and quadrupole magnets, we may get potential expressions for all other multipole magnets. The results up to fifth order are compiled in Table 6.6.

Each expression for the magnetic potential is composed of both the real and the imaginary contribution. Since both components differ only by a rotational angle, real magnets are generally aligned such that only one or the other component appears. Only due to alignment errors may the other component appear as a field error which can be treated as a perturbation.

\footnotetext{
${ }^{2}$ Consistent with the definitions of magnet strengths, the underlined quantities represent the magnet strengths of rotated multipole magnets.
} 
Table 6.5 Correspondence between the potential coefficients and multipole strength parameters

\begin{tabular}{|c|c|c|c|c|c|c|c|c|c|c|}
\hline & & & & & $A_{00}$ & & & & & \\
\hline & & & & $A_{10}$ & & $A_{01}$ & & & & \\
\hline & & & $A_{20}$ & & $A_{11}$ & & $A_{02}$ & & & \\
\hline & & $A_{30}$ & & $A_{21}$ & & $A_{12}$ & & $A_{03}$ & & \\
\hline & $A_{40}$ & & $A_{31}$ & & $A_{22}$ & & $A_{13}$ & & $A_{04}$ & \\
\hline \multirow[t]{7}{*}{$A_{50}$} & & $A_{41}$ & & $A_{32}$ & & $A_{23}$ & & $A_{14}$ & & $A_{05}$ \\
\hline & & & & & 企 & & & & & \\
\hline & & & & & 0 & & & & & \\
\hline & & & & $-\kappa_{y}$ & & $\kappa_{x}$ & & & & \\
\hline & & & $-\underline{k}$ & & $k$ & & $\underline{k}$ & & & \\
\hline & & $-\underline{m}$ & & $m$ & & $\underline{m}$ & & $-m$ & & \\
\hline & $-\underline{r}$ & & $r$ & & $\underline{r}$ & & $r$ & & $-\underline{r}$ & \\
\hline$-\underline{d}$ & & $d$ & & $\underline{d}$ & & $-d$ & & $-\underline{d}$ & & $d$ \\
\hline
\end{tabular}

Table 6.6 Magnetic multipole potentials

\begin{tabular}{l|l}
\hline Dipole & $-\frac{e}{p_{0}} V_{1}=-\kappa_{y} x+\kappa_{x} y$ \\
\hline Quadrupole & $-\frac{e}{p_{0}} V_{2}=-\frac{1}{2} \underline{k}\left(x^{2}-y^{2}\right)+k x y$, \\
\hline Sextupole & $-\frac{e}{p_{0}} V_{3}=-\frac{1}{6} \underline{m}\left(x^{3}-3 x y^{2}\right)+\frac{1}{6} m\left(3 x^{2} y-y^{3}\right)$, \\
\hline Octupole & $-\frac{e}{p_{0}} V_{4}=-\frac{1}{24} \underline{r}\left(x^{4}-6 x^{2} y^{2}+y^{4}\right)+\frac{1}{24} r\left(x^{3} y-x y^{3}\right)$, \\
\hline Decapole & $-\frac{e}{p_{0}} V_{5}=-\frac{1}{120} \underline{d}\left(x^{5}-10 x^{3} y^{2}+5 x y^{4}\right)+\frac{1}{120} d\left(5 x^{4} y-10 x^{2} y^{3}+y^{5}\right)$ \\
\hline
\end{tabular}

\subsubsection{Kinematic Terms}

Having identified the pure multipole components, we concentrate now on using the recursion formula for other terms which so far have been neglected. First, we note that coefficients of the same order $n$ on the left-hand side of (6.65) must be split into two parts to distinguish pure multipole components $A_{j k, n}$ of order $n$ from the $n$ th-order terms $A_{j k}^{*}$ of lower-order multipoles which we label by an asterisk * . Since we have already derived the pure multipole terms, we explore (6.65) for the $A^{*}$ coefficients only

$$
A_{p, q+2}^{*}+A_{p+2, q}^{*}=\text { r.h.s. of }(6.65)
$$

For the predetermined coefficients $A_{10}, A_{01}$ and $A_{11}$ there are no corresponding terms $A^{*}$ since that would require indices $p$ and $q$ to be negative. For $p=0$ and $q=0$ we have

$$
A_{02}^{*}+A_{20}^{*}=-\kappa_{0 x} A_{10}-\kappa_{0 y} A_{01}=0 .
$$


This solution is equivalent to (6.70) and does not produce any new field terms. The next higher-order terms for $p=0$ and $q=1$ or for $p=1$ and $q=0$ are determined by the equations

$$
\begin{aligned}
& A_{03}^{*}+A_{21}^{*}=-\kappa_{0 x} g-\kappa_{0 y} \underline{g}-\kappa_{x}^{\prime \prime}=C, \\
& A_{12}^{*}+A_{30}^{*}=-\kappa_{0 y} g+\kappa_{0 x} \underline{g}+\kappa_{y}^{\prime \prime}=D,
\end{aligned}
$$

where we set in preparation for the following discussion the right-hand sides equal to the as yet undetermined quantities $C$ and $D$. Since we have no lead how to separate the coefficients we set

$$
\begin{aligned}
& A_{21}^{*}=f C, \quad A_{03}^{*}=(1-f) C, \\
& A_{12}^{*}=g D, \quad A_{30}^{*}=(1-g) D,
\end{aligned}
$$

where $0 \leq(f, g) \leq 1$ and $f=g$. The indeterminate nature of this result is an indication that these terms may depend on the actual design of the magnets.

Trying to interpret the physical meaning of these terms, we assume a magnet with a pure vertical dipole field in the center of the magnet, $B_{y}(0,0,0) \neq 0$, but no horizontal or finite longitudinal field components, $B_{x}(0,0,0)=0$ and $B_{z}(0,0,0)=$ 0 . Consistent with these assumptions the magnetic potential is

$$
\begin{aligned}
\frac{e c}{\beta E} V(x, y, z) & =-A_{01} y-\frac{1}{2} A_{21}^{*} x^{2} y-\frac{1}{2} A_{12}^{*} x y^{2} \\
& -\frac{1}{6} A_{30}^{*} x^{3}-\frac{1}{6} A_{03}^{*} y^{3}+O(4) .
\end{aligned}
$$

From (6.73) we get $D \equiv 0, C=-B_{y}^{\prime \prime}$ and with (6.74) $A_{12}^{*}=A_{30}^{*}=0$. The magnetic-field potential reduces therefore to

$$
\frac{e c}{\beta E} V(x, y, z)=-\kappa_{x} y+\frac{1}{2} f \kappa_{x}^{\prime \prime} x^{2} y+\frac{1}{6}(1-f) \kappa_{x}^{\prime \prime} y^{3}
$$

and the magnetic-field components are

$$
\begin{aligned}
& \frac{e c}{\beta E} B_{x}=-f \kappa_{x}^{\prime \prime} x y, \\
& \frac{e c}{\beta E} B_{y}=+\kappa_{x}-\frac{1}{2} f \kappa_{x}^{\prime \prime} x^{2}-\frac{1}{2}(1-f) \kappa_{x}^{\prime \prime} y^{2} .
\end{aligned}
$$

The physical origin of these terms becomes apparent if we investigate the two extreme cases for which $f=0$ or $f=1$ separately. For $f=0$ the magnetic fields in these cases are $\left(\frac{e c}{\beta E} B_{x}=0, \frac{e c}{\beta E} B_{y}=\kappa_{x}-\frac{1}{2} \kappa_{x}^{\prime \prime} y^{2}\right)$ and $\left(\frac{e c}{\beta E} B_{x}=-\kappa_{x}^{\prime \prime} x y, \frac{e c}{\beta E} B_{y}=\kappa_{x}-\frac{1}{2} \kappa_{x}^{\prime \prime} x^{2}\right)$ for $f=1$. Both cases differ only in the $\kappa_{x}^{\prime \prime}$-terms describing the magnet fringe field. In the case of a straight bending magnet $\left(B_{y} \neq 0\right)$ with infinitely wide poles in the $x$-direction, the horizontal field 


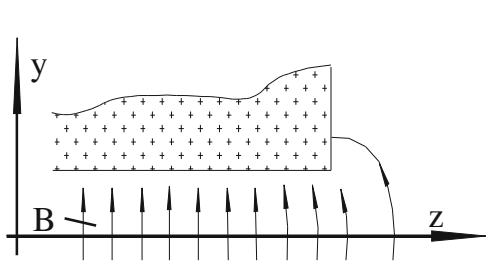

side view

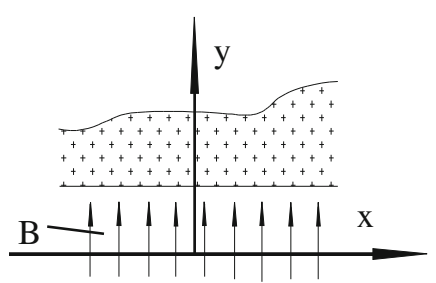

frontal view

Fig. 6.16 Dipole end field configuration for $f=0$

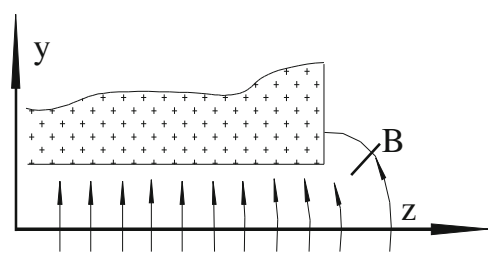

side view

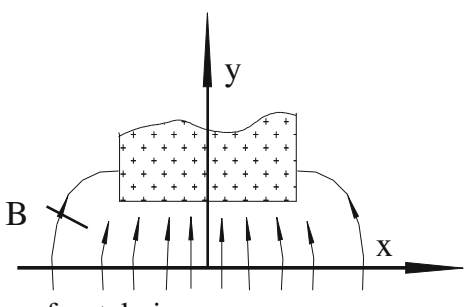

frontal view

Fig. 6.17 Dipole end field configuration for $0<f<1$

component $B_{x}$ must vanish consistent with $f=0$. The field configuration in the fringe field region is of the form shown in Fig. 6.16 and independent of $x$.

Conversely, the case $0<f<1$ describes the field pattern in the fringe field of a bending magnet with poles of finite width in which case finite horizontal field components $B_{x}$ appear off the symmetry planes. The fringe fields not only bulge out of the magnet gap along $z$ but also spread horizontally due to the finite pole width as shown in Fig. 6.17, thus creating a finite horizontal field component off the midplane. While it is possible to identify the origin of these field terms, we are not able to determine the exact value of the factor $f$ in a general way but may apply three-dimensional magnet codes to determine the field configuration numerically. The factor $f$ is different for each type of magnet depending on its actual mechanical dimensions.

Following general practice in beam dynamics and magnet design, however, we ignore these effects of finite pole width, since they are specifically kept small by design, and we may set $f=g=0$. In this approximation we get

$$
A_{21}^{*}=A_{12}^{*}=0
$$

and

$$
\begin{aligned}
& A_{03}^{*}=-\kappa_{x} k-\kappa_{y} \underline{k}-\kappa_{x}^{\prime \prime}, \\
& A_{30}^{*}=-\kappa_{y} k+\kappa_{x} \underline{k}+\kappa_{x}^{\prime \prime} .
\end{aligned}
$$

Similar effects of finite pole sizes appear for all multipole terms. As before, we set $f=0$ for lack of accurate knowledge of the actual magnet design and assume 
that these terms are very small by virtue of a careful magnet design within the good field region provided for the particle beam. For the fourth-order terms we have therefore with $A_{22}^{*} \equiv 0$ and

$$
\begin{gathered}
A_{40}^{*}=\kappa_{x} \underline{m}-\kappa_{y} m-4 \kappa_{x} \kappa_{y} k+4 \kappa_{x}^{2} \underline{k}+\underline{k}^{\prime \prime}+2 \kappa_{x} \kappa_{y}^{\prime \prime}+2 \kappa_{x}^{\prime} \kappa_{y}^{\prime}, \\
A_{04}^{*}=\kappa_{y} m-\kappa_{x} \underline{m}-4 \kappa_{x} \kappa_{y} k-4 \kappa_{y}^{2} \underline{k}-\underline{k}^{\prime \prime}-2 \kappa_{y} \kappa_{x}^{\prime \prime}-2 \kappa_{y}^{\prime} \kappa_{x}^{\prime} .
\end{gathered}
$$

In the case $p=q$, we expect $A_{i j}=A_{j i}$ from symmetry and get

$$
\begin{array}{r}
2 A_{13}^{*}=2 A_{31}^{*}=-\kappa_{x} m-\kappa_{y} \underline{m}+2 \kappa_{x}^{2} k+2 \kappa_{y}^{2} k-k^{\prime \prime} \\
+2 \kappa_{y} \kappa_{y}^{\prime \prime}-2 \kappa_{x} \kappa_{x}^{\prime \prime}-\kappa_{x} \kappa_{x}^{\prime}+\kappa_{y} \kappa_{y}^{\prime} .
\end{array}
$$

With these terms we have finally determined all coefficients of the magnetic potential up to fourth order. Higher-order terms can be derived along similar arguments. Using these results, the general magnetic-field potential up to fourth order is from (6.63)

$$
\begin{aligned}
-\frac{e c}{\beta E} V(x, y, z)= & +A_{10} x+A_{01} y \\
& +\frac{1}{2} A_{20} x^{2}+\frac{1}{2} A_{02} y^{2}+A_{11} x y \\
& +\frac{1}{6} A_{30} x^{3}+\frac{1}{2} A_{21} x^{2} y+\frac{1}{2} A_{12} x y^{2}+\frac{1}{6} A_{03} y^{3} \\
+ & \frac{1}{6} A_{30}^{*} x^{3}+\frac{1}{6} A_{03}^{*} y^{3} \\
& +\frac{1}{24} A_{40} x^{4}+\frac{1}{6} A_{31} x^{3} y+\frac{1}{4} A_{22} x^{2} y^{2}+\frac{1}{6} A_{13} x y^{3} \\
& +\frac{1}{24} A_{04} y^{4}+\frac{1}{24} A_{40}^{*} x^{4}+\frac{1}{6} A_{31}^{*} x y\left(x^{2}+y^{2}\right)+\frac{1}{24} A_{04}^{*} y^{4}+O(5) .
\end{aligned}
$$

From the magnetic potential we obtain the magnetic field expansion by differentiation with respect to $x$ or $y$ for $B_{x}$ and $B_{y}$, respectively. Up to third order we obtain the transverse field components in energy independent formulation

$$
\begin{aligned}
\frac{e c}{\beta E} B_{x}=- & \kappa_{y}-\underline{k} x+k y \\
& -\frac{1}{2} \underline{m}\left(x^{2}-y^{2}\right)+m x y+\frac{1}{2}\left(-\kappa_{y} k+\kappa_{x} \underline{k}+\kappa_{y}^{\prime \prime}\right) x^{2} \\
& -\frac{1}{6} \underline{r}\left(x^{3}-3 x y^{2}\right)-\frac{1}{6} r\left(y^{3}-3 x^{2} y\right) \\
& -\frac{1}{12}\left(\kappa_{x} m+\kappa_{y} \underline{m}+2 \kappa_{x}^{2} k+2 \kappa_{y}^{2} k+k^{\prime \prime}-\kappa_{y} \kappa_{y}^{\prime \prime}\right. \\
& \left.+\kappa_{x} \kappa_{x}^{\prime \prime}+\kappa_{x}^{\prime 2}-\kappa_{y}^{\prime 2}\right)\left(3 x^{2} y+y^{3}\right) \\
& +\frac{1}{6}\left(\kappa_{x} \underline{m}-\kappa_{y} m-4 \kappa_{x} \kappa_{y} k+4 \kappa_{x}^{2} \underline{k}\right. \\
& \left.+\underline{k}^{\prime \prime}+2 \kappa_{x} \kappa_{y}^{\prime \prime}+2 \kappa_{x}^{\prime} \kappa_{y}^{\prime}\right) x^{3}+\mathcal{O}(4)
\end{aligned}
$$


and

$$
\begin{aligned}
\frac{e c}{\beta E} B_{y}=+ & \kappa_{x}+k \underline{k y}+k x \\
& +\frac{1}{2} m\left(x^{2}-y^{2}\right)+\underline{m} x y-\frac{1}{2}\left(\kappa_{x} k+\kappa_{y} \underline{k}+\kappa_{x}^{\prime \prime}\right) y^{2} \\
& +\frac{1}{6} r\left(x^{3}-3 x y^{2}\right)-\frac{1}{6} \underline{r}\left(y^{3}-3 x^{2} y\right) \\
& -\frac{1}{12}\left(\kappa_{x} m+\kappa_{y} \underline{m}+2 \kappa_{x}^{2} k+2 \kappa_{y}^{2} k+k^{\prime \prime}-\kappa_{y} \kappa_{y}^{\prime \prime}\right. \\
& \left.+\kappa_{x} \kappa_{x}^{\prime \prime}+\kappa_{x}^{\prime 2}-\kappa_{y}^{\prime 2}\right)\left(x^{3}+3 x y^{2}\right) \\
& +\frac{1}{6}\left(\kappa_{y} m-\kappa_{x} \underline{m}-4 \kappa_{x} \kappa_{y} k-4 \kappa_{y}^{2} \underline{k}\right. \\
& \left.-\underline{k}^{\prime \prime}-2 \kappa_{y} \kappa_{x}^{\prime \prime}-2 \kappa_{x}^{\prime} \kappa_{y}^{\prime}\right) y^{3}+\mathcal{O}(4),
\end{aligned}
$$

where $m=\frac{e}{p} s_{3}$ and $r=\frac{e}{p} s_{4}$. The third component of the gradient in a curvilinear coordinate system is $B_{z}=-\frac{1}{h} \frac{\partial V}{\partial z}$ and collecting all terms up to second order we get

$$
\begin{aligned}
\frac{e c}{\beta E} B_{z}=+\kappa_{x}^{\prime} y-\kappa_{y}^{\prime} x+ & \left(\kappa_{y} \kappa_{y}^{\prime}-\kappa_{x} \kappa_{x}^{\prime}+k^{\prime}\right) x y \\
& +\left(\kappa_{x} \kappa_{y}^{\prime}-\frac{1}{2} \underline{k}^{\prime}\right) x^{2}-\left(\kappa_{y} \kappa_{x}^{\prime}-\frac{1}{2} \underline{k}^{\prime}\right) y^{2}+\mathcal{O}(3) .
\end{aligned}
$$

Upon closer inspection of (6.84)-(6.86) it becomes apparent that most terms originate from a combination of different multipoles. These equations describe the general fields in any magnet, yet in practice, special care is taken to limit the number of fundamentally different field components present in any one magnet. In fact most magnet are designed as single multipoles like dipoles or quadrupoles or sextupoles etc. A beam transport system utilizing only such magnets is also called a separated-function lattice since bending and focusing is performed in different types of magnets. A combination of bending and focusing, however, is being used for some special applications and a transport system composed of such combinedfield magnets is called a combined-function lattice. Sometimes even a sextupole term is incorporated in a magnet together with the dipole and quadrupole fields. Rotated magnets, like rotated sextupoles $\underline{s_{3}}$ and octupoles $\underline{s_{4}}$ are either not used or in the case of a rotated quadrupole the chosen strength is generally weak and its effect on the beam dynamics is treated by perturbation methods.

No mention has been made about electric field patterns. However, since the Laplace equation for electrostatic fields in material free areas is the same as for magnetic fields we conclude that the electrical potentials are expressed by (6.82) as well and the electrical multipole field components are also given by (6.84)(6.86) after replacing the magnetic field $\left(B_{x}, B_{y}, B_{z}\right)$ by electric-field components $\left(E_{x}, E_{y}, E_{z}\right)$. 
Fig. 6.18 Frenet-Serret coordinate system

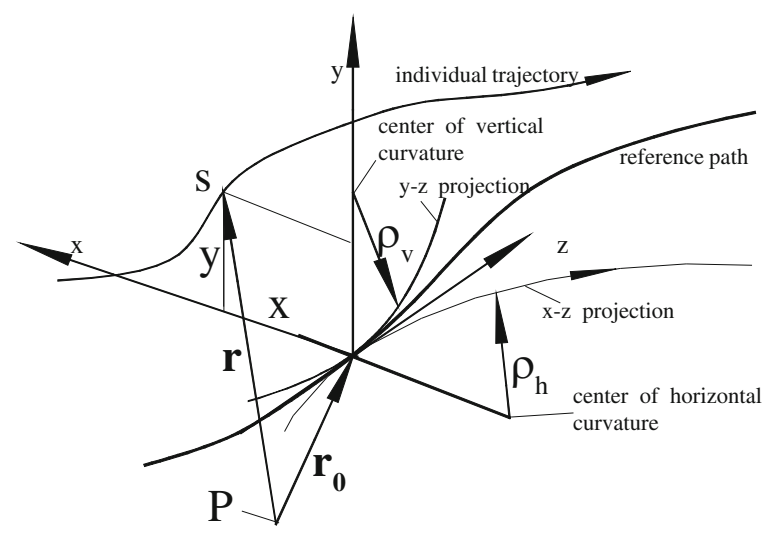

\subsection{Third-Order Differential Equation of Motion*}

Equations of motions have been derived in Chap. 5 for the transverse $(x, z)$ and $(y, z)$ planes up to second order which is adequate for most applications. Sometimes, however, it might be desirable to use equations of motion in higher order of precision or to investigate perturbations of higher order. A curvilinear Frenet-Serret coordinate system moving along the curved trajectory of the reference particle $\boldsymbol{r}_{0}(z)$, was used and we generalize this system to include curvatures in both transverse planes as shown in Fig. 6.18.

In this $(x, y, z)$-coordinate system, a particle at the location $s$ and under the influence of a Lorentz force follows a path described by the vector $\boldsymbol{r}$ as shown in Fig. 6.18. The change in the momentum vector per unit time is due only to a change in the direction of the momentum while the magnitude of the momentum remains unchanged in a static magnetic fields. Therefore $\boldsymbol{p}=p \mathrm{~d} \boldsymbol{r} / \mathrm{d} s$ where $p$ is the value of the particle momentum and $\mathrm{d} \boldsymbol{r} / \mathrm{d} s$ is the unit vector along the particle trajectory. With $\frac{\mathrm{d} p}{\mathrm{~d} \tau}=\frac{\mathrm{d} p}{\mathrm{~d} s} \beta c$, where $\tau=\frac{s}{\beta c}$, the particle velocity $\boldsymbol{v}_{s}=\frac{\mathrm{d} \boldsymbol{r}}{\mathrm{d} \tau}=\frac{\mathrm{d} r}{\mathrm{~d} s} \beta c$, and we obtain the differential equation describing the particle trajectory under the influence of a Lorentz force $\mathbf{F}_{\mathrm{L}}$. From $\frac{\mathrm{d} \boldsymbol{p}}{\mathrm{d} \tau}=\mathbf{F}_{\mathrm{L}}=e\left[\boldsymbol{v}_{s} \times \boldsymbol{B}\right]$ we get

$$
\frac{\mathrm{d}^{2} \boldsymbol{r}}{\mathrm{d} s^{2}}=\frac{e c}{\beta E}\left[\frac{\mathrm{d} \boldsymbol{r}}{\mathrm{d} s} \times \boldsymbol{B}\right]
$$

and to evaluate (6.87) further, we note that

$$
\frac{\mathrm{d} \boldsymbol{r}}{\mathrm{d} s}=\frac{\mathrm{d} \boldsymbol{r} / \mathrm{d} z}{\mathrm{~d} s / \mathrm{d} z}=\frac{\boldsymbol{r}^{\prime}}{\boldsymbol{s}^{\prime}}
$$

and

$$
\frac{\mathrm{d}^{2} \boldsymbol{r}}{\mathrm{d} s^{2}}=\frac{1}{s^{\prime}} \frac{\mathrm{d}}{\mathrm{d} z}\left(\frac{\boldsymbol{r}^{\prime}}{s^{\prime}}\right) .
$$


With this, the general equation of motion is from (6.87)

$$
\frac{\mathrm{d}^{2} \boldsymbol{r}}{\mathrm{d} z^{2}}-\frac{1}{2 s^{\prime 2}} \frac{\mathrm{d} \boldsymbol{r}}{\mathrm{d} z} \frac{\mathrm{d} s^{\prime 2}}{\mathrm{~d} z}=\frac{e c}{\beta E} s^{\prime}\left[\frac{\mathrm{d} \boldsymbol{r}}{\mathrm{d} z} \times \boldsymbol{B}\right] .
$$

In the remainder of this section, we will re-evaluate this equation in terms of more simplified parameters. From Fig. 6.18 or (4.21) we have $\boldsymbol{r}=\boldsymbol{r}_{0}+x \boldsymbol{u}_{x}+y \boldsymbol{u}_{y}$, where the vectors $\boldsymbol{u}_{x}, \boldsymbol{u}_{y}$ and $\boldsymbol{u}_{z}$ are the unit vectors defining the curvilinear coordinate system. To completely evaluate (6.89), the second derivative $\mathrm{d}^{2} \boldsymbol{r} / \mathrm{d} z^{2}$ must be derived from (4.23) with $\mathrm{d} \boldsymbol{u}_{z}=-\kappa_{x} \boldsymbol{u}_{x} \mathrm{~d} z-\kappa_{y} \boldsymbol{u}_{y} \mathrm{~d} z$ and $h=1+\kappa_{x} x+\kappa_{y} y$ for

$$
\frac{\mathrm{d}^{2} \boldsymbol{r}}{\mathrm{d} z^{2}}=\left(x^{\prime \prime}-\kappa_{x} h\right) \boldsymbol{u}_{x}+\left(y^{\prime \prime}-\kappa_{y} h\right) \boldsymbol{u}_{y}+\left(2 \kappa_{x} x^{\prime}+2 \kappa_{y} y^{\prime}+\kappa_{x}^{\prime} x+\kappa_{y}^{\prime} y\right) \boldsymbol{u}_{z},
$$

and (6.89) becomes with (4.23) and $s^{\prime 2}=r^{\prime 2}$

$$
\begin{aligned}
& \left(x^{\prime \prime}-\kappa_{x} h-\frac{x^{\prime}}{2 s^{\prime 2}} \frac{\mathrm{d} s^{\prime 2}}{\mathrm{~d} z}\right) \boldsymbol{u}_{x}+\left(y^{\prime \prime}-\kappa_{y} h-\frac{y^{\prime}}{2 s^{\prime 2}} \frac{\mathrm{d} s^{\prime 2}}{\mathrm{~d} z}\right) \boldsymbol{u}_{y} \\
& \quad+\left(2 \kappa_{x} x^{\prime}+2 \kappa_{y} y^{\prime}+\kappa_{x}^{\prime} x+\kappa_{y}^{\prime} y-\frac{h}{s^{\prime 2}} \frac{\mathrm{d} s^{\prime 2}}{\mathrm{~d} z}\right) \boldsymbol{u}_{z}=\frac{e c}{\beta E} s^{\prime}\left[\frac{\mathrm{d} \boldsymbol{r}}{\mathrm{d} z} \times \boldsymbol{B}\right] .
\end{aligned}
$$

Here the quantities $\kappa_{x}$ and $\kappa_{y}$ are the curvatures defining the ideal particle trajectory or the curvilinear coordinate system. This is the general equation of motion for a charged particles in a magnetic field $\boldsymbol{B}$. So far no approximations have been made. For practical use we may separate the individual components and get the differential equations for transverse motion

$$
\begin{aligned}
x^{\prime \prime}-\kappa_{x} h-\frac{1}{2} \frac{x^{\prime}}{s^{\prime 2}} \frac{\mathrm{d} s^{\prime 2}}{\mathrm{~d} z} & =\frac{e c}{\beta E} s^{\prime}\left[y^{\prime} B_{z}-h B_{y}\right], \\
y^{\prime \prime}-\kappa_{y} h-\frac{1}{2} \frac{y^{\prime}}{s^{\prime 2}} \frac{\mathrm{d} s^{\prime 2}}{\mathrm{~d} z} & =\frac{e c}{\beta E} s^{\prime}\left[h B_{x}-x^{\prime} B_{z}\right] .
\end{aligned}
$$

Chromatic effectsoriginate from the momentum factor $\frac{e c}{\beta E}$ which is different for particles of different energies. We expand this factor into a power series in $\delta$

$$
\frac{e c}{\beta E}=\frac{e}{p_{0}}\left(1-\delta+\delta^{2}-\delta^{3}+\ldots\right)
$$

where $\delta=\Delta p / p_{0}$ and $c p_{0}=\beta E_{0}$ is the ideal particle momentum. A further approximation is made when we expand $s^{\prime}$ to third order in $x$ and $y$ while restricting the discussion to paraxial rays with $x^{\prime} \ll 1$ and $y^{\prime} \ll 1$

$$
s^{\prime} \approx h+\frac{1}{2}\left(x^{\prime 2}+y^{\prime 2}\right)\left(1-\kappa_{x} x-\kappa_{y} y\right)+\ldots .
$$


Evaluating the derivative $\mathrm{d} s^{2} / \mathrm{d} z^{2}$ second-order derivatives $x^{\prime \prime}$ and $y^{\prime \prime}$ appear which, neglecting fourth-order terms, can here be replaced by the unperturbed equations of motion $x^{\prime \prime}+\left(\kappa_{x}^{2}+k\right) x=0$ and $y^{\prime \prime}+\left(\kappa_{y}^{2}+k\right) y=0$. For the magnetic field components, we insert in $(6.92 \mathrm{a}),(6.92 \mathrm{~b})$ expressions $(6.84)-(6.86)$ while making use of (6.93) and (6.94). Keeping all terms up to third order in $x, y, x^{\prime}, y^{\prime}$ and $\delta$, we finally obtain equations of motion for a particle with charge $e$ in an arbitrary magnetic field derivable from a scalar potential. For the horizontal and vertical plane the general equations of motion in a curvilinear coordinate system and including chromatic terms up to third-order in $(x, y, \delta)$ are (6.95) and (6.96), respectively.

$$
\begin{aligned}
x^{\prime \prime}+\left(\kappa_{x}^{2}+k\right) x & =\kappa_{x} \delta-\kappa_{x} \delta^{2}+\kappa_{x} \delta^{3}-\left(\underline{k}+\kappa_{x} \kappa_{y}\right) y \\
& -\left(\underline{m}+2 \kappa_{x} \underline{k}+2 \kappa_{y} k+2 \kappa_{x}^{2} \kappa_{y}\right) x y-\frac{1}{2} m\left(x^{2}-y^{2}\right) \\
& -\left(\kappa_{x}^{3}+2 \kappa_{x} k\right) x^{2}-\left(\kappa_{x} \kappa_{y}^{2}-\frac{1}{2} \kappa_{x} k+\frac{2}{3} \kappa_{y} \underline{k}-\frac{1}{2} \kappa_{x}^{\prime \prime}\right) y^{2} \\
& +\frac{1}{2} \kappa_{x}\left(x^{\prime 2}-y^{\prime 2}\right)+\kappa_{x}^{\prime}\left(x x^{\prime}+y y^{\prime}\right)+\kappa_{y}^{\prime}\left(x^{\prime} y-x y^{\prime}\right)+\kappa_{y} x^{\prime} y^{\prime} \\
& -\frac{1}{6} r x\left(x^{2}-3 y^{2}\right)+\frac{1}{6} \underline{r} y\left(y^{2}-3 x^{2}\right) \\
& +\frac{1}{12}\left(\kappa_{y} \underline{m}-11 \kappa_{x} m+2 \kappa_{y}^{2} k-10 \kappa_{x}^{2} k+k^{\prime \prime}-\kappa_{y} \kappa_{y}^{\prime \prime}\right. \\
& \left.\quad+\kappa_{x} \kappa_{x}^{\prime \prime}-\kappa_{y}^{\prime 2}+\kappa_{x}^{\prime 2}\right) x^{3} \\
& -\left(2 \kappa_{x} \underline{m}+\kappa_{y} m+\kappa_{x}^{2} \underline{k}+2 \kappa_{x} \kappa_{y} k\right) x^{2} y \\
& +\frac{1}{4}\left(5 \kappa_{x} m-7 \kappa_{y} \underline{m}+6 \kappa_{x}^{2} k+k^{\prime \prime}-\kappa_{y} \kappa_{y}^{\prime \prime}-2 \kappa_{y}^{2} k\right. \\
& \left.\quad+5 \kappa_{x} \kappa_{x}^{\prime \prime}+\kappa_{x}^{\prime 2}-\kappa_{y}^{\prime 2}-\kappa_{x} \kappa_{y} \underline{k}\right) x y^{2} \\
& +\frac{1}{6}\left(10 \kappa_{x} \kappa_{y} k+8 \kappa_{x}^{\prime \prime} \kappa_{y}+\kappa_{x} \underline{m}+4 \kappa_{y}^{2} \underline{k}+\underline{k}^{\prime \prime}+2 \kappa_{x}^{\prime} \kappa_{y}^{\prime}+5 \kappa_{y} m\right) y^{3} \\
& -\left(2 \kappa_{x}^{2}+\frac{3}{2} k\right) x x^{\prime 2}-\left(\kappa_{x}^{\prime} \kappa_{y}+\kappa_{x} \kappa_{y}^{\prime}\right) x x^{\prime} y-\kappa_{x} \kappa_{x}^{\prime} x^{2} x^{\prime} \\
& -\frac{1}{2} \underline{k}^{\prime} x^{2} y^{\prime}-\kappa_{y} \kappa_{y}^{\prime} x^{\prime} y^{2}-\kappa_{x} \kappa_{y} x x^{\prime} y^{\prime}-\frac{1}{2}\left(k \underline{k}+3 \kappa_{x} \kappa_{y}\right) x^{\prime 2} y \\
& +k^{\prime} x y y^{\prime}-\frac{1}{2}\left(k+\kappa_{x}^{2}\right) x y^{\prime 2}-\left(2 \kappa_{y}^{2}-\underline{k}\right) x^{\prime} y y^{\prime}+\frac{1}{2} \underline{k}^{\prime} y^{2} y^{\prime}-\frac{1}{2} \underline{k} y y^{\prime 2} \\
& +\left(2 \kappa_{x}^{2}+k\right) x \delta+\left(2 \kappa_{x} \kappa_{y}+\underline{k}\right) y \delta-\kappa_{x}^{\prime} y y^{\prime} \delta+\kappa_{y}^{\prime} x y^{\prime} \delta \\
& +\frac{1}{2} \kappa_{x}\left(x^{\prime 2}+y^{\prime 2}\right) \delta+\left(\frac{3}{2} \kappa_{y} \underline{k}+\kappa_{x} \kappa_{y}^{2}-\frac{1}{2} \kappa_{x} k-\frac{1}{2} \kappa_{x}^{\prime \prime}-\frac{1}{2} m\right) y^{2} \delta \\
& +\left(\frac{1}{2} m+2 \kappa_{x} k+\kappa_{x}^{3}\right) x^{2} \delta+\left(\underline{m}+2 \kappa_{x}^{2} \kappa_{y}+2 \kappa_{y} k+2 \kappa_{x} \underline{k}\right) x y \delta \\
& -\left(k+2 \kappa_{x}^{2}\right) x \delta^{2}-\left(\underline{k}+2 \kappa_{x} \kappa_{y}\right) y \delta^{2}+\mathcal{O}(4) \\
& \\
& \\
&
\end{aligned}
$$$$
\begin{aligned}
y^{\prime \prime}+\left(\kappa_{y}^{2}-k\right) y & =+\kappa_{y} \delta-\kappa_{y} \delta^{2}+\kappa_{y} \delta^{3}-\left(\underline{k}+\kappa_{x} \kappa_{y}\right) x \\
& +\left(m-2 \kappa_{y} \underline{k}+2 \kappa_{x} k-2 \kappa_{x} \kappa_{y}^{2}\right) x y-\frac{1}{2} \underline{m}\left(x^{2}-y^{2}\right)
\end{aligned}
$$ 


$$
\begin{aligned}
& -\left(\kappa_{y}^{3}-2 \kappa_{y} k\right) y^{2}-\left(\kappa_{x}^{2} \kappa_{y}+\frac{1}{2} \kappa_{y} k+\frac{3}{2} \kappa_{x} \underline{k}-\frac{1}{2} \kappa_{y}^{\prime \prime}\right) x^{2} \\
& -\frac{1}{2} \kappa_{y}\left(x^{\prime 2}-y^{\prime 2}\right)+\kappa_{y}^{\prime}\left(x x^{\prime}+y y^{\prime}\right)-\kappa_{x}^{\prime}\left(x^{\prime} y-x y^{\prime}\right)+\kappa_{x} x^{\prime} y^{\prime} \\
& -\frac{1}{6} r y\left(y^{2}-3 x^{2}\right)-\frac{1}{6} r x\left(x^{2}-3 y^{2}\right) \\
& -\frac{1}{12}\left(\kappa_{x} m-11 \kappa_{y} \underline{m}+2 \kappa_{x}^{2} k-10 \kappa_{y}^{2} k+k^{\prime \prime}-\kappa_{y} \kappa_{y}^{\prime \prime}\right. \\
& \left.+\kappa_{x} \kappa_{x}^{\prime \prime}+\kappa_{x}^{\prime 2}-\kappa_{y}^{\prime 2}\right) y^{3} \\
& +\left(2 \kappa_{y} m+\kappa_{x} \underline{m}-\kappa_{y}^{2} \underline{k}+2 \kappa_{x} \kappa_{y} k\right) x y^{2} \\
& -\frac{1}{4}\left(5 \kappa_{y} \underline{m}-7 \kappa_{x} m+6 \kappa_{y}^{2} k+k^{\prime \prime}+\kappa_{x} \kappa_{x}^{\prime \prime}-2 \kappa_{x}^{2} k\right. \\
& \left.-5 \kappa_{y} \kappa_{y}^{\prime \prime}-\kappa_{y}^{\prime 2}+\kappa_{x}^{\prime 2}+\kappa_{x} \kappa_{y} \underline{k}\right) x^{2} y \\
& +\frac{1}{6}\left(-10 \kappa_{x} \kappa_{y} k+8 \kappa_{x} \kappa_{y}^{\prime \prime}-\kappa_{y} m+4 \kappa_{x}^{2} \underline{k}+\underline{k}^{\prime \prime}+2 \kappa_{x}^{\prime} \kappa_{y}^{\prime}-5 \kappa_{x} \underline{m}\right) x^{3} \\
& -\left(2 \kappa_{y}^{2}-\frac{1}{2} k-\underline{k}\right) y y^{\prime 2}-\left(\kappa_{x} \kappa_{y}^{\prime}+\kappa_{x}^{\prime} \kappa_{y}\right) x y y^{\prime}-\kappa_{y} \kappa_{y}^{\prime} y^{2} y^{\prime} \\
& -\frac{1}{2} \underline{k}^{\prime} x^{\prime} y^{2}-\kappa_{x} \kappa_{x}^{\prime} x^{2} y^{\prime}-\kappa_{x} \kappa_{y} x^{\prime} y y^{\prime}-\frac{1}{2}\left(\underline{k}+3 \kappa_{x} \kappa_{y}\right) x y^{\prime 2} \\
& -k^{\prime} x x^{\prime} y+\frac{1}{2}\left(k-\kappa_{y}^{2}\right) x^{\prime 2} y-\left(2 \kappa_{x}^{2}+k\right) x x^{\prime} y^{\prime}+\frac{1}{2} k^{\prime} x^{2} x^{\prime}-\frac{1}{2} \underline{k} x x^{\prime 2} \\
& +\left(2 \kappa_{y}^{2}-k\right) y \delta+\left(2 \kappa_{x} \kappa_{y}+\underline{k}\right) x \delta-\kappa_{y}^{\prime} x x^{\prime} \delta+\kappa_{x}^{\prime} x^{\prime} y \delta \\
& +\frac{1}{2} \kappa_{y}\left(x^{\prime 2}+y^{\prime 2}\right) \delta+\left(\frac{3}{2} \kappa_{x} \underline{k}+\kappa_{x}^{2} \kappa_{y}+\frac{1}{2} \kappa_{y} k-\frac{1}{2} \kappa_{y}^{\prime \prime}+\frac{1}{2} \underline{m}\right) x^{2} \delta \\
& +\left(-\frac{1}{2} \underline{m}-2 \kappa_{y} k+\kappa_{y}^{3}\right) y^{2} \delta-\left(m-2 \kappa_{x} \kappa_{y}^{2}+2 \kappa_{x} k-2 \kappa_{y} \underline{k}\right) x y \delta \\
& +\left(k-2 \kappa_{y}^{2}\right) y \delta^{2}-\left(\underline{k}+2 \kappa_{x} \kappa_{y}\right) x \delta^{2}+\mathcal{O}(4) \text {. }
\end{aligned}
$$

In spite of our attempt to derive a general and accurate equation of motion, we note that some magnet boundaries are not correctly represented. The natural bending magnet is of the sector type and wedge or rectangular magnets require the introduction of additional corrections to the equations of motion which are not included here. This is also true for cases where a beam passes off center through a quadrupole, in which case theory assumes a combined function sector magnet and corrections must be applied to model correctly a quadrupole with parallel pole faces. The magnitude of such corrections is, however, in most cases very small. Equation (6.95) shows an enormous complexity which in real beam transport lines, becomes very much relaxed due to proper design and careful alignment of the magnets. Nonetheless (6.95) and (6.96) for the vertical plane, can be used as a reference to find and study the effects of particular perturbation terms. In a special beam transport line one or the other of these perturbation terms may become significant and can now be dealt with separately. This may be the case where strong multipole effects from magnet fringe fields cannot be avoided or because large beam sizes and divergences are important and necessary. The possible significance of any perturbation term must be evaluated for each beam transport system separately. 
In most beam transport lines the magnets are built in such a way that different functions like bending, focusing etc., are not combined thus eliminating all terms that depend on those combinations like $\kappa_{x} \kappa_{y}, \kappa_{x} k$ or $m \kappa_{x}$ etc. As long as the terms on the right-hand sides are small we may apply perturbation methods to estimate the effects on the beam caused by these terms. It is interesting, however, to try to identify the perturbations with aberrations known from light optics.

Chromatic terms $\kappa_{x}\left(\delta-\delta^{2}+\delta^{3}\right)$, for example, are constant perturbations for off momentum particles causing a shift of the equilibrium orbit which ideally is the trivial solution $x \equiv 0$ of the differential equation $x^{\prime \prime}+\left(k+\kappa_{x}^{2}\right) x=0$. Of course, this is not quite true since $\kappa_{x}$ is not a constant but the general conclusion is still correct. This shift is equal to $\Delta x=\kappa_{x}\left(\delta-\delta^{2}+\delta^{3}\right) /\left(k+\kappa_{x}^{2}\right)$ and is related to the dispersion function $D$ by $D=\Delta x / \delta$. In light optics this corresponds to the dispersion of colors of a beam of white light (particle beam with finite energy spread) passing through a prism (bending magnet). We may also use a different interpretation for this term. Instead of a particle with an energy deviation $\delta$ in an ideal magnet $\kappa_{x}$ we can interpret this term as the perturbation of a particle with the ideal energy by a magnetic field that deviates from the ideal value. In this case, we replace $\kappa_{x}\left(\delta-\delta^{2}-\delta^{3}\right)$ by $-\Delta \kappa_{x}$ and the shift in the ideal orbit is then called an orbit distortion. Obviously, here and in the following paragraphs the interpretations are not limited to the horizontal plane alone but apply also to the vertical plane caused by similar perturbations. Terms proportional to $x^{2}$ cause geometric aberrations, where the focal length depends on the amplitude $x$ while terms involving $x^{\prime}$ lead to the well-known phenomenon of astigmatism or a combination of both aberrations. Additional terms depend on the particle parameters in both the vertical and horizontal plane and therefore lead to more complicated aberrations and coupling.

Terms depending also on the energy deviation $\delta$, on the other hand, give rise to chromatic aberrations which are well known from light optics. Specifically, the term $\left(k+2 \kappa_{x}^{2}\right) x \delta$ is the source for the dependence of the focal length on the particle momentum. Some additional terms can be interpreted as combinations of aberrations described above.

It is interesting to write down the equations of motion for a pure quadrupole system where only $k \neq 0$ in which case (6.95) becomes

$$
\begin{aligned}
x^{\prime \prime}+k x & =k x\left(\delta-\delta^{2}-\delta^{3}\right) \\
& -\frac{1}{12} k^{\prime \prime} x\left(x^{2}+3 y^{2}\right)-\frac{3}{2} k x x^{\prime 2}+k x^{\prime} y y^{\prime}+k^{\prime} x y y^{\prime}+\mathcal{O}(4) .
\end{aligned}
$$

We note that quadrupoles produce only second order chromatic aberrations and geometric perturbations only in third order. 


\subsection{Longitudinal Field Devices}

General field equations have been derived in this chapter with the only restriction that there be no solenoid fields, which allowed us to set $A_{00}=0$ in (6.66), and concentrate on transverse fields only. Longitudinal fields like those produced in a solenoid magnet are used in beam transport systems for very special purposes and their effect on beam dynamics cannot be ignored. We assume now that the lowestorder coefficient $A_{00}$ in the potential (6.63) does not vanish

$$
A_{00}(z) \neq 0
$$

Longitudinal fields do not cause transverse beam deflection although there can be some amplitude dependent focusing or coupling. We may therefore choose a cartesian coordinate system along such fields by setting $\kappa_{x}=\kappa_{y}=0$, and the recursion formula $(6.65)$ reduces to

$$
A_{02}+A_{20}=-A_{00}^{\prime \prime}
$$

Again, we have a solution where $A_{02}+A_{20}=0$, which is a rotated quadrupole as derived in (6.25) and can be ignored here. The additional component of the field is $A_{02}^{*}+A_{20}^{*}=-A_{00}^{\prime \prime}$ and describes the endfields of the solenoid. For reasons of symmetry with respect to $x$ and $y$ we have $A_{02}^{*}=A_{20}^{*}$ and

$$
A_{02}^{*}=A_{20}^{*}=-\frac{1}{2} A_{00}^{\prime \prime} \text {. }
$$

With this, the potential (6.63) for longitudinal fields is

$$
-V_{\mathrm{s}}(x, y, z)=A_{00}-\frac{1}{4} A_{00}^{\prime \prime}\left(x^{2}+y^{2}\right)=A_{00}-\frac{1}{4} A_{00}^{\prime \prime} r^{2},
$$

where we have made use of rotational symmetry. The longitudinal field component becomes from (6.101) in linear approximation

$$
B_{\mathrm{z}}=+A_{00}^{\prime}
$$

and the transverse components

$$
\begin{aligned}
& B_{r}=-\frac{1}{2} A_{00}^{\prime \prime} r=-\frac{1}{2} B_{\mathrm{z}}^{\prime} r, \\
& B_{\varphi}=0 .
\end{aligned}
$$

The azimuthal field component obviously vanishes because of symmetry. Radial field components appear whenever the longitudinal field strength varies as is the case in the fringe field region at the end of a solenoid shown in Fig. 6.19.

The strength $B_{0}$ in the center of a long solenoid magnet can be calculated in the same way we determined dipole and higher-order fields utilizing Stokes' theorem. 
Fig. 6.19 Solenoid field

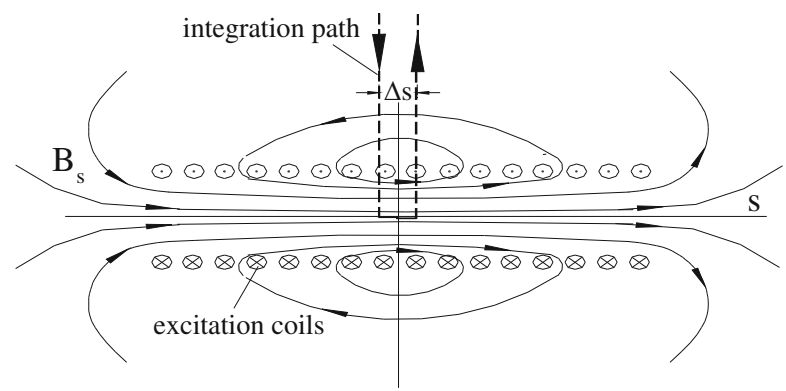

The integral $\oint \boldsymbol{B} \mathrm{d} z$ is performed along a path as indicated in Fig. 6.19. The only contribution to the integral comes from the integral along the field at the magnet axis. All other contributions vanish because the integration path cuts field lines at a right angles, where $\boldsymbol{B} \mathrm{d} z=0$ or follows field lines to infinity where $B_{z}=0$. We have therefore

$$
\oint \boldsymbol{B} \mathrm{d} z=B_{0} \Delta z=\mu_{0} \mu_{\mathrm{r}} J \Delta z
$$

where $J$ is the total solenoid current per unit length. The solenoid field strength is therefore given by

$$
B_{0}(x=0, y=0)=\mu_{0} \mu_{\mathrm{r}} J .
$$

The total integrated radial field $\int B_{r} \mathrm{~d} z$ can be evaluated from the central field for each of the fringe regions. We imagine a cylinder concentric with the solenoid axis and with radius $r$ to extend from the solenoid center to a region well outside the solenoid. In the center of the solenoid a total magnetic flux of $\pi r^{2} B_{0}$ enters this cylinder. It is clear that along the infinitely long cylinder the flux will exit the surface of the cylinder through radial field components. We have therefore

$$
\pi r^{2} B_{0}=\int_{0}^{\infty} 2 \pi r B_{r}(r) \mathrm{d} z
$$

where we have set $z=0$ at the center of the solenoid. The integrated radial field per fringe field is then

$$
\int_{0}^{\infty} B_{r}(r) \mathrm{d} z=-\frac{1}{2} B_{0} r .
$$

The linear dependence of the integrated radial fields on the distance $r$ from the axis constitutes linear focusing capabilities of solenoidal fringe fields. Such solenoid focusing is used, for example, around a conversion target to catch a highly divergent positron beam. The positron source is generally a small piece of a heavy 
metal like tungsten placed in the path of a high energy electron beam. Through an electromagnetic cascade, positrons are generated and emerge from a point like source into a large solid angle. If the target is placed in the center of a solenoid the radial positron motion couples with the longitudinal field to transfer the radial particle momentum into azimuthal momentum. At the end of the solenoid, the azimuthal motion couples with the radial field components of the fringe field to transfer azimuthal momentum into longitudinal momentum. In this idealized picture a divergent positron beam emerging from a small source area is transformed or focused into a quasi-parallel beam of larger cross section. Such a focusing device is called a $\lambda / 4$-lens, since the particles follow one quarter of a helical trajectory in the solenoid.

In other applications large volume solenoids are used as part of elementary particles detectors in high energy physics experiments performed at colliding-beam facilities. The strong influence of these solenoidal detector fields on beam dynamics in a storage ring must be compensated in most cases. In still other applications solenoid fields are used just to contain a particle beam within a small circular aperture like that along the axis of a linear accelerator.

\subsection{Periodic Wiggler Magnets}

Particular arrays or combinations of magnets can produce desirable results for a variety of applications. A specially useful device of this sort is a wiggler magnet [7] which is composed of a series of short bending magnets with alternating field excitation. Most wiggler magnets are used as sources of high brightness photon beams in synchrotron radiation facilities and are often also called undulators. There is no fundamental difference between both. We differentiate between a strong field wiggler magnet and an undulator, which is merely a wiggler magnet at low fields, because of the different synchrotron radiation characteristics. As long as we talk about magnet characteristics in this text, we make no distinction between both types of magnets. Wiggler magnets are used for a variety of applications to either produce coherent or incoherent photon beams in electron accelerators, or to manipulate electron beam properties like beam emittance and energy spread. To compensate anti-damping in a combined function synchrotron a wiggler magnet including a field gradient has been used for the first time to modify the damping partition numbers [8]. In colliding-beam storage rings wiggler magnets are used to increase the beam emittance for maximum luminosity [9]. In other applications, a very small beam emittance is desired as is the case in damping rings for linear colliders or synchrotron radiation sources which can be achieved by employing damping wiggler magnets in a different way [10].

Wiggler magnets are generally designed as flat magnets as shown in Fig. 6.20 [7] with field components only in one plane or as helical wiggler magnets [1113] where the transverse field component rotates along the magnetic axis. In this discussion, we concentrate on flat wigglers which are used in growing numbers to 
Fig. 6.20 Permanent magnet wiggler showing the magnetization direction of individual blocks (schematic)

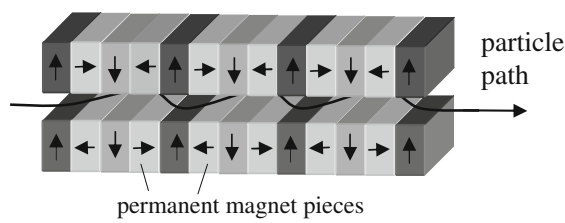

generate, for example, intense beams of synchrotron radiation from electron beams, to manipulate beam parameters or to pump a free electron laser.

\subsubsection{Wiggler Field Configuration}

Whatever the application may be, the wiggler magnet deflects the electron beam transversely in an alternating fashion without introducing a net deflection on the beam. Wiggler magnets are generally considered to be insertion devices installed in a magnet free straight section of the lattice and not being part of the basic magnet lattice. To minimize the effect of wiggler fields on the particle beam, the integrated magnetic field through the whole wiggler magnet must be zero

$$
\int_{\text {wiggler }} B_{\perp} \mathrm{d} z=0 .
$$

Since a wiggler magnet is a straight device, we use a fixed cartesian coordinate system $(x, y, z)$ with the $z$-axis parallel to the wiggler axis to describe the wiggler field, rather than a curvilinear system that would follow the oscillatory deflection of the reference path in the wiggler. The origin of the coordinate system is placed in the middle of one of the wiggler magnets. The whole magnet may be composed of $N$ equal and symmetric pole pieces placed along the $z$-axis at a distance $\lambda_{\mathrm{p}} / 2$ from pole center to pole center as shown in Fig. 6.21. Each pair of adjacent wiggler poles forms one wiggler period with a period length $\lambda_{\mathrm{p}}$ and the whole magnet is composed of $N / 2$ periods. Since all periods are assumed to be the same and the beam deflection is compensated within each period no net beam deflection occurs for the complete magnet.

Upon closer inspection of the precise beam trajectory we observe a lateral displacement of the beam within a wiggler magnet. To compensate this lateral beam displacement, the wiggler magnet should begin and end with only a half pole of length $\lambda_{\mathrm{p}} / 4$ to allow the beams to enter and exit the wiggler magnet parallel with the unperturbed beam path.

The individual magnets comprising a wiggler magnet are in general very short and the longitudinal field distribution differs considerable from a hard-edge model. In fact most of the field will be fringe fields. We consider only periodic fields which can be expanded into a Fourier series along the axis including a strong fundamental 
Fig. 6.21 Field distribution in a wiggler magnet

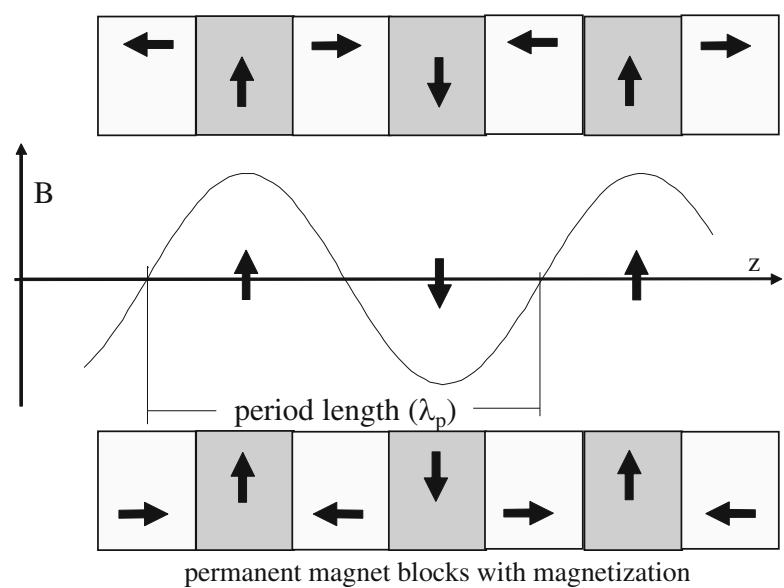

component with a period length $\lambda_{\mathrm{p}}$ and higher harmonics expressed by the ansatz [14]

$$
B_{y}=B_{0} \sum_{n \geq 0} b_{2 n+1}(x, y) \cos \left[(2 n+1) k_{\mathrm{p}} z\right],
$$

where the wave number $k_{\mathrm{p}}=2 \pi / \lambda_{\mathrm{p}}$. The functions $b_{i}(x, y)$ describe the variation of the field amplitude orthogonal to the beam axis for the harmonic $i$. The content of higher harmonics is greatly influenced by the particular design of the wiggler magnet and the ratio of the period length to the pole gap aperture. For very long periods relative to the pole aperture the field profile approaches that of a hard-edge dipole field with a square field profile along the $z$-axis. For very short periods compared to the pole aperture, on the other hand, we find only a significant amplitude for the fundamental period and very small perturbations due to higher harmonics.

We may derive the full magnetic field from Maxwell's equations based on a sinusoidal field along the axis. Each field harmonic may be determined separately due to the linear superposition of fields. To eliminate a dependence of the magnetic field on the horizontal variable $x$, we assume a pole width which is large compared to the pole aperture. The fundamental field component is then

$$
B_{y}(y, z)=B_{0} b_{1}(y) \cos k_{\mathrm{p}} z
$$

Maxwell's curl equation is in the wiggler aperture $\boldsymbol{\nabla} \times \boldsymbol{B}=0, \frac{\partial B_{z}}{\partial y}=\frac{\partial B_{y}}{\partial z}$ and with (6.110) we have

$$
\frac{\partial B_{z}}{\partial y}=\frac{\partial B_{y}}{\partial z}=-B_{0} b_{1}(y) k_{\mathrm{p}} \sin k_{\mathrm{p}} z .
$$


Integration of (6.111) with respect to $z$ gives the vertical field component

$$
B_{y}=-B_{0} k_{\mathrm{p}} b_{1}(y) \int_{0}^{z} \sin k_{\mathrm{p}} \bar{z} \mathrm{~d} \bar{z} .
$$

We have not yet determined the $y$-dependence of the amplitude function $b_{1}(y)$. From $\nabla \boldsymbol{B}=0$ and the independence of the field on the horizontal position we get with (6.110)

$$
\frac{\partial B_{z}}{\partial z}=-\frac{\partial B_{y}}{\partial y}=-B_{0} \frac{\partial b_{1}(y)}{\partial y} \cos k_{\mathrm{p}} z .
$$

Forming the second derivatives $\partial^{2} B_{z} /(\partial y \partial z)$ from (6.111), (6.113) we get for the amplitude function the differential equation

$$
\frac{\partial^{2} b_{1}(y)}{\partial y^{2}}=k_{\mathrm{p}}^{2} b_{1}(y),
$$

which can be solved by the hyperbolic functions

$$
b_{1}(y)=a \cosh k_{\mathrm{p}} y+b \sinh k_{\mathrm{p}} y .
$$

Since the magnetic field is symmetric with respect to $y=0$ and $b_{1}(0)=1$, the coefficients are $a=1$ and $b=0$. Collecting all partial results, the wiggler magnetic field is finally determined by the components

$$
\begin{aligned}
& B_{x}=0, \\
& B_{y}=B_{0} \cosh k_{\mathrm{p}} y \cos k_{\mathrm{p}} z, \\
& B_{z}=-B_{0} \sinh k_{\mathrm{p}} y \sin k_{\mathrm{p}} z,
\end{aligned}
$$

where $B_{z}$ is obtained by integration of (6.111) with respect to $y$.

The hyperbolic dependence of the field amplitude on the vertical position introduces higher-order field-errors which we determine by expanding the hyperbolic functions

$$
\begin{aligned}
& \cosh k_{\mathrm{p}} y=1+\frac{\left(k_{\mathrm{p}} y\right)^{2}}{2 !}+\frac{\left(k_{\mathrm{p}} y\right)^{4}}{4 !}+\frac{\left(k_{\mathrm{p}} y\right)^{6}}{6 !}+\frac{\left(k_{\mathrm{p}} y\right)^{8}}{8 !}+\ldots, \\
& \sinh k_{\mathrm{p}} y=+\left(k_{\mathrm{p}} y\right)+\frac{\left(k_{\mathrm{p}} y\right)^{3}}{3 !}+\frac{\left(k_{\mathrm{p}} y\right)^{5}}{5 !}+\frac{\left(k_{\mathrm{p}} y\right)^{7}}{7 !}+\ldots
\end{aligned}
$$

Typically the vertical gap in a wiggler magnet is much smaller than the period length or $y \ll \lambda_{\mathrm{p}}$ to avoid drastic reduction of the field strength. Due to the fast convergence of the series expansions (6.117) only a few terms are required to obtain an accurate expression for the hyperbolic function within the wiggler 
aperture. The expansion (6.117) displays higher-order field components explicitly which, however, do not have the form of higher-order multipole fields and we cannot treat these fields just like any other multipole perturbation but must consider them separately.

To determine the path distortion due to wiggler fields, we follow the reference trajectory through one quarter period starting at a symmetry plane in the middle of a pole. At the starting point $z=0$ in the middle of a wiggler pole the beam direction is parallel to the reference trajectory and the deflection angle at a downstream point $z$ is given by

$$
\begin{aligned}
\vartheta(z) & =\frac{e}{p} \int_{0}^{z} B_{y}(\bar{z}) \mathrm{d} \bar{z}=\frac{e}{p} B_{0} \cosh k_{\mathrm{p}} y \int_{0}^{z} \cos k_{\mathrm{p}} \bar{z} \mathrm{~d} \bar{z} \\
& =\frac{e}{p} B_{0} \frac{1}{k_{\mathrm{p}}} \cosh k_{\mathrm{p}} y \sin k_{\mathrm{p}} z .
\end{aligned}
$$

The maximum deflection angle is equal to the deflection angle for a quarter period or half a wiggler pole and is from (6.119) for $y=0$ and $k_{\mathrm{p}} z=\pi / 2$

$$
\theta=\frac{e}{p} B_{0} \frac{\lambda_{\mathrm{p}}}{2 \pi} .
$$

This deflection angle is used to define the wiggler strength parameter

$$
K=\beta \gamma \theta=\frac{c e}{2 \pi m c^{2}} B_{0} \lambda_{\mathrm{p}}
$$

where $m c^{2}$ is the particle rest energy and $\gamma$ the particle energy in units of the rest energy. In more practical units this strength parameter is

$$
K=C_{K} B_{0}(\mathrm{~T}) \lambda_{\mathrm{p}}(\mathrm{cm}) \approx B_{0}(\mathrm{~T}) \lambda_{\mathrm{p}}(\mathrm{cm})
$$

where

$$
C_{K}=\frac{c e}{2 \pi m c^{2}}=0.93373 \mathrm{~T}^{-1} \mathrm{~cm}^{-1}
$$

The parameter $K$ is a characteristic wiggler constant defining the wiggler strength and is not to be confused with the general focusing strength $K=\kappa^{2}+k$. Coming back to the distinction between wiggler and undulator magnet, we speak of a wiggler magnet if $K \gg 1$ and of an undulator if $K \ll 1$. Of course, many applications happen in a gray zone of terminology when $K \approx 1$. 


\subsection{Electrostatic Quadrupole}

A different focusing device based on electrostatic fields can be designed very much along the strategy for a magnetic quadrupole. We pick the first term on the r.h.s. of (6.25) and modify the expression to reflect the beam rigidity (6.12) for electric fields

$$
V_{2}(x, y)=-R_{\mathrm{b}} \beta A_{20} \frac{1}{2}\left(x^{2}-y^{2}\right)=-g \frac{1}{2}\left(x^{2}-y^{2}\right),
$$

where the field gradient, $g=\partial E_{x} / \partial x$. Such a device can be constructed by placing metallic surfaces in the form of a hyperbola

$$
x^{2}-y^{2}= \pm R=\text { const. }
$$

where $R$ is the aperture radius of the device as shown in Fig. 6.22 (left)

The potential of the four electrodes is alternately $V= \pm \frac{1}{2} g R^{2}$. This design can be somewhat simplified by replacing the hyperbolic metal surfaces by equivalently sized metallic tubes as shown in Fig. 6.22 (right). Numerical computer simulation programs can be used to determine the degradation of the quadrupole field due to this simplification.
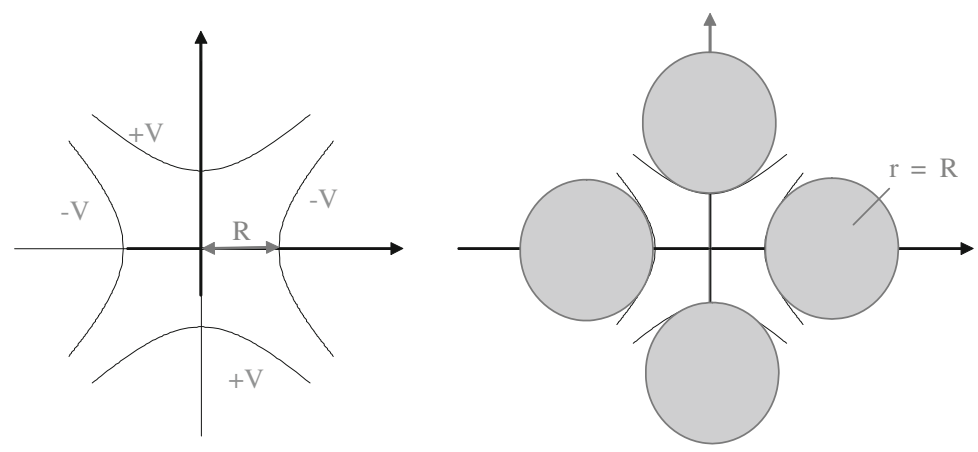

Fig. 6.22 Electric field quadrupole, ideal pole profile (left), and an example of a practical approach with cylindrical metallic tubes (right) 


\section{Problems}

6.1 (S). Show that the electrical power in the excitation coil is independent of the number of turns. Show also that the total electrical power in a copper coil depends only on the total weight of the copper used and the current density.

6.2 (S). Design an electrostatic quadrupole which provides a focal length of $10 \mathrm{~m}$ in the horizontal plane for particles with a kinetic energy of $10 \mathrm{MeV}$. The device shall have an aperture with a diameter of $10 \mathrm{~cm}$ and an effective length of $0.1 \mathrm{~m}$. What is the form of the electrodes, their orientation and potential?

6.3 (S). In the text, we have derived the fields from a scalar potential. We could also derive the magnetic fields from a vector potential $\boldsymbol{A}$ through the differentiation $\boldsymbol{B}=\boldsymbol{\nabla} \times \boldsymbol{A}$. For purely transverse magnetic fields, show that only the longitudinal component $A_{z} \neq 0$ must be non zero. Derive the vector potential for a dipole and quadrupole field and compare with the scalar potential. What is the difference between the scalar potential and the vector potential?

6.4 (S). Derive the pole profile (aperture radius $r=1 \mathrm{~cm}$ ) for a combined function magnet including a dipole field to produce for a particle beam of energy $E=$ $50 \mathrm{GeV}$ a bending radius of $\rho=300 \mathrm{~m}$, a focusing strength $k=0.45 \mathrm{~m}^{-2}$ and a sextupole strength of $m=23.0 \mathrm{~m}^{-3}$.

6.5 (S). Strong mechanical forces exist between the magnetic poles when a magnet is energized. Are these forces attracting or repelling the poles? Why? Consider a dipole magnet $\ell=1 \mathrm{~m}$ long, a pole width $w=0.2 \mathrm{~m}$ and a field of $B=1.5 \mathrm{~T}$. Estimate the total force between the two magnet poles?

6.6 (S). Following the derivation of (5.7) for a bending magnet, derive a similar expression for the electrical excitation current in A-turns of a quadrupole with an aperture radius $R$ and a desired field gradient $g$. What is the total excitation current necessary in a quadrupole with an effective length of $\ell=1 \mathrm{~m}$ and $R=3 \mathrm{~cm}$ to produce a focal length of $f=50 \mathrm{~m}$ for particles with an energy of $c p=500 \mathrm{GeV}$ ?

6.7 (S). Consider a coil in the aperture of a magnet as shown in Fig. 6.14. All $n$ windings are made of very thin wires and are located exactly on the radius $R$. We rotate now the coil about its axis at a rotation frequency $v$. Such rotating coils are used to measure the multipole field components in a magnet. Show analytically that the recorded signal is composed of harmonics of the rotation frequency $v$. What is the origin of the harmonics?

6.8 (S). Explain why a quadrupole with finite pole width does not produce a pure quadrupole field. What are the other allowed multipole field components ignore mechanical tolerances and why?

6.9 (S). Through magnetic measurements the following vertical magnetic multipole field components in a quadrupole are determined. At $x=1.79 \mathrm{~cm}$ and $y=0$ $\mathrm{cm}: B_{2}=0.3729 \mathrm{~T}, B_{3}=1.25 \times 10^{-4} \mathrm{~T}, B_{4}=0.23 \times 10^{-4} \mathrm{~T}, B_{5}=0.36 \times 10^{-4} \mathrm{~T}$, 
$B_{6}=0.726 \times 10^{-4} \mathrm{~T}, B_{7}=0.020 \times 10^{-4} \mathrm{~T}, B_{8}=0.023 \times 10^{-4} \mathrm{~T}, B_{9}=0.0051 \times 10^{-4}$ T, $B_{10}=0.0071 \times 10^{-4} \mathrm{~T}$. Calculate the relative multipole strengths at $x=1 \mathrm{~cm}$ normalized to the quadrupole field at $1 \mathrm{~cm}$. Why do the 12-pole and 20-pole components stand out with respect to the other multipole components?

6.10 (S). Derive the equation for the pole profile of an iron dominated upright octupole with a bore radius $R$. Ignore longitudinal variations. To produce a field of $0.2 \mathrm{~T}$ at the pole tip $(R=3 \mathrm{~cm})$ what total current per coil is required?

6.11 (S). Calculate and design the current distribution for a pure air coil, superconducting dipole magnet to produce a field of $B_{0}=5 \mathrm{~T}$ in an aperture of radius $R=3 \mathrm{~cm}$ without exceeding an average current density of $\hat{\jmath}=1,000 \mathrm{~A} / \mathrm{mm}^{2}$.

6.12. Derive an expression for the current distribution in air coils to produce a combination of a dipole, quadrupole and sextupole field. Express the currents in terms of fields and field gradients.

\section{References}

1. G.E. Fischer, Iron dominated magnets, in AIP Conference Proceedings, vol. 153 (American Institute of Physics, New York, 1987), p. 1047

2. J.T. Tanabe, Iron Dominated Electromagnets (World Scientific, Singapore, 2005)

3. R. Perin, S. van der Meer, Technical Report, CERN 67-7, CERN, Geneva (1967)

4. K. Halbach, Technical Report, UCRL-17436, LLNL, Lawrence Livermore National Laboratory (1967)

5. K.L. Brown, Adv. Part. Phys. 1, 71 (1967)

6. G. Leleux, An $o(n \log n / \log \log n)$ sorting algorithm. Technical Report, SOC/ALIS 18, Départment du Synchrotron Saturn, Saclay (1969)

7. H. Motz, J. Appl. Phys. 22, 527 (1951).

8. K. Robinson, G.A. Voss, in Proceedings of the International Symposium Electron and Positron Storage Rings (Presses Universitaires de France, Paris, 1966), p. III-4

9. J.M. Paterson, J.R. Rees, H. Wiedemann, Technical Report, PEP-Note 125, Stanford Linear Accelerator Center, Stanford (1975)

10. H. Wiedemann, Nucl. Instrum. Methods A266, 24 (1988)

11. W.R. Smythe, Static and Dynamic Electricity (McGraw-Hill, New York, 1950)

12. L.R. Elias, W.M. Fairbanks, J.M.J. Madey, H.A. Schwettmann, T.J. Smith, Phys. Rev. Lett. 36, 717 (1976)

13. B.M. Kincaid, J. Appl. Phys. 48, 2684 (1977)

14. L. Smith, Effects of wigglers and ubdulators on beam dynamics. Technical Report, ESG Techn. Note 24, SLAClawrence bertkeley Laboratory (1986) 


\section{Part III \\ Beam Dynamics}




\section{Chapter 7 \\ Single Particle Dynamics}

The general equations of motion, characterized by an abundance of perturbation terms on the right-hand side of, for example, (6.95), (6.96) have been derived in the previous chapter. If these perturbation terms were allowed to become significant in real beam transport systems, we would face almost insurmountable mathematical problems trying to describe the motion of charged particles in a general way. For practical mathematical reasons it is therefore important to design components for particle beam transport systems such that undesired terms appear only as small perturbations. With a careful design of beam guidance magnets and accurate alignment of these magnets we can indeed achieve this goal.

Most of the perturbation terms are valid solutions of the Laplace equation describing higher order fields components. Virtually all these terms can be minimized to the level of perturbations by proper design of beam transport magnets. Specifically, we will see that the basic goals of beam dynamics can be achieved by using only two types of magnets, bending magnets and quadrupoles which sometimes are combined into one magnet. Beam transport systems, based on only these two lowest order magnet types, are called linear systems and the resulting theory of particle dynamics in the presence of only such magnets is referred to as linear beam dynamics or linear beam optics.

In addition to the higher order magnetic field components, we also find purely kinematic terms in the equations of motion due to large amplitudes or due to the use of curvilinear coordinates. Some of these terms are generally very small for particle trajectories which stay close to the reference path such that divergences are small, $x^{\prime} \ll 1$ and $y^{\prime} \ll 1$. The lowest order kinematic effects resulting from the use of a curvilinear coordinate system, however, cannot generally be considered small perturbations. One important consequence of this choice for the coordinate system is that the natural bending magnet is a sector magnet which has very different beam dynamics properties than a rectangular magnet which would be the natural magnet

This chapter has been made Open Access under a CC BY 4.0 license. For details on rights and licenses please read the Correction https://doi.org/10.1007/978-3-319-18317-6_28

(C) The Author(s) 2015

H. Wiedemann, Particle Accelerator Physics, Graduate Text in Physics, https://doi.org/10.1007/978-3-319-18317-6_7 
type for a Cartesian coordinate system. While a specific choice of a coordinate system will not change the physics, we must expect that some features are expressed easier or more complicated in one or the other coordinate system. We have chosen to use the curvilinear system because it follows the ideal path of the particle beam and offers a simple direct way to express particle trajectories deviating from the ideal path. In a fixed Cartesian coordinate system we would have to deal with geometric expressions relating the points along the ideal path to an arbitrary reference point. The difference becomes evident for a simple trajectory like a circle of radius $r$ and center at $\left(x_{0}, y_{0}\right)$ which in a fixed orthogonal coordinate system would be expressed by $\left(x-x_{0}\right)^{2}+\left(y-y_{0}\right)^{2}=r^{2}$. In the curvilinear coordinate system this equation reduces to the simple identity $x(z)=0$.

\subsection{Linear Beam Transport Systems}

The theory of beam dynamics based on quadrupole magnets for focusing is called strong focusing beam dynamics in contrast to the case of weak focusing, which utilizes the focusing of sector magnets in combination with a small gradient in the bending magnet profile. Such focusing is employed in circular accelerators like betatrons or some cyclotrons and the first generation of synchrotrons. The invention of strong focusing by Christofilos [1] and independently by Courant et al. [2] changed quickly the way focusing arrangements for large particle accelerators are determined. One of the main attraction for this kind of focusing was the ability to greatly reduce the magnet aperture needed for the particle beam since the stronger focusing confines the particles to a much smaller cross section compared to weak focusing. A wealth of publications and notes have been written during the fifties to determine and understand the intricacies of strong focusing, especially the rising problems of alignment and field tolerances as well as those of resonances. Particle stability conditions from a mathematical point of view have been investigated by Moser [3].

In this chapter, we will discuss the theory of linear charged particle beam dynamics and apply it to the development of beam transport systems, the characterization of particle beams, and to the derivation of beam stability criteria. The bending and focusing function may be performed either in separate magnets or be combined within a synchrotron magnet. The arrangement of magnets in a beam transport system, called the magnet lattice, is often referred to as a separated function or combined function lattice depending on whether the lattice makes use of separate dipole and quadrupole magnets or uses combined function magnets, respectively.

Linear equations of motion can be extracted from (6.95), (6.96) to treat beam dynamics in first or linear approximation. For simplicity and without restricting generality we assume the bending of the beam to occur only in one plane, the $x$-plane. The linear magnetic fields for bending and quadrupole magnets are 
expressed by

$$
\begin{aligned}
& B_{x}=-g y, \\
& B_{y}=B_{y 0}+g x,
\end{aligned}
$$

where $B_{y 0}$ is the dipole field and $g$ the gradient of the quadrupole field. With these field components we obtain from (6.95), (6.96) the equations of motion in the approximation of linear beam dynamics

$$
\begin{aligned}
x^{\prime \prime}+\left(k_{0}+\kappa_{0 x}^{2}\right) x & =0, \\
y^{\prime \prime}-k_{0} y & =0 .
\end{aligned}
$$

Both, the focusing from the bending magnet and that from a quadrupole may be combined into one parameter

$$
K(z)=k_{0}(z)+\kappa_{0 x}^{2}(z)
$$

So far no distinction has been made between combined or separated function magnets and the formulation of the equations of motion based on the magnet strength parameter $K$ as defined in (7.3), is valid for both types of magnets. For separated function magnets either $k_{0}$ or $\kappa_{0 x}$ is set to zero while for combined function magnets both parameters are nonzero.

\subsubsection{Nomenclature}

Focusing along a beam transport line is performed by discrete quadrupoles placed to meet specific particle beam characteristics required at the end or some intermediate point of the beam line. The dependence of the magnet strength on $z$ is, therefore, a priori indeterminate and is the subject of lattice design in accelerator physics. To describe focusing lattices simple symbols are used to point out location and sometimes relative strength of magnets. In this text we will use symbols from Fig. 7.1 for bending magnets, quadrupoles, and sextupoles or multipoles.

All magnets are symbolized by squares along the $z$-axis and the length of the symbol may represent the actual magnetic length. The symbol for pure dipole magnets is a square centered about the $z$-axis while bending magnets with a gradient are shifted vertically to indicate the sign of the focusing. Positive squares are used to indicate horizontal focusing and negative squares for horizontal defocusing quadrupoles. 
Fig. 7.1 Symbols for magnets in lattice design and typical distributions of magnets along a beam transport line

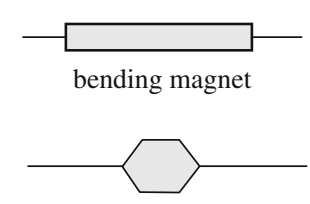

sextupole/multipole

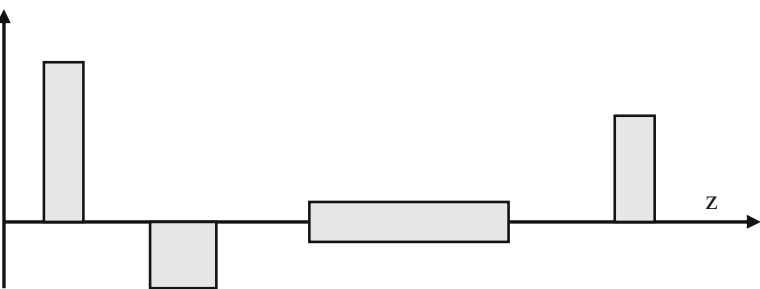

Using such symbols, a typical beam transport line may have general patterns like that shown in Fig 7.1. The sequence of magnets and their strength seems random and is mostly determined by external conditions to be discussed later. More regular magnet arrangements occur for circular accelerators or very long beam transport lines composed of periodic sections.

\subsection{Matrix Formalism in Linear Beam Dynamics}

The seemingly arbitrary distribution of focusing parameters in a beam transport system makes it impossible to formulate a general solution of the differential equations of motion (7.2). To describe particle trajectories analytically through a beam transport line composed of drift spaces, bending magnets, and quadrupoles, we will derive mathematical tools which consist of partial solutions and can be used to describe complete particle trajectories.

In this section we will derive and discuss the matrix formalism [4] as a method to describe particle trajectories. This method makes use of the fact that the magnet strength parameters are constant at least within each individual magnet. The equations of motion become very simple since the restoring force $K$ is constant and the solutions have the form of trigonometric functions. The particle trajectories may now be described by analytical functions at least within each uniform element of a transport line including magnet free drift spaces.

These solutions can be applied to any arbitrary beam transport line, where the focusing parameter $K$ changes in a step like function as shown in Fig. 7.1. Cutting this beam line into its smaller elements so that $K=$ const. in each of these pieces 
we will be able to follow the particle trajectories analytically step by step through the whole transport system. This is the model generally used in particle beam optics and is called the hard edge model.

In reality, however, since nature does not allow sudden changes of physical quantities (natura non facit saltus) the hard edge model is only an approximation, although for practical purposes a rather good one. In a real magnet the field strength does not change suddenly from zero to full value but rather follows a smooth transition from zero to the maximum field. Sometimes, the effects due to this smooth field transition or fringe field are important and we will derive the appropriate corrections later in this section. For now, we continue using the hard edge model for beam transport magnets and keep in mind that in some cases a correction may be needed to take into account the effects of a smooth field transition at the magnet edges.

Using this approximation, where $1 / \rho_{0}$ and $k$ are constants, and ignoring perturbations, the equation of motion is reduced to that of a harmonic oscillator,

$$
u^{\prime \prime}+K_{u} u=0, \quad \text { where } \quad K_{u}=k_{u 0}+\kappa_{0 u}^{2}=\text { const } .
$$

The principal solutions have been derived in Sect. 5.5.1 and are expressed in matrix formulation by

$$
\left(\begin{array}{c}
u(z) \\
u^{\prime}(z)
\end{array}\right)=\left(\begin{array}{ll}
C_{u}(z) & S_{u}(z) \\
C_{u}^{\prime}(z) & S_{u}^{\prime}(z)
\end{array}\right)\left(\begin{array}{c}
u_{0} \\
u_{0}^{\prime}
\end{array}\right),
$$

where $u$ may be used for either $x$ or $y$. We have deliberately separated the motion in both planes since we do not consider coupling. Formally, we could combine the two $2 \times 2$ transformation matrices for each plane into one $4 \times 4$ matrix describing the transformation of all four coordinates

$$
\left(\begin{array}{c}
x(z) \\
x^{\prime}(z) \\
y(z) \\
y^{\prime}(z)
\end{array}\right)=\left(\begin{array}{cccc}
C_{x}(z) & S_{x}(z) & 0 & 0 \\
C_{x}^{\prime}(z) & S_{x}^{\prime}(z) & 0 & 0 \\
0 & 0 & C_{y}(z) & S_{y}(z) \\
0 & 0 & C_{y}^{\prime}(z) & S_{y}^{\prime}(z)
\end{array}\right)\left(\begin{array}{c}
x_{0} \\
x_{0}^{\prime} \\
y_{0} \\
y_{0}^{\prime}
\end{array}\right) .
$$

Obviously the transformations are still completely decoupled but in this form we could include coupling effects, where, for example, the $x$-motion depends also on the $y$-motion and vice versa. This can be further generalized to include any particle parameter like the longitudinal position of a particle with respect to a reference particle, or the energy of a particle, the spin vector, or any particle coordinate that may depend on other coordinates. In the following paragraphs we will restrict the discussion to linear $(2 \times 2)$ transformation matrices for a variety of beam line elements. 


\subsubsection{Driftspace}

In a driftspace of length $\ell$ or in a weak bending magnet, where $\kappa_{0 x}^{2} \ll 1$ and $k_{0}=0$, the focusing parameter $K=0$ and the solution of (7.4) in matrix formulation can be expressed by

$$
\left(\begin{array}{l}
u(z) \\
u^{\prime}(z)
\end{array}\right)=\left(\begin{array}{ll}
1 & \ell \\
0 & 1
\end{array}\right)\left(\begin{array}{l}
u_{0} \\
u_{0}^{\prime}
\end{array}\right)
$$

A more precise derivation of the transformation matrices for bending magnets of arbitrary strength will be described later in this chapter. Any drift space of length $\ell=z-z_{0}$, therefore, is represented by the simple transformation matrix

$$
\mathcal{M}_{\mathrm{d}}(\ell \mid 0)=\left(\begin{array}{ll}
1 & \ell \\
0 & 1
\end{array}\right) \text {. }
$$

We recognize the expected features of a particle trajectory in a field free drift space. The amplitude $u$ changes only if the trajectory has an original non vanishing slope $u_{0}^{\prime} \neq 0$ while the slope itself does not change at all.

\subsubsection{Quadrupole Magnet}

For a pure quadrupole the bending term $\kappa_{0 x}=0$ and the field gradient or quadrupole strength $k(z) \neq 0$ can be positive as well as negative. With these assumptions we solve again (7.4) and determine the integration constants by initial conditions. For $k>0$ we get the transformation for a focusing quadrupole

$$
\left(\begin{array}{c}
u(z) \\
u^{\prime}(z)
\end{array}\right)=\left(\begin{array}{cc}
\cos \psi & \frac{1}{\sqrt{k}} \sin \psi \\
-\sqrt{k} \sin \psi & \cos \psi
\end{array}\right)\left(\begin{array}{c}
u\left(z_{0}\right) \\
u^{\prime}\left(z_{0}\right)
\end{array}\right),
$$

where $\psi=\sqrt{k}\left(z-z_{0}\right.$. This equation is true for any section within the quadrupole as long as both points $z_{0}$ and $z$ are within the active length of the quadrupole.

For a full quadrupole of length $\ell$ and strength $k$ we set $\varphi=\sqrt{k \ell}$ and the transformation matrix for a full quadrupole in the focusing plane is

$$
\mathcal{M}_{\mathrm{QF}}(\ell \mid 0)=\left(\begin{array}{cc}
\cos \varphi & \frac{1}{\sqrt{k}} \sin \varphi \\
-\sqrt{k} \sin \varphi & \cos \varphi
\end{array}\right) .
$$


Fig. 7.2 Example of a beam transport line (schematic)

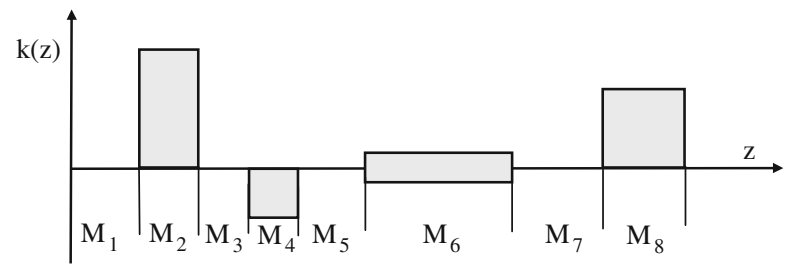

Similarly, we get in the other plane with $k<0$ the solution for a defocusing quadrupole

$$
\left(\begin{array}{c}
u(z) \\
u^{\prime}(z)
\end{array}\right)=\left(\begin{array}{cc}
\cosh \psi & \frac{1}{\sqrt{|k|}} \sinh \psi \\
\sqrt{|k|} \sinh \psi & \cosh \psi
\end{array}\right)\left(\begin{array}{c}
u\left(z_{0}\right) \\
u^{\prime}\left(z_{0}\right)
\end{array}\right),
$$

where $\psi=\sqrt{|k|}\left(z-z_{0}\right)$. The transformation matrix in the defocusing plane through a complete quadrupole of length $\ell$ with $\varphi=\sqrt{|k|} \ell$ is therefore

$$
\mathcal{M}_{\mathrm{QD}}(\ell \mid 0)=\left(\begin{array}{cc}
\cosh \varphi & \frac{1}{\sqrt{|k|}} \sinh \varphi \\
\sqrt{|k|} \sinh \varphi & \cosh \varphi
\end{array}\right) .
$$

These transformation matrices make it straight forward to follow a particle through a transport line. Any arbitrary sequence of drift spaces, bending magnets and quadrupole magnets can be represented by a series of transformation matrices $\mathcal{M}_{i}$. The transformation matrix for the whole composite beam line is then just equal to the product of the individual matrices. For example, by multiplying all matrices along the path in Fig. 7.2 the total transformation matrix $\mathcal{M}$ for the eight magnetic elements of this example is determined by the product

$$
\mathcal{M}=\mathcal{M}_{8} \ldots \mathcal{M}_{4} \mathcal{M}_{3} \mathcal{M}_{2} \mathcal{M}_{1}
$$

and the particle trajectory transforms through the whole composite transport line like

$$
\left(\begin{array}{c}
u(z) \\
u^{\prime}(z)
\end{array}\right)=\mathcal{M}\left(z \mid z_{0}\right)\left(\begin{array}{c}
u\left(z_{0}\right) \\
u^{\prime}\left(z_{0}\right)
\end{array}\right),
$$

where the starting point $z_{0}$ in this case is at the beginning of the drift space $\mathcal{M}_{1}$ and the end point $z$ is at the end of the magnet $\mathcal{M}_{8}$. 


\subsubsection{Thin Lens Approximation}

As will become more apparent in the following sections, this matrix formalism is widely used to calculate trajectories for individual particle or for a virtual particle representing the central path of a whole beam. The repeated multiplication of matrices, although straightforward, is very tedious and therefore, most beam dynamics calculations are performed on digital computers. In some cases, however, it is desirable to analytically calculate the approximate properties of a small set of beam elements. For these cases it is sufficient to use what is called the thin lens approximation. In this approximation it is assumed that the length of a quadrupole magnet is small compared to its focal length $(\ell \ll f)$ and we set

$$
\ell \rightarrow 0
$$

while keeping the focal strength constant,

$$
f^{-1}=+k l=\text { const } .
$$

This result is analogous to geometric light optics, where we assume the glass lenses to be infinitely thin. As a consequence $\varphi=\sqrt{k} \ell \rightarrow 0$ and the transformation matrices $(7.10),(7.12)$ are the same in both planes except for the sign of the focal length

$$
\left(\begin{array}{c}
u(z) \\
u^{\prime}(z)
\end{array}\right)=\left(\begin{array}{cc}
1 & \ell \\
-\frac{1}{f} & 1
\end{array}\right)\left(\begin{array}{l}
u_{0} \\
u_{0}^{\prime}
\end{array}\right)
$$

where

$$
\begin{aligned}
& f^{-1}=k \ell>0 \text { in the focusing plane } \\
& f^{-1}=k \ell<0 \text { in the defocusing plane. }
\end{aligned}
$$

The transformation matrix has obviously become very simple and exhibits only the focusing property in form of the focal length. Quite generally one may regard for single as well as composite systems the matrix element $M_{21}$ as the element that expresses the focal strength of the transformation.

In thin lens approximation it is rather easy to derive focusing properties of simple compositions of quadrupoles. A quadrupole doublet composed of two quadrupole magnets separated by a drift space of length $L$ is described by the total transformation matrix

$$
\begin{aligned}
\mathcal{M}_{\mathrm{db}}(L \mid 0) & =\left(\begin{array}{cc}
1 & 0 \\
-\frac{1}{f_{2}} & 1
\end{array}\right)\left(\begin{array}{ll}
1 & L \\
0 & 1
\end{array}\right)\left(\begin{array}{cc}
1 & 0 \\
-\frac{1}{f_{1}} & 1
\end{array}\right) \\
& =\left(\begin{array}{cc}
1-L / f_{1} & L \\
-1 / f^{*} & 1-L / f_{2}
\end{array}\right),
\end{aligned}
$$


Fig. 7.3 Reversed lattice

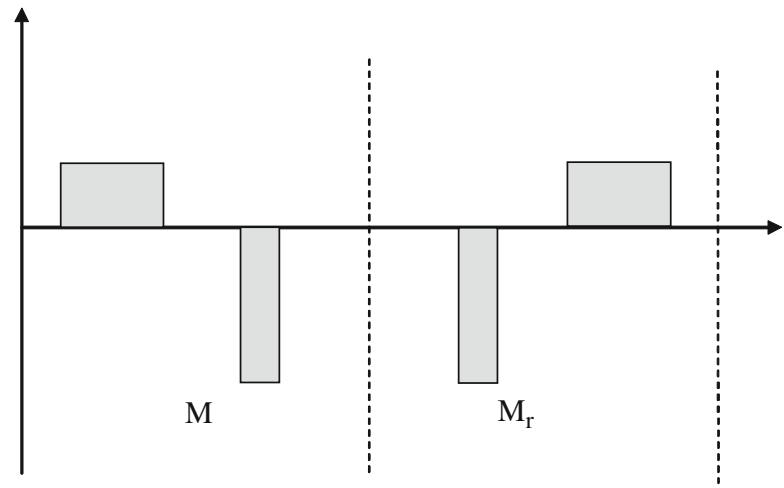

where we find the well known expression from geometric paraxial light optics

$$
\frac{1}{f^{*}}=\frac{1}{f_{1}}+\frac{1}{f_{2}}-\frac{L}{f_{1} f_{2}} .
$$

Such a doublet can be made focusing in both planes if, for example, the quadrupole strengths are set such that $f_{1}=-f_{2}=f$. The total focal length then is $f^{*}=$ $+L / f^{2}>0$ in both the horizontal and the vertical plane.

This simple result, where the focal length is the same in both planes, is a valid solution only in thin lens approximation. For a doublet of finite length quadrupoles the focal length in the horizontal plane is always different from that in the vertical plane as can be verified by using the transformations (7.10), (7.12) to calculate the matrix $\mathcal{M}_{\mathrm{db}}$. Since individual matrices are not symmetric with respect to the sign of the quadrupole field, the transformation matrices for the horizontal plane $\mathcal{M}_{\mathrm{db}, \mathrm{x}}$ and the vertical plane $\mathcal{M}_{\mathrm{db}, \mathrm{y}}$ must be calculated separately and turn out to be different. In special composite cases, where the quadrupole distribution is symmetric as shown in Fig. 7.3, the matrices for both of the two symmetric half sections are related in a simple way. If the matrix for one half of the symmetric beam line is

$$
\mathcal{M}=\left(\begin{array}{ll}
a & b \\
c & d
\end{array}\right)
$$

then the reversed matrix for the second half of the beam line is

$$
\mathcal{M}_{\mathrm{r}}=\left(\begin{array}{ll}
d & b \\
c & a
\end{array}\right)
$$

and the total symmetric beam line has the transformation matrix

$$
\mathcal{M}_{\text {tot }}=\mathcal{M}_{\mathrm{r}} \mathcal{M}=\left(\begin{array}{cc}
a d+b c & 2 b d \\
2 a c & a d+b c
\end{array}\right) \text {. }
$$


Fig. 7.4 Symmetric quadrupole triplet

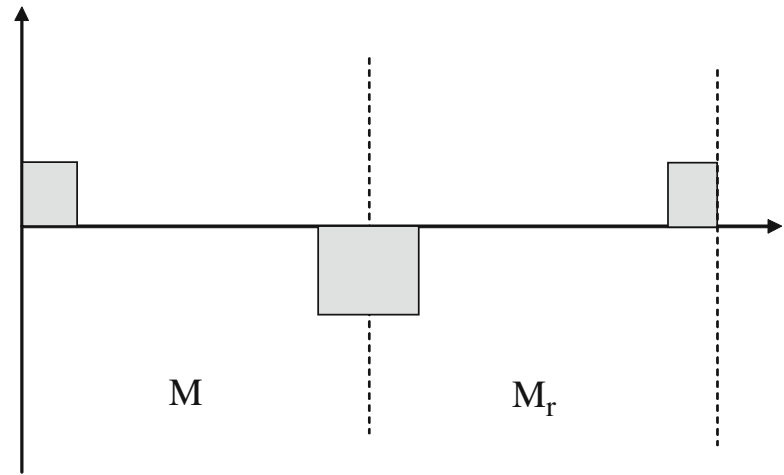

We made no particular assumptions for the lattice shown in Fig. 7.3 except for symmetry and the relations (7.21), (7.22) are true for any arbitrary but symmetric beam line.

The result for the reversed matrix is not to be confused with the inverse matrix, where the direction of the particle path is also reversed. The inverses matrix of (7.21) is

$$
\mathcal{M}_{\mathrm{i}}=\left(\begin{array}{cc}
d & -b \\
-c & a
\end{array}\right)
$$

Going through an arbitrary section of a beam line and then back to the origin again results in a total transformation matrix equal to the unity matrix

$$
\mathcal{M}_{\text {tot }}=\mathcal{M}_{\mathrm{i}} \mathcal{M}=\left(\begin{array}{ll}
1 & 0 \\
0 & 1
\end{array}\right)
$$

These results allow us now to calculate the transformation matrix $\mathcal{M}_{\text {tr }}$ for a symmetric quadrupole triplet. With (7.19), (7.24) the transformation matrix of a quadrupole triplet as shown in Fig. 7.4 is

$$
\mathcal{M}_{\mathrm{tr}}=\mathcal{M}_{\mathrm{r}} \mathcal{M}=\left(\begin{array}{cc}
1-2 L^{2} / f^{2} & 2 L(1+L / f) \\
-1 / f^{*} & 1-2 L^{2} / f^{2}
\end{array}\right),
$$

where $f^{*}$ is defined by (7.20) with $f_{1}=-f_{2}=f$.

Such a triplet is focusing in both planes as long as $f>L$. Symmetric triplets as shown in Fig. 7.4 have become very important design elements of long beam transport lines or circular accelerators since such a triplet can be made focusing in both planes and can be repeated arbitrarily often to provide a periodic focusing structure called a FODO-channel. The acronym is derived from the sequence of focusing (F) and defocusing (D) quadrupoles separated by non-focusing elements (O) like a drift space or a bending magnet. 


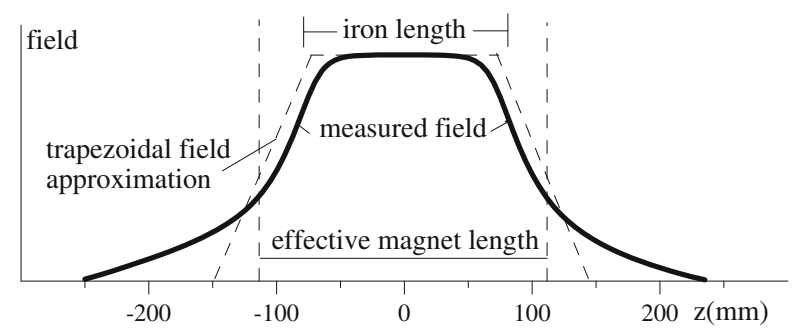

Fig. 7.5 Field profile in a real quadrupole with a bore radius of $R=3 \mathrm{~cm}$ and an iron length of $\ell_{\text {iron }}=15.9 \mathrm{~cm}$

\subsubsection{Quadrupole End Field Effects}

In defining the transformation through a quadrupole we have assumed the strength parameter $k(z)$ to be a step function with a constant nonzero value within the quadrupole and zero outside. Such a hard edge field distribution is only approximately true for a real quadrupole. The strength parameter in a real quadrupole magnet varies in a gentle way from zero outside the quadrupole to a maximum value in the middle of the quadrupole. In Fig. 7.5 the measured gradient of a real quadrupole along the axis is shown.

The field extends well beyond the length of the iron core and the effective magnetic length, defined by

$$
\ell_{\mathrm{eff}}=\frac{\int g \mathrm{~d} z}{g_{0}},
$$

where $g_{0}$ is the field gradient in the middle of the quadrupole, is longer than the iron length by about the radius of the bore aperture

$$
\ell_{\text {eff }} \approx \ell_{\text {iron }}+R
$$

This is the effective or hard edge magnet length $\ell_{0}$ with strength $k$. The real field distribution can be approximated by a trapezoid such that $\int g \mathrm{~d} z$ is the same in both cases (see Fig. 7.5). To define the trapezoidal approximation we assume a fringe field extending over a length equal to the bore radius $R$ as shown in Fig. 7.5. End field effects must therefore be expected specifically in quadrupoles with large bore radii and short iron cores. It is interesting to investigate as to what extend the transformation characteristics for a real quadrupole differ from the hard edge model. The real transformation matrix can be obtained by slicing the whole axial quadrupole field distribution in thin segments of varying strength. Treating these segments as short hard edge quadrupoles the full transformation matrix is the product of the matrices for all segments. 


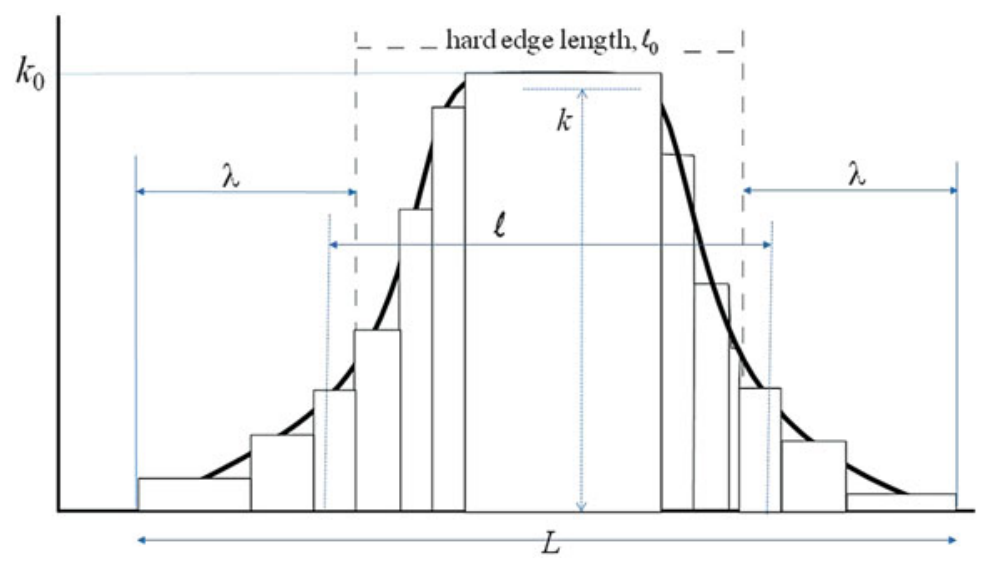

Fig. 7.6 Decomposition of an actual quadrupole field profile into segments of hard edge quadrupoles. $\left(k_{0}, \ell_{0}\right.$ are for the hard edge model, $k, \ell$ for the hard edge model with real fringe fields, $\lambda$ and $L$ are used for mathematical evaluation only)

While it is possible to obtain an accurate transformation matrix this way the variations of the matrix elements due to this smooth field distribution turn out to be mostly small and in practice, therefore, the hard edge model is used to develop beam transport lattices. Nonetheless after a satisfactory solution has been found, these real transformation matrices should be used to check the solution and possibly make small adjustment to the idealized hard edge model design.

In this section, we will discuss an analytical estimate of the correction to be expected for a real field distribution [5] by looking for the "effective" hard edge model parameters $(k, \ell)$ which result in a transformation matrix equal to the transformation matrix for the corresponding real quadrupole. The transformation matrix for the real quadrupole be

$$
\mathcal{M}_{\mathrm{Q}}=\left(\begin{array}{cc}
C & S \\
C^{\prime} & S^{\prime}
\end{array}\right),
$$

where the matrix elements are the result of multiplying all "slice" matrices for the quadrupole segments as shown in Fig. 7.6 over the length $L$.

We assume now that this real quadrupole can be represented by a hard edge model quadrupole of length $\ell$ with adjacent drift spaces $\lambda$ as indicated in Fig. 7.6. The transformation through this system for a focusing quadrupole is given by [5]

$$
\begin{aligned}
& \left(\begin{array}{ll}
1 & \lambda \\
0 & 1
\end{array}\right)\left(\begin{array}{cc}
\cos \varphi & \frac{1}{\sqrt{k}} \sin \varphi \\
-\sqrt{k} \sin \varphi & \cos \varphi
\end{array}\right)\left(\begin{array}{ll}
1 & \lambda \\
0 & 1
\end{array}\right) \\
& =\left(\begin{array}{cc}
\cos \varphi-\sqrt{k} \lambda \sin \varphi & 2 \lambda \cos \varphi-\frac{1}{\sqrt{k}} \sin \varphi \\
-\sqrt{k} \sin \varphi & \cos \varphi-\sqrt{k} \lambda \sin \varphi
\end{array}\right)
\end{aligned}
$$


with $\varphi=\sqrt{k} \ell_{0}$. This hard edge transformation matrix must be the same as the actual matrix (7.29) and we will use this equality to determine the effective quadrupole parameters $k, \ell$. First, we note that the choice of the total length $L=$ $\ell_{0}+2 \lambda$ is arbitrary as long as it extends over the whole field profile, and both, the "slices" and hard edge matrices extend over the whole length $L$ by employing drift spaces if necessary. Equating (7.29) and (7.30) we can compose two equations which allow us to determine the effective parameters $k, \ell$ from known quantities

$$
\begin{aligned}
& C_{\mathrm{f}}-\frac{1}{2} L C_{\mathrm{f}}^{\prime}=\cos \varphi_{\mathrm{f}}+\frac{1}{2} \varphi_{\mathrm{f}} \sin \varphi_{\mathrm{f}}, \\
& C_{\mathrm{f}}^{\prime} \ell_{\mathrm{f}}=-\varphi_{\mathrm{f}} \sin \varphi_{\mathrm{f}} .
\end{aligned}
$$

Here we have added the index ${ }_{\mathrm{f}}$ to indicate a focusing quadrupole. The first of these equations can be solved for $\varphi_{\mathrm{f}}$ since the quantities $C_{\mathrm{f}}, C_{\mathrm{f}}^{\prime}$, and $L$ are known. The second equation then is solved for $\ell_{\mathrm{f}}$ and $k_{\mathrm{f}}=\varphi_{\mathrm{f}}^{2} / \ell_{\mathrm{f}}$. Two parameters are sufficient to equate the $2 \times 2$ matrices (7.29), (7.30) since two of the four equations are redundant for symmetry reasons, $M_{11}=M_{22}=C=S^{\prime}$, and the determinant of the matrices on both sides must be unity. Similarly, we get for a defocusing quadrupole

$$
\begin{aligned}
& C_{\mathrm{d}}-\frac{1}{2} L C_{\mathrm{d}}^{\prime}=\cosh \varphi_{\mathrm{d}}-\frac{1}{2} \varphi_{\mathrm{d}} \sinh \varphi_{\mathrm{d}}, \\
& C_{\mathrm{d}}^{\prime} \ell_{\mathrm{d}}=-\varphi_{\mathrm{d}} \sinh \varphi_{\mathrm{d}} .
\end{aligned}
$$

Equations (7.31) and (7.32) define a hard edge representation of a real quadrupole. However, we note that the effective quadrupole length $\ell$ and strength $k$ are different from the customary definition, where $k_{0}$ is the actual magnet strength in the middle of the quadrupole and the magnet length is defined by $\ell_{0}=\frac{1}{k_{0}} \int k(z) \mathrm{d} z$. We also observe that the effective values $\ell$ and $k$ are different for the focusing and defocusing plane. Since the endfields are not the same for all quadrupoles but depend on the design parameters of the magnet we cannot determine the corrections in general. In practical cases, however, it turns out that the corrections $\Delta k=k-k_{0}$ and $\Delta \ell=$ $\ell-\ell_{0}$ are small for quadrupoles which are long compared to the aperture and are larger for short quadrupoles with a large aperture. In fact the differences $\Delta k$ and $\Delta \ell$ turn out to have opposite polarity and the thin lens focal length error $\Delta k \Delta \ell$ is generally very small.

As an example, we use the quadrupole of Fig. 7.5 and calculate the corrections due to end field effects. We calculate the total transformation matrix for the real field profile as discussed above by approximating the actual field distribution by a series of hard edge "slice" matrices in both planes as a function of the focusing strength $k_{0}$ and solve (7.31), (7.32) for the effective parameters $\left(k_{\mathrm{f}}, \ell_{\mathrm{f}}\right)$ and $\left(k_{\mathrm{d}}, \ell_{\mathrm{d}}\right)$, respectively. In Fig. 7.7 these relative fringe field corrections to the quadrupole strength $\Delta k / k_{0}$ and to the quadrupole length $\Delta \ell / \ell_{0}$ are shown as functions of the strength $k_{0}$. The effective quadrupole length is longer and the effective quadrupole strength is lower than the pure hard edge values. In addition the corrections are different in both planes. Depending on the sensitivity of the beam transport system these corrections may have to be included in the final optimization. 
Fig. 7.7 Fringe field correction for the quadrupole of Fig. 7.5 with a bore radius of $R=3.0 \mathrm{~cm}$ and a steel length of $\ell_{\text {iron }}=15.9 \mathrm{~cm}$

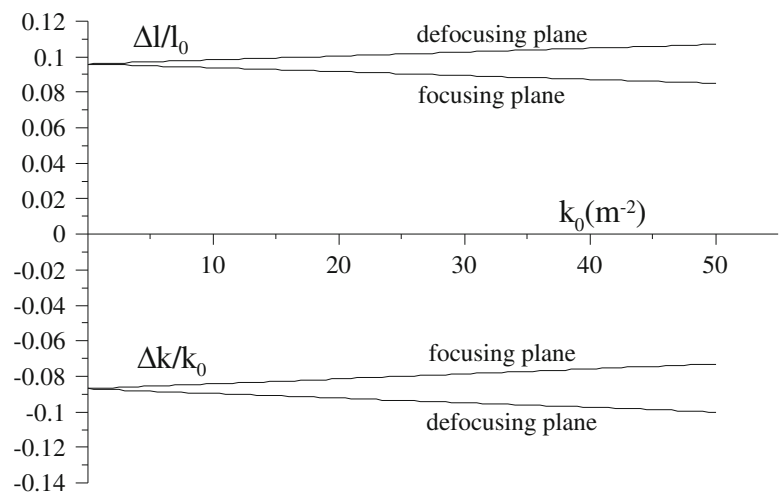

\subsection{Focusing in Bending Magnets}

Bending magnets have been treated so far just like drift spaces as far as focusing properties are concerned. This is a good approximation for weak bending magnets which bend the beam only by a small angle. In cases of larger deflection angles, however, we observe focusing effects which are due to the particular type of magnet and its end fields. In Chap. 6 we discussed the geometric focusing term $\kappa^{2}$ which appears in sector magnets only. Other focusing terms are associated with bending magnets and we will discuss in this section these effects in a systematic way. Specifically, the focusing of charged particles crossing end fields at oblique angles will be discussed.

The linear theory of particle beam dynamics uses a curvilinear coordinate system following the path of the reference particle and it is assumed that all magnetic fields are symmetric about this path. The "natural" bending magnet in this system is one, where the ideal path of the particles enters and exits normal to the magnet pole faces. Such a magnet is called a sector magnet as shown in Fig. 7.8. The total deflection of a particle depends on the distance of the particle path from the ideal path in the deflecting plane which, for simplicity, we assume to be in the horizontal $x$-plane. Particles following a path at a larger distance from the center of curvature than the ideal path travel a longer distance through this magnet and, therefore, are deflected by a larger angle than a particle on the ideal path. Correspondingly, a particle passing through the magnet closer to the center of curvature is deflected less.

This asymmetry leads to a focusing effect which is purely geometric in nature. On the other hand, we may choose to use a magnet such that the ideal path of the particle beam does not enter the magnet normal to the pole face but rather at an angle. Such a configuration has an asymmetric field distribution about the beam axis and therefore leads to focusing effects. We will discuss the effects of fringe fields in more detail in Sect. 7.3.2. 


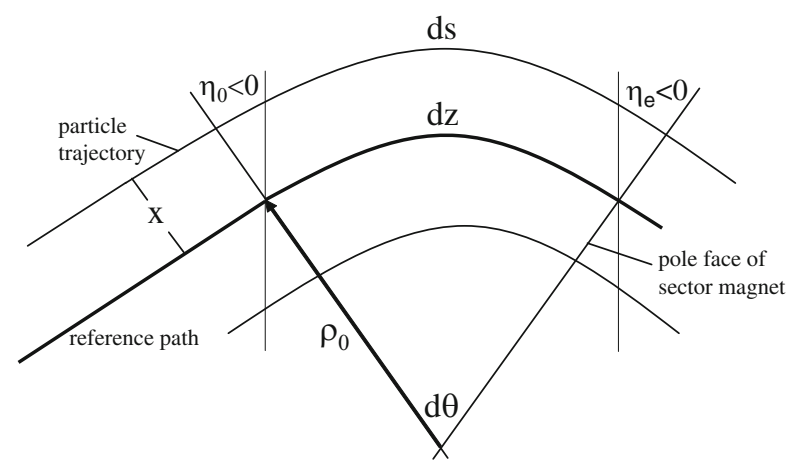

Fig. 7.8 Focusing in a sector magnet, where $\eta_{0}=\eta_{\mathrm{e}}=0$

\subsubsection{Sector Magnets}

The degree of focusing in a sector magnet can be evaluated in any infinitesimal sector of such a magnet by calculating the deflection angle as a function of the particle position $x$. With the notation from Fig. 7.8 we get for the deflection angle while keeping only linear terms in $x$

$$
\mathrm{d} \theta=\kappa_{0} \mathrm{~d} \sigma=\kappa_{0}\left(1+\kappa_{0} x\right) \mathrm{d} z
$$

The first term on the r.h.s. merely defines the ideal path, while the second $x$ dependent term of the deflection angle in (7.33) describes the particle motion in the vicinity of the ideal path. With respect to the curvilinear coordinate system following the ideal path we get the additional deflection

$$
\delta \theta=\kappa_{0}^{2} x \mathrm{~d} z
$$

This correction is to be included in the differential equation of motion as an additional focusing term

$$
\Delta x^{\prime \prime}=-\frac{\delta \theta}{\mathrm{d} z}=-\kappa_{0}^{2} x
$$

to the straight quadrupole focusing leading to the equation of motion

$$
x^{\prime \prime}+\left(k+\kappa_{0}^{2}\right) x=0
$$

which is identical to the result obtained in Sect. 5.3.

The differential equation (7.36) has the same form as that for a quadrupole and therefore the solutions must be of the same form. Using this similarity we replace $k$ 
by $\left(k+\kappa_{0}^{2}\right)$ and obtain immediately the transformation matrices for a general sector magnet. For $K=k+\kappa_{0}^{2}>0$ and

$$
\Theta=\sqrt{K} \ell
$$

we get from (7.10) the transformation matrix

$$
\mathcal{M}_{\text {sy }, \mathrm{f}}(\ell \mid 0)=\left(\begin{array}{cc}
\cos \Theta & \frac{1}{\sqrt{K}} \sin \Theta \\
-\sqrt{K} \sin \Theta & \cos \Theta
\end{array}\right),
$$

where $\ell$ is the arc length of the sector magnet and where both the focusing term $k$ and the bending term $\kappa_{0}$ may be nonzero. Such a magnet is called a synchrotron magnet since this magnet type was first used for lattices of synchrotrons.

For the defocusing case, where $K=k+\kappa_{0}^{2}<0$ and $\Theta=\sqrt{|K|} \ell$, we get from (7.12)

$$
\mathcal{M}_{\text {sy }, \mathrm{d}}(\ell \mid 0)=\left(\begin{array}{cc}
\cosh \Theta & \frac{1}{\sqrt{|K|}} \sinh \Theta \\
\sqrt{|K|} \sinh \Theta & \cosh \Theta
\end{array}\right) .
$$

Note that the argument $\Theta$ is equal to the deflection angle $\theta$ only in the limit $k \rightarrow 0$ because these transformation matrices include bending as well as focusing in the same magnet. Obviously, in the nondeflecting plane $\kappa_{0}=0$ and such a magnet acts just like a quadrupole with strength $k$ and length $\ell$.

A subset of general sector magnets are pure dipole sector magnets, where we eliminate the focusing by setting $k=0$ and get the pure dipole strength $K=\kappa_{0}^{2}>0$. The transformation matrix for a pure sector magnet of length $\ell$ and bending angle $\theta=\kappa_{0} \ell$ in the deflecting plane becomes from (7.38)

$$
\mathcal{M}_{\mathrm{s}, \rho}(\ell \mid 0)=\left(\begin{array}{cc}
\cos \theta & \rho_{0} \sin \theta \\
-\kappa_{0} \sin \Theta & \cos \Theta
\end{array}\right) \text {. }
$$

If we also let $\kappa_{0} \rightarrow 0$ we arrive at the transformation matrix of a sector magnet in the nondeflecting plane

$$
\mathcal{M}_{\mathrm{s}, 0}(\ell \mid 0)=\left(\begin{array}{ll}
1 & \ell \\
0 & 1
\end{array}\right)
$$

which has the form of a drift space. A pure dipole sector magnet therefore behaves in the non-deflecting plane just like a drift space of length $\ell$. Note that $\ell$ is the arc length of the magnet while the engineering magnet length might be given as the straight length between entry and exit point. 


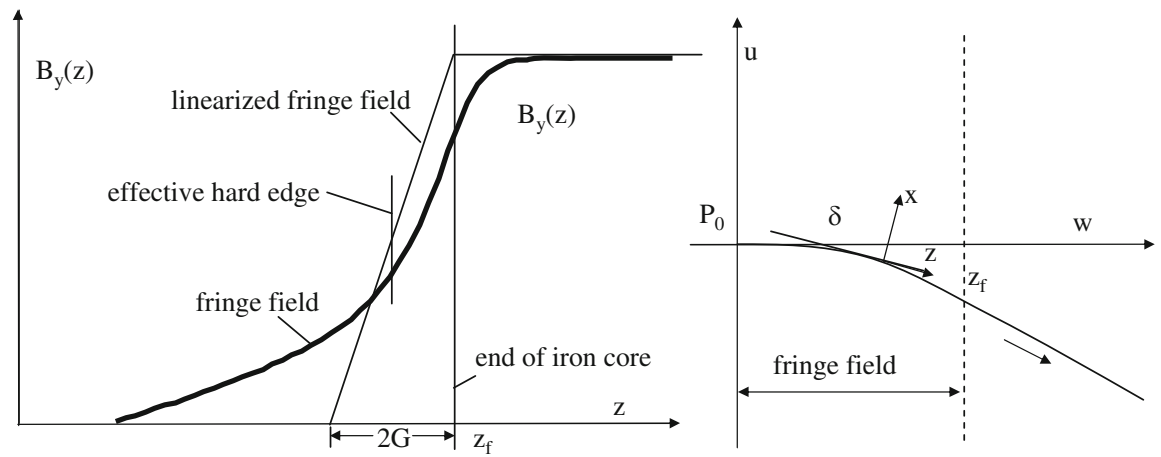

Fig. 7.9 End field profile in a dipole magnet and fringe field focusing

\subsubsection{Fringe Field Effects}

The results obtained above are those for a hard edge model and do not reflect modifications caused by the finite extend of the fringe fields. The hard edge model is again an idealization and for a real dipole we consider the gradual transition of the field from the maximum value to zero outside the magnet. The extend of the dipole fringe field is typically about equal to the gap height or distance between the magnet poles.

We assume magnet poles which are very wide compared to the gap height and therefore transverse field components in the deflecting plane, here $B_{x}$, can be neglected. At the entrance into a magnet the vertical field component $B_{y}$ increases gradually from the field free region to the maximum value in the middle of the magnet (Fig. 7.9). We will discuss the effects on the particle dynamics caused by this fringe field and compare it with the results for a hard edge model magnet.

For the following discussion we consider both a fixed orthogonal Cartesian coordinate system $(u, v, w)$, used in the fringe area, as well as a moving curvilinear system $(x, y, z)$. The origin of the fixed coordinate system is placed at the point $P_{0}$ where the field starts to rise (Fig. 7.9). At this point both coordinate systems coincide. The horizontal field component vanishes for reasons of symmetry

$$
B_{u}=0
$$

and the vertical field component in the fringe region may be described by

$$
B_{v}=F(w) .
$$

With Maxwell's curl equation $\partial B_{w} / \partial v-\partial B_{v} / \partial w=0$ we get after integration the longitudinal field component $B_{w}=\int\left(\partial B_{v} / \partial w\right) \mathrm{d} v$ or

$$
B_{w}=y \frac{\partial F(w)}{\partial w},
$$


where $y=v$ and where a linear fringe field (see Fig. 7.9) was assumed with $\partial F(w) / \partial w=$ const. These field components must be expressed in the curvilinear coordinate system $(x, y, z)$. Within the fringe field $B_{w}(z)$ can be split into $B_{x}$ and $B_{z}$ as shown in Fig. 7.9. The horizontal field component is then $B_{x}=B_{w} \sin \delta$ where $\delta$ is the deflection angle at the point $z$ defined by

$$
\delta=\frac{e}{p_{0}} \int_{0}^{z} F(\bar{z}) \mathrm{d} \bar{z} .
$$

With

$$
B_{w}=y \frac{\partial F(w)}{\partial w}=y \frac{\partial F(w)}{\partial z} \frac{\mathrm{d} z}{\mathrm{~d} w} \approx y \frac{\partial F(z)}{\partial z} \frac{1}{\cos \delta}
$$

we get

$$
B_{x}(z)=y F^{\prime}(z) \tan \delta,
$$

where $F^{\prime}(z)=\mathrm{d} F / \mathrm{d} z$. The vertical fringe field component is with $\partial B_{x} / \partial y-$ $\partial B_{y} / \partial x=0$ and integration

$$
B_{y}(z)=B_{y 0}+x F^{\prime}(z) \tan \delta .
$$

The longitudinal field component is from (7.46) and with $B_{z}=B_{w} \cos \delta$

$$
B_{z}(z)=y F^{\prime}(z) .
$$

The field components of the fringe field depend linearly on the transverse coordinates and therefore fringe field focusing [6] must be expected. With the definition of the focal length from (7.3) we get

$$
\frac{1}{f}=\int_{0}^{z \mathrm{f}} K(\bar{z}) \mathrm{d} \bar{z},
$$

where $K(z)$ is the focusing strength parameter defined in (). In the deflecting plane the fringe field focusing is with $k(z)=\left(e / p_{0}\right) \partial B_{y}(z) / \partial x$ and (7.48)

$$
\frac{1}{f_{x}}=\int_{0}^{z \mathrm{f}}\left(\kappa^{\prime} \tan \delta+\kappa^{2}\right) \mathrm{d} \bar{z},
$$

where we have set $\kappa(z)=\left(e / p_{0}\right) F(z)$. For small deflection angles $\delta$ in the fringe field $\tan \delta \approx \delta=\int_{0}^{z_{\mathrm{f}}} \kappa \mathrm{d} \bar{z}$ and after integration of (7.48) by parts through the full fringe field we get the focal length while neglecting higher order terms in $\delta_{\mathrm{f}}$

$$
\frac{1}{f_{x}}=\kappa_{0} \delta_{\mathrm{f}},
$$


where $\kappa_{0}=1 / \rho_{0}$ is the curvature in the central part of the magnet and $\delta_{\mathrm{f}}$ is the total deflection angle in the fringe field region.

This result does not deviate from that of the hard edge model, where for a small deflection angle $\delta$ we have from (7.40) $1 / f_{x} \approx \kappa_{0} \delta$ agreeing with (7.52). We obtain therefore the convenient result that in the deflecting plane of a sector magnet there is no need to correct the focusing because of the finite extend of the fringe field.

\subsubsection{Finite Pole Gap}

In the vertical plane this situation is different since we expect vertical focusing from (7.47) while there is no focusing in the approximation of a hard edge model. Using the definition (7.50) of the focal length in the vertical plane gives with $K(z)=-k(z)$ and (7.47)

$$
\frac{1}{f_{y}}=-\int_{0}^{z_{\mathrm{f}}} \kappa^{\prime} \tan \delta \mathrm{d} \bar{z} \approx-\int_{0}^{z_{\mathrm{f}}} \kappa^{\prime}(\bar{z}) \delta(\bar{z}) \mathrm{d} \bar{z}
$$

The fringe field of a sector magnet therefore leads to a defocusing effect which depends on the particular field profile. We may approximate the fringe field by a linear fit over a distance approximately equal to the pole gap $2 G$ which is a good approximation for most real dipole magnets. We neglect the nonlinear part of the fringe field and approximate the slope of the field strength by $\kappa^{\prime}=\kappa_{0} / 2 G=$ const. The focal length for the full fringe field of length $z_{\mathrm{f}}=2 G$ is therefore with $\kappa(z)=$ $\kappa^{\prime} z, 0 \leq z \leq z_{\mathrm{f}}$ and

$$
\delta(z)=\int_{0}^{z} \kappa^{\prime} \bar{z} \mathrm{~d} \bar{z}=\frac{\kappa_{0}}{4 G} z^{2}
$$

given by

$$
\frac{1}{f_{y}}=-\int_{0}^{2 G} \kappa^{\prime} \delta(\bar{z}) \mathrm{d} \bar{z}=-\frac{1}{3} \kappa_{0}^{2} G=-\frac{1}{3} \kappa_{0} \delta_{\mathrm{f}}
$$

where

$$
\delta_{\mathrm{f}}=\kappa_{0} G
$$

This is the focusing due to the fringe field at the entrance of a sector magnet. At the exit we have the same effect since the sign change of $\kappa^{\prime}$ is compensated by the need to integrate now from full field to the field free region which is just opposite to the case in the entrance fringe field. Both end fields of a sector magnet provide a small vertical defocusing. We note that this defocusing is quadratic in nature, since $\delta_{\mathrm{f}} \propto \kappa_{0}$ and therefore independent of the sign of the deflection. 
With these results we may now derive a corrected transformation matrix for a sector magnet by multiplying the hard edge matrix (7.41)on either side with thin length fringe field focusing

$$
\left(\begin{array}{cc}
1 & 0 \\
-\frac{1}{f_{y}} & 1
\end{array}\right)\left(\begin{array}{ll}
1 & \ell \\
0 & 1
\end{array}\right)\left(\begin{array}{cc}
1 & 0 \\
-\frac{1}{f_{y}} & 1
\end{array}\right)
$$

and get with (7.55) and $\theta=\ell / \rho_{0}$ for the transformation matrix in the vertical, non-deflecting plane of a sector magnet instead of (7.41)

$$
\mathcal{M}_{\mathrm{s}, 0}(\ell \mid 0)=\left(\begin{array}{cc}
1+\frac{1}{3} \theta \delta_{\mathrm{f}} & \ell \\
\frac{2}{3} \frac{\delta_{\mathrm{f}}}{\rho_{0}}-\frac{1}{9} \frac{\delta_{\mathrm{f}}^{2}}{\rho_{0}^{2}} \ell & 1+\frac{1}{3} \theta \delta_{\mathrm{f}}
\end{array}\right) .
$$

The second order term in the $M_{21}$-matrix element can be ignored for practical purposes but is essential to keep the determinant equal to unity.

\subsubsection{Wedge Magnets}

In a more general case compared to a sector magnet we will allow the reference path of the particle beam to enter and exit the magnet at an arbitrary angle with the pole face. Figure 7.10 shows such a wedge magnets and we will derive its transformation matrices. First, we note that the fringe field effect is not different from the previous case of a sector magnet except that now the angle $\delta(z)$ must be replaced by a new angle $\eta+\delta(z)$ where the pole rotation angle $\eta$ and the sign convention is defined in Fig. 7.10.

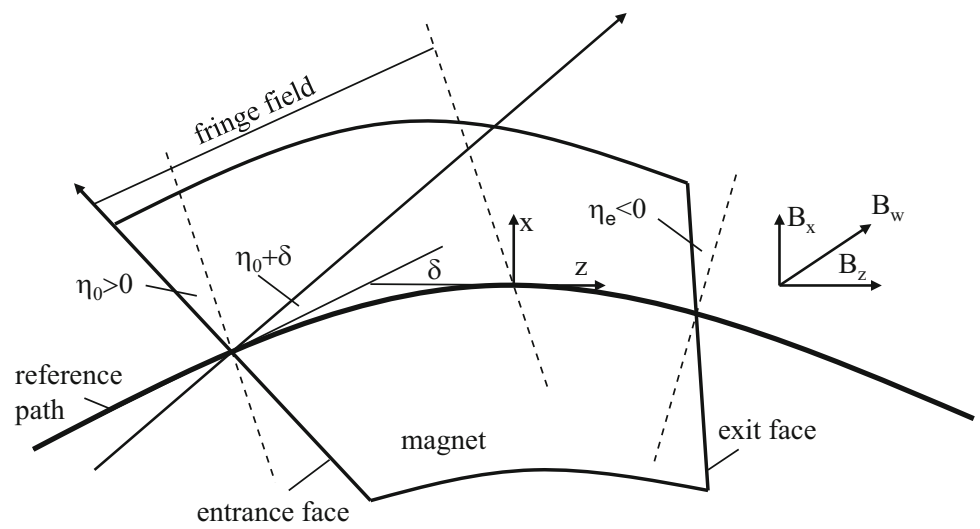

Fig. 7.10 Fringe field focusing in wedge magnets 
Different from the case of a sector magnet, we cannot replace the tangent in (7.51) by its argument since the angle $\eta$ may be large to prohibit such an approximation. As a further consequence of a large value of $\eta$, we must take into account the actual path length in the fringe field. To calculate the focal length $f_{x}$, we have instead of (7.51)

$$
\frac{1}{f_{x}}=\int_{0}^{z_{\mathrm{f}}}\left[\kappa^{\prime} \tan (\eta+\delta)+\kappa^{2}\right] \mathrm{d} \bar{z}
$$

Expanding for small angles $\delta \ll 1$ we get $\tan (\eta+\delta) \approx \tan \eta+\delta$. This approximation is true only as long as $\delta \tan \eta \ll 1$ or for entrance angles $\eta$ not too close to $90^{\circ}$ and the argument in the integral (7.59) becomes $\kappa^{\prime} \tan \eta+\kappa^{\prime} \delta+\kappa^{2}$. In addition to the terms for a sector magnet, a new term $\left(\kappa^{\prime} \tan \eta\right)$ appears and the focal length of the fringe field is

$$
\frac{1}{f_{x}}=\int_{0}^{z_{\mathrm{f}}} \kappa^{\prime} \tan \eta \mathrm{d} \bar{z}+\kappa_{0} \delta_{\mathrm{f}}=\kappa_{0} \tan \eta+\kappa_{0} \delta_{\mathrm{f}},
$$

where the integral extends over the whole fringe field. Since to first order the path length through the fringe field is

$$
z_{\mathrm{f}}=\frac{2 G}{\cos \eta}
$$

where $2 G$ is the pole gap height, we have

$$
\delta_{\mathrm{f}}=\int_{0}^{2 G / \cos \eta} \kappa \mathrm{d} \bar{z}
$$

The term $\kappa_{0} \delta_{\mathrm{f}}$ describes again the well-known focusing of a sector magnet in the deflecting plane while the term $\kappa_{0} \tan \eta$ provides the correction necessary for nonnormal entry of the beam path into the magnet. For the case shown in Fig. 7.10, where $\eta>0$, we obtain beam focusing in the deflecting plane from the fringe field. Similarly, we get a focusing or defocusing effect at the exit fringe field depending on the sign of the pole rotation. The complete transformation matrix of a wedge magnet in the horizontal deflecting plane is obtained by multiplying the matrix of a sector magnet with thin lens matrices to take account of edge focusing. For generality, however, we must assume that the entrance and the exit angle may be different. We will therefore distinguish between the edge focusing for the entrance angle $\eta=\eta_{0}$ and that for the exit angle $\eta=\eta_{\mathrm{e}}$ and get for the transformation matrix in the deflecting plane

$$
\mathcal{M}_{\mathrm{w}, \rho}(\ell, 0)=\left[\begin{array}{cc}
1 & 0 \\
-\frac{1}{\rho_{0}} \tan \eta_{\mathrm{e}} & 1
\end{array}\right]\left[\begin{array}{cc}
\cos \theta & \rho_{0} \sin \theta \\
-\frac{1}{\rho_{0}} \sin \theta & \cos \theta
\end{array}\right]\left[\begin{array}{cc}
1 & 0 \\
-\frac{1}{\rho_{0}} \tan \eta_{0} & 1
\end{array}\right] \text {. }
$$


In the vertical plane the focal length is similar to (7.53) and for not too large angles $\eta$

$$
\frac{1}{f_{y}}=-\int_{0}^{z_{\mathrm{f}}} \kappa^{\prime} \tan (\eta+\delta) \mathrm{d} \bar{z} \approx-\kappa_{0} \tan \eta-\int_{0}^{z_{\mathrm{f}}} \kappa^{\prime} \delta \mathrm{d} \bar{z}
$$

Again we have the additional focusing term which is now focusing in the vertical plane for $\eta<0$. For a linear fringe field the focal length is in analogy to (7.55)

$$
\frac{1}{f_{y}}=-\kappa_{0} \tan \eta+\frac{1}{3} \kappa_{0} \delta_{\mathrm{f}}
$$

where

$$
\delta_{\mathrm{f}}=\int_{0}^{2 G / \cos \eta} \kappa \mathrm{d} \bar{z}=\kappa^{\prime} \frac{2 G^{2}}{\cos ^{3} \eta}=\frac{\kappa_{0} G}{\cos ^{2} \eta},
$$

since $\kappa(z) \approx \kappa^{\prime} z$ and $\kappa^{\prime}=\kappa_{0} /(G / \cos \eta)$. The complete transformation matrix in the vertical plane for a horizontally deflecting wedge magnet becomes then

$$
\mathcal{M}_{\mathrm{w}, 0}(\ell, 0)=\left[\begin{array}{ccc}
1 & 0 \\
-\frac{1}{\rho_{0}}\left(\tan \eta_{\mathrm{e}}+\frac{1}{3} \delta_{\mathrm{f}_{\mathrm{e}}}\right) & 1
\end{array}\right]\left[\begin{array}{ll}
1 & \ell \\
0 & 1
\end{array}\right]\left[\begin{array}{cc}
1 & 0 \\
-\frac{1}{\rho_{0}}\left(\tan \eta_{0}+\frac{1}{3} \delta_{\mathrm{f}_{0}}\right) & 1
\end{array}\right] .
$$

Equations (7.63) and (7.67) are for bending magnets with arbitrary entrance and exit angles $\eta_{0}$ and $\eta_{\mathrm{e}}$. We note specifically that the transformation in the nondeflecting plane becomes different from a simple drift space and find a focusing effect due to the magnet fringe fields which depends on the entrance and exit angles between particle trajectory and pole face.

This general derivation of the focusing properties of a wedge magnet must be taken with caution where the pole face rotations are very large. In spite of the finite pole rotation angles we have assumed that the particles enter the fringe field at the same location $z$ along the beam line independent of the transverse particle amplitude $x$. Similarly, the path length of the trajectory in such a wedge magnet depends on the particle amplitude $x$ and slope $x^{\prime}$. Obviously these are second order effects but may become significant in special cases.

\subsubsection{Rectangular Magnet}

A particular case of a wedge magnet is the rectangular magnet which has parallel end faces. If we install this magnet symmetrically about the intended particle trajectory the entrance and exit angles equal to half the bending angle as shown in Fig. 7.11. 


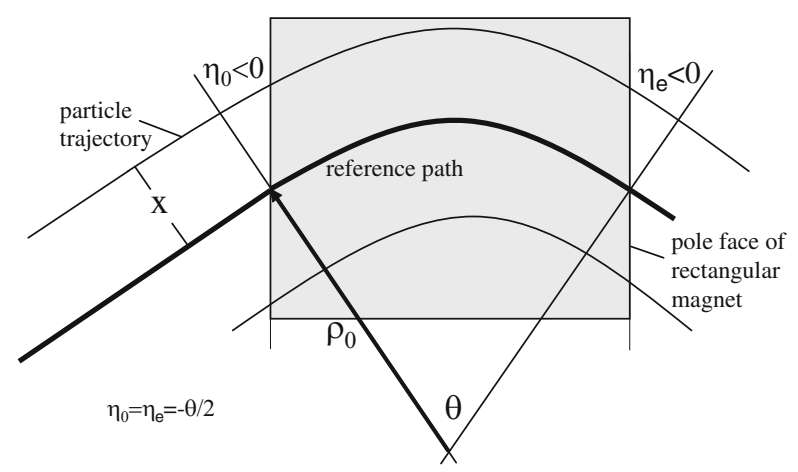

Fig. 7.11 Rectangular magnet

For a deflection angle $\theta, \eta_{0}=\eta_{\mathrm{e}}=-\theta / 2$ and the transformation matrix in the deflecting plane is from (7.63)

$$
\begin{aligned}
M_{\mathrm{r}, \rho}(\ell \mid 0) & =\left(\begin{array}{cc}
1 & 0 \\
-\frac{\tan \eta_{\mathrm{e}}}{\rho_{0}} & 1
\end{array}\right)\left(\begin{array}{cc}
\cos \theta & \rho_{0} \sin \theta \\
-\frac{\sin \theta}{\rho_{0}} & \cos \theta
\end{array}\right)\left(\begin{array}{cc}
1 & 0 \\
-\frac{\tan \eta_{0}}{\rho_{0}} & 1
\end{array}\right) \\
& =\left(\begin{array}{cc}
1 & \rho_{0} \sin \theta \\
0 & 1
\end{array}\right) .
\end{aligned}
$$

A rectangular dipole magnet transforms in the deflecting plane like a drift space of length $\rho_{0} \sin \theta$ and does not focus the beam. Note, that the "magnet length" $\ell$ defined by the deflection angle $\theta=\ell / \rho_{0}$ is the arc length and is related to the straight magnet length $L$ by

$$
L=2 \rho_{0} \sin \frac{\theta}{2}=2 \rho_{0} \sin \frac{\ell}{2 \rho_{0}} .
$$

In the vertical plane we observe a focusing with the focal length

$$
\frac{1}{f_{y}}=+\frac{1}{\rho_{0}}\left(\tan \frac{\theta}{2}-\frac{\delta_{\theta / 2}}{3}\right) \text {. }
$$

From (7.66) $\delta_{\theta / 2}=G /\left[\rho_{0} \cos (\theta / 2)\right]$ and with (7.69) $\delta_{\theta / 2}=2 G \tan (\theta / 2) / L$. Inserting this in (7.70), we obtain for the transformation matrix of a rectangular bending magnet in the nondeflecting plane

$$
\mathcal{M}_{\mathrm{r}, 0}(\ell \mid 0)=\left(\begin{array}{cc}
1 & 0 \\
-\frac{1}{f_{y}} & 1
\end{array}\right)\left(\begin{array}{ll}
1 & \ell \\
0 & 1
\end{array}\right)\left(\begin{array}{cc}
1 & 0 \\
-\frac{1}{f_{y}} & 1
\end{array}\right)=\left(\begin{array}{cc}
1-\frac{\ell}{f_{y}} & \ell \\
-\frac{2}{f_{y}}+\frac{\ell}{f_{y}^{2}} & 1-\frac{\ell}{f_{y}}
\end{array}\right),
$$


where

$$
\frac{1}{f_{y}}=\frac{1}{\rho_{0}}\left(1-\frac{2 G}{3 L \cos (\theta / 2)}\right) \tan \left(\frac{\theta}{2}\right) .
$$

In a rectangular dipole magnet we find just the opposite edge focusing properties compared to a sector magnet. The focusing in the deflecting plane of a sector magnet has shifted to the vertical plane in a rectangular magnet and focusing is completely eliminated in the deflecting plane. Because of the finite extend of the fringe field, however, the focusing strength is reduced by the fraction $2 G /[3 L \cos (\theta / 2)]$ where $2 G$ is the gap height and $L$ the straight magnet length.

\subsubsection{Focusing in a Wiggler Magnet}

The derivation of fringe field focusing in ordinary dipole magnets as discussed in previous sections can be directly applied to wiggler magnets. The beam path in a wiggler magnet is generally not parallel to the reference trajectory $z$ because of the transverse deflection in the wiggler field and follows a periodic sinusoidal form along the reference path. For this reason the field component $B_{z}$ appears to the particle partially as a transverse field $B_{\xi}=B_{z} \tan \vartheta \approx B_{z} \vartheta$, where we use for a moment $\xi$ as an auxiliary transverse coordinate normal to and in the plane of the actual wiggling beam path. We also assume that the wiggler deflection angle is small, $\vartheta \ll 1$. The field component $B_{\xi}$ can be expressed with (6.116), (6.117) more explicitly by

$$
\frac{e}{p} B_{\xi}=-\left[\frac{1}{\rho_{0}} \sin \left(k_{\mathrm{p}} z\right)\right]^{2} \frac{\sinh \left(k_{\mathrm{p}} y\right) \cosh \left(k_{\mathrm{p}} y\right)}{k_{\mathrm{p}}}
$$

where $1 / \rho_{0}=\frac{e}{p} B_{0}$ is the inverse bending radius in the center of a wiggler pole at which point the field reaches the maximum value $B_{0}$. With the expansions (6.119) we have finally

$$
\frac{e}{p} B_{\xi}=-\left[\frac{1}{\rho_{0}} \sin \left(k_{\mathrm{p}} z\right)\right]^{2}\left(y+\frac{2}{3} k_{\mathrm{p}}^{2} y^{3}+\ldots\right) .
$$

The linear $y$-dependence is similar to that found to produce vertical focusing in wedge magnets. Since the wiggler field appears quadratically in (7.73) $B_{\xi}(z)=$ $B_{\xi}(-z)$ and $B_{\xi}\left(B_{0}\right)=B_{\xi}\left(-B_{0}\right)$. In other words, the transverse field has the same sign along all wiggler poles independent of the polarity of the vertical main wiggler field. The integrated field gradient per wiggler half pole is from (7.74)

$$
k_{y} \ell=-\frac{1}{\rho_{0}^{2}} \int_{0}^{\lambda_{\mathrm{p}} / 4} \sin ^{2} k_{\mathrm{p}} z \mathrm{~d} z=-\frac{1}{8} \frac{\lambda_{\mathrm{p}}}{\rho_{0}^{2}}
$$


Fig. 7.12 Wiggler magnet with parallel pole end faces

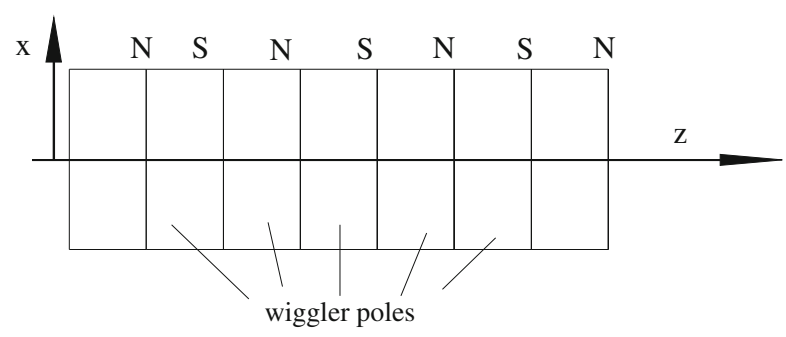

where $\ell$ is the effective length of the focusing element and $k_{\mathrm{p}}=\frac{2 \pi}{\lambda_{\mathrm{p}}}$. The integrated equivalent quadrupole strength or inverse focal length for each half pole is

$$
k_{y} \ell-\frac{1}{f_{y}}=-\frac{1}{8}\left(\frac{e B_{0}}{p_{0}}\right)^{2} \lambda_{\mathrm{p}}=-\frac{\lambda_{\mathrm{p}}}{8 \rho_{0}^{2}} .
$$

For $N$ wiggler poles we have $2 N$ times the focusing strength and the focal length of the total wiggler magnet of length $L_{\mathrm{w}}=\frac{1}{2} N \lambda_{\mathrm{p}}$ expressed in units of the wiggler strength parameter $K$ becomes

$$
\frac{1}{f_{y}}=\frac{K^{2}}{2 \gamma^{2}} k_{\mathrm{p}}^{2} L_{\mathrm{w}} .
$$

Tacitly, a rectangular form of the wiggler poles has been assumed (Fig. 7.12) and consistent with our sign convention, we find that wiggler fringe fields cause focusing in the nondeflecting plane. Within the approximation used there is no corresponding focusing effect in the deflecting plane. This is the situation for most wiggler magnets or poles except for the first and last half pole where the beam enters the magnetic field normal to the pole face.

A reason to possibly use wiggler magnets with rotated pole faces like wedge magnets originates from the fact that the wiggler focusing is asymmetric and not part of the lattice focusing and may therefore need to be compensated. For moderately strong wiggler fields the asymmetric focusing in both planes can mostly be compensated by small adjustments of lattice quadrupoles. The focusing effect of strong wiggler magnets, however, may generate a significant perturbation of the lattice focusing structure or create a situation where no stable solution for betatron functions exists anymore. The severity of this problem can be reduced by designing the wiggler poles as wedge magnets in such a way as to split the focusing equally between both the horizontal and vertical plane. In this case local correction can be applied efficiently in nearby lattice quadrupoles.

We will therefore discuss the focusing and transformation matrix through a wiggler pole in the case of arbitrary entry and exit angles. To derive the complete and general transformation matrices, we note that the whole wiggler field can be 
Fig. 7.13 Wiggler magnet with wedge shaped poles

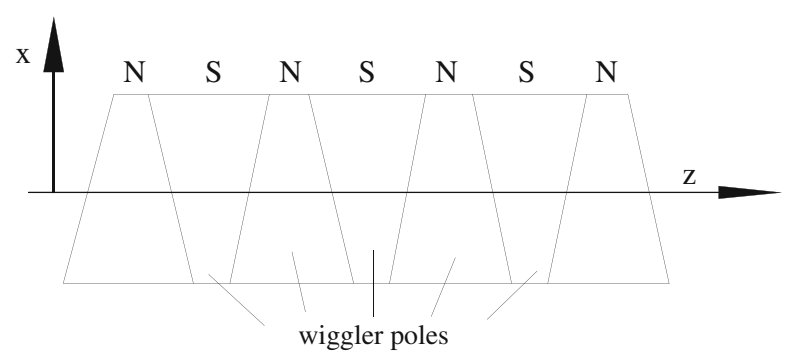

treated in the same way as the fringe field of ordinary magnets. The focal length of one half pole in the horizontal deflecting plane is from (7.60)

$$
\frac{1}{f_{x}}=\int_{0}^{\lambda_{\mathrm{p}} / 4} \kappa_{x}^{\prime} \eta \mathrm{d} z+\kappa_{x 0} \delta_{\mathrm{f}},
$$

where the pole face rotation angle $\eta$ has been assumed to be small and of the order of the wiggler deflection angle per pole (Fig. 7.13). With $\kappa_{x}=\kappa_{x 0} \cos k_{\mathrm{p}} z$ the field slope is

$$
\kappa_{x}^{\prime}=\kappa_{x 0} k_{\mathrm{p}} \sin k_{\mathrm{p}} z
$$

and after integration of (7.78), the focal length for the focusing of a wiggler half pole is

$$
\frac{1}{f_{x}}=\kappa_{x 0}\left(\delta_{\mathrm{f}}+\eta\right),
$$

where $\delta_{\mathrm{f}}$ is given by (7.56) and in the case of a wiggler magnet is equal to the deflection angle of a half pole. In the case of a rectangular wiggler pole $\eta=-\delta_{\mathrm{f}}$ and the focusing in the deflecting plane vanishes as we would expect. In the nondeflecting plane (7.53) applies and the focal length is for small angles $\eta$ and $\delta$

$$
\frac{1}{f_{y}}=-\int_{0}^{\lambda_{\mathrm{p}} / 4} \kappa_{x}^{\prime}[\eta+\delta(\bar{z})] \mathrm{d} \bar{z} .
$$

The focal length per wiggler half pole is after integration

$$
\frac{1}{f_{y}}=-\kappa_{x 0}\left(\eta+\delta_{\mathrm{f}}\right)-\frac{\pi}{4} \kappa_{x 0} \delta_{\mathrm{f}} .
$$

Here again setting $\eta=-\delta_{\mathrm{f}}$ restores the result obtained in (7.77).

The focusing in each single wiggler pole is rather weak and we may apply thin lens approximation to derive the transformation matrices. For this we consider the focusing to occur in the middle of each wiggler pole with drift spaces of length $\lambda_{\mathrm{p}} / 4$ 
on each side. With $2 / f$ being the focal length of a full pole in either the horizontal plane (7.80) or vertical plane (7.82) the transformation matrix for each wiggler pole is finally

$$
\begin{aligned}
\mathcal{M}_{\text {pole }} & =\left(\begin{array}{cc}
1 & \lambda_{\mathrm{p}} / 4 \\
0 & 1
\end{array}\right)\left(\begin{array}{cc}
1 & 0 \\
-2 / f & 1
\end{array}\right)\left(\begin{array}{cc}
1 & \lambda_{\mathrm{p}} / 4 \\
0 & 1
\end{array}\right) \\
& =\left(\begin{array}{cc}
1-\frac{\lambda_{\mathrm{p}}}{2 f} & \frac{\lambda_{\mathrm{p}}}{f}\left(1-\frac{\lambda_{\mathrm{p}}}{4 f}\right) \\
-\frac{2}{f} & 1-\frac{\lambda_{\mathrm{p}}}{2 f}
\end{array}\right) \approx\left(\begin{array}{cc}
1 & \frac{1}{2} \lambda_{\mathrm{p}} \\
-\frac{2}{f} & 1
\end{array}\right),
\end{aligned}
$$

where the approximation $\lambda_{\mathrm{p}} \ll f$ was used. For a wiggler magnet of length $L_{\mathrm{w}}=$ $\frac{1}{2} N \lambda_{\mathrm{p}}$, we have $N$ poles and the total transformation matrix is

$$
\mathcal{M}_{\text {wiggler }}=\mathcal{M}_{\text {pole }}^{N}
$$

This transformation matrix can be applied to each plane and any pole rotation angle $\eta$. Specifically, we set $\eta=-K / \gamma$ for a rectangular pole shape and $\eta=0$ for pole rotations orthogonal to the path like in sector magnets.

\subsubsection{Hard-Edge Model of Wiggler Magnets}

Although the magnetic properties of wiggler magnets are well understood and easy to apply it is nonetheless often desirable to describe the effects of wiggler magnets in the form of hard-edge models. This is particularly true when numerical programs are to be used which do not include the feature of properly modeling a sinusoidal wiggler field. On the other hand accurate modeling is important since frequently strong wiggler magnets are to be inserted into a beam transport lattice.

For the proper modeling of linear wiggler magnet properties we choose three conditions to be fulfilled. The deflection angle for each pole should be the same as that for the equivalent hard-edge model. Similarly the edge focusing must be the same. Finally, like any other bending magnet in an electron circular accelerator, a wiggler magnet also contributes to quantum excitation and damping of the beam emittance and beam energy spread. The quantum excitation is in first approximation proportional to the third power of the curvature while the damping scales like the square of the curvature similar to focusing.

Considering now a wiggler field

$$
B(z)=B_{0} \sin k_{\mathrm{p}} z
$$

we try to model the field for a half pole with parallel endpoles by a hard-edge magnet. Three conditions should be met. The deflection angle of the hard-edge model of length $\ell$ and field $B$ must be the same as that for a wiggler half pole, 
Fig. 7.14 Hard edge model for a wiggler magnet period

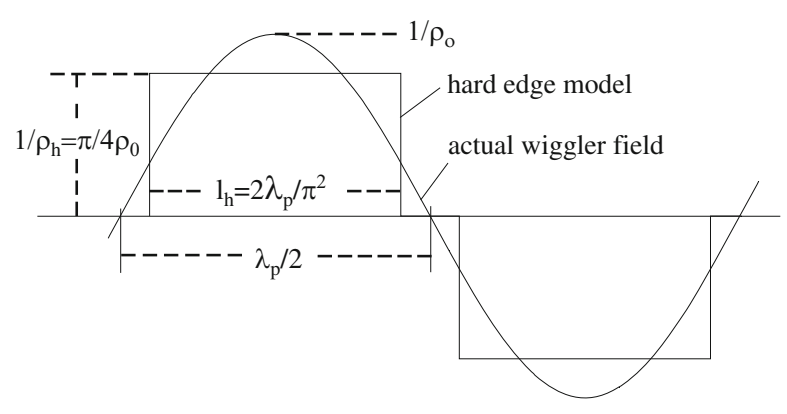

or

$$
\theta=\frac{\ell_{\mathrm{h}}}{\rho_{\mathrm{h}}}=\frac{e}{p_{0}} \int_{\text {halfpole }} B_{y}(z) \mathrm{d} z=\frac{\lambda_{\mathrm{p}}}{2 \pi \rho_{0}} .
$$

Here we use $\rho_{\mathrm{h}}$ for the bending radius of the equivalent hard-edge model and $\rho_{0}$ for the bending radius at the peak wiggler field $B_{0}$. The edge focusing condition can be expressed by

$$
\frac{1}{f}=\frac{\ell_{\mathrm{h}}}{\rho_{\mathrm{h}}^{2}}=\frac{1}{\rho_{0}^{2}} \int_{\text {halfpole }} \sin ^{2} k_{\mathrm{p}} z \mathrm{~d} z=\frac{\lambda_{\mathrm{p}}}{8 \rho_{0}^{2}} .
$$

Modeling a wiggler field by a single hard-edge magnet requires in linear beam optics only two conditions to be met which can be done with the two parameters $B(z)$ and $\ell$ available. From (7.86), (7.87) we get therefore the hard-edge magnet parameters (Fig. 7.14)

$$
\rho_{\mathrm{h}}=\frac{4}{\pi} \rho_{0} \quad \text { and } \quad \ell_{\mathrm{h}}=\frac{2}{\pi^{2}} \lambda_{\mathrm{p}}
$$

For a perfect modeling of the equilibrium energy spread and emittance due to quantum excitation in electron storage rings we would also like the cubic term to be the same

$$
\frac{\ell_{\mathrm{h}}}{\rho_{\mathrm{h}}^{3}} \stackrel{?}{=} \frac{1}{\rho_{0}^{3}} \int_{\text {halfpole }} \sin ^{3} k_{\mathrm{p}} z \mathrm{~d} z=\frac{\lambda_{\mathrm{p}}}{3 \pi \rho_{0}^{3}} .
$$

Since we have no more free parameters available, we can at this point only estimate the mismatch. With (7.87), (7.88) we get from (7.89) the inequality

$$
\frac{1}{3 \pi} \neq \frac{\pi}{32}
$$

which indicates that the quantum excitation from wiggler magnets is not correctly treated although the error is only about $8 \%$. 
Similarly, one could decide that the quadratic and cubic terms must be equal while the deflection angle is let free. This would be a reasonable assumption since the total deflection angle of a wiggler is compensated anyway. In this case the deflection angle would be underestimated by about $8 \%$. Where these mismatches are not significant, the simple hard-edge model (7.89) can be applied. For more accuracy the sinusoidal wiggler field must be segmented into smaller hard-edge magnets.

\subsection{Elements of Beam Dynamics}

The most basic elements of a beam transport line are drift spaces, bending magnets and focusing magnets or quadrupoles. Obviously, in a drift space of length $\ell$ the electric or magnetic field vanishes. Bending magnets act as beam guidance devices while quadrupoles will focus the beam. In the following section, we will discuss building blocks made up of bending magnets and quadrupoles, which exhibit features known from light optics thus justifying our extensive use of terminology from optics in particle beam dynamics.

\subsubsection{Building Blocks for Beam Transport Lines}

With special arrangements of bending and focusing magnets it is possible to construct lattice sections with particular properties. We may desire a lattice section with specific chromatic properties, achromatic or isochronous sections. In the next paragraphs we will discuss such lattice elements with special properties.

\section{General Focusing Properties}

The principal solutions and some elements of transformation matrices through an arbitrary beam transport line can reveal basic beam optical properties of this beam line. A close similarity to paraxial light optics is found in the matrix element $C^{\prime}(z)$. As shown schematically in Fig. 7.15, parallel trajectories $\left(u_{0}^{\prime}=0\right)$ are deflected by the focusing system through the matrix element $C^{\prime}(z)$ and emerge with a slope $u^{\prime}(z)=C^{\prime}(z) u_{0}$.

From light optics we know that $-u_{0} / u^{\prime}(z)$ is defined as the focal length of the system. In analogy, we define therefore also a focal length $f$ for a composite focusing system by setting

$$
f^{-1}=C^{\prime}(z)
$$

The focal point is defined by the condition $u\left(z_{\mathrm{f}}\right)=0$ and is, therefore, located where the cosine like solution becomes zero or $C\left(z_{\mathrm{f}}\right)=0$. 


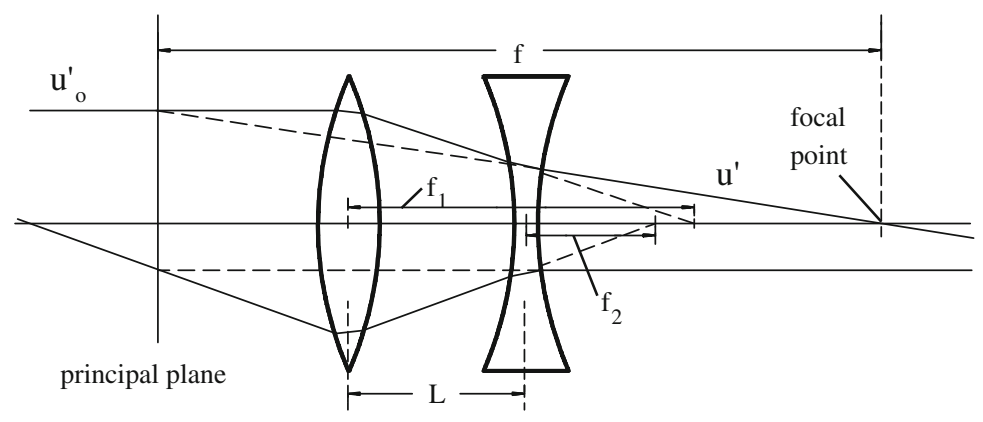

Fig. 7.15 Focusing in a quadrupole doublet

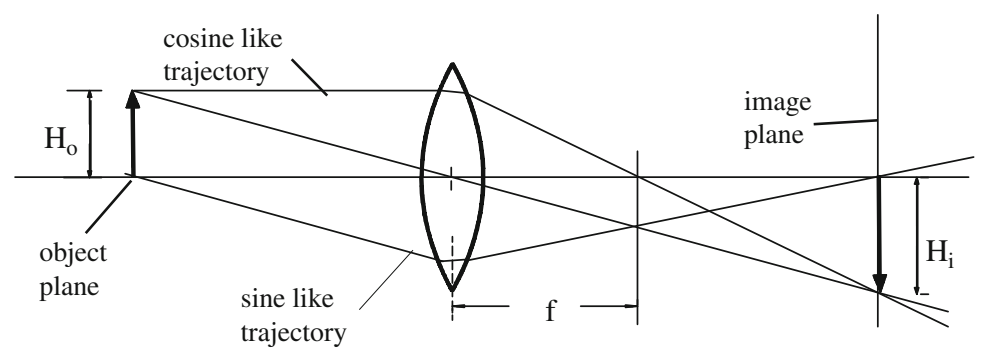

Fig. 7.16 Point to point imaging

More similarities with paraxial light optics can be identified. Point to point imaging, for example, is defined in particle beam optics by the sine like function $S(z)$, starting at the object plane at $z=z_{0}$. The image point is located where the sine-like function crosses again the reference axis or where $S\left(z_{i}+z_{0}\right)=0$ as shown in Fig. 7.16.

By definition such a section of a beam transport system has a betatron phase advance of $180^{\circ}$. The beam size or object size $H_{0}$ at $z_{0}$ is transformed by the cosine like function to become at the image point $H\left(z_{i}\right)=\left|C\left(z_{i}+z_{0}\right)\right| H_{0}$ and the magnification of the beam optical system is given by the absolute value of the cosine like function at the image point

$$
M=\left|C\left(z_{i}+z_{0}\right)\right| .
$$

\section{Chromatic Properties}

Very basic features can be derived for the chromatic characteristics of a beam transport line. In (5.81), we have already derived the dispersion function

$$
D(z)=S(z) \int_{0}^{z} \kappa(\tilde{z}) C(\tilde{z}) \mathrm{d} \tilde{z}-C(z) \int_{0}^{z} \kappa(\tilde{z}) S(\tilde{z}) \mathrm{d} \tilde{z} .
$$


From this expression we conclude that there is dispersion only if at least one of the two integrals in (7.93) is nonzero. That means only dipole fields can cause a dispersion as a consequence of the linear chromatic perturbation term $\kappa \delta$. All other perturbation terms in (6.95), (6.96) are of higher order in $\delta$ or depend on the transverse particle coordinates and therefore contribute only to higher order corrections of the dispersion function.

Specifically, we find from (5.26) the lowest order chromatic quadrupole perturbation to be $k x \delta$. Since any arbitrary particle trajectory is composed of an energy independent part $x_{\beta}$ and an energy dependent part $D \delta$, expressed by $x=x_{\beta}+D \delta$, we find the lowest chromatic quadrupole perturbation to the dispersion function to be the second order term $k D \delta^{2}$ which does not contribute to linear dispersion.

While some dispersion cannot be avoided in beam transport systems where dipole magnets are used, it is often desirable to remove this dispersion at least in some parts of the beam line. As a condition for that to happen at say $z=z_{\mathrm{d}}$, we require that $D\left(z_{\mathrm{d}}\right)=0$. From (7.93) this can be achieved if

$$
\frac{S\left(z_{\mathrm{d}}\right)}{C\left(z_{\mathrm{d}}\right)}=\frac{\int_{0}^{z_{\mathrm{d}}} \kappa(\tilde{z}) S(\tilde{z}) \mathrm{d} \tilde{z}}{\int_{0}^{z_{\mathrm{d}}} \kappa(\tilde{z}) C(\tilde{z}) \mathrm{d} \tilde{z}},
$$

a condition that can be met by proper adjustments of the focusing structure.

\section{Achromatic Lattices}

A much more interesting case is the one, where we require both the dispersion and its derivative to vanish, $D\left(z_{\mathrm{d}}\right)=0$ and $D^{\prime}\left(z_{\mathrm{d}}\right)=0$. In this case we have no dispersion function downstream from the point $z=z_{\mathrm{d}}$ up to the point, where the next dipole magnet creates a new dispersion function. The conditions for this to happen are

$$
\begin{aligned}
& D\left(z_{\mathrm{d}}\right)=0=-S\left(z_{\mathrm{d}}\right) I_{\mathrm{c}}+C\left(z_{\mathrm{d}}\right) I_{\mathrm{s}}, \\
& D^{\prime}\left(z_{\mathrm{d}}\right)=0=-S^{\prime}\left(z_{\mathrm{d}}\right) I_{\mathrm{c}}+C^{\prime}\left(z_{\mathrm{d}}\right) I_{\mathrm{s}},
\end{aligned}
$$

where we have set $I_{\mathrm{c}}=\int_{0}^{z_{\mathrm{d}}} \kappa C \mathrm{~d} \tilde{z}$ and $I_{\mathrm{s}}=\int_{0}^{z_{\mathrm{d}}} \kappa S \mathrm{~d} \tilde{z}$. We can solve (7.95) for $I_{\mathrm{c}}$ or $I_{\mathrm{s}}$ and get

$$
\begin{aligned}
& {\left[C\left(z_{\mathrm{d}}\right) S^{\prime}\left(z_{\mathrm{d}}\right)-S\left(z_{\mathrm{d}}\right) C^{\prime}\left(z_{\mathrm{d}}\right)\right] I_{\mathrm{c}}=0,} \\
& {\left[C\left(z_{\mathrm{d}}\right) S^{\prime}\left(z_{\mathrm{d}}\right)-S\left(z_{\mathrm{d}}\right) C^{\prime}\left(z_{\mathrm{d}}\right)\right] I_{\mathrm{s}}=0 .}
\end{aligned}
$$

Since $C\left(z_{\mathrm{d}}\right) S^{\prime}\left(z_{\mathrm{d}}\right)-S\left(z_{\mathrm{d}}\right) C^{\prime}\left(z_{\mathrm{d}}\right)=1$, the conditions for a vanishing dispersion function are

$$
\begin{aligned}
& I_{\mathrm{c}}=\int_{0}^{z_{\mathrm{d}}} \kappa(\tilde{z}) C(\tilde{z}) \mathrm{d} \tilde{z}=0, \\
& I_{\mathrm{s}}=\int_{0}^{z_{\mathrm{d}}} \kappa(\tilde{z}) S(\tilde{z}) \mathrm{d} \tilde{z}=0 .
\end{aligned}
$$


A beam line is called a first order achromat or short an achromat if and only if both conditions (7.97) are true. The physical characteristics of an achromatic beam line is that at the end of the beam line, the position and the slope of a particle trajectory is independent of the energy.

\subsubsection{Isochronous Systems}

For the accelerating process we will find that the knowledge of the path length is of great importance. The path length $L$ of any arbitrary particle trajectory can be derived by integration to give

$$
L=\int \mathrm{d} s=\int_{0}^{L_{0}} \frac{\mathrm{d} s}{\mathrm{~d} \tilde{z}} \mathrm{~d} \tilde{z}=\int_{0}^{L_{0}} \sqrt{x^{\prime 2}+y^{\prime 2}+\left(1+\kappa_{x} x\right)^{2}} \mathrm{~d} \tilde{z},
$$

where $L_{0}$ is the length of the beam line along the ideal reference path. For simplicity we have ignored a vertical deflection of the beam. The path length variation due to a vertical bend would be similar to that for a horizontal bend and can therefore be easily derived form this result. Since $x^{\prime}, y^{\prime}$ and $\kappa_{x} x$ are all small compared to unity, we may expand the square root and get in keeping only second order terms

$$
L=\int_{0}^{L_{0}}\left[1+\kappa_{x} x+\frac{1}{2}\left(x^{\prime 2}+y^{\prime 2}+\kappa_{x}^{2} x^{2}\right)\right] \mathrm{d} \tilde{z}+\mathcal{O}(3) .
$$

Utilizing (5.83) we get from (7.99) for the path length difference

$$
\begin{aligned}
\left(L-L_{0}\right)_{\text {sector }}= & x_{0} \int_{0}^{L_{0}} \kappa_{x}(\tilde{z}) C(\tilde{z}) \mathrm{d} \tilde{z}+x_{0}^{\prime} \int_{0}^{L_{0}} \kappa_{x}(\tilde{z}) S(\tilde{z}) \mathrm{d} \tilde{z} \\
& +\delta \int_{0}^{L_{0}} \kappa_{x}(\tilde{z}) D(\tilde{z}) \mathrm{d} \tilde{z}
\end{aligned}
$$

The variation of the path length has two contributions. For $\delta=0$ the path length varies due to the curvilinear coordinate system, where dipole fields exist. This is a direct consequence of the coordinate system which selects a sector magnet as its natural bending magnet. The ideal path enters and exits this type of dipole magnet normal to its pole face as shown in Fig. 7.17. It becomes obvious from Fig. 7.17 that the path length difference depends on the particle position with respect to the reference path and is in linear approximation

$$
\mathrm{d} \ell=\ell-\ell_{0}=\left(\rho_{0}+x\right) \mathrm{d} \varphi-\rho_{0} \mathrm{~d} \varphi .
$$




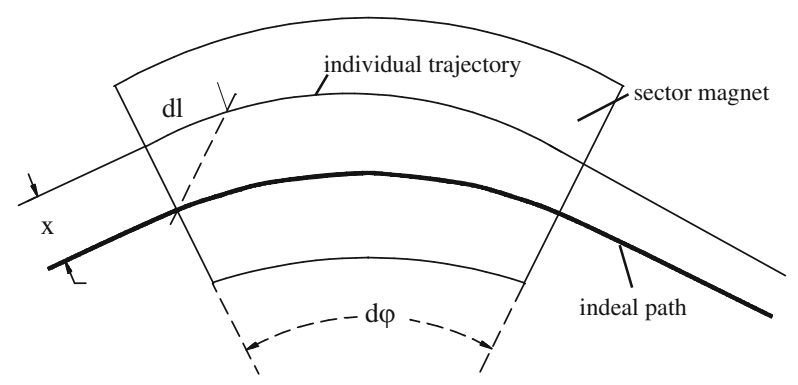

Fig. 7.17 Path length in a sector magnet

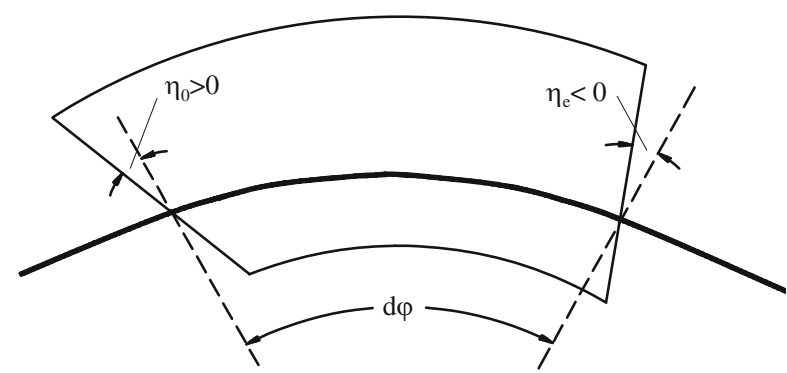

Fig. 7.18 Path length in a wedge magnet

Figure 7.18 displays the general situation for a wedge magnet with arbitrary entrance and exit pole face angles. The path length differs from that in a sector magnet on either end of the magnet. The first integral in (7.100) therefore must be modified to take into account the path length elements in the fringe field. For a wedge magnet we have therefore instead of (7.100)

$$
\begin{aligned}
\left(L-L_{0}\right)_{\text {wedge }}=x_{0} & \int_{0}^{L_{0}} \kappa_{x}(\tilde{z}) C(\tilde{z}) \mathrm{d} \tilde{z} \\
& +\left[C\left(z_{0}\right) x_{0}+\rho_{0}\right] \eta_{0}+\left[C\left(z_{\mathrm{e}}\right) x_{0}+\rho_{0}\right] \eta_{e} \\
& -x_{0} C\left(z_{0}\right) \tan \eta_{0}-x_{0} C\left(z_{\mathrm{e}}\right) \tan \eta_{\mathrm{e}} \\
& +x_{0}^{\prime} \int_{0}^{L_{0}} \kappa_{x}(\tilde{z}) S(\tilde{z}) \mathrm{d} \tilde{z}+\delta \int_{0}^{L_{0}} \kappa_{x}(\tilde{z}) D(\tilde{z}) \mathrm{d} \tilde{z} \\
\approx(L & \left.-L_{0}\right)_{\text {sector }}+\mathcal{O}(2) .
\end{aligned}
$$

Here $\left[C(z) x_{0}+\rho_{0}\right] \eta$ is the arc length through the wedge-like deviations from a sector magnet which must be compensated by the decrease or increase $C(z) x_{0} \tan \eta$ in the adjacent drift space. For small edge angles both terms compensate well and the total path length of a wedge magnet is similar to that of a sector magnet. In general we therefore ignore path length variations in wedge magnets with respect to 
sector magnets as well as those in the adjacent drift spaces. For large edge angles, however, this assumption should be reconsidered.

Equation (7.100) imposes quite severe restrictions on the focusing system if the path length is required to be independent of initial condition and the energy. Since the parameters $x_{0}, x_{0}^{\prime}$ and $\delta$ are independent parameters for different particles, all three integrals in (7.100) must vanish separately. An isochronous beam transport line must therefore be a first order achromat (7.97) with the additional condition that $\int \kappa_{x} D \mathrm{~d} \tilde{z}=0$.

For highly relativistic particles $(\beta \approx 1)$ and this condition is equivalent to being an isochronous beam line. In general, any beam line becomes isochronous if we require the time of flight rather than the path length to be equal for all particles. In this case we have to take into account the velocity of the particles as well as its variation with energy. The variation of the particle velocity with energy introduces in (7.100) an additional chromatic correction and the variation of the time of flight becomes

$$
\beta c\left(T-T_{0}\right)=x_{0} I_{\mathrm{c}}+x_{0}^{\prime} I_{\mathrm{s}}+\delta\left(I_{\mathrm{d}}-\gamma^{-2}\right) .
$$

In straight beam lines, where no bending magnets are involved, (7.103) vanishes and higher than linear terms must be considered. From (7.99) it is obvious that the bending independent terms are quadratic in nature and therefore isochronicity cannot be achieved exactly since

$$
\beta c \Delta T=\int_{0}^{L_{0}}\left(x^{\prime 2}+y^{\prime 2}\right) \mathrm{d} \tilde{z}>0 .
$$

This integral is positive for any particle oscillating with a finite betatron amplitude. A straight beam transport line is therefore an isochronous transport system only in first order.

\section{Problems}

7.1 (S). Sketch a quadrupole doublet and draw the sine- and cosine-like trajectories through the quadrupole doublet to the focal point for the horizontal and vertical plane. Verify that (7.20) is indeed true. (hint: first define from where to where you need to measure the combined focal length $f$ ).

7.2 (S). Consider a thin quadrupole doublet with a drift space of $1 \mathrm{~m}$ between them. The quadrupole strengths are to be adjusted to make a focal point in both planes at a point $5 \mathrm{~m}$ from the second quadrupole. Determine the quadrupole strengths and calculate the combined doublet focal length in both planes. Sketch the doublet focusing and define in this sketch the calculated combined focal lengths. 
7.3 (S). Consider a quadrupole doublet made of thin lenses. a) Calculate the focal length of a quadrupole doublet with $\left|f_{1}\right|=\left|f_{2}\right|=5 \mathrm{~m}$ and a distance between the magnets of $d=1 \mathrm{~m}$. Plot for this doublet the focal length as a function of particle momentum $-5 \%<\Delta p / p<5 \%$. b) Use a parallel beam of radius $r_{0}$ and maximum divergence $r^{\prime}$ and calculate the beam radius $r$ at the focal point of this doublet. c) Plot the magnification $r / r_{0}$ as a function of momentum $-5 \%<\Delta p / p<5 \%$. What is the chromatic aberration $\left(r-r_{0}\right) / r_{0}$ of the spot size?

7.4 (S). Sector and rectangular magnets have opposite focusing properties. Determine the geometry of a wedge magnet with equal focusing in both planes (ignore the gap effect).

7.5 (S). In an arbitrary open beam transport line, we assume that at the point $z_{0}$ the particle beam is kicked in the horizontal or vertical plane by the deflection angle $\vartheta$. What is the betatron amplitude for the beam at any point $z$ downstream from $z_{0}$ ? To maximize the betatron amplitude at $z$ how should the lattice functions, betatron function and/or phase, be chosen at $z_{0}$ and $z$ ?

7.6 (S). Design a beam bump within three cells of a symmetric FODO lattice $\frac{1}{2} \mathrm{QF}_{1}$ $\mathrm{QD}_{1}-\mathrm{QF}_{2}-\mathrm{QD}_{2}-\mathrm{QF}_{3}-\mathrm{QD}_{3}-\frac{1}{2} \mathrm{QF}_{4}$ with a betatron phase advance $\psi_{\mathrm{F}}=90^{\circ}$ per cell. Further assume there are special coils in the quadrupoles to produce dipole fields which can be used to deflect the beam. a) Construct a symmetric beam bump which starts at $\mathrm{QF}_{1}$, ends at $\mathrm{QF}_{4}$ and reaches an amplitude $A_{\mathrm{M}}=2 \mathrm{~cm}$ in the center of $\mathrm{QD}_{2}$. How many trim coils need to be activated? b) Derive the relative kick angles required to construct the beam bump and calculate the beam displacement in each quadrupole. Is $A_{\mathrm{M}}$ the maximum amplitude of the beam bump? Why? Why not? (hint: do not use betatron and phase functions, but use thin lens approximation)

7.7. a) Design a symmetric thin lens triplet with a focal point for both planes at the same point $z=z_{\mathrm{f}}$. b) Calculate and plot the betatron function for the quadrupole triplet and some drift space extending beyond the focal point. The value for the betatron function be $\beta=8 \mathrm{~m}$ at the entrance to the triplet $z=0$ where we also assume $\alpha=0$ c) Derive the phase advance in one plane between $z=0$ and $z=z_{\mathrm{f}}$ both from the elements of the transformation matrix and by integrating the betatron function. Both method should give the same results. (note: do the integration roughly from the drawing of the betatron function with linear interpolation).

\section{References}

1. N. Christofilos, US Patent No 2,736,766 (1950)

2. E.D. Courant, M.S. Livingston, H.S. Snyder, Phys. Rev. 88, 1190 (1952)

3. J. Moser, Stabilitätsverhalten kanonischer differentialgleichungssysteme. Nachr. der Akad. der Wiss. Göttingen IIa(6), 87 (1951)

4. E.D. Courant, H.S. Snyder, Appl. Phys. 3, 1 (1959)

5. K.G. Steffen, High Energy Beam Optics (Wiley, New York, 1965)

6. R.F.K. Herzog, Acta Phys. Austriaca 4, 431 (1951) 



\section{Chapter 8 \\ Particle Beams and Phase Space}

The solution of the linear equations of motion allows us to follow a single charged particle through an arbitrary array of magnetic elements. Often, however, it is necessary to consider a beam of many particles and it would be impractical to calculate the trajectory for every individual particle. We, therefore, look for some representation of the whole particle beam.

To learn more about the collective motion of particles, we observe their dynamics in phase space. Each particle at any point along a beam transport line is represented by a point in six-dimensional phase space with coordinates $\left(x, p_{x}, y, p_{y}, s, E\right)$ where $p_{x} \approx p_{0} x^{\prime}$ and $p_{y} \approx p_{0} y^{\prime}$ are the transverse momenta with $c p_{0}=\beta E_{0}, s$ the coordinate along the individual trajectory, $E_{0}$ the ideal particle energy and $E$ the particle energy. Instead of the energy $E$ often the momentum $c p$ or the momentum deviation from the ideal momentum $\Delta p=p-p_{0}$ or the relative momentum deviation $\Delta p / p_{0}$ may be used. We use the momentum to study particle dynamics in the presence of magnetic field. In accelerating systems, like linear accelerators, the use of the particle's kinetic energy is more convenient. Similarly, when the beam energy stays constant, we use instead of the transverse momenta rather the slope of the trajectories $x^{\prime}, y^{\prime}$ which are proportional to the transverse momenta and are generally very small so we may set $\sin x^{\prime} \approx x^{\prime}$, etc.

The coupling between the horizontal and vertical plane is being ignored in linear beam dynamics or treated as a perturbation as is the coupling between transverse and longitudinal motion. Only the effect of energy errors on the trajectory will be treated in this approximation. First, however, we set $\Delta E=0$ and represent the beam by its particle distribution in the horizontal $\left(x, x^{\prime}\right)$ or vertical $\left(y, y^{\prime}\right)$-phase space separately. Because of the absence of coupling between degrees of freedom in this approximation we may split the six-dimensional phase space into three independent two-dimensional phase planes.

This chapter has been made Open Access under a CC BY 4.0 license. For details on rights and licenses please read the Correction https://doi.org/10.1007/978-3-319-18317-6_28 


\subsection{Beam Emittance}

Particles in a beam occupy a certain region in phase space which is called the beam emittance and we define three independent two-dimensional beam emittances. Their numerical values multiplied by $\pi$ are equal to the area occupied by the beam in the respective phase plane. The beam emittance is a measure of the transverse or longitudinal temperature of the beam and depends on the source characteristics of a beam or on other effects like quantized emission of photons into synchrotron radiation and its related excitation and damping effects.

A simple example of a beam emittance and its boundaries is shown in Fig. 8.1, where particles emerge from a disk with radius $w$ and where the direction of the particle trajectories can be anywhere within $\pm 90^{\circ}$ with respect to the surface of the source. The proper phase space representation of this beam at the surface of the source is shown in Fig. 8.1(left). All particles are contained in a narrow strip within the boundaries $x_{\max }= \pm w$ but with a large distribution of transverse momenta $\left(p_{x}=p_{0} \tan x^{\prime}\right)$.

Any real beam emerging from its source will be clipped by some aperture limitations of the vacuum chamber. We assume a simple iris as the aperture limitation located at a distance $d$ from the source and an opening with a radius of $R=w$. The fact that we choose the iris aperture to be the same as the size of the source is made only to simplify the arguments. Obviously many particles emerging from the source will be absorbed at the iris. The part of the beam which passes the iris occupies a phase space area at the exit of the iris like the shaded area shown in Fig. 8.1 (right). Among all particles emerging from the source with an amplitude $x= \pm w$ only those will pass the iris for which the slope of the trajectory is between $x^{\prime}=0$ and $x^{\prime}=\mp 2 w / \ell$. This beam now has a measurable beam emittance as determined by the source and iris aperture.
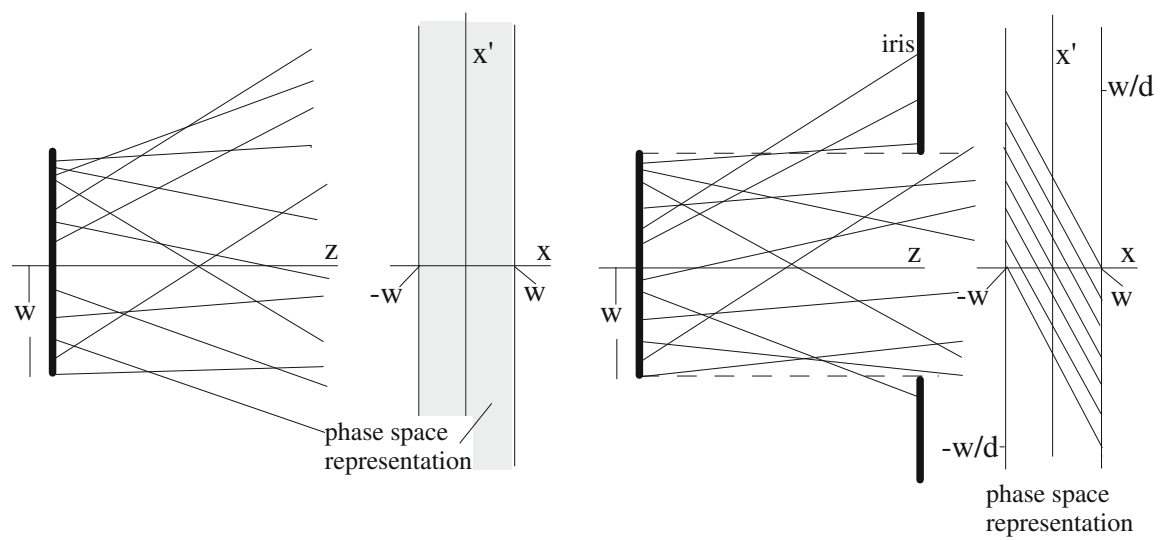

Fig. 8.1 Beam from a diffuse source in real space and in phase space (left). Reduction of phase space (shaded area) due to beam restriction by an iris aperture (right) 
The concept of describing a particle beam in phase space will become very powerful in beam dynamics since we can prove that the density of particles in phase space does not change along a beam transport line, where the forces acting on particles can be derived from macroscopic electric and magnetic fields. In other words particles that are within a closed boundary in phase space at one point of the beam line stay within that boundary. This is Liouville's theorem which we will prove for the fields used in beam dynamics.

\subsubsection{Liouville's Theorem*}

In Chap. 7 we have learned to follow individual particles through an arbitrary beam transport line made up of drift spaces, dipole and quadrupole magnets. Since this is true for any particle with known initial parameters $\left(x_{0}, x_{0}^{\prime}, y_{0}, y_{0}^{\prime}\right)$ it is in principle possible to calculate trajectories along a beam line for a large number of particles forming a particle beam. This is impractical, and we are therefore looking for more simple mathematical methods to describe the beam as a whole. To this end, we make use of methods in statistical mechanics describing the evolution of a large number of particles forming a particle beam.

Liouville's theorem is of specific importance in this respect and we will use it extensively to describe the properties of a particle beam as a whole. This theorem states that under the influence of conservative forces the particle density in phase space stays constant. Since $((7.1),(7.2))$ is equivalent to the equation of a free harmonic oscillator, we know that the motion of many particles in phase space follow Liouville's theorem. A more direct proof of the validity of Liouville's theorem in particle beam dynamics can be obtained by observing the time evolution of an element in the six-dimensional phase space.

If $\Psi$ is the particle density in phase space, the number of particles within a sixdimensional, infinitesimal element is

$$
\Psi\left(x, y, z, p_{x}, p_{y}, p_{z}\right) \mathrm{d} x \mathrm{~d} y \mathrm{~d} z \mathrm{~d} p_{x} \mathrm{~d} p_{y} \mathrm{~d} p_{z} .
$$

The phase space current created by the motion of these particles is

$$
j=\left(\Psi \dot{x}, \Psi \dot{y}, \Psi \dot{z}, \Psi \dot{p}_{x}, \Psi \dot{p}_{y}, \Psi \dot{p}_{z}\right)
$$

where the time derivatives are to be taken with respect to a time $\tau$ measured along the trajectory of the phase space element. This time is to be distinguished from the reference time $t$ along the reference orbit in the same way as we distinguish between the coordinates $s$ and $z$. We set therefore $\dot{x}=\mathrm{d} x / \mathrm{d} \tau$, etc. The phase space current must satisfy the continuity equation

$$
\boldsymbol{\nabla} \boldsymbol{j}+\frac{\partial \Psi}{\partial \tau}=0
$$


From this, we get with (8.2) and the assumption that the particle location does not depend on its momentum and vice versa

$$
\begin{aligned}
-\frac{\partial \Psi}{\partial \tau} & =\nabla_{r}(\Psi \dot{\boldsymbol{r}})+\nabla_{p}(\Psi \dot{\boldsymbol{p}}) \\
& =\dot{\boldsymbol{r}} \nabla_{r} \Psi+\Psi\left(\nabla_{r} \dot{\boldsymbol{r}}\right)+\dot{\boldsymbol{p}} \nabla_{p} \Psi+\Psi\left(\nabla_{p} \dot{\boldsymbol{p}}\right),
\end{aligned}
$$

where $\nabla_{r}=\left(\frac{\partial}{\partial x}, \frac{\partial}{\partial y}, \frac{\partial}{\partial z}\right)$ and $\nabla_{p}=\left(\frac{\partial}{\partial p_{x}}, \frac{\partial}{\partial p_{y}}, \frac{\partial}{\partial p_{z}}\right)$. The time derivative of the space vector $\mathbf{r}$

$$
\frac{\dot{\boldsymbol{r}}}{c}=\frac{c \boldsymbol{p}}{\sqrt{c^{2} p^{2}+m^{2} c^{4}}},
$$

does not depend on the location $\mathbf{r}$, and we have therefore

$$
\nabla_{r} \dot{r}=0
$$

From the Lorentz force equation we get

$$
\nabla_{p} \dot{\boldsymbol{p}}=e \nabla_{p}[\dot{\boldsymbol{r}} \times \boldsymbol{B}]=e \boldsymbol{B}\left(\nabla_{p} \times \dot{\boldsymbol{r}}\right)-e \dot{\boldsymbol{r}}\left(\nabla_{p} \times \boldsymbol{B}\right) .
$$

The magnetic field $\boldsymbol{B}$ does not depend on the particle momentum $\boldsymbol{p}$ and therefore the second term on the right hand side of (8.7) vanishes. For the first term, we find $\nabla_{p} \times \dot{\boldsymbol{r}}=0$ because $\left(\nabla_{p} \times \dot{\boldsymbol{r}}\right)_{x}=\frac{\partial \dot{z}}{\partial p_{y}}-\frac{\partial \dot{y}}{\partial p_{z}}$ and $\frac{\partial \dot{z}}{\partial p_{y}}=c \frac{\partial}{\partial p_{y}} \frac{p_{z}}{\sqrt{p^{2}+m^{2} c^{2}}}=\frac{c p_{y} p_{z}}{\left(p^{2}+m^{2} c^{2}\right)^{3 / 2}}=$ $\frac{\partial \dot{y}}{\partial p_{z}}$, where we have used $p^{2}=p_{x}^{2}+p_{y}^{2}+p_{z}^{2}$. We get a similar result for the other components and have finally for (8.7)

$$
\nabla_{p} \dot{\boldsymbol{p}}=0 \text {. }
$$

With these results, we find from (8.4) the total time derivative of the phase space density $\Psi$ to vanish

$$
\frac{\partial \Psi}{\partial \tau}+\nabla_{r} \Psi \dot{\boldsymbol{r}}+\nabla_{p} \Psi \dot{\boldsymbol{p}}=\frac{\mathrm{d} \Psi}{\mathrm{d} \tau}=0,
$$

proving the invariance of the phase space density $\Psi$.

Independent from general principles of classical mechanics we have shown the validity of Liouville's theorem for the motion of charged particles under the influence of Lorentz forces. This is obviously also true for that part of the Lorentz force that derives from an electrical field since

$$
\nabla_{p} \dot{\boldsymbol{p}}=e \nabla_{p} \boldsymbol{E}=0
$$

because the electric field $\boldsymbol{E}$ does not depend on the particle momentum. 
The same result can be derived in a different way from the property of the Wronskian in particle beam dynamics. For that, we assume that the unit vectors $\boldsymbol{u}_{1}, \boldsymbol{u}_{2} \ldots, \boldsymbol{u}_{6}$ form a six-dimensional, orthogonal coordinate system. The determinant formed by the components of the six vectors $\boldsymbol{x}_{1}, \boldsymbol{x}_{2}, \ldots, \boldsymbol{x}_{6}$ in this system is equal to the volume of the six-dimensional polygon defined by the vectors $\boldsymbol{x}_{i}$. The components of the vectors $\boldsymbol{x}_{i}$ with respect to the base vectors $\boldsymbol{u}_{j}$ are $x_{i j}$ and the determinant is

$$
D=\left|\begin{array}{llllll}
x_{11} & x_{12} & x_{13} & x_{14} & x_{15} & x_{16} \\
x_{21} & x_{22} & x_{23} & \ldots & \ldots & \ldots \\
x_{31} & x_{32} & \ldots & \ldots & \ldots & \ldots \\
x_{41} & \ldots & \ldots & \ldots & \ldots & \ldots \\
x_{51} & \ldots & \ldots & \ldots & \ldots & \ldots \\
x_{61} & \ldots & \ldots & \ldots & \ldots & x_{66}
\end{array}\right|=\left|x_{1}, x_{2}, \boldsymbol{x}_{3}, \boldsymbol{x}_{4}, \boldsymbol{x}_{5}, \boldsymbol{x}_{6}\right|
$$

We will derive the transformation characteristics of this determinant considering a transformation

$$
y_{i}=\mathcal{M} x_{\mathrm{j}}
$$

where $\mathcal{M}=\left(a_{\mathrm{ij}}\right)$ and the determinant (8.11) then transforms like

$$
\begin{aligned}
\left|\boldsymbol{y}_{1}, \boldsymbol{y}_{2} \ldots, \boldsymbol{y}_{6}\right| & =\left|\sum_{j_{1}=1}^{6} a_{1 j_{1}} \boldsymbol{x}_{j_{1}}, \sum_{j_{1}=1}^{6} a_{2 j_{2}} \boldsymbol{x}_{j_{2}}, \ldots \sum_{j_{1}=1}^{6} a_{6 j_{6}} \boldsymbol{x}_{j_{6}}\right| \\
& =\sum^{6} a_{1 j_{1}} a_{2 j_{2}} \ldots a_{6 j_{6}}\left|\boldsymbol{x}_{j_{1}}, \boldsymbol{x}_{j_{2}}, \ldots \boldsymbol{x}_{j_{6}}\right| .
\end{aligned}
$$

The determinant $\left|\boldsymbol{x}_{j_{1}}, \boldsymbol{x}_{j_{2}}, \ldots \boldsymbol{x}_{j_{6}}\right|$ is equal to zero if two or more of the indices $j_{i}$ are equal and further the determinant changes sign if two indices are interchanged. These rules lead to

$$
\left|\boldsymbol{y}_{1}, \boldsymbol{y}_{2} \ldots, \boldsymbol{y}_{6}\right|=\sum_{j_{i}=1}^{6} \epsilon_{j_{1 j_{2} \ldots j_{6}}} a_{1 j_{1}} a_{2 j_{2}} \ldots a_{6 j_{6}}\left|\boldsymbol{x}_{1}, \boldsymbol{x}_{2}, \ldots, \boldsymbol{x}_{6}\right|,
$$

where

$$
\epsilon_{j_{1}, j_{2} \ldots j_{6}}=\left\{\begin{array}{cl}
+1 & \text { for even permutations of the indices } j_{i} \\
-1 & \text { for odd permutations of the indices } j_{i} \\
0 & \text { if any two indices are equal. }
\end{array}\right.
$$


The sum $\sum_{j_{i}=1}^{6} \epsilon_{j_{1} j_{2} \ldots j_{6}} a_{1 j_{1}} a_{2 j_{2}} \ldots a_{6 j_{6}}$ is just the determinant of the transformation matrix $\mathcal{M}$ and finally we get

$$
\left|\boldsymbol{y}_{1}, \boldsymbol{y}_{2} \ldots, \boldsymbol{y}_{6}\right|=|\mathcal{M}|\left|\boldsymbol{x}_{1}, \boldsymbol{x}_{2}, \ldots, \boldsymbol{x}_{6}\right|
$$

For a particle beam transport line, however, we know that $|\mathcal{M}|$ is the Wronskian with

$$
W=|\mathcal{M}|=1
$$

If we now identify this six-dimensional space with the six-dimensional phase space, we see from (8.16) and (8.17) that the phase space under the class of transformation matrices considered in beam dynamics is constant. Conversely, if $W \neq 1$, we would get a change in phase space.

\subsubsection{Transformation in Phase Space}

Liouville's theorem provides a powerful tool to describe a beam in phase space. Knowledge of the area occupied by particles in phase space at the beginning of a beam transport line will allow us to determine the location and distribution of the beam at any other place along the transport line without having to calculate the trajectory of every individual particle.

In the previous paragraph, we found that the phase space density is a constant under the assumed forces. There are three space and three momentum coordinates. In beam dynamics, we often use trajectory slopes instead of transverse momenta. Similar relations exist for other coordinates. Using slopes instead of momenta preserves the phase space density only as long as $p_{0}$ is a constant, which is true in most beam dynamics calculations. We distinguish therefore two definitions of the beam emittance, the normalized emittance $\epsilon_{\mathrm{n}}$ based on space-momentum phase space and the geometric emittance $\epsilon$ based on space-slope phase space. Both are related by

$$
\epsilon_{\mathrm{n}}=\beta \gamma \epsilon
$$

where $\gamma$ is the relativistic factor and $\beta=v / c$ the relative particle velocity.

In beam dynamics it has become customary to surround all particles of a beam in phase space by an ellipse called the phase ellipse (Fig. 8.2) described by

$$
\gamma x^{2}+2 \alpha x x^{\prime}+\beta x^{\prime 2}=\epsilon,
$$

where $\alpha, \beta, \gamma$ and $\epsilon$ are ellipse parameters. This seemingly arbitrary boundary will soon gain physical significance. The area enclosed by the ellipse is called the 
Fig. 8.2 Phase space ellipse

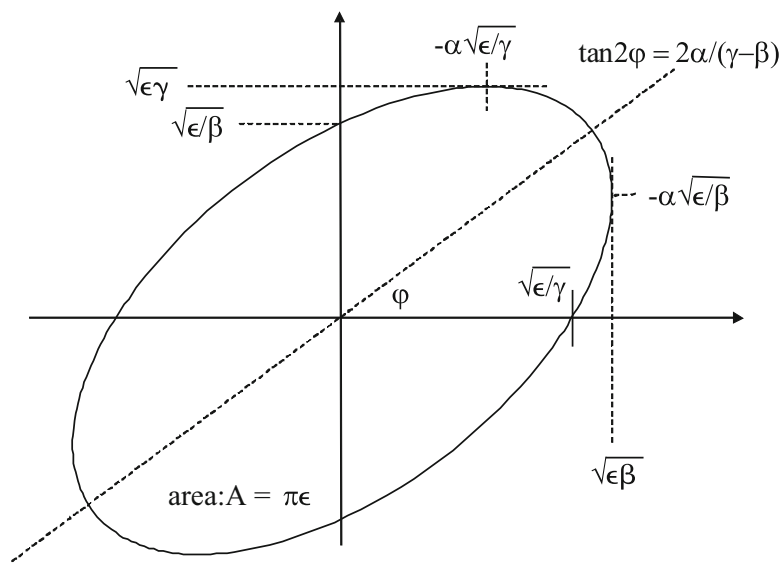

geometric beam emittance $\epsilon^{1}$ defined by

$$
\int_{\text {ellipse }} \mathrm{d} x \mathrm{~d} x^{\prime}=\pi \epsilon,
$$

while the parameters $\alpha, \beta$ and $\gamma$ determine the shape and orientation of the ellipse. This characterization of the beam emittance by the area of an ellipse seems at first arbitrary although practical. Later in Sect.8.2, we will see that all particles travel along their individual ellipses in phase space. If we now choose that or those particles on the largest phase ellipse within a particular beam, we know that all other particles within that ellipse will stay within that ellipse. We are thereby able to describe the collective behavior of a beam formed by many particles by the dynamics of a single particle.

Since all particles enclosed by a phase ellipse stay within that ellipse, we only need to know how the ellipse parameters transform along the beam line to be able to describe the whole particle beam. Let the equation

$$
\gamma_{0} x_{0}^{2}+2 \alpha_{0} x_{0} x_{0}^{\prime}+\beta_{0} x_{0}^{\prime 2}=\epsilon
$$

be the equation of the phase ellipse at the starting point $z=0$ of the beam line. Any particle trajectory transforms from the starting point $z=0$ to any other point $z \neq 0$ by the transformation $\left(\begin{array}{c}x(z) \\ x^{\prime}(z)\end{array}\right)=\left(\begin{array}{cc}C(z) & S(z) \\ C^{\prime}(z) & S^{\prime}(z)\end{array}\right)\left(\begin{array}{c}x_{0} \\ x_{0}^{\prime}\end{array}\right)$. Solving for $x_{0}$ and $x_{0}^{\prime}$ and inserting into (8.20), we get after sorting of coefficients and stopping to show

\footnotetext{
${ }^{1}$ The literature is not always uniform in the representation of numerical values for the beam emittance. Often the beam emittance is quoted in units of $\pi-\mathrm{mm}$-mrad and it is not always clear if the factor $\pi$ is included in the numerical value or not. We define in this book the beam emittance as the beam phase space area divided by $\pi$ in accordance with Hamiltonian dynamics.
} 
explicitly the $(z)$-dependence

$$
\begin{aligned}
\epsilon=( & \left.C^{\prime 2} \beta_{0}-2 S^{\prime} C^{\prime} \alpha_{0}+S^{\prime 2} \gamma_{0}\right) x^{2} \\
& +2\left(-C C^{\prime} \beta_{0}+S^{\prime} C \alpha_{0}+S C^{\prime} \alpha_{0}-S S^{\prime} \gamma_{0}\right) x x^{\prime} \\
& +\left(C^{2} \beta_{0}-2 S C \alpha_{0}+S^{2} \gamma_{0}\right) x^{\prime 2} .
\end{aligned}
$$

This equation can be brought into the form (8.18) by replacing the coefficients in (8.21) with

$$
\begin{aligned}
& \gamma=C^{\prime 2} \beta_{0}-2 S^{\prime} C^{\prime} \alpha_{0}+S^{\prime 2} \gamma_{0}, \\
& \alpha=-C C^{\prime} \beta_{0}+\left(S^{\prime} C+S C^{\prime}\right) \alpha_{0}-S S^{\prime} \gamma_{0}, \\
& \beta=C^{2} \beta_{0}-2 S C \alpha_{0}+S^{2} \gamma_{0} .
\end{aligned}
$$

The resulting ellipse equation still has the same area $\pi \epsilon$ as we would expect, but due to different parameters $\gamma, \alpha, \beta$, the new ellipse has a different orientation and shape. During a transformation along a beam transport line the phase ellipse will continuously change its form and orientation but not its area. In matrix formulation the ellipse parameters, which are also called Twiss parameters [11], transform from (8.22) like

$$
\left(\begin{array}{l}
\beta(z) \\
\alpha(z) \\
\gamma(z)
\end{array}\right)=\left(\begin{array}{ccc}
C^{2} & -2 C S & S^{2} \\
-C C^{\prime} & C S^{\prime}+C^{\prime} S-S S^{\prime} \\
C^{\prime 2} & -2 C^{\prime} S^{\prime} & S^{\prime 2}
\end{array}\right)\left(\begin{array}{c}
\beta_{0} \\
\alpha_{0} \\
\gamma_{0}
\end{array}\right)
$$

The orientation, eccentricity and area of an ellipse is defined by three parameters, while (8.20) includes four parameters $\alpha, \beta, \gamma$ and $\epsilon$. Since the area is defined by $\epsilon$ we expect the other three parameters to be correlated. From geometric properties of an ellipse we find that correlation to be

$$
\beta \gamma-\alpha^{2}=1 .
$$

So far, we have used only the $\left(x, x^{\prime}\right)$-phase space, but the results are valid also for the $\left(y, y^{\prime}\right)$-phase space. Equation (8.23) provides the tool to calculate beam parameters anywhere along the beam line from the initial values $\beta_{0}, \alpha_{0}, \gamma_{0}$.

The phase ellipse in a drift space, for example, becomes distorted in a clock wise direction without changing the slope of any particle as shown in Fig. 8.3. If the drift space is long enough a convergent beam transforms eventually into a divergent beam, while the angular envelope $A=x_{\max }^{\prime}=\sqrt{\epsilon \gamma}$ stays constant. The point $z_{\mathrm{w}}$ at which the beam reaches its minimum size is determined by $\alpha\left(z_{\mathrm{w}}\right)=0$ and we get from (8.23) for the location of a beam waist in a drift section.

$$
\ell=z_{\mathrm{W}}-z_{0}=\frac{\alpha_{0}}{\gamma_{0}} .
$$




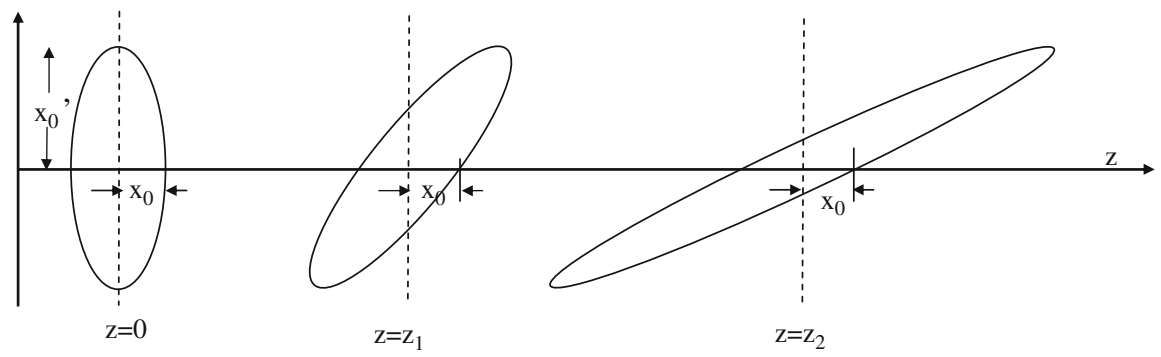

Fig. 8.3 Transformation of a phase space ellipse at different locations along a drift section

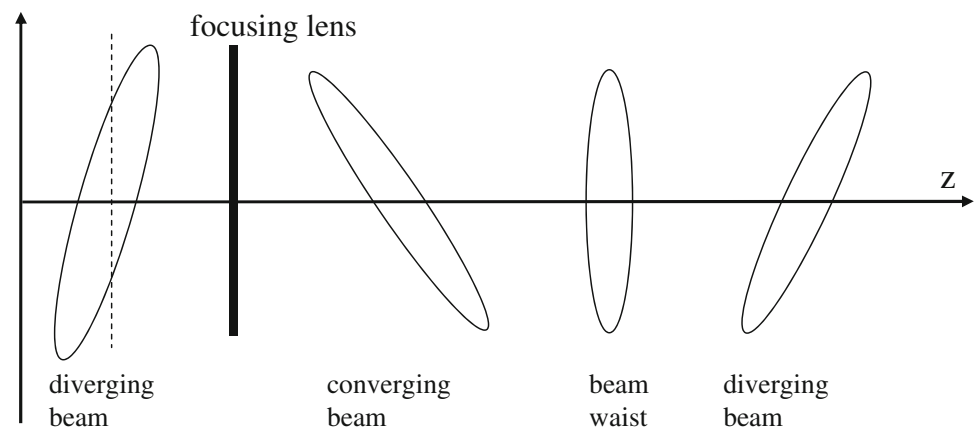

Fig. 8.4 Transformation of a phase ellipse due to a focusing quadrupole. The phase ellipse is shown at different locations along a drift space downstream from the quadrupole

This point of minimum beam size is up or downstream of $z=z_{0}$ depending on the sign of $\alpha_{0}$ being negative or positive, respectively.

More formally, the transformation through a simple drift space of length $\ell$ is

$$
\left(\begin{array}{l}
\beta(\ell) \\
\alpha(\ell) \\
\gamma(\ell)
\end{array}\right)=\left(\begin{array}{ccc}
1 & -2 \ell & \ell^{2} \\
0 & 1 & -\ell \\
0 & 0 & 1
\end{array}\right)\left(\begin{array}{l}
\beta_{0} \\
\alpha_{0} \\
\gamma_{0}
\end{array}\right),
$$

which describes, for example, the transition of a convergent phase ellipse to a divergent phase ellipse as shown in Fig. 8.4. Particles in the upper half of the phase ellipse move from left to right and particles in the lower half from right to left. During the transition from the convergent to divergent phase ellipse we find an upright ellipse which describes the beam at the location of a waist. The form and orientation of the phase ellipse tells us immediately the characteristics beam behavior. Convergent beams are characterized by a rotated phase ellipse extending from the left upper quadrant to the lower right quadrant while a divergent beam spreads from the left lower to the right upper quadrant. A symmetric phase ellipse signals the location of a waist or symmetry point. 
A divergent beam fills, after some distance, the whole vacuum chamber aperture and in order not to lose beam a focusing quadrupole must be inserted. During the process of focusing a diverging beam entering a focusing quadrupole reaches a maximum size and then starts to converge again. This transformation, generated by a focusing quadrupole is shown in Fig. 8.4, where we recognize slopes of particle trajectories to reverse signs thus forming a convergent beam.

After this step, the beam may develop as shown for a drift space until the next focusing quadrupole is required. In reality this focusing scenario is complicated by the fact that we need also vertical focusing which requires the insertion of defocusing quadrupoles as well.

\subsubsection{Beam Matrix}

Particle beams are conveniently described in phase space by enclosing their distribution with ellipses. Transformation rules for such ellipses through a beam transport system have been derived for a two-dimensional phase space and we expand here the discussion of phase space transformations to more dimensions. The equation for an $n$-dimensional ellipse can be written in the form

$$
\boldsymbol{u}^{T} \boldsymbol{\sigma}^{-1} \boldsymbol{u}=1,
$$

where the symmetric matrix $\sigma$ is still to be determined, $\boldsymbol{u}^{T}$ is the transpose of the coordinate vector $\boldsymbol{u}$ defined by

$$
\boldsymbol{u}=\left(\begin{array}{c}
x \\
x^{\prime} \\
y \\
y^{\prime} \\
\tau \\
\delta \\
\vdots
\end{array}\right) .
$$

The volume of this $n$-dimensional ellipse is

$$
V_{n}=\frac{\pi^{n / 2}}{\Gamma(1+n / 2)} \sqrt{\operatorname{det} \sigma},
$$

where $\Gamma$ is the gamma function. Applying (8.27) to the two dimensional phase space, we get for the ellipse equation

$$
\sigma_{11} x^{2}+2 \sigma_{12} x x^{\prime}+\sigma_{22} x^{\prime 2}=1
$$


and comparison with (8.18) defines the beam matrix with well known beam parameters as

$$
\sigma=\left(\begin{array}{ll}
\sigma_{11} & \sigma_{12} \\
\sigma_{21} & \sigma_{22}
\end{array}\right)=\epsilon^{2}\left(\begin{array}{cc}
\beta & -\alpha \\
-\alpha & \gamma
\end{array}\right)
$$

Since only three of the four parameters in the beam matrix $\sigma$ are independent, we find that $\sigma_{21}=\sigma_{12}$. This identification of the beam matrix can be expanded to six or arbitrary many dimensions including, for example, spin or coupling terms which we have so far neglected. The two-dimensional "volume" or phase space area is

$$
V_{2}=\pi \sqrt{\operatorname{det} \sigma}=\pi \sqrt{\sigma_{11} \sigma_{22}-\sigma_{12}^{2}}=\pi \epsilon
$$

consistent with the earlier definition of beam emittance, since $\beta \gamma-\alpha^{2}=1$.

The definition of the beam matrix elements are measures of the particle distribution in phase space. As such, we would expect different definitions for different distributions. Since most particle beams have a Gaussian or bell shaped distribution, however, we adopt a uniform definition of beam matrix elements. The betatron oscillation amplitude for a particular particle and its derivative is described by

$$
\begin{aligned}
x_{i} & =a_{i} \sqrt{\beta} \cos \left(\psi+\psi_{i}\right), \\
x_{i}^{\prime} & =a_{i} \frac{\beta^{\prime}}{2 \sqrt{\beta}} \cos \left(\psi+\psi_{i}\right)-a_{i} \frac{1}{\sqrt{\beta}} \sin \left(\psi+\psi_{i}\right) .
\end{aligned}
$$

We form now average values of all particles within a well defined fraction of a beam and get

$$
\begin{aligned}
\left\langle x_{i}^{2}\right\rangle & =\left\langle a_{i}^{2} \cos ^{2}\left(\psi+\psi_{i}\right)\right\rangle \beta=\frac{1}{2}\left\langle a_{i}^{2}\right\rangle \beta=\epsilon \beta, \\
\left\langle x_{i}^{\prime 2}\right\rangle & =\left\langle a_{i}^{2}\right\rangle \frac{\alpha^{2}}{\beta} \frac{1}{2}+\left\langle a_{i}^{2}\right\rangle \frac{1}{\beta} \frac{1}{2}=\frac{1}{2}\left\langle a_{i}^{2}\right\rangle \frac{1+\alpha^{2}}{\beta}=\epsilon \gamma, \\
\left\langle x_{i} x_{i}^{\prime}\right\rangle & =-\left\langle a_{i}^{2}\right\rangle \alpha \frac{1}{2}=-\epsilon \alpha,
\end{aligned}
$$

where we have assumed a Gaussian particle distribution and a beam emittance defined by $\epsilon=\left\langle a_{i}^{2} \sin ^{2}\left(\psi-\psi_{i}\right)\right\rangle$. This definition describes that part of the beam which is within one standard deviation of the distribution in multidimensional phase space. The beam matrix elements are finally defined by

$$
\begin{aligned}
& \sigma_{11}=\left\langle x_{i}^{2}\right\rangle=\epsilon \beta, \\
& \sigma_{22}=\left\langle x_{i}^{\prime 2}\right\rangle=\epsilon \gamma, \\
& \sigma_{12}=\left\langle x_{i} x_{i}^{\prime}\right\rangle=-\epsilon \alpha .
\end{aligned}
$$


With this definition the beam emittance can be expressed by

$$
\epsilon^{2}=\sigma_{11} \sigma_{22}-\sigma_{12}^{2}=\left\langle x_{i}^{2}\right\rangle\left\langle x_{i}^{\prime 2}\right\rangle-\left\langle x_{i} x_{i}^{\prime}\right\rangle^{2}
$$

This definition is generally accepted also for any arbitrary particle distribution. Specifically, beams from linear accelerators or proton and ion beams can have arbitrary distributions.

Similar to the two-dimensional case, we look for the evolution of the $n$ dimensional phase ellipse along a beam transport line. With $\mathcal{M}\left(P_{1} \mid P_{2}\right)$ the $n \times n$ transformation matrix from point $P_{0}$ to $P_{1}$ we get $\boldsymbol{u}_{1}=\mathcal{M}\left(P_{1} \mid P_{0}\right) \boldsymbol{u}_{0}$ and the equation of the phase ellipse at point $P_{1}$ is

$$
\left(\mathcal{M}^{-1} \boldsymbol{u}_{1}\right)^{T} \boldsymbol{\sigma}_{0}^{-1}\left(\mathcal{M}^{-1} \boldsymbol{u}_{1}\right)=\boldsymbol{u}_{1}^{T} \boldsymbol{\sigma}_{1}^{-1} \boldsymbol{u}_{1}=1
$$

With $\left(\mathcal{M}^{T}\right)^{-1} \sigma_{0}^{-1} \mathcal{M}^{-1}=\left[\mathcal{M} \sigma_{0} \mathcal{M}^{T}\right]^{-1}$ the beam matrix transforms therefore like

$$
\sigma_{1}=\mathcal{M} \sigma_{0} \mathcal{M}^{T}
$$

This formalism will be useful for the experimental determination of beam emittances.

\section{Measurement of the Beam Emittance}

The ability to manipulate in a controlled and measurable way the orientation and form of the phase ellipse with quadrupoles gives us the tool to experimentally determine the emittance of a particle beam. Since the beam emittance is a measure of both the beam size and beam divergence, we cannot directly measure its value. While we are able to measure the beam size with the use of a fluorescent screen, for example, the beam divergence cannot be measured directly. If, however, the beam size is measured at different locations or under different focusing conditions such that different parts of the ellipse will be probed by the beam size monitor, the beam emittance can be determined.

Utilizing the definition of the beam matrix in (8.31) we have

$$
\sigma_{11} \sigma_{22}-\sigma_{12}^{2}=\epsilon^{2}
$$

and the beam emittance can be measured, if we find a way to determine the beam matrix. To determine the beam matrix $\sigma_{0}$ at point $P_{0}$, we consider downstream from $P_{0}$ a beam transport line with some quadrupoles and beam size monitors like fluorescent screens at three places $P_{1}$ to $P_{3}$. From (8.23) and (8.31) we get for the 
beam sizes $\sigma_{i, 11}$ at locations $P_{i}$ three relations of the form ${ }^{2}$

$$
\sigma_{\mathrm{i}, 11}=C_{\mathrm{i}}^{2} \sigma_{0,11}+2 S_{\mathrm{i}} C_{\mathrm{i}} \sigma_{0,12}+S_{\mathrm{i}}^{2} \sigma_{0,22}
$$

which we may express in matrix formulation by

$$
\left(\begin{array}{l}
\sigma_{1,11} \\
\sigma_{2,11} \\
\sigma_{3,11}
\end{array}\right)=\left(\begin{array}{lll}
C_{1}^{2} & 2 C_{1} S_{1} & S_{1}^{2} \\
C_{2}^{2} & 2 C_{2} S_{2} & S_{2}^{2} \\
C_{3}^{2} & 2 C_{3} S_{3} & S_{3}^{2}
\end{array}\right)\left(\begin{array}{l}
\sigma_{0,11} \\
\sigma_{0,12} \\
\sigma_{0,22}
\end{array}\right)=\mathcal{M}_{\sigma}\left(\begin{array}{l}
\sigma_{0,11} \\
\sigma_{0,12} \\
\sigma_{0,22}
\end{array}\right),
$$

where $C_{i}$ and $S_{i}$ are elements of the transformation matrix from point $P_{0}$ to $P_{i}$ and $\sigma_{i, j k}$ are elements of the beam matrix at $P_{i}$. Equation (8.44) can be solved for the beam matrix elements $\sigma_{i, j k}$ at $P_{0}$

$$
\left(\begin{array}{l}
\sigma_{0,11} \\
\sigma_{0,12} \\
\sigma_{0,22}
\end{array}\right)=\left(\mathcal{M}_{\sigma}^{T} \mathcal{M}_{\sigma}\right)^{-1} \mathcal{M}_{\sigma}^{T}\left(\begin{array}{l}
\sigma_{1,11} \\
\sigma_{2,11} \\
\sigma_{3,11}
\end{array}\right)
$$

where the matrix $\mathcal{M}_{\sigma}$ is known from the parameters of the beam transport line between $P_{0}$ and $P_{\mathrm{i}}$ and $\mathcal{M}_{\sigma}^{T}$ is the transpose of it. The solution vector can be used in (8.42) to calculate finally the beam emittance.

This procedure to measure the beam emittance is straight forward but requires three beam size monitors at appropriate locations such that the measurements can be conducted with the desired resolution. A much simpler procedure makes use of only one beam size monitor at $P_{1}$ and one quadrupole between $P_{0}$ and $P_{1}$. We vary the strength of the quadrupole and measure the beam size at $P_{1}$ as a function of the quadrupole strength. These beam size measurements as a function of quadrupole strength are equivalent to the measurements at different locations discussed above and we can express the results of $n$ beam size measurements by the matrix equation

$$
\left(\begin{array}{c}
\sigma_{1,11} \\
\sigma_{2,11} \\
\vdots \\
\sigma_{n, 11}
\end{array}\right)=\left(\begin{array}{ccc}
C_{1}^{2} & 2 C_{1} S_{1} & S_{1}^{2} \\
C_{2}^{2} & 2 C_{2} S_{2} & S_{2}^{2} \\
\vdots & \vdots & \vdots \\
C_{n}^{2} & 2 C_{n} S_{n} & S_{n}^{2}
\end{array}\right)\left(\begin{array}{l}
\sigma_{0,11} \\
\sigma_{0,12} \\
\sigma_{0,22}
\end{array}\right)=\mathcal{M}_{\sigma, n}\left(\begin{array}{c}
\sigma_{0,11} \\
\sigma_{0,12} \\
\sigma_{0,22}
\end{array}\right)
$$

\footnotetext{
${ }^{2}$ Note: the sign of the cross term is different from (8.23) because $\sigma_{12}=-\alpha$.
} 
This method of emittance measurement is also known as quad scan. Like in (8.45) the solution is from simple matrix multiplications

$$
\left(\begin{array}{c}
\sigma_{0,11} \\
\sigma_{0,12} \\
\sigma_{0,22}
\end{array}\right)=\left(\mathcal{M}_{\sigma, n}^{T} \mathcal{M}_{\sigma, n}\right)^{-1} \mathcal{M}_{\sigma, n}^{T}\left(\begin{array}{c}
\sigma_{1,11} \\
\sigma_{2,11} \\
\vdots \\
\sigma_{n, 11}
\end{array}\right)
$$

An experimental procedure has been derived which allows us to determine the beam emittance through measurements of beam sizes as a function of focusing. Practically, the evaluation of (8.47) is performed by measuring the beam size $\sigma_{1,11}(k)$ at $P_{1}$ as a function of the quadrupole strength $k$ and comparing the results with the theoretical expectation

$$
\sigma_{1,11}(k)=C^{2}(k) \sigma_{0,11}+2 C(k) S(k) \sigma_{0,12}+S^{2}(k) \sigma_{0,22}
$$

By fitting the parameters $\sigma_{0,11}, \sigma_{0,12}$ and $\sigma_{0,22}$ to match the beam size measurements, one can determine the beam emittance from (8.42). However, this procedure does not guarantee automatically a measurement with the desired precision. To accurately fit three parameters we must be able to vary the beam size considerably such that the nonlinear variation of the beam size with quadrupole strength becomes quantitatively significant. An analysis of measurement errors indicates that the beam size at $P_{0}$ should be large and preferable divergent. In this case variation of the quadrupole strength will dramatically change the beam size at $P_{1}$ from a large value when the quadrupole is off, to a narrow focal point and again to a large value by over focusing.

A most simple arrangement consists of a single quadrupole and a screen at a distance $d$. Assuming that the length $\ell_{\mathrm{q}}$ of the quadrupole is $\ell_{\mathrm{q}} \ll d$, we can use thin lens approximation and the total transformation matrix is then

$$
\left(\begin{array}{cc}
1-d / f & d \\
-1 / f & 1
\end{array}\right)=\left(\begin{array}{ll}
1 & d \\
0 & 1
\end{array}\right)\left(\begin{array}{cc}
1 & 0 \\
-1 / f & 1
\end{array}\right)
$$

Equation (8.48) becomes

$$
\sigma_{1,11}(k)=\left(1-d \ell_{\mathrm{q}} k\right)^{2} \sigma_{0,11}+2\left(1-d \ell_{\mathrm{q}} k\right) d \sigma_{0,12}+d^{2} \sigma_{0,22}
$$

or after reordering

$$
\begin{gathered}
\sigma_{1,11}(k)=\left(d^{2} \ell_{\mathrm{q}}^{2} \sigma_{0,11}\right) k^{2}+\left(-2 d \ell_{\mathrm{q}} \sigma_{0,11}-2 d^{2} \ell_{\mathrm{q}} \sigma_{0,12}\right) k \\
+\left(\sigma_{0,11}+2 d \sigma_{0,12}+d^{2} \sigma_{0,22}\right) .
\end{gathered}
$$


Fitting $\sigma_{1,11}(k)$ with a parabola $\left(a k^{2}+b k+c\right)$ will determine the whole beam matrix $\sigma_{0}$ by

$$
\begin{aligned}
\sigma_{0,11} & =\frac{a}{d^{2} \ell_{\mathrm{q}}^{2}}, \\
\sigma_{0,12} & =\frac{-b-2 d \ell_{\mathrm{q}} \sigma_{0,11}}{2 d^{2} \ell_{\mathrm{q}}}, \\
\sigma_{0,22} & =\frac{c-\sigma_{0,11}-2 d \sigma_{0,12}}{d^{2}} .
\end{aligned}
$$

The beam matrix not only defines the beam emittance but also the betatron functions at the beginning of the quadrupole in this measurement. We gain with this measurement a full set of initial beam parameters $\left(\alpha_{0}, \beta_{0}, \gamma_{0}^{\prime}, \epsilon\right)$ and may now calculate beam parameters at any point along the transport line.

\subsection{Betatron Functions}

The trajectory of a particle through an arbitrary beam transport system can be determined by repeated multiplication of transformation matrices through each of the individual elements of the beam line. This method is convenient especially for computations on a computer but it does not reveal many properties of particle trajectories. For deeper insight, we attempt to solve the equation of motion analytically. The differential equation of motion is

$$
u^{\prime \prime}+k(z) u=0
$$

where $u$ stands for $x$ or $y$ and $k(z)$ is an arbitrary function of $z$ resembling the particular distribution of focusing along a beam line. For a general solution of (8.52) we apply the method of variation of integration constants and use an ansatz with a $z$-dependent amplitude and phase

$$
u(z)=\sqrt{\epsilon} \sqrt{\beta(z)} \cos \left[\psi(z)-\psi_{0}\right]
$$

which is similar to the solution of a harmonic oscillator with a constant coefficient $k$. The quantities $\epsilon$ and $\psi_{0}$ are integration constants. From (8.53) we form first and second derivatives with the understanding that $\beta=\beta(z), \psi=\psi(z)$, etc.

$$
\begin{aligned}
& u^{\prime}= \sqrt{\epsilon} \frac{\beta^{\prime}}{2 \sqrt{\beta}} \cos \left(\psi-\psi_{0}\right)-\sqrt{\epsilon} \sqrt{\beta} \sin \left(\psi-\psi_{0}\right) \psi^{\prime}, \\
& u^{\prime \prime}=\sqrt{\epsilon} \frac{\beta \beta^{\prime \prime}-\frac{1}{2} \beta^{\prime 2}}{2 \beta^{3 / 2}} \cos \left(\psi-\psi_{0}\right)-\sqrt{\epsilon} \frac{\beta^{\prime}}{\sqrt{\beta}} \sin \left(\psi-\psi_{0}\right) \psi^{\prime} \\
&-\epsilon \sqrt{\beta} \sin \left(\psi-\psi_{0}\right) \psi^{\prime \prime}-\sqrt{\epsilon} \sqrt{\beta} \cos \left(\psi-\psi_{0}\right) \psi^{\prime 2},
\end{aligned}
$$


and insert into (8.52). The sum of all coefficients of the sine and cosine terms respectively must vanish separately to make the ansatz (8.53) valid for all phases $\psi$. From this, we get the two conditions:

$$
\frac{1}{2}\left(\beta \beta^{\prime \prime}-\frac{1}{2} \beta^{2}\right)-\beta^{2} \psi^{\prime 2}+\beta^{2} k=0
$$

and

$$
\beta^{\prime} \psi^{\prime}+\beta \psi^{\prime \prime}=0 \text {. }
$$

Equation (8.56) can be integrated immediately since $\beta^{\prime} \psi+\beta \psi^{\prime \prime}=\left(\beta \psi^{\prime}\right)^{\prime}$ for

$$
\beta \psi^{\prime}=\text { const }=1,
$$

where a specific normalization of the phase function has been chosen by selecting the integration constant to be equal to unity. From (8.57) we get for the phase function

$$
\psi(z)=\int_{0}^{z} \frac{\mathrm{d} \bar{z}}{\beta(\bar{z})}+\psi_{0} .
$$

Knowledge of the function $\beta(z)$ along the beam line obviously allows us to compute the phase function. Inserting (8.57) into (8.55) we get the differential equation for the function $\beta(z)$

$$
\frac{1}{2} \beta \beta^{\prime \prime}-\frac{1}{4} \beta^{2}+\beta^{2} k=1,
$$

which becomes with $\alpha=-\frac{1}{2} \beta^{\prime}$ and $\gamma=\left(1+\alpha^{2}\right) / \beta$

$$
\beta^{\prime \prime}+2 k \beta-2 \gamma=0 .
$$

The justification for the definition of $\gamma$ becomes clear below, when we make the connection to ellipse geometry and (8.24).With $\alpha^{\prime}=-\frac{1}{2} \beta^{\prime \prime}$ this is equivalent to

$$
\alpha^{\prime}=k \beta-\gamma .
$$

Before we solve (8.60) we try to determine the physical nature of the functions $\beta(z), \alpha(z)$, and $\gamma(z)$. To do that, we note first that any solution that satisfies (8.60) together with the phase function $\psi(z)$ can be used to make (8.53) a real solution of the equation of motion (8.52). From that solution and the derivative (8.54) we eliminate the phase $\left(\psi-\psi_{0}\right)$ and obtain a constant of motion which is also called the Courant-Snyder invariant [4]

$$
\gamma u^{2}+2 \alpha u u^{\prime}+\beta u^{\prime 2}=\epsilon .
$$


This invariant expression is equal to the equation of an ellipse with the area $\pi \epsilon$ which we have encountered in the previous section and the particular choice of the letters $\beta, \alpha, \gamma, \epsilon$ for the betatron functions and beam emittance becomes now obvious. The physical interpretation of this invariant is that of a single particle traveling in phase space along the contour of an ellipse with the parameters $\beta, \alpha$, and $\gamma$. Since these parameters are functions of $z$ however, the form of the ellipse is changing constantly but, due to Liouville's theorem, any particle starting on that ellipse will stay on it. The choice of an ellipse to describe the evolution of a beam in phase space is thereby more than a mathematical convenience. We may now select a single particle to define a phase ellipse and know that all particles with lesser betatron oscillation amplitudes will stay within that ellipse. The description of an ensemble of particles forming a beam have thereby been reduced to that of a single particle.

The ellipse parameter functions or Twiss parameters $\beta, \alpha, \gamma$ and the phase function $\psi$ are called the betatron functions or lattice functions or Twiss functions and the oscillatory motion of a particle along the beam line (8.53) is called the betatron oscillation. This oscillation is quasi periodic with varying amplitude and frequency.

To demonstrate the close relation to the solution of a harmonic oscillator, we use the betatron and phase function to perform a coordinate transformation

$$
(u, z) \quad \longrightarrow \quad(w, \psi)
$$

by setting

$$
w(\psi)=\frac{u(z)}{\sqrt{\beta(z)}} \quad \text { and } \quad \psi=\int_{0}^{z} \frac{\mathrm{d} \bar{z}}{\beta(\bar{z})},
$$

where $u(z)$ stands for $x(z)$ and $y(z)$ respectively. The new coordinates $(w, \psi)$ are called normalized coordinates and equation of motion (8.52) transforms to

$$
\frac{\mathrm{d}^{2} w}{\mathrm{~d} \psi^{2}}+w^{2}=0
$$

which indeed is the equation of a harmonic oscillator with angular frequency one. This identity will be very important for the treatment of perturbing driving terms that appear on the right hand side of (8.65) which will be discussed in more detail in Sect. 8.3.1.

So far, we have tacitly assumed that the betatron function $\beta(z)$ never vanishes or changes sign. This can be shown to be true by setting $q(z)=\sqrt{\beta(z)}$ and inserting into (8.59). With $\beta^{\prime}=2 q q^{\prime}$ and $\beta^{\prime \prime}=2\left(q^{\prime 2}+q q^{\prime \prime}\right)$ we get the differential equation

$$
q^{\prime \prime}+k q-\frac{1}{q^{3}}=0 \text {. }
$$


The term $1 / q^{3}$ prevents a change of sign of $q(z)$. Letting $q>0$ vary toward zero $q^{\prime \prime} \approx 1 / q^{3} \rightarrow \infty$. This curvature, being positive, will become arbitrarily large and eventually turns the function $q(z)$ around before it reaches zero. Similarly, the function $q(z)$ stays negative along the whole beam line if it is negative at one point. Since the sign of the betatron function is not determined and does not change, it has became customary to use only the positive solution.

The beam emittance parameter $\epsilon$ appears as an amplitude factor in the equation for the trajectory of an individual particle. This amplitude factor is equal to the beam emittance only for particles traveling on an ellipse that just encloses all particles in the beam. In other words, a particle traveling along a phase ellipse with amplitude $\sqrt{\epsilon}$ defines the emittance of that part of the total beam which is enclosed by this ellipse or for all those particles whose trajectories satisfy

$$
\beta u^{\prime 2}+2 \alpha u u^{\prime}+\gamma u^{2} \leq \epsilon_{u} .
$$

Since it only leads to confusion to use the letter $\epsilon$ as an amplitude factor we will from now on use it only when we want to define the whole beam and set $\sqrt{\epsilon}=a$ for all cases of individual particle trajectories.

\subsubsection{Beam Envelope}

To describe the beam and beam sizes as a whole, a beam envelope equation can be defined. All particles on the beam emittance defining ellipse follow trajectories described by

$$
x_{i}(z)=\sqrt{\epsilon} \sqrt{\beta(z)} \cos \left[\psi(z)+\delta_{i}\right],
$$

where $\delta_{i}$ is an arbitrary phase constant for the particle $i$. By selecting at every point along the beam line that particle $i$ for which $\cos \left[\psi(z)+\delta_{i}\right]= \pm 1$, we can construct an envelope of the beam containing all particles

$$
E(z)= \pm \sqrt{\epsilon} \sqrt{\beta(z)} .
$$

Here the two signs indicate only that there is an envelope an either side of the beam center. We note that the beam envelope is determined by the beam emittance $\epsilon$ and the betatron function $\beta(z)$. The beam emittance is a constant of motion and resembles the transverse "temperature" of the beam. The betatron function reflects exterior forces from focusing magnets and is highly dependent on the particular arrangement of quadrupole magnets. It is this dependence of the beam envelope on the focusing structure that lets us design beam transport systems with specific properties like small or large beam sizes at particular points. 


\subsection{Beam Dynamics in Terms of Betatron Functions}

Properties of betatron functions can now be used to calculate the parameters of individual particle trajectories anywhere along a beam line. Any particle trajectory can be described by

$$
u(z)=a \sqrt{\beta} \cos \psi+b \sqrt{\beta} \sin \psi
$$

and the amplitude factors $a$ and $b$ can be determined by setting at $z=0$

$$
\begin{aligned}
\psi=0, & \beta=\beta_{0}, \quad u(0)=u_{0}, \\
\alpha=\alpha_{0}, & u^{\prime}(0)=u_{0}^{\prime}
\end{aligned}
$$

With these boundary conditions we get

$$
\begin{aligned}
& a=\frac{1}{\sqrt{\beta_{0}}} u_{0}, \\
& b=\frac{\alpha_{0}}{\sqrt{\beta_{0}}} u_{0}+\sqrt{\beta_{0}} u_{0}^{\prime},
\end{aligned}
$$

and after insertion into (8.70) the particle trajectory and its derivative is

$$
\begin{gathered}
u(z)=\sqrt{\frac{\beta}{\beta_{0}}}\left(\cos \psi+\alpha_{0} \sin \psi\right) u_{0}+\sqrt{\beta \beta_{0}} \sin \psi u_{0}^{\prime}, \\
u^{\prime}(z)=\frac{1}{\sqrt{\beta_{0} \beta}}\left[\left(\alpha_{0}-\alpha\right) \cos \psi-\left(1+\alpha \alpha_{0}\right) \sin \psi\right] u_{0} \\
+\sqrt{\frac{\beta_{0}}{\beta}}(\cos \psi-\alpha \sin \psi) u_{0}^{\prime},
\end{gathered}
$$

or in matrix formulation

$$
\left(\begin{array}{cc}
C(z) & S(z) \\
C^{\prime}(z) & S^{\prime}(z)
\end{array}\right)=\left(\begin{array}{cc}
\sqrt{\frac{\beta}{\beta_{0}}}\left(\cos \psi+\alpha_{0} \sin \psi\right) & \sqrt{\beta \beta_{0}} \sin \psi \\
\frac{\alpha_{0}-\alpha}{\sqrt{\beta \beta_{0}}} \cos \psi-\frac{1+\alpha \alpha_{0}}{\sqrt{\beta \beta_{0}}} \sin \psi & \sqrt{\frac{\beta_{0}}{\beta}}(\cos \psi-\alpha \sin \psi)
\end{array}\right) .
$$

Knowledge of the betatron functions along a beam line allows us to calculate individual particle trajectories. The betatron functions can be obtained by either solving numerically the differential equation (8.59) or by using the matrix formalism (8.23) to transform phase ellipse parameters. Since the ellipse parameters in (8.23) and the betatron functions are equivalent, we have found a straightforward way to calculate their values anywhere once we have initial values at the start of the beam line. This method is particularly convenient when using computers to perform matrix multiplication. 
Transformation of the betatron functions becomes very simple in a drift space where the transformation matrix is

$$
\left(\begin{array}{cc}
C(z) & S(z) \\
C^{\prime}(z) & S^{\prime}(z)
\end{array}\right)=\left(\begin{array}{ll}
1 & z \\
0 & 1
\end{array}\right)
$$

The betatron functions at the point $z$ are from (8.26)

$$
\begin{aligned}
& \beta(z)=\beta_{0}-2 \alpha_{0} z+\gamma_{0} z^{2}, \\
& \alpha(z)=\alpha_{0}-\gamma_{0} z, \\
& \gamma(z)=\gamma_{0},
\end{aligned}
$$

with initial values $\beta_{0}, \alpha_{0}, \gamma_{0}$ taken at the beginning of the drift space.

We note that $\gamma(z)=$ const. in a drift space. This result can be derived also from the differential equation (8.60) which for $k=0$ becomes $\beta^{\prime \prime}=2 \gamma$ and the derivative with respect to $z$ is $\beta^{\prime \prime \prime}=2 \gamma^{\prime}$. On the other hand, we calculate from the first equation (8.76) the third derivative of the betatron function with respect to $z$ to be $\beta^{\prime \prime \prime}=0$. Obviously both results are correct only if the $\gamma$-function is a constant in a drift space where $k=0$.

The location of a beam waist is defined by $\alpha=0$ and occurs from (8.76) at $z_{\mathrm{w}}=\alpha_{0} / \gamma_{0}$. The betatron function increases quadratically with the distance from the beam waist (see Fig. 8.5) and can be expressed by

$$
\beta\left(z-z_{\mathrm{w}}\right)=\beta_{\mathrm{w}}+\frac{\left(z-z_{\mathrm{w}}\right)^{2}}{\beta_{\mathrm{w}}},
$$

where $\beta_{\mathrm{w}}$ is the value of the betatron function at the waist and $z-z_{\mathrm{W}}$ is the distance from the waist. From (8.77) we note that the magnitude of the betatron function away from the waist reaches large values for both large and small betatron functions at the waist. We may therefore look for conditions to obtain the minimum value for the betatron function anywhere in a drift space of length $2 L$. For this we take the derivative of $\beta$ with respect to $\beta_{\mathrm{w}}$ and get from $\left(\mathrm{d} \beta / \mathrm{d} \beta_{\mathrm{w}}=0\right)$

$$
\beta_{\mathrm{w}, \mathrm{opt}}=L
$$

Fig. 8.5 Betatron function in a drift space

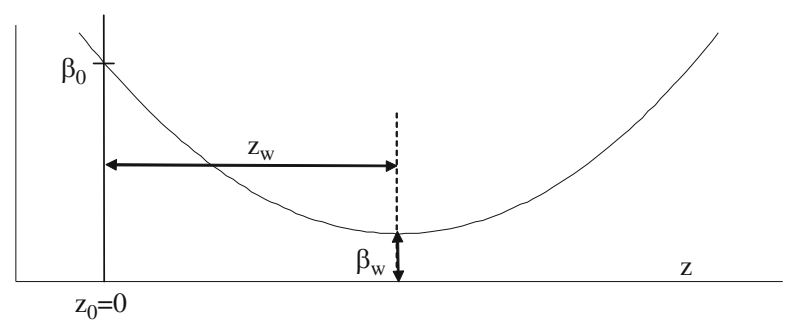


At either end of the drift space we have then

$$
\beta(L)=2 \beta_{\mathrm{w}, \mathrm{opt}}
$$

This is the optimum solution for the betatron function on either side of a drift space with length $2 L$ resulting in a minimum aperture requirement along a drift space of length $L$. The phase advance in a drift space is from (8.77)

$$
\psi(L)=\int_{0}^{L} \frac{\mathrm{d} \bar{z} / \beta_{\mathrm{w}}}{1+\left(\bar{z} / \beta_{\mathrm{w}}\right)^{2}}=\arctan \frac{L}{\beta_{\mathrm{w}}} \rightarrow \frac{\pi}{2} \quad \text { for } \quad \frac{L}{\beta_{\mathrm{w}}} \rightarrow \infty .
$$

The phase advance through a drift space of length $2 L$ is therefore never larger than $\pi$ and actually never quite reaches that value

$$
\Delta \psi_{\mathrm{drift}}<\pi
$$

\subsubsection{Beam Dynamics in Normalized Coordinates}

The form and nomenclature of the differential equation (8.52) resembles very much that of a harmonic oscillator and indeed this is not accidental since in both cases the restoring force increases linearly with the oscillation amplitude. In particle beam dynamics we find an oscillatory solution with varying amplitude and frequency and by a proper coordinate transformation we are able to make the motion of a particle look mathematically exactly like that of a harmonic oscillator. This kind of formulation of beam dynamics will be very useful in the evaluation of perturbations on particle trajectories since all mathematical tools that have been developed for harmonic oscillators will be available for particle beam dynamics.

We introduce Floquet's coordinates, or normalized coordinates through the transformation

$$
w=\frac{u}{\sqrt{\beta}}
$$

and

$$
\varphi(z)=\int_{0}^{z} \frac{\mathrm{d} \bar{z}}{v \beta(\bar{z})} .
$$

Note, that we used in here a different normalization than that selected in (8.57) to adapt more appropriately to the issues to be discussed here. With this transformation we get for the first derivative

$$
u^{\prime}=\dot{w} \frac{\sqrt{\beta}}{v \beta}+w \frac{\beta^{\prime}}{2 \sqrt{\beta}}=\frac{1}{v \sqrt{\beta}} \dot{w}-\frac{\alpha}{\sqrt{\beta}} w
$$


and for the second derivative

$$
u^{\prime \prime}=\frac{\ddot{w}}{v^{2} \beta^{3 / 2}}-w \frac{\alpha^{\prime}}{\sqrt{\beta}}-w \frac{\alpha^{2}}{\beta^{3 / 2}},
$$

where dots indicate derivatives with respect to the phase $\dot{w}=\mathrm{d} w / \mathrm{d} \varphi$, etc. We insert these expressions into (8.52) and get the general equation of motion expressed in normalized coordinates

$$
u^{\prime \prime}+k u=\frac{1}{v^{2} \beta^{3 / 2}}[\ddot{w}+\underbrace{\left(\frac{1}{2} \beta \beta^{\prime \prime}-\alpha^{2}+k \beta^{2}\right)}_{=1} v^{2} w]=p(x, y, z),
$$

where the right-hand side represents a general perturbation term $p(x, y, z)$ which was neglected so far. The square bracket is equal to unity according to (8.59) and the equation of motion takes the simple form of a harmonic oscillator with some perturbation

$$
\ddot{w}+v^{2} w-v^{2} \beta^{3 / 2} p(x, y, z)=0 .
$$

This nonlinear equation of motion can be derived from the Hamiltonian

$$
\mathcal{H}=\frac{1}{2} \dot{w}^{2}+\frac{1}{2} v^{2} w^{2}-v^{2} \beta^{3 / 2} \sum_{k=1}^{n} \beta^{\frac{k-1}{2}} \frac{p_{k}}{k} w^{k},
$$

where coupling has been ignored and

$$
p(x, z)=\sum_{k=1}^{n} p_{k} x^{k-1}=\sum_{k=1}^{n} p_{k} \beta^{\frac{k-1}{2}} w^{k-1},
$$

where $p_{k}$ is a perturbation of order $k$. Later, we will perform another canonical transformation to action-angle variables, which brings the Hamiltonian into a convenient form to exhibit effects of perturbations.

Since the parameter $v$ is constant, we have in the case of vanishing perturbations $p_{n} \equiv 0$ the exact equation of a harmonic oscillator and particles perform in this representation periodic sine-like oscillations with the frequency $v$

$$
w=w_{0} \cos (\psi+\delta) .
$$

The transformation matrix in these variables is given by

$$
\mathcal{M}(z \mid 0)=\left(\begin{array}{cc}
C(\psi) & S(\psi) \\
C^{\prime}(\psi) & S^{\prime}(\psi)
\end{array}\right)=\left(\begin{array}{cc}
\cos (\psi) & \sin (\psi) \\
-\sin (\psi) & \cos (\psi)
\end{array}\right)
$$

as can easily be derived from (8.90). 
Fig. 8.6 Ideal phase ellipse in normalized coordinates

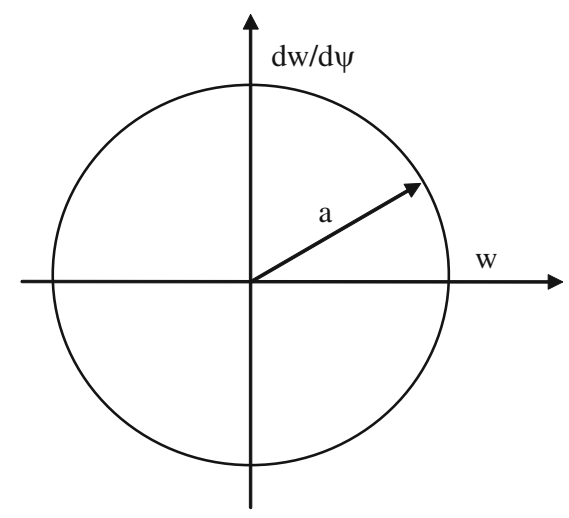

The use of normalized coordinates not only allows us to treat particle beam dynamics equivalent to a harmonic oscillator but is also convenient in the discussions of perturbations or aberrations. In phase space each particle performs closed trajectories in the form of an ellipse which we called the phase ellipse. In Cartesian coordinates this ellipse, however, continuously changes its shape and orientation and correlations between two locations are not always obvious. If we use normalized coordinates, the unperturbed phase ellipse becomes an invariant circle as shown in Fig. 8.6.

From (8.82) we get with $u(z)=a \sqrt{\beta(z)} \cos \psi(z)$ where $\psi(z)=v \varphi(z)$

$$
\begin{aligned}
w(\varphi) & =\frac{u}{\sqrt{\beta}}=a \cos \psi, \\
\frac{\mathrm{d} w}{\mathrm{~d} \psi} & =\sqrt{\beta} u^{\prime}+\frac{\alpha}{\sqrt{\beta}} u=-a \sin \psi,
\end{aligned}
$$

and after elimination of the phase the Courant-Snyder invariant becomes

$$
w^{2}+\left(\frac{\mathrm{d} w}{\mathrm{~d} \psi}\right)^{2}=a^{2},
$$

where $a$ is the betatron oscillation amplitude.

The equation of motion (8.87) is now ready to be transformed into action-angle variables. The constancy of the action $J$ is now synonymous with the CourantSnyder invariant (5.59) or the constancy of the beam emittance.

$$
J=\frac{1}{2} v\left(\gamma u^{2}+2 \alpha u u^{\prime}+\beta u^{\prime 2}\right)=\frac{1}{2} v \epsilon .
$$

In $(\psi, J)$ phase-space, the particle moves along a circle with radius $J$ at a revolution frequency $v$. The motion is uniform, periodic and stable. Including the independent variable $\varphi$ to form a three-dimensional phase-space, we find a particle 


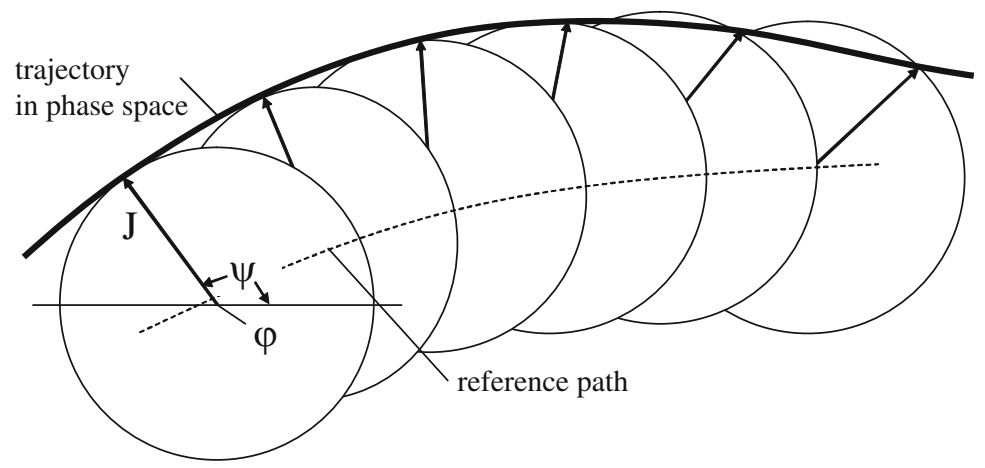

Fig. 8.7 Unperturbed particle trajectories in $(\psi, J, \varphi)$ phase-space

to spiral along the surface of a torus as shown in Fig. 8.7. The ensemble of all particles oscillating with the same amplitude $J$ follow spirals occupying the full surface of the torus.

This result is not particularly interesting in itself since it only corroborates what we have found earlier for harmonic oscillators with simpler mathematical tools. The circle in $(\psi, J)$-phase space, however, provides us with a reference against which to compare perturbed motions and derive stability criteria. Indeed, we will later use canonical transformations to eliminate well-known linear motions, like the circular motion of an unperturbed harmonic oscillator in $(\psi, J)$-space to exhibit more clearly the effects of perturbation only. Including perturbations into the Hamiltonian (5.57) allows the determination of perturbed tunes and study resonance phenomena. Having defined canonical variables for the system, we also will be able to study the evolution of particle beams by applying Vlasov's equation in Sect. 12.1. The Fokker-Planck equation finally will allow us to determine beam parameters even in the presence of statistical events.

We have chosen the betatron phase $\psi$ as the independent variable and the particles cover one full turn along the phase "ellipse" for each betatron oscillation. This is a convenient way of representation in beam transport systems, yet, for circular accelerators we find it more useful to define $\varphi=\psi / v$ as the independent variable in which case the particle rotation frequency in phase space is the same as that in the ring. This is particularly convenient when we discuss field and alignment perturbations which occur periodically in a ring and allow the application of Fourier techniques.

\subsection{Dispersive Systems}

Beam guidance and focusing is performed by applying Lorentz forces and the effects of these fields on particle trajectories depend on the momentum of the particles. So far, we have derived beam dynamics for particles with ideal momenta 
for which the beam transport system is designed. To properly describe the dynamics of a real particle beam we must include chromatic effects caused by an error in the beam energy or by a spread of energies within the particle beam. In Sect. 5.5.4; the perturbation due to a momentum error has been derived and expressed in terms of a dispersion. Continuing the formulation of beam dynamics in terms of transformation matrices we derive in this section transformation matrices for particles with a momentum error.

\subsubsection{Analytical Solution}

The dispersion function has been derived as a special solution to a chromatic perturbation term in (5.81) where

$$
D(z)=\int_{0}^{z} \kappa(\bar{z})[S(z) C(\bar{z})-C(z) S(\bar{z})] \mathrm{d} \bar{z}
$$

describes the dispersion function in a beam transport line. There is no contribution to the dispersion function unless there is at least one bending magnet in the beam line. Knowledge of the location and strength of bending magnets, together with the principal solutions of the equations of motion, we may calculate the dispersion anywhere along the beam transport line by integration of (8.96).

Similar to the matrix formalism for betatron oscillations we would also like to apply the same formalism for the dispersion function. For this we note that the particle deviation $u$ from the reference path is composed of the betatron motion and a displacement due to an energy error $u=u_{\beta}+u_{\delta}$. The transformation matrix is therefore a composite of both contributions and can be expressed by

$$
\left(\begin{array}{c}
u(z) \\
u^{\prime}(z) \\
\delta
\end{array}\right)=\mathcal{M}\left(\begin{array}{c}
u_{\beta}\left(z_{0}\right) \\
u_{\beta}^{\prime}\left(z_{0}\right) \\
\delta
\end{array}\right)+\mathcal{M}\left(\begin{array}{c}
u_{\delta}\left(z_{0}\right) \\
u_{\delta}^{\prime}\left(z_{0}\right) \\
\delta
\end{array}\right)
$$

where $\mathcal{M}$ is the $3 \times 3$ transformation matrix, $\delta$ the relative momentum error and $u_{\delta}(z)=D(z) \delta$ and $u_{\delta}^{\prime}(z)=D^{\prime}(z) \delta$ the displacement and slope, respectively, of the reference path for particles with a momentum error $\delta$. Equation (8.97) can also be applied to the dispersion function alone by setting the betatron oscillation amplitudes to zero and the momentum error $\delta=1$ for

$$
\left(\begin{array}{c}
D(z) \\
D^{\prime}(z) \\
1
\end{array}\right)=\mathcal{M}\left(\begin{array}{c}
D\left(z_{0}\right) \\
D^{\prime}\left(z_{0}\right) \\
1
\end{array}\right)
$$


By determining the transformation matrices for individual bending magnets, we are in a position to calculate in matrix formulation the dispersion function anywhere along a beam transport line.

In the deflecting plane of a pure sector magnet the principal solutions are with $K=\kappa^{2}=1 / \rho^{2}$

$$
\left(\begin{array}{cc}
C(z) & S(z) \\
C^{\prime}(z) & S^{\prime}(z)
\end{array}\right)=\left(\begin{array}{cc}
\cos (\kappa z) & \rho \sin (\kappa z) \\
-\kappa \sin (\kappa z) & \cos (\kappa z)
\end{array}\right)
$$

With $\rho=$ const we get from (8.96) and (8.99) for the dispersion function within the magnet

$$
\begin{aligned}
D(z) & =\sin (\kappa z) \int_{0}^{z} \cos (\kappa \bar{z}) \mathrm{d} \bar{z}-\cos (\kappa z) \int_{0}^{z} \sin (\kappa \bar{z}) \mathrm{d} \bar{z} \\
& =\rho_{0}[1-\cos (\kappa z)] \\
D^{\prime}(z) & =\sin (\kappa z) .
\end{aligned}
$$

Particles with momentum error $\delta$ follow an equilibrium path given by $D(z) \delta$ which can be determined experimentally by observing the beam path for two different values of the beam momentum $\delta_{1}$ and $\delta_{2}$. The difference of the two paths divided by the momentum difference is the dispersion function $D(z)=\Delta u /\left(\delta_{2}-\delta_{1}\right)$. In practical applications this is done either by changing the beam energy or by changing the strength of the bending magnets. In circular electron accelerators, however, only the first method will work since the electrons always adjust the energy through damping to the energy determined by the magnetic fields. In circular electron accelerators, we determine the dispersion function by changing the rffrequency which enforces a change in the particle energy as we will discuss later in Chap. 9.

\subsubsection{3 × 3-Transformation Matrices}

From (8.99) and (8.100) we may form now $3 \times 3$-transformation matrices. In the deflecting plane of a pure sector magnet of arc length $\ell$ such a transformation matrix is

$$
\mathcal{M}_{\mathrm{s}, \rho}(\ell \mid 0)=\left(\begin{array}{ccc}
\cos \theta & \rho \sin \theta & \rho(1-\cos \theta) \\
-\frac{1}{\rho} \sin \theta & \cos \theta & \sin \theta \\
0 & 0 & 1
\end{array}\right)
$$


where $\theta=\ell / \rho$ is the deflection angle of the magnet. In the non deflecting plane, the magnet behaves like a drift space with $\frac{1}{\rho}=0, k=0$ and arc length $\ell$

$$
\mathcal{M}_{\mathrm{s}, 0}(\ell \mid 0)=\left(\begin{array}{ccc}
C(z) & S(z) & 0 \\
C^{\prime}(z) & S^{\prime}(z) & 0 \\
0 & 0 & 1
\end{array}\right)
$$

For a synchrotron magnet of the sector type we get from (7.38) in analogy to (8.100), replacing $\kappa$ by $\sqrt{K}$ and with $\Theta=\sqrt{k+\kappa^{2}} \ell$ and $\kappa=1 / \rho$ for the case of a focusing synchrotron magnet

$$
\mathcal{M}_{\text {sy }, \mathrm{f}}(\ell \mid 0)=\left(\begin{array}{ccc}
\cos \Theta & \frac{\sin \Theta}{\sqrt{K}} & \frac{1-\cos \Theta}{\rho K} \\
-\sqrt{K} \sin \Theta & \cos \Theta & \frac{\sin \Theta}{\rho \sqrt{K}} \\
0 & 0 & 1
\end{array}\right)
$$

and for a defocusing synchrotron magnet

$$
\mathcal{M}_{\mathrm{sy}, \mathrm{d}}(\ell \mid 0)=\left(\begin{array}{ccc}
\cosh \Theta & \frac{\sinh \Theta}{\sqrt{|K|}} & \frac{\cosh \Theta-1}{\rho_{0}|K|} \\
\sqrt{|K|} \sinh \Theta & \cosh \Theta & \frac{\sinh \Theta}{\rho_{0} \sqrt{|K|}} \\
0 & 0 & 1
\end{array}\right)
$$

where $\Theta=\sqrt{\left|k+\kappa_{0}^{2}\right|} \ell$.

In case of a rectangular magnet without field gradient, we multiply the matrix for a sector magnet by the transformation matrices for endfield-focusing. Since these end effects act like quadrupoles we have no new contribution to the dispersion and the transformation matrices for each endfield are

$$
\mathcal{M}_{\mathrm{e}}=\left(\begin{array}{ccc}
1 & 0 & 0 \\
\kappa \tan (\theta / 2) & 1 & 0 \\
0 & 0 & 1
\end{array}\right)
$$

With these endfield matrices the chromatic transformation matrix for a rectangular bending magnet in the deflecting plane is obtained from (8.103) with $\mathcal{M}_{\mathrm{r}, \rho}=$ $\mathcal{M}_{\mathrm{e}} \mathcal{M}_{\text {sy, } \rho} \mathcal{M}_{\mathrm{e}}$ for $k=0$

$$
\mathcal{M}_{\mathrm{r}, \rho}(\ell \mid 0)=\left(\begin{array}{ccc}
1 & \rho \sin \theta & \rho(1-\cos \theta) \\
0 & 1 & 2 \tan (\theta / 2) \\
0 & 0 & 1
\end{array}\right)
$$

Similarly, we can derive the transformation matrices for rectangular synchrotron magnets. 
Only bending magnets create a dispersion. Therefore the transformation matrices of other magnets or drift spaces are extended to $3 \times 3$ matrices by adding a third column and row with all elements equal to zero and $M_{33}=1$.

\subsubsection{Linear Achromat}

Frequently it is necessary in beam transport systems to deflect a particle beam. If this is done in an arbitrary way an undesirable finite dispersion function will remain at the end of the deflecting section. Special magnet arrangements exist which allow to bend a beam without generating a residual dispersion. Such magnet systems composed of only bending magnets and quadrupoles are called linear achromats.

Consider, for example, an off momentum particle travelling along the ideal path of a straight beam line. At some location, we insert a bending magnet and the off-momentum particle will be deflected by a different angle with respect to particles with correct momenta. The difference in the deflection angle appears as a displacement in phase space from the center to a finite value $\Delta \dot{w}=\delta D(z) / \sqrt{\beta}$. From here on, the off momentum reference path follows the dispersion function $D(z) \delta$ and the particle performs betatron oscillations in the form of circles until another bending magnet further modifies or compensates this motion (Fig. 8.8).

In case a second bending magnet is placed half a betatron oscillation downstream from the first causing the same deflection angle the effect of the first magnet can be compensated completely and the particle continues to move along the ideal path again. A section of a beam transport line with this property is called an achromat.

Figure 8.9 displays an achromatic section proposed by Panofsky [10] which may be used as a building block for curved transport lines or circular accelerators. This section is composed of a symmetric arrangement of two bending magnets with a

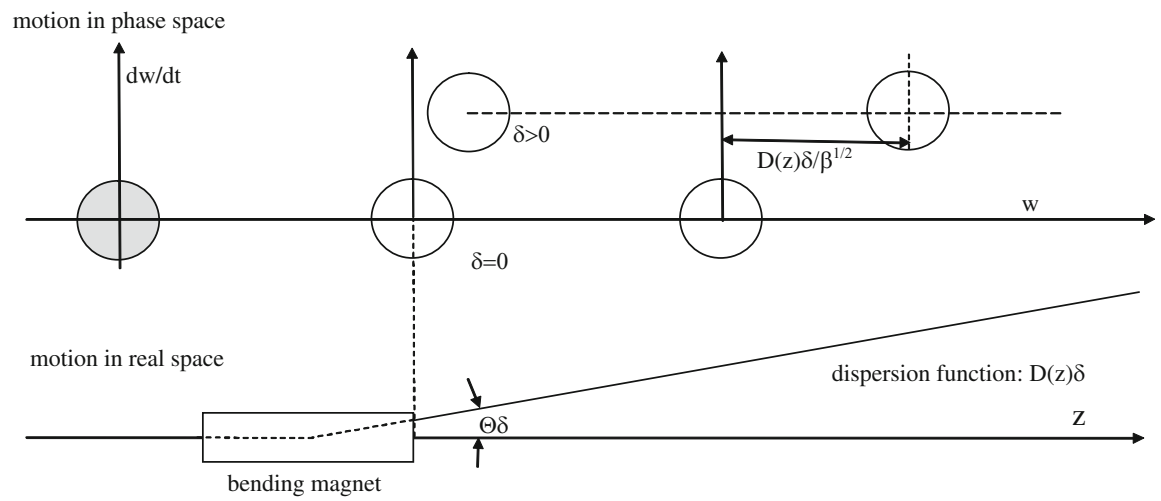

Fig. 8.8 Trajectory of an off momentum particle through a chromatic beam transport section 


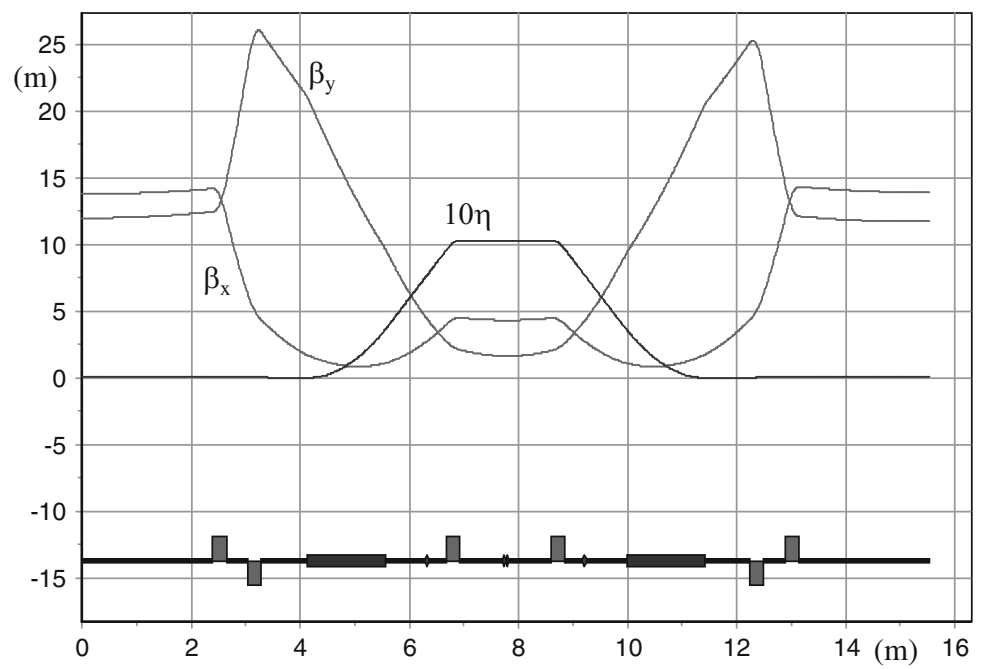

Fig. 8.9 Double bend achromat $[3,10]$

quadrupole in the center and is also know as a double bend achromat or a ChasmanGreen lattice [3].

General conditions for linear achromats have been discussed in Sect. 7.4 and we found that the integrals

$$
I_{\mathrm{S}}=\int_{0}^{z} \kappa(\bar{z}) S(\bar{z}) \mathrm{d} \bar{z}=0
$$

and

$$
I_{\mathrm{c}}=\int_{0}^{z} \kappa(\bar{z}) C(\bar{z}) \mathrm{d} \bar{z}=0,
$$

must vanish for a lattice section to become achromatic. For a double bend achromat this can be accomplished by a single parameter or quadrupole if adjusted such that the betatron phase advance between the vertex points of the bending magnet is $180^{\circ}$. A variation of this lattice, the triple bend achromat $[5,8]$, is shown in Fig. 8.10, where a third bending magnet is inserted for practical reasons to provide more locations to install sextupoles for chromatic corrections. Magnet arrangements as shown in Figs. 8.9 and 8.10 are dispersion free deflection units or linear achromats. This achromat is focusing only in the deflecting plane but defocusing in the nondeflecting plane which must be compensated by external quadrupole focusing or, since there are no special focusing requirements for the nondeflecting plane, by either including a field gradient in the pole profile of the bending magnets [6] or additional quadrupoles between the bending magnets. In a beam transport line this 


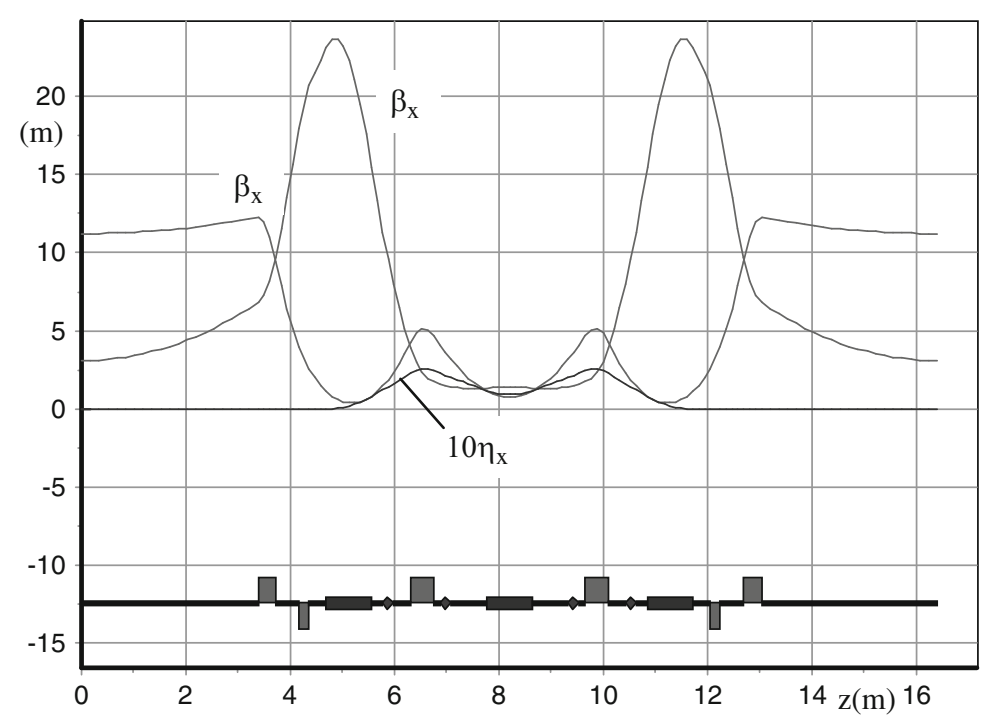

Fig. 8.10 Triple bend achromat [5]

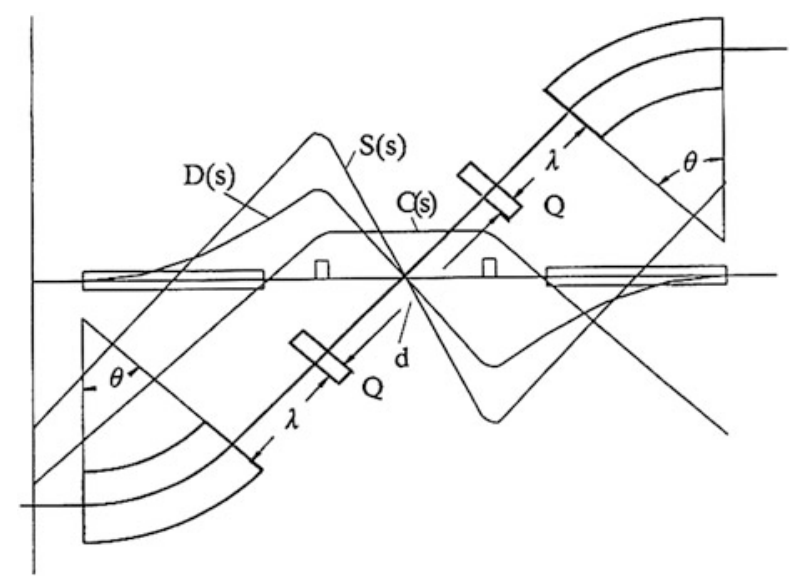

Fig. 8.11 Achromatic beam translation

achromat can be used for diagnostic purposes to measure the energy and energy spread of a particle beam as will be discussed in more detail in Sect. 8.4.5

A further variation of the lattice in Fig. 8.9 has been proposed by Steffen [10] to generate an achromatic beam translation as shown in Fig. 8.11. In this case, the total phase advance must be $360^{\circ}$ because the integral $I_{\mathrm{c}}$ would not vanish anymore for reasons of symmetry. We use therefore stronger focusing to make $I_{\mathrm{c}}$ vanish because both the bending angle and the cosine like function change sign. 
Achromatic properties are obtained again for parameters meeting the condition [10]

$$
\rho \tan (\theta / 2)+\lambda=\frac{1}{\sqrt{k}} \frac{d \sqrt{k} \cos \varphi+2 \sin \varphi}{d \sqrt{k} \sin \varphi-2 \cos \varphi},
$$

where $\varphi=\sqrt{k} \ell$ and $k, \ell$ the quadrupole strength and length, respectively. The need for beam translation occurs frequently during the design of beam transport lines. Solutions exist to perform such an achromatic translation but the required focusing is much more elaborate and may cause significantly stronger aberrations compared to a simple one directional beam deflection of the double bend achromat type.

Utilizing symmetric arrangements of magnets, deflecting achromats can be composed from bending magnets only [10]. One version has become particularly important for synchrotron radiation sources, where wiggler magnets are used to produce high intensity radiation. Such triple bend achromat are composed of a row of alternately deflecting bending magnets which do not introduce a net deflection on the beam. Each unit or period of such a wiggler magnet (Fig. 8.12) is a linear achromat.

The transformation of the dispersion through half a wiggler unit is the superposition of the dispersion function from the first magnet at the end of the second magnet plus the contribution of the dispersion from the second magnet. In matrix formulation and for hard edge rectangular magnets the dispersion at the end of half a wiggler period is

$$
\left(\begin{array}{l}
D_{w} \\
D_{w}^{\prime}
\end{array}\right)=\left(\begin{array}{l}
-\rho_{0}(1-\cos \theta) \\
-2 \tan (\theta / 2)
\end{array}\right)+\left(\begin{array}{cc}
1 & \ell_{w} \\
0 & 1
\end{array}\right)\left(\begin{array}{l}
\rho_{0}(1-\cos \theta) \\
2 \tan (\theta / 2)
\end{array}\right),
$$

where $\rho>0, \theta=\ell_{\mathrm{w}} \rho$ and $\ell_{\mathrm{w}}$ the length of one half wiggler pole (see Fig. 8.12). Evaluation of (8.110) gives the simple result

$$
\begin{aligned}
& D_{\mathrm{w}}=2 \ell_{\mathrm{w}} \tan (\theta / 2), \\
& D_{\mathrm{w}}^{\prime}=0 .
\end{aligned}
$$

The dispersion reaches a maximum in the middle of the wiggler period and vanishes again for reasons of symmetry at the end of the period. For sector magnets

Fig. 8.12 Wiggler achromat

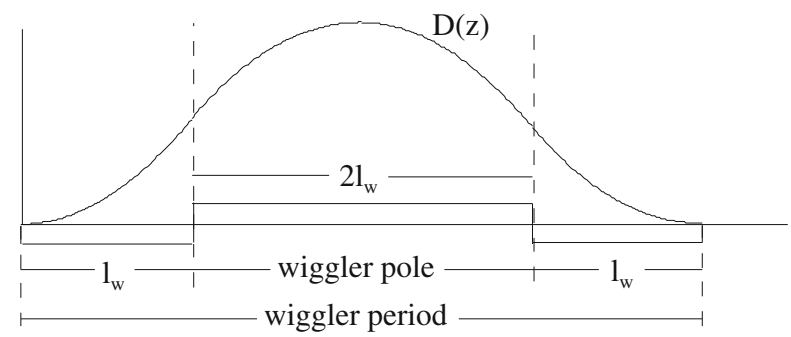


we would have obtained the same results. Each full wiggler period is therefore from a beam optics point of view a linear achromat. Such an arrangement can also be used as a spectrometer by placing a monitor in the center, where the dispersion is large. For good momentum resolution, however, beam focusing must be provided in the deflecting plane upstream of the bending magnets to produce a small focus at the beam monitors as will be discussed in the next section.

The examples of basic lattice designs discussed in this section are particularly suited for analytical treatment. In practice, modifications of these basic lattices are required to meet specific boundary conditions making, however, analytical treatment much more complicated. With the availability of computers and numerical lattice design codes, it is prudent to start with basic lattice building blocks and then use a fitting program for modifications to meet particular design goals.

\subsubsection{Spectrometer}

Although the dispersion has been treated as a perturbation it is a highly desired feature of a beam line to determine the energy or energy distribution of a particle beam. Such a beam line is called a spectrometer for which many different designs exist. A specially simple and effective spectrometer can be made with a single $180^{\circ}$ sector magnet $[2,9]$. For such a spectrometer, the transformation matrix is from (8.101)

$$
\mathcal{M}\left(180^{\circ}\right)=\left(\begin{array}{ccc}
-1 & 0 & 2 \rho_{0} \\
0 & -1 & 0 \\
0 & 0 & -1
\end{array}\right) \text {. }
$$

In this spectrometer all particles emerging from a small target (Fig. 8.13) are focused to a point again at the exit of the magnet. The focal points for different energies, however, are separated spatially due to dispersion. Mathematically, this is

Fig. 8.13 Hundred and eighty degree spectrometer

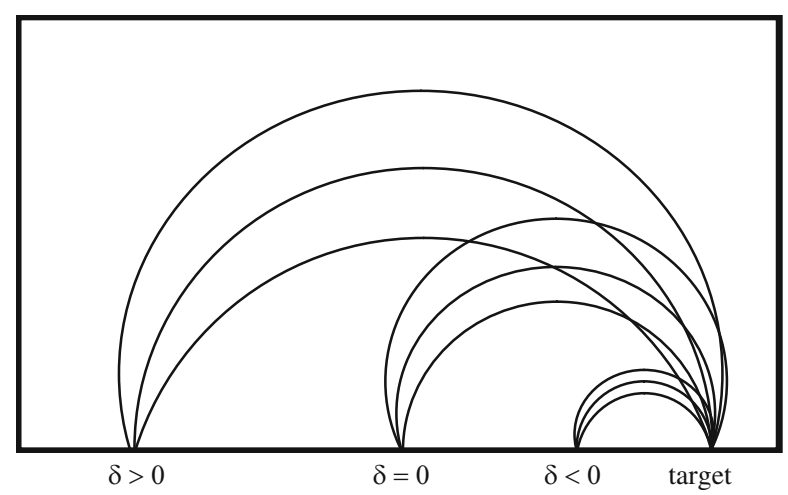


evident since the particle trajectories at the end of the magnet are given by

$$
x=-x_{0}+2 \rho \delta
$$

where $x_{0}$ is the starting point of a particle within the target, and showing different positions $x$ for different energies $\delta$. The energy dispersion of the secondary particles is $\Delta x=2 \rho \delta$.

The image point is independent of $x_{0}^{\prime}$ and only proportional to $\delta$ with a large proportionality factor which allows a large energy resolution. While this spectrometer seems to have almost ideal features it is also an example of the limitations of perturbation methods. For larger values of $\delta$ of the order of several percent higher order terms cannot be neglected anymore. Inclusion of such terms, for example, will first tilt and then bend the focal plane at the end of the magnet.

More sophisticated spectrometers including focusing to accept large emittance beams have been devised with special efforts to reduce the effects of aberrations. It is not the intend of this text to discuss in detail such designs. More comprehensive overviews for spectrometers with further references can be found for example in $[1,10]$. In the treatment of this spectrometer we have ignored the nondeflecting plane. Since there is no focusing, particles are widely spread out in this plane at the end of the magnet. Practical versions of this spectrometer, therefore, include a focusing term in the nondeflecting plane in such a way that the resulting focusing is the same in both planes [7].

\subsubsection{Measurement of Beam Energy Spectrum}

Frequently it is desirable to determine experimentally the particle energy and energy spread. Basically only one bending magnet is needed to perform this experiment. The finite beam size of the monochromatic part of the beam will greatly influence the resolution of the energy measurement. Optimum resolution is achieved if some focusing is included and the measurement is performed at a location, where the beam size is small while the dispersion is large. In Fig. 8.14 particle beams at two different energies are shown in phase space, where both beam centers are separated by the dispersion and its slope.

In reality no such separation exists since we have a spread of energies rather than two distinct energies. This energy spread is mixed with the spread in phase space of the beam emittance and beams of different energies can only be separated completely if the relative energy difference is at least

$$
\delta_{\min }=\frac{\delta p}{p_{0}} \geq \frac{2 E_{\mathrm{b}}}{D}=2 \frac{\sqrt{\epsilon \beta}}{D},
$$




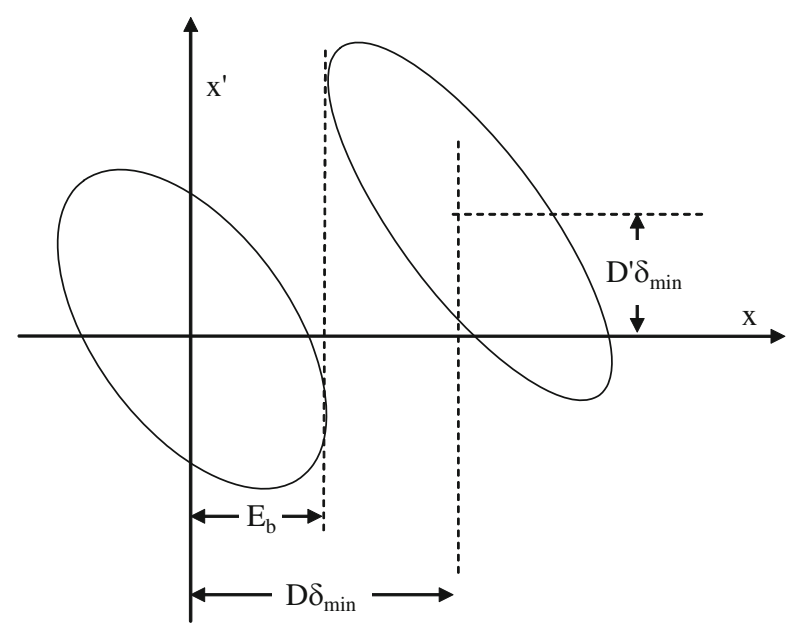

Fig. 8.14 Momentum resolution in phase space

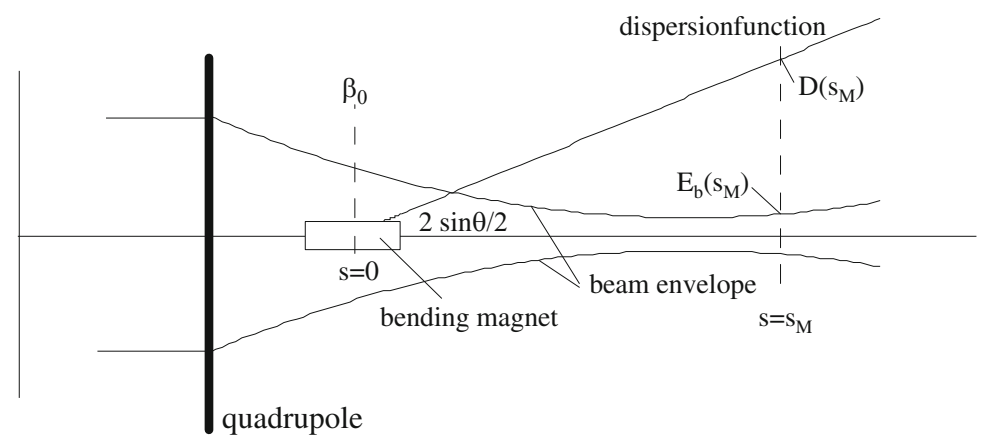

Fig. 8.15 Measurement of the momentum spectrum

where $E_{\mathrm{b}}=\sqrt{\epsilon \beta}$ is the beam envelope. To maximize the energy resolution the beam size $E_{\mathrm{b}}$ should be small and the dispersion $D(z)$ large. From Fig. 8.14 we note therefore, that for a given beam emittance and dispersion the energy resolution can be improved significantly if the measurement is performed at or close to a beam waist, where $\beta$ reaches a minimum.

To derive mathematical expressions for the energy resolution and conditions for the maximum energy resolution $1 / \delta_{\min }$ we assume a beam line as shown in Fig. 8.15 with the origin of the coordinate system $z=0$ in the center of the bending magnet. The salient features of this beam line is the quadrupole followed by a bending magnet. With this sequence of magnets we are able to focus the particle beam in the deflection plane while leaving the dispersion unaffected. In case of a reversed magnet sequence the dispersion function would be focused as well compromising the energy resolution. 
Transforming the dispersion (8.100) back from the end of the sector bending magnet to the middle of the magnet we get the simple result

$$
\left(\begin{array}{l}
D_{0} \\
D_{0}^{\prime}
\end{array}\right)=\left(\begin{array}{cc}
\cos \frac{\theta}{2} & -\rho_{0} \sin \frac{\theta}{2} \\
\frac{1}{\rho_{0}} \sin \frac{\theta}{2} & \cos \frac{\theta}{2}
\end{array}\right)\left(\begin{array}{l}
\rho_{0}(1-\cos \theta) \\
\sin \theta
\end{array}\right)=\left(\begin{array}{c}
0 \\
2 \sin \frac{\theta}{2}
\end{array}\right),
$$

The dispersion appears to originate in the middle of the magnet with a slope $D_{0}^{\prime}=2 \sin \theta / 2$. At a distance $z$ from the middle of the bending magnet the betatron function is given by $\beta(z)=\beta_{0}-2 \alpha_{0} z+\gamma_{0} z^{2}$ where $\left(\beta_{0}, \alpha_{0}, \gamma_{0}\right)$ are the Twiss functions in the middle of the bending magnet, and the dispersion is $D(z)=2 \sin (\theta / 2) z$. Inserting these expressions into (8.114) we can find the location $z_{\mathrm{M}}$ for maximum momentum resolution by differentiating $\delta_{\min }$ with respect to $z$. Solving $\mathrm{d} \delta_{\min } / \mathrm{d} z=0$ for $z$ we get

$$
z_{\mathrm{M}}=\frac{\beta_{0}}{\alpha_{0}}
$$

and the maximum momentum resolution is

$$
\delta_{\min }^{-1}=\frac{\sqrt{\beta_{0}} \sin (\theta / 2)}{\sqrt{\epsilon}} .
$$

The best momentum resolution for a beam with emittance $\epsilon$ is achieved if both the bending angle $\theta$ and the betatron function $\beta_{0}$ in the middle of the bending magnet are large. From condition (8.116), we also find $\alpha_{0}>0$ which means that the beam must be converging to make a small spot size at the observation point downstream of the bending magnet. With (8.76) we find that $z_{\mathrm{M}}=\beta_{0} / \alpha_{0}=-\beta_{\mathrm{M}} / \alpha_{\mathrm{M}}$ and from the beam envelope $E_{\mathrm{b}}^{2}=\epsilon \beta_{\mathrm{M}}$ at $z=z_{\mathrm{M}}$ we get the derivative $2 E_{\mathrm{b}} E_{\mathrm{b}}^{\prime}=\epsilon \beta_{\mathrm{M}}^{\prime}=-2 \epsilon \alpha_{\mathrm{M}}$. With this and $D / D^{\prime}=z$, the optimum place to measure the energy spread of a particle beam is at

$$
z_{\mathrm{M}}=\frac{D\left(z_{\mathrm{M}}\right)}{D^{\prime}\left(z_{\mathrm{M}}\right)}=\frac{E_{\mathrm{b}}\left(z_{\mathrm{M}}\right)}{E_{\mathrm{b}}^{\prime}\left(z_{\mathrm{M}}\right)}
$$

It is interesting to note that the optimum location $z_{\mathrm{M}}$ is not at the beam waist, where $\beta(z)$ reaches a minimum, but rather somewhat beyond the beam waist, where $D / \sqrt{\beta}$ is maximum.

At this point we may ask if it is possible through some clever beam focusing scheme to improve this resolution. Analogous to the previous derivation we look for the maximum resolution $\delta_{\min }^{-1}=D(z) /[2 \sqrt{\epsilon \beta(z)}]$. The dispersion is expressed in terms of the principal solution $D(z)=S(z) D^{\prime}(0)$ and $D^{\prime}(z)=S^{\prime}(z) D^{\prime}(0)$ since $D(0)=0$. The betatron function is given by $\beta(z)=C^{2}(z) \beta_{0}-2 C(z) S(z) \alpha_{0}+$ $S^{2}(z) \gamma_{0}$ and the condition for maximum resolution turns out to be $\alpha / \beta=-D^{\prime} / D$. 
With this, we get the resolution

$$
\delta_{\min }^{-1}=\frac{D(z)}{2 \sqrt{\epsilon \beta}}=\frac{S(z) D_{0}^{\prime}}{2 \sqrt{\epsilon \beta}}=\frac{\sin (\theta / 2)}{\sqrt{\epsilon \beta}} S(z)
$$

and finally with $S(z)=\sqrt{\beta_{0} \beta(z)} \sin \psi(z)$

$$
\delta_{\min }^{-1}=\frac{\sqrt{\beta_{0}} \sin (\theta / 2)}{\sqrt{\epsilon}} \sin \psi(z) \leq \frac{\sqrt{\beta_{0}} \sin (\theta / 2)}{\sqrt{\epsilon}},
$$

which is at best equal to result (8.117) for $\psi(z)=90^{\circ}$. The momentum resolution is never larger than in the simple setup of Fig. 8.15 no matter how elaborate a focusing lattice is employed.

If more than one bending magnet is used the resolution may be increased if the betatron phases between the magnets $\psi\left(z_{i}\right)$ and the place of the measurement $\psi\left(z_{\mathrm{M}}\right)$ are chosen correctly. The resolution then is

$$
\delta_{\min }^{-1}=\frac{1}{\sqrt{\epsilon}} \sum_{i} \sqrt{\beta_{0 i}} \sin \left(\theta_{i} / 2\right) \sin \left[\psi\left(z_{\mathrm{M}}\right)-\psi\left(z_{i}\right)\right],
$$

where the sum is taken over all magnets $i$. Such an energy resolving system is often used in beam transport lines to filter out a small energy band of a particle beam with a larger energy spread. In this case a small slit is placed at the place for optimum momentum resolution $\left(z=z_{\mathrm{M}}\right)$. Of course, for highly relativistic electrons the momentum spectrum is virtually equal to the energy spectrum.

This discussion is restricted to linear beam optics which does not address problems caused by nonlinear effects and geometric as well as chromatic aberrations.

\subsubsection{Path Length and Momentum Compaction}

The existence of different reference paths implies that the path length between two points of a beam transport line may be different as well for different particle momenta. We will investigate this since the path length is of great importance as will be discussed in detail in Chap. 9. In preparation for this discussion, we derive here the functional dependencies of the path length on momentum and focusing lattice.

The path length along a straight section of the beam line depends on the angle of the particle trajectory with the reference path. In this chapter we are interested only in linear beam dynamics and may neglect such second order corrections to the path length. The only linear contribution to the path length comes from the curved sections of the beam transport line. The total path length is therefore given by

$$
L=\int(1+\kappa x) \mathrm{d} z
$$


We evaluate (8.122) along the reference path, where $x=D(z) \delta$. First we find the expected result $L_{0}=\int \mathrm{d} z$ for $\delta=0$, which is the ideal design length of the beam line or the design circumference of a circular accelerator. The deviation from this ideal length is then

$$
\Delta L=\delta \int \kappa(z) D(z) \mathrm{d} z
$$

The variation of the path length with momentum is determined by the momentum compaction factor, defined by

$$
\alpha_{\mathrm{c}}=\frac{\Delta L / L_{0}}{\delta} \quad \text { with } \quad \delta=\frac{\Delta p}{p} .
$$

Its numerical value can be calculated with (8.123) and is

$$
\alpha_{\mathrm{c}}=\frac{1}{L_{0}} \int_{0}^{L_{0}} \kappa(z) D(z) \mathrm{d} z=\left\langle\frac{D(z)}{\rho}\right\rangle .
$$

In this approximation the path length variation is determined only by the dispersion function in bending magnets and the path length depends only on the energy of the particles. To prepare for the needs of longitudinal phase focusing in Chap. 9, we will not only consider the path length but also the time it takes a particle to travel along that path. If $L$ is the path length, the travel time is given by

$$
\tau=\frac{L}{c \beta} .
$$

Here $\beta=v / c$ is the velocity of the particle and is not to be confused with the betatron function. The variation of $\tau$ gives by logarithmic differentiation

$$
\frac{\Delta \tau}{\tau}=\frac{\Delta L}{L}-\frac{\Delta \beta}{\beta} .
$$

With $\Delta L / L=\alpha_{\mathrm{c}} \delta$ and $c p=\beta E$ we get $\mathrm{d} p / p=\mathrm{d} \beta / \beta+\mathrm{d} E / E$ and with $\mathrm{d} E / E=$ $\beta^{2} \mathrm{~d} p / p$ we can solve for $\mathrm{d} \beta / \beta=\left(1 / \gamma^{2}\right) \mathrm{d} p / p$, where $\gamma=E / m c^{2}$ is the relativistic factor. From (8.127) we have then

$$
\frac{\Delta \tau}{\tau}=-\left(\frac{1}{\gamma^{2}}-\alpha_{c}\right) \frac{\mathrm{d} p}{p}=-\eta_{\mathrm{c}} \frac{\mathrm{d} p}{p}
$$

and call the combination

$$
\eta_{\mathrm{c}}=\left(\frac{1}{\gamma^{2}}-\alpha_{\mathrm{c}}\right)
$$


the momentum compaction. The energy

$$
\gamma_{\mathrm{t}}=\frac{1}{\sqrt{\alpha_{\mathrm{c}}}}
$$

for which the momentum compaction vanishes is called the transition energy which will play an important role in phase focusing. Below transition energy the arrival time is determined by the actual velocity of the particles while above transition energy the particle speed is so close to the speed of light that the arrival time of a particle with respect to other particles depends more on the path length than on its speed. For a circular accelerator we may relate the time $\tau_{\mathrm{r}}$ a particle requires to follow a complete orbit to the revolution frequency $\omega_{\mathrm{r}}$ and get from (8.128)

$$
\frac{\mathrm{d} \omega_{\mathrm{r}}}{\omega_{\mathrm{r}}}=-\frac{\mathrm{d} \tau_{\mathrm{r}}}{\tau_{\mathrm{r}}}=\eta_{\mathrm{c}} \frac{\mathrm{d} p}{p} .
$$

For particles above transition energy this quantity is negative which means a particle with a higher energy needs a longer time for one revolution than a particle with a lower energy. This is because the dispersion function causes particles with a higher energy to follow an equilibrium orbit with a larger average radius compared to the radius of the ideal orbit.

By special design of the lattice one could generate an oscillating dispersion function in such a way as to make the momentum compaction $\eta_{\mathrm{c}}$ to vanish. Such a transport line or circular accelerator would be isochronous to the approximation used here. Due to higher order aberrations, however, there are nonlinear terms in the dispersion function which together with an energy spread in the beam cause a spread of the revolution frequency compromising the degree of isochronicity. These higher order corrections are discussed later in Chap. 9.4.1.

\section{Problems}

8.1 (S). Particle trajectories in phase space follow the shape of an ellipse. Derive a transformation of the phase space coordinates $\left(u, u^{\prime}\right)$ to coordinates $(w, \dot{w})$ such that the particle trajectories are circles with the radius $\beta \epsilon$.

8.2 (S). Use (8.18) for the phase ellipse and prove that the area enclosed by the ellipse is indeed equal to $\pi \epsilon$.

8.3 (S). Show that the transformation of the beam matrix (8.41) is consistent with the transformation of the lattice functions.

8.4 (S). Sometimes two FODO channels of different parameters must be matched. Show that a lattice section can be designed with a phase advance of $\Delta \psi_{x}=\Delta \psi_{y}=$ $\pi / 2$, which will provide the desired matching of the betatron functions from the 
symmetry point of one FODO channel to the symmetry point of the other channel. Such a matching section is also called a quarter wavelength transformer. Does this transformer also work for curved FODO channels, where the dispersion is finite?

8.5. Construct a beam bump like in problem 7.6 but now use betatron and phase functions for the solution. What are the criteria for either $A_{\mathrm{M}}$ being the maximum displacement or not? For which phase $\psi_{\mathrm{M}}$ would the dipole fields be minimum? Is there a more economic solution for a symmetric beam bump with an amplitude $A_{\mathrm{M}}$ in the center of $\mathrm{QD}_{2}$ ?

8.6. Consider a ring made from an even number of FODO cells. To provide component free space we cut the ring along a symmetry line through the middle of two quadrupoles on opposite sides of the ring and insert a drift space of length $\ell_{d}$. Derive the transformation matrix for this ring and compare with that of the unperturbed ring. What is the tune change of the accelerator. The betatron functions will be modified. Derive the new value of the horizontal betatron function at the symmetry point in units of the unperturbed betatron function. Is there a difference to whether the free section is inserted in the middle of a focusing or defocusing quadrupole? How does the $\eta$-function change?

8.7. Consider a regular FODO lattice, where some bending magnets are eliminated to provide magnet free spaces and to reduce the $\eta$-function in the straight section. How does the minimum value of the $\eta$-function scale with the phase per FODO cell. Show if conditions exist to match the $\eta$-function perfectly in the straight section of this lattice?

\section{References}

1. A.P. Banford, The Transport of Charged Particle Beams (Spon, London, 1966)

2. E.E. Chambers, R. Hofstadter, in CERN Symposium. CERN 56-25, Geneva (1956), p. 106

3. R. Chasman, K. Green, E. Rowe, IEEE Trans. NS 22, 1765 (1975)

4. E.D. Courant, H.S. Snyder, Appl. Phys. 3, 1 (1959)

5. D. Einfeld, G. Mülhaupt, Nucl. Instrum. Methods 172, 55 (1980)

6. A. Jackson, in 1987 IEEE Particle Accelerator Conference, Dubna, 1963, p. 365

7. D.L. Judd, S.A. Bludman, Nucl. Instrum. Methods 1, 46 (1956)

8. E. Rowe, IEEE Trans. NS 28, 3145 (1981)

9. K. Siegbahn, S. Svartholm, Nature 157, 872 (1946)

10. K.G. Steffen, High Energy Beam Optics (Wiley, New York, 1965), p. 117

11. R.Q. Twiss, N.H. Frank, Rev. Sci. Instrum. 20, 1 (1949) 


\section{Chapter 9 \\ Longitudinal Beam Dynamics}

In previous chapters we have concentrated the discussion on the interaction of transverse electrical and magnetic fields with charged particles and have derived appropriate formalisms to apply this interaction to the design of beam transport systems. The characteristics of these transverse fields is that they allow to guide charged particles along a prescribed path but do not contribute directly to the energy of the particles through acceleration. For particle acceleration we must generate fields with nonvanishing force components in the direction of the desired acceleration. Such fields are called longitudinal fields or accelerating fields. In a very general way we describe in this section the interaction of longitudinal electric fields with charged particles to derive the process of particle acceleration, its scaling laws, and its stability limits.

The usefulness and application of electric fields to accelerate charged particles depends greatly on the temporal variations of these fields. Accelerating fields can be static or pulsed or they may be electromagnetic fields oscillating at high frequencies. Conceptually, the most simple way to accelerate charged particles is through a static field applied to two electrodes as shown in Fig. 9.1. In this case, the total kinetic energy a particle can gain while traveling from one electrode to the other is equal to the product of the particle charge and the voltage between the electrodes.

Electric breakdown phenomena, however, limit the maximum applicable voltage and thereby the maximum energy gain. Nonetheless, this method is intriguingly simple and efficient compared to other accelerating methods and therefore still plays a significant role among modern particle accelerators, for example, in particle sources. Electrostatic acceleration schemes are specifically useful for low energy particles for which other methods of acceleration would be inefficient. Higher voltages and particle energies can be reached if the electric fields are applied in the form of very short pulses. Application of electro-static high voltages to accelerate particles is limited to some 10 million volts due to high voltage breakdown.

This chapter has been made Open Access under a CC BY 4.0 license. For details on rights and licenses please read the Correction https://doi.org/10.1007/978-3-319-18317-6_28 
Fig. 9.1 Principle of electrostatic accelerators

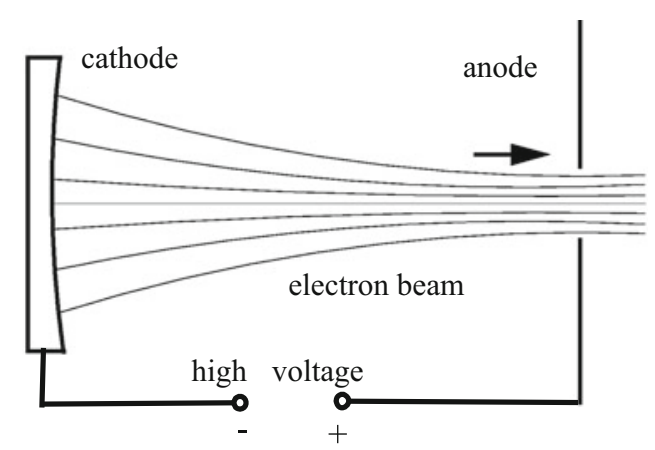

For higher particle energies different acceleration methods must be used. The most common and efficient way to accelerate charged particles to high energies is to use high frequency electromagnetic fields in specially designed accelerating structures. Acceleration to high energies occurs while charged particles either pass once through many or many times through one or few accelerating structures each excited to electric field levels below the break down threshold. In this section, we concentrate the discussion on charged particle acceleration by electromagnetic radio frequency fields.

\subsection{Longitudinal Particle Motion}

Application of radio frequency in short rf-fields has become exceptionally effective for the acceleration of charged particles. Both, fields and particle motion can be synchronized in an effective way to allow the acceleration of charged particles in principle to arbitrary large energies were it not for other limitations.

The first idea and experiment for particle acceleration with radio frequency fields has been published by Ising [1] although he did not actually succeed to accelerate particles due to an inefficient approach to rf-technology. Later Wideroe [2] introduced the concept of generating the accelerating fields in resonating rfcavities and was able to accelerate heavy ions. Original papers describing these and other early developments of particle acceleration by rf-fields are collected in a monogram edited by Livingston [3].

To study the interaction of electromagnetic rf-fields with charged particles, we assume a plane electromagnetic wave of frequency $\omega$ propagating in the $z$-direction. A free electromagnetic wave does not have a longitudinal electric field component and therefore a special physical environment, called the accelerating structure, must be provided to generate accelerating field components in the direction of propagation. As will be discussed later in Sect. 18.1 this is achieved by proper choice of boundary conditions. To study particle dynamics in longitudinal fields, 
we assume that we were able to generate rf-fields with an electric field component along the path of the particles expressed by $v \approx c$

$$
\boldsymbol{E}(z, t)=\boldsymbol{E}_{0} \mathrm{e}^{\mathrm{i}(\omega t-k z)}=\boldsymbol{E}_{0} \mathrm{e}^{\mathrm{i} \psi},
$$

where the phase $\psi=\omega t-k z$. The particle momentum changes at a rate equal to the electric force exerted on the particle by the rf-field

$$
\frac{\mathrm{d} \boldsymbol{p}}{\mathrm{d} t}=e \boldsymbol{E}(\psi)=\frac{\mathrm{d}}{\mathrm{d} t}(\gamma m c \boldsymbol{\beta}) .
$$

Multiplying this with the particle velocity we get the rate of change of the kinetic energy, $\mathrm{d} E_{\mathrm{kin}}=c \beta \mathrm{d} p$. Integration of (9.2) with respect to the time becomes unnecessarily complicated for general fields because of the simultaneous variation of the electric field and particle velocity with time. We therefore integrate (9.2) with respect to the longitudinal coordinate and obtain instead of the momentum gain the increase in the kinetic or total energy for the complete accelerating structure

$$
\Delta E=\left(\gamma-\gamma_{0}\right) m c^{2}=e \int \boldsymbol{E}(\psi) \mathrm{d} z,
$$

where $\gamma_{0} m c^{2}$ is the energy of the particle before acceleration. Of course, the trick to integrate the electric field through the accelerating section rather than over time following the particle is only a conceptual simplification and the time integration will have to be executed at some point. Generally this is done when the particular accelerating section, the fields, and the synchronization is known.

Travelling electromagnetic waves are used in linear accelerators and the accelerating structure is designed such that the phase velocity of the wave is equal to the velocity of the particles to be accelerated. In this case, the particle travels along the structure in synchronism with the wave and is therefore accelerated or decelerated at a constant rate. Maximum acceleration is obtained if the particles ride on the crest of the wave.

In a standing wave accelerating section the electric field has the form

$$
\boldsymbol{E}(z, t)=\boldsymbol{E}_{0}(z) \mathrm{e}^{\mathrm{i} \omega t+\delta},
$$

where $\delta$ is the phase at the moment the particle enters the accelerating section at $t=0$. When we refer to an accelerating voltage $V$ in a standing wave cavity we mean to say a particle traveling close to the speed of light through the cavity will gain a maximum kinetic energy of $e V$ while passing the cavity center at the moment the field reaches its crest. Such a particle would enter the cavity some time before the field reaches a maximum and will exit when the field is decaying again. For slower particles the energy gain would be lower because of the longer transit time. 


\subsubsection{Longitudinal Phase Space Dynamics}

Successful particle acceleration depends on stable and predictable interaction of charged particles and electromagnetic fields. Because oscillating rf-fields are used, special criteria must be met to assure systematic particle acceleration rather than random interaction with rf-fields producing little or no acceleration. The constructive interaction of particles and waves have been investigated in 1945 independently by Veksler [4] and McMillan [5] leading to the discovery of the fundamental principle of phase focusing. In this subsection, we will derive the physics of phase focusing and apply it to the design of particle accelerators.

The degree of acceleration depends on the momentary phase $\psi$ of the field as seen by the particle while travelling through or with an electromagnetic field. Straight superposition of an electromagnetic wave and charged particle motion will not necessarily lead to a net acceleration. In general, the particles are either too slow or too fast with respect to the phase velocity of the wave and the particle will, during the course of interaction with the electromagnetic wave, integrate over a range of phases and may gain little or no net energy from the electric fields. Therefore, special boundary conditions for the accelerating rf-wave must be met such that maximum or at least net acceleration can be achieved. This can be done by exciting and guiding the electromagnetic waves in specially designed accelerating structures designed such that the phase velocity of the electromagnetic wave is equal to the particle velocity. Only then can we choose a specific phase and integration of (9.3) becomes straightforward for particles travelling in the direction of propagation of the electromagnetic waves.

For practical reasons, specifically in circular accelerators, particle acceleration occurs in short, straight accelerating sections placed along the particle path. In this case no direct traveling wave exists between adjacent accelerating sections and specific synchronicity conditions must be met for the fields in different accelerating sections to contribute to particle acceleration as desired. For the purpose of developing a theory of stable particle acceleration we may imagine an rf-wave traveling along the path of the particle with a phase velocity equal to the particle velocity and an amplitude which is zero everywhere except in discrete accelerating cavities.

To ensure proper synchronization one could assume that every rf-cavity is powered by its own microwave source. This is done often in high power rf-cavities, but is, for example, impractical in linear accelerators. For the case of individual power sources the phase of the rf-field can be chosen in each cavity such that its voltage reaches the desired value at the moment the particles pass through. The synchronisation for many cavities fed by one power source is more complicated and we will discuss in the following paragraphs how to accomplish this.

We consider a number of rf-cavities powered by a single microwave source. To derive the synchronicity conditions, we consider first two accelerating sections separated by the distance $L$ as shown in Fig.9.2. Once the proper operating conditions are known for two sections a third section may be added by applying 
Fig. 9.2 Discrete accelerating sections

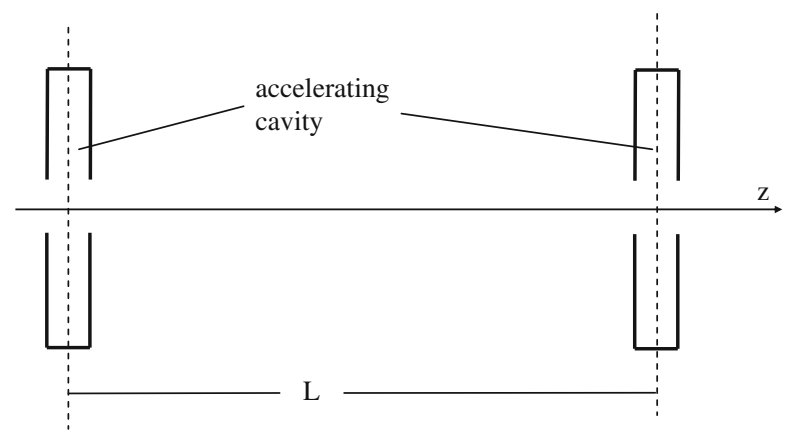

the same synchronicity condition between each pair of cavities. The successive accelerating sections need not necessarily be a physically different sections but could be the same section or the same sections passed through by the particles at periodic time intervals. For example, the distance $L$ between successive accelerating sections may be equal to the circumference of a circular accelerator.

For systematic acceleration the phase of the rf-fields in each of the accelerating sections must reach specific values at the moment the particles arrive. If the phase of the fields in each of $N$ accelerating sections is adjusted to be the same at the time of arrival of the particles, the total acceleration is $N$ times the acceleration in each individual section. This phase is called the synchronous phase $\psi_{\mathrm{s}}$ defined by

$$
\psi_{\mathrm{s}}=\omega t-k z=\mathrm{const}
$$

where $\omega$ is the oscillating frequency of the electromagnetic field. The time derivative of (9.5) vanishes and the synchronicity condition is

$$
\dot{\psi}_{\mathrm{s}}=\omega-k \beta c=0 \text {, }
$$

since $\mathrm{d} z / \mathrm{d} t=\beta c$. This condition can be met if we set

$$
k=\frac{2 \pi}{L}
$$

and the frequency of the electromagnetic field is then from (9.6)

$$
\omega_{1}=k_{1} \beta c=\frac{2 \pi}{L} \beta c=\frac{2 \pi}{\Delta T},
$$

where $\omega_{1}$ is the lowest frequency satisfying the synchronicity condition and $\Delta T$ is the time needed for particles with velocity $\beta c$ to travel the distance $L$. This equation relates the time of travel between successive accelerating sections with the frequency of the accelerating rf-fields in a conditional way to assure systematic 
particle acceleration and the relation (9.8) is therefore called the synchronicity condition.

However, any integer multiple of the frequency $\omega_{1}$ satisfies the synchronicity condition as well and we may instead of (9.8) define permissible frequencies of the accelerating rf-fields by

$$
\omega_{h}=h \omega_{1}=k_{h} \beta c=\frac{2 \pi}{L} h \beta c=\frac{2 \pi}{\Delta T} h,
$$

where $h$ is an integer called the harmonic number with $k_{h}=h k_{1}$.

The synchronicity condition must be fulfilled for any spatial arrangement of the accelerating structures which are powered by a single microwave source to get the maximum acceleration. To illuminate the principle, we assume here, for example, a series of short, equidistant accelerating gaps or accelerating sections along the path of a particle. Let each of these gaps be excited by its own power source to produce an accelerating rf-field at some random phase. The synchronicity condition (9.8) is fulfilled if the rf-frequency is the same in each of these gaps, which are separated by an integer multiple of rf-wavelength. However, it does not require each accelerating gap to have the same rf-phase at the arrival time of the particles. Each cavity in a set of accelerating cavities oscillating at the same frequency may be tuned to an arbitrary rf-phase and the synchronicity condition still would be met. From a practical point of view, however, it is inefficient to choose arbitrary phases and it is more reasonable to adjust the phase in each cavity to the optimum phase desired for maximum acceleration.

The assumption that the rf-frequency of all cavities be the same is unnecessarily restrictive considering that any harmonic of the fundamental frequency is acceptable. Therefore, a set of accelerating cavities in a circular accelerator, for example, may include cavities resonating at any harmonic of $\omega_{1}$. This is sometimes done to achieve specific effects (e.g. bunch lengthening), but in the absence of such requirements makes only complicates the Rf-system.

A straightforward application of the synchronicity condition can be found in the design of the Wideroe linear accelerator structure [2] as shown in Fig. 9.3. Here the fields are generated by an external rf-source and applied to a series of metallic drift tubes. Accelerating fields build up at gaps between the tubes while the tubes themselves serve as a field screens for particles during the time the electric fields is changing sign and would be decelerating. The length of the field free drift tubes is determined by the velocity of the particles and is $L=c \beta T_{\mathrm{rf}}$ where $T_{\mathrm{rf}}$ is the period of the rf-field. As the particle energy increases so does the velocity $c \beta$ and the length $L$ of the tube must increase too. Only when the particles become highly relativistic will the distance between field free drift sections become a constant together with the velocity of the particles. Structures with varying drift lengths are generally found in low energy proton or ion accelerators based on the Alvarez structure [6], which is a technically more efficient version of the Wideroe structure. 


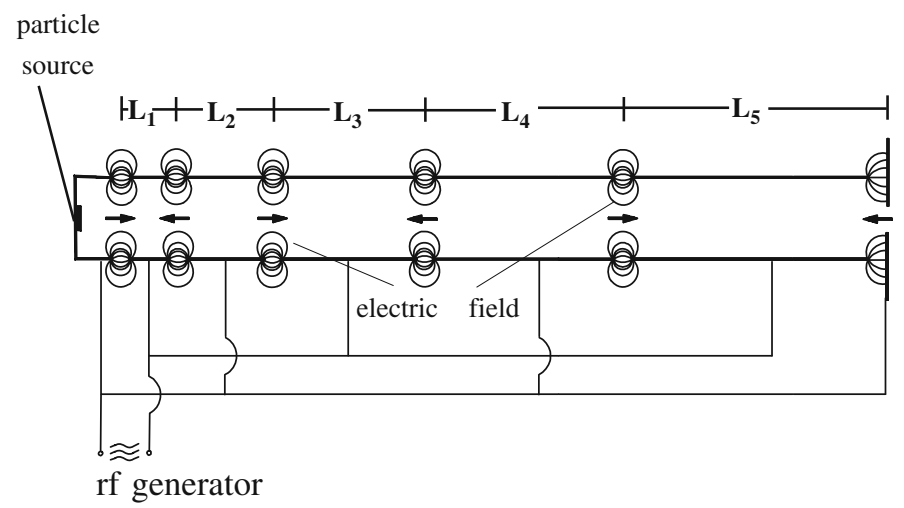

Fig. 9.3 Wideroe linac structure

For electrons it is much easier to reach relativistic energies where the velocity is sufficiently constant such that in general no longitudinal variation of the accelerating structure is needed. In circular accelerators, we cannot adjust the distance between cavities or the circumference as the particle velocity $\beta$ increases. The synchronicity condition therefore must be applied differently. From (9.9) we find the rf-frequency to be related to the particle velocity and distances between cavities. Consequently we have the relation

$$
\beta \lambda_{\text {rf }} h=L,
$$

which requires that the distance between any pair of accelerating cavities be an integer multiple of $\beta \lambda_{\text {rf }}$. Since $L$ and $h$ are constants, this condition requires that the rf-frequency be changed during acceleration proportional to the particle velocity $\beta$. Only for particles reaching relativistic energies, when $\beta \approx 1$, will the distance between cavities approach an integer multiple of the rf-wave length and the circumference $C$ must then meet the condition

$$
C=\beta h \lambda_{\mathrm{rf}}
$$

\subsection{Equation of Motion in Phase Space}

So far, we have assumed that both the particle velocity $\beta$ and the wave number $k$ are constant. This is not a valid general assumption. For example, we cannot assume that the time of flight from one gap to the next is the same for all particles. For low energy particles we have a variation of the time of flight due to the variation of the particle velocities for different particle momenta. The wave number $k$ or the distance between accelerating sections need not be the same 
for all particles either. A momentum dependent path length between accelerating sections exists if the lattice between such sections includes bending magnets. As a consequence, the synchronicity condition must be modified to account for such chromatic effects.

Removing the restriction of a constant wave number $k$, we obtain by a variation of (9.6)

$$
\Delta \dot{\psi}=\dot{\psi}-\dot{\psi}_{\mathrm{s}}=-\Delta(k \beta c)=-c k \Delta \beta-\beta c \frac{\partial k}{\partial p} \frac{\partial p}{\partial t} \Delta t,
$$

where

$$
k=k_{\mathrm{h}}=h \frac{2 \pi}{L_{0}}=\frac{2 \pi}{\lambda_{\mathrm{rf}}}=h \frac{\omega}{\beta c},
$$

and $L_{0}$ is the distance between accelerating gaps along the ideal path. The synchronous phase is kept constant $\psi_{\mathrm{s}}=$ const or $\dot{\psi}_{\mathrm{s}}=0$ and serves as the reference phase against which all deviations are measured.

The momentum dependence of the wave number comes from the fact that the path length $L$ between accelerating gaps may be different from $L_{0}$ for off momentum particles. The variation of the wave number with particle momentum is therefore

$$
\left.\frac{\partial k}{\partial p}\right|_{0}=\left.\frac{\partial k}{\partial L} \frac{\partial L}{\partial p}\right|_{0}=-\left.\frac{k_{\mathrm{h}}}{L_{0}} \frac{\partial L}{\partial p}\right|_{0}=-\frac{k_{\mathrm{h}}}{p_{0}} \alpha_{\mathrm{c}},
$$

where $\alpha_{\mathrm{c}}$ is the momentum compaction factor. We evaluate the momentum compaction factor starting from the path length $L=\int_{0}^{L_{0}}\left(1+\frac{x}{\rho}\right) \mathrm{d} z$. For transverse particle motion $x=x_{\beta}+\eta\left(\Delta p / p_{0}\right)$ and employing average values of the integrands the integral becomes

$$
L=L_{0}+\left\langle\frac{x_{\beta}}{\rho}\right\rangle L_{0}+\left\langle\frac{\eta}{\rho}\right\rangle \frac{\Delta p}{p_{0}} L_{0} .
$$

Because of the oscillatory character of the betatron motion $\left\langle\kappa_{x} x_{\beta}\right\rangle=0$. The relative path length variation is $\frac{\Delta L}{L_{0}}=\left\langle\frac{\eta}{\rho}\right\rangle \frac{\Delta p}{p_{0}}=\alpha_{\mathrm{c}} \frac{\Delta p}{p_{0}}$ and the momentum compaction factor becomes

$$
\alpha_{\mathrm{c}}=\left\langle\frac{\eta}{\rho}\right\rangle
$$

The momentum compaction factor increases only in curved sections where $\rho \neq 0$ and the path length is longer or shorter for higher energy particles depending on the dispersion function being positive or negative, respectively. For a linear accelerator the momentum compaction factor vanishes since the length of a straight line does 
not depend on the momentum. With $(\partial p / \partial t) \Delta t=\Delta p$ and $m c \gamma^{3} \Delta \beta=\Delta p$ we get finally for (9.12) with (9.14) and after some manipulation

$$
\dot{\psi}=-\beta c k_{\mathrm{h}}\left(\gamma^{-2}-\alpha_{\mathrm{c}}\right) \frac{\Delta c p}{c p_{0}} .
$$

The term $\gamma^{-2}$ in (9.17) appears together with the momentum compaction factor $\alpha_{\mathrm{c}}$ and therefore has the same physical relevance. This term represents the variation of the particle velocity with energy. Therefore, even in a linear accelerator where $\alpha_{\mathrm{c}}=0$, the time of flight between accelerating gaps is energy dependent as long as particles are still nonrelativistic.

After differentiation of (9.17) with respect to the time, we get the equation of motion in the longitudinal direction describing the variation of the phase with respect to the synchronous phase $\psi_{\mathrm{s}}$ for particles with a total momentum deviation $\Delta p$

$$
\ddot{\psi}+\frac{\partial}{\partial t}\left(\beta c k_{\mathrm{h}} \eta_{\mathrm{c}} \frac{\Delta c p}{c p_{0}}\right)=0 .
$$

In most practical applications, parameters like the particle velocity $\beta$ or the energy vary only slowly during acceleration compared to the rate of change of the phase and we consider them for the time being as constants. The slow variation of these parameters constitutes an adiabatic variation of external parameters for which Ehrenfest's theorem holds. The equation of motion in the potential of the rf-field becomes in this approximation

$$
\ddot{\psi}+\frac{\beta c k_{\mathrm{h}} \eta_{\mathrm{c}}}{c p_{0}} \frac{\partial}{\partial t} \Delta c p=0 .
$$

Integration of the electrical fields along the accelerating sections returns the kinetic energy gain per turn

$$
e \int_{L} \boldsymbol{E}(\psi) \mathrm{d} \mathbf{z}=e V(\psi),
$$

where $V(\psi)$ is the total particle accelerating voltage seen by particles along the distance $L$. For particles with the ideal energy and following the ideal orbit the acceleration is $e V\left(\psi_{\mathrm{s}}\right)$ where $\psi_{\mathrm{s}}$ is the synchronous phase.

Acceleration, however, is not the only source for energy change of particles. There are also gains or losses from, for example, interaction with the vacuum chamber environment, external fields like free electron lasers, synchrotron radiation or anything else exerting longitudinal forces on the particle other than accelerating fields. We may separate all longitudinal forces into two classes, one for which the energy change depends only on the phase of the accelerating fields $V(\psi)$ and the other where the energy change depends only on the energy of the particle $U(E)$ 
itself. The total energy gain $\Delta E$ per unit time or per turn is the composition of both types of external effects

$$
\Delta E=e V(\psi)-U(E)
$$

where $U(E)$ is the energy dependent loss per turn due, for example, to synchrotron radiation.

\subsubsection{Small Oscillation Amplitudes}

For arbitrary variations of the accelerating voltage with time we cannot further evaluate the equation of motion unless the discussion is restricted to small variations in the vicinity of the synchronous phase. While the ideal particle arrives at the accelerating cavities exactly at the synchronous phase $\psi_{\mathrm{s}}$, most other particles in a real beam arrive at slightly different phases. For small deviations $\varphi$ from the synchronous phase,

$$
\varphi=\psi-\psi_{\mathrm{s}}
$$

we can expand the accelerating voltage into a Taylor series at $\psi=\psi_{\mathrm{s}}$ and get for the average rate of change of the particle energy with respect to the energy of the synchronous particle from (9.20)

$$
\frac{\mathrm{d}}{\mathrm{d} t} \Delta E=\frac{1}{T_{0}}\left[e V\left(\psi_{\mathrm{s}}\right)+\left.e \frac{\mathrm{d} V}{\mathrm{~d} \psi}\right|_{\psi_{\mathrm{s}}} \varphi-U\left(E_{0}\right)-\left.\frac{\mathrm{d} U}{\mathrm{~d} E}\right|_{E_{0}} \Delta E\right],
$$

where the particle energy $E=E_{0}+\Delta E$ and $T_{0}$ is the time of flight between adjacent cavities for the reference particle

$$
T_{0}=\frac{L_{0}}{\beta c} \text {. }
$$

At equilibrium $e V\left(\psi_{\mathrm{s}}\right)=U\left(E_{0}\right)$, and since $\beta \Delta c p=\Delta E$, we get with (9.23) and $\ddot{\varphi}=\ddot{\psi}$ from (9.19) the equation of motion or phase equation

$$
\ddot{\varphi}+\left.\frac{c k_{\mathrm{h}} \eta_{\mathrm{c}}}{c p_{0} T_{0}} e \frac{\mathrm{d} V}{\mathrm{~d} \psi}\right|_{\psi_{\mathrm{s}}} \varphi+\left.\frac{1}{T_{0}} \frac{\mathrm{d} U}{\mathrm{~d} E}\right|_{E_{0}} \frac{\Delta c p}{c p_{0}}=0 .
$$

With (9.17) and $\psi=\psi_{\mathrm{s}}+\varphi$ Eq. (9.25) becomes the differential equation of motion for small phase oscillations

$$
\ddot{\varphi}+2 \alpha_{z} \dot{\varphi}+\Omega^{2} \varphi=0,
$$


where the damping decrement $\alpha_{z}$ is defined by

$$
\alpha_{z}=-\left.\frac{1}{2 T_{0}} \frac{\mathrm{d} U}{\mathrm{~d} E}\right|_{E_{0}}
$$

and the synchrotron frequency by

$$
\Omega^{2}=\left.\frac{c k_{\mathrm{h}} \eta_{\mathrm{c}}}{c p_{0} T_{0}} e \frac{\mathrm{d} V}{\mathrm{~d} \psi}\right|_{\psi_{\mathrm{s}}} .
$$

Particles orbiting in a circular accelerator perform longitudinal oscillations with the frequency $\Omega$. These phase oscillations are damped or antidamped depending on the sign of the damping decrement. Damping occurs only if there is an energy loss which depends on the particle energy itself as in the case of synchrotron radiation. In most cases of accelerator physics we find the damping time to be much longer than the phase oscillation period and we may therefore discuss the phase equation while ignoring damping terms. Whenever damping becomes of interest, we will include this term again.

This phase equation is valid only for small oscillation amplitudes because only the linear term has been used in the expansion for the rf-voltage. For larger amplitudes this approximation cannot be made anymore and direct integration of the differential equation is necessary. The small amplitude approximation, however, is accurate to describe most of the fundamental features of phase oscillations. At large amplitudes, the nonlinear terms will introduce a change in the phase oscillation frequency and finally a limit to stable oscillations to be discussed later in this chapter.

The phase equation has the form of the equation of motion for a damped harmonic oscillator and we will look for conditions leading to a positive frequency and stable phase oscillations. Because the phase equation was derived first for synchrotron accelerators the oscillations are also called synchrotron oscillations and are of fundamental importance for beam stability in all circular accelerators based on rf-acceleration. For real values of the oscillation frequency we find that particles which deviate from the synchronous phase are subjected to a restoring force leading to harmonic oscillations about the equilibrium or synchronous phase. From the equation of motion (9.25) it becomes clear that phase focusing is proportional to the derivative of the accelerating voltage rather than to the accelerating voltage itself and is also proportional to the momentum compaction $\eta_{\mathrm{c}}$.

To gain further insight into the phase equation and determine stability criteria, we must make an assumption for the waveform of the accelerating voltage. In most cases, the rf-accelerating fields are created in resonant cavities and therefore the accelerating voltage can be expressed by a sinusoidal waveform

$$
V(\psi)=\hat{V}_{0} \sin \psi
$$


and expanded about the synchronous phase to get with $\psi=\psi_{\mathrm{s}}+\varphi$

$$
V\left(\psi_{\mathrm{s}}+\varphi\right)=\hat{V}_{0}\left(\sin \psi_{\mathrm{s}} \cos \varphi+\sin \varphi \cos \psi_{\mathrm{s}}\right) .
$$

Keeping only linear terms in $\varphi$ the phase equation is

$$
\ddot{\varphi}+\Omega^{2} \varphi=0,
$$

where the synchrotron oscillation frequency becomes now

$$
\Omega^{2}=\frac{c k_{\mathrm{h}} \eta_{\mathrm{c}}}{c p_{0} T_{0}} e \hat{V}_{0} \cos \psi_{\mathrm{s}}
$$

A particle passing periodically through localized and synchronized accelerating fields along its path performs synchrotron oscillations with the frequency $\Omega$ about the synchronous phase.

In circular accelerators we have frequently the situation that several rf-cavities are employed to provide the desired acceleration. The reference time $T_{0}$ is most conveniently taken as the revolution time and the rf-voltage $\hat{V}_{0}$ is the total accelerating voltage seen by the particle while orbiting around the ring once. The rf-frequency is an integer multiple of the revolution frequency,

$$
f_{\mathrm{rf}}=h f_{\mathrm{rev}},
$$

where the integer $h$ is the harmonic number and the revolution frequency is with the circumference $C$

$$
f_{\mathrm{rev}}=\frac{1}{T_{0}}=\frac{C}{c \beta} .
$$

From (9.32) the synchrotron frequency is in more practical units

$$
\Omega^{2}=\omega_{\mathrm{rev}}^{2} \frac{h \eta_{\mathrm{c}} e \hat{V}_{0} \cos \psi_{\mathrm{s}}}{2 \pi \beta c p_{0}} .
$$

Similar to the betatron oscillation tunes, we define the synchrotron oscillation tune or short the synchrotron tune as the ratio

$$
v_{\mathrm{s}}=\frac{\Omega}{\omega_{\mathrm{rev}}} .
$$

For real values of the synchrotron oscillation frequency the phase equation assumes the simple form

$$
\varphi=\hat{\varphi} \cos \left(\Omega t+\chi_{\mathrm{i}}\right),
$$



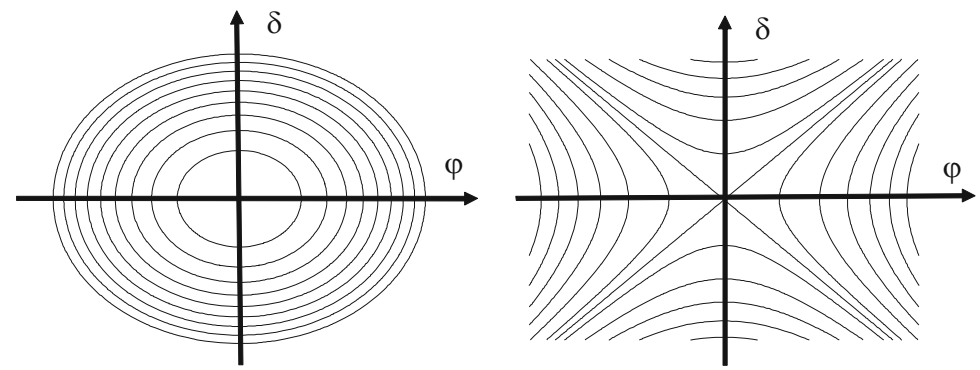

Fig. 9.4 Synchrotron oscillations in phase space for stable motion $\left(\Omega^{2}>0\right)$ (left) and for unstable motion $\left(\Omega^{2}<0\right)($ right $)$

where $\chi_{\mathrm{i}}$ is an arbitrary phase function for the particle $i$ at time $t=0$. With $\dot{\psi}=\dot{\varphi}$ we find from (9.17), (9.32) the relation between the momentum and phase deviation for real values of the synchrotron oscillation frequency $v_{s} \approx 0.001-0.01$

$$
\delta=\frac{\Delta c p}{c p_{0}}=-\frac{\dot{\varphi}}{h \omega_{\mathrm{rev}} \eta_{\mathrm{c}}}=\frac{\Omega \hat{\varphi}}{h \omega_{\mathrm{rev}} \eta_{\mathrm{c}}} \sin \left(\Omega t+\chi_{\mathrm{i}}\right) .
$$

The particle momentum deviation, being the conjugate variable to the phase, also oscillates with the synchrotron frequency about the ideal momentum. Both, the phase and momentum oscillations describe the particle motion in longitudinal phase spaceas shown in Fig. 9.4 for stable and unstable synchrotron oscillations, respectively. At the time $t_{0}$ when in (9.38) the phase $\Omega t_{0}+\chi_{\mathrm{i}}=0$ and we expect the momentum deviation to be zero while the phase reaches the maximum value $\hat{\varphi}$. Thus both oscillations are $90^{\circ}$ out of phase. Particles with a negative momentum compaction $\eta_{\mathrm{c}}<0$ move clockwise in phase space about the reference point while a positive momentum compaction causes the particles to rotate counter clockwise.

The same process that has led to phase focusing will also provide the focusing of the particle momentum. Any particle with a momentum different from the ideal momentum will undergo oscillations at the synchrotron frequency which are described by $\delta=-\hat{\delta} \sin \left(\Omega t+\chi_{\mathrm{i}}\right)$, where the maximum momentum deviation is related to the maximum phase excursion $\hat{\varphi}$ by

$$
\hat{\delta}=\left|\frac{\Omega}{h \omega_{\text {rev }} \eta_{\mathrm{c}}}\right| \hat{\varphi}
$$

By inverse deduction we may express the momentum equation similar to the phase equation (9.31) and get with $\Delta p / p_{0}=\delta$ the differential equation for the momentum deviation

$$
\frac{\mathrm{d}^{2} \delta}{\mathrm{d} t^{2}}+\Omega^{2} \delta=0
$$


Similar to the transverse particle motion, we eliminate from (9.37), (9.38) the argument of the trigonometric functions to obtain an invariant of the form

$$
\frac{\delta^{2}}{\hat{\delta}^{2}} \pm \frac{\varphi^{2}}{\hat{\varphi}^{2}}=1 \quad \text { with } \quad \hat{\delta}=\frac{\Omega}{h \omega_{\text {rev }}} \hat{\varphi}
$$

where the sign is chosen to indicate stable or unstable motion depending on whether the synchrotron oscillation frequency $\Omega$ is real or imaginary respectively. The trajectories for both cases are shown in Fig. 9.4. Clearly, the case of imaginary values of the synchrotron oscillation frequency leads to exponential growth in the oscillation amplitude.

\subsubsection{Phase Stability}

The synchrotron oscillation frequency must be real and the right-hand side of (9.32) must therefore be positive to obtain stable solutions for phase oscillations. All parameters in (9.32) are positive quantities except for the momentum compaction $\eta_{\mathrm{c}}$ and the phase factor $\cos \psi_{\mathrm{s}}$. For low particle energies the momentum compaction is in general positive because $\gamma^{-2}>\alpha_{\mathrm{c}}$ but becomes negative for higher particle energies. The energy at which the momentum compaction changes sign is called the transition energydefined by

$$
\gamma_{\mathrm{tr}}=\frac{1}{\sqrt{\alpha}_{\mathrm{c}}}
$$

Since the momentum compaction factor for circular accelerators is approximately equal to the inverse horizontal tune $\alpha_{\mathrm{c}} \approx v_{x}^{-2}$, we conclude that the transition energy $\gamma_{\text {tr }}$ is of the order of the tune and therefore in general a small number reaching up to the order of a hundred for very large accelerators. For electrons, the transition energy is of the order of a few $\mathrm{MeV}$ and for protons in the $\mathrm{GeV}$ regime. In circular electron accelerators the injection energy always is selected to be well above the transition energy and no stability problems occur during acceleration since the transition energy is not crossed. Not so for protons. Proton linear accelerators with an energy of the order of $10 \mathrm{GeV}$ or higher are very costly and therefore protons and ions in general must be injected into a circular accelerator below transition energy.

The synchronous rf-phase must be selected depending on the particle energy being below or above the transition energy. Stable phase focusing can be obtained in either case if the rf-synchronous phase is chosen as follows

$$
\begin{array}{lll}
0<\psi_{\mathrm{s}}<\frac{\pi}{2} & \text { for } & \gamma<\gamma_{\mathrm{tr}} \\
\frac{\pi}{2}<\psi_{\mathrm{s}}<\pi & \text { for } & \gamma>\gamma_{\mathrm{tr}}
\end{array}
$$


In a proton accelerator with an injection energy below transition energy the rfphase must be changed very quickly when the transition energy is being crossed. Often the technical difficulty of this sudden change in the rf-phase is ameliorated by the use of pulsed quadrupoles [7, 8], which is an efficient way of varying momentarily the momentum compaction factor by perturbing the dispersion function. A sudden change of a quadrupole strength can lower the transition energy below the actual energy of the particle. This helpful "perturbation" lasts for a small fraction of a second while the particles are still being accelerated and the rf-phase is changed. By the time the quadrupole pulse terminates, the rf-phase has been readjusted and the particle energy is now above the unperturbed transition energy.

In general, we find that a stable phase oscillation for particles under the influence of accelerating fields can be obtained by properly selecting the synchronous phase $\psi_{\mathrm{s}}$ in conjunction with the sign of the momentum compaction such that

$$
\Omega^{2}>0
$$

This is the principle of phase focusing [5] and is a fundamental process to obtain stable particle beams in circular high-energy accelerators. An oscillating accelerating voltage together with a finite momentum compaction produces a stabilizing focusing force in the longitudinal degree of freedom just as transverse magnetic or electric fields can produce focusing forces for the two transverse degrees of freedom. With the focusing of transverse amplitudes we found a simultaneous focusing of its conjugate variable, the transverse momentum. The same occurs in the longitudinal phase where the particle energy or the energy deviation from the ideal energy is the conjugate variable to the time or phase of a particle. Both variables are related by (9.17) and a focusing force not only exists for the phase or longitudinal particle motion but also for the energy keeping the particle energy close to the ideal energy.

Focusing conditions have been derived for all six degrees of freedom where the source of focusing originates either from the magnet lattice for transverse motion or from a combination of accelerating fields and a magnetic lattice property for the energy and phase coordinate. The phase stability can be seen more clearly by observing the particle trajectories in phase space. Equation (9.31) describes the motion of a pendulum with the frequency $\Omega$ which, for small amplitudes $\sin \varphi \approx \varphi$ becomes equal to the equation of motion for a linear harmonic oscillator and can be derived from the Hamiltonian

$$
\mathcal{H}=\frac{1}{2} \dot{\varphi}^{2}+\frac{1}{2} \Omega^{2} \varphi^{2}
$$

Small amplitude oscillations in phase space are shown in Fig.9.4 and we note the confinement of the trajectories to the vicinity of the reference point. In case of unstable motion the trajectories quickly lead to unbound amplitudes in energy and phase (Fig. 9.4 right). 


\section{Large Oscillation Amplitudes}

For larger oscillation amplitudes we cannot anymore approximate the trigonometric function $\sin \varphi \approx \varphi$ by its argument. Following the previous derivation for the equation of motion (9.31) we get now

$$
\ddot{\varphi}=-\Omega^{2} \sin \varphi,
$$

which can be derived from the Hamiltonian

$$
\mathcal{H}=\frac{1}{2} \dot{\varphi}^{2}-\Omega^{2} \cos \varphi
$$

being identical to that of a mechanical pendulum. As a consequence of our ability to describe synchrotron motion by a Hamiltonian and canonical variables, we expect the validity of the Poincaré integral

$$
J_{1}=\int_{z} \mathrm{~d} \dot{\varphi} \mathrm{d} \varphi=\text { const }
$$

under canonical transformations. Since the motion of particles during synchrotron oscillations can be described as a series of canonical transformations [9], we find the particle density in the $(\varphi, \dot{\varphi})$ phase space to be a constant of motion. The same result has been used in transverse phase space and the area occupied by this beam in phase space has been called the beam emittance. Similarly, we define an emittance for the longitudinal phase space. Different choices of canonical variables can be defined as required to emphasize the physics under discussion. Specifically we find it often convenient to use the particle momentum instead of $\dot{\varphi}$ utilizing the relation (9.17).

Particle trajectories in phase space can be derived directly from the Hamiltonian by plotting solutions of (9.47) for different values of the "energy" $\mathcal{H}$ of the system. These trajectories, well known from the theory of harmonic oscillators, are shown in Fig. 9.5 for the case of a synchronous phase $\psi_{\mathrm{s}}=\pi$.

The trajectories in Fig. 9.5 are of two distinct types. In one type the trajectories are completely local and describe oscillations about equilibrium points separated by $2 \pi$ along the abscissa. For the other type the trajectories are not limited to a particular area in phase and the particle motion assumes the characteristics of libration. This phenomenon is similar to the two cases of possible motion of a mechanical pendulum or a swing. At small amplitudes we have periodic motion about the resting point of the swing. For increasing amplitudes, however, that oscillatory motion could become a libration when the swing continues to go over the top. The lines separating the regime of libration from the regime of oscillation are called separatrices.

Particle motion is stable inside the separatrices due to the focusing properties of the potential well which in this representation is just the $\cos \varphi$-term in (9.47). The area within separatrices is commonly called an rf-bucket describing a place where 


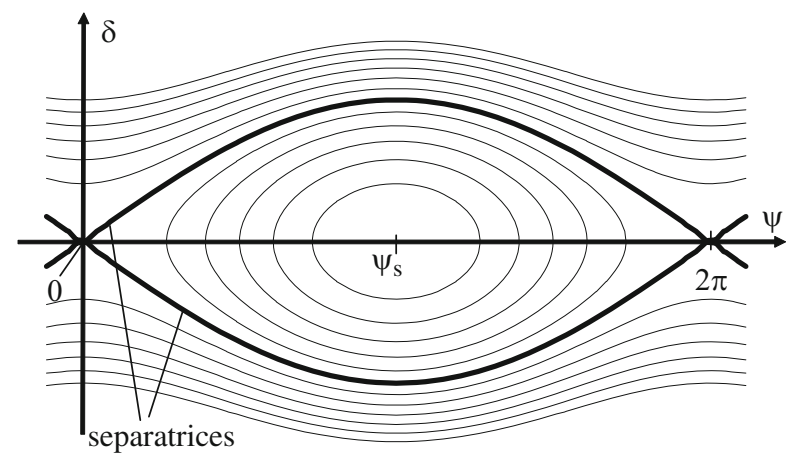

Fig. 9.5 Phase space diagrams for a synchronous phase $\psi_{\mathrm{s}}=\pi$

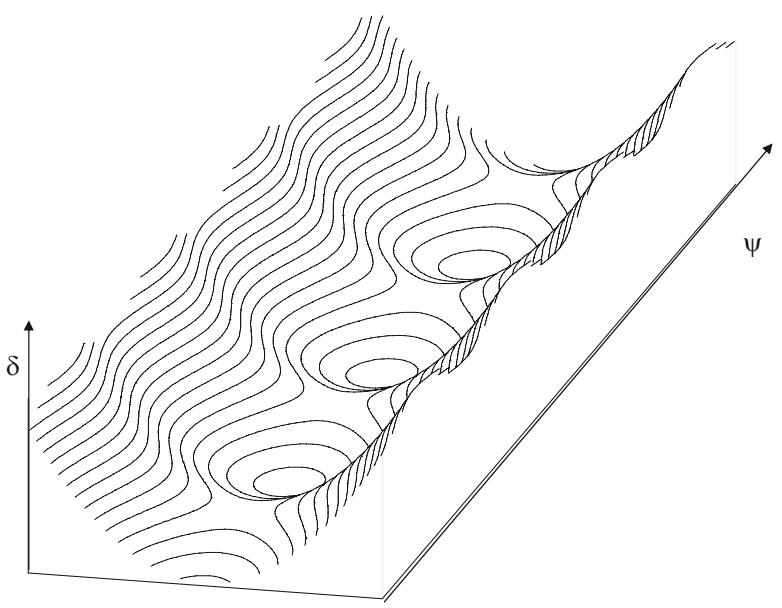

Fig. 9.6 Potential well for stationary rf buckets, $\psi_{\mathrm{s}}=\pi$

particles are in stable motion. In Fig. 9.6 the Hamiltonian (9.47) is shown in a threedimensional representation with contour lines representing the equipotential lines. The stable potential wells, within the separatrices, keeping the particles focused toward the equilibrium position, are clearly visible.

Inside the separatrices the average energy gain vanishes due to oscillatory phase motion of the particles. This is obvious from (9.30) which becomes for $\psi_{\mathrm{s}}=\pi$

$$
V(\psi)=\hat{V}_{0} \sin \psi=\hat{V}_{0} \sin \left(\psi_{\mathrm{s}}+\varphi\right)=\hat{V}_{0} \sin \varphi
$$

averaging to zero since the average phase $\langle\varphi\rangle=0$.

The area within such separatrices is called a stationary rf-bucket. Such buckets, while not useful for particle acceleration, provide the necessary potential well to produce stable bunched particle beams in facilities where the particle energy need not be changed as for example in a proton or ion storage ring where bunched 
beams are desired. Whenever particles must receive energy from accelerating fields, may it be for straight acceleration or merely to compensate for energy losses like synchrotron radiation, the synchronous phase must be different from zero. As a matter of fact, due to the principle of phase focusing, particles within the regime of stability automatically oscillate about the appropriate synchronous phase independent of their initial parameters.

In the discussion of large amplitude oscillations we have tacitly assumed that the synchrotron oscillation frequency remains constant and equal to (9.32) yet, we also note that the frequency is proportional to the variation of the rf-voltage with phase and we have included in the definition of the synchrotron frequency only linear terms so far. Specifically, we note in Fig. 9.5 that the trajectories in phase space are elliptical only for small amplitudes but are periodically distorted for larger amplitudes. This distortion leads to a spread of the synchrotron oscillation frequency.

\subsubsection{Acceleration of Charged Particles}

In the preceding paragraph we have arbitrarily assumed that the synchronous phase be zero $\psi_{\mathrm{s}}=0$ and as a result of this choice we obtained stationary, non-accelerating rf-buckets. No particle acceleration occurs since the particles pass through the cavities when the fields are zero. Whenever particle acceleration is required a finite synchronous phase must be chosen. The average energy gain per revolution is then

$$
\Delta E=V\left(\psi_{\mathrm{s}}\right)=\hat{V}_{0} \sin \psi_{\mathrm{s}}
$$

Beam dynamics and stability becomes much different for $\psi_{\mathrm{s}} \neq 0$. From (9.19), we get with (9.21), (9.30), (9.32) a phase equation more general than (9.46)

$$
\ddot{\varphi}+\frac{\Omega^{2}}{\cos \psi_{\mathrm{s}}}\left[\sin \left(\psi_{\mathrm{s}}+\varphi\right)-\sin \psi_{\mathrm{s}}\right]=0,
$$

or after expanding the trigonometric term into its components

$$
\ddot{\varphi}+\frac{\Omega^{2}}{\cos \psi_{\mathrm{s}}}\left(\sin \psi_{\mathrm{s}} \cos \varphi+\sin \varphi \cos \psi_{\mathrm{s}}-\sin \psi_{\mathrm{s}}\right)=0 .
$$

This equation can also be derived directly from the Hamiltonian for the dynamics of phase motion

$$
\frac{1}{2} \dot{\varphi}^{2}-\frac{\Omega^{2}}{\cos \psi_{\mathrm{s}}}\left[\cos \left(\psi_{\mathrm{s}}+\varphi\right)-\cos \psi_{\mathrm{s}}+\varphi \sin \psi_{\mathrm{s}}\right]=\mathcal{H}
$$




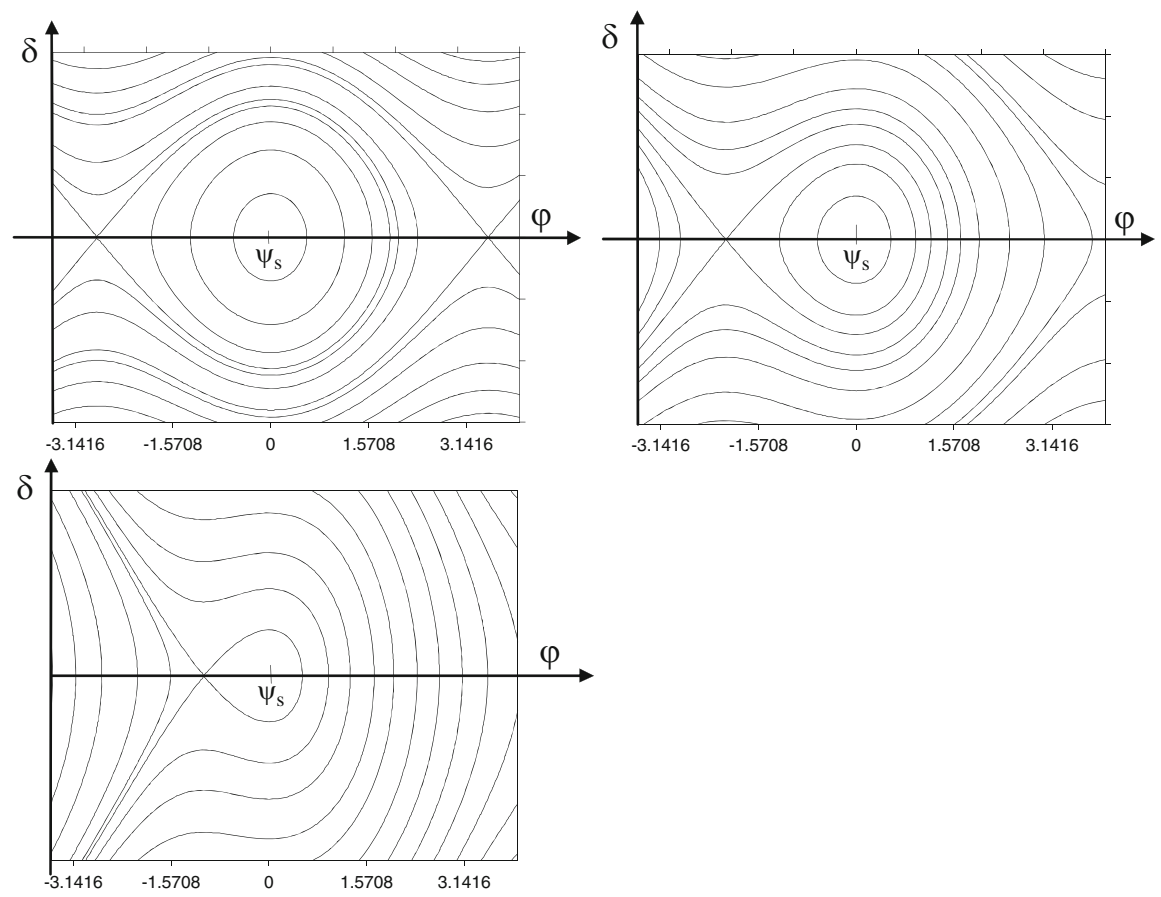

Fig. 9.7 Phase space diagrams for particles above transition energy $\left(\gamma>\gamma_{\mathrm{tr}}\right)$, synchronous phases of $\psi_{\mathrm{s}}=\pi / 3$ (top left), $5 \pi / 6$ (top right), $2 \pi / 3$ (bottom)

The phase space trajectories or diagrams differ now considerably from those in Fig. 9.5 depending on the value of the synchronous phase $\psi_{\mathrm{s}}$. In Fig. 9.7 phase space diagrams are shown for different values of the synchronous phase and a negative value for the momentum compaction $\eta_{\mathrm{c}}$.

We note clearly the reduction in stable phase space area as the synchronous phase is increased or as the particle acceleration is increased. Outside the phase stable areas the particles follow unstable trajectories leading to continuous energy loss or gain depending on the sign of the momentum compaction. Equation (9.53) describes the particle motion in phase space for arbitrary values of the synchronous phase and we note that this equation reduces to $(9.45)$ if we set $\psi_{\mathrm{s}}=\pi$. The energy gain for the synchronous particle at $\psi=\psi_{\mathrm{s}}$ becomes from (9.18)

$$
\Delta E=e \int \boldsymbol{E}\left(\psi_{\mathrm{s}}\right) \mathrm{d} z .
$$

We obtain a finite energy gain or loss whenever the synchronous phase in accelerating sections is different from an integer multiple of $180^{\circ}$ assuming that all accelerating sections obey the synchronicity condition. The form of (9.54) actually is more general insofar as it integrates over all fields encountered along the path of 

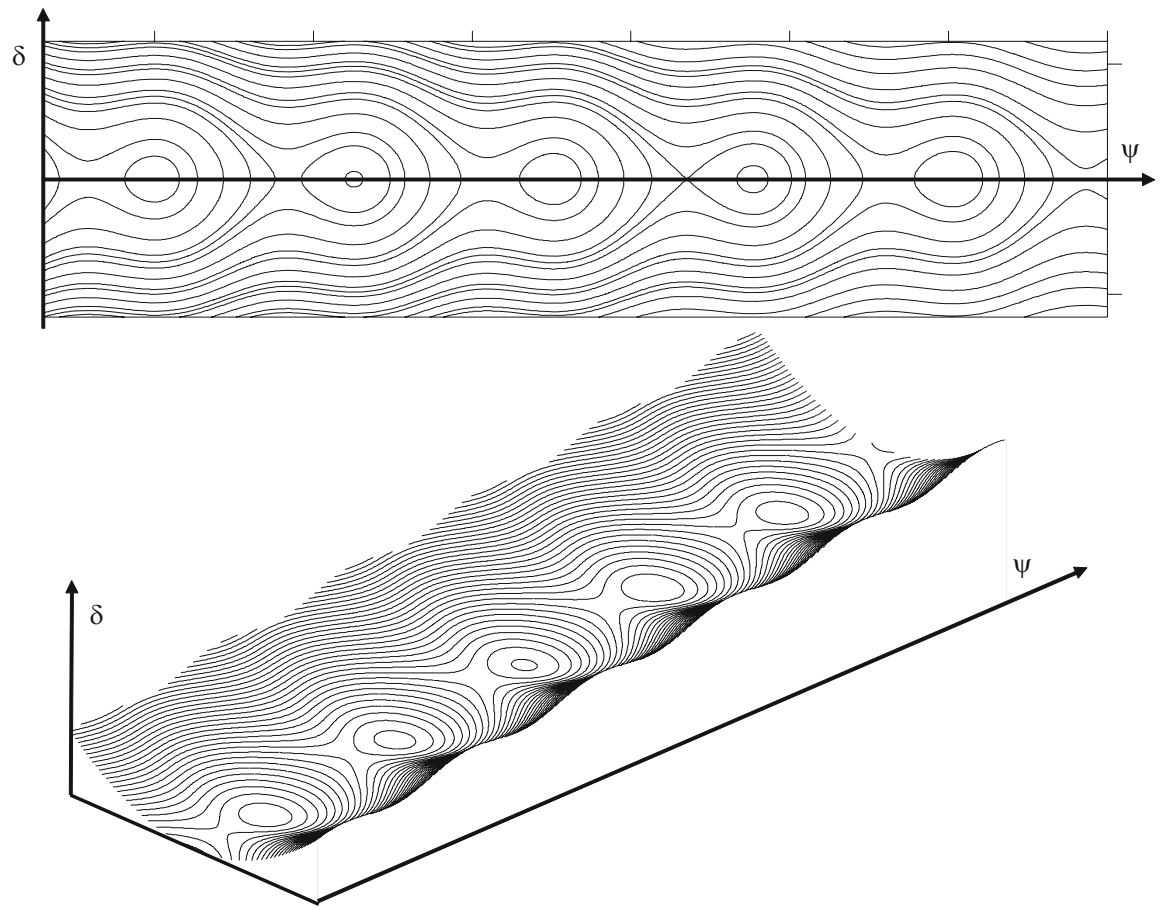

Fig. 9.8 3D rendition of a potential well for moving if buckets $\psi_{\mathrm{s}} \neq 0$

the particle. In case some accelerating sections are not synchronized, the integral collects all contributions as determined by the phase of the rf-wave at the time the particle arrives at a particular section whether it be accelerating or decelerating. The synchronicity condition merely assures that the acceleration in all accelerating sections is the same for each turn.

Particle trajectories in phase space are determined by the Hamiltonian (9.53), which is similar to (9.47) except for the linear term in $\varphi$. Due to this term, the potential well is now tilted (Fig. 9.8) compared to the stationary case (Fig. 9.6). We still have quadratic minima in the potential well function to provide stable phase oscillations, but particles escaping over the maxima of the potential well will be lost because they continuously loose or gain energy as can be seen by following such trajectories in Fig. 9.9. This is different from the case of stationary buckets where such a particle would just wander from bucket to bucket while staying close to the ideal energy at the center of the buckets. Phase stable regions in case of finite values of the synchronous phase are called moving rf-buckets.

The situation is best demonstrated by the three diagrams in Fig. 9.9 showing the accelerating field, the potential, and the phase space diagram as a function of the phase for different synchronous phases. 


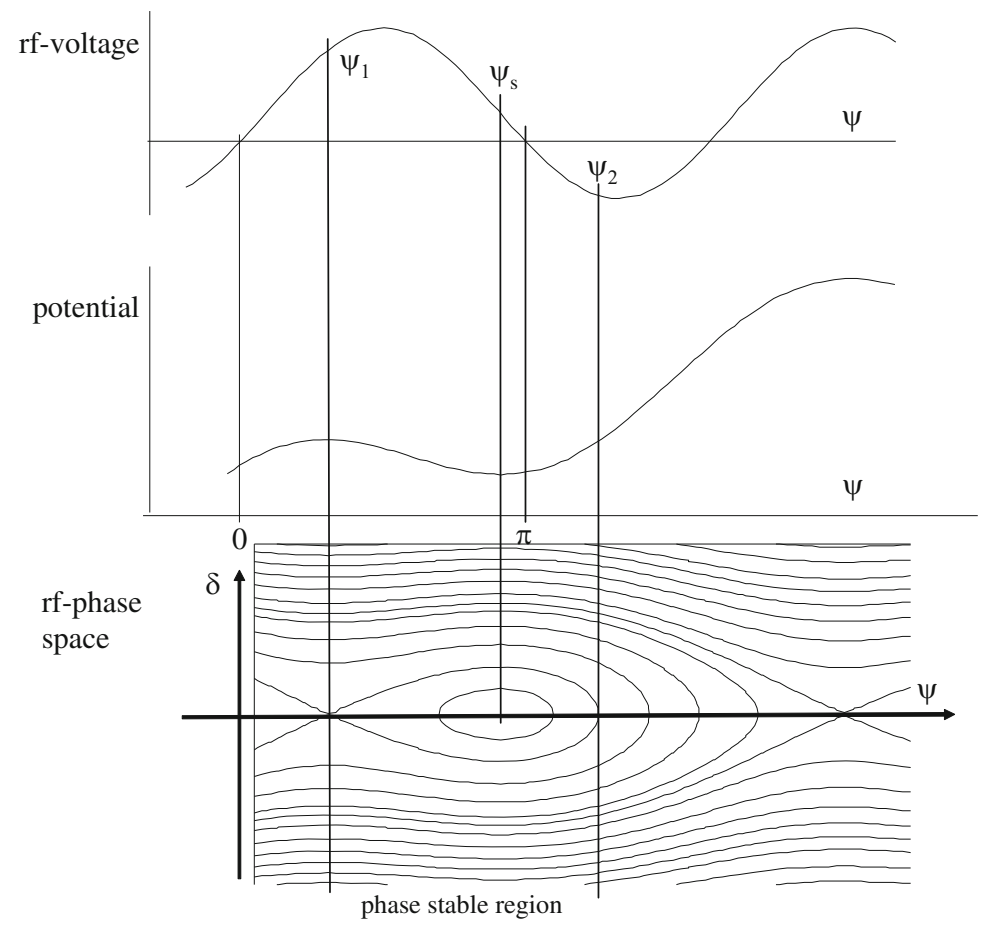

Fig. 9.9 Phase space focusing for moving rf buckets displaying the phase relationship of accelerating field, potential, and rf bucket

In this particular case we have assumed that the particle energy is above transition energy and that the synchronous phase is such that $\cos \psi_{\mathrm{s}}<0$ to obtain stable synchrotron oscillations. The center of the bucket is located at the synchronous phase $\psi_{\mathrm{s}}$ and the longitudinal stability range is limited by the phases $\psi_{1}$ and $\psi_{2}$. In the next section we will derive analytical expressions for the longitudinal stability limit and use the results to determine the momentum acceptance of the bucket as well.

While both phases, $\psi_{\mathrm{s}}$ as well as $\pi-\psi_{\mathrm{s}}$, would supply the desired energy gain only one phase provides stability for the particles. The stable phase is easily chosen by noting that the synchrotron oscillation frequency $\Omega$ must be real and therefore $\eta_{\mathrm{c}} \cos \psi_{\mathrm{s}}>0$. Depending on such operating conditions the rf-bucket has different orientations as shown in Fig. 9.10.

We still can choose whether the electric field should accelerate or decelerate the beam by choosing the sign of the field. For the decelerating case which, for example, is of interest for free electron lasers, the "fish" like buckets in the phase space diagram are mirror imaged. 


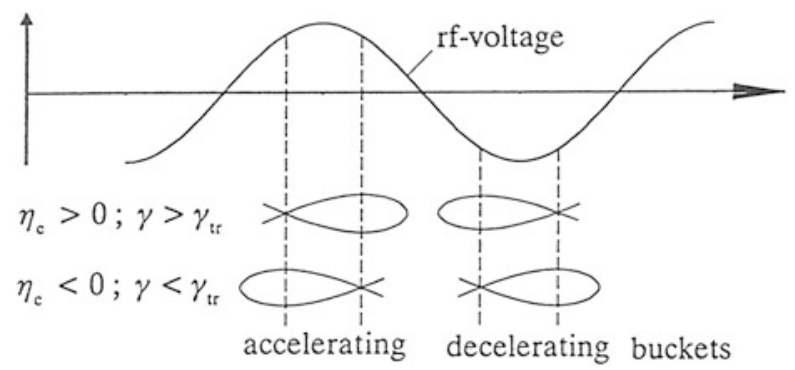

Fig. 9.10 Relationship between $\mathrm{rf}$ phase and orientation of moving rf buckets for accelerating as well as decelerating fields

\subsection{Longitudinal Phase Space Parameters}

We will here investigate in more detail specific properties and parameters of longitudinal phase space motion. From these parameters it will be possible to define stability criteria.

\subsubsection{Separatrix Parameters}

During the discussions of particle dynamics in longitudinal phase space we found specific trajectories in phase space, called separatrices which separate the phase stable region from the region where particles follow unstable trajectories leading away from the synchronous phase and from the ideal momentum. Within the phase stable region particles perform oscillations about the synchronous phase and the ideal momentum. This "focal point" in the phase diagram is called a stable fixed point (sfp). The unstable fixed point (ufp) is located where the two branches of the separatrix cross. The location of fixed points can be derived from the two conditions:

$$
\frac{\partial \mathcal{H}}{\partial \dot{\psi}}=0 \quad \text { and } \quad \frac{\partial \mathcal{H}}{\partial \psi}=0 .
$$

From the first condition, we find with (9.53) that $\dot{\psi}_{\mathrm{f}}=0$ independent of any other parameter. All fixed points are therefore located along the $\psi$-axis of the phase diagram as shown in Fig. 9.11.

The second condition leads to the actual location of the fixed points $\psi_{\mathrm{f}}$ on the $\psi$-axis and is with $\psi=\psi_{\mathrm{s}}+\varphi$

$$
\sin \psi_{\mathrm{f}}-\sin \psi_{\mathrm{s}}=0 .
$$


Fig. 9.11 Characteristic bucket and separatrix parameters

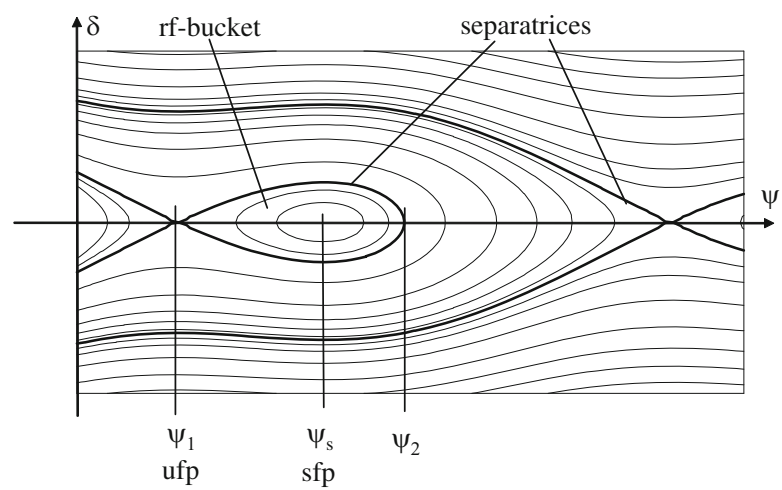

This equation can be solved for $\psi_{\mathrm{f}}=\psi_{\mathrm{s}}$ or $\psi_{\mathrm{f}}=\pi-\psi_{\mathrm{s}}$ and the coordinates of the fixed points are

$$
\begin{array}{ll}
\left(\psi_{\mathrm{sf}}, \dot{\psi}_{\mathrm{sf}}\right)=\left(\psi_{\mathrm{s}}, 0\right) & \text { for the stable fixed point, sfp, and } \\
\left(\psi_{\mathrm{uf}}, \dot{\psi}_{\mathrm{uf}}\right)=\left(\pi-\psi_{\mathrm{s}}, 0\right) & \text { for the unstable fixed point, ufp. }
\end{array}
$$

The distinction between a stable and unstable fixed point is made through the existence of a minimum or maximum in the potential at these points respectively. In Fig. 9.9, this distinction becomes obvious where we note the stable fixed points in the center of the potential minima and the unstable fixed points at the saddle points. The maximum stable phase elongation or bunch length is limited by the separatrix and the two extreme points $\psi_{1}$ and $\psi_{2}$ which we will determine in Sect. 9.3.3.

\subsubsection{Momentum Acceptance}

Particles on trajectories just inside the separatrix reach maximum deviations in phase and momentum from the ideal values in the course of performing synchrotron oscillations. A characteristic property of the separatrix therefore is the definition of the maximum phase or momentum deviation a particle may have and still undergo stable synchrotron oscillations. The value of the maximum momentum deviation is called the momentum acceptance of the accelerator. To determine the numerical value of the momentum acceptance, we use the coordinates of the unstable fixed point (9.57) and calculate the value of the Hamiltonian for the separatrix which is from (9.53) with $\psi_{\mathrm{uf}}=\psi_{\mathrm{s}}+\varphi_{\mathrm{uf}}=\pi-\psi_{\mathrm{s}}$ and $\dot{\psi}_{\mathrm{uf}}=0$

$$
\mathcal{H}_{f}=\frac{\Omega^{2}}{\cos \psi_{\mathrm{s}}}\left[2 \cos \psi_{\mathrm{s}}-\left(\pi-2 \psi_{\mathrm{s}}\right) \sin \psi_{\mathrm{s}}\right] .
$$

Following the separatrix from this unstable fixed point, we eventually reach the location of maximum distance from the ideal momentum. Since $\dot{\varphi}$ is proportional 
to $\Delta p / p_{0}$, the location of the maximum momentum acceptance can be obtained through a differentiation of (9.53) with respect to $\varphi$

$$
\dot{\varphi} \frac{\partial \dot{\varphi}}{\partial \varphi}-\Omega^{2} \frac{\sin \psi_{\mathrm{s}}-\sin \left(\psi_{\mathrm{s}}+\varphi\right)}{\cos \psi_{\mathrm{s}}}=0 .
$$

At the extreme points where the momentum reaches a maximum or minimum, $\partial \dot{\varphi} / \partial \varphi=0$ which occurs at the phase

$$
\sin \left(\psi_{\mathrm{s}}+\varphi\right)=\sin \psi_{\mathrm{s}} \quad \text { or } \quad \varphi=0
$$

This is exactly the condition we found in (9.56) for the location of the stable fixed points and is independent of the value of the Hamiltonian. The maximum momentum deviation or momentum acceptance $\dot{\varphi}_{\text {acc }}$ occurs therefore for all trajectories at the phase of the stable fixed points $\psi=\psi_{\mathrm{s}}$. We equate at this phase (9.58) with (9.53) to derive an expression for the maximum momentum acceptance

$$
\frac{1}{2} \dot{\varphi}_{\mathrm{acc}}^{2}=\Omega^{2}\left[2-\left(\pi-2 \psi_{\mathrm{s}}\right) \tan \psi_{\mathrm{s}}\right]
$$

In accelerator physics it is customary to define an over voltage factor. This factor is equal to the ratio of the maximum rf-voltage in the cavities to the desired energy gain in the cavity $U_{0}$

$$
q=\frac{e V_{0}}{U_{0}}=\frac{1}{\sin \psi_{\mathrm{s}}}
$$

and can be used to replace trigonometric functions of the synchronous phase. To solve (9.61), we use the expression

$$
\frac{1}{2} \pi-\psi_{\mathrm{s}}=\arccos \frac{1}{q}
$$

derived from the identity $\cos \left(\frac{1}{2} \pi-\psi_{\mathrm{s}}\right)=\sin \psi_{\mathrm{s}}$, replace the synchrotron oscillation frequency $\Omega$ by its representation (9.35) and get with (9.17) the momentum acceptance for a moving bucket

$$
\left(\frac{\Delta p}{p_{0}}\right)_{\mathrm{acc}}^{2}=\frac{e V_{0} \sin \psi_{\mathrm{s}}}{\pi h\left|\eta_{\mathrm{c}}\right| c p_{0}} 2\left(\sqrt{q^{2}-1}-\arccos \frac{1}{q}\right) .
$$

The function

$$
F(q)=2\left(\sqrt{q^{2}-1}-\arccos \frac{1}{q}\right)
$$

is shown in Fig. 9.12 as a function of the over voltage factor $q$. 
Fig. 9.12 Over voltage function $F(q)$

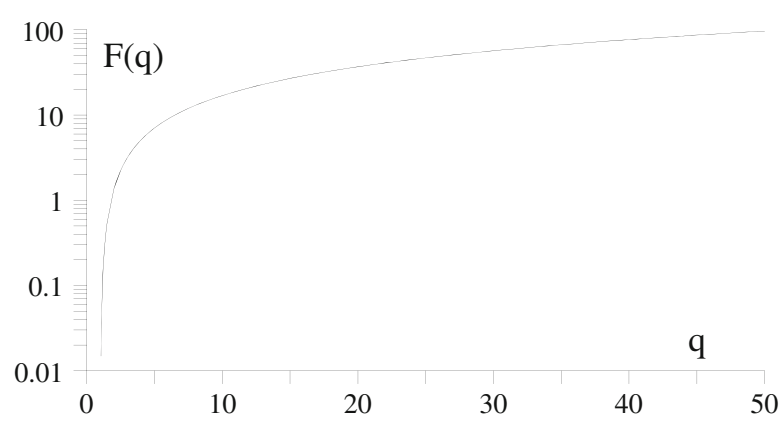

The synchronous phase is always different from zero or $\pi$ when charged particles are to be accelerated. In circular electron and very high-energy proton accelerators the synchronous phase must be nonzero even without acceleration to compensate for synchrotron radiation losses. In low and medium energy circular proton or heavy ion storage rings no noticeable synchrotron radiation occurs and the synchronous phase is either $\psi_{\mathrm{s}}=0$ or $\pi$ depending on the energy being below or above the transition energy. In either case $\sin \psi_{\mathrm{s}}=0$ which, however, does not necessarily lead to a vanishing momentum acceptance since the function $F(q)$ approaches the value $2 q$ and the factor $\sin \psi_{\mathrm{s}} F(q) \rightarrow 2$ in (9.64) while $q \rightarrow \infty$. Therefore stable buckets for protons and heavy ions can be produced with a finite energy acceptance. The maximum momentum acceptance for such stationary buckets is from (9.64)

$$
\left(\frac{\Delta p}{p_{0}}\right)_{\max , \text { stat. }}^{2}=\frac{2 e V_{0}}{\pi h\left|\eta_{\mathrm{c}}\right| c p_{0}}
$$

Note that this expression for the maximum momentum acceptance appears to be numerically inconsistent with (9.39) for $\hat{\varphi}=\pi$, because (9.39) has been derived for small oscillations only $(\hat{\varphi} \ll \pi)$. From Fig. 9.11, we note that the aspect ratios of phase space ellipses change while going from bucket center towards the separatrices. The linear proportionality between maximum momentum deviation and maximum phase of (9.39) becomes distorted for large values of $\hat{\varphi}$ such that the acceptance of the rf-bucket is reduced by the factor $2 / \pi$ from the value of (9.39).

The momentum acceptance is further reduced for moving buckets as the synchronous phase increases. In circular accelerators, where the required energy gain for acceleration or compensation of synchrotron radiation losses per turn is $U_{0}$, the momentum acceptance is

$$
\left(\frac{\Delta p}{p_{0}}\right)_{\text {max }, \text { moving }}^{2}=\frac{U_{0}}{\pi h\left|\eta_{\mathrm{c}}\right| c p_{0}} F(q)=\frac{F(q)}{2 q}\left(\frac{\Delta p}{p_{0}}\right)_{\text {max,static }}^{2} .
$$

The reduction $F(q) / 2 q$ in momentum acceptance is solely a function of the synchronous phase and is shown in Fig. 9.13 for the case $\gamma>\gamma_{\text {tr }}$. 
Fig. 9.13 Reduction factor of the momentum acceptance $F(q) / 2 q$ as a function of the synchronous phase

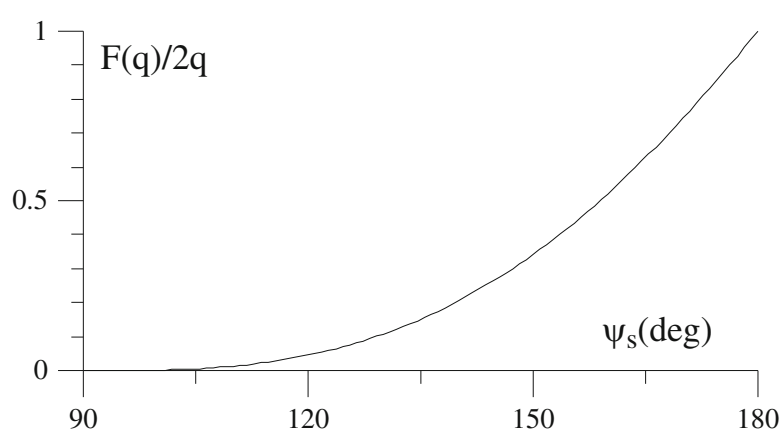

Overall, the momentum acceptance depends on lattice and rf-parameters and scales proportional to the square root of the rf-voltage in the accelerating cavities. Strong transverse focusing decreases the momentum compaction thereby increasing the momentum acceptance while high rf-frequencies diminish the momentum acceptance. Very high frequency accelerating systems based, for example, on high intensity lasers to produce high accelerating fields are expected to have a rather small momentum acceptance and work therefore best with quasi-monoenergetic beams.

It is often customary to use other parameters than the momentum as the coordinates in longitudinal phase space. The most common parameter is the particle energy deviation $\Delta E / \omega_{\mathrm{rf}}$ together with the phase. In these units, we get for the stationary bucket instead of (9.66)

$$
\left.\frac{\Delta E}{\omega_{\mathrm{rf}}}\right|_{\text {max,stat. }}=\sqrt{\frac{2 e V_{0} E_{0} \beta}{\pi h\left|\eta_{\mathrm{c}}\right| \omega_{\mathrm{rf}}^{2}}},
$$

which is measured in $\mathrm{eV}$-sec. Independent of the conjugate coordinates used, the momentum acceptance for moving rf-buckets can be measured in units of a stationary rf-bucket, where the proportionality factor depends only on the synchronous phase.

\subsubsection{Bunch Length}

During the course of synchrotron oscillations, particles oscillate between extreme values in momentum and phase with respect to the reference point and both modes of oscillation are out of phase by $90^{\circ}$. All particles of a beam perform incoherent phase oscillations about a common reference point and generate thereby the appearance of a steady longitudinal distribution of particles, which we call a 
particle bunch. The total bunch length is twice the maximum longitudinal excursion of particles from the bunch center defined by

$$
\frac{\ell}{2}= \pm \frac{c}{h \omega_{\mathrm{rev}}} \hat{\varphi}= \pm \frac{\lambda_{\mathrm{rf}}}{2 \pi} \hat{\varphi}
$$

where $\hat{\varphi}$ is the maximum phase deviation.

In circular electron accelerators the rf-parameters are generally chosen to generate a bucket which is much larger than the core of the beam. Statistical emission of synchrotron radiation photons generates a Gaussian particle distribution in phase space and therefore the rf-acceptance is adjusted to provide stability far into the tails of this distribution. To characterize the beam, however, only the core (one standard deviation) is used. In the case of bunch length or energy deviation we consider therefore only the situation for small oscillation amplitudes. In this approximation the bunch length becomes with (9.39)

$$
\frac{\ell}{2}= \pm\left.\frac{c\left|\eta_{\mathrm{c}}\right|}{\Omega} \frac{\Delta p}{p_{0}}\right|_{\max }
$$

or with (9.35)

$$
\frac{\ell}{2}= \pm\left.\frac{c \sqrt{2 \pi}}{\omega_{\mathrm{rev}}} \sqrt{\frac{\eta_{\mathrm{c}} c p_{0}}{h e \hat{V} \cos \psi_{\mathrm{s}}}} \frac{\Delta p}{p_{0}}\right|_{\max }
$$

The bunch length in a circular electron accelerator depends on a variety of rf-and lattice parameters. It is inversely proportional to the square root of the rf-voltage and frequency. A high frequency and rf-voltage can be used to reduce the bunch length of which only the rf-voltage remains a variable once the system is installed. Practical considerations, however, limit the range of bunch length adjustment this way. The momentum compaction is a lattice function and theoretically allows the bunch length to adjust to any small value. For high-energy electron rings $\eta_{\mathrm{c}} \approx-\alpha_{\mathrm{c}}$ and by arranging the focusing such that the dispersion functions changes sign, the momentum compaction factor of a ring can become zero or even negative. Rings for which $\eta_{\mathrm{c}}=0$ are called isochronous rings [10]. By adjusting the momentum compaction to zero, phase focusing is lost similar to the situation going through transition in proton accelerators and total beam loss may occur. In this case, however, nonlinear, higher order effects become dominant which must be taken into consideration. If on the other hand the momentum compaction is adjusted to very small values, beam instability may be avoidable. [11] The benefit of an isochronous or quasi-isochronous ring would be that the bunch length in an electron storage ring could be made very small. This is important, for example, to either create short synchrotron radiation pulses or maximize the efficiency of a free electron laser by preserving the micro bunching at the laser wavelength as the electron beam orbits in the storage ring. 


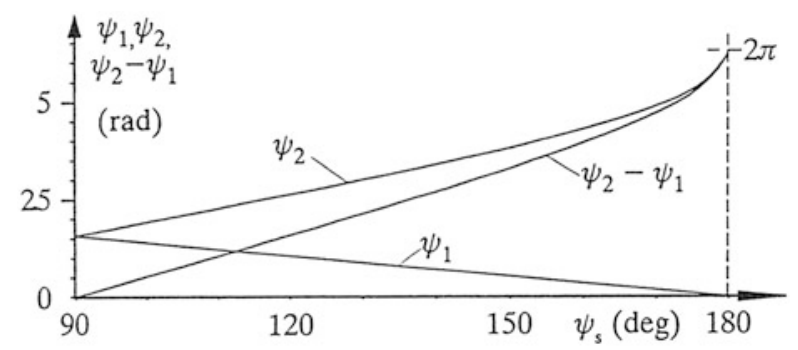

Fig. 9.14 Maximum phases limiting the extend of moving buckets

In a circular proton or ion accelerator we need not be concerned with the preservation of Gaussian tails and therefore the whole rf-bucket could be filled with the beam proper at high density. In this case, the bunch length is limited by the extreme phases $\psi_{1}$ and $\psi_{2}$ of the separatrix. Because the longitudinal extend of the separatrix depends on the synchronous phase, we expect the bunch length to depend also on the synchronous phase. One limit is given by the unstable fixed point at $\psi_{1}=\pi-\psi_{\mathrm{s}}$. The other limit must be derived from (9.53), where we replace $\mathcal{H}$ by the potential of the separatrix from (9.58). Setting $\dot{\varphi}=0$, we get for the second limit of stable phases the transcendental equation

$$
\cos \psi_{1,2}+\psi_{1,2} \sin \psi_{\mathrm{s}}=\left(\pi-\psi_{\mathrm{s}}\right) \sin \psi_{\mathrm{s}}-\cos \psi_{\mathrm{s}}
$$

This equation has two solutions $\bmod (2 \pi)$ of which $\psi_{1}$ is one solution and the other is $\psi_{2}$. Both solutions and their difference are shown in Fig. 9.14 as functions of the synchronous phase.

The bunch length of proton beams is therefore determined only by

$$
\ell_{p}=\frac{\lambda_{\mathrm{rf}}}{2 \pi}\left(\psi_{2}-\psi_{1}\right)
$$

Different from the electron case, we find the proton bunch length to be directly proportional to the rf-wavelength. On the other hand, there is no direct way of compressing a proton bunch by raising or lowering the rf-voltage. This difference stems from the fact that electrons radiate and adjust by damping to a changed rfbucket while non-radiating particles do not have this property. However, applying adiabatic rf-voltage variation we may modify the bunch length as will be discussed in Sect. 9.3.5.

\subsubsection{Longitudinal Beam Emittance}

Separatrices distinguish between unstable and stable regions in the longitudinal phase space. The area of stable phase space in analogy to transverse phase space 
is called the longitudinal beam emittance; however, it should be noted that the definition of longitudinal emittance as used in the accelerator physics community often includes the factor $\pi$ in the numerical value of the emittance and is therefore equal to the real phase space area. To calculate the longitudinal emittance, we evaluate the integral $\oint p \mathrm{~d} q$ where $p$ and $q$ are the conjugate variables describing the synchrotron oscillation.

Similar to transverse beam dynamics we distinguish again between beam acceptance and beam emittance. The acceptance is the maximum value for the beam emittance to be able to pass through a transport line or accelerator components. In the longitudinal phase space the acceptance is the area enclosed by the separatrices. Of course, we ignore here other possible acceptance limitations which are not related to the parameters of the accelerating system. The equation for the separatrix can be derived by equating (9.53) with (9.58) which gives with (9.17) and (9.35)

$$
\left(\frac{\Delta c p}{c p_{0}}\right)^{2}=\frac{e V_{0}}{\pi h\left|\eta_{\mathrm{c}}\right| c p_{0}}\left[\cos \varphi+1+\left(2 \psi_{\mathrm{s}}+\varphi-\pi\right) \sin \psi_{\mathrm{s}}\right] \text {. }
$$

We define a longitudinal beam emittance by

$$
\epsilon_{\varphi}=\int_{S} \frac{\Delta E}{\omega_{\mathrm{rf}}} \mathrm{d} \varphi
$$

where the integral is to be taken along a path $S$ tightly enclosing the beam in phase space. Only for $\psi_{\mathrm{s}}=n \pi$ can this integral be solved analytically. The maximum value of the beam emittance so defined is the acceptance of the system. Numerically, the acceptance of a stationary bucket can be calculated by inserting (9.74) into (9.75) and integration along the enclosing separatrices resulting in

$$
\epsilon_{\varphi, \text { acc }}=8 \sqrt{\frac{2 e V_{0} E_{0} \beta}{\pi h\left|\eta_{\mathrm{c}}\right| \omega_{\mathrm{rf}}^{2}}}
$$

Comparison with the momentum acceptance (9.75) shows the simple relation that the longitudinal acceptance is eight times the energy acceptance

$$
\epsilon_{\varphi, \text { acc }}=\left.8 \frac{\Delta E}{\omega_{\mathrm{rf}}}\right|_{\max , \text { stat }} .
$$

For moving rf-buckets, the integration (9.75) must be performed numerically between the limiting phases $\psi_{1}$ and $\psi_{2}$. The resulting acceptance in percentage of the acceptance for the stationary rf-bucket is shown in Fig. 9.15 as a function of the synchronous phase angle.

The acceptance for $\psi_{\mathrm{s}}<180^{\circ}$ is significantly reduced imposing some practical limits on the maximum rate of acceleration for a given maximum rf-voltage. During the acceleration cycle, the magnetic fields in the lattice magnets are increased 


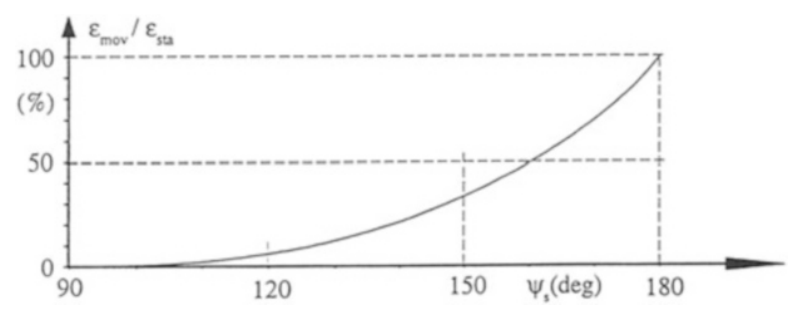

Fig. 9.15 Acceptance of moving $\mathrm{rf}$ buckets in units of the acceptance of a stationary $\mathrm{rf}$ bucket

consistent with the available maximum rf-voltage and by virtue of the principle of phase focusing the particles will keep close to the synchronous phase whenever the rate of energy increase is slow compared to the synchrotron oscillation frequency which is always the case. In high-energy electron synchrotrons or storage rings the required "acceleration" is no more a free parameter but is mainly determined by the energy loss due to synchrotron radiation and a stable beam can be obtained only if sufficient rf-voltage is supplied to provide the necessary acceptance.

\subsubsection{Phase Space Matching}

In transverse phase space a need for matching exists while transferring a beam from one accelerator to another accelerator. Such matching conditions exist also in longitudinal phase space. In the absence of matching part of the beam may be lost due to lack of overlap with the rf-bucket or severe phase space dilution may occur if a beam is injected unmatched into a too large rf-bucket. In the case of electrons a mismatch generally has no detrimental effect on the beam unless part or all of the beam exceeds rf-bucket limitations. Because of synchrotron radiation and concomitant damping, electrons always assume a Gaussian distribution about the reference phase and ideal momentum. The only matching then requires that the rf-bucket is large enough to enclose the Gaussian distribution far into the tails of 7-10 standard deviations.

In proton and heavy ion accelerators such damping is absent and careful phase space matching during the transfer of particle beams from one accelerator to another is required to preserve beam stability and phase space density. A continuous monochromatic beam, for example, being injected into an accelerator with too large an rf-bucket as shown in Fig. 9.16 will lead to a greatly diluted emittance.

This is due to the fact that the synchrotron oscillation is to some extend nonlinear and the frequency changes with oscillation amplitude with the effect that for all practical purposes the beam eventually occupies all available phase space. This does not conflict with Liouville's theorem, since the microscopic phase space is preserved albeit fragmented and spread through filamentation over the whole bucket. 


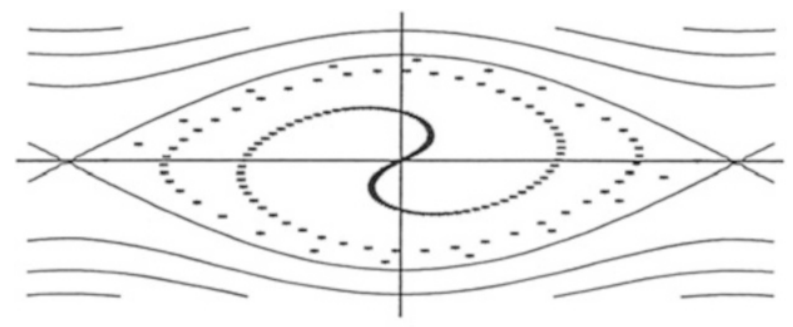

Fig. 9.16 Phase space filamentation after a few synchrotron oscillations

The situation is greatly altered if the rf-voltage is reduced and adjusted to just cover the energy spread in the beam. Not all particles will be accepted, specifically those in the vicinity of the unstable fixed points, but all particles that are injected inside the rf-bucket remain there and the phase space density is not diluted. The acceptance efficiency is equal to the bucket overlap on the beam in phase space. A more sophisticated capturing method allows the capture of almost all particles in a uniform longitudinal distribution by turning on the rf-voltage very slowly [12], a procedure which is also called adiabatic capture.

Other matching problems occur when the injected beam is not continuous. A beam from a booster synchrotron or linear accelerator may be already bunched but may have a bunch length which is shorter than the rf-wavelength or we may want to convert a bunched beam with a significant momentum spread into an unbunched beam with small momentum spread. Whatever the desired modification of the distribution of the beam in phase space may be, there are procedures to allow the change of particular distributions while keeping the overall emittance constant.

For example, to accept a bunched beam with a bunch length shorter than the rf-wavelength in the same way as a continuous beam by matching only the momentum acceptance would cause phase space filamentation as shown in Fig. 9.17. In a proper matching procedure the rf-voltage would be adjusted such that a phase space trajectory surrounds closely the injected beam (Fig. 9.17 left). In mathematical terms, we would determine the bunch length $\hat{\varphi}$ of the injected beam and following (9.70) would adjust the rf-voltage such that the corresponding momentum acceptance $\hat{\delta}=\left(\Delta p / p_{0}\right)_{\max }$ matches the momentum spread in the incoming beam. If no correct matching is done and the beam is injected like shown in (Fig. 9.17 right), then the variation of synchrotron oscillation frequency with amplitude would cause filamentation and dilution of beam phase space. Effectively, this simulates in real space a larger effective emittance.

Equation (9.70) represents a relation between the maximum momentum deviation and phase deviation for small amplitude phase space trajectories which allows us to calculate the bunch length as a function of external parameters. Methods have been discussed in transverse particle dynamics which allow the manipulation of conjugate beam parameters in phase space while keeping the beam emittance constant. Specifically, within the limits of constant phase space we were able to 

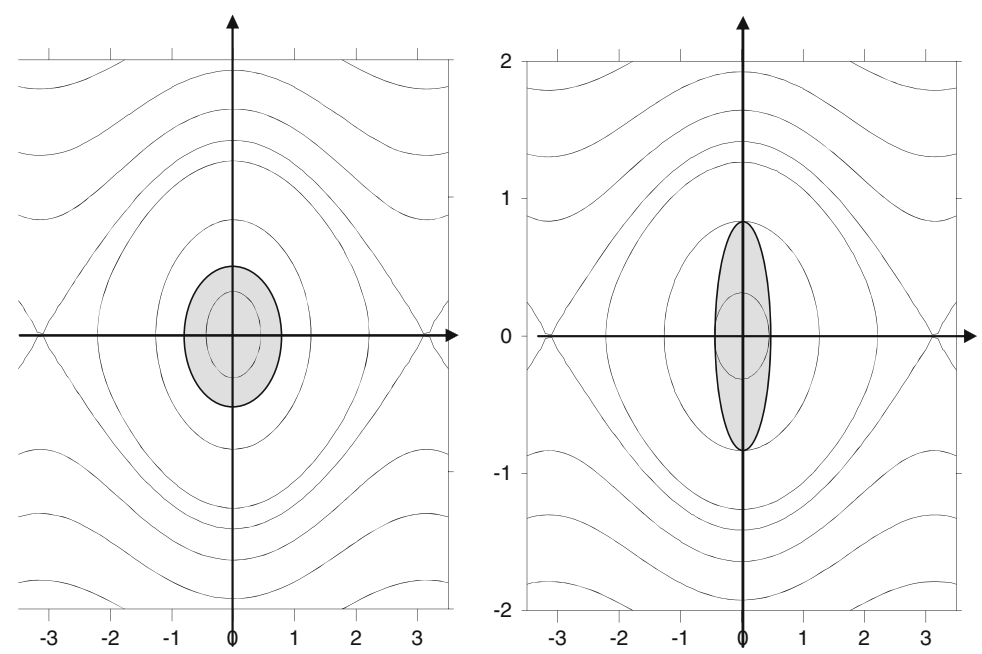

Fig. 9.17 Mismatch for a bunched beam (right). Proper match for a bunched beam (left)

exchange beam size and transverse momentum or beam divergence by appropriate focusing arrangements to produce,for example, a wide parallel beam or a small beam focus.

Similarly, we are able to manipulate within the limits of a constant longitudinal beam emittance the bunch length and momentum spread. The focusing device in this case is the voltage in accelerating cavities. Assume, for example, a particle bunch with a very small momentum spread but a long bunch length as shown in Fig. 9.18 left. To transform such a bunch into a short bunch we would suddenly increase the rf-voltage in a time short compared to the synchrotron oscillation period. The whole bunch then starts to rotate within the new bucket (Fig.9.18 middle) exchanging bunch length for momentum spread. After a quarter synchrotron oscillation period, the bunch length has reached its shortest value and starts to increase again through further rotation of the bunch unless the rf-voltage is suddenly increased a second time to stop the phase space rotation of the bunch (Fig. 9.18 right). The rf-voltage therefore must be increased to such a value that all particles on the bunch boundary follow the same phase space trajectory.

This phase space manipulation can be conveniently expressed by repeated application of (9.39). The maximum momentum deviation $\left(\widehat{\Delta p} / p_{0}\right)_{0}$ and the maximum phase deviation $\hat{\varphi}_{0}$ for the starting situation in Fig. 9.18 (left) are related by

$$
\left.\frac{\widehat{\Delta p}}{p_{0}}\right|_{0}=\frac{\Omega_{0}}{h \omega_{\mathrm{rev}}\left|\eta_{\mathrm{c}}\right|} \hat{\varphi}_{0},
$$

where $\Omega_{0}$ is the starting synchrotron oscillation frequency for the rf-voltage $V_{0}$. To start bunch rotation the rf-voltage is increased to $V_{1}$ (Fig.9.18 middle) and after 


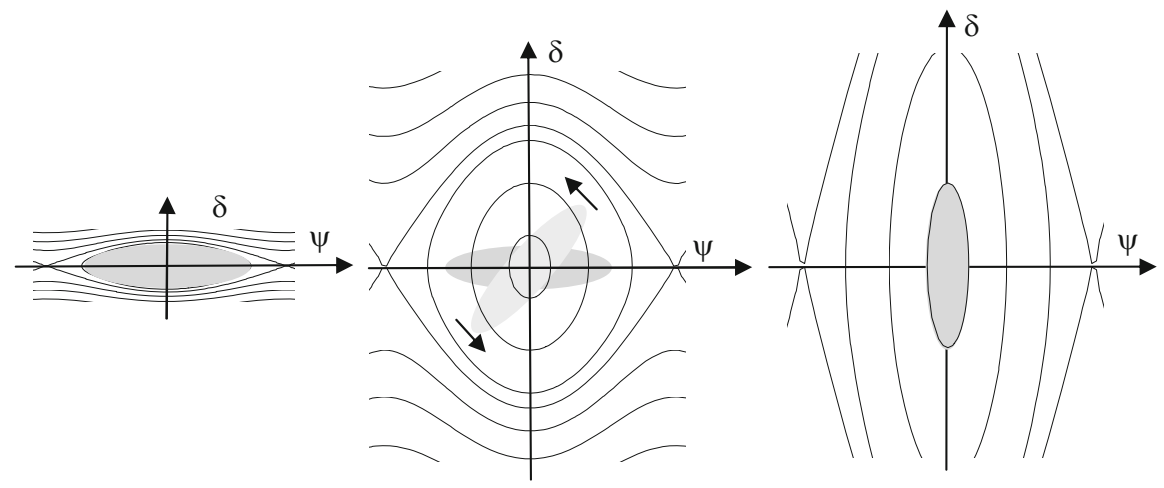

Fig. 9.18 Phase space rotation

a quarter synchrotron oscillation period at the frequency $\Omega_{1} \propto \sqrt{V_{1}}$ the phase deviation $\hat{\varphi}_{0}$ has transformed into the momentum deviation

$$
\left.\frac{\widehat{\Delta p}}{p_{0}}\right|_{1}=\frac{\Omega_{1}}{h \omega_{\mathrm{rev}}\left|\eta_{\mathrm{c}}\right|} \hat{\varphi}_{0}
$$

At the same time the original momentum error $\widehat{\Delta p} /\left.p_{0}\right|_{0}$ has become a phase error $\hat{\varphi}_{1}$ given by

$$
\left.\frac{\widehat{\Delta p}}{p_{0}}\right|_{0}=\frac{\Omega_{1}}{h \omega_{\text {rev }}\left|\eta_{\mathrm{c}}\right|} \hat{\varphi}_{1} .
$$

Now we need to stop further phase space rotation of the whole bunch. This can be accomplished by increasing a second time the rf-voltage during a time short compared to the synchrotron oscillation period in such a way that the new bunch length or $\hat{\varphi}$ is on the same phase space trajectory as the new momentum spread $\widehat{\Delta p} /\left.p_{0}\right|_{1}$ (Fig. 9.18 right). The required rf-voltage is then determined by

$$
\left.\frac{\widehat{\Delta p}}{p_{0}}\right|_{1}=\frac{\Omega_{2}}{h \omega_{\mathrm{rev}}\left|\eta_{\mathrm{c}}\right|} \hat{\varphi}
$$

while solving $\Omega_{2}$ for the voltage $V_{2}$. We take the ratio of (9.77) and (9.80) to get

$$
\frac{\hat{\varphi}_{1} \Omega_{2}}{\hat{\varphi}_{0} \Omega_{0}}=\frac{\widehat{\Delta p} /\left.p_{0}\right|_{1}}{\widehat{\Delta p} /\left.p_{0}\right|_{0}}
$$


and replace the ratio of the momentum spreads by the ratio of (9.78) and (9.79). With $\Omega_{i} \propto \sqrt{V_{i}}$ and $\ell \propto \hat{\varphi}$ we get finally the scaling law for the reduction of the bunch length

$$
\frac{\ell_{1}}{\ell_{0}}=\left(\frac{V_{0}}{V_{2}}\right)^{\frac{1}{4}}
$$

In other words the bunch length can be reduced by increasing the rf-voltage in a two step process and the bunch length reduction scales like the fourth power of the rfvoltage. This phase space manipulation is symmetric in the sense that a beam with a large momentum spread and a short bunch length can be converted into a bunch with a smaller momentum spread at the expense of the bunch length by reducing the rf-voltage in two steps.

The bunch length manipulation described here is correct and applicable only for non-radiating particles. For radiating particles like electrons, the bunch manipulation is easier due to damping effects. Equation (9.39) still holds, but the momentum spread is independently determined by synchrotron radiation and the bunch length therefore scales simply proportional to the square root of the rf-voltage.

\subsection{Higher-Order Phase Focusing}

The principle of phase focusing is fundamental for beam stability in circular accelerators and we find the momentum compaction factor to be a controlling quantity. Since the specific value of the momentum compaction determines critically the beam stability, it is interesting to investigate the consequences to beam stability as the momentum compaction factor varies. Specifically, we will discuss the situation where the linear momentum compaction factor is reduced to very small values and higher-order terms become significant. This is, for example, of interest in proton or ion accelerators going through transition energy during acceleration, or as we try to increase the quadrupole focusing in electron storage rings to obtain a small beam emittance, or when we intentionally reduce the momentum compaction to reduce the bunch length. In extreme cases, the momentum compaction factor becomes zero at transition energy or in an isochronous storage ring where the revolution time is made the same for all particles independent of the momentum. The linear theory of phase focusing would predict beam loss in such cases due to lack of phase stability. To accurately describe beam stability when the momentum compaction factor is small or vanishes, we cannot completely ignore higher-order terms. Some of the higher-order effects on phase focusing will be discussed here. There are two main contributions to the higher-order momentum compaction factor, one from the dispersion function and the other from the momentum dependent path length. First, we derive the higher-order contributions to the dispersion function, and then apply the results to the principle of phase focusing to determine the perturbation on the beam stability. 


\subsubsection{Dispersion Function in Higher Order}

The first-order change in the reference path for off energy particles is proportional to the relative momentum error. The proportionality factor is a function of the position $s$ and is called the dispersion function. This result is true only in linear beam dynamics. We will now derive chromatic effects on the reference path in higher order to allow a more detailed determination of the chromatic stability criteria. The linear differential equation for the normalized dispersion function is

$$
\frac{\mathrm{d}^{2} w_{0}}{\mathrm{~d} \varphi^{2}}+v^{2} w_{0}=v^{2} \beta^{\frac{3}{2}} \kappa=v^{2} F(\varphi)
$$

where $\varphi$ is the betatron phase, $w_{0}=\eta_{0} / \sqrt{\beta}, \beta(z)$ the betatron function and $\eta(z)$ the undisturbed dispersion function. The periodic solution of (9.84) is called the normalized dispersion function $w_{0}(\varphi)=\sum_{n=-\infty}^{+\infty} \frac{v^{2} F_{n} \mathrm{e}^{\mathrm{i} n \varphi}}{v^{2}-n^{2}}$, and

$$
F(\varphi)=\beta^{\frac{3}{2}} \kappa=\sum F_{n} \mathrm{e}^{\mathrm{i} n \varphi} .
$$

This linear solution includes only the lowest-order chromatic error term from the bending magnets and we must therefore include higher-order chromatic terms into the differential equation of motion. To do that we use the general differential equation of motion while ignoring all coupling terms

$$
\begin{aligned}
x^{\prime \prime}+\left(\kappa^{2}+k\right) x & =+\kappa \delta-\kappa \delta^{2}+\kappa \delta^{3}-\frac{1}{2} m(1-\delta) x^{2}+\kappa^{\prime} x x^{\prime} \\
& -\left(\kappa^{3}+2 \kappa k\right)(1-\delta) x^{2}+\frac{1}{2} \kappa(1-\delta) x^{\prime 2} \\
& +\left(2 \kappa^{2}+k\right) x \delta-\left(k+2 \kappa^{2}\right) x \delta^{2}+O(4),
\end{aligned}
$$

where $\kappa=1 / \rho$. We are only interested in the chromatic solution with vanishing betatron oscillation amplitudes and insert for the particle position therefore

$$
x_{\eta}=\eta_{0} \delta+\eta_{1} \delta^{2}+\eta_{2} \delta^{3}+\mathcal{O}(4) .
$$

Due to the principle of linear superposition separate differential equations exist for each component $\eta_{i}$ by collecting on the right-hand side terms of equal power in $\delta$. For the terms linear in $\delta$, we find the well-known differential equation for the dispersion function

$$
\eta_{0}^{\prime \prime}+K(z) \eta_{0}=\kappa=\sum_{n} F_{0 n} \mathrm{e}^{\mathrm{i} n \varphi}
$$


where we also express the perturbations by its Fourier expansion. The terms quadratic in $\delta$ form the differential equation

$$
\begin{aligned}
\eta_{1}^{\prime \prime}+K(z) \eta_{1}= & -\sum_{n} F_{0 n} \mathrm{e}^{\mathrm{i} n \varphi} \\
& -\frac{1}{2} m \eta_{0}^{2}-\left(\kappa^{3}+2 \kappa k\right) \eta_{0}^{2}+\frac{1}{2} \kappa \eta_{0}^{\prime 2}+\kappa^{\prime} \eta_{0} \eta_{0}^{\prime}+\left(2 \kappa^{2}+k\right) \eta_{0} \\
= & -\sum_{n} F_{0 n} \mathrm{e}^{\mathrm{i} n \varphi}+\sum_{n} F_{1 n} \mathrm{e}^{\mathrm{i} n \varphi},
\end{aligned}
$$

and terms cubic in $\delta$ are determined by

$$
\begin{aligned}
\eta_{2}^{\prime \prime}+K(z) \eta_{2}=+ & \sum_{n} F_{0 n} \mathrm{e}^{\mathrm{i} n \varphi}-\sum_{n} F_{1 n} \mathrm{e}^{\mathrm{i} n \varphi} \\
& -m \eta_{0} \eta_{1}-2\left(\kappa^{3}+2 \kappa k\right) \eta_{0} \eta_{1}+\left(2 \kappa^{2}+k\right) \eta_{1} \\
& +\kappa \eta_{0}^{\prime} \eta_{1}^{\prime}+\kappa^{\prime}\left(\eta_{0} \eta_{1}^{\prime}+\eta_{0}^{\prime} \eta_{1}\right)+\kappa^{\prime} \eta_{0} \eta_{0}^{\prime} \\
= & +\sum_{n} F_{0 n} \mathrm{e}^{\mathrm{i} n \varphi}-\sum_{n} F_{1 n} \mathrm{e}^{\mathrm{i} n \varphi}+\sum_{n} F_{2 n} \mathrm{e}^{\mathrm{i} n \varphi}
\end{aligned}
$$

We note that the higher-order dispersion functions are composed of the negative lower-order solutions plus an additional perturbation. After transformation of these differential equations into normalized variables, $w=\eta / \sqrt{\beta}$, etc., we get with $j=$ $0,1,2$ differential equations of the form

$$
\ddot{w}_{j}(\varphi)+v_{0}^{2} w_{j}(\varphi)=v_{0}^{2} \beta^{3 / 2} F(z)=v_{0}^{2} \sum_{m=0}^{m=j} \sum_{n=-\infty}^{n=\infty}(-1)^{m+j} \beta^{3 / 2} F_{m n} \mathrm{e}^{\mathrm{i} n \varphi},
$$

where we have expressed the periodic perturbation on the r.h.s. by an expanded Fourier series. Noting that the dispersion functions $w_{j}(\varphi)$ are periodic, we try the ansatz

$$
w_{j}(\varphi)=\sum_{n} w_{j n} \mathrm{e}^{\mathrm{i} n \varphi},
$$

and insertion into (9.91) allows to solve for the individual Fourier coefficients $w_{j n}$ by virtue of the orthogonality of the exponential functions $\mathrm{e}^{\mathrm{i} n \varphi}$. We get for the dispersion functions up to second order and reverting to the ordinary $\eta$-function

$$
\eta_{0}(\varphi)=+\beta^{2}(\varphi) \sum_{n} \frac{F_{0 n} \mathrm{e}^{\mathrm{i} n \varphi}}{v^{2}-n^{2}},
$$




$$
\begin{aligned}
& \eta_{1}(\varphi)=-\beta^{2}(\varphi) \sum_{n} \frac{F_{0 n} \mathrm{e}^{\mathrm{i} n \varphi}}{v^{2}-n^{2}}+\beta^{2}(\varphi) \sum_{n} \frac{F_{1 n} \mathrm{e}^{\mathrm{i} n \varphi}}{v^{2}-n^{2}} \\
& \eta_{2}(\varphi)=+\beta^{2}(\varphi) \sum_{n} \frac{F_{0 n} \mathrm{e}^{\mathrm{i} n \varphi}}{v^{2}-n^{2}}-\beta^{2}(\varphi) \sum_{n} \frac{F_{1 n} \mathrm{e}^{\mathrm{i} n \varphi}}{v^{2}-n^{2}}+\beta^{2}(\varphi) \sum_{n} \frac{F_{2 n} \mathrm{e}^{\mathrm{i} n \varphi}}{v^{2}-n^{2}}
\end{aligned}
$$

The solutions of the higher-order differential equations have the same integerresonance behavior as the linear solution for the dispersion function. The higherorder corrections will become important for lattices where strong sextupoles are required in which cases the sextupole terms may be the major perturbations to be considered. Other perturbation terms depend mostly on the curvature $\kappa$ in the bending magnets and, therefore, maybe small for large rings or beam-transport lines with weak bending magnets.

\subsubsection{Path Length in Higher Order}

The path length together with the velocity of particles governs the time of arrival at the accelerating cavities from turn to turn and therefore defines the stability of a particle beam. Generally, only the linear dependence of the path length on particle momentum is considered. We find, however, higher-order chromatic contributions of the dispersion function to the path length as well as momentum independent contributions due to the finite angle of the trajectory with respect to the ideal orbit during betatron oscillations.

The path length for a particular trajectory from point $z_{0}$ to point $z$ in our curvilinear coordinate system can be derived from the integral $L=\oint_{0}^{z} \mathrm{~d} s$, where $s$ is the coordinate along the particular trajectory. This integral can be expressed by

$$
L=\oint \sqrt{(1+\kappa x)^{2}+x^{\prime 2}+y^{\prime 2}} \mathrm{~d} z,
$$

where the first term of the integrand represents the contribution to the path length due to curvature generated by bending magnets while the second and third term are contributions due to finite horizontal and vertical betatron oscillations. For simplicity, we ignore vertical bending magnets. Where this simplification cannot be made, it is straight forward to extend the derivation of the path length in higher order to include bending and betatron oscillations in the vertical plane as well. We expand (9.94) up to second order and get for the path length variation $\Delta L=L-L_{0}$

$$
\Delta L=\oint\left(\kappa x+\frac{1}{2} \kappa^{2} x^{2}+\frac{1}{2} x^{\prime 2}+\frac{1}{2} y^{\prime 2}\right) \mathrm{d} z+\mathcal{O}(3)
$$


The particle amplitudes are composed of betatron oscillation, orbit distortions and off energy orbits

$$
\begin{aligned}
& x=x_{\beta}+x_{0}+\eta_{0} \delta+\eta_{1} \delta^{2}+\ldots, \\
& y=y_{\beta}+y_{0},
\end{aligned}
$$

where $\left(x_{\beta}, y_{\beta}\right)$ describe the betatron oscillations and $\left(\eta_{0}, \eta_{1}, \ldots\right)$ are the linear and higher-order dispersion functions derived in Sect. 9.4.1. The quantities $\left(x_{0}, y_{0}\right)$ describe the deviation of the actual orbit from the ideal orbit or orbit distortion due to magnetic field and alignment errors.

Evaluating the integral (9.95), we note that the oscillatory character of $\left(x_{\beta}, y_{\beta}\right)$ causes all terms linear in $\left(x_{\beta}, y_{\beta}\right)$ to vanish while averaging over many turns. The orbit distortions $\left(x_{0}, y_{0}\right)$ are statistical in nature since the correction in a real accelerator is done such that $\left\langle x_{0}\right\rangle=0$ and $\left\langle x_{0}^{\prime}\right\rangle=0$. Betatron oscillations and orbit distortions are completely independent and therefore cross terms like $\left\langle x_{\beta} x_{0}\right\rangle$ vanish. The dispersion function $\eta_{0}$ and the higher-order term $\eta_{1}$ are unique periodic solutions of the inhomogeneous equation of motion. For the betatron oscillations we assume a nonresonant tune which causes terms like $\left\langle x_{\beta} \eta_{0}\right\rangle$ to vanish as well. With these results the path length variation is

$$
\begin{aligned}
\Delta L & \approx \frac{1}{2} \oint\left(x_{\beta}^{\prime 2}+y_{\beta}^{\prime 2}+x_{0}^{\prime 2}+y_{0}^{\prime 2}+\kappa^{2} x_{\beta}^{2}+\kappa^{2} x_{0}^{2}\right) \mathrm{d} z \\
& +\delta \oint \kappa \eta_{0} \mathrm{~d} z+\delta^{2} \oint\left(\kappa \eta_{1}+\frac{1}{2} \kappa^{2} \eta_{0}^{2}+\frac{1}{2} \eta_{0}^{\prime 2}\right) \mathrm{d} z .
\end{aligned}
$$

There are three main contributions of which two are of chromatic nature. The finite transverse betatron oscillations as well as orbit distortions contribute to a second order increase in the path length of the beam transport system which is of non-chromatic nature. Equation (9.97) can be simplified by using the explicit expressions for the particle motion $x_{\beta}(z)=\sqrt{\epsilon_{x} \beta_{x}(z)} \sin \psi_{x}(z)$ and $x_{\beta}^{\prime}(z)=\sqrt{\epsilon_{x} / \beta_{x}(z)}\left[\cos \psi_{x}(z)-\alpha_{x}(z) \sin \psi_{x}(z)\right]$. Forming the square $x_{\beta}^{\prime 2}=\epsilon_{x} / \beta_{x}(z)\left(\cos ^{2} \psi_{x}-\alpha_{x} \sin 2 \psi_{x}+\alpha_{x}^{2} \sin ^{2} \psi_{x}\right)$ and averaging over all phases $\psi_{x}$

$$
\begin{aligned}
\oint x_{\beta}^{\prime 2} \mathrm{~d} z & =\epsilon_{x} \oint \frac{1}{\beta_{x}}\left(\cos ^{2} \psi_{x}+\alpha_{x}^{2} \sin ^{2} \psi_{x}\right) \mathrm{d} z \\
& =\epsilon_{x} \oint\left(\frac{\cos 2 \psi_{x}}{\beta_{x}}+\gamma_{x} \sin ^{2} \psi_{x}\right) \mathrm{d} z \approx \frac{1}{2} \epsilon_{x} \oint \gamma_{x} \mathrm{~d} z
\end{aligned}
$$

where we used the simplifying expression $\left\langle\sin ^{2} \psi_{x}\right\rangle \approx \frac{1}{2}$. Similarly, we get $\oint y_{\beta}^{\prime 2} \mathrm{~d} z \approx \frac{1}{2} \epsilon_{y} \oint \gamma_{y} \mathrm{~d} z$, and $\oint \kappa^{2} x_{\beta}^{2} \mathrm{~d} z \approx \frac{1}{2} \epsilon_{x} \oint \kappa^{2} \beta_{x} \mathrm{~d} z$. The integrals are taken 
over the entire beam transport line of length $L_{0}$ and using average values for the integrands, the path-length variation is

$$
\begin{aligned}
\frac{\Delta L}{L_{0}} & =\frac{1}{4}\left(\epsilon_{x}\left\langle\gamma_{x}\right\rangle+\epsilon_{y}\left\langle\gamma_{y}\right\rangle+\epsilon_{x}\left\langle\kappa^{2} \beta_{x}\right\rangle\right) \\
& +\frac{1}{2}\left\langle x_{0}^{\prime 2}\right\rangle+\frac{1}{2}\left\langle y_{0}^{\prime 2}\right\rangle+\frac{1}{2}\left\langle\kappa^{2} x_{0}^{2}\right\rangle \\
& +\alpha_{\mathrm{c}} \delta+\left(\left\langle\kappa \eta_{1}\right\rangle+\frac{1}{2}\left\langle\kappa^{2} \eta_{0}^{2}\right\rangle+\frac{1}{2}\left\langle{\eta_{0}^{\prime}}^{2}\right\rangle\right) \delta^{2}+\mathcal{O}(3) .
\end{aligned}
$$

In this expression for the path-length variation we find separate contributions due to betatron oscillations, orbit distortion and higher-order chromatic effects. We have used the emittance $\epsilon$ as the amplitude factor for betatron oscillation and get therefore a path length spread within the beam due to the finite beam emittance $\epsilon$. Note specifically that for an electron beam this emittance scales by the factor $n_{\sigma}^{2}$ to include Gaussian tails, where $n_{\sigma}$ is the oscillation amplitude in units of the standard amplitude $\sigma$. For whole beam stability a total emittance of $\epsilon_{\text {tot }}=7^{2} \epsilon-10^{2} \epsilon$ should be considered. For stable machine conditions, the contribution of the orbit distortion is the same for all particles and can therefore be corrected by an adjustment of the rf-frequency. We include these terms here, however, to allow the estimation of allowable tolerances for dynamic orbit changes.

\subsubsection{Higher Order Momentum Compaction Factor}

The longitudinal phase stability in a circular accelerator depends on the value of the momentum compaction $\eta_{\mathrm{c}}$, which actually regulates the phase focusing to obtain stable particle motion. This parameter is not a quantity that can be chosen freely in the design of a circular accelerator without jeopardizing other desirable design goals. If, for example, a small beam emittance is desired in an electron storage ring, or if for some reason it is desirable to have an isochronous ring where the revolution time for all particles is the same, the momentum compaction should be made to become very small. This in itself does not cause instability unless the momentum compaction approaches zero and higher-order chromatic terms modify phase focusing to the extent that the particle motion becomes unstable. To derive conditions for the loss of phase stability, we evaluate the path length variation (9.99) with momentum in higher order

$$
\frac{\Delta L}{L_{0}}=\alpha_{\mathrm{c}} \delta+\alpha_{1} \delta^{2}+\xi+\mathcal{O}(3),
$$

where $\xi$ represents the momentum independent term

$$
\xi=\frac{1}{4}\left(\epsilon_{x}\left\langle\gamma_{x}\right\rangle+\epsilon_{y}\left\langle\gamma_{y}\right\rangle+\epsilon_{x}\left\langle\kappa^{2} \beta_{x}\right\rangle\right)
$$


and

$$
\alpha_{1}=\left\langle\kappa \eta_{1}\right\rangle+\frac{1}{2}\left\langle\kappa^{2} \eta_{0}^{2}\right\rangle+\frac{1}{2}\left\langle\eta_{0}^{\prime 2}\right\rangle
$$

is the non-linear momentum compaction factor.

From the higher order dispersion and path length we may now derive the value of the higher order momentum compaction factor. First we note that we are not interested in oscillatory terms. Therefore $(9.93 \mathrm{~b})$ reduces to

$$
\eta_{1}(\varphi)=-\frac{\beta^{2}(\varphi)}{v^{2}} F_{00}+\frac{\beta^{2}(\varphi)}{v^{2}} F_{10}
$$

where

$$
\begin{aligned}
& F_{00}=\langle\kappa\rangle \text { and } \\
& F_{10}=\left\langle-\frac{1}{2} m \eta_{0}^{2}-\left(\kappa^{3}+2 \kappa k\right) \eta_{0}^{2}+\frac{1}{2} \kappa \eta_{0}^{\prime 2}+\kappa^{\prime} \eta_{0} \eta_{0}^{\prime}+\left(2 \kappa^{2}+k\right) \eta_{0}\right\rangle .
\end{aligned}
$$

Furthermore

$$
\left\langle\kappa \eta_{1}\right\rangle=\left\langle\kappa \frac{\beta^{2}(\varphi)}{v^{2}}\right\rangle\left(-F_{00}+F_{10}\right),
$$

where the average is to be taken over one superperiod of the accelerator. The other terms in (9.102) and (9.101) are straight forward. With the knowledge of the higher order momentum compaction factor we are now able to consider higher order phase motion.

\subsubsection{Higher-Order Phase Space Motion}

Following the derivation of the linear phase equation, we note that it is the variation of the revolution time with momentum rather than the path-length variation that affects the synchronicity condition. With the expanded momentum compaction $\eta_{\mathrm{c}}=$ $\frac{1}{\gamma^{2}}-\alpha_{\mathrm{c}}$ we get the differential equation for the phase oscillation to second order

$$
\frac{\partial \psi}{\partial t}=-\omega_{\mathrm{rf}}\left(\eta_{\mathrm{c}} \delta-\alpha_{1} \delta^{2}-\xi\right)
$$

and for the momentum oscillation

$$
\frac{\partial \delta}{\partial t}=\frac{e V_{\mathrm{rf}}}{T_{0} c p_{0}}\left(\sin \psi-\sin \psi_{\mathrm{s}}\right) .
$$




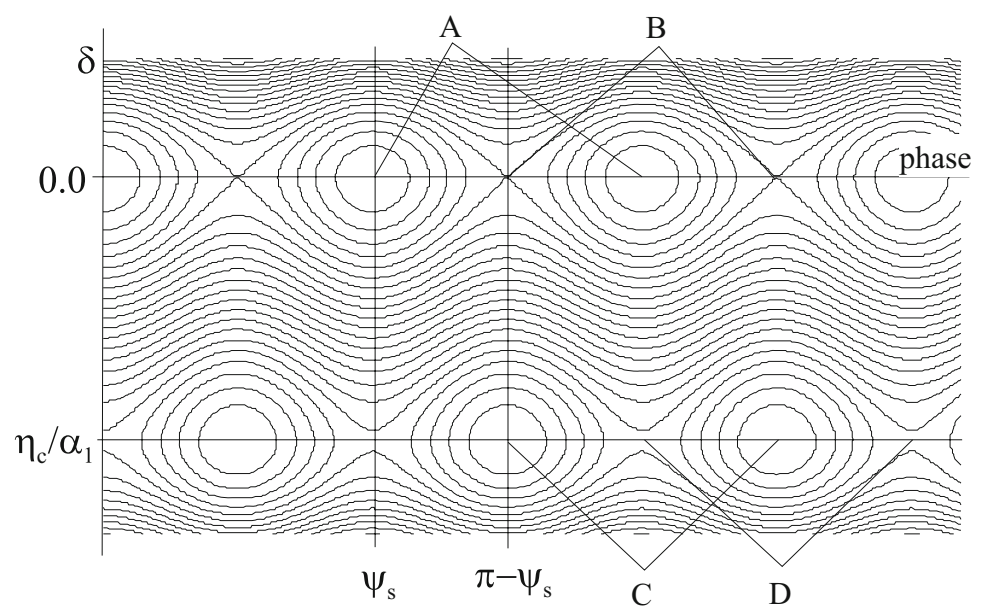

Fig. 9.19 Second-order longitudinal phase space for $\psi_{\mathrm{s}}=0, \xi=0$ and weak perturbation $\alpha_{1} / \eta_{\mathrm{c}}=-3.0$

In linear approximation, where $\alpha_{1}=0$ and $\xi=0$, a single pair of fixed points and separatrices exist in phase space. These fixed points can be found from the condition that $\dot{\psi}=0$ and $\dot{\delta}=0$ and they lie on the abscissa for $\delta=0$. The stable fixed point is located at $\left(\psi_{\mathrm{sf}}, \delta_{\mathrm{sf}}\right)=\left(\psi_{\mathrm{s}}, 0\right)$ defining the center of the rfbucket where stable phase oscillations occur. The unstable fixed point at $\left(\psi_{\mathrm{uf}}, \delta_{\mathrm{uf}}\right)=$ $\left(\pi-\psi_{\mathrm{s}}, 0\right)$ defines the crossing point of the separatrices separating the trajectories of oscillations from those of librations.

Considering also higher-order terms in the theory of phase focusing leads to a more complicated pattern of phase space trajectories. Setting (9.106) equal to zero we note that the abscissae of the fixed points are at the same location as for the linear case

$$
\psi_{1 \mathrm{f}}=\psi_{\mathrm{s}} \quad \text { and } \quad \psi_{2 \mathrm{f}}=\pi-\psi_{\mathrm{s}} .
$$

The energy coordinates of the fixed points, however, are determined by solving (9.105) for $\dot{\psi}=0$ or

$$
\eta_{\mathrm{c}} \delta-\alpha_{1} \delta^{2}-\xi=0
$$

with the solutions

$$
\delta_{\mathrm{f}}=+\frac{\eta_{\mathrm{c}}}{2 \alpha_{1}}(1 \pm \sqrt{1-\Gamma})
$$



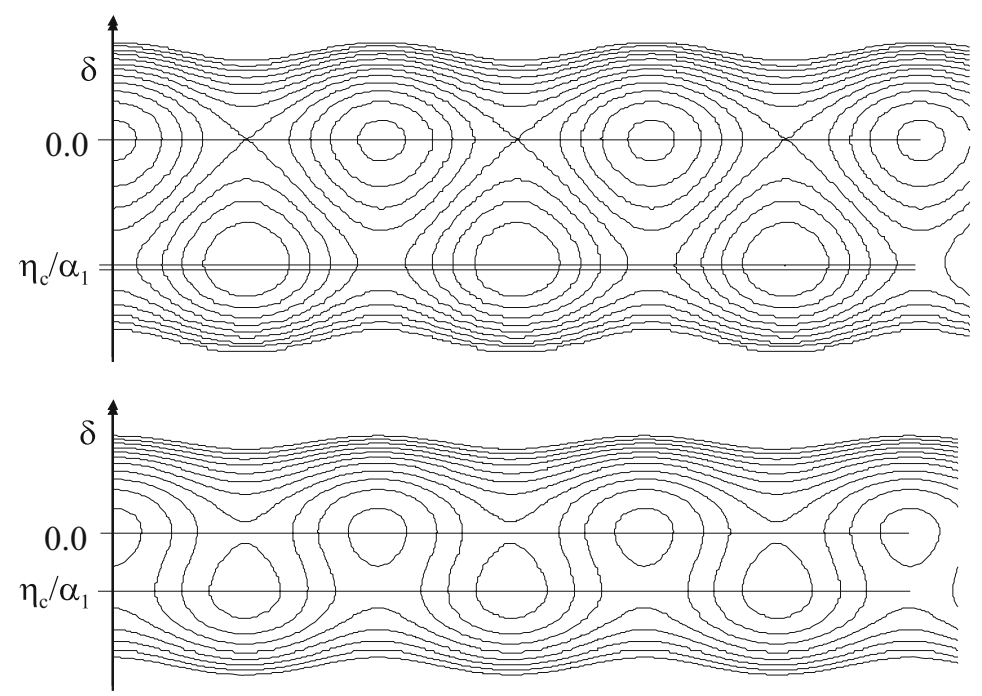

Fig. 9.20 Higher-order longitudinal phase space diagrams for $\psi_{\mathrm{s}}=0, \xi=0$ and strong perturbation $\alpha_{1} / \eta_{\mathrm{c}}=-6.0$ (top) and $\alpha_{1} / \eta_{\mathrm{c}}=-13.5$ (bottom)

where

$$
\Gamma=\frac{4 \xi \alpha_{1}}{\eta_{\mathrm{c}}^{2}}
$$

Due to the quadratic character of (9.108), we get now two layers of fixed points with associated areas of oscillation and libration. In Figs. 9.19, 9.20, the phase diagrams are shown for increasing values of $\alpha_{1}$ while for now we set the momentum independent perturbation $\xi=0$. Numerically, the contour lines have been calculated from the Hamiltonian (9.114) with $\Delta / 2 \eta_{\mathrm{c}}=0.005$, where $\Delta$ is defined in (26199). The appearance of the second layer of stable islands and the increasing perturbation of the original rf-buckets is obvious. There is actually a point [Fig. 9.20 (top)] where the separatrices of both island layers merge. We will use this merging of the separatrices later to define a tolerance limit for the perturbation on the momentum acceptance.

The coordinates of the fixed points in the phase diagram are determined from (9.116), (9.117) and are for the linear fixed points in the first layer

$$
\begin{aligned}
& \text { point A: } \psi_{\mathrm{A}}=\psi_{\mathrm{s}}, \quad \delta_{\mathrm{A}}=\frac{\eta_{\mathrm{c}}}{2 \alpha_{1}}(1-\sqrt{1-\Gamma}) \text {, } \\
& \text { point B: } \psi_{\mathrm{B}}=\pi-\psi_{\mathrm{s}}, \quad \delta_{\mathrm{B}}=\frac{\eta_{\mathrm{c}}}{2 \alpha_{1}}(1-\sqrt{1-\Gamma}) \text {. }
\end{aligned}
$$




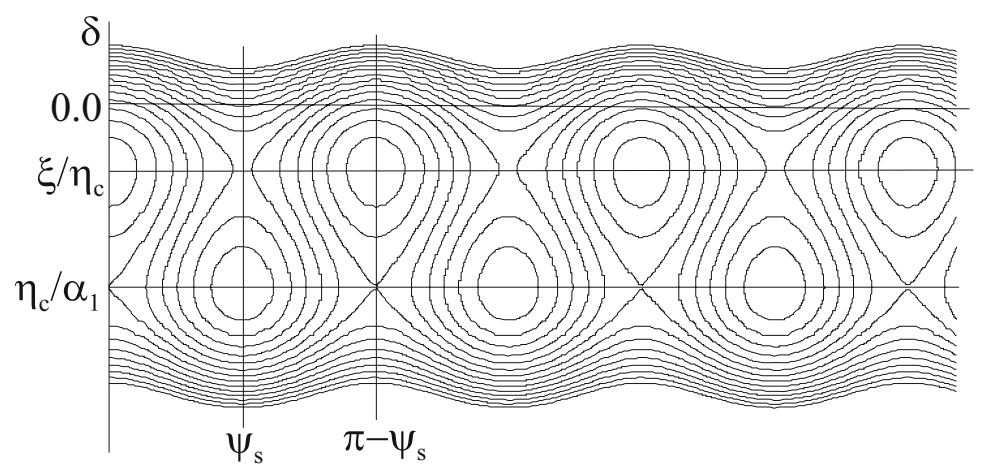

Fig. 9.21 Second-order longitudinal phase space for the same parameters as Fig. 9.20 (top), but now with $2 \xi / \eta_{\mathrm{c}}=-0.125$

The momenta of these fixed points are at $\delta=0$ for $\Gamma=0$ consistent with earlier discussions. As orbit distortions and betatron oscillations increase, however, we note a displacement of the equilibrium momentum as $\Gamma$ increases.

The fixed points of the second layer of islands or rf-buckets are displaced both in phase and in momentum with respect to the linear fixed points such that the stable and unstable fixed points are interchanged. The locations of the second layer of fixed points are

$$
\begin{array}{ll}
\text { point C: } \quad \psi_{\mathrm{C}}=\psi_{\mathrm{s}}, & \delta_{\mathrm{C}}=\frac{\eta_{\mathrm{c}}}{2 \alpha_{1}}(1+\sqrt{1-\Gamma}), \\
\text { point D: } \quad \psi_{\mathrm{D}}=\pi-\psi_{\mathrm{s}}, \quad \delta_{\mathrm{D}}=\frac{\eta_{\mathrm{c}}}{2 \alpha_{1}}(1+\sqrt{1-\Gamma}) .
\end{array}
$$

The dependence of the coordinates for the fixed points on orbit distortions and the amplitude of betatron oscillations becomes evident from (9.121), (9.124). Specifically, we note a shift in the reference momentum of the beam by $\xi / \eta_{\mathrm{c}}$ as the orbit distortion increases as demonstrated in the examples shown in Figs. 9.21, 9.22, 9.23c, d. Betatron oscillations, on the other hand, cause a spread of the beam momentum in the vicinity of the fixed points. This readjustment of the beam momentum is a direct consequence of the principle of phase focusing whereby the particle follows a path such that the synchronicity condition is met. The phase space diagram of Fig. 9.19 is repeated in Fig. 9.21 with a parameter $2 \xi / \eta_{\mathrm{c}}=-0.125$ and in Fig. 9.22 with the further addition of a finite synchronous phase of $\psi_{\mathrm{s}}=0.7$ rad. In addition to the shift of the reference momentum a significant reduction in the momentum acceptance compared to the regular rf-buckets is evident in both diagrams.

As long as the perturbation is small and $\left|\alpha_{1}\right| \ll\left|\eta_{\mathrm{c}}\right|$, the new fixed points are located far away from the reference momentum and their effect on the particle dynamics can be ignored. The situation becomes very different whenever the linear momentum compaction becomes very small or even zero due to strong 


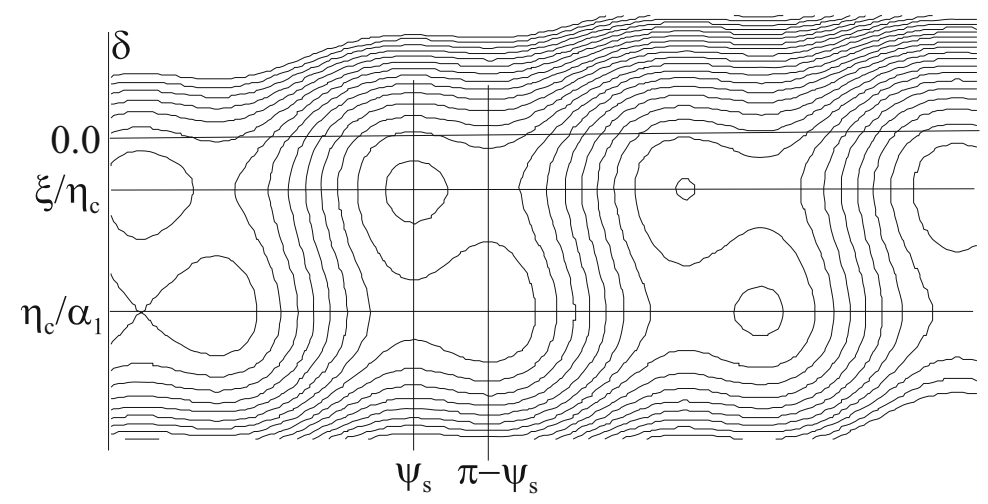

Fig. 9.22 Higher-order longitudinal phase space diagrams for $\psi_{\mathrm{s}}=0.7,2 \xi / \eta_{\mathrm{c}}=-0.125$ and a weak perturbation $\alpha_{1} / \eta_{\mathrm{c}}=-3.0$

quadrupole focusing during momentum ramping through transition or in the case of the deliberate design of a low $\alpha$-lattice for a quasi isochronous storage ring. In these cases higher order perturbations become significant and cannot be ignored. We cannot assume anymore that the perturbation term $\alpha_{1}$ is negligibly small and the phase dynamics may very well become dominated by perturbations.

The perturbation $\alpha_{1}$ of the momentum compaction factor depends on the perturbation of the dispersion function and is therefore also dependent on the sextupole distribution in the storage ring. Given sufficient sextupole families it is at least in principle possible to adjust the parameter $\alpha_{1}$ to zero or a small value by a proper distribution of sextupoles.

\subsubsection{Stability Criteria}

Stability criteria for phase oscillations under the influence of higher order momentum compaction terms can be derived from the Hamiltonian. The nonlinear equations of motion (9.105), (9.106) can be derived from the Hamiltonian

$$
H=\frac{e V_{\mathrm{rf}}}{T_{0} c p_{0}}\left[\cos \psi-\cos \psi_{\mathrm{s}}+\left(\psi-\psi_{\mathrm{s}}\right) \sin \psi_{\mathrm{s}}\right]+\omega_{\mathrm{rf}}\left(\xi \delta-\frac{1}{2} \eta_{\mathrm{c}} \delta^{2}+\frac{1}{3} \alpha_{1} \delta^{3}\right)
$$

To eliminate inconsequential factors for the calculation of phase space trajectories, we simplify (9.113) to

$$
\tilde{H}=\Delta\left[\cos \psi-\cos \psi_{\mathrm{s}}+\left(\psi-\psi_{\mathrm{s}}\right) \sin \psi_{\mathrm{s}}\right]+2 \frac{\xi}{\eta_{\mathrm{c}}} \delta-\delta^{2}+\frac{2}{3} \frac{\alpha_{1}}{\eta_{\mathrm{c}}} \delta^{3},
$$


where

$$
\Delta=\frac{2 e V_{\mathrm{rf}}}{T_{0} c p_{0} \omega_{\mathrm{rf}} \eta_{\mathrm{c}}} .
$$

We may use (9.114) to calculate phase space trajectories and derive stability conditions for various combinations of the parameters $\Delta$, the perturbation of the momentum compaction $\alpha_{1}$, and the synchronous phase $\psi_{\mathrm{s}}$ (Figs. 9.19, 9.20, 9.21, and 9.22). In Fig. 9.23, the phase diagrams of Figs. 9.19, 9.20, 9.21, and 9.22 are displayed now as three-dimensional surfaces plots with the same parameters. Starting from the linear approximation where only regular rf-buckets appear along the $\psi$-axis, we let the ratio $\alpha_{1} / \eta_{\mathrm{c}}$ increase and find the second set of rf-buckets to move in from large relative momentum errors $\delta_{\mathrm{f}}$ toward the main rf-buckets. A significant modification of the phase diagrams occurs when the perturbation reaches such values that the separatrices of both sets of buckets merge as shown in Fig. 9.20 (top). A further increase of the perturbation quickly reduces the momentum

a)

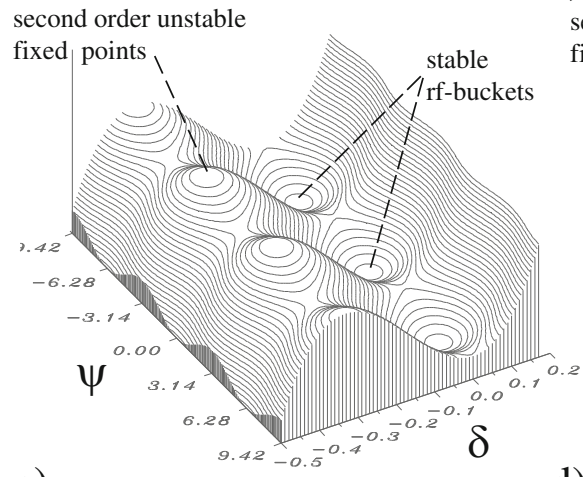

c)

second order unstable fixed points

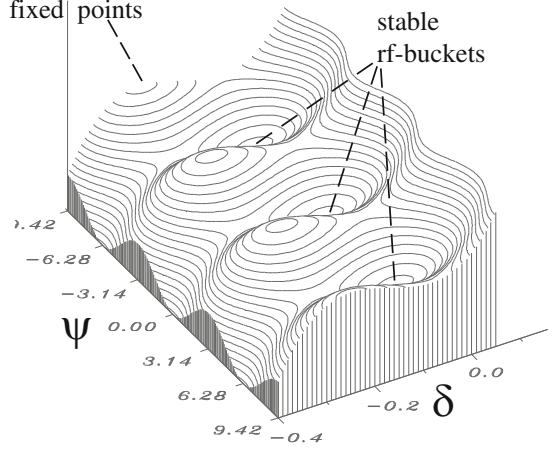

b)

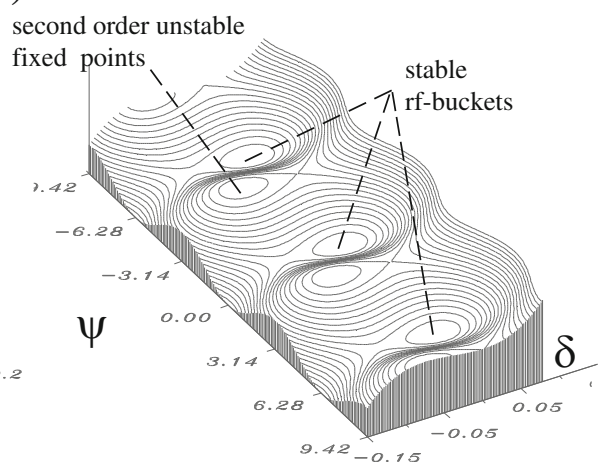

d)

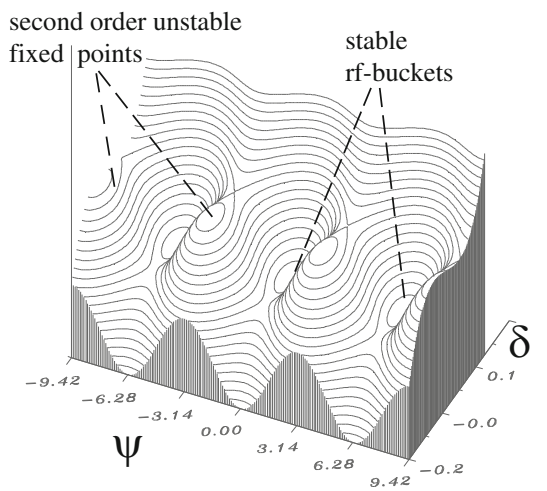

Fig. 9.23 Three dimensional rendition of Figs. 9.19(a), 9.20(b), 9.21(c) and 9.22(d) 
acceptance of the rf-system as can be noticed by comparing Figs. 9.20 (top) and (bottom) or Figs. 9.23 (top) and 9.23 (bottom). The effect of the momentum shift when $\xi \neq 0$ becomes obvious in Figs. 9.21, 9.22, 9.23c, $\mathrm{d}$ as well as the effect of a finite synchronous phase in Fig. 9.23d.

From these qualitative observations we derive a threshold of allowable perturbation $\alpha_{1}$ above which the momentum acceptance of the system becomes significantly reduced. From Figs. 9.20 (top) we take the condition for momentum stability when the separatrices of both sets of buckets merge which occurs when the Hamiltonian for both separatrices or for the fixed points (B) and (C) are equal and

$$
\tilde{H}\left(\pi-\psi_{\mathrm{s}}, \delta_{\mathrm{B}}\right)=\tilde{H}\left(\psi_{\mathrm{s}}, \delta_{\mathrm{C}}\right)
$$

Equation (9.116) becomes in the form of (9.114)

$$
\begin{aligned}
& \Delta\left(-2 \cos \psi_{\mathrm{s}}+\left(\pi-2 \psi_{\mathrm{s}}\right) \sin \psi_{\mathrm{s}}\right)-\delta_{\mathrm{B}}^{2}+\frac{2}{3} \frac{\alpha_{1}}{\eta_{\mathrm{c}}} \delta_{\mathrm{B}}^{3}+2 \frac{\xi}{\eta_{\mathrm{c}}} \delta_{\mathrm{B}} \\
& =-\delta_{\mathrm{C}}^{2}+\frac{2}{3} \frac{\alpha_{1}}{\eta_{\mathrm{c}}} \delta_{\mathrm{C}}^{3}+2 \frac{\xi}{\eta_{\mathrm{c}}} \delta_{\mathrm{C}} .
\end{aligned}
$$

Comparing (9.115) with the results of linear theory, we note that the maximum unperturbed momentum acceptance is related to the parameter $\Delta$ by

$$
\Delta=\frac{1}{F(q) \sin \psi_{\mathrm{s}}}\left(\frac{\Delta p}{p_{0}}\right)_{\max }^{2} \frac{\left|\eta_{\mathrm{c}}\right|}{\eta_{\mathrm{c}}}
$$

where $\frac{1}{q}=\sin \psi_{\mathrm{s}}$ and $F(q)$ is defined in (9.65). Equation (9.117) can be solved for the maximum momentum acceptance

$$
\left(\frac{\Delta p}{p_{0}}\right)_{\max }^{2}=\frac{\eta_{\mathrm{c}}}{\left|\eta_{\mathrm{c}}\right|}\left(\delta_{\mathrm{C}}^{2}-\delta_{\mathrm{B}}^{2}\right)+\frac{2}{3} \frac{\alpha_{1}}{\left|\eta_{\mathrm{c}}\right|}\left(\delta_{\mathrm{C}}^{3}-\delta_{\mathrm{B}}^{3}\right)+2 \frac{\xi}{\left|\eta_{\mathrm{c}}\right|}\left(\delta_{\mathrm{C}}-\delta_{\mathrm{B}}\right) .
$$

Using the expression (9.109) for the coordinates of the fixed points (9.119) eventually becomes with (9.110)

$$
\left(\frac{\Delta p}{p_{0}}\right)_{\max }^{2}=\frac{\eta_{\mathrm{c}}^{2}}{3 \alpha_{1}^{2}}(1-\Gamma)^{3 / 2}
$$

and the stability criterion that the nonlinear perturbation not reduce the momentum acceptance is finally expressed by

$$
\alpha_{1} \leq \frac{\left|\eta_{\mathrm{c}}\right|}{\sqrt{3}} \frac{(1-\Gamma)^{3 / 4}}{\left(\frac{\Delta p}{p_{0}}\right)_{\text {desired }}}
$$


From this criterion we note that the momentum independent perturbation $\Gamma$ can further limit the momentum acceptance until there is for $\Gamma \geq 1$ no finite momentum acceptance left at all.

The momentum shift and the momentum acceptance as well as stability limits can be calculated analytically as a function of $\alpha_{1}$ and the momentum independent term $\Gamma$. As long as the perturbation is small and (9.121) is fulfilled we calculate the momentum acceptance for the linear rf-buckets from the value of the Hamiltonian (9.114). For stronger perturbations, where the separatrices of both layers of rfbuckets have merged and are actually exchanged (Fig. 9.20), a different value of the Hamiltonian must be chosen. The maximum stable synchrotron oscillation in this case is not anymore defined by the separatrix through fixed point B but rather by the separatrix through fixed point $\mathrm{C}$. In the course of synchrotron oscillations a particle reaches maximum momentum deviations from the reference momentum at the phase $\psi=\psi_{\mathrm{s}}$. We have two extreme momentum deviations, one at the fixed point (C), and the other half a synchrotron oscillation away. Both points have the same value of the Hamiltonian (9.114) and are related by

$$
2 \frac{\xi}{\eta_{\mathrm{c}}} \hat{\delta}-\hat{\delta}^{2}+\frac{2}{3} \frac{\alpha_{1}}{\eta_{\mathrm{c}}} \hat{\delta}^{3}=2 \frac{\xi}{\eta_{\mathrm{c}}} \delta_{\mathrm{C}}-\delta_{\mathrm{C}}^{2}+\frac{2}{3} \frac{\alpha_{1}}{\eta_{\mathrm{c}}} \delta_{\mathrm{C}}^{3}
$$

We replace $\delta_{\mathrm{C}}$ from (9.112) and obtain a third-order equation for the maximum momentum acceptance $\hat{\delta}$

$$
2 \frac{\xi}{\eta_{\mathrm{c}}} \hat{\delta}-\hat{\delta}^{2}+\frac{2}{3} \frac{\alpha_{1}}{\eta_{\mathrm{c}}} \hat{\delta}^{3}=-\frac{\eta_{\mathrm{c}}}{6 \alpha_{1}^{2}}\left[1+(1-\Gamma)^{3 / 2}-\frac{3}{2} \Gamma\right] .
$$

This third-order equation can be solved analytically and has the solutions

$$
\begin{aligned}
& \hat{\delta}_{1}=\frac{\eta_{\mathrm{c}}}{2 \alpha_{1}}(1-2 \sqrt{1-\Gamma}), \\
& \hat{\delta}_{2,3}=\frac{\eta_{\mathrm{c}}}{2 \alpha_{1}}(1+\sqrt{1-\Gamma}) .
\end{aligned}
$$

Two of the three solutions are the same and define the momentum at the crossing of the separatrix at the fixed point (C) while the other solution determines the momentum deviation half a synchrotron oscillation away from the fixed point (C). We plot these solutions in Fig. 9.24 together with the momentum shift of the reference momentum at the fixed point (A). As long as there is no momentum independent perturbation $(\Gamma=0)$ the momentum acceptance is given by

$$
-2<-\frac{2 \alpha_{1}}{\eta_{\mathrm{c}}} \delta_{i}<1
$$

The asymmetry of the momentum acceptance obviously reflects the asymmetry of the separatrix. For $\alpha_{1} \rightarrow 0$ the momentum acceptance in (9.120) diverges, which is a reminder that we consider here only the case where the perturbation $\alpha_{1}$ exceeds 
Fig. 9.24 Higher-order momentum acceptance

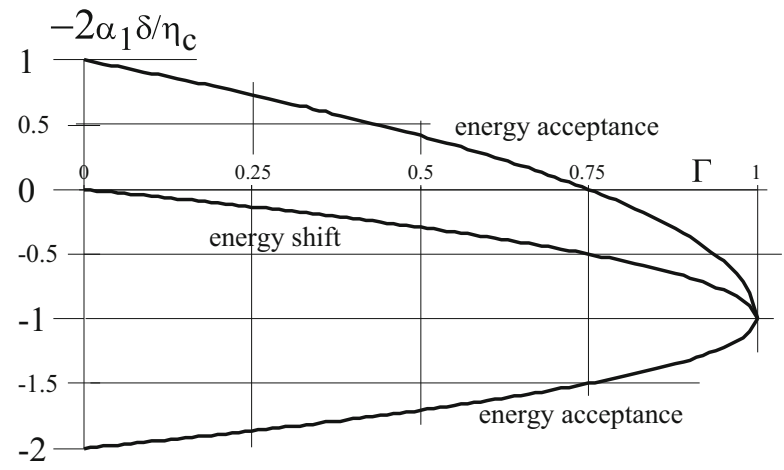

the limit (9.121). In reality the momentum acceptance does not increase indefinitely but is limited by other criteria, for example, by the maximum rf-voltage available. The momentum acceptance limits of (9.124) are further reduced by a finite beam emittance when $\Gamma \neq 0$ causing a spread in the revolution time. All beam stability is lost as $\Gamma$ approaches unity and the stability criterion for stable synchrotron motion in the presence of betatron oscillations is defined by

$$
\frac{4 \xi \alpha_{1}}{\eta_{\mathrm{c}}^{2}}<1,
$$

where the parameter $\xi$ is defined by $(9.101)$.

In evaluating the numerical value of $\xi$ we must consider the emittances $\epsilon_{x, y}$ as amplitude factors. In case of a Gaussian electron beam in a storage ring, for example, a long quantum lifetime can be obtained only if particles with betatron oscillation amplitudes up to at least seven standard values are stable. For such particles the emittance is $\epsilon=7^{2} \epsilon_{\sigma}$, where $\epsilon_{\sigma}$ is the beam emittance for one standard deviation. Similarly, the momentum acceptance must be large enough to include a momentum deviation of $\delta_{\max } \geq 7 \sigma_{E} / E_{0}$.

In general, the stability criteria can be met especially if sextupole magnets are adjusted that the linear perturbation $\alpha_{1}$ of the momentum compaction is made small. In this case, however, we must consider dynamic stability of the beam and storage ring to prevent $\alpha_{1}$ to vary more than the stability criteria allow. Any dynamic variation $\Delta \alpha_{1}$ must meet the condition

$$
\Delta \alpha_{1}<\frac{\eta_{\mathrm{c}}^{2}}{4 \xi} .
$$

Even if the quadratic term $\alpha_{1}$ is made to approach zero we still must consider the momentum shift due to non-chromatic terms when $\xi \neq 0$. From (9.111) we have for the momentum shift $\delta_{0}$ of the stable fixed point $\mathrm{A}$

$$
\delta_{0}=\frac{\eta_{\mathrm{c}}}{2 \alpha_{1}}(1-\sqrt{1-\Gamma}),
$$


where $\Gamma$ is small when $\alpha_{1} \rightarrow 0$ and the square root can be expanded. In this limit the momentum shift becomes

$$
\delta_{0} \rightarrow \frac{\xi}{\eta_{\mathrm{c}}} \quad \text { for } \quad \alpha_{1} \rightarrow 0 .
$$

To achieve low values of the momentum compaction, it is therefore also necessary to reduce the particle beam emittance. Case studies of isochronous lattices show, however, that this might be very difficult because the need to generate both positive and negative values for the dispersion function generates large values for the slopes of the dispersion leading to rather large beam emittances.

Adjusting the quadratic term $\alpha_{1}$ to zero finally brings us back to the situation created when the linear momentum compaction was reduced to small values. One cannot ignore higher-order terms anymore. In this case we would expect that the quadratic and cubic perturbations of the momentum compaction will start to play a significant role since $\eta_{\mathrm{c}} \approx 0$ and $\alpha_{1} \approx 0$. The quadratic term $\alpha_{3}$ will introduce a spread of the momentum compaction due to the momentum spread in the beam while the cubic term $\alpha_{4}$ introduces a similar spread to the linear term $\alpha_{1}$.

\section{Problems}

9.1 (S). A $500 \mathrm{MHz}$ rf-system is supposed to be used in a Wideroe type linac to accelerate protons from a $1 \mathrm{MeV}$ Van de Graaf accelerator. Determine the length of the first three drift tubes for an accelerating voltage at the gaps of $0.5 \mathrm{MeV}$ while assuming that the length of the tubes shall not be less than $15 \mathrm{~cm}$. Describe the operating conditions from an rf-frequency point of view.

9.2 (S). A proton beam with a finite energy spread is injected at an energy of $200 \mathrm{MeV}$ into a storage ring in $n_{\mathrm{b}}$ equidistant short bunches while the rf-system in the storage ring is turned off. Derive an expression for the debunching time or the time it takes for the bunched proton beam to spread out completely.

9.3 (S). The momentum acceptance in a synchrotron is reduced as the synchronous phase is increased. Derive a relationship between the maximum acceleration rate and momentum acceptance. How does this relationship differ for protons and radiating electrons?

9.4 (S). Derive an expression for and plot the synchrotron frequency as a function of oscillation amplitude within the separatrices. What is the synchrotron frequency at the separatrices?

9.5 (S). Sometimes it is desirable to produce short bunches, even only temporary in a storage ring either to produce short X-ray pulses or for quick ejection from a damping ring into a linear collider. By a sudden change of the rf-voltage the bunch can be made to rotate in phase space. Determine analytically the shortest possible 
bunch length as a function of the rf-voltage increase considering a finite energy spread. For how many turns would the short bunch remain within $50 \%$ of its shortest value?

9.6. Calculate the synchrotron oscillation frequency for a $9 \mathrm{GeV}$ proton booster. The maximum momentum is $c p_{\max }=8.9 \mathrm{GeV}$ the harmonic number $h=84$, the rf-voltage $V_{\mathrm{rf}}=200 \mathrm{kV}$, transition energy $\gamma_{\mathrm{tr}}=5.4$ and rf-frequency at maximum momentum $f_{\mathrm{rf}}=52.8 \mathrm{MHz}$. Calculate and plot the rf and synchrotron oscillation frequency as a function of momentum from an injection momentum of $400 \mathrm{MeV}$ to a maximum momentum of $8.9 \mathrm{GeV}$ while the synchronous phase is $\psi_{\mathrm{s}}=45^{\circ}$. What is the momentum acceptance at injection and at maximum energy? How long does the acceleration last?

9.7. Specify a synchrotron of your choice made up of FODO cells for the acceleration of relativistic particles. Assume an rf-system to provide an accelerating voltage equal to $10^{-4}$ of the maximum particle energy in the synchrotron. During acceleration the synchrotron oscillation tune shall remain less than $v_{\mathrm{s}}<0.02$. What are the numerical values for the rf-frequency, harmonic number, rf-voltage, synchronous phase angle and acceleration time in your synchrotron? In case of a proton synchrotron determine the change in the bunch length during acceleration.

\section{References}

1. G. Ising, Arkiv för Matematik, Astronomi och Fysik 18, 1 (1924)

2. R. Wideroe, Archiev für Elektrotechnik 21, 387 (1928)

3. M.S. Livingston (ed.), The Development of High-Energy Accelerators (Dover, New York, 1966)

4. V.I. Veksler, DAN(USSR) 44, 393 (1944)

5. E.M. McMillan, Phys. Rev. 68, 143 (1945)

6. L.W. Alvarez, Phys. Rev. 70, 799 (1946)

7. K. Johnsen, in CERN Symposium on High Energy Accelerators (CERN, Geneva, 1956), p. 295

8. G.K. Green, in CERN Symposium on High Energy Accelerators (CERN, Geneva, 1956), p. 103

9. H. Goldstein, Classical Mechanics (Addison-Wesley, Reading, 1950)

10. D. Deacon, Theory of the Isochronous Storage Ring Laser. PhD thesis, Stanford University (1979)

11. C. Pellegrini, D. Robin, Nucl. Instrum. Methods A301, 27 (1991)

12. C.G. Lilliequist, K.R. Symon, Technical Report MURA-491, MURA, Chicago (1959) 


\section{Chapter 10 \\ Periodic Focusing Systems}

The fundamental principles of charged particle beam dynamics as discussed in previous chapters can be applied to almost every beam transport need. Focusing and bending devices for charged particles are based on magnetic or electric fields which are specified and designed in such a way as to allow the application of fundamental principles of beam optics leading to predictable results.

Beam transport systems can be categorized into two classes: The first group includes beam transport lines which are designed to guide charged particle beams from point $A$ to point $B$. In the second class, we find beam transport systems or magnet lattices forming circular accelerators. The physics of beam optics is the same in both cases but in the design of actual solutions different boundary conditions apply. Basic linear building blocks in a beam transport line are the beam deflecting bending magnets, quadrupoles to focus the particle beam, and field free drift spaces between magnets. Transformation matrices have been derived in Chap. 7 and we will apply these results to compose more complicated beam transport systems. The arrangement of magnets along the desired beam path is called the magnet lattice or short the lattice.

Beam transport lines can consist of an irregular array of magnets or a repetitive sequence of a group of magnets. Such a repetitive magnet sequence is called a periodic magnet lattice, or short periodic lattice and if the magnet arrangement within one period is symmetric this lattice is called a symmetric magnet lattice, or short a symmetric lattice. By definition a circular accelerator lattice is a periodic lattice with the circumference being the period length. To simplify the design and theoretical understanding of beam dynamics it is customary, however, to segment the full circumference of a circular accelerator into identical sectors which are repeated a number of times to form the complete ring. Such sectors are called superperiods and define usually most salient features of the accelerator in contrast to much smaller periodic segments called cells, which include only a few magnets.

In this chapter, we concentrate on the study of periodic focusing structures. For long beam transport lines and specifically for circular accelerators it is prudent to

This chapter has been made Open Access under a CC BY 4.0 license. For details on rights and licenses please read the Correction https://doi.org/10.1007/978-3-319-18317-6_28 
consider focusing structures that repeat periodically. In this case, one can apply beam dynamics properties of one periodic lattice structure as many times as necessary with known characteristics. In circular particle accelerators such periodic focusing structures not only simplify the determination of beam optics properties in a single turn but we will also be able to predict the stability criteria for particles orbiting an indefinite number of revolutions around the ring.

To achieve focusing in both planes, we will have to use both focusing and defocusing quadrupoles in a periodic sequence such that we can repeat a lattice period any number of times to form an arbitrary long beam line which provides the desired focusing in both planes.

\subsection{FODO Lattice}

The most simple periodic lattice would be a sequence of equidistant focusing quadrupoles of equal strength. This arrangement is unrealistic with magnetic quadrupole fields which do not focus in both the horizontal and vertical plane in the same magnet. The most simple and realistic compromise is therefore a periodic lattice like the symmetric quadrupole triplet which was discussed in Sect. 7.2.3. and is shown schematically in Fig. 10.1.

Each half of such a lattice period is composed of a focusing $(\mathrm{F})$ and a defocusing (D) quadrupole with a drift space $(\mathrm{O})$ in between forming a FODO sequence. Combining such a sequence with its mirror image as shown in Fig. 10.1 results in a periodic lattice which is called a FODO lattice or a FODO channel. By starting the period in the middle of a quadrupole and continuing to the middle of the next quadrupole of the same sign not only a periodic lattice but also a symmetric lattice is defined. Such an elementary unit of focusing is called a lattice unit or in this case a FODO cell. The FODO lattice is the most widely used lattice especially in high energy accelerator systems because of its simplicity, flexibility, and its beam dynamical stability.

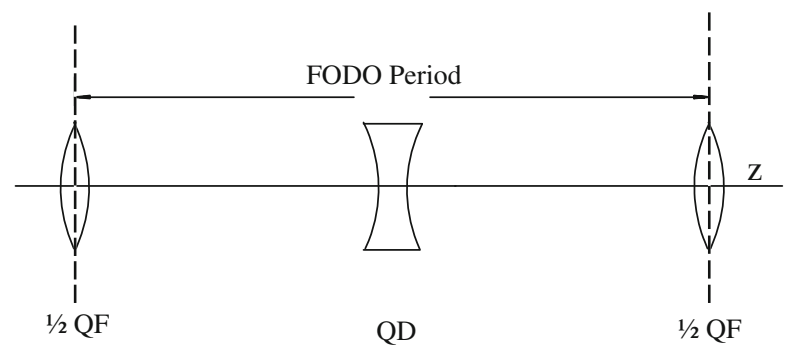

Fig. 10.1 FODO-lattice ( $Q F$ focusing quadrupole, $Q D$ defocusing quadrupole) 


\subsubsection{Scaling of FODO Parameters}

To determine the properties and stability criteria for a FODO period we restrict ourselves to thin lens approximation, where we neglect the finite length of the quadrupoles. The FODO period can be expressed symbolically by the sequence $\frac{1}{2} \mathrm{QF}-\mathrm{L}-\mathrm{QD}-\mathrm{L}-\frac{1}{2} \mathrm{QF}$, where the symbol $L$ represents a drift space of length $L$ and the symbols QF and QD are focusing or defocusing quadrupoles, respectively. In either case we have a triplet structure for which the transformation matrix has been derived in Sect. 7.2.3

$$
\mathcal{M}_{\mathrm{FODO}}=\left(\begin{array}{cc}
1-2 \frac{L^{2}}{f^{2}} & 2 L\left(1+\frac{L}{f}\right) \\
-\frac{1}{f^{*}} & 1-2 \frac{L^{2}}{f^{2}}
\end{array}\right) .
$$

Here $f_{\mathrm{f}}=-f_{\mathrm{d}}=f, 1 / f^{*}=2(1-L / f) L / f^{2}$ and is called a symmetric FODO lattice.

From the transformation matrix (10.1) we can deduce an important property for the betatron function. The diagonal elements are equal as they always are in any symmetric lattice. Comparison of this property with elements of the transformation matrix expressed in terms of betatron functions (8.74) shows that the solution of the betatron function is periodic and symmetric since $\alpha=0$ both at the beginning and the end of the lattice period. We therefore have symmetry planes in the middle of the quadrupoles for the betatron functions in the horizontal as well as in the vertical plane. The betatron functions then have the general periodic and symmetric form as shown in Fig. 10.2.

From (8.22) and (10.1), we can derive the analytical expression for the periodic and symmetric betatron function by setting $\beta_{0}=\beta, \alpha_{0}=0$ and $\gamma_{0}=1 / \beta$ and get

$$
\beta=\left(1-2 \frac{L^{2}}{f^{2}}\right)^{2} \beta+4 L^{2}\left(1+\frac{L}{f}\right)^{2} \frac{1}{\beta},
$$

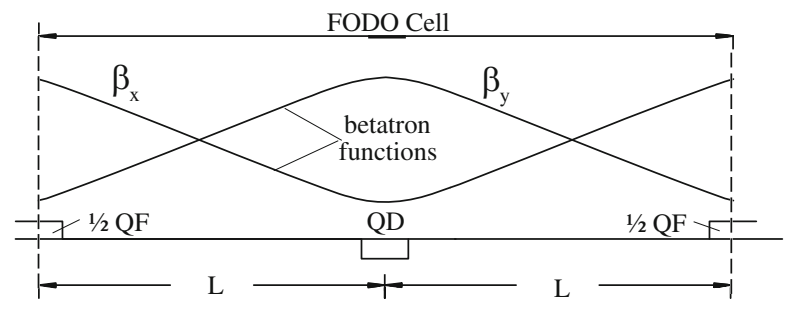

Fig. 10.2 Periodic betatron functions in a FODO channel 
where $f>0$ and $\beta$ is the value of the betatron function in the middle of the focusing quadrupole $\mathrm{QF}$. Solving for $\beta$, we get

$$
\beta^{+}=L \frac{\frac{f}{L} \frac{f}{L+1}}{\sqrt{\frac{f^{2}}{L^{2}-1}}}=L \frac{\kappa(\kappa+1)}{\sqrt{\kappa^{2}-1}},
$$

where we define the FODO parameter $\kappa$ by

$$
\kappa=\frac{f}{L}>1
$$

and set $\beta=\beta^{+}$to indicate the solution in the center of the focusing quadrupole. The FODO parameter $\kappa$ is used only here and should not be identified with our general use of this letter being the curvature. Had we started at the defocusing quadrupole we would have to replace $f$ by $-f$ and get analogous to (10.3) for the value of the betatron function in the middle of the defocusing quadrupole

$$
\beta^{-}=L \frac{\kappa(\kappa-1)}{\sqrt{\kappa^{2}-1}} .
$$

These are the solutions for both the horizontal and the vertical plane. In the middle of the horizontally focusing quadrupole QF $(f>0)$ we have $\beta_{x}=\beta^{+}$and $\beta_{y}=\beta^{-}$and in the middle of the horizontally defocusing quadrupole QD $(f<0)$, we have $\beta_{x}=\beta^{-}$and $\beta_{y}=\beta^{+}$. From the knowledge of the betatron functions at one point in the lattice, it is straightforward to calculate the value at any other point by proper matrix multiplications as discussed earlier. In open arbitrary beam transport lines the initial values of the betatron functions are not always known and there is no process other than measurements of the actual particle beam in phase space to determine the values of the betatron functions as discussed in Sect. 8.1.3. The betatron functions in a periodic lattice in contrast are completely determined by the requirement that the solution be periodic with the periodicity of the lattice. It is not necessary that the focusing lattice be symmetric to obtain a unique, periodic solution. Equation (8.22) can be used for any periodic lattice requiring only the equality of the betatron functions at the beginning and at the end of the periodic structure. Of course, not any arbitrary although periodic arrangement of quadrupoles will lead to a viable solution and we must therefore derive conditions for periodic lattices to produce stable solutions.

The betatron phase for a FODO cell can be derived by applying (8.74) to a symmetric lattice. With $\alpha_{0}=\alpha=0$ and $\beta_{0}=\beta$ this matrix is

$$
\left(\begin{array}{cc}
\cos \phi & \beta \sin \phi \\
-\frac{1}{\beta} \sin \phi & \cos \phi
\end{array}\right),
$$


where $\phi$ is the betatron phase advance through a full symmetric period. Since the matrix (10.6) must be equal to the matrix (10.1) the phase must be

$$
\cos \phi=1-2 \frac{L^{2}}{f^{2}}=\frac{\kappa^{2}-2}{\kappa^{2}}
$$

or

$$
\sin \frac{\phi}{2}=\frac{1}{\kappa}
$$

For the solution (10.8) to be real the parameter $\kappa$ must be larger than unity, a result which also becomes obvious from (10.3), (10.5). This condition is equivalent to stating that the focal length of half a quadrupole in a FODO lattice must be longer than the distance to the next quadrupole.

The solutions for periodic betatron functions depend strongly on the quadrupole strengths. Specifically, we observe that (10.3) has minimum characteristics for $\beta^{+}$. Taking the derivative $\mathrm{d} \beta^{+} / \mathrm{d} \kappa=0,(10.3)$ becomes

$$
\kappa_{0}^{2}-\kappa_{0}-1=0,
$$

which can be solved for

$$
\kappa_{0}=\frac{1}{2} \pm \sqrt{\frac{1}{4}+1}=1.6180
$$

The optimum phase advance per FODO cell is therefore

$$
\phi_{0} \approx 76.345^{\circ}
$$

The maximum value of the betatron function reaches a minimum for a FODO lattice with a phase advance of about $76.3^{\circ}$ per cell. Since beam sizes scale with the square root of the betatron functions, a lattice with this phase advance per cell requires the minimum beam aperture.

This criteria, however, is true only for a flat beam when $\epsilon_{x} \gg \epsilon_{y}$ or $\epsilon_{y} \gg \epsilon_{x}$. For a round beam with uniform particle distribution in phase space $\epsilon_{x} \approx \epsilon_{y}$ and we get for the maximum beam acceptance by minimizing the beam diameter or $E_{x}^{2}+E_{y}^{2} \sim$ $\beta_{x}+\beta_{y}$, where $E_{x}$ and $E_{y}$ are the beam envelopes in the horizontal and vertical plane, respectively (Fig. 10.3). This minimum is determined by $\mathrm{d}\left(\beta_{x}+\beta_{y}\right) / \mathrm{d} \kappa=0$, or for

$$
\kappa_{\mathrm{opt}}=\sqrt{2}
$$

and the optimum betatron phase per cell is then from (10.8)

$$
\phi_{\mathrm{opt}}=90^{\circ}
$$


Fig. 10.3 Maximum beam acceptance of a FODO lattice with a circular aperture of radius $R$ and where $E_{x, y}=\sqrt{\epsilon_{x, y} \beta_{x, y}}$

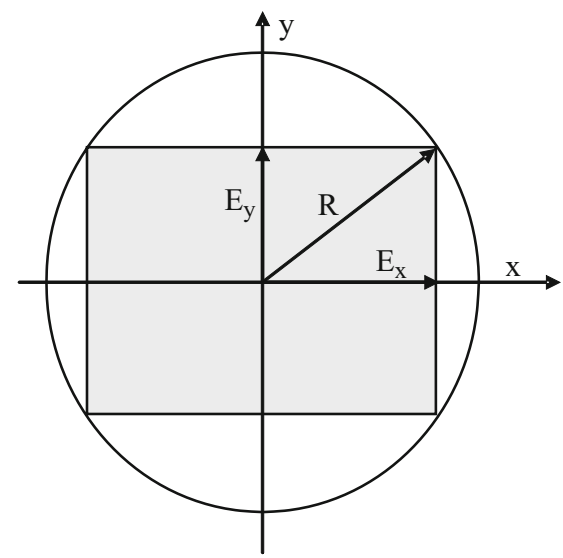

This solution requires the minimum radial aperture $R$ in quadrupoles for a beam with equal beam emittances in both planes $\epsilon_{x}=\epsilon_{y}=\epsilon$. The betatron functions in the middle of the quadrupoles are then simply

$$
\begin{aligned}
& \beta_{\mathrm{opt}}^{+}=L(2+\sqrt{2}), \\
& \beta_{\mathrm{opt}}^{-}=L(2-\sqrt{2}) .
\end{aligned}
$$

The beam envelopes are $E_{x}=\sqrt{\epsilon \beta_{\mathrm{opt}}^{+}}$and $E_{y}=\sqrt{\epsilon \beta_{\mathrm{opt}}^{-}}$and the maximum beam emittance to fit an aperture of radius $R$ or the acceptance of the aperture can be determined from

$$
E_{x}^{2}+E_{y}^{2}=R^{2}=\epsilon\left(\beta^{+}+\beta^{-}\right)_{\mathrm{opt}} .
$$

From (10.14) we find $\left(\beta^{+}+\beta^{-}\right)_{\text {opt }}=4 L$ and the acceptance of a FODO channel with an aperture radius $R$ becomes

$$
\epsilon_{\max }=\frac{R^{2}}{4 L}
$$

Of course, this definition of the acceptance is true only for a monochromatic beam. In a real beam we must include the dispersion and energy spread in the beam to find the optimum acceptance. Also there are other particle distributions for which this optimisation may not be quite accurate.

With this optimum solution we may develop general scaling laws for the betatron functions in a FODO lattice. The values of the betatron functions need not be known at all points of a periodic lattice to characterize the beam optical properties. It is 
Fig. 10.4 Scaling of horizontal and vertical betatron functions in a FODO lattice

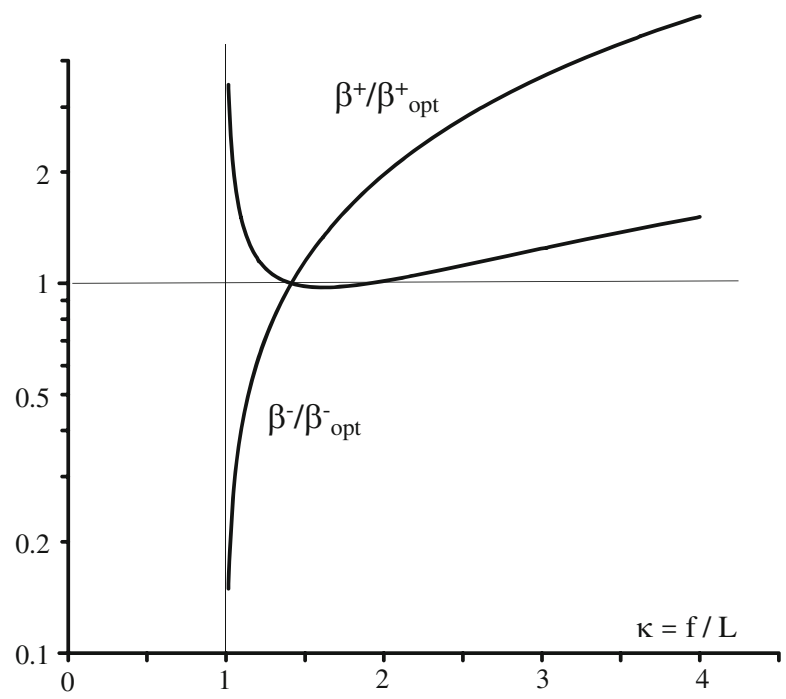

sufficient to know these values at characteristic points like the symmetry points in a FODO channel, where the betatron functions reach maximum or minimum values. From $(10.3),(10.14)$ the betatron functions at these symmetry points are given by

$$
\begin{aligned}
\frac{\beta^{+}}{\beta_{\mathrm{opt}}} & =\frac{\kappa(\kappa+1)}{(2+\sqrt{2}) \sqrt{\kappa^{2}-1}} \\
\frac{\beta^{-}}{\beta_{\mathrm{opt}}} & =\frac{\kappa(\kappa-1)}{(2-\sqrt{2}) \sqrt{\kappa^{2}-1}}
\end{aligned}
$$

The scaling of the betatron function is independent of $\mathrm{L}$ and depends only on the ratio of the focal length to the distance between quadrupoles $\kappa=f / L$. In Fig. 10.4 the betatron functions $\beta^{+}$and $\beta^{-}$are plotted as a function of the FODO parameter $\kappa$.

The distance $L$ between quadrupoles is still a free parameter and can be adjusted to the needs of the particular application. We observe, however, that the maximum value of the betatron function varies linear with $L$ and the maximum beam size in a FODO lattice scales like $\sqrt{L}$.

\subsubsection{Betatron Motion in Periodic Structures}

For the design of circular accelerators it is of fundamental importance to understand the long term stability of the beam over many revolutions. Specifically we need to know if the knowledge of beam dynamics in one periodic unit can be extrapolated to many periodic units. 


\section{Stability Criterion}

The periodic solution for one FODO cell has been derived in the last section and we expect that such periodic focusing cells can be repeated indefinitely. Following the classic paper by Courant and Snyder [1], we will derive the stability conditions for an indefinite number of periodic but not necessarily symmetric focusing cells. The structure of the cells can be arbitrary but must be periodic. If $\mathcal{M}(z+2 L \mid z)$ is the transformation matrix for one cell, we have for $N$ cells

$$
\mathcal{M}(z+N 2 L \mid z)=[\mathcal{M}(z+2 L \mid z)]^{N} .
$$

Stable solutions are obtained if all elements of the total transformation matrix stay finite as $N$ increases indefinitely. To find the conditions for this we calculate the eigenvalues $\lambda$ of the characteristic matrix equation. The eigenvalues $\lambda$ are a measure for the magnitude of the matrix elements and therefore finite values for the eigenvalues will be the indication that the transformation matrix stays finite as well. The characteristic matrix equation

$$
(\mathcal{M}-\lambda \mathcal{I}) x=0
$$

where $\mathcal{I}$ is the unity matrix. For nontrivial values of the eigenvectors $(x \neq 0)$ the determinant

$$
|\mathcal{M}-\lambda \mathcal{I}|=\left|\begin{array}{cc}
C-\lambda & S \\
C^{\prime} & S^{\prime}-\lambda
\end{array}\right|=0
$$

must vanish and with $C S^{\prime}-S C^{\prime}=1$ the eigenvalue equation is

$$
\lambda^{2}-\left(C+S^{\prime}\right) \lambda+1=0 .
$$

The solutions are

$$
\lambda_{1,2}=\frac{1}{2}\left(C+S^{\prime}\right) \pm \sqrt{\frac{1}{4}\left(C+S^{\prime}\right)^{2}-1}
$$

or with the substitution $\frac{1}{2}\left(C+S^{\prime}\right)=\cos \phi$

$$
\lambda_{1,2}=\cos \phi \pm \mathrm{i} \sin \phi=\mathrm{e}^{\mathrm{i} \phi} .
$$

The betatron phase $\phi$ must be real or the trace of the matrix $\mathcal{M}$ must be

$$
\operatorname{Tr}\{\mathcal{M}\}=C+S^{\prime} \leq 2 .
$$


On the other hand, the transformation matrix for a full lattice period is

$$
\mathcal{M}=\left(\begin{array}{cc}
\cos \phi+\alpha \sin \phi & \beta \sin \phi \\
-\gamma \sin \phi & \cos \phi-\alpha \sin \phi
\end{array}\right)
$$

which can be expressed with $\mathcal{J}=\left(\begin{array}{cc}\alpha & \beta \\ -\gamma & -\alpha\end{array}\right)$ by

$$
\mathcal{M}=\mathcal{I} \cos \phi+\mathcal{J} \sin \phi .
$$

This matrix has the form of Euler's formula for a complex exponential. Since the determinant of $\mathcal{M}$ is unity we get $\gamma \beta-\alpha^{2}=1$ or $\mathcal{J}^{2}=-\mathcal{I}$. Similar to Moivre's formula, for $N$ equal periods

$$
\mathcal{M}^{N}=(\mathcal{I} \cos \phi+\mathcal{J} \sin \phi)^{N}=\mathcal{I} \cos (N \phi)+\mathcal{J} \sin (N \phi)
$$

and the trace for $N$ periods is bounded if $\cos \phi<1$ or if (10.24) holds or if

$$
\operatorname{Tr}\left(\mathcal{M}^{N}\right)=2 \cos (N \phi) \leq 2 .
$$

This result is called the stability criterion for periodic beam transport lattices. We note that the trace of the transformation matrix $\mathcal{M}$ does not depend on the reference point $z$. To show this we consider two different reference points $z_{1}$ and $z_{2}$, where $z_{1}<z_{2}$, for which the following identities hold

$$
\mathcal{M}\left(z_{2}+2 L \mid z_{1}\right)=\mathcal{M}\left(z_{2} \mid z_{1}\right) \mathcal{M}\left(z_{1}+2 L \mid z_{1}\right)=\mathcal{M}\left(z_{2}+2 L \mid z_{2}\right) \mathcal{M}\left(z_{2} \mid z_{1}\right)
$$

and solving for $\mathcal{M}\left(z_{2}+2 L \mid z_{2}\right)$ we get

$$
\mathcal{M}\left(z_{2}+2 L \mid z_{2}\right)=\mathcal{M}\left(z_{2} \mid z_{1}\right) \mathcal{M}\left(z_{1}+2 L \mid z_{1}\right) \mathcal{M}^{-1}\left(z_{2} \mid z_{1}\right) .
$$

This is a similarity transformation and therefore, both transformation matrices $\mathcal{M}\left(z_{2}+2 L \mid z_{2}\right)$ and $\mathcal{M}\left(z_{1}+2 L \mid z_{1}\right)$ have the same trace and eigenvalues independent of the choice of the location $z$.

\subsubsection{General FODO Lattice}

So far we have considered FODO lattices, where both quadrupoles have equal strength, $f_{1}=-f_{2}=f$. Since we made no use of this in the derivation of the stability criterion for betatron functions we expect that stability can also be obtained 
for unequal quadrupoles strengths. In this case the transformation matrix of half a FODO cell is

$$
\mathcal{M}_{\frac{1}{2}}=\left(\begin{array}{cc}
1 & 0 \\
-\frac{1}{f_{2}} & 1
\end{array}\right)\left(\begin{array}{ll}
1 & L \\
0 & 1
\end{array}\right)\left(\begin{array}{cc}
1 & 0 \\
-\frac{1}{f_{1}} & 1
\end{array}\right)=\left(\begin{array}{cc}
1-\frac{L}{f_{1}} & L \\
-\frac{1}{f^{*}} & 1-\frac{L}{f_{2}}
\end{array}\right),
$$

where $1 / f^{*}=+1 / f_{1}+1 / f_{2}-L /\left(f_{1} f_{2}\right)$. Multiplication with the reverse matrix gives for the full transformation matrix of the FODO cell

$$
\mathcal{M}=\left(\begin{array}{cc}
1-2 \frac{L}{f^{*}} & 2 L\left(1-\frac{L}{f_{2}}\right) \\
-\frac{2}{f^{*}} 1-\frac{L}{f_{1}} & 1-2 \frac{L}{f^{*}}
\end{array}\right) .
$$

The stability criterion

$$
\operatorname{Tr}\{\mathcal{M}\}=\left|2-\frac{4 L}{f^{*}}\right|<2
$$

is equivalent to

$$
0<\frac{L}{f^{*}}<1
$$

To determine the region of stability in the $(u, v)$-plane, where $u=L / f_{1}$ and $v=L / f_{2}$ we get from (10.34) the condition

$$
0<u+v-u v<1
$$

where $u$ and $v$ can be positive or negative. Solving the second inequality for either $u$ or $v$ we find the conditions $|u|<1$ and $|v|<1$. With this, the first inequality can be satisfied only if $u$ and $v$ have different signs. The boundaries of the stability region are therefore given by the four equations

$$
\begin{aligned}
& |u|=1, \quad|v|=\frac{|u|}{1+|u|}, \\
& |v|=1,|u|=\frac{|v|}{1+|v|},
\end{aligned}
$$

defining the stability region shown in Fig. 10.5 which is also called the necktie diagram because of its shape. Due to the full symmetry in $|u|$ and $|v|$ the shaded area in Fig. 10.5 is the stability region for both the horizontal and vertical plane.

For convenience, we used the thin lens approximation to calculate the necktie diagram. Nothing fundamentally will, however, change when we use the transformation matrices for real quadrupoles of finite length except for a small variation of the stability boundaries depending on the degree of deviation from the thin lens approximation. With the general transformation matrix for a full FODO period 
Fig. 10.5 Necktie diagram

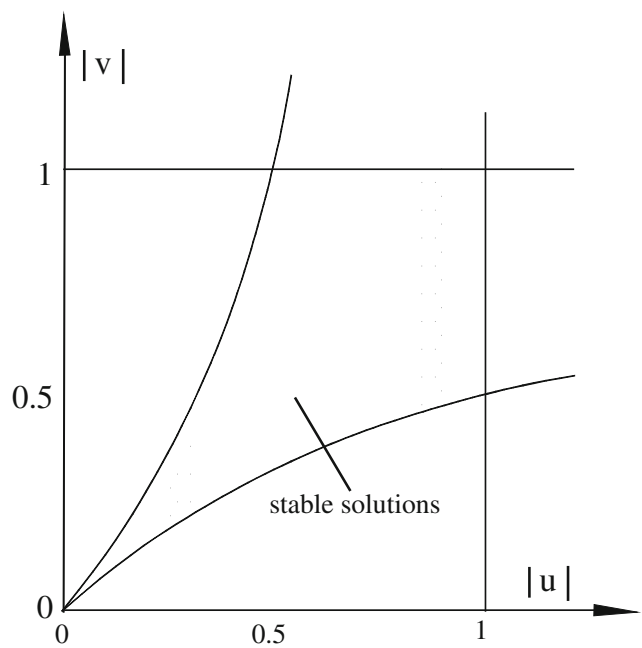

$\mathcal{M}=\left(\begin{array}{cc}C & S \\ C^{\prime} & S^{\prime}\end{array}\right)$ the periodic solution for the betatron function is $\beta^{2}=\frac{S^{2}}{1-C^{2}}$ and the stability condition

$$
\operatorname{Tr} \mathcal{M}=\left|C+S^{\prime}\right|<2 .
$$

The stability diagram has still the shape of a necktie although the boundaries are slightly curved (Fig. 10.5).

A general transformation matrix for half a FODO cell can be obtained in matrix formalism with $\psi=\sqrt{k} \ell$ by multiplying the matrices

$$
\begin{aligned}
& \mathcal{M}_{\frac{1}{2}}=\left(\begin{array}{cc}
\cosh \psi_{2} & \frac{\ell_{2}}{\psi_{2}} \sinh \psi_{2} \\
\frac{\psi_{2}}{\ell_{2}} \sinh \psi_{2} & \cosh \psi_{2}
\end{array}\right)\left(\begin{array}{ll}
1 & L \\
0 & 1
\end{array}\right) \\
& \times\left(\begin{array}{cc}
\cos \psi_{1} & \frac{\ell_{1}}{\psi_{1}} \sin \psi_{1} \\
-\frac{\psi_{1}}{\ell_{1}} \sin \psi_{1} & \cos \psi_{1}
\end{array}\right),
\end{aligned}
$$

where now $L$ is not the half cell length but just the drift space between two adjacent quadrupoles of finite length and the indices refer to the first and the second half quadrupole, respectively. From this we get the full period transformation matrix by multiplication with the reverse matrix

$$
\mathcal{M}=\left(\begin{array}{cc}
C & S \\
C^{\prime} & S^{\prime}
\end{array}\right)=\mathcal{M}_{\frac{1}{2}, \mathrm{r}} \mathcal{M}_{\frac{1}{2}}
$$

Obviously the mathematics becomes elaborate although straight forward and it is prudent to use computers to find the desired results. 
Table 10.1 FODO cell parameters

\begin{tabular}{l|l|l|l|l}
\hline Example & $\# 1$ & $\# 2$ & $\# 3$ & $\# 4$ \\
\hline Energy, E $(\mathrm{GeV})$ & 10 & 50 & 4 & 20,000 \\
\hline Half cell length, $\mathrm{L}(\mathrm{m})$ & 6.0 & 2.6 & 3.6 & 114.25 \\
\hline Quadrupole length, $\ell_{\mathrm{q}}(\mathrm{m})$ & 0.705 & 1.243 & 0.15 & 3.64 \\
\hline Bending magnet length, $\ell_{\mathrm{b}}(\mathrm{m})$ & 3.55 & 2.486 & 2.5 & 99.24 \\
\hline Phase advance per cell, $\psi$ & 101.4 & 108.0 & 135.0 & 90.0 \\
\hline Quadrupole strength,$k\left(\mathrm{~m}^{-2}\right)$ & $\cdots$ & $\cdots$ & $\cdots$ & $\cdots$ \\
\hline Lattice type $^{\mathrm{b}}(\mathrm{FODO})$ & $\mathrm{sf}$ & $\mathrm{cf}$ & $\mathrm{sf}$ & sf \\
\hline
\end{tabular}

${ }^{\text {a }}$ These parameters will be determined in Problem 6.1

${ }^{\mathrm{b}} s f$ separated function, $c f$ combined function lattice

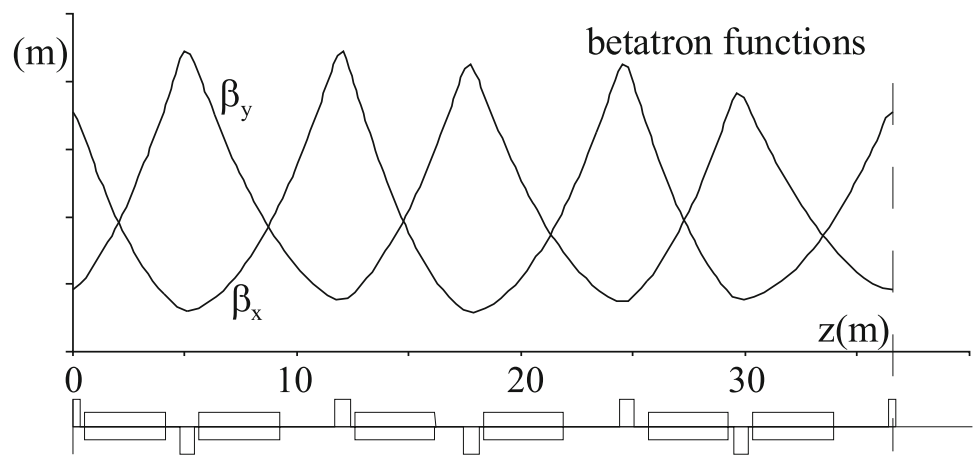

Fig. 10.6 FODO lattice for one octant of a synchrotron $[2,3]$

As reference examples to study and discuss a variety of accelerator physics issues in this text, we consider different FODO lattices (Table 10.1) which are of some but definitely not exhaustive practical interest. Other periodic lattices are of great interest as well specifically for synchrotron radiation sources but are less accessible to analytical discussions than a FODO lattice. All examples except \#2 are separated function lattices.

Example \#1 is that for a $10 \mathrm{GeV}$ electron synchrotron at DESY [2,3] representing a moderately strong focusing lattice with a large stability range as is commonly used if no extreme beam parameters are required as is the case for synchrotrons used to inject into storage rings. Figure 10.6 shows the betatron functions for this lattice. We note small deviations from a regular FODO lattice which is often required to make space for other components. Such deviations from a regular lattice cause only small perturbations in the otherwise periodic betatron functions. As example \#2 we use the lattice for the long curved beam transport lines leading the $50 \mathrm{GeV}$ beam from the linac to the collision area at the Stanford Linear Collider [4]. This lattice exhibits the greatest deviation from a thin lens FODO channel as shown in Fig. 10.7. Example \#3 resembles a theoretical lattice for an extremely small beam emittance used to study fundamental limits of beam stability and control of aberrations [7]. Lattices 


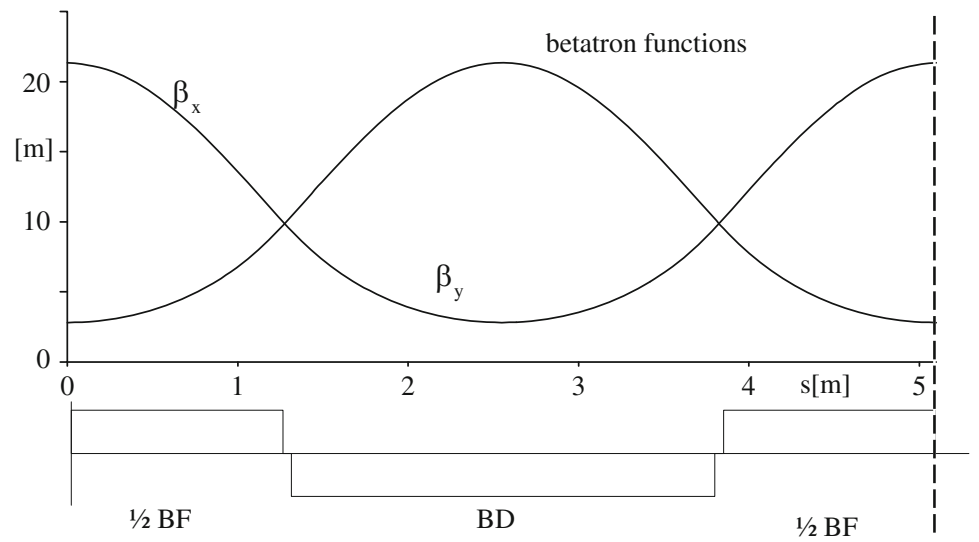

Fig. 10.7 FODO cell for a linear collider transport line [5, 6] (example \#2 in Table 10.1)

for future very high energy hadron colliders in the TeV range use rather long FODO cells leading to large values of the betatron and dispersion functions and related high demands on magnet field and alignment tolerances. Arc lattice parameters for the $20 \mathrm{TeV}$ Superconducting Super Collider, SSC are compiled as example \#4.

\subsection{Beam Dynamics in Periodic Closed Lattices}

In the previous section, we discussed the beam dynamics in a FODO lattice and we will use such periodic lattices to construct a closed path for circular accelerators like synchrotrons and storage rings. The term "circular" is used in this context rather loosely since such accelerators are generally composed of both circular and straight sections giving the ring the appearance of a circle, a polygon or racetrack. Common to all these rings is the fact that the reference path must be a closed path so that the total circumference of the ring constitutes a periodic lattice that repeats turn for turn.

\subsubsection{Hill's Equation}

The motion of particles or more massive bodies in periodic external fields has been studied extensively by astronomers in the last century specially in connection with the three body problem. In particle beam dynamics we find the equation of motion in periodic lattices to be similar to those studied by the astronomer Hill. We will discuss in this chapter the equation of motion, called Hill's equation its solutions and properties. 
Particle beam dynamics in periodic systems is determined by the equation of motion

$$
u^{\prime \prime}+K(z) u=0
$$

where $K(z)$ is periodic with the period $L_{\mathrm{p}}$

$$
K(z)=K\left(z+L_{\mathrm{p}}\right)
$$

The length of a period $L_{\mathrm{p}}$ may be the circumference of the circular accelerator lattice or the length of a superperiod repeating itself several times around the circumference. The differential equation (10.39) with the periodic coefficient (10.40) has all the characteristics of a Hill's differential equation [8]. The solutions of Hill's equation and their properties have been formulated in Floquet's theorem

- two independent solutions exist of the form

$$
\begin{aligned}
& u_{1}(z)=w(z) \mathrm{e}^{\mathrm{i} \mu z / L_{\mathrm{p}}} \\
& u_{2}(z)=w^{*}(z) \mathrm{e}^{-\mathrm{i} \mu z / L_{\mathrm{p}}}
\end{aligned}
$$

- $w^{*}(z)$ is the complex conjugate solution to $w(z)$. For all practical cases of beam dynamics we have only real solutions and $w^{*}(z)=w(z)$;

- the function $w(z)$ is unique and periodic in $z$ with period $L_{\mathrm{p}}$

$$
w\left(z+L_{\mathrm{p}}\right)=w(z)
$$

- $\mu$ is a characteristic coefficient defined by

$$
\cos \mu=\frac{1}{2} \operatorname{Tr}\left[\mathcal{M}\left(z+L_{\mathrm{p}} \mid z\right)\right]
$$

- the trace of the transformation matrix $\mathcal{M}$ is independent of $z$

$$
\operatorname{Tr}\left[\mathcal{M}\left(z+L_{\mathrm{p}} \mid z\right]\right) \neq f(z)
$$

- the determinant of the transformation matrix is equal to unity

$$
\operatorname{det} \mathcal{M}=1
$$

- the solutions remain finite for

$$
\frac{1}{2} \operatorname{Tr}\left[\mathcal{M}\left(z+L_{\mathrm{p}} \mid z\right)\right]<1
$$

The amplitude function $w(z)$ and the characteristic coefficient $\mu$ can be correlated to quantities we have derived earlier using different methods. The transformation 
of a trajectory $u$ through one lattice period of length $L_{\mathrm{p}}$ must be equivalent to the multiplication by the transformation matrix (10.25) for that period which gives

$$
u\left(z+L_{\mathrm{p}}\right)=(\cos \psi+\alpha \sin \psi) u(z)+\beta \sin \psi u^{\prime}(z),
$$

where $u$ stands for any of the two solutions (10.41) and $\psi$ is the betatron phase advance for the period. From $(10.41),(10.42)$ we get on the other hand

$$
u\left(z+L_{\mathrm{p}}\right)=u(z) \mathrm{e}^{ \pm \mathrm{i} \mu}=u(z)(\cos \mu \pm \mathrm{i} \sin \mu)
$$

Comparing the coefficients for the sine and cosine terms we get

$$
\cos \psi=\cos \mu \quad \text { or } \quad \psi=\mu
$$

and

$$
\alpha u(z)+\beta u^{\prime}(z)= \pm \mathrm{i} u(z) .
$$

The first equality can be derived also from (10.25) and (10.43). Equation (10.50) can be further simplified by a logarithmic differentiation

$$
\frac{u^{\prime \prime}}{u^{\prime}}-\frac{u^{\prime}}{u}=-\frac{\beta^{\prime}}{\beta}-\frac{\alpha^{\prime}}{ \pm \mathrm{i}-\alpha} .
$$

On the other hand, we can construct from (10.39), (10.50) the expression

$$
\frac{u^{\prime \prime}}{u^{\prime}}-\frac{u^{\prime}}{u}=\frac{-K \beta}{ \pm \mathrm{i}-\alpha}-\frac{ \pm \mathrm{i}-\alpha}{\beta} .
$$

and equating the r.h.s. of both expressions (10.51) and (10.52), we find

$$
\left(1-\alpha^{2}-K \beta^{2}+\alpha^{\prime} \beta-\alpha \beta^{\prime}\right) \pm \mathrm{i}\left(2 \alpha+\beta^{\prime}\right)=0,
$$

where all functions in brackets are real as long as we have stability. Both brackets must be equal to zero separately with the solutions

$$
\beta^{\prime}=-2 \alpha
$$

and

$$
\alpha^{\prime}=K \beta-\gamma
$$

Equation (10.54) can be used in (10.50) for

$$
\frac{u^{\prime}}{u}=\frac{ \pm \mathrm{i}-\alpha}{\beta}= \pm \frac{\mathrm{i}}{\beta}+\frac{1}{2} \frac{\beta^{\prime}}{\beta},
$$


and after integration

$$
\log \frac{u}{u_{0}}= \pm \mathrm{i} \int_{0}^{z} \frac{\mathrm{d} \zeta}{\beta}+\frac{1}{2} \log \frac{\beta}{\beta_{0}}
$$

where $u_{0}=u\left(z_{0}\right)$ and $\beta_{0}=\beta\left(z_{0}\right)$ for $z=z_{0}$. Solving for $u(z)$ we get the well known solution

$$
u(z)=a \sqrt{\beta(z)} \mathrm{e}^{ \pm \mathrm{i} \psi},
$$

where $a=u_{0} / \sqrt{\beta_{0}}$ and

$$
\psi\left(z-z_{0}\right)=\int_{z_{0}}^{z} \frac{\mathrm{d} \zeta}{\beta(\zeta)}
$$

With $\psi\left(L_{\mathrm{p}}\right)=\mu$ and

$$
\sqrt{\beta(z)}=\frac{w(z)}{a}
$$

we find the previous definitions of the betatron functions to be consistent with the coefficients of Floquet's solutions in a periodic lattice. In the next section we will apply the matrix formalism to determine the solutions of the betatron functions in periodic lattices.

\subsubsection{Periodic Betatron Functions}

Having determined the existence of stable solutions for particle trajectories in periodic lattices we will now derive periodic and unique betatron functions. For this we take the transformation matrix of a full lattice period

$$
\mathcal{M}\left(z+L_{\mathrm{p}} \mid z\right)=\left(\begin{array}{cc}
C & S \\
C^{\prime} & S^{\prime}
\end{array}\right)
$$

and construct the transformation matrix for betatron functions.

$$
\left(\begin{array}{l}
\beta \\
\alpha \\
\gamma
\end{array}\right)=\left(\begin{array}{ccc}
C^{2} & -2 C S & S^{2} \\
-C C^{\prime} & C S^{\prime}+C^{\prime} S & -S S^{\prime} \\
C^{\prime 2} & -2 C^{\prime} S^{\prime} & S^{\prime 2}
\end{array}\right)\left(\begin{array}{l}
\beta_{0} \\
\alpha_{0} \\
\gamma_{0}
\end{array}\right)=\mathcal{M}_{\beta}\left(\begin{array}{l}
\beta_{0} \\
\alpha_{0} \\
\gamma_{0}
\end{array}\right) .
$$

Because of the quadratic nature of the matrix elements, we find the same result in case of a $180^{\circ}$ phase advance for the lattice segment. Any such lattice segment with a phase advance of an integer multiple of $180^{\circ}$ is neutral to the transformation 
of lattice functions. This feature can be used to create irregular insertions in a lattice that do not disturb the lattice functions outside the insertions.

To obtain from (10.62) a general periodic solution for the betatron functions we simply solve the eigenvector equation

$$
\left(\mathcal{M}_{\beta}-\mathcal{I}\right) \boldsymbol{\beta}=0
$$

The solution can be obtained from the component equations of (10.63)

$$
\begin{aligned}
\left(C^{2}-1\right) \beta-2 S C \alpha+S^{2} \gamma & =0, \\
C C^{\prime} \beta-\left(S^{\prime} C+C S^{\prime}-1\right) \alpha+S S^{\prime} \gamma & =0, \\
C^{\prime 2} \beta-2 S^{\prime} C^{\prime} \alpha+\left(S^{\prime 2}-1\right) \gamma & =0 .
\end{aligned}
$$

A particular simple solution is obtained if the periodic lattice includes a symmetry point. In this case, we define this symmetry point as the start of the periodic lattice with $\alpha=0$, and get the simple solutions

$$
\beta^{2}=\frac{S^{2}}{1-C^{2}}, \quad \alpha=0, \quad \gamma=\frac{1}{\beta} .
$$

The transformation matrix for a superperiod or full circumference of a ring becomes then simply from (8.74)

$$
\mathcal{M}=\left(\begin{array}{cc}
\cos \mu & \beta \sin \mu \\
-\frac{1}{\beta} \sin \mu & \cos \mu
\end{array}\right)
$$

where $\mu$ is the phase advance for the full lattice period. The solutions are stable as long as the trace of the transformation matrix meets the stability criterion (10.37) or as long as $\mu \neq n \pi$, where $n$ is an integer.

Different from an open transport line, well determined and unique starting values for the periodic betatron functions exist in a closed lattice due to the periodicity requirement allowing us to determine the betatron function anywhere else in the lattice. Although (10.65) allows both a positive and a negative solution for the betatron function, we choose only the positive solution for the definition of the betatron function.

Stable periodic solutions for asymmetric but periodic lattices, where $\alpha \neq 0$, can be obtained in a straightforward way from (10.64) as long as the determinant $\left|\mathcal{M}_{\mathrm{p}}-\mathcal{I}\right| \neq 0$.

The betatron phase for a full turn around a circular accelerator of circumference $C$ is from (10.59)

$$
\mu\left(L_{C}\right)=\int_{z}^{z+L_{C}} \frac{\mathrm{d} \zeta}{\beta(\zeta)}
$$


If we divide this equation by $2 \pi$ we get a quantity $v$ which is equal to the number of betatron oscillations executed by particles traveling once around the ring. This number is called the tune or operating point of the circular accelerator. Since there are different betatron functions in the horizontal plane and in the vertical plane, we also get separate tunes in a circular accelerator for both planes

$$
v_{x, y}=\frac{1}{2 \pi} \oint \frac{\mathrm{d} \zeta}{\beta_{x, y}(\zeta)}
$$

This definition is equivalent to having chosen the integration constant in (8.57) equal to $1 / 2 \pi$ instead of unity. Yet another normalization can be obtained by choosing $1 / v$ for the integration constant in (8.57), in which case the phase defined as

$$
\varphi(z)=\frac{\psi(z)}{v}=\int_{0}^{z} \frac{\mathrm{d} \zeta}{v \beta(\zeta)}
$$

varies between 0 and $2 \pi$ along the circumference of a ring lattice. This normalization will become convenient when we try to decompose periodic field errors in the lattice into Fourier components to study their effects on beam stability.

Equation (10.68) can be used to get an approximate expression for the relationship between the betatron function and the tune. If $\bar{\beta}$ is the average value of the betatron function around the ring then $\mu\left(L_{C}\right)=2 \pi v \approx L_{C} / \bar{\beta} \approx 2 \pi R / \bar{\beta}$ or

$$
\bar{\beta}=\frac{R}{v} .
$$

This equation is amazingly accurate for most rings and is therefore a useful tool for a quick estimate of the average betatron function or for the tunes often referred to as the smooth approximation.

In a circular accelerator three tunes are defined for the three degrees of freedom, the horizontal, vertical and longitudinal motion. In Fig. 10.8 the measured frequency

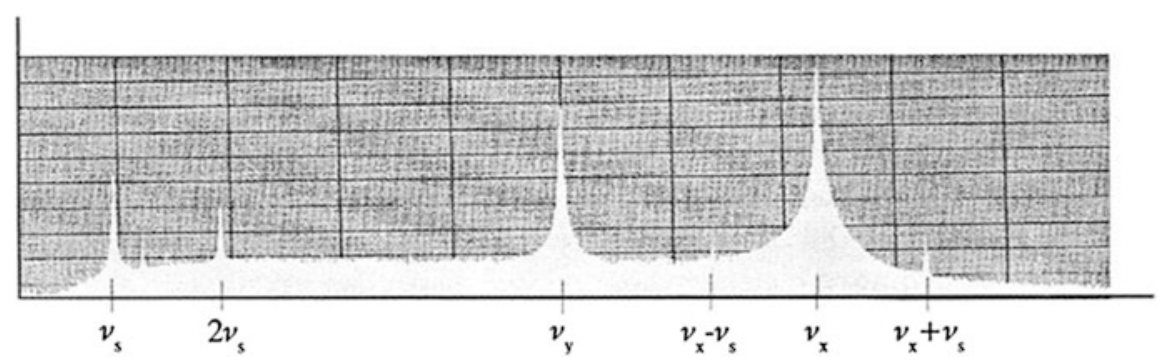

Fig. 10.8 Frequency spectrum from a circulating particle beam, $v_{\mathrm{s}}$ synchrotron tune, $v_{x}, v_{y}$ betatron tunes, $v_{x} \pm v_{y}$ satellites 
spectrum is shown for a particle beam in a circular accelerator. The electric signal from an isolated electrode in the vacuum chamber is recorded and connected to a frequency analyzer. The signal amplitude depends on the distance of the passing beam to the electrode and therefore includes the information of beam oscillations as a modulation of the revolution frequency.

Synchrotron oscillations can also be detected with electrodes and the signal from synchrotron oscillations appears on a spectrum analyzer as sidebands to harmonics of the revolution frequency. Analogous to the transverse motion, a longitudinal tune $v_{\mathrm{s}}$ is defined as the number of oscillations per revolution or as the synchrotron tune.

We note a number of frequencies in the observed spectrum of the storage ring SPEAR as shown in Fig. 10.8. At the low frequency end two frequencies indicate the longitudinal tune $v_{\mathrm{s}}$ and its first harmonic at $2 v_{\mathrm{s}}$. The two large signals are the horizontal and vertical tunes of the accelerator. Since the energy oscillation affects the focusing of the particles, we also observe two weak satellite frequencies on one of the transverse tunes at a distance of $\pm v_{\mathrm{s}}$. The actual frequencies observed are not directly equal to $v \omega_{0}$, where $\omega_{0}$ is the revolution frequency, but are only equal to the non-integral part of the tune $\Delta v \omega_{0}$, where $\Delta v$ is the distance to the integer nearest to $v$.

\subsubsection{Periodic Dispersion Function}

The dispersion function can be periodic if the lattice is periodic. In this section we will determine the periodic solution of the dispersion function first for the simple lattice building block of a FODO channel and then for general but periodic lattice segments.

\section{Scaling of the Dispersion in a FODO Lattice}

Properties of a FODO lattice have been discussed in detail for a monochromatic particle beam only and no chromatic effects have been taken into account. To complete this discussion we now include chromatic effects which cause, in linear approximation, a dispersion proportional to the energy spread in the beam and are caused by bending magnets. We have used the transformation matrix for a symmetric quadrupole triplet as the basic FODO cell. The bending magnet edge focusing was ignored and so were chromatic effects. In the following we still ignore the quadratic edge focusing effects of the bending magnets, but we cannot ignore any longer linear effects of energy errors. For simplicity we assume again thin lenses for the quadrupoles and get for the chromatic transformation matrix through half a 
FODO cell, $\frac{1}{2} \mathrm{QF}-\mathrm{B}-\frac{1}{2} \mathrm{QD}$ with (8.101) and assuming small deflection angles

$$
\mathcal{M}_{\frac{1}{2} \text { FODO }}=\left(\begin{array}{ccc}
1 & 0 & 0 \\
1 / f & 1 & 0 \\
0 & 0 & 1
\end{array}\right)\left(\begin{array}{ccc}
1 & L & \frac{1}{2 \rho_{0}} L^{2} \\
0 & 1 & \frac{L}{\rho_{0}} \\
0 & 0 & 1
\end{array}\right)\left(\begin{array}{ccc}
1 & 0 & 0 \\
-1 / f & 1 & 0 \\
0 & 0 & 1
\end{array}\right)
$$

or after multiplication

$$
\mathcal{M}_{\frac{1}{2} \text { FODO }}=\left(\begin{array}{ccc}
1-\frac{L}{f} & L & \frac{1}{2 \rho_{0}} L^{2} \\
-\frac{L}{f^{2}} & 1+\frac{L}{f} & \frac{L}{\rho_{0}}\left(1+\frac{L}{2 f}\right) \\
0 & 0 & 1
\end{array}\right) .
$$

The absolute value of the focal length $f$ is the same for both quadrupoles but since we start at the symmetry point in the middle of a quadrupole this focal length is based only on half a quadrupole. We have also assumed that the deflection angle of the bending magnet is small, $\theta \ll 1$, in analogy to thin lens approximation for quadrupoles. Lastly, we assumed that the bending magnets occupy the whole drift space between adjacent quadrupoles. This is not quite realistic but allows us an analytical and reasonable accurate approach.

In Sect. 8.4 dispersive elements of transformation matrices have been derived. In periodic lattices, however, we look for a particular solution which is periodic with the periodicity of the focusing lattice and label the solution by $\eta(z)$ or the $\eta$-function in distinction from the ordinary, generally non-periodic dispersion function $D(z)$. The typical form of the periodic dispersion function in a FODO lattice is shown in Fig. 10.9.

In addition to being periodic, this $\eta$-function must be symmetric with respect to the symmetry points in the middle of the FODO quadrupoles, where the derivative of the $\eta$-function vanishes. The transformation through one half FODO cell is

$$
\left(\begin{array}{c}
\eta^{-} \\
0 \\
1
\end{array}\right)=\mathcal{M}_{\frac{1}{2} \text { FODO }}\left(\begin{array}{c}
\eta^{+} \\
0 \\
1
\end{array}\right),
$$

Fig. 10.9 Dispersion function in FODO cells (example $\sharp 1$ in Table 10.1)

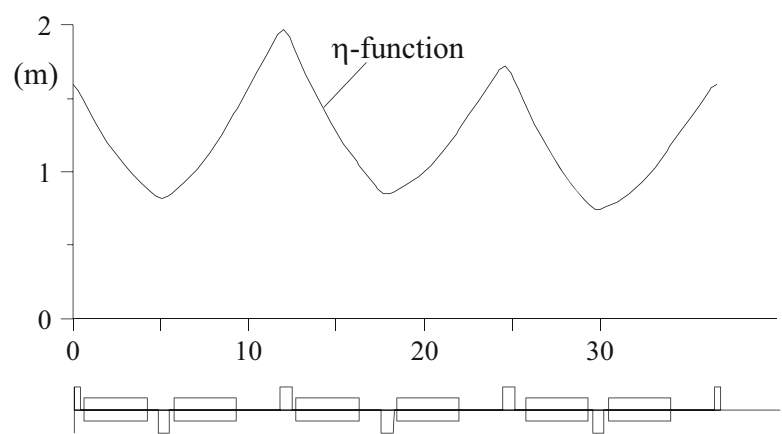


where we have set $\delta=1$ in accordance with the definition of dispersion functions and deflection in the horizontal plane.

In the particular arrangement of quadrupoles chosen in (10.71) the focusing quadrupole is the first element and the dispersion function reaches a maximum value $\eta^{+}$there. In the center of the defocusing quadrupole the dispersion function is reduced to a minimum value $\eta^{-}$. The opposite sequence of quadrupoles would lead to similar results. From (10.72) we get with $\eta^{\prime+}=\eta^{\prime-}=0$ the two equations

$$
\begin{aligned}
& \eta^{-}=\left(1-\frac{L}{f}\right) \eta^{+}+\frac{L^{2}}{2 \rho_{0}}, \\
& 0=-\frac{L}{f^{2}} \eta^{+}+\frac{L}{\rho_{0}}\left(1+\frac{L}{2 f}\right) .
\end{aligned}
$$

Solving (10.73) for the periodic dispersion function in the middle of the FODO quadrupoles, where $\eta^{\prime}=0$, we get in the focusing or defocusing quadrupole respectively

$$
\begin{aligned}
& \eta^{+}=\frac{f^{2}}{\rho_{0}}\left(1+\frac{L}{2 f}\right)=\frac{L^{2}}{2 \rho_{0}} \kappa(2 \kappa+1) \\
& \eta^{-}=\frac{f^{2}}{\rho_{0}}\left(1-\frac{L}{2 f}\right)=\frac{L^{2}}{2 \rho_{0}} \kappa(2 \kappa-1),
\end{aligned}
$$

where $\kappa=f / L$.

As mentioned before, in this approximation the bending magnet is as long as the length of half the FODO cell since the quadrupoles are assumed to be thin lenses and no drift spaces have been included between the quadrupoles and the bending magnet. The bending radius $\rho_{0}$, therefore, is equal to the average bending radius in the FODO lattice. From the known values of the dispersion function at the beginning of the FODO lattice we can calculate this function anywhere else in the periodic cell. Similar to the discussion in Sect. 10.1, we chose an optimum reference lattice, where

$$
\kappa_{0}=\sqrt{2},
$$

and

$$
\begin{aligned}
& \eta_{0}^{+}=\frac{L^{2}}{2 \rho}(4+\sqrt{2}), \\
& \eta_{0}^{-}=\frac{L^{2}}{2 \rho}(4-\sqrt{2}) .
\end{aligned}
$$

In Fig. 10.10 the values of the dispersion functions, normalized to those for the optimum FODO lattice in the middle of the FODO quadrupoles, are plotted versus the FODO cell parameter $\kappa$.

From Fig. 10.10 we note a diminishing dispersion function in a FODO cell as the betatron phase per cell or the focusing is increased $(f \rightarrow 0)$. This result will be important later for the design of storage rings for specific applications requiring either large or small beam emittances. The procedure to determine the dispersion functions in a FODO cell is straightforward and can easily be generalized to real 
Fig. 10.10 Scaling of the dispersion function in a FODO lattice

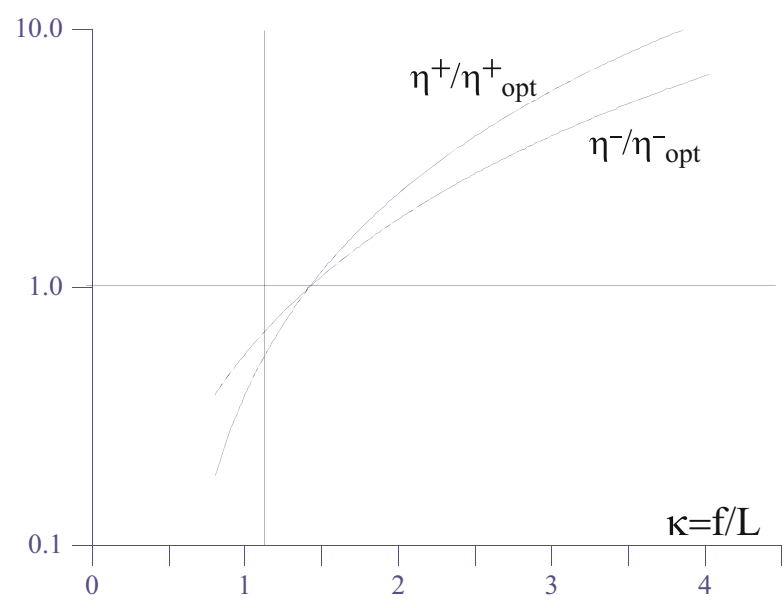

FODO lattices with finite quadrupole length and shorter bending magnets although it may be desirable to perform the matrix multiplications on a computer. For exploratory designs of accelerators structures, however, the thin lens approximation is a powerful and fairly accurate design tool.

\section{General Solution for the Periodic Dispersion}

In the previous section the dispersion function for a periodic and symmetric FODO lattice was derived. Many periodic lattice structures, however, are neither symmetric nor are they pure FODO structures and therefore we need to derive the periodic dispersion function in a more general form. To do this, we include in the equation of motion also the linear energy error term from, for example, (5.46)

$$
u^{\prime \prime}+K(z) u=\kappa_{0}(z) \delta .
$$

For particles having the ideal energy $(\delta=0)$ the right hand side vanishes and the solutions are composed of betatron oscillations and the trivial solution

$$
u_{0}(z) \equiv 0
$$

This trivial solution of (10.77) is clearly periodic and represents what is called in beam transport systems the ideal path and in circular accelerators the equilibrium orbit or closed orbit about which particles perform betatron oscillations. The expression for the ideal equilibrium orbit is this simple since we decided to use a curvilinear coordinate system which follows the design orbit (10.78) as determined by the placement of bending magnets and quadrupoles. 
For off momentum particles $(\delta \neq 0)$ the ideal path or closed orbit is displaced. Ignoring for a moment the $z$-dependence of $K$ and $\kappa_{0}$, this systematic displacement of the orbit is of the order of

$$
\Delta u=\frac{\kappa_{0}}{K} \delta
$$

as suggested by (10.77). In a real circular accelerator we expect a similar although $z$-dependent displacement of the equilibrium orbit for off momentum particles. Only one equilibrium orbit exists for each particle energy in a given closed lattice. If there were two solutions $u_{1}$ and $u_{2}$ of (10.77) we could write for the difference

$$
\left(u_{1}-u_{2}\right)^{\prime \prime}+K(z)\left(u_{1}-u_{2}\right)=0,
$$

which is the differential equation for betatron oscillations. Different solutions for the same energy, therefore, differ only by energy independent betatron oscillations which are already included in the general solution as the homogeneous part of the differential equation (10.77). Therefore, in a particular circular lattice only one unique equilibrium orbit or closed orbit exists for each energy.

Chromatic transformation matrices have been derived in Sect. 8.4. If we apply these $3 \times 3$-matrices to a circular lattice and calculate the total transformation matrix around the whole ring, we will be able to determine a self-consistent solution for equilibrium orbits. Before we calculate the periodic equilibrium orbits, we note that the solutions of (10.77) are proportional to the momentum deviation $\delta$. We therefore define the generalized periodic dispersion function as the equilibrium orbit for $\delta=1$ which we call the $\eta$-function. The transformation matrix for a periodic lattice of length $L_{\mathrm{p}}$ is

$$
\mathcal{M}\left(z+L_{\mathrm{p}} \mid z\right)=\left(\begin{array}{ccc}
C\left(z+L_{\mathrm{p}}\right) & S\left(z+L_{\mathrm{p}}\right) & D\left(z+L_{\mathrm{p}}\right) \\
C^{\prime}\left(z+L_{\mathrm{p}}\right) & S^{\prime}\left(z+L_{\mathrm{p}}\right) & D^{\prime}\left(z+L_{\mathrm{p}}\right) \\
0 & 0 & 1
\end{array}\right)
$$

and we get for the $\eta$-function with $\eta\left(z+L_{\mathrm{p}}\right)=\eta(z), \eta^{\prime}\left(z+L_{\mathrm{p}}\right)=\eta^{\prime}(z)$

$$
\begin{aligned}
& \eta(z)=C\left(z+L_{\mathrm{p}}\right) \eta(z)+S\left(z+L_{\mathrm{p}}\right) \eta^{\prime}(z)+D\left(z+L_{\mathrm{p}}\right), \\
& \eta^{\prime}(z)=C^{\prime}\left(z+L_{\mathrm{p}}\right) \eta(z)+S^{\prime}\left(z+L_{\mathrm{p}}\right) \eta^{\prime}(z)+D^{\prime}\left(z+L_{\mathrm{p}}\right) .
\end{aligned}
$$

These two equations can be solved for $\eta(z)$ and $\eta^{\prime}(z)$, the periodic dispersion function at the point $z$. The equilibrium orbit for any off momentum particle can be derived from this solution by multiplying with $\delta$

$$
u_{\delta}(z)=\eta(z) \delta .
$$


In a more formal way the periodic solution for the dispersion function can be derived from (10.82) while we drop the arguments for increased clarity

$$
\begin{aligned}
(C-1) \eta+S \eta^{\prime}+D & =0, \\
C^{\prime} \eta+\left(S^{\prime}-1\right) \eta^{\prime}+D^{\prime} & =0,
\end{aligned}
$$

which, in vector notation is

$$
\left(\mathcal{M}_{\eta}-\mathcal{I}\right) \eta=0
$$

where $\mathcal{M}_{\eta}$ is defined by $(10.81)$ and $\eta=\left(\eta, \eta^{\prime}, 1\right)$. The periodic dispersion function is therefore the eigenvector of the eigenvalue equation (10.85).

A particularly simple result is obtained again if the point $z$ is chosen at a symmetry point, where $\eta_{\text {sym }}^{\prime}=0$. In this case the dispersion function at the symmetry point is

$$
\eta_{\mathrm{sym}}=\frac{D}{1-C} \quad \text { and } \quad \eta_{\mathrm{sym}}^{\prime}=0
$$

Once the values of the $\eta$-functions are known at one point it is straightforward to obtain the values at any other point in the periodic lattice by matrix multiplication.

We may also try to derive an analytical solution for the periodic dispersion from the differential equation

$$
\eta^{\prime \prime}+K \eta=\kappa
$$

The solution is again the composition of the solutions for the homogeneous and the inhomogeneous differential equation. First, we transform (10.87) into normalized coordinates $w_{\eta}=\eta / \sqrt{\beta}$ and $\mathrm{d} \varphi=\mathrm{d} z /(v \beta)$. In these coordinates (10.87) becomes

$$
\frac{\mathrm{d}^{2} w_{\eta}}{\mathrm{d} \varphi^{2}}+v^{2} w_{\eta}=v^{2} \beta^{3 / 2} \kappa=v^{2} F(\varphi)
$$

An analytical solution to (10.88) has been derived in Sect. 5.5.4 and we have accordingly

$$
\begin{aligned}
& w_{\eta}(\varphi)=w_{0 \eta} \cos \nu \varphi+\frac{\dot{w}_{0 \eta}}{v} \sin \nu \varphi \\
& +v \int_{0}^{\varphi} F(\tau) \sin v(\varphi-\tau) \mathrm{d} \tau, \\
& \frac{\dot{w}_{\eta}}{v}(\varphi)=-w_{0 \eta} \sin \nu \varphi+\frac{\dot{w}_{0 \eta}}{\nu} \cos \nu \varphi \\
& +v \int_{0}^{\varphi} F(\tau) \cos v(\varphi-\tau) \mathrm{d} \tau,
\end{aligned}
$$

where we have set $\dot{w}=\frac{\mathrm{d}}{\mathrm{d} \varphi} w(\varphi)$. To select a periodic solution, we set

$$
w_{\eta}(2 \pi)=w_{\eta}(0)=w_{0 \eta} \quad \text { and } \quad \dot{w}_{\eta}(2 \pi)=\dot{w}_{0 \eta} .
$$


Inserting these boundary conditions into (10.89) to determine $\left(w_{0 \eta}, \dot{w}_{0 \eta}\right)$ and use the results in the first equation of (10.89) to get the general periodic solution for the normalized dispersion function after some manipulations

$$
w_{\eta}(\varphi)=\frac{v}{2 \sin \pi v} \int_{\varphi}^{\varphi+2 \pi} F(\tau) \cos [v(\varphi-\tau+\pi)] \mathrm{d} \tau .
$$

Now we return to the original variables $(\eta, z)$, and get from (10.90) the equation for the periodic dispersion or $\eta$-function

$$
\eta(z)=\frac{\sqrt{\beta(z)}}{2 \sin \pi v} \int_{z}^{z+L_{\mathrm{p}}} \frac{\sqrt{\beta(\zeta)}}{\rho(\zeta)} \cos v[\varphi(z)-\varphi(\zeta)+\pi] \mathrm{d} \zeta
$$

This solution shows clearly that the periodic dispersion function at any point $z$ depends on all bending magnets in the ring. We also observe a fundamental resonance phenomenon which occurs should the tune of the ring approach an integer in which case finite equilibrium orbits for off momentum particles do not exist anymore. To get stable equilibrium orbits, the tune of the ring must not be chosen to be an integer or in accelerator terminology an integer resonance must be avoided

$$
v \neq n
$$

where $n$ is an integer.

This is consistent with the solution (10.86) demanding that $\left|C\left(z+L_{\mathrm{p}}\right)\right|$ be less than unity. Since $C$ is the matrix element for the total ring we have $C=\cos 2 \pi v$ which obviously is equal to +1 only for integer values of the tune $v$. While (10.89) is not particularly convenient to calculate the dispersion function, it clearly exhibits the resonance character and will be very useful later in some other context, for example, if we want to determine the effect of a single bending magnet.

Another way to solve the differential equation (10.88) will be considered to introduce a powerful mathematical method useful in periodic systems. We note that the perturbation term $F(z)=\beta^{3 / 2}(z) \kappa(z)$ is a periodic function with the period $L_{\mathrm{p}}$ or $2 \pi$ using normalized coordinates. The perturbation term can therefore be expanded into a Fourier series

$$
\beta^{3 / 2} \kappa=\sum F_{n} \mathrm{e}^{\mathrm{i} n \varphi}
$$

where

$$
F_{n}=\frac{1}{2 \pi} \oint \beta^{3 / 2} \kappa \mathrm{e}^{-\mathrm{i} n \varphi} \mathrm{d} \varphi
$$


or if we go back to regular variables

$$
F_{n}=\frac{1}{2 \pi \nu} \oint \frac{\sqrt{\beta(\zeta)}}{\rho(\zeta)} \mathrm{e}^{-\mathrm{i} n \varphi(\zeta)} \mathrm{d} \zeta
$$

Similarly, we may expand the periodic $\eta$-function into a Fourier series

$$
w_{\eta}(\varphi)=\sum W_{\eta n} \mathrm{e}^{\mathrm{i} n \varphi} .
$$

Using both (10.93), (10.96) in (10.88) we get

$$
\left(-n^{2}+v^{2}\right) \sum W_{\eta n} \mathrm{e}^{-\mathrm{i} n \varphi}=v^{2} \sum F_{n} \mathrm{e}^{-\mathrm{i} n \varphi},
$$

which can be solved for the Fourier coefficients $W_{\eta n}$ of the periodic dispersion function

$$
W_{\eta n}=\frac{v^{2} F_{n}}{v^{2}-n^{2}}
$$

The periodic solution of the differential equation (10.88) is finally

$$
w_{\eta}(\varphi)=\sum_{n=-\infty}^{+\infty} \frac{v^{2} F_{n} \mathrm{e}^{\mathrm{i} n \varphi}}{v^{2}-n^{2}} .
$$

It is obvious again, that the tune must not be an integer to avoid a resonance. This solution is intrinsically periodic since $\varphi$ is periodic and the relation to (10.90) can be established by replacing $F_{n}$ by its definition (10.94). Using the property $F_{-n}=F_{n}$ we get for a symmetric lattice and with formula GR[1.445.6 $]^{1}$

$$
\begin{aligned}
w_{\eta}(\varphi) & =\sum_{n=-\infty}^{+\infty} \frac{\mathrm{e}^{\mathrm{i} n \varphi} \frac{v}{2 \pi} \oint \kappa(\zeta) \sqrt{\beta(\zeta)} \mathrm{e}^{-\mathrm{i} i \zeta} \mathrm{d} \zeta}{v^{2}-n^{2}} \\
& =\frac{\nu}{\pi} \oint \kappa(\zeta) \sqrt{\beta(\zeta)}\left[\frac{1}{2 v^{2}}+\sum_{n=1}^{\infty} \frac{\cos n(\zeta-\varphi)}{v^{2}-n^{2}}\right] \mathrm{d} \zeta \\
& =\frac{1}{2 \sin v \pi} \oint \kappa(\zeta) \sqrt{\beta(\zeta)} \cos (\nu[\varphi-\zeta+\pi]) \mathrm{d} \zeta
\end{aligned}
$$

which is the same as (10.90) since $\mathrm{d} \tau=\nu \beta \mathrm{d} \zeta$. For an asymmetric lattice the proof is similar albeit somewhat more elaborate. Solution (10.100) expresses the dispersion

\footnotetext{
${ }^{1}$ We will abbreviate in this way formulas from the Table of Integrals, Series and Products, I.S. Gradshteyn/I.M. Ryzhik, 4th edition.
} 
function as the combination of a constant and a sum of oscillatory terms. Evaluating the non-oscillatory part of the integral, we find the average value of the dispersion or $\eta$-function,

$$
\langle\eta\rangle \approx \frac{\langle\beta\rangle}{v_{0}} .
$$

This result by itself is of limited usefulness but can be used to obtain an estimate for the momentum compaction factor $\alpha_{\mathrm{c}}$ defined analogous to (8.125) by

$$
\alpha_{\mathrm{c}}=\frac{1}{L_{\mathrm{p}}} \oint \frac{\eta(z)}{\rho(z)} \mathrm{d} z \approx\left\langle\frac{\eta}{\rho}\right\rangle .
$$

A good approximation for the momentum compaction factor is therefore $\alpha_{\mathrm{c}} \approx$ $\langle\beta\rangle /(\rho \nu)$ and with (10.70) integrated only over the arcs of the ring

$$
\alpha_{\mathrm{c}} \approx \frac{1}{v^{2}}
$$

Thus we find the interesting result that the transition energy $\gamma_{t}$ is approximately equal to the horizontal tune of a circular accelerator

$$
\gamma_{\mathrm{t}} \approx v_{x}
$$

As a cautionary note for circular accelerators with long straight sections, only the tune of the arc sections should be used here since straight sections do not contribute to the momentum compaction factor but can add significantly to the tune.

\subsubsection{Periodic Lattices in Circular Accelerators}

Circular accelerators and long beam transport lines can be constructed from fundamental building blocks like FODO cells or other magnet sequences which are then repeated many times. Any cell or lattice unit for which a periodic solution of the lattice functions can be found may be used as a basic building block for a periodic lattice. Such units need not be symmetric but the solution for a symmetric lattice segment is always periodic.

FODO cells as elementary building blocks for larger beam transport lattices may lack some design features necessary to meet the objectives of the whole facility. In a circular accelerator we need for example some component free spaces along the orbit to allow the installation of experimental detectors or other machine components like accelerating sections, injection magnets or synchrotron radiation producing insertion devices. A lattice made up of standard FODO cells with bending magnets would not provide such spaces. 
The lattice of a circular accelerator therefore exhibits generally more complexity than that of a simple FODO cell. Often, a circular accelerator is made up of a number of superperiods which may be further subdivided into segments with special features like dispersion suppression section, achromatic sections, insertions, matching sections or simple focusing and bending units like FODO cells. To illustrate basic lattice design concepts, we will discuss specific lattice solutions to achieve a variety of objectives.

\section{Synchrotron Lattice}

For a synchrotron whose sole function is to accelerate particles the problem of free space can be solved quite easily. Most existing synchrotrons are based on a FODO lattice recognizing its simplicity, beam dynamical stability and efficient use of space. To provide magnet free spaces, we merely eliminate some of the bending magnets. As a consequence the whole ring lattice is composed of curved as well as straight FODO cells. The elimination of bending magnets must, however, be done thoughtfully since the dispersion function depends critically on the distribution of the bending magnets. Random elimination of bending magnets may lead to an uncontrollable perturbation of the dispersion function.

Often it is desirable to have the dispersion function vanish or at least be small in magnet free straight sections to simplify injection and avoid possible instabilities if rf-cavities are placed where the dispersion function is finite. The general approach to this design goal is, for example, to use regular FODO cells for the arcs followed by a dispersion matching section, where the dispersion function is brought to zero or at least to a small value leading finally to a number of bending magnet free straight FODO cells. As an example such a lattice is shown in Fig. 10.11 for a $3.5 \mathrm{GeV}$ synchrotron [9].

Figure 10.11 shows one quadrant of the whole ring and we clearly recognize three different lattice segments including seven arc FODO half cells, two half

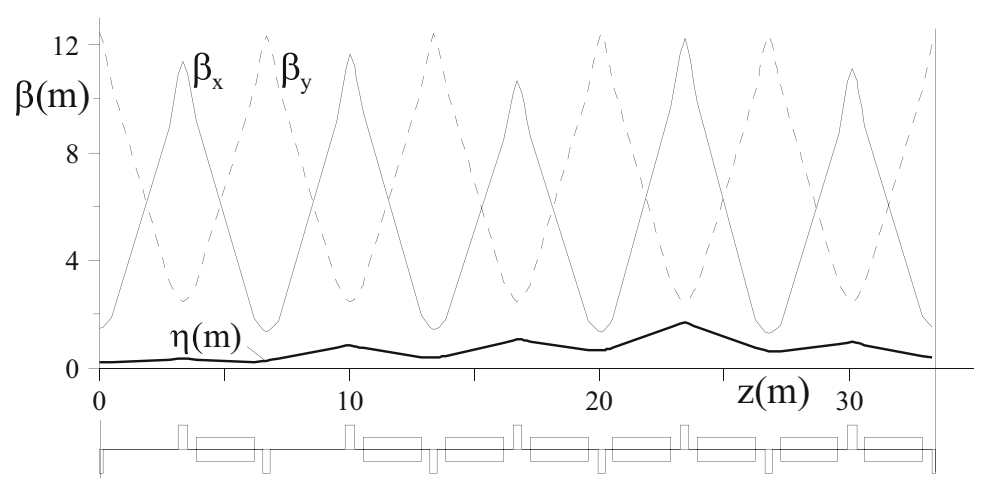

Fig. 10.11 Typical FODO lattice for a separated function synchrotron 
cells to match the dispersion function and one half cell for installation of other machine components. Such a quadrant is mirror reflected at one or the other end to form one of two ring lattice superperiods. In this example the ring consists of two superperiods although another ring could be composed by a different number of superperiods. A specific property of the lattice shown in Fig. 10.11 is, as far as focusing is concerned, that the whole ring is made up of equal FODO cells with only two quadrupole families QF and QD. The betatron functions are periodic and are not significantly affected by the presence or omission of bending magnets which are assumed to have negligible edge focusing. By eliminating bending magnets in an otherwise unperturbed FODO lattice, we obtain magnet free spaces equal to the length of the bending magnets which are used for the installation of accelerating components, injection magnets, and beam monitoring equipment.

\section{Phase Space Matching}

Periodic lattices like FODO channels exhibit unique solutions for the betatron and dispersion functions. In realistic accelerator designs, however, we will not be able to restrict the lattice to periodic cells only. We will find the need for a variety of lattice modifications which necessarily require locally other than periodic solutions. Within a lattice of a circular accelerator, for example, we encountered the need to provide some magnet free spaces, where the dispersion function vanishes. In colliding beam facilities it is desirable to provide for a very low value of the betatron function at the beam collision point to maximize the luminosity. These and other lattice requirements necessitate a deviation from the periodic cell structure.

Beam transport lines are in most cases not based on periodic focusing. If such transport lines carry beam to be injected into a circular accelerator or must carry beam from such an accelerator to some other point, we must consider proper matching conditions at locations, where lattices of different machines or beam transport systems meet $[10,11]$. Joining arbitrary lattices may result in an inadequate over lap of the phase ellipse for the incoming beam with the acceptance of the downstream lattice as shown in Fig. 10.12a.

a)

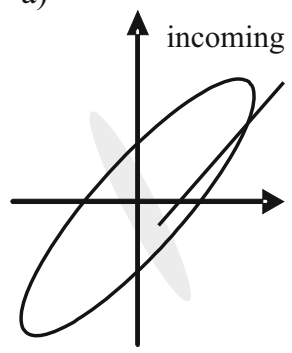

b)

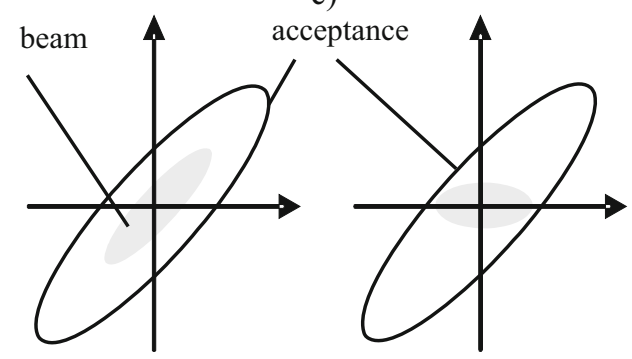

Fig. 10.12 Matching conditions in phase space: mismatch (a), perfect match (b), efficient match $(\mathbf{c})$ 
For a perfect match of two lattices, all lattice functions must be the same at the joining point as shown in Fig. 10.12b

$$
\left(\beta_{x}, \alpha_{x}, \beta_{y}, \alpha_{y}, \eta, \eta^{\prime}\right)_{1}=\left(\beta_{x}, \alpha_{x}, \beta_{y}, \alpha_{y}, \eta, \eta^{\prime}\right)_{2}
$$

In this case, the phase ellipse at the end of lattice ${ }_{1}$ is similar to the acceptance ellipse at the entrance of lattice ${ }_{2}$ (see Fig. 10.12). To avoid dilution of particles in phase space perfect matching is desired in proton and ion beam transport systems and accelerators. For electrons this is less critical because electron beams regain the appropriate phase ellipse through synchrotron radiation and damping. The main goal of matching an electron beam is to assure that the emittance of the incoming beam is fully accepted by the downstream lattice as shown in Fig. 10.12b, c. Perfect matching of all lattice functions and acceptances with beam emittance, however, provides the most economic solution since no unused acceptance exist. Matching of the dispersion function $\left(\eta, \eta^{\prime}\right)$ in addition also assures that phase ellipses for off momentum particles match as well.

Matching in circular accelerators is much more restrictive than that between independent lattices. In circular accelerators a variety of lattice segments for different functions must be tied together to form a periodic magnet structure. To preserve the periodic lattice functions, we must match them exactly between different lattice segments. Failure of perfect matching between lattice segments can lead to lattice functions which are vastly different from design goals or do not exist at all.

In general there are six lattice functions to be matched requiring six variables or quadrupoles in the focusing structure of the upstream lattice to produce a perfect match. Matching quadrupoles must not be too close together in order to provide some independent matching power for individual quadrupoles. As an example, the betatron functions can be modified most effectively if a quadrupole is used at a location, where the betatron function is large and not separated from the matching point by multiples of $\pi$ in betatron phase. Most independent matching conditions for both the horizontal and vertical betatron functions are created if matching quadrupoles are located where one betatron function is much larger than the other allowing almost independent control of matching condition.

It is impossible to perform such general matching tasks by analytic methods and a number of numerical codes are available to solve such problems. Frequently used matching codes are TRANSPORT [12], or MAD [13]. Such programs are an indispensable tool for lattice design and allow the fitting of any number of lattice functions to desired values including boundary conditions to be met along the matching section.

\section{Dispersion Matching}

A very simple, although not perfect, method to reduce the dispersion function in magnet free straight sections is to eliminate one or more bending magnets close 
to but not at the end of the arc and preferably following a focusing quadrupole, QF. In this arrangement of magnets the dispersion function reaches a smaller value compared to those in regular FODO cells with a slope that becomes mostly compensated by the dispersion generated in the last bending magnet. The match is not perfect but the dispersion function is significantly reduced, where this is desirable, and magnet free sections can be created in the lattice. This method requires no change in the quadrupole or bending magnet strength and is therefore also operationally very simple as demonstrated in the example of a synchrotron lattice shown in Fig. 10.11. We note the less than perfect matching of the dispersion function which causes a beating of an originally periodic dispersion function. In the magnet free straight sections, however, the dispersion function is considerably reduced compared to the values in the regular FODO cells.

More sophisticated matching methods must be employed, where a perfect match of the dispersion function is required. Matching of the dispersion to zero requires the adjustment of two parameters, $\eta=0$ and $\eta^{\prime}=0$, at the beginning of the straight section. This can be achieved by controlling some of the upstream quadrupoles. Compared to a simple two parameter FODO lattice (Fig. 10.11) this variation requires a more complicated control system and additional power supplies to specially control the matching quadrupoles. This dispersion matching process disturbs the betatron functions which must be separately controlled and matched by other quadrupoles in dispersion free sections. Such a matching method is utilized in a number of storage rings with a special example shown in Fig. 10.13 [14].

Here, we note the perfect matching of the dispersion function as well as the associated perturbation of the betatron function requiring additional matching. Quadrupoles QFM and QDM are adjusted such that $\eta=0$ and $\eta^{\prime}=0$ in the straight section. In principle this could be done even without eliminating a bending magnet, but the strength of the dispersion matching quadrupoles would significantly deviate from that of the regular FODO quadrupoles and cause a large distortion of the betatron function in the straight section. To preserve a symmetric lattice, the

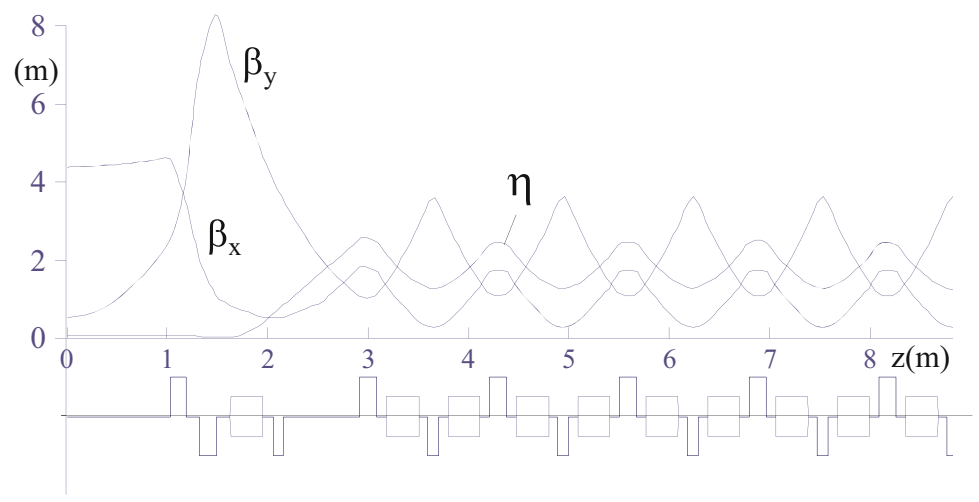

Fig. 10.13 Lattice for a $1.2 \mathrm{GeV}$ low emittance damping ring 
betatron function must be matched with the quadrupoles Q1 and Q2 to get $\alpha_{x}=0$ and $\alpha_{y}=0$ at the symmetry points of the lattice.

\section{Dispersion Suppressor}

A rather elegant method of dispersion matching has been developed by Keil [15]. Noting that dispersion matching requires two parameters he chooses to vary the last bending magnets at the end of the arcs rather than quadrupoles. The great advantage of this method is to leave the betatron functions and the tunes undisturbed at least as long as we may ignore the end field focusing of the bending magnets which is justified in large high energy accelerators. This dispersion suppressor consists of four FODO half cells following directly the regular FODO cells at a focusing quadrupole QF as shown in Fig. 10.14. The strength of the bending magnets are altered into two types with a total bending angle of all four magnets to be equal to two regular bending magnets.

The matching conditions can be derived analytically from the transformation matrix for the full dispersion suppressor as a function of the individual magnet parameters. An algebraic manipulation program has been used to derive a result that is surprisingly simple. If $\theta$ is the bending angle for regular FODO cell bending magnets and $\psi$ the betatron phase for a regular FODO half cell, the bending angles $\theta_{1}$ and $\theta_{2}$ are determined by [15]

$$
\theta_{1}=\theta\left(1-\frac{1}{4 \sin ^{2} \psi}\right)
$$

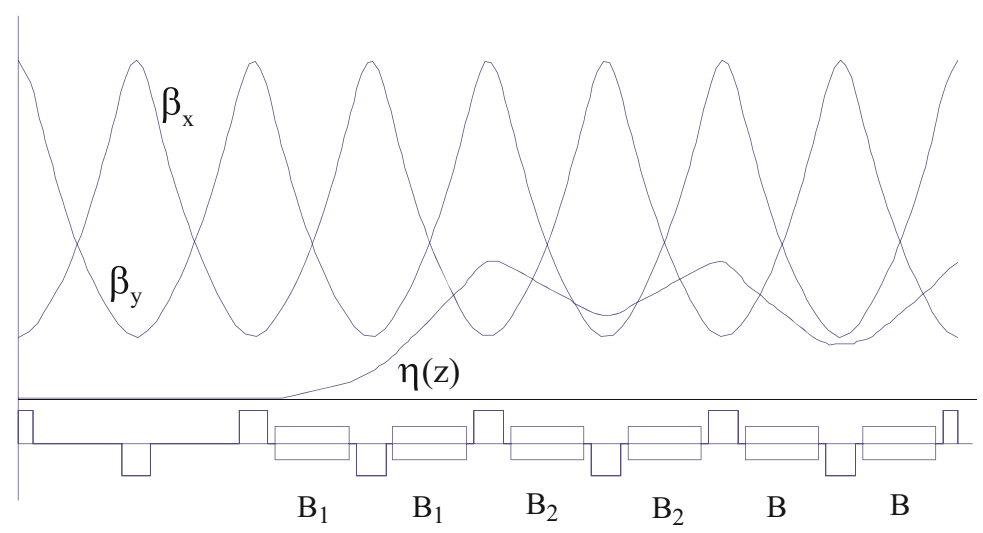

Fig. 10.14 Dispersion suppressor lattice 
and

$$
\theta_{2}=\theta\left(\frac{1}{4 \sin ^{2} \psi}\right),
$$

where

$$
\theta=\theta_{1}+\theta_{2}
$$

This elegant method requires several FODO cells to match the dispersion function and is therefore most appropriately used in large systems. Where a compact lattice is important, matching by quadrupoles as discussed earlier might be more space efficient.

\section{Magnet Free Insertions}

An important part of practical lattice design is to provide magnet free spaces which are needed for the installation of other essential accelerator components or experimental facilities. Methods to provide limited magnet free spaces by eliminating bending magnets in FODO lattices have been discussed earlier. Often, however, much larger magnet free spaces are required and procedures to provide such sections need to be formulated.

The most simple and straight forward approach is to use a set of quadrupoles and focus the lattice functions $\beta_{x}, \beta_{y}$ and $\eta$ into a magnet free section such that the derivatives $\alpha_{x}, \alpha_{y}$ and $\eta^{\prime}$ vanish in the center of this section. This method is commonly applied to interaction areas in colliding beam facilities to provide optimum beam conditions for maximum luminosity at the collision point. A typical example is shown in Fig. 10.15.

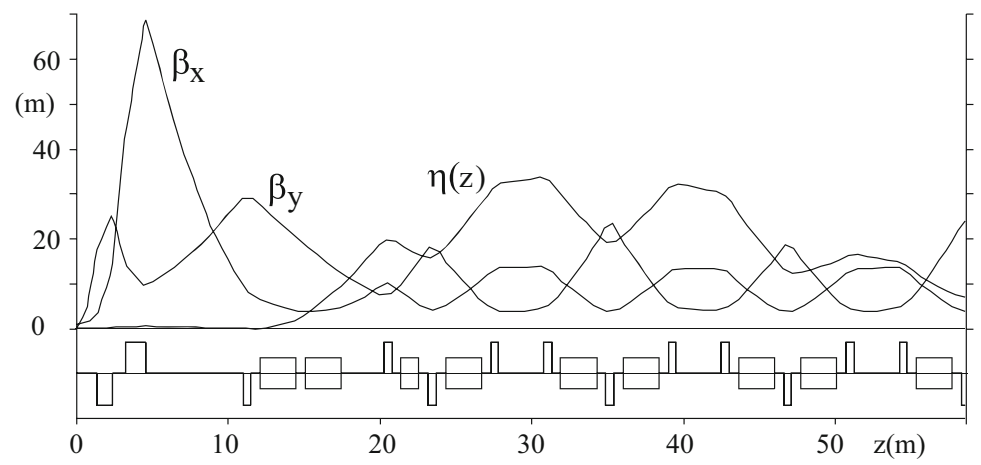

Fig. 10.15 Lattice of the SPEAR storage ring 
Fig. 10.16 Lattice of the ADONE storage ring

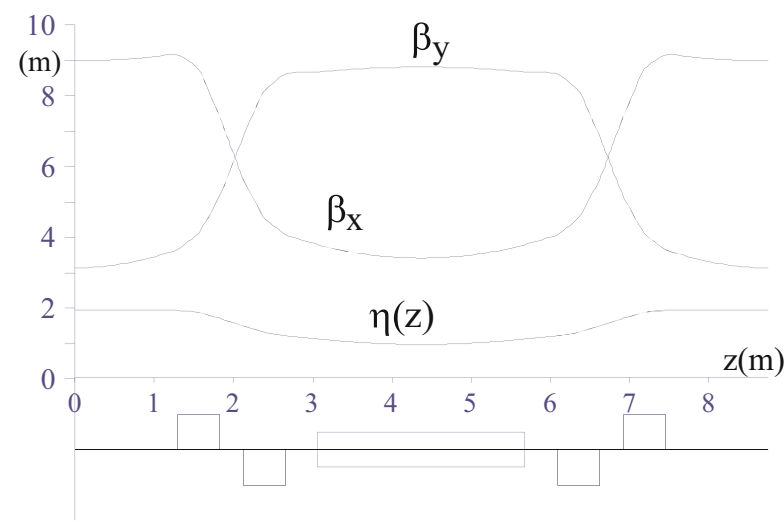

Another scheme to provide magnet free spaces is exercised in the SPEAR lattice (Fig. 10.15) where the FODO structure remains unaltered except that the FODO cells have been separated in the middle of the QF quadrupoles. A separation in the middle of the QD quadrupoles would have worked as well. Since the middle of FODO quadrupoles are symmetry points a modest separation can be made with minimal perturbation to the betatron functions and no perturbation to the dispersion function since $\eta^{\prime}=0$ in the middle of FODO quadrupoles.

A more general design approach to provide magnet free spaces in a periodic lattice is exercised in the storage ring shown in Fig. 10.16 [16] or the storage ring as shown in Fig. 10.15 [17]. In the ADONE lattice the quadrupoles of a FODO lattice are moved together to form doublets and alternate free spaces are filled with bending magnets or left free for the installations of other components.

\section{Collins Insertion}

A simple magnet free insertion for dispersion free segments of the lattice has been proposed by Collins [18]. The proposed insertion consists of a focusing and a defocusing quadrupole of equal strength with a long drift space in between as shown in Fig. 10.17. In thin lens approximation, the transformation matrix for the insertion is

$$
\mathcal{M}_{\text {ins }}=\left(\begin{array}{ll}
1 & d \\
0 & 1
\end{array}\right)\left(\begin{array}{cc}
1 & 0 \\
1 / f & 1
\end{array}\right)\left(\begin{array}{ll}
1 & D \\
0 & 1
\end{array}\right)\left(\begin{array}{cc}
1 & 0 \\
-1 / f & 1
\end{array}\right)\left(\begin{array}{ll}
1 & d \\
0 & 1
\end{array}\right) .
$$

This insertion matrix must be equated with the transformation matrix for this same insertion expressed in terms of lattice functions at the insertion point with the regular lattice

$$
\mathcal{M}_{\text {ins }}=\left(\begin{array}{cc}
\cos \psi+\alpha \sin \psi & \beta \sin \psi \\
-\frac{1+\alpha^{2}}{\beta} \sin \psi & \cos \psi-\alpha \sin \psi
\end{array}\right) .
$$




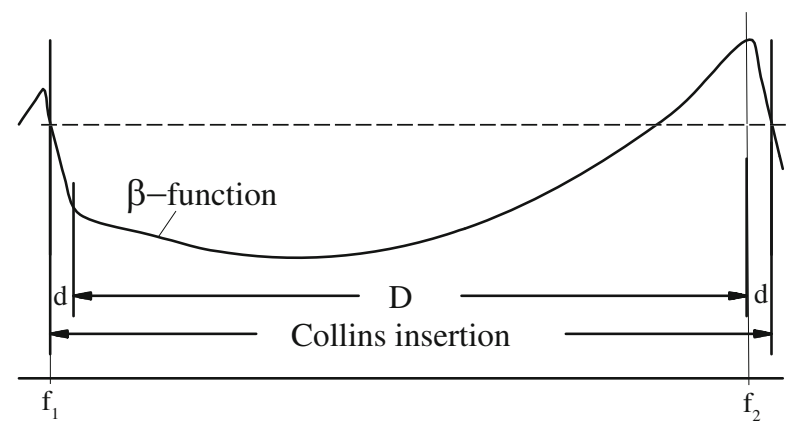

Fig. 10.17 Collins insertion

Both matrices provide three independent equations to be solved for the drift lengths $d$ and $D$ and for the focal length $f$ of the quadrupoles. After multiplications of all matrices we equate matrix elements and get

$$
D=\frac{\alpha^{2}}{\gamma}, \quad d=\frac{1}{\gamma}, \quad \text { and } \quad f=-\frac{\alpha}{\gamma} .
$$

These relations are valid for both planes only if $\alpha_{x}=-\alpha_{y}$. Generally, this is not the case for arbitrary lattices but for a weak focusing FODO lattice this condition is met well. We note that this design provides an insertion of length $D$ which is proportional to the value of the betatron functions at the insertion point and requires that $\alpha \neq 0$.

Of course any arbitrary insertion with a unity transformation matrix $\mathcal{I}$ in both planes is a valid solution as well. Such solutions can in principle always be enforced by matching with a sufficient number of quadrupoles. If the dispersion function and its derivative is zero such an insertion may also have a transformation matrix of $-\mathcal{I}$. This property of insertions is widely used in computer designs of insertions when fitting routines are available to numerically adjust quadrupole strength such that desired lattice features are met including the matching of the lattice functions to the insertion point. A special version of such a solution is the low beta insertion for colliding beam facilities.

\section{Low Beta Insertions}

In colliding beam facilities long magnet free straight sections are required to allow the installation of high energy particle detectors. In the center of these sections, where two counter rotating particle beams collide, the betatron functions must reach very small values forming a narrow beam waist. This requirement allows to minimize the destructive beam-beam effect when two beams collide and thereby maximize the luminosity of the colliding beam facility [19]. 


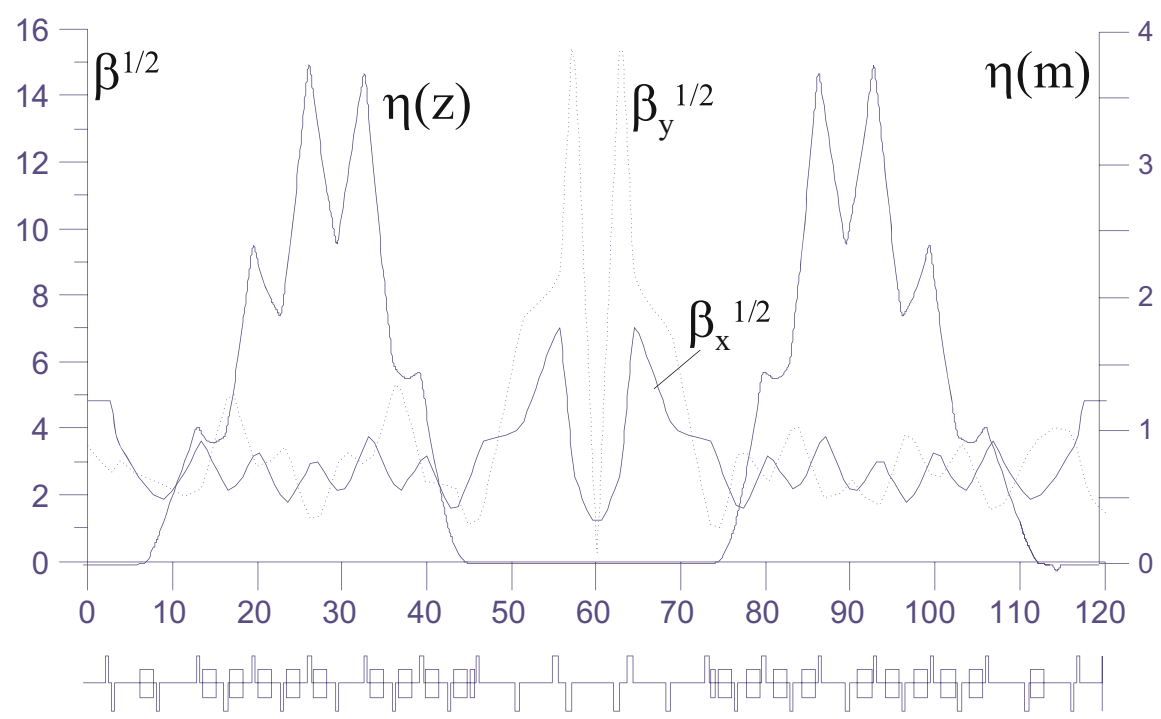

Fig. 10.18 Lattice functions of a colliding beam storage ring [21]. Shown is half the circumference with the collision point, low beta and vanishing dispersion in the center

An example for the incorporation of such a low beta insertion is shown in Fig. 10.18 representing one of many variations of a low beta insertion in colliding beam facilities [20]. The special challenge in this matching problem is to provide a very small value for the betatron functions at the collision point. To balance the asymmetry of the focusing in the closest quadrupoles the betatron functions in both planes are generally not made equally small but the vertical betatron function is chosen smaller than the horizontal to maximize the luminosity. The length of the magnet free straight section is determined by the maximum value for the betatron function that can be accepted in the first vertically focusing quadrupole. The limit may be determined by just the physical aperture available or technically possible in these insertion quadrupoles or by the chromaticity and ability to correct and control chromatic and geometric aberrations.

The maximum value of the betatron function at the entrance to the first quadrupole, the minimum value at the collision point, and the magnet free section are correlated by the equation for the betatron function in a drift space. Assuming symmetry about the collision point, the betatron functions develop from there like

$$
\beta(z)=\beta^{*}+\frac{z^{2}}{\beta^{*}},
$$

where $\beta^{*}$ is the value of the betatron function at the symmetry point, $z$ the distance from the collision point and $2 L_{\text {ins }}$ the full length of the insertion between the innermost quadrupoles. 
The distance $L$ tended to be quite large to allow the installation of large particle detectors for high energy physics experiment. As a consequence, the betatron function became very large in the first quadrupoles causing severe perturbations and limitations in particle dynamics. This, of course, created a limit in the achievable luminosity. In new colliding beam facilities, like B-factories, the lowbeta creating quadrupoles are incorporated deeply into the detectors, thus reducing $L$ and the maximum value for the betatron functions. This compromise resulted in significantly higher luminosity of colliding beams.

\subsection{FODO Lattice and Acceleration*}

So far we have ignored the effect of acceleration in beam dynamics. In specific cases, however, acceleration effects must be considered specifically if the particle energy changes significantly along the beam line. In linear accelerators such a need occurs at low energies when we try to accelerate a large emittance beam through the small apertures of the accelerating sections. For example, when a positron beam is to be created the positrons emerging from a target within a wide solid angle are focused into the small aperture of a linear accelerator. After some initial acceleration in the presence of a solenoid field along the accelerating structure it is desirable to switch over to more economic quadrupole focusing. Even at higher energies when the beam diameter is much smaller than the aperture strong focusing is still desired to minimize beam break up instabilities.

\subsubsection{Lattice Structure}

A common mode of focusing uses a FODO lattice in conjunction with the linac structure. We may, however, not apply the formalism developed for FODO lattices without modifications because the particle energy changes significantly along the lattice. A thin lens theory has been derived by Helm [22] based on a regular FODO channel in the particle reference system. Due to Lorentz contraction the constant quadrupole separations $L^{*}$ in the particle system become increasing distances in the laboratory system as the beam energy increases. To show this quantitatively, we consider a FODO channel installed along a linear accelerator and starting at the energy $\gamma_{0}$ with a constant cell half length $\tilde{L}=\gamma_{0} L^{*}$. The tick-marks along the scale in Fig. 10.19 indicate the locations of the quadrupoles and the distances between magnets in the laboratory system are designated by $L_{1}, L_{2} \ldots$

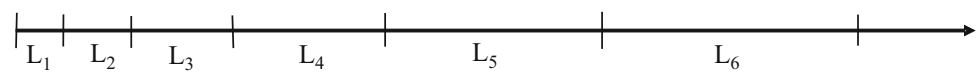

Fig. 10.19 FODO channel and acceleration 
With the acceleration $\alpha$ in units of the rest energy per unit length and $\gamma_{0}$ the particle energy at the center of the first quadrupole, the condition to have a FODO channel in the particle system is

$$
L^{*}=\int_{0}^{L_{1}} \frac{\mathrm{d} z}{1+\frac{\alpha z}{\gamma_{0}}}=\frac{\gamma_{0}}{\alpha} \ln \left(1+\frac{\alpha L_{1}}{\gamma_{0}}\right) .
$$

The quantity $2 L^{*}$ is the length of a FODO cell in the particle system and $L_{1}$ is the distance between the first and second quadrupole in the laboratory system. Solving for $L_{1}$ we get

$$
L_{1}=L^{*} \frac{\mathrm{e}^{\kappa}-1}{\kappa}
$$

where

$$
\kappa=\frac{\alpha}{\gamma_{0}} L^{*}
$$

At the same time the beam energy has increased from $\gamma_{0}$ to

$$
\gamma_{1}=\gamma_{0}+\alpha L_{1}
$$

Equation (10.113) can be applied to any of the downstream distances between quadrupoles. The $n^{\text {th }}$ distance $L_{n}$, for example, is determined by an integration from $z_{n-1}$ to $z_{n}$ or equivalently from 0 to $L_{n}$

$$
L^{*}=\int_{0}^{L_{n}} \frac{\mathrm{d} z}{1+\frac{\alpha z}{\gamma_{n-1}}}=\frac{\gamma_{n-1}}{\alpha} \ln \left(1+\frac{\alpha L_{n}}{\gamma_{n-1}}\right) .
$$

While solving for $L_{n}$, we express the energy $\gamma_{n-1}$ by addition of the energy gains $\gamma_{n-1}=\sum_{i}^{n-1} \Delta \gamma_{i}=\alpha \sum_{i}^{n-1} L_{i}$ and taking the distances $L_{i}$ from expressions (10.114) and (10.117) we get for $\kappa \ll 1$

$$
L_{n}=L^{*} \frac{\mathrm{e}^{\kappa}-1}{\kappa} \mathrm{e}^{(n-1) \kappa} .
$$

In thin lens approximation, the distances between successive quadrupoles increase exponentially in the laboratory system like (10.118) to resemble the focusing properties of a regular FODO channel with a cell length $2 L^{*}$ in the particle system under the influence of an accelerating field.

Such FODO channels are used to focus large emittance particle beams in linear accelerators as is the case for positron beams in positron linacs. For strong focusing as is needed for low energies where the beam emittance is large, the thin lens approximation, however, is not accurate enough and a more exact formulation of the transformation matrices must be applied [23], which we will derive here in some detail. 


\subsubsection{Transverse Beam Dynamics and Acceleration}

Transverse focusing can be significantly different along a linear accelerator due to the rapid changing particle energy compared to a fixed energy transport line and the proper beam dynamics must be formulated in the presence of longitudinal acceleration. To derive the correct equations of motion we consider the particle dynamics in the presence of the complete Lorentz force including electrical fields

$$
\dot{\boldsymbol{p}}=e \boldsymbol{E}+e[\dot{\boldsymbol{r}} \times \boldsymbol{B}] .
$$

To solve this differential equation we consider a straight beam transport line with quadrupoles aligned along the $z$-coordinate as we would have in a linear accelerator. The accelerating fields are assumed to be uniform with a finite component only along the $z$-coordinate. At the location $\boldsymbol{r}=(x, y, z)$, the fields can be expressed by $\boldsymbol{E}=(0,0, \alpha / e)$ and $\boldsymbol{B}=(g x, g y, 0)$, where the acceleration $\alpha$ is defined by

$$
\alpha=e|\boldsymbol{E}|
$$

To evaluate (10.119), we express the time derivative of the momentum, $\dot{\boldsymbol{p}}=$ $\gamma m \dot{r}$ by

$$
\dot{\boldsymbol{p}}=\dot{\gamma} m \dot{\boldsymbol{r}}+\gamma m \ddot{\boldsymbol{r}},
$$

From $c \dot{p}=\dot{E} / \beta$ we find that $\dot{\gamma}=\alpha \beta / m c^{2}$ and (10.121) becomes for the $x$ component

$$
c \dot{p}_{x}=\alpha \beta m \dot{x}+\frac{1}{c} E \ddot{x}
$$

In this subsection, we make ample use of quantities $\alpha, \beta, \gamma$ being acceleration and relativistic parameters which should not be confused with the lattice functions, which we will not need here. Bowing to convention, we refrain from introducing new labels.

The variation of the momentum with time can be expressed also with the Lorentz equation (10.119) and with the specified fields, we get

$$
\dot{p}_{x}=-c e \beta g x
$$

We replace the time derivatives in $(10.122)$ by derivatives with respect to the independent variable $z$

$$
\begin{aligned}
& \dot{x}=\beta c x^{\prime}, \\
& \ddot{x}=\beta^{2} c^{2} x^{\prime \prime}+\frac{\alpha}{\gamma^{3} m} x^{\prime},
\end{aligned}
$$


and after insertion into (10.122) and equating with (10.123) the equation of motion becomes

$$
\frac{\mathrm{d}^{2} x}{\mathrm{~d} z^{2}}+\frac{\alpha}{\beta^{2} E} \frac{\mathrm{d} x}{\mathrm{~d} z}+\frac{c e g}{\beta E} x=0
$$

where we used the relation $\beta^{2}+1 / \gamma^{2}=1$. With $\frac{\alpha}{\beta}=\frac{\mathrm{d} c p / \mathrm{d} z}{c p_{0}}$ and defining the quantity

$$
\eta_{0}=\frac{\mathrm{d} p / \mathrm{d} z}{p_{0}}=\frac{\alpha}{\beta c p_{0}}
$$

we get for the equation of motion in the horizontal plane, $u=x$

$$
\frac{\mathrm{d}^{2} u}{\mathrm{~d} z^{2}}+\frac{\eta_{0}}{1+\eta_{0} z} \frac{\mathrm{d} u}{\mathrm{~d} z}+\frac{k_{0}}{1+\eta_{0} z} u=0
$$

introducing the quadrupole strength $k_{0}=\frac{e g}{p_{0}}$. Equation (10.127) is valid also for the vertical plane $u=y$ if we only change the sign of the quadrupole strength $k_{0}$. Equation (10.127) is a Bessel's differential equation, which becomes obvious by defining a new independent variable

$$
\xi=\frac{2 \beta}{\eta_{0}} \sqrt{k_{0}\left(1+\eta_{0} z\right)}
$$

transforming (10.127) into

$$
\frac{\mathrm{d}^{2} u}{\mathrm{~d} \xi^{2}}+\frac{1}{\xi} \frac{\mathrm{d} u}{\mathrm{~d} \xi}+u=0
$$

which is the equation of motion in the presence of both transverse and longitudinal fields.

\section{Analytical Solutions}

The solutions of the differential equation (10.129) are Bessel's functions of the first and second kind in zero order

$$
u(z)=C_{1} I_{0}(\xi)+C_{2} Y_{0}(\xi)
$$

In terms of initial conditions $\left(u_{0}, u_{0}^{\prime}\right)$ for $z=0$ we can express the solutions in matrix formulation

$$
\left(\begin{array}{c}
u(z) \\
u^{\prime}(z)
\end{array}\right)=\pi \frac{\sqrt{k}}{\eta_{0}}\left(\begin{array}{cc}
-I_{0} & Y_{0} \\
\frac{\sqrt{k} I_{1}}{\sqrt{1+\eta_{0} z}} & \frac{\sqrt{k} Y_{1}}{\sqrt{1+\eta_{0} z}}
\end{array}\right)\left(\begin{array}{cc}
Y_{10} & \frac{Y_{00}}{\sqrt{k}} \\
I_{10} & \frac{I_{00}}{\sqrt{k}}
\end{array}\right)\left(\begin{array}{c}
u_{0} \\
u_{0}^{\prime}
\end{array}\right) .
$$


Here we defined $Z_{\mathrm{i}}=Z_{\mathrm{i}}\left(\frac{2 \beta}{\eta_{0}} \sqrt{k\left(1+\eta_{0} z\right)}\right)$ and $Z_{\mathrm{i} 0}=Z_{\mathrm{i} 0}\left(\frac{2 \beta}{\eta_{0}} \sqrt{k}\right)$ where $Z_{\mathrm{i}}$ stands for either of the Bessel's functions $I_{\mathrm{i}}$ or $Y_{\mathrm{i}}$ and $i=0,1$.

\section{Transformation Matrices}

The transformation matrix for a drift space can be obtained from (10.131) by letting $k \rightarrow 0$, but it is much easier to just integrate (10.127) directly with $k=0$. We get from (10.127) $\frac{u^{\prime \prime}}{u^{\prime}}=-\frac{\eta_{0}}{1+\eta_{0} z}$, and after logarithmic integration $u^{\prime}=\frac{1}{1+\eta_{0} z}+$ const. After still another integration

$$
u=u_{0}+\frac{u_{0}^{\prime}}{\eta_{0}} \log \left(1+\eta_{0} z\right)
$$

or for a drift space of length $L$

$$
\left(\begin{array}{c}
u(L) \\
u^{\prime}(L)
\end{array}\right)=\left(\begin{array}{cc}
1 & \frac{1}{\eta_{0}} \log \left(1+\eta_{0} L\right) \\
0 & \frac{1}{1+\eta_{0} L}
\end{array}\right)\left(\begin{array}{l}
u_{0} \\
u_{0}^{\prime}
\end{array}\right) .
$$

For most practical purposes we may assume that $2 \frac{\sqrt{k}}{\eta_{0}} \gg 1$ and may, therefore, use asymptotic expressions for the Bessel's functions. In this approximation the transformation matrix of a focusing quadrupole of length $\ell$ is

$$
\begin{aligned}
& \mathcal{M}_{\mathrm{f}}=\left(\begin{array}{cr}
\sigma \cos \Delta \xi & \frac{\sigma}{\sqrt{k}} \sin \Delta \xi \\
-\sigma^{3} \sqrt{k} \sin \Delta \xi & \sigma^{3} \cos \Delta \xi
\end{array}\right) \\
& +\left(\begin{array}{cc}
\frac{\sigma}{8}\left(\frac{3}{\xi_{0}}+\frac{1}{2}\right) \sin \Delta \xi & \frac{\sigma}{8 \sqrt{k}} \frac{\Delta \xi}{\xi_{0} \xi \ell} \cos \Delta \xi \\
\frac{3 \sigma^{3}}{8} \frac{\Delta \xi}{\xi_{0} \xi_{\ell}} \sqrt{k} \cos \Delta \xi & -\frac{\sigma^{3}}{8}\left(\frac{1}{\xi_{0}}+\frac{3}{2}\right) \sin \Delta \xi
\end{array}\right),
\end{aligned}
$$

where

$$
\sigma^{4}=\frac{1}{1+\eta_{0} \ell}
$$

and with $\Delta \xi=\xi_{\ell}-\xi_{0}$,

$$
\begin{aligned}
& \xi_{0}=\frac{2}{\eta_{0}} \sqrt{k_{0}} \quad \text { and } \\
& \xi_{\ell}=\frac{2}{\eta_{0}} \sqrt{k\left(1+\eta_{0} \ell\right)} .
\end{aligned}
$$


Similarly we get for a defocusing quadrupole

$$
\begin{aligned}
\mathcal{M}_{\mathrm{d}}=\left(\begin{array}{cc}
\sigma \cosh \Delta \xi & \frac{\sigma}{\sqrt{k}} \sinh \Delta \xi \\
-\sigma^{3} \sqrt{k} \sinh \Delta \xi & \sigma^{3} \cosh \Delta \xi
\end{array}\right) \\
+\left(\begin{array}{cc}
\frac{\sigma}{8}\left(\frac{3}{\xi_{0}}+\frac{1}{2}\right) \sinh \Delta \xi & \frac{\sigma}{8 \sqrt{k}} \frac{\Delta \xi}{\xi_{0} \xi_{\ell}} \cosh \Delta \xi \\
\frac{3 \sigma^{3}}{8} \frac{\Delta \xi}{\xi_{0} \xi_{\ell}} \sqrt{k} \cosh \Delta \xi & -\frac{\sigma^{3}}{8}\left(\frac{1}{\xi_{0}}+\frac{3}{2}\right) \sinh \Delta \xi
\end{array}\right),
\end{aligned}
$$

These transformation matrices can be further simplified for low accelerating fields noting that $\frac{\eta_{0} \ell}{4} \ll 1$. In this case $\xi_{\ell}-\xi_{0} \approx \sqrt{k} \ell=\psi$ and with

$$
\Delta=\frac{1}{8}\left(\frac{3}{\xi_{0}}+\frac{1}{\xi_{\ell}}\right) \approx \frac{1}{8}\left(\frac{3}{\xi_{\ell}}+\frac{1}{\xi_{0}}\right)
$$

we get for a focusing quadrupole the approximate transformation matrix

$$
\begin{aligned}
\mathcal{M}_{\mathrm{f}}=\left(\begin{array}{cc}
\sigma & 0 \\
0 & \sigma^{3}
\end{array}\right)[ & \left(\begin{array}{cc}
\cos \psi & \frac{1}{\sqrt{k}} \sin \psi \\
-\sqrt{k} \sin \psi & \cos \psi
\end{array}\right) \\
& \left.+\left(\begin{array}{cc}
\Delta \sin \psi & 0 \\
0 & -\Delta \sin \psi
\end{array}\right)\right] .
\end{aligned}
$$

and similar for a defocusing quadrupole

$$
\begin{aligned}
\mathcal{M}_{\mathrm{d}}=\left(\begin{array}{cc}
\sigma & 0 \\
0 & \sigma^{3}
\end{array}\right)[ & \left(\begin{array}{cc}
\cosh \psi & \frac{1}{\sqrt{k_{0}}} \sinh \psi \\
-\sqrt{k_{0}} \sinh \psi & \cosh \psi
\end{array}\right) \\
& \left.+\left(\begin{array}{cc}
\Delta \sinh \psi & 0 \\
0 & -\Delta \sinh \psi
\end{array}\right)\right] .
\end{aligned}
$$

Finally, the transformation matrix for a drift space of length $L$ in an accelerating system can be derived from either (10.140) or (10.141) by letting $k \rightarrow 0$ for

$$
\mathcal{M}_{0}=\left(\begin{array}{cc}
1 & -\frac{1}{\eta_{0}} \log \sigma^{4} \\
0 & \sigma^{4}
\end{array}\right)
$$

where $\sigma^{4}=1 /\left(1+\eta_{0} L\right)$ in agreement with (10.122). In the limit of vanishing accelerating fields $\eta_{0} \rightarrow 0$ and we obtain back the well-known transformation matrices for a drift space. Similarly, we may test (10.140) and (10.141) for consistency with regular transformation matrices.

In Eqs. (10.140)-(10.142) we have the transformation matrices for all elements to form a FODO channel in the presence of acceleration. We may now apply all formalisms used to derive periodic betatron, dispersion functions or beam envelopes 
as derived in Sect. 10.1 for regular FODO cells. Considering one half cell we note that the quadrupole strength $k$ of the first half quadrupole is determined by the last half quadrupole of the previous FODO half cell. We have therefore two variables left, the half cell drift length $L$ and the strength $k_{1}$ of the second half quadrupole of the FODO half cell, to fit the lattice functions to a symmetric solution by requiring that $\alpha_{x}=0$ and $\alpha_{y}=0$.

\section{Adiabatic Damping}

Transformation matrices derived in this section are not phase space conserving because their determinant is no more equal to unity. The determinant for a drift space with acceleration is, for example,

$$
\operatorname{det} \mathcal{M}_{0}=\sigma^{4}=\frac{1}{1+\eta_{0} z}
$$

which is different from unity if there is a finite acceleration. The two-dimensional $\left(x, x^{\prime}\right)$-phase space, for example, is not invariant anymore. For example, the area of a rhombus in phase space, defined by the two vectors $\boldsymbol{x}_{0}=(x, 0)$ and $\boldsymbol{x}_{0}^{\prime}=\left(0, x_{0}^{\prime}\right)$, is reduced according to $(10.143)$ to

$$
\left|\boldsymbol{x}, \boldsymbol{x}^{\prime}\right|=\frac{1}{1+\eta_{0} z}\left|\boldsymbol{x}_{0}, \boldsymbol{x}_{0}^{\prime}\right|
$$

and the beam emittance, defined by $\boldsymbol{x}$ and $\boldsymbol{x}^{\prime}$, is therefore not preserved in the presence of accelerating fields. This phenomenon is known as adiabatic damping under which the beam emittance varies like

$$
\epsilon=\frac{1}{1+\eta_{0} z} \epsilon_{0}=\frac{p_{0}}{p} \epsilon_{0} .
$$

where $\eta_{0} \Delta z=\Delta E / E_{0}$ is the relative energy gain along the length $\Delta z$ of the accelerator. From this we see immediately that the normalized phase space area $c p \epsilon$ is conserved in full agreement with Liouville's theorem. In beam transport systems where the particle energy is changing it is therefore more convenient and dynamically correct to use the truly invariant normalized beam emittance defined by

$$
\epsilon_{\mathrm{n}}=\beta \gamma \epsilon
$$

This normalized emittance remains constant even when the particle energy is changing due to external electric fields. In the presence of dissipating processes like synchrotron radiation, scattering or damping, however, even the normalized beam emittance changes because Liouville's theorem of the conservation of phase space is not valid anymore. 
From (10.144) we obtain formally the constancy of the normalized beam emittance by multiplying with the momenta $p_{0}$ and $p=p_{0}\left(1+\eta_{0} z\right)$ for

$$
\left|\boldsymbol{x},\left(1+\eta_{0} z\right) p_{0} \boldsymbol{x}^{\prime}\right|=\left|\boldsymbol{x}_{0}, p_{0} \boldsymbol{x}_{\mathbf{0}}{ }^{\prime}\right|
$$

or with the transverse momenta $p_{0} \boldsymbol{x}^{\prime}=\boldsymbol{p}_{0 x}$ and $\left(1+\eta_{0} z\right) p_{0} \boldsymbol{x}^{\prime}=\boldsymbol{p}_{x}$

$$
\left|\boldsymbol{x}, \boldsymbol{p}_{x}\right|=\left|\boldsymbol{x}_{0}, \boldsymbol{p}_{0 x}\right|=\text { const. }
$$

This can be generalized to a six-dimensional phase space, remembering that in this case $\operatorname{det}\left(\mathcal{M}_{0}\right)=\left(\frac{1}{1+\eta_{0} z}\right)^{3}$ since the matrix has the form $\left(\begin{array}{cc}1 & -\frac{1}{\eta_{0}} \log \sigma^{4} \\ 0 & \sigma^{4}\end{array}\right)$

$$
\mathcal{M}_{0}=\left(\begin{array}{cccccc}
1-\frac{4}{\eta_{0}} \log \sigma^{4} & 0 & 0 & 0 & 0 \\
0 & \sigma^{4} & 0 & 0 & 0 & 0 \\
0 & 0 & 1 & -\frac{4}{\eta_{0}} \log \sigma^{4} & 0 & 0 \\
0 & 0 & 0 & \sigma^{4} & 0 & 0 \\
0 & 0 & 0 & 0 & 1 & A \\
0 & 0 & 0 & 0 & 0 & \sigma^{4}
\end{array}\right),
$$

where $A$ is an $\mathrm{rf}$ related quantity irrelevant for our present arguments. For the six-dimensional phase space with coordinates $\boldsymbol{x}, \boldsymbol{p}_{x}, \boldsymbol{y}, \boldsymbol{p}_{y}, \boldsymbol{\tau}, \boldsymbol{\Delta E}$, where $\boldsymbol{p}_{x}, \boldsymbol{p}_{y}$ are the transverse momenta, $\tau$ the longitudinal position of the particle with respect to a reference particle and $\Delta \boldsymbol{E}$ the energy deviation we get finally with $\left|\boldsymbol{x}_{0}, \boldsymbol{p}_{0 x}, \boldsymbol{y}_{0}, \boldsymbol{p}_{0 y}, \boldsymbol{\tau}_{0}, \Delta \boldsymbol{E}_{0}\right|=\left(\begin{array}{cccccc}x_{0} & 0 & 0 & 0 & 0 & 0 \\ 0 & p_{0 x} & 0 & 0 & 0 & 0 \\ 0 & 0 & y_{0} & 0 & 0 & 0 \\ 0 & 0 & 0 & p_{0 y} & 0 & 0 \\ 0 & 0 & 0 & 0 & \tau_{0} & 0 \\ 0 & 0 & 0 & 0 & 0 & \sigma^{4}\end{array}\right)$

$$
\left|\boldsymbol{x}, \boldsymbol{p}_{x}, \boldsymbol{y}, \boldsymbol{p}_{y}, \boldsymbol{\tau}, \Delta E\right|=\left|\boldsymbol{x}_{0}, \boldsymbol{p}_{0 x}, \boldsymbol{y}_{0}, \boldsymbol{p}_{0 y}, \boldsymbol{\tau}_{0}, \Delta \boldsymbol{E}_{0}\right|=\mathrm{const} .
$$

These results do not change if we had included focusing in the transformation matrix. From (10.140), (10.141), we see immediately that the determinants for both matrices are

$$
\operatorname{det}\left(\mathcal{M}_{\mathrm{f}}\right) \approx \operatorname{det}\left(\mathcal{M}_{\mathrm{d}}\right) \approx \sigma^{4}
$$

ignoring small terms proportional to $\Delta$. 


\section{Problems}

\section{Use thin lens approximation unless otherwise noted.}

10.1 (S). Produce a conceptual design for a separated function proton synchrotron to be used to accelerate protons from a kinetic energy of $10-150 \mathrm{GeV} / \mathrm{c}$. The circular vacuum chamber aperture has a radius of $R=20 \mathrm{~mm}$ and is supposed to accommodate a beam with a uniform beam emittance of $\epsilon=5 \mathrm{~mm}$ mrad in both planes and a uniform momentum spread of $\sigma_{E} / E= \pm 0.1 \%$. The peak magnetic bending field is $B=1.8 \mathrm{~T}$ at $150 \mathrm{GeV} / \mathrm{c}$.

10.2 (S). Specify a FODO cell to be used as the basic lattice unit for a $50 \mathrm{GeV}$ synchrotron or storage ring. The quadrupole aperture for the beam shall have a radius of $R=3 \mathrm{~cm}$. Adjust parameters such that a Gaussian beam with an emittance of $\epsilon=5 \mathrm{~mm}$ mrad in the horizontal plane, of $\epsilon=0.5 \mathrm{~mm}$ mrad in the vertical plane and an energy spread of $\Delta E / E_{0}=0.01$ would fit within the quadrupole aperture. Ignore wall thickness of the vacuum chamber.

(a) Considering the magnetic field limitations of conventional magnets, adjust bending radius, focal length and if necessary cell length to stay within realistic limits for conventional magnets.

(b) What is the dipole field and the pole tip field of the quadrupoles? Adjust the total number of cells such that there is an even number of FODO cells and the tunes are far away from an integer or half integer resonance?

10.3 (S). Consider a ring composed of an even number $2 n_{\mathrm{c}}$ of FODO cells. To provide two component free spaces, we cut the ring at a symmetry line through the middle of two quadrupoles on opposite sides of the ring and insert a drift space of length $2 \ell$ which is assumed to be much shorter than the value of the betatron function at this symmetry point $\ell \ll \beta_{0}$. Derive the transformation matrix for this ring and compare with that of the unperturbed ring. What is the tune change of the accelerator. The betatron functions will be modified. Derive the new value of the horizontal betatron function at the symmetry point in units of the unperturbed betatron function. Is there a difference to whether the free section is inserted in the middle of a focusing or defocusing quadrupole? How does the $\eta$-function change?

10.4 (S). Sometimes two FODO channels of different parameters must be matched. Show that a lattice section can be designed with a phase advance of $\Delta \psi_{x}=\Delta \psi_{y}=$ $\pi / 2$, which will provide the desired matching of the betatron functions from the symmetry point of one FODO cell to the symmetry point of the other cells. Such a matching section is also called a quarter wavelength transformer and is applicable to any matching of symmetry points. Does this transformer also work for curved FODO channels, where the dispersion is finite?

10.5 (S). The quadrupole lattice of the synchrotron in Fig. 10.11 forms a pure FODO lattice. Yet the horizontal betatron function shows some beating perturbation while the vertical betatron function is periodic. What is the source of perturbation 
for the horizontal betatron function? An even stronger perturbation is apparent for the dispersion function. Explain why the dispersion function is perturbed.

10.6. For one example determine the real quadrupole length required to produce the quoted betatron phase advances per FODO cell in Table 10.1. Compare with thin lens quadrupole strengths.

10.7. Calculate the values of the betatron functions in the center of the quadrupoles for $\sharp 1$ and $\sharp 2$ FODO cells in Table 10.1 and compare with the actual thick lens betatron functions in Figs. 10.6 and 10.7. Discuss the difference.

10.8. The original lattice of Problem 10.4 is to be expanded to include dispersion free cells. Incorporate into the lattice two symmetric dispersion suppressors based on the FODO lattice of the ring following the scheme shown in Fig. 10.14. Adjust the bending magnet strength to retain a total bending angle of $2 \pi$ in the ring. Incorporate the two dispersion suppressors symmetrically into the ring and make a schematic sketch of the lattice.

10.9. In the dispersion free region of Problem 10.8 introduce a symmetric Collins insertion to provide a long magnet free section of the ring. Determine the parameters of the insertion magnets and drift spaces. Use thin lens approximation to calculate a few values of the betatron functions in the Collins insertions and plot betatron and dispersion functions through the Collins insertion.

10.10. For the complete ring lattice of Problem 10.9 make a parameter list including such parameters as circumference, revolution time, number of cells, tunes (use simple numerical integration to calculate the phase advance in the Collins insertion), max. beam sizes, magnet types, length, strengths, etc.

10.11. The fact that a Collins straight section can be inserted into any transport line without creating perturbations outside the insertion makes these insertions also a periodic lattice. A series of Collins straight sections can be considered as a periodic lattice composed of quadrupole doublets and long drift spaces in between. Construct a circular accelerator by inserting bending magnets into the drift spaces $d$ and adjusting the drift spaces to $D=5 \mathrm{~m}$. What is the phase advance per period? Calculate the periodic $\eta$-function and make a sketch with lattice and lattice functions for one period.

10.12. Consider a regular FODO lattice as shown in Fig. 10.11, where some bending magnets are eliminated to provide magnet free spaces and to reduce the $\eta$-function in the straight section. How does the minimum value of the $\eta$-function scale with the phase per FODO cell. Show if conditions exist to match the $\eta$-function perfectly in the straight section of this lattice?

10.13. How many protons would produce a circulating beam of $1 \mathrm{~A}$ in the ring of Problem 10.1? Calculate the total power stored in that beam at $150 \mathrm{GeV} / \mathrm{c}$. By how many degrees could one liter of water be heated up by this energy? The proton beam emittance be $\epsilon_{x, y}=5 \mathrm{~mm} \mathrm{mrad}$ at the injection energy of $10 \mathrm{GeV} / \mathrm{c}$. Calculate the average beam width at $150 \mathrm{GeV} / \mathrm{c}$ along the lattice and assume this beam to hit 
because of a sudden miss-steering a straight piece of vacuum chamber at an angle of $10 \mathrm{mrad}$. If all available beam energy is absorbed in a $1 \mathrm{~mm}$ thick steel vacuum chamber by how much will the strip of steel heat up? Will it melt? (specific heat $c_{\mathrm{Fe}}=0.11 \mathrm{cal} / \mathrm{g} /{ }^{\circ} \mathrm{C}$, melting temperature $T_{\mathrm{Fe}}=1528^{\circ} \mathrm{C}$.

\section{References}

1. E.D. Courant, H.S. Snyder, Appl. Phys. 3, 1 (1959)

2. G. Hemmie, Die zukunft des synchrotrons (desy ii). Technical Report DESY M-82-18, DESY, DESY, Hamburg (1982)

3. J. Rossbach, F. Willeke, Desy-ii optical design of a new 10 gev electron positron synchrotron. Technical Report DESY M-83-03, DESY, DESY, Hamburg (1983)

4. Slac linear collider, conceptual design report. Technical Report SLAC-229, SLAC, Stanford, CA (1981)

5. S. Kheifets, T. Figuth, K.L. Brown, A.W. Chao, J.J. Murray, R.V. Servranckx, H. Wiedemann, G.E. Fischer, in 13th International Conference on High Energy Accelerators, Novosibirsk, USSR (1986)

6. G.E. Fischer, W. Davis-White, T. Figuth, H. Wiedemann, in 12th International Conference on High Energy Accelerators (Fermilab, Chicago, IL, 1983)

7. L. Emery A wiggler-based ultra-low-emittance damping ring lattice and its chromatic correction. Ph.D. thesis, Stanford University, Stanford, CA (1990)

8. J.J. Stoker, Nonlinear Vibrations (Interscience, New York, 1950)

9. H. Wiedemann, M. Baltay, J. Voss, K. Zuo, C. Chavis, R. Hettel, J. Sebek, H.D. Nuhn, J. Safranek, L. Emery, M. Horton, J. Weaver, J. Haydon, T. Hostetler, R.Ortiz, M. Borland, S. Baird, W. Lavender, P. Kung, J. Mello, W. Li, H. Morales, L. Baritchi, P. Golceff, T. Sanchez, R. Boyce, J. Cverino, D. Mostowfi, D.F. Wang, D. Baritchi, G. Johnson, C. Wermelskirchen, B. Youngman, C. Jach, J. Yang, R. Yotam, in Proceedings of 1991 IEEE Particle Accelerator Conference, San Francisco. IEEE Cat. No. 91CH3038-7, p. 801 (1991)

10. H.G. Hereward, K. Johnsen, P. Lapostolle, in Proceedings of CERN Symposium on High Energy Physics, CERN, p. 179 (1956)

11. A.J. Lichtenberg, K.W. Robinson, Technical Report CEA 13, Harvard University-CEA, Cambridge, MA (1956)

12. K.L. Brown, D.C. Carey, CH. Iselin, F. Rothacker, Technical Report SLAC-75, CERN 73-16, SLAC-91, CERN-80-4, CERN,FNAL,SLAC (1972)

13. F.C. Iselin, J. Niederer, Technical Report CERN/LEP-TH/88-38, CERN, CERN, Geneva (1988)

14. G.E. Fischer, W. Davis-White, T. Figuth, H. Wiedemann, in Proceedings of 12th International Conference on High Energy Accelerator (Fermilab, Chicago, 1983)

15. E. Keil, Theoretical Aspects of the Behaviour of Beams in Accelerators and Storage Rings, vol. CERN 77-13 (CERN, Geneva, 1986), p. 29

16. M. Bassetti, M. Preger, Technical Report Int. Note T-14, Laboratori Nationali di Frascati, Frascati, Italy (1972)

17. M. Lee, P. Morton, J. Rees, B. Richter, Technical Report, Stanford Linear Accelerator Center (1971)

18. T.L. Collins, Technical Report CEA 86, Harvard University-CEA, Cambridge, MA (1961)

19. K. Robinson, G.A. Voss, Technical Report CEAL-1029, Harvard University, Cambridge, USA (1966)

20. K. Robinson, G.A. Voss, in Proceedings of International Symposium on Electron and Positron Storage Rings, Paris, France (Presses Universitaires de France, 1966), p. III-4 
21. Summary of the preliminary design of beijing 2.2/2.8 gev electron positron collider (bepc). Technical Report, IHEP Academica Sinica, Beijing, PRC (1982)

22. R. Helm, Technical Report SLAC-2, SLAC, Stanford, CA (1962)

23. H. Wiedemann, Strong focusing in linear accelerators. Technical Report DESY Report 68/5, DESY, DESY, Hamburg (1968) 


\section{Part IV \\ Beam Parameters}




\section{Chapter 11 \\ Particle Beam Parameters}

Particle beams are characterized by a set of quantifying parameters being either constants of motion or functions varying from point to point along a beam transport line. The parameters may be a single particle property like the betatron function which is the same for all particles within a beam or quantities that are defined only for a collection of particles like beam sizes or beam intensity. We will define and derive expressions for such beam parameters and use them to characterize particle beams and develop methods for manipulation of such parameters.

\subsection{Definition of Beam Parameters}

Particle beams and individual particles are characterized by a number of parameters which we use in beam dynamics. We will define such parameters first before we discuss the determination of their numerical value.

\subsubsection{Beam Energy}

Often we refer to the energy of a particle beam although we actually describe only the nominal energy of a single particle within this beam. Similarly, we speak of the beam momentum, beam kinetic energy or the velocity of the beam, when we mean to say that the beam is composed of particles with nominal values of these quantities. We found in earlier chapters that the most convenient quantity to characterize the "energy" of a particle is the momentum for transverse beam dynamics and the kinetic energy for acceleration. To unify the nomenclature it has become common to use the term energy for both quantities noting that the quantity of pure momentum should be multiplied with the velocity of light $(c p)$ to become

This chapter has been made Open Access under a CC BY 4.0 license. For details on rights and licenses please read the Correction https://doi.org/10.1007/978-3-319-18317-6_28 
dimensionally correct. Thus, the particle momentum is expressed in the dimension of an energy without being numerically identical either to the total energy or the kinetic energy but approaching both for highly relativistic energies.

\subsubsection{Time Structure}

A true collective beam parameter is the time structure of the particle stream. We make the distinction between a continuous beam being a continuous flow of particles and a bunched beam. Whenever particles are accelerated by means of rf-fields a bunched beam is generated, while continuous beams can in general be sustained only by dc accelerating fields or when no acceleration is required as may be true for a proton beam in a storage ring. A pulsed beam consists of a finite number of bunches or a continuous stream of particles for a finite length of time. For example, a beam pulse from a linear accelerator is made up of a finite string of micro bunches generated by rf-accelerating fields.

\subsubsection{Beam Current}

The beam intensity or beam current is expressed in terms of an electrical current using the common definition of the ratio of the electrical charge passing by a current monitor per unit time. For bunched beams the time span during which the charge is measured can be either shorter than the duration of the bunch or the beam pulse or may be long compared to both. Depending on which time scale we use, we define the bunch current or peak current, the pulse current or the average current respectively.

In Fig. 11.1 the general time structure of bunched beams is shown. The smallest unit is the microbunch, which is separated from the next microbunch by the wavelength of the accelerating rf-field or a multiple thereof. The microbunch current or peak current $\hat{I}$ is defined as the total microbunch charge $q$ divided by the microbunch duration $\tau_{\mu}$,

$$
\hat{I}=\frac{q}{\tau_{\mu}} .
$$

The micro pulse duration must be specially defined to take a nonuniform charge distribution of the particular accelerator into account. A series of microbunches form a beam pulse which is generally determined by the duration of the rf-pulse. In a conventional S-band electron linear accelerator the rf-pulse duration is of the order of a few micro seconds while a superconducting linac can produce a continuous stream of microbunches thus eliminating the pulse structure of the beam. An electrostatic accelerator may produce pulsed beams if the accelerating voltage is applied only for short time intervals. The pulse current $I_{\mathrm{p}}$ is defined as the average 
a)

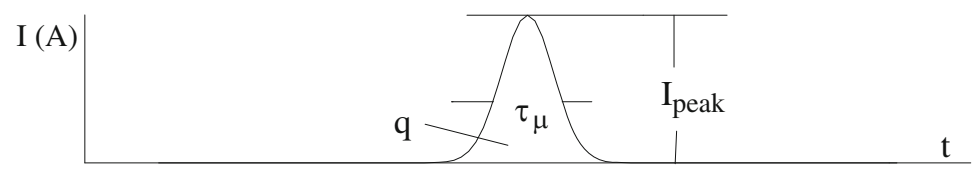

b)

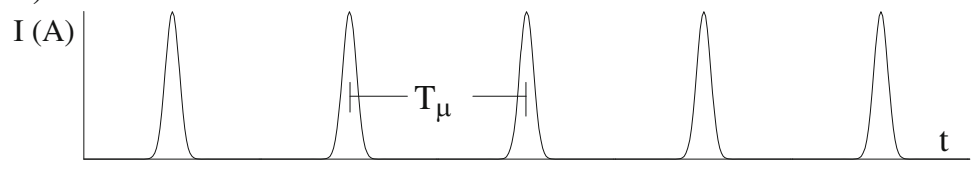

c)

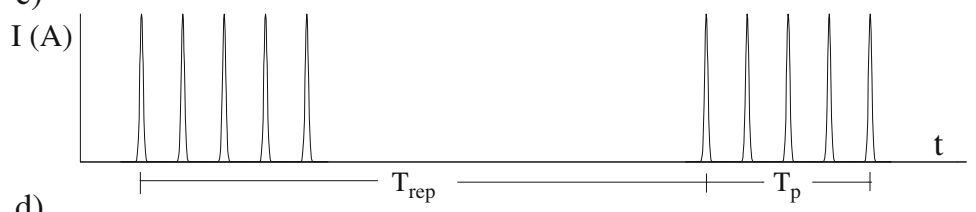

d)

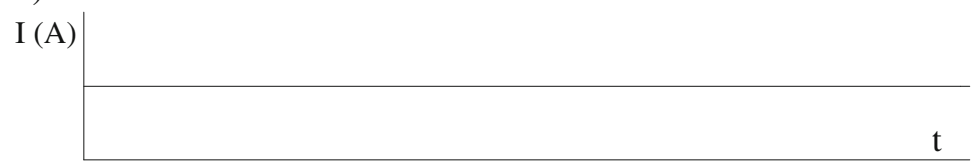

Fig. 11.1 Definitions for time structure and pulse currents. (a) Peak current, $\hat{I}=q / \tau_{\mu}$, where $\tau_{\mu}$ is the microbunch duration and $q$ the charge per microbunch. (b) Pulse current $I_{\mathrm{p}}=\hat{I} \tau_{\mu} / T_{\mu}=q / T_{\mu}$, where $T_{\mu}$ is the micro-bunch period. (c) Average current $\langle I\rangle=I_{\mathrm{p}} T_{\mathrm{p}} v_{\text {rep }}$, with $T_{\mathrm{p}}$ the pulse duration and $v_{\text {rep }}$ the pulse repetition rate. (d) Continuous beam current

current during the duration of the pulse. If the duration of the micro bunch is $\tau_{\mu}$ and the time between successive microbunches $T_{\mu}$ the pulse current is

$$
I_{\mathrm{p}}=\hat{I} \frac{\tau_{\mu}}{T_{\mu}}=\frac{q}{T_{\mu}} .
$$

The average beam current, finally, is the beam current averaged over a complete cycle of the particular accelerator.

$$
\langle I\rangle=I_{\mathrm{p}} \frac{T_{\mathrm{p}}}{T_{\mathrm{r}}}=\frac{q}{T_{\mathrm{r}}} \frac{T_{\mathrm{p}}}{T_{\mu}}=\frac{n_{\mu} q}{T_{\text {rep }}},
$$

where $n_{\mu}$ is the number of microbunches per pulse and $q$ the charge in a microbunch. In a beam transport line, this is the total charge passing by per unit time, where the unit time is as long as the distance between beam pulses. In a circular accelerator it is, for example, the total circulating charge divided by the revolution time. For the experimenter using particles from a cycling synchrotron accelerator the average current is the total charge delivered to the experiment during a time long compared to the cycling time divided by that time. 
The "beam on-beam off" time is measured by the duty factor defined as the fraction of actual beam time to total time at the experimental station. Depending on the application, it is desirable to have a high duty factor where the particles come more uniformly distributed in time compared to a low duty factor where the same number of particles come in short bursts.

\subsubsection{Beam Dimensions}

Of great importance for the design of particle accelerators is the knowledge of beam size parameters like transverse dimensions, bunch length and energy spread as well as the particle intensity distribution in six-dimensional phase space. In this respect, electron beams may behave different from beams of heavier particles like protons which is a consequence of synchrotron radiation and effects of quantized emission of photons on the dynamic parameters of the electrons. Where such radiation effects are negligible beams of any kind of particles evolve the same way along a beam line. Specifically, we have seen that in such cases the beam emittances are a constant of motion and the beam sizes are therefore modulated only by the variation of the betatron and dispersion functions as determined by the focusing structure. The particle distribution stays constant while rotating in phase space. This is true for the transverse as well as for the longitudinal and energy parameters.

A linear variation of beam emittance with energy is introduced when particles are accelerated or decelerated. We call this variation adiabatic damping, where the beam emittance scales inversely proportional with the particle momentum and the transverse beam size, divergence, bunch length and energy spread scale inversely to the square root of the particle momentum. This adiabatic damping actually is not a true damping process where the area in phase space is reduced. It rather reflects the particular definition of beam emittance with respect to the canonical dimensions of phase space. In transverse beam dynamics we are concerned with geometric parameters and a phase space element would be expressed by the product $\Delta u \Delta u^{\prime}$. Liouville's theorem, however, requires the use of canonical variables, momentum and position, and the same phase space element is $\Delta u \Delta p_{u}$, where $\Delta p_{u}=p_{0} u^{\prime}$ and $u$ is any of the three degrees of freedom. Acceleration increases the particle momentum $p_{0}$ and as a consequence the geometric emittance $\Delta u \Delta u^{\prime}$ must be reduced to keep the product $\Delta u \Delta p_{u}$ constant. This reduction of the geometric emittance by acceleration is called adiabatic damping and occurs in all three degrees of freedom.

More consistent with Liouville's theorem of constant phase space density is the normalized emittance defined by

$$
\epsilon_{n}=\beta \gamma \epsilon
$$


where $\gamma$ is the particle energy in units of the rest energy and $\beta=v / c$. This normalized emittance obviously has the appropriate definition to stay constant under the theorem of Liouville.

It is often difficult and not practical to define a beam emittance for the whole beam. Whenever the beam is fuzzy at the edges it may not make sense to include all particles into the definition of the beam emittance and provide expensive aperture for the fuzzy part of the beam. Relativistic electron beams in circular accelerators are particularly fuzzy due to the quantized emission of synchrotron radiation and as a consequence the particle distribution transforms into a Gaussian distribution. Later, we will discuss the evolution of the beam emittance due to statistical effects in great detail and derive the particle distribution from the Fokker-Planck equation. The electron beam emittance is defined for that part of the beam which is contained within one standard unit of the Gaussian distribution. this is true also for any other parameter which assumes a Gaussian distribution like beam size, divergence, energy spread, phase etc.

The beam emittance for particle beams is primarily defined by the characteristic source parameters and source energy. Given perfect matching between different accelerators and beam lines during subsequent acceleration, this source emittance is reduced inversely proportional to the particle momentum by adiabatic damping and stays constant in terms of normalized emittance. This describes accurately the ideal situation for proton and ion beams, for nonrelativistic electrons and electrons in linear accelerators as long as statistical effects are absent. A variation of the emittance occurs in the presence of statistical effects in the form of collisions with other particles or emission of synchrotron radiation and we will concentrate here in more detail on the evolution of beam emittances in highly relativistic electron beams.

Statistical processes cause a spreading of particles in phase space or a continuous increase of beam emittance. In cases where this diffusion is due to the particle density, the emittance increase may decrease significantly because the scattering occurrence drops to lower and lower values as the particle density decreases. Such a case appears in intra-beam scattering [1-3], where particles within the same bunch collide and exchange energy. Specifically when particles exchange longitudinal momentum into transverse momentum and gain back the lost longitudinal momentum from the accelerating cavities. The beam "heats" up transversely which becomes evident in the increased beam emittance and beam sizes.

Statistical perturbations due to synchrotron radiation, however, lead to truly equilibrium states where the continuous excitation due to quantized emission of photons is compensated by damping. Discussing first the effect of damping will prepare us to combine the results with statistical perturbations leading to an equilibrium state of the beam dimensions. 


\subsection{Damping}

Emission of synchrotron radiation causes the appearance of a reaction force on the emitting particle which must be taken into account to accurately describe particle dynamics. In doing so, we note from the theory of synchrotron radiation that the energy lost into synchrotron radiation is lost through the emission of many photons and we may assume that the energy loss is continuous. Specifically, we assume that single photon emissions occur fast compared to the oscillation period of the particle such that we may treat the effect of the recoil force as an impulse.

In general, we must consider the motion of a particle in all three degrees of freedom or in six-dimensional phase space. The appearance of damping stems from the emission of synchrotron radiation in general, but the physics leading to damping in the longitudinal degree of freedom is different from that in the transverse degrees of freedom. The rate of energy loss into synchrotron radiation depends on the particle energy itself being high at high energies and low at low energies. As a consequence, a particle with a higher than ideal energy will loose more energy to synchrotron radiation than the ideal particle and a particle with lower energy will loose less energy. The combined result is that the energy difference between such three particles has been reduced, an effect that shows up as damping of the beam energy spread. With the damping of the energy spread, we observe also a damping of its conjugate variable, the longitudinal phase or bunch length.

In the transverse plane we note that the emission of a photon leads to a loss of longitudinal as well as transverse momentum since the particle performs betatron oscillations. The total lost momentum is, however, replaced in the cavity only in the longitudinal direction. Consequently, the combined effect of emission of a photon and the replacement of the lost energy in accelerating cavities leads to a net loss of transverse momentum or transverse damping.

Although damping mechanisms are different for transverse and longitudinal degrees of freedom, the total amount of damping is limited and determined by the amount of synchrotron radiation. This correlation of damping decrements in all degrees of freedom was derived first by Robinson [4] for general accelerating fields as long as they are not so strong that they would appreciably affect the particle orbit.

\subsubsection{Robinson Criterion}

Following Robinson's idea we will derive what is now known as Robinson's damping criterion by observing the change of a six dimensional vector in phase space due to synchrotron radiation and acceleration. The components of this vector are the four transverse coordinates $\left(x, x^{\prime}, y, y^{\prime}\right)$, the energy deviation $\Delta E$, and the longitudinal phase deviation from the synchronous phase $\varphi=\psi-\psi_{\mathrm{s}}$. Consistent with smooth approximation a continuous distribution of synchrotron radiation along the orbit is assumed as well as continuous acceleration to compensate energy losses. 
During the short time $\mathrm{d} t$ the six-dimensional vector

$$
\boldsymbol{u}=\left(x, x^{\prime}, y, y^{\prime}, \varphi, \delta E\right)
$$

will change by an amount proportional to $\mathrm{d} t$. We may expand the transformations into a Taylor series keeping only linear terms and express the change of the phase space vector in form of a matrix transformation

$$
\Delta \boldsymbol{u}=\boldsymbol{u}_{1}-\boldsymbol{u}_{0}=\mathrm{d} t \mathcal{M} \boldsymbol{u}_{0}
$$

From the eigenvalue equation for this transformation matrix,

$$
\mathcal{M} \boldsymbol{u}_{j}=\lambda_{j} \boldsymbol{u}_{j}
$$

where $\boldsymbol{u}_{j}$ are the eigenvectors, $\lambda_{i}$ the eigenvalues being the roots of the characteristic equation $\operatorname{det}(\mathcal{M}-\lambda \mathcal{I})=0$ and $\mathcal{I}$ the unity matrix. From (11.6) we get

$$
\boldsymbol{u}_{1}=(1+\mathcal{M} \mathrm{d} t) \boldsymbol{u}_{0}=\left(1+\lambda_{j} \mathrm{~d} t\right) \boldsymbol{u}_{0} \approx \boldsymbol{u}_{0} \mathrm{e}^{\lambda_{j} \mathrm{~d} t}
$$

Since the eigenvectors must be real the eigenvalues come in conjugate complex pairs

$$
\lambda_{j}=\alpha_{i} \pm \mathrm{i} \beta_{i}
$$

where $i=1,2,3$ and

$$
\sum_{j=1}^{j=6} \lambda_{j}=2 \sum_{i=1}^{i=3} \alpha_{i}
$$

The quantities $\alpha_{i}$ cause exponential damping or excitation of the eigenvectors depending on whether they are negative or positive, while the $\beta_{i}$ contribute only a frequency shift of the oscillations.

Utilizing the transformation matrix $\mathcal{M}$, we derive expressions for the eigenvalues by evaluating the expression $\left.\frac{\mathrm{d}}{\mathrm{d} \tau} \operatorname{det}(\tau \mathcal{M}-\lambda \mathcal{I})\right|_{\tau=0}$ in two different ways. With $\mathcal{M}=$ $\lambda_{j} \mathcal{I}$ we get

$$
\frac{\mathrm{d}}{\mathrm{d} \tau} \operatorname{det}\left[\left(\tau \lambda_{i}-\lambda\right) \mathcal{I}\right]_{\tau=0}=\left.\frac{\mathrm{d}}{\mathrm{d} \tau} \prod_{j=1}^{j=6}\left(\tau \lambda_{j}-\lambda\right)\right|_{\tau=0}=-\lambda^{5} \sum_{j=1}^{j=6} \lambda_{j} .
$$


On the other hand, we may execute the differentiation on the determinant directly and get

$$
\begin{aligned}
& \left.\frac{\mathrm{d}}{\mathrm{d} \tau} \operatorname{det}(\tau \mathcal{M}-\geq \mathcal{I})\right|_{\tau=0}= \\
& \left|\begin{array}{cccc}
m_{11} & m_{12} & m_{13} & \cdots \\
\tau m_{21} & \tau m_{22}-\lambda & \tau m_{23} & \ldots \\
\tau m_{31} & \tau m_{32} & \tau m_{33}-\lambda & \ldots \\
\ldots & \cdots & \ldots & \cdots
\end{array}\right|_{\tau=0} \\
& +\left|\begin{array}{cccc}
\tau m_{11}-\lambda & \tau m_{12} & \tau m_{13} & \cdots \\
m_{21} & m_{22} & m_{23} & \cdots \\
\tau m_{31} & \tau m_{32} & \tau m_{33}-\lambda & \cdots \\
\ldots & \cdots & \cdots & \cdots
\end{array}\right|_{\tau=0}+\cdots \\
& =-\lambda^{5} m_{11} \cdots-\lambda^{5} m_{66}=-\lambda^{5} \sum_{j=1}^{j=6} m_{j j} .
\end{aligned}
$$

Comparing (11.9) and (11.10) we note with (11.8) the relation

$$
\sum_{j-1}^{j=6} \lambda_{j}=\sum_{j-1}^{j=6} m_{j j}=2 \sum_{i=1}^{i=3} \alpha_{i}
$$

between eigenvalues, matrix elements, and damping decrements. To further identify the damping we must determine the transformation. The elements $m_{11}, m_{33}$, and $m_{55}$ are all zero because the particle positions $(x, y, \varphi)$ are not changed by the emission of a photon or by acceleration during the time $\mathrm{d} t$.

$$
m_{11}=0 \quad m_{33}=0 \quad m_{55}=0 .
$$

The slopes, however, will change. Since synchrotron radiation is emitted in the forward direction we have no direct change of the particle trajectory due to the emission process. We ignore at this point the effects of a finite radiation opening angle $\theta= \pm 1 / \gamma$ and show in connection with the derivation of the vertical beam emittance that this effect is negligible while determining damping. Acceleration will change the particle direction because the longitudinal momentum is increased while the transverse momentum stays constant (see Fig. 11.2).

As shown in Fig. 11.2, a particle with a total momentum $p_{0}$ and a transverse momentum $p_{0 t}$ due to betatron oscillation emits a photon of energy $\varepsilon_{\gamma}$. This process leads to a loss of momentum of $-\Delta p=\varepsilon_{\gamma} / \beta$, where $\beta=v / c$ and a loss of transverse momentum. Acceleration will again compensate for this energy loss. During acceleration the momentum is increased by $\Delta p_{\mathrm{rf}}=+\left(P_{\mathrm{rf}} / c \beta\right) \mathrm{d} t$, where $P_{\mathrm{rf}}$ is the rf-power to the beam. The transverse momentum during this acceleration 
Fig. 11.2 Reduction of the transverse momentum of trajectories by acceleration. For simplicity we assume here that the energy loss $-\Delta \mathrm{p}$ due to the emission of a photon is immediately compensated by accelerating fields in a rf-cavity $\left(\Delta p_{\text {rf }}\right)$

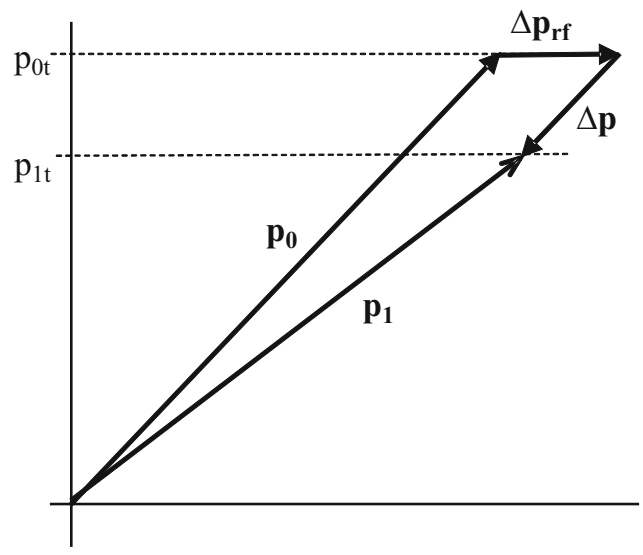

is not changed and we have therefore $\left(p_{0}-\Delta p\right) u_{0}^{\prime}=\left[p_{0}-\Delta p+\left(P_{\mathrm{rf}} / c \beta\right) \mathrm{d} t\right] u_{1}^{\prime}$, where $u_{0}^{\prime}$ and $u_{1}^{\prime}$ are the slopes of the particle trajectory before and after acceleration, respectively. With $u^{\prime}=\dot{u} / \beta c$ and $c p_{0}=\beta E_{0}$ we have to first order in $\Delta p$ and $P_{\mathrm{rf}} \mathrm{d} t$

$$
\dot{u}_{1}=\frac{E_{0}}{E_{0}+P_{\mathrm{rf}} \mathrm{d} t} \dot{u}_{0} \approx\left(1-\frac{P_{\mathrm{rf}} \mathrm{d} t}{E_{0}}\right) \dot{u}_{0} .
$$

From (11.7) we get with (11.13) using average values for the synchrotron radiation power around the ring and with $u=x$ or $y$

$$
m_{22}=-\frac{\left\langle P_{\gamma}\right\rangle}{E_{0}} \quad \text { and } \quad m_{44}=-\frac{\left\langle P_{\gamma}\right\rangle}{E_{0}}
$$

where we note that in the absence of acceleration the rf-power is equal to the nominal synchrotron radiation power $\left\langle P_{\gamma}\right\rangle=U_{0} / T_{0}$. The energy variation of the particle is the combination of energy loss $-P_{\gamma} \mathrm{d} t$ and gain $P_{\mathrm{rf}} \mathrm{d} t$. With

$$
P_{\gamma}(E)=P_{\gamma}\left(E_{0}\right)+\left.\frac{\partial P_{\gamma}}{\partial E}\right|_{0} \Delta E_{0} \quad \text { and } \quad P_{\mathrm{rf}}(\psi)=P_{\mathrm{rf}}\left(\psi_{\mathrm{s}}\right)+\left.\frac{\partial P_{\mathrm{rf}}}{\partial \psi}\right|_{\psi_{\mathrm{s}}} \varphi,
$$

where $\varphi=\psi-\psi_{\mathrm{s}}$ we get

$$
\begin{aligned}
\Delta E_{1} & =\Delta E_{0}-\left\langle P_{\gamma}(E)\right\rangle \mathrm{d} t+P_{\mathrm{rf}}(\psi) \mathrm{d} t \\
& =\Delta E_{0}-\left.\frac{\partial\left\langle P_{\gamma}\right\rangle}{\partial E}\right|_{0} \Delta E \mathrm{~d} t+\left.\frac{\partial P_{r f}}{\partial \psi}\right|_{\psi_{\mathrm{s}}} \varphi \mathrm{d} t
\end{aligned}
$$


because $P_{\gamma}\left(E_{0}\right)=P_{\mathrm{rf}}\left(\psi_{\mathrm{s}}\right)$. Equation (11.15) exhibits two more elements of the transformation matrix

$$
m_{65}=\left.\frac{\partial P_{\mathrm{rf}}}{\partial \psi}\right|_{\psi_{\mathrm{s}}} \quad \text { and } \quad m_{66}=-\left.\frac{\partial\left\langle P_{\gamma}\right\rangle}{\partial E}\right|_{0} .
$$

We have now all elements necessary to determine the damping decrements. From (11.12), (11.14), (11.16) we get the sum of the damping decrements

$$
\sum_{i=1}^{i=3} \alpha_{i}=\frac{1}{2} \sum_{j=1}^{j=6} m_{j j}=-\frac{\left\langle P_{\gamma}\right\rangle}{E_{0}}-\left.\frac{1}{2} \frac{\partial\left\langle P_{\gamma}\right\rangle}{\partial E}\right|_{0},
$$

which depends only on the synchrotron radiation power and the particle energy. This result was first derived by Robinson [4] and is known as Robinson's damping criterion.

We may separate the damping decrements. For a plane circular accelerator without vertical bending magnets and coupling, the vertical damping decrement $\alpha_{y}=\alpha_{2}$ can be extracted. Since the vertical motion is not coupled to either the horizontal or the synchrotron oscillations, we get from (11.14) and (11.17)

$$
\alpha_{y}=-\frac{1}{2} \frac{\left\langle P_{\gamma}\right\rangle}{E_{0}} \text {. }
$$

The damping decrement for synchrotron oscillations has been derived in (9.27) and is

$$
\alpha_{z}=-\left.\frac{1}{2} \frac{\mathrm{d}\left\langle P_{\gamma}\right\rangle}{\mathrm{d} E}\right|_{0} .
$$

The horizontal damping decrement finally can be derived from Robinson's damping criterion (11.17) and the two known decrements (11.18), (11.19) to be

$$
\alpha_{x}=-\frac{1}{2} \frac{\left\langle P_{\gamma}\right\rangle}{E_{0}}-\left.\frac{1}{2} \frac{\partial\left\langle P_{\gamma}\right\rangle}{\partial E}\right|_{0}+\left.\frac{1}{2} \frac{\mathrm{d}\left\langle P_{\gamma}\right\rangle}{\mathrm{d} E}\right|_{0}
$$

We may further evaluate the total and partial differential of the synchrotron radiation power $P_{\gamma}$ with energy $E$. The synchrotron radiation power is proportional to the square of the particle energy $E$ and magnetic field $B$ at the source of radiation and the partial differential is therefore

$$
\left.\frac{\partial P_{\gamma}}{\partial E}\right|_{0}=2 \frac{\left\langle P_{\gamma}\right\rangle}{E_{0}}
$$


The total differential of the synchrotron radiation power depends not only on the particle energy directly but also on the variation of the magnetic field with energy as seen by the particle. A change in the particle energy causes a shift in the particle orbit where the $\eta$-function is nonzero and this shift may move the particle to a location with different field strength. To include all energy dependent contributions, we inspect the definition of the average synchrotron radiation power $\left\langle P_{\gamma}\right\rangle=\frac{1}{T_{0}} \oint P_{\gamma} \mathrm{d} \tau$ and noting that for highly relativistic particles $c \mathrm{~d} \tau=\mathrm{d} z=\left(1+\frac{\eta}{\rho} \frac{\Delta E}{E_{0}}\right) \mathrm{d} z$ the average radiation power becomes

$$
\left\langle P_{\gamma}\right\rangle=\frac{1}{c T_{0}} \oint P_{\gamma}\left(1+\frac{\eta}{\rho} \frac{\Delta E}{E_{0}}\right) \mathrm{d} z .
$$

Differentiating (11.22) with respect to the energy

$$
\left.\frac{\mathrm{d}\left\langle P_{\gamma}\right\rangle}{\mathrm{d} E}\right|_{0}=\frac{1}{c T_{0}} \oint\left[\left.\frac{\mathrm{d} P_{\gamma}}{\mathrm{d} E}\right|_{0}+P_{\gamma} \frac{\eta}{\rho E_{0}}\right] \mathrm{d} z,
$$

where

$$
\left.\frac{\mathrm{d} P_{\gamma}}{\mathrm{d} E}\right|_{0}=2 \frac{P_{\gamma}}{E_{0}}+2 \frac{P_{\gamma}}{B_{0}} \frac{\mathrm{d} B}{\mathrm{~d} x} \frac{\mathrm{d} x}{\mathrm{~d} E}=2 \frac{P_{\gamma}}{E_{0}}+2 \frac{P_{\gamma}}{E_{0}} \rho k \eta .
$$

Collecting all components, the synchrotron oscillation damping decrement (11.19) is finally

$$
\alpha_{\mathrm{z}}=-\left.\frac{1}{2} \frac{\mathrm{d}\left\langle P_{\gamma}\right\rangle}{\mathrm{d} E}\right|_{0}=-\frac{1}{2} \frac{\left\langle P_{\gamma}\right\rangle}{E_{0}}(2+\vartheta)
$$

where we used $\left\langle P_{\gamma}\right\rangle \propto \oint \kappa^{2} \mathrm{~d} z$ and $P_{\gamma 0} \propto \kappa^{2}$ with $\kappa=1 / \rho$

$$
\vartheta=\frac{\oint \kappa^{3} \eta\left(1+2 \rho^{2} k\right) \mathrm{d} z}{\oint \kappa^{2} \mathrm{~d} z}
$$

Similarly, we get from (11.20) for the horizontal damping decrement

$$
\alpha_{x}=-\frac{1}{2} \frac{\left\langle P_{\gamma}\right\rangle}{E_{0}}(1-\vartheta) .
$$


In summary the damping decrements for betatron and synchrotron oscillations can be expressed by

$$
\begin{aligned}
\alpha_{z}=-\frac{1}{2} \frac{\left\langle P_{\gamma}\right\rangle}{E}(2+\vartheta) & =-\frac{1}{2} \frac{\left\langle P_{\gamma}\right\rangle}{E} J_{z}, \\
\alpha_{x}=-\frac{1}{2} \frac{\left\langle P_{\gamma}\right\rangle}{E}(1-\vartheta) & =-\frac{1}{2} \frac{\left\langle P_{\gamma}\right\rangle}{E} J_{x}, \\
\alpha_{y}=-\frac{1}{2} \frac{\left\langle P_{\gamma}\right\rangle}{E} & =-\frac{1}{2} \frac{\left\langle P_{\gamma}\right\rangle}{E} J_{y},
\end{aligned}
$$

where the factors $J_{i}$ are the damping partition numbers,

$$
\begin{aligned}
& J_{z}=2+\vartheta, \\
& J_{x}=1-\vartheta, \\
& J_{y}=1 .
\end{aligned}
$$

Robinson's damping criterion can be expressed by

$$
\sum_{i} J_{i}=4
$$

In more practical quantities, the damping decrements can be obtained with (24.35) from

$$
\alpha_{u}=-\frac{1}{3} r_{\mathrm{e}} c \gamma^{3}\left\langle\frac{1}{\rho^{2}}\right\rangle J_{u} .
$$

Damping occurs in circular electron accelerators in all degrees of freedom. In transverse planes particles oscillate in the potential created by quadrupole focusing and any finite amplitude is damped by synchrotron radiation damping. Similarly, longitudinal synchrotron oscillations are contained by a potential well created by the rf-fields and the momentum compaction and finite deviations of particles in energy and phase are damped by synchrotron radiation damping. We note that the synchrotron oscillation damping is twice as strong as transverse damping.

All oscillation amplitudes $a_{u}$ in six dimensional phase space are damped $(\alpha<0)$ or anti-damped $(\alpha>0)$ like

$$
a_{u}=a_{0 u} \mathrm{e}^{\alpha_{u} t}
$$

and the damping or rise times are

$$
\tau_{u}=\frac{1}{a_{u}}
$$


In a particular choice of lattice, damping rates can be shifted between different degrees of freedom and special care must be exercised when combined function magnets or strong sector magnets are introduced into a ring lattice.

Both the synchrotron and betatron oscillation damping can be modified by a particular choice of lattice. From (11.25) we note the contribution $\kappa^{3} \eta$ which is caused by sector magnets. Particles with higher energies follow a longer path in a sector magnet and therefore radiate more. Consequently synchrotron damping is increased with $\vartheta$. This term vanishes for rectangular magnets and must be modified appropriately for wedge magnets. For a rectangular magnet

$$
\vartheta_{\text {rect }}=\frac{\oint 2 \kappa \eta k \mathrm{~d} z}{\oint \kappa^{2} \mathrm{~d} z}
$$

and for wedge magnets

$$
\vartheta_{\text {wedge }}=\frac{\sum_{i}\left[\kappa^{2} \theta_{0} \eta_{0}+\int 2(\kappa \eta k) \mathrm{d} z+\kappa^{2} \theta_{\mathrm{e}} \eta_{\mathrm{e}}\right]_{i}}{\oint \kappa^{2} \mathrm{~d} z},
$$

where we add all contributions from all magnets $i$ in the ring. The edge angles at the entrance $\theta_{0}$ and exit $\theta_{\mathrm{e}}$ are defined to be positive going from a rectangular magnet toward a sector magnet.

The second term in the nominator of (11.25) becomes significant for combined function magnets and vanishes for separated function magnets. Specifically. a strong focusing gradient $(k>0)$ combined with beam deflection can contribute significantly to $\vartheta$. For $\vartheta=1$ all damping in the horizontal plane is lost and anti-damping or excitation of betatron oscillations appears for $\vartheta>1$. This occurs, for example, in older combined function synchrotrons. At low energies, however, the beam in such lattices is still stable due to strong adiabatic damping and only at higher energies when synchrotron radiation reduces acceleration will horizontal anti-damping take over and dictate an upper limit to the feasibility of such accelerators. Conversely, vertical focusing $(k<0)$ can be implemented into bending magnets such that the horizontal damping is actually increased since $\vartheta<0$. However, there is a limit for the stability of synchrotron oscillations for $\vartheta=2$.

\subsection{Particle Distribution in Longitudinal Phase Space}

The particle distribution in phase space is rarely uniform. To determine the required aperture in a particle transport system avoiding excessive losses we must, however, know the particle distribution. Proton and ion beams involve particle distributions which due to Liouville's theorem do not change along a beam transport system, except for the variation of the betatron and dispersion function. The particle distribution can therefore be determined by measurements of beam transmission 
through a slit for varying openings. If this is done at two points about $90^{\circ}$ apart in betatron phase space, angular as well as spatial distribution can be determined.

This procedure can be applied also to electrons in a transport system. The distribution changes, however, significantly when electrons are injected into a circular accelerator. We will discuss the physics behind this violation of Liouville's theorem and determine the resulting electron distribution in phase space.

Relativistic electron and positron beams passing through bending magnets emit synchrotron radiation, a process that leads to quantum excitation and damping. As a result the original beam emittance at the source is completely replaced by an equilibrium emittance that is unrelated to the original source characteristics. Postponing a rigorous treatment of statistical effects to Chap. 12 we concentrate here on a more visual discussion of the reaction of synchrotron radiation on particle and beam parameters.

\subsubsection{Energy Spread}

Statistical emission of photons causes primarily a change of particle energy leading to an energy spread within the beam. To evaluate the effect of quantized emission of photons on the beam energy spread, we observe particles undergoing synchrotron oscillations so that a particle with an energy deviation $A_{0}$ at time $t_{0}$ will have an energy error at time $t$ of

$$
A(t)=A_{0} \mathrm{e}^{\mathrm{i} \Omega\left(t-t_{0}\right)}
$$

Emission of a photon with energy $\varepsilon$ at time $t_{1}$ causes a perturbation and the particle continues to undergo synchrotron oscillations but with a new amplitude

$$
A_{1}=A_{0} \mathrm{e}^{\mathrm{i} \Omega\left(t-t_{0}\right)}-\varepsilon \mathrm{e}^{\mathrm{i} \Omega\left(t-t_{1}\right)}
$$

The change in oscillation amplitude due to the emission of one photon of energy $\varepsilon$ can be derived from (11.36) by multiplying with its imaginary conjugate for

$$
A_{1}^{2}=A_{0}^{2}+\varepsilon^{2}-2 \varepsilon A_{0} \cos \left[\Omega\left(t_{1}-t_{0}\right)\right] .
$$

Because the times at which photon emission occurs is random we have for the average increase in oscillation amplitude due to the emission of a photon of energy $\varepsilon$

$$
\left\langle\Delta A^{2}\right\rangle=\left\langle A_{1}^{2}-A_{0}^{2}\right\rangle=\varepsilon^{2}
$$

The rate of change in amplitude per unit time due to this statistical or quantum excitation while averaging around the ring is

$$
\left\langle\left.\frac{\mathrm{d} A^{2}}{\mathrm{~d} t}\right|_{\mathrm{q}}\right\rangle_{z}=\int_{0}^{\infty} \varepsilon^{2} \dot{n}(\varepsilon) \mathrm{d} \epsilon=\left\langle\dot{\mathcal{N}}_{\mathrm{ph}}\left\langle\varepsilon^{2}\right\rangle\right\rangle_{z}
$$


where $\dot{n}(\varepsilon)$ is the number of photons of energy $\varepsilon$ emitted per unit time and energy bin $\mathrm{d} \varepsilon$. This can be equated to the total photon flux $\dot{\mathcal{N}}_{\text {ph }}$ multiplied by the average square of the photon energy and again taking the average along the orbit.

Damping causes a reduction in the synchrotron oscillation amplitude and with $A=A_{0} \mathrm{e}^{\alpha_{s} t}$ and the synchrotron oscillation damping time $\tau_{z}=1 /\left|\alpha_{z}\right|(11.27)$

$$
\left\langle\left.\frac{\mathrm{d} A^{2}}{\mathrm{~d} t}\right|_{\mathrm{d}}\right\rangle_{z}=-\frac{2}{\tau_{z}}\left\langle A^{2}\right\rangle
$$

Both quantum excitation and damping lead to an equilibrium state

$$
\left\langle\dot{\mathcal{N}}_{\mathrm{ph}}\left\langle\varepsilon^{2}\right\rangle\right\rangle_{z}-\frac{2}{\tau_{z}}\left\langle A^{2}\right\rangle=0
$$

or solving for $\left\langle A^{2}\right\rangle$

$$
\left\langle A^{2}\right\rangle=\frac{1}{2} \tau_{z}\left\langle\dot{\mathcal{N}}_{\mathrm{ph}}\left\langle\varepsilon^{2}\right\rangle\right\rangle_{z}
$$

Due to the central limit theorem of statistics the energy distribution caused by the statistical emission of photons assumes a Gaussian distribution with the standard root mean square energy spread $\sigma_{\varepsilon}^{2}=\frac{1}{2}\left\langle A^{2}\right\rangle$. The photon spectrum will be derived in Part 22.6 and the integral in (11.39) can be evaluated to give [5]

$$
\dot{\mathcal{N}}_{\text {ph }}\left\langle\varepsilon^{2}\right\rangle=\frac{55}{24 \sqrt{3}} P_{\gamma} \varepsilon_{\mathrm{c}}
$$

Replacing the synchrotron radiation power $P_{\gamma}$ by its expression in (24.34) and the critical photon energy $\varepsilon_{\mathrm{c}}=\hbar \omega_{\mathrm{c}}$ by (24.49) we get

$$
\dot{\mathcal{N}}_{\text {ph }}\left\langle\varepsilon^{2}\right\rangle=\frac{55}{32 \pi \sqrt{3}}\left[c C_{\gamma} \hbar c\left(m c^{2}\right)^{4} \gamma^{7} \kappa^{3}\right]
$$

with $C_{\gamma}=\frac{4 \pi}{3} \frac{r_{\mathrm{e}}}{\left(\mathrm{mc}^{2}\right)^{3}}=8.8460 \times 10^{-5} \mathrm{~m} / \mathrm{GeV}^{3}$ and the equilibrium energy spreadbecomes finally with (11.27) and (24.34)

$$
\frac{\sigma_{\varepsilon}^{2}}{E^{2}}=\frac{\tau_{z}}{4 E^{2}}\left\langle\dot{\mathcal{N}}_{\mathrm{ph}}\left\langle\varepsilon^{2}\right\rangle\right\rangle_{z}=C_{\mathrm{q}} \frac{\gamma^{2}}{J_{z}} \frac{\left\langle\kappa^{3}\right\rangle_{z}}{\left\langle\kappa^{2}\right\rangle_{z}}
$$

where

$$
C_{\mathrm{q}}=\frac{55}{32 \sqrt{3}} \frac{\hbar c}{m c^{2}}=3.84 \times 10^{-13} \mathrm{~m}
$$

for electrons and positrons. The equilibrium energy spread in an electron storage ring depends only on the beam energy and the bending radius. 


\subsubsection{Bunch Length}

The conjugate coordinate to the energy deviation is the phase and a spread of particle energy appears also as a spread in phase or as a longitudinal particle distribution and an equilibrium bunch length. The bunch length is

$$
\sigma_{\ell}=\frac{c\left|\eta_{c}\right|}{\Omega} \frac{\sigma_{\varepsilon}}{E_{0}}
$$

and replacing the synchrotron oscillation frequency by its expression (9.35) we get finally for the equilibrium bunch length in a circular electron accelerator

$$
\sigma_{\ell}=\frac{\sqrt{2 \pi} c}{\omega_{\mathrm{rev}}} \sqrt{\frac{\eta_{\mathrm{c}} E_{0}}{h e \hat{V} \cos \psi_{\mathrm{s}}}} \frac{\sigma_{\varepsilon}}{E_{0}} .
$$

The equilibrium electron bunch length can be varied by varying the rf-voltage and scales like $\sigma_{\ell} \propto 1 / \sqrt{\hat{V}}$ which is a much stronger dependence than the scaling obtained for non-radiating particles in Sect. 9.3.5. A very small bunch length can be obtained by adjusting the momentum compaction to a small value including zero. As the momentum compaction approaches zero, but second order terms must be considered which has been discussed in detail in Sect.9.4.4. An electron storage ring where the momentum compaction is adjusted to be zero or close to zero is called an isochronous ring [6] or a quasi isochronous ring [7]. Such rings do not yet exist at this time but are intensely studied and problems are being solved in view of great benefits for research in high energy physics, synchrotron radiation sources and free electron lasers to produce short particle or light pulses.

\subsection{Transverse Beam Emittance}

The sudden change of particles energy due to the quantized emission of photons also causes a change in the characteristics of transverse particle motion. Neither position nor the direction of the particle trajectory is changed during the forward emission of photons. From beam dynamics, however, we know that different reference trajectories exist for particles with different energies. Two particles with energies $c p_{1}$ and $c p_{2}$ follow two different reference trajectories separated at the position $z$ along the beam transport line by a distance

$$
\Delta x(z)=\eta(z) \frac{c p_{1}-c p_{2}}{c p_{0}},
$$

where $\eta(z)$ is the dispersion function and $c p_{0}$ the reference energy. Although particles in general do not exactly follow these reference trajectories, they do 
perform betatron oscillations about these trajectories. The sudden change of the particle energy causes a sudden change in the reference path and thereby a sudden change in the betatron oscillation amplitude.

\subsubsection{Equilibrium Beam Emittance}

Postponing again a rigorous discussion of the evolution of phase space due to statistical perturbations to the next chapter, we follow here a more intuitive path to determine the equilibrium transverse beam emittance. Similar to the discussion leading to the equilibrium energy spread we will observe perturbations to the transverse motion caused by photon emission. In the case of longitudinal quantum excitation it was sufficient to consider the effect of photon emission on the particle energy alone since the particle phase is not changed by this process.

As a particle emits a photon it will not change its actual position and direction. However, the position of a particle with respect to the ideal reference orbit is the combination of its betatron oscillation amplitude and a chromatic contribution due to a finite energy deviation and dispersion. Variation of the particle position $u=$ $u_{\beta}+\eta\left(\Delta E / E_{0}\right)$, and direction $u^{\prime}=u_{\beta}^{\prime}+\eta^{\prime}\left(\Delta E / E_{0}\right)$ due $\varepsilon$ is described by

$$
\begin{aligned}
\delta u=0=\delta u_{\beta}+\eta \frac{\epsilon}{E} & \text { or } \delta u_{\beta}=-\eta \frac{\epsilon}{E}, \\
\delta u^{\prime}=0=\delta u_{\beta}^{\prime}+\eta^{\prime} \frac{\epsilon}{E} & \text { or } \delta u_{\beta}^{\prime}=-\eta^{\prime} \frac{\epsilon}{E} .
\end{aligned}
$$

We note the sudden changes in the betatron amplitudes and slopes because the sudden energy loss leads to a simultaneous change in the reference orbit. This perturbation will modify the phase ellipse the particles move on. The variation of the phase ellipse $\gamma u^{2}+2 \alpha u u^{\prime}+\beta u^{\prime 2}=a^{2}$ is expressed by

$$
\gamma \delta\left(u_{\beta}^{2}\right)+2 \alpha \delta\left(u_{\beta} u_{\beta}^{\prime}\right)+\beta \delta\left(u_{\beta}^{\prime 2}\right)=\delta\left(a^{2}\right)
$$

and inserting the relations (11.50) we get terms of the form $\delta\left(u_{\beta}^{2}\right)=\left(u_{\beta 0}+\delta u_{\beta}\right)^{2}-$ $u_{\beta 0}^{2}$ etc. Emission of photons can occur at any betatron phase and we therefore average over all phases. As a consequence, all terms depending linearly on the betatron amplitude and its derivatives or variations thereof vanish. The average variation of the phase ellipse or oscillation amplitude $a$ due to the emission of photons with energy $\varepsilon$ becomes then

$$
\left\langle\delta a^{2}\right\rangle=\frac{\varepsilon^{2}}{E_{0}^{2}} \mathcal{H}(z),
$$


where

$$
\mathcal{H}(z)=\beta \eta^{\prime 2}+2 \alpha \eta \eta^{\prime}+\gamma \eta^{2}
$$

We average again over all photon energies, multiply by the total number of photons emitted per unit time and integrate over the whole ring to get the variation of the oscillation amplitude per turn

$$
\Delta\left\langle a^{2}\right\rangle=\frac{1}{c E_{0}^{2}} \oint \dot{N}_{\mathrm{ph}}\left\langle\varepsilon^{2}\right\rangle \mathcal{H}(z) \mathrm{d} z .
$$

The rate of change of the oscillation amplitude is then with $z=c t$

$$
\left.\frac{\mathrm{d}\left\langle a^{2}\right\rangle}{\mathrm{d} t}\right|_{\mathrm{q}}=\frac{1}{E_{0}^{2}}\left\langle\dot{N}_{\mathrm{ph}}\left\langle\varepsilon^{2}\right\rangle \mathcal{H}(z)\right\rangle_{z},
$$

where the index $z$ indicates averaging around the ring. This quantum excitation of the oscillation amplitude is compensated by damping for which we have similar to $(11.40)$

$$
\left.\left\langle\frac{\mathrm{d} a^{2}}{\mathrm{~d} t}\right\rangle\right|_{\mathrm{d}}=2 \alpha_{x}\left\langle a^{2}\right\rangle
$$

Equilibrium is reached when quantum excitation and damping are of equal strength which occurs for

$$
\frac{\sigma_{u}^{2}}{\beta_{u}}=\frac{\tau_{u}}{4 E^{2}}\left\langle\dot{N}_{\mathrm{ph}}\left\langle\varepsilon^{2}\right\rangle \mathcal{H}_{u}\right\rangle_{z} .
$$

Here we have used the definition of the standard width of a Gaussian particle distribution

$$
\sigma_{u}^{2}=\left\langle u^{2}(z)\right\rangle=\left\langle\frac{1}{2} a^{2} \beta_{u}\right\rangle
$$

with the betatron function $\beta_{u}$ and $u=x$ or $y$. With (11.27), (11.44) and (24.34) we get finally

$$
\epsilon_{u}=\frac{\sigma_{u}^{2}}{\beta_{u}}=C_{\mathrm{q}} \frac{\gamma^{2}}{J_{u}} \frac{\left\langle\kappa^{3} \mathcal{H}_{u}\right\rangle}{\left\langle\kappa^{2}\right\rangle},
$$

which we define as the equilibrium beam emittance of a relativistic electron in a circular accelerator. 


\subsubsection{Emittance Increase in a Beam Transport Line}

In (11.53) we decided to integrate the quantum excitation over a complete turn of a circular accelerator. This should not be taken as a restriction but rather as an example. If we integrate along an open beam transport line we would get the increase of the beam emittance along this beam line. This becomes important for very high energy linear colliders where beams are transported along the linear accelerator and some beam transport system in the final focus section just ahead of the collision point. Any dipole field along the beam path contributes to an increase of the beam emittance, whether it be real dipole magnets, dipole field errors, path displacements in a quadrupole, or small correction magnets for beam steering. Since there is no damping, the emittance growth is therefore in both planes from (11.53) and (11.57)

$$
\Delta \epsilon_{u}=\frac{1}{2 c E_{0}^{2}} \int \dot{N}_{\mathrm{ph}}\left\langle\varepsilon^{2}\right\rangle \mathcal{H}_{u}(z) \mathrm{d} z .
$$

The function $\mathcal{H}$ is now evaluated with the dispersion functions $D_{u}(z)$ instead of the periodic $\eta$-function with contributions from any dipole field. Since such fields can occur in both planes there is an emittance increase in both planes as well. With (11.44) the increase in beam emittance is finally

$$
\Delta \epsilon_{u}=\frac{55 C_{\gamma} \hbar c\left(m c^{2}\right)^{2}}{64 \pi \sqrt{3}} \gamma^{5} \int \kappa^{3} \mathcal{H}_{u} \mathrm{~d} z
$$

where the integration is taken along the beam line. The perturbation of the beam emittance in a beam transport line increases with the fifth power of the particle energy. At very high energies we expect therefore a significant effect of dipole errors on the beam emittance even if the basic beam transport line is straight.

So far, we have not yet distinguished between the horizontal and vertical plane since the evolution of the phase space does not depend on the particular degree of freedom. The equilibrium beam emittance, however, depends on machine parameters and circular accelerators are not constructed symmetrically. Specifically, accelerators are mostly constructed in a plane and therefore there is no deflection in the plane normal to the ring plane. Assuming bending only occurs in the horizontal plane, we may use (11.58) directly as the result for the horizontal beam emittance $u=x$ only.

\subsubsection{Vertical Beam Emittance}

In the vertical plane, the bending radius $\rho_{v} \rightarrow \infty$ and the vertical beam emittance reduces to zero by virtue of damping. Whenever we have ideal conditions like this it is prudent to consider effects that we may have neglected leading to less than ideal 
results. In this case, we have neglected the fact that synchrotron radiation photons are emitted not strictly in the forward direction but rather into a small angle $\pm 1 / \gamma$. Photons emitted at a slight angle exert a recoil on the particle normal to the direction of the trajectory. A photon emitted at an angle $\theta$ with respect to the direction of the trajectory and an azimuth $\phi$ causes a variation of the vertical slope by

$$
\delta y^{\prime}=-\theta \cos \phi \frac{\varepsilon}{E_{0}},
$$

while the position is not changed $\delta y=0$. This leads to a finite beam emittance which can be derived analogous to the general derivation above

$$
\frac{\sigma_{y}^{2}}{\beta_{y}}=\frac{\tau_{y}}{4 E^{2}}\left\langle\dot{N}_{\mathrm{ph}}\left\langle\varepsilon^{2} \theta^{2} \cos ^{2} \phi\right\rangle \beta_{y}\right\rangle_{z} .
$$

We set

$$
\left\langle\varepsilon^{2} \theta^{2} \cos ^{2} \phi\right\rangle \approx\left\langle\varepsilon^{2}\right\rangle\left\langle\theta^{2}\right\rangle\left\langle\cos ^{2} \phi\right\rangle \approx\left\langle\varepsilon^{2}\right\rangle \frac{1}{2 \gamma^{2}}
$$

and get finally for the fundamental lower limit of the vertical beam emittance

$$
\frac{\sigma_{y}^{2}}{\beta_{y}}=\epsilon_{y}=C_{\mathrm{q}} \frac{\bar{\beta}_{y}}{2 J_{y}} \frac{\left\langle\kappa^{3}\right\rangle}{\left\langle\kappa^{2}\right\rangle} .
$$

Very roughly $\epsilon_{y} / \epsilon_{x}=1 / \gamma^{2} \ll 1$ and it is therefore justified to neglect this term in the calculation of the horizontal beam emittance. This fundamental lower limit of the equilibrium beam emittance is of the order of $10^{-13} \mathrm{~m}$, assuming the betatron function and the bending radius to be of similar magnitude, and therefore indeed very small compared to actual achieved beam emittances in real accelerators. In reality, we observe a larger beam emittance in the vertical plane due to coupling or due to vertical steering errors which create a small vertical dispersion and, consequently, a small yet finite vertical beam emittance. As a practical rule the vertical beam emittance is of the order of one percent or less of the horizontal beam emittance due to field and alignment tolerances of the accelerator magnets. For very small horizontal beam emittances, however, this percentage may increase because the vertical beam emittance due to vertical dipole errors becomes more significant.

Sometimes it is necessary to include vertical bending magnets in an otherwise horizontal ring. In this case the vertical dispersion function is finite and so is $\mathcal{H}_{y}(z)$. The vertical emittance is determined by evaluating (11.58) while using the vertical dispersion function. Note, however, that all bending magnets must be included in the calculation of equilibrium beam emittances because for quantum excitation it is immaterial whether the energy loss was caused in a horizontally or vertically bending magnet. The same is true for the damping term in the denominator. Differences in the horizontal and vertical beam emittance come from the different betatron and $\eta$-functions at the location of the radiation source. 


\subsubsection{Beam Sizes}

Beam parameters like width, height, length, divergence, and energy spread are not all fixed independent quantities, but rather depend on emittances and lattice and rf-parameters. These multiple dependencies allow the adjustment of beam parameters, within limits, to be optimum for the intended application. In this section we will discuss such dependencies.

A particle beam at any point of a beam transport line may be represented by a few phase ellipses for different particle momenta as shown in Fig. 11.3. The phase ellipses for different momenta are shifted proportional to the dispersion function at that point and its derivative. Generally, the form and orientation of the ellipses are slightly different too due to chromatic aberrations in the focusing properties of the beam line. For the definition of beam parameters we need therefore the knowledge of the lattice functions including chromatic aberrations and the beam emittance and momentum spread.

The particle beam width or beam height is determined by the emittance, betatron function, dispersion function and energy spread. The betatron and dispersion functions vary along a beam transport line and depend on the distribution of the beam focusing elements. The beam sizes are therefore also functions of the location along the beam line. From the focusing lattice these functions can be derived and the beam sizes be calculated.

The beam size of a particle beam is generally not well defined since the boundaries of a beam tends to be fuzzy. We may be interested in the beam size that defines all of a particle beam. In this case we look for that phase ellipse that

Fig. 11.3 Distribution of beam ellipses for a beam with finite emittance and momentum spread (schematic). The variation in the shape of the phase ellipses for different energies reflect the effect of chromatic aberrations

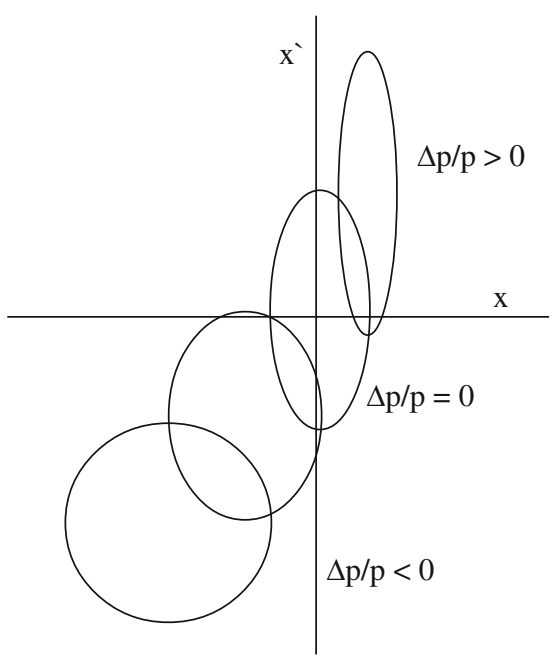


encloses all particles and obtain the beam size in the form of the beam envelope. The beam half-width or half-height of this beam envelope is defined by

$$
u_{\beta}(z)=\sqrt{\epsilon_{u} \beta_{u}(z)}
$$

with $u=(x, y)$. If there is also a finite momentum spread within the beam particles the overall beam size or beam envelope is increased by the dispersion

$$
u_{\eta}(z)=\eta_{u}(z) \frac{\Delta c p}{c p_{0}}
$$

and the total beam size is

$$
u_{\mathrm{tot}}(z)=u_{\beta}(z)+u_{\eta}(z)=\sqrt{\epsilon_{u} \beta_{u}(z)}+\eta_{u}(z) \frac{\Delta c p}{c p_{0}} .
$$

This definition of the beam size assumes a uniform particle distribution within the beam and is used mostly to determine the acceptance of a beam transport system. The acceptance of a beam transport system is defined as the maximum emittance a beam may have and still pass through the vacuum chambers of a beam line. In Fig. 11.3 this would be the area of that ellipse that encloses the whole beam including off momentum particles. In practice, however, we would choose a larger acceptance to allow for errors in the beam path.

Since the lattice functions vary along a beam line the required aperture to let a beam with the maximum allowable emittance pass is not the same everywhere along the system. To characterize the aperture variation consistent with the acceptance, a beam stay clear area, BSC, is defined as the required material free aperture of the beam line.

The beam parameters for a Gaussian particle distributions are defined as the standard values of the Gaussian distribution $\sigma_{x}, \sigma_{x^{\prime}}, \sigma_{y}, \sigma_{y^{\prime}}, \sigma_{\delta}, \sigma_{\ell}$, where most designations have been defined and used in previous chapters and where $\sigma_{\delta}=\sigma_{\epsilon} / c p_{0}$ and $\sigma_{\ell}$ the bunch length. Quoting beam sizes for any particle type in units of $\sigma$ can be misleading specifically in connection with beam intensities. For example, a beam with a horizontal and vertical size of one sigma has a cross section of $2 \sigma_{x} 2 \sigma_{y}$ and includes only $46.59 \%$ of the beam. This is accepted for electron beams with Gaussian distribution but for proton beams intensities are often given for $\sqrt{6} \sigma$ 's to cover most of the beam. In Table 11.1 the fraction of the total beam intensity is compiled for a few generally used units of beam size measurement and for beam size, cross section, and volume. The beam size for Gaussian beams is thereby

$$
\sigma_{\mathrm{u}, \mathrm{tot}}=\sqrt{\epsilon_{u} \beta_{u}(z)+\eta^{2}(z) \sigma_{\delta}^{2}}
$$

Four parameters are required to determine the beam size in each plane although in most cases the vertical dispersion vanishes. 
Table 11.1 Fraction of total beam intensity

\begin{tabular}{l|l|l|l}
\hline & One-dimension (\%) & Two-dimension (\%) & Three-dimension (\%) \\
\hline $1 \sigma$ & 68.26 & 46.59 & 31.81 \\
\hline $2 \sigma$ & 95.44 & 91.09 & 86.93 \\
\hline$\sqrt{6} \sigma$ & 98.56 & 97.14 & 95.74 \\
\hline
\end{tabular}

\subsubsection{Beam Divergence}

The angular distribution of particles within a beam depends on the rotation of the phase ellipse and we define analogous to the beam size an angular beam envelope by

$$
\sigma_{\mathrm{u}^{\prime}, \mathrm{tot}}=\sqrt{\epsilon_{u} \gamma_{u}(z)+\eta^{\prime 2}(z) \sigma_{\delta}^{2}}
$$

Again, there is a contribution from the betatron motion, from a finite momentum spread and from associated chromatic aberration. The horizontal and vertical beam divergencies are also determined by four parameters in each plane.

\subsection{Variation of the Damping Distribution}

Robinson's criterion provides an expression for the overall damping in six-dimensional phase space without specifying the distribution of damping in the three degrees of freedom. In accelerators we make an effort to decouple the particle motion in the three degrees of freedom as much as possible and as a result we try to optimize the beam parameters in each plane separately from the other planes for our application. Part of this optimization is the adjustment of damping and as a consequence of beam emittances to desired values. Robinson's criterion allows us to modify the damping in one plane at the expense of damping in another plane. This shifting of damping is done by varying damping partition numbers.

From the definition of the $\vartheta$ parameter is clear that damping partition numbers can be modified depending on whether the accelerator lattice is a combined function or a separated function lattice. Furthermore, we may adjust virtually any distribution between partition numbers by choosing a combination of gradient and separated function magnets.

\subsubsection{Damping Partition and Rf-Frequency}

Actually such "gradients" can be introduced even in a separated function lattice. If the rf-frequency is varied the beam will follow a path that meets the synchronicity condition. Increasing the rf-frequency, for example, leads to a shorter wavelength 
and therefore the total path length in the ring need to be shorter. As a consequence of the principle of phase stability the beam energy is reduced and the beam follows a lower energy equilibrium orbit with the same harmonic number as the reference orbit for the reference energy. Decreasing the rf-frequency leads just to the opposite effect. The off momentum orbits pass systematically off center through quadrupoles which therefore function like combined function gradient magnets.

To quantify this effect we use only the second term in the expression (11.25) for $\vartheta$. The first term, coming from sector magnets, will stay unaffected. Displacement of the orbit in the quadrupoles will cause a bending with a bending radius

$$
\frac{1}{\rho_{\mathrm{q}}}=k \delta x
$$

An rf-frequency shift causes a momentum change of

$$
\frac{\Delta p}{p_{0}}=-\frac{1}{\alpha_{\mathrm{c}}} \frac{\Delta f_{\mathrm{rf}}}{f_{\mathrm{rf}}},
$$

which in turn causes a shift in the equilibrium orbit of

$$
\delta x=\eta \frac{\Delta p}{p_{0}}=-\frac{\eta}{\alpha_{\mathrm{c}}} \frac{\Delta f_{\mathrm{rf}}}{f_{\mathrm{rf}}}
$$

and the bending radius of the shifted orbit in quadrupoles is

$$
\frac{1}{\rho_{\mathrm{q}}}=k \delta x=k \eta \frac{\Delta p}{p_{0}}=-k \frac{\eta}{\alpha_{\mathrm{c}}} \frac{\Delta f_{\mathrm{rf}}}{f_{\mathrm{rf}}} .
$$

Inserted into the second term of (11.25), we get

$$
\Delta \vartheta=-\frac{1}{\alpha_{\mathrm{c}}} \frac{\oint 2 k^{2} \eta^{2} \mathrm{~d} z}{\oint \frac{1}{\rho_{0}^{2}} \mathrm{~d} z} \frac{\Delta f_{\mathrm{rf}}}{f_{\mathrm{rf}}},
$$

where $\rho_{0}$ is the bending radius of the ring bending magnets All quantities in (11.72) are fixed properties of the lattice and changing the rf-frequency leads just to the expected effect. Specifically, we note that all quadrupoles contribute additive irrespective of their polarity. We may apply this to a simple isomagnetic FODO lattice where all bending magnets and quadrupoles have the same absolute strength respectively with $\oint \mathrm{d} z / \rho_{0}^{2}=2 \pi / \rho_{0}$. Integration of the nominator in (11.72) leads to

$$
\oint 2 k^{2} \eta^{2} \mathrm{~d} z=2 k^{2}\left(\eta_{\max }^{2}+\eta_{\min }^{2}\right) l_{\mathrm{q}} 2 n_{\mathrm{c}}
$$

where $l_{\mathrm{q}}$ is half the quadrupole length in a FODO lattice, $\eta_{\max }$ and $\eta_{\min }$ the values of the $\eta$-function in the focusing QF and defocusing QD quadrupoles, respectively, 
and $n_{\mathrm{c}}$ the number of FODO cells in the ring. With all this the variation of the $\vartheta$ parameter

$$
\Delta \vartheta=-n_{\mathrm{c}} \frac{2 \rho_{0}}{\pi \alpha_{\mathrm{c}} l_{\mathrm{q}}} \frac{\eta_{\max }^{2}+\eta_{\min }^{2}}{f^{2}} \frac{\Delta f_{\mathrm{rf}}}{f_{\mathrm{rf}}}
$$

Here we have used the focal length $f^{-1}=k l_{\mathrm{q}}$. Replacing in (11.73) the $\eta$ functions by the expressions (10.74) derived for a FODO lattice, we recall the relation $f=\kappa L$ and get finally [8]

$$
\Delta \vartheta=-\frac{\rho_{0}}{\rho} \frac{1}{\alpha_{\mathrm{c}}} \frac{L}{l_{\mathrm{q}}}\left(4 \kappa^{2}+1\right) \frac{\Delta f_{\mathrm{rf}}}{f_{\mathrm{rf}}},
$$

where $\rho$ is the average bending radius in the FODO cell. The variation of the $\vartheta$ parameter in a FODO lattice is the more sensitive to rf-frequency variations the longer the cell compared to the quadrupole length and the weaker the focusing. For other lattices the expressions may not be as simple as for the FODO lattice but can always be computed numerically by integrations and evaluation of (11.72).

By varying the rf-frequency and thereby the horizontal and longitudinal damping partition number we have found a way to either increase or decrease the horizontal beam emittance. The adjustments, however, are limited. To decrease the horizontal beam emittance we would increase the horizontal partition number and at the same time the longitudinal partition number would be reduced. The limit is reached when the longitudinal motion becomes unstable or in practical cases when the partition number drops below about half a unit. Other more practical limits may occur before stability limits are reached if, for example, the momentum change becomes too large to fit the beam into the vacuum chamber aperture.

\subsection{Variation of the Equilibrium Beam Emittance}

In circular electron accelerators the beam emittance is determined by the emission of synchrotron radiation and the resulting emittance is not always equal to the desired value. In such situations methods to alter the equilibrium emittance are desired and we will discuss in the next sections such methods which may be used to either increase or decrease the beam emittance.

\subsubsection{Beam Emittance and Wiggler Magnets}

The beam emittance in an electron storage ring can be greatly modified by the use of wiggler magnets both to increase [9] or to decrease the beam emittance. A decrease in beam emittance has been noted by Tazzari [10] while studying the effect of a 
number of wiggler magnets in a low emittance storage ring design. Manipulation of the beam emittance in electron storage rings has become of great interest to obtain extremely small beam emittances and we will therefore derive systematic scaling laws for the effect of wiggler magnets on the beam emittance as well as on the beam energy spread $[10,11]$.

The particle beam emittance in a storage ring is the result of two competing effects, the quantum excitation caused by the quantized emission of photons and the damping effect. Both effects lead to an equilibrium beam emittance observed in electron storage rings.

Independent of the value of the equilibrium beam emittance in a particular storage ring, it can be further reduced by increasing the damping without also increasing the quantum excitation. More damping can be established by causing additional synchrotron radiation through the installation of deflecting dipole magnets like strong wigglers magnets. In order to avoid quantum excitation of the beam emittance, however, the placement of wiggler magnets has to be chosen carefully. As discussed earlier, an increase of the beam emittance through quantum excitation is caused only when synchrotron radiation is emitted at a place in the storage ring where the dispersion function is finite. Emittance reducing wiggler magnets therefore must be placed in areas around the storage ring where the dispersion vanishes to minimize quantum excitation. To calculate the modified equilibrium beam emittance, we start from (11.54) and get with (11.44) and (11.57) an expression for the quantum excitation of the emittance which can be expanded to include wiggler magnets

$$
\left.\frac{\mathrm{d} \epsilon}{\mathrm{d} t}\right|_{\mathrm{q}, 0}=\frac{2}{3} r_{\mathrm{e}} C_{\mathrm{q}} \gamma^{5}\left\langle\kappa^{3} \mathcal{H}\right\rangle_{0},
$$

The quantity $\mathcal{H}$ is evaluated for the plane for which the emittance is to be determined, $E$ is the particle energy, and $\rho$ the bending radius of the regular ring magnets. The average \langle\rangle is to be taken for the whole ring and the index ${ }_{0}$ indicates that the average $\left\langle\kappa^{3} \mathcal{H}\right\rangle_{0}$ be taken only for the ring proper without wiggler magnets.

Since the contributions of different magnets, specifically, of regular storage ring magnets and wiggler magnets are independent of each other, we may use the results of the basic ring lattice and add to the regular quantum excitation and damping the appropriate additions due to the wiggler magnets,

$$
\left.\frac{\mathrm{d} \epsilon}{\mathrm{d} t}\right|_{\mathrm{q}, \mathrm{w}}=\frac{2}{3} r_{\mathrm{e}} C_{\mathrm{q}} \gamma^{5}\left[\left\langle\kappa^{3} \mathcal{H}\right\rangle_{0}+\left\langle\kappa^{3} \mathcal{H}\right\rangle_{\mathrm{w}}\right] .
$$

Both, ring magnets and wiggler magnets produce synchrotron radiation and contribute to damping of the transverse particle oscillations. Again, we may consider both contributions separately and adding the averages we get the combined rate of emittance damping from (11.55) and (11.27)

$$
\left.\frac{\mathrm{d} \epsilon}{\mathrm{d} t}\right|_{\mathrm{d}, \mathrm{w}}=-\frac{2}{3} r_{\mathrm{e}} c \epsilon_{\mathrm{w}} J_{u} \gamma^{3}\left[\left\langle\kappa^{2}\right\rangle_{0}+\left\langle\kappa^{2}\right\rangle_{\mathrm{w}}\right],
$$


where $\epsilon_{\mathrm{w}}$ is the beam emittance with wiggler magnets and $J_{u}$ the damping partition number with $u=x, y$. The equilibrium beam emittance is reached when the quantum excitation rate and the damping rates are of equal magnitude. We add therefore (11.76) and (11.77) and solve for the emittance

$$
\epsilon_{\mathrm{w}}=C_{\mathrm{q}} \frac{\gamma^{2}}{J_{x}} \frac{\left\langle\kappa^{3} \mathcal{H}\right\rangle_{0}+\left\langle\kappa^{3} \mathcal{H}\right\rangle_{\mathrm{w}}}{\left\langle\kappa^{2}\right\rangle_{0}+\left\langle\kappa^{2}\right\rangle_{\mathrm{w}}} .
$$

With $\epsilon_{0}$ being the unperturbed beam emittance the relative emittance change due to the presence of wiggler magnets is

$$
\frac{\epsilon_{\mathrm{w}}}{\epsilon_{0}}=\frac{1+\left\langle\kappa^{3} \mathcal{H}\right\rangle_{\mathrm{w}} /\left\langle\kappa^{3} \mathcal{H}\right\rangle_{0}}{1+\left\langle\kappa^{2}\right\rangle_{\mathrm{w}} /\left\langle\kappa^{2}\right\rangle_{0}} .
$$

Making use of the definition of average parameter values we get with the circumference of the storage ring $C=2 \pi R$

$$
\begin{aligned}
& \left\langle\kappa^{3} \mathcal{H}\right\rangle_{0}=\frac{1}{C} \oint\left|\kappa_{0}^{3}\right| \mathcal{H} \mathrm{d} z, \quad\left\langle\kappa^{3} \mathcal{H}\right\rangle_{\mathrm{w}}=\frac{1}{C} \oint\left|\kappa_{\mathrm{w}}^{3}\right| \mathcal{H} \mathrm{d} z, \\
& \left\langle\kappa^{2}\right\rangle_{0}=\frac{1}{C} \oint \kappa_{0}^{2} \mathrm{~d} z, \quad \text { and } \quad\left\langle\kappa^{2}\right\rangle_{\mathrm{w}}=\frac{1}{C} \oint \kappa_{\mathrm{w}}^{2} \mathrm{~d} z \text {. }
\end{aligned}
$$

Evaluation of these integrals for the particular storage ring and wiggler magnet employed gives from (11.79) the relative change in the equilibrium beam emittance. We note that the quantum excitation term scales like the cube while the damping scales only quadratically with the wiggler curvature. This feature leads to the effect that the beam emittance is always reduced for small wiggler fields and increases only when the third power terms become significant.

Concurrent with a change in the beam emittance a change in the momentum spread due to the wiggler radiation can be derived similarly,

$$
\frac{\sigma_{\epsilon \mathrm{w}}^{2}}{\sigma_{\epsilon 0}^{2}}=\frac{1+\left\langle\kappa^{3}\right\rangle_{\mathrm{w}} /\left\langle\kappa^{3}\right\rangle_{0}}{1+\left\langle\kappa^{2}\right\rangle_{\mathrm{w}} /\left\langle\kappa^{2}\right\rangle_{0}} .
$$

Closer inspection of (11.79) and (11.81) reveals basic rules and conditions for the manipulations of beam emittance and energy spread. If the ring dispersion function is finite in the wiggler section strong quantum excitation may occur depending on the magnitude of the wiggler magnet bending radius $\rho_{\mathrm{w}}$. This situation is desired if the beam emittance must be increased [9]. If wiggler magnets are placed into a storage ring lattice were the ring dispersion function vanishes, only the small dispersion function due to the wiggler magnets must be considered for the calculation of $\left\langle\mathcal{H}_{\mathrm{w}}\right\rangle$ and therefore only little quantum excitation occurs. In this case the beam emittance can be reduced since the wiggler radiation contributes more strongly to damping and we call such magnets damping wigglers [10,11]. Whenever wiggler magnets are used which are stronger than the ordinary ring magnets $\rho_{\mathrm{w}}<\rho_{0}$ the momentum spread in the beam is increased. This is true for virtually all cases of interest. 
Conceptual methods to reduce the beam emittance in a storage ring have been derived which are based on increased synchrotron radiation damping while avoiding quantum excitation effects. Optimum lattice parameters necessary to achieve this will be derived in the next section.

\subsubsection{Damping Wigglers}

The general effects of wiggler magnet radiation on the beam emittance has been described and we found that the beam emittance can be reduced if the wiggler is placed where $\eta=0$ to eliminate quantum excitation. This assumption, however, is not quite correct. Even though we have chosen a place, where the storage ring dispersion function vanishes, the quantum excitation factor $\mathcal{H}_{\mathrm{w}}$ is not exactly zero once the wiggler magnets are turned on because they create their own dispersion function. To calculate this dispersion function, we assume a sinusoidal wiggler field [11]

$$
B(z)=B_{\mathrm{w}} \cos k_{\mathrm{p}} z
$$

where $k_{\mathrm{p}}=2 \pi / \lambda_{\mathrm{p}}$ and $\lambda_{\mathrm{p}}$ the wiggler period length (Fig. 11.4). The differential equation for the dispersion function is then

$$
\eta^{\prime \prime}=\kappa=\kappa_{\mathrm{w}} \cos k_{\mathrm{p}} z
$$

which can be solved by

$$
\begin{aligned}
& \eta(z)=\frac{\kappa_{\mathrm{w}}}{k_{\mathrm{p}}^{2}}\left(1-\cos k_{\mathrm{p}} z\right), \\
& \eta^{\prime}(z)=\frac{\kappa_{\mathrm{w}}}{k_{\mathrm{p}}} \sin k_{\mathrm{p}} z,
\end{aligned}
$$

where we have assumed that the wiggler magnet is placed in a dispersion free location $\eta_{0}=\eta_{0}^{\prime}=0$. With this solution, the first two Eqs. (11.80) can be evaluated. To simplify the formalism we ignore the $z$-dependence of the lattice functions within the wiggler magnet setting $\alpha_{x}=0$ and $\beta_{x}=$ const. Evaluating the integrals (11.80), we note that the absolute value of the bending radius must be used along the integration path because the synchrotron radiation does not depend on the sign of the deflection. With this in mind, we evaluate the integrals $\int_{0}^{\lambda_{\mathrm{p}} / 2}\left|\kappa^{3}\right| \eta^{2} \mathrm{~d} z$

Fig. 11.4 Dispersion function in one period of a wiggler magnet

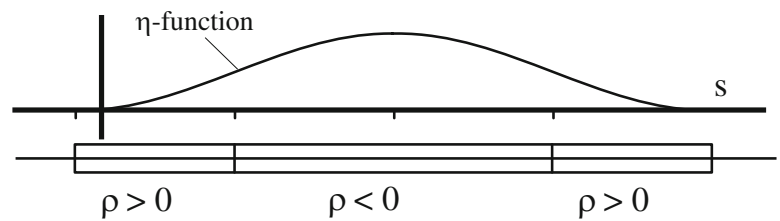


and $\int_{0}^{\lambda_{\mathrm{p}} / 2}\left|\kappa^{3}\right| \eta^{\prime 2} \mathrm{~d} z$. For each half period of the wiggler magnet the contribution to the integral is

$$
\Delta \int_{0}^{\lambda_{\mathrm{p}} / 2}\left|\kappa^{3}\right| \mathcal{H} \mathrm{d} z=\frac{12}{5} \frac{1}{\beta_{x}} \frac{\kappa_{\mathrm{w}}^{5}}{k_{\mathrm{p}}^{5}}+\frac{4}{15} \frac{\kappa_{\mathrm{w}}^{5} \beta_{x}}{k_{\mathrm{p}}^{3}} \approx \frac{4}{15} \frac{\kappa_{\mathrm{w}}^{5} \beta_{x}}{k_{\mathrm{p}}^{3}},
$$

where the approximation $\lambda_{\mathrm{p}} \ll \beta_{x}$ was used. For the whole wiggler magnet with $N_{\mathrm{w}}$ periods the total quantum excitation integral is with the deflection angle per wiggler half pole $\Theta_{\mathrm{w}}=\kappa_{\mathrm{w}} / k_{\mathrm{p}}$

$$
\int_{\mathrm{w}}\left|\kappa_{\mathrm{w}}^{3}\right| \mathcal{H} \mathrm{d} z \approx N_{\mathrm{w}} \frac{8}{15} \frac{\beta_{x}}{\rho_{\mathrm{w}}^{2}} \Theta_{\mathrm{w}}^{3}
$$

Similarly, the damping integral for the total wiggler magnet is

$$
\int_{\mathrm{w}} \kappa^{2} \mathrm{~d} z=\pi N_{\mathrm{w}} \kappa_{\mathrm{w}} \Theta_{\mathrm{w}}
$$

Inserting expressions (11.80), (11.86), (11.87) into (11.79), we get for the emittance ratio

$$
\frac{\epsilon_{\mathrm{xw}}}{\epsilon_{x 0}}=\frac{1+\frac{4}{15 \pi} N_{\mathrm{w}} \frac{\beta_{x}}{\left\langle\mathcal{H}_{0}\right\rangle} \frac{\rho_{0}^{2}}{\rho_{\mathrm{w}}^{2}} \Theta_{\mathrm{w}}^{3}}{1+\frac{1}{2} N_{\mathrm{w}} \frac{\rho_{0}}{\rho_{\mathrm{w}}} \Theta_{\mathrm{w}}}
$$

where $\left\langle\mathcal{H}_{0}\right\rangle$ is the average value of $\mathcal{H}$ in the ring bending magnets excluding the wiggler magnets. We note from (11.88) that the beam emittance indeed can be reduced by wiggler magnets if $\Theta_{\mathrm{w}}$ is kept small. For easier numerical calculation we replace $\left\langle\mathcal{H}_{0}\right\rangle$ by the unperturbed beam emittance which is in the limit $\rho_{\mathrm{w}} \rightarrow \infty$

$$
\left\langle\mathcal{H}_{0}\right\rangle=\frac{J_{x} \rho_{0} \epsilon_{\mathrm{x} 0}}{C_{\mathrm{q}} \gamma^{2}}
$$

and get instead of (11.88)

$$
\frac{\epsilon_{x \mathrm{w}}}{\epsilon_{x 0}}=\frac{1+\frac{4 C_{\mathrm{q}}}{15 \pi J_{x}} N_{\mathrm{w}} \frac{\beta_{x}}{\epsilon_{x 0} \rho_{\mathrm{w}}} \gamma^{2} \frac{\rho_{0}}{\rho_{\mathrm{w}}} \Theta_{\mathrm{w}}^{3}}{1+\frac{1}{2} N_{\mathrm{w}} \frac{\rho_{0}}{\rho_{\mathrm{w}}} \Theta_{\mathrm{w}}}
$$

The beam emittance is reduced by wiggler magnets whenever the condition

$$
\frac{8}{15 \pi} \frac{C_{\mathrm{q}}}{J_{x}} \frac{\beta_{x}}{\epsilon_{0} \rho_{\mathrm{w}}} \gamma^{2} \Theta_{\mathrm{w}}^{2} \leq 1
$$


is fulfilled. For large numbers of wiggler poles $N_{\mathrm{w}} \rightarrow \infty$ the beam emittance reaches asymptotically a lower limit given by

$$
\epsilon_{x \mathrm{w}} \rightarrow \frac{8}{15 \pi} \frac{C_{\mathrm{q}}}{J_{x}} \frac{\beta_{x}}{\rho_{\mathrm{w}}} \gamma^{2} \Theta_{\mathrm{w}}^{2} .
$$

In this limit the ultimate beam emittance is independent of the unperturbed beam emittance. This derivation did not include any perturbation of the original lattice functions due to focusing effects by the wiggler poles. Such perturbations are either small or must be compensated such that our assumptions still are valid.

For many wiggler poles the increase in momentum spread also reaches an asymptotic limit which is given from (11.81)

$$
\frac{\sigma_{\epsilon \mathrm{W}}^{2}}{\sigma_{\epsilon o}^{2}} \rightarrow \frac{\rho_{0}}{\rho_{\mathrm{w}}}=\frac{B_{\mathrm{w}}}{B_{0}},
$$

where $B_{0}$ is the magnetic field strength in the ring magnets. Beam stability and acceptance problems may occur if the beam momentum spread is allowed to increase too much and therefore inclusion of damping wigglers must be planned with some caution.

\subsection{Robinson Wiggler*}

The horizontal betatron motion in a combined function synchrotron FODO lattice is not damped because $\vartheta>1$. Beam stability in a synchrotron therefore exists only during acceleration when the anti-damping is over compensated by adiabatic damping, and the maximum energy achievable in a combined function synchrotron is determined when the quantum excitation becomes too large to be compensated by adiabatic damping. In an attempt, at the Cambridge Electron Accelerator CEA, to convert the synchrotron into a storage ring the problem of horizontal beam instability was solved by the proposal [12] to insert a damping wiggler consisting of a series of poles with alternating fields and gradients designed such that the horizontal partition number becomes positive and $-2<\vartheta<1$.

Such magnets can be used generally to vary the damping partition numbers without having to vary the rf-frequency and thereby moving the beam away from the center of the beam line.

\subsubsection{Damping Partition and Synchrotron Oscillation}

The damping partition number and damping depend on the relative momentum spread of the whole beam. During synchrotron oscillations, significant momentum deviations can occur, specifically, in the tails of a Gaussian distribution. Such 
momentum deviations, although only temporary, can lead to reduced damping or outright anti-damping [8]. To quantify this effect, we write (11.72) in the form

$$
\Delta \vartheta=\frac{\oint 2 k^{2} \eta^{2} \mathrm{~d} z}{\oint \kappa_{\mathrm{a}}^{2} \mathrm{~d} z} \frac{\Delta p}{p_{0}}=C_{0} \frac{\Delta p}{p_{0}} .
$$

The momentum deviation is not a constant but rather oscillates with the synchrotron oscillation frequency,

$$
\frac{\Delta p}{p_{0}}=\left.\frac{\Delta p}{p_{0}}\right|_{\max } \sin \Omega t=\delta_{\max } \sin \Omega t,
$$

where $\Omega$ is the synchrotron oscillation frequency. The damping partition number oscillates as well (11.94) and the damping decrement is therefore

$$
\frac{1}{\tau}=\frac{1}{\tau_{x 0}}\left(1-C_{0} \delta_{\max } \sin \Omega t\right) .
$$

If the perturbation is too large we have anti-damping during part of the synchrotron oscillation period. As a consequence the beam is "breathing" in its horizontal and longitudinal dimensions while undergoing synchrotron oscillations. To quantify this, we calculate similar to (11.56) the total rate of change of the betatron oscillation amplitude $a^{2}$, as defined by the phase space ellipse $\gamma u^{2}+$ $2 \alpha u u^{\prime}+\beta u^{\prime 2}=a^{2}$, composed of quantum excitation and modified damping

$$
\frac{\mathrm{d}\left\langle a^{2}\right\rangle}{\mathrm{d} t}=\frac{\left\langle\dot{N}_{\mathrm{ph}}\left\langle\epsilon_{\gamma}^{2}\right\rangle \mathcal{H}\right\rangle}{E_{0}^{2}}-\frac{2\left\langle a^{2}\right\rangle}{\tau} .
$$

The amplitude $a^{2}$ has the dimension of an emittance but we are interested here in the maximum amplitude which can be expressed in terms of a betatron amplitude by $a^{2}=u_{\max }^{2} / \beta_{u}$. Replacing the varying damping time by $\tau^{-1}=$ $\tau_{0}^{-1}\left(1-\delta_{\max } C_{0} \sin \Omega t\right)(11.97)$ becomes

$$
\frac{\mathrm{d}\left\langle u_{\max }^{2}\right\rangle}{\left\langle u_{\max }^{2}\right\rangle}=\frac{2}{\tau_{0}} \delta_{\max } C_{0} \sin \Omega t \mathrm{~d} t,
$$

which can be readily integrated to give

$$
\left\langle u_{\max }^{2}\right\rangle=\left\langle u_{\max , 0}^{2}\right\rangle \exp \left[\frac{2 \delta_{\max } C_{0}}{\Omega \tau_{0}}(1-\cos \Omega t)\right] \text {. }
$$

A particle with a betatron amplitude $u_{\max , 0}$ will, during the course of a synchrotron oscillation period, reach amplitudes as large as $u_{\max }$. The effect is the largest for particles with large energy oscillations. On the other hand, the effect on the core of the beam is generally very small since $\delta_{\max }$ is small. 


\subsubsection{Can We Eliminate the Beam Energy Spread?}

To conclude the discussions on beam manipulation we try to conceive a way to eliminate the energy spread in a particle beam. From beam dynamics we know that the beam particles can be sorted according to their energy by introducing a dispersion function. The distance of a particle from the reference axis is proportional to its energy and given by

$$
x_{\delta}=D \delta \text {, }
$$

where $D$ is the value of the dispersion at the location under consideration and $\delta=\Delta E / E_{0}$ the energy error. For simplicity we make no difference between energy and momentum during this discussion. We consider now a cavity excited at a higher mode such that the accelerating field is zero along the axis, but varies linearly with the distance from the axis. If now the accelerating field, or after integration through the cavity, the accelerating voltage off axis is

$$
e V_{\mathrm{rf}}\left(x_{\delta}\right)=-\frac{x_{\delta}}{D} E_{0},
$$

we have just compensated the energy spread in the beam. The particle beam has become monochromatic, at least to the accuracy assumed here. In reality the dispersion of the beam is not perfect due to the finite beam emittance.

We will discuss cavity modes and find that the desired mode exists indeed and the lowest order of such modes is the $\mathrm{TM}_{110}$-mode. So far we seem to have made no mistake and yet, Liouville's theorem seems to be violated because this scheme does not change the bunch length and the longitudinal emittance has been indeed reduced by application of macroscopic fields.

The problem is that we are by now used to consider transverse and longitudinal phase space separate. While this separation is desirable to manage the mathematics of beam dynamics, we must not forget, that ultimately beam dynamics occurs in six-dimensional phase space. Since Liouville's theorem must be true, its apparent violation warns us to observe changes in other phase space dimensions. In the case of beam monochromatization we notice that the transverse beam emittance has been increased. The transverse variation of the longitudinal electric field causes by virtue of Maxwell's equations the appearance of transverse magnetic fields which deflect the particles transversely thus increasing the transverse phase space at the expense of the longitudinal phase space.

This is a general feature of electromagnetic fields which is known as the Panofsky-Wenzel theorem [13] stating that transverse acceleration occurs whenever there is a transverse variation of the longitudinal accelerating field. We will discuss this in more detail in Sect.22.1.4. So, indeed we may monochromatize a particle beam with the use of a $\mathrm{TM}_{110}$-mode, but only at the expense of an increase in the transverse beam emittance. 


\subsection{Beam Life Time}

Particles travelling along a beam transport line or orbiting in a circular accelerator can be lost due to a variety of causes. We ignore the trivial cases of beam loss due to technical malfunctioning of beam line components or losses caused by either complete physical obstruction of the beam line or a mismatch of vacuum chamber aperture and beam dimensions. For a well designed beam transport line or circular accelerator we distinguish two main classes for particle loss which are losses due to scattering and losses due to instabilities. While particle losses due to scattering with other particles is a single particle effect leading to a gradual loss of beam intensity, instabilities can lead to catastrophic loss of part or all of the beam. In this chapter we will concentrate on single particle losses due to interactions with residual gas atoms.

The effect of particle scattering on the beam parameters is different in a beam transport line compared to a circular accelerator especially compared to storage rings. Since a beam passes through transport lines only once, we are not concerned about beam life time but rather with the effect of particle scattering on the transverse beam size. For storage rings, in contrast, we consider both the effect of scattering on the beam emittance as well as the overall effect on the beam lifetime. Since long lifetimes of the order of many hours are desired in storage rings even small effects can accumulate to reduce beam performance significantly. In proton rings continuous scattering with residual gas atoms or with other protons of the same beam can change the beam parameters considerably for lack of damping. Even for electron beams, where we expect the effects of scattering to vanish within a few damping times, we may observe an increase in beam emittance. This is specifically true due to intra beam scattering for dense low emittance beams at low energies when damping is weak.

Collisions of particles with components of residual gas atoms, losses due to a finite acceptance limited by the physical or dynamic aperture, collisions with other particles of the same beam, or with synchrotron radiation photons can lead to absorption of the scattered particles or cause large deflections leading to instable trajectories and eventual particle loss. The continuous loss of single particles leads to a finite beam lifetime and may in severe cases require significant hardware modifications or a different mode of operation to restore a reasonable beam lifetime.

Each of these loss mechanisms has a particular parameter characterizing and determining the severity of the losses. Scattering effects with residual gas atoms are clearly dominated by the vacuum pressure while scattering effects with other particles in the same beam depend on the particle density. Some absorption of particles at the vacuum chamber walls will always occur due to the Gaussian distribution of particles in space. Even for non-radiating proton beams which are initially confined to a small cross section, we observe the development of a halo of particles outside the beam proper due to intra beam scattering. The expansion of this halo is obviously limited by the vacuum chamber aperture. In circular accelerators this aperture limitation may not only be effected by solid vacuum chambers but also by "soft walls" due to stability limits imposed by the dynamic aperture. 
Longitudinal phase or energy oscillations are limited either by the available rf-parameters determining the momentum acceptance or by the transverse acceptance at locations, where the dispersion function is nonzero whichever is more restrictive. A momentum deviation or spread translates at such locations into a widening of the beam and particle loss occurs if the momentum error is too large to fit within the stable aperture. Transverse oscillation amplitudes are limited by the transverse acceptance as limited by the vacuum chamber wall or by aberrations due to nonlinear fields.

\subsubsection{Beam Lifetime and Vacuum}

Particle beams are generally confined within evacuated chambers to avoid excessive scattering on residual gas atoms. Considering multiple Coulomb scattering alone the rms radial scattering angle of particles with momentum $p$ and velocity $\beta$ passing through a scattering material of thickness $L$ can be described by $[14,15]$

$$
\vartheta_{\mathrm{rms}}=Z \frac{20_{\mathrm{MeV}}}{\beta c p} \sqrt{\frac{L}{L_{\mathrm{r}}}},
$$

where $Z$ is the charge multiplicity of the particle and $L_{\mathrm{r}}$ the radiation length of the scattering material. We may integrate (11.101) and get the beam radius $r$ of a pencil beam after passing through a scatterer of thickness $L$

$$
r \approx Z \frac{40_{\mathrm{MeV}} L}{3 \beta c p} \sqrt{\frac{L}{L_{\mathrm{r}}}} .
$$

The beam emittance generated by scattering effects is then in both the horizontal and vertical plane just the product of the projections of the distance $r$ of the particles from the reference path and the radial scattering angles $\vartheta$ onto the respective plane. From (11.101), (11.102) the beam emittance growth due to Coulomb scattering in a scatterer of length $L$ is then

$$
\epsilon_{x, y}(\operatorname{rad~m})=Z^{2} \frac{2}{3}\left(\frac{14(\mathrm{MeV})}{\beta c p}\right)^{2} \frac{L^{2}(\mathrm{~m})}{L_{\mathrm{r}}(\mathrm{m})} .
$$

For atmospheric air the radiation length is $L_{\mathrm{r}}=300.5 \mathrm{~m}$ and a pencil electron beam with a momentum of say $c p=1,000 \mathrm{MeV}$ passing through $20 \mathrm{~m}$ of atmospheric air would grow through scattering to a beam diameter of $6.9 \mathrm{~cm}$ or to a beam emittance of about $177 \mathrm{mradmm}$ in each plane. This is much too big an increase in beam size to be practical in a $20 \mathrm{~m}$ beam transport line let alone in a circular accelerator or storage ring, where particles are expected to circulate at nearly the speed of light for many turns like in a synchrotron or for many hours in a storage ring. 
To avoid beam blow up due to scattering we obviously need to provide an evacuated environment to the beam with a residual gas pressure which must be the lower the longer the beam is supposed to survive scattering effects. This does not mean that beam transport in atmospheric pressure must be avoided at all cost. Sometimes it is very useful to let a beam pass though air to provide free access for special beam monitoring devices specifically at the end of a beam transport line before the beam is injected into a circular accelerator. Obviously, this can be done only if the scattering effects through very thin metallic windows and the short length of atmospheric air will not spoil the beam emittance too much.

\section{Elastic Scattering}

As particles travel along an evacuated pipe they occasionally collide with atoms of the residual gas. These collisions can be either on nuclei or electrons of the residual gas atoms. The physical nature of the collision depends on the mass of the colliding partners. Particles heavier than electrons suffer mostly an energy loss in collisions with the atomic shell electrons while they lose little or no energy during collisions with massive nuclei but are merely deflected from their path by elastic scattering. The lighter electrons in contrast suffer both deflection as well as energy losses during collisions.

In this section we concentrate on the elastic scattering process, where the energy of the fast particle is not changed. For the purpose of calculating particle beam lifetimes due to elastic or Coulomb scattering we ignore screening effects by shell electrons and mathematical divergence problems at very small scattering angles. The scattering process therefore is described by the classical Rutherford scattering with the differential cross section per atom

$$
\frac{\mathrm{d} \sigma}{\mathrm{d} \Omega}=\frac{1}{4 \pi \epsilon_{0}}\left(\frac{z Z e^{2}}{2 \beta c p}\right)^{2} \frac{1}{\sin ^{4}(\theta / 2)},
$$

where $z$ is the charge multiplicity of the incident particle $e Z$ the charge of the heavy scattering nucleus, $\theta$ the scattering angle with respect to the incident path, $\Omega$ the solid angle with $\mathrm{d} \Omega=\sin \theta \mathrm{d} \theta \mathrm{d} \varphi$, and $\varphi$ the polar angle.

To determine the particle beam lifetime or the particle loss rate we will calculate the rate of events for scattering angles larger than a maximum value of $\hat{\theta}$ which is limited by the acceptance of the beam transport line. Any particle being deflected by an angle larger than this maximum scattering angle will be lost. We integrate the scattering cross section over all angles greater than $\hat{\theta}$ up to the maximum scattering angle $\pi$. With $n$ scattering centers or atoms per unit volume and $N$ beam particles the loss rate is

$$
-\frac{\mathrm{d} N}{\mathrm{~d} t}=2 \pi c \beta n N \int_{\hat{\theta}}^{\pi} \frac{\mathrm{d} \sigma}{\mathrm{d} \Omega} \sin \theta \mathrm{d} \theta
$$


Under normal conditions at $0{ }^{\circ} \mathrm{C}$ and a gas pressure of $760 \mathrm{~mm}$ mercury the number of scattering centers in a homogeneous gas is equal to twice Avogadro's number $\mathcal{A}$ and becomes for an arbitrary gas pressure $P$

$$
n=2 \mathcal{A} \frac{P(\text { Torr })}{760}=2 \times 2.68675 \times 10^{19} \frac{P(\text { Torr })}{760} .
$$

The factor 2 comes from the fact that homogeneous gases are composed of two atomic molecules, where each atom acts as a separate scattering center. This assumption would not be true for single atomic noble gases which we do not consider here, but will be included in a later generalization. The integral on the r.h.s. of (11.105) becomes with (11.104)

$$
\int_{\hat{\theta}}^{\pi} \frac{\sin \theta d \theta}{\sin ^{4}(\theta / 2)}=\frac{2}{\tan ^{2}(\hat{\theta} / 2)} .
$$

Dividing (11.105) by $N$ we find an exponential decay of beam intensity with time

$$
N=N_{0} \mathrm{e}^{-t / \tau}
$$

where the decay time constant or beam lifetime is

$$
\tau^{-1}=c \beta 2 \mathcal{A} \frac{P(\text { Torr })}{760}\left(\frac{z Z e^{2}}{2 \beta c p}\right)^{2} \frac{4 \pi}{\tan ^{2}(\hat{\theta} / 2)} .
$$

The maximum acceptable scattering angle $\hat{\theta}$ is limited by the acceptance $\epsilon_{A}$ of the beam transport line. A particle being scattered by an angle $\theta$ at a location where the betatron function has the value $\beta_{\theta}$ reaches a maximum betatron oscillation amplitude of $a=\sqrt{\beta_{a} \beta_{\theta}} \theta$ elsewhere along the beam transport line where the betatron function is $\beta_{a}$. The minimum value of $A^{2} / \beta_{A}$ along the ring lattice, where $A$ is the vacuum chamber aperture or the limit of the dynamic aperture whichever is smaller, is equal to the ring acceptance

$$
\epsilon_{A}=\left.\frac{A^{2}}{\beta_{A}}\right|_{\min } .
$$

For simplicity we ignore here the variation of the betatron function and take an average value $\langle\beta\rangle$ at the location of the scattering event and get finally for the maximum allowable scattering angle

$$
\hat{\theta}^{2}=\frac{\epsilon_{A}}{\langle\beta\rangle}
$$

This angle is generally rather small and we may set $\tan (\hat{\theta} / 2) \approx(\hat{\theta} / 2)$. Utilizing these definitions and approximations we obtain for the lifetime of a beam made up 
of singly charged particles $z=1$ due to elastic Coulomb scattering expressed in more practical units

$$
\tau_{\mathrm{cs}} \text { (hours) }=10.25 \frac{(c p)^{2}\left(\mathrm{GeV}^{2}\right) \epsilon_{\mathrm{A}}(\mathrm{mm} \mathrm{mrad})}{\langle\beta(\mathrm{m})\rangle P(\mathrm{nTorr})},
$$

where we have assumed that the residual gas composition is equivalent to nitrogen gas $\mathrm{N}_{2}$ with $Z^{2} \approx 49$. The Coulomb scattering lifetime is proportional to the ring acceptance or proportional to the square of the aperture $A$ where $A^{2} / \beta$ is a minimum.

The particle loss due to Coulomb scattering is most severe at low energies and increases with the acceptance of the beam transport line. Furthermore, the beam lifetime depends on the focusing in the transport line through the average value of the betatron function. If instead of averaging the betatron function we integrate the contributions to the beam lifetime along the transport line we find that the effect of the scattering event depends on the betatron function at the location of the collision and the probability that such a collision occurs at this location depends on the gas pressure there. Therefore, it is prudent to not only minimize the magnitude of the betatron functions alone but rather minimize the product $\beta P$ along the transport line. Specifically, where large values of the betatron function cannot be avoided, extra pumping capacity should be provided to reach locally a low vacuum pressure for long Coulomb scattering lifetime.

We have made several simplifications and approximations by assuming a homogeneous gas and assuming that the maximum scattering angle be the same in all directions. In practical situations, however, the acceptance need not be the same in the vertical and horizontal plane. First we will derive the beam lifetime for nonisotropic aperture limits. We assume that the apertures in the horizontal and vertical plane allow maximum scattering angles of $\hat{\theta}_{x}$ and $\hat{\theta}_{y}$. Particles are then lost if the scattering angle $\theta$ into a polar angle $\varphi$ exceeds the limits

$$
\theta>\frac{\hat{\theta}_{x}}{\cos \varphi} \text { and } \quad \theta>\frac{\hat{\theta}_{y}}{\sin \varphi} .
$$

The horizontal aperture will be relevant for all particles scattered into a polar angle between zero and $\arctan \left(\hat{\theta}_{y} / \hat{\theta}_{x}\right)$ while particles scattered into a polar angle of $\arctan \left(\hat{\theta}_{y} / \hat{\theta}_{x}\right)$ and $\pi / 2$ will be absorbed by the vertical aperture whenever the scattering angle exceeds this limit. We calculate the losses in only one quadrant of the polar variable and multiply the result by 4 since the scattering and absorption process is symmetric about the polar axis. The integral (11.107) becomes in this case

$$
\begin{aligned}
\int_{\hat{\theta}}^{\pi} \frac{\sin \theta \mathrm{d} \theta \mathrm{d} \varphi}{\sin ^{4}(\theta / 2)} & =4 \int_{0}^{\arctan \left(\hat{\theta}_{y} / \hat{\theta}_{x}\right)} \mathrm{d} \varphi \int_{\hat{\theta}_{x} / \cos \varphi}^{\pi} \frac{\sin \theta \mathrm{d} \theta}{\sin ^{4}(\theta / 2)} \\
& +4 \int_{\arctan \left(\hat{\theta}_{y} / \hat{\theta}_{x}\right)}^{\pi / 2} \mathrm{~d} \varphi \int_{\hat{\theta}_{y} / \sin \varphi}^{\pi} \frac{\sin \theta \mathrm{d} \theta}{\sin ^{4}(\theta / 2)} .
\end{aligned}
$$


The solutions of the integrals are similar to that in (11.107) and we get

$$
\begin{gathered}
\int_{\hat{\theta}}^{\pi} \frac{\sin \theta \mathrm{d} \theta \mathrm{d} \varphi}{\sin ^{4}(\theta / 2)}=\frac{8}{\hat{\theta}_{y}^{2}}\left[\pi+\left(R^{2}+1\right) \sin (2 \arctan R)\right. \\
+2(R-1) \arctan R],
\end{gathered}
$$

where $R=\hat{\theta}_{y} / \hat{\theta}_{x}$.

Using (11.115) instead of (11.107) in (11.109) gives a more accurate expression for the beam lifetime due to Coulomb scattering. We note that for $R=1$ we do not get exactly the lifetime (11.109) but find a lifetime that is larger by a factor of $1+\pi / 2$. This is because we used a rectangular aperture in (11.115) compared to a circular aperture in (11.107). The beam lifetime (11.112) becomes now for a rectangular acceptance

$$
\tau_{\mathrm{cs}}(\text { hours })=10.25 \frac{2 \pi}{F(R)} \frac{(c p)^{2}\left(\mathrm{GeV}^{2}\right) \epsilon_{\mathrm{A}}(\mathrm{mm} \mathrm{mrad})}{\langle\beta(\mathrm{m})\rangle P(\text { nTorr })} .
$$

The function $F(R)$

$$
F(R)=\left[\pi+\left(R^{2}+1\right) \sin (2 \arctan R)+2\left(R^{2}-1\right) \arctan R\right.
$$

is shown in Fig. 11.5. For some special cases the factor $2 \pi / F(R)$ assumes the values

\begin{tabular}{cccc}
\hline shape of aperture & round & square & rectangular \\
ratio: $R=\hat{\theta}_{y} / \hat{\theta}_{x}$ & 1.00 & 1.00 & $0 \rightarrow 1$ \\
$2 \pi / F(R)$ & 1.00 & 1.22 & $2 \rightarrow 1.22$ \\
\hline
\end{tabular}

Fig. 11.5 Function $F(R)$ to determine the acceptance for Coulomb scattering

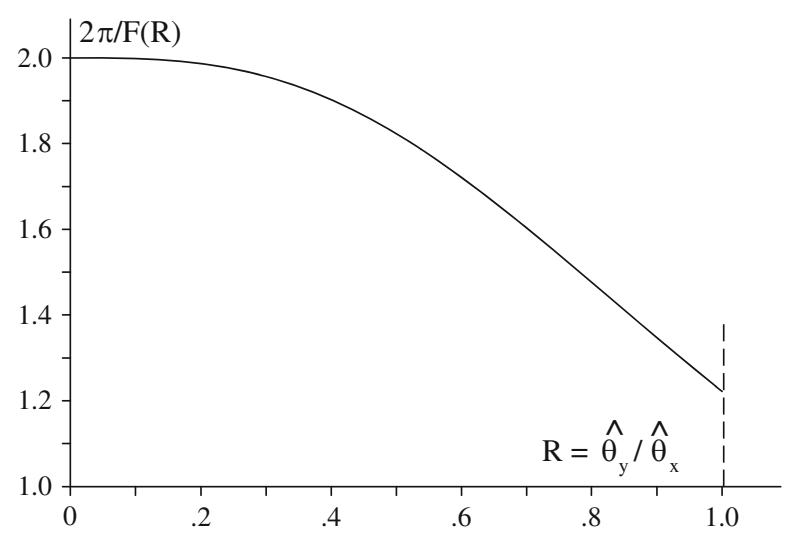


Tacitly we have assumed that the vertical acceptance is smaller than the horizontal acceptance which in most cases is true. In cases, where $\hat{\theta}_{y}>\hat{\theta}_{x}$, we may use the same equations with $x$ and $y$ exchanged.

Particles performing large amplitude betatron oscillations form a Coulomb scattering halo around the beam proper. In case of an electron storage ring the particle intensity in this halo reaches an equilibrium between the constant supply of scattered electrons and synchrotron radiation damping.

The deviation of the particle density distribution from a Gaussian distribution due to scattering can be observed and measured. In Fig. 11.6 beam lifetime measurements are shown for an electron beam in a storage ring as a function of a variable ring acceptance as established by a movable scraper. The abscissa is the actual position of the scraper during the beam lifetime measurement, while the variable for the ordinate is the aperture for which a pure Gaussian particle distribution would give the same beam lifetime.

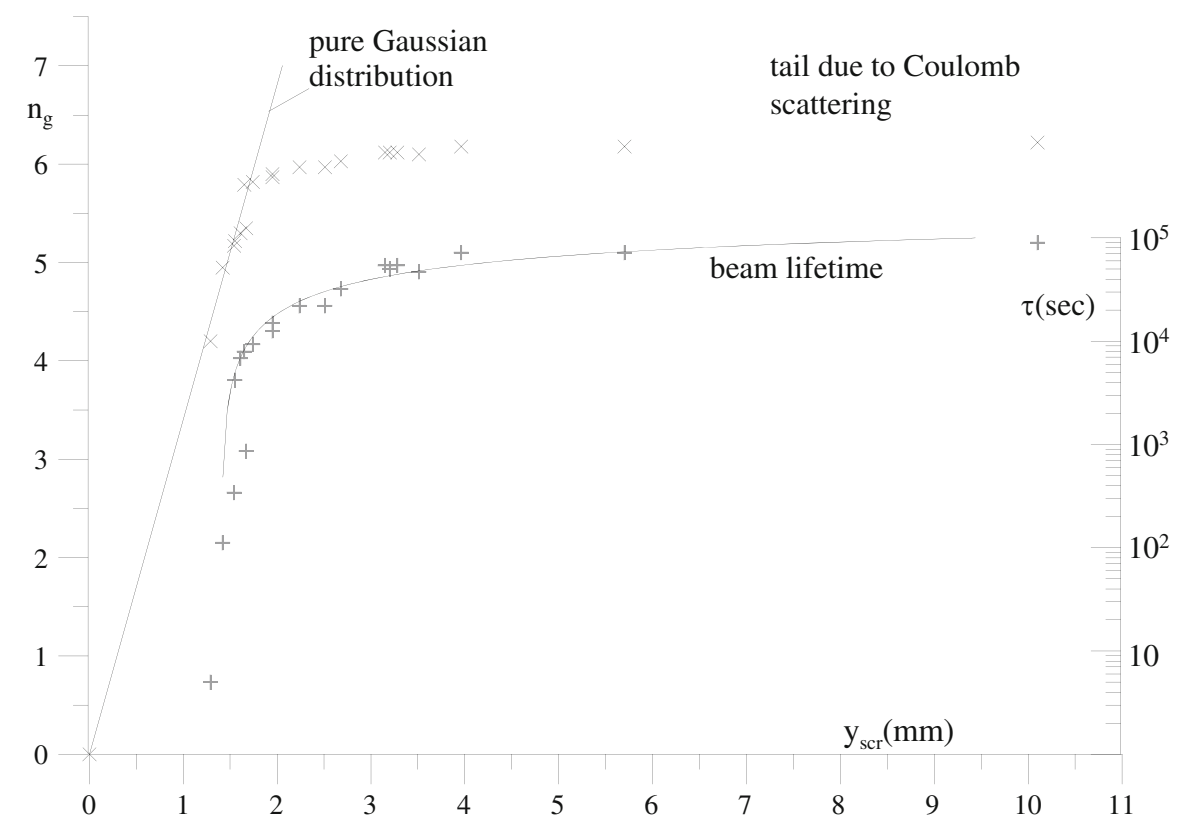

Fig. 11.6 Measurement of beam lifetime in an electron storage ring with a movable scraper. The curve on the left shows the Coulomp scattering halo for amplitudes larger than $6 \sigma$ indicating a strong deviation from Gaussian particle distribution. the curve on the right shows the beam life time as a function of scraper position 
If the particle distribution had been purely Gaussian the measured points would lie along a straight line. In reality, however, we observe an overpopulation of particles in the tails of the distribution for amplitudes larger than about $6 \sigma$ forcing the scraper to be located farther away from the beam center to get a beam lifetime equal to that of a pure Gaussian distribution. This overpopulation or halo at large amplitudes is due to elastic Coulomb scattering on the residual gas atoms.

Since the acceptance of the storage ring is proportional to the square of the aperture at the scraper, we expect the beam lifetime due to Coulomb scattering to vary proportional to the square of the scraper position. This is shown in Fig. 11.7 for good vacuum and poor vacuum conditions. In the case of poor vacuum we find a saturation of the beam lifetime at large scraper openings which indicates that the scraper is no longer the limiting aperture in the ring. This measurement therefore allows an accurate determination of the physical ring acceptance or the dynamic aperture whichever is smaller.

So far we have assumed the residual gas to consist of homogeneous two atom molecules. This is not an accurate description of the real composition of the residual gas although on average the residual gas composition is equivalent to a nitrogen gas. Where the effects of a more complex gas composition becomes important, we apply (11.109) to each different molecule and atom of the residual gas and we replace the relevant factor $P Z^{2}$ by a summation over all gas components. If $P_{i}$ is

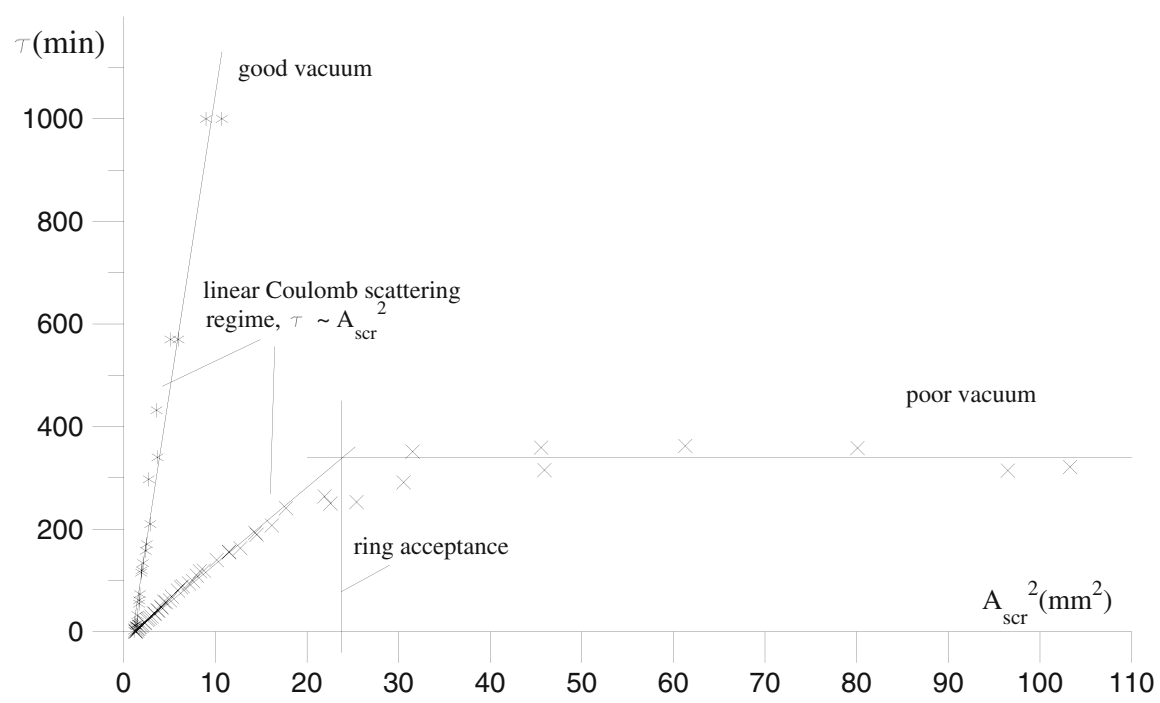

Fig. 11.7 Beam lifetime in an electron storage ring as a function of the acceptance. The transition of the curve on the right from a linear dependence of beam lifetime on the acceptance to a constant life time occurs when the acceptance due to the scraper position is equal to the ring acceptance 
the partial pressure of the molecules $i$ and $Z_{j}$ the atomic number of the atom $j$ in the molecule $i$ we replace in (11.109)

$$
P Z^{2} \rightarrow \sum_{i, j} P_{i} Z_{j}^{2}
$$

and sum over all atoms $i$ in the molecule $j$.

\section{Inelastic Scattering}

Charged particles passing through matter become deflected by strong electrical fields from the atomic nuclei. This deflection constitutes an acceleration and the charged particles lose energy through emission of radiation which is called bremsstrahlung. If this energy loss is too large such that the particle energy error becomes larger than the storage ring energy acceptance the particle gets lost. We are therefore interested to calculate the probability for such large energy losses to estimate the beam lifetime.

The probability to suffer a relative energy $\operatorname{loss} \delta=\mathrm{d} E / E_{0}$ due to such an inelastic scattering process has been derived by Bethe and Heitler [16, 17]. For extreme relativistic particles and full screening this probability per unit thickness of matter is [17]

$$
\mathrm{d} P=2 \bar{\Phi} n \frac{\mathrm{d} \delta}{\delta}(1-\delta)\left[\left(\frac{2-2 \delta+\delta^{2}}{1-\delta}-\frac{2}{3}\right) 2 \ln \frac{183}{Z^{1 / 3}}+\frac{2}{9}\right]
$$

where $n$ is the number of atoms per unit volume and the factor $\bar{\phi}$ is with the fine structure constant $\alpha=1 / 137$

$$
\bar{\Phi}=r_{\mathrm{e}}^{2} Z^{2} \alpha
$$

We integrate this probability over all energy losses larger than the energy acceptance of the storage ring $\delta \geq \delta_{\text {acc }}$ and get after some manipulation and setting $\delta_{\text {acc }} \ll 1$

$$
\begin{aligned}
P & =2 \bar{\Phi} n \int_{\delta_{\mathrm{acc}}}^{1} \frac{\mathrm{d} \delta}{\delta}(1-\delta)\left[\left(\frac{2-2 \delta+\delta^{2}}{1-\delta}-\frac{2}{3}\right) 2 \ln \frac{183}{Z^{1 / 3}}+\frac{2}{9}\right] \\
& \approx \frac{3}{4}\left(-\ln \delta_{\mathrm{acc}}\right)\left(4 \ln \frac{183}{Z^{1 / 3}}+\frac{3}{9}\right) n \bar{\Phi}
\end{aligned}
$$


The radiation length $L_{\mathrm{r}}$ is defined as the distance over which the particle energy has dropped to $1 / e$ due to inelastic scattering. For highly relativistic particles this length is given by [17]

$$
\frac{1}{L_{\mathrm{r}}}=\bar{\Phi} n\left(4 \ln \frac{183}{Z^{1 / 3}}+\frac{2}{9}\right) .
$$

Combining (11.121) and (11.122), we find the simple solution that the probability for a particle to suffer a relative energy loss of more than $\delta_{\text {acc }}$ per radiation length is

$$
P_{\mathrm{rad}}=-\frac{4}{3} \ln \delta_{\mathrm{acc}} .
$$

To calculate the beam lifetime or beam decay rate due to bremsstrahlung we note that the probability for a particle loss per unit time is equal to the beam decay rate or equal to the inverse of the beam lifetime. The bremsstrahlung lifetime is therefore

$$
\tau_{\mathrm{bs}}^{-1}=-\frac{1}{N_{0}} \frac{\mathrm{d} N}{\mathrm{~d} t}=P \frac{c}{L_{\mathrm{r}}}=-\frac{4}{3} \frac{c}{L_{\mathrm{r}}} \ln \delta_{\mathrm{acc}} .
$$

The radiation length for gases are usually expressed for a standard temperature of $20^{\circ} \mathrm{C}$ and a pressure of 760 Torr. Under vacuum conditions the radiation length of the residual gas is therefore increased by the factor $760 / P_{\text {Torr }}$. We recognize again the complex composition of the residual gas and define an effective radiation length by

$$
\frac{1}{L_{\mathrm{r}, \mathrm{eff}}}=\sum_{i} \frac{1}{L_{\mathrm{r}, i}}
$$

where $L_{\mathrm{r}, i}$ is the radiation length for gas molecules of type $i$. The beam lifetime due to bremsstrahlung for a composite residual gas is from (11.124), (11.125)

$$
\tau_{\mathrm{bs}}^{-1}=-\frac{4}{3} c \sum_{i} \frac{1}{760} \frac{\tilde{P}_{i}}{L_{\mathrm{r}, i}} \ln \delta_{\mathrm{acc}},
$$

where $\tilde{P}_{i}$ is the residual partial gas pressure for gas molecules of type $i$. Although the residual gas of ultra high vacuum systems rarely includes a significant amount of nitrogen gas, the average value for $\left\langle Z^{2}\right\rangle$ of the residual gas components is approximately 50 or equivalent to nitrogen gas. For all practical purposes we may therefore assume the residual gas to be nitrogen with a radiation length under normal conditions of $L_{\mathrm{r}, \mathrm{N}_{2}}=290 \mathrm{~m}$ and scaling to the actual vacuum pressure $P_{\mathrm{vac}}$ we get for the beam lifetime

$$
\tau_{\mathrm{bs}}^{-1}\left(\text { hours }^{-1}\right)=0.00653 P_{\text {vac }}(\text { nTorr }) \ln \frac{1}{\delta_{\text {acc }}} .
$$


Basically the bremsstrahlung lifetime depends only on the vacuum pressure and the energy acceptance and the product of beam lifetime and vacuum pressure is a function of the energy acceptance $\delta_{\text {acc }}=\Delta \gamma / \gamma$,

$$
\tau_{\mathrm{bs}}(\text { hour }) P(\text { nTorr })=\frac{153.14}{\ln (\gamma / \Delta \gamma)} .
$$

In tabular form we get:

$$
\begin{aligned}
& \delta_{\mathrm{acc}}=\Delta \gamma / \gamma \quad 0.0050 .0100 .0150 .0200 .025 \\
& \tau \text { (hr) } P \text { (nTorr) } 28.9033 .2536 .4639 .1541 .51
\end{aligned}
$$

There are many more forms of interaction possible between energetic particles and residual gas atoms. Chemical, atomic, and nuclear reactions leading to the formation of new molecules like ozone, ionization of atoms or radioactive products contribute further to energy loss of the beam particles and eventual loss from the beam. These effects, however, are very small compared to Coulomb scattering or bremsstrahlung losses and may therefore be neglected in the estimation of beam lifetime.

\subsubsection{Ultra High Vacuum System}

Accelerated particles interact strongly with residual gas atoms and molecules by elastic and inelastic collisions. To minimize particle loss due to such collisions we provide an evacuated beam pipe along the desired beam path. For open beam transport systems high vacuum of $10^{-5}-10^{-7}$ Torr is sufficient. This is even sufficient for pulsed circular accelerators like synchrotrons, where the particles remain only for a short time. In storage rings, however, particles are expected to circulate for hours and therefore ultra high vacuum conditions must be created.

\section{Thermal Gas Desorption}

To reach very low gas pressures in the region of $10^{-10}-10^{-11}$ Torr in the regime of ultra high vacuum (UHV) we must consider the continuous desorption of gas molecules from the walls due to thermal desorption. Gas molecules adsorbed on the chamber surface are in thermal equilibrium with the environment and the thermal energy of the molecules assumes a statistically determined Boltzmann distribution. This distribution includes a finite probability for molecules to gain a large enough amount of energy to overcome the adsorption energy and be released from the wall.

The total gas flow from the wall due to this thermal gas desorption depends mostly on the preparation of the material. While for carefully cleaned surfaces the thermal desorption coefficient may be of the order of $10^{-12}-10^{-13} \mathrm{Torr} 1 \mathrm{t} / \mathrm{sec} / \mathrm{cm}^{2}$ a bakeout to $140-300^{\circ} \mathrm{C}$ can reduce this coefficient by another order of magnitude. 


\section{Synchrotron Radiation Induced Desorption}

In high-energy electron or positron accelerators a significant amount of energy is emitted in form of synchrotron radiation. This radiation is absorbed by vacuum chamber walls and causes not only a heating effect of the chamber walls but also the desorption of gas molecules adsorbed on the surface.

The physical process of photon induced gas desorption evolves in two steps [18]. First a photon hitting the chamber walls causes a secondary electron emission with the probability $\eta_{\mathrm{e}}(\varepsilon)$, where $\varepsilon$ is the photon energy. Secondly, the emission as well as the subsequent absorption of that photoelectron can desorb neutral atoms from the chamber surface with the probability $\eta_{\mathrm{d}}$. To calculate the total desorption in a storage ring, we start from the differential synchrotron radiation photon flux (24.56) which we integrate over the ring circumference and write now in the form

$$
\frac{\mathrm{d} N(\varepsilon)}{\mathrm{d} t}=\frac{8 \pi \alpha}{9} \gamma \frac{I_{\mathrm{b}}}{e} \frac{\Delta \omega}{\omega} S(\zeta),
$$

where $\varepsilon=\hbar \omega$ is the photon energy, $I_{\mathrm{b}}$ the beam current, $E$ the beam energy and $S(\zeta)$ a mathematical function defined by (24.57).

The photoelectron current $\dot{N}_{\mathrm{e}}$ results from the folding of (11.129) with the photoelectron emission coefficient $\eta_{\mathrm{e}}(\omega)$ for the material used to construct the vacuum chamber and the integration over all photon energies,

$$
\dot{N}_{\mathrm{e}}=\frac{8 \pi \alpha}{9 e m c^{2}} E I_{\mathrm{b}} \int_{0}^{\infty} \frac{\eta_{\mathrm{e}}(\omega)}{\omega} S\left(\frac{\omega}{\omega_{c}}\right) \mathrm{d} \omega .
$$

The photoelectron emission coefficient depends on the choice of the material for the vacuum chamber. Figure 11.8 displays the photoelectron coefficient for aluminum as a function of photon energy [19].

Fig. 11.8 Photon electron coefficient $\eta_{\mathrm{e}}$ for aluminum [19]

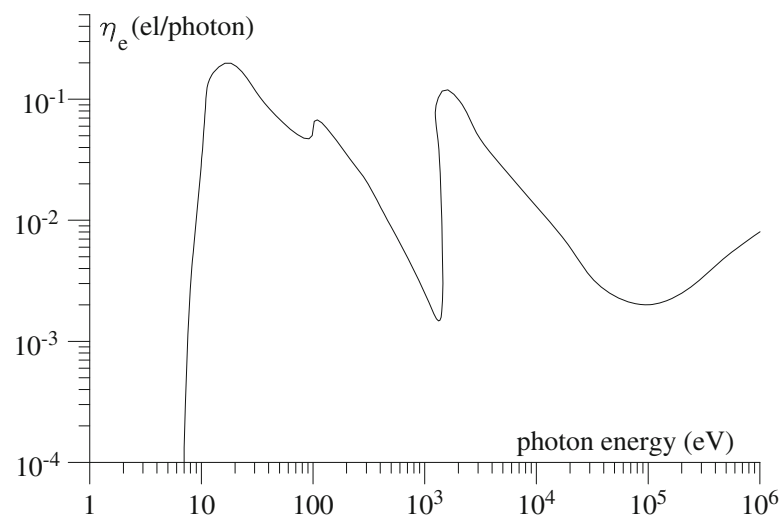


We note there are virtually no photoelectrons for photon energies of less than $10 \mathrm{eV}$. At $1,460 \mathrm{eV}$ the $\mathrm{K}$-edge of aluminum causes a sharp increase of the coefficient followed by a monotonous decrease for higher photon energies.

The photoelectron coefficient depends not only on the material of the photon absorber but also on the incident angle. The probability to release an electron from the surface is increased for shallow incidence of the photon. The enhancement factor $F(\Theta)$ represents the increase in the photoelectron-emission coefficient $\eta_{\mathrm{e}}(\epsilon)$ due to a non normal incidence of a photon on the surface, where $\Theta$ is the angle between the photon trajectory and the plane to the absorbing surface. For angles close to normal incidence $\left(\Theta=90^{\circ}\right)$ the enhancement factor scales like the inverse of the sine of the angle

$$
F(\Theta)=\frac{1}{\sin \Theta} .
$$

For small angles, however, the enhancement factor falls off from the inverse sine dependence as has been determined by measurements [20] and reaches a maximum value of about seven for small angles. The gas production is determined by the desorption rate $Q$, defined as the total number of neutral atoms released along the circumference from the chamber surface,

$$
Q=2 \frac{22.4 \times 760}{6 \times 10^{23}} \dot{N}_{\mathrm{e}} \eta_{\mathrm{d}},
$$

where $Q$ is expressed in Torr $1 \mathrm{t} / \mathrm{sec}$ and $\eta_{\mathrm{d}}$ is the desorption coefficient. The factor 2 is due to the fact that a photo electron can desorb an atom while leaving or arriving at a surface. With (11.132) we get the average vacuum pressure $\langle P\rangle$ from

$$
\langle P\rangle=\frac{Q}{S},
$$

where $S$ is the total installed pumping speed in the storage ring. For a reasonably accurate estimate of the photon flux we may use the small argument approximation (24.60) for photon energies $\varepsilon \leq \varepsilon_{\mathrm{c}}$. Photons of higher energies generally do not contribute significantly to the desorption since there are only few. To obtain the photon flux we therefore need to integrate only from $10 \mathrm{eV}$ to $\epsilon \approx \epsilon_{\mathrm{c}}$ the differential photon flux (24.60) folded with the photoelectron-emission coefficient $\eta_{\mathrm{e}}(\varepsilon)$.

The desorption coefficient $\eta_{\mathrm{d}}$ is largely determined by the treatment of the vacuum chamber like baking, beam cleaning, argon discharge cleaning, etc. For example in the aluminum chamber of the storage ring SPEAR [21] the desorption coefficient at $1.5 \mathrm{GeV}$ was initially about $\eta_{\mathrm{d}} \approx 5 \times 10^{-3}$ then $5 \times 10^{-4}$ after 1 month of operation, $10^{-4}$ after 2 months of operation and reached about $3 \times 10^{-6}$ after about 1 year of operation. These numbers are not to be viewed too generally, since the cleaning process depends strongly on the particular preparation of the surfaces. However, following well established cleaning procedures and handling of ultra high vacuum components these numbers can be of general guidance consistent with observations on other storage rings. 
Laboratory measurements [19] show the following relationship between photoelectron current $I_{\text {phe }}=e \dot{N}_{\mathrm{e}}$, desorption coefficient $\eta_{\mathrm{d}}$ and total integrated beam time of a vacuum system

$$
\eta_{\mathrm{d}}=7 \times 10^{-5} \frac{I_{\mathrm{phe}}(\mathrm{A})}{t(\mathrm{hr})^{0.63}} .
$$

New vacuum chambers release much gas when the first synchrotron radiation strikes the surface, but cleans quickly as the radiation cleaning continues.

\section{Problems}

11.1 (S). The Rf-frequency of a storage ring is $500 \mathrm{MHz}$. Every bucket is filled with particles. What is the time difference between successive bunches?

11.2 (S). Calculate the synchrotron damping time for a $3 \mathrm{GeV}$ storage ring with a bending radius of $\rho=10 \mathrm{~m}$ and pure rectangular dipole magnets. Assume $100 \%$ bending magnet fill factor. What is the synchrotron damping time in this ring? How long does it take to radiate away all its energy?

11.3. Consider a circular electron storage ring of your choice and specify beam energy, current, ring circumference and average vacuum chamber dimensions. Calculate the total thermal gas desorption and the total required pumping capacity in the ring. Now add synchrotron radiation and estimate the increase of pumping speed needed after say $100 \mathrm{Ah}$ of beam operation. Plot the average gas pressure as a function of integrated beam time.

11.4. An electron beam circulating in a $1.5 \mathrm{GeV}$ storage ring emits synchrotron radiation. The rms emission angle of photons is $1 / \gamma$ about the forward direction of the particle trajectory. Determine the photon phase space distribution at the source point and at a distance of $10 \mathrm{~m}$ away while ignoring the finite particle beam emittance. Now assume a Gaussian particle distribution with a horizontal beam emittance of $\epsilon_{x}=1.5 \times 10^{-7} \mathrm{rad} \mathrm{m}$. Fold both the photon and particle distributions and determine the photon phase space distribution $10 \mathrm{~m}$ away from the source point if the electron beam size is $\sigma_{x}=1.225 \mathrm{~mm}$, the electron beam divergence $\sigma_{x^{\prime}}=0.1225 \mathrm{mrad}$ and the source point is a symmetry point of the storage ring. Assume the dispersion function to vanish at the source point. For what minimum photon wavelength would the vertical electron beam size appear diffraction limited if the emittance coupling is $10 \%$ ?

11.5. Consider an electron beam in an isomagnetic $6 \mathrm{GeV}$ storage ring with a bending radius of $\rho=20 \mathrm{~m}$. Calculate the rms energy spread $\sigma_{\varepsilon} / E_{0}$ and the synchrotron oscillation damping time $\tau_{s}$. 


\section{References}

1. B. Touschek, in Proceedings of 1963 Summer Study on Storage Rings, Acceleration and Experiment at Super High Energies, vol. BNL-Report 7534 (Brookhaven BNL, New York, 1956), p. 171

2. A. Piwinski, in 9th International Conference on High Energy Accelerators (Stanford Linear Accelerator Center, Stanford, 1974), p. 405

3. J.D. Bjorken, S.K. Mtingwa, Part. Accel. 13, 115 (1983)

4. K.W. Robinson, Phys. Rev. 111, 373 (1958)

5. M. Sands, Phys. Rev. 97, 470 (1955)

6. D. Deacon, Theory of the isochronous storage ring laser. Ph.D. thesis, Stanford University, Stanford, 1979

7. C. Pellegrini, D. Robin, Nucl. Instrum. Methods A301, 27 (1991)

8. H. Wiedemann, Technical Report, PEP-Note 48, Stanford Linear Accelerator Center, Stanford (1973)

9. J.M. Paterson, J.R. Rees, H. Wiedemann, Technical Report, PEP-Note 125, Stanford Linear Accelerator Center, Stanford (1975)

10. S. Tazzari, Electron Storage Rings for the Production of Synchrotron Radiation Lecture Notes of Physics, vol. 296 (Springer, Berlin/Heidelberg, 1986), p. 140

11. H. Wiedemann, Low Emittance Storage Ring Design. Lecture Notes of Physics, vol. 296 (Springer, Berlin/Heidelberg, 1986), p. 390

12. K. Robinson, G.A. Voss, in Proceedings of the International Conference on Electron and Positron Storage Rings (Presses Universitaires de France, Paris, 1966), p. III-4

13. W.K.H. Panofsky, W.A. Wenzel, Rev. Sci. Instrum. 27, 967 (1956)

14. V.L. Highland, Nucl. Instrum. Methods 129, 497 (1975)

15. V.L. Highland, Nucl. Instrum. Methods 161, 171 (1979)

16. H. Bethe, Proc. Camb. Phil. Soc. 30, 524 (1934)

17. W. Heitler, The Quantum Theory of Radiation (Clarendon, Oxford, 1954)

18. E. Garwin, in Proceedings of the 4th International Vacuum Congress (Institute of Physics and the Physical Society, London, 1968), p. 131

19. J. Kouptsidis, A.G. Mathewson, Technical Report, DESY 76/49, DESY, Hamburg (1976)

20. O. Gröbner, A.G. Mathewson, P. Strubin, Technical Report, LEP-VAC/AGM/sm, CERN, Geneva (1983)

21. D. Bostic, U. Cummings, N. Dean, B. Jeong, J. Jurow, IEEE Trans. Nucl. Sci. 22, 1540 (1975) 


\section{Chapter 12 \\ Vlasov and Fokker-Planck Equations*}

Mathematical tools have been derived in previous chapters to describe the dynamics of singly charged particles in electromagnetic fields. While the knowledge of single-particle dynamics is essential for the development of particle beam transport systems, we are still missing a formal treatment of the behavior of multiparticle beams. In principle a multiparticle beam can be described simply by calculating the trajectories of every single particle within this beam, a procedure that is obviously too inefficient to be useful for the description of any real beam involving a very large number of particles.

In this paragraph we will derive concepts to describe the collective dynamics of a beam composed of a large number of particles and its evolution along a transport line utilizing statistical methods that lead to well defined descriptions of the total beam parameters. Mathematical problems arise only when we have a particle beam with neither few particles nor very many particles. Numerical methods must be employed if the number of particles are of importance and where statistical methods would lead to incorrect results.

The evolution of a particle beam has been derived based on Liouville's theorem assuring the constancy of the particle density in phase space. However, this concept has not allowed us to determine modifications of particle distributions due to external forces. Particle distributions are greatly determined by particle source parameters, quantum effects due to synchrotron radiation, nonlinear magnetic fields, collisions with other particles in the same beam, with particles in another beam or with atoms of the residual gases in the beam environment to name only a few phenomena that could influence that distribution. In this chapter, we will derive mathematical methods that allow the determination of particle distributions under the influence of various external electromagnetic forces.

This chapter has been made Open Access under a CC BY 4.0 license. For details on rights and licenses please read the Correction https://doi.org/10.1007/978-3-319-18317-6_28 


\subsection{The Vlasov Equation}

To study the development of a particle beam along a transport line, we will concentrate on the evolution of a particle density distribution function $\Psi(\boldsymbol{r}, \boldsymbol{p}, t)$ in six-dimensional phase space where every particle is represented by a single point. We consider a volume element of phase space that is small enough that we may assume the particle density to be constant throughout that element and determine its evolution in time. In doing so, we will further assume a large, statistically significant number of particles in each volume element and only a slow variation of the particle density from one volume element to any adjacent volume element. To simplify the equations, we restrict the following discussion to two-dimensional phase space $\left(w, p_{w}\right)$ and use exclusively normalized coordinates. The derivation is exactly the same for other coordinates.

The dynamics of a collection of particles can be studied by observing the evolution of their phase space. Specifically, we may choose a particular phase space element and follow it along its path taking into account the forces acting on it. To do this, we select a phase space element in form of a rectangular box defined by the four corner points $P_{i}$ in Fig. 12.1.

At the time $t$ these corners have the coordinates

$$
\begin{aligned}
& P_{1}\left(w, p_{w}\right), \\
& P_{2}\left(w+\Delta w, p_{w}\right), \\
& P_{3}\left(w+\Delta w, p_{w}+\Delta p_{w}\right), \\
& P_{4}\left(w, p_{w}+\Delta p_{w}\right) .
\end{aligned}
$$

A short time $\Delta t$ later, this rectangular box will have moved and may be deformed into a new form of a quadrilateral $\left(\mathrm{Q}_{1}, \mathrm{Q}_{2}, \mathrm{Q}_{3}, \mathrm{Q}_{4}\right)$ as shown in Fig. 12.1. In determining the volume of the new box at time $t+\Delta t$ we will assume the conservation of particles allowing no particles to be generated or getting lost. To keep the derivation general the rate of change in the conjugate variables is defined by

$$
\begin{aligned}
& \dot{w}=f_{w}\left(w, p_{w}, t\right), \\
& \dot{p}_{w}=g_{w}\left(w, p_{w}, t\right),
\end{aligned}
$$

Fig. 12.1 Two-dimensional motion of a rectangle in phase space

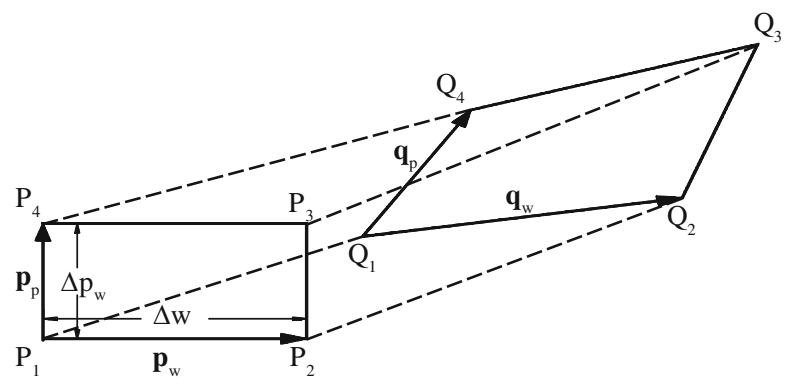


where $\dot{w}=\mathrm{d} w / \mathrm{d} t$ and $\dot{p}_{w}=\mathrm{d} p_{w} / \mathrm{d} t$ and the time interval $\Delta t$ is small enough to allow linear expansion of the particle motion. In other words, the time interval shall be chosen such that no physical parameters of the dynamical system change significantly and a Taylor's expansion up to linear terms can be applied. The new corners of the volume element are then given by

$$
\begin{aligned}
& Q_{1}\left[w+f_{w}\left(w, p_{w}, t\right) \Delta t, p_{w}+g_{w}\left(w, p_{w}, t\right) \Delta t\right], \\
& Q_{2}\left[w+\Delta w+f_{w}\left(w+\Delta w, p_{w}, t\right) \Delta t,\right. \\
& \left.p_{w}+g_{w}\left(w+\Delta w, p_{w}, t\right) \Delta t\right], \\
& Q_{3}\left[w+\Delta w+f_{w}\left(w+\Delta w, p_{w}+\Delta p_{w}, t\right) \Delta t,\right. \\
& \left.p_{w}+\Delta p_{w}+g_{w}\left(w+\Delta w, p_{w}+\Delta p_{w}, t\right) \Delta t\right], \\
& Q_{4}\left[w+f_{w}\left(w, p_{w}+\Delta p_{w}, t\right) \Delta t,\right. \\
& \left.p_{w}+\Delta p_{w}+g_{w}\left(w, p_{w}+\Delta p_{w}, t\right) \Delta t\right] .
\end{aligned}
$$

The goal of our discussion is now to derive an expression for the particle density $\Psi\left(w, p_{w}, t\right)$ after a time $\Delta t$. Because of the conservation of particles we have

$$
\Psi\left(w+f_{w} \Delta t, p_{w}+g_{w} \Delta t, t+\Delta t\right) \Delta A_{Q}=\Psi\left(w, p_{w}, t\right) \Delta A_{P},
$$

where $\Delta A_{P}$ and $\Delta A_{Q}$ are the areas in phase space as defined by the corner points $P_{i}$ and $Q_{i}$, respectively. From Fig. 12.1 and (12.1) we derive an expression for the phase space areas which are at the starting time $t$

$$
\Delta A_{P}=\Delta w \Delta p_{w}
$$

and at the time $t+\Delta t$ from (12.3)

$$
\Delta A_{Q}=\Delta w \Delta p_{w}\left[1+\left(\frac{\partial f_{w}}{\partial w}+\frac{\partial g_{w}}{\partial p_{w}}\right) \Delta t\right],
$$

where Taylor's expansions have been used for the functions $f_{w}$ and $g_{w}$ retaining only linear terms. To prove (12.6) we note that the area $\Delta A_{P}$ has the form of a quadrilateral with its sides determined by two vectors and the area, therefore, is equal to the determinant formed by these two vectors. In our case these vectors are $\boldsymbol{p}_{w}=(\Delta w, 0)$ pointing from $P_{1}$ to $P_{2}$ and $\boldsymbol{p}_{p}=\left(0, \Delta p_{w}\right)$ pointing from $P_{1}$ to $P_{4}$. The area therefore is

$$
\left|\boldsymbol{p}_{w}, \boldsymbol{p}_{p}\right|=\left|\begin{array}{cc}
\Delta w & 0 \\
0 & \Delta p_{w}
\end{array}\right|=\Delta w \Delta p_{w}=\Delta A_{P}
$$

in agreement with (12.5). A time interval $\Delta t$ later these vectors will have changed as determined by (12.2). Each of the corner points $P_{i}$ is moving although with different 
speed thus distorting the rectangle $P_{i}$ into the shape $Q_{i}$ of Fig. 12.1. To calculate the new vectors defining the distorted area we expand the functions $f_{w}$ and $g_{w}$ in a Taylor's series at the point $\left(w, p_{w}\right)$. While, for example, the $w$-component of the movement of point $P_{1}$ along the $w$ coordinate is given by $f_{w} \Delta t$ the same component for $P_{2}$ changes by $f_{w} \Delta t+\frac{\partial f_{w}}{\partial w} \Delta w \Delta t$. The $w$-component of the vector $\boldsymbol{q}_{w}=Q_{1}-Q_{2}$ therefore becomes $\Delta w+\frac{\partial f_{w}}{\partial w} \Delta w \Delta t$. Similarly, we can calculate the $p$-component of this vector as well as both components for the vector $\boldsymbol{q}_{p}=Q_{1}-Q_{4}$. The phase space area of the distorted rectangle $\left(Q_{1}, Q_{2}, Q_{3}, Q_{4}\right)$ at time $t+\Delta t$ with these vector components is then given by

$$
\left|\boldsymbol{q}_{w}, \boldsymbol{q}_{p}\right|=\left|\begin{array}{cc}
\Delta w+\frac{\partial f_{w}}{\partial w} \Delta w \Delta t & \frac{\partial f_{w}}{\partial p_{w}} \Delta p_{w} \Delta t \\
\frac{\partial g_{w}}{\partial w} \Delta w \Delta t & \Delta p_{w}+\frac{\partial g_{w}}{\partial p_{w}} \Delta p_{w} \Delta t
\end{array}\right|=\Delta A_{Q} .
$$

Dropping second-order terms in $\Delta t$ we get indeed the expression (12.6). Obviously, the phase space volume does not change if

$$
\frac{\partial f_{w}}{\partial w}+\frac{\partial g_{w}}{\partial p_{w}}=0
$$

in agreement with the result obtained in Chap. 8, where we have assumed that the Lorentz force is the only force acting on the particle. In this paragraph, however, we have made no such restrictions and it is this generality that allows us to derive, at least in principle, the particle distribution under the influence of any forces. Equation (12.9) tells us that there is no damping if the velocity $\dot{w}=f_{w}$ is independent of the position and the forces $\dot{p}=g_{w}$ are independent of the momentum.

The factor

$$
\left[1+\left(\frac{\partial f_{w}}{\partial w}+\frac{\partial g_{w}}{\partial p_{w}}\right) \Delta t\right]
$$

in (12.6) is the general Wronskian of the transformation and is not necessarily equal to unity. We have such an example in the form of adiabatic damping. Indeed we have damping or anti-damping whenever the Wronskian is different from unity.

To illustrate this, we use the example of a damped harmonic oscillator, which is described by the second-order differential equation $\ddot{w}+2 \alpha_{w} \dot{w}+\omega_{0}^{2} w=0$, or in form of a set of two linear differential equations

$$
\begin{aligned}
\dot{w}=\omega_{0} p_{w} & =f_{w}\left(w, p_{w}, t\right), \\
\dot{p}_{w}=-\omega_{0} w-2 \alpha_{w} p_{w} & =g_{w}\left(w, p_{w}, t\right) .
\end{aligned}
$$

From this we find indeed the relation

$$
\frac{\partial f_{w}}{\partial w}+\frac{\partial g_{w}}{\partial p_{w}}=-2 \alpha_{w}
$$


where $\alpha_{w}$ is the damping decrement ${ }^{1}$ of the oscillator. We have obtained on a general basis that the phase space density for harmonic oscillators will vary only if damping forces are present. Here we use the term damping in a very general way including excitation depending on the sign of the damping decrement $\alpha_{w}$. The designation $\alpha_{w}$ for the damping decrement may potentially lead to some confusion with the same use for the betatron function $\alpha=-\frac{1}{2} \beta^{\prime}$. However, we choose here to rather require some care than introduce against common use new designations for the damping decrement or the betatron functions. We also note that for all cases where the damping time is long compared to the oscillation time, and we consider here only such cases, the damping occurs for both conjugate trajectories.

The derivation in two-dimensional phase space can easily be generalized to sixdimensional phase space with the generalized volume element

$$
\Delta V_{P}=\Delta r \Delta p
$$

at time $t$ and a time interval $\Delta t$ later

$$
\Delta V_{Q}=\Delta \boldsymbol{r} \Delta \boldsymbol{p}\left[1+\nabla_{r} \boldsymbol{f} \Delta t+\nabla_{p} \boldsymbol{g} \Delta t\right] .
$$

The Nabla operators are defined by

$$
\nabla_{r}=\left(\frac{\partial}{\partial w}, \frac{\partial}{\partial v}, \frac{\partial}{\partial u}\right) \quad \text { and } \quad \nabla_{p}=\left(\frac{\partial}{\partial p_{w}}, \frac{\partial}{\partial p_{v}}, \frac{\partial}{\partial p_{u}}\right)
$$

where $(w, v, u)$ are normalized variables and the vector functions $\boldsymbol{f}$ and $\boldsymbol{g}$ are defined by the components $\boldsymbol{f}=\left(f_{w}, f_{v}, f_{u}\right)$ and $\boldsymbol{g}=\left(g_{w}, g_{v}, g_{u}\right)$.

Equation (12.4) can now be reduced further after applying a Taylor's expansion to the density function $\Psi$. With (12.5), (12.6) and keeping only linear terms

$$
\frac{\partial \Psi}{\partial t}+f_{w} \frac{\partial \Psi}{\partial w}+g_{w} \frac{\partial \Psi}{\partial p_{w}}=-\left(\frac{\partial f_{w}}{\partial w}+\frac{\partial g_{w}}{\partial p_{w}}\right) \Psi .
$$

It is straightforward to generalize this result again to six-dimensional phase space

$$
\frac{\partial \Psi}{\partial t}+f \nabla_{r} \Psi+g \nabla_{p} \Psi=-\left(\nabla_{r} f+\nabla_{p} g\right) \Psi,
$$

which is called the Vlasov equation. If there is no damping the r.h.s. of the Vlasov equation vanishes and we have

$$
\frac{\partial \Psi}{\partial t}+f \nabla_{r} \Psi+g \nabla_{p} \Psi=0
$$

\footnotetext{
${ }^{1}$ The letter $\alpha_{u}$ is used here for the damping decrement. Since in beam dynamics $\alpha_{u}$ is also used to identify a lattice function, a mixup of the quantities could occur. We have chosen not to use a different nomenclature, however, since this choice is too deeply entrenched in the community. With some care, confusion can be avoided.
} 
This is simply the total time derivative of the phase space density $\Psi$ telling us that in the absence of damping it remains a constant of motion. The preservation of the phase space density is Liouville's theorem and we have demonstrated in this paragraph the validity of this theorem for a Hamiltonian system with vanishing dissipating forces $\left(\nabla_{r} \boldsymbol{f}+\nabla_{p} \boldsymbol{g}\right)=0$.

Equation (12.18) describes the evolution of a multiparticle system in phase space where the physics of the particular particle dynamics is introduced through the functions $\boldsymbol{f}(\boldsymbol{r}, \boldsymbol{p}, t)$ and $\boldsymbol{g}(\boldsymbol{r}, \boldsymbol{p}, t)$. The definition of these functions in (12.2) appears similar to that for the Hamiltonian equations of motion. In case $\boldsymbol{r}$ and $\boldsymbol{p}$ are canonical variables we may indeed derive these functions from the Hamiltonian

$$
\begin{gathered}
\dot{\boldsymbol{r}}=\nabla_{p} \mathcal{H}=\boldsymbol{f}, \\
\dot{\boldsymbol{p}}=-\nabla_{r} \mathcal{H}=\boldsymbol{g},
\end{gathered}
$$

where $\mathcal{H}$ is the Hamiltonian of the system. We are therefore, at least in principle, able to solve the evolution of a multiparticle system in phase space if its Hamiltonian is known. It should be emphasized, however, that the variables $(w, p)$ need not be canonical to be used in the Vlasov equation.

It is interesting to apply the Vlasov equation to simple one-dimensional harmonic oscillators with vanishing perturbation. Introducing the canonical variable $p$ through $\dot{w}=v p$, the Hamiltonian becomes $\mathcal{H}_{0}=\frac{1}{2} v p^{2}+\frac{1}{2} v w^{2}$ and the equations of motion are

$$
\begin{aligned}
& \dot{w}=+\frac{\partial \mathcal{H}_{0}}{\partial p}=v p=f, \\
& \dot{p}=-\frac{\partial \mathcal{H}_{0}}{\partial w}=-v w=g .
\end{aligned}
$$

It is customary for harmonic oscillators and similarly for particle beam dynamics to use the oscillation phase as the independent or "time" variable. Since we have not made any specific use of the real time in the derivation of the Vlasov equation, we choose here the phase as the "time" variable. For the simple case of an undamped harmonic oscillator $\frac{\partial f}{\partial w}=0$ and $\frac{\partial g}{\partial p}=0$ and consequently the Vlasov equation becomes from (12.16) with (12.20)

$$
\frac{\partial \Psi}{\partial \varphi}+v p \frac{\partial \Psi}{\partial w}-v w \frac{\partial \Psi}{\partial p}=0 .
$$

In cylindrical phase space coordinates $(w=r \cos \theta, p=r \sin \theta, \varphi)$ this reduces to the simple equation

$$
\frac{\partial \Psi}{\partial \varphi}-v \frac{\partial \Psi}{\partial \theta}=0
$$


Fig. 12.2 Beam motion in phase space

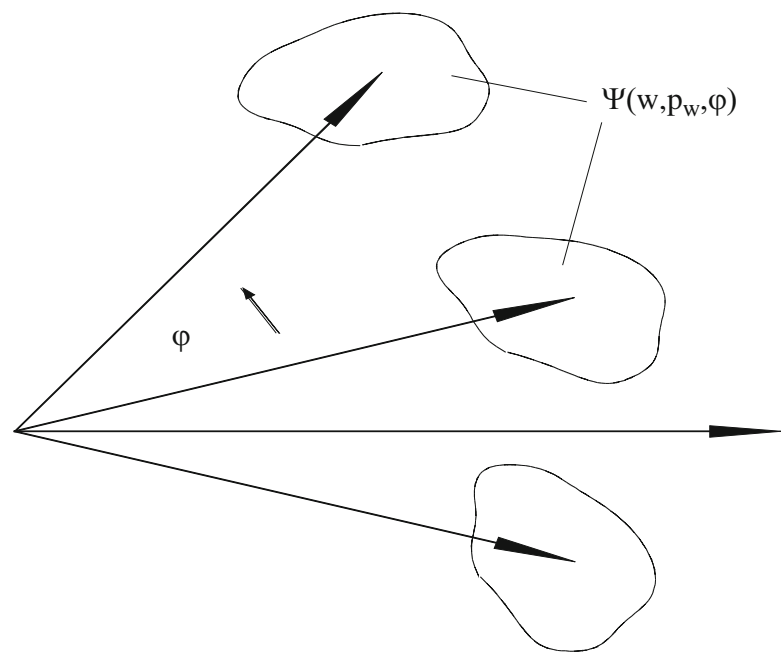

Any differentiable function with the argument $(r, \theta+\nu \varphi)$ can be a solution of (12.22) describing the evolution of the particle density $\Psi$ with time

$$
\Psi\left(w, p_{w}, \varphi\right)=F(r, \theta+v \varphi)
$$

Any arbitrary particle distribution in $\left(w, p_{w}\right)$-phase space merely rotates about the center with the frequency $v$ and remains otherwise unchanged as shown in Fig. 12.2. This is just another way of saying that an ensemble of many particles behaves like the sum of all individual particles since any interaction between particles as well as damping forces have been ignored. In $\left(x, x^{\prime}\right)$-phase space this rotation is deformed into a "rotation" along elliptical trajectories. The equation of motion in $\left(w, p_{w}\right)$ phase space is solved by $r=$ const indicating that the amplitude $r$ is a constant of motion. In $\left(x, x^{\prime}\right)$-phase space we set $w=x / \sqrt{\beta}$ and $p=\sqrt{\beta} x^{\prime}+\frac{\alpha}{\sqrt{\beta}} x$ and get from $r^{2}=w^{2}+p_{w}^{2}$ for this constant of motion

$$
\beta x^{\prime 2}+2 \alpha x x^{\prime}+\gamma x^{2}=\text { const }
$$

which is the Courant-Snyder invariant. The Vlasov equation allows us to generalize this result collectively to all particles in a beam. Any particular particle distribution a beam may have at the beginning of the beam transport line or circular accelerator will be preserved as long as damping or other statistical effects are absent. 


\subsubsection{Betatron Oscillations and Perturbations}

The Vlasov equation will prove to be a useful tool to derive particle beam parameters. Specifically, it allows us to study the influence of arbitrary macroscopic fields on particle density in phase space and on the characteristic frequency of particle motion. To demonstrate this, we expand the example of the harmonic oscillator to include also perturbation terms. For such a perturbed system the equation of motion is

$$
\ddot{w}+v_{0}^{2} w=v_{0}^{2} \beta^{\frac{3}{2}} \sum_{n>0} p_{n} \beta^{\frac{n}{2}} w^{n}
$$

where the coefficients $p_{n}$ are the strength parameters for the $n$th order perturbation term and the amplitude $w$ is the normalized betatron oscillation amplitude. The Vlasov equation allows us to calculate the impact of these perturbation terms on the betatron frequency. We demonstrate this first with a linear perturbation term $(n=1)$ caused by a gradient field error $p_{1}=-\delta k$ in a quadrupole. In this case the equation of motion is from (12.25)

$$
\ddot{w}+v_{0}^{2} w=-v_{0}^{2} \beta^{2} \delta k w
$$

or

$$
\ddot{w}+v_{0}^{2}\left(1+\beta^{2} \delta k\right) w=0 .
$$

This second-order differential equation can be replaced by two first-order differential equations which is in general the most straight forward way to obtain the functions (12.2)

$$
\begin{aligned}
& \dot{w}=v_{0} \sqrt{1+\beta^{2} \delta k} p, \\
& \dot{p}=-v_{0} \sqrt{1+\beta^{2} \delta k} w .
\end{aligned}
$$

Here it is assumed that the betatron function $\beta$ and the quadrupole field error $\delta k$ are uniformly distributed along the beam line and therefore can be treated as constants. This approach is justified since we are interested only in the average oscillation frequency of the particles and not in fast oscillating terms. The desired result can be derived directly from (12.28) without any further mathematical manipulation by comparison with (12.20). From there the oscillating frequency for the perturbed system is given by

$$
v=v_{0} \sqrt{1+\beta^{2} \delta k} \approx v_{0}\left(1+\frac{1}{2} \beta^{2} \delta k\right),
$$


for small perturbations. The betatron frequency shift can be expressed by the lowest order harmonic of the Fourier expansion for the periodic perturbation function $v_{0} \beta^{2} \delta k$ to give

$$
2 \pi v_{0}\left(\beta^{2} \delta k\right)_{0}=\oint v_{0} \beta^{2} \delta k \mathrm{~d} \varphi=\oint \beta \delta k \mathrm{~d} z
$$

making use of the definition for the betatron phase $\mathrm{d} \varphi=\mathrm{d} z / \nu_{0} \beta$. The tune shift $\delta v$ due to quadrupole field errors is therefore from (12.29)

$$
\delta v=v-v_{0}=\frac{1}{4 \pi} \oint \beta \delta k \mathrm{~d} z
$$

in agreement with (15.64). Again, the Vlasov equation confirms this result for all particles irrespective of the distribution in phase space. This procedure can be expanded to any order of perturbation. From the differential equation (12.25) one gets in analogy to the equations of motion (12.28)

$$
\begin{gathered}
\dot{w}=v_{0} \sqrt{1-\beta^{3 / 2} \sum_{n>0} p_{n} \beta^{n / 2} w^{n-1}} p, \\
\dot{p}=-v_{0} \sqrt{1-\beta^{3 / 2} \sum_{n>0} p_{n} \beta^{n / 2} w^{n-1}} w .
\end{gathered}
$$

For small perturbations the solution for the unperturbed harmonic oscillator $w(\varphi)=w_{0} \sin \left(v_{0} \varphi+\delta\right)$ may be used where $\delta$ is an arbitrary phase constant. The tune shift $\Delta v=v-v_{0}$ is thus while integrating over all perturbations around a circular accelerator

$$
\Delta v=-\sum_{n>0} \frac{1}{4 \pi} \oint p_{n} \beta^{\frac{n+1}{2}} w_{0}^{n-1} \sin ^{n-1}\left[v_{0} \varphi(z)+\delta\right] \mathrm{d} z
$$

where we have changed the independent variable from $\varphi$ to $z$ by $\mathrm{d} z=v_{0} \beta \mathrm{d} \varphi$.

Not all perturbation terms contribute to a tune variation. All even terms $n=2 m$, where $m$ is an integer, integrate, for example, to zero in this approximation and a sextupole field therefore does not contribute to a tune shift or tune spread. This conclusion must be modified, however, due to higher-order approximations which become necessary when perturbations cannot be considered small anymore. Furthermore, we find from (12.33) that the tune shift is independent of the particle oscillation amplitude only for quadrupole field errors $n=1$. For higher-order multipoles the tune shift becomes amplitude dependent resulting in a tune spread within the particle beam rather than a coherent tune shift for all particles of the beam. 
In a particular example, the tune spread caused by a single octupole $(n=3)$ in a circular accelerator is given by

$$
\Delta v_{3}=-\frac{\epsilon_{w}}{8 \pi} \oint p_{3} \beta^{2} \mathrm{~d} z
$$

where $w_{0}^{2}=\epsilon_{w}$ is the emittance of the beam. Similar results can be found for higherorder multipoles.

\subsubsection{Damping}

At the beginning of this section we have decided to ignore damping and have used the undamped Vlasov equation (12.18). Damping or anti-damping effects do, however, occur in real systems and it is interesting to investigate if the Vlasov equation can be used to derive some general insight into damped systems as well. For a damped oscillator we use (12.11), (12.12) to form the Vlasov equation in the form of (12.16). Instead of the phase we now use the real time as the independent variable to allow the intuitive definition of the damping decrement as the relative decay of the oscillation amplitude with time

$$
\frac{\partial \Psi}{\partial t}+\omega_{0} p_{w} \frac{\partial \Psi}{\partial w}-\left(\omega_{0} w+2 \alpha_{w} p_{w}\right) \frac{\partial \Psi}{\partial p_{w}}=+2 \alpha_{w} \Psi .
$$

This partial differential equation can be solved analytically in a way similar to the solution of the undamped harmonic oscillator by using cylindrical coordinates. For very weak damping we expect a solution close to (12.23) where the amplitude $r$ in phase space was a constant of motion. For a damped oscillator we try to form a similar invariant from the solution of a damped harmonic oscillator

$$
w=w_{0} \mathrm{e}^{-\alpha_{w} t} \cos \sqrt{\omega_{0}^{2}-\alpha_{w}^{2}} t=r \mathrm{e}^{-\alpha_{w} t} \cos \theta .
$$

With the conjugate component $\omega_{0} p_{w}=\dot{w}$, we form the expression

$$
\frac{\omega_{0} p_{w}+\alpha_{w} w}{\sqrt{\omega_{0}^{2}-\alpha_{w}^{2}}}=-w_{0} \mathrm{e}^{-\alpha_{w} t} \sin \sqrt{\omega_{0}^{2}-\alpha_{w}^{2}} t=-r \mathrm{e}^{-\alpha_{w} t} \sin \theta
$$

and eliminate the phase $\theta$ from (12.36), (12.37) keeping only terms linear in the damping decrement $\alpha_{w}$ to obtain the "invariant"

$$
r^{2} \mathrm{e}^{-2 \alpha_{w} t}=w^{2}+p_{w}^{2}+2 \frac{\alpha_{w}}{\omega_{0}} w p_{w}
$$


Obviously if we set $\alpha_{w}=0$ we have the invariant of the harmonic oscillator. The time dependent factor due to finite damping modifies this "invariant". However, for cases where the damping time is long compared to the oscillation period we may still consider (12.38) a quasi invariant. The phase coordinate $\theta$ can be derived from (12.36), (12.37) as a function of $w$ and $p_{w}$ as may be verified by insertion into the differential equation (12.35). The solution for the phase space density of a damped oscillator is of the form

$$
\Psi\left(w, p_{w}, t\right)=\mathrm{e}^{2 \alpha_{w} t} F(r, \Phi),
$$

where $F(r, \Phi)$ is any arbitrary but differentiable function of $r$ and $\Phi$ and the phase $\Phi$ is defined by

$$
\Phi=\theta+\sqrt{\omega_{0}^{2}-\alpha_{w}^{2}} t=\arctan \left(+\frac{\omega_{0} p_{w}+\alpha_{w} w}{\sqrt{\omega_{0}^{2}-\alpha_{w}^{2} w}}\right)+\sqrt{\omega_{0}^{2}-\alpha_{w}^{2}} t
$$

For very weak damping $\alpha_{w} \rightarrow 0$ and the solution (12.39) approaches (12.23) where $\alpha_{w}=0$ and $\nu \varphi=\omega_{0} t$ as expected. Therefore even for finite damping a particle distribution rotates in phase space although with a somewhat reduced rotation frequency due to damping. The particle density $\Psi$, however, changes exponentially with time due to the factor $\mathrm{e}^{2 \alpha_{w} t}$. For damping $\alpha_{w}>0$, we get an increase in the phase space density at the distance $R$ from the beam center. At the same time the real particle oscillation amplitudes $\left(w, p_{w}\right)$ are being reduced proportional to $\mathrm{e}^{-\alpha_{w} t}$ and the increase in the phase space density at $R$ reflects the concentration of particles in the beam center from larger amplitudes due to damping.

In conclusion we found that in systems where velocity dependent forces exist, we have damping $\left(\alpha_{w}>0\right)$ or anti-damping $\left(\alpha_{w}<0\right)$ of oscillation amplitudes. As has been discussed such forces do exist in accelerators leading to damping. Mostly, however, the Vlasov equation is applied to situations where particles interact with self or external fields that can lead to instabilities. It is the task of particle beam dynamics to determine the nature of such interactions and to derive the circumstances under which the damping coefficient $\alpha_{w}$, if not zero, is positive for damping or negative leading to beam instability.

\subsection{Damping of Oscillations in Electron Accelerators}

In electron accelerators we are concerned mainly with damping effects caused by the emission of synchrotron radiation. All six degrees of freedom for particle motion are damped. Damping of energy oscillations occurs simply from the fact that the synchrotron radiation power is energy dependent. Therefore a particle 
with a higher energy than the reference particle radiates more and a particle with less energy radiates less. The overall effect is that the energy deviation is reduced or damped. Damping of the transverse motion is principally a geometric effect. The photons of synchrotron radiation are emitted into the direction of the particle motion. Therefore part of the energy loss is correlated to a loss in transverse momentum. On the other hand, the lost energy is restored through accelerating fields with longitudinal components only. The overall effect of an energy loss during the course of betatron oscillations is therefore a loss of transverse momentum which leads to a reduction in the transverse oscillation amplitude, an effect we call damping. In the next section, we will discuss the physics leading to damping and derive the appropriate damping decrement for different modes of oscillations.

\subsubsection{Damping of Synchrotron Oscillations}

In a real beam particles are spread over a finite distribution of energies close to the reference energy. The magnitude of this energy spread is an important parameter to be considered for both beam transport systems as well as for experimental applications of particle beams. In general, an energy spread as small as possible is desired to minimize chromatic aberrations and for improved accuracy of experimental observation. We will therefore derive the parametric dependence of damping and discuss methods to reduce the energy spread within a particle beam.

To do this, we consider a beam of electrons being injected with an arbitrary energy distribution into a storage ring ignoring incidental beam losses during the injection process due to a finite energy acceptance. Particles in a storage ring undergo synchrotron oscillations which are oscillations about the ideal momentum and the ideal longitudinal position. Since energy and time or equivalently energy and longitudinal position are conjugate phase space variables, we will investigate both the evolution of the energy spread as well as the longitudinal distribution or bunch length of the particle beam.

The evolution of energy spread or bunch length of the particle beam will depend very much on the nature of particles and their energy. For heavy particles like protons or ions there is no synchrotron radiation damping and therefore the phase space for such beams remains constant. As a consequence, the energy spread or bunch length also stays a constant. A similar situation occurs for electrons or positrons at very low energies since synchrotron radiation is negligible. Highly relativistic electrons, however, produce intense synchrotron radiation leading to a strong damping effect.

The damping decrement $\alpha_{w}$ is defined in the Vlasov equation by

$$
\frac{\partial f}{\partial w}+\frac{\partial g}{\partial p}=-2 \alpha_{w}
$$


Fig. 12.3 Longitudinal particle position

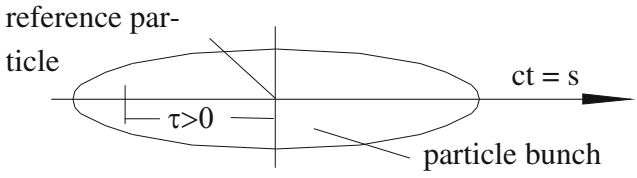

and can be calculated with the knowledge of the functions $f$ and $g$. For the conjugate variables $\left(w, p_{w}\right)$ we use the time deviation of a particle with respect to the synchronous particle $w=\tau$ as shown in Fig. 12.3 and the difference of the particle's energy $E$ from the synchronous or reference energy $E_{0}$ and set $p_{w}=\epsilon=E-E_{0}$.

Since $f=\frac{\mathrm{d} \tau}{\mathrm{d} t}=\dot{\tau}$ and $g=\frac{\mathrm{d} \epsilon}{\mathrm{d} t}=\dot{\epsilon}$ we have to determine the rate of change for the conjugate variables. The rate of change of $\tau$ is from (9.17) with $c p_{0} \approx E_{0}$

$$
\frac{\mathrm{d} \tau}{\mathrm{d} t}=-\eta_{\mathrm{c}} h \frac{\epsilon}{E_{0}},
$$

where we have replaced the phase by the time $\dot{\psi}=c \beta h k_{0} \dot{\tau}$ and the relative momentum error by the relative energy error since we consider here only highly relativistic particles. The latter replacement is a matter of convenience since we will be using the energy gain in accelerating fields.

The energy rate of change $\dot{\epsilon}$ is the balance of the energy gained in accelerating fields and the energy lost due to synchrotron radiation or other losses

$$
\dot{\epsilon}=\frac{1}{T}\left[e V_{\mathrm{rf}}\left(\tau_{\mathrm{s}}+\tau\right)-U\left(E_{\mathrm{s}}+\epsilon\right)\right] .
$$

Here $T$ is the time it takes the particles to travel the distance $L$. The energy gain within the distance $L$ for a particle traveling a time $\tau$ behind the reference or synchronous particle is $e V_{\mathrm{rf}}\left(\tau_{\mathrm{s}}+\tau\right)$ and $U$ is the energy loss to synchrotron radiation along the same distance of travel. here we assume the energy gain or loss to be distributed evenly over the length of $L$.

Before we go on, we apply these expressions to the simple situation of a linear accelerator of length $L$ where the momentum compaction factor vanishes $\alpha_{\mathrm{c}}=0$ and where there is no energy loss due to synchrotron radiation $U \equiv 0$. Furthermore, we ignore for now other energy losses and have with $\eta_{\mathrm{c}}=1 / \gamma^{2}$

$$
\begin{aligned}
& f=\dot{\tau}=\frac{1}{\beta^{2} \gamma^{2}} \frac{\epsilon}{E}, \\
& g=\dot{\epsilon}=\frac{1}{T} e V_{\mathrm{rf}}\left(\tau_{\mathrm{s}}+\tau\right) .
\end{aligned}
$$

Inserted into (12.41) we find the damping decrement to vanish which is consistent with the constancy of phase space. From the Vlasov equation we learn that in the absence of damping the energy spread $\epsilon$ stays constant as the particle beam gets accelerated. 
The Vlasov equation still can be used to also describe adiabatic damping but we need to use the relative energy spread as one of the variables. Instead of the second equation (12.44) we have then with $\delta=\frac{\epsilon}{E}$

$$
g=\frac{\mathrm{d}}{\mathrm{d} t} \delta=\frac{\frac{\epsilon}{E_{t}}-\frac{\epsilon}{E_{0}}}{\Delta t},
$$

where $E_{0}$ and $E_{t}$ are the energies time $t_{0}$ and $t=t_{0}+\mathrm{d} t$, respectively. We choose the time interval $\mathrm{d} t$ small enough so that the energy increase $\mathrm{d} E=a \mathrm{~d} t \ll E_{0}$ and get

$$
g=-\frac{\epsilon}{E_{t}} \frac{a}{E_{0}} .
$$

The damping decrement becomes from (12.41) with $\delta=\frac{\epsilon}{E}$ and $\partial f / \partial \tau=0$

$$
\frac{\partial g}{\partial \delta}=-\frac{a}{E_{0}}=-2 \alpha_{w}=\frac{1}{\delta} \frac{\mathrm{d} \delta}{\mathrm{d} t}
$$

and after integration

$$
\int \frac{\mathrm{d} \delta}{\delta}=\ln \frac{\delta}{\delta_{0}}=-\int \frac{a}{E_{0}} \mathrm{~d} t=-\int \frac{\mathrm{d} E}{E_{0}}=+\ln \frac{E_{0}}{E_{t}}
$$

or

$$
\frac{\delta}{\delta_{0}}=\frac{E_{0}}{E_{t}} .
$$

The relative energy spread in the beam is reduced during acceleration inversely proportional to the energy. The reduction of the relative energy spread is called adiabatic damping. This name is unfortunate in the sense that it does not actually describe a damping effect in phase space as we just found out but rather describes the variation of the relative energy spread with energy which is merely a consequence of the constant phase space density or Liouville's theorem.

Returning to the general case (12.43) we apply a Taylor's expansion to the rfvoltage in (12.44) and get for terms on the r.h.s. keeping only linear terms

$$
\begin{aligned}
& e V_{\mathrm{rf}}\left(\tau_{\mathrm{s}}+\tau\right)=e V_{\mathrm{rf}}\left(\tau_{\mathrm{s}}\right)+\left.e \frac{\partial V_{\mathrm{rf}}}{\partial \tau}\right|_{\tau_{\mathrm{s}}} \tau, \\
& -U\left(E_{\mathrm{s}}+\epsilon\right)=-U\left(E_{\mathrm{s}}\right)-\left.\frac{\partial U}{\partial E}\right|_{E_{\mathrm{s}}} \epsilon .
\end{aligned}
$$


Since the energy gain from the rf-field $e V_{\mathrm{rf}}\left(\tau_{\mathrm{s}}\right)$ for the synchronous particle just compensates its energy loss $U\left(E_{\mathrm{s}}\right)$, we have instead of (12.43) now

$$
\dot{\epsilon}=\frac{1}{T}\left[e \dot{V}_{\mathrm{rf}}\left(\tau_{\mathrm{s}}\right) \tau-\left.\frac{\partial U}{\partial E}\right|_{E_{\mathrm{s}}} \epsilon\right]
$$

where we have set $\dot{V}_{\text {rf }}=\frac{\partial V_{\text {rf }}}{\partial \tau}$. The synchrotron oscillation damping decrement can now be derived from (12.41) with (12.44), (12.52) to give

$$
\alpha_{\mathrm{s}}=+\left.\frac{1}{2} \frac{1}{T} \frac{\partial U}{\partial E}\right|_{E_{\mathrm{s}}} .
$$

We will now derive the damping decrement for the case that the energy loss is only due to synchrotron radiation. The energy loss along the transport line $L$ is given by

$$
U_{\mathrm{s}}=\frac{1}{c} \int_{0}^{L} P_{\gamma} \mathrm{d} s
$$

where $P_{\gamma}$ is the synchrotron radiation power and the integration is taken along the actual particle trajectory $s$. If $\rho(z)$ is the bending radius along $z$, we have $\frac{\mathrm{d} s}{\mathrm{~d} z}=1+\frac{x}{\rho}$. With $x=x_{\beta}+\eta \frac{\epsilon}{E_{\mathrm{s}}}$ and averaging over many betatron oscillations, we get $\left\langle x_{\beta}\right\rangle=0$ and

$$
\frac{\mathrm{d} s}{\mathrm{~d} z}=1+\frac{\eta}{\rho} \frac{\epsilon}{E}
$$

This asymmetric averaging of the betatron oscillation only is permissible if the synchrotron oscillation frequency is much lower than the betatron oscillation frequency as is the case in circular accelerators. With $\mathrm{d} s=\left[1+(\eta / \rho)\left(\epsilon / E_{\mathrm{s}}\right)\right] \mathrm{d} z$ in (12.54), the energy loss for a particle of energy $E_{\mathrm{s}}+\epsilon$ is

$$
U_{\mathrm{s}}\left(E_{\mathrm{s}}+\epsilon\right)=\frac{1}{c} \int_{\mathrm{L}} P_{\gamma}\left(1+\frac{\eta}{\rho} \frac{\epsilon}{E_{\mathrm{S}}}\right) \mathrm{d} z
$$

or after differentiation with respect to the energy

$$
\left.\frac{\partial U_{\mathrm{s}}}{\partial E}\right|_{E_{\mathrm{s}}}=\frac{1}{c} \int_{\mathrm{L}}\left[\frac{\mathrm{d} P_{\gamma}}{\mathrm{d} E}+P_{\gamma} \frac{\eta}{\rho} \frac{1}{E_{\mathrm{s}}}\right]_{E_{\mathrm{s}}} \mathrm{d} z .
$$

The synchrotron radiation power is proportional to the square of the energy and the magnetic field $P_{\gamma} \sim E_{\mathrm{s}}^{2} B_{0}^{2}$ which we use in the expansion

$$
\frac{\mathrm{d} P_{\gamma}}{\mathrm{d} E}=\frac{\partial P_{\gamma}}{\partial E}+\frac{\partial P_{\gamma}}{\partial B_{0}} \frac{\partial B}{\partial E}=2 \frac{P_{\gamma}}{E_{\mathrm{s}}}+2 \frac{P_{\gamma}}{B} \frac{\partial B}{\partial x} \frac{\partial x}{\partial E} .
$$


The variation of the synchrotron radiation power with energy depends directly on the energy but also on the magnetic field if there is a field gradient $\frac{\partial B}{\partial x}$ and a finite dispersion function $\eta=E_{\mathrm{s}} \frac{\partial x}{\partial E}$. The magnetic field as well as the field gradient is to be taken at the reference orbit. Collecting all these terms and setting $\frac{1}{B_{0}} \frac{\partial B}{d x}=\rho k$ we get for (12.57)

$$
\begin{aligned}
\left.\frac{\partial U_{\mathrm{s}}}{\partial E}\right|_{E_{\mathrm{s}}} & =\left.\frac{1}{c} \int_{L}\left(2 \frac{P_{\gamma}}{E_{\mathrm{s}}}+2 \frac{P_{\gamma}}{E_{\mathrm{s}}} \rho k \eta+\frac{P_{\gamma}}{E_{\mathrm{s}}} \frac{\eta}{\rho}\right)\right|_{E_{\mathrm{s}}} \mathrm{d} z \\
& =\frac{U_{\mathrm{s}}}{E_{\mathrm{s}}}\left[2+\left.\frac{1}{c U_{\mathrm{s}}} \int_{L} P_{\gamma} \eta\left(\frac{1}{\rho}+2 \rho k\right)\right|_{E_{\mathrm{s}}} \mathrm{d} z\right],
\end{aligned}
$$

where we have made use of $U_{\mathrm{s}}=\frac{1}{c} \int_{L} P_{\gamma}\left(E_{\mathrm{s}}\right) \mathrm{d} z$. Recalling the expressions for the synchrotron radiation power and energy loss $P_{\gamma}=C_{\gamma} E_{\mathrm{s}}^{4} / \rho^{2}$ and $U_{\mathrm{s}}=$ $C_{\gamma} E_{\mathrm{s}}^{4} \int \mathrm{d} z / \rho^{2}$, we may simplify (12.59) for

$$
\left.\frac{\partial U}{\partial E}\right|_{E_{\mathrm{s}}}=\frac{U_{\mathrm{s}}}{E_{\mathrm{s}}}(2+\vartheta)
$$

where the $\vartheta$-parameter has been introduced in (11.25). We finally get from (12.53) with (12.60) the damping decrement for synchrotron oscillations

$$
\alpha_{\epsilon}=\frac{U_{\mathrm{s}}}{2 T E_{\mathrm{s}}}(2+\vartheta)=\frac{U_{\mathrm{s}}}{2 T E_{\mathrm{s}}} J_{\epsilon}=\frac{\left\langle P_{\gamma}\right\rangle}{2 E_{\mathrm{s}}} J_{\epsilon},
$$

in full agreement with results obtained earlier. Since all parameters except $\vartheta$ are positive we have shown that the synchrotron oscillations for radiating particles are damped. A potential situation for anti-damping can be created if $\vartheta<-2$.

To calculate the damping decrement, we assume accelerating fields evenly distributed around the ring to restore the lost energy. In practice this is not true since only few rf-cavities in a ring are located at one or more places around the ring. As long as the revolution time around the ring is small compared to the damping time, however, we need not consider the exact location of the accelerating cavities and may assume an even and uniform distribution around the ring.

\subsubsection{Damping of Vertical Betatron Oscillations}

Particles orbiting in a circular accelerator undergo transverse betatron oscillations. These oscillations are damped in electron rings due to the emission of synchrotron radiation. First we will derive the damping decrement for the vertical betatron oscillation. In a plane accelerator with negligible coupling this motion is independent 
of other oscillations. This is not the case for the horizontal betatron motion which is coupled to the synchrotron oscillation due to the presence of a finite dispersion function. We will therefore derive the vertical damping decrement first and then discuss a very general theorem applicable for the damping in circular accelerators. This theorem together with the damping decrement for the synchrotron and vertical betatron oscillations will enable us to derive the horizontal damping in a much simpler way than would be possible in a more direct way.

In normalized coordinates the functions $f$ and $g$ are for the vertical plane

$$
\begin{aligned}
& \frac{\mathrm{d} w}{\mathrm{~d} \varphi}=+v p=f(w, p, \varphi), \\
& \frac{\mathrm{d} p}{\mathrm{~d} \varphi}=-v w=g(w, p, \varphi),
\end{aligned}
$$

where $v=v_{y}, w=y / \sqrt{\beta_{y}}, \frac{1}{v_{y}} \frac{\mathrm{d} w}{\mathrm{~d} \varphi}=\sqrt{\beta_{y}} y^{\prime}-\frac{1}{2} \frac{\beta_{y}^{\prime}}{\sqrt{\beta_{y}}} y$ and $v_{y} \varphi=\psi_{y}$ is the vertical betatron phase.

Due to the emission of a synchrotron radiation photon alone the particle does not change its position $y$ nor its direction of propagation $y^{\prime}$. With this we derive now the damping within a path element $\Delta z$ which includes the emission of photons as well as the appropriate acceleration to compensate for that energy loss. Just after the emission of the photon but before the particle interacts with accelerating fields let the transverse momentum and total energy be $p_{\perp}$ and $E_{\mathrm{s}}$,respectively. The slope of the particle trajectory is therefore (Fig. 12.4)

$$
y_{0}^{\prime}=\frac{c p_{\perp}}{\beta E_{\mathrm{s}}} .
$$

Energy is transferred from the accelerating cavity to the particle at the rate of the synchrotron radiation power $P_{\gamma}$ and the particle energy increases in the cavity of length $\Delta z$ from $E_{\mathrm{S}}$ to $E_{\mathrm{s}}+P_{\gamma} \frac{\Delta z}{\beta c}$ and the slope of the particle trajectory becomes at the exit of the cavity of length $\Delta z$ due to this acceleration

$$
y_{1}^{\prime}=\frac{c p_{\perp}}{\beta E_{\mathrm{s}}+P_{\gamma} \frac{\Delta z}{c}} \approx \frac{c p_{\perp}}{\beta E_{\mathrm{s}}}\left(1-\frac{P_{\gamma}}{\beta E_{\mathrm{s}}} \frac{\Delta z}{c}\right) .
$$

Fig. 12.4 Acceleration and damping

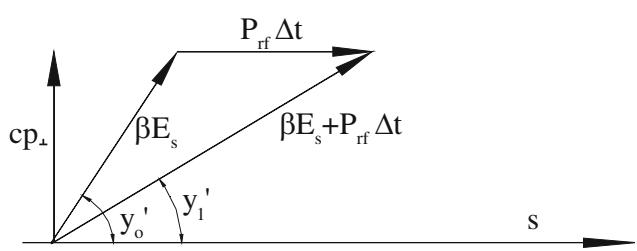


We are now in a position to express the functions $f$ and $g$ in terms of physical parameters. The function $f$ is expressed by

$$
f=\frac{\Delta w}{\Delta \varphi}=\frac{y_{1}-y_{0}}{\sqrt{\beta_{y}} \Delta \varphi}=\frac{y_{0}^{\prime}}{\sqrt{\beta_{y}}} \frac{\Delta z}{\Delta \varphi}=v \sqrt{\beta_{y}} y_{0}^{\prime},
$$

where we made use of $\Delta \varphi=\Delta z /(\nu \beta)$. The damping decrement will depend on the derivation $\frac{\mathrm{d} f}{\mathrm{~d} w}$ which can be seen from (12.66) to vanish since $f$ does not depend on $w$

$$
\frac{\partial f}{\partial w}=0
$$

The variation of the conjugate variable $p$ with phase is from (12.62)

$$
\frac{\Delta p}{\Delta \varphi}=\frac{\frac{\mathrm{d} w_{1}}{\mathrm{~d} \varphi}-\frac{\mathrm{d} w_{0}}{\mathrm{~d} \varphi}}{\nu \Delta \varphi}
$$

From linear beam dynamics, we find

$$
\frac{\mathrm{d} w_{1}}{\mathrm{~d} \varphi}-\frac{\mathrm{d} w_{0}}{\mathrm{~d} \varphi}=\sqrt{\beta_{y}}\left(y_{1}^{\prime}-y_{0}^{\prime}\right)-\frac{1}{2} \frac{\beta_{y}^{\prime}}{\sqrt{\beta_{y}}}\left(y_{1}-y_{0}\right)
$$

and get with (12.65), (12.66)

$$
g(w, p, \varphi)=\frac{\Delta p}{\Delta \varphi}=\frac{-\sqrt{\beta_{y}} \frac{P_{\gamma}}{\beta c E_{\mathrm{s}}} \Delta z y_{0}^{\prime}+F(y)}{\nu \Delta \varphi} .
$$

The function $F(y)$ is a collection of $y$-dependent terms that become irrelevant for our goal. Damping will be determined by the value of the derivative $\frac{\partial g}{\partial p}$ which with $y_{0}^{\prime}=\frac{1}{\sqrt{\beta_{y}}} \frac{\mathrm{d} w}{\mathrm{~d} \varphi}+\frac{1}{2} \beta_{y}^{\prime} \frac{1}{\beta_{y}} y_{0}$ becomes

$$
\frac{\partial g}{\partial p}=v \frac{\partial g}{\partial \frac{\mathrm{d} w}{\mathrm{~d} \varphi}}=\frac{P_{\gamma}}{\beta c E_{\mathrm{s}}} \frac{\Delta z}{\Delta \varphi}
$$

In the derivation of (12.71) we have used the betatron phase as the "time" and get therefore the damping per unit betatron phase advance. Transforming to the real time with $\frac{\Delta z}{\beta c \Delta \varphi}=\frac{T_{\text {rev }}}{2 \pi}$ and (12.41)

$$
\frac{\partial g}{\partial p}=\frac{P_{\gamma}}{E_{\mathrm{s}}} \frac{T_{\mathrm{rev}}}{2 \pi}=-2 \alpha_{y} \frac{T_{\mathrm{rev}}}{2 \pi}
$$


and solving for the vertical damping decrement

$$
\alpha_{y}=-\frac{\left\langle P_{\gamma}\right\rangle}{2 E_{\mathrm{s}}} .
$$

In this last equation, we have used the average synchrotron radiation power which is the appropriate quantity in case of a non-isomagnetic ring. The damping of the vertical betatron function is proportional to the synchrotron radiation power. This fact can be used to increase damping when so desired by increasing the synchrotron radiation power from special magnets in the lattice structure.

\subsubsection{Robinson's Damping Criterion}

The general motion of charged particles extends over all six degrees of freedom in phase space and therefore the particle motion is described in six-dimensional phase space as indicated in the general Vlasov equation (12.17). It is, however, a fortunate circumstance that it is technically possible to construct accelerator components in such a fashion that there is only little or no coupling between different pairs of conjugate coordinates. As a consequence, we can generally treat horizontal betatron oscillations separate from the vertical betatron oscillations and both of them separate from synchrotron oscillations. Coupling effects that do occur will be treated as perturbations. There is some direct coupling via the dispersion function between synchrotron and particularly the horizontal betatron oscillations but the frequencies are very different with the synchrotron oscillation frequency being in general much smaller than the betatron oscillation frequency. Therefore in most cases the synchrotron oscillation can be ignored while discussing transverse oscillations and we may average over many betatron oscillations when we discuss synchrotron motion.

A special property of particle motion in six-dimensional phase space must be introduced allowing us to make general statements about the overall damping effects in a particle beam. We start from the Vlasov equation (12.17)

$$
\frac{\partial \Psi}{\partial t}+f \nabla_{r} \Psi+g \nabla_{p} \Psi=-\left(\nabla_{r} f+\nabla_{p} g\right) \Psi
$$

and define a total damping decrement $\alpha_{\mathrm{t}}$ by setting

$$
\nabla_{r} f+\nabla_{p} g=-2 \alpha_{\mathrm{t}}
$$

The total damping decrement is related to the individual damping decrements of the transverse and longitudinal oscillations but the precise dependencies are not yet obvious. In the derivation of (12.17), we have expanded the functions $f$ and $g$ in a Taylor series neglecting all terms of second or higher order in time and got as a 
result the simple expression (12.75) for the overall damping. Upon writing (12.75) in component form, we find from the components of the 1.h.s. that the overall damping decrement $\alpha_{\mathrm{t}}$ is just the sum of all three individual damping decrements and we may therefore set

$$
\nabla_{r} \boldsymbol{f}+\nabla_{p} \boldsymbol{g}=-2 \alpha_{\mathrm{t}}=-2\left(\alpha_{x}+\alpha_{y}+\alpha_{\epsilon}\right)
$$

From this equation and the linearity of the functions $f$ and $g$ describing the physics of the dynamical system general characteristics of the damping process can be derived. The damping decrement does not depend on the dynamic variables of the particles and coupling terms do not contribute to damping. The damping rate is therefore the same for all particles within a beam. In the following paragraphs, we will discuss in more detail the general characteristics of synchrotron radiation damping. Specifically, we will determine the functions $\boldsymbol{f}$ and $\boldsymbol{g}$ and derive an expression for the total damping.

We consider a small section of a beam transport line or circular accelerator including all basic processes governing the particle dynamics. These processes are focusing, emission of photons and acceleration. All three processes are assumed to occur evenly along the beam line. The six-dimensional phase space to be considered is

$$
\left(x, x^{\prime}, y, y^{\prime}, \tau, \epsilon\right) .
$$

During the short time $\Delta t$ some of the transverse coordinates change and it is those changes that determine eventually the damping rate. Neither the emission of a synchrotron radiation photon nor the absorption of energy in the accelerating cavities causes any change in the particle positions $x, y$, and $\tau$. Indicating the initial coordinates by the index 0 and setting $\beta c \Delta t=\Delta z$ we get for the evolution of the particle positions within the length element $\Delta z$ in the three space dimensions

$$
\begin{aligned}
& x=x_{0}+x_{0}^{\prime} \Delta z, \\
& y=y_{0}+y_{0}^{\prime} \Delta z, \\
& \tau=\tau_{0}+\eta_{\mathrm{c}} \frac{\epsilon_{0}}{E_{\mathrm{s}}} \frac{\Delta z}{\beta c} .
\end{aligned}
$$

The conjugate coordinates vary in a somewhat more complicated way. First we note that the Vlasov equation does not require the conjugate coordinates to be canonical variables. Indeed this derivation will become simplified if we do not use canonical variables but use the slopes of the particle trajectories with the reference path and the energy deviation. The change of the slopes due to focusing is proportional to the oscillation amplitude and vanishes on average. Emission of a synchrotron radiation photon occurs typically within an angle of $\pm 1 / \gamma$ causing a small transverse kick to the particle trajectory. In general, however, this transverse kick will be very small and we may assume for all practical purposes the slope 
of the transverse trajectory not to be altered by photon emission. Forces parallel to the direction of propagation of the particles can be created, however, through the emission of synchrotron radiation photons. In this case, the energy or energy deviation of the particle will be changed like

$$
\epsilon=\epsilon_{0}-P_{\gamma} \frac{\Delta z}{\beta c}+P_{\mathrm{rf}} \frac{\Delta z}{\beta c} .
$$

Here we use the power $P_{\gamma}$ to describe the synchrotron radiation energy loss rate a particle may suffer during the time $\beta c \Delta t=\Delta z$. No particular assumption has been made about the nature of the energy loss except that during the time $\Delta t$ it be small compared to the particle energy. To compensate this energy loss the particles become accelerated in rf-cavities. The power $P_{\mathrm{rf}}$ is the energy flow from the cavity to the particle beam, not to be confused with the total power the rf-source delivers to the cavity.

The transverse slopes $x^{\prime}$ and $y^{\prime}$ are determined by the ratio of the transverse to the longitudinal momentum $u^{\prime}=p_{u} / p_{z}$ where $u$ stands for $x$ or $y$, respectively. During acceleration in the rf-cavity the transverse momentum does not change but the total kinetic energy increases from $E_{\mathrm{s}}$ to $E_{\mathrm{s}}+P_{\mathrm{rf}} \frac{\Delta z}{\beta c}$ and the transverse slope of the trajectory is reduced after a distance $\Delta z$ to

$$
u^{\prime}=\frac{c p_{u}}{c p_{z}+P_{\mathrm{rf}} \beta \frac{\Delta z}{\beta c}} \approx u_{0}^{\prime}-\frac{P_{\mathrm{rf}}}{E_{\mathrm{s}}} \frac{\Delta z}{\beta c} u_{0}^{\prime} .
$$

Explicitly, the transverse slopes vary now like

$$
\begin{aligned}
& x^{\prime}=x_{0}^{\prime}-\frac{P_{\mathrm{rf}}}{E_{\mathrm{s}}} \frac{\Delta z}{\beta c} x_{0}^{\prime}, \\
& y^{\prime}=y_{0}^{\prime}-\frac{P_{\mathrm{rf}}}{E_{\mathrm{s}}} \frac{\Delta z}{\beta c} y_{0}^{\prime} .
\end{aligned}
$$

All ingredients are available now to formulate expressions for the functions $\boldsymbol{f}$ and $\boldsymbol{g}$ in component form

$$
\begin{aligned}
\boldsymbol{f} & =\left(x_{0}^{\prime}, y_{0}^{\prime}, \eta_{\mathrm{c}} \frac{\epsilon}{E_{\mathrm{s}}}\right), \\
\boldsymbol{g} & =\left(-\frac{P_{\mathrm{rf}}}{E_{\mathrm{s}}} x_{0}^{\prime},-\frac{P_{\mathrm{rf}}}{E_{\mathrm{s}}} y_{0}^{\prime},-P_{\gamma}+P_{\mathrm{rf}}\right) .
\end{aligned}
$$

With these expressions we evaluate (12.76) and find that $\nabla_{r} f=0$. For the determination of $\nabla_{p} g$ we note that the power $P_{\mathrm{rf}}$ from the cavity is just equal to the average radiation power $\left\langle P_{\gamma}\right\rangle$ and the derivative of the radiation power with respect to the particle energy is

$$
-\frac{\partial P_{\gamma}}{\partial \epsilon}=-2 \frac{P_{\gamma}}{E_{\mathrm{s}}}
$$


Finally, we note that the rf-power $P_{\mathrm{rf}}$ is equal to the average radiation power $\left\langle P_{\gamma}\right\rangle$ and get from (12.76)

$$
\alpha_{x}+\alpha_{y}+\alpha_{\epsilon}=2 \frac{\left\langle P_{\gamma}\right\rangle}{E_{\mathrm{s}}} .
$$

The sum of all damping decrements is a constant, a result which has been derived first by Robinson [1] and is known as the Robinson criterion. The total damping depends only on the synchrotron radiation power and the particle energy and variations of magnetic field distribution in the ring keeping the radiation power constant will not affect the total damping rate but may only shift damping from one degree of freedom to another.

\subsubsection{Damping of Horizontal Betatron Oscillations}

With the help of the Robinson criterion, the damping decrement for the horizontal betatron oscillation can be derived by simple subtraction. Inserting (12.61), (12.75) into (12.84) and solving for the horizontal damping decrement we get

$$
\alpha_{x}=\frac{\left\langle P_{\gamma}\right\rangle}{2 E_{\mathrm{s}}}(1-\vartheta) \text {. }
$$

The damping decrements derived from the Vlasov equation agree completely with the results obtained in Sect. 11.2 by very different means.

No matter what type of magnet lattice we use, the total damping depends only on the synchrotron radiation power and the particle energy. We may, however, vary the distribution of the damping rates through the $\vartheta$-parameter to different oscillation modes by proper design of the focusing and bending lattice in such a way that one damping rate is modified in the desired way limited only by the onset of anti-damping in another mode. Specifically, this is done by introducing gradient bending magnets with a field gradient such as to produce the desired sign of the $\vartheta$ parameter.

\subsection{The Fokker-Planck Equation}

From the discussions of the previous section it became clear that the Vlasov equation is a useful tool to determine the evolution of a multiparticle system under the influence of forces depending on the physical parameters of the system through differentiable functions. If, however, the dynamics of a system in phase space depends only on its instantaneous physical parameters where the physics of the particle dynamics cannot be expressed by differentiable functions, the Vlasov 
equation will not be sufficient to describe the full particle dynamics. A process which depends only on the state of the system at the time $t$ and not on its history is called a Markoff process.

In particle beam dynamics we have frequently the appearance of such processes where forces are of purely statistical nature like those caused, for example, by the quantized emission of synchrotron radiation photons or by collisions with other particles within the same bunch or residual gas atoms. To describe such a situation we still have variations of the coordinates per unit time similar to those in (12.2) but we must add a term describing the statistical process and we set therefore

$$
\begin{gathered}
\dot{w}=f_{w}\left(w, p_{w}, t\right)+\sum \xi_{i} \delta\left(t-t_{i}\right), \\
\dot{p}_{w}=g_{w}\left(w, p_{w}, t\right)+\sum \pi_{i} \delta\left(t-t_{i}\right),
\end{gathered}
$$

where $\xi_{i}$ and $\pi_{i}$ are instantaneous statistical changes in the variables $w$ and $p_{w}$ with a statistical distribution in time $t_{i}$ and where $\delta\left(t-t_{i}\right)$ is the Dirac delta function. The probabilities $P_{w}(\xi)$ and $P_{p}(\pi)$ for statistical occurrences with amplitudes $\xi$ and $\pi$ be normalized and centered

$$
\begin{aligned}
& \int P_{w}(\xi) \mathrm{d} \xi=1, \quad \int P_{w}(\xi) \xi \mathrm{d} \xi=0, \\
& \int P_{p}(\pi) \mathrm{d} \pi=1, \quad \int P_{p}(\pi) \pi \mathrm{d} \pi=0 .
\end{aligned}
$$

The first equations normalize the probability amplitudes and the second equations are true for symmetric statistical processes. The sudden change in the amplitude by $\Delta w_{i}$ or in momentum by $\Delta p_{w i}$ due to one such process is given by

$$
\begin{aligned}
\Delta w_{i} & =\int \xi_{i} \delta\left(t-t_{i}\right) \mathrm{d} t=\xi_{i}, \\
\Delta p_{w i} & =\int \pi_{i} \delta\left(t-t_{i}\right) \mathrm{d} t=\pi_{i} .
\end{aligned}
$$

Analogous to the discussion of the evolution of phase space in the previous section, we will now formulate a similar evolution including statistical processes. At the time $t+\Delta t$, the particle density in phase space is taken to be $\Psi\left(w, p_{w}, t+\Delta t\right)$ and we intend to relate this to the particle density at time $t$. During the time interval $\Delta t$ there are finite probabilities $P_{w}(\xi), P_{p}(\pi)$ that the amplitude $(w-\xi)$ or the momentum $\left(p_{w}-\pi\right)$ be changed by a statistical process to become $w$ or $p_{w}$ at time $t$. This definition of the probability function also covers the cases where particles during the time $\Delta t$ either jump out of the phase space area $\Delta A_{P}$ or appear in the phase space area $\Delta A_{Q}$.

To determine the number of particles ending up within the area $\Delta A_{Q}$, we look at all area elements $\Delta A_{P}$ which at time $t$ are a distance $\Delta w=\xi$ and $\Delta p_{w},=\pi$ away from the final area element $\Delta A_{Q}$ at time $t+\Delta t$. As a consequence of our 
assumption that the particle density is only slowly varying in phase space, we may assume that the density $\Psi$ is uniform within the area elements $\Delta A_{P}$ eliminating the need for a local integration. We may now write down the expression for the phase space element and the particle density at time $t+\Delta t$ by integrating over all values of $\xi$ and $\pi$

$$
I=\Delta A_{P} \int_{-\infty}^{+\infty} \int_{-\infty}^{+\infty} \Psi\left(w-\xi, p_{w}-\pi, t\right) P_{w}(\xi) P_{p}(\pi) \mathrm{d} \xi \mathrm{d} \pi,
$$

where we used the abbreviation $I=\Psi\left(w+f_{w} \Delta t, p_{w}+g_{w} \Delta t, t+\Delta t\right) \Delta A_{Q}$. The volume elements $\Delta A_{P}$ and $\Delta A_{Q}$ are given by (12.5), (12.6), respectively. The statistical fluctuations may in general be of any magnitude. In particle beam dynamics, however, we find that the fluctuations with reasonable probabilities are small compared to the values of the variables $w$ and $p_{w}$. The phase space density can therefore be expanded into a Taylor series where we retain linear as well as quadratic terms in $\xi$ and $\pi$

$$
\begin{aligned}
\Psi\left(w-\xi, p_{w}-\pi, t\right) & =\Psi_{0}-\xi \frac{\partial \Psi_{0}}{\partial w}-\pi \frac{\partial \Psi_{0}}{\partial p_{w}} \\
& +\frac{1}{2} \xi^{2} \frac{\partial^{2} \Psi_{0}}{\partial w^{2}}+\frac{1}{2} \pi^{2} \frac{\partial^{2} \Psi_{0}}{\partial p_{w}^{2}}+\xi \pi \frac{\partial^{2} \Psi_{0}}{\partial w \partial p_{w}}
\end{aligned}
$$

where $\Psi_{0}=\Psi\left(w, p_{w}, t\right)$ and we finally get for the integrals with (12.88)

$$
I=\Psi_{0}+\frac{1}{2} \frac{\partial^{2} \Psi_{0}}{\partial w^{2}} \int \xi^{2} P_{w}(\xi) \mathrm{d} \xi+\frac{1}{2} \frac{\partial^{2} \Psi_{0}}{\partial p_{w}^{2}} \int \pi^{2} P_{p}(\pi) \mathrm{d} \pi
$$

For simplicity, we leave off the integration limits which are still from $-\infty$ to $+\infty$. If we now set $\mathcal{N}$ to be the number of statistical occurrences per unit time we may simplify the quadratic terms on the r.h.s. of (12.92) by setting

$$
\begin{aligned}
\frac{1}{2} \int \xi^{2} P_{w}(\xi) \mathrm{d} \xi & =\frac{1}{2}\left\langle\mathcal{N}_{\xi} \xi^{2}\right\rangle \Delta t, \\
\frac{1}{2} \int \pi^{2} P_{p}(\pi) \mathrm{d} \pi & =\frac{1}{2}\left\langle\mathcal{N}_{\pi} \pi^{2}\right\rangle \Delta t,
\end{aligned}
$$

and get similarly to the derivation of the Vlasov equation in Sect. 12.1

$$
\begin{aligned}
\frac{\partial \Psi_{0}}{\partial t}+f_{w} \frac{\partial \Psi_{0}}{\partial w}+g_{w} \frac{\partial \Psi_{0}}{\partial p_{w}} & =-\left(\frac{\partial f_{w}}{\partial w}+\frac{\partial g_{w}}{\partial p_{w}}\right) \Psi_{0} \\
& +\frac{1}{2}\left\langle\mathcal{N}_{\xi} \xi^{2}\right\rangle \frac{\partial^{2} \Psi_{0}}{\partial w^{2}}+\frac{1}{2}\left\langle\mathcal{N}_{\pi} \pi^{2}\right\rangle \frac{\partial^{2} \Psi_{0}}{\partial p_{w}^{2}}
\end{aligned}
$$


This partial differential equation is identical to the Vlasov equation except for the statistical excitation terms and is called the Fokker-Planck equation [2]. We define diffusion coefficients describing the flow in $\xi$ and $\pi$ space by

$$
\begin{aligned}
& D_{\xi}=\frac{1}{2}\left\langle\mathcal{N}_{\sim \xi^{2}}\right\rangle, \\
& D_{\pi}=\frac{1}{2}\left\langle\mathcal{N}_{\approx} \pi^{2}\right\rangle,
\end{aligned}
$$

and the Fokker-Planck equation becomes finally

$$
\frac{\partial \Psi}{\partial t}+f_{w} \frac{\partial \Psi}{\partial w}+g_{w} \frac{\partial \Psi}{\partial p_{w}}=2 \alpha_{w} \Psi+D_{\xi} \frac{\partial^{2} \Psi}{\partial w^{2}}+D_{\pi} \frac{\partial^{2} \Psi}{\partial p_{w}^{2}} .
$$

For the case of damped oscillators the Fokker-Planck equation can be derived similar to (12.35) and is

$$
\frac{\partial \Psi}{\partial t}+\omega_{0} p_{w} \frac{\partial \Psi}{\partial w}-\left(\omega_{0} w+2 \alpha_{w} p_{w}\right) \frac{\partial \Psi}{\partial p_{w}}=2 \alpha_{w} \Psi+D_{\xi} \frac{\partial^{2} \Psi}{\partial w^{2}}+D_{\pi} \frac{\partial^{2} \Psi}{\partial p_{w}^{2}} .
$$

This form of the Fokker-Planck equation will be very useful to describe a particle beam under the influence of diffusion processes. In the following section, we will derive general solutions which will be applicable to specific situations in accelerator physics.

\subsubsection{Stationary Solution of the Fokker-Planck Equation}

A unique stationary solution exists for the particle density distribution described by the partial differential equation (12.98). To derive this solution we transform (12.98) to cylindrical coordinates $\left(w, p_{w}\right) \rightarrow(r, \theta)$ with $w=r \cos \theta$ and $p_{w}=r \sin \theta$ and note terms proportional to derivatives of the phase space density with respect to the angle $\theta$. One of these terms $\omega_{0} \Psi_{\theta}$ exists even in the absence of diffusion and damping and describes merely the betatron motion in phase space while the other terms depend on damping and diffusion. The diffusion terms will introduce a statistical mixing of the phases $\theta$ and after some damping times any initial azimuthal variation of the phase space density will be washed out. We are here only interested in the stationary solution and therefore set all derivatives of the phase space density with respect to the phase $\theta$ to zero. In addition we find it necessary to average square terms of $\cos \theta$ and $\sin \theta$. With these assumptions the Fokker-Planck Equation (12.98) becomes after some manipulations in the new coordinates

$$
\frac{\mathrm{d} \Psi}{\mathrm{d} t}=2 \alpha_{w} \Psi+\left(\alpha_{w} r+\frac{D}{r}\right) \frac{\partial \Psi}{\partial r}+D \frac{\partial^{2} \Psi}{\partial r^{2}},
$$


where we have defined a total diffusion coefficient

$$
D=\frac{1}{2}\left(D_{\xi}+D_{\pi}\right)
$$

Equation (12.100) has some similarity with, for example, wave equations in quantum mechanics which are solved by the method of separation of variables and we expect the stationary solution for the phase space density to be of the form $\Psi(r, t)=\sum_{n} F_{n}(t) G_{n}(r)$. The solution $G_{n}(r)$ must meet some particular boundary conditions. Specifically, at time $t=0$, we may have any arbitrary distribution of the phase space density $G_{n 0}(r)$. Furthermore, we specify that there be a wall at $r=R$ beyond which the phase space density drops to zero and consequently, the boundary conditions are

$$
\begin{aligned}
& G_{n}(r<R)=G_{n 0}(r), \\
& G_{n}(r>R)=0 .
\end{aligned}
$$

By the method of separation of the constants we find for the functions $F_{n}(t)$

$$
F_{n}(t)=\text { const. } \mathrm{e}^{-\alpha_{n} t},
$$

where the quantity $-\alpha_{n}$ is the separation constant. The general form of the solution for (12.100) may now be expressed by a series of orthogonal functions or eigenmodes of the distribution $G_{n}(r)$ which fulfill the boundary conditions (12.102)

$$
\Psi(r, t)=\sum_{n \geq 0} c_{n} G_{n}(r) \mathrm{e}^{-\alpha_{n} t} .
$$

The amplitudes $c_{n}$ in (12.104) are determined such as to fit the initial density distribution

$$
\Psi_{0}(r, t=0)=\sum_{n \geq 0} c_{n} G_{n 0}(r)
$$

With the ansatz (12.104) we get from (12.100) for each of the eigenmodes the following second-order differential equation:

$$
\frac{\partial^{2} G_{n}}{\partial r^{2}}+\left(\frac{1}{r}+\frac{\alpha_{w}}{D} r\right) \frac{\partial G_{n}}{\partial r}+\frac{\alpha_{w}}{D}\left(2+\frac{\alpha_{n}}{\alpha_{w}}\right) G_{n}=0 .
$$

All terms with a coefficient $\alpha_{n}>0$ vanish after some time due to damping (12.103). Negative values for the damping decrements $\alpha_{n}<0$ define instabilities which we will not consider here. Stationary solutions, therefore require the separation constants to be zero $\alpha_{n}=0$. Furthermore, all solutions $G_{n}$ must vanish at the boundary $r=R$ where $R$ may be any value including infinity if there are no physical boundaries at all to limit the maximum particle oscillation amplitude. In the latter 
case where there are no walls, the differential equation (12.106) can be solved by the stationary distribution

$$
\Psi(r, t)=\sum_{\substack{n \geq 0 \\ \alpha_{n}=0}} c_{n} G_{n}(r) \propto \exp \left(-\frac{\alpha_{w}}{2 D} r^{2}\right),
$$

which can easily be verified by backinsertion into (12.106). The solution for the particle distribution in phase space under the influence of damping $\alpha_{w}$ and statistical fluctuations $D$ is a Gaussian distribution with the standard width

$$
\sigma_{r}=\sqrt{\frac{D}{\alpha_{w}}}
$$

Normalizing the phase space density the stationary solution of the FokkerPlanck equation for a particle beam under the influence of damping and statistical fluctuations is

$$
\Psi(r)=\frac{1}{\sqrt{2 \pi} \sigma_{r}} \mathrm{e}^{-r^{2} / 2 \sigma_{r}^{2}}
$$

Eigenfunctions for which the eigenvalues $\alpha_{n}$ are not zero, are needed to describe an arbitrary particle distribution, e.g., a rectangular distribution at time $t=0$. The Fokker-Planck equation, however, tells us that after some damping times these eigensolutions have vanished and the Gaussian distribution is the only stationary solution left. The Gaussian distribution is not restricted to the $r$-space alone. The particle distribution in equilibrium between damping and fluctuations is also Gaussian in the normalized phase space $\left(w, p_{w}\right)$ as well as in real space. With $r^{2}=w^{2}+p_{w}^{2}$ we get immediately for the density distribution in $\left(w, p_{w}\right)$-space

$$
\Psi\left(w, p_{w}\right)=\frac{1}{2 \pi \sigma_{w} \sigma_{p_{w}}} \mathrm{e}^{-w^{2} / 2 \sigma_{w}^{2}} \mathrm{e}^{-p_{w}^{2} / 2 \sigma_{p w}^{2}},
$$

where we have set $\sigma_{w}=\sigma_{p_{w}}=\sqrt{\frac{D}{\alpha_{w}}}$. The standard deviation in $w$ and $p_{w}$ is the same as for $r$ which is to be expected since all three quantities have the same dimension and are linearly related.

In real space we have for $u=x$ or $y$ by definition $u=\sqrt{\beta_{u}} w$ and $p=\frac{\dot{w}}{v}$ where $\dot{w}=\frac{\mathrm{d} w}{\mathrm{~d} \varphi}$. On the other hand, $p=\sqrt{\beta_{x}} x^{\prime}-\frac{\beta^{\prime}}{2 \sqrt{\beta}} x$ and inserted into (12.107) we get the density distribution in real space

$$
\Psi\left(u, u^{\prime}\right) \propto \exp \left(-\frac{\gamma_{u} u^{2}-\beta_{u}^{\prime} u u^{\prime}+\beta_{u} u^{\prime 2}}{2 \sigma_{w}^{2}}\right) .
$$


This distribution describes the particle distribution in real phase space where particle trajectories follow tilted ellipses. Note that we carefully avoid replacing the derivative of the betatron function with $\beta^{\prime}=-2 \alpha$ because this would lead to a definite confusion between the damping decrement and the betatron function. To further reduce confusion we also use the damping times $\tau_{i}=\alpha_{i}^{-1}$. Integrating the distribution (12.111) for all values of the angles $u^{\prime}$, for example, gives the particle distribution in the horizontal or vertical midplane. Using the mathematical relation $\int_{\infty}^{\infty} \mathrm{e}^{-p^{2} x^{2} \pm q x} \mathrm{~d} x=\frac{\sqrt{\pi}}{p} \mathrm{e}^{q^{2} /\left(4 p^{2}\right)}$ [3], we get

$$
\Psi(u)=\frac{1}{\sqrt{2 \pi} \sqrt{\beta_{u}} \sigma_{w}} \mathrm{e}^{-u^{2} / 2 \sigma_{u}^{2}},
$$

where the standard width of the horizontal Gaussian particle distribution is

$$
\sigma_{u}=\sqrt{\beta} \sigma_{w}=\sqrt{\beta} \sqrt{\tau_{u} D_{u}}
$$

The index ${ }_{u}$ has been added to the diffusion and damping terms to indicate that these quantities are in general different in the horizontal and vertical plane. The damping time depends on all bending magnets, vertical and horizontal, but only on the damping-partition number for the plane under consideration. Similar distinction applies to the diffusion term.

In a similar way, we get the distribution for the angles by integrating (12.111) with respect to $u$

$$
\Psi\left(u^{\prime}\right)=\frac{\sqrt{\beta}}{\sqrt{2 \pi} \sqrt{1+\frac{1}{4} \beta^{\prime 2} \sigma_{w}}} \exp \left[-\frac{\beta u^{\prime 2}}{2\left(1+\frac{1}{4} \beta^{\prime 2}\right) \sigma_{w}^{2}}\right],
$$

where the standard width of the angular distribution is

$$
\sigma_{u}^{\prime}=\sqrt{\frac{4+\beta^{\prime 2}}{4 \beta}} \sigma_{w}=\sqrt{\frac{4+\beta^{\prime 2}}{4 \beta}} \sqrt{\tau_{u} D_{u}} .
$$

We have not made any special assumption as to the horizontal or vertical plane and find in (12.112)-(12.115) the solutions for the particle distribution in both planes.

In the longitudinal phase space the equations of motion are mathematically equal to Eq. (12.11). First we define new variables

$$
\dot{w}=-\frac{\Omega_{\mathrm{s} 0}}{\eta_{\mathrm{c}}} \dot{\tau},
$$


where $\Omega_{\mathrm{s} 0}$ is the synchrotron oscillation frequency, $\eta_{\mathrm{c}}$ the momentum compaction and $\tau$ the longitudinal deviation of a particle from the reference particle. The conjugate variable we define by

$$
p=-\frac{\dot{\epsilon}}{E_{0}},
$$

where $\epsilon$ is the energy deviation from the reference energy $E_{0}$. After differentiation of (12.52) and making use of (12.53) and the definition of the synchrotron oscillation frequency, we use these new variables and obtain the two first-order differential equations

$$
\begin{aligned}
& \dot{w}=+\Omega_{\mathrm{s}} p, \\
& \dot{p}=-\Omega_{\mathrm{s}} w-2 \alpha_{\epsilon} p .
\end{aligned}
$$

These two equations are of the same form as (12.11) and the solution of the longitudinal Fokker-Planck equation is therefore similar to (12.112)-(12.115). The energy distribution within a particle beam under the influence of damping and statistical fluctuations becomes with $p=\delta=\epsilon / E_{0}$

$$
\Psi(\delta)=\frac{1}{\sqrt{2 \pi} \sigma_{\delta}} \mathrm{e}^{-\delta^{2} / 2 \sigma_{\delta}^{2}},
$$

where the standard value for the energy spread in the particle beam is defined by

$$
\frac{\sigma_{\epsilon}}{E_{0}}=\sqrt{\tau_{\epsilon} D_{\epsilon}}
$$

In a similar way, we get for the conjugate coordinate $\tau$ with $w=\frac{\Omega_{\mathrm{s}}}{\eta_{\mathrm{c}}} \tau$ the distribution

$$
\Psi(\tau)=\frac{1}{\sqrt{2 \pi} \sigma_{\tau}} \mathrm{e}^{-\tau^{2} / 2 \sigma_{\tau}^{2}} .
$$

The standard width of the longitudinal particle distribution is finally

$$
\sigma_{\tau}=\frac{\left|\eta_{\mathrm{c}}\right|}{\Omega_{\mathrm{s}}} \sqrt{\tau_{\epsilon} D_{\epsilon}} .
$$

The deviation in time $\tau$ of a particle from the synchronous particle is equivalent to the distance of these two particles and we may therefore define the standard value for the bunch length from (12.123) by

$$
\sigma_{\ell}=c \beta \frac{\left|\eta_{\mathrm{c}}\right|}{\Omega_{\mathrm{s}}} \sqrt{\tau_{\epsilon} D_{\epsilon}} .
$$


By application of the Fokker-Planck equation to systems of particles under the influence of damping and statistical fluctuations, we were able to derive expressions for the particle distribution within the beam. In fact, we were able to determine that the particle distribution is Gaussian in all six degrees of freedom. Since such a distribution does not exhibit any definite boundary for the beam, it becomes necessary to define the size of the distributions in all six degrees of freedom by the standard value of the Gaussian distribution. Specific knowledge of the nature for the statistical fluctuations are required to determine the numerical values of the beam sizes.

In Chap. 13 we will apply these results to determine the equilibrium beam emittance in an electron positron storage ring where the statistical fluctuations are generated by quantized emission of synchrotron radiation photons.

\subsubsection{Particle Distribution within a Finite Aperture}

The particle distribution in an electron beam circulating in a storage ring is a Gaussian if we ignore the presence of walls containing the beam. All other modes of particle distribution are associated with a finite damping time and vanish therefore after a short time. In a real storage ring we must, however, consider the presence of vacuum chamber walls which cut off the Gaussian tails of the particle distribution. Although the particle intensity is very small in the far tails of a Gaussian distribution, we cannot cut off those tails too tight without reducing significantly the beam lifetime. Due to quantum excitation, we observe a continuous flow of particles from the beam core into the tails and back by damping toward the core. A reduction of the aperture into the Gaussian distribution absorbs therefore not only those particles which populate these tails at a particular moment but also all particles which reach occasionally large oscillation amplitudes due to the emission of a high energy photon. The absorption of particles due to this effect causes a reduction in the beam lifetime which we call the quantum lifetime.

The presence of a wall modifies the particle distribution especially close to the wall. This modification is described by normal mode solutions with a finite damping time which is acceptable now because any aperture less than an infinite aperture absorbs beam particles thus introducing a finite beam lifetime. Cutting off Gaussian tails at large amplitudes will not affect the Gaussian distribution in the core and we look therefore for small variations of the Gaussian distribution which become significant only quite close to the wall. Instead of (12.107) we try the ansatz

$$
\Psi(r, t)=\mathrm{e}^{-\frac{\alpha_{W}}{2 D} r^{2}} g(r) \mathrm{e}^{-\alpha t}
$$

where $1 / \alpha$ is the time constant for the distribution, with the boundary condition that the particle density be zero at the aperture or acceptance defining wall $r=A$ or

$$
\Psi(A, t)=0 .
$$


Equation (12.125) must be a solution of (12.100) and back insertion of (12.125) into (12.100) gives the condition on the function $g(r)$

$$
g^{\prime \prime}+\left(\frac{1}{r}-\frac{r}{\sigma^{2}}\right) g^{\prime}+\frac{\alpha}{\alpha_{w} \sigma^{2}} g=0 .
$$

Since $g(r)=1$ in case there is no wall, we expand the correction into a power series

$$
g(r)=1+\sum_{k \geq 1} C_{k} x^{k}, \quad \text { where } \quad x=\frac{r^{2}}{2 \sigma^{2}} .
$$

Inserting (12.128) into (12.127) and collecting terms of equal powers in $r$ we derive the coefficients

$$
C_{k}=\frac{1}{(k !)^{2}} \prod_{p=1}^{p=k}(p-1-X) \approx-\frac{(k-1) !}{(k !)^{2}} X,
$$

where $X=\frac{\alpha}{2 \alpha_{w}} \ll 1$. The approximation $X \ll 1$ is justified since we expect the vacuum chamber wall to be far away from the beam center such that the expected quantum lifetime $1 / \alpha$ is long compared to the damping time $1 / \alpha_{w}$ of the oscillation under consideration. With these coefficients (12.128) becomes

$$
g(r)=1-\frac{\alpha}{2 \alpha_{w}} \sum_{k \geq 1} \frac{1}{k k !} x^{k} .
$$

For $x=A^{2} /\left(2 \sigma^{2}\right) \gg 1$ where $A$ is the amplitude or amplitude limit for the oscillation $w$, the sum in (12.130) can be replaced by an exponential function

$$
\sum_{k \geq 1} \frac{1}{k k !} x^{k} \approx \frac{\mathrm{e}^{x}}{x}
$$

From the condition $g(A)=0$ we finally get for the quantum lifetime $\tau_{\mathrm{q}}=1 / \alpha$

$$
\tau_{\mathrm{q}}=\frac{1}{2} \tau_{w} \frac{\mathrm{e}^{x}}{x}
$$

where

$$
x=\frac{A^{2}}{2 \sigma^{2}} .
$$

The quantum lifetime $\tau_{\mathrm{q}}$ is related to the damping time. To make the quantum life time very large of the order of 50 or more hours, the aperture must be at least about $7 \sigma_{w}$ in which case $x=24.5$ and $\mathrm{e}^{x} / x=1.8 \times 10^{9}$. 
The aperture $A$ is equal to the transverse acceptance of a storage ring for a onedimensional oscillation like the vertical betatron oscillation while longitudinal or energy oscillations are limited through the maximum energy acceptance allowed by the rf-voltage. Upon closer look, however, we note a complication for horizontal betatron oscillations and synchrotron oscillations because of the coupling from energy oscillation into transverse position due to a finite dispersion function. We also have assumed that $\alpha /\left(2 \alpha_{w}\right) \ll 1$ which is not true for tight apertures of less than one sigma. Both of these situations have been investigated in detail $[4,5]$ and the interested reader is referred to those references.

Specifically, if the acceptance $A$ of a storage ring is defined at a location where there is also a finite dispersion function, Chao [4] derives a combined quantum lifetime of

$$
\tau=\frac{\mathrm{e}^{n^{2} / 2}}{\sqrt{2 \pi} \alpha_{x} n^{3}} \frac{1}{(1+r) \sqrt{r(1-r)}},
$$

where $n=A / \sigma_{\mathrm{T}}, \sigma_{\mathrm{T}}^{2}=\sigma_{x}^{2}+\eta^{2} \sigma_{\delta}^{2}, r=\eta^{2} \sigma_{\delta}^{2} / \sigma_{\mathrm{T}}^{2}, A$ the transverse aperture, $\eta$ the dispersion function at the same location where the aperture is $A, \sigma_{x}$ the transverse beam size and $\sigma_{\delta}=\sigma_{\epsilon} / E$ the standard relative energy width in the beam.

\subsubsection{Particle Distribution in the Absence of Damping}

To obtain a stationary solution for the particle distribution it was essential that there were eigensolutions with vanishing eigenvalues $\alpha_{n}=0$. As a result, we obtained an equilibrium solution where the statistical fluctuations are compensated by damping. In cases where there is no damping, we would expect a different solution with particles spreading out due to the effect of diffusion alone. This case can become important in very high energy electron positron linear colliders where an extremely small beam emittance must be preserved along a long beam transport line. The differential equation (12.106) becomes in this case

$$
\frac{\partial^{2} G_{n}}{\partial r^{2}}+\frac{1}{r} \frac{\partial G_{n}}{\partial r}+\frac{\alpha_{n}}{D} G_{n}=0 .
$$

We will assume that a beam with a Gaussian particle distribution is injected into a damping free transport line and we therefore look for solutions of the form

$$
\Psi_{n}(r, t)=c_{n} G_{n}(r) \mathrm{e}^{-\alpha_{n} t},
$$

where

$$
G_{n}(r)=\mathrm{e}^{-r^{2} / 2 \sigma_{0}^{2}}
$$


with $\sigma_{0}$ being the beam size at $t=0$. We insert (12.137) into (12.135) and obtain an expression for the eigenvalues $\alpha_{n}$

$$
\alpha_{n}=\frac{2 D}{\sigma_{0}^{2}}-\frac{D}{\sigma_{0}^{4}} r^{2} .
$$

The time dependent solution for the particle distribution now becomes

$$
\Psi(r, t)=A \exp \left(-\frac{2 D}{\sigma_{0}^{2}} t\right) \exp \left[\left(-\frac{r^{2}}{2 \sigma_{0}^{2}}\right)\left(1-\frac{2 D}{\sigma_{0}^{2}} t\right)\right] .
$$

Since nowhere a particular mode is used we have omitted the index $n$. The solution (12.139) exhibits clearly the effect of the diffusion in two respects. The particle density decays exponentially with the decrement $2 D / \sigma_{0}^{2}$. At the same time the distribution remains to be Gaussian although being broadened by diffusion. The time dependent beam size $\sigma$ is given by

$$
\sigma^{2}(t)=\frac{\sigma_{0}^{2}}{1-\frac{2 D}{\sigma_{0}^{2}} t} \approx \sigma_{0}^{2}\left(1+\frac{2 D}{\sigma_{0}^{2}} t\right)
$$

where we have assumed that the diffusion term is small $\left(2 D / \sigma_{0}^{2}\right) t \ll 1$. Setting $\sigma^{2}=\sigma_{u}^{2}=\epsilon_{u} \beta_{u}$ for the plane $u$ where $\beta_{u}$ is the betatron function at the observation point of the beam size $\sigma_{u}$. The time dependent beam emittance is

$$
\epsilon_{u}=\epsilon_{u 0}+\frac{2 D}{\beta_{u}} t
$$

or the rate of change

$$
\frac{\mathrm{d} \epsilon_{u}}{\mathrm{~d} t}=\frac{2 D}{\beta_{u}}=\frac{D_{\xi}+D_{\pi}}{\beta_{u}} .
$$

Due to the diffusion coefficient $D$ we obtain a continuous increase of the beam emittance in cases where no damping is available.

The Fokker-Planck diffusion equation provides a tool to describe the evolution of a particle beam under the influence of conservative forces as well as statistical processes. Specifically, we found that such a system has a stationary solution in cases where there is damping. The stationary solution for the particle density is a Gaussian distribution with the standard width of the distribution $\sigma$ given by the diffusion constant and the damping decrement.

In particular, the emission of photons due to synchrotron radiation has the properties of a Markoff process and we find therefore the particle distribution to be Gaussian. Indeed we will see that this is true in all six dimensions of phase space. 
Obviously not every particle beam is characterized by the stationary solution of the Fokker-Planck equation. Many modes contribute to the particle distribution and specifically at time $t=0$ the distribution may have any arbitrary form. However, it has been shown that after a time long compared to the damping times only one nontrivial stationary solution is left, the Gaussian distribution.

\section{Problems}

12.1 (S). Derive from the Vlasov equation an expression for the synchrotron frequency while ignoring damping. A second rf-system with different frequency can be used to change the synchrotron tune. Determine a system that would reduce the synchrotron tune for the reference particle to zero while providing the required rf-voltage at the synchronous phase. What is the relationship between both voltages and phases? Is the tune shift the same for all particles?

12.2 (S). Formulate an expression for the equilibrium bunch length in a storage ring with two rf-systems of different frequencies to control bunch length.

12.3 (S). Energy loss of a particle beam due to synchrotron radiation provides damping. Show that energy loss due to interaction with an external electromagnetic field does not provide beam damping.

12.4 (S). An arbitrary particle distribution of beam injected into a storage ring damps out while a Gaussian distribution evolves with a standard width specific to the ring design. What happens if a beam from another storage ring with a different Gaussian distribution is injected? Explain why this beam changes its distribution to the ring specific Gaussian distribution.

12.5 (S). Consider a $1.5 \mathrm{GeV}$ electron storage ring with a bending field of $1.5 \mathrm{~T}$. The circumference may be covered to $60 \%$ by bending magnets. Let the bremsstrahlung lifetime be $100 \mathrm{~h}$, the Coulomb scattering lifetime $50 \mathrm{~h}$ and the Touschek lifetime $60 \mathrm{~h}$. Calculate the total beam lifetime including quantum excitation as a function of aperture. How many "sigma's" $(A / \sigma)$ must the apertures be in order not to reduce the beam lifetime by more than $10 \%$ due to quantum excitation?

12.6. To reduce coupling instabilities between bunches of a multibunch beam it is desirable to give each bunch a different synchrotron tune. This can be done, for example, by employing two rf-systems operating at harmonic numbers $h$ and $h+1$. Determine the ratio or required rf-voltages to split the tunes between successive bunches by $\Delta v / v_{\mathrm{s}}$.

12.7. Attempt to damp out the energy spread of a storage ring beam in the following way. At a location where the dispersion function is finite one could insert a $\mathrm{TM}_{110^{-}}$ mode cavity. Such a cavity produces accelerating fields which vary linear with the transverse distance of a particle from the reference path. This together with a 
linear change in particle energy due to the dispersion would allow the correction of the energy spread in the beam. Derive the complete Vlasov equation for such an arrangement and discuss the six-dimensional dynamics. Show that it impossible to achieve a monochromatic stable beam.

12.8. Derive an expression for the diffusion due to elastic scattering of beam particles on residual gas atoms. How does the equilibrium beam emittance of an electron beam scale with gas pressure and beam energy? Determine an expression for the required gas pressure to limit the emittance growth of a proton or ion beam to no more than $1 \%$ per hour and evaluate numerical for a proton emittance of $10^{-9} \mathrm{rad}-\mathrm{m}$ at an energy of $300 \mathrm{GeV}$. Is this a problem if the achievable vacuum pressure is $1 \mathrm{nTorr}$ ? Concentrating the allowable scattering to one location of $10 \mathrm{~cm}$ length (gas jet as a target) in a ring of $4 \mathrm{~km}$ circumference, calculate the tolerable pressure of the gas jet.

12.9. For future linear electron colliders it may be desirable to provide a switching of the beams from one experimental detector to another. Imagine a linear collider system with two experimental stations separated transversely by $50 \mathrm{~m}$. To guide the beams from the linear accelerators to the experimental stations use translating FODO cells and determine the parameters required to keep the emittance growth of a beam to less than $10 \%$ (beam emittance $10^{-11}$ rad-m at $500 \mathrm{GeV}$ ).

\section{References}

1. K.W. Robinson, Phys. Rev. 111, 373 (1958)

2. H. Risken, The Fokker-Planck Equation (Springer, Berlin/Heidelberg, 1989)

3. I.S. Gradshteyn, I.M. Ryzhik, Table of Integrals, Series, and Products, 4th edn. (Academic, New York, 1965)

4. A.W. Chao, in Physics of High Energy Particle Accelerators, vol. AIP 87, p. 395, ed. by M. Month, M. Dienes (American Institute of Physics, New York, 1982)

5. Y.H. Chin, Quantum lifetime. Technical Report DESY Report 87-062, DESY, DESY, Hamburg (1987) 


\section{Chapter 13 \\ Equilibrium Particle Distribution*}

The wide variety of particle beam applications require often very specific beam characteristics in terms of say cross section, divergence, energy spread or pulse structure. To a large extend such parameters can be adjusted by particular application of focusing and other forces. In this chapter, we will discuss some of these methods of beam optimization and manipulation.

\subsection{Particle Distribution in Phase Space}

The beam emittance of particle beams is primarily defined by characteristic source parameters and source energy. Given perfect matching between different accelerators and beam lines during subsequent acceleration, this source emittance is reduced inversely proportional to the particle momentum by adiabatic damping and stays constant in terms of normalized emittance. This describes accurately the situation for proton and ion beams, for nonrelativistic electrons and electrons in linear accelerators.

The beam emittance for relativistic electrons, however, evolves fundamentally different in circular accelerators. Relativistic electron and positron beams passing through bending magnets emit synchrotron radiation, a process that leads to quantum excitation and damping. As a result, the original beam emittance at the source is completely replaced by an equilibrium emittance that is unrelated to the source characteristics.

This chapter has been made Open Access under a CC BY 4.0 license. For details on rights and licenses please read the Correction https://doi.org/10.1007/978-3-319-18317-6_28 


\subsubsection{Diffusion Coefficient and Synchrotron Radiation}

Emission of a photon causes primarily a change of the particle energy but the characteristics of the particle motion is changed as well. Neither position nor the direction of the particle trajectory is changed during the emission of photons. From beam dynamics, however, we know that different reference trajectories exist for particles with different energies. Two particles with energies $c p_{0}$ and $c p_{1}$ follow two different reference trajectories separated at the position $z$ along the beam transport line by a distance

$$
\Delta x=\eta(z) \frac{c p_{1}-c p_{0}}{c p_{0}}
$$

where $\eta(z)$ is the dispersion function and $c p_{0}$ the reference energy. Although particles in general do not exactly follow these reference trajectories they do perform betatron oscillations about them. The sudden change of the particle energy during the emission of a photon leads to a sudden change in the reference path and thereby to a sudden change in the betatron oscillation amplitude.

Following the discussion of the Fokker-Planck equation in Chap. 12, we may derive a diffusion coefficient from these sudden changes in the coordinates. Using normalized coordinates $w=x / \sqrt{\beta}$, the change in the betatron amplitude at the moment a photon of energy $\epsilon_{\gamma}$ is emitted becomes

$$
\Delta w=\xi=-\frac{\eta(z)}{\sqrt{\beta_{x}}} \frac{\epsilon_{\gamma}}{E_{0}} .
$$

Similarly, the conjugate coordinate $\dot{w}=\sqrt{\beta_{x}} x_{\beta}^{\prime}+\alpha_{x} x_{\beta}$ changes by

$$
\Delta \dot{w}=\pi=-\sqrt{\beta_{x}} \eta^{\prime} \frac{\epsilon_{\gamma}}{E_{0}}-\frac{\alpha_{x}}{\sqrt{\beta_{x}}} \eta \frac{\epsilon_{\gamma}}{E_{0}} .
$$

The frequency at which these statistical variations occur is the same for $\xi$ and $\pi$ and is equal to the number of photons emitted per unit time

$$
\mathcal{N}_{\xi}=\mathcal{N}_{\pi}=\mathcal{N}
$$

From (13.2), (13.3) we get

$$
\xi^{2}+\pi^{2}=\left(\frac{\epsilon_{\gamma}}{E_{0}}\right)^{2}\left[\frac{\eta^{2}}{\beta_{x}}+\left(\sqrt{\beta_{x}} \eta^{\prime}+\frac{\alpha_{x}}{\sqrt{\beta_{x}}} \eta\right)^{2}\right]=\left(\frac{\epsilon_{\gamma}}{E_{0}}\right)^{2} \mathcal{H},
$$


where we have defined a special lattice function

$$
\mathcal{H}=\beta_{x} \eta^{\prime 2}+2 \alpha_{x} \eta \eta^{\prime}+\gamma_{x} \eta^{2}
$$

We are interested in the average value of the total diffusion coefficient (12.101)

$$
D=\frac{1}{2}\left\langle\mathcal{N}\left(\xi^{2}+\pi^{2}\right)\right\rangle_{z}=\frac{1}{2 E_{0}^{2}}\left\langle\mathcal{N}\left\langle\epsilon_{\gamma}^{2}\right\rangle \mathcal{H}\right\rangle_{z},
$$

where the average $\langle\cdots\rangle_{z}$ is to be taken along the whole transport line or the whole circumference of a circular accelerator. Since photon emission does not occur outside of bending magnets, the average is taken only along the length of the bending magnets. To account for the variation in photon energies, we use the rms value of the photon energies $\left\langle\epsilon_{\gamma}^{2}\right\rangle$. The theory of synchrotron radiation is discussed in much detail in Chap. 23 and we take in the following paragraph only relevant results of this theory.

The number of photons emitted per unit time with frequencies between $\omega$ and $\omega+\mathrm{d} \omega$ is simply the spectral radiation power at this frequency divided by the photon energy $\hbar \omega$. Here, we consider only bending magnet radiation and treat radiation from insertion devices as perturbations. Of course, this approach must be modified if a significant part of radiation comes from non-bending magnet radiation. The spectral photon flux from a single electron is from (25.132) with the synchrotron radiation power $(24.34)$

$$
\frac{\mathrm{d} n(\omega)}{\mathrm{d} \omega}=\frac{1}{\hbar \omega} \frac{\mathrm{d} P(\omega)}{\mathrm{d} \omega}=\frac{P_{\gamma}}{\hbar \omega_{c}^{2}} \frac{9 \sqrt{3}}{8 \pi} \int_{\zeta}^{\infty} K_{5 / 3}(x) \mathrm{d} x,
$$

where $\zeta=\omega / \omega_{c}$ and the critical photon energy defined in (24.49). The total photon flux is by integration over all frequencies

$$
\mathcal{N}=\frac{P_{\gamma}}{\hbar \omega_{c}} \frac{9 \sqrt{3}}{8 \pi} \int_{0}^{\infty} \int_{\zeta}^{\infty} K_{5 / 3}(x) \mathrm{d} x \mathrm{~d} \zeta
$$

which becomes with $\mathrm{GR}(6.561 .16)$ and $\Gamma(1 / 6) \Gamma(1 / 6)=5 \pi / 3$ after integration by parts from $\operatorname{AS}(6.1 .17)$

$$
\mathcal{N}=\frac{P_{\gamma}}{\hbar \omega_{c}} \frac{9 \sqrt{3}}{8 \pi} \int_{0}^{\infty} K_{5 / 3}(\zeta) \mathrm{d} \zeta=\frac{15 \sqrt{3}}{8} \frac{P_{\gamma}}{\hbar \omega_{c}} .
$$

The rms value of the photon energy $\left\langle\epsilon_{\gamma}^{2}\right\rangle$ can be derived in the usual way from the spectral distribution $n(\omega)$ by

$$
\left\langle\epsilon_{\gamma}^{2}\right\rangle=\frac{\hbar^{2}}{\mathcal{N}} \int_{0}^{\infty} \omega^{2} n(\omega) \mathrm{d} \omega=\frac{9 \sqrt{3} P_{\gamma} \hbar \omega_{\mathrm{c}}}{8 \pi \mathcal{N}} \int_{0}^{\infty} \zeta^{2} \int_{\zeta}^{\infty} K_{5 / 3}(x) \mathrm{d} x \mathrm{~d} \zeta
$$


and is after integration by parts

$$
\left\langle\epsilon_{\gamma}^{2}\right\rangle=\frac{P_{\gamma} \hbar \omega_{c}}{\mathcal{N}} \frac{9 \sqrt{3}}{8 \pi} \frac{1}{3} \int_{0}^{\infty} \zeta^{3} K_{5 / 3}(\zeta) \mathrm{d} \zeta .
$$

The integral of the modified Bessel's function in (13.12) is from GR[6.561.16] $4 \Gamma\left(2+\frac{5}{6}\right) \Gamma\left(2-\frac{5}{6}\right)$ where we use again $\mathrm{AS}(6.1 .17)$ for $\Gamma\left(\frac{5}{6}\right) \Gamma\left(\frac{1}{6}\right)=2 \pi$. Collecting all terms

$$
\mathcal{N}\left\langle\epsilon_{\gamma}^{2}\right\rangle=\frac{55}{24 \sqrt{3}} P_{\gamma} \hbar \omega_{c}
$$

and the diffusion coefficient (13.7) becomes

$$
D=\frac{1}{2}\left\langle\mathcal{N}\left(\xi^{2}+\pi^{2}\right)\right\rangle_{z}=\frac{55}{48 \sqrt{3}} \frac{\left\langle P_{\gamma} \hbar \omega_{c} \mathcal{H}\right\rangle_{z}}{E_{0}^{2}}
$$

The stationary solution for the Fokker-Planck equation has been derived describing the equilibrium particle distribution in phase space under the influence of quantum excitation and damping. In all six dynamical degrees of freedom the equilibrium distribution is a Gaussian distribution and the standard value of the distribution width is determined by the damping time and the respective diffusion coefficient. In this chapter, we will be able to calculate quantitatively the diffusion coefficients and from that the beam parameters.

\subsubsection{Quantum Excitation of Beam Emittance}

High energy electron or positron beams passing through a curved beam transport line suffer from quantum excitation which is not compensated by damping since there is no acceleration. In Sect. 12.3.3 we have discussed this effect and found the transverse beam emittance to increase linear with time (12.142) and we get with (13.14)

$$
\frac{\mathrm{d} \epsilon_{x}}{c \mathrm{~d} t}=\frac{\mathrm{d} \epsilon_{x}}{\mathrm{~d} z}=\frac{55}{24 \sqrt{3}} \frac{r_{\mathrm{e}} \hbar c}{m c^{2}} \gamma^{5}\left\langle\frac{\mathcal{H}}{\rho^{3}}\right\rangle_{z} .
$$

There is a strong energy dependence of the emittance increase along the beam transport line and the effect becomes significant for high beam energies as proposed for linear collider systems. Since the emittance blow up depends on the lattice function $\mathcal{H}$, we would choose a very strong focusing lattice to minimize the dilution of the beam emittance. For this reason, the beam transport system for the linear collider at the Stanford Linear Accelerator Center [1] is composed of very strongly focusing combined bending magnets. 
Particle distributions become modified each time we inject a beam into a circular accelerator with significant synchrotron radiation. Arbitrary particle distributions can be expected from injection systems before injection into a circular accelerator. If the energy in the circular accelerator is too small to produce significant synchrotron radiation the particular particle distribution is preserved according to Liouville's theorem while all particles merely rotate in phase space as discussed in Sect. 12.1. As the beam energy is increased or if the energy is sufficiently high at injection to generate significant synchrotron radiation, all modes in the representation of the initial particle distribution vanish within a few damping times while only one mode survives or builds up which is the Gaussian distribution with a standard width given by the diffusion constant and the damping time. In general, any deviation from this unique equilibrium solution and be it only a mismatch to the correct orientation of the beam in phase space will persist for a time not longer than a few damping times.

\subsection{Equilibrium Beam Emittance}

In circular electron accelerators, as in electron storage rings, quantum excitation is counteracted by damping. Since quantum excitation is not amplitude dependent but damping is, there is an equilibrium beam emittance when both effects are equally strong. In the presence of quantum fluctuations Liouville's theorem is not applicable strictly anymore. In the case of an electron beam in equilibrium the phase space density for a beam in equilibrium is preserved, although in a different way. While Liouville's theorem is based on Hamiltonian mechanics and demands that no particle should escape its phase space position we allow in the case of an electron beam in equilibrium that a particle may escape its phase space position but be replaced instantly by another particle due to damping.

\subsubsection{Horizontal Equilibrium Beam Emittance}

The horizontal beam size is related to damping and diffusion coefficient from (12.113) like

$$
\frac{\sigma_{x}^{2}}{\beta_{x}}=\tau_{x} D_{x} .
$$

Damping times have been derived in Sect. 12.2 and with (13.7) the horizontal beam size $\sigma_{x}$ at a location where the value of the betatron function is $\beta_{x}$ becomes

$$
\frac{\sigma_{x}^{2}}{\beta_{x}}=\frac{\left\langle\mathcal{N}\left\langle\epsilon_{\gamma}^{2}\right\rangle \mathcal{H}\right\rangle_{z}}{2 E_{0} J_{x}\left\langle P_{\gamma}\right\rangle_{z}} .
$$


The ratio $\sigma_{x}^{2} / \beta_{x}$ is consistent with our earlier definition of the beam emittance. For a particle beam which is in equilibrium between quantum excitation and damping, this ratio is defined as the equilibrium beam emittance being equivalent to the beam emittance for all particles within one standard value of the Gaussian distribution. For further simplification, we make use of the expression (13.13) and get with the radiation power (24.34) and the critical frequency (24.49) the horizontal beam emittance equation

$$
\epsilon_{x}=C_{\mathrm{q}} \gamma^{2} \frac{\left\langle\mathcal{H} /\left|\rho^{3}\right|\right\rangle_{z}}{J_{x}\left\langle 1 / \rho^{2}\right\rangle_{z}},
$$

where we adopted Sands' [2] definition of a quantum excitation constant

$$
C_{\mathrm{q}}=\frac{55}{32 \sqrt{3}} \frac{\hbar c}{m c^{2}}=3.84 \times 10^{-13} \mathrm{~m} .
$$

The equilibrium beam emittance scales like the square of the beam energy and depends further only on the bending radius and the lattice function $\mathcal{H}$. From the definition of $\mathcal{H}$ the horizontal equilibrium beam emittance depends on the magnitude of the dispersion function and can therefore be adjusted to small or large values depending on the strength of the focusing for the dispersion function.

\subsubsection{Vertical Equilibrium Beam Emittance}

The vertical beam emittance follows from (13.18) considering that the dispersion function and therefore $\mathcal{H}$ vanishes. Consequently, the equilibrium vertical beam emittance seems to be zero because there is only damping but no quantum excitation. In this case we can no longer ignore the fact that the photons are emitted into a finite although very small angle about the forward direction of particle propagation. Each such emission causes both a loss in the particle energy and a transverse recoil deflecting the particle trajectory. The photons are emitted typically within an angle $1 / \gamma$ generating a transverse kick without changing the betatron oscillation amplitude. With $\delta y=0$ and $\delta y^{\prime}=\frac{1}{\gamma} \frac{\epsilon_{\gamma}}{E_{0}}$, we get for the statistical variations

$$
\begin{aligned}
& \xi^{2}=0 \\
& \pi^{2}=\beta_{y} \frac{1}{\gamma^{2}}\left(\frac{\epsilon_{\gamma}}{E_{0}}\right)^{2} .
\end{aligned}
$$

Following a derivation similar to that for the horizontal beam emittance, we get for the vertical beam emittance equation

$$
\epsilon_{y}=C_{\mathrm{q}} \frac{\left\langle\beta_{y} /\left|\rho^{3}\right|\right\rangle_{z}}{J_{y}\left\langle 1 / \rho^{2}\right\rangle_{z}} .
$$


This is the fundamentally lower limit of the equilibrium beam emittance due to the finite emission angle of synchrotron radiation. For an isomagnetic ring the vertical beam emittance

$$
\epsilon_{y}=C_{\mathrm{q}} \frac{\left\langle\beta_{y}\right\rangle_{z}}{J_{y}|\rho|}
$$

does not depend on the particle energy but only on the bending radius and the average value of the betatron function. In most practical circular accelerator designs, both the bending radius and the betatron function are of similar magnitude and the fundamental emittance limit therefore is of the order of $C_{\mathrm{q}}=10^{-13}$ radian meter, indeed very small compared to actually achieved beam emittances.

The assumption that the vertical dispersion function vanishes in a flat circular accelerator is true only for an ideal ring. Dipole field errors, quadrupole misalignments and any other source of undesired dipole fields create a vertical closed orbit distortion and an associated vertical dispersion function. This vertical dispersion function, often called spurious dispersion function, is further modified by orbit correction magnets but it is not possible to completely eliminate it because the location of dipole errors are not known.

Since the diffusion coefficient $D$ is quadratic in the dispersion function (13.7) we get a contribution to the vertical beam emittance from quantum excitation similar to that in the horizontal plane. Indeed, this effect on the vertical beam emittance is much larger than that due to the finite emission angle of photons discussed above and is therefore together with coupling the dominant effect in the definition of the vertical beam emittance.

The contribution to the vertical beam emittance is in analogy to the derivation leading to (13.18)

$$
\Delta \epsilon_{y}=C_{\mathrm{q}} \gamma^{2} \frac{\left\langle\mathcal{H}_{y} /\left|\rho^{3}\right|\right\rangle_{z}}{J_{y}\left\langle 1 / \rho^{2}\right\rangle_{z}}
$$

where

$$
\frac{\mathcal{H}_{y}}{|\rho|^{3}}=\left\langle\frac{\beta_{y} \eta_{y}^{\prime 2}+2 \alpha_{y} \eta_{y} \eta_{y}^{\prime}+\gamma_{y} \eta_{y}^{2}}{|\rho|^{3}}\right\rangle_{z} .
$$

To minimize this effect, orbit correction schemes must be employed which not only correct the equilibrium orbit but also the perturbation to the dispersion function. Of course, the same effect with similar magnitude occurs also in the horizontal plane but is in general negligible compared to ordinary quantum excitation. 


\subsection{Equilibrium Energy Spread and Bunch Length}

The statistical processes caused by the emission of synchrotron radiation photons affect not only the four transverse dimensions of phase space but also the energytime phase space. Particles orbiting in a circular accelerator emit photons with a statistical distribution of energies while only the average energy loss is replaced in the accelerating cavities.

\subsubsection{Equilibrium Beam Energy Spread}

This leaves a residual statistical distribution of the individual particle energies which we have derived in Sect. 12.3 to be Gaussian just like the transverse particle distribution with a standard width given by (12.121). The conjugate coordinate is the "time" $w=\frac{\Omega}{\eta_{c}} \tau$ where $\tau$ is the deviation in time of a particle from the synchronous particle, and $\epsilon$ the energy deviation of a particle from the reference energy $E_{0}$.

The emission of a photon will not change the position of the particle in time and therefore $\xi=0$. The conjugate coordinate being the particle energy will change due to this event by the magnitude of the photon energy and we have $\pi=\epsilon_{\gamma} / E_{0}$. Comparing with (13.5), we note that we get the desired result analogous to the transverse phase space by setting $\mathcal{H}=1$ and using the correct damping time for longitudinal motion. The equilibrium energy spread becomes then from (12.121) in analogy to (13.18)

$$
\frac{\sigma_{\epsilon}^{2}}{E_{0}^{2}}=C_{\mathrm{q}} \gamma^{2} \frac{\left\langle\left|1 / \rho^{3}\right|\right\rangle_{z}}{J_{\epsilon}\left\langle 1 / \rho^{2}\right\rangle_{z}},
$$

which in a separated function lattice depends only on the particle energy and the bending radius. In a fully or partially combined function lattice, the partition number $J_{\epsilon}$ can be modified providing a way to vary the energy spread.

\subsubsection{Equilibrium Bunch Length}

There is also a related equilibrium distribution in the longitudinal dimension which defines the length of the particle bunch. This distribution is also Gaussian and the standard bunch length is from (12.123), (12.124)

$$
\sigma_{\ell}=c \beta \frac{\left|\eta_{\mathrm{c}}\right|}{\Omega_{\mathrm{s}}} \frac{\sigma_{\epsilon}}{E_{0}} .
$$


The equilibrium bunch length not only depends on the particle energy and the bending radius but also on the focusing lattice through the momentum compaction factor and the partition number as well as on rf-parameters included in the synchrotron oscillation frequency $\Omega_{\mathrm{s}}$. To exhibit the scaling, we introduce lattice and rf-parameters into (13.26) to get with (13.25) and the definition of the synchrotron frequency (9.32) an expression for the equilibrium bunch length

$$
\sigma_{\ell}^{2}=\frac{2 \pi C_{\mathrm{q}}}{\left(m c^{2}\right)^{2}} \frac{\eta_{c} E_{0}^{3} R^{2}}{J_{\epsilon} h e \hat{V}_{0} \cos \psi_{\mathrm{s}}} \frac{\left\langle\left|1 / \rho^{3}\right|\right\rangle_{z}}{\left\langle 1 / \rho^{2}\right\rangle_{z}},
$$

where $R$ is the average radius of the ring. The bunch length can be modified through more parameters than any other characteristic beam parameter in the sixdimensional phase space. Lattice design affects the resulting bunch length through the momentum compaction factor and the partition number. Strong focusing results in a small value for the momentum compaction factor and a small bunch length. Independent of the strength of the focusing, the momentum compaction factor can in principle be adjusted to any value including zero and negative values by allowing the dispersion function to change sign along a circular accelerator because the momentum compaction factor is the average of the dispersion function $\alpha_{\mathrm{c}}=\langle\eta / \rho\rangle$. In this degree of approximation the bunch length could therefore be reduced to arbitrarily small values by reducing the momentum compaction factor. However, close to the transition energy phase focusing to stabilize synchrotron oscillations is lost.

Introduction of gradient magnets into the lattice modifies the partition numbers as we have discussed in Sect. 12.2.1. As a consequence, both, the energy spread and bunch length increase or decrease at the expense of the opposite effect on the horizontal beam emittance. The freedom to adjust any of these three beam parameters in this way is therefore limited but nonetheless an important means to make small adjustments if necessary. Obviously, the rf-frequency as well as the rf-voltage have a great influence on the bunch length. The bunch length scales inversely proportional to the square root of the rf-frequency and is shorter for higher frequencies. Generally, no strong reasons exist to choose a particular rf-frequency but might become more important if control of the bunch length is important for the desired use of the accelerator. The bunch length is also determined by the rate of change of the rf-voltage in the accelerating cavities at the synchronous phase

$$
\dot{V}\left(\psi_{\mathrm{s}}\right)=\left.\frac{\mathrm{d}}{\mathrm{d} \psi} \hat{V} \sin \psi\right|_{\psi=\psi_{\mathrm{s}}}=\hat{V} \cos \psi_{\mathrm{s}} .
$$

For a single frequency rf-system the bunch length can be shortened when the rf-voltage is increased. To lengthen the bunch the rf-voltage can be reduced up to a point where the rf-voltage would fail to provide a sufficient energy acceptance. 


\subsection{Phase-Space Manipulation}

The distribution of particles in phase space is given either by the injector characteristics and injection process or in the case of electron beams by the equilibrium of quantum excitation due to synchrotron radiation and damping. The result of these processes are not always what is desired and it is therefore useful to discuss some method to modify the particle distribution in phase space within the validity of Liouville's theorem.

\subsubsection{Exchange of Transverse Phase-Space Parameters}

In beam dynamics we are often faced with the desire to change the beam size in one of the six phase-space dimensions. Liouville's theorem tells us that this is not possible with macroscopic fields unless we let another dimension vary as well so as not to change the total volume in six-dimensional phase space.

A very simple example of exchanging phase-space dimensions is the increase or decrease of one transverse dimension at the expense of its conjugate coordinate. A very wide and almost parallel beam, for example, can be focused to a small spot size where, however, the beam divergence has become very large. Obviously, this process can be reversed too and we describe such a process as the rotation of a beam in phase space or as phase-space rotation.

A more complicated but often very desirable exchange of parameters is the reduction of beam emittance in one plane at the expense of the emittance in the other plane. Is it, for example, possible to reduce say the vertical beam emittance to zero at the expense of the horizontal emittance? Although Liouville's theorem would allow such an exchange other conditions in Hamiltonian theory will not allow this kind of exchange in multidimensional phase space. The condition of symplecticity is synonymous with Liouville's theorem only in one dimension. For $n$ dimensions the symplecticity condition imposes a total of $n(2 n-1)$ conditions on the dynamics of particles in phase space [3]. These conditions impose an important practical limitation on the exchange of phase space between different degrees of freedom. Specifically, it is not possible to reduce the smaller of two phase-space dimensions further at the expense of the larger emittance, or if the phase space is the same in two dimensions neither can be reduced at the expense of the other.

\subsubsection{Bunch Compression}

Longitudinal phase space can be exchanged also by special application of magnetic and rf-fields. Specifically, we often face the problem to compress the bunch to a very short length at the expense of energy spread. 
For linear colliders the following problem exists. Very small transverse beam emittances can be obtained only in storage rings specially designed for low equilibrium beam emittances. Therefore, an electron beam is injected from a conventional source into a damping ring specially designed for low equilibrium beam emittance. After storage for a few damping times the beam is ejected from the damping ring again and transferred to the linear accelerator to be further accelerated. During the damping process in the damping ring, however, the bunch length will also reach its equilibrium value which in practical storage rings is significantly longer than could be accepted in, for example, an S-band or X-band linear accelerator. The bunch length must be shortened.

This is done in a specially designed beam transport line between the damping ring and linear accelerator consisting of a non-isochronous transport line and an accelerating section installed at the beginning of this line (Fig. 13.1).

The accelerating section is phased such that the center of the bunch or the reference particle does not see any field while the particles ahead of the reference particle are accelerated and the particles behind are decelerated. Following this accelerating section, the particles travel through a curved beam transport system with a finite momentum compaction factor $\alpha_{\mathrm{c}}=\frac{1}{L_{0}} \int_{0}^{L_{0}} \frac{\eta}{\rho} \mathrm{d} z$ where $L_{0}$ is the length of the beam transport line. Early particles within a bunch, having been accelerated, follow a longer path than the reference particles in the center of the bunch while the decelerated particles being late with respect to the bunch center follow a shortcut. All particles are considered highly relativistic and the early particles fall back toward the bunch center while late particles catch up with the bunch center. If the parameters of the beam transport system are chosen correctly the bunch length reaches its minimum value at the desired location at, for example, the entrance of the linear accelerator. From that point on the phase-space rotation is halted because of lack of momentum compaction in a straight line. Liouville's theorem is not violated because the energy spread in the beam has been increased through the phase dependent acceleration in the bunch-compression system.

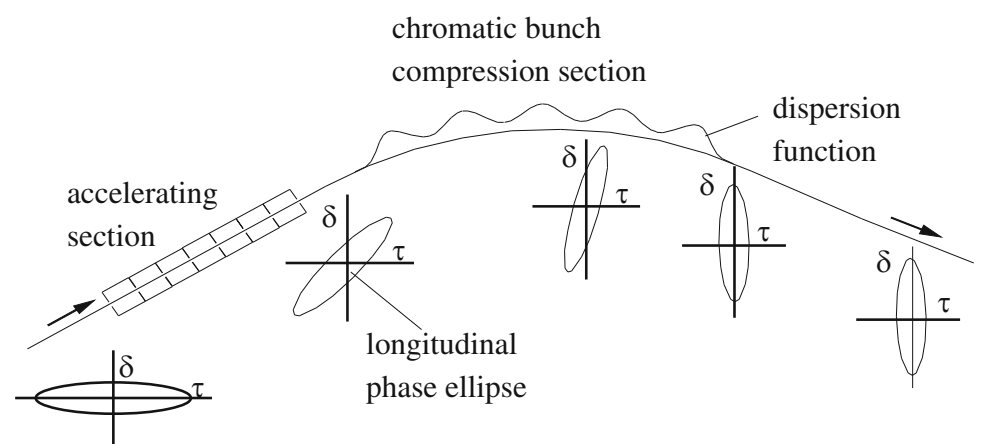

Fig. 13.1 Bunch-compressor system (schematic) 
Formulating this bunch compression in more mathematical terms, we start from a particle distribution in longitudinal phase space described by the phase ellipse

$$
\hat{\tau}_{0}^{2} \epsilon^{2}+\hat{\epsilon}_{0}^{2} \tau^{2}=\hat{\tau}_{0}^{2} \hat{\epsilon}_{0}^{2}=a^{2}
$$

where $a$ is the longitudinal emittance and $\tau$ is the particle location along the bunch measured from the bunch center such that $\tau>0$ if the particle trails the bunch center. In the first step of bunch compression, we apply an acceleration

$$
\Delta \epsilon=-e V_{0} \sin \omega_{\mathrm{rf}} \tau \approx-e V_{0} \omega_{\mathrm{rf}} \tau
$$

The particle energy is changed according to its position along the bunch. Replacing $\epsilon$ in (13.29) by $\epsilon+\Delta \epsilon$ and sorting we get

$$
\hat{\tau}_{0}^{2} \epsilon^{2}-2 \hat{\tau}_{0}^{2} e V_{0} \omega_{\mathrm{rf}} \epsilon \tau+\left(\hat{\tau}_{0}^{2} e^{2} V_{0}^{2} \omega_{\mathrm{rf}}^{2}+\hat{\epsilon}_{0}^{2}\right) \tau^{2}=a^{2},
$$

where the appearance of the cross term indicates the rotation of the ellipse. The second step is the actual bunch compression in a non-isochronous transport line of length $L$ and momentum compaction $\Delta z / L=\eta_{\mathrm{c}} \epsilon /\left(c p_{0}\right)$. Traveling though this beam line, a particle experiences a shift in time of

$$
\Delta \tau=\frac{\Delta z}{\beta c}=\frac{\eta_{\mathrm{c}} L}{\beta c} \frac{\epsilon}{c p_{0}} .
$$

Again, the time $\tau$ in (13.31) is replaced by $\tau+\Delta \tau$ to obtain the phase ellipse at the end of the bunch compressor of length $L$. The shortest bunch length occurs when the phase ellipse becomes upright. The coefficient for the cross term must therefore be zero giving a condition for minimum bunch length

$$
e V_{0}=-\frac{c p_{0} \beta c}{L \eta_{\mathrm{c}} \omega_{\mathrm{rf}}} .
$$

From the remaining coefficients of $\epsilon^{2}$ and $\tau^{2}$, we get the bunch length after compression

$$
\hat{\tau}=\frac{\hat{\epsilon}_{0}}{e V_{\mathrm{rf}} \omega_{\mathrm{rf}}}
$$

and the energy spread

$$
\hat{\epsilon}=\hat{\tau}_{0} \omega_{\mathrm{rf}} e V_{\mathrm{rf}},
$$

where we used the approximation $\hat{\tau}_{0} e V_{0} \omega_{\mathrm{rf}} \gg \hat{\epsilon}_{0}$. This is justified because we must accelerate particles at the tip of the bunch by much more than the original energy 
spread to obtain efficient bunch compression. Liouville's theorem is obviously kept intact since

$$
\hat{\epsilon} \hat{\tau}=\hat{\epsilon}_{0} \hat{\tau}_{0}
$$

For tight bunch compression, a particle beam with small energy spread is required as well as an accelerating section with a high rf-voltage and frequency. Of course, the same parameters contribute to the increase of the energy spread which can become the limiting factor in bunch compression. If this is the case, one could compress the bunch as much as is acceptable followed by acceleration to higher energies to reduce the energy spread by adiabatic damping, and then go through a bunch compression again.

The momentum compaction factor $\alpha_{\mathrm{c}}=\frac{1}{L_{0}} \int \frac{\eta}{\rho} \mathrm{d} z$ is often referred to as the $R_{56}$ of the compression lattice. This designation comes from the TRANSPORT nomenclature where a $6 \times 6$-transformation matrix is defined for the variables $\left(x, x^{\prime}, y, y^{\prime}, s, \delta\right)$. Here $s$ is the individual particle path length and $\delta$ the relative energy deviation. The correlation of $s$ with $\delta$ is the $R_{56}$ element and in linear approximation $s=s_{0}+R_{56} \delta$. Recalling the definition of the momentum compaction factor $\alpha_{\mathrm{c}}=\frac{\Delta L / L_{0}}{\Delta p / p_{0}}$ we recognize the identity $\alpha_{\mathrm{c}}=\frac{1}{L_{0}} \int \frac{\eta}{\rho} \mathrm{d} z=\frac{R_{56}}{L_{0}}$.

\subsubsection{Alpha Magnet}

Bunch compression requires two steps. First, an accelerating system must create a correlation between particle energy and position. Then, we utilize a non-isochronous magnetic transport line to rotate the particle distribution in phase space until the desired bunch length is reached.

The first step can be eliminated in the case of an electron beam generated in an rf-gun. Here the electrons emerge from a cathode which is inserted into an rf-cavity [4]. The electrons are accelerated immediately where the acceleration is a strong function of time because of the rapidly oscillating field. In Fig. 13.2 the result from computer simulations of the particle distribution in phase space [5] is shown for an electron beam from a $3 \mathrm{GHz}$ rf-gun [6, 7] (Fig. 13.3).

For bunch compression we use an alpha magnet which got its name from the alpha like shape of the particle trajectories. This magnet is made from a quadrupole split in half where the other half is simulated by a magnetic mirror plate at the vertical midplane. While ordinarily a particle beam would pass through a quadrupole along the axis or parallel to this axis this is not the case in an alpha magnet. The particle trajectories in an alpha magnet have very unique properties which were first recognized by Enge [8]. Most obvious is the fact that the entrance and exit point can be the same for all particles independent of energy. The same is true also for the total deflection angle. Borland [9] has analyzed the particle dynamics in an alpha magnet in detail and we follow his derivation here. Particles 


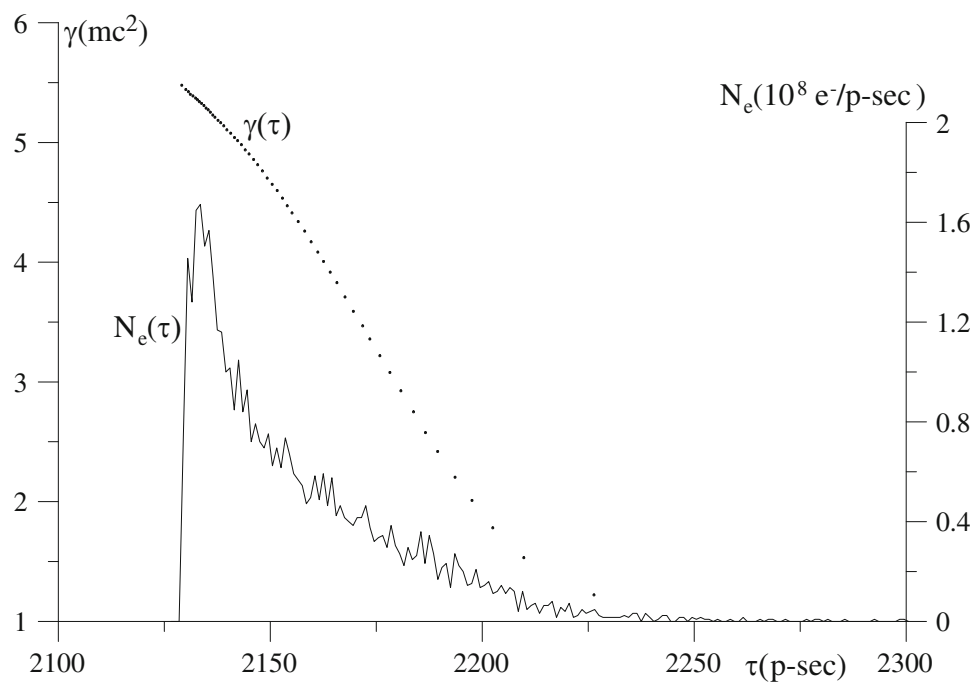

Fig. 13.2 Particle distribution in phase space for an electron beam from an $\mathrm{rf}$ gun with thermionic cathode

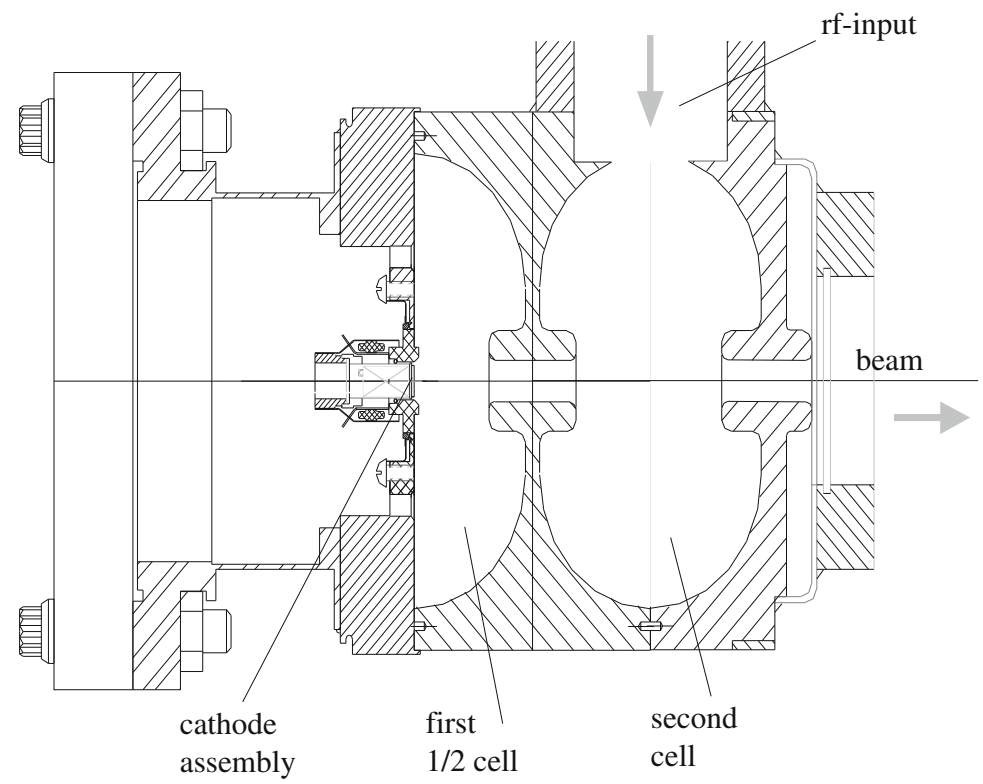

Fig. 13.3 Cross section of a microwave electron gun $[6,7]$

entering the alpha magnet fall under the influence of the Lorentz force

$$
\boldsymbol{F}_{\mathrm{L}}=e \boldsymbol{E}+e[\boldsymbol{v} \times \boldsymbol{B}],
$$


Fig. 13.4 Alpha magnet and particle trajectories

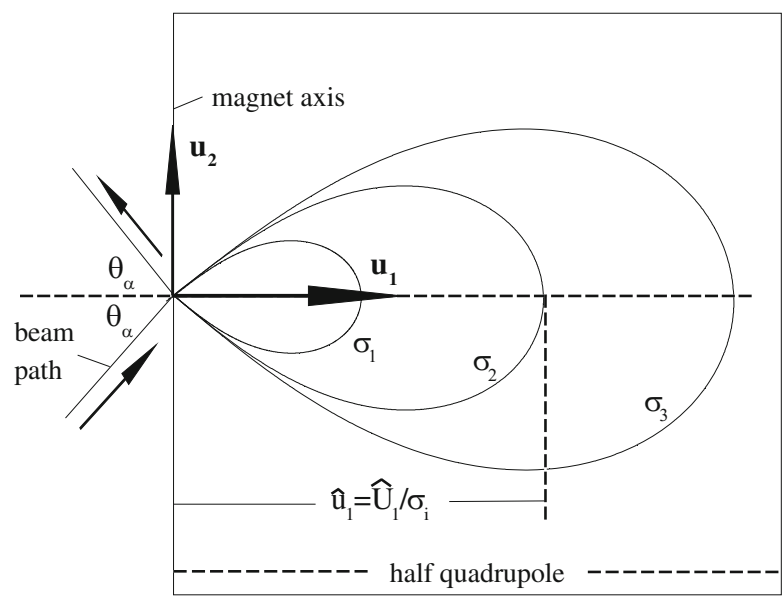

where we ignore the electrical field. Replacing the magnetic field by its gradient $\boldsymbol{B}=\left(g u_{3}, 0, g u_{1}\right)$, we get in the coordinate system of Fig. 13.4 the equation of motion,

$$
\frac{\mathrm{d}^{2} \boldsymbol{u}}{\mathrm{d} z^{2}}=-\sigma^{2}\left[\frac{\mathrm{d} \boldsymbol{u}}{\mathrm{d} z} \times \boldsymbol{u}\right]
$$

where the scaling factor

$$
\sigma^{2}\left(\mathrm{~m}^{-2}\right)=\frac{e g}{m c^{2} \beta \gamma}=5.86674 \times 10^{6} \frac{g(\mathrm{~T} / \mathrm{m})}{\beta \gamma},
$$

and the coordinate vector $\boldsymbol{u}=\left(u_{1}, u_{2}, u_{3}\right)$.

By introducing normalized coordinates $\boldsymbol{U}=\sigma \boldsymbol{u}$ and path length $S=\sigma z$, Eq. (13.38) becomes

$$
\frac{\mathrm{d}^{2} \boldsymbol{U}}{\mathrm{d} S^{2}}=-\left[\frac{\mathrm{d} \boldsymbol{U}}{\mathrm{d} S} \times\left(U_{3}, 0, U_{1}\right)\right] .
$$

The remarkable feature of (13.40) is the fact that it does not exhibit any dependence on the particle energy or the magnetic field. One solution for (13.40) is valid for all operating conditions and beam energies. The alpha shaped trajectories are similar to each other and scale with energy and field gradient according to the normalization introduced above.

Equation (13.40) can be integrated numerically and in doing so, Borland obtains for the characteristic parameters of the normalized trajectory in an alpha magnet [9]

$$
\begin{aligned}
& \theta_{\alpha}=0.71052 \quad \mathrm{rad}, S_{\alpha}=4.64210 \text {, } \\
& =40.70991 \mathrm{deg}, \hat{U}_{1}=1.81782 \text {, }
\end{aligned}
$$


where $\theta_{\alpha}$ is the entrance and exit angle with respect to the magnet face, $S_{\alpha}$ is the normalized path length and $\hat{U}_{1}$ is the apex of the trajectory in the alpha magnet. We note specifically that the entrance and exit angle $\theta_{\alpha}$ is independent of beam energy and magnetic field. It is therefore possible to construct a beam transport line including an alpha magnet.

Upon introducing the scaling factor (13.39), (13.41) becomes equation

$$
\begin{aligned}
& s_{\alpha}(\mathrm{m})=\frac{S_{\alpha}}{\sigma}=0.19165 \sqrt{\frac{\beta \gamma}{g(\mathrm{~T} / \mathrm{m})}}, \\
& \hat{u}_{1}(\mathrm{~m})=\frac{\hat{U}_{1}}{\sigma}=0.07505 \sqrt{\frac{\beta \gamma}{g(\mathrm{~T} / \mathrm{m})}} .
\end{aligned}
$$

Bunch compression occurs due to the functional dependence of the path length on the particle energy. Taking the derivative of (13.42) with respect to the particle momentum $\tilde{p}_{0}=\beta \gamma$, one gets the compression equation

$$
\frac{\mathrm{d} s_{\alpha}(\mathrm{m})}{\mathrm{d} \tilde{p}_{0}}=\frac{0.07505}{\sqrt{2 g(\mathrm{~T} / \mathrm{m}) \tilde{p}_{0}}} .
$$

For bunch compression, higher momentum particles must arrive first because they follow a longer path and therefore fall back with respect to later particles. For example, an electron beam with the phase-space distribution from Fig. 13.2 becomes compressed as shown in Fig. 13.5.

Because of the small longitudinal emittance of the beam it is possible to generate very short electron bunches of some $100 \mathrm{f}-\mathrm{sec}$ (rms) duration which can be used to produce intense coherent far infrared radiation [10].

Fig. 13.5 Particle distribution in longitudinal phase space after compression in an alpha magnet

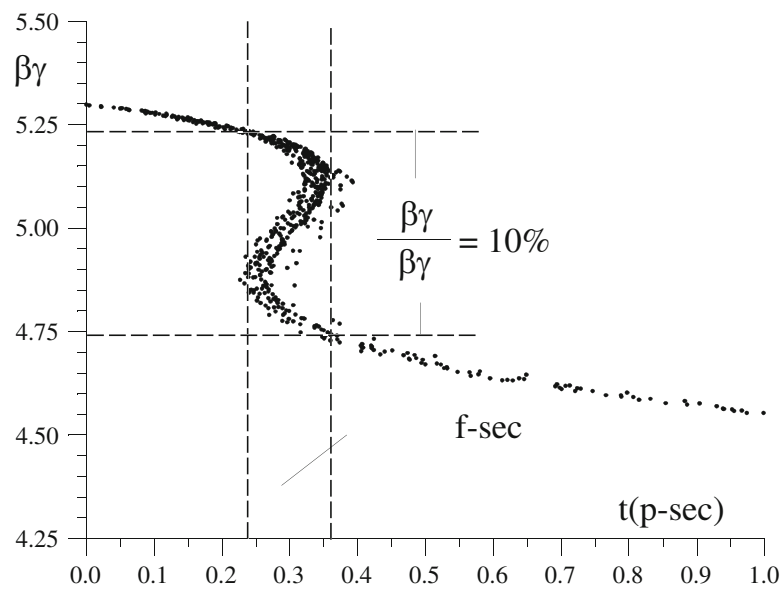




\subsection{Polarization of a Particle Beam}

For high energy physics experimentation, it is sometimes important to have beams of transversely or longitudinally polarized particles. It is possible, for example, to create polarized electron beams by photoemission from GaAs cathodes [11]. From a beam dynamics point of view, we are concerned with the transport of polarized beams through a magnet system and the resulting polarization status. The magnetic moment vector of a particle rotates about a magnetic field vector. An electron with a component of a longitudinal polarization traversing a vertical dipole field would experience a rotation of the longitudinal polarization about the vertical axis. On the other hand, the vertical polarization would not be affected while passing through a horizontally bending magnet. This situation is demonstrated in Fig. 13.6.

Similarly, longitudinal polarization is not affected by a solenoid field. In linear collider facilities, specific spin rotators are introduced to manipulate the electron spin in such a way as to preserve beam polarization and obtain the desired spin direction at an arbitrarily located collision point along the beam transport line. For the preservation of beam polarization, it is important to understand and formulate spin dynamics.

Electron and positron beams circulating for a long time in a storage ring can become polarized due to the reaction of continuous emission of transversely polarized synchrotron radiation. The evolution of the polarization has been studied in detail by several researchers [12-15] and the polarization time is given by [15]

$$
\frac{1}{\tau_{\mathrm{pol}}}=\frac{5 \sqrt{3}}{8} \frac{r_{\mathrm{c}} c^{2} \hbar \gamma^{5}}{m c^{2} \rho^{3}}
$$

with a theoretically maximum achievable polarization of $92.38 \%$. The polarization time is a strong function of beam energy and is very long for low energies. At

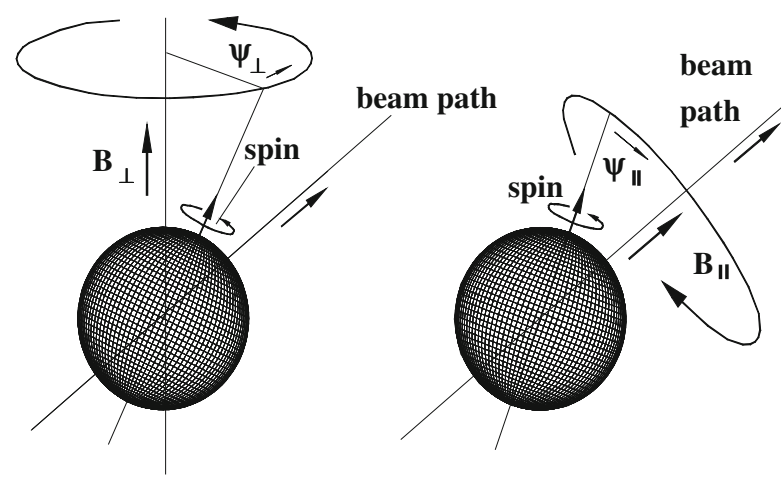

Fig. 13.6 Precession of the particle spin in a transverse or longitudinal magnetic field 
energies of several $\mathrm{GeV}$, however, this time becomes short compared to the storage time of an electron beam in a storage ring.

This build up of polarization is counteracted by nonlinear magnetic field errors which cause precession of the spin depending on the betatron amplitude and energy of the particle thus destroying polarization. Again, we must understand spin dynamics to minimize this depolarization. Simple relations exist for the rotation of the spin while the particle passes through a magnetic field. To rotate the spin by a magnetic field, there must be a finite angle between the spin direction and that of the magnetic field. The spin rotation angle about the axis of a transverse field depends on the angle between the spin direction $\sigma_{\mathrm{s}}\left(\left|\boldsymbol{\sigma}_{\mathrm{s}}\right|=1\right)$ and magnetic field $\boldsymbol{B}_{\perp}$ and is given by [14]

$$
\psi_{\perp}=C_{\perp}\left(1+\frac{1}{\gamma}\right)\left|\sigma_{\mathrm{s}} \times \boldsymbol{B}_{\perp}\right| \ell
$$

where

$$
\begin{aligned}
\eta_{\mathrm{g}} & =\frac{g-2}{2}=0.00115965 \\
C_{\perp} & =\frac{e \eta_{\mathrm{g}}}{m c^{2}}=0.0068033\left(\mathrm{~T}^{-1} \mathrm{~m}^{-1}\right)
\end{aligned}
$$

$g$ the gyromagnetic constant and $\boldsymbol{B}_{\perp} \ell$ the integrated transverse magnetic field strength. Apart from a small term $1 / \gamma$, the spin rotation is independent of the energy. In other words, a spin component normal to the field direction can be rotated by $90^{\circ}$ while passing though a magnetic field of $2.309 \mathrm{Tm}$ and it is therefore not important at what energy the spin is rotated.

Equation (13.45) describes the situation in a flat storage ring with horizontal bending magnets only unless the polarization of the incoming beam is strictly vertical. Any horizontal or longitudinal polarization component would precess while the beam circulates in the storage ring. As long as this spin is the same for all particles the polarization would be preserved. Unfortunately, the small energy dependence of the precession angle and the finite energy spread in the beam would wash out the polarization. On the other hand the vertical polarization of a particle beam is preserved in an ideal storage ring. Field errors, however, may introduce a depolarization effect. Horizontal field errors from misalignments of magnets, for example, would rotate the vertical spin. Fortunately, the integral of all horizontal field components in a storage ring is always zero along the closed orbit and the net effect on the vertical polarization is zero. Nonlinear fields, however, do not cancel and must be minimized to preserve the polarization.

A transverse spin can also be rotated about the longitudinal axis of a solenoid field and the rotation angle is

$$
\psi_{\|}=\frac{e}{E}\left(1+\eta_{\mathrm{g}} \frac{\gamma}{1+\gamma}\right)\left|\sigma_{\mathrm{s}} \times \boldsymbol{B}_{\|}\right| \ell
$$


In a solenoid field it is therefore possible to rotate a horizontal polarization into a vertical polarization, or vice versa. Spin rotation in a longitudinal field is energy dependent and such spin rotations should therefore be done at low energies if possible.

The interplay between rotations about a transverse axis and the longitudinal axis is responsible for a spin resonance which destroys whatever beam polarization exists. To show this, we assume a situation where the polarization vector precesses just by $2 \pi$, or an integer multiple $n$ thereof, while the particle circulates once around the storage ring. In this case $\psi_{\perp}=n 2 \pi, e B_{\perp} \ell / E=2 \pi$, and we get from (13.45)

$$
n=\eta_{\mathrm{g}}(1+\gamma)
$$

For $n=1$, resonance occurs at a beam energy of $E=440.14 \mathrm{MeV}$. At this energy any small longitudinal field interacts with the polarization vector at the same phase, eventually destroying any transverse polarization. This resonance occurs at equal energy intervals of

$$
E_{n}(\mathrm{MeV})=440.14+440.65(n-1)
$$

and can be used in storage rings as a precise energy calibration by observing the loss of polarization due to spin-resonances at $E_{n}$ while the beam energy is changed.

In Fig. 13.7 spin dynamics is shown for the case of a linear collider where a longitudinally polarized beam is desired at the collision point. For example, a longitudinally polarized beam is generated at the source and accelerated in a linear accelerator. No rotation of the polarization direction occurs because no magnetic fields are involved yet. At an energy of $1.2 \mathrm{GeV}$ the beam is transferred to a damping ring to reduce the beam emittance. To preserve polarization in the damping ring

Fig. 13.7 Spin manipulation during beam transfer from linear accelerator to damping ring and back

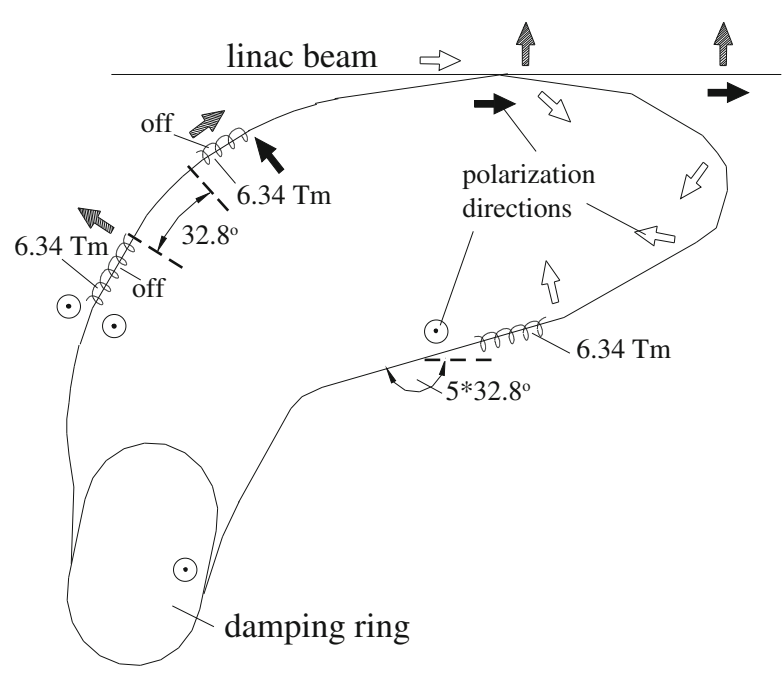


the polarization must be vertical. In Fig. 13.7, we assume a longitudinal polarized beam coming out of the linear accelerator. A series of transverse fields amounting to $5 \times 2.309 \mathrm{Tm}$ creating a total deflection angle of $5 \times 32.8^{\circ}$. The longitudinal spin from the linac is rotated by $5 \times 90^{\circ}$ to a horizontal spin as shown in Fig. 13.7 by the open arrows. A solenoid of $6.34 \mathrm{Tm}$ rotates this spin about the beam axis to become a vertical spin which survives the storage time in the damping ring. After emittance reduction in a few damping times the beam is ejected again with vertical spin. Now we have to decide which spin orientation we need at the collision point of the linear collider. there are two cases. In the first case (left side of the ejected beam in Fig. 13.7 a solenoid of $6.34 \mathrm{Tm}$ rotates the spin to become transverse followed by a $32.8^{\circ}$ bending section to make the spin longitudinal. This beam is injected back into the linac for collisions with longitudinal spin. of course, any bending downstream must be carefully implemented to preserve the spin. In the second case we turn the first solenoid after ejection off and the beam with vertical spin arrives at the second solenoid unaffected by the $32.8^{\circ}$ bend. in the second solenoid the spin is rotated to become a horizontal spin which is then reinjected into the linac. Note in both cases there is some spin rotation after the second solenoid yet in both cases the effect on the spin is just what is desired to have a transverse or longitudinal spin in the linac.

To rotate the longitudinal into a horizontal spin, followed by a solenoid field which rotates the horizontal into a vertical spin, is used in the transport line to the damping ring to obtain the desired vertical spin orientation. This orientation is in line with all magnets in the damping ring and the polarization can be preserved.

To obtain the desired rotation in the beam transport magnets at a given energy, the beam must be deflected by a specific deflection angle which is from (13.45)

$$
\theta=\frac{e}{\beta E} B_{\perp} \ell=\frac{\psi_{\perp}}{\eta_{\mathrm{g}}} \frac{1}{1+\gamma} .
$$

Coming out of the damping ring the beam passes through a combination of two solenoids and two transverse field sections. Depending on which solenoid is turned on, we end up with a longitudinal or transverse polarization at the entrance of the linac. By the use of both solenoids any polarization direction can be realized.

\section{Problems}

13.1 (S). Show that the horizontal damping partition number is negative in a fully combined function FODO lattice as employed in older synchrotron accelerators. Why, if there is horizontal anti-damping in such synchrotrons, is it possible to retain beam stability during acceleration? What happens if we accelerate a beam and keep it orbiting in the synchrotron at some higher energy? 
13.2. Use the high energy, linear part of the particle distribution in Fig. 13.2 and specify an alpha magnet to achieve best bunch compression at the observation point $2 \mathrm{~m}$ downstream from the magnet. By how much is the bunchlength increased if you now also include the variation of velocities. What are the alpha magnet parameters? Sketch the particle distribution at the entrance and exit of the alpha magnet.

13.3. Specify relevant parameters for an electron storage ring made of FODO cells with the goal to produce a very short equilibrium bunch length of $\sigma_{\ell}=1 \mathrm{~mm}$. Use superconducting cavities for bunch compression with a maximum field of $10 \mathrm{MV} / \mathrm{m}$ and a total length of not more than $10 \%$ of the ring circumference.

13.4. Describe spin rotations in bending magnets in matrix formulation.

13.5. Consider an electron storage ring for an energy of $30 \mathrm{GeV}$ and a bending radius of $\rho=500 \mathrm{~m}$ and calculate the polarization time. The vertical polarization will be perturbed by spurious dipole fields. Use statistical methods to calculate the rms change of polarization direction per unit time and compare with the polarization time. Determine the alignment tolerances to get a polarized beam.

\section{References}

1. H. Wiedemann, Basic lattice for the slc. Technical Report, Int. Report, Stanford Linear Accelerator Center, Stanford (1979)

2. M. Sands, The physics of electron storage rings, an introduction, in Physics with Intersecting Storage Rings (Academic, New York, 1971)

3. H. Weyl, The Classical Groups (Princeton University Press, Princeton/New York, 1946)

4. S.P. Kapitza, V.N. Melekhin, The Microtron (Harwood Academic, London, 1987)

5. H. Wiedemann, Nucl. Instrum. Methods A266, 24 (1988)

6. E. Tanabe, M. Borland, M.C. Green, R.H. Miller, L.V. Nelson, J.N. Weaver, H. Wiedemann, in 14th Meeting on Linear Accelerators (Nara, 1989), p. 140

7. M. Borland, M.C. Geen, R.H. Miller, L.V. Nelson, E. Tanabe, J.N. Weaver, H. Wiedemann, in Proceedings of Linearf Accelerator Conference (Los Alamos National Laboratory, Albuquerque, 1990)

8. H.A. Enge, Rev. Sci. Instrum. 34, 385 (1963)

9. M. Borland, A High-Brightness Thermionic Electron Gun, Ph.D. thesis, Stanford University, Stanford, 1991

10. P.H. Kung, H.C. Lihn, D. Bocek, H. Wiedemann, Phys. Rev. Lett. 73, 967 (1994)

11. D.C. Schultz, J. Clendenin, J. Frisch, E. Hoyt, L. Klaisner, M. Wood, D. Wright, M. Zoloterev, in Proceedings of 3rd European Particle Accelerator Conference, ed. by H. Henke, H. Homeyer, Ch. Petiot-Jean-Genaz (Edition Frontiere, Gif-sur Yvette, 1992), p. 1029

12. Ya.S. Derbenev, A.M. Kondratenko, Sov. Phys. JETP 37, 968 (1973)

13. A.A. Sokolov, I.M. Ternov, Synchrotron Radiation (Pergamon, Oxford, 1968)

14. V.N. Baier, Radiative polarization of electrons in storage rings, in Physics with Intersecting Storage Rings, ed. by B. Touschek (Academic, New York, 1971)

15. A.W. Chao, in Physics of High Energy Particle Accelerators, ed. by M. Month, M. Dienes, vol. 87 (American Institute of Physics, New York, 1982), p. 395 



\section{Chapter 14 \\ Beam Emittance and Lattice Design}

The task of lattice design for proton and ion beams can be concentrated to a pure particle beam optics problem. Transverse as well as longitudinal emittances of such beams are constants of motion and therefore do not depend on the particular design of the beam transport or ring lattice. This situation is completely different for electron and positron beams in circular accelerators where the emission of synchrotron radiation determines the particle distribution in six-dimensional phase space. The magnitude and characteristics of synchrotron radiation effects can, however, be manipulated and influenced by an appropriate choice of lattice parameters. We will discuss optimization and scaling laws for the transverse beam emittance of electron or positron beams in circular accelerators.

Originally electron storage rings have been designed, optimized and constructed for the sole use as colliding beam facilities for high energy physics. The era of electron storage rings for experimentation at the very highest particle energies has, however, reached a serious limitation due to excessive energy losses into synchrotron radiation. Of course, such a limitation does not exist for proton and ion beams with particle energies up to the order of some tens of TeV's and storage rings are therefore still the most powerful and productive research tool in high-energy physics. At lower and medium-energies electron storage rings with specially high luminosity still serve as an important research tool in high energy physics to study more subtle phenomena which could not be detected on earlier storage rings with lower luminosity like $\tau$ - and $B$-factories.

To overcome the energy limitation in electron colliding beam facilities, the idea of linear colliders which avoids energy losses into synchrotron radiation $[1,2]$ becomes increasingly attractive to reach ever higher center of mass energies for high-energy physics. Even though electron storage rings are displaced by this development as the central part of a colliding beam facility they play an important role for linear colliders in the form of damping rings to prepare very small emittance particle beams.

This chapter has been made Open Access under a CC BY 4.0 license. For details on rights and licenses please read the Correction https://doi.org/10.1007/978-3-319-18317-6_28 
The single purpose of electron storage rings for high-energy physics has been replaced by a multitude of applications of synchrotron radiation from such rings in a large variety of basic and applied research disciplines. It is therefore appropriate to discuss specific design and optimization criteria for electron storage rings.

Synchrotron radiation sources have undergone significant transitions and modifications over past years. Originally, most experiments with synchrotron radiation were performed parasitically on high energy physics colliding beam storage rings. Much larger photon fluxes could be obtained from such sources compared to any other source available. The community of synchrotron radiation users grew rapidly and so did the variety of applications and fields. By the time the usefulness of storage rings for high energy physics was exhausted some of these facilities were turned over to the synchrotron radiation community as fully dedicated radiation sources. Those are called first generation synchrotron radiation sources. They were not optimized for minimum beam emittance and maximum photon beam brightness. Actually, the optimization for high energy physics called for a maximum beam emittance to maximize collision rates for elementary particle events. The radiation sources were mostly bending magnets although the development and use of insertion devices started in these rings. Typically, the beam emittance is in the 100s of nanometer.

As the synchrotron radiation community further grew, funds became available to construct dedicated radiation facilities. Generally, these rings were designed as bending magnet sources but with reduced beam emittance $(\leq 100 \mathrm{~nm})$ to increase photon brightness. The design emittances were much smaller than those in first generation rings but still large by present day standards. The use of insertion devices did not significantly affect the storage ring designs yet. These rings are called second generation rings.

Third generation synchrotron radiation sources were and are being designed, constructed and operated now. These rings are specifically designed for insertion device radiation with minimum beam emittances below $20 \mathrm{~nm}$ down to $0.5 \mathrm{~nm}$ for maximum photon beam brightness. As such, they exhibit a large number of magnetfree insertion straight sections.

Finally, fourth generation synchrotron radiation sources are the latest development for synchrotron radiation sources. Such sources are based on linear accelerator technology and the principle of single pass FELs where a high energy and high quality electron beam passing through a long undulator produces coherent undulator radiation in the $\mathrm{X}$-ray regime.

Whatever the applications, in most cases it is the beam emittance which will ultimately determine the usefulness of the storage ring design for a particular application. We will derive and discuss physics and scaling laws for the equilibrium beam emittance in storage rings while using basic phenomena and processes of accelerator physics as derived in previous sections. 


\subsection{Equilibrium Beam Emittance in Storage Rings}

The equilibrium beam emittance in electron storage rings is determined by the counteracting effects of quantum excitation and damping as has been discussed earlier. Significant synchrotron radiation occurs only in bending magnets and the radiation from each bending magnet contributes independently to both quantum excitation and damping. The contribution of each bending magnet to the equilibrium beam emittance can be determined by calculating the average values for $\left\langle\left|\kappa^{3}\right| \mathcal{H}\right\rangle$ and $\left\langle\kappa^{2}\right\rangle$ by

$$
\left\langle|\kappa|^{3} \mathcal{H}\right\rangle_{z}=\frac{1}{C} \int_{0}^{C}\left|\kappa^{3}(z)\right| \mathcal{H}(z) \mathrm{d} z,
$$

where $\mathcal{H}$ is defined by (11.52) and $C$ is the circumference of the storage ring. Obviously, this integral receives contributions only where there is a finite bending radius and therefore the total integral is just the sum of individual integrals over each bending magnet.

\subsubsection{FODO Lattice}

We consider here briefly the FODO lattice because of its simplicity and its ability to give us a quick feeling for the scaling of beam emittance with lattice parameters. The beam emittance can be manipulated at design time by adjusting $\langle\mathcal{H}\rangle$ to the desired value. To calculate the average value $\langle\mathcal{H}\rangle$ in a FODO lattice is somewhat elaborate. Here, we are interested primarily in the scaling of the beam emittance with FODO lattice parameters. Recollecting the results for the symmetric solutions of the lattice functions in a FODO lattice (10.3), (10.5), (10.74) we notice the following scaling laws

$$
\begin{aligned}
\beta & \propto L, \\
\beta^{\prime} & \propto L^{0}, \\
\eta & \propto L^{2} / \rho, \\
\eta^{\prime} & \propto L / \rho,
\end{aligned}
$$

where $L$ is the distance between the centers of adjacent quadrupoles. All three terms in the function $\mathcal{H}(z)=\gamma(z) \eta^{2}+2 \alpha(z) \eta \eta^{\prime}+\beta(z) \eta^{\prime 2}$ scale in a similar fashion like

$$
\{\mathcal{H}(z)\}=\left\{\frac{1}{L} \frac{L^{4}}{\rho} ; L^{0} \frac{L^{2}}{\rho} \frac{L}{\rho} ; L \frac{L^{2}}{\rho}\right\} \propto \frac{L^{3}}{\rho^{2}}
$$


and the equilibrium emittance for a FODO lattice scales then like

$$
\epsilon_{x}=C_{\mathrm{q}} \gamma^{2} \frac{\left\langle\mathcal{H} / \rho^{3}\right\rangle}{\left\langle 1 / \rho^{2}\right\rangle} \propto \gamma^{2} \frac{L^{3}}{\rho^{3}} \propto \gamma^{2} \Theta^{3},
$$

where $\Theta=\ell_{\mathrm{b}} / \rho$ is the deflection angle in each bending magnet. The proportionality factor depends on the beam focusing. A minimum can be reached for a focal length of $|f| \approx 1.06 \mathrm{~L}$ in each half-quadrupole resulting in a minimum beam emittance achievable in a FODO lattice given in practical units by

$$
\epsilon(\operatorname{rad} \mathrm{m}) \approx 10^{-11} E^{2}(\mathrm{GeV}) \Theta^{3}\left(\mathrm{deg}^{3}\right)
$$

where $\varphi=2 \pi / N_{\mathrm{M}}, N_{\mathrm{M}}$ the number of bending magnets in the ring and $N_{\mathrm{M}} / 2$ the total number of FODO cells in the ring. This result is significant because it exhibits a general scaling law of the beam emittance proportional to the square of the beam energy and the cube of the deflecting angle in each bending magnet, which is valid for all lattice types. The coefficients, though, vary for different lattices. While the beam energy is primarily driven by the desired photon spectrum, we find that high brightness photon beams from low emittance electron beams require a storage ring design composed of many lattice cells with a small deflection angle per magnet. Of course, there are some limits on how far one can go with this concept due to other limitations, not the least being size and cost of the ring which both grow with the number of lattice cells.

\subsubsection{Minimum Beam Emittance}

While the cubic dependence of the beam emittance on the bending angle is a significant design criterion, we discuss here a more detailed optimization strategy. The emittance is determined by the beam energy, the bending radius and the $\mathcal{H}$-function. Generally, we have no choice on the beam energy which is mostly determined by the desired critical photon energy of bending magnet and insertion device radiation or cost. Similarly, the bending radius is defined by the ring geometry, desired spectrum etc. Interestingly, it is not the bending radius but rather the bending angle which influences the equilibrium beam emittance. The main process to minimize the beam emittance is to adjust the focusing such that the lattice functions in the bending magnets generate a minimum value for $\left\langle\mathcal{H}_{\mathrm{b}}\right\rangle_{z}$. The equilibrium beam emittance (13.18) depends only on the lattice function $\mathcal{H}_{\mathrm{b}}(z)$ inside bending magnets. Independent of any lattice type, we may therefore consider this function only within bending magnets. For the purpose of this discussion we assume a regular periodic lattice, where all bending magnets are the same and all lattice functions within each bending magnet are the same. That allows us to concentrate our discussion just on one bending magnet. The contribution of any individual bending magnet to the beam emittance can be determined by calculation 
of the average value for

$$
\left\langle\mathcal{H}_{\mathrm{b}}\right\rangle_{z}=\frac{1}{\ell_{\mathrm{b}}} \int_{0}^{\ell_{\mathrm{b}}} \mathcal{H}_{\mathrm{b}}(z) \mathrm{d} z
$$

where $\ell_{\mathrm{b}}$ is the length of the bending magnet and the bending radius is assumed to be constant within a magnet. From here on, we ignore the index $x$ since we assume a flat storage ring in the horizontal plane. All lattice functions are therefore to be taken in the horizontal plane.

In evaluating the integral (14.1) we must include all contributions. The emission of photons depends only on the bending radius regardless of whether the bending occurs in the horizontal or vertical plane. Since for the calculation of equilibrium beam emittances only the energy loss due to the emission of photons is relevant it does not matter in which direction the beam is bent. The effect of the emission of a photon on the particle trajectory, however, is different for both planes because dispersion functions are different resulting in a different quantum excitation factor $\mathcal{H}$. For a correct evaluation of the equilibrium beam emittances in the horizontal and vertical plane (14.1) should be evaluated for both planes by determining $\mathcal{H}_{x}$ and $\mathcal{H}_{y}$ separately but including in both calculations all bending magnets in the storage ring.

The integral in (14.1) can be evaluated for each magnet if the values of the lattice functions at the beginning of the bending magnet are known. With these initial values the lattice functions at any point within the bending magnet can be calculated assuming a pure dipole magnet. With the definitions of parameters from Fig. 14.1, we find the following expressions for the lattice functions in a bending magnet where $z$ is the distance from the entrance of the magnet

$$
\begin{aligned}
& \beta(z)=\beta_{0}-2 \alpha_{0} z+\gamma_{0} z^{2}, \\
& \alpha(z)=\alpha_{0}-\gamma_{0} z, \\
& \gamma(z)=\gamma_{0}, \\
& \eta(z)=\eta_{0}+\eta_{0}^{\prime} z+\rho(1-\cos \theta), \\
& \eta^{\prime}(z)=\eta_{0}^{\prime}+\sin \theta .
\end{aligned}
$$

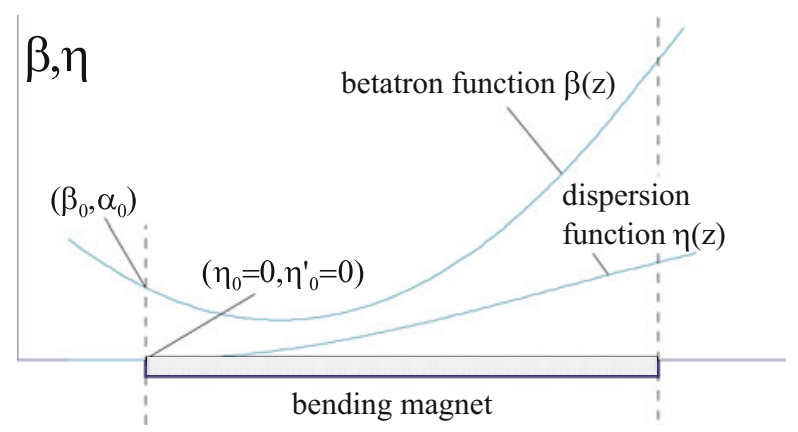

Fig. 14.1 Lattice functions in a bending magnet 
Here the deflection angle is $\theta=z / \rho$ and $\beta_{0}, \alpha_{0}, \gamma_{0}, \eta_{0}, \eta_{0}^{\prime}$ are the values of the lattice functions at the beginning of the magnet. Before we use these equations we assume lattices where $\eta_{0}=\eta_{0}^{\prime}=0$. The consequences of this assumption will be discussed later. Inserting (14.10) into (14.1) we get for small deflection angles after integration over one dipole magnet

$$
\left\langle\mathcal{H}_{\mathrm{b}}\right\rangle_{\mathrm{z}}=\frac{1}{3} \Theta^{2} \beta_{0}-\alpha_{0} \rho \frac{1}{4} \Theta^{3}+\gamma_{0} \rho^{2} \frac{1}{20} \Theta^{4}+\mathcal{O}\left(\Theta^{5}\right),
$$

where we have assumed the bending radius to be constant within the length $\ell_{\mathrm{b}}$ of the magnet. In a storage ring with dipole magnets of different strength, contributions from all magnets must be added to give the average quantum excitation term for the whole ring of length $C$

$$
\left\langle\left|\kappa^{3}\right| \mathcal{H}_{\mathrm{b}}\right\rangle_{z}=\frac{1}{C} \sum_{i}\left\langle\left|\kappa^{3}\right| \mathcal{H}_{\mathrm{b}, i}\right\rangle_{z} \ell_{\mathrm{b}, i}
$$

where we sum over all magnets $i$ with length $\ell_{\mathrm{b}, i}$. In an isomagnetic ring the factor $\left\langle\left|\kappa^{3}\right| \mathcal{H}_{\mathrm{b}} /\left\langle\kappa^{2}\right\rangle\right\rangle_{z}$ becomes simply $|\kappa|\left\langle\mathcal{H}_{\mathrm{b}}\right\rangle_{z}$ and the equilibrium beam emittance is

$$
\epsilon_{\text {iso }}=C_{\mathrm{q}} \frac{\gamma^{2}}{J_{x}}|\kappa|\left\langle\mathcal{H}_{\mathrm{b}}\right\rangle_{z} .
$$

Inserting (14.11) into (14.13) we get for the beam emittance in the lowest order of approximation

$$
\epsilon_{\text {iso }}=C_{\mathrm{q}} \gamma^{2} \Theta^{3}\left[\frac{1}{3} \frac{\beta_{0}}{\ell_{\mathrm{b}}}-\frac{1}{4} \alpha_{0}+\frac{1}{20} \gamma_{0} \ell_{\mathrm{b}}\right]+\mathcal{O}\left(\Theta^{4}\right),
$$

where $\gamma_{0}=\gamma\left(z_{0}\right)$ is one of the lattice functions not to be confused with the particle energy $\gamma$.

Here we have assumed a separate function lattice where the damping partition number $J_{x}=1$. For strong bending magnets or sector magnets this assumption is not always justified due to focusing in the bending magnets and the damping partition number should be corrected accordingly.

The result (14.14) shows clearly a cubic dependence of the beam emittance on the deflection angle $\Theta$ of the bending magnets which is a general lattice property since we have not yet made any assumption on the lattice type yet. Equation (14.14) exhibits minima with respect to both $\alpha_{0}$ and $\beta_{0}$. We solve the derivation $\partial\langle\mathcal{H}\rangle / \partial \alpha_{0}=0$ for $\alpha_{0}$ and the derivative $\partial\langle\mathcal{H}\rangle / \partial \beta_{0}=0$ for $\beta_{0}$ and get the optimum values for the Twiss functions at the entrance to the bending magnet

$$
\begin{aligned}
\beta_{0, \mathrm{opt}} & =\sqrt{\frac{12}{5}} \ell_{\mathrm{b}}, \\
\alpha_{0, \mathrm{opt}} & =\sqrt{15}
\end{aligned}
$$


and the minimum value for $\langle\mathcal{H}\rangle$ is

$$
\langle\mathcal{H}\rangle_{\min }=\frac{\Theta^{3} \rho}{4 \sqrt{15}} .
$$

With this, the minimum obtainable beam emittance in any lattice is from (13.18)

$$
\epsilon_{\mathrm{dba}, \min } \approx C_{\mathrm{q}} \gamma^{2} \frac{\left\langle\mathcal{H}_{\mathrm{b}}(z) / \rho^{3}\right\rangle_{z}}{\left\langle 1 / \rho^{2}\right\rangle_{z}} \approx C_{\mathrm{q}} \gamma^{2} \frac{\Theta^{3}}{4 \sqrt{15}}
$$

The results are very simple for small deflection angles but for angles larger than about $30^{\circ}$ per bending magnet the error for $\langle\mathcal{H}\rangle_{\min }$ exceeds $10 \%$ and higher order terms must be included.

For simplicity, we assumed that the dispersion functions $\eta_{0}=0$ and $\eta_{0}=0$. This a desirable feature, because it means that the dispersion function is also zero in the insertion devices (ID) of a synchrotron radiation source. A finite dispersion function in IDs can lead to an undesirable increase of the beam emittance.

In summary it has been demonstrated that for certain optimum lattice functions in the bending magnets the equilibrium beam emittance becomes a minimum. No assumption about a particular lattice has been made. Another observation is that the beam emittance is proportional to the third power of the magnet deflection angle and proportional to the square of the beam energy. Therefore many small deflection magnets interspersed within quadrupoles should be used to achieve a small beam emittance. Low emittance storage rings, therefore, are characterized by many short magnet lattice cells.

This approach has been used for a number of third generation synchrotron light sources. However, soon it was apparent that modification of the dispersion function could produce even smaller beam emittance in spite of the effect of IDs. Only, as it became possible in recent years to reach sub-nm beam emittances with sufficient dynamic aperture did the choice of finite dispersions in the IDs become undesirable again.

\subsection{Absolute Minimum Emittance}

In the previous section we found conditions which lead to a minimum beam emittance in an isomagnetic ring

$$
\epsilon_{x}=C_{\mathrm{q}} \gamma^{2} \frac{1}{\rho \ell} \int_{-\frac{1}{2} \ell}^{\frac{1}{2} \ell} \mathcal{H}(z) \mathrm{d} z
$$

The $\mathcal{H}$-function in (14.1) is a nonlinear function of $z$ and therefore any asymmetry of the Twiss functions lead to larger values of the $\mathcal{H}$-integral. We may 
therefore assume that a symmetric $\mathcal{H}$-function may actually give the smallest value for the integral and emittance [3]. Following Teng we first discuss the case when $\eta_{0}=\eta_{0}^{\prime}=0$, and $\alpha_{x 0}=0$ at the center of the bending magnet. Note that this condition is different from the previous assumption for the Twiss functions. At a distance $z$ from the magnet center the Twiss functions are for small deflection angles $\left(\Theta \lesssim 30^{\circ}\right)$

$$
\begin{aligned}
& \beta(z)=\beta_{0}+\frac{z^{2}}{\beta_{0}}, \quad \alpha(z)=-\frac{z}{\beta_{0}}, \quad \gamma(z)=\gamma_{0} \\
& \eta(z) \approx \eta_{0}+\frac{1}{2} \frac{z^{2}}{\rho}, \quad \eta^{\prime}(z)=\frac{z}{\rho} .
\end{aligned}
$$

The $\mathcal{H}$-function (11.52) becomes then with $\ell$ the length of the bending magnet $\langle\mathcal{H}\rangle_{z}=\frac{1}{\ell} \int_{0}^{\ell}\left(\beta \eta^{\prime 2}+2 \alpha \eta \eta^{\prime}+\gamma \eta^{2}\right) \mathrm{d} z$, where the three integrals are

$$
\begin{aligned}
\frac{1}{\ell} \int_{0}^{\ell}\left(\beta \eta^{\prime 2}\right) \mathrm{d} z & =\frac{\beta_{0}}{3 \rho^{2}} \frac{\ell^{3}}{4}+\frac{1}{5} \frac{1}{\beta_{0} \rho^{2}} \frac{\ell^{5}}{16}, \\
\frac{1}{\ell} \int_{0}^{\ell}\left(2 \alpha \eta \eta^{\prime}\right) \mathrm{d} z & =-\frac{4}{3} \frac{\eta_{0}}{\beta_{0} \rho} \frac{\ell^{3}}{8}-\frac{8}{5 \beta_{0} \rho^{2}} \frac{\ell^{5}}{32}, \\
\frac{1}{\ell} \int_{0}^{\ell}\left(\gamma \eta^{2}\right) \mathrm{d} z & =\frac{\eta_{0}^{2}}{\beta_{0}} \ell+\frac{2}{3} \frac{\eta_{0}}{\beta_{0} \rho} \frac{\ell^{3}}{8}+\frac{2}{20 \beta_{0} \rho^{2}} \frac{\ell^{5}}{32}
\end{aligned}
$$

to result finally in

$$
\int \mathcal{H}(z) \mathrm{d} z=\frac{\eta_{0}^{2}}{\beta_{0}} \ell+\left(\frac{\beta_{0}}{12 \rho^{2}}-\frac{\eta_{0}}{12 \beta_{0} \rho}\right) \ell^{3}+\frac{1}{320 \beta_{0} \rho^{2}} \ell^{5} .
$$

First, we consider the case in which we set $\eta_{0}=0$ and

$$
\int \mathcal{H}_{1}(z) \mathrm{d} z=\frac{\beta_{0}}{12 \rho^{2}} \ell^{3}+\frac{1}{320 \beta_{0} \rho^{2}} \ell^{5}=\frac{\ell^{4}}{\rho^{2}}\left(\frac{\beta_{0}}{12 \ell}+\frac{\ell}{320 \beta_{0}}\right) .
$$

This integral has a minimum versus $\beta_{0}$ and from $\frac{\partial}{\partial \beta_{0}} \int \mathcal{H}_{1}(z) \mathrm{d} z=0$ the optimum value for $\beta_{0}$ is

$$
\frac{\beta_{0}}{\ell}=\sqrt{\frac{3}{80}}
$$


With this optimum $\int \mathcal{H}_{1}(z) \mathrm{d} z=\frac{1}{8 \sqrt{15}} \frac{\ell^{4}}{\rho^{3}}$ and the minimum beam emittance is

$$
\epsilon_{x}=C_{\mathrm{q}} \gamma^{2} \frac{\Theta^{3}}{J_{x}} \frac{1}{8 \sqrt{15}}
$$

This result has been derived by L. Teng in [3] and immediately rejected as "absolute minimum but useless". This judgement was based on the realization that the dispersion function at either end of the bending magnet is not zero and must therefore be of finite value at the insertion straight section too. This is not good as discussed above because insertion devices will enhance the emittance where $\eta \neq 0$ and will also lead to an increased effective emittance for the synchrotron radiation users. This becomes a serious problem for very small beam emittances as can be obtained now about 30 years after his note. However there is a way out.

If we cut one bending magnet in a cell into two pieces and install them as the first and last bending magnet we get a zero dispersion function for all straight sections without change of the beam emittance. There may be an arbitrary number of such bending magnets between those half-magnets and there are enough quadrupoles between the last bending magnet and the center of the straight section to match the horizontal betatron function to any desired value while the dispersion function is now zero in the IDs. The vertical betatron function does not contribute to the emittance and may be matched any way possible within reason. Within the unit cell we expect a periodic matching section between magnets. Incidentally, the same result can be obtained if we set $\alpha_{0}=0$ in (14.11) and look again for the optimum $\beta_{0}$. However, we must replace the total deflection angle by its half.

Just to be complete in this discussion we assume for a moment that $\eta_{0} \neq 0$

$$
\int \mathcal{H}_{2}(z) \mathrm{d} z=\frac{\eta_{0}^{2}}{\beta_{0}} \ell+\frac{\beta_{0}}{12 \rho^{2}} \ell^{3}+\frac{1}{320 \beta_{0} \rho^{2}} \ell^{5}-\frac{\eta_{0}}{12 \beta_{0} \rho} \ell^{3} .
$$

From $\frac{\partial}{\partial \beta_{0}} \int \mathcal{H}_{2}(z) \mathrm{d} z=0$ the optimum betatron function is

$$
\frac{\beta_{0}^{2}}{\ell^{2}}=\frac{3}{80}+\frac{12 \eta_{0}^{2} \rho^{2}}{\ell^{4}}-\frac{\eta_{0} \rho}{\ell^{2}} .
$$

Furthermore there is also an optimum dispersion function and evaluating $\frac{\partial}{\partial \eta_{0}} \int \mathcal{H}_{2}(z) \mathrm{d} z=0$ we get an optimum dispersion function in the middle of the bending magnet of

$$
\eta_{0}=\frac{\ell^{2}}{24 \rho}=\frac{\ell}{24} \Theta
$$


for which $\int \mathcal{H}_{2}(z) \mathrm{d} z=\frac{1}{12 \sqrt{15}} \frac{\ell^{4}}{\rho^{3}}$ and the minimum beam emittance

$$
\epsilon_{x}=C_{\mathrm{q}} \gamma^{2} \frac{\Theta^{3}}{J_{x}} \frac{1}{12 \sqrt{15}}
$$

which is even smaller.

The reduction in emittance by a factor $3 / 2$ looks desirable but now we have again a finite although small dispersion function in the long straight section. The dispersion function scales like the square root of the betatron functions and for a betatron phase of $90^{\circ}$, for example, the dispersion function $\eta^{*}$ in the middle of the straight section is $\eta^{*}=\sqrt{\frac{\beta^{*}}{\beta_{0}}} \eta_{0}=\frac{\sqrt{\beta^{*} \ell}}{124} \sqrt[4]{\frac{5}{3}}$. For the users the effective emittance $\epsilon_{\text {eff }}=\epsilon_{0} \sqrt{1+\frac{\eta^{* 2} \delta^{2}}{\epsilon_{0} \beta^{*}}}$, where the relative energy spread is $\delta^{2}=C_{\mathrm{q}} \gamma^{2} \frac{\Theta^{3}}{J_{\mathrm{s}} \rho}=\epsilon_{0} \frac{12 \sqrt{15}}{\rho \Theta^{3}}$. Finally, the effective beam emittance is

$$
\frac{\epsilon_{\text {eff }}}{\epsilon_{0}}=\sqrt{1+\frac{5}{12} \frac{1}{\Theta^{3}}} .
$$

To keep the effective beam emittance close to the natural emittance the deflection angle in the bending magnets must be large. In other words, the effective beam emittance for finite values of the dispersion function in insertion devices is much larger for modern low emittance storage rings with small deflection angles per bending magnet. for an emittance increase of a factor $\sqrt{2}$ the deflection angle per bending magnet must be $\Theta>0.75$ or $42.8^{\circ}$.

\subsection{Beam Emittance in Periodic Lattices}

To achieve a small particle beam emittance a number of different basic magnet storage ring lattice units are available and in principle most any periodic lattice unit can be used to achieve as small a beam emittance as desired. More practical considerations, however, will limit the minimum beam emittance achievable in a lattice. While all lattice types to be discussed have been used in existing storage rings and work well at medium to large beam emittances, differences in the characteristics of particular lattice types become more apparent as the desired equilibrium beam emittance is pushed to very small values.

Of the large variety of magnet lattices that have been used in existing storage rings the most commonly used ones are based on the double bend achromat (DBA) and derivatives thereof. In the DBA lattice the straight sections are separated by two bending magnets forming an achromat. In more recent years this approach has been modified into a multi-bend achromat where several bending magnets form an achromat between the straight sections. This trend was stimulated by the desire to minimize the beam emittance ever more while utilizing the $\Theta^{3}$ scaling. However, 
at the same time the ring circumference would grow equally because of the higher number of long insertion straight sections unless there are several bending magnets between straight sections, thus limiting circumference and costs.

\subsubsection{The Double Bend Achromat Lattice (DBA)}

The double bend achromat or DBA lattice is designed to make full use of the minimization of beam emittance by the proper choice of lattice functions as discussed earlier. In Fig. 14.2 the basic layout of this lattice is shown.

A set of two or three quadrupoles provides the matching of the lattice functions into the bending magnet to achieve the minimum beam emittance. The central part of the lattice between the bending magnets may consist of one or more quadrupoles and its only function is to focus the dispersion function such that it is matched again to zero at the end of the next bending magnet resulting necessarily in a phase advance from bending magnet to bending magnet of close to $180^{\circ}$. This lattice type has been proposed first by Panofsky [4] and later by Chasman and Green [5] as an optimized lattice for a synchrotron radiation source. In Fig. 14.3 an example of a synchrotron light source based on this type of lattice is shown representing the solution of the design study for the European Synchrotron Radiation Facility ESRF [6].

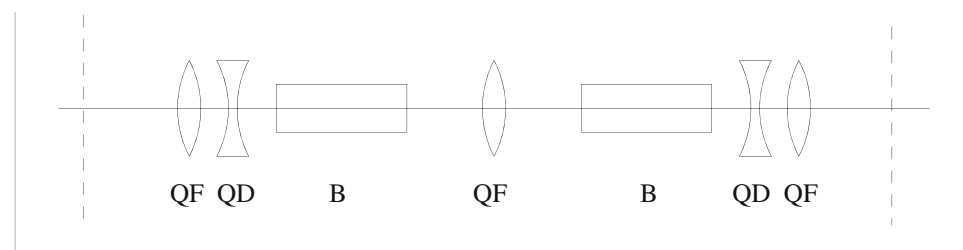

Fig. 14.2 Double bend achromat (DBA) lattice (schematic) first proposed by Panofsky [4]

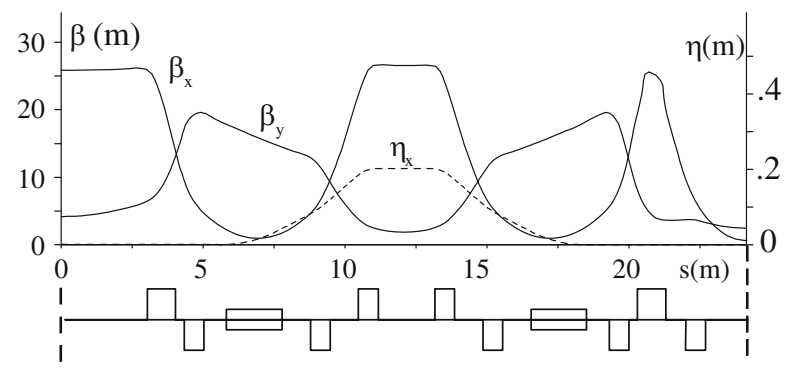

Fig. 14.3 European synchrotron radiation facility, ESRF [6] (one half of 16 superperiods). The lattice is asymmetric to provide a mostly parallel beam in one insertion and a small beam cross section in the other 
The ideal minimum beam emittance (14.17) in this lattice type for small bending angles and an isomagnetic ring with $J_{x}=1$ is

$$
\epsilon_{\mathrm{DBA}}=\frac{C_{\mathrm{q}}}{4 \sqrt{15}} \gamma^{2} \Theta^{3}
$$

or in more practical units

$$
\epsilon_{\mathrm{DBA}}(\mathrm{rad} \mathrm{m})=5.036 \times 10^{-13} E^{2}\left(\mathrm{GeV}^{2}\right) \Theta^{3}\left(\mathrm{deg}^{3}\right) .
$$

This lattice type can be very useful for synchrotron light sources where many component and dispersion free straight sections are required for the installation of insertion devices. For damping rings this lattice type is not quite optimum since it is a rather "open" lattice with a low bending magnet fill factor and consequently a long damping time. Other more compact lattice types must be pursued to achieve in addition to a small beam emittance also a short damping time.

\subsubsection{The FODO Lattice}

The FODO lattice, shown schematically in Fig. 14.4 is the most commonly used and best understood lattice in storage rings optimized for high-energy physics colliding beam facilities where large beam emittances are desired. This choice is obvious considering that the highest beam energies can be achieved while maximizing the fill factor of the ring with bending magnets.

This lattice provides the most space for bending magnets compared to other lattices. The usefulness of the FODO lattice, however, is not only limited to highenergy large emittance storage rings. By using very short cells very low beam emittances can be achieved as has been demonstrated in the first low emittance storage ring designed [7] and constructed [8] as a damping ring for the linear collider SLC to reach an emittance of $11 \times 10^{-9} \mathrm{~m}$ at $1 \mathrm{GeV}$.

The lattice functions in a FODO structure have been derived and discussed in detail and are generally determined by the focusing parameters of the quadrupoles.

Fig. 14.4 FODO lattice (schematic)

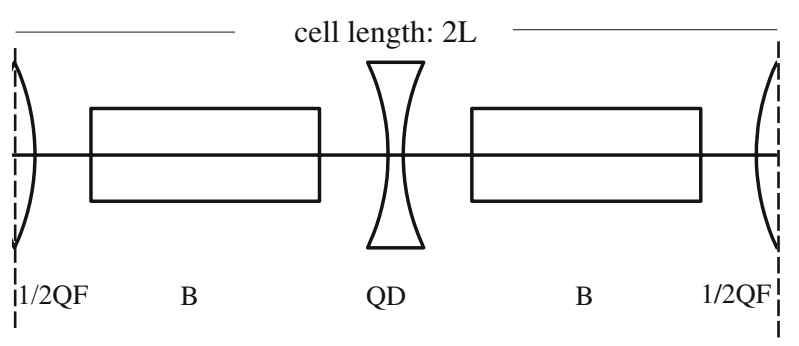


Fig. 14.5 Electron beam emittance of a FODO lattice as a function of the betatron phase advance per half cell in the deflecting plane

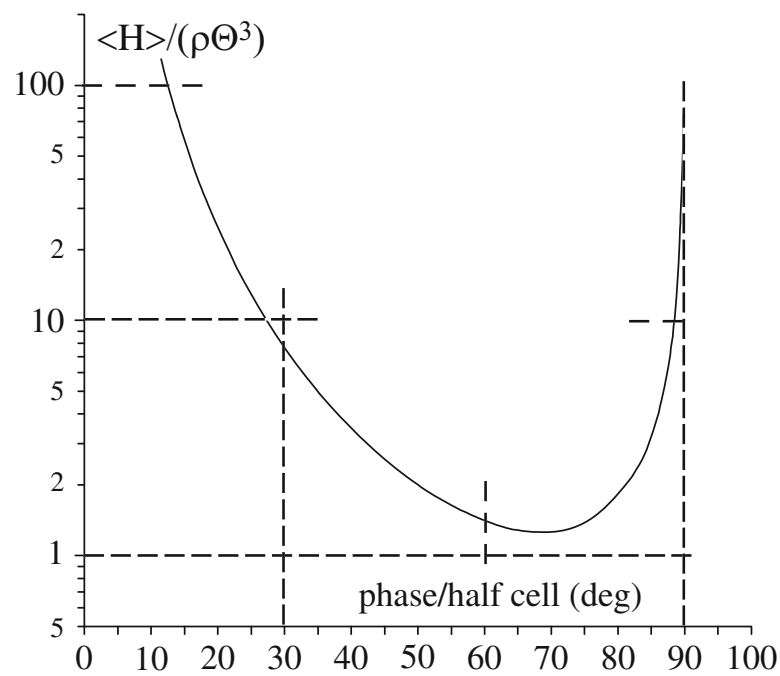

Since FODO cells are not achromatic the dispersion function is in general not zero at either end of the bending magnets.

The beam emittance can be derived analytically in thin lens approximation by integrating the quantum excitation factor along the bending magnets. The result is shown in Fig. 14.5 where the function $\left[\langle\mathcal{H}\rangle /\left(\rho \Theta^{3}\right)\right]$ is plotted as a function of the betatron phase advance per FODO half cell which is determined by the focal length of the quadrupoles.

The beam emittance for an isomagnetic FODO lattice is given by [9]

$$
\epsilon_{\mathrm{FODO}}=C_{\mathrm{q}} \gamma^{2} \Theta^{3} \frac{\ell_{\mathrm{b}}}{\ell_{\mathrm{b}, 0}} \frac{\langle\mathcal{H}\rangle}{\rho \Theta^{3}},
$$

where $\ell_{\mathrm{b}, 0}$ is the actual effective length of one bending magnet and $2 \ell_{\mathrm{b}}$ the length of a FODO cell. From Fig. 14.5 it becomes apparent that the minimum beam emittance is reached for a betatron phase of about $136.8^{\circ}$ per FODO cell. In this case $\langle\mathcal{H}\rangle /\left(\rho \Theta^{3}\right) \approx 1.25$ and the minimum beam emittance in such a FODO lattice in practical units is

$$
\epsilon_{\mathrm{FODO}}(\operatorname{radm})=97.53 \times 10^{-13} \frac{\ell_{\mathrm{b}}}{\ell_{\mathrm{b}, 0}} E^{2}\left(\mathrm{GeV}^{2}\right) \Theta^{3}\left(\mathrm{deg}^{3}\right)
$$

Comparing the minimum beam emittance achievable in various lattice types the FODO lattice seems to be the least appropriate lattice to achieve small beam emittances. This, however, is only an analytical distinction. FODO cells can be made much shorter than the lattice units of other structures and for a given circumference many more FODO cells can be incorporated than for any other lattice. As a consequence, the deflection angles per FODO cell can be much smaller. For very 
low emittance storage ring, therefore, it is not a priori obvious that one lattice is better than another. However, additional requirements like number of desired insertion straight sections for a particular application must be included in the decision for the optimum storage ring lattice.

\subsubsection{Optimum Emittance for Colliding Beam Storage Rings}

The single most important parameter of colliding beam storage rings is the luminosity and most of the design effort for such facilities is aimed at maximizing the collision rate. As a consequence of the beam-beam effect, the beam emittance must be chosen to be as large as possible for maximum luminosity as will be discussed in Sect. 21.2.2. Since for most high energy storage rings a FODO lattice is employed it is clear that for maximum emittance the phase advance per cell should be kept low as indicated in Fig. 14.5. Of course, there is a practical limit given by increasing magnet apertures and associated costs.

In linear colliders there is no beam stability concern due to the beam-beam effect like in a storage ring and a much smaller beam cross section can be chosen. The limit here is the total beam-beam disruption due to the large electromagnetic fields at the surface of the colliding beams. Strong synchrotron radiation introduce, for example, significant energy losses which jeopardize the analysis of high energy physics events.

\section{Problems}

14.1 (S). Derive an approximate expression of the beam emittance in an isomagnetic FODO lattice as a function of phase per cell and determine the minimum value of the emittance. Use a lattice which is symmetric in both planes and assume that the bending magnets are as long as the half cells $\left(\ell_{\mathrm{b}}=L\right)$.

14.2 (S). Consider a storage ring made of FODO cells at an energy of your choice. How many bending magnets or half cells do you need to reach a beam emittance of no more than $\epsilon_{x}=5 \cdot 10^{-9} \mathrm{~m}$ ?

\section{References}

1. M. Tigner. Nuovo Cimento 37, 1228 (1965)

2. J.E. Augustin, N. Dikanski, Y. Derbenev, J. Rees, B. Richter, A. Skrinski, M. Tigner, H. Wiedemann, in Proceedings of the Workshop on Possibilities and Limitations of Accelerators and Detectors (1979), p. 87

3. L. Teng, Technical Report, TM-1269, Fermi Nat. Lab., Chicago (1984) 
4. K.G. Steffen, High Energy Beam Optics (Wiley, New York, 1965), p. 117

5. R. Chasman, K. Green, E. Rowe, IEEE Trans. Nucl. Sci. 22, 1765 (1975)

6. Design report of the european synchrotron radiation facility (esrf). Technical report, Grenoble (1985)

7. H. Wiedemann, Scaling of damping rings for colliding linac beam systems. in 11th International Conference on High Energy Accelerators (CERN/Birkhäuser, Geneva/Basel, 1980), p 693

8. G.E. Fischer, W. Davis-White, T. Figuth, H. Wiedemann, in Proceedings of 12th International Conference High Energy Accelerators (Fermilab, Chicago, 1983)

9. R. Helm, H. Wiedemann, Technical Report, PEP-Note 303, Stanford Linear Accelerator Center, Stanford (1979) 


\section{Part V \\ Perturbations}




\section{Chapter 15 \\ Perturbations in Beam Dynamics}

The study of beam dynamics under ideal conditions is the first basic step toward the design of a beam transport system. In the previous sections we have followed this path and have allowed only the particle energy to deviate from its ideal value. In a real particle beam line or accelerator we may, however, not assume ideal and linear conditions. More sophisticated beam transport systems require the incorporation of nonlinear sextupole fields to correct for chromatic aberrations. Deviations from the desired field configurations can be caused by transverse or longitudinal misplacements of magnets with respect to the ideal beam path. Of similar concern are errors in the magnetic field strength, undesirable field effects caused in the field configurations at magnet ends, or higher order multipole fields resulting from design, construction, and assembly tolerances. Still other sources of errors may be beam-beam perturbations, magnetic detectors for high energy physics experiments, insertion devices in beam transport systems or accelerating sections, which are not part of the magnetic lattice configurations. The impact of such errors is magnified in strong focusing beam transport systems as has been recognized soon after the invention of the strong focusing principle. Early overviews and references can be found for example in $[1,2]$.

A horizontal bending magnet has been characterized as a magnet with only a vertical field component. This is true as long as this magnet is perfectly aligned, in most cases perfectly level. Small rotations about the magnet axis result in the appearance of horizontal field components which must be taken into account for beam stability calculations.

We also assumed that the magnetic field in a quadrupole or higher multipole vanishes at the center of magnet axis. Misalignments of any multipole generates all lower order perturbations which is known as "spill-down".

In addition, any multipole can be rotated by a small angle with respect to the reference coordinate system. As a result we observe the appearance of a small component of a rotated or skew quadrupole causing coupling of horizontal and vertical betatron oscillations.

This chapter has been made Open Access under a CC BY 4.0 license. For details on rights and licenses please read the Correction https://doi.org/10.1007/978-3-319-18317-6_28 
Although such misalignments and field errors are unintentional and undesired, we have to deal with their existence since there is no way to avoid such errors in a real environment. The particular effects of different types of errors on beam stability will be discussed. Tolerance limits on these errors as well as corrective measures must be established to avoid destruction of the particle beam. Common to all these perturbations from ideal conditions is that they can be considered small compared to forces of linear elements. We will therefore discuss mathematical perturbation methods that allow us to determine the effects of perturbations and to apply corrective measures for beam stability. The equations of motion with perturbations are

$$
u^{\prime \prime}+\left(k+\kappa_{u}^{2}\right) u=p_{r s n}(z) x^{r} y^{n-s} \quad \text { where } u=x \text { or } y
$$

and $r, s=0,1,2 \ldots$ with $r+s+1=n$ and $n$ the order of perturbation. In the remainder of this text whenever $r=0$ or $s=0$ we use $p_{n}$ instead of $p_{r s n}{ }^{1}$

\subsection{Magnet Field and Alignment Errors}

First we consider field errors created by magnet misalignments like displacements or rotations from the ideal positions. Such magnet alignment errors, however, are not the only cause for field errors. External sources like the earth magnetic field, the fields of nearby electrical current carrying conductors, magnets connected to vacuum pumps or ferromagnetic material in the vicinity of beam transport magnets can cause similar field errors. For example electrical power cables connected to other magnets along the beam transport line must be connected such that the currents in all cables are compensated. This occurs automatically for cases, where the power cables to and from a magnet run close together. In circular accelerators one might, however, be tempted to run the cables around the ring only once to save the high material and installation costs. This, however, causes an uncompensated magnetic field in the vicinity of cables which may reach as far as the particle beam pipe. The economic solution is to seek electrical current compensation among all magnet currents by running electrical currents in different directions around the ring. Careful design of the beam transport system can in most cases minimize the impact of such field perturbations while at the same time meeting economic goals.

Multipole errors in magnets are not the only cause for perturbations. For beams with large divergence or a large cross section, kinematic perturbation terms may have to be included. Such terms are neglected in paraxial beam optics discussed here, but will be derived in detail later.

\footnotetext{
${ }^{1}$ Note that for this definition $p_{0}=0$ and therefore there is no conflict with the momentum $p_{0}$.
} 


\subsubsection{Self Compensation of Perturbations}

The linear superposition of individual dipole contributions to the dispersion function can be used in a constructive way. Any contribution to the dispersion function by a short magnet can be eliminated again by a similar magnet located $180^{\circ}$ in betatron phase downstream from the first magnet. If the betatron function at the location of both magnets is the same, the magnet strengths are the same too. For quantitative evaluation we assume two dipole errors introducing a beam deflection by the angles $\theta_{1}$ and $\theta_{2}$ at locations with betatron functions of $\beta_{1}$ and $\beta_{2}$ and betatron phases $\psi_{1}$ and $\psi_{2}$, respectively. Since the dispersion function or fractions thereof evolve like a sine like function, we find for the variation of the dispersion function at a phase $\psi(z) \geq \psi_{2}$

$$
\Delta D(z)=\theta_{1} \sqrt{\beta \beta_{1}} \sin \left[\psi(z)-\psi_{1}\right]+\theta_{2} \sqrt{\beta \beta_{2}} \sin \left[\psi(z)-\psi_{2}\right] .
$$

For the particular case where $\theta_{1}=\theta_{2}$ and $\beta_{1}=\beta_{2}$ we find

$$
\Delta D(z)=0 \text { for } \quad \psi_{2}-\psi_{1}=(2 n+1) \pi .
$$

If $\theta_{1}=-\theta_{2}$

$$
\Delta D(z)=0 \text { for } \psi_{2}-\psi_{1}=2 n \pi,
$$

where $n$ is an integer. This property of the dispersion function can be used in periodic lattices if, for example, a vertical displacement of the beam line is desired. In this case we would like to deflect the beam vertically and as soon as the beam reaches the desired elevation a second dipole magnet deflects the beam by the same angle but opposite sign to level the beam line parallel to the horizontal plane again. In an arbitrary lattice such a beam displacement can be accomplished without creating a residual dispersion outside the beam deflecting section if we place two vertical or rotated bending magnets of opposite sign at locations separated by a betatron phase of $2 \pi$.

Similarly, a deflection in the same direction by two dipole magnets does not create a finite dispersion outside the deflecting section if both dipoles are separated by a betatron phase of $(2 n+1) \pi$. This feature is important to simplify beamtransport lattices since no additional quadrupoles are needed to match the dispersion function.

Sometimes it is necessary to deflect the beam in both the horizontal and vertical direction. This can be done in a straightforward way by a sequence of horizontal and vertical bending sections leading, however, to long beam lines. In a more compact version, we combine the beam deflection in both planes within one or a set of magnets. To obtain some vertical deflection in an otherwise horizontally deflecting beam line, we may rotate a whole arc section about the beam axis at the start of this section to get the desired vertical deflection. Some of the horizontal deflection 
is thereby transformed into the vertical plane. At the start of such a section we introduce by the rotation of the coordinate system a sudden change in all lattice functions. Specifically, a purely horizontal dispersion function is coupled partly into a vertical dispersion. If we rotate the beam line and coordinate system back at a betatron phase of $2 n \pi$ downstream from the start of rotation, the coupling of the dispersion function as well as that of other lattice functions is completely restored. For that to work without further matching, however, we require that the rotated part of the beam line has a phase advance of $2 n \pi$ in both planes as, for example, a symmetric FODO lattice would have. This principle has been used extensively for the terrain following beam transport lines of the SLAC Linear Collider to the collision point.

\subsection{Dipole Field Perturbations}

Dipole fields are the lowest order magnetic fields and therefore also the lowest order field perturbations. The equation of motion (15.1) is in this case

$$
u^{\prime \prime}+\left(k+\kappa_{u}^{2}\right) u=p_{1}(z),
$$

where $p_{1}(z)$ represents any dipole field error, whether it be chromatic or not. In trying to establish expressions for dipole errors due to field or alignment errors, we note that the bending fields do not appear explicitly anymore in the equations of motions because of the specific selection of the curvilinear coordinate system and it is therefore not obvious in which form dipole field errors would appear in the equation of motion (15.5). In (6.95) or (6.96) we note, however, a dipole field perturbation due to a particle with a momentum error $\delta$. This chromatic term $\kappa_{x 0} \delta$ is similar to a dipole field error as seen by a particle with the momentum $\beta E_{0}(1+\delta)$. For particles with the ideal energy we may therefore replace the chromatic term $\kappa \delta$ by a field error $-\Delta \kappa$. Perturbations from other sources may be obtained by variations of magnet positions $(\Delta x, \Delta y)$ or magnet strengths. Up to second order, the horizontal dipole perturbation terms due to magnet field $(\Delta \kappa)$ and alignment errors $(\Delta x, \Delta y)$ are from $(6.95)$

$$
\begin{array}{r}
p_{1,, x}(z)=-\Delta \kappa_{x 0}+\left(\kappa_{x 0}^{2}+k_{0}\right) \Delta x+\left(2 \kappa_{x 0} \Delta \kappa_{x 0}+\Delta k\right) \Delta x \\
-\frac{1}{2} m\left(\Delta x^{2}-2 x_{\mathrm{c}} \Delta x-\Delta y^{2}+2 y_{\mathrm{c}} \Delta y\right)+\mathcal{O}(3),
\end{array}
$$

where we used $x=x_{\beta}+x_{\mathrm{c}}-\Delta x$ and $y=y_{\beta}+y_{\mathrm{c}}-\Delta y$ with $\left(x_{\beta}, y_{\beta}\right)$ the betatron oscillations and $\left(x_{\mathrm{c}}, y_{\mathrm{c}}\right)$ the closed orbit deviation in the magnet. In the presence of multipole magnets the perturbation depends on the displacement of the beam with respect to the center of multipole magnets. 
There is a similar expression for vertical dipole perturbation terms and we get from (6.96) ignoring vertical bending magnets $\left(\kappa_{y_{0}}=0\right)$ but not vertical dipole errors, $\Delta \kappa_{y 0} \neq 0$,

$$
p_{1, y}(z)=-\Delta \kappa_{y 0}-k_{0} \Delta y-m\left(x_{\mathrm{c}} \Delta y+y_{\mathrm{c}} \Delta x\right)+\mathcal{O}(3) .
$$

Such dipole field errors deflect the beam from its ideal path and we are interested to quantify this perturbation and to develop compensating methods to minimize the distortions of the beam path. In an open beam transport line the effect of dipole field errors on the beam path can be calculated within the matrix formalism.

A dipole field error at point $z_{\mathrm{k}}$ deflects the beam by an angle $\theta$. If $\mathbf{M}\left(z_{\mathrm{m}} \mid z_{\mathrm{k}}\right)$ is the transformation matrix of the beam line between the point $z_{\mathrm{k}}$, where the kick occurs, and the point $z_{\mathrm{m}}$, where we observe the beam position, we find a displacement of the beam center line, for example, in the $x$-plane by

$$
\Delta x=M_{12} \theta,
$$

where $M_{12}$ is the element of the transformation matrix in the first row and the second column. Due to the linearity of the equation of motion, effects of many kicks caused by dipole errors can be calculated by summation of individual beam center displacements at the observation point $z_{\mathrm{m}}$ for each kick. The displacement of a beam at the location $z_{\mathrm{m}}$ due to many dipole field errors is then given by

$$
\Delta x\left(z_{\mathrm{m}}\right)=\sum_{\mathrm{k}} M_{12}\left(z_{\mathrm{m}} \mid z_{\mathrm{k}}\right) \theta_{\mathrm{k}}
$$

where $\theta_{\mathrm{k}}$ are kicks due to dipole errors at locations $z_{\mathrm{k}}<z_{\mathrm{m}}$ and $M_{12}\left(z_{\mathrm{m}} \mid z_{\mathrm{k}}\right)$ the $M_{12}$-matrix element of the transformation matrix from the perturbation at $z_{\mathrm{k}}$ to the monitor at $z_{\mathrm{m}}$.

Generally, we do not know the strength and location of errors. Statistical methods are applied therefore to estimate the expectation value for beam perturbation and displacement. With $M_{12}\left(z_{\mathrm{m}} \mid z_{\mathrm{k}}\right)=\sqrt{\beta_{\mathrm{m}} \beta_{\mathrm{k}}} \sin \left(\psi_{\mathrm{m}}-\psi_{\mathrm{k}}\right)$ we calculate the root-meansquare of (15.9) noting that the phases $\psi_{\mathrm{k}}$ are random and cross terms involving different phases cancel. With $\left\langle\theta_{\mathrm{k}}^{2}\right\rangle=\sigma_{\theta}^{2}$ and $\left\langle\Delta u^{2}\right\rangle=\sigma_{u}^{2}$ we get finally from (15.9) the expectation value of the path distortion $\sigma_{u}$ at $z_{\mathrm{m}}$ due to statistical errors with a standard value $\sigma_{\theta}$

$$
\sigma_{u}=\sqrt{\beta_{\mathrm{m}}\left\langle\beta_{\mathrm{k}}\right\rangle} \sqrt{N_{\theta}} \sigma_{\theta}
$$

where $\left\langle\beta_{\mathrm{k}}\right\rangle$ is the average betatron function at the location of errors and $N_{\theta}$ the number of dipole field errors. Random angles are not obvious, but if we identify the potential sources, we may be in a better position to estimate $\sigma_{\theta}$. For example, alignment errors $\sigma_{\Delta u}$ of quadrupoles are related to $\sigma_{\theta}$ by $\sigma_{\theta}=k \ell_{\mathrm{q}} \sigma_{\Delta u}$, where $\frac{1}{f}=$ $k \ell_{\mathrm{q}}$ are the inverse focal lengths of the quadrupoles. 


\subsubsection{Dipole Field Errors and Dispersion Function}

The dispersion function of a beam line is determined by the strength and placement of dipole magnets. As a consequence, dipole field errors also contribute to the dispersion function and we determine such contributions to the dispersion function due to dipole field errors. First, we note from the general expression for the linear dispersion function that the effect of dipole errors adds linearly to the dispersion function by virtue of the linearity of the equation of motion. We may therefore calculate separately the effect of dipole errors and add the results to the ideal solution for the dispersion function.

\subsubsection{Perturbations in Open Transport Lines}

While these properties are useful for specific applications, general beam dynamics requires that we discuss the effects of errors on the dispersion function in a more general way. To this purpose we use the general equation of motion up to linear terms in $\delta$ and add constant perturbation terms. In the following discussion we use only the horizontal equation of motion, but the results can be immediately applied to the vertical plane as well. The equation of motion with only linear chromatic terms and a quadratic sextupole term is then

$$
x^{\prime \prime}+\left(k+\kappa_{x}^{2}\right) x=k x \delta-\frac{1}{2} m x^{2}(1-\delta)-\Delta \kappa_{x}(1-\delta)+\mathcal{O}(2) .
$$

We observe two classes of perturbation terms, the ordinary chromatic terms and those due to field errors. Taking advantage of the linearity of the solution we decompose the particle position into four components

$$
x=x_{\beta}+x_{\mathrm{c}}+\eta_{x} \delta+v_{x} \delta,
$$

where $x_{\beta}$ is the betatron motion, $x_{\mathrm{c}}$ the distorted beam path or orbit, $\eta_{x}$ the ideal dispersion function and $v_{x}$ the perturbation of the dispersion that derives from field errors. The individual parts of the solution then are determined by the following set of differential equations:

$$
\begin{aligned}
x_{\beta}^{\prime \prime}+\left(k+\kappa_{x}^{2}\right) x_{\beta} & =-\frac{1}{2} m x_{\beta}^{2}+m x_{\beta} x_{\mathrm{c}}, \\
x_{\mathrm{c}}^{\prime \prime}+\left(k+\kappa_{x}^{2}\right) x_{\mathrm{c}} & =-\Delta \kappa_{x}-\frac{1}{2} m x_{\mathrm{c}}^{2}, \\
\eta_{x}^{\prime \prime}+\left(k+\kappa_{x}^{2}\right) \eta_{x} & =\kappa_{x}, \\
v_{x}^{\prime \prime}+\left(k+\kappa_{x}^{2}\right) v_{x} & =+\Delta \kappa_{x}+\frac{1}{2} m x_{\mathrm{c}}^{2}+k x_{\mathrm{c}}-m x_{\mathrm{c}} \eta_{x} .
\end{aligned}
$$


In the ansatz (15.12) we have ignored the energy dependence of the betatron function since it will be treated separately as an aberration and has no impact on the dispersion. We have solved (15.13a)-(15.13c) before and concentrate therefore on the solution of (15.13d). Obviously, the field errors cause distortions of the beam path $x_{\mathrm{c}}$ which in turn cause additional variations of the dispersion function. The principal solutions are

$$
\begin{gathered}
C(z)=\sqrt{\beta(z) / \beta_{0}} \cos \left[\psi(z)-\psi_{0}\right], \\
S(z)=\sqrt{\beta(z) \beta_{0}} \sin \left[\psi(z)-\psi_{0}\right],
\end{gathered}
$$

and the Greens function becomes

$$
G(z, \sigma)=S(z) C(\sigma)-S(\sigma) C(z)=\sqrt{\beta(z) \beta(\sigma)} \sin [\psi(z)-\psi(\sigma)]
$$

With this the solution of $(15.13 \mathrm{~d})$ is

$$
\begin{aligned}
& v_{x}(z)=-x_{\mathrm{c}}(z) \\
& \quad+\sqrt{\beta_{x}(z)} \int_{0}^{z}\left(k-m \eta_{x}\right) \sqrt{\beta_{x}(\zeta)} x_{\mathrm{c}}(\zeta) \sin \left[\psi_{x}(z)-\psi_{x}(\zeta)\right] \mathrm{d} \zeta .
\end{aligned}
$$

Here, we have split off the solution for the two last perturbation terms in (15.13d) which, apart from the sign, is exactly the orbit distortion (15.13b). In a closed lattice we look for a periodic solution of (15.17), which can be written in the form

$$
\begin{aligned}
v_{x}(z)=- & x_{\mathrm{c}}(z)+\frac{\sqrt{\beta_{x}(z)}}{2 \sin \pi v_{x}} \\
& \times \int_{z}^{z+L_{\mathrm{p}}}\left(k-m \eta_{x}\right) \sqrt{\beta_{x}(\zeta)} x_{\mathrm{c}}(\zeta) \cos \left\{v_{x}\left[\varphi_{x}(z)-\varphi_{x}(\zeta)+\pi\right]\right\} \mathrm{d} \zeta,
\end{aligned}
$$

where $x_{\mathrm{c}}(z)$ is the periodic solution for the distorted orbit and $L_{\mathrm{p}}$ the length of the orbit. In the vertical plane we have exactly the same solution except for a change in sign for some terms

$$
\begin{aligned}
v_{y}(z)=- & y_{\mathrm{c}}(z)-\frac{\sqrt{\beta_{y}(z)}}{2 \sin \pi v_{y}} \\
& \times \int_{z}^{z+L_{\mathrm{p}}}\left(k-m \eta_{x}\right) \sqrt{\beta_{y}(\zeta)} y_{\mathrm{c}}(\zeta) \cos \left[v_{y}\left(\varphi_{y}(z)-\varphi_{y}(\zeta)+\pi\right)\right] \mathrm{d} \zeta .
\end{aligned}
$$

For reasons of generality we have included here sextupoles to permit chromatic corrections in long curved beam lines with bending magnets. The slight asymmetry due to the term $m \eta_{x}$ in the vertical plane derives from the fact that in real 
accelerators only one orientation of the sextupoles is used. Due to this orientation the perturbation in the horizontal plane is $-\frac{1}{2} m x^{2}(1-\delta)$ and in the vertical plane $m x y(1-\delta)$. In both cases we get the term $m \eta_{x}$ in the solution integrals.

Again we may ask how this result varies as we add acceleration to such a transport line. Earlier in this section we found that the path distortion is independent of acceleration under certain periodic conditions. By the same arguments we can show that the distortion of the dispersions (15.18) and (15.19) are also independent of acceleration and the result of this discussion can therefore be applied to any periodic focusing channel.

\subsubsection{Existence of Equilibrium Orbits}

Particles orbiting around a circular accelerator perform in general betatron oscillations about the equilibrium orbit and we will discuss properties of this equilibrium orbit. Of fundamental interest is of course that such equilibrium orbits exist at all. We will not try to find conditions for the existence of equilibrium orbits in arbitrary electric and magnetic fields but restrict this discussion to fields with midplane symmetry as they are used in particle beam systems. The existence of equilibrium orbits can easily be verified for particles like electrons and positrons because these particles radiate energy in form of synchrotron radiation as they orbit around the ring.

We use the damping process to find the eventual equilibrium orbit in the presence of arbitrary dipole perturbations. To do this, we follow an orbiting particle starting with the parameters $x=0$ and $x^{\prime}=0$. This choice of initial parameters will not affect the generality of the argument since any other value of initial parameters is damped independently because of the linear superposition of betatron oscillations.

As an electron orbits in a circular accelerator it will encounter a number of kicks from dipole field errors or field errors due to a deviation of the particle energy from the ideal energy. After one turn the particle position is the result of the superposition of all kicks the particle has encountered in that turn. Since each kick leads to a particle oscillation given by

$$
x(z)=\sqrt{\beta(z) \beta_{\theta}} \theta \sin \left[v \varphi(z)-v \varphi_{\theta}\right]
$$

we find for the superposition of all kicks in one turn

$$
x(z)=\sqrt{\beta(z)} \sum_{i} \sqrt{\beta_{i}} \theta_{i} \sin \left[v \varphi(z)-v \varphi_{i}\right],
$$

where the index $i$ indicates the location of the kicks. We ask ourselves now what is the oscillation amplitude after many turns. For that we add up the kicks from all past turns and include damping effects expressed by the factor $\mathrm{e}^{-k T_{0} / \tau}$ on the particle oscillation amplitude, where $T_{0}$ is the revolution time, $k T_{0}$ is the time passed since 
the kick occurred $k$ turns ago, and $\tau$ the damping time. The contribution to the betatron oscillation due to kicks $k$ turns ago, is then given by

$$
\Delta x_{k}(z)=\sqrt{\beta(z)} \mathrm{e}^{-k T_{0} / \tau} \sum_{i} \sqrt{\beta_{i}} \theta_{i} \sin \left[2 \pi v k+v \varphi(z)-v \varphi_{i}\right] .
$$

Adding the contributions from all past turns results in the position $x(z)$ of the particle

$$
x(z)=\sum_{k=0}^{\infty} \sqrt{\beta(z)} \mathrm{e}^{-k T_{0} / \tau} \sum_{i} \sqrt{\beta_{i}} \theta_{i} \sin \left[2 \pi \nu k+\nu \varphi(z)-\nu \varphi_{i}\right] .
$$

After some rearranging (15.22) becomes

$$
x(z)=C_{\theta} \sum_{k=0}^{\infty} \mathrm{e}^{-k T_{0} / \tau} \sin (2 \pi \nu k)+S_{\theta} \sum_{k=0}^{\infty} \mathrm{e}^{-k T_{0} / \tau} \cos (2 \pi \nu k),
$$

where

$$
\begin{aligned}
C_{\theta} & =\sum_{i} \sqrt{\beta(z) \beta_{i}} \theta_{i} \cos \left[\varphi(z)-\varphi_{i}\right], \\
S_{\theta} & =\sum_{i} \sqrt{\beta(z) \beta_{i}} \theta_{i} \sin \left[\varphi(z)-\varphi_{i}\right] .
\end{aligned}
$$

With the definition $q=\mathrm{e}^{-T_{0} / \tau}$ we use the mathematical identities

$$
\sum_{k=0}^{\infty} \mathrm{e}^{-k T_{0} / \tau} \sin (2 \pi \nu k)=\frac{q \sin 2 \pi \nu}{1-2 q \cos 2 \pi \nu+q^{2}}
$$

and

$$
\sum_{k=0}^{\infty} \mathrm{e}^{-k T_{0} / \tau} \cos (2 \pi \nu k)=\frac{1-q \cos 2 \pi \nu}{1-2 q \cos 2 \pi \nu+q^{2}}
$$

and get finally instead of (15.23)

$$
x(z)=\frac{C_{\theta} q \sin 2 \pi \nu+S_{\theta}(1-q \cos 2 \pi \nu)}{1-2 q \cos 2 \pi v+q^{2}} .
$$

The revolution time is generally much shorter than the damping time $T_{0} \ll \tau$ and therefore $q \approx 1$. In this approximation we get after some manipulation and using (15.24)

$$
x(z)=\frac{\sqrt{\beta(z)}}{2 \sin \pi v} \sum_{i} \sqrt{\beta_{i}} \theta_{i} \cos \left[v \varphi(z)-v \varphi_{i}+v \pi\right] .
$$


Equation (15.28) describes the particle orbit reached by particles after some damping times. The solution does not include anymore any reference to earlier turns and kicks except those in one turn and the solution therefore is a steady state solution defined as the equilibrium orbit .

The cause and nature of the kicks $\theta_{i}$ is undefined and can be any perturbation, random or systematic. A particular set of such errors are systematic errors in the deflection angle for particles with a momentum error $\delta$ for which $\theta_{i}=\kappa_{i} \ell_{i} \delta$ is the deflection angle of the bending magnet $i$. These errors are equivalent to those that led to the dispersion or $\eta$-function. Indeed, setting $\eta(z)=x(z) / \delta$ in (15.28) we get the solution (10.91) for the $\eta$ function. The trajectories $x(z)=\eta(z) \delta$ therefore are the equilibrium orbits for particles with a relative momentum deviation $\delta=\Delta p / p_{0}$ from the ideal momentum $p_{0}$.

In the next subsection we will discuss the effect of random dipole field errors $\theta_{i}$ on the beam orbit. These kicks, since constant in time, are still periodic with the periodicity of the circumference and lead to a distorted orbit which turns out to be again equal to the equilibrium orbit found here.

To derive the existence of equilibrium orbits we have made use of the damping of particle oscillations. Since this damping derives from the energy loss of particles due to synchrotron radiation we have proof only for equilibrium orbits for radiating particles like electrons and positrons. The result obtained applies also to any other charged particle. The damping time may be extremely long, but is not infinite and a particle will eventually reach the equilibrium orbit. The concept of equilibrium orbits is therefore valid even though a proton or ion will never reach that orbit in a finite time but will oscillate about it.

\subsubsection{Closed Orbit Distortion}

The solution (15.28) for the equilibrium orbit can be derived also directly by solving the equation of motion. Under the influence of dipole errors the equation of motion is

$$
u^{\prime \prime}+K(z) u=p_{1}(z)
$$

where the dipole perturbation $p_{0}(z)$ is independent of coordinates $(x, y)$ and energy error $\delta$. This differential equation has been solved earlier in Sect.5.5.4, where a dipole field perturbation was introduced as an energy error of the particle. Therefore, we can immediately write down the solution for an arbitrary beam line for which the principal solutions $C(z)$ and $S(z)$ are known

$$
u(z)=C(z) u_{0}+S(z) u_{0}^{\prime}+P(z) \delta
$$


with

$$
P(z)=\int_{0}^{z} p_{1}(\zeta)[S(z) C(\zeta)-S(\zeta) C(z)] \mathrm{d} \zeta
$$

The result (15.30) can be interpreted as a composition of betatron oscillations with initial values $\left(u_{0}, u_{0}^{\prime}\right)$ and a superimposed perturbation $P(z)$ which defines the equilibrium trajectory for the betatron oscillations. In (15.31) we have assumed that there is no distortion at the beginning of the beam line, $P(0)=0$. If there were already a perturbation of the reference trajectory from a previous beam line, we define a new reference path by linear superposition of new perturbations to the continuation of the perturbed path from the previous beam line section. The particle position $\left(u_{0}, u_{0}^{\prime}\right)$ is composed of the betatron oscillation $\left(u_{0 \beta}, u_{0 \beta}^{\prime}\right)$ and the perturbation of the reference path $\left(u_{0 \mathrm{c}}, u_{0 \mathrm{c}}^{\prime}\right)$. With $u_{0}=u_{0 \beta}+u_{0 \mathrm{c}}$ and $u_{0}^{\prime}=u_{0 \beta}^{\prime}+u_{0 \mathrm{c}}^{\prime}$ we get

$$
u(z)=\left[u_{0 \beta} C(z)+u_{0 \beta}^{\prime} S(z)\right]+\left[u_{0 \mathrm{c}} C(z)+u_{0 \mathrm{c}}^{\prime} S(z)\right]+P(z) .
$$

In a circular accelerator we look for a self-consistent periodic solution. Because the differential equation (15.29) is identical to that for the dispersion function, the solution must be similar to (10.91) and is called the closed orbit, reference orbit or equilibrium orbit given by

$$
u_{\mathrm{c}}(z)=\frac{\sqrt{\beta(z)}}{2 \sin \pi v} \oint_{z}^{z+C} p_{1}(\zeta) \sqrt{\beta(\zeta)} \cos [v \varphi(z)-v \varphi(\zeta)+v \pi] \mathrm{d} \zeta
$$

where $C$ is the circumference of the accelerator. We cannot anymore rely on a superperiodicity of length $L_{\mathrm{p}}$ since the perturbations $p_{r s n}(\zeta)$ due to misalignment or field errors are statistically distributed over the whole ring. Again the integer resonance character discussed earlier for the dispersion function is obvious, indicating there is no stable orbit if the tune of the circular accelerator is an integer. The influence of the integer resonance is noticeable even when the tune is not quite an integer. From (15.33) we find a perturbation $p_{1}(z)$ to have an increasing effect the closer the tune is to an integer value. The similarity of the closed orbit and the dispersion function in a circular accelerator is deeper than merely mathematical. The dispersion function defines closed orbits for energy deviating particles approaching the real orbit (15.33) as $\delta \rightarrow 0$.

Up to second order the horizontal and vertical dipole perturbation terms due to magnet field and alignment errors are given by (15.6) and (15.7). In the presence of multipole magnets the perturbation depends on the displacement of the beam with respect to the center of multipole magnets.

A vertical closed orbit distortion is shown in Fig. 15.1 for the PEP storage ring. Here, a Gaussian distribution of horizontal and vertical alignment errors with an rms error of $0.05 \mathrm{~mm}$ in all quadrupoles has been simulated. In spite of the statistical 


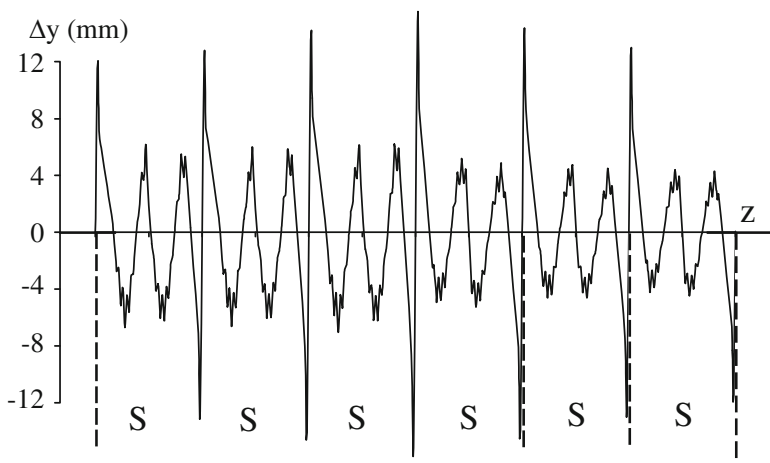

S: superperiod

Fig. 15.1 Simulation of the closed orbit distortion in the sixfold symmetric PEP lattice due to statistical misalignments of quadrupoles by an amount $\langle\Delta x\rangle_{\mathrm{rms}}=\langle\Delta y\rangle_{\mathrm{rms}}=0.05 \mathrm{~mm}$

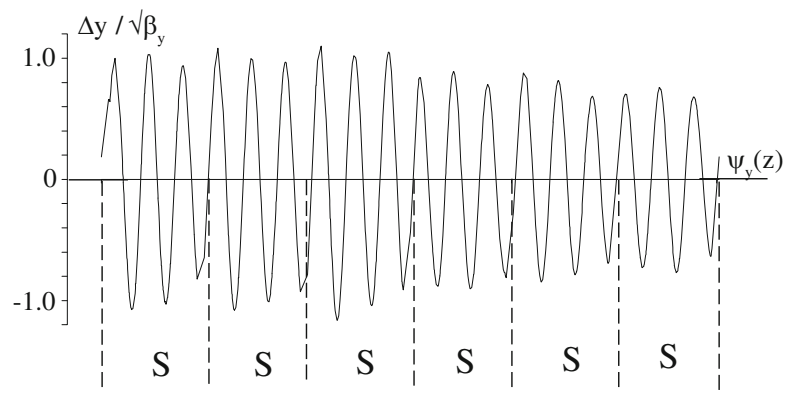

Fig. 15.2 Closed orbit distortion of Fig. 15.2 in normalized coordinates as a function of the betatron phase $\varphi$

distribution of errors a strong oscillatory character of the orbit is apparent and counting oscillations we find 18 oscillations being equal to the vertical tune of PEP as we would expect from the denominator of (15.33).

We also note large values of the orbit distortion adjacent to the interaction points (dashed lines), where the betatron function becomes large, again in agreement with expectations from (15.33) since $u_{\mathrm{c}} \propto \sqrt{\beta}$. A more regular representation of the same orbit distortion can be obtained if we plot the normalized closed orbit $u_{\mathrm{c}}(z) / \sqrt{\beta(z)}$ as a function of the betatron phase $\psi(z)$ shown in Fig. 15.2. In this representation the strong harmonic close to the tune becomes evident while the statistical distribution of perturbations appears mostly in the amplitude of the normalized orbit distortion.

For the sake of simplicity terms of third or higher order as well as terms associated with nonlinear magnets have been neglected in both Eqs. (15.6) and (15.7). All terms in (15.6) and (15.7) are considered small perturbations and can therefore be treated individually and independent of other perturbations terms. Sextupole and 
higher multipole perturbations depend on the orbit itself and to get a self-consistent periodic solution of the distorted orbit, iteration methods must be employed.

Solutions for equilibrium orbits can be obtained by inserting the perturbation (15.6) or (15.7) into (15.33). First, we will concentrate on a situation, where only one perturbing kick exists in the whole lattice, assuming the perturbation to occur at $z=z_{\mathrm{k}}$ and to produce a kick $\theta_{\mathrm{k}}=\int p_{1}(\zeta) \mathrm{d} \zeta$ in the particle trajectory. The orbit distortion at a location $z<z_{\mathrm{k}}$ in the lattice is from (15.33)

$$
u_{0}(z)=\frac{1}{2} \sqrt{\beta(z) \beta\left(z_{\mathrm{k}}\right)} \theta_{\mathrm{k}} \frac{\cos \left[v \pi-v \varphi\left(z_{\mathrm{k}}\right)+v \varphi(z)\right]}{\sin \pi v} .
$$

If on the other hand we look for the orbit distortion downstream from the perturbation $z>z_{\mathrm{k}}$ the integration must start at $z$, follow the ring to $z=C$ and then further to $z=z+C$. The kick, therefore, occurs at the place $C+z_{\mathrm{k}}$ with the phase $\varphi(C)+\varphi\left(z_{\mathrm{k}}\right)=2 \pi+\varphi\left(z_{u}\right)$ and the orbit is given by

$$
u_{0}(z)=\frac{1}{2} \sqrt{\beta(z) \beta\left(z_{\mathrm{k}}\right)} \theta_{\mathrm{k}} \frac{\cos \left[v \pi-v \varphi(z)+v \varphi\left(z_{\mathrm{k}}\right)\right]}{\sin \pi v} .
$$

This mathematical distinction of cases $z<z_{\mathrm{k}}$ and $z>z_{\mathrm{k}}$ is a consequence of the integration starting at $z$ and ending at $z+C$ and is necessary to account for the discontinuity of the slope of the equilibrium orbit at the location of the kick. At the point $z=z_{\mathrm{k}}$ obviously both equations are the same. In Fig. 15.3 the normalized distortion of the ideal orbit due to a single dipole kick is shown. In a linear lattice this distortion is independent of the orbit and adds in linear superposition. If, however, sextupoles or other coupling or nonlinear magnets are included in the lattice, the distortion due to a single or multiple kick depends on the orbit itself and selfconsistent solutions can be obtained only by iterations.

In cases where a single kick occurs at a symmetry point of a circular accelerator we expect the distorted orbit to also be symmetric about the kick. This is expressed in the asymmetric phase terms of both equations. Indeed, since $\varphi\left(z_{\mathrm{k}}\right)-\varphi(z)=$ $\Delta \varphi$ for $z_{\mathrm{k}}>z$ and $\varphi(z)-\varphi\left(z_{\mathrm{k}}\right)=\Delta \varphi$ for $z>z_{\mathrm{k}}$ the orbit distortion extends symmetrically in either direction from the location of the kick.

Fig. 15.3 Distorted orbit due to a single dipole kick for a tune just above an integer (left) and for a tune below an integer (right)
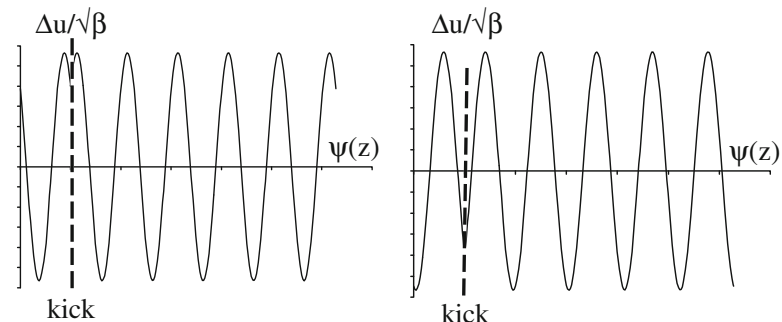
The solution for the perturbed equilibrium orbit is specially simple at the place where the kick occurs. With $\varphi(z)=\varphi\left(z_{k}\right)$ the orbit distortion is

$$
u_{\mathrm{k}}=\frac{1}{2} \beta_{\mathrm{k}} \theta_{\mathrm{k}} \cot \pi v .
$$

In situations where a short bending magnet like an orbit correction magnet and a beam position monitor are at the same place or at least close together we may use these devices to measure the betatron function at that place $z_{\mathrm{k}}$ by measuring the tune $v$ of the ring and the change in orbit $u_{\mathrm{k}}$ due to a kick $\theta_{\mathrm{k}}$. Equation (15.36) can then be solved for the betatron function $\beta_{\mathrm{k}}$ at the location $z_{\mathrm{k}}$. This procedure can obviously be applied in both planes to experimentally determine $\beta_{x}$ as well as $\beta_{y}$.

\subsubsection{Statistical Distribution of Dipole Field and Alignment Errors}

In a real circular accelerator a large number of field and misalignment errors of unknown location and magnitude must be expected. If the accelerator is functional we may measure the distorted orbit with the help of beam position monitors and apply an orbit correction as discussed later in this section. During the design stage, however, we need to know the sensitivity of the ring design to such errors in order to determine alignment tolerances and the degree of correction required. In the absence of detailed knowledge about errors we use statistical methods to determine the most probable equilibrium orbit. All magnets are designed, fabricated, and aligned within statistical tolerances, which are determined such that the distorted orbit allows the beam to stay within the vacuum pipe without loss. An expectation value for the orbit distortion can be derived by calculating the root-mean-square of (15.33)

$$
\begin{aligned}
u_{0}^{2}(z)=\frac{\beta(z)}{4 \sin ^{2} \pi v} & \oint_{z}^{z+C} \oint_{z}^{z+C} p_{1}(\sigma) p_{1}(\tau) \sqrt{\beta(\sigma)} \sqrt{\beta(\tau)} \\
& \times \cos \left[v\left(\varphi_{z}-\varphi_{\sigma}+\pi\right)\right] \cos \left[v\left(\varphi_{z}-\varphi_{\tau}+\pi\right)\right] \mathrm{d} \sigma \mathrm{d} \tau
\end{aligned}
$$

where for simplicity $\varphi_{z}=\varphi(z)$ etc. This double integral can be evaluated by expanding the cosine functions to separate the phases $\varphi_{\sigma}$ and $\varphi_{\tau}$. We get terms like $\cos \nu \varphi_{\sigma} \cos \nu \varphi_{\tau}$ and $\sin \nu \varphi_{\sigma} \sin \nu \varphi_{\tau}$ or mixed terms. All these terms tend to cancel except when $\sigma=\tau$ since both the perturbations and their locations are statistically distributed in phase. Only for $\sigma=\tau$ will we get quadratic terms that contribute to a finite expectation value for the orbit distortion

$$
\begin{aligned}
& \left\langle p_{1}^{2}(\tau)\left[\cos ^{2} v\left(\varphi_{z}+\pi\right) \cos ^{2} v \varphi_{\tau}+\sin ^{2} v\left(\varphi_{z}+\pi\right) \sin ^{2} v \varphi_{\tau}\right]\right\rangle \\
& =\left\langle p_{1}^{2}(\tau)\right\rangle\left[\cos ^{2} v\left(\varphi_{z}+\pi\right)\left\langle\cos ^{2} v \varphi_{\tau}\right\rangle+\sin ^{2} v\left(\varphi_{z}+\pi\right)\left\langle\sin ^{2} v \varphi_{\tau}\right\rangle\right] \\
& =\left\langle p_{1}^{2}(\tau)\right\rangle \frac{1}{2}
\end{aligned}
$$


and with this (15.37) becomes

$$
\left\langle u_{0}^{2}(z)\right\rangle=\frac{\beta(z)}{8 \sin ^{2} \pi v} \sum_{i}\left\langle p_{1}^{2}\left(\sigma_{i}\right) \beta\left(\sigma_{i}\right) \ell_{i}^{2}\right\rangle,
$$

where the integrals have been replaced by a single sum over all perturbing fields of length $\ell_{i}$. This can be done since we assume that the betatron phase does not change much over the length of individual perturbations. Equation (15.38) gives the expectation value for the orbit distortion at the point $z$ and since the errors are statistically distributed we get from the central limit theorem a Gaussian distribution of the orbit distortions with the standard deviation $\sigma_{u}^{2}(z)=\left\langle u_{0}^{2}(z)\right\rangle$ from (15.38). In other words if an accelerator is constructed with tolerances $\left\langle p_{1}^{2}\left(\sigma_{i}\right)\right\rangle$ there is a $68 \%$ probability that the orbit distortions are of the order $\sqrt{\left\langle u_{0}^{2}(z)\right\rangle}$ as calculated from (15.38) and a $98 \%$ probability that they are not more than twice that large.

As an example, we consider a uniform beam transport line, where all quadrupoles have the same strength and the betatron functions are periodic like in a FODO channel. This example seems to be very special since hardly any practical beam line has these properties, but it is still a useful example and may be used to simulate more general beam lines for a quick estimate of alignment tolerances. Assuming a Gaussian distribution of quadrupole misalignments with a standard deviation $\sigma_{\Delta u}$ and quadrupole strength $k$, the perturbations are $p_{1}(z)=k \sigma_{\Delta u}$ and the expected orbit distortion is

$$
\sqrt{\left\langle u_{0}^{2}(z)\right\rangle}=\sqrt{\beta(z)} A \sigma_{\Delta u},
$$

where $A$ is called the error amplification factor defined by

$$
A^{2}=\frac{N}{8 \sin ^{2} \pi v}\left\langle\left(k \ell_{\mathrm{q}}\right)^{2} \beta\right\rangle \approx \frac{N}{8 \sin ^{2} \pi v} \frac{\bar{\beta}}{f^{2}},
$$

$\left\langle\left(k \ell_{\mathrm{q}}\right)^{2} \beta\right\rangle$ is taken as the average value for the expression in all $N$ misaligned quadrupoles, $f$ is the focal length of the quadrupoles, and $\bar{\beta}$ the average betatron function.

The expectation value for the maximum value of the orbit distortion $\left\langle\hat{u}_{0}^{2}(z)\right\rangle$ is larger. In (15.38) we have averaged the trigonometric functions

$$
\left\langle\cos ^{2} v \varphi(\tau)\right\rangle=\left\langle\sin ^{2} v \varphi(\tau)\right\rangle=\frac{1}{2}
$$

and therefore

$$
\left\langle\hat{u}_{0}^{2}\right\rangle=2\left\langle u_{0}^{2}(z)\right\rangle
$$

These methods obviously require a large number of misalignments to become statistically accurate. While this is not always the case for shorter beam lines it is 
still useful to perform such calculations. In cases where the statistical significance is really poor, one may use 100 or more sets of random perturbations and apply them to the beam line or ring lattice. This way a better evaluation of the distribution of possible perturbations is possible.

Clearly, the tolerance requirements increase as the average value of betatron functions, the quadrupole focusing, or the size of the accelerator or number of magnets $N$ is increased. No finite orbit can be achieved if the tune is chosen to be an integer value. Most accelerators work at tunes which are about one quarter away from the next integer to maximize the trigonometric denominator $|\sin \pi v| \approx 1$. From a practical standpoint we may wonder what compromise to aim for between a large aperture and tight tolerances. It is good practice to avoid perturbations as reasonable as possible and then, if necessary, enlarge the magnet aperture to accommodate distortions which are too difficult to avoid. As a practical measure it is possible to restrict the uncorrected orbit distortion in most cases to $5-10 \mathrm{~mm}$ and provide magnet apertures that will accommodate this.

What happens if the expected orbit distortions are larger than the vacuum aperture which is virtually sure to happen at least during initial commissioning of more sensitive accelerators? In this case one relies on fluorescent screens or electronic monitoring devices located along the beam line, which are sensitive enough to detect even small beam intensities passing by only once. By empirically employing corrector magnets the beam can be guided from monitor to monitor thus establishing a path and eventually a closed orbit. Once all monitors receive a signal, more sophisticated and computerized orbit control mechanism may be employed.

\subsubsection{Dipole Field Errors in Insertion Devices}

Periodic magnet arrays like wiggler and undulator magnets are used often in synchrotron radiation sources to produce specific radiation characteristics. The requirement for such insertion devices is that the total deflection angle be zero as to not affect the closed orbit in the storage ring

$$
\int_{-\infty}^{+\infty} B_{\perp} \mathrm{d} z=0 .
$$

In reality that is not possible because of manufacturing tolerances. A real trajectory through an undulator may look like shown in Fig. 15.4 [3].

From Fig. 15.4 it is obvious that a particle entering the undulator on axis will exit the magnet with a large distance from the axis and with a significant angle. Both will contribute to the orbit distortions. The problem here is that this orbit distortion is gap dependent and as the experimental user changes the gap the orbit changes all around the storage ring moving at the same time the source position for all other users. It is therefore imperative to correct this distortion before it can affect the orbit. 
Fig. 15.4 Trajectory through an undulator without any special corrections [3]

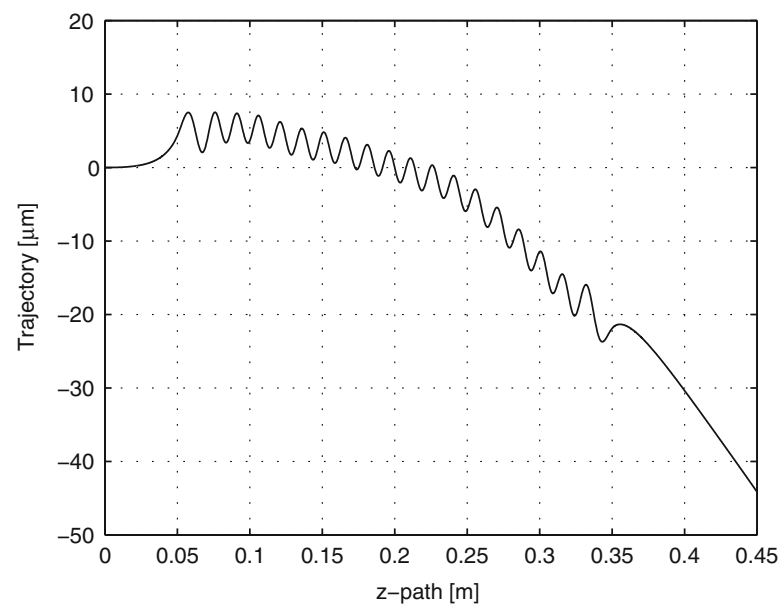

There are two quantities which must be corrected, both being called the first and second integral

$$
\begin{aligned}
& I_{1}=\int B \mathrm{~d} z=0 \text { and } \\
& I_{2}=\int \mathrm{d} z^{\prime} \int B \mathrm{~d} z=0 .
\end{aligned}
$$

Both integrals should be zero because $I_{1}$ is proportional to the exit angle and $I_{2}$ proportional to the position at the undulator exit. Both errors should and can be corrected by a steering magnet before the entrance and right after the undulator exit. By adjusting the entrance steering magnet the exit angle can be varied and the exit beam displacement can be made to be zero. After adjusting the exit beam position to zero the angle still may be wrong which can be adjusted to zero with the exit steering magnet. With this correction the undulator effect on the orbit is eliminated. Unfortunately, the first and second integral can be in a permanent magnet device gap-dependent. Therefore, before using the undulator the steering corrections must be determined experimentally as a function of gap size. This information is stored in the control computer and as the gap size is changed by the user the computer will also change the steering field such that the orbit stays constant during change of the gap. This procedure is know as feed-forward. With this correction the undulkator has become a true insertion device from an accelerator physics point of view.

We notice, however, in Fig. 15.4 that the oscillating trajectory within the undulator is not along a straight line. In the particular case of Fig. 15.4 the trajectory resembles an arc which can reduce the radiation characteristics especially for higher harmonics. This can be corrected by two long coils one each around the full array of poles. This coil can deflect the beam on a dipole trajectory such that it compensates the average curvature within the undulator. Of course if this is done then the 
correction of the first and second integral must be repeated again. The long coil current is also gap dependent and for successful feed-forward three tables must be prepared for the computer control of undulator gap changes.

\subsubsection{Closed Orbit Correction}

Due to magnetic field and alignment errors a distorted equilibrium orbit is generated as discussed in the previous section. Specifically for distinct localized dipole field errors at position $z_{k}$

$$
u_{0}(z)=\frac{\sqrt{\beta(z)}}{2 \sin \pi \nu} \sum_{k} \sqrt{\beta_{k}} \theta_{k} \cos \left[\nu \varphi(z)-v \varphi_{k}+v \pi\right] .
$$

Since orbit distortions reduce the available aperture for betatron oscillations and can change other beam parameters it is customary in accelerator design to include a special set of magnets for the correction of distorted orbits. These orbit correction magnets produce orbit kicks and have, therefore, the same effect on the orbit as dipole errors. However, now the location and the strength of the kicks are known. Before we try to correct an orbit it must have been detected with the help of beam position monitors. The position of the beam in these monitors is the only direct information we have about the distorted orbit. From the set of measured orbit distortions $u_{i}$ at the $m$ monitors $i$ we form a vector

$$
\boldsymbol{u}_{m}=\left(u_{1}, u_{2}, u_{3}, \ldots, u_{m}\right)
$$

and use the correctors to produce additional "orbit distortions" at the monitors through carefully selected kicks $\theta_{k}$ in orbit correction magnets which are also called trim magnets. For $n$ corrector magnets the change in the orbit at the monitor $i$ is

$$
\Delta u_{i}=\frac{\sqrt{\beta_{i}}}{2 \sin \pi v} \sum_{k=1}^{n} \sqrt{\beta_{k}} \theta_{k} \cos \left[v\left(\varphi_{i}-\varphi_{k}+\pi\right)\right],
$$

where the index $k$ refers to the corrector at $z=z_{k}$. The orbit changes at the beam position monitors due to the corrector kicks can be expressed in a matrix equation

$$
\Delta \mathbf{u}_{m}=\mathcal{M} \boldsymbol{\theta}_{n}
$$

where $\boldsymbol{\Delta} \mathbf{u}_{m}$ is the vector formed from the orbit changes at all $m$ monitors, $\boldsymbol{\theta}_{n}$ the vector formed by all kicks in the $n$ correction magnets, and $\mathcal{M}$ the response matrix $\mathcal{M}=\left(\mathcal{M}_{i k}\right)$ with

$$
\mathcal{M}_{i k}=\frac{\sqrt{\beta_{i} \beta_{k}}}{2 \sin \pi v} \cos \left[v\left(\varphi_{i}-\varphi_{k}+\pi\right)\right] .
$$


The distorted orbit can be corrected at least at the position monitors with corrector kicks $\theta_{k}$ chosen such that $\Delta \mathbf{u}_{m}=-\mathbf{u}_{m}$ or

$$
\boldsymbol{\theta}_{n}=-\mathcal{M}^{-1} \mathbf{u}_{m}
$$

Obviously, this equation can be solved exactly if $n=m$ and also for $n>m$ if not all correctors are used. Additional conditions could be imposed in the latter case like minimizing the corrector strength.

While an orbit correction according to (15.48) is possible it is not always the optimum way to do it. A perfectly corrected orbit at the monitors still leaves finite distortions between the monitors. To avoid large orbit distortions between monitors sufficiently many monitors and correctors must be distributed along the beam line. A more sophisticated orbit correction scheme would only try to minimize the sum of the squares of the orbit distortions at the monitors

$$
\left(\mathbf{u}_{m}-\Delta \mathbf{u}_{m}\right)_{\min }^{2}=\left(\mathbf{u}_{m}-\mathcal{M} \boldsymbol{\theta}_{n}\right)_{\min }^{2}
$$

thus avoiding extreme corrector settings due to an unnecessary requirement for perfect correction at monitor locations.

This can be achieved for any number of monitors $m$ and correctors $n$ although the quality of the resulting orbit depends greatly on the actual number of correctors and monitors. To estimate the number of correctors and monitors needed we remember the similarity of dispersion function and orbit distortion. Both are derived from similar differential equations. The solution for the distorted orbit, therefore, can also be expressed by Fourier harmonics similar to (10.99). With $F_{n}$ being the Fourier harmonics of $-\beta^{3 / 2}(z) \Delta \kappa(z)$, the distorted orbit is

$$
u_{0}(z)=\sqrt{\beta(z)} \sum_{n=-\infty}^{+\infty} \frac{v^{2} F_{n} \mathrm{e}^{i n \varphi}}{v^{2}-n^{2}}
$$

which exhibits a resonance for $v=n$. The harmonic spectrum of the uncorrected orbit $u_{0}(z)$ has therefore also a strong harmonic content for $n \approx v$. To obtain an efficient orbit correction both the beam position monitor and corrector distribution around the accelerator must have a strong harmonic close to the tune $v$ of the accelerator. It is, therefore, most efficient to distribute monitors and correctors uniformly with respect to the betatron phase $\psi(z)$ rather than uniform with $z$ and use at least about four units of each per betatron wave length.

With sufficiently many correctors and monitors the orbit can be corrected in different ways. One could excite all correctors in such a way as to compensate individual harmonics in the distorted orbit as derived from beam position measurement. Another simple and efficient way is to look for the first corrector that most efficiently reduces the orbit errors then for the second most efficient and so on. This latter method is most efficient since the practicality of other methods can be greatly influenced by errors of the position measurements as well as field errors in the 
Fig. 15.5 Orbit of Fig. 15.2 before and after correction

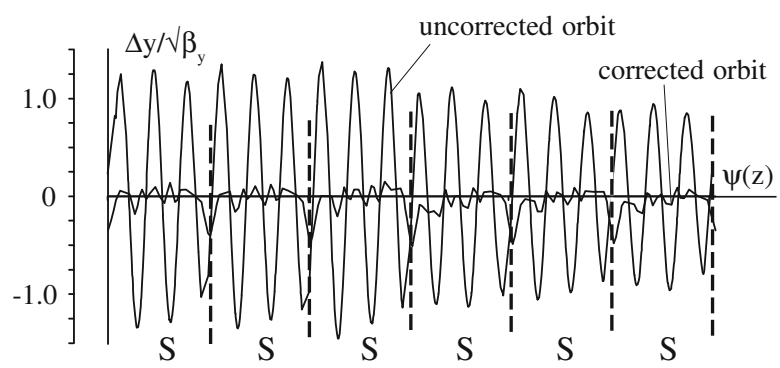

correctors. The single most effective corrector method can be employed repeatedly to obtain an acceptable orbit. Of similar practical effectiveness is the method of beam bumps. Here, a set of three to four correctors are chosen and powered in such a way as to produce a beam bump compensating an orbit distortion in that area. This method is a local orbit correction scheme while the others are global schemes.

As a practical example, we show the vertical orbit in the storage ring PEP before and after correction (Fig. 15.5) in normalized units. The orbit distortions are significantly reduced and the strong harmonic close to the betatron frequency has all but disappeared. Even in normalized units the orbit distortions have now a statistical appearance and further correction would require many more correctors. The peaks at the six interaction points of the lattice, indicated by dashed lines, are actually small orbit distortions and appear large only due to the normalization to a very small value of the betatron function $\beta_{y}$ at the interaction point.

\subsubsection{Response Matrix}

In the last section we found the relation of the beam position response at each position monitor (BPM) due to a change in any of the steering magnets in the circular accelerator. The matrix made up of these relations (15.47) is called the response matrix. The elements of this response matrix gives us an inside look at perturbations, calibration errors and alignment and field tolerances. Each element of the response matrix is defined by the movement of the beam at a particular beam position monitor due to a known change in a particular steering magnet. That response is made up of all fields encountered by the beam from the steerer to the BPM, all ideal bending, quadrupole and sextupole fields, but also all undesired fields originating from manufacturing tolerances, alignment errors, field errors, stray fields etc. Also included are calibration and alignment errors (offset) of BPMs and steering magnets. Therefore these response matrix elements contain all the information of perturbations which we would like to know. Unfortunately such errors are not spelled out in clear text but must be assumed. To find such errors one uses a computer program like LOCO $[4,5]$ which allows the user to choose a specific source of suspected errors. The program then uses such errors and tries 
a fit to measured response matrix data. Usually most if not all errors are found to be corrected in a series of different approximations. Of course, the measurement of some 5,000 to 10,000 response matrix elements takes time. It is a very repetitive measurement which is best left to a computer. Such programs are part of the Accelerator Tool box (AT) [6] which includes many more routines to analyse and optimize electron storage rings.

\subsubsection{Orbit Correction with Single Value Decomposition (SVD)}

Space age developments have had their impact on accelerator physics too. Transmission of pictures from space craft and communication in general push for methods to get the information with a minimum of data transfers. Mathematicians found a way to invert a big matrix and determine the most significant eigenvalues. In accelerator physics, we know from the last section that a series of approximations based on the most significant corrector leads to a greatly improved closed orbit. Now, with the new approach, which is called Singe Value Decomposition (SVD), we get the desired result in one application. The method of SVD provides us with the matrix inversion (15.48) such that all steering corrections are listed in order of magnitude.

\section{Single Value Decomposition (SVD)}

Assume a $n \times m$ matrix $\mathcal{A}$ like the one in (15.46). This matrix can be decomposed into three matrices such that

$$
\mathcal{A}=\mathcal{U} \mathcal{W} \mathcal{V}^{\mathrm{T}}
$$

Here, the columns of $\mathcal{U}$ are the eigenvectors of $\mathcal{A} \mathcal{A}^{\mathrm{T}}$ and $\mathcal{V}$ is made up of rows which are the eigenvectors of $\mathcal{A}^{\mathrm{T}} \mathcal{A}$. Finally, $\mathcal{W}$ is a diagonal matrix with elements being the "singular values" equal to the square root of the eigenvalues of both $\mathcal{A} \mathcal{A}^{\mathrm{T}}$ and $\mathcal{A}^{\mathrm{T}} \mathcal{A}$. The inverse $\mathcal{A}^{-1}$ is

$$
\mathcal{A}^{-1}=\mathcal{V} \mathcal{W}^{-1} \mathcal{U}^{\mathrm{T}}
$$

and the desired corrector strength are from (15.48)

$$
\boldsymbol{\theta}_{n}=-\mathcal{A}^{-1} \mathbf{u}_{m}
$$

where $\theta_{1}$ is the strongest and $\boldsymbol{\theta}_{n}$ the weakest corrector. 

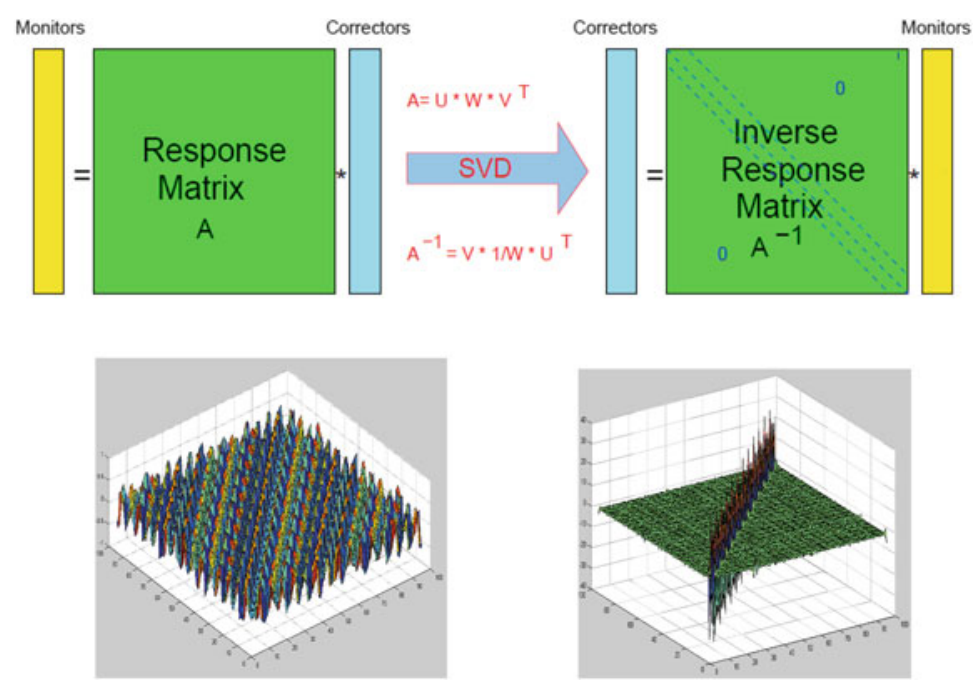

Fig. 15.6 3-D graph of the horizontal response matrix elements before correction (left) and after applying SVD (right). The values are shown versus the number of steering magnets and the number of BPMs each close to 100

More visually, we show the process in some graphs from the synchrotron light source PLS-II in Pohang, Korea. ${ }^{2}$ The response matrix before correction is shown in Fig. 15.6 in a 3-D graph (lower left) where the two axis are the number of steering magnets and number of BPMs.

There are somewhat regular oscillation in the values of the matrix elements visible, which are due to the repetition of the betatron oscillation in each superperiods. Applying SVD the inverse response matrix is of diagonal form and shown in Fig. 15.6 (lower right). The strength of the steering magnets to correct the closed orbit is finally shown in Fig. 15.7 where it is quite obvious that only a few correctors are very effective. Of the 96 corrector magnets installed in PLS-II only about 30 to 50 are effective. All others do not contribute to orbit correction but rather fight each other and therefore should not be used.

While it is not known which corrector magnets and BPMs are the most effective a sufficient number of both, about 6 per betatron oscillation, should be installed. Eventually, correction of the orbit defines the most effective correctors and BPMs, about 4 each per betatron oscillation. In the vertical plane the process is exactly the same with some variation of numbers due to the different tune.

\footnotetext{
${ }^{2}$ I thank Dr. Sheungwan Shin, PLS-II, Pohang, Korea for providing the data and graphs.
} 


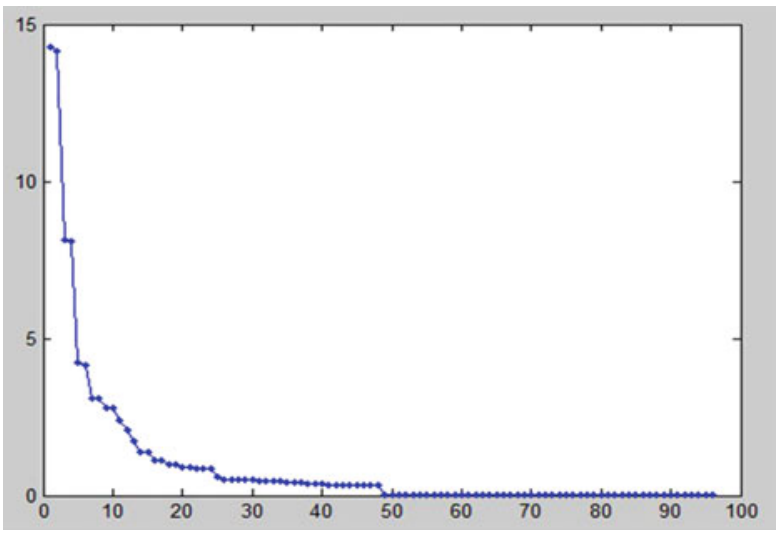

Fig. 15.7 Strength of the corrector magnets ordered from most to least effective

\subsection{Quadrupole Field Perturbations}

The dipole perturbation terms cause a shift in the beam path or closed orbit without affecting the focusing properties of the beam line. The next higher perturbation terms which depend linearly on the transverse particle offset from the ideal orbit will affect focusing because these perturbations act just like quadrupoles. Linear perturbation terms are of the form

$$
u^{\prime \prime}+\left(k_{u}+\kappa_{u}^{2}\right) u=p_{2}(z) u,
$$

where $u$ stand for $x$ and $y$, respectively. More quantitatively, these linear perturbations are from (15.6) and (15.7)

$$
\begin{aligned}
& p_{2, x}(z)=-\Delta\left(k_{x}+\kappa_{x}^{2}\right) x-m \Delta x x+\ldots \\
& p_{2, y}(z)=+\Delta k_{y} y+m \Delta x y+\ldots
\end{aligned}
$$

As a general feature, we recognize the "feed down" from misalignments of higher order multipoles. A misaligned sextupole, for example, generates dipole as well as gradient fields. Any misaligned multipole produces fields in all lower orders.

Quadrupole fields determine the betatron function as well as the phase advance or tune in a circular accelerator. We expect therefore that linear field errors will modify these parameters and we will derive the effect of gradient errors on lattice functions and tune. 


\subsubsection{Betatron Tune Shift}

Gradient field errors have a first order effect on the betatron phase and tune. Specifically in circular accelerators we have to be concerned about the tune not to deviate too much from stable values to avoid beam loss. The effect of a linear perturbation on the tune can be easily derived in matrix formulation for one single perturbation. For simplicity we choose a symmetry point in the lattice of a circular accelerator and insert on either side of this point a thin half-lens perturbation with the transformation matrix

$$
\mathcal{M}_{\mathrm{p}}=\left(\begin{array}{cr}
1 & 0 \\
-1 / f & 1
\end{array}\right)
$$

where $f^{-1}=-\frac{1}{2} \int p_{2}(z) \mathrm{d} z$ and $p_{2}(z)$ is the total perturbation. Combining this with the transformation of an ideal ring (8.74) with $\beta=\beta_{0}, \alpha=\alpha_{0}=0$ and $\psi_{0}=2 \pi v_{0}$

$$
\mathcal{M}_{0}=\left(\begin{array}{cc}
C(z) & S(z) \\
C^{\prime}(z) & S^{\prime}(z)
\end{array}\right)=\left(\begin{array}{cc}
\cos \psi_{0} & \beta_{0} \sin \psi_{0} \\
-\frac{1}{\beta_{0}} \sin \psi_{0} & \cos \psi_{0}
\end{array}\right)
$$

we get for the trace of the total transformation matrix $\mathcal{M}=\mathcal{M}_{\mathrm{p}} \mathcal{M}_{0} \mathcal{M}_{\mathrm{p}}$

$$
\operatorname{Tr} \mathcal{M}=2 \cos \psi_{0}-2 \frac{\beta_{0}}{f} \sin \psi_{0},
$$

where $\beta_{0}$ is the unperturbed betatron function at the location of the perturbation and $\psi_{0}=2 \pi v_{0}$ the unperturbed phase advance per turn. The trace of the perturbed ring is $\operatorname{Tr} \mathcal{M}=2 \cos \psi$ and we have therefore

$$
\cos \psi=\cos \psi_{0}-\frac{\beta_{0}}{f} \sin \psi_{0} .
$$

With $\psi=2 \pi \nu=2 \pi \nu_{0}+2 \pi \delta \nu$ and $\cos \psi=\cos \psi_{0} \cos 2 \pi \delta \nu-\sin \psi_{0} \sin 2 \pi \delta \nu$ we get for small perturbations the tune shift

$$
\delta v=\frac{1}{2 \pi} \frac{\beta_{0}}{f}=-\frac{\beta_{0}}{4 \pi} \int p_{2}(z) \mathrm{d} z .
$$

For more than a single gradient error one would simply add the individual contribution from each error to find the total tune shift. The same result can be obtained from the perturbed equation of motion (15.54). To show this, we introduce normalized coordinates $w=u / \sqrt{\beta}$ and $\varphi=\int \frac{\mathrm{d} z}{\nu \beta}$ and (15.54) becomes

$$
\ddot{w}+v_{0}^{2} w=v_{0}^{2} \beta^{2}(z) p_{2}(z) w .
$$


For simplicity, we drop the index ${ }_{u}$ and recognize that all equations must be evaluated separately for $x$ and $y$. Since both the betatron function $\beta(z)$ and perturbations $p_{2}(z)$ are periodic, we may Fourier expand the coefficient of $v_{0} w$ on the r.h.s. and get for the lowest, non-oscillating harmonic

$$
F_{0}=\frac{1}{2 \pi} \int_{0}^{2 \pi} v_{0} \beta^{2} p_{2}(z) \mathrm{d} \varphi=\frac{1}{2 \pi} \oint \beta(z) p_{2}(z) \mathrm{d} z
$$

Inserting this into (15.60) and collecting terms linear in $w$ we get

$$
\ddot{w}+\left(v_{0}^{2}-v_{0} F_{0}\right) w=0
$$

and the new tune $v=v_{0}+\delta v$ is determined by

$$
v^{2}=v_{0}^{2}-v_{0} F_{0} \approx v_{0}^{2}+2 v_{0} \delta v
$$

Solving for $\delta v$ gives the linear tune perturbation

$$
\delta v=-\frac{1}{2} F_{0}=-\frac{1}{4 \pi} \oint \beta(z) p_{2}(z) \mathrm{d} z
$$

in complete agreement with the result obtained in (15.59). The tune shift produced by a linear perturbation has great diagnostic importance. By varying the strength of an individual quadrupole and measuring the tune shift it is possible to derive the value of the betatron function in this quadrupole.

The effect of linear perturbations contributes in first approximation only to a static tune shift. In higher approximation, however, we note specific effects which can affect beam stability and therefore must be addressed in more detail. To derive these effects we solve (15.60) with the help of a Green's function as discussed in Sect. 5.5.4 and obtain the perturbation

$$
P(\varphi)=\int_{0}^{\varphi} v_{0} \beta^{2}(\chi) p_{2}(\chi) w(\chi) \sin \left[v_{0}(\varphi-\chi)\right] \mathrm{d} \chi
$$

where we have made use of the principal solutions. We select a particular, unperturbed trajectory, $w(\chi)=w_{0} \cos (v \chi)$ with $\dot{w}_{0}=0$ and get the perturbed particle trajectory

$$
w(\nu \varphi)=w_{0} \cos \left(v_{0} \varphi\right)+w_{0} v_{0} \int_{0}^{\varphi} \beta^{2} p_{2} \cos \left(v_{0} \chi\right) \sin \left[v_{0}(\varphi-\chi)\right] \mathrm{d} \chi,
$$

where $\beta=\beta(\chi)$ and $p_{2}=p_{2}(\chi)$. If, on the other hand, we consider the perturbations to be a part of the lattice, we would describe the same trajectory by

$$
w(\varphi)=w_{0} \cos \nu \varphi
$$


Both solutions must be equal. Specifically the phase advance per turn must be the same and we get from (15.66), (15.67) after one turn $\varphi=2 \pi$ for the perturbed tune $v=v_{0}+\delta v$

$\cos 2 \pi\left(v_{0}+\delta \nu\right)=\cos 2 \pi v_{0}+v_{0} \int_{0}^{2 \pi} \beta^{2}(\varphi) p_{2}(\varphi) \cos \left(v_{0} \varphi\right) \sin \left[v_{0}(2 \pi-\varphi)\right] \mathrm{d} \varphi$,

which can be solved for the tune shift $\mathrm{d} \nu$. Obviously the approximation breaks down for large values of the perturbation as soon as the r.h.s. becomes larger than unity. For small perturbations, however, we expand the trigonometric functions and get

$$
\begin{aligned}
\delta v & =-\frac{1}{4 \pi} \oint \beta(z) p_{2}(z) \mathrm{d} z \\
& -\frac{1}{4 \pi \sin 2 \pi v_{0}} \oint \beta(z) p_{2}(z) \sin \left\{2 v_{0}[\pi-\varphi(z)]\right\} \mathrm{d} z .
\end{aligned}
$$

The first term is the average tune shift which has been derived before, while the second term is of oscillatory nature averaging to zero over many turns if the tune of the circular accelerator is not equal to a half integer or multiples thereof. We have found hereby a second resonance condition to be avoided which occurs for half integer values of the tunes

$$
v_{0} \neq \frac{1}{2} n
$$

This resonance is called a half integer resonance and causes divergent solutions for the lattice functions.

\subsubsection{Optics Perturbation Due to Insertion Devices}

The use of insertion devices in synchrotron light sources can introduce significant focusing perturbations. Undulators and wiggler magnets are a series of dipole magnets with end effects causing vertical focusing. This focusing will perturb the periodic betatron function in the storage ring and with it all correction that have been made. Because the perturbation scales like the square of the magnet field it is for most undulators too small to be significant. However, wiggler magnet may cause some problems. Like for orbit correction we do not like to spread the correction of the betatron functions all around the ring. To localize the correction to the vicinity of the insertion device we consider only the closest lattice quadrupoles on either side of the wiggler magnet which are not yet beyond the next insertion device. To minimize the number of quadrupoles needed for correction we start away from the wiggler where we expect the betatron functions to stay unperturbed, say in the middle of the next long straight section. Starting from there we adjust quadrupoles such that in the 
middle of the wiggler magnet $\alpha_{x, y}=0$ and $\eta^{\prime}=0$. That requires three quadrupoles for matching. If we match from the middle of the wiggler magnet to the middle of the next straight section we would need six quadrupoles to match $\beta_{x, y}, \alpha_{x, y}, \eta$ and $\eta^{\prime}$. For optimum localization of the perturbation one should use the three quadrupole closest to the wiggler magnet. This correction does not take care of the perturbations in the betatron phase. If two more quadrupoles are available one could try to use them for tune correction. This, however, is not always possible and one might therefore use two quadrupole families for the whole ring to readjust the tunes to the original value. Because there usually are many superperiods, the effect of a small tune correction is distributed around the ring and causes little variation in the beatron functions. The corrections in the quadrupoles are again for a permanent magnet wiggler gapdependent and must be determined before general use to establish feed-forward of the computer control of the wiggler.

\subsubsection{Resonances and Stop Band Width}

Calculating the tune shift from (15.68) we noticed that there is no solution if the perturbation is too large such that the absolute value of the r.h.s. becomes larger than unity. In this case the tune becomes imaginary leading to ever increasing betatron oscillation amplitudes and beam loss. This resonance condition occurs not only at a half integer resonance but also in a finite vicinity. The region of instability is called the stop band and the width of unstable tune values is called the stop band width which can be calculated by using a higher approximation for the perturbed solution. Following the arguments of Courant and Snyder [1] we note that the perturbation (15.65) depends on the betatron oscillation $w(\varphi)$ itself and we now use in the perturbation integral the first order approximation (15.66) rather than the unperturbed solution to calculate the perturbation (15.65). Then instead of (15.68) we get with

$$
v_{0} \beta^{2}(\varphi) p_{2}(\varphi)=g(\varphi)
$$

$$
\begin{aligned}
\cos 2 \pi\left(v_{0}+\delta \nu\right)-\cos 2 \pi v_{0}= & +\int_{0}^{2 \pi} g(\varphi) \cos \left(v_{0} \varphi\right) \sin v_{0}(2 \pi-\varphi) \mathrm{d} \varphi \\
& +v_{0} \int_{0}^{2 \pi} g(\chi) \sin v_{0}(2 \pi-\chi) \\
& \times \int_{0}^{\chi} g(\zeta) \cos \left(v_{0} \zeta\right) \sin v_{0}(\chi-\zeta) \mathrm{d} \zeta \mathrm{d} \chi
\end{aligned}
$$

This expression can be used to calculate the stop band width due to gradient field errors which we will do for the integer resonance $v_{0}=n+\delta v$ and for the half 
integer resonance $v_{0}=n+1 / 2+\delta v$ where $n$ is an integer and $\delta v$ the deviation of the tune from these resonances. To evaluate the first integral $I_{1}$, on the r.h.s. of (15.72) we make use of the relation

$$
\cos \left(v_{0} \varphi\right) \sin \left[v_{0}(2 \pi-\varphi)\right]=\frac{1}{2} \sin \left(2 \pi v_{0}\right)+\frac{1}{2} \sin \left[2 v_{0}(\pi-\varphi)\right]
$$

and get with $\mathrm{d} z=v_{0} \beta \mathrm{d} \varphi$ and

$$
\oint \beta(z) p_{2}(z) \mathrm{d} z=\int_{0}^{2 \pi} g(\varphi) \mathrm{d} \varphi=2 \pi F_{0}
$$

from (15.62) for the first integral

$$
I_{1}=\pi F_{0} \sin \left(2 \pi \nu_{0}\right)+\frac{1}{2} \int_{0}^{2 \pi} g(\varphi) \sin \left[2 \nu_{0}(\pi-\varphi)\right] \mathrm{d} \varphi .
$$

The second term of the integral $I_{1}$ has oscillatory character averaging to zero over many turns and with $\delta \ll 1$

$$
I_{1}=\pi F_{0} \sin 2 \pi v_{0} \approx\left\{\begin{array}{ll}
2 \pi^{2} F_{0} \delta \nu & \text { for } v_{0}=n+\delta \nu \\
-2 \pi^{2} F_{0} \delta \nu & \text { for } v_{0}=n+\frac{1}{2}+\delta \nu
\end{array} .\right.
$$

The second integral $I_{2}$ in (15.72) can best be evaluated while expressing the trigonometric functions in their exponential form. Terms like $\mathrm{e}^{ \pm \mathrm{i} v(2 \pi-2 \chi)}$ or $\mathrm{e}^{ \pm \mathrm{i} v(2 \pi-2 \varsigma)}$ vanish on average over many turns. With

$$
\int_{0}^{2 \pi} f(\chi) \mathrm{d} \chi \int_{0}^{\chi} f(\zeta) \mathrm{d} \zeta=\frac{1}{2} \int_{0}^{2 \pi} f(\chi) \mathrm{d} \chi \int_{0}^{2 \pi} f(\zeta) \mathrm{d} \zeta
$$

we get for the second integral

$$
\begin{aligned}
I_{2}=-\frac{\nu_{0}}{16} \int_{0}^{2 \pi} & g(\chi) \int_{0}^{2 \pi} g(\zeta) \\
& \times\left\{\left(\mathrm{e}^{\mathrm{i} 2 \pi \nu_{0}}+\mathrm{e}^{-\mathrm{i} 2 \pi \nu_{0}}\right)-\left[\mathrm{e}^{\mathrm{i} 2 \nu_{0}(\pi-\chi+\zeta)}+\mathrm{e}^{-\mathrm{i} 2 \nu_{0}(\pi-\chi+\zeta)}\right]\right\} \mathrm{d} \zeta \mathrm{d} \chi
\end{aligned}
$$

Close to the integer resonance $v_{0}=n+\delta v$ and

$$
\begin{aligned}
I_{2, n}=-\frac{\nu_{0}}{16} \int_{0}^{2 \pi} & g(\chi) \int_{0}^{2 \pi} g(\zeta) \\
& \times\left\{\left(\mathrm{e}^{\mathrm{i} 2 \pi \delta v}+\mathrm{e}^{-\mathrm{i} 2 \pi \delta v}\right)-\left[\mathrm{e}^{\mathrm{i} 2 n(\zeta-\chi)}+\mathrm{e}^{-i 2 n(\zeta-\chi)}\right]\right\} \mathrm{d} \zeta \mathrm{d} \chi
\end{aligned}
$$


while in the vicinity of the half integer resonance $v_{0}=n+\frac{1}{2}+\delta v$

$$
\begin{aligned}
I_{2, n+\frac{1}{2}}=-\frac{\nu_{0}}{16} \int_{0}^{2 \pi} g(\chi) \int_{0}^{2 \pi} g(\zeta)\{ & -\left(\mathrm{e}^{\mathrm{i} 2 \pi \delta \nu}+\mathrm{e}^{-\mathrm{i} 2 \pi \delta \nu}\right) \\
+ & {\left.\left[\mathrm{e}^{\mathrm{i} 2\left(n+\frac{1}{2}\right)(\zeta-\chi)}+\mathrm{e}^{-\mathrm{i} 2\left(n+\frac{1}{2}\right)(\zeta-\chi)}\right]\right\} \mathrm{d} \zeta \mathrm{d} \chi . }
\end{aligned}
$$

The integralscan now be expressed in terms of Fourier harmonics of $v_{0} \beta^{2}(\varphi) p_{1}(\varphi)$, where the amplitudes of the harmonics $F_{q}$ with integer $q>0$ are given by

$$
\left|F_{q}\right|^{2}=F_{q} F_{q}^{*}=\frac{\nu_{0}}{\pi^{2}} \int_{0}^{2 \pi} g(\chi) \mathrm{e}^{-\mathrm{i} q \chi} \mathrm{d} \chi \int_{0}^{2 \pi} g(\zeta) \mathrm{e}^{\mathrm{i} q \zeta} \mathrm{d} \zeta
$$

For $F_{0}$ we have from the Fourier transform the result

$$
F_{0}=\langle g(\varphi)\rangle=v_{0}\left\langle\beta^{2}(\varphi) p_{2}(\varphi)\right\rangle
$$

and we get for (15.78) with this and ignoring terms quadratic in $\delta v$

$$
I_{2, n} \approx \frac{1}{8} \pi^{2}\left(F_{2 n}^{2}-4 F_{0}^{2} \cos 2 \pi \delta \nu\right)
$$

and for (9.57)

$$
I_{2, n+\frac{1}{2}} \approx-\frac{1}{8} \pi^{2}\left(F_{2 n+1}^{2}-4 F_{0}^{2} \cos 2 \pi \delta \nu\right)
$$

At this point we may collect the results and get on the 1.h.s. of (15.72) for $v_{0}=n+\delta v$

$$
\cos 2 \pi\left(v_{0}+\delta \nu\right)-\cos 2 \pi v_{0}=\cos 2 \pi\left(v_{0}+\delta \nu\right)-1+2 \pi^{2} \delta \nu^{2} .
$$

This must be equated with the r.h.s. which is the sum of integrals $I_{1}$ and $I_{2}$ and with $F_{0}^{2} \cos 2 \pi \delta v=\mathcal{O}\left(\delta^{4} v\right)$

$$
\cos 2 \pi\left(v_{0}+\delta v\right)-1=-2 \pi^{2} \delta v^{2}+2 \pi^{2} F_{0} \delta v+\frac{1}{8} \pi^{2}\left(F_{2 n}^{2}-4 F_{0}^{2}\right) .
$$

The boundaries of the stop band on either side of the integer resonance $v_{0} \approx$ $n$ can be calculated from the condition that $\cos 2 \pi\left(\nu_{0}+\delta \nu\right) \leq 1$ which has two solutions $\delta v_{1,2}$. From (15.83) we get therefore

$$
\delta v^{2}-F_{0} \delta v=\frac{1}{16}\left(\left|F_{2 n}\right|^{2}-4 F_{0}^{2}\right)
$$

and solving for $\delta v$

$$
\delta v_{1,2}=\frac{1}{2} F_{0} \pm \frac{1}{4}\left|F_{2 n}\right|
$$


the stop band width is finally

$$
\Delta v=\delta v_{1}-\delta v_{2}=\frac{1}{2}\left|F_{2 n}\right|=\frac{1}{2 \pi} \oint \beta(z) p_{2}(z) \mathrm{e}^{-\mathrm{i} 2 n \zeta(z)} \mathrm{d} z .
$$

The stop band width close to the integer tune $v \approx n$ is determined by the second harmonic of the Fourier spectrum for the perturbation. The vicinity of the resonance for which no stable betatron oscillations exist increases with the value of the gradient field error and with the value of the betatron function at the location of the field error. For the half integer resonance $v_{0} \approx n+\frac{1}{2}$, the stop band width has a similar form

$$
\Delta v_{\frac{1}{2}}=\frac{1}{2}\left|F_{2 n+1}\right|=\frac{1}{2 \pi} \int_{0}^{2 \pi} \beta(z) p_{2}(z) \mathrm{e}^{-\mathrm{i}(2 n+1) \zeta(z)} \mathrm{d} z .
$$

The lowest order Fourier harmonic $n=0$ determines the static tune shift while the resonance width depends on higher harmonics. The existence of finite stop bands is not restricted to linear perturbation terms only. Nonlinear, higher order perturbation terms lead to higher order resonances and associated stop bands. In such cases one would replace in (15.60) the linear perturbation $\beta^{\frac{1}{2}} p_{2}(z) w$ by the $n$th order nonlinear perturbation $\beta^{n / 2} p_{n}(z) w^{n-1}$ and basically go through the same derivation. Later in this chapter, we will use a different way to describe resonance characteristics caused by higher order perturbations. At this point we note only that perturbations of order $n$ are weighted by the $n / 2$ power of the betatron function at the location of the perturbation and increased care must be exercised, where large values of the betatron functions cannot be avoided. Undesired fields at such locations must be minimized.

\subsubsection{Perturbation of Betatron Function}

The existence of linear perturbation terms causes not only the tunes but also betatron functions to vary around the ring or along a beam line. This variation, also called beta-beat can be derived by observing the perturbation of a particular trajectory like for example the sine-like solution given by

$$
S_{0}\left(z_{0} \mid z\right)=\sqrt{\beta(z)} \sqrt{\beta_{0}} \sin v_{0}\left[\varphi(z)-\varphi_{0}\right] .
$$

The sine-like function or trajectory in the presence of linear perturbation terms is by the principle of linear superposition the combination of the unperturbed solution (8.74) and perturbation (5.75)

$$
\begin{aligned}
S\left(z_{0} \mid z\right)=\sqrt{\beta(z)} \sqrt{\beta_{0}} & \sin v_{0} \varphi(z) \\
& +\sqrt{\beta(z)} \int_{z_{0}}^{z} p_{2}(\zeta) \sqrt{\beta(\zeta)} S_{0}\left(z_{0} \mid \zeta\right) \sin v_{0}[\varphi(z)-\varphi(\zeta)] \mathrm{d} \zeta .
\end{aligned}
$$


Following the sinusoidal trajectory for the whole ring circumference or length of a superperiod $L_{\mathrm{p}}$, we have with $z=z_{0}+L_{\mathrm{p}}, \beta\left(z_{0}+L_{\mathrm{p}}\right)=\beta\left(z_{0}\right)=\beta_{0}$ and $\varphi\left(z_{0}+L_{\mathrm{p}}\right)=2 \pi+\varphi_{0}$

$$
\begin{aligned}
S\left(z_{0} \mid z_{0}+L_{\mathrm{p}}\right) & =\beta_{0} \sin 2 \pi v_{0}+\beta_{0} \oint_{z_{0}}^{z_{0}+L_{\mathrm{p}}} \beta(\zeta) p_{1}(\zeta) \\
& \times \sin v_{0}\left[\varphi(\zeta)-\varphi_{0}\right] \sin \left[v_{0}\left(2 \pi+\varphi_{0}-\varphi(\zeta)\right)\right] d \zeta
\end{aligned}
$$

The difference due to the perturbation from the unperturbed trajectory (15.87) at $z=z_{0}+L_{\mathrm{p}}$ is

$$
\begin{aligned}
\Delta S & =S\left(z_{0} \mid z_{0}+L_{\mathrm{p}}\right)-S_{0}\left(z_{0} \mid z_{0}+L_{\mathrm{p}}\right) \\
& =\beta_{0} \int_{z_{0}}^{z_{0}+L_{\mathrm{p}}} \beta(\zeta) p_{2}(\zeta) \sin \left[v_{0}\left(\varphi_{\zeta}-\varphi_{0}\right)\right] \sin \left[v_{0}\left(2 \pi+\varphi_{0}-\varphi_{\zeta}\right)\right] \mathrm{d} \zeta
\end{aligned}
$$

where we abbreviated $\varphi\left(z_{0}\right)=\varphi_{0}$ etc.

The variation of the sine like function can be derived also from the variation of the $M_{12}$ element of the transformation matrix for the whole ring

$$
\Delta S=\Delta(\beta \sin 2 \pi \nu)=\Delta \beta \sin 2 \pi v_{0}+\beta_{0} 2 \pi \Delta \nu \cos 2 \pi v_{0}
$$

We use (15.64) for the tune shift $\delta v=-\frac{1}{2} F_{0}$, equate (15.90) with (15.89) and solve for $\Delta \beta / \beta$. After some manipulations, where we replace temporarily the trigonometric functions by their exponential expressions, the variation of the betatron function becomes at $\varphi(z)$

$$
\frac{\Delta \beta(z)}{\beta(z)}=\frac{1}{2 \sin 2 \pi v_{0}} \oint \beta(\zeta) p_{2}(\zeta) \cos \left[2 v_{0}(\varphi(z)-\varphi(\zeta)+\pi)\right] \mathrm{d} \zeta
$$

The perturbation of the betatron function shows clearly resonance character and a half integer tune must be avoided. We observe a close similarity with the solution (10.91) of the dispersion function or the closed orbit (15.28). Setting $\mathrm{d} \zeta=v_{0} \beta(\zeta) \mathrm{d} \varphi$, we find by comparison that the solution for the perturbed betatron function can be derived from a differential equation similar to a modified equation (10.88)

$$
\frac{\mathrm{d}^{2}}{\mathrm{~d} \varphi^{2}}\left(\frac{\Delta \beta}{\beta}\right)+\left(2 v_{0}\right)^{2} \frac{\Delta \beta}{\beta}=\left(2 v_{0}\right)^{2} \frac{1}{2} \beta^{2}(z) p_{2}(z) .
$$

Expanding the periodic function $v_{0} \beta^{2} p_{2}=\sum_{q} F_{q} \mathrm{e}^{\mathrm{i} q \varphi}$ we try the periodic ansatz

$$
\frac{\Delta \beta}{\beta}=\sum_{q} B_{q} F_{q} \mathrm{e}^{\mathrm{i} q \varphi}
$$


and get from (15.92)

$$
\sum_{q}\left[-q^{2}+\left(2 v_{0}\right)^{2}\right] B_{q} F_{q} \mathrm{e}^{\mathrm{i} q \varphi}=2 v_{0} \sum_{q} F_{q} \mathrm{e}^{\mathrm{i} q \varphi}
$$

This can be true for all values of the phase $\varphi$ only if the coefficients of the exponential functions vanish separately for each value of $q$ or if

$$
B_{q}=\frac{2 v_{0}}{\left(2 v_{0}\right)^{2}-q^{2}} .
$$

Inserting into the periodic ansatz (15.93) the perturbation of the betatron function in another form is

$$
\frac{\Delta \beta}{\beta}=\frac{v_{0}}{2} \sum_{q} \frac{F_{q} \mathrm{e}^{\mathrm{i} q \varphi}}{v_{0}^{2}-(q / 2)^{2}} .
$$

Again we recognize the half inter resonance leading to an infinitely large perturbation of the betatron function. In the vicinity of the half integer resonance $v_{0} \approx n+\frac{1}{2}=q / 2$ the betatron function can be expressed by the resonant term only

$$
\frac{\Delta \beta}{\beta} \approx \frac{1}{2}\left|F_{2 n+1}\right| \frac{\cos (2 n+1) \varphi}{v_{0}-\left(n+\frac{1}{2}\right)}
$$

and with $\left|F_{2 n+1}\right|=2 \Delta v_{\frac{1}{2}}$ from (15.86) we get again the perturbation of the betatron function (15.91). The beat factor for the variation of the betatron function is define by

$$
B F=1+\left(\frac{\Delta \beta}{\beta_{0}}\right)_{\max }=1+\frac{\Delta \nu_{2 n+1}}{2 v_{0}-(2 n+1)},
$$

where $\Delta v_{2 n+1}$ is the half integer stop band width. The beating of the betatron function is proportional to the stop band width and therefore depends greatly on the value of the betatron function at the location of the perturbation. Even if the tune is chosen safely away from the next resonance, a linear perturbation at a large betatron function may still cause an unacceptable beat factor. It is generally prudent to design lattices in such a way as to avoid large values of the betatron functions. As a practical note, any value of the betatron function which is significantly larger than the quadrupole distances should be considered large. For many beam transport problems this is easier said than done. Therefore, where large betatron functions cannot be avoided or must be included to meet our design goals, results of perturbation theory warn us to apply special care for beam line component design, alignment and to minimize undesirable stray fields. 


\subsection{Chromatic Effects in a Circular Accelerator}

Energy independent perturbations as discussed in previous sections can have a profound impact on the stability of particle beams in the form of perturbations of the betatron function or through resonances. Any beam transport line must be designed and optimized with these effects in mind since it is impossible to fabricate ideal magnets and align them perfectly. Although such field and alignment errors can have a destructive effect on a beam, it is the detailed understanding of these effects that allow us to minimize or even avoid such problems by a careful design within proven technology.

To complete the study of perturbations, we note that a realistic particle beam is never quite mono-energetic and includes a finite distribution of particle energies. Bending as well as focusing is altered if the particle momentum is not the ideal momentum. We already derived the momentum dependent reference path in transport lines involving bending magnets. Beyond this basic momentum dependent effect we observe other chromatic aberrations which contribute in a significant way to the perturbations of lattice functions. The effect of chromatic aberrations due to a momentum error is the same as that of a corresponding magnet field error and for beam stability we must include chromatic aberrations.

\subsubsection{Chromaticity}

Perturbations of beam dynamics can occur in beam transport systems even in the absence of magnet field and alignment errors. Deviations of particle energies from the ideal design energy cause perturbations in the solutions of the equations of motion. We have already derived the variation of the equilibrium orbit for different energies. Energy related or chromatic effects can be derived also for other lattice functions. Focusing errors due to an energy error cause such particles to be imaged at different focal points causing a blur of the beam spot. In a beam transport system, where the final beam spot size is of great importance as, for example, at the collision point of linear colliders, such a blur causes a severe degradation of the attainable luminosity. In circular accelerators we have no such direct imaging task but note that the tune of the accelerator is determined by the overall focusing and tune errors occur when the focusing system is in error.

In this chapter we will specifically discuss effects of energy errors on tunes of a circular accelerator and means to compensate for such chromatic aberrations. The basic means of correction are applicable to either circular or open beam transport systems if, for the latter case, we only replace the tune by the phase advance of the transport line in units of $2 \pi$. The control of these chromatic effects in circular accelerators is important for two reasons, to avoid loss of particles due to tune shifts into resonances and to prevent beam loss due to an instability, which we call the head tail instability to be discussed in more detail in Sect. 22.5. 
Fig. 15.8 Chromatic focusing errors

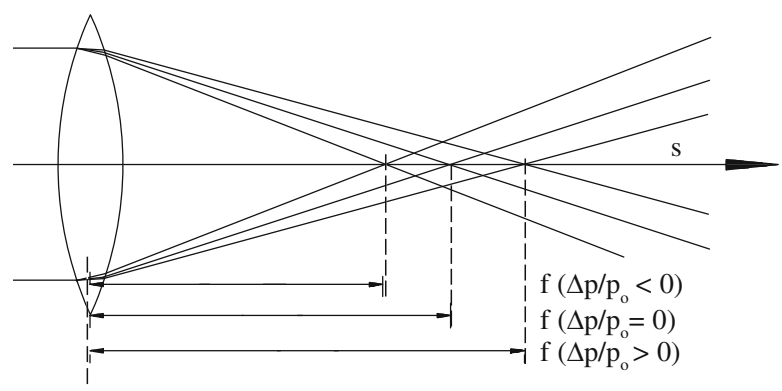

Fig. 15.9 Chromaticity correction with sextupoles

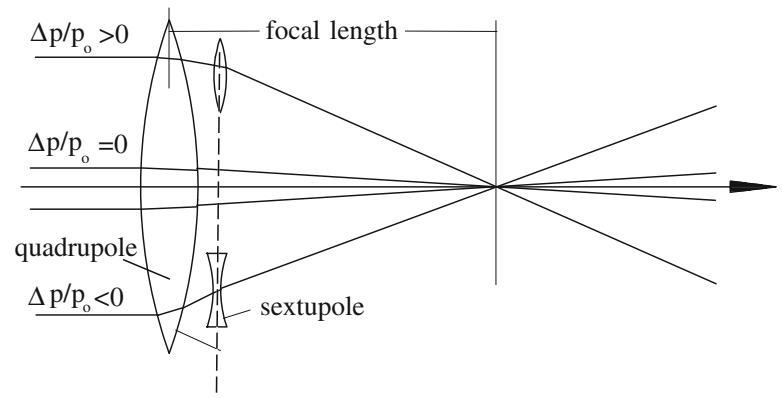

The lowest order chromatic perturbation is caused by the variation of the focal length of the quadrupoles with energy (Fig. 15.8). This kind of error is well known from light optics, where a correction of this chromatic aberration can at least partially be obtained by the use of different kinds of glasses for the lenses in a composite focusing system.

In particle beam optics no equivalent approach is possible. To still correct for the chromatic perturbations we remember that particles with different energies can be separated by the introduction of a dispersion function. Once the particles are separated by energy we apply different focusing corrections depending on the energy of the particles. Higher energy particles are focused less than ideal energy particles and lower energy particles are over focused. For a correction of these focusing errors we need a magnet which is focusing for higher energy particles and defocusing for lower energy particles (Fig. 15.9). A sextupole has just that property.

The variation of tunes with energy is called the chromaticity and is defined by

$$
\xi=\frac{\Delta v}{\Delta p / p_{0}} .
$$

The chromaticity derives from second and higher order perturbations in $(x, y, \delta)$ and the relevant equations of motion are from

$$
\begin{aligned}
& x^{\prime \prime}+k x=k x \delta-\frac{1}{2} m\left(x^{2}-y^{2}\right), \\
& y^{\prime \prime}-k x=-k y \delta+m x y .
\end{aligned}
$$


Setting $x=x_{\beta}+\eta_{x} \delta$ and $y=y_{\beta}$, assuming that $\eta_{y} \equiv 0$, we retain only betatron oscillation terms involving $x_{\beta}$ or $y_{\beta}$ to derive chromatic tune shifts. In doing so we note three types of chromatic perturbation terms, those depending on the betatron motion only, those depending on the momentum error only, and terms depending on both. With these expansions (15.98) becomes

$$
\begin{aligned}
x_{\beta}^{\prime \prime}+k x_{\beta} & =k x_{\beta} \delta-m \eta_{x} x_{\beta} \delta-\frac{1}{2} m\left(x_{\beta}^{2}-y_{\beta}^{2}\right)+\mathcal{O}(3), \\
y_{\beta}^{\prime \prime}-k y_{\beta} & =-k y_{\beta} \delta+m \eta_{x} y_{\beta} \delta+m x_{\beta} y_{\beta}+\mathcal{O}(3) .
\end{aligned}
$$

We ignore for the time being non chromatic terms of second order which will be discussed later as geometric aberrations and get

$$
\begin{aligned}
x_{\beta}^{\prime \prime}+k x_{\beta} & =\left(k-m \eta_{x}\right) x_{\beta} \delta, \\
y_{\beta}^{\prime \prime}-k y_{\beta} & =-\left(k-m \eta_{x}\right) y_{\beta} \delta .
\end{aligned}
$$

The perturbation terms now are linear in the betatron amplitude and therefore have the character of a gradient error. From Sect. 15.3 we know that these types of errors lead to a tune shift which by comparison with (15.64) becomes in terms of a phase shift

$$
\begin{aligned}
& \Delta \psi_{x}=-\frac{1}{2} \delta \oint \beta_{x}\left(k-m \eta_{x}\right) \mathrm{d} z, \\
& \Delta \psi_{y}=+\frac{1}{2} \delta \oint \beta_{y}\left(k-m \eta_{x}\right) \mathrm{d} z
\end{aligned}
$$

Equations (15.101) are applicable for both circular and open beam lines. Using the definition of the chromaticity for circular accelerators we have finally

$$
\begin{aligned}
& \xi_{x}=-\frac{1}{4 \pi} \oint \beta_{x}\left(k-m \eta_{x}\right) \mathrm{d} z, \\
& \xi_{y}=+\frac{1}{4 \pi} \oint \beta_{y}\left(k-m \eta_{x}\right) \mathrm{d} z .
\end{aligned}
$$

Similar to the definition of tunes the chromaticities are also an integral property of the circular accelerator lattice. Setting the sextupole strength $m$ to zero one gets the natural chromaticities determined by focusing terms only

$$
\begin{aligned}
& \xi_{x 0}=-\frac{1}{4 \pi} \oint \beta_{x} k \mathrm{~d} z, \\
& \xi_{y 0}=+\frac{1}{4 \pi} \oint \beta_{y} k \mathrm{~d} z .
\end{aligned}
$$

The natural chromaticities are always negative which is to be expected since focusing is less effective for higher energy particles $(\delta>0)$ and therefore the number of betatron oscillations is reduced.

For a thin lens symmetric FODO lattice the calculation of the chromaticity becomes very simple. With the betatron function $\beta^{+}$at the center of a focusing quadrupole of strength $k^{+}=k$ and $\beta^{-}$at the defocusing quadrupole of strength 
$k^{-}=k$, the chromaticity of one FODO half cell is

$$
\xi_{x 0}=-\frac{1}{4 \pi}\left(\beta^{+} \int k^{+} \mathrm{d} z+\beta^{-} \int k^{-} \mathrm{d} z\right)=-\frac{\beta^{+}-\beta^{-}}{4 \pi} \int k \mathrm{~d} z .
$$

With $\beta^{+}(10.3)$ and $\beta^{-}(10.5)$ and $\int k \mathrm{~d} z=1 / f=1 /(\kappa L)$, where $\kappa$ is the FODO strength parameter and $L$ the length of a FODO half cell, we get the chromaticity per FODO half-cell in a more practical formulation

$$
\xi_{x 0}=-\frac{1}{2 \pi} \frac{1}{\sqrt{\kappa^{2}-1}}=-\frac{1}{\pi} \tan \left(\frac{1}{2} \psi_{x}\right),
$$

where $\psi_{x}$ is the horizontal betatron phase for the full FODO cell. The same result can be obtained for the vertical plane.

The natural chromaticity for each $90^{\circ}$ FODO cell is therefore equal to $1 / \pi$. Although this value is rather small, the total chromaticity for the complete lattice of a storage ring or synchrotron, made up of many FODO cells, can become quite large. For the stability of a particle beam and the integrity of the imaging process by quadrupole focusing it is important that the natural chromaticity be corrected.

It is interesting at this point to discuss for a moment the chromatic effect if, for example, all bending magnets have a systematic field error with respect to other magnets. In an open beam transport line the beam would follow an off momentum path as determined by the difference of the beam energy and the bending magnet "energy". Any chromatic aberration from quadrupoles as well as sextupoles would occur just as discussed.

In a circular accelerator the effect of systematic field errors might be different. We consider, for example, the case where we systematically change the strength of all bending magnets. In an electron storage ring, the particle beam would automatically stay at the ideal design orbit with the particle energy being defined by the strength of the bending magnets. The strength of the quadrupoles and sextupole magnets, however, would now be systematically too high or too low with respect to the bending magnet field and particle energy. Quadrupoles introduce therefore a chromatic tune shift proportional to the natural chromaticity while the sextupoles are ineffective because the beam orbit leads through magnet centers. Changing the strength of the bending magnets by a fraction $\Delta$ in an electron circular accelerator and measuring the tune change $\Delta v$ one can determine experimentally the natural chromaticity $\left(\xi_{0}=-\Delta v / \Delta\right)$ of the ring. In Fig. 15.10 the measurement of the tunes as a function of the bending magnet current is shown for the storage ring SPEAR. From the slope of the graphs we derive the natural chromaticities of the SPEAR storage ring as $\xi_{x}=-11.4$ and $\xi_{y}=-11.7$.

In a proton accelerator the beam energy must be changed through acceleration or deceleration together with a change of the bending magnet strength to keep the beam on the reference orbit before this measurement can be performed. 
Fig. 15.10 Experimental determination of the natural chromaticity in a storage ring by measuring the tunes as a function of the excitation current $I=I_{0}+\Delta I$ in the bending magnets

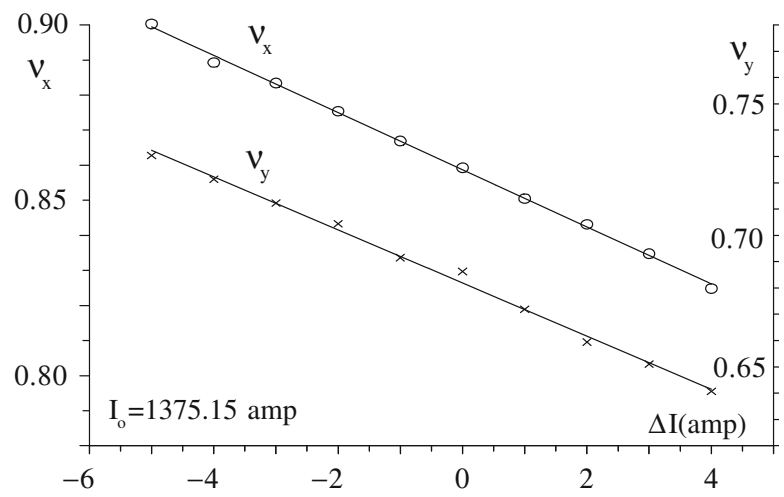

\subsubsection{Chromaticity Correction}

Equations (15.102) clearly suggest the usefulness of sextupole magnets for chromatic correction. Sextupoles must be placed along the orbit of a circular accelerator or along a beam transport line at locations, where the dispersion function does not vanish, $\eta_{x} \neq 0$. A single sextupole is sufficient, in principle, to correct the chromaticity for the whole ring or transport line but its strength may exceed technical limits or cause problems of geometric aberrations to the beam stability. This is due to the nonlinear nature of sextupole fields which causes dynamic instability for large amplitudes for which the sextupole field is no more a perturbation. The largest betatron oscillation amplitude which is still stable in the presence of nonlinear fields is called the dynamic aperture. To maximize the dynamic aperture it is prudent to distribute many chromaticity correcting sextupoles along the beam line or circular accelerator.

To correct both the horizontal and the vertical chromaticity two different groups of sextupoles are required. For a moment we assume that there be only two sextupoles. To calculate the required strength of these sextupoles for chromaticity correction we use thin lens approximation and replacing integrals in (15.102) by a sum the corrected chromaticities are

$$
\begin{aligned}
& \xi_{x}=\xi_{x 0}+\frac{1}{4 \pi}\left(m_{1} \eta_{x 1} \beta_{x 1}+m_{2} \eta_{x 2} \beta_{x 2}\right) \ell_{\mathrm{s}}=0, \\
& \xi_{y}=\xi_{y 0}+\frac{1}{4 \pi}\left(m_{1} \eta_{x 1} \beta_{y 1}+m_{2} \eta_{x 2} \beta_{y 2}\right) \ell_{\mathrm{s}}=0 .
\end{aligned}
$$

Here we assume that two different sextupoles, each of length $\ell_{s}$, are available at locations $z_{1}$ and $z_{2}$. Solving for the sextupole strengths we get from (15.106)

$$
\begin{aligned}
& m_{1} \ell_{\mathrm{s}}=-\frac{4 \pi}{\eta_{x 1}} \frac{\xi_{x 0} \beta_{y 2}-\xi_{y 0} \beta_{x 2}}{\beta_{x 1} \beta_{y 2}-\beta_{x 2} \beta_{y 1}}, \\
& m_{2} \ell_{\mathrm{s}}=-\frac{4 \pi}{\eta_{x 2}} \frac{\xi_{x 0} \beta_{y 1}-\xi_{y 0} \beta_{x 1}}{\beta_{x 1} \beta_{y 2}-\beta_{x 2} \beta_{y 1}} .
\end{aligned}
$$


It is obvious that the dispersion function at sextupoles should be large to minimize sextupoles strength. It is also clear that the betatron functions must be different preferably with $\beta_{x} \gg \beta_{y}$ at the $m_{1}$ sextupole and $\beta_{x} \ll \beta_{y}$ at the $m_{2}$ sextupole to avoid "fighting" between sextupoles leading to excessive strength requirements.

In general this approach based on only two sextupoles in a ring to correct chromaticities leads to very strong sextupoles causing both magnetic design problems and strong higher order aberrations. A more gentle correction uses two groups or families of sextupoles with individual magnets distributed more evenly around the circular accelerator and the total required sextupole strength is spread over all sextupoles. In cases of severe aberrations, as discussed later, we will need to subdivide all sextupoles into more than two families for a more sophisticated correction of chromaticities. Instead of (15.106) we write for the general case of chromaticity correction

$$
\begin{aligned}
& \xi_{x}=\xi_{x 0}+\frac{1}{4 \pi} \sum_{i} m_{i} \eta_{x i} \beta_{x i} \ell_{\mathrm{s} i} \\
& \xi_{y}=\xi_{y 0}+\frac{1}{4 \pi} \sum_{i} m_{i} \eta_{x i} \beta_{y i} \ell_{\mathrm{s} i},
\end{aligned}
$$

where the sum is taken over all sextupoles. In the case of a two family correction scheme we still can solve for $m_{1}$ and $m_{2}$ by grouping the terms into two sums.

The chromaticity of a circular accelerator as defined in this section obviously does not take care of all chromatic perturbations. Since the function $\left(k-m \eta_{x}\right)$ in (15.100) is periodic, we can Fourier analyze it and note that the chromaticity only describes the effect of the non-oscillating lowest order Fourier component (15.103). All higher order components are treated as chromatic aberrations. In Sect. 17.2 we will discuss in more detail such higher order chromatic and geometric aberrations.

\subsubsection{Chromaticity in Higher Approximation}

So far we have used only quadrupole and sextupole fields to define and calculate the chromaticity. From the general equations of motion we know, however, that many more perturbation terms act just like sextupoles and therefore cannot be omitted without further discussion. To derive the relevant equations of motion from (6.95), (6.96) we set $x=x_{\beta}+\eta_{x} \delta$ and $y=y_{\beta}+\eta_{y} \delta$ where we keep for generality the symmetry between vertical and horizontal plane. Neglecting, however, coupling terms we get with perturbations quadratic in $(x, y, \delta)$ but at most linear in $\delta$ and after separating the dispersion function a differential equation of the form $\left(u=x_{\beta}\right.$ or $\left.y_{\beta}\right)$

$$
u_{\beta}^{\prime \prime}+K u_{\beta}=-\Delta K u_{\beta} \delta-\Delta L u_{\beta}^{\prime} \delta
$$


where

$$
\begin{aligned}
K_{x}= & \kappa_{x}^{2}+k, \\
K_{y}= & \kappa_{y}^{2}-k, \\
-\Delta K_{x}= & 2 \kappa_{x}^{2}+k-\left(m+2 \kappa_{x}^{3}+4 \kappa_{x} k\right) \eta_{x} \\
& \quad-\left(\underline{m}+2 \kappa_{x} \underline{k}+2 \kappa_{y} k\right) \eta_{y}+\kappa_{x}^{\prime} \eta_{x}^{\prime}-\kappa_{y}^{\prime} \eta_{y}^{\prime}, \\
-\Delta K_{y}= & 2 \kappa_{y}^{2}-k+\left(m-2 \kappa_{y} \underline{k}+2 \kappa_{x} k\right) \eta_{x} \\
& \quad+\left(\underline{m}-2 \kappa_{y}^{3}+4 \kappa_{y} k\right) \eta_{y}-\kappa_{x}^{\prime} \eta_{x}^{\prime}+\kappa_{y}^{\prime} \eta_{y}^{\prime}, \\
-\Delta L_{x}= & -\Delta L_{y}=+\kappa_{x}^{\prime} \eta_{x}+\kappa_{y}^{\prime} \eta_{y}+\kappa_{x} \eta_{x}^{\prime}+\kappa_{y} \eta_{y}^{\prime} \\
= & +\frac{\mathrm{d}}{\mathrm{d} z}\left(\kappa_{x} \eta_{x}+\kappa_{y} \eta_{y}\right) .
\end{aligned}
$$

The perturbation terms (15.109) depend on the betatron oscillation amplitude as well as on the slope of the betatron motion. If by some manipulation we succeed in transforming (15.109) into an equation with terms proportional only to $u$ we obtain immediately the chromaticity. We try a transformation of the form $u=v f(z)$ where $f(z)$ is a still to be determined function of $z$. With $u^{\prime}=v^{\prime} f+v f^{\prime}$ and $u^{\prime \prime}=v^{\prime \prime} f+$ $2 v^{\prime} f^{\prime}+v f^{\prime \prime}(15.109)$ becomes

$$
v^{\prime \prime} f+2 v^{\prime} f^{\prime}+v f^{\prime \prime}+K v f+\Delta K v f \delta+\Delta L v^{\prime} f \delta+\Delta L v f^{\prime} \delta=0 .
$$

Now we introduce a condition defining the function $f$ such that in (15.115) the coefficients of $v^{\prime}$ vanish. This occurs if

$$
2 f^{\prime}=-\Delta L f \delta .
$$

To first order in $\delta$ this equation can be solved by

$$
f=1+\frac{1}{2} \delta\left(\kappa_{x} \eta_{x}+\kappa_{y} \eta_{y}\right)
$$

and (15.115) becomes

$$
v^{\prime \prime}+\left[K+\left(f^{\prime \prime}+\delta \Delta K\right)\right] v=0 .
$$

The chromaticity in this case is $\xi=\frac{1}{4 \pi} \oint\left(\frac{f^{\prime \prime}}{\delta}+\Delta K\right) \beta \mathrm{d} z$, which becomes with $\frac{2 f^{\prime \prime}}{\delta}=\frac{\mathrm{d}^{2}}{\mathrm{~d} z^{2}}\left(\kappa_{x} \eta_{x}+\kappa_{y} \eta_{y}\right)$ and $(15.112)$

$$
\begin{aligned}
\xi_{x} & =\frac{1}{4 \pi} \oint\left(\frac{f^{\prime \prime}}{\delta}+\Delta K_{x}\right) \beta_{x} \mathrm{~d} z \\
& =\frac{1}{4 \pi} \oint \frac{1}{2} \frac{\mathrm{d}^{2}}{\mathrm{~d} z^{2}}\left(\kappa_{x} \eta_{x}+\kappa_{y} \eta_{y}\right) \beta_{x} \mathrm{~d} z
\end{aligned}
$$




$$
\begin{aligned}
& -\frac{1}{4 \pi} \oint \beta_{x}\left[\left(2 \kappa_{x}^{2}+k\right)+\kappa_{x}^{\prime} \eta_{x}^{\prime}-\kappa_{y}^{\prime} \eta_{y}^{\prime}\right. \\
& \left.-\left(m+2 \kappa_{x}^{3}+4 \kappa_{x} k\right) \eta_{x}-\left(\underline{m}+2 \kappa_{x} \underline{k}+2 \kappa_{y} k\right) \eta_{y}\right] \mathrm{d} z .
\end{aligned}
$$

The first integral can be integrated twice by parts to give $\oint \frac{1}{2}\left(\kappa_{x} \eta_{x}+\kappa_{y} \eta_{y}\right) \beta^{\prime \prime} \mathrm{d} z$. Using $\frac{1}{2} \beta^{\prime \prime}=\gamma_{x}-K \beta$, and (15.119) the horizontal chromaticity is finally

$$
\begin{aligned}
\xi_{x}=\frac{1}{4 \pi} \oint[-( & \left.+2 \kappa_{x}^{2}\right)-\kappa_{x}^{\prime} \eta_{x}^{\prime}-\kappa_{y}^{\prime} \eta_{y}^{\prime} \\
& \left.+\left(m+\kappa_{x}^{3}+3 \kappa_{x} k\right) \eta_{x}+\left(\underline{m}+2 \kappa_{x} \underline{k}+\kappa_{y} k\right) \eta_{y}\right] \beta_{x} \mathrm{~d} z \\
& +\frac{1}{4 \pi} \oint\left(\kappa_{x} \eta_{x}+\kappa_{y} \eta_{y}\right) \gamma_{x} \mathrm{~d} z .
\end{aligned}
$$

A similar expression can be derived for the vertical chromaticity

$$
\begin{aligned}
\xi_{y}=\frac{1}{4 \pi} \oint\left[\left(-2 \kappa_{y}^{2}+k\right)+\kappa_{x}^{\prime} \eta_{x}^{\prime}-\kappa_{y}^{\prime} \eta_{y}^{\prime}\right. & \\
& \left.-\left(m+2 \kappa_{x} \underline{k}+\kappa_{y} k\right) \eta_{x}-\left(\underline{m}-\kappa_{y}^{3}+3 \kappa_{y} k\right) \eta_{y}\right] \beta_{y} \mathrm{~d} z \\
& +\frac{1}{4 \pi} \oint\left(\kappa_{x} \eta_{x}+\kappa_{y} \eta_{y}\right) \gamma_{y} \mathrm{~d} z .
\end{aligned}
$$

In deriving the chromaticity we used the usual curvilinear coordinate system for which the sector magnet is the natural bending magnet. For rectangular or wedge magnets the chromaticity must be determined from (15.121) by taking the edge focusing into account. Generally, this is done by applying a delta function focusing at the edges of dipole magnets with a focal length of

$$
\frac{1}{f_{x}}=\frac{1}{\rho} \tan \epsilon \int \delta\left(z_{\text {edge }}\right) \mathrm{d} z .
$$

Similarly, we proceed with all other terms which include focusing.

The chromaticity can be determined experimentally simply by measuring the tunes for a beam circulating with a momentum slightly different from the lattice reference momentum. In an electron ring, this is generally not possible since any momentum deviation of the beam is automatically corrected by radiation damping within a short time. To sustain an electron beam at a momentum different from the reference energy, we must change the frequency of the accelerating cavity. Due to the mechanics of phase focusing, a particle beam follows such an orbit that the particle revolution time in the ring is an integer multiple of the rf-oscillation period in the accelerating cavity. By proper adjustment of the rf-frequency the beam orbit is centered along the ideal orbit and the beam momentum is equal to the ideal momentum as determined by the actual magnetic fields. 
If the rf-frequency is raised, for example, the oscillation period becomes shorter and the revolution time for the beam must become shorter too. This is accomplished only if the beam momentum is changed in such a way that the particles now follow a new orbit that is shorter than the ideal reference orbit. Such orbits exist for particles with momenta less than the reference momentum. The relation between revolution time and momentum deviation is a lattice property expressed by the momentum compaction which we write now in the form

$$
\frac{\Delta f_{\mathrm{rf}}}{f_{\mathrm{rf}}}=-\eta_{\mathrm{c}} \frac{\Delta c p}{c p_{0}} .
$$

Through the knowledge of the lattice and momentum compaction we can relate a relative change in the rf-frequency to a change in the beam momentum. Measurement of the tune change due to the momentum change determines immediately the chromaticity.

\subsubsection{Non-linear Chromaticity*}

The chromaticity of a circular accelerator is defined as the linear rate of change of the tunes with the relative energy deviation $\delta$. With the increased amount of focusing that is being applied in modern circular accelerators, especially in storage rings, to obtain specific particle beam properties like very high energies in large rings or a small emittance, the linear chromaticity term is no longer sufficient to describe the chromatic dynamics of particle motion. Quadratic and cubic terms in $\delta$ must be considered to avoid severe stability problems for particles with energy error. Correcting the chromaticity with only two families of sextupoles we could indeed correct the linear chromaticity but the nonlinear chromaticity may be too severe to permit stable beam operation.

We derive the nonlinear chromaticity from the equation of motion expressed in normalized coordinates and including up to third-order chromatic focusing terms

$$
\ddot{w}+v_{00}^{2} w=v_{00}^{2} \beta^{2} p_{2}(\varphi) w=\left(a \delta+b \delta^{2}+c \delta^{3}\right) w,
$$

where the coefficients $a, b, c$ are perturbation functions up to third order in $\delta$ and linear in the amplitude $w$, and where $v_{00}$ is the unperturbed tune. From (6.95) and (6.96) these perturbations are

$$
\begin{aligned}
& a=v_{00}^{2} \beta^{2}\left[\left(k+2 \kappa_{x}^{2}\right)+m \eta_{x}+\ldots\right], \\
& b=v_{00}^{2} \beta^{2}\left[-\left(k+2 \kappa_{x}^{2}\right)-m \eta_{x}+\ldots\right], \\
& c=v_{00}^{2} \beta^{2}\left[\left(k+2 \kappa_{x}^{2}\right)+m \eta_{x}+\ldots\right] .
\end{aligned}
$$


This equation defines nonlinear terms for the chromaticity which have been solved for the quadratic term [7] and for the cubic term [8, 9]. While second and third-order terms become significant in modern circular accelerators, higherorder terms can be recognized by numerical particle tracking but are generally insignificant.

Since the perturbations on the r.h.s. of (15.124) are periodic for a circular accelerator we may Fourier expand the coefficients

$$
\begin{aligned}
& a(\varphi)=a_{0}+\sum_{n \neq 0} a_{n} \mathrm{e}^{\mathrm{i} n \varphi}, \\
& b(\varphi)=b_{0}+\sum_{n \neq 0} b_{n} \mathrm{e}^{\mathrm{i} n \varphi}, \\
& c(\varphi)=c_{0}+\sum_{n \neq 0} c_{n} \mathrm{e}^{\mathrm{i} n \varphi} .
\end{aligned}
$$

From the lowest-order harmonics of the perturbations we get immediately the first approximation of nonlinear chromaticities

$$
v_{0}^{2}=v_{00}^{2}-\delta\left(a_{0}+b_{0} \delta+c_{0} \delta^{2}\right)
$$

or

$$
v_{0}^{2}=v_{00}^{2}\left(1-\beta^{2} \int_{0}^{2 \pi} p_{1}(\varphi) \mathrm{d} \varphi\right)
$$

With this definition we reduce the equation of motion (15.124) to

$$
\ddot{w}+v_{00}^{2} w=v_{0}^{2} \beta^{2} \delta\left(\sum_{n>0} 2 a_{n} \cos n \varphi+\delta \sum_{n>0} 2 b_{n} \cos n \varphi+\delta^{2} \sum_{n>0} 2 c_{n} \cos n \varphi\right) w
$$

The remaining perturbation terms on the r.h.s. look oscillatory and therefore seem not to contribute to an energy dependent tune shift. In higher-order approximation, however, we find indeed resonant terms which do not vanish but contribute to a systematic tune shift. Such higher-order tune shifts cannot be ignored in all cases and therefore an analytical expression for this chromatic tune shift will be derived. To solve the differential equation (15.131), we consider the r.h.s. as a small perturbation with $\delta$ serving as the smallness parameter. Mathematical methods for a solution have been developed and are based on a power series in $\delta$. We apply this method to both the cosine and sine like principal solution and try the ansatz

$$
C(\varphi)=\sum_{n \geq 0} C_{n}(\varphi) \delta^{n} \quad \text { and } \quad S(\varphi)=\sum_{n \geq 0} S_{n}(\varphi) \delta^{n}
$$

Concentrating first on the cosine like solution we insert (15.132) into (15.131) and sort for same powers in $\delta$ noting that each term must vanish separately to make the 
ansatz valid for all values of $\delta$. The result is a set of differential equations for the individual solution terms

$$
\begin{aligned}
\ddot{C}_{0}+v_{0}^{2} C_{0} & =0 \\
\ddot{C}_{1}+v_{0}^{2} C_{1} & =v_{0}^{2} \beta^{2}(\varphi) p_{2}(\varphi) C_{0}, \\
& \ldots \\
& \ldots \\
\ddot{C}_{n}+v_{0}^{2} C_{n} & =v_{0}^{2} \beta^{2}(\varphi) p_{2}(\varphi) C_{n-1},
\end{aligned}
$$

where derivatives $\ddot{C}_{i}$ are taken with respect to the phase $\varphi$, e.g. $\ddot{C}_{i}=\partial^{2} C_{i} / \partial \varphi^{2}$. These are defining equations for the functions $C_{0}, C_{1}, \cdots C_{n}$ with $C_{i}=C_{i}(\varphi)$ and each function depending on a lower-order solution. The lowest-order solutions are the principal solutions of the unperturbed motion

$$
C_{0}(\varphi)=\cos v_{0} \varphi \quad \text { and } \quad S_{0}(\varphi)=\frac{1}{v_{0}} \sin v_{0} \varphi .
$$

The differential equations (15.133) can be solved with the Green's Function method which we have applied earlier to deal with perturbation terms. All successive solutions can now be derived from the unperturbed solutions through

$$
\begin{aligned}
C_{n+1}(\varphi) & =\frac{1}{v_{0}} \int_{0}^{\varphi} \beta(\zeta) p_{2}(\zeta) \sin \left[v_{0}(\zeta-\varphi)\right] C_{n}(\zeta) \mathrm{d} \zeta \\
S_{n+1}(\varphi) & =\frac{1}{v_{0}} \int_{0}^{\varphi} \beta(\zeta) p_{2}(\zeta) \sin \left[v_{0}(\zeta-\varphi)\right] S_{n}(\zeta) \mathrm{d} \zeta
\end{aligned}
$$

With the unperturbed solution $C_{0}$ we get for $C_{1}$

$$
C_{1}(\varphi)=\frac{1}{v_{0}} \int_{0}^{\varphi} \beta(\zeta) p_{2}(\zeta) \sin \left[v_{0}(\zeta-\varphi)\right] \cos \left(v_{0} \zeta\right) \mathrm{d} \zeta
$$

and utilizing this solution $C_{2}$ becomes

$$
\begin{aligned}
C_{2}(\varphi)=\frac{1}{v_{0}^{2}} \int_{0}^{\varphi} \beta(\zeta) & p_{2}(\zeta) \sin \left[v_{0}(\zeta-\varphi)\right] \cos \left(v_{0} \zeta\right) \\
& \times \int_{0}^{\zeta} \beta(\zeta) p_{2}(\xi) \sin \left[v_{0}(\xi-\zeta)\right] \cos \left(v_{0} \xi\right) \mathrm{d} \xi \mathrm{d} \zeta
\end{aligned}
$$

Further solutions are derived following this procedure although the formulas get quickly rather elaborate. With the cosine and sine like solutions we can formulate the transformation matrix for the whole ring

$$
\mathcal{M}=\left(\begin{array}{cc}
C(2 \pi) & S(2 \pi) \\
\dot{C}(2 \pi) & \dot{S}(2 \pi)
\end{array}\right)
$$


and applying Floquet's theorem, the tune of the circular accelerator can be determined from the trace of the transformation matrix

$$
2 \cos 2 \pi \nu=C(2 \pi)+\dot{S}(2 \pi)
$$

where $\dot{S}=\mathrm{d} S / \mathrm{d} \varphi$. With the ansatz (15.132) this becomes

$$
2 \cos 2 \pi \nu=\sum_{n \geq 0} C_{n}(2 \pi) \delta^{n}+\sum_{n \geq 0} \dot{S}_{n}(2 \pi) \delta^{n},
$$

Retaining only up to third-order terms in $\delta$, we finally get after some manipulations with (15.135)

$$
\begin{aligned}
& \cos 2 \pi v=\cos 2 \pi v_{0}-\frac{1}{2 v_{0}} \sin 2 \pi v_{0} \int_{0}^{2 \pi} p_{2}(\zeta) \mathrm{d} \zeta \\
& +\frac{1}{2 v_{0}^{2}} \int_{0}^{2 \pi} \int_{0}^{\zeta} p_{1}(\zeta) p_{2}(\beta) \sin \left[v_{0}(\zeta-\beta-2 \pi)\right] \\
& \times \sin \left[v_{0}(\beta-\alpha)\right] \mathrm{d} \beta \mathrm{d} \alpha \\
& +\frac{1}{2 v_{0}^{3}} \int_{0}^{2 \pi} \int_{0}^{\zeta} \int_{0}^{\xi} p_{2}(\zeta) p_{2}(\xi) p(\gamma) \sin \left[v_{0}(\zeta-\gamma-2 \pi)\right] \\
& \quad \times \sin \left[v_{0}(\xi-\zeta)\right] \sin \left[v_{0}(\gamma-\xi)\right] \mathrm{d} \gamma \mathrm{d} \xi \mathrm{d} \zeta
\end{aligned}
$$

These integrals can be evaluated analytically and (15.141) becomes after some fairly lengthy but straightforward manipulations

$$
\begin{aligned}
\cos 2 \pi v=\cos 2 \pi & v_{0}-\delta^{2}\left(\frac{\pi \sin 2 \pi v_{0}}{2 v_{0}} \sum_{n>0} \frac{a_{n}^{2}}{n^{2}-4 v_{0}^{2}}\right) \\
& -\delta^{3}\left(\frac{\pi \sin 2 \pi v_{0}}{v_{0}} \sum_{n>0} \frac{a_{n}^{2} b_{n}^{2}}{n^{2}-4 v_{0}^{2}}\right) \\
- & \delta^{3}\left\{\left(\frac{\pi \sin 2 \pi v_{0}}{4 v_{0}} \sum_{s>0} \sum_{t>0} \frac{a_{s} b_{t}}{t^{2}-4 v_{0}^{2}}\right)\right. \\
& \left.\times\left[a_{s+t} \frac{1+\frac{4 v_{0}^{2}}{t(s+t)}}{(s+t)^{2}-4 v_{0}^{2}}+a_{|s-t|} \frac{1-\frac{4 v_{0}^{2}}{t(s-t)}}{(s+t)^{2}-4 v_{0}^{2}}\right]\right\}+\mathcal{O}\left(\delta^{4}\right) .
\end{aligned}
$$

This expression defines the chromatic tune shift up to third order. Note that the tune $v_{0}$ is not the unperturbed tune but already includes the lowest-order approximation of the chromaticity (15.129). The relevant perturbations here are linear in the betatron amplitude and drive therefore half-integer resonances as is 
Fig. 15.11 Variation of the vertical tune with energy in the storage ring PEP if the chromaticities are corrected by only two families of sextupoles

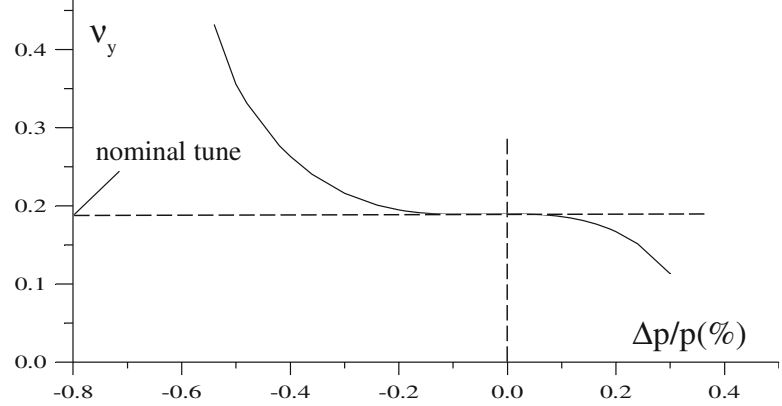

obvious from (15.142). The main contribution to the perturbation observed here are from the quadrupole and sextupole terms

$$
p_{2}(\varphi)=v_{0}^{2} \beta^{2}\left(k-m \eta_{x}\right)\left(\delta-\delta^{2}+\delta^{3} \ldots\right) .
$$

In large storage rings the nonlinear chromaticity becomes quite significant as demonstrated in Fig. 15.11. Here the tune variation with energy in the storage ring PEP is shown both for the case where only two families of sextupoles are used to compensate for the natural chromaticities [8]. Since in this ring an energy acceptance of at least $\pm 1 \%$ is required, we conclude from Fig. 15.11 that insufficient stability is available because of the nonlinear chromaticity terms shifting the tunes for off-momentum particles to an integer resonance within the desired energy acceptance.

For circular accelerators or rings with a large natural chromaticity it is important to include in the calculation of the nonlinear chromaticity higher-order terms of the dispersion function $\eta_{x}$. Following the discussion in Sect.9.4.1 we set in (15.143)

$$
\eta_{x}(\varphi)=\eta_{x 0}+\eta_{1} \delta+\eta_{2} \delta^{2}+\ldots
$$

and find the Fourier components $a_{n}$ and $b_{n}$ in (15.142) defined by

$$
\begin{aligned}
v_{0}^{2} \beta^{2}\left(k-m \eta_{x 0}\right) & =\sum_{n \geq 0} 2 a_{n} \cos n \varphi, \\
-v_{0}^{2} \beta^{2}\left(k-m \eta_{x 0}+m \eta_{1}\right) & =\sum_{n \geq 0} 2 b_{n} \cos n \varphi .
\end{aligned}
$$

Nonlinear energy terms in the $\eta$-function can sometimes become quite significant and must be included to improve the accuracy of analytical expressions for the nonlinear chromaticity. In such cases more sophisticated methods of chromaticity correction are required to control nonlinear chromaticities as well. One procedure is to distribute sextupoles in more than two families while keeping their total strength to retain the desired chromaticity. Using more than two families of sextupoles 
allows us to manipulate the strength of specific harmonics $a_{n}$ such as to minimize the nonlinear chromaticities. Specifically, we note in (15.142) that the quadratic chromaticity term originates mainly from the resonant term $\frac{a_{n}^{2}}{n^{2}-4 v_{0}^{2}}$. This term can be minimized by a proper distribution of sextupoles suppressing the $n^{\text {th }}$-harmonic of the chromaticity function $v^{2} \beta^{2}(k-m \eta)$. Special computer programs like PATRICIA [8] calculate the contribution of each sextupole to the Fourier coefficients $a_{n}$ and provide thereby the information required to select optimum sextupole locations and field strength to minimize quadratic and cubic chromaticities.

\subsection{Kinematic Perturbation Terms*}

The rules of linear beam dynamics allow the design of beam transport systems with virtually any desired beam characteristics. Whether such characteristics actually can be achieved depends greatly on our ability or lack thereof to control the source and magnitude of perturbations. Only the lowest-order perturbation terms were discussed in the realm of linear, paraxial beam dynamics. With the continued sophistication of accelerator design and increased demand on beam quality it becomes more and more important to also consider higher-order magnetic field perturbations as well as kinematic perturbation terms.

The effects of such terms in beam-transport lines, for example, may compromise the integrity of a carefully prepared very low emittance beam for linear colliders or may contribute to nonlinear distortion of the chromaticity in circular accelerators and associated reduced beam stability. Studying nonlinear effects we will not only consider nonlinear fields but also the effects of linear fields errors in higher order, whether it be higher-order perturbation terms or higher-order approximations for the equations of motion. The sources and physical nature of perturbative effects must be understood to determine limits to beam parameters and to design correcting measures.

Perturbations of beam dynamics not only occur when there are magnetic field and alignment errors present. During the derivation of the general equation of motion in Chap. 5 we encountered in addition to general multipole fields a large number of kinematic perturbation terms or higher-order field perturbations which appear even for ideal magnets and alignment. Generally, such terms become significant for small circular accelerators or wherever beams are deflected in high fields generating bending radii of order unity or less. If, in addition, the beam sizes are large the importance of such perturbations is further aggravated. In many cases well-known aberration phenomena from light optics can be recognized.

Of the general equations of motion, we consider terms up to third order for ideal linear upright magnets and get the equation of motion in the horizontal and 
deflecting plane

$$
\begin{aligned}
x^{\prime \prime}+\left(\kappa_{x}^{2}+k\right) x= & -\kappa_{x}^{3} x^{2}+2 \kappa_{x} k x^{2}+\left(\frac{1}{2} \kappa_{x} k+\frac{1}{2} \kappa_{x}^{\prime \prime}\right) y^{2} \\
& +\frac{1}{2} \kappa_{x}\left(x^{\prime 2}-y^{\prime 2}\right)+\kappa_{x}^{\prime}\left(x x^{\prime}+y y^{\prime}\right) \\
& +\frac{1}{12}\left(-10 \kappa_{x}^{2} k+k^{\prime \prime}+\kappa_{x} \kappa_{x}^{\prime \prime}+\kappa_{x}^{\prime 2}\right) x^{3}-\left(2 \kappa_{x}^{2}+\frac{3}{2} k\right) x x^{\prime 2} \\
& +\frac{1}{4}\left(+6 \kappa_{x}^{2} k+k^{\prime \prime}+5 \kappa_{x} \kappa_{x}^{\prime \prime}+\kappa_{x}^{\prime 2}\right) x y^{2} \\
& -\kappa_{x} \kappa_{x}^{\prime} x^{2} x^{\prime}+k^{\prime} x y y^{\prime}-\frac{1}{2} k x y^{\prime 2}+\frac{1}{2} \kappa_{x}^{2} x y^{\prime 2} \\
& +\kappa_{x} \delta-\kappa_{x} \delta^{2}+\kappa_{x} \delta^{3}+\left(2 \kappa_{x}^{2}+k\right) x \delta-\kappa_{x}^{\prime} y y^{\prime} \delta \\
& +\frac{1}{2} \kappa_{x}\left(x^{\prime 2}+y^{\prime 2}\right) \delta+\left(-\frac{1}{2} \kappa_{x} k-\frac{1}{2} \kappa_{x}^{\prime \prime}\right) y^{2} \delta \\
& +\left(2 \kappa_{x} k+\kappa_{x}^{3}\right) x^{2} \delta-\left(k+2 \kappa_{x}^{2}\right) x \delta^{2}+\mathcal{O}(4) .
\end{aligned}
$$

In the nondeflecting or vertical plane the equation of motion is

$$
\begin{aligned}
y^{\prime \prime}-k y= & +2 \kappa_{x} k x y-\kappa_{x}^{\prime}\left(x^{\prime} y-x y^{\prime}\right)+\kappa_{x} x^{\prime} y^{\prime} \\
& -\frac{1}{12}\left(+2 \kappa_{x}^{2} k+k^{\prime \prime}+\kappa_{x} \kappa_{x}^{\prime \prime}+\kappa_{x}^{\prime 2}\right) y^{3} \\
& -\frac{1}{4}\left(k^{\prime \prime}+\kappa_{x} \kappa_{x}^{\prime \prime}-2 \kappa_{x}^{2} k+\kappa_{x}^{\prime 2}\right) x^{2} y \\
& +\frac{1}{2} k y y^{\prime 2}-\kappa_{x} \kappa_{x}^{\prime} x^{2} y^{\prime} \\
& -k^{\prime} x x^{\prime} y+\frac{1}{2} k x^{\prime 2} y-\left(2 \kappa_{x}^{2}+k\right) x x^{\prime} y^{\prime} \\
& -k y \delta+\kappa_{x}^{\prime} x^{\prime} y \delta-2 \kappa_{x} k x y \delta+k y \delta^{2}+\mathcal{O}(4) .
\end{aligned}
$$

It is quite clear from these equations that most perturbations become significant only for large amplitudes and oblique particle trajectories or for very strong magnets. The lowest-order quadrupole perturbations are of third order in the oscillation amplitudes and therefore become significant only for large beam sizes. Secondorder perturbations occur only in combined-function magnets and for particle trajectories traversing a quadrupole at large amplitudes or offsets from the axis. Associated with the main fields and perturbation terms are also chromatic variations thereof and wherever beams with a large energy spread must be transported such perturbation terms become important. Specifically, the quadrupole terms $k x \delta$ and $k y \delta$ determine the chromatic aberration of the focusing system and play a significant role in the transport of beams with large momentum spread. In most cases of beam dynamics, all except the linear chromatic terms can be neglected.

Evaluating the effect of perturbations on a particle beam, we must carefully select the proper boundary conditions for bending magnets. Only for sector magnets is the field boundary normal to the reference path and occurs therefore at the same location 
$z$ independent of the amplitude. Generally, this is not true and we must adjust the integration limits according to the particle oscillation amplitudes $x$ and $y$ and actual magnet boundary just as we did in the derivation of linear transformation matrices for rectangular or wedge magnets.

\subsection{Perturbation Methods in Beam Dynamics}

In this chapter, mathematical procedures have been developed to evaluate the effect of specific perturbations on beam dynamics parameters. It is the nature of perturbations that they are unknown and certain assumptions as to their magnitude and distribution have to be made. Perturbations can be systematic, statistical but periodic or just statistical and all can have a systematic or statistical time dependence.

Systematic perturbations in most cases become known through careful magnetic measurements and evaluation of the environment of the beam line. By construction magnet parameters may be all within statistical tolerances but systematically off the design values. This is commonly the case for the actual magnet length. Such deviations generally are of no consequences since the assumed magnet length in the design of a beam-transport line is arbitrary within limits. After the effective length of any magnet type to be included in a beam line is determined by magnetic measurements, beam optics calculations need to be repeated to reflect the variation in length. Similarly, deviations of the field due to systematic errors in the magnet gap or bore radius can be cancelled by experimental calibration of the fields with respect to the excitation current. Left are then only small statistical errors in the strength and length within each magnet type.

One of the most prominent systematic perturbation is an energy error a particle may have with respect to the ideal energy. We have treated this perturbation in much detail leading to dispersion or $\eta$-functions and chromaticities.

Other sources of systematic field errors come from the magnetic field of ion pumps or rf-klystrons, from earth magnetic field, and current carrying cables along the beam line. The latter source can be substantial and requires some care in the choice of the direction the electrical current flows such that the sum of currents in all cables is mostly if not completely compensated. Further sources of systematic field perturbations originate from the vacuum chamber if the permeability of the chamber or welding material is too high, if eddy currents exist in cycling accelerators or due to persistent currents in superconducting materials which are generated just like eddy currents during the turn on procedure. All these effects are basically accessible to measurements and compensatory measures in contrast to statistical perturbations as a result of fabrication tolerances. 


\subsubsection{Periodic Distribution of Statistical Perturbations}

Whatever statistical perturbations exist in circular accelerators, we know that these perturbations are periodic, with the ring circumference being the period length. The perturbation can therefore always be expressed by a Fourier series. The equation of motion in the presence of, for example, dipole field errors is in normalized coordinates

$$
\ddot{w}+v_{0}^{2} w=-v_{0}^{2} \beta^{3 / 2} \Delta \kappa .
$$

The dipole perturbation $\beta^{3 / 2} \Delta \kappa$ is periodic and can be expressed by the Fourier series

$$
\beta^{3 / 2} \Delta \kappa=\sum_{n} F_{n} \mathrm{e}^{\mathrm{i} n \varphi}
$$

where $v_{0} \varphi$ is the betatron phase and the Fourier harmonics $F_{n}$ are given by

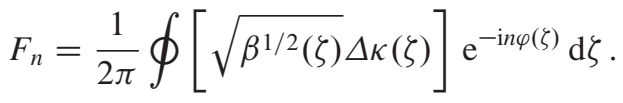

The location of the errors is not known and we may therefore only calculate the expectation value for the perturbation by multiplying (15.151) with its complex conjugate. In doing so, we note that each localized dipole perturbation deflects the beam by an angle $\theta$ and replace therefore the integral in (15.151) by a sum over all perturbations. With $\int \Delta \kappa \mathrm{d} \zeta \approx \theta$ we get for $F_{n} F_{n}^{*}=\left|F_{n}\right|^{2}$

$$
\left|F_{n}\right|^{2}=\frac{1}{4 \pi^{2}}\left[\sum_{k} \beta_{k} \theta_{k}^{2}+\sum_{k \neq j} \sqrt{\beta_{k} \beta_{j}} \theta_{k} \theta_{j} \mathrm{e}^{-\mathrm{i} n\left(\varphi_{k}-\varphi_{j}\right)}\right],
$$

where $\beta_{k}$ is the betatron function at the location of the dipole perturbation. The second sum in (15.152) vanishes in general, since the phases for the perturbations are randomly distributed.

For large circular accelerators composed of a regular lattice unit like FODO cells we may proceed further in the derivation of the effects of perturbations letting us determine the field and alignment tolerances of magnets. For simplicity, we assume that the lattice magnets are the source of dipole perturbations and that the betatron functions are the same at all magnets. Equation (15.152) then becomes

$$
\left|F_{n}\right|^{2}=\frac{1}{4 \pi^{2}} N_{m} \beta_{m} \sigma_{\theta}^{2}
$$

where $\sigma_{\theta}$ is the expectation value for the statistical deflection angle due to dipole perturbations. In a little more sophisticated approach, we would separate all magnets 
into groups with the same strength and betatron function and (15.153) becomes

$$
\left|F_{n}\right|^{2}=\frac{1}{4 \pi^{2}} \sum_{m} N_{m} \beta_{m} \sigma_{\theta, m}^{2},
$$

where the sum is taken over all groups of similar perturbations and $N_{m}$ is the number of perturbations within the group $m$. In a pure FODO lattice, for example, obvious groups would be all QF's, all QD's and all bending magnets. From now on we will, however, not distinguish between such groups anymore to simplify the discussion.

Periodic dipole perturbations cause a periodic orbit distortion which is from (15.149)

$$
w(\varphi)=-\sum_{n} \frac{v_{0}^{2} F_{n}}{\left(v_{0}^{2}-n^{2}\right)} \mathrm{e}^{\mathrm{i} n \varphi} .
$$

The expectation value for the orbit distortion is obtained by multiplying (15.155) with it's complex conjugate and we get with $w(\varphi)=u(z) / \sqrt{\beta(z)}$

$$
u u^{*}=\beta(z) v^{4}\left|F_{n}\right|^{2} \sum_{n=-\infty}^{+\infty} \frac{\mathrm{e}^{\mathrm{i} i \varphi}}{\left(v^{2}-n^{2}\right)} \sum_{m=-\infty}^{+\infty} \frac{\mathrm{e}^{-i m \varphi}}{\left(v^{2}-m^{2}\right)} .
$$

The sums can be replaced by $-\frac{\pi \cos v(\pi-\varphi)}{v \sin v \pi}$ and we get finally for the expectation value of the orbit distortion $\sigma_{u}$ at locations with a betatron function $\beta$

$$
\sigma_{u}^{2}=\beta \frac{N \bar{\beta} \sigma_{\theta}^{2}}{8 \sin ^{2} \pi v},
$$

where $\bar{\beta}$ is the average betatron function at the locations of perturbations. This result is in full agreement with the result (15.39) for misaligned quadrupoles, where $\sigma_{\theta}=\sigma_{\mathrm{u}} / f, \sigma_{\mathrm{u}}$ the statistical quadrupole misalignment and $f$ the focal length of the quadrupole.

This procedure is not restricted to dipole errors only but can be applied to most any perturbation occurring in a circular accelerator. For this we determine which quantity we would like to investigate, be it the tunes, the chromaticity, perturbation of the dispersion functions, or any other beam parameter. Variation of expressions for such quantities due to variations of magnet parameters and squaring such variation we get the perturbation of the quantity under investigation. Generally, perturbation terms of order $n$ in normalized coordinates are expressed by

$$
P_{n}(z)=v_{0}^{2} \beta^{3 / 2} \beta^{n / 2} p_{n}(z) w^{n-1} .
$$

Because the perturbations are assumed to be small, we may replace the oscillation amplitudes $w^{n}$ in the perturbation term by their principle unperturbed solutions. 
Considering that the beam position $w$ is a composite of, for example, betatron oscillation $w_{\beta}$, orbit distortion $w_{\mathrm{c}}$, and energy error $w_{\eta}$ we set

$$
w=w_{\beta}+w_{\mathrm{c}}+w_{\eta}
$$

and note that any higher-order perturbation contributes to the orbit, the eta-function, the tunes, betatron functions, and other beam parameters. Orbit distortions in sextupoles of strength $m$, for example, produce the perturbations

$$
P_{2}(z)=\frac{1}{2} v_{0}^{2} \beta^{5 / 2} m w^{2}
$$

which for $w_{\eta}=0$ can be decomposed into three components

$$
\begin{aligned}
& P_{20}(z)=\frac{1}{2} v_{0}^{2} \beta^{5 / 2} m w_{\mathrm{c}}^{2}, \\
& P_{21}(z)=v_{0}^{2} \beta^{5 / 2} m w_{\mathrm{c}} w_{\beta}, \\
& P_{22}(z)=\frac{1}{2} v_{0}^{2} \beta^{5 / 2} m w_{\beta}^{2} .
\end{aligned}
$$

The perturbation $P_{20}$ causes an orbit distortion and since the perturbations are randomly distributed the contribution to the orbit according to (15.157) is

$$
\sigma_{u}^{2}=\beta_{u} \frac{N_{\mathrm{s}} \beta_{u \mathrm{~s}} \sigma_{\theta}^{2}}{8 \sin ^{2} \pi v_{u}},
$$

where $N_{\mathrm{s}}$ is the number of sextupoles, $\beta_{u \mathrm{~s}}$ the value of the betatron function and $\sigma_{\mathrm{c}}$ the rms orbit distortion at the sextupoles, $\sigma_{\theta}=\frac{1}{2} m \sigma_{\mathrm{c}}^{2} \ell_{\mathrm{s}}$ and $\ell_{\mathrm{s}}$ is the effective sextupole deflection and length, respectively. In cases of very strong sextupoles iteration methods must be applied since the orbit perturbation depends on the orbit. Similarly, we could have set $w_{\mathrm{c}}=0$ to calculate the perturbation of the $\eta$-function due to sextupole magnets.

The linear perturbation $P_{21}$ in $(15.161)$ causes a statistical tune shift and a perturbation of the betatron function. Following the derivation of tune shifts in Sect. 15.3, we find the expectation value for the tune shift to be

$$
\left\langle\delta^{2} v\right\rangle=\frac{1}{16 \pi^{2}} \sum_{k} \beta_{k} m_{k} \ell_{k}\left\langle u_{0}^{2}\right\rangle_{k},
$$

where $\left\langle u_{0}^{2}\right\rangle$ is the random misalignment of the sextupole magnets or random orbit distortions in the sextupoles.

We find the interesting result, that sextupoles contribute to a tune error only if there is a finite orbit distortion or misalignment $u_{0}$, while a finite betatron oscillation amplitude of a particle in the same sextupoles does not contribute to a tune shift. Similarly, we may use the effects of systematic errors to get expressions for the 
probable variation of the betatron function (15.91) due to gradient errors from misaligned sextupoles.

In the approximation of small perturbations, we are able to determine the expectation value for the effect of statistical errors on a particular beam parameter or lattice function. This formalism is used particularly when we are interested to define tolerances for magnetic field quality and magnet alignment by calculating backwards from the allowable perturbation of beam parameters to the magnitude of the errors. Some specific statistical effects will be discussed in subsequent sections.

\subsubsection{Periodic Perturbations in Circular Accelerators}

Alignment and field errors in circular accelerators not only cause a distortion of the orbit but also a perturbation of the $\eta$-functions. Although these perturbations occur in both the horizontal and vertical plane, we will discuss only the effect in the vertical plane. While the derivations are the same for both planes the errors contribute only to a small perturbation of the already existing horizontal $\eta$-function while the ideal vertical $\eta$-function vanishes, and therefore the perturbation can contribute a large variation of beam parameters. This is specifically true for electron storage ring where the vertical beam emittance is very small and a finite vertical $\eta$-function may increase this emittance considerably.

Similar to (15.11) we use the equation of motion

$$
y^{\prime \prime}-k y=+\Delta \kappa_{y}-\Delta \kappa_{y} \delta-k y \delta+m x y
$$

with the decomposition

$$
y=y_{\mathrm{c}}+v_{y} \delta
$$

and get in normalized coordinates $\tilde{y}=y / \sqrt{\beta_{y}}$, while ignoring the betatron motion, the differential equations for the orbit distortion $\tilde{y}_{\mathrm{c}}$

$$
\ddot{\tilde{y}}_{\mathrm{c}}+v_{y}^{2} \tilde{y}_{\mathrm{c}}=+v_{y}^{2} \beta_{y}^{3 / 2}\left(\Delta \kappa_{y}+m x_{\mathrm{c}} y_{\mathrm{c}}\right)
$$

and for the perturbation of the $\eta$-function $\tilde{v}_{y}=v_{y} / \sqrt{\beta_{y}}$

$$
\ddot{\tilde{v}}_{y}+v_{y}^{2} \tilde{v}_{y}=-v_{y}^{2} \beta_{y}^{3 / 2} \Delta \kappa_{y}+v_{y}^{2} \beta_{y}^{2}\left(k-m \eta_{x}\right) \tilde{y}_{\mathrm{c}} .
$$

First, we note in a linear lattice where $m=0$ that the differential equations for both the closed orbit distortion and the $\eta$-function perturbation are the same except for a sign in the perturbation. Therefore, in analogy to (15.157)

$$
\left\langle v_{y}^{2}(z)\right\rangle=\frac{\beta(z) \bar{\beta}_{\theta}}{8 \sin ^{2} \pi v} \sum_{i} \sigma_{i \theta}^{2} .
$$


The perturbation of the $\eta$-function becomes more complicated in strong focusing lattices, where the chromaticity is corrected by sextupole fields. In this case, we note that all perturbation terms on the r.h.s. are periodic and we express them in Fourier series

$$
v^{2} \beta_{y}^{3 / 2} \Delta \kappa_{y}=\sum_{n=-\infty}^{n=+\infty} F_{n} \mathrm{e}^{\mathrm{i} n \varphi}
$$

with

$$
F_{n}=\frac{v^{2}}{2 \pi} \int_{0}^{2 \pi} \beta^{3 / 2} \Delta \kappa_{y} \mathrm{e}^{-\mathrm{i} n \tau} \mathrm{d} \tau
$$

and

$$
v^{2} \beta_{y}^{2}\left(k-m \eta_{x}\right)=\sum_{n=-\infty}^{n=+\infty} A_{n} \mathrm{e}^{\mathrm{i} n \varphi}
$$

with

$$
A_{n}=\frac{v^{2}}{2 \pi} \int_{0}^{2 \pi} \beta^{2}\left(k-m \eta_{x}\right) \mathrm{e}^{-\mathrm{i} n \tau} \mathrm{d} \tau
$$

We also make use of the periodicity of the perturbation of the $\eta$-function and set

$$
\tilde{v}_{y}=\sum_{n=-\infty}^{n=+\infty} E_{n} \mathrm{e}^{\mathrm{i} n \varphi}
$$

Inserting (15.169)-(15.173) into (15.167) we get with the periodic solution of the closed orbit

$$
\begin{gathered}
\tilde{y}_{\mathrm{c}}(\varphi)=\sum_{n} \frac{F_{n}}{v^{2}-n^{2}} \mathrm{e}^{\mathrm{i} n \varphi} \\
\sum_{n}\left[\left(v^{2}-n^{2}\right) E_{n}+F_{n}\right] \mathrm{e}^{\mathrm{i} n \varphi}-\sum_{m, r} \frac{A_{m} F_{r}}{v^{2}-n^{2}} \mathrm{e}^{\mathrm{i}(m+r) \varphi}=0 .
\end{gathered}
$$

Noting that this equation must be true for all phases $\varphi$ all terms with the same exponential factor must vanish separately and we may solve for the harmonics of the $\eta$-function

$$
E_{n}=-\frac{F_{n}}{v^{2}-n^{2}}+\sum_{r} \frac{A_{n-r} F_{r}}{\left(v^{2}-n^{2}\right)\left(v^{2}-r^{2}\right)}
$$


The perturbation of the $\eta$-function is therefore

$$
\tilde{v}_{y}(\varphi)=-\tilde{y}_{\mathrm{c}}(\varphi)+\sum_{n, r} \frac{A_{n-r} F_{r}}{\left(v^{2}-n^{2}\right)\left(v^{2}-r^{2}\right)} \mathrm{e}^{\mathrm{i} n \varphi},
$$

We extract from the double sum on the r.h.s. of (15.177) all terms with $n=r$ and get from those terms the expression $A_{0} \sum_{n} \frac{F_{n}}{\left(v^{2}-n^{2}\right)^{2}} \mathrm{e}^{\mathrm{i} n \varphi}$. The coefficient $A_{0}$, however, is just the natural chromaticity $A_{0}=2 \xi_{0 y} / v_{y}$ and the perturbation of the $\eta$-function is from (15.177)

$$
\tilde{v}_{y}(\varphi)=-\tilde{y}_{\mathrm{c}}(\varphi)+\frac{2 \xi_{y}}{v_{y}} \sum_{n} \frac{F_{n} \mathrm{e}^{\mathrm{i} n \varphi}}{\left(v^{2}-n^{2}\right)^{2}}+\sum_{n \neq r} \frac{A_{n-r} F_{r} \mathrm{e}^{\mathrm{i} n \varphi}}{\left(v^{2}-n^{2}\right)\left(v^{2}-r^{2}\right)}
$$

By correcting the orbit distortion and compensating the chromaticity, we are able to greatly reduce the perturbation of the vertical $\eta$-function. All terms with $r=0$ vanish for a truly random distribution of misalignment errors since $F_{0}=0$. Taking the quadrupole lattice as fixed we find the remaining terms to depend mainly on the distribution of the orbit correction $F_{r}$ and sextupole positions $A_{i}$. For any given sextupole distribution the orbit correction must be done such as to eliminate as much as possible all harmonics of the orbit in the vicinity of the tunes $r \not v_{y}$ and to center the corrected orbit such that $F_{0}=0$.

Furthermore, we note that some care in the distribution of the sextupoles must be exercised. While this distribution is irrelevant for the mere correction of the natural chromaticities, higher harmonics of the chromaticity function must be held under control as well. The remaining double sum is generally rather small since the resonance terms have been eliminated and either $v-n$ or $v-r$ is large. However, in very large rings or very strong focusing rings this contribution to the perturbation of the $\eta$-function may still be significant.

\subsubsection{Statistical Methods to Evaluate Perturbations}

In an open beam-transport line the perturbation effect at a particular point depends only on the upstream perturbations. Since perturbations cannot change the position but only the slope of particle trajectories, we merely transform the random kick angle $\theta_{k}$ from the location of the perturbation to the observation point. Adding all perturbations upstream of the observation point we get with $\psi=\psi(z)$

$$
\begin{aligned}
& u(z)=\sqrt{\beta(z)} \sum_{\substack{k \\
\psi_{k}<\psi(z)}} \sqrt{\beta_{k}} \sin \left(\psi-\psi_{k}\right) \theta_{k}, \\
& u^{\prime}(z)=\frac{1}{\sqrt{\beta(z)}} \sum_{\substack{k \\
\psi_{k}<\psi(z)}} \sqrt{\beta_{k}} \cos \left(\psi-\psi_{k}\right) \theta_{k} .
\end{aligned}
$$


The expectation value for the position of the beam center at the observation point becomes from the first equation (15.179) noting the complete independence of the perturbations

$$
\sigma_{u}(z)=\sqrt{\beta(z)} \frac{1}{2} N \sqrt{\langle\beta\rangle} \sigma_{\theta} .
$$

Random variations of the beam position are customarily corrected by special steering magnets if such correction is required at all. In long beam-transport systems like those required in linear colliders a mere correction of the beam position at the collision point, for example, may not be acceptable. Specifically, nonlinear perturbations lead to an incoherent increase of the beam size which can greatly reduce the usefulness of the colliding-beam system. In the next subsection we will therefore discuss general perturbations in beam-transport lines and their effect on the beam cross section.

\subsection{Control of Beam Size in Transport Lines}

For the transport of beams with a very small beam size or beam emittance like in a linear collider facilities we are specially concerned about the impact of any kind of perturbation on the integrity of a small beam emittance. Errors can disturb the beam size in many ways. We have discussed already the effect of dipole errors on the dispersion. The distortion of the dispersion causes an increase in the beam size due to the energy spread in the beam. Quadrupole field errors affect the value of betatron functions and therefore the beam size. Vertical orbit distortions in sextupoles give rise to vertical-horizontal coupling. In this section we will try to evaluate these effects on the beam size.

We use the equations of motion (6.95), (6.96) up to second order in $x, y$ and $\delta$, and assume the curvature to be small of the order or less than $(x, y, \delta)$. This is a proper assumption for high-energy beam transport lines like in linear colliders. For lower-energy beam lines very often this assumption is still correct and where a better approximation is needed more perturbation terms must be considered. For the horizontal plane we get

$$
\begin{aligned}
x^{\prime \prime}+\left(\kappa_{x}^{2}+k\right) x= & \kappa_{x} \delta-\kappa_{x} \delta^{2}-k y-\frac{1}{2} m\left(x^{2}-y^{2}\right)(1-\delta) \\
& -\Delta \kappa_{x}(1-\delta)+k x \delta+\Delta k x(1-\delta)+O(3)
\end{aligned}
$$

and for the vertical plane

$$
\begin{aligned}
y^{\prime \prime}-k y= & \kappa_{y} \delta-\kappa_{y} \delta^{2}-\underline{k} x+m x y(1-\delta)-k y \delta \\
& -\Delta \kappa_{y}(1-\delta)-\Delta k y(1-\delta)+\mathcal{O}(3)
\end{aligned}
$$


In these equations rotated magnets $\left(\kappa_{y}, \underline{k}, \underline{m}\right)$ are included as small quantities because rotational alignment errors of upright magnets cause rotated error fields although no rotated magnets per se are used in the beam line. For the solution of (15.181) and (15.182) we try the ansatz

$$
\begin{aligned}
& x=x_{\beta}+x_{\mathrm{c}}+\eta_{x} \delta+v_{x} \delta+w_{x} \delta^{2}, \\
& y=y_{\beta}+y_{\mathrm{c}}+\eta_{y} \delta+v_{y} \delta+w_{y} \delta^{2} .
\end{aligned}
$$

Here we define $\left(x_{\beta}, y_{\beta}\right)$ as the betatron oscillations, $\left(x_{\mathrm{c}}, y_{\mathrm{c}}\right)$ the orbit distortions, $\left(\eta_{x}, \eta_{y}\right)$ the dispersion function, $\left(v_{x}, v_{y}\right)$ the perturbations of the dispersion functions due to magnetic field errors, and $\left(w_{x}, w_{y}\right)$ the first-order chromatic perturbation of the dispersion functions $\left(\eta_{\text {tot }}=\eta+v+w \delta+\ldots\right)$. This ansatz leads to the following differential equations in the horizontal plane where we assume the bending radii to be large and $\kappa_{x}, \kappa_{y}$ are therefore treated as small quantities

$$
\begin{aligned}
x_{\beta}^{\prime \prime}+k x_{\beta}= & -\underline{k} y_{\beta}-\frac{1}{2} m\left(x_{\beta}^{2}-y_{\beta}^{2}\right)-m\left(x_{\beta} x_{\mathrm{c}}-y_{\beta} y_{\mathrm{c}}\right)+\Delta k x_{\beta}, \\
x_{\mathrm{c}}^{\prime \prime}+k x_{\mathrm{c}}= & -\Delta \kappa_{x}+\Delta k x_{\mathrm{c}}-\underline{k} y_{\mathrm{c}}-\frac{1}{2} m\left(x_{\mathrm{c}}^{2}-y_{\mathrm{c}}^{2}\right), \\
\eta_{x}^{\prime \prime}+k \eta_{x}= & +\kappa_{x}, \\
v_{x}^{\prime \prime}+k v_{x}= & -\underline{k} v_{y}-m\left(x_{\beta}+x_{\mathrm{c}}\right)\left(\eta_{x}+v_{x}\right)+m\left(y_{\beta}+y_{\mathrm{c}}\right)\left(\eta_{y}+v_{y}\right) \\
& +\Delta k\left(x_{\mathrm{c}}+x_{\beta}\right)+\Delta \kappa_{x}+\Delta k\left(\eta_{x}+v_{x}\right) \\
& +k x_{\beta}+k x_{\mathrm{c}}+\frac{1}{2} m\left(x_{\mathrm{c}}^{2}-y_{\mathrm{c}}^{2}\right)+\underline{k} y_{\mathrm{c}}, \\
w_{x}^{\prime \prime}+k w_{x}= & -\kappa_{x}-\frac{1}{2} m\left(\eta_{x}^{2}+2 \eta_{x} v_{x}-2 \eta_{y} v_{y}-v_{y}^{2}\right)+k\left(\eta_{x}+v_{x}\right) \\
= & +m\left(x_{\mathrm{c}} \eta_{x}+x_{\mathrm{c}} v_{x}-y_{\mathrm{c}} \eta_{y}-y_{\mathrm{c}} v_{y}\right)+\left(\eta_{x}+v_{x}\right) x_{\beta}-v_{y} y_{\beta} .
\end{aligned}
$$

Similarly, we get for the vertical plane

$$
\begin{aligned}
y_{\beta}^{\prime \prime}-k y_{\beta}= & -k x_{\beta}+m x_{\beta} y_{\beta}-\Delta k y_{\beta}+m\left(x_{\mathrm{c}} y_{\beta}+x_{\beta} y_{\mathrm{c}}\right), \\
y_{\mathrm{c}}^{\prime \prime}-k y_{\mathrm{c}}= & -\Delta \kappa_{y}-\Delta k y_{\mathrm{c}}-\underline{k} x_{\mathrm{c}}+m x_{\mathrm{c}} y_{\mathrm{c}}, \\
\eta_{y}^{\prime \prime}-k \eta_{y}= & +\kappa_{y}, \\
v_{y}^{\prime \prime}-k v_{y}= & +\Delta \kappa_{y}-\underline{k}\left(\eta_{y}+v_{y}\right)+m\left(x_{\beta}+x_{\mathrm{c}}\right)\left(\eta_{y}+v_{y}\right) \\
& +m\left(\eta_{x}+v_{x}\right)\left(y_{\beta}+y_{\mathrm{c}}\right)+\Delta k\left(y_{\beta}+y_{\mathrm{c}}\right)+\underline{k} x_{\mathrm{c}}-m x_{\mathrm{c}} y_{\mathrm{c}} \\
& -k\left(y_{\beta}+y_{\mathrm{c}}\right)-\Delta k\left(\eta_{y}+v_{y}\right), \\
w_{y}^{\prime \prime}-k w_{y}= & -\kappa_{y}+k\left(\eta_{y}+v_{y}\right) \\
= & +m\left(\eta_{x} \eta_{y}+\eta_{x} v_{y}+v_{x} \eta_{y}+v_{x} v_{y}\right) .
\end{aligned}
$$

The solution of all these differential equations is, if not already known, straightforward. We consider every perturbation to be localized as a thin element causing just a kick which propagates along the beam line. If $\beta_{j}$ is the betatron function at the observation point and $\beta_{i}$ that at the point of the perturbation $p_{n i}$ the solutions 
of (15.184), (15.185) have the form

$$
u_{j}=\sqrt{\beta_{j}} \sum_{i} \sqrt{\beta_{i}} \sin \psi_{j i} \int p_{n i} \mathrm{~d} z
$$

The kick due to the perturbation is $\theta_{i}=\int p_{n i} \mathrm{~d} z$, where the integral is taken along the perturbation assumed to be short. To simplify the equations to follow we define the length $\ell_{i}=\theta_{i} /\left\langle p_{n i}\right\rangle$. Since most errors derive from real magnets, this length is identical with that of the magnet causing the perturbation and $\psi_{j i}=\psi_{j}-\psi_{i}$ is the betatron phase between perturbation and observation point. A closer look at (15.184) and (15.185) shows that many perturbations depend on the solution itself requiring an iterative solution process. Here we will, however, concentrate only an the first iteration.

Ignoring coupling terms we have in (15.184) two types of perturbations, statistically distributed focusing errors $\Delta k$ and geometric aberration effects due to sextupoles. We assume here that the beam line is chromatically corrected by the use of self-correcting achromats in which case the term $\frac{1}{2} m\left(x_{\beta}^{2}-y_{\beta}^{2}\right)$ is self-canceling. The expectation value for the betatron oscillation amplitude due to errors is then

$$
x_{\beta}^{2}(z)=\beta_{x}(z) \sum_{i} \beta_{x i}\left\langle\left(p_{n i} \ell_{i}\right)^{2}\right\rangle \sin ^{2} \psi_{j i}
$$

or

$$
\left\langle x_{\beta}^{2}(z)\right\rangle=\beta_{x}(z) \bar{\beta}_{x}\left\langle\underline{k}^{2} y_{\beta}^{2}+\Delta k^{2} x_{\beta}^{2}+m^{2}\left(x_{\beta}^{2} x_{\mathrm{c}}^{2}+y_{\beta}^{2} y_{\mathrm{c}}^{2}\right)\right\rangle \frac{1}{2} N_{\mathrm{M}} \ell^{2},
$$

where $\bar{\beta}_{x}$ is the average value of the betatron functions at the errors, $N_{\mathrm{M}}$ the number of perturbed magnets and $\ell$ the magnet length. With $\underline{k}=k \alpha$, where $\alpha$ is the rotational error, we get

$$
\begin{aligned}
\left\langle x_{\beta}^{2}(z)\right\rangle=\frac{1}{2} \beta_{x}(z) \bar{\beta}_{x} N_{\mathrm{M}} k^{2} \ell^{2} & \\
\times & {\left[\sigma_{\alpha}^{2} \sigma_{y}^{2}+\sigma_{k}^{2} \sigma_{x}^{2}+\frac{m^{2}}{k^{2}}\left(\sigma_{x}^{2} \sigma_{y \mathrm{c}}^{2}-\sigma_{y}^{2} \sigma_{y \mathrm{c}}^{2}\right)\right] . }
\end{aligned}
$$

We have assumed the errors to have a Gaussian distribution with standard width $\sigma$. Therefore, $\sigma_{\alpha}^{2}=\left\langle\alpha^{2}\right\rangle, \sigma_{k}^{2}=\left\langle(\Delta k / k)^{2}\right\rangle, \sigma_{x \mathrm{c}}=\left\langle x_{\mathrm{c}}^{2}\right\rangle$, etc., and $\sigma_{y}, \sigma_{x}$ the standard beam size for the Gaussian particle distribution. Since $\left\langle x_{\beta}^{2}(z)\right\rangle / \beta(z)=\Delta \epsilon_{x}$ is the increase in beam emittance and $\sigma_{x}^{2}=\epsilon_{x} \bar{\beta}_{x}, \sigma_{y}^{2}=\epsilon_{y} \bar{\beta}_{y}$ we get for a round beam for which $\epsilon_{x}=\epsilon_{y}$ and the average values for the betatron functions are the same $\left(\bar{\beta}_{x}=\bar{\beta}_{y}\right)$

$$
\frac{\Delta \epsilon_{x}}{\epsilon_{x}}=\frac{1}{2} \bar{\beta}^{2} N_{\mathrm{M}} k^{2} \ell^{2}\left[\sigma_{\alpha}^{2}+\sigma_{k}^{2}+\frac{m^{2}}{k^{2}}\left(\sigma_{x \mathrm{c}}^{2}+\sigma_{y \mathrm{c}}^{2}\right)\right] .
$$


To keep the perturbation of the beam small the alignment $\sigma_{\alpha}$ and magnet field quality $\sigma_{k}$ must be good and the focusing weak which, however, for other reasons is not desirable. For a chromatically corrected beam line we have $k / m=\bar{\eta}_{x}$, which can be used in (15.190). The perturbation of the vertical beam emittance follows exactly the same results because we used a round beam.

The expectation value for the shift of the beam path is derived from (15.184a), (15.185b) with (15.186) in a similar way as for the betatron oscillations

$$
\left\langle x_{\mathrm{c}}^{2}(z)\right\rangle=\frac{1}{2} \beta_{x}(z) \bar{\beta}_{x} N_{\mathrm{M}} \ell^{2}\left[\left\langle\Delta \kappa_{x}^{2}\right\rangle+k^{2} \sigma_{k}^{2}\left\langle x_{\mathrm{c}}^{2}\right\rangle+k^{2} \sigma_{\alpha}^{2}\left\langle y_{\mathrm{c}}^{2}\right\rangle\right] .
$$

This expression for the path distortion, however, is not to be used to calculate the perturbation of the dispersion. In any properly operated beam line one expects this path distortion to be corrected leading to a smaller residual value depending on the correction scheme applied and the resolution of the monitors. With some effort the path can be corrected to better than $1 \mathrm{~mm}$ rms which should be used to evaluate path dependent perturbation terms in (15.184), (15.185). In the vertical plane we get

$$
\left\langle y_{\mathrm{c}}^{2}(z)\right\rangle=\frac{1}{2} \beta_{y}(z) \bar{\beta}_{y} N_{\mathrm{M}} \ell^{2}\left[\left\langle\Delta \kappa_{y}^{2}\right\rangle+k^{2} \sigma_{k}^{2}\left\langle y_{\mathrm{c}}^{2}\right\rangle+k^{2} \sigma_{\alpha}^{2}\left\langle x_{\mathrm{c}}^{2}\right\rangle\right] .
$$

The perturbation of the dispersion is with (15.184d) and (15.186)

$$
v_{x}(z)=-x_{\mathrm{c}}(z)+\sqrt{\beta_{x}(z)} \sum_{i} \sqrt{\beta_{x i}} p_{x i} \ell_{i} \sin \psi_{x j i}
$$

In (15.184d) we note the appearance of the same perturbation terms as for the path distortion apart from the sign and we therefore separate that solution in (15.193). The perturbations left are then

$$
p_{x i}=\left(k-m \eta_{x}\right)\left(x_{\beta}+x_{\mathrm{c}}\right)+m\left(y_{\beta}+y_{\mathrm{c}}\right) \eta_{y}+\Delta k \eta_{x}+\cdots
$$

In this derivation the betatron phase $\psi_{j i}$ does not depend on the energy since the chromaticity is corrected. Without this assumption, we would get another contribution to $v_{x}$ from the beam-path distortion. We also note that the chromaticity factor $\left(k-m \eta_{x}\right)$ can to first order be set to zero for chromatically corrected beam lines. The expectation value for the distortion of the dispersion is finally given by

$$
\left\langle v_{x}^{2}(z)\right\rangle=x_{\mathrm{c}}^{2}(z)+\frac{1}{2} \beta_{x}(z) \bar{\beta}_{x} N_{\mathrm{M}} \ell^{2}\left[\left\langle\Delta k^{2}\right\rangle \bar{\eta}_{x}^{2}+m^{2} \bar{\eta}_{y}^{2}\left\langle y_{\beta}^{2}\right\rangle+m^{2} \bar{\eta}_{y}^{2}\left\langle y_{\mathrm{c}}^{2}\right\rangle\right]
$$

or with some manipulation

$$
\left\langle v_{x}^{2}(z)\right\rangle=\left\langle x_{\mathrm{c}}^{2}(z)\right\rangle+\frac{1}{2} \beta_{x}(z) \bar{\beta}_{x} N_{M} k^{2} \ell^{2}\left[\sigma_{k}^{2} \bar{\eta}_{x}^{2}+\left(\frac{\bar{\eta}_{y}}{\bar{\eta}_{x}}\right)^{2}\left(\bar{\beta}_{y} \epsilon_{y}+\sigma_{y \mathrm{c}}^{2}\right)\right]
$$


The perturbation of the dispersion function is mainly caused by quadrupole field errors while the second term vanishes for a plane beam line where $\eta_{y}=0$. In principle, the perturbation can be corrected if a specific path distortion is introduced which would compensate the perturbation at the point $z$ as can be seen from (15.193).

In the vertical plane we proceed just the same and get instead of (15.193)

$$
v_{y}(z)=-y_{\mathrm{c}}(z)+\sqrt{\beta_{y}(z)} \sum_{i} \sqrt{\beta_{y i}}\left(p_{y i} \ell_{i}\right) \sin \psi_{y j i}
$$

with

$$
\begin{aligned}
p_{y i}=-( & \left.-m \eta_{x}\right)\left(y_{\beta}+y_{\mathrm{c}}\right)+m v_{x}\left(y_{\beta}+y_{\mathrm{c}}\right) \\
& +m\left(\eta_{y}+v_{y}\right)\left(x_{\beta}+x_{\mathrm{c}}\right)-\Delta k\left(\eta_{y}+v_{y}\right)-\underline{k}\left(\eta_{y}+v_{y}\right) .
\end{aligned}
$$

Again due to chromaticity correction we have $\left(k-m \eta_{x}\right) \approx 0$ and get for the expectation value of $\left\langle v_{y}^{2}\right\rangle$ in first approximation with $v_{y} \equiv 0$ in (15.198) and the average values $\bar{\eta}_{x}$ and $\bar{v}_{x}$

$$
\begin{aligned}
\left\langle v_{y}^{2}(z)\right\rangle=\left\langle y_{\mathrm{c}}^{2}(z)\right\rangle & +\frac{1}{2} \beta_{y}(z) \bar{\beta}_{y} N_{\mathrm{M}} k^{2} \ell^{2} \\
& \times\left[\left(\sigma_{k}^{2}+\sigma_{\alpha}^{2}+\frac{\left\langle x_{\mathrm{c}}^{2}\right\rangle}{\bar{\eta}_{x}^{2}}\right) \bar{\eta}_{y}^{2}+\frac{\bar{v}_{x}^{2}}{\bar{\eta}_{x}^{2}}\left(\beta_{y} \epsilon_{y}+\left\langle y_{\mathrm{c}}^{2}\right\rangle\right)\right] .
\end{aligned}
$$

For a plane beam line where $\eta_{y} \equiv 0$, we clearly need to go through a further iteration to include the perturbation of the dispersion which is large compared to $\eta_{y}=0$. In this approximation, we also set $y_{\mathrm{c}}(z)=0$ and

$$
\left\langle v_{y}^{2}(z)\right\rangle=\frac{\bar{v}_{x}^{2}}{\bar{\eta}_{x}^{2}}\left(\beta_{y} \epsilon_{y}+\left\langle y_{\mathrm{c}}^{2}\right\rangle\right) .
$$

Using this in a second iteration gives finally for the variation of the vertical dispersion function due to field and alignment errors

$$
\begin{aligned}
\left\langle v_{y}^{2}(z)\right\rangle=\left\langle y_{\mathrm{c}}^{2}(z)\right\rangle+\frac{1}{2} \beta_{y}(z) \bar{\beta}_{y} N_{\mathrm{M}} k^{2} \ell^{2}\left(\sigma_{k}^{2}+\sigma_{\alpha}^{2}+\frac{\left\langle x_{\mathrm{c}}^{2}\right\rangle}{\bar{\eta}_{x}^{2}}\right) & \\
\times & {\left[\left(\bar{\eta}_{y}^{2}+\bar{v}_{y}^{2}\right)+\frac{\bar{v}_{y}^{2}}{\bar{\eta}_{x}^{2}} \beta_{x} \epsilon_{x}+\frac{\bar{v}_{x}^{2}}{\bar{\eta}_{x}^{2}}\left(\beta_{y} \epsilon_{y}+\left\langle y_{\mathrm{c}}^{2}\right\rangle\right)\right] . }
\end{aligned}
$$

This second-order dispersion due to dipole field errors is generally small but becomes significant in linear-collider facilities where extremely small beam emittances must be preserved along beam lines leading up to the collision point. 


\section{Problems}

15.1 (S). Use the perturbation terms $P_{22}(z)$ in (15.161) and show that pure betatron oscillations in sextupoles do not cause a tune shift in first approximation. Why is there a finite tune shift for the $P_{21}(z)$-term?

15.2 (S). Show analytically that the dispersion function for a single bending magnet with a bending angle $\theta$ seems approximately to emerge from the middle of the magnet with a slope $D^{\prime}=\theta$.

15.3 (S). Use the lattice of example \#3 in Table 10.1 and introduce vertical rms misalignments of all quadrupoles by $\langle\delta x\rangle_{\mathrm{rms}}=0.1 \mathrm{~mm}$. Calculate the vertical rms dispersion function. Then, add also rotational alignment errors of the bending magnets by $\langle\delta \alpha\rangle_{\text {rms }}=0.17 \mathrm{mrad}$ and calculate again the vertical rms dispersion.

15.4 (S). Use two bending magnets separated by a drift space of length $\ell$. Both bending magnets are of equal but opposite strength. Such a deflection arrangement causes a parallel displacement $d$ of the beam path. Show that in this case the contribution to the dispersion at the end of the second bending magnet is $D=-d$ and $D^{\prime}=0$.

15.5 (S). For the rings in Problems 15.6 or 15.7 calculate the rms tolerance on the quadrupole strength to avoid the integer or half integer resonance. What is the corresponding tolerance on the quadrupole length? To avoid gradient fields in bending magnets the pole profiles must be aligned parallel with respect to the horizontal midplane. What is the angular tolerance for parallelism of the poles?

15.6. Use parameters of example \#4 in Table 10.1 for a FODO lattice and construct a full ring. Adjust the quadrupole strength such that both tunes are an integer plus a quarter. Calculate the rms alignment tolerance on the quadrupoles required to keep the beam within $\sigma_{x}=0.1 \mathrm{~mm}$ and $\sigma_{x}=0.1 \mathrm{~mm}$ of the ideal orbit. What is the amplification factor? Determine the rms deflection tolerance of the bending magnets to keep the beam within $0.1 \mathrm{~mm}$ of the ideal orbit. A rotation of the bending magnets about its axis creates vertical orbit distortions. If the magnets are aligned to a rotational tolerance of $\sigma_{\alpha}=0.17 \mathrm{mrad}$ (this is about the limit of conventional alignment techniques) what is the expectation value for the vertical orbit distortion?

15.7. Repeat the calculation of Problem 15.6 with the lattice example \#1 in Table 10.1. The alignment tolerances are much relaxed with respect to the ring in Problem 15.6. What are the main three contributions influencing the tolerance requirements? Make general recommendations to relax tolerances.

15.8. Design an electrostatic quadrupole with an aperture radius of $3 \mathrm{~cm}$ which is strong enough to produce a tune split of $\delta v=0.01$ between a counter rotating particle and antiparticle beam at an energy of $3 \mathrm{GeV}$. Assume the quadrupole to be placed at a location with a betatron function of $\beta=10 \mathrm{~m}$. How long must the quadrupole be if the electric field strength is to be limited to no more than $15 \mathrm{kV} / \mathrm{cm}$ ? 
15.9. Consider a long straight beam-transport line for a beam with an emittance of $\epsilon=10^{-12}$ rad-m from the end of a $500 \mathrm{GeV}$ linear collider linac toward the collision point. Use a FODO channel with $\beta_{\max }=5 \mathrm{~m}$ and determine statistical tolerances for transverse and rotational alignment and strength tolerances for the FODO cell quadrupoles to prevent the beam emittance from dilution by more than $10 \%$.

15.10. Use parameters of example \#4 in Table 10.1 for a FODO lattice and construct a full ring. Adjust the quadrupole strength such that both tunes are an integer plus a quarter. Calculate the rms alignment tolerance on the quadrupoles required to keep the beam within $\sigma_{x}=0.1 \mathrm{~mm}$ and $\sigma_{x}=0.1 \mathrm{~mm}$ of the ideal orbit. What is the amplification factor? Determine the rms deflection tolerance of the bending magnets to keep the beam within $0.1 \mathrm{~mm}$ of the ideal orbit. A rotation of the bending magnets about its axis creates vertical orbit distortions. If the magnets are aligned to a rotational tolerance of $\sigma_{\alpha}=0.17 \mathrm{mrad}$ (this is about the limit of conventional alignment techniques) what is the expectation value for the vertical orbit distortion?

15.11. Consider statistical transverse alignment errors of the quadrupoles in the large hadron collider lattice example \#4 in Table 10.1 of $\langle\delta x\rangle_{\mathrm{rms}}=0.1 \mathrm{~mm}$. What is the rms path distortion at the end of one turn? Determine the allowable rotational alignment error of the bending magnets to produce a vertical path distortion of no more than that due to quadrupole misalignments. How precise must the bending magnet fields be aligned to not contribute more path distortion than the quadrupole misalignments.

15.12. Repeat the calculation of Problem 15.10 with the lattice example \#1 in Table 10.1. The alignment tolerances are much relaxed with respect to the ring in Problem 15.10. What are the main three contributions influencing the tolerance requirements? Make general recommendations to relax tolerances.

15.13. Calculate the expectation value for the integer and half integer stop band width of the ring in Problem 15.5. Gradient errors introduce a perturbation of the betatron functions. What is the probable perturbation of the betatron function for the case in Problem 15.5?

15.14. Consider a FODO cell equal to examples \#1, \#2, and \#4 in Table 10.1, adjust the phase advance per cell to equal values and calculate the natural chromaticities. Insert thin sextupoles into the center of the quadrupoles and adjust to zero chromaticities. How strong are the sextupoles? Why must the sextupoles for lattice \#2 be so much stronger compared with lattice \#4 even though the chromaticity per cell is about the same?

15.15. Consider the transformation of phase ellipses through one full FODO cell of the examples in Problem 15.14. Let the emittance for the phase ellipses be $\epsilon=$ $10 \mathrm{~mm}$ mrad. First transform the equation for the phase ellipse into a circle by setting $u=x$ and $v=\alpha_{x}+\beta x^{\prime}$. Transform the phase circle from the center of the $\mathrm{QF}$ through one full FODO cell to the center of the next QF ignoring any sextupole terms. Repeat this transformation but include now the sextupole in the first QF only 
as calculated in Problem 15.14. Discuss the distortions of the phase circle for the three different FODO lattices.

\section{References}

1. E.D. Courant, H.S. Snyder, Appl. Phys. 3, 1 (1959)

2. G. Lüders, Nuovo Cimento Suppl. 2, 1075 (1955)

3. S. Chunjarean, Supercond. Sci. Technol. 24, 055013 (2011)

4. J. Safranek, Experimental determination of storage ring optics using orbit response measurements. NIMA 388, 27-36 (1997)

5. J. Safranek, G. Portmann, A. Terebilo, C. Steier, Matlab-based loco, in European Particle Accelerator Conference, EPAC 2002, Paris, France, p. 1184 (2002)

6. A. Terebilo, Accelerator toolbox for matlab. Technical Report SLAC-PUB-8732, Stanford University, SLAC (2001)

7. N. Vogt-Nielsen, Expansion of the characteristic exponents and the floquet solutions for the linear homogeneous second order differential equation with periodic coefficients. Technical Report MURA/NVN/3, MURA, Chicago, IL (1956)

8. H. Wiedemann, Chromaticity correction in large storage rings. Technical Report PEP-Note 220, Stanford Linear Accelerator Center, Stanford, CA (1976)

9. P.L. Morton, Derivation of nonlinear chromaticity by higher-order smooth approximation. Technical Report PEP Note-221, SLAC, Stanford, CA (1976) 


\section{Chapter 16 \\ Resonances}

Particle resonances in circular accelerators occur as a result of perturbation terms involving particular Fourier harmonics. That approach is based on the common knowledge that periodic perturbations of a harmonic oscillator can cause a resonance when the perturbation frequency is equal to an eigenfrequency of the oscillator.

\subsection{Lattice Resonances}

Perturbation terms in the equation of motion can lead to a special class of beam instabilities called resonances, which occur if perturbations act on a particle in synchronism with its oscillatory motion. While such a situation is conceivable in a very long beam transport line composed of many periodic sections, the appearance of resonances is generally restricted to circular accelerators. There, perturbations occur periodically at every turn and we may Fourier analyze the perturbation with respect to the revolution frequency. If any of the harmonics of the perturbation terms coincides with the eigenfrequency of the particles a resonance can occur and particles may get lost. Such resonances caused by field imperfections of the magnet lattice are also called structural resonances or lattice resonances. We have already come across two such resonances, the integer and the half-integer resonances.

The characteristics of these two resonances is that the equilibrium orbit and the overall focusing is not defined. Any small dipole or quadrupole error would therefore lead to particle loss as would any deviation of the particle position and energy from ideal values. Since these resonances are caused by linear field errors, we call them also linear resonances. Higher order resonances are caused in a similar way by nonlinear fields which are considered to be field errors with respect to the ideal linear lattice even though we may choose to specifically include such magnets,

This chapter has been made Open Access under a CC BY 4.0 license. For details on rights and licenses please read the Correction https://doi.org/10.1007/978-3-319-18317-6_28 
like sextupoles or octupoles, into the lattice to compensate for particular beam dynamics problems.

\subsubsection{Resonance Conditions}

In this section the characteristics of resonances in circular accelerators will be derived starting from the equation of motion in normalized coordinates with only the $n$th order multipole perturbation term. This is no restriction of generality since in linear approximation each multipole perturbation having its own resonance structure will be superimposed to that of other multipole perturbations. On the other hand, the treatment of only a single multipole perturbation will reveal much clearer the nature of the resonance. The equation of motion in normalized horizontal coordinates for an $n$ th-order perturbation is from (15.1)

$$
\ddot{w}+v_{0 x}^{2} w=v_{0 x}^{2} \beta_{x}^{3 / 2} \beta_{x}^{r / 2} \beta_{y}^{s / 2} p_{r s}(\varphi) w^{r} v^{s}
$$

where $v_{0 x}$ is the unperturbed horizontal tune and $r, s=0,1,2 \ldots$ integers with $r+$ $s=n-1$. A similar equation holds for vertical oscillations by replacing $w(\varphi), v_{0 x}, x$ with $v(\varphi)$, etc. and vice versa. The perturbations can be any appropriate term in the equations of motion (6.95) and (6.96), however, we will consider primarily perturbation terms which occur most frequently in circular accelerators and are due to rotated quadrupole or nonlinear multipole fields. The general treatment of resonances for other perturbations is not fundamentally different and is left to the interested reader. From Chap. 9 we extract the dominant perturbation terms of order $n=r+s+1$ in normalized coordinates and compile them in Table 16.1 ordered by perturbations of order $r$ and $s$. To keep track of all types of perturbations requires the use of many indices. We will keep the discussion more simple by ignoring coupling, which will be treated separately, and concentrate only on a single order

Table 16.1 Lowest order perturbation terms

\begin{tabular}{|c|c|c|c|}
\hline \multicolumn{2}{|c|}{ Order } & \multirow[b]{2}{*}{$v_{x 0}^{2} \sqrt{\beta_{x}^{(r+3)} \beta_{y}^{s}} p_{r s, x}(\varphi) w^{r} v^{s}$} & \multirow[b]{2}{*}{$v_{y 0}^{2} \beta_{x}^{r / 2} \beta_{y}^{(s+3) / 2} p_{r s, y}(\varphi)$} \\
\hline$r$ & $s$ & & \\
\hline 0 & 1 & $-v_{x 0}^{2} \beta_{x}^{3 / 2} \beta_{y}^{1 / 2} \underline{k v}$ & \\
\hline 1 & 0 & & $-v_{y 0}^{2} \beta_{x}^{1 / 2} \beta_{y}^{3 / 2} k w$ \\
\hline 2 & 0 & $-v_{x 0}^{2} \beta_{x}^{5 / 2} \frac{1}{2} m w^{2}$ & \\
\hline 2 & 2 & & $-v_{y 0}^{2} \beta_{x}^{1 / 2} \beta_{y}^{2} m w v$ \\
\hline 0 & 2 & $+v_{x 0}^{2} \beta_{x}^{3 / 2} \beta_{y} \frac{1}{2} m v^{2}$ & \\
\hline 4 & 1 & $-v_{x 0}^{2} \beta_{x}^{3} \frac{1}{6} r w^{3}$ & \\
\hline 2 & 1 & & $+v_{y 0}^{2} \beta_{x} \beta_{y}^{2} \frac{1}{2} r w^{2} v$ \\
\hline 1 & 2 & $+v_{x 0}^{2} \beta_{x}^{2} \beta_{y} \frac{1}{2} r w v^{2}$ & \\
\hline 0 & 3 & & $-v_{y 0}^{2} \beta_{y}^{3} \frac{1}{6} r v^{3}$ \\
\hline
\end{tabular}


of perturbation. For simplicity we drop from here on the index ${ }_{x}$ and set $\beta_{x}=\beta$. Equation (16.1) becomes then

$$
\ddot{w}+v_{0}^{2} w=v_{0}^{2} \beta^{n / 2+1} p_{n}(\varphi) w^{n-1},
$$

All perturbations are periodic in $\varphi$ and can be expanded into a Fourier series

$$
q_{n}(\varphi)=v_{0}^{2} \beta^{n / 2+1} p_{n}(\varphi)=\sum_{m} q_{n m} \mathrm{e}^{\mathrm{i} m \varphi}
$$

Since the perturbation is supposed to be small, we will insert the unperturbed oscillation $w_{0}$ on the right-hand side of (16.2). The general form of the unperturbed betatron oscillation can be written like

$$
w_{0}(\varphi)=a \mathrm{e}^{\mathrm{i} \nu_{0} \varphi}+b \mathrm{e}^{-\mathrm{i} \nu_{0} \varphi}
$$

where $a$ and $b$ are arbitrary constants and we may now express the amplitude factor in the perturbation term $w^{n-1}(\varphi)$ by a sum of exponential terms which we use on the right hand side of (16.2) as a first approximation

$$
w^{n-1}(\varphi) \approx w_{0}^{n-1}(\varphi)=\sum_{|l| \leq n-1} W_{l} \mathrm{e}^{-\mathrm{i} l_{0} \varphi} .
$$

Inserting both (16.3) and (16.5) into (16.2) we get for the equation of motion

$$
\ddot{w}+v_{0}^{2} w=\sum_{l, m} W_{l} q_{n m} \mathrm{e}^{-\mathrm{i}\left(m+l v_{0}\right) \varphi} .
$$

The solution of this equation includes resonant terms whenever there is a perturbation term with a frequency equal to the eigenfrequency $v_{0}$ of the oscillator. The resonance condition is therefore ( $m, n, l$ are integers)

$$
m+l v_{0}=v_{0} \quad \text { with } \quad|l| \leq n-1 .
$$

From earlier discussions we expect to find here the integer resonance caused by dipole errors $n=1$. In this case the index $l$ can only be $l=0$ and we get from (16.7)

$$
v_{0}=m
$$

which is indeed the condition for an integer resonance.

Magnetic gradient field errors $(n=2)$ can cause both a half integer-resonance as well as an integer resonance. The index $l$ can have the values $l=0$ and $l= \pm 1$. Note however that not all coefficients $W_{l}$ necessarily are nonzero. In this particular case, the coefficient for $l=0$ is indeed zero as becomes obvious by inspection 
of (16.4). The resonance conditions for these second order resonances are

$$
\begin{array}{ll}
m+v_{0}=v_{0} \rightarrow m=0 & \rightarrow \text { tune shift at any tune } \\
m-v_{0}=v_{0} \rightarrow m=2 v_{0} & \rightarrow \text { integer and half integer resonance, } \\
m=v_{0} & \rightarrow \text { no resonance because } W_{0}=0 .
\end{array}
$$

Among the resonance conditions (16.9) we notice that for $m=0$ the effect of the perturbation on the particle motion exists independent of the particular choice of the tune $v_{0}$. The perturbation includes a nonvanishing average value $q_{20}$ which in this particular case represents the average gradient error of the perturbation. Like any other gradient field in the lattice, this gradient error also contributes to the tune and therefore causes a tune shift. From (16.2) we find the new tune to be determined by $v^{2}=v_{0}^{2}\left[1-\left\langle\beta^{2} p_{2}\right\rangle_{\varphi}\right]$ and the tune shift is $\delta v \approx-\frac{1}{2} v_{0}\left\langle\beta^{2} p_{2}\right\rangle_{\varphi}$ in agreement with our earlier result in Sect. 15.3.1.

Third order resonances $(n=3)$ can be driven by sextupole fields and the index $l$ can have values

$$
l=-2,-1,0,+1,+2 .
$$

Here we note that $W_{1}=W_{-1}=0$ and therefore no resonances occur for $l= \pm 1$. The resonance for sextupole field perturbations are then

$$
\begin{array}{ll}
m-2 v_{0}=v_{0} \rightarrow m=3 v_{0} & \rightarrow \text { third order resonance, } \\
m=v_{0} \rightarrow m=v_{0} & \rightarrow \text { integer resonance, } \\
m+2 v_{0}=v_{0} \rightarrow m=-v_{0} & \rightarrow \text { integer resonance. }
\end{array}
$$

Sextupole fields can drive third order resonances at tunes of

$$
v_{0}=r+\frac{1}{3} \quad \text { or } \quad v_{0}=r-\frac{1}{3},
$$

where $r$ is an integer. They also can drive integer resonances.

Finally we derive resonance conditions for octupole fields $(n=4)$ where

$$
l=-3,-2,-1,0,+1,+2,+3
$$

and again some values of $l$ do not lead to a resonance since the amplitude coefficient $W_{q}$ is zero. For octupole terms this is the case for $l=0$ and $l= \pm 2$. The remaining resonance terms are then

$$
\begin{array}{ll}
m-3 v_{0}=v_{0} \rightarrow m=4 v_{0} & \rightarrow \text { quarter integer resonance, } \\
m-v_{0}=v_{0} \rightarrow m=2 v_{0} & \rightarrow \text { half integer resonance, } \\
m+v_{0}=v_{0} \rightarrow m=0 & \rightarrow \text { tune spread at any tune, } \\
m+3 v_{0}=v_{0} \rightarrow m=-2 v_{0} & \rightarrow \text { half integer resonance. }
\end{array}
$$


The resonance condition for $m=0$ leads to a shift in the oscillation frequency. Different from gradient errors, however, we find the tune shift generated by octupole fields to be amplitude dependent $v^{2}=v_{0}^{2}\left[1-\left\langle\beta^{3} p_{4} w^{2}\right\rangle_{\varphi}\right]$. The amplitude dependence of the tune shift causes an asymmetric tune spread to higher or lower values depending on the sign of the perturbation term $p_{4}$ while the magnitude of the shift is determined by the oscillation amplitude of the particle.

The general resonance condition for betatron oscillations in one plane can be expressed by

$$
|m|=(|l| \pm 1) v_{0},
$$

where $|l| \leq n-1$ and the value $|l|+1$ is the order of resonance. The index $m$ is equal to the order of the Fourier harmonic of the perturbation and we call therefore these resonances structural or lattice resonances to distinguish them from resonances caused, for example, by externally driven oscillating fields.

The maximum order of resonances in this approximation depends on the order of nonlinear fields present. An $n$ th-order multipole field can drive all resonances up to $n^{\text {th }}$-order with driving amplitudes that depend on the actual multipole field strength and locations within the lattice. Generally, the higher the order $n$ the weaker is the resonance. In electron circular accelerators radiation damping makes higher order resonances ineffective. This is not the case for proton or ion beams which accumulate any effect leading, if not to beam loss, then to beam dilution or emittance blow-up.

The term resonance is used very generally to include also effects which do not necessarily lead to a loss of the beam. Such "resonances" are characterized by $m=0$ and are independent of the tune. In the case of gradient errors this condition was shown to lead to a stable shift in tune for the whole beam. Unless this tune shift moves the beam onto another resonance the beam stability is not affected. Similarly, octupole fields introduce a spread of tunes in the beam proportional to the square of the oscillation amplitude. Again no loss of particles occurs unless the tune spread reaches into the stop band of a resonance. By induction we conclude that all even perturbation terms, where $n$ is an even integer, lead to some form of tune shift or spread. No such tune shifts occur for odd order perturbations in the approximation used here. Specifically we note that dipoles, sextupoles or decapoles etc. do not lead to a tune shift for weak perturbations. Later, however, we will discuss the Hamiltonian resonance theory and find, for example, that strong sextupole perturbations can indeed cause a tune spread in higher order.

In this derivation of resonance parameters we have expanded the perturbations into Fourier series and have assumed the full circular accelerator lattice as the expansion period. In general, however, a circular accelerator is composed of one or more equal superperiods. For a circular lattice composed of $N$ superperiods the Fourier expansion has nonzero coefficients only every $N$ th-harmonic and therefore 
the modified resonance conditions are

$$
|j| N=(|l| \pm 1) v_{0}
$$

where $j$ is an integer. A high super-periodicity actually eliminates many resonances and is therefore a desirable design feature for circular accelerator lattices. The integer and half-integer resonances, however, will always be present independent of the super-periodicity because the equilibrium orbits and the betatron functions respectively are not defined. On the other hand, integer and half-integer resonances driven by multipole perturbations may be eliminated in a high periodicity lattice with the overall effect of a reduced stop band width. It should be noted here, that the reduction of the number of resonances works only within the applied approximation. "Forbidden" resonances may be driven through field and alignment errors which compromise the high lattice periodicity or by strong non-linearities and coupling creating resonant driving terms in higher order approximation. Nevertheless, the forbidden resonances are weaker in a lattice of high periodicity compared to a low periodicity lattice.

\subsubsection{Coupling Resonances}

Betatron motion in a circular accelerator occurs in both the horizontal and vertical plane. Perturbations can be present which depend on the betatron oscillation amplitude in both planes. Such terms are called coupling terms. The lowest order coupling term is caused by a rotated quadrupole or by the rotational misalignment of regular quadrupoles. In general we have in the horizontal plane the equation of motion from (16.1)

$$
\ddot{w}+v_{0 x}^{2} w=v_{0 x}^{2} \beta_{x}^{3 / 2} \beta_{x}^{r / 2} \beta_{y}^{s / 2} p_{r s}(\varphi) w^{r} v^{s},
$$

where $r, s$ are integers and $w, v$ describe betatron oscillations in the horizontal and vertical plane, respectively. Again we use the unperturbed solutions $w_{0}(\varphi)$ and $v_{0 x}(\varphi)$ of the equations of motion in the form (16.4) and express the higher order amplitude terms in the perturbation by the appropriate sums of trigonometric expressions:

$$
q_{r s}(\varphi)=v_{0 x}^{2} \beta_{x}^{3 / 2} \beta_{x}^{r / 2} \beta_{y}^{s / 2} p_{r s}(\varphi)=\sum_{m} q_{r s m} \mathrm{e}^{\mathrm{i} m \varphi},
$$

and similar to (16.5)

$$
\begin{aligned}
& w^{r-1}(\varphi)=\sum_{|l| \leq r-1} W_{l} \mathrm{e}^{\mathrm{i} l \nu_{0 x} \varphi}, \\
& v^{s-1}(\varphi)=\sum_{|\ell| \leq s-1} V_{\ell} \mathrm{e}^{\mathrm{i} \ell \nu_{0 y} \varphi} .
\end{aligned}
$$


Insertion into (16.17) gives after some sorting

$$
\ddot{w}+v_{0 x}^{2} w=\sum q_{r s m} W_{l} V_{\ell} \mathrm{e}^{\mathrm{i}\left[\left(m+l v_{0 x}+\ell v_{0 y}\right) \varphi\right]},
$$

where $m, l$ and $\ell$ are integers. The resonance condition is

$$
m+l v_{0 x}+\ell v_{0 y}=v_{0 x},
$$

and the quantity

$$
|l|+|\ell|+1 \leq n
$$

designates the order of the coupling resonances. Again, for a super-periodicity $N$ we replace $m$ by $j N$, where $j$ is an integer. As an example, we discuss a perturbation term caused by a rotated quadrupole for which the equation of motion is

$$
\ddot{w}+v_{0}^{2} w=q_{01}(\varphi) v \text {. }
$$

In this case we have $n=2$ and $r=0$ and the lowest order resonance condition with $l=0$ and $\ell= \pm 1$ is from $(16.21)$

$$
m+\ell v_{0 y}=v_{0 x}
$$

Resonance occurs for

$$
|m|=v_{0 x}+v_{0 y} \quad \text { and } \quad|m|=v_{0 x}-v_{0 y} .
$$

There is no coupling resonance for $\ell=0$ since $V_{0}=0$. The resonances identified in (16.25) are called linear coupling resonances or linear sum resonance (left) and linear difference resonance (right), respectively.

Delaying proof for a later discussion we note at this point that the sum resonance can lead to a loss of beam while the difference does not cause a loss of beam but rather leads to an exchange of horizontal and vertical betatron oscillations. In circular accelerator design we therefore adjust the tunes such that a sum resonance is avoided.

\subsubsection{Resonance Diagram}

The resonance condition (16.15) has been derived for horizontal motion only, but a similar equation can be derived for the vertical motion. Both resonance conditions can be written in a more symmetric way

$$
l \nu_{0 x}+\ell v_{0 y}=j N,
$$


Fig. 16.1 Resonance diagram for a ring with superperiodicity one, $N=1$

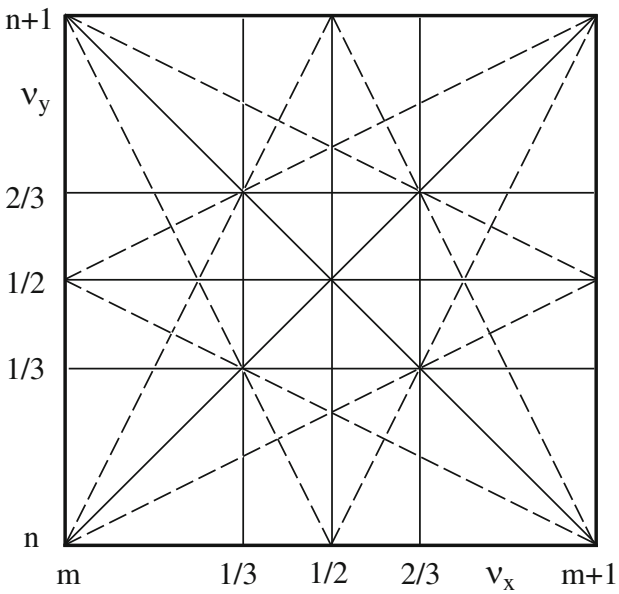

where $l, \ell, j$ are integers and $|l|+|\ell|$ is the order of the resonance. Plotting all lines (16.26) for different values of $l, \ell, j$ in a $\left(v_{y}, v_{x}\right)$ diagram produces what is called a resonance diagram. In Fig. 16.1 an example of a resonance diagram for $N=1$ is shown displaying all resonances up to third order with $|l|+|\ell| \leq 3$.

The operating points for a circular accelerator are chosen to be clear of any of these resonances. It should be noted here that the resonance lines are not mathematically thin lines in the resonance diagram but rather exhibit some "thickness" which is called the stop band width. This stop band width depends on the strength of the resonance as was discussed earlier.

Not all resonances are of the same strength and generally get weaker with increasing order. While a particle beam would not survive on an integer or a halfinteger resonance all other resonances are basically survivable, at least for electron beams. For proton or ion beams higher order resonance must be avoided to prevent beam dilution. Only in particular cases, where strong multipole field perturbations cause a higher order resonance, may we observe beam loss. This is very likely to be the case for third order resonances in rings, where strong sextupole magnets are employed to correct for chromatic aberrations.

The beneficial effect of a high super-periodicity or symmetry $N$ in a circular accelerator becomes apparent in such a resonance diagram because the density of resonance lines is reduced by the factor $N$ and the area of stability between resonances to operate the accelerator becomes proportionately larger. In Fig. 16.2, the resonance diagram for a ring with super-periodicity four $(N=4)$ is shown and the reduced number of resonances is obvious. Wherever possible a high symmetry in the design of a circular accelerator should be attempted. Conversely, breaking a high order of symmetry can lead to a reduction in stability if not otherwise compensated. 
Fig. 16.2 Resonance diagram for a ring with superperiodicity four, $N=4$

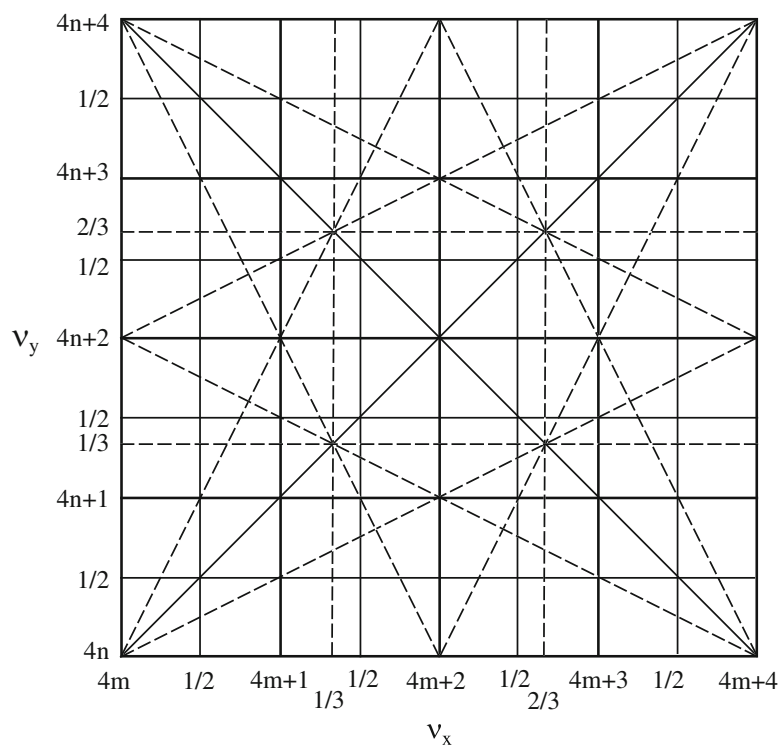

\subsection{Hamiltonian Resonance Theory*}

In the realm of Hamiltonian resonance theory we will be able to derive not only obvious resonant behavior but also resonant dynamics which does not necessarily lead to a loss of the beam but to a significant change of beam parameters. We also will be able to determine the strength of resonances, effectiveness, escape mechanisms and more.

\subsubsection{Non-linear Hamiltonian}

While simple Fourier expansions of perturbations around a circular accelerator allow us to derive the locations of lattice resonances in the tune diagram, we can obtain much deeper insight into the characteristics of resonances through the application of the Hamiltonian theory of linear and nonlinear oscillators. Soon after the discovery of strong focusing, particle dynamicists noticed the importance of perturbations with respect to beam stability and the possibility of beam instability even in the presence of very strong focusing.

Extensive simulations and development of theories were pursued in an effort to understand beam stability in high-energy proton synchrotrons then being designed at the Brookhaven National Laboratory and CERN. The first Hamiltonian theory of linear and non linear perturbations has been published by Schoch [1] which includes also references to early attempts to solve perturbation problems. A modern, consistent and complete theory of all resonances has been developed, for example, 
by Guignard [2]. In this text, we will concentrate on main features of resonance theory and point the interested reader for more details to these references.

Multipole perturbations have been discussed as the source of resonances and we will discuss in this chapter the Hamiltonian resonance theory. The equation of motion under the influence of an $n$ th-order perturbation is in normalized coordinates and in the horizontal plane without coupling (see Table 16.1)

$$
\ddot{w}+v_{0}^{2} w=q_{n}(\varphi) w^{n-1},
$$

which can be also derived from the nonlinear Hamiltonian

$$
H_{w}=\frac{1}{2} \dot{w}^{2}+\frac{1}{2} v_{0}^{2} w^{2}+\bar{q}_{n}(\varphi)\left(\frac{v_{0}}{2}\right)^{n / 2} w^{n} .
$$

Here we introduced

$$
\bar{q}_{n}(\varphi)=-q_{n}(\varphi) \frac{1}{n}\left(\frac{\nu_{0}}{2}\right)^{-n / 2}
$$

for future convenience.

To discuss resonance phenomena it is useful to perform a canonical transformation from the coordinates $(w, \dot{w})$ to action-angle variables $(J, \psi)$ which can be derived from the generating function (5.52) and the new Hamiltonian expressed in action-angle variables is

$$
H=v_{0} J+\bar{q}_{n}(\varphi) J^{n / 2} \cos ^{n}(\psi-\vartheta) .
$$

The action-angle variables take on the role of an "energy" and frequency of the oscillatory system. Due to the phase dependent perturbation $p_{n}(\varphi)$ the oscillation amplitude $J$ is no more a constant of motion and the circular motion in phase space becomes distorted as shown in Fig. 16.3 for a sextupolar perturbation. The oscillator frequency $\dot{\psi}=\partial \psi / \partial \varphi=v$ is similarly perturbed and can be derived from the second Hamiltonian equation of motion

$$
\frac{\partial H}{\partial J}=\dot{\psi}=v_{0}+\frac{n}{2} \bar{q}_{n}(\varphi) J^{n / 2-1} \cos ^{n}(\psi-\vartheta) .
$$

Perturbation terms seem to modify the oscillator frequency $v_{0}$ but because of the oscillatory trigonometric factor it is not obvious if there is a net shift or spread in the tune. We therefore expand the perturbation $\bar{q}_{n}(\varphi)$ as well as the trigonometric factor $\cos ^{n} \psi$ to determine its spectral content. The distribution of the multipole perturbations in a circular accelerator is periodic with a periodicity equal to the length of a superperiod or of the whole ring circumference and we are therefore 
Fig. 16.3 Nonlinear perturbation of phase-space motion

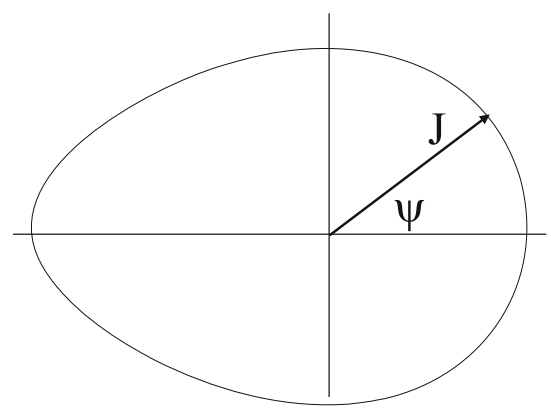

able to expand the perturbation $\bar{q}_{n}(\varphi)$ into a Fourier series

$$
\bar{q}_{n}(\varphi)=\sum_{l} \bar{q}_{n l} \mathrm{e}^{-\mathrm{i} l N \varphi}
$$

where $N$ is the super-periodicity of the circular accelerator. We also expand the trigonometric factor in (16.31) into exponential functions, while dropping the arbitrary phase $\vartheta$

$$
\cos ^{n} \psi=\sum_{|m| \leq n} c_{n m} \mathrm{e}^{\mathrm{i} m \psi}
$$

and get

$$
\begin{aligned}
\bar{q}_{n}(\varphi) \cos ^{n} \psi & =\sum_{l} \bar{q}_{n l} \mathrm{e}^{-\mathrm{i} 1 N \varphi} \sum_{|m| \leq n} c_{n m} \mathrm{e}^{\mathrm{i} m \psi} \\
& =\sum_{\substack{l \\
|m| \leq n}} c_{n m} \bar{q}_{n l} \mathrm{e}^{\mathrm{i}(m \psi-l N \varphi)} \\
& =c_{n 0} \bar{q}_{n 0}+\sum_{\substack{l \geq 0 \\
0<m \leq n}} 2 c_{n m} \bar{q}_{n l} \cos (m \psi-l N \varphi) .
\end{aligned}
$$

In the last equation, the perturbation $\bar{q}_{n}(\varphi)$ is expanded about a symmetry point merely to simplify the expressions of resonant terms. For asymmetric lattices the derivation is similar but includes extra terms. We have also separated the nonoscillatory term $c_{n 0} p_{n 0}$ from the oscillating terms to distinguish between systematic frequency shifts and mere periodic variations of the tune. The Hamiltonian (16.30) now becomes with (16.34)

$$
H=v_{0} J+c_{n 0} \bar{q}_{n 0} J^{n / 2}+J^{n / 2} \sum_{\substack{l \geq 0 \\ 0<m \leq n}} 2 c_{n m} \bar{q}_{n l} \cos (m \psi-l N \varphi)
$$


The third term on the r.h.s. consists mostly of fast oscillating terms which in this approximation do not lead to any specific consequences. For the moment we will ignore these terms and remember to come back later in this chapter. The shift of the oscillator frequency due to the lowest-order perturbation becomes obvious and may be written as

$$
\frac{\partial H}{\partial J}=\dot{\psi}=v_{0}+\frac{n}{2} c_{n 0} \bar{q}_{n 0} J^{n / 2-1}+\text { oscillatory terms. }
$$

Since $c_{n 0} \neq 0$ for even values of $n$ only, we find earlier results confirmed, where we observed the appearance of amplitude-dependent tune shifts and tune spreads for even-order perturbations. Specifically we notice, that there is a coherent amplitude independent tune shift for all particles within a beam in case of a gradient field perturbation with $n=2$ and an amplitude dependent tune spread within a finite beam size for all other higher- and even-order multipole perturbations.

To recapitulate the canonical transformation of the normalized variables to action-angle variables has indeed eliminated the angle coordinate as long as we neglect oscillatory terms. The angle variable therefore is in this approximation a cyclic variable and the Hamiltonian formalism tells us that the conjugate variable, in this case the amplitude $J$ is a constant of motion or an invariant. This is an important result which we obtained by simple application of the Hamiltonian formalism confirming our earlier expectation to isolate constants of motion.

This has not been possible in a rigorous way since we had to obtain approximate invariants by neglecting summarily all oscillatory terms. In certain circumstances this approximation can lead to totally wrong results. To isolate these circumstances we pursue further canonical transformations to truly separate from the oscillating terms all non-oscillating terms of order $n / 2$ while the rest of the oscillating terms are transformed to a higher order in the amplitude $J$.

\subsubsection{Resonant Terms}

Neglecting oscillating terms is justified only in such cases where these terms oscillate rapidly. Upon closer inspection of the arguments in the trigonometric functions we notice however that for each value of $m$ in (16.35) there exists a value $l$ which causes the phase

$$
m_{r} \psi_{r} \approx m \psi-l N \varphi
$$

to vary only slowly possibly leading to a resonance. The condition for the occurrence of such a resonance is $\psi_{r} \approx 0$ or with $\psi \approx v_{0} \varphi$

$$
m_{r} v_{0} \approx r N
$$


where we have set $l=r$ to identify the index for which the resonance condition (16.38) is met. The index $m_{r}$ is the order of the resonance and can be any integer $1 \leq m_{\mathrm{r}} \leq n$.

The effects of resonances do not only appear when the resonance condition is exactly fulfilled for $\psi_{\mathrm{r}}=0$. Significant changes in the particle motion can be observed when the particle oscillation frequency approaches the resonance condition. We therefore keep all terms which vary slowly compared to the betatron frequency $\dot{\psi}$.

After isolating resonant terms we may now neglect all remaining fast oscillating terms with $m \neq m_{r}$. Later we will show that these terms can be transformed to higher order and are therefore of no consequence to the order of approximation of interest. Keeping only resonant terms defined by (16.38) we get from (16.35) the $n$ th-order Hamiltonian in normalized coordinates

$$
H=v_{0} J+c_{n 0} \bar{q}_{n 0} J^{n / 2}+J^{n / 2} \sum_{\substack{r \\ 0<m_{r} \leq n}} 2 c_{n m_{r}} \bar{q}_{n r} \cos \left(m_{r} \psi_{r}\right) .
$$

The value of $m_{r}$ indicates the order of the resonance and we note that the maximum order of resonance driven by a multipole of order $n$ is not higher than $n$. A dipole field therefore can drive only an integer resonance, a quadrupole field up to a half-integer resonance, a sextupole up to a third-order resonance, an octupole up to a quarter resonance and so forth although not all allowed resonances become real. We know for example already that a sextupole does not drive a tune shift or a quarter integer resonance in the approximation used so far. As we have noticed before, whenever we derive mathematical results we should keep in mind that such results are valid only within the approximation under consideration. It is, for example, known [3] that sextupoles can also drive quarter integer resonances through higherorder terms. In nonlinear particle beam dynamics any statement about stability or instability must be accompanied by a statement defining the order of approximation made to allow independent judgement for the validity of a result to a particular problem.

The interpretation of the Hamiltonian (16.39) becomes greatly simplified after another canonical transformation to eliminate the appearance of the independent variable $\varphi$. We thereby transform to a coordinate system that moves with the reference particle, thus eliminating the linear motion that we already know. The new coordinates rotate once per revolution and thereby eliminate the linear rotation in phase space that we know already. This can be achieved by a canonical similarity transformation from the coordinates $(J, \psi)$ to $\left(J_{1}, \psi_{1}\right)$ which we derive from the generating function

$$
G_{1}=J_{1}\left(\psi-\frac{r N \varphi}{m_{r}}\right)
$$


From this we get the relations between the old and new coordinates

$$
\frac{\partial G_{1}}{\partial J_{1}}=\psi_{1}=\psi-\frac{r N}{m_{r}} \varphi
$$

and

$$
\frac{\partial G_{1}}{\partial \psi}=J=J_{1}
$$

The quantity $\psi_{1}$ now describes the phase deviation of a particle from that of the reference particle. Since the generating function depends on the independent variable $\varphi$ we get for the new Hamiltonian $H_{1}=H+\partial G_{1} / \partial \varphi$ or

$$
H_{1}=\left(v_{0}-\frac{r N}{m_{r}}\right) J_{1}+c_{n 0} \bar{q}_{n 0} J_{1}^{n / 2}+2 c_{n m_{\mathrm{r}}} \bar{q}_{n r} J_{1}^{n / 2} \cos \left(m_{\mathrm{r}} \psi_{1}+r N \varphi\right) \text {, }
$$

where we have retained for simplicity only the highest-order resonant term $\left(m_{r}=n\right)$. With $\dot{\psi}=(\mathrm{d} \psi / \mathrm{d} \varphi)=v$ and (16.38) a resonance condition occurs whenever

$$
v_{0} \approx \frac{r N}{m_{r}}=v_{r}
$$

Setting $\Delta v_{r}=v_{0}-v_{r}$ for the distance of the tune $v_{0}$ from the resonance tune $v_{r}$ the Hamiltonian becomes with all perturbation terms

$$
H=\Delta v_{\mathrm{r}} J_{1}+\sum_{n} c_{n 0} \bar{q}_{n 0} J_{1}^{n / 2}+\sum_{n} J_{1}^{n / 2} \sum_{\substack{r \\ 0<m_{r} \leq n}} 2 c_{n m_{r}} \bar{q}_{n r} \cos \left(m_{r} \psi_{1}\right) .
$$

The coefficients $c_{n 0}$ are defined by (16.33) and the harmonic amplitudes of the perturbations are defined by the Fourier expansion (16.32). The resonance order $r$ and integer $m_{r}$ depend on the ring tune and are selected such that (16.44) is approximately met. A selection of most common multipole perturbations are compiled in Table 16.1 and picking an $n$ th-order term we get from (16.29) the expression for $\bar{q}_{n r}$.

In the course of the mathematical derivation we started out in (16.28) with only one multipole perturbation of order $n$. For reasons of generality, however, all orders of perturbation $n$ have been included again in (16.45). We will, however, not deal with the complexity of this multi-resonance Hamiltonian nor do we need to in order to investigate the character of individual resonances. Whatever the number of actual resonances may be in a real system the effects are superpositions of individual resonances. We will therefore investigate in more detail single resonances and discuss superpositions of more than one resonance later in this chapter. 


\subsubsection{Resonance Patterns and Stop-Band Width}

Equation (16.45) can be used to calculate the stop band width of resonances and to explore resonance patterns which are a superposition of particle trajectories $H=$ const in $\left(\psi_{1}, J_{1}\right)$ phase-space. Depending on the nature of the problem under study, we may use selective terms from both sums in (16.45). Specifically to illustrate characteristic properties of resonances, we will use from the first sum the term $c_{40} \bar{q}_{40}$, which originates from an octupole field. This is the lowest order term that provides some beam stability as we will see. From the second sum we choose a single $n$ th-order term driving the $r$ th-order resonance and get the simplified Hamiltonian

$$
H_{1}=\Delta v_{\mathrm{r}} J_{1}+c_{40} \bar{q}_{40} J_{1}^{2}+2 c_{n m_{r}} \bar{q}_{n r} J_{1}^{n / 2} \cos \left(m_{r} \psi_{1}\right)=\text { const. }
$$

To further simplify the writing of equations and the discussion of results we divide (16.46) by $2 c_{n m_{r}} \bar{q}_{n r} J_{10}^{n / 2}$, where the amplitude $J_{10}$ is an arbitrary reference amplitude of a particle at the starting point. Defining an amplitude ratio or beat factor

$$
R=\frac{J_{1}}{J_{10}},
$$

and considering only resonances of order $m_{r} \approx n,(16.46)$ becomes

$$
R \Delta+R^{2} \Omega+R^{n / 2} \cos n \psi_{1}=\mathrm{const}
$$

where the detuning from the resonance is

$$
\Delta=\frac{\Delta v_{r}}{2 c_{n m_{r}} \bar{q}_{n r} J_{10}^{n / 2-1}}
$$

and the tune-spread parameter

$$
\Omega=\frac{c_{40} \bar{q}_{40}}{2 c_{n m_{r}} \bar{q}_{n r} J_{10}^{n / 2-2}} .
$$

This expression has been derived first by Schoch [1] for particle beam dynamics. Because the ratio $R$ describes the variation of the oscillation amplitude in units of the starting amplitude $J_{0}$ we call the quantity $R$ the beat factor of the oscillation.

Before we discuss stop-bands and resonance patterns we make some general observations concerning particle stability. The stability of particle motion in the vicinity of resonances depends strongly on the distance of the tune from the nearest $n$ th-order resonance and on the tune-spread parameter $\Omega$. When both parameters $\Delta$ and $\Omega$ vanish we have no stability for any finite oscillation amplitude, since (16.48) 
can be solved for all values of $\psi_{1}$ only if $R=0$. For a finite tune-spread parameter $\Omega \neq 0$ while $\Delta=0$ (16.48) becomes $R^{2}\left(\Omega+R^{n / 2-2} \cos n \psi_{1}\right)=$ const and resonances of order $n>4$ exhibit some range of stability for amplitudes $R^{n / 2-2}<$ $|\Omega|$. Oscillations in the vicinity of, for example, a quarter resonance are all stable for $|\Omega|>1$ and all unstable for smaller values of the tune-spread parameter $|\Omega|<1$. A finite tune-spread parameter $\Omega$ appears in this case due to an octupolar field and has a stabilizing effect at least for small amplitudes.

For very small oscillation amplitudes $(R \rightarrow 0)$ the oscillating term in (16.48) becomes negligible for $n>4$ compared to the detuning term and the particle trajectory approaches the form of a circle with radius $R$. This well behaved character of particle motion at small amplitudes becomes distorted for resonances of order $n=2$ and $n=3$ in case of small detuning and a finite tune spread parameter. We consider $\Delta=0$ and have

$$
R^{2} \Omega+R^{n / 2} \cos n \psi=\text { const, }
$$

where $n=2$ or $n=3$. For very small amplitudes the quadratic term is negligible and the dominant oscillating term alone is unstable. The amplitude for a particle starting at $R \approx 0$ and $\psi_{1}=0$ grows to large amplitudes as $\psi_{1}$ increases, reaching values which make the quadratic tune-spread term dominant before the trigonometric term becomes negative. The resulting trajectory in phase space becomes a figure of eight for the half-integer resonance as shown in Fig. 16.4.

In the case of a third-order resonance small-amplitude oscillations behave similarly and follow the outline of a clover leave as shown in Fig. 16.5.

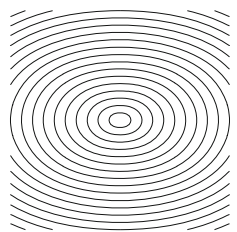

$\Delta=-5, \Omega=0$

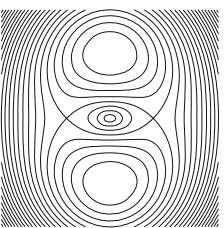

$\Delta=-5, \Omega=1$

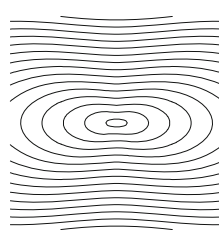

$\Delta=-2, \Omega=0$

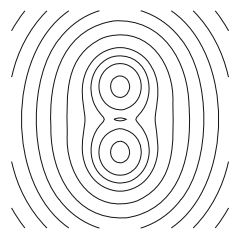

$\Delta=-2, \Omega=1$

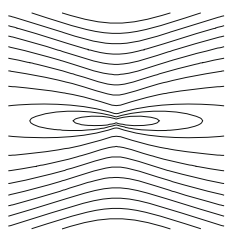

$\Delta=-1.1, \Omega=0 \quad \Delta=0, \Omega=0$

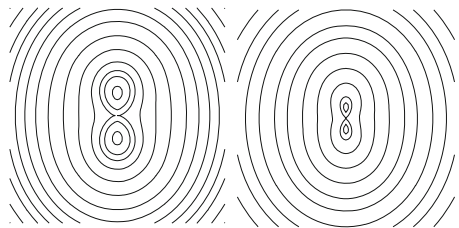

$\Delta=-1.1, \Omega=1$

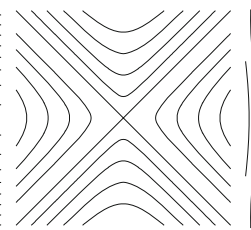

$\Delta=0, \Omega=1$

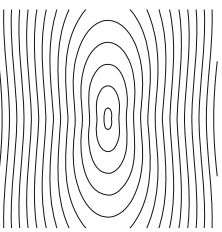

$\Delta=2, \Omega=0$

Fig. $16.4\left(R, \psi_{1}\right)$ phase-space motion for a half-integer resonance. Top row from left to right: $\Omega=0$ and $\Delta=(-5,-2,-1.1,0,2)$; bottom row: $\Omega=1$ and $\Delta=(-5,-2,-1.1,0,2)$ 

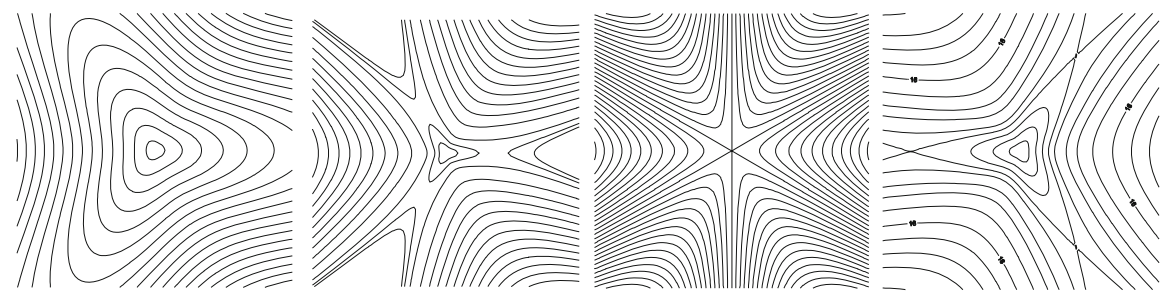

$$
\Delta=-6, \Omega=0
$$

$$
\Delta=-2, \Omega=0
$$$$
\Delta=0, \Omega=0
$$

$\Delta=3, \Omega=0$
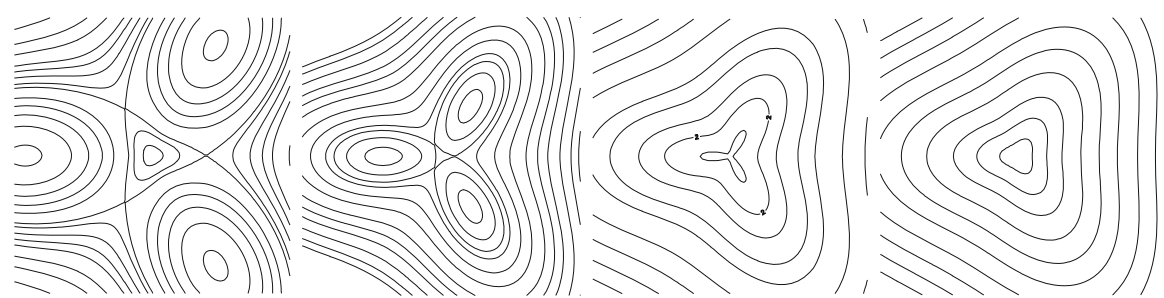

$$
\Delta=-6, \Omega=1
$$

$\Delta=-2, \Omega=1$

$\Delta=0, \Omega=1$

$\Delta=3, \Omega=1$

Fig. 16.5 $\left(R, \psi_{1}\right)$ phase-space motion for a third-order resonance. Top row from left to right: $\Omega=0, \Delta=(-6,-2,0,3)$; bottom row: $\Omega=1, \Delta=(-6,-2,0,3)$

\subsubsection{Half-Integer Stop-Band}

A more detailed discussion of (16.45) will reveal that instability due to resonances does not only happen exactly at resonant tunes. Particle oscillations become unstable within a finite vicinity of resonance lines in the resonance diagram and such areas of instability are known as stop-bands. The most simple case occurs for $\Omega=0$ and a half-integer resonance, where $n=2$ and

$$
R\left(\Delta+\cos 2 \psi_{1}\right)=\text { const } .
$$

For this equation to be true for all values of the angle variable $\psi_{1}$ we require that the quantity in the brackets does not change sign while $\psi_{1}$ varies from 0 to $2 \pi$. This condition cannot be met if $|\Delta| \leq 1$. To quantify this we observe a particle starting with an amplitude $J=J_{0}$ at $\psi_{1}=0$ and (16.52) becomes

$$
R \Delta+R \cos 2 \psi_{1}=\Delta+1 .
$$

Now we calculate the variation of the oscillation amplitude $R$ as the angle variable $\psi_{1}$ increases. The beat factor $R$ reaches its maximum value at $2 \psi_{1}=\pi$ and is

$$
R_{\max }=\frac{\Delta+1}{\Delta-1}>0
$$


The variation of the amplitude $R$ is finite as long as $\Delta>1$. If $\Delta<0$, we get a similar stability condition

$$
R_{\max }=\frac{|\Delta|-1}{|\Delta|+1}>0
$$

and stability occurs for $\Delta<-1$. The complete resonance stability criterion for the half-integer resonance is therefore

$$
|\Delta|>1
$$

Beam instability due to a half-integer resonance $(n=2)$ occurs within a finite vicinity $\Delta v_{r}= \pm 2 c_{2 r} \bar{q}_{2 r}$ as defined by (16.49) and the total stop-band width for a half-integer resonance becomes

$$
\Delta v_{\text {stop }}^{(2)}= \pm 2 c_{2 m_{r}} \bar{q}_{2 r}
$$

The width of the stop-band increases with the strength of the perturbation but does not depend on the oscillation amplitude $J_{0}$. However, for higher-order resonances the stop band width does depend on the oscillation amplitudes as will be discussed later.

To observe the particle trajectories in phase space, we calculate the contour lines for (16.48) setting $n=2$ and obtain patterns as shown in Fig. 16.4. Here the particle trajectories are plotted in the $(\psi, J)$ phase space for a variety of detuning parameters $\Delta$ and tune-spread parameters $\Omega$. Such diagrams are called resonance patterns. The first row of Fig. 16.4 shows particle trajectories for the case of a half-integer resonance with a vanishing tune-spread parameter $\Omega=0$. As the detuning $\Delta$ is increased we observe a deformation of particle trajectories but no appearance of a stable island as long as $|\Delta|<1$. Although we show mostly resonance patterns for negative values of the detuning $\Delta<0$ the patterns look exactly the same for $\Delta>0$ except that they are rotated by $90^{\circ}$. For $|\Delta|>1$ the unstable trajectories part vertically from the origin and allow the appearance of a stable island that grows as the detuning grows. In the second row of resonance patterns, we have included a finite tune-spread parameter of $\Omega=1$ which leads to a stabilization of all large amplitude particle trajectories. Only for small amplitudes do we still recognize the irregularity of a figure of eight trajectory as mentioned above.

\subsubsection{Separatrices}

The appearance of island structures as noticeable from the resonance patterns is a common phenomenon and is due to tune-spread terms of even order like that of an octupole field. In Fig. 16.6 common features of resonance patterns are shown and 


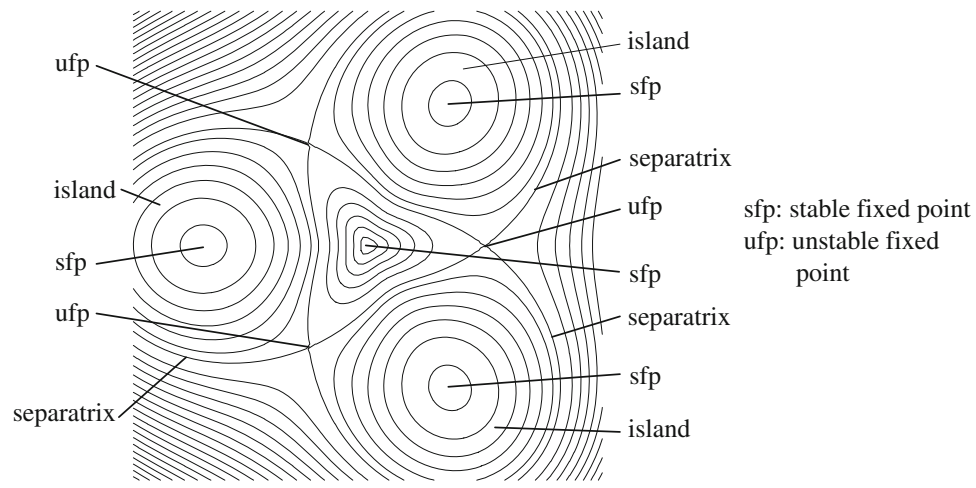

Fig. 16.6 Common features of resonance patterns

we note specifically the existence of a central stable part and islands surrounding the central part.

The boundaries of the areas of stable motion towards the islands are called separatrices. These separatrices also separate the area of stable motion from that for unstable motion. The crossing points of these separatrices, as well as the center of the islands, are called fixed points of the dynamic system and are defined by the conditions

$$
\frac{\partial H_{1}}{\partial \psi_{1}}=0 \quad \text { and } \quad \frac{\partial H_{1}}{\partial J_{1}}=0 .
$$

Application of these conditions to (16.46) defines the location of the fixed points and we find from the first equation (16.58) the azimuthal positions $\psi_{1}=\psi_{\mathrm{f}}$ of the fixed points from

$$
\sin \left(m_{r} \psi_{1 \mathrm{f}}\right)=0
$$

or

$$
m_{r} \psi_{1 \mathrm{f} k}=k \pi
$$

where $k$ is an integer number in the range $0<k<2 m_{r}$. From the second equation (16.58) we get an expression for the radial location of the fixed points $J_{\mathrm{f} k}$

$$
\Delta v_{\mathrm{r}}+2 c_{40} q_{40} J_{\mathrm{f} k}+\frac{n}{2} 2 c_{n m_{r}} \bar{q}_{n r} J_{\mathrm{f} k}^{n / 2-1} \cos (\pi k)=0 .
$$

There are in principle $2 m_{r}$ separate fixed points in each resonance diagram. Closer inspections shows that alternately every second fixed point is a stable fixed point or an unstable fixed point, respectively. The unstable fixed points coincide with the crossing points of separatrices and exist even in the absence of octupole terms. 


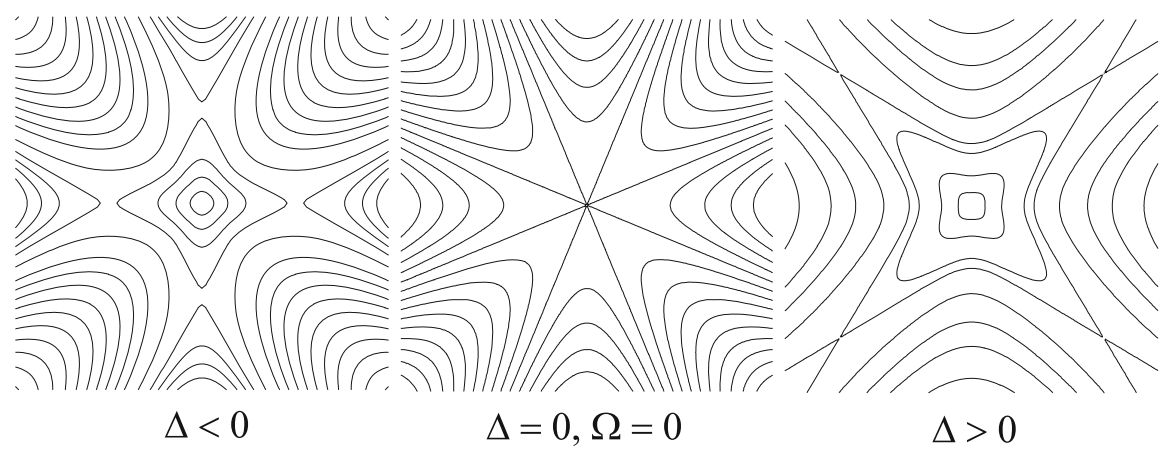

Fig. 16.7 Fourth-order resonance patterns. From left to right: $\Omega=0,(\Delta<0, \Delta=0, \Delta>0)$

Stable fixed points define the center of stable islands and, except for the primary stable fixed point at the origin of the phase diagram, exist only in the presence of a tune spread caused by octupole like terms $c_{n 0} q_{n 0} J^{n / 2}$ in (16.43), which contribute to beam stability. Trajectories that were unstable without the octupole term become closed trajectories within an island area centered at stable fixed points. This island structure is characteristic for resonances since the degree of symmetry is equal to the order of the resonance (see Fig. 16.7).

\subsubsection{General Stop-Band Width}

From the discussion of the half-integer resonance, it became apparent that certain conditions must be met to obtain stability for particle motion. Specifically we expect instability in the vicinity of resonances and we will try to determine quantitatively the area of instability or stop-band width for general resonances. Similar to (16.53) we look for stable solutions from

$$
R \Delta+R^{n / 2} \cos n \psi_{1}=\Delta \pm 1
$$

which describes a particle starting with an amplitude $R=1$. Equation (16.62) must be true along all points of the trajectory and for reasons of symmetry the particle oscillation amplitude approaches again the starting amplitude for $\psi_{1}=0$ as $\psi_{1} \rightarrow$ $2 \pi / n$. Solving for $\Delta$ we get real solutions for $R$ only if

$$
\Delta^{+} \geq-\frac{R^{n / 2}-1}{R-1} \Longrightarrow-\frac{1}{2} n \quad \text { for } \quad R \approx 1
$$


where the index ${ }^{+}$indicates the sign to be used on the r.h.s. of (16.62). Similarly, following a particle starting with $R=1$ at $\psi_{1}=\pi / n$ to $\psi_{1}=3 \pi / n$ we get the condition

$$
\Delta^{-} \leq \frac{1}{2} n .
$$

The total $n$ th-order stop-band width is therefore with (16.49)

$$
\Delta v_{\text {stop }}^{(n)}=n\left|c_{n m_{r}} \bar{q}_{n r}\right| J_{0}^{n / 2-1}
$$

indicating that stable particle motion is possible only for tunes outside this stopband. The stop-band width of nonlinear resonances $(n>2)$ is strongly amplitude dependent and special effort must be exercised to minimize higher-order perturbations. Where higher-order magnetic fields cannot be eliminated it is prudent to minimize the value of the betatron functions at those locations.

Where higher-order magnetic fields cannot be eliminated it is prudent to minimize the value of the betatron functions at those locations. Such a case occurs, for example, in colliding-beam storage rings, where the strongly nonlinear field of one beam perturbs the trajectories of particles in the other beam. This effect is well known as the beam-beam effect.

Through a series of canonical transformations and redefinitions of parameters we seem to have parted significantly from convenient laboratory variables and parameters. We will therefore convert (16.65) back to variables we are accustomed to use. We set $l=r$ and $m_{r}=n$ where $r \approx \frac{n}{N} \nu_{0}$ and tacitly ignored lower-order resonances $m_{r}<n$. From (16.32) we find the Fourier components

$$
\bar{q}_{n r}=\frac{1}{2 \pi} \int_{0}^{2 \pi} \bar{q}_{n}(\varphi) \mathrm{e}^{\mathrm{i} r N \varphi} \mathrm{d} \varphi
$$

and from (16.33) we have $c_{n n}=\frac{1}{2^{n}}$. The amplitude factor $J_{0}^{n / 2-1}$ is replaced by (8.95) which becomes with (5.54a), (5.54b) and $\psi_{1}=0$

$$
J_{0}=\frac{1}{2} v_{0} w_{0}^{2}=\frac{1}{2} v_{0} \frac{x_{0}^{2}}{\beta} .
$$

Finally, we recall the definition (16.29) $q_{n}(\varphi)=-\frac{1}{n} \bar{q}_{n}(\varphi)\left(\frac{\nu_{0}}{2}\right)^{-n / 2}$ and get for the $n$ th-order stop-band width

$$
\Delta v_{\text {stop }}^{(n)}=\frac{w_{0}^{n-2}}{2^{n-1} \pi v_{0}}\left|\int_{0}^{2 \pi} \bar{q}_{n}(\varphi) \mathrm{e}^{\mathrm{i} r N \varphi} \mathrm{d} \varphi\right|,
$$

where $\bar{q}_{n}$ is the $n$ th-order perturbation from Table 16.1. This result is general and includes our earlier finding for the half-integer resonance. For resonances of order $n>2$ the stop-band width increases with amplitude limiting the stability of particle 


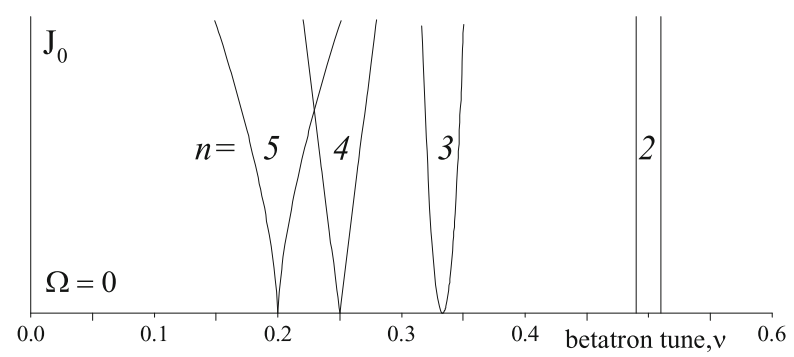

Fig. 16.8 Stop-band width as a function of the amplitude $J_{0}$ for resonances of order $n=2,3,4,5$ and detuning parameter $\Omega=0$

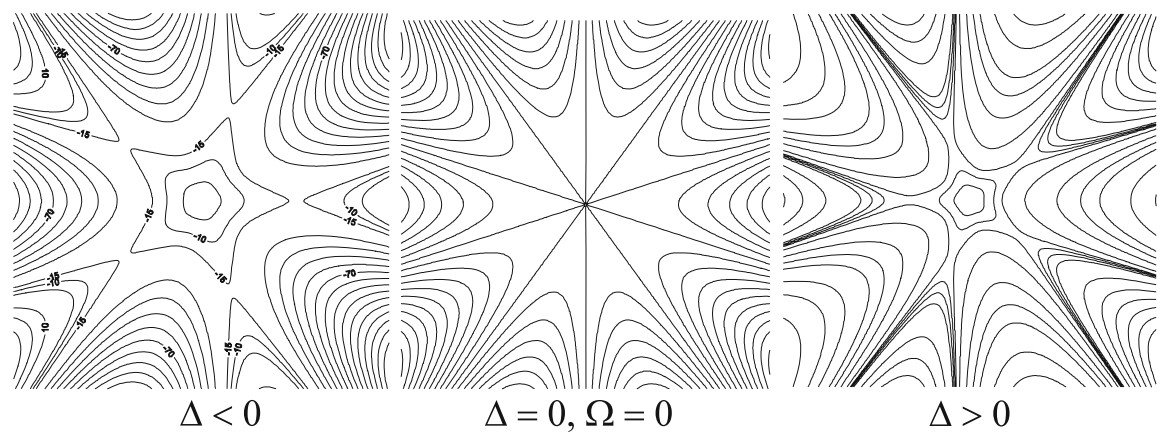

Fig. 16.9 Fifth-order resonance patterns. From left to right: $\Omega=0,(\Delta<0, \Delta=0, \Delta>0)$

beams to the vicinity of the axis (Fig. 16.8). The introduction of sufficiently strong octupole terms can lead to a stabilization of resonances and we found, for example, that the quarter resonance is completely stabilized if $\Omega \geq 1$. For resonances of order $n>4$, however, the term $R^{n / 2} \cos n \psi_{1}$ becomes dominant for large values of the amplitude and resonance therefore cannot be avoided.

Figure 16.9 shows, for example, a stable area for small amplitudes at the fifthorder resonance, as we would expect, but at larger amplitudes the motion becomes unstable.

\subsection{Third-Order Resonance}

The third-order resonance plays a special role in accelerator physics and we will therefore discuss this resonance in more detail. The special role is generated by the need to use sextupoles for chromaticity correction. While such magnets are beneficial in one respect, they may introduce third-order resonances that need to be avoided or at least kept under control. Sometimes the properties of a third-order 
resonance are also used constructively to eject particles at the end of a synchrotron acceleration cycle slowly over many turns.

In the absence of octupole fields the Hamiltonian for the third-order resonance is from (16.46) for $n=3$ and $q_{40}=0$

$$
H_{1}=\Delta v_{1 / 3} J_{1}+\bar{q}_{3 r} J_{1}^{3 / 2} \cos 3 \psi_{1} .
$$

We expand $\cos 3 \psi_{1}=\cos ^{3} \psi_{1}-3 \cos \psi_{1} \sin ^{2} \psi_{1}$ and return to normalized coordinates

$$
w=\sqrt{\frac{2 J_{1}}{v_{0}}} \cos \psi_{1}, \quad \text { and } \quad \dot{w}=\sqrt{2 v_{0} J_{1}} \sin \psi_{1} .
$$

In these coordinates the Hamiltonian reveals the boundaries of the stable region from the unstable resonant region. Introducing the normalized coordinates into (16.69), we get the Hamiltonian

$$
H_{1}=\Delta v_{1 / 3} \frac{v_{0}}{2}\left(w^{2}+\frac{\dot{w}^{2}}{v_{0}^{2}}\right)+\bar{q}_{3 r} \frac{v_{0}^{3 / 2}}{2^{3 / 2}}\left(w^{3}-3 w \frac{\dot{w}^{2}}{v_{0}^{2}}\right)
$$

Dividing by $\bar{q}_{3 r}\left(\frac{\nu_{0}}{2}\right)^{3 / 2}$ and subtracting a constant term $\frac{1}{2} W_{0}^{3}$, where

$$
W_{0}=\frac{4}{3} \frac{\Delta v_{1 / 3}}{\bar{q}_{3 r} \sqrt{2 v_{0}}}
$$

the Hamiltonian assumes a convenient form to exhibit the boundaries between the stable and unstable area

$$
\begin{aligned}
\tilde{H}_{1} & =\frac{3}{2} W_{0}\left(w^{2}+\frac{\dot{w}^{2}}{v_{0}^{2}}\right)+\left(w^{3}-3 w \frac{\dot{w}^{2}}{v_{0}^{2}}\right)-\frac{1}{2} W_{0}^{3} \\
& =\left(w-\frac{1}{2} W_{0}\right)\left(w-\sqrt{3} \frac{\dot{w}}{v_{0}}+W_{0}\right)\left(w+\sqrt{3} \frac{\dot{w}}{v_{0}}+W_{0}\right) .
\end{aligned}
$$

This Hamiltonian has three linear solutions for $\tilde{H}_{1}=0$ defining the separatrices. The resonance plot for (16.73) is shown in Fig. 16.10 where we have assumed that $W_{0}$ is positive. For a given distribution of the sextupoles $\bar{q}_{3 r}$ the resonance pattern rotates by $180^{\circ}$ while moving the tune from one side of the resonance to the other. Clearly, there is a stable central part bounded by separatrices. The area of the central part depends on the strength and distribution of the sextupole fields summarized by $\bar{q}_{3 r}$ and the distance $\Delta v_{1 / 3}$ of the tune from the third-order resonance. 


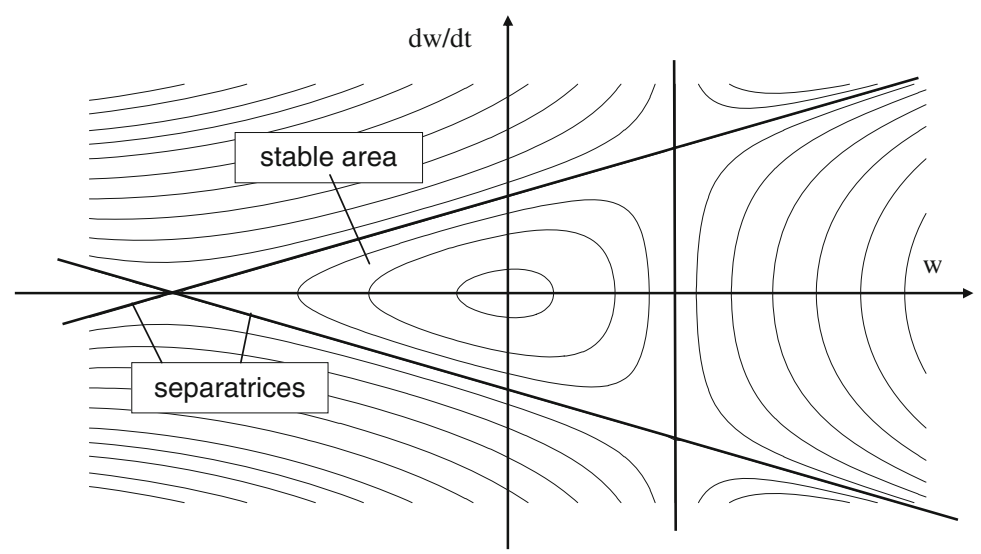

Fig. 16.10 Third-order resonance

The higher-order field perturbation $\bar{q}_{3 r}$ depends on the distribution of the sextupoles around the circular accelerator. In the horizontal plane

$$
\bar{q}_{3}(\varphi)=-v_{0}^{2} \beta^{5 / 2} \frac{1}{2} m .
$$

or with (16.29)

$$
q_{3}(\varphi)=\frac{1}{3} \sqrt{2 v_{0}} \beta^{5 / 2} m .
$$

The Fourier components of this perturbation are given by

$$
q_{3 r}=\frac{1}{2 \pi} \int_{0}^{2 \pi} q_{3}(\varphi) \mathrm{e}^{\mathrm{i} r N \varphi} \mathrm{d} \varphi
$$

and the perturbation term becomes finally with $m_{r}=3$ and $c_{33}=\frac{1}{8}$ from (16.33)

$$
\bar{q}_{3 r}=\frac{\sqrt{2 \nu_{0}}}{24 \pi} \int_{0}^{2 \pi} \beta^{5 / 2} m \mathrm{e}^{\mathrm{i} r N \varphi} \mathrm{d} \varphi
$$

where $\varphi=\int_{0}^{z} \frac{\mathrm{d} \zeta}{\nu_{0} \beta}, m=m(\varphi)$ is the sextupole distribution and $\beta=\beta(\varphi)$ the horizontal betatron function. From this expression, it becomes clear that the perturbation and with it the stable area in phase space depends greatly on the distribution of the sextupoles around the ring. Minimizing the $r$ th Fourier component obviously benefits beam stability. 


\subsubsection{Particle Motion in Phase Space}

It is interesting to study particle motion close to a resonance in some more detail by deriving the equations of motion from the Hamiltonian (16.69). The phase variation is

$$
\frac{\partial H_{1}}{\partial J_{1}}=\frac{\partial \psi_{1}}{\partial \varphi}=\Delta v_{1 / 3}+\frac{3}{2} \bar{q}_{3 r} J_{1}^{1 / 2} \cos 3 \psi_{1}
$$

Now, we follow a particle as it orbits the ring and observe its coordinates every time it passes by the point with phase $\varphi_{0}$ or $\psi_{0}$, which we assume for convenience to be zero. Actually, we observe the particle only every third turn, since we are not interested in the rotation of the resonance pattern in phase space by $120^{\circ}$ every turn.

For small amplitudes the first term is dominant and we note that the particles move in phase space clockwise or counter clockwise depending on $\Delta v_{1 / 3}$ being negative or positive, respectively. The motion becomes more complicated in the vicinity and outside the separatrices, where the second term is dominant. For a particle starting at $\psi_{1}=0$ the phase $\psi_{1}$ increases or decreases from turn to turn and asymptotically approaches $\psi_{1}= \pm 30^{\circ}$ depending on the perturbation $\bar{q}_{3 r}$ being positive or negative, respectively. The particles therefore move clockwise or counter clockwise and the direction of this motion is reversed, whenever we move into an adjacent area separated by separatrices because the trigonometric term has changed sign.

To determine exactly the position of a particle after $3 q$ turns we have with $\psi(q)=$ $3 q \cdot 2 \pi v_{0}$

$$
\psi_{1}(q)=2 \pi\left(3 v_{0}-r N\right) q
$$

With this phase expression we derive the associated amplitude $J_{1 q}$ from the Hamiltonian (16.69) and may plot the particle positions for successive triple turns $3 q=0,3,6,9, \ldots$ in a figure similar to Fig. 16.10. The change in the oscillation amplitude is from the second Hamiltonian equation of motion

$$
\frac{\partial H_{1}}{\partial \psi_{1}}=-\frac{\partial J_{1}}{\partial \varphi}=-3 \bar{q}_{3 r} J_{1}^{3 / 2} \sin 3 \psi_{1}
$$

and is very small in the vicinity of $\psi_{1} \approx 0$ or even multiples of $30^{\circ}$ (for $w>$ separatrix in Fig. 16.10). For $\psi_{1}$ being equal to odd multiples of $30^{\circ}$, on the other hand, the oscillation amplitude changes rapidly as shown in Fig. 16.10 on the left side beyond the crossing point of the separatrixes or beyond the unstable point. 


\section{Problems}

16.1 (S). Consider a simple optimized FODO lattice forming a circular ring. Calculate the natural chromaticity (ignore focusing in bending magnets) and correct the chromaticities to zero by placing thin sextupoles in the center of the quadrupoles. Calculate and plot the horizontal third-order stop-band width as a function of the horizontal tune.

16.2 (S). Show that in (16.33) the coefficients $c_{n 0}$ are non-zero only for even values of $n$.

16.3 (S). Show that in (16.33) the coefficients $c_{n n}=\frac{1}{2^{n}}$.

16.4. Plot a resonance diagram up to fourth order for the PEP lattice with tunes $v_{x}=21.28$ and $v_{y}=18.16$ and a super-periodicity of $N=6$ or any other circular accelerator lattice with multiple super-periodicity. Choose the parameters of the diagram such that a resonance environment for the above tunes of at least \pm 3 ( \pm half the number of superperiods) integers is covered.

16.5. Choose numerical values for parameters of a single multipole in the Hamiltonian (16.45) and plot a resonance diagram $\mathcal{H}(J, \psi)=$ const. Determine the stability limit for your choice of parameters. What would the tolerance on the multipole field perturbation be if you require a stability for an emittance as large as $\epsilon=100 \mathrm{~mm}$ $\operatorname{mrad}$ ?

16.6. Take the lattice of Problem 16.1 and adjust its tune to the third-order resonance so that the unstable fixed point on the symmetry axis are $5 \mathrm{~cm}$ from the beam center. Determine the equations for the separatrices. Choose a point $P$ just outside the stable area and close to the crossing of two separatrices along the symmetry axis. Where in the diagram would a particle starting at $P$ be after 3,6 , and 9 turns? At what amplitude could you place a $5 \mathrm{~mm}$ thin septum magnet to eject the beam from the accelerator?

\section{References}

1. A. Schoch, Theory of linear and nonlinear perturbations of betatron oscillations in alternating gradient synchrotrons. Technical Report CERN 57-23, CERN, CERN, Geneva (1957)

2. G. Guignard, The general theory of all sum and difference resonances in a three dimensional magnetic field in a synchrotron. Technical Report CERN 76-06, CERN, CERN, Geneva (1976)

3. S.Ohnuma, Quarter integer resonance by sextupoles. Technical Report TM-448, FERMI Lab, Batavia, IL (1973) 


\section{Chapter 17 \\ Hamiltonian Nonlinear Beam Dynamics*}

Deviations from linear beam dynamics in the form of perturbations and aberrations play an important role in accelerator physics. Beam parameters, quality and stability are determined by our ability to correct and control such perturbations. Hamiltonian formulation of nonlinear beam dynamics allows us to study, understand and quantify the effects of geometric and chromatic aberrations in higher order than discussed so far. Based on this understanding we may develop correction mechanisms to achieve more and more sophisticated beam performance. We will first discuss higher-order beam dynamics as an extension to the linear matrix formulation followed by specific discussions on aberrations. Finally, we develop the Hamiltonian perturbation theory for particle beam dynamics in accelerator systems.

\subsection{Higher-Order Beam Dynamics}

Chromatic and geometric aberrations appear specifically in strong focusing transport systems designed to preserve carefully prepared beam characteristics. As a consequence of correcting chromatic aberrations by sextupole magnets, nonlinear geometric aberrations are introduced. The effects of both types of aberrations on beam stability must be discussed in some detail. Based on quantitative expressions for aberrations, we will be able to determine criteria for stability of a particle beam.

\subsubsection{Multipole Errors}

The general equations of motion (6.95), (6.96) exhibit an abundance of driving terms which depend on second or higher-order transverse particle coordinates $\left(x, x^{\prime}, y, y^{\prime}\right)$ or linear and higher-order momentum errors $\delta$. Magnet alignment and

This chapter has been made Open Access under a CC BY 4.0 license. For details on rights and licenses please read the Correction https://doi.org/10.1007/978-3-319-18317-6_28 
field errors add another multiplicity to these perturbation terms. Although the designers of accelerator lattices and beam guidance magnets take great care to minimize undesired field components and avoid focusing systems that can lead to large transverse particle deviations from the reference orbit, we cannot completely ignore such perturbation terms.

In previous sections we have discussed the effect of some of these terms and have derived basic beam dynamics features as the dispersion function, orbit distortions, chromaticity and tune shifts as a consequence of particle momentum errors or magnet alignment and field errors. More general tools are required to determine the effect of any arbitrary driving term on the particle trajectories. In developing such tools we will assume a careful design of the accelerator under study in layout and components so that the driving terms on the r.h.s. of (6.95), (6.96) can be treated truly as perturbations. This may not be appropriate in all circumstances in which cases numerical methods need to be applied. For the vast majority of accelerator physics applications it is, however, appropriate to treat these higher-order terms as perturbations.

This assumption simplifies greatly the mathematical complexity. Foremost, we can still assume that the general equations of motion are linear differential equations. We may therefore continue to treat every perturbation term separately as we have done so before and use the unperturbed solutions for the amplitude factors in the perturbation terms. The perturbations are reduced to functions of the location $z$ along the beam line and the relative momentum error $\delta$ only and such differential equations can be solved analytically as we will see. Summing all solutions for the individual perturbations finally leads to the composite solution of the equation of motion in the approximation of small errors.

The differential equations of motion (6.95), (6.96) can be expressed in a short form by

$$
u^{\prime \prime}+K(z) u=\sum_{\mu, \nu, \sigma, \rho, \tau \geq 0} p_{\mu \nu \sigma \rho \tau}(z) x^{\mu} x^{\prime \nu} y^{\sigma} y^{\prime \rho} \delta^{\tau}
$$

where $u=x$ or $u=y$ and the quantities $p_{\mu \nu \sigma \rho \tau}(z)$ represent the coefficients of perturbation terms. The same form of equation can be used for the vertical plane but we will restrict the discussion to only one plane neglecting coupling effects.

Some of the perturbation terms $p_{\mu \nu \sigma \rho \tau}$ can be related to aberrations known from geometrical light optics. Linear particle beam dynamics and Gaussian geometric light optics works only for paraxial beams where the light rays or particle trajectories are close to the optical axis or reference path. Large deviations in amplitude, as well as fast variations of amplitudes or large slopes, create aberrations in the imaging process leading to distortions of the image known as spherical aberrations, coma, distortions, curvature and astigmatism. While corrections of such aberrations are desired, the means to achieve corrections in particle beam dynamics are different from those used in light optics. Much of the theory of particle beam dynamics is devoted to diagnose the effects of aberrations on particle beams and to develop and apply such corrections. 
The transverse amplitude $x$ can be separated into its components which under the assumptions made are independent from each other

$$
x=x_{\beta}+x_{0}+x_{\delta}+\sum x_{\mu \nu \sigma \rho \tau}
$$

The first three components of solution (17.2) have been derived earlier and are associated with specific lowest order perturbation terms:

$x_{\beta}(z)$ is the betatron oscillation amplitude and general solution of the homogeneous differential equation of motion with vanishing perturbations $p_{\mu \nu \sigma \rho \tau}=0$ for all indices.

$x_{\mathrm{c}}(z)$ is the orbit distortion and is a special solution caused by amplitude and momentum independent perturbation terms like dipole field errors or displacements of quadrupoles or higher multipoles causing a dipole-field error. The relevant perturbations are characterized by $\mu=\nu=\sigma=\rho=\tau=0$ but otherwise arbitrary values for the perturbation $p_{00000}$. Note that in the limit $p_{00000} \rightarrow 0$ we get the ideal reference path or reference orbit $x_{\mathrm{c}}(z)=0$.

$x_{\delta}(z)$ is the chromatic equilibrium orbit for particles with an energy different from the ideal reference energy, $\delta \neq 0$, and differs from the reference orbit with or without distortion $x_{\mathrm{c}}(z)$ by the amount $x_{\delta}(z)$ which is proportional to the dispersion function $\eta(z)$ and the relative momentum deviation $\delta, x_{\delta}(z)=\eta(z) \delta$. In this case $\mu=\nu=\sigma=\rho=0$ and $\tau=1$.

All other solutions $x_{\mu \nu \sigma \rho \tau}$ are related to remaining higher-order perturbations. The perturbation term $p_{10000}$, for example, acts just like a quadrupole and may be nothing else but a quadrupole field error causing a tune shift and a variation in the betatron oscillations. Other terms, like $p_{00100}$ can be correlated with linear coupling or with chromaticity if $p_{10001} \neq 0$. Sextupole terms $p_{20000}$ are used to compensate chromaticities, in which case the amplitude factor $x^{2}$ is expressed by the betatron motion and chromatic displacement

$$
x^{2} \approx\left(x_{\beta}+x_{\delta}\right)^{2}=\left(x_{\beta}+\eta \delta\right)^{2} \Longrightarrow 2 \eta x_{\beta} \delta .
$$

The $x_{\beta}^{2}$-term, which we neglected while compensating the chromaticity, is the source for geometric aberrations due to sextupolar fields becoming strong for large oscillation amplitudes and the $\eta^{2} \delta^{2}$-term contributes to higher-order solution of the $\eta$-function. We seem to make arbitrary choices about which perturbations to include in the analysis. Generally therefore only such perturbations are included in the discussion which are most appropriate to the problem to be investigated and solved. If, for example, we are only interested in the orbit distortion $x_{\mathrm{c}}$, we ignore in lowest order of approximation the betatron oscillation $x_{\beta}$ and all chromatic and higher-order terms. Should, however, chromatic variations of the orbit be of interest one would evaluate the corresponding component separately. On the other hand, if we want to calculate the chromatic variation of betatron oscillations, we need to include the betatron oscillation amplitudes as well as the off momentum orbit $x_{\delta}$. 
In treating higher-order perturbations we make an effort to include all perturbations that contribute to a specific aberration to be studied or to define the order of approximation used if higher-order terms are to be ignored. A careful inspection of all perturbation terms close to the order of approximation desired is prudent to ensure that no significant term is missed. Conversely such an inspection might very well reveal correction possibilities. An example is the effect of chromaticity which is generated by quadrupole field errors for off momentum particles but can be compensated by sextupole fields at locations where the dispersion function is finite. Here the problem is corrected by fields of a different order from those causing the chromaticity.

To become more quantitative we discuss the analytical solution of (17.1). Since in our approximation this solution is the sum of all partial solutions for each individual perturbation term, the problem is solved if we find a general solution for an arbitrary perturbation. The solution of, for example, the horizontal equation of motion

$$
x^{\prime \prime}+K(z) x=p_{\mu \nu \sigma \rho \tau} x^{\mu} x^{\prime \nu} y^{\sigma} y^{\prime \rho} \delta^{\tau}
$$

can proceed in two steps. First we replace the oscillation amplitudes on the r.h.s. by their most significant components

$$
\begin{aligned}
& x^{\mu} \rightarrow\left(x_{\beta}+x_{0}+x_{\delta}\right)^{\mu}, \quad x^{\prime \nu} \rightarrow\left(x_{\beta}^{\prime}+x_{0}^{\prime}+x_{\delta}^{\prime}\right)^{\nu}, \\
& y^{\sigma} \rightarrow\left(y_{\beta}+y_{0}+y_{\delta}\right)^{\sigma}, \quad y^{\prime \rho} \rightarrow\left(y_{\beta}^{\prime}+y_{0}^{\prime}+y_{\delta}^{\prime}\right)^{\rho} .
\end{aligned}
$$

As discussed before, in a particular situation only those components are eventually retained that are significant to the problem. Since most accelerators are constructed in the horizontal plane we may set the vertical dispersion $y_{\delta}=0$. The decomposition (17.5) is inserted into the r.h.s of (17.4) and again only terms significant for the particular problem and to the approximation desired are retained. The solution $x_{\mu \nu \sigma \rho \tau}$ can be further broken down into components each relating to only one individual perturbation term. Whatever number of perturbation terms we decide to keep, the basic differential equation for the perturbation is of the form

$$
P^{\prime \prime}+K(z) P=p\left(x_{\beta}, x_{\beta}^{\prime}, x_{\mathrm{c}}, x_{\mathrm{c}}^{\prime}, x_{\delta}, x_{\delta}^{\prime}, y_{\beta}, y_{\beta}^{\prime}, y_{\mathrm{c}}, y_{\mathrm{c}}^{\prime}, y_{\delta}, y_{\delta}^{\prime}, \delta, z\right) \text {, }
$$

for which we have discussed the solution in Sect. 5.5.4. Following these steps we may calculate, at least in principle, the perturbations $P(z)$ for any arbitrary higherorder driving term $p(z)$. In praxis, however, even principal solutions of particle trajectories in composite beam transport systems can be expressed only in terms of the betatron functions. Since the betatron functions cannot be expressed in a convenient analytical form, we are unable to obtain an analytical solution and must therefore employ numerical methods. 


\subsubsection{Non-linear Matrix Formalism}

In linear beam dynamics this difficulty has been circumvented by the introduction of transformation matrices, a principle which can be used also for beam transport systems including higher-order perturbation terms. This non-linear matrix formalism was developed by Karl Brown [1-3] and we follow his reasoning in the discussion here. The solution to (17.1) can be expressed in terms of initial conditions. Similar to discussions in the context of linear beam dynamics we solve (17.6) for individual lattice elements only where $K(z)=$ const. In this case (5.75) can be solved for any piecewise constant perturbation along a beam line. Each solution depends on initial conditions at the beginning of the magnetic element and the total solution can be expressed in the form

$$
\begin{aligned}
& x(z)=c_{110} x_{0}+c_{120} x_{0}^{\prime}+c_{130} \delta_{0}+c_{111} x_{0}^{2}+c_{112} x_{0} x_{0}^{\prime}+\ldots, \\
& x^{\prime}(z)=c_{210} x_{0}+c_{220} x_{0}^{\prime}+c_{230} \delta_{0}+c_{211} x_{0}^{2}+c_{212} x_{0} x_{0}^{\prime}+\ldots,
\end{aligned}
$$

where the coefficients $c_{i j k}$ are functions of $z$. The nomenclature of the indices becomes obvious if we set $x_{1}=x, x_{2}=x^{\prime}$, and $x_{3}=\delta$. The coefficient $c_{i j k}$ then determines the effect of the perturbation term $x_{j} x_{k}$ on the variable $x_{i}$. In operator notation we may write

$$
c_{i j k}=\left\langle x_{i} \mid x_{j 0} x_{k 0}\right\rangle
$$

The first-order coefficients are the principal solutions

$$
\begin{aligned}
& c_{110}(z)=C(z), \quad c_{210}(z)=C^{\prime}(z), \\
& c_{120}(z)=S(z), \quad c_{220}(z)=S^{\prime}(z), \\
& c_{130}(z)=D(z), \quad c_{230}(z)=D^{\prime}(z) .
\end{aligned}
$$

Before continuing with the solution process, we note that the variation of the oscillation amplitudes $\left(x^{\prime}, y^{\prime}\right)$ are expressed in a curvilinear coordinate system generally used in beam dynamics. This definition, however, is not identical to the intuitive assumption that the slope $x^{\prime}$ of the particle trajectory is equal to the angle $\Theta$ between the trajectory and reference orbit. In a curvilinear coordinate system the slope $x^{\prime}=\mathrm{d} x / \mathrm{d} z$ is a function of the amplitude $x$. To clarify the transformation, we define angles between the trajectory and the reference orbit by

$$
\frac{\mathrm{d} x}{\mathrm{~d} s}=\Theta \quad \text { and } \quad \frac{\mathrm{d} y}{\mathrm{~d} s}=\Phi,
$$

where

$$
\mathrm{d} s=(1+\kappa x) \mathrm{d} z
$$


In linear beam dynamics there is no numerical difference between $x^{\prime}$ and $\Theta$ which is a second-order effect nor is there a difference in straight parts of a beam transport line where $\kappa=0$. The relation between both definitions is from (17.10), (17.11)

$$
\Theta=\frac{x^{\prime}}{1+\kappa x} \quad \text { and } \quad \Phi=\frac{y^{\prime}}{1+\kappa x},
$$

where $x^{\prime}=\mathrm{d} x / \mathrm{d} z$ and $y^{\prime}=\mathrm{d} y / \mathrm{d} z$. We will use these definitions and formulate second-order transformation matrices in a Cartesian coordinate system $(x, y, z)$. Following Brown's notation [1], we may express the nonlinear solutions of (17.4) in the general form

$$
u_{i}=\sum_{j=1}^{3} c_{i j 0} u_{j 0}+\sum_{\substack{j=1 \\ k=1}}^{3} T_{i j k}(z) u_{j 0} u_{k 0},
$$

with

$$
\left(u_{1}, u_{2}, u_{3}\right)=(x, \Theta, \delta),
$$

where $z$ is the position along the reference particle trajectory. Nonlinear transformation coefficients $T_{i j k}$ are defined similar to coefficients $\mathrm{c}_{i j k}$ in (17.8) by

$$
T_{i j k}=\left\langle u_{i} \mid u_{j 0} u_{k 0}\right\rangle,
$$

where the coordinates are defined by (17.14). In linear approximation both coefficients are numerically the same and we have

$$
\left(\begin{array}{lll}
c_{110} & c_{120} & c_{130} \\
c_{210} & c_{220} & c_{230} \\
c_{310} & c_{320} & c_{330}
\end{array}\right)=\left(\begin{array}{ccc}
C(z) & S(z) & D(z) \\
C^{\prime}(z) & S^{\prime}(z) & D^{\prime}(z) \\
0 & 0 & 1
\end{array}\right) .
$$

Earlier in this section we decided to ignore coupling effects which could be included easily in (17.13) if we set for example $x_{4}=y$ and $x_{5}=y^{\prime}$ and expand the summation in (17.13) to five indices. For simplicity, however, we will continue to ignore coupling.

The equations of motion (6.95), (6.96) are expressed in curvilinear coordinates and solving (5.75) results in coefficients $c_{i j k}$ which are different from the coefficients $T_{i j k}$ if one or more variables are derivatives with respect to $z$. In the equations of motion all derivatives are transformed like (17.12) generating a $\Theta$-term as well as an $x \Theta$-term. If, for example, we were interested in the perturbations to the particle amplitude $x$ caused by perturbations proportional to $x_{0} \Theta_{0}$, we are looking for the coefficient $T_{112}=\left\langle x \mid x_{0} \Theta_{0}\right\rangle$. Collecting from (6.95) only second-order perturbation 
terms proportional to $x x^{\prime}$, we find

$$
x=c_{112} x_{0} x_{0}^{\prime}=c_{112} x_{0} \Theta_{0}+\mathcal{O}(3) .
$$

An additional second-order contribution appears as a spill over from the linear transformation

$$
x=c_{120} x_{0}^{\prime}=c_{120}\left(1+\kappa_{x} x_{0}\right) \Theta_{0} .
$$

Collecting all $x_{0} \Theta_{0}$-terms, we get finally

$$
T_{112}=c_{112}+c_{120} \kappa_{x}=c_{112}+\kappa_{x} S(z) .
$$

To derive a coefficient like $T_{212}=\left\langle\Theta \mid x_{0} \Theta_{0}\right\rangle$ we also have to transform the derivative of the particle trajectory at the end of the magnetic element. First, we look for all contributions to $x^{\prime}$ from $x_{0} x_{0}^{\prime}$-terms which originate from $x^{\prime}=c_{220} x_{0}^{\prime}+$ $c_{212} x_{0} x_{0}^{\prime}$. Setting in the first term $x_{0}^{\prime}=\Theta_{0}\left(1+\kappa_{x} x_{0}\right)$ and in the second term $x_{0} x_{0}^{\prime} \approx$ $x_{0} \Theta_{0}$, we get with $c_{220}=S^{\prime}(z)$ and keeping again only second-order terms

$$
x^{\prime}=\left[c_{212}+\kappa_{x} S^{\prime}(z)\right] x_{0} \Theta_{0} .
$$

On the 1.h.s. we replace $x^{\prime}$ by $\Theta\left(1+\kappa_{x} x\right)$ and using the principal solutions we get

$$
x \Theta \approx\left(C_{x} x_{0}+S_{x} \Theta_{0}\right)\left(C_{x}^{\prime} x_{0}+S_{x}^{\prime} \Theta_{0}\right)=\left(C_{x} S_{x}^{\prime}+C_{x}^{\prime} S_{x}\right) x_{0} \Theta_{0}
$$

keeping only the $x_{0} \Theta$-terms. Collecting all results, the second-order coefficient for this perturbation becomes

$$
T_{212}=\left\langle\Theta \mid x_{0} \Theta_{0}\right\rangle=c_{212}+\kappa_{x} S^{\prime}(z)-\kappa_{x}\left(C_{x} S_{x}^{\prime}+C_{x}^{\prime} S_{x}\right)
$$

In a similar way we can derive all second-order coefficients $T_{i j k}$. Equations (17.13) define the transformation of particle coordinates in second order through a particular magnetic element. For the transformation of quadratic terms we may ignore the third order difference between the coefficients $c_{i j k}$ and $T_{i j k}$ and get

$$
\begin{aligned}
& x^{2}=\left(C_{x} x_{0}+S_{x} x_{0}^{\prime}+D_{x} \delta_{0}\right)^{2}, \\
& x x^{\prime}=\left(C_{x} x_{0}+S_{x} x_{0}^{\prime}+D_{x} \delta_{0}\right)\left(C_{x}^{\prime} x_{0}+S_{x}^{\prime} x_{0}^{\prime}+D_{x}^{\prime} \delta_{0}\right) \\
& x \delta=\left(C_{x} x_{0}+S_{x} x_{0}^{\prime}+D_{x} \delta_{0}\right) \delta_{0} \\
& \vdots \quad \text { etc. }
\end{aligned}
$$


All transformation equations can now be expressed in matrix form after correctly ordering equations and coefficients and a general second-order transformation matrix can be formulated in the form

$$
\left(\begin{array}{c}
x \\
\Theta \\
\delta \\
x^{2} \\
x \Theta \\
x \delta \\
\Theta^{2} \\
\Theta \delta \\
\delta^{2}
\end{array}\right)=\mathcal{M}\left(\begin{array}{c}
x_{0} \\
\Theta_{0} \\
\delta_{0} \\
x_{0}^{2} \\
x_{0} \Theta_{0} \\
x_{0} \delta_{0} \\
\Theta_{0}^{2} \\
\Theta_{0} \delta_{0} \\
\delta_{0}^{2}
\end{array}\right),
$$

where we have ignored the $y$-plane. The second-order transformation matrix is then

$$
\begin{gathered}
\mathcal{M}= \\
\left(\begin{array}{ccccccccc}
C & S & D & T_{111} & T_{112} & T_{116} & T_{122} & T_{126} & T_{166} \\
C^{\prime} & S^{\prime} & D^{\prime} & T_{211} & T_{212} & T_{216} & T_{222} & T_{226} & T_{266} \\
0 & 0 & 1 & 0 & 0 & 0 & 0 & 0 & 0 \\
0 & 0 & 0 & C^{2} & 2 C S & 2 C D & S^{2} & 2 S D & D^{2} \\
0 & 0 & 0 & C C^{\prime} & C S^{\prime}+C^{\prime} S & C D^{\prime}+C^{\prime} D & S S^{\prime} & S D^{\prime}+S^{\prime} D & D D^{\prime} \\
0 & 0 & 0 & 0 & 0 & C & 0 & S & D \\
0 & 0 & 0 & C^{\prime 2} & 2 C^{\prime} S^{\prime} & 2 C^{\prime} D & S^{\prime 2} & 2 S^{\prime} D^{\prime} & D^{\prime 2} \\
0 & 0 & 0 & 0 & 0 & C^{\prime} & 0 & S^{\prime} & D^{\prime} \\
0 & 0 & 0 & 0 & 0 & 0 & 0 & 0 & 1
\end{array}\right)
\end{gathered}
$$

with $C=C_{x}, S=S_{x}, \ldots$ etc.

A similar equation can be derived for the vertical plane. If coupling effects are to be included the matrix could be further expanded to include also such terms. While the matrix elements must be determined individually for each magnetic element in the beam transport system, we may in analogy to linear beam dynamics multiply a series of such matrices to obtain the transformation matrix through the whole composite beam transport line. As a matter of fact the transformation matrix has the same appearance as (17.24) for a single magnet or a composite beam transport line and the magnitude of the nonlinear matrix elements will be representative of imaging errors like spherical and chromatic aberrations.

To complete the derivation of second-order transformation matrices we derive, as an example, an expression of the matrix element $T_{111}$ from the equation of motion (6.95). To obtain all $x_{0}^{2}$-terms, we look in (6.95) for perturbation terms proportional to $x^{2}, x x^{\prime}$ and $x^{\prime 2}$, replace these amplitude factors by principal solutions 
and collect only terms quadratic in $x_{0}$ to get the relevant perturbation

$$
p(z)=\left[-\left(\frac{1}{2} m+2 \kappa_{x} k+\kappa_{x}^{3}\right) C_{x}^{2}+\frac{1}{2} \kappa_{x} C_{x}^{\prime 2}+\kappa_{x}^{\prime} C_{x} C_{x}^{\prime}\right] x_{0}^{2} .
$$

First, we recollect that the theory of nonlinear transformation matrices is based on the constancy of magnet strength parameters and we set therefore $\kappa_{x}^{\prime}=0$. Where this is an undue simplification like in magnet fringe fields one could approximate the smooth variation of $\kappa_{x}$ by a step function. Inserting (17.26) into (5.75) the secondorder matrix element

$$
\begin{aligned}
c_{111} & =T_{111} \\
& =-\left(\frac{1}{2} m+2 \kappa_{x} k+\kappa_{x}^{3}\right) \int_{0}^{z} C_{x}^{2}(\zeta) G(z, \zeta) \mathrm{d} \zeta-\frac{1}{2} \kappa_{x} \int_{0}^{z} C_{x}^{\prime 2}(\zeta) G(z, \zeta) \mathrm{d} \zeta .
\end{aligned}
$$

The integrands are powers of trigonometric functions and can be evaluated analytically. In a similar way we may now derive any second-order matrix element of interest. A complete list of all second order matrix elements can be found in [1].

This formalism is valuable whenever the effect of second-order perturbations must be evaluated for particular particle trajectories. Specifically, it is suitable for nonlinear beam simulation studies where a large number of particles representing the beam are to be traced through nonlinear focusing systems to determine, for example, the particle distribution and its deviation from linear beam dynamics at a focal point. This formalism is included in the program TRANSPORT [4] allowing the determination of the coefficients $T_{i j k}$ for any beam transport line and providing fitting routines to eliminate such coefficients by proper adjustment and placement of nonlinear elements like sextupoles.

\subsection{Aberrations}

From light optics we are familiar with the occurrence of aberrations which cause the distortion of optical images. We have repeatedly noticed the similarity of particle beam optics with geometric or paraxial light optics and it is therefore not surprising that there is also a similarity in imaging errors. Aberrations in particle beam optics can cause severe stability problems and must therefore be controlled.

We distinguish two classes of aberrations, geometric aberrations and for off momentum particles chromatic aberrations. The geometric aberrations become significant as the amplitude of betatron oscillations increases while chromatic aberration results from the variation of the optical system parameters for different colors of the light rays or in our case for different particle energies. For the discussion of salient features of aberration in particle beam optics we study the equation of motion in the horizontal plane and include only bending magnets, quadrupoles and sextupole magnets. The equation of motion in this case becomes 
in normalized coordinates

$$
\ddot{w}+v_{0}^{2} w=v_{0}^{2} \beta^{3 / 2} \kappa \delta+v_{0}^{2} \beta^{2} k w \delta-\frac{1}{2} v_{0}^{2} \beta^{5 / 2} m w^{2},
$$

where $\beta=\beta_{x}$. The particle deviation $w$ from the ideal orbit is composed of two contributions, the betatron oscillation amplitude $w_{\beta}$ and the shift in the equilibrium orbit for particles with a relative momentum error $\delta$. This orbit shift $w_{\delta}$ is determined by the normalized dispersion function at the location of interest $\left(w_{\delta}=\tilde{\eta} \delta=\frac{\eta}{\sqrt{\beta}} \delta\right)$ and the particle position can be expressed by the composition

$$
w=w_{\beta}+w_{\delta}=w_{\beta}+\tilde{\eta} \delta .
$$

Inserting (17.29) into (17.28) and employing the principle of linear superposition (17.28) can be separated into two differential equations, one for the betatron motion and one for the dispersion function neglecting quadratic or higher-order terms in $\delta$. The differential equation for the dispersion function is then

$$
\ddot{\tilde{\eta}}+v_{0}^{2} \tilde{\eta}=v_{0}^{2} \beta^{1 / 2} \kappa+v_{0}^{2} \beta^{2} k \tilde{\eta} \delta-\frac{1}{2} v_{0}^{2} \beta^{5 / 2} m \tilde{\eta}^{2} \delta,
$$

which has been solved earlier in Sect. 9.4.1. All other terms include the betatron oscillation $w_{\beta}$ and contribute therefore to aberrations of betatron oscillations expressed by the differential equation

$$
\ddot{w}_{\beta}+v_{0}^{2} w_{\beta}=v_{0}^{2} \beta^{2} k w_{\beta} \delta-v_{0}^{2} \beta^{2} m \eta w_{\beta} \delta-\frac{1}{2} v_{0}^{2} \beta^{5 / 2} m w_{\beta}^{2} .
$$

The third term in (17.31) is of geometric nature causing a perturbation of beam dynamics at large betatron oscillation amplitudes and, as will be discussed in Sect. 17.3, also gives rise to an amplitude dependent tune shift. This term appears as an isolated term in second order and no local compensation scheme is possible. Geometric aberrations must therefore be expected whenever sextupole magnets are used to compensate for chromatic aberrations.

The first two terms in (17.31) represent the natural chromaticity from quadrupoles and the compensation by sextupole magnets, respectively. Whenever it is possible to compensate the chromaticity at the location where it occurs both terms would cancel for $m \eta=k$. Since the strength changes sign for both magnets going from one plane to the other the compensation is correct in both planes. This method of chromaticity correction is quite effective in long beam transport systems with many equal lattice cells. An example of such a correction scheme are the beam transport lines from the SLAC linear accelerator to the collision point of the Stanford Linear Collider, SLC, [5]. This transport line consists of a dense sequence of strong magnets forming a combined function FODO channel (for parameters see example \#2 in Table 10.1). In these magnets dipole, quadrupole and sextupole components are combined in the pole profile and the chromaticity compensation occurs locally. 
This method of compensation, however, does not work generally in circular accelerators because of special design criteria which often require some parts of the accelerator to be dispersion free and the chromaticity created by the quadrupoles in these sections must then be corrected elsewhere in the lattice. Consequently both chromaticity terms in (17.31) do not cancel anymore locally and can be adjusted to cancel only globally.

The consequence of these less than perfect chromaticity correction schemes is the occurrence of aberrations through higher-order effects. We get a deeper insight for the effects of these aberrations in a circular accelerator by noting that the coefficients of the betatron oscillation amplitude $w_{\beta}$ for both chromatic perturbations are periodic functions in a circular accelerator and can therefore be expanded into a Fourier series. Only non-oscillatory terms of these expansions cancel if the chromaticity is corrected while all other higher harmonics still appear as chromatic aberrations.

\subsubsection{Geometric Aberrations}

Geometric perturbations from sextupole fields scale proportional to the square of the betatron oscillation amplitude leading to a loss of stability for particles oscillating at large amplitudes. From the third perturbation term in (17.31) we expect this limit to occur at smaller amplitudes in circular accelerators where either the betatron functions are generally large or where the focusing and therefore the chromaticity and required sextupole correction is strong or where the tunes are large. Most generally this occurs in large proton and electron colliding-beam storage rings or in electron storage rings with strong focusing.

\section{Compensation of Nonlinear Perturbations}

In most older circular accelerators the chromaticity is small and can be corrected by two families of sextupoles. Although in principle only two sextupole magnets for the whole ring are required for chromaticity compensation, this is in most cases impractical since the strength of the sextupoles becomes too large exceeding technical limits or leading to loss of beam stability because of intolerable geometric aberrations. For chromaticity compensation we generally choose a more even distribution of sextupoles around the ring and connect them into two families compensating the horizontal and vertical chromaticity, respectively. This scheme is adequate for most not too strong focusing circular accelerators. Where beam stability suffers from geometric aberrations more sophisticated sextupole correction schemes must be utilized.

To analyze the geometric aberrations due to sextupoles and develop correction schemes we follow a particle along a beam line including sextupoles. Here we understand a beam line to be an open system from a starting point to an image point 
Fig. 17.1 Linear particle motion in phase space

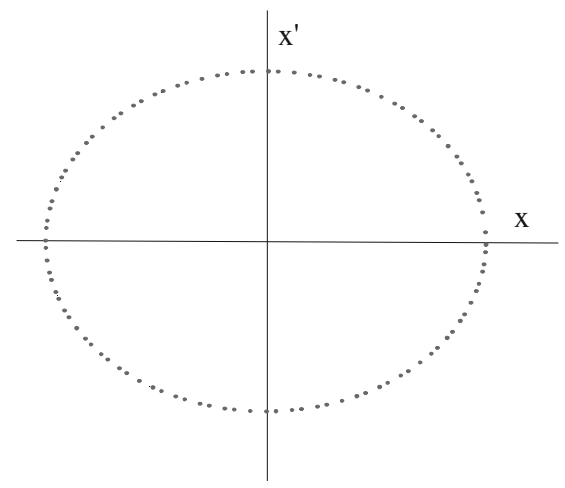

Fig. 17.2 Typical phase space motion in the presence of nonlinear fields

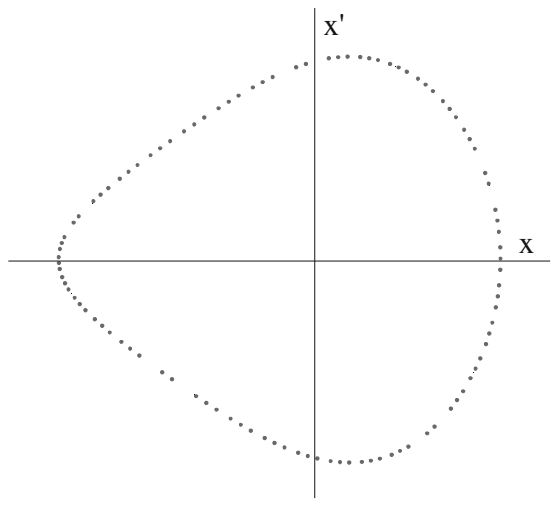

at the end or one full circumference of a circular accelerator. Following any particle through the beam line and ignoring for the moment nonlinear fields we expect the particle to move along an ellipse in phase space as shown in Fig. 17.1. Travelling through the length of a circular accelerator with phase advance $\psi=2 \pi v_{0}$ a particle moves $v_{0}$ revolutions around the phase ellipse in Fig. 17.1.

Including nonlinear perturbations due to, for example, sextupole magnets the phase space trajectory becomes distorted from the elliptical form as shown in Fig. 17.2. An arbitrary distribution of sextupoles along a beam line can cause large variations of the betatron oscillation amplitude leading to possible loss of particles on the vacuum chamber wall even if the motion is stable in principle. The PEP storage ring [6] was the first storage ring to require a more sophisticated sextupole correction [7] beyond the mere compensation of the two chromaticities because geometric aberrations were too strong to give sufficient beam stability. Chromaticity correction with only two families of sextupoles in PEP would have produced large amplitude dependent tune shifts leading to reduced beam stability.

Such a situation can be greatly improved with additional sextupole families [7] to minimize the effect of these nonlinear perturbation. Although individual 
perturbations may not be reduced much by this method the sum of all perturbations can be compensated to reduce the overall perturbation to a tolerable level.

In this sextupole correction scheme the location and strength of the individual sextupoles are selected such as to minimize the perturbation of the particle motion in phase space at the end of the beam transport line. Although this correction scheme seems to work in not too extreme cases it is not sufficient to guarantee beam stability. This scheme works only for one amplitude due to the nonlinearity of the problem and in cases where sextupole fields are no longer small perturbations we must expect a degradation of this compensation scheme for larger amplitudes. As the example of PEP shows, however, an improvement of beam stability can be achieved beyond that obtained by a simple two family chromaticity correction. Clearly, a more formal analysis of the perturbation and derivation of appropriate correction schemes are desirable.

\section{Sextupoles Separated by a $-\mathcal{I}$-Transformation}

A chromaticity correction scheme that seeks to overcomes this amplitude dependent aberration has been proposed by Brown and Servranckx [8]. In this scheme possible sextupole locations are identified in pairs along the beam transport line such that each pair is separated by a negative unity transformation

$$
-\mathcal{I}=\left(\begin{array}{cccc}
-1 & 0 & 0 & 0 \\
0 & -1 & 0 & 0 \\
0 & 0 & -1 & 0 \\
0 & 0 & 0 & -1
\end{array}\right)
$$

Placing sextupoles of equal strength at these two locations we get an additive contribution to the chromaticity correction. The effect of geometric aberrations, however, is canceled for all particle oscillation amplitudes. This can be seen if we calculate the transformation matrix through the first sextupole, the $-\mathcal{I}$ section, and then through the second sextupole. The sextupoles are assumed to be thin magnets inflicting kicks on particle trajectories by the amount

$$
\Delta x^{\prime}=-\frac{1}{2} m_{0} \ell_{\mathrm{s}}\left(x^{2}-y^{2}\right),
$$

and

$$
\Delta y^{\prime}=-m_{0} \ell_{\mathrm{s}} x y
$$


where $\ell_{\mathrm{s}}$ is the sextupole length. We form a $4 \times 4$ transformation matrix through a thin sextupole and get

$$
\begin{aligned}
\left(\begin{array}{c}
x \\
x^{\prime} \\
y \\
y^{\prime}
\end{array}\right) & =\mathcal{M}_{\mathrm{s}}\left(x_{0}, y_{0}\right)\left(\begin{array}{l}
x_{0} \\
x_{0}^{\prime} \\
y_{0} \\
y_{0}^{\prime}
\end{array}\right) \\
& =\left(\begin{array}{cccc}
1 & 0 & 0 & 0 \\
-\frac{1}{2} m_{0} \ell_{\mathrm{s}} x_{0} & 1 & \frac{1}{2} m_{0} \ell_{\mathrm{s}} x_{0} & 0 \\
0 & 0 & 1 & 0 \\
0 & 0 & m_{0} \ell_{\mathrm{s}} x_{0} & 1
\end{array}\right)\left(\begin{array}{l}
x_{0} \\
x_{0}^{\prime} \\
y_{0} \\
y_{0}^{\prime}
\end{array}\right)
\end{aligned}
$$

To evaluate the complete transformation we note that in the first sextupole the particle coordinates are $\left(x_{0}, y_{0}\right)$ and become after the $-\mathcal{I}$-transformation in the second sextupole $\left(-x_{0},-y_{0}\right)$. The transformation matrix through the complete unit is therefore

$$
\mathcal{M}_{t}=\mathcal{M}_{\mathrm{s}}\left(x_{0}, y_{0}\right)(-\mathcal{I}) \mathcal{M}_{\mathrm{s}}\left(-x_{0},-y_{0}\right)
$$

Independent of the oscillation amplitude we observe a complete cancellation of geometric aberrations in both the horizontal and vertical plane. This correction scheme has been applied successfully to the final focus system of the Stanford Linear Collider [9], where chromatic as well as geometric aberrations must be controlled and compensated to high accuracy to allow the focusing of a beam to a spot size at the collision point of only a few micrometer.

The effectiveness of this correction scheme and its limitations in circular accelerators has been analyzed in more detail by Emery [10] and we will discuss some of his findings. As an example, we use strong focusing FODO cells for an extremely low emittance electron storage ring [10] and investigate the beam parameters along this lattice. Any other lattice could be used as well since the characteristics of aberrations is not lattice dependent although the magnitude may be. The particular FODO lattice under discussion as shown in Fig. 17.3 is a thin lens lattice with $90^{\circ}$ cells, a distance between quadrupoles of $L_{\mathrm{q}}=3.6 \mathrm{~m}$ and an

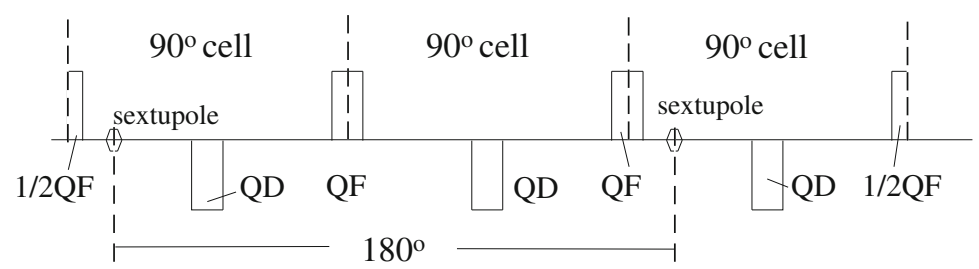

Fig. 17.3 FODO lattice and chromaticity correction 
a)

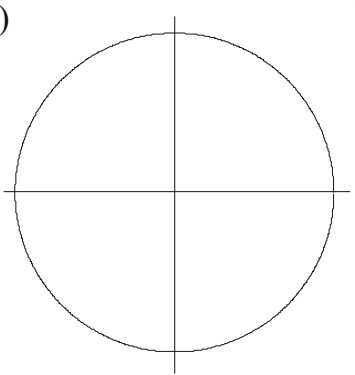

d)

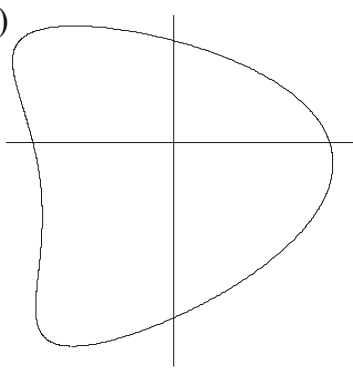

b)

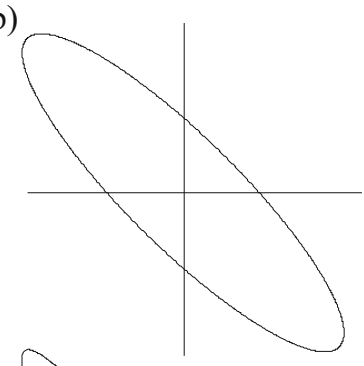

e)

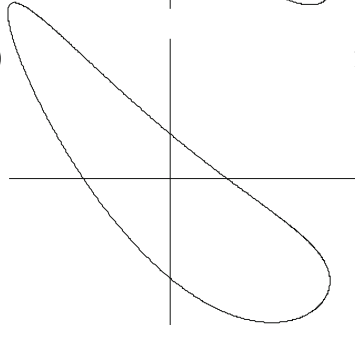

c)

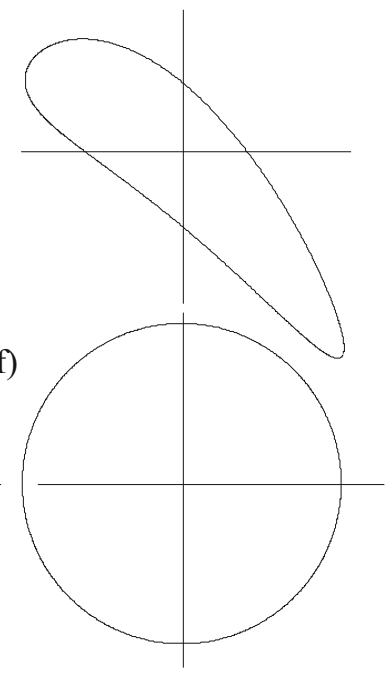

Fig. 17.4 Phase ellipses along a FODO channel including nonlinear aberrations due to thin sextupole magnets separated by exactly $180^{\circ}$ in betatron phase (consult text for details)

integrated half quadrupole strength of $\left(k \ell_{\mathrm{q}}\right)^{-1}=\sqrt{2} L_{\mathrm{q}}$. The horizontal and vertical betatron functions at the symmetry points are 12.29 and $2.1088 \mathrm{~m}$ respectively. Three FODO cells are shown in Fig. 17.3 including one pair of sextupoles separated by $180^{\circ}$ in betatron phase space. We choose a phase ellipse for an emittance of $\epsilon=200 \mathrm{~mm}-\mathrm{mrad}$ which is an upright ellipse at the beginning of the FODO lattice, Fig. 17.4a. Due to quadrupole focusing the ellipse becomes tilted at the entrance to the first sextupole, Fig. 17.4b. The thin lens sextupole introduces a significant angular perturbation (Fig. 17.4c) leading to large lateral aberrations in the quadrupole QF (Fig. 17.4d). At the entrance to the second sextupole the distorted phase ellipse is rotated by $180^{\circ}$ and all aberrations are compensated again by this sextupole, Fig. 17.4e. Finally, the phase ellipse at the end of the third FODO cell is again an upright ellipse with no distortions left, Fig. 17.4f. The range of stability therefore extends to infinitely large amplitudes ignoring any other detrimental effects.

The compensation of aberrations works as long as the phase advance between sextupoles is exactly $180^{\circ}$. A shift of the second sextupole by a few degrees or a quadrupole error resulting in a similar phase error between the sextupole pair would greatly reduce the compensation. In Fig. 17.5 the evolution of the phase ellipse from Fig. 17.4 is repeated but now with a phase advance between the sextupole pair of only $175^{\circ}$. A distortion of the phase ellipse due to aberrations can be observed which may build up to instability as the particles pass through many similar cells. Emery has analyzed numerically this degradation of stability and finds empirically 

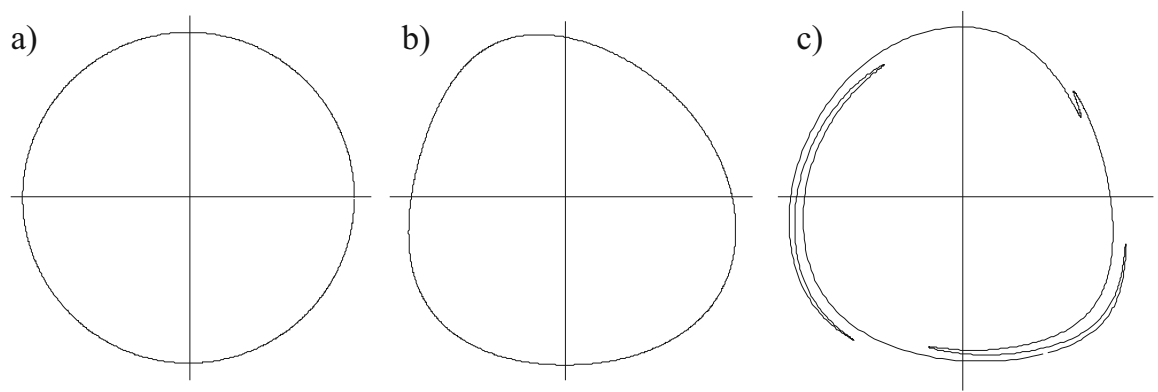

Fig. 17.5 Thin sextupole magnets separated by $175^{\circ}$ in betatron phase space. The unperturbed phase ellipse (a) becomes slightly perturbed (b) at the end of the first triple FODO cell (Fig.17.3, and more so after passing through many such triplets (c)

a)

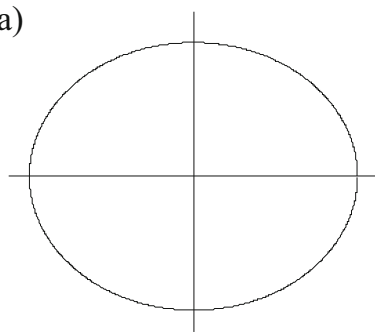

b)

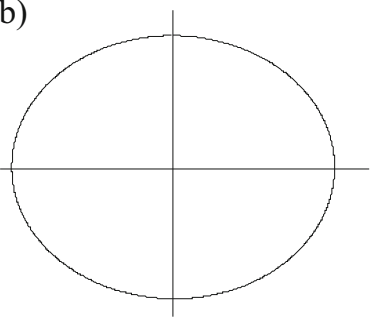

c)

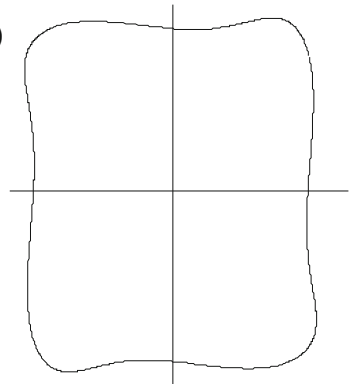

Fig. 17.6 Phase ellipses along a FODO channel including nonlinear aberrations due to finite length sextupole magnets placed exactly 180 degrees apart. Phase ellipse (a) transforms to (b) after one FODO triplet cell and to (c) after passage through many such cells

the maximum stable betatron amplitude to scale with the phase error like $\Delta \varphi^{-0.52}$ [10]. The sensitivity to phase errors together with unavoidable quadrupole field errors and orbit errors in sextupoles can significantly reduce the effectiveness of this compensation scheme.

The single most detrimental arrangement of sextupoles compared to the perfect compensation of aberrations is to interleave sextupoles which means to place other sextupoles between two pairs of compensating sextupoles [8]. Such interleaved sextupoles introduce amplitude dependent phase shifts leading to phase errors and reduced compensation of aberrations. This limitation to compensate aberrations is present even in a case without apparent interleaved sextupoles as shown in Fig. 17.6 for the following reason.

The assumption of thin magnets is sometimes convenient but, as Emery points out, can lead to erroneous results. For technically realistic solutions, we must allow the sextupoles to assume a finite length and find, as a consequence, a loss of complete compensation for geometric aberrations because sextupoles of finite length are only one particular case of interleaved sextupole arrangements. If we consider the sextupoles made up of thin slices we still find that each slice of the first 
sextupole has a corresponding slice exactly $180^{\circ}$ away in the second sextupoles. However, other slices are interleaved between such ideal pairs of thin slices. In Fig. 17.6 the sequence of phase ellipses from Fig. 17.4 is repeated with the only difference of using now a finite length of $0.3 \mathrm{~m}$ for the sextupoles. From the last phase ellipse it becomes clear that the aberrations are not perfectly compensated as was the case for thin sextupoles. Although the $-\mathcal{I}$-transformation scheme to eliminate geometric aberrations is not perfectly effective for real beam lines it is still prudent to arrange sextupoles in that way, if possible, to minimize aberrations and apply additional corrections.

\subsubsection{Filamentation of Phase Space}

Some distortion of the unperturbed trajectory in phase space due to aberrations is inconsequential to beam stability as long as this distortion does not build up and starts growing indefinitely. A finite or infinite growth of the beam emittance enclosed within a particular particle trajectory in phase space may at first seem impossible since we deal with macroscopic, non-dissipating magnetic fields where Liouville's theorem must hold. Indeed numerical simulations indicate that the total phase space occupied by the beam does not seem to increase but an originally elliptical boundary in phase space can grow, for example, tentacles like a spiral galaxy leading to larger beam sizes without actually increasing the phase space density. This phenomenon is called filamentation of the phase space and can evolve like shown in Fig. 17.7.

For particle beams this filamentation is as undesirable as an increase in beam emittance or beam loss. We will therefore try to derive the causes for beam filamentation in the presence of sextupole non-linearities which are the most important non-linearities in beam dynamics. In this discussion we will follow the ideas developed by Autin [11] which observes the particle motion in action-angle phase space under the influence of nonlinear fields.

a)

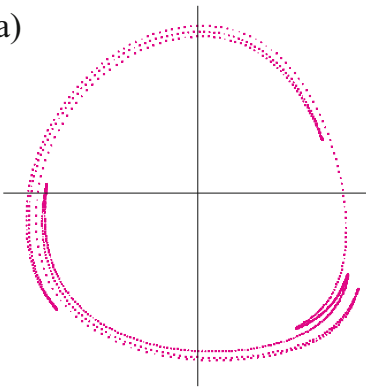

b)

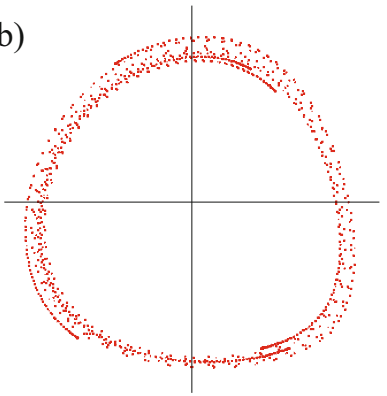

c)

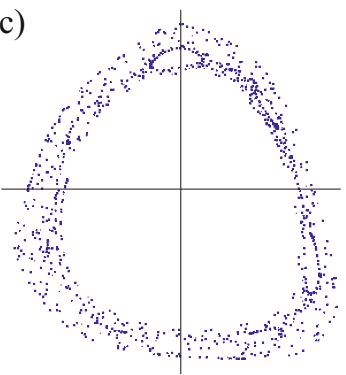

Fig. 17.7 Filamentation of phase space after passage through an increasing number of FODO cells 
For simplicity of expression, we approximate the nonlinear sextupoles by thin magnets. This does not restrict our ability to study the effect of finite length sextupoles since we may always represent such sextupoles by a series of thin magnets. A particle in a linear lattice follows a circle in action-angle phase space with a radius equal to the action $J_{0}$. The appearance of a nonlinearity along the particle trajectory will introduce an amplitude variation $\Delta J$ to the action which is from the Courant-Snyder invariant for both the horizontal and vertical plane

$$
\begin{aligned}
& \Delta J_{x}=v_{x 0} w \Delta w+\frac{1}{v_{x 0}} \dot{w} \Delta \dot{w}=\frac{1}{v_{x 0}} \dot{w} \Delta \dot{w}, \\
& \Delta J_{y}=v_{y 0} v \Delta v+\frac{1}{v_{y 0}} \dot{v} \Delta \dot{v}=\frac{1}{v_{y 0}} \dot{v} \Delta \dot{v},
\end{aligned}
$$

since $\Delta w=\Delta v=0$ for a thin magnet. Integration of the equations of motion in normalized coordinates over the "length" $\ell$ of the thin magnet produces a variation of the slopes

$$
\begin{aligned}
& \Delta \dot{w}=v_{x 0} \sqrt{\beta_{x}} \frac{1}{2} m \ell\left(x^{2}-y^{2}\right), \\
& \Delta \dot{v}=-v_{y 0} \sqrt{\beta_{y}} m \ell x y .
\end{aligned}
$$

We insert (17.38) into (17.37) and get after transformation into action-angle variables and linearization of the trigonometric functions the variation of the action

$$
\begin{aligned}
& \Delta J_{x}=\frac{m \ell}{4} \sqrt{\frac{2 J_{x} \beta_{x}}{v_{x 0}}}\left\{\left(J_{x} \beta_{x}-2 J_{y} \beta_{y} \frac{v_{x}}{v_{y}}\right) \sin \psi_{x}+J_{x} \beta_{x} \sin 3 \psi_{x}\right. \\
& \left.-J_{y} \beta_{y} \frac{v_{x}}{v_{y}}\left[\sin \left(\psi_{x}+2 \psi_{y}\right)+\sin \left(\psi_{x}-2 \psi_{y}\right)\right]\right\}, \\
& \Delta J_{y}=\frac{m \ell}{2} \sqrt{\frac{2 J_{x} \beta_{x}}{v_{x 0}}} J_{y} \beta_{y}\left[\sin \left(\psi_{x}+2 \psi_{y}\right)-\sin \left(\psi_{x}-2 \psi_{y}\right)\right] .
\end{aligned}
$$

Since the action is proportional to the beam emittance, (17.39) allow us to study the evolution of beam filamentation over time. The increased action from (17.39) is due to the effect of one nonlinear sextupole magnet and we obtain the total growth of the action by summing over all turns and all sextupoles. To sum over all turns we note that the phases in the trigonometric functions increase by $2 \pi \nu_{0, x, y}$ every turn and we have for the case of a single sextupole after an infinite number of turns expressions of the form

$$
\sum_{n=0}^{\infty} \sin \left[\left(\psi_{x j}+2 \pi v_{x 0} n\right)+2\left(\psi_{y j}+2 \pi v_{y 0} n\right)\right],
$$

where $\psi_{x j}$ and $\psi_{y j}$ are the phases at the location of the sextupole $j$. Such sums of trigonometric functions are best solved in the form of exponential functions. In this case the sine function terms are equivalent to the imaginary part of the exponential functions

$$
\mathrm{e}^{\mathrm{i}\left(\psi_{x j}+2 \psi_{y j}\right)} \mathrm{e}^{\mathrm{i} 2 \pi\left(v_{x 0}+2 v_{y 0}\right) n}
$$


The second factor forms an infinite geometric series and the imaginary part of the sum is therefore

$$
\operatorname{Im} \frac{\mathrm{e}^{\mathrm{i}\left(\psi_{x j}+2 \psi_{y j}\right)}}{1-\mathrm{e}^{\mathrm{i} 2 \pi\left(v_{x 0}+2 v_{y 0}\right)}}=\frac{\cos \left[\left(\psi_{x j}-\pi v_{x 0}\right)+2\left(\psi_{y j}-\pi v_{y 0}\right)\right]}{2 \sin \left[\pi\left(v_{x 0}+2 v_{y 0}\right)\right]} .
$$

This solution has clearly resonant character leading to an indefinite increase of the action if $v_{x 0}+2 v_{y 0}$ is an integer. Similar results occur for the other three terms and Autin's method to observe the evolution of the action coordinate over many turns allows us to identify four resonances driven by sextupolar fields which can lead to particle loss and loss of beam stability if not compensated. Resonant growth of the apparent beam emittance occurs according to (17.39) for

$$
\begin{array}{ccc}
v_{x 0}=q_{1}, & \text { or } & v_{x 0}+2 v_{y 0}=q_{3}, \\
3 v_{x 0}=q_{2}, & \text { or } & v_{x 0}-2 v_{y 0}=q_{4},
\end{array}
$$

where the $q_{i}$ are integers. In addition to the expected integer and third integer resonance in the horizontal plane, we find also two third order coupling resonances in both planes where the sum resonance leads to beam loss while the difference resonance only initiates an exchange of the horizontal and vertical emittances. The asymmetry is not fundamental and is the result of our choice to use only upright sextupole fields.

So far we have studied the effect of one sextupole on particle motion. Since no particular assumption was made as to the location and strength of this sextupole, we conclude that any other sextupole in the ring would drive the same resonances and we obtain the beam dynamics under the influence of all sextupoles by adding the individual contributions. In the expressions of this section we have tacitly assumed that the beam is observed at the phase $\psi_{x 0, y 0}=0$. If this is not the desired location of observation the phases $\psi_{x j}$ need to be replaced by $\psi_{x j}-\psi_{x 0}$, etc., where the phases $\psi_{x j, y j}$ define the location of the sextupole $j$. Considering all sextupoles in a circular lattice we sum over all such sextupoles and get, as an example, for the sum resonance used in the derivation above from (17.39)

$$
\Delta J_{x, v_{x}+2 v_{y}}=-\sum_{j} \frac{m_{j} \ell_{j}}{4} \sqrt{\frac{2 J_{x} \beta_{x j}}{v_{x 0}}} J_{y} \beta_{y j} \frac{v_{x}}{v_{y}} \sin \left(\psi_{x j}+2 \psi_{y j}\right) .
$$

Similar expressions exist for other resonant terms. Equation (17.44) indicates a possibility to reduce the severity of driving terms for the four resonances. Sextupoles are primarily inserted into the lattice where the dispersion function is nonzero to compensate for chromaticities. Given sufficient flexibility these sextupoles can be arranged to avoid driving these resonances. Additional sextupoles may be located in dispersion free sections and adjusted to compensate or at least minimize the four resonance driving terms without affecting the chromaticity correction. The perturbation $\Delta J$ is minimized by distributing the sextupoles such that the resonant 
driving terms in (17.39) are as small as possible. This is accomplished by harmonic correction which is the process of minimization of expressions

$$
\begin{aligned}
\sum_{j} m_{j} \ell_{j} \beta_{x}^{3 / 2} \mathrm{e}^{\mathrm{i} \psi_{x j}} & \rightarrow 0, \\
\sum_{j} m_{j} \ell_{j} \beta_{x}^{3 / 2} \mathrm{e}^{\mathrm{i} 3 \psi_{x j}} & \rightarrow 0, \\
\sum_{j} m_{j} \ell_{j} \beta_{x}^{1 / 2} \beta_{y} \mathrm{e}^{\mathrm{i} \psi_{x j}} & \rightarrow 0, \\
\sum_{j} m_{j} \ell_{j} \beta_{x}^{1 / 2} \beta_{y} \mathrm{e}^{\mathrm{i}\left(\psi_{x j}+2 \psi_{y j}\right)} & \rightarrow 0, \\
\sum_{j} m_{j} \ell_{j} \beta_{x}^{1 / 2} \beta_{y} \mathrm{e}^{\mathrm{i}\left(\psi_{x j}-2 \psi_{y j}\right)} & \rightarrow 0 .
\end{aligned}
$$

The perturbations of the action variables in (17.39) cancel perfectly if we insert sextupoles in pairs at locations which are separated by a $-\mathcal{I}$ transformation as discussed previously in this chapter. The distribution of sextupoles in pairs is therefore a particular solution to (17.45) for the elimination of beam filamentation and specially suited for highly periodic lattices while (17.45)-(17.49) provide more flexibility to achieve similar results in general lattices and sextupole magnets of finite length.

Cancellation of resonant terms does not completely eliminate all aberrations caused by sextupole fields. Because of the existence of nonlinear sextupole fields the phases $\psi_{j}$ depend on the particle amplitude and resonant driving terms are therefore canceled only to first order. For large amplitudes we expect increasing deviation from the perfect cancellation leading eventually to beam filamentation and beam instability. Maximum stable oscillation amplitudes in $(x, y)$-space due to nonlinear fields form the dynamic aperture which is to be distinguished from the physical aperture of the vacuum chamber. This dynamic aperture is determined by numerical tracking of particles. Given sufficiently large physical apertures in terms of linear beam dynamics the goal of correcting nonlinear aberrations is to extend the dynamic aperture to or beyond the physical aperture. Methods discussed above to increase the dynamic aperture have been applied successfully to a variety of particle storage rings, especially by Autin [11] to the antiproton cooling ring ACOL, where a particularly large dynamic aperture is required.

\subsubsection{Chromatic Aberrations}

Correction of natural chromaticities is not a complete correction of all chromatic aberrations. For sensitive lattices nonlinear chromatic perturbation terms must be included. Both linear as well as nonlinear chromatic perturbations have been discussed in detail in Sect.9.4.1. Such terms lead primarily to gradient errors and therefore the sextupole distribution must be chosen such that driving terms for half 
integer resonances are minimized. Together with tune shifts due to gradient field errors we observe also a variation of the betatron function. Chromatic gradient errors in the presence of sextupole fields are

$$
p_{1}(z)=(k-m \eta) \delta
$$

and the resulting variation of the betatron function has been derived in Sect. 15.3. For the perturbation (17.50) the linear variation of the betatron function with momentum is from (15.91)

$$
\frac{\Delta \beta(z)}{\beta_{0}}=\frac{\delta}{2 \sin 2 \pi v_{0}} \int_{z}^{z+L} \beta(k-m \eta) \cos \left[2 \nu_{0}\left(\varphi_{z}-\varphi_{\zeta}+2 \pi\right)\right] \mathrm{d} \zeta,
$$

where $L$ is the length of the superperiod, $\varphi_{z}=\varphi(z)$ and $\varphi_{\zeta}=\varphi(\zeta)$. The same result can be expressed in the form of a Fourier expansion for $N_{\mathrm{s}}$ superperiods in a ring lattice by

$$
\frac{\Delta \beta}{\beta}=\delta \frac{v_{0}}{4 \pi} \sum_{q} \frac{F_{q} \mathrm{e}^{\mathrm{i} N_{\mathrm{s}} q \varphi}}{v_{0}^{2}-\left(N_{\mathrm{s}} q / 2\right)^{2}}
$$

where

$$
F_{q}=\frac{v_{0}}{2 \pi} \int_{0}^{2 \pi} \beta^{2}(k-m \eta) \mathrm{e}^{\mathrm{i} N_{\mathrm{s}} q \varphi} \mathrm{d} \varphi
$$

Both expressions exhibit the presence of half integer resonances and we must expect the area of beam stability in phase space to be reduced for off momentum particles because of the increased strength of the resonances. Obviously, this perturbation does not appear in cases where the chromaticity is corrected locally so that $(k-m \eta) \equiv 0$ but few such cases exist. To minimize the perturbation of the betatron function, we look for sextupole distributions such that the Fourier harmonics are as small as possible by eliminating excessive "fighting" between sextupoles and by minimizing the resonant harmonic $q=2 v_{0}$. Overall, however, it is not possible to eliminate this beta-beat completely. With a finite number of sextupoles the beta-beat can be adjusted to zero only at a finite number of points along the beam line.

In colliding-beam storage rings, for example, we have specially sensitive sections just adjacent to the collision points. To maximize the luminosity the lattice is designed to produce small values of the betatron functions at the collision points and consequently large values in the adjacent quadrupoles. In order not to further increase the betatron functions there and make the lattice more sensitive to errors, one might choose to seek sextupole distributions such that the beta-beat vanishes at the collision point and its vicinity. 
Having taken care of chromatic gradient errors we are left with the variation of geometric aberrations as a function of particle momentum. Specifically, resonance patterns vary and become distorted as the particle momentum is changed. Generally this should not cause a problem as long as the dynamic aperture can be optimized to exceed the physical aperture. A momentum error will introduce only a small variation to the dynamic aperture as determined by geometric aberrations for on momentum particles only. If, however, the dynamic aperture is limited by some higher-order resonances even a small momentum change can cause a big difference in the stable phase space area.

Analytical methods are useful to minimize detrimental effects of geometric and chromatic aberrations due to nonlinear magnetic fields. We have seen how by careful distribution of the chromaticity correcting sextupoles, resonant beam emittance blow up and excessive beating of the betatron functions for off momentum particles can be avoided or at least minimized within the approximations used. In Sect. 17.3, we will also find that sextupolar fields can produce strong tune shifts for larger amplitudes leading eventually to instability at nearby resonances. Here again a correct distribution of sextupoles will have a significant stabilizing effect. Although there are a number of different destabilizing effects, we note that they are driven by only a few third order resonances. Specifically, in large circular lattices a sufficient number of sextupoles and locations for additional sextupoles are available for an optimized correction scheme. In small rings such flexibility often does not exist and therefore the sophistication of chromaticity correction is limited. Fortunately, in smaller rings the chromaticity is much smaller and some of the higher-order aberrations discussed above are very small and need not be compensated. Specifically, the amplitude dependent tune shift is generally negligible in small rings while it is this effect which limits the dynamic aperture in most cases of large circular accelerators.

The optimization of sextupole distribution requires extensive analysis of the linear lattice and it is best to use a numerical program to do the well known but cumbersome work. At present the program OPA [12] is widely used. This program uses a linear lattice and adjusts the sextupoles such that chromaticities and some harmonics are corrected. With the new sextupole strengths the dynamic aperture can be obtained in the same program.

In trying to solve aberration problems in beam dynamics we are, however, mindful of approximations made and terms neglected for lack of mathematical tools to solve analytically the complete nonlinear dynamics in realistic accelerators. The design goals for circular accelerators become more and more demanding on our ability to control nonlinear aberrations. On one hand the required cross sectional area in the vicinity of the ideal orbit for a stable beam remains generally constant for most designs but the degree of aberrations is increased in an attempt to reach very special beam characteristics. As a consequence, the nonlinear perturbations become stronger and the limits of dynamic aperture occur for smaller amplitudes compared to less demanding lattices and require more and more sophisticated methods of chromaticity correction and control of nonlinear perturbations. 


\subsubsection{Particle Tracking}

No mathematical methods are available yet to calculate analytically the limits of the dynamic aperture for any but the most simple lattices. High order approximations are required to treat strong aberrations in modern circular accelerator designs. The most efficient way to determine beam stability characteristics for a particular lattice design is to perform numerical particle tracking studies.

Perturbations of localized nonlinear fields on a particle trajectory are easy to calculate and tracking programs follow single particles along their path incorporating any nonlinear perturbation encountered. Since most nonlinear fields are small, we may use thin lens approximation and passage of a particle through a nonlinear field of any order inflicts therefore only a deflection on the particle trajectory. During the course of tracking the deflections of all non-linearities encountered are accumulated for a large number of turns and beam stability or instability is judged by the particle surviving the tracking or not, respectively. The basic effects of nonlinear fields in numerical tracking programs are therefore reduced to what actually happens to particles travelling through such fields producing results in an efficient way. Of course from an intellectual point of view such programs are not completely satisfactory since they serve only as tools providing little direct insight into actual causes for limitations to the dynamic aperture and instability.

The general approach to accelerator design is to develop first a lattice in linear approximation meeting the desired design goals followed by an analytical approach to include chromaticity correcting sextupoles in an optimized distribution. Further information about beam stability and dynamic aperture can at this point only be obtained from numerical tracking studies. Examples of widely used computer programs to perform such tracking studies are in historical order PATRICIA [7], RACETRACK [13], OPA [12] and more.

Tracking programs generally require as input an optimized linear lattice and allow then particle tracking for single particles as well as for a large number of particles simulating a full beam. Nonlinear fields of any order can be included as thin lenses in the form of isolated multipole magnets like sextupoles or a multipole errors of regular lattice magnets. The multipole errors can be chosen to be systematic or statistical and the particle momentum may have a fixed offset or may be oscillating about the ideal momentum due to synchrotron oscillations.

Results of such computer studies contribute information about particle dynamics which is not available otherwise. The motion of single particles in phase space can be observed together with an analysis of the frequency spectrum of the particle under the influence of all nonlinear fields included and at any desired momentum deviation.

Further information for the dynamics of particle motion can be obtained from the frequency spectrum of the oscillation. An example of this is shown in Fig. 17.8 as a function of oscillation amplitudes. For small amplitudes we notice only the fundamental horizontal betatron frequency $v_{x}$. As the oscillation amplitude is increased this basic frequency is shifted toward lower values while more frequencies 


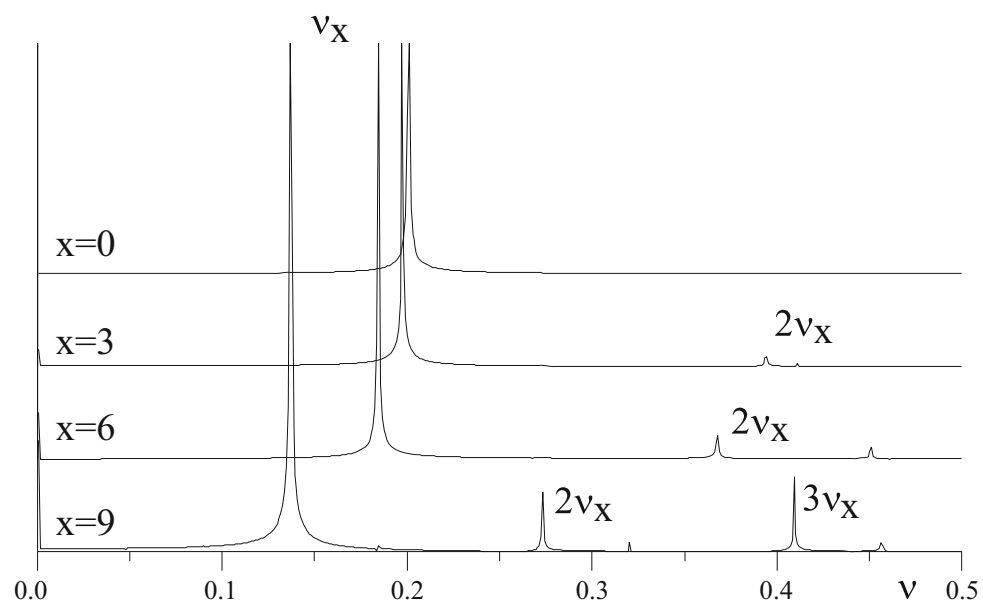

Fig. 17.8 Frequency spectrum for betatron oscillations with increasing amplitudes (x) as determined by particle tracking with PATRICIA

appear. We note the appearance of higher harmonics of $v_{x}$ due to the nonlinear nature of motion.

The motion of a particle in phase space and its frequency spectrum as a result of particle tracking can give significant insight into the dynamics of a single particle. For the proper operation of an accelerator, however, we also need to know the overall stability of the particle beam. To this purpose we define initial coordinates of a large number of particles distributed evenly over a cross section normal to the direction of particle propagation to be tested for stability. All particles are then tracked for many turns and the surviving particles are displayed over the original cross section at the beginning of the tracking thus defining the area of stability or dynamic aperture.

\subsection{Hamiltonian Perturbation Theory}

The Hamiltonian formalism has been applied to derive tune shifts and to discuss resonance phenomena. This was possible by a careful application of canonical transformation to eliminate, where possible, cyclic variables from the Hamiltonian and obtain thereby an invariant of the motion. We have also learned that this "elimination" process need not be perfect. During the discussion of resonance theory, we observed that slowly varying terms function almost like cyclic variables giving us important information about the stability of the motion.

During the discussion of the resonance theory, we tried to transform perturbation terms to a higher order in oscillation amplitude than required by the approximation desired and where this was possible we could then ignore such higher-order fastoscillating terms. This procedure was successful for all terms but resonant terms. 
In this section we will ignore resonant terms and concentrate on higher-order terms which we have ignored so far [14]. By application of a canonical identity transformation we try to separate from fast oscillating terms those which vary only slowly. To that goal, we start from the nonlinear Hamiltonian (16.30)

$$
H=v_{0} J+p_{n}(\varphi) J^{n / 2} \cos ^{n} \psi
$$

Fast-oscillating terms can be transformed to a higher order by a canonical transformation which can be derived from the generating function

$$
G_{1}=\psi J_{1}+g(\psi, \varphi) J_{1}^{n / 2}
$$

where the function $g(\psi, \varphi)$ is an arbitrary but periodic function in $\psi$ and $\varphi$ which we will determine later. From (17.55) we get for the new angle variable $\psi_{1}$ and the old action variable $J$

$$
\begin{aligned}
& \psi_{1}=\frac{\mathrm{d} G_{1}}{\mathrm{~d} J_{1}}=\psi+\frac{n}{2} g(\psi, \varphi) J^{n / 2-1}, \\
& J=\frac{\mathrm{d} G_{1}}{\mathrm{~d} \psi}=J_{1}+\frac{\partial g}{\partial \psi} J_{1}^{n / 2-1},
\end{aligned}
$$

and the new Hamiltonian is

$$
H_{1}=H+\frac{\mathrm{d} G_{1}}{\mathrm{~d} \varphi}=H+\frac{\partial g(\psi, \varphi)}{\partial \varphi} J_{1}^{n / 2} .
$$

We replace now the old variables $(\psi, J)$ in the Hamiltonian by the new variables $\left(J_{1}, \psi_{1}\right)$ and expand

$$
J^{n / 2}=\left(J_{1}+\frac{\partial g}{\partial \psi} J_{1}^{n / 2}\right)^{n / 2}=J_{1}^{n / 2}+\frac{n}{2} \frac{\partial g}{\partial \psi} J_{1}^{n-1}+\cdots
$$

With (17.56), (17.58) the Hamiltonian (17.57) becomes

$$
\begin{aligned}
H_{1} & =v_{0} J_{1}+J_{1}^{n / 2}\left[v_{0} \frac{\partial g}{\partial \psi}+p_{n}(\varphi) \cos ^{n} \psi+\frac{\partial g}{\partial \varphi}\right] \\
& +J_{1}^{n-1}\left[\frac{n}{2} p_{n}(\varphi) \cos ^{n} \psi \frac{\partial g}{\partial \psi}\right]+\mathcal{O}\left(J_{1}^{n+1 / 2}\right) .
\end{aligned}
$$

All terms of order $n+1 / 2$ or higher in the amplitude $J$ as well as quadratic terms in $g(\psi, \varphi)$ or derivations thereof have been neglected. We still must express all terms of the Hamiltonian in the new variables and define therefore the function

$$
Q(\psi, \varphi)=v_{0} \frac{\partial g}{\partial \psi}+p_{n}(\varphi) \cos ^{n} \psi+\frac{\partial w}{\partial \varphi} .
$$


In the approximation of small perturbations we have $\psi_{1} \approx \psi$ or $\psi_{1}=\psi+\Delta \psi$ and may expand (17.60) like

$$
\begin{aligned}
Q_{1}\left(\psi_{1}, \varphi\right) & =Q(\psi, \varphi)+\frac{\partial Q}{\partial \psi} \Delta \psi \\
& =Q(\psi, \varphi)+\frac{n}{2} g\left(\psi_{1}, \varphi\right) J_{1}^{n / 2-1} \frac{\partial Q}{\partial \psi},
\end{aligned}
$$

where we used the first equation of (17.56). The Hamiltonian can be greatly simplified if we make full use of the periodic but otherwise arbitrary function $g\left(\psi_{1}, \varphi\right)$. With (17.62) we obtain from (17.59)

$$
\begin{aligned}
H_{1} & =v_{0} J_{1}+J_{1}^{n / 2} Q_{1}\left(\psi_{1}, \varphi\right) \\
& +\frac{n}{2} J_{1}^{n-1}\left[p_{n}(\varphi) \cos ^{n} \psi_{1} \frac{\partial w}{\partial \psi}-g(\psi, \varphi) \frac{\partial Q}{\partial \psi}\right]+\cdots
\end{aligned}
$$

and we will derive the condition that

$$
Q(\psi, \varphi)=0
$$

First we set

$$
\cos ^{n} \psi_{1}=\sum_{m=-n}^{n} a_{n m} \mathrm{e}^{\mathrm{i} m \psi_{1}}
$$

and try an expansion of $g\left(\psi_{1}, \varphi\right)$ in a similar way by setting

$$
g\left(\psi_{1}, \varphi\right)=\sum_{m=-n}^{n} g_{m}(\varphi) \mathrm{e}^{\mathrm{i} m\left(\psi_{1}-v_{0} \varphi\right)}
$$

where the function $g$ obviously is still periodic in $\psi$ and $\varphi$ as long as $g_{m}(\varphi)$ is periodic. With

$$
\frac{\partial g}{\partial \psi_{1}}=\sum_{m=-n}^{n} g_{m}(\varphi) \mathrm{i} m \mathrm{e}^{\mathrm{i} m\left(\psi_{1}-\nu_{0} \varphi\right)}
$$

and

$$
\frac{\partial g}{\partial \varphi}=\sum_{m=-n}^{n}\left[\frac{\partial g_{m}}{\partial \varphi}-\mathrm{i} v_{0} m g_{m}(\varphi)\right] \mathrm{e}^{\mathrm{i} m\left(\psi_{1}-v_{0} \varphi\right)}
$$


we get instead of (17.60)

$$
\begin{aligned}
& Q\left(\psi_{1}, \varphi\right) \approx Q(\psi, \varphi)= \\
& \begin{aligned}
\mathrm{i} v_{0} \sum_{m=-n}^{n} m g_{m} \mathrm{e}^{\mathrm{i} m\left(\psi_{1}-\nu_{0} \varphi\right)}+p_{n}(\varphi) \sum_{m=-n}^{n} a_{n m} \mathrm{e}^{\mathrm{i} m \psi_{1}} \\
+\sum_{m=-n}^{n}\left(\frac{\partial g_{m}}{\partial \varphi}-\mathrm{i} \nu_{0} m g_{m}\right) \mathrm{e}^{\mathrm{i} m\left(\psi_{1}-v_{0} \varphi\right)}=0
\end{aligned}
\end{aligned}
$$

noting from (17.62) that the difference $\Delta Q=Q\left(\psi_{1}, \varphi\right)-Q(\psi, \varphi)$ contributes nothing to the term of order $J_{1}^{n / 2}$ for $n>2$. The imaginary terms cancel and we get

$$
Q\left(\psi_{1}, \varphi\right) \approx p_{n}(\varphi) \sum_{m=-n}^{n} a_{n m} \mathrm{e}^{\mathrm{i} m \psi}+\sum_{m=-n}^{n} \frac{\partial g_{m}}{\partial \varphi} \mathrm{e}^{\mathrm{i} m\left(\psi-v_{0} \varphi\right)}=0
$$

This equation must be true for all values of $\varphi$ and therefore the individual terms of the sums must vanish independently

$$
p_{n}(\varphi) a_{n m}+\frac{\partial g_{m}}{\partial \varphi} \mathrm{e}^{-\mathrm{i} m \nu_{0} \varphi}=0
$$

for all values of $m$. After integration we have

$$
g_{m}(\varphi)=g_{m 0}-a_{n m} \int_{0}^{\varphi} p_{n}(\phi) \mathrm{e}^{\mathrm{i} m \nu_{0} \phi} \mathrm{d} \phi
$$

and since the coefficients $g_{m}(\varphi)$ must be periodic $\left[g_{m}(\varphi)=g_{m}\left(\varphi+\frac{2 \pi}{N}\right)\right]$ where $N$ is the super-periodicity, we are able to eventually determine the function $g\left(\psi_{1}, \varphi\right)$. With

$$
g_{m}(\varphi) \mathrm{e}^{\mathrm{i} m\left(\psi_{1}-v_{0} \varphi\right)}=g_{m}\left(\varphi+\frac{2 \pi}{N}\right) \mathrm{e}^{\mathrm{i} m\left(\psi_{1}-v_{0} \varphi-\frac{2 \pi}{N} \nu_{0}\right)}
$$

and (17.71) we have

$$
\begin{aligned}
g_{m 0} \mathrm{e}^{\mathrm{i} m\left(\psi-\nu_{0} \varphi\right)}-a_{n m} \mathrm{e}^{\mathrm{i} m\left(\psi-\nu_{0} \varphi\right)} \int_{0}^{\varphi} p_{n}(\bar{\phi}) \mathrm{e}^{\mathrm{i} m \nu_{0} \bar{\phi}} \mathrm{d} \bar{\phi} & \\
& =\mathrm{e}^{\mathrm{i} m\left(\psi-\nu_{0} \varphi-\frac{2 \pi}{N} \nu_{0}\right)}\left(g_{m 0}-a_{n m}\right) \int_{0}^{\varphi+\frac{2 \pi}{N}} p_{n}(\bar{\phi}) \mathrm{e}^{\mathrm{i} m \nu_{0} \bar{\phi}} \mathrm{d} \bar{\phi} .
\end{aligned}
$$


Solving for $g_{m 0}$ we get

$$
g_{m 0}\left(1-\mathrm{e}^{\mathrm{i} m \frac{2 \pi}{N} \nu_{0}}\right)=a_{n m} \int_{0}^{\frac{2 \pi}{N}} p_{n}(\bar{\phi}) \mathrm{e}^{\mathrm{i} m \nu_{0} \bar{\phi}} \mathrm{d} \bar{\phi} .
$$

A solution for $g_{m 0}$ exists only if there are no perturbations and $p(\varphi) \equiv 0$ or if $\left(1-\mathrm{e}^{\mathrm{i} m \frac{2 \pi}{N} \nu_{0}}\right) \neq 0$. In other words we require the condition

$$
m v_{0} \neq q N
$$

where $q$ is an integer number. The canonical transformation (17.55) leads to the condition (17.64) only if the particle oscillation frequency is off resonance. We have therefore the result that all nonresonant perturbation terms can be transformed to higher-order terms in the oscillation amplitudes while the resonant terms lead to phenomena discussed earlier. From (17.73) we derive $g_{m 0}$, obtain the function $g_{m}(\varphi)$ from (17.71) and finally the function $g\left(\psi_{1}, \varphi\right)$ from (17.66). Since $Q\left(\psi_{1}, \varphi\right)=0$, the Hamiltonian is from (17.63)

$$
\begin{aligned}
H_{1}=v_{0} & J_{1}+J_{1}^{n / 2} Q_{1}\left(\psi_{1}, \varphi\right) \\
& +\frac{n}{2} J_{1}^{n-1}\left[p_{n}(\varphi) \cos ^{n} \psi_{1} \frac{\partial g}{\partial \psi_{1}}-g\left(\psi_{1}, \varphi\right) \frac{\partial Q}{\partial \psi_{1}}\right]+\cdots
\end{aligned}
$$

Nonresonant terms appear only in order $J_{1}^{n-1}$. As long as such terms can be considered small we conclude that the particle dynamics is determined by the linear tune $v_{0}$, a tune shift or tune spread caused by perturbations and resonances. Note that the Hamiltonian (17.75) is not the complete form but addresses only the nonresonant case of particle dynamics while the resonant case of the Hamiltonian has been derived earlier.

We will now continue to evaluate (17.75) and note that the product

$$
g\left(\psi_{1}, \varphi\right) \frac{\partial Q\left(\psi_{1}, \varphi\right)}{\partial \psi_{1}}=0
$$

in this approximation and get

$$
T(\psi, \varphi)=\frac{n}{2} p_{n}(\varphi) \cos ^{n} \psi \frac{\partial g}{\partial \psi},
$$

where we have dropped the index on $\psi$ and set from now on $\psi_{1}=\psi$ which is not to be confused with the variable $\psi$ used before the transformation (17.55). Using the Fourier spectrum for the perturbations and summing over all but resonant terms 
$q \neq q_{\mathrm{r}}$ we get from $(17.73)$

$$
\begin{aligned}
g_{m 0}\left(1-\mathrm{e}^{\mathrm{i} m \frac{2 \pi}{N} \nu_{0}}\right) & =a_{n m} \sum_{q \neq q_{\mathrm{r}}} \int_{0}^{\frac{2 \pi}{N}} p_{n q} \mathrm{e}^{\mathrm{i}\left(m \nu_{0}-q N\right) \varphi} \mathrm{d} \varphi \\
& =a_{n m} \sum_{q \neq q_{\mathrm{r}}} p_{n q} \frac{\mathrm{e}^{\mathrm{i} m \frac{2 \pi}{N} \nu_{0}}-1}{\mathrm{i}\left(m \nu_{0}-q N\right)},
\end{aligned}
$$

or

$$
g_{m 0}=\mathrm{i} a_{n m} \sum_{q \neq q_{\mathrm{r}}} \frac{p_{n q}}{m v_{0}-q N} .
$$

Note that we have excluded in the sums the resonant terms $q=q_{\text {r }}$ where $m_{r} v_{0}-q_{\mathrm{r}} N=0$. These resonant terms include also terms $q=0$ which do not cause resonances of the ordinary type but lead to tune shifts and tune spreads. After insertion into (17.71) and some manipulations we find

$$
\begin{aligned}
g_{m}(\varphi) & =\mathrm{i} a_{n m} \sum_{q \neq q_{\mathrm{r}}} \frac{p_{n q}}{m v_{0}-q N}-a_{n m} \sum_{q \neq q_{\mathrm{r}}} \int_{0}^{\varphi} p_{n q} \mathrm{e}^{\mathrm{i}\left(m v_{0}-q N\right) \phi} \mathrm{d} \phi \\
& =\mathrm{i} a_{n m} \sum_{q \neq q_{\mathrm{r}}} p_{n q} \frac{\mathrm{e}^{\mathrm{i}\left(m v_{0}-q N\right) \varphi}}{m v_{0}-q N}
\end{aligned}
$$

and with (17.66)

$$
g(\psi, \varphi)=\mathrm{i} \sum_{m=-n}^{n} \sum_{q \neq q_{\mathrm{r}}} \frac{a_{n m} p_{n q}}{m v_{0}-q N} \mathrm{e}^{\mathrm{i} m \psi} \mathrm{e}^{-\mathrm{i} q N) \varphi} .
$$

From (17.77) we get with (17.65) and (17.81)

$$
T(\psi, \varphi)=\mathrm{i} \frac{n}{2} \sum_{q \neq q_{\mathrm{r}}} p_{n q} \mathrm{e}^{-\mathrm{i} q N \varphi} \sum_{m=-n}^{n} a_{n m} \mathrm{e}^{\mathrm{i} m \psi} m g(\psi, \varphi)
$$

This function $T(\psi, \varphi)$ is periodic in $\psi$ and $\varphi$ and we may apply a Fourier expansion like

$$
T(\psi, \varphi)=\sum_{r} \sum_{s \neq \frac{r v_{0}}{N}} T_{r s} \mathrm{e}^{\mathrm{i}(r \psi-s N \varphi)}
$$


where the coefficients $T_{r s}$ are determined by

$$
T_{r s}=\frac{N}{4 \pi^{2}} \int_{0}^{2 \pi} \mathrm{e}^{-\mathrm{i} r \psi} \mathrm{d} \psi \int_{0}^{2 \pi / N} \mathrm{e}^{\mathrm{i} s N \varphi} T(\psi, \varphi) \mathrm{d} \varphi .
$$

To evaluate (17.84) it is most convenient to perform the integration with respect to the betatron phase $\psi$ before we introduce the expansions with respect to $\varphi$. Using (17.65), (17.66), (17.77), we get from (17.84) after some reordering

$$
\begin{aligned}
T_{r s}=\mathrm{i} & \frac{n N}{4 \pi} \sum_{m=-n}^{n} m \int_{0}^{2 \pi} \sum_{j=-n}^{n} \frac{a_{n j}}{2 \pi} \mathrm{e}^{\mathrm{i}(j+m-r) \psi} \mathrm{d} \psi \\
& \times \int_{0}^{2 \pi / N} p_{n}(\varphi) g_{m}(\varphi) \mathrm{e}^{\mathrm{i}\left(m v_{0}-s N\right) \varphi} \mathrm{d} \varphi .
\end{aligned}
$$

The integral with respect to $\psi$ is zero for all values $j+m-r \neq 0$ and therefore equal to $a_{n, r-m}$

$$
T_{r s}=\mathrm{i} \frac{n N}{4 \pi} \sum_{m=-n}^{m} m a_{m, r-m} \int_{0}^{2 \pi / N} p_{n}(\varphi) g_{m}(\varphi) \mathrm{e}^{-\mathrm{i}\left(m \nu_{0}-s N\right) \varphi} \mathrm{d} \varphi
$$

Expressing the perturbation $p_{n}(\varphi)$ by its Fourier expansion and replacing $g_{m}(\varphi)$ by (17.80), (17.85) becomes

$$
T_{r s}=-\frac{n}{2} \sum_{m=-n}^{n} m a_{m, r-m} a_{n, m} \sum_{q \neq q_{\mathrm{r}}} \frac{p_{n, s-q} p_{n, q}}{m v_{0}-q N} .
$$

With this expression we have fully defined the function $T(\psi, \varphi)$ and obtain for the non-resonant Hamiltonian (17.75)

$$
H=v_{0} J+J^{n-1} \sum_{r} \sum_{s \neq \frac{r}{N} \nu_{0}} T_{r s} \mathrm{e}^{\mathrm{i}(r \psi-s N \varphi)} .
$$

We note in this result a higher-order amplitude dependent tune spread which has a constant contribution $T_{00}$ as well as oscillatory contributions.

Successive application of appropriate canonical transformations has lead us to derive detailed insight into the dynamics of particle motion in the presence of perturbations. Of course, every time we applied a canonical transformation of variables it was in the hope of obtaining a cyclic variable. Except for the first transformation to action-angle variables, this was not completely successful. However, we were able to extract from perturbation terms depending on both 
action-angle variables such elements that do not depend on the angle variable. As a result, we are now able to determine to a high order of approximation shifts in the betatron frequency caused by perturbations as well as the occurrence and nature of resonances.

Careful approximations and simplifications had to be made to keep the mathematical formulation manageable. Specifically we had to restrict the perturbation theory in this section to one order of multipole perturbation and we did not address effects of coupling between horizontal and vertical betatron oscillations.

From a more practical view point one might ask to what extend this higherorder perturbation theory is relevant for the design of particle accelerators. Is the approximation sufficient or is it more detailed than needed? As it turns out so often in physics we find the development of accelerator design to go hand in hand with the theoretical understanding of particle dynamics. Accelerators constructed up to the late sixties were designed with moderate focusing and low chromaticities requiring no or only very weak sextupole magnets. In contrast more modern accelerators require much stronger sextupole fields to correct for the chromaticities and as a consequence, the effects of perturbations, in this case third-order perturbations, become more and more important. The ability to control the effects of such perturbations actually limits the performance of particle accelerators. For example, in colliding-beam storage rings the strongly nonlinear fields introduced by the beambeam effect limit the attainable luminosity while a lower limit on the attainable beam emittance for synchrotron light sources or damping rings is determined by strong sextupole fields.

\subsubsection{Tune Shift in Higher Order}

In (16.36) we found the appearance of tune shifts due to even order multipole perturbations only. Third-order sextupole fields, therefore, would not affect the tunes. This was true within the degree of approximation used at that point. In this section, however, we have derived higher-order tune shifts and should therefore discuss again the effect of sextupolar fields on the tune.

Before we evaluate the sextupole terms, however, we like to determine the contribution of a quadrupole perturbation to the higher-order tune shift. In lower order we have derived earlier a coherent tune shift for the whole beam. We use (17.86) and calculate $T_{00}$ for $n=2$

$$
T_{00}=\sum_{q \neq q_{\mathrm{r}}} p_{2, q} p_{2,-q} \sum_{m=-2}^{2} \frac{m a_{2, m} a_{2,-m}}{m v_{0}-q N} .
$$


With $4 a_{2,2}=a_{2,-2}=2 a_{2,0}=1$ and $a_{2,1}=a_{2,-1}=0$ the term in the bracket becomes

$$
\frac{-2}{-2 v_{0}-q N}+\frac{2}{2 v_{0}-q N}=\frac{2 q N}{\left(2 v_{0}\right)^{2}-(q N)^{2}}
$$

and (17.88) is simplified to

$$
T_{00}=-\sum_{q \neq q_{\mathrm{r}}} p_{2, q} p_{2,-q} \frac{2 q N}{\left(2 v_{0}\right)^{2}-(q N)^{2}} .
$$

In this summation we note the appearance of the index $q$ in pairs as a positive and a negative value. Each such pair cancels and therefore

$$
T_{00,2}=0,
$$

where the index 2 indicates that this coefficient was evaluated for a secondorder quadrupole field. This result is not surprising since all quadrupole fields contribute directly to the tune and formally a quadrupole field perturbation cannot be distinguished from a "real" quadrupole field.

In a similar way we derive the $T_{00}$ coefficient for a third-order multipole or a sextupolar field. From (17.86) we get for $n=3$

$$
T_{00,3}=-\frac{3}{2} \sum_{q \neq q_{\mathrm{r}}} p_{3, q} p_{3,-q} \sum_{m=-3}^{3} \frac{m a_{3, m} a_{3,-m}}{m \nu_{0}-q N} .
$$

Since $\cos ^{3} \psi$ is an even function we have $a_{3, m}=a_{3,-m}, a_{3,1}=\frac{3}{8}$ and $a_{3,3}=\frac{1}{8}$. The second sum in (17.91) becomes now

$$
\begin{aligned}
\frac{1}{64}\left(\frac{3}{3 v_{0}+q N}+\frac{q}{v_{0}+q N}+\frac{q}{v_{0}-q N}\right. & \left.+\frac{3}{3 v_{0}-q N}\right) \\
& =\frac{1}{64}\left(\frac{18 v_{0}}{v_{0}^{2}-(q N)^{2}}+\frac{18 v_{0}}{\left(3 v_{0}\right)^{3}-(q N)^{2}}\right),
\end{aligned}
$$

and after separating out the terms for $q=0,(17.91)$ becomes

$$
\begin{aligned}
T_{00,3}=- & \frac{15}{32 v_{0}} p_{3,0}^{2} \\
& \quad-\frac{27 v_{0}}{64} \sum_{q \neq q_{\mathrm{r}}} p_{3, q} p_{3,-q}\left[\frac{1}{v_{0}^{2}-(q N)^{2}}+\frac{1}{\left(3 v_{0}\right)^{3}-(q N)^{2}}\right] .
\end{aligned}
$$


This expression in general is nonzero and we found, therefore, that sextupole fields indeed, contribute to a tune shift although in a high order of approximation. This tune shift can actually become very significant for strong sextupoles and for tunes close to an integer or third integer resonances. Although we have excluded resonances $\left(q=q_{\mathrm{r}}\right)$, terms close to resonances become important. Obviously, the tunes should be chosen such as to minimize both terms in the bracket of (17.92). This can be achieved with $v_{0}=q N+\frac{1}{2} N$ and $3 v_{0}=r N+\frac{1}{2} N$ where $q$ and $r$ are integers. Eliminating $v_{0}$ from both equations we get the condition $3 q-r+1=0$ or $r=3 q+1$. With this we finally get from the two tune conditions the relation $2 v_{0}=(2 q+1) N$ or

$$
v_{\mathrm{opt}}=\frac{2 q+1}{2} N
$$

Of course, an additional way to minimize the tune shift is to arrange the sextupole distribution in such a way as to reduce strong harmonics in (17.92). In summary, we find for the non-resonant Hamiltonian in the presence of sextupole fields.

$$
H_{3}=v_{0} J+T_{00,3} J^{2}+\text { higher order terms }
$$

and the betatron oscillation frequency or tune is given by

$$
v=v_{0}+2 T_{00,3} J
$$

In this higher-order approximation of beam dynamics we find that sextupole fields cause an amplitude dependent tune shift in contrast to our earlier first-order conclusion

$$
\frac{\Delta v}{v_{0}}=\frac{v-v_{0}}{v_{0}}=T_{00,3}\left(\gamma u^{2}+2 u u^{\prime}+\beta u^{\prime 2}\right)=T_{00,3} \epsilon,
$$

where we have used (5.59) with $\epsilon$ the emittance of a single particle oscillating with a maximum amplitude $a^{2}=\beta \epsilon$. We have shown through higher-order perturbation theory that odd order nonlinear fields like sextupole fields, can produce amplitude dependent tune shifts which in the case of sextupole fields are proportional to the square of the betatron oscillation amplitude and therefore similar to the tune shift caused by octupole fields. In a beam where particles have different betatron oscillation amplitudes this tune shift leads to a tune spread for the whole beam.

In practical accelerator designs requiring strong sextupoles for chromaticity correction it is mostly this tune shift which moves large amplitude particles onto a resonance thus limiting the dynamic aperture. Since this tune shift is driven by the integer and third-order resonance, it is imperative in such cases to arrange the sextupoles such as to minimize this driving term for geometric aberration. 


\section{Problems}

17.1 (S). Derive the expression for the second-order matrix element $T_{166}$ and give a physical interpretation for this term.

17.2 (S). Show that the perturbation proportional to $x_{0}^{2}$ is $p\left(z \mid x_{0}^{2}\right)=$ $\left[\left(-\frac{1}{2} m-\kappa^{3}-2 \kappa k\right) C+\frac{1}{2} \kappa C^{\prime 2}\right] x_{0}^{2}$, where $C=C(z)=\cos \sqrt{k} z$ and $C^{\prime}=C^{\prime}(z)$ and the second-order matrix element

$T_{111}=\left(-\frac{1}{2} m-\kappa^{3}-2 \kappa k\right) \frac{1}{3 k}\left[k S^{2}+(1-C)\right]+\frac{1}{6} \kappa\left[2(1-C)-k S^{2}\right]$.

17.3 (S). Consider a large circular accelerator made of many FODO cells with a phase advance of $90^{\circ}$ per cell. Locate chromaticity correcting sextupoles in the center of each quadrupole and calculate the magnitude for one of the five expressions (17.45)-(17.49). Now place non-interleaved sextupole in pairs $180^{\circ}$ apart and calculate the same two expressions for the new sextupole distribution.

17.4 (S). Use the lattice of Problem 17.3 and determine the tunes of the ring. Are the tunes the best choices for the super-periodicity of the ring to avoid resonance driven sextupole aberrations? How would you go about improving the situation?

17.5. Expand the second-order transformation matrix to include path length terms relevant for the design of an isochronous beam transport system and derive expressions for the matrix elements. Which elements must be adjusted and how would you do this? Which parameters would you observe to control your adjustment?

17.6. Sextupoles are used to compensate for chromatic aberrations at the expense of geometric aberrations. Derive a condition for which the geometric aberration has become as large as the original chromatic aberration. What is the average perturbation of geometric aberrations on the betatron motion? Try to formulate a "rule of thumb" stability criteria for the maximum sextupole strength. Is it better to place a chromaticity correcting sextupole at a high beta location (weak sextupole) or at a low beta location (weak aberration)?

17.7. Consider both sextupole distributions of Problem 17.3 and form a phasor diagram of one of expressions (17.45)-(17.49) for the first four or more FODO cells. Discuss desirable features of the phasor diagram and explain why the $-\mathcal{I}$ correction scheme works well. A phasor diagram is constructed by adding vectorially each term of an expression (17.45)-(17.49) going along a beam line.

17.8. The higher-order chromaticity of a lattice may include a strong quadratic term. What dependence on energy would one expect in this case for the beta beat? Why? Can your findings be generalized to higher-order terms? 


\section{References}

1. K.L. Brown, R. Belbeoch, P. Bounin, Rev. Sci. Instrum. 35, 481 (1964)

2. K.L. Brown, The adjustable phase planar helical undulator, in 5th International Conference on High Energy Accelerators, Frascati, Italy (1965)

3. K.L. Brown, Adv. Part. Phys. 1, 71 (1967)

4. K.L. Brown, D.C. Carey, C.H. Iselin, F. Rothacker, Technical Report SLAC-75, CERN 73-16, SLAC-91, CERN-80-4, CERN,FNAL,SLAC (1972)

5. Slac linear collider, conceptual design report. Technical Report SLAC-229, SLAC, Stanford, CA (1981)

6. Pep technical design report. Technical Report SLAC-189, LBL-4299, SLAC, Stanford, CA (1976)

7. H. Wiedemann, Chromaticity correction in large storage rings. Technical Report PEP-Note 220, Stanford Linear Accelerator Center, Stanford, CA (1976)

8. K.L. Brown, R.V. Servranckx, in 11th International Conference on High Energy Accelerators. Stanford linear Accelerator Center, Birkäuser, Basel (1980)

9. J.J. Murray, K.L. Brown, T. Fieguth, in 1987 IEEE Particle Accelerators Conference, Washington. IEEE Cat. No. 87CH2387-9 (1987)

10. L. Emery, A wiggler-based ultra-low-emittance damping ring lattice and its chromatic correction. Ph.D. thesis, Stanford University, Stanford, CA (1990)

11. B. Autin, The cern anti-proton collector. Technical Report CERN 74-2, CERN, CERN, Geneva (1974)

12. A. Streun, Opa. Available from PSI (2010)

13. A. Wrulich, in Proceedings of Workshop on Accelerator Orbit and Partickle Tracking Programs. Technical Report BNL-317615, BNL, Brookhaven, NY (1982)

14. F.T. Cole, Longitudinal motion in circular accelerators, in Physics of Particle Accelerators, vol. AIP 153, ed. by M. Month, M. Dienes (The American Institute of Physics, New York, 1987), p. 44 


\section{Part VI \\ Acceleration}




\section{Chapter 18 \\ Charged Particle Acceleration}

Particle acceleration by rf-fields has been discussed, for example, in considerable detail in $[1,2]$ where relationships between longitudinal phase oscillation and beam stability are derived and discussed. The accelerating fields were assumed to be available in resonant cavities, but we ignored conditions that must be met to generate such fields and ensure positive energy transfer to the particle beam. In this chapter, we will discuss relevant characteristics of rf-cavities and study the interaction of the rf-generator with accelerating cavity and beam.

It is not the intention here to develop a general microwave theory but we will restrict ourselves rather to such aspects which are of importance for particle accelerator physics. Considerable performance limits occur in accelerators by technical limitations in various accelerator systems as, for example, the rf-system and it is therefore useful for the accelerator designer to have a basic knowledge of such limits.

\subsection{Rf-Waveguides and Cavities}

Commonly, high frequency rf-fields are used to accelerate charged particles and the interaction of such electromagnetic waves with charged particles has been discussed earlier together with the derivation of synchronization conditions to obtain continuous particle acceleration. In doing so plane rf-waves have been used ignoring the fact that such fields do not have electrical field components in the direction of particle and wave propagation. Although this assumption has not made the results obtained so far obsolete, a satisfactory description of the wave-particle interaction must include the establishment of appropriate field configurations.

Electromagnetic waves useful for particle acceleration must exhibit field components in the direction of particle propagation which in our coordinate system is the $z$-direction. The synchronization condition can be achieved in two ways. First, an

This chapter has been made Open Access under a CC BY 4.0 license. For details on rights and licenses please read the Correction https://doi.org/10.1007/978-3-319-18317-6_28 
electromagnetic wave travels along the direction of the desired particle acceleration with a phase velocity which is equal to the velocity of the particle. In this case, a particle starting, say, at the crest of the wave where the field strength is largest, would be continuously accelerated at the maximum rate as it moves along with the wave. Another way of particle acceleration occurs from electromagnetic fields created in rf-cavities placed at particular locations along the particle path. In this case, the phase velocity of the wave is irrelevant. For positive particle acceleration the phase of the electromagnetic field must be adjusted such that the integrated acceleration is positive, while the particle passes through the cavity. Obviously, if the velocity of the particle or the length of the cavity is such that it takes several oscillation periods for a particle to traverse the cavity no efficient acceleration is possible.

\subsubsection{Wave Equation}

To generate electromagnetic field components in the direction of wave propagation we cannot use free plane waves, but must apply specific boundary conditions by properly placing conducting metallic surfaces to modify the electromagnetic wave into the desired form. The theory of electromagnetic waves, waveguides and modes is well established and we repeat here only those aspects relevant to particle acceleration. For more detailed reading consult, for example, [3, 4]. Maxwell's equations for our application in a charge free environment are

$$
\begin{array}{ll}
\nabla(\epsilon \boldsymbol{E})=0, & \nabla \times \boldsymbol{E}=-\frac{\mathrm{d} \boldsymbol{B}}{\mathrm{d} t}, \\
\nabla \boldsymbol{B}=0, & c^{2} \nabla \times \boldsymbol{B}=\epsilon \mu \frac{\mathrm{d} \boldsymbol{E}}{\mathrm{d} t},
\end{array}
$$

and we look for solutions in the form of rf-fields oscillating with frequency $\omega$ and $\boldsymbol{U}=\boldsymbol{U}_{0} \mathrm{e}^{\mathrm{i} \omega t}$ where $\boldsymbol{U}=\boldsymbol{E}$ or $\boldsymbol{B}$. A uniform medium is assumed which need not be a vacuum but may have a dielectric constant $\epsilon$ and a magnetic permeability $\mu$. Maxwell's curl equations become then

$$
\begin{aligned}
& \nabla \times \boldsymbol{E}=-\mathrm{i} \omega \boldsymbol{B}, \\
& c^{2} \nabla \times \boldsymbol{B}=\mathrm{i}>\mu \omega \boldsymbol{E} .
\end{aligned}
$$

Eliminating the magnetic or electric field strength from both equations and using the vector relation $\nabla \times(\nabla \times \boldsymbol{a})=\nabla(\nabla \boldsymbol{a})-\nabla^{2} \boldsymbol{a}$ we get the respective wave equations

$$
\begin{aligned}
\nabla^{2} \boldsymbol{E}+k^{2} \boldsymbol{E} & =0, \\
\nabla^{2} \boldsymbol{B}+k^{2} \boldsymbol{B} & =0,
\end{aligned}
$$

where

$$
k=\epsilon \mu \frac{\omega^{2}}{c^{2}} .
$$


In the case of a plane wave propagating along the $z$-axis the transverse partial derivatives vanish $\frac{\partial}{\partial x}=\frac{\partial}{\partial y}=0$, since field parameters of a plane wave do not vary transverse to the direction of propagation. The differential equation (18.3) for the electrical field component then becomes $\left(\frac{\partial^{2}}{\partial z^{2}}+k^{2}\right) \boldsymbol{E}=0$ and the solution is

$$
\boldsymbol{E}=\boldsymbol{E}_{0} \mathrm{e}^{\mathrm{i}(\omega t-k z)}
$$

For real values of the wave number $k$ the solutions of (18.3) describe waves propagating with the phase velocity

$$
v_{\mathrm{ph}}=\frac{z}{t}=\frac{c}{\sqrt{\epsilon \mu}} \leq c .
$$

An imaginary component of $k$, on the other hand, would lead to an exponential damping term for the fields, a situation that occurs, for example, in a conducting surface layer where the fields decay exponentially over a distance of the skin depth. Between conducting boundaries, the wave number is real and describes propagating waves of arbitrary frequencies. As has been noted before, however, such plane waves lack electrical field components in the direction of propagation. In the following section, we will therefore derive conditions to obtain from (18.3) waves with longitudinal field components.

\subsubsection{Rectangular Waveguide Modes}

Significant modification of wave patterns can be obtained from the proximity of metallic boundaries. To demonstrate this, we evaluate the electromagnetic field of a wave propagating along the axis of a rectangular metallic pipe or rectangular waveguide as shown in Fig. 18.1. Since we are interested in getting a finite value for the $z$-component of the electrical field we try the ansatz

$$
E_{z}=\psi_{x}(x) \psi_{y}(y) \psi_{z}(z)
$$

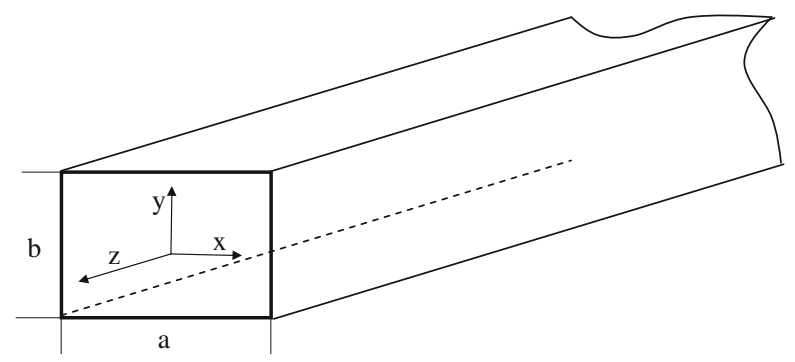

Fig. 18.1 Rectangular waveguide 
and look for boundary conditions that are required to obtain nonvanishing longitudinal fields. Insertion into (18.3) gives

$$
\frac{\psi_{x}^{\prime \prime}(x)}{\psi_{x}(x)}+\frac{\psi_{y}^{\prime \prime}(y)}{\psi_{y}(y)}+\frac{\psi_{z}^{\prime \prime}(z)}{\psi_{z}(z)}=-\epsilon \mu \frac{\omega^{2}}{c^{2}}=-k^{2},
$$

where the r.h.s. is a constant while the functions $\psi_{u}(u)$ are functions of the variable $u=x, y$, or $z$. In order that this equation be true for all values of the coordinates, the ratios $\frac{\psi_{u}^{\prime \prime}(u)}{\psi_{u}(u)}$ must be constant and we may write (18.8) in the form

$$
k_{x}^{2}+k_{y}^{2}+k_{z}^{2}=k^{2}
$$

Differentiating (18.7) twice with respect to $z$ results in the differential equation for the z-component of the electrical field

$$
\frac{\mathrm{d}^{2} E_{z}}{\mathrm{~d} z^{2}}=-k_{z}^{2} E_{z},
$$

which can be solved readily. The wavenumber $k_{z}$ must be real for propagating waves and with the definition

$$
k_{\mathrm{c}}^{2}=k_{x}^{2}+k_{y}^{2}
$$

we get with (18.9)

$$
k_{z}^{2}=k^{2}-k_{\mathrm{c}}^{2} .
$$

The solution (18.7) of the wave equation for the $z$-component of the electrical field is then finally

$$
E_{z}=E_{0 z} \psi_{x}(x) \psi_{y}(y) \mathrm{e}^{\mathrm{i}\left(\omega t-k_{z} z\right)} .
$$

The nature of the parameters in this equation will determine if the wave fields are useful for acceleration of charged particles. The phase velocity is given by

$$
v_{\mathrm{ph}}=\frac{\omega}{k_{z}}=\frac{\omega}{\sqrt{k^{2}-k_{\mathrm{c}}^{2}}} .
$$

An electromagnetic wave in a rectangular metallic pipe is propagating only if the phase velocity is real or $k>k_{\mathrm{c}}$ and the quantity $k_{\mathrm{c}}$ is therefore called the cutoff wave number. For frequencies with a wave number less than the cutoff value the phase velocity becomes imaginary and the wave decays exponentially like $\exp \left(-\sqrt{\left|k^{2}-k_{\mathrm{c}}^{2}\right|} z\right)$. 
Conducting boundaries modify electromagnetic waves in such a way that finite longitudinal electric field components can be produced which, at least in principle, can be used for particle acceleration. Although we have found solutions seemingly suitable for particle acceleration, we cannot use such an electromagnetic wave propagating in a smooth rectangular pipe to accelerate particles. Inserting (18.9) into (18.14) the phase velocity of a traveling waveguide mode in a rectangular pipe becomes

$$
v_{\mathrm{ph}}=\frac{c}{\sqrt{\epsilon \mu} \sqrt{1-\left(k_{\mathrm{c}} / k\right)^{2}}}
$$

and is with $k>k_{\mathrm{c}}$ in vacuum or air $(\epsilon \approx \mu \approx 1)$ larger than the velocity of light. There can be no net acceleration since the wave rolls over the particles, which cannot move faster than the speed of light. This problem occurs in a smooth pipe of any cross section. We must therefore seek for modifications of a smooth pipe in such a way that the phase velocity is reduced or to allow a standing wave pattern, in which case the phase velocity does not matter anymore. The former situation occurs for traveling wave linac structures, while the latter is specially suited for single accelerating cavities.

For a standing wave pattern $k_{z}=0$ or $k=k_{\mathrm{c}}$ and with (18.9), (18.11) the cutofffrequency is

$$
\omega_{\mathrm{c}}=\frac{c k_{\mathrm{c}}}{\sqrt{\epsilon \mu}} .
$$

To complete the solution (18.13) for transverse dimensions, we apply boundary conditions to the amplitude functions $\psi_{x}$ and $\psi_{y}$. The rectangular waveguide with a width $a$ in the $x$-direction and a height $b$ in the $y$-direction (Fig. 18.1) be aligned along the $z$-axis. Since the tangential component of the electrical field must vanish at conducting surfaces, the boundary conditions are

$$
\begin{array}{ll}
\psi_{x}(x)=0 & \text { for } \quad x= \pm \frac{1}{2} a \\
\psi_{y}(y)=0 & \text { for } \quad y= \pm \frac{1}{2} b .
\end{array}
$$

The solutions must be cosine functions to meet the boundary conditions and the complete solution (18.13) for the longitudinal electric field can be expressed by

$$
E_{z}=E_{0} \cos \frac{m \pi x}{a} \cos \frac{n \pi y}{b} \mathrm{e}^{\mathrm{i}\left(\omega t-k_{z} z\right)},
$$

where $m \geq 1$ and $n \geq 1$ are integers defining transverse field modes. The trigonometric functions are eigenfunctions of the differential equation (18.10) with boundary conditions (18.17) and the integers $m$ and $n$ are eigenvalues. In a similar way we get an expression for the $z$-component of the magnetic field strength $B_{z}$. The boundary conditions require that the tangential magnetic field component at a 
conducting surface is the same inside and outside the conductor which is equivalent to the requirement that

$$
\left.\frac{\partial B_{z}}{\partial x}\right|_{x= \pm \frac{1}{2} a}=0 \quad \text { and }\left.\quad \frac{\partial B_{z}}{\partial y}\right|_{y= \pm \frac{1}{2} b}=0
$$

These boundary conditions can be met by sine functions and the $z$-component of the magnetic field strength is therefore in analogy to (18.18) given by

$$
B_{z}=B_{0} \sin \frac{m \pi x}{a} \sin \frac{n \pi y}{b} \mathrm{e}^{\mathrm{i}\left(\omega t-k_{z} z\right)} .
$$

The cutoff frequency is the same for both the electrical and magnetic field component and is closely related to the dimension of the wave guide. With the definition (18.11) the cutoff frequency can be determined from

$$
k_{\mathrm{c}}^{2}=+k_{x}^{2}+k_{y}^{2}=\left(\frac{m \pi}{a}\right)^{2}+\left(\frac{n \pi}{b}\right)^{2} .
$$

All information necessary to complete the determination of field components have been collected. Using (18.3), (18.18) the component equations are with $\frac{\partial}{\partial z}=$ $-\mathrm{i} k_{z}$

$$
\begin{array}{ll}
-\mathrm{i} \omega B_{x}=\frac{\partial E_{z}}{\partial y}+\mathrm{i} k_{z} E_{y}, & \mathrm{i} \epsilon \mu \frac{\omega}{c} E_{x}=c \frac{\partial B_{z}}{\partial y}+\mathrm{i} k_{z} c B_{y}, \\
-\mathrm{i} \omega B_{y}=-\mathrm{i} k_{z} E_{x}-\frac{\partial E_{z}}{\partial x}, & \mathrm{i} \epsilon \mu \frac{\omega}{c} E_{y}=-\mathrm{i} k_{z} c B_{x}-c \frac{\partial B_{z}}{\partial x}, \\
-\mathrm{i} \omega B_{z}=\frac{\partial E_{y}}{\partial x}-\frac{\partial E_{x}}{\partial y}, & \mathrm{i} \epsilon \mu \frac{\omega}{c} E_{z}=c \frac{\partial B_{y}}{\partial x}-c \frac{\partial B_{x}}{\partial y} .
\end{array}
$$

From the first four equations we may extract expressions for the transverse field components $E_{x}, E_{y}, B_{x}, B_{y}$ as functions of the known z-components

$$
\begin{aligned}
& E_{x}=-\mathrm{i} \frac{1}{k_{\mathrm{c}}^{2}}\left(k_{z} \frac{\partial E_{z}}{\partial x}+\frac{\omega}{c} c \frac{\partial B_{z}}{\partial y}\right), \\
& E_{y}=\mathrm{i} \frac{1}{k_{\mathrm{c}}^{2}}\left(-k_{z} \frac{\partial E_{z}}{\partial y}+\frac{\omega}{c} c \frac{\partial B_{z}}{\partial x}\right), \\
& c B_{x}=\mathrm{i} \frac{1}{k_{\mathrm{c}}^{2}}\left(k_{z} c \frac{\partial B_{z}}{\partial x}+\epsilon \mu \frac{\omega}{c} \frac{\partial E_{z}}{\partial y}\right), \\
& c B_{y}=-\mathrm{i} \frac{1}{k_{\mathrm{c}}^{2}}\left(k_{z} c \frac{\partial B_{z}}{\partial y}+\epsilon \mu \frac{\omega}{c} \frac{\partial E_{z}}{\partial x}\right),
\end{aligned}
$$

where

$$
k_{z}^{2}=k^{2}-\left(\frac{m \pi}{a}\right)^{2}-\left(\frac{n \pi}{b}\right)^{2}
$$

and $k^{2}=\epsilon \mu \omega^{2} / c^{2}$.

By application of proper boundary conditions at the conducting surfaces of a rectangular waveguide we have derived expressions for the $z$-component of the 
electromagnetic fields and are able to formulate the remaining field components in terms of the $z$-component. Two fundamentally different field configurations can be distinguished depending on whether we choose $E_{z}$ or $B_{z}$ to vanish. All field configurations, for which $E_{z}=0$, form the class of transverse electrical modes or short TE-modes. Similarly, all fields for which $B_{z}=0$ form the class of transverse magnetic modes or short TM-modes. Each class of modes consists of all modes obtained by varying the indices $m$ and $n$. The particular choice of these mode integers is commonly included in the mode nomenclature and we speak therefore of $\mathrm{TM}_{m n}$ or $\mathrm{TE}_{m n}$-modes. For the remainder of this chapter we will concentrate only on the transverse magnetic or TM-modes, since TE-modes are useless for particle acceleration. The lowest order TM-mode is the $\mathrm{TM}_{11}$-mode producing the $z$-component of the electrical field, which is maximum along the $z$-axis of the rectangular waveguide and falls off from there like a cosine function to reach zero at the metallic surfaces. Such a mode would be useful for particle acceleration if it were not for the phase velocity being larger than the speed of light. In the next subsection we will see how this mode may be used anyway. The next higher mode, the $\mathrm{TM}_{21}$-mode would have a similar distribution in the vertical plane but exhibits a node along the $x$-axis.

Before we continue the discussion on field configurations we note that electromagnetic waves with frequencies above cutoff frequency $\left(k>k_{\mathrm{c}}\right)$ propagate along the axis of the rectangular waveguide. A waveguide wavelength can be defined by

$$
\lambda_{\mathrm{z}}=\frac{2 \pi}{\sqrt{k^{2}-k_{\mathrm{c}}^{2}}}>\lambda,
$$

which is always longer than the free space wavelength $\lambda=2 \pi / k$ and

$$
\frac{1}{\lambda^{2}}=\frac{1}{\lambda_{\mathrm{z}}^{2}}+\frac{1}{\lambda_{\mathrm{c}}^{2}},
$$

where $\lambda_{\mathrm{c}}=2 \pi / k_{\mathrm{c}}$.

The frequency of this traveling electromagnetic wave is from (18.25) with (18.9), (18.16)

$$
\omega=\omega_{\mathrm{c}} \sqrt{1+\frac{k_{\mathrm{z}}^{2}}{k_{\mathrm{c}}^{2}}} .
$$

Electromagnetic energy travels along the waveguide with a velocity known as the group velocity defined by

$$
v_{\mathrm{g}}=\frac{\mathrm{d} \omega}{\mathrm{d} k_{\mathrm{z}}}=\frac{c}{\sqrt{\mu \epsilon}} \sqrt{1-\frac{k_{\mathrm{c}}^{2}}{k^{2}}}<\frac{c}{\sqrt{\mu \epsilon}}<c .
$$


In contrast to the phase velocity, the group velocity is always less than the speed of light as it should be. Rectangular waveguides are mostly used to transport high frequency microwaves from the generator to the accelerating cavity. The bandwidth of waveguides is rather broad and the mechanical tolerances relaxed. Small variation in dimension due to pressurization or evacuation to eliminate field breakdown do generally not matter.

\subsubsection{Cylindrical Waveguide Modes}

For accelerating cavities we try to reach the highest fields possible at a well defined wavelength. Furthermore, accelerating cavities must be operated under vacuum. These requirements result in very tight mechanical tolerances which can be met much easier in round rf-cavities. Analogous to the rectangular case we derive therefore field configurations in cylindrical cavities (Fig. 18.2). The derivation of the field configuration is similar to that for rectangular waveguides although now the wave equation (18.3) is expressed in cylindrical coordinates $(r, \varphi, z)$ and we get for the z-component of the electrical field

$$
\frac{\partial^{2} E_{z}}{\partial r^{2}}+\frac{1}{r} \frac{\partial E_{z}}{\partial r}+\frac{1}{r^{2}} \frac{\partial^{2} E_{z}}{\partial \varphi^{2}}+\frac{\partial^{2} E_{z}}{\partial z^{2}}+k^{2} E_{z}=0 .
$$

with

$$
k^{2}=\epsilon \mu \frac{\omega^{2}}{c^{2}} .
$$

In a stationary configuration the field is expected to be periodic in $\varphi$ while the $z$-dependence is the same as for rectangular waveguides. Using the derivatives $\frac{\partial}{\partial \varphi}=$

Fig. 18.2 Cylindrical resonant cavity (pill box cavity)

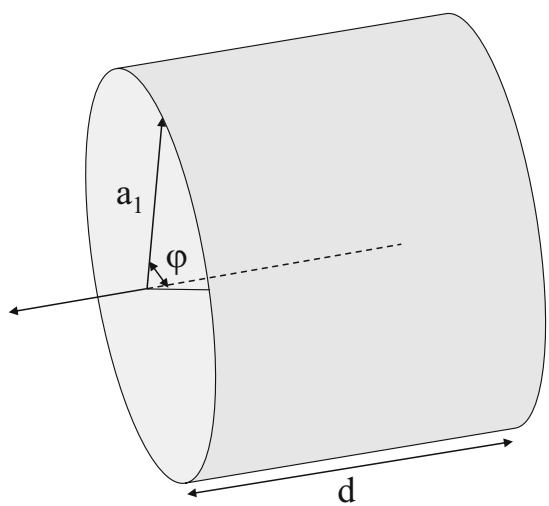


$-\mathrm{i} m$, where $m$ is an integer eigenvalue, and $\frac{\partial}{\partial z}=-\mathrm{i} k_{z}$, we get from (18.29) for the $z$-component of the electric field

$$
\frac{\partial^{2} E_{z}}{\partial r^{2}}+\frac{1}{r} \frac{\partial E_{z}}{\partial r}+\left(k_{\mathrm{c}}^{2}-\frac{m^{2}}{r^{2}}\right) E_{z}=0,
$$

where $k_{\mathrm{c}}^{2}=k^{2}-k_{z}^{2}$ consistent with its previous definition. This differential equation can be solved with Bessel's functions in the form [5]

$$
E_{z}=E_{0} \mathrm{~J}_{m}\left(k_{\mathrm{c}} r\right) \mathrm{e}^{\mathrm{i}\left(\omega t-m \varphi-k_{z} z\right)},
$$

which must meet the boundary condition $E_{z}=0$ for $r=a$, where $a$ is the radius of the cylindrical waveguide. The location of the cylindrical boundaries are determined by the roots of Bessel's functions of order $m$. For the lowest order $m=0$ the first root $a_{1}$ is (see Fig. 18.3)

$$
k_{\mathrm{c}} a_{1}=2.405 \quad \text { or at a radius } \quad a_{1}=\frac{2.405}{k_{\mathrm{c}}} .
$$

Fig. 18.3 Electromagnetic field pattern for a $\mathrm{TM}_{010}$-mode in a circular waveguide. Three dimensional field configuration (a) and radial dependence of fields (b)
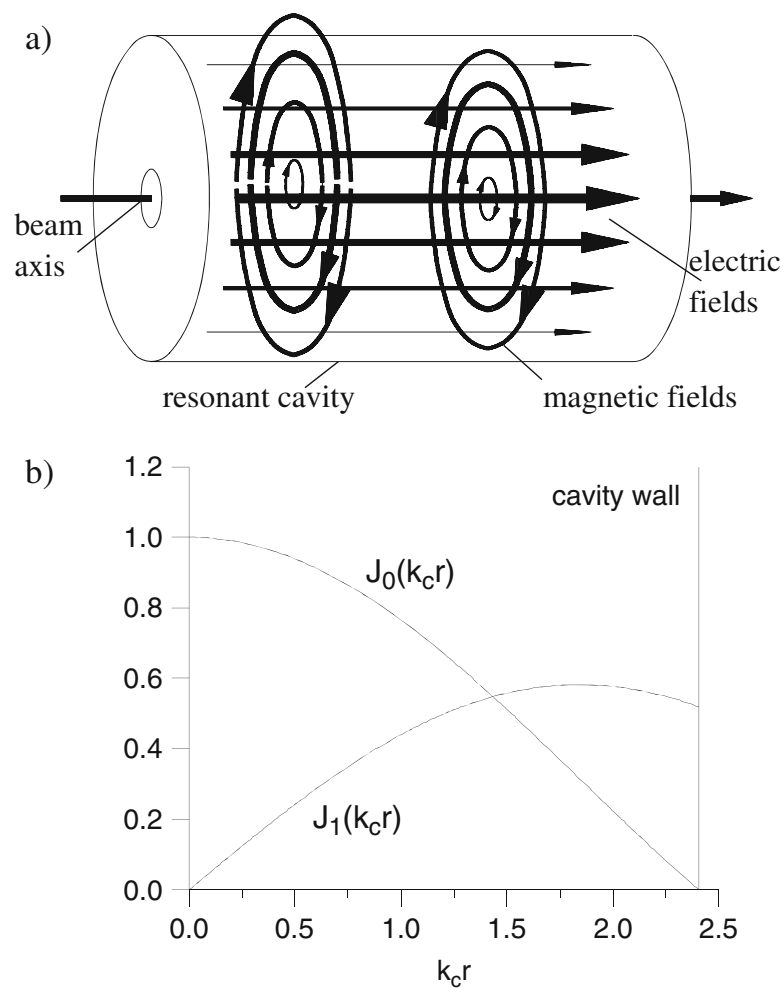
To define a cylindrical cavity two counter propagating waves are created by adding end caps at $z= \pm \frac{1}{2} d$ and with $\mathrm{i} k_{z}=\frac{\pi p}{d}$ and (18.12)

$$
k_{\mathrm{c}}^{2}=\epsilon \mu \frac{\omega^{2}}{c^{2}}-\frac{\pi^{2} p^{2}}{d^{2}} .
$$

Solving for the resonance frequency $\omega$ of the lowest order or the $\mathrm{TM}_{010}$-mode, we get with (18.33), $m=0$ and $p=0$

$$
\omega_{010}=\frac{c}{\sqrt{\epsilon \mu}} \frac{2.405}{a_{1}}
$$

and the $z$-component of the electrical field is

$$
E_{z}=E_{z, 010} J_{0}\left(2.405 \frac{r}{a_{1}}\right) \mathrm{e}^{\mathrm{i} \omega_{010} t}
$$

The waveguide wavenumber

$$
k_{\mathrm{z}}^{2}=k^{2}-k_{\mathrm{c}}^{2}
$$

must be positive in order to obtain a travelling wave rather than a wave decaying exponentially along the waveguide $\left(k_{\mathrm{z}}^{2}<0\right)$. Solving for $k_{z}$ we get with $\omega_{\mathrm{c}}=c k_{\mathrm{c}}$

$$
k_{z}^{2}=k^{2}\left(1-\frac{\omega_{\mathrm{c}}^{2}}{\omega^{2}}\right) \text {. }
$$

The cutoff frequency is determined by the diameter of the waveguide and limits the propagation of electromagnetic waves in circular waveguides to wavelengths which are less than the diameter of the pipe. To determine the phase velocity of the wave we set $\psi=\omega t-k_{z} z=$ const and get from the derivative $\dot{\psi}=\omega-k_{z} \dot{z}=0$ the phase velocity

$$
v_{\mathrm{ph}}=\dot{z}=\frac{\omega}{k_{z}}
$$

Inserting (18.38) into (18.39) we get again a phase velocity which exceeds the velocity of light and therefore any velocity a material particle can reach. We were able to modify plane electromagnetic waves in such a way as to produce the desired longitudinal electric field component but note that these fields are not yet suitable for particle acceleration because the phase rolls over the particles and the net acceleration is zero. To make such electromagnetic waves useful for particle acceleration further modifications of the waveguide are necessary to slow down the phase velocity.

To complete our discussion we determine also the group velocity which is the velocity of electromagnetic energy transport along the waveguide. The 
group velocity $v_{\mathrm{g}}$ is defined by

$$
v_{\mathrm{g}}=\frac{\mathrm{d} \omega}{\mathrm{d} k_{z}} .
$$

Differentiating (18.38) with respect to $k_{z}$ we get

$$
k_{z}=\frac{\omega}{c^{2}} \frac{\mathrm{d} \omega}{\mathrm{d} k_{z}}
$$

or with (18.39) the group velocity

$$
v_{\mathrm{g}}=\frac{\mathrm{d} \omega}{\mathrm{d} k_{z}}=\frac{c^{2} k_{z}}{\omega}=c \frac{c}{v_{\mathrm{ph}}}<c,
$$

since $v_{\mathrm{ph}}>c$.

\section{TM-Mode Field Components in Cylindrical Waveguides}

Similar to the case of rectangular waveguides we can derive all field components. Since we know $E_{z}$ and $B_{z}$ we may use (18.2), expressed in cylindrical coordinates, to determine all other field components. Furthermore, we note from (18.32) that $\frac{\partial}{\partial \varphi}=-\mathrm{i} m$ and $\frac{\partial}{\partial z}=-\mathrm{i} k_{z}$. Maxwell's equations (18.2) in cylindrical coordinates are in component form

$$
\begin{array}{cc}
\mathrm{i} \frac{m}{r} E_{z}-\mathrm{i} k_{z} E_{\varphi}=\mathrm{i} \omega B_{r}, & \mathrm{i} \frac{m}{r} c B_{z}-\mathrm{i} k_{z} c B_{\varphi}=\mathrm{i} \epsilon \mu \frac{\omega}{c} E_{r}, \\
\mathrm{i} k_{z} E_{r}+\frac{\partial E_{z}}{\partial r}=+\mathrm{i} \omega B_{\varphi}, & -\mathrm{i} k_{z} c B_{r}-c \frac{\partial B_{z}}{\partial r}=-\mathrm{i} \epsilon \mu \frac{\omega}{c} E_{\varphi}, \\
\frac{1}{r} E_{\varphi}+\frac{\partial E_{\varphi}}{\partial r}+\mathrm{i} \frac{m}{r} E_{r}=-\mathrm{i} \omega B_{z}, & \frac{1}{r} c B_{\varphi}+c \frac{\partial B_{\varphi}}{\partial r}+\mathrm{i} \frac{m}{r} c B_{r}=-\mathrm{i} \epsilon \mu \frac{\omega}{c} E_{z},
\end{array}
$$

These equations can be used to define individual field components. For example, from the first equation (left) and the second equation (right) we may eliminate $B_{r}$ and solve for $E_{\varphi}$ in terms of $E_{z}$ and $B_{z}$. We are mostly interested in TM-mode fields where $B_{z}$. Conversely, for TE-modes $E_{z}=0$. The field components applicable to both modes are given by (18.44). For the TM-modes $B_{0}=0$ and for TE-modes $E_{0}=0$.

$$
\begin{aligned}
& E_{r}=-\frac{k_{z}}{k_{\mathrm{c}}^{2}}\left(\mathrm{i} \frac{\partial E_{z}}{\partial r}+\frac{1}{k_{z}} \frac{m}{r} \omega B_{z}\right), \\
& E_{\varphi}=\frac{1}{k_{\mathrm{c}}^{2}}\left(-\frac{m}{r} k_{z} E_{z}+\mathrm{i} \omega \frac{\partial B_{z}}{\partial r}\right) \\
& E_{z}=E_{0} J_{m}\left(k_{\mathrm{c}} r\right) \mathrm{e}^{\mathrm{i}\left(\omega t-m \varphi-k_{z} z\right)}, \\
& c B_{r}=\frac{1}{k_{\mathrm{c}}^{2}}\left(-\mathrm{i} k_{z} c \frac{\partial B_{z}}{\partial r}+\epsilon \mu \frac{\omega}{c} \frac{m}{r} E_{z}\right), \\
& c B_{\varphi}=-\frac{1}{k_{\mathrm{c}}^{2}}\left(k_{z} \frac{m}{r} c B_{z}+\mathrm{i} \epsilon \mu \frac{\omega}{c} \frac{\partial E_{z}}{\partial r}\right), \\
& c B_{z}=B_{0} J_{m}\left(k_{\mathrm{c}} r\right) \mathrm{e}^{\mathrm{i}\left(\omega t-m \varphi-k_{z} z\right)} .
\end{aligned}
$$




\subsection{Rf-Cavities}

\subsubsection{Square Cavities}

The waveguide modes are not yet ready to be used for particle acceleration because of excessive phase velocities. This problem can be solved by considering two waves travelling in opposite directions on the same axis of the waveguide. Both fields have the form of (18.18) and the superposition of both waves gives

$$
E_{z}=2 E_{0} \cos \frac{m \pi x}{a} \cos \frac{n \pi y}{b} \cos \frac{p \pi z}{d} \mathrm{e}^{\mathrm{i} \omega t},
$$

where $d$ is defined by

$$
d=\frac{\pi p}{k_{z}}
$$

and $p$ is an integer.

The superposition of two equal but opposite waves form a standing wave with nodes half a waveguide length apart. Closing off the waveguide at such nodes points with a metallic surface fulfills automatically all boundary conditions. The resulting rectangular box forms a resonant cavity enclosing a standing electro-magnetic wave which can be used for particle acceleration.In analogy to the waveguide mode terminology we extend the nomenclature to cavities by adding a third index for the eigenvalue $p$. The lowest cavity mode is the $\mathrm{TM}_{110}$-mode. The indices $m$ and $n$ cannot be zero because of the boundary conditions for $E_{z}$. For $p=0$ we find $E_{z}$ to be constant along the axis of the cavity varying only with $x$ and $y$.The boundary conditions are met automatically at the end caps since with $p=0$ also $k=0$ and the transverse field components vanish everywhere. The electrical field configuration for the $\mathrm{TM}_{110}$-mode consists therefore of a finite $E_{z}$-component being constant only along $z$ and falling off transversely from a maximum value to zero at the walls. In practical applications rectangular boxes are rarely used as accelerating cavities. There are, however, special applications like beam position monitors where rectangular cavities are preferred.

\subsubsection{Cylindrical Cavity}

Similarly, we may form a cylindrical cavity by two counter propagating waves. By adding endcaps at $z= \pm \frac{1}{2} d$ standing waves are established and with $k_{z}=\frac{p \pi}{d}$ we get from (18.37)

$$
k_{\mathrm{c}}^{2}=\epsilon \mu \frac{\omega^{2}}{c^{2}}-\frac{p^{2} \pi^{2}}{d^{2}} .
$$


Solving for the resonance frequency $\omega$ of the lowest order or the $\mathrm{TM}_{010}$-mode, we get with (18.33), $m=0$ and $p=0$

$$
\omega_{010}=\frac{c}{\sqrt{\epsilon \mu}} \frac{2.405}{a_{1}}
$$

and the $z$-component of the electrical field is

$$
E_{z}=2 E_{z, 010} \mathrm{~J}_{0}\left(2.405 \frac{r}{a_{1}}\right) \cos \left(\omega_{010} t\right)
$$

The resonance frequency is inversely proportional to the radius of the cavity and to keep the size of accelerating cavities manageable, short wave radio frequencies are chosen. For electron linear accelerators a wavelength of $\lambda=10 \mathrm{~cm}$ is often used corresponding to a frequency of $2997.93 \mathrm{MHz}$ and a cavity radius of $a_{1}=3.83 \mathrm{~cm}$. For storage rings a common frequency is $499.65 \mathrm{MHz}$ or $\lambda=60 \mathrm{~cm}$ and the radius of the resonance cavity is $a_{1}=22.97 \mathrm{~cm}$. The size of the cavities is in both cases quite reasonable. For much lower rf-frequencies the size of a resonant cavity becomes large. Where such low frequencies are desired the diameter of a cavity can be reduced at the expense of efficiency by loading it with magnetic material like ferrite with a permeability $\mu>1$ as indicated by (18.48). This technique also allows the change of the resonant frequency during acceleration to synchronize with low energy protons, for example, which have not yet reached relativistic energies. To keep the rf-frequency synchronized with the revolution frequency, the permeability of the magnetic material in the cavity can be changed by an external electrical current. The drawback of using materials like ferrites is that they are lossy in electromagnetic fields, get hot and produce significant outgassing in vacuum environments.

The nomenclature for different modes is similar to that for rectangular waveguides and cavities. The eigenvalues are equal to the number of field maxima in $\varphi, r$ and $z$ and are indicated as indices in this order. The $\mathrm{TM}_{010}$-mode, therefore exhibits only a radial variation of field strength independent of $\varphi$ and $z$. Again, we distinguish TM-modes and TE-modes but continue to consider only TM-modes for particle acceleration. Electrical fields in such a cavity have all the necessary properties for particle acceleration. Small openings along the $z$-axis allow the beam to pass through the cavity and gain energy from the accelerating field. Cylindrical cavities can be excited in many different modes with different frequencies. For particle acceleration the dimensions of the cavity are chosen such that at least one resonant frequency satisfies the synchronicity condition of the circular accelerator. In general this is the frequency of the $\mathrm{TM}_{010}$-mode which is also called the fundamental cavity mode or frequency.

From the expressions (18.44) we find that the lowest order TM-mode does not include transverse electrical field components since $k_{z}=0$ and $m=0$. The only 
transverse field is the azimuthal magnetic field which is with (18.49)

$$
\frac{c}{\sqrt{\epsilon \mu}} B_{\varphi}=-\mathrm{i} E_{z, 010} \mathrm{~J}_{1}\left(2.405 \frac{r}{a_{1}}\right) \mathrm{e}^{\mathrm{i} \omega_{010} t} .
$$

\subsubsection{Energy Gain}

The kinetic energy gained in such a cavity can be obtained by integrating the time dependent field along the particle path. The cavity center be located at $z=0$ and a particle entering the cavity at time $\omega_{010} t=-\pi / 2$ or at $z=-d / 2$ may encounter the phase $\delta$ of the microwave field. The electric field along the $z$-axis as seen by the particle travelling with velocity $v$ has the form $E_{z}=E_{z 0} \sin \left(\omega \frac{z}{v}+\delta\right)$ and we get for the kinetic energy gain of a particle passing through the cavity with velocity $v$

$$
\Delta E_{\mathrm{kin}}=e E_{z 0} \int_{-\frac{1}{2} d}^{\frac{1}{2} d} \cos \left(\omega \frac{z}{v}+\delta\right) \mathrm{d} z .
$$

In general, the change in the particle velocity is small during passage of one rf-cavity and the integral is a maximum for $\delta=\pi / 2$ when the field reaches a maximum at the moment the particle is half way through the cavity. Defining an accelerating cavity voltage

$$
V_{\mathrm{rf}}=E_{z 0} d=E_{010} d
$$

the kinetic energy gain is after integration

$$
\Delta E_{\mathrm{kin}}=e V_{\mathrm{rf}} \frac{\sin \frac{\omega d}{2 v}}{\frac{\omega d}{2 v}}=e V_{\mathrm{cy}},
$$

where we have defined an effective cavity voltage and the transit-time factor is

$$
T=\frac{\sin \frac{\omega d}{2 v}}{\frac{\omega d}{2 v}} .
$$

The transit-time factor provides the correction on the particle acceleration due to the time variation of the field while the particles traverse the cavity. In a resonant pill box cavity (Fig. 18.4a) we have $d=\lambda / 2$ and the transit-time factor for a particle traveling approximately at the speed of light is

$$
T_{\text {pillbox }}=\frac{2}{\pi}<1
$$




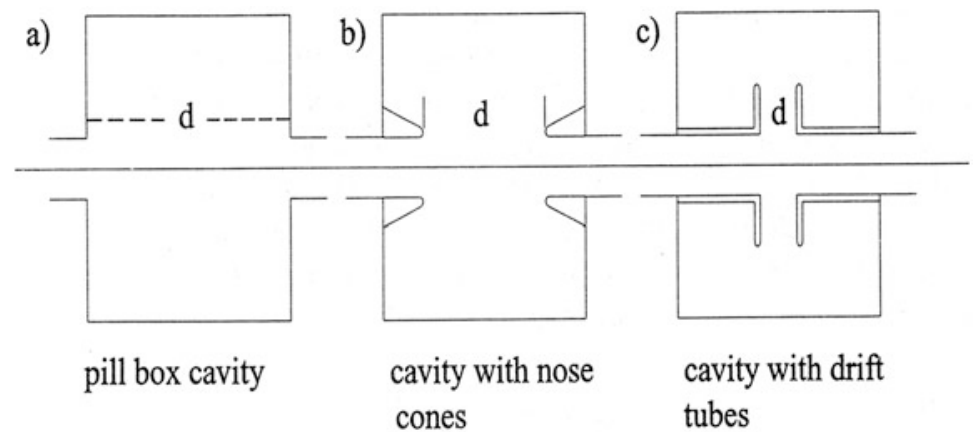

Fig. 18.4 Resonant cavities with drift tubes (schematic). (a) Pill box cavity. (b) Cavity with nose cones. (c) Cavity with drift tubes

As the cavity length or the active accelerating gap in the cavity is reduced, the transient time factor can be increased. The simple pill box cavity may be modified by adding nose cones (Fig. 18.4b) or by adding drift tubes at the entrance and exit of the cavity as shown in Fig. 18.4c. In this case the parameter $d$ in (18.54) is the active accelerating gap.

For small velocities $(v \ll c)$ the transit time factor and thereby the energy gain is small or maybe even negative. Maximum energy gain is obtained for particles travelling at or close to the speed of light. Externally driven accelerating cavity

\subsubsection{Rf-Cavity as an Oscillator}

Accelerator cavities can be described as damped oscillators with external excitation. Damping occurs due to energy losses in the walls of the cavity and transfer of energy to the particle beam while an external rf-power source is connected to the cavity to sustain the rf-fields. Many features of an accelerating cavity can be expressed in well-known terms of a damped, externally excited harmonic oscillator which is described in the form

$$
\ddot{x}+2 \alpha \dot{x}+\omega_{0}^{2} x=D \mathrm{e}^{\mathrm{i} \omega t},
$$

where $\alpha$ is the damping decrement, $\omega_{0}$ the unperturbed oscillator frequency and $D$ the amplitude of the external driving force with frequency $\omega$. The equilibrium solution can be expressed in the form $x=A \mathrm{e}^{\mathrm{i} \omega t}$, where the complex amplitude $A$ is determined after insertion of this ansatz into (18.56)

$$
A=\frac{D}{\omega_{0}^{2}-\omega^{2}+\mathrm{i} 2 \alpha \omega}=a \mathrm{e}^{\mathrm{i} \Psi}
$$


Fig. 18.5 Resonance curve for a damped oscillator

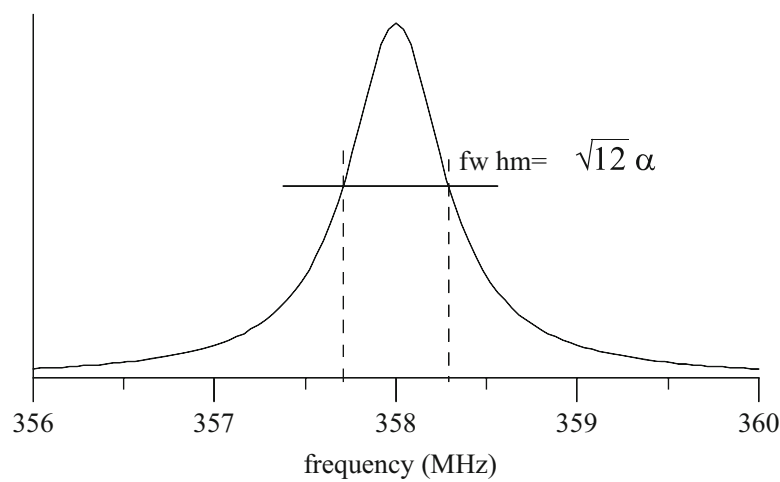

The angle $\Psi$ is the phase shift between the external excitation and the oscillator and the amplitude $a=\operatorname{Re}(A)$ is from (18.57)

$$
a=\frac{D}{\sqrt{\left(\omega_{0}^{2}-\omega^{2}\right)^{2}+4 \alpha^{2} \omega^{2}}},
$$

Plotting the oscillation amplitude $a$ as a function of the excitation frequency $\omega$, we get the resonance curve for the oscillator as shown in Fig. 18.5. The resonance frequency at which the oscillator reaches the maximum amplitude depends on the damping and is

$$
\omega_{\mathrm{r}}=\sqrt{\omega_{0}^{2}-2 \alpha^{2}}
$$

For an undamped oscillator the resonance amplitude becomes infinite but is finite whenever there is damping. The oscillator can be excited within a finite distance from the resonance frequency and the width of the resonance curve at half maximum amplitude is

$$
\Delta \omega_{\frac{1}{2}} \approx \pm 2 \sqrt{3} \alpha \quad \text { for } \quad \alpha \ll \omega_{\mathrm{r}}
$$

If there were no external excitation to sustain the oscillation, the amplitude would decay like $a \propto \mathrm{e}^{-\alpha t}$. The energy of the oscillator scales like $W \propto A^{2}$ and the energy loss per unit time $P=-\mathrm{d} W / \mathrm{d} t=2 \alpha W$, which can be used to determine the quality factor of this oscillator as defined in (18.80)

$$
Q=\frac{\omega_{\mathrm{r}}}{2 \alpha} .
$$


The quality factor is reduced as damping increases. For the case of an accelerating cavity, we expect therefore a higher $Q$-value called the unloaded $Q_{0}$ when there is no beam, and a reduced quality factor called loaded $Q$ when there is a beam extracting energy from the cavity. The time constant for the decay of oscillation amplitudes or the cavity damping time is

$$
t_{\mathrm{d}}=\frac{1}{\alpha}=\frac{2 Q}{\omega_{\mathrm{r}}}
$$

and the field amplitude decays to $1 /$ e during $Q / \pi$ oscillations.

Coming back to the equation of motion (18.56) for this oscillator, we have the solution

$$
x(t)=a \mathrm{e}^{\mathrm{i}(\omega t+\Psi)}
$$

noting that the oscillator assumes the same frequency as the external excitation but is out of synchronism by the phase $\Psi$. The magnitude and sign of this phase shift depends on the excitation frequency and can be derived from (18.57) in the form

$$
\omega_{\mathrm{r}}^{2}-\omega^{2}+\mathrm{i} 2 \alpha \omega=\frac{D}{a} \mathrm{e}^{-\mathrm{i} \Psi}=\frac{D}{a}(\cos \Psi-\mathrm{i} \sin \Psi) .
$$

Both the real and imaginary parts must separately be equal and we get for the phase shift between excitation and oscillator

$$
\cot \Psi=\frac{\omega^{2}-\omega_{\mathrm{r}}^{2}}{2 \alpha \omega} \approx 2 Q \frac{\omega-\omega_{\mathrm{r}}}{\omega_{\mathrm{r}}},
$$

where we have made use of (18.61) and the approximation $\omega \approx \omega_{\mathrm{r}}$. For excitation at the resonance frequency we find the oscillator to lag behind the driving force by $\frac{1}{2} \pi$ and is almost in phase or totally out of phase for very low or very high frequencies, respectively. In rf-jargon this phase shift is called the tuning angle.

\subsubsection{Cavity Losses and Shunt Impedance}

Radio frequency fields can be enclosed within conducting surfaces only because electrical surface currents are induced by these fields which provide the shielding effect. For a perfect conductor with infinite surface conductivity these currents would be lossless and the excitation of such a cavity would persist indefinitely. This situation is achieved to a considerable degree, albeit not perfect, in superconducting cavities. In warm cavities constructed of copper or aluminum the finite resistance of the material causes surface currents to produce heating losses leading to a depletion of field energy. To sustain a steady field in the cavity, radio frequency 
power must be supplied continuously. The surface currents in the conducting cavity boundaries can be derived from Maxwell's curl equation or Ampere's law (18.2). In cylindrical coordinates this vector equation becomes for the lowest order TM-mode in component form

$$
\begin{aligned}
-\frac{\partial B_{\varphi}}{\partial z} & =\mu_{0} \mu j_{r}, \\
0 & =j_{\varphi}, \\
\frac{\partial r B_{\varphi}}{r \partial r} & =\frac{B_{\varphi}}{r}+\frac{\partial B_{\varphi}}{\partial r}=\mu_{0} \mu j_{z}+\mathrm{i} \frac{\epsilon \mu}{c^{2}} \omega E_{z} .
\end{aligned}
$$

Because we do not consider perfectly but only well conducting boundaries, we expect fields and surface currents to penetrate somewhat into the conducting material. The depth of penetration of fields and surface currents into the conductor is well-known as the skin depth [3]

$$
\delta_{\mathrm{s}}=\sqrt{\frac{2}{\mu_{0} \mu_{\mathrm{w}} \omega \sigma_{\mathrm{w}}}},
$$

where $\sigma_{\mathrm{w}}$ is the conductivity of the cavity wall and $\mu_{\mathrm{w}}$ the permeability of the wall material. The azimuthal magnetic field component induces surface currents in the cylindrical walls as well as in the end caps. In both cases the magnetic field decays within a skin depth from the surface inside the conductor. The first Eq. (18.65) applies to the end caps and the integral through the skin depth is

$$
\left.\int_{S}^{S+\delta_{\mathrm{s}}} \frac{\partial B_{\varphi}(r)}{\partial z} \mathrm{~d} z \approx B_{\varphi}(r)\right|_{S} ^{S+\delta_{\mathrm{s}}} \approx-B_{\varphi}(r, S),
$$

since $B_{\varphi}\left(r, S+\delta_{\mathrm{s}}\right) \approx 0$ just under the surface $S$ of the wall. We integrate also the third Eq. (18.65) at the cylindrical walls and get for the first term $\int B_{\varphi} / r \mathrm{~d} r \approx B_{\varphi} \delta_{\mathrm{s}} / a_{1}$, which is negligible small, while the second term has a form similar to (18.67). The electrical term $E_{z}$ vanishes because of the boundary condition and the surface current densities for the cylindrical wall and end caps, respectively, are therefore related to the magnetic fields by

$$
\begin{aligned}
& \mu_{0} \mu j_{z} \delta_{\mathrm{s}}=B_{\varphi}\left(a_{1}, z\right), \\
& \mu_{0} \mu j_{r} \delta_{\mathrm{s}}=B_{\varphi}\left(r, \pm \frac{1}{2} d\right) .
\end{aligned}
$$

The cavity losses per unit wall surface area are given by

$$
\frac{\mathrm{d} P_{\mathrm{cy}}}{\mathrm{d} S}=\tilde{r}_{\mathrm{s}} j_{\mathrm{s}}^{2},
$$


where $j_{\mathrm{s}}$ is the surface current density and $\tilde{r}_{\mathrm{s}}$ is the surface resistance given by

$$
\tilde{r}_{\mathrm{s}}=\sqrt{\frac{\mu_{0} \mu_{\mathrm{w}} \omega}{2 \sigma_{\mathrm{w}}}} .
$$

With $j_{\mathrm{s}}=j_{r, z} \delta_{\mathrm{s}},(18.50),(18.66)$ and the integration of (18.69) is performed over all inside surfaces of the cavity to give

$$
P_{\mathrm{cy}}=\frac{1}{4} \epsilon_{0} \omega \delta_{\mathrm{s}} \epsilon \frac{\mu_{\mathrm{w}}}{\mu} E_{010}^{2} \int_{S} \mathrm{~J}_{1}^{2}\left(2.405 \frac{r}{a_{1}}\right) \mathrm{d} S,
$$

where $\epsilon$ and $\mu$ is the dielectric constant and permeability of the material inside the cavity, respectively and $\mu_{\mathrm{w}}$ the wall permeability. Evaluating the integral over all surfaces, we get for the cylindrical wall the integral value $2 \pi a_{1} d J_{1}^{2}(2.405)$. For each of the two end caps the integral $2 \pi \int_{0}^{a_{1}} \mathrm{~J}_{1}^{2}\left(2.405 \frac{r}{a_{1}}\right) r \mathrm{~d} r$ must be evaluated and is from integration tables [6]

$$
2 \pi \int_{0}^{a_{1}} \mathrm{~J}_{1}^{2}\left(2.405 \frac{r}{a_{1}}\right) r \mathrm{~d} r=\pi a_{1}^{2} \mathrm{~J}_{1}^{2}(2.405) .
$$

The total cavity wall losses become finally with $V_{\mathrm{rf}}=E_{010} d$ from (18.52)

$$
P_{\mathrm{cy}}=\frac{1}{2} \pi \epsilon_{0} \omega \delta_{\mathrm{s}} \epsilon \frac{\mu_{\mathrm{w}}}{\mu} V_{\mathrm{rf}}^{2} J_{1}^{2}(2.405) \frac{a_{1}\left(a_{1}+d\right)}{d^{2}} .
$$

It is convenient to separate fixed cavity parameters from adjustable parameters. Once the cavity is constructed, the only adjustable parameter is the strength of the electrical field $E_{010}$ or the effective cavity voltage $V_{\text {cy }}$. Expressing the cavity losses in terms of an impedance, we get from (18.73) and (18.53)

$$
P_{\mathrm{cy}}=\frac{V_{\mathrm{cy}}^{2}}{2 R_{\mathrm{s}}}
$$

where the cavity shunt impedance including transient time factor is defined by ${ }^{1}$

$$
R_{\mathrm{s}}=\frac{1}{\pi \epsilon_{0}} \frac{1}{\omega \delta_{\mathrm{s}} \epsilon} \frac{\mu}{\mu_{\mathrm{w}}} \frac{d^{2}}{a_{1}\left(a_{1}+d\right)} \frac{1}{\mathrm{~J}_{1}^{2}(2.405)}\left(\frac{\sin \frac{\omega d}{2 v}}{\frac{\omega d}{2 v}}\right)^{2} .
$$

The factor of 2 in (18.74) results from the fact that on average the rf-voltage is $\left\langle V_{\mathrm{cy}}^{2}=\hat{V}_{\mathrm{cy}}^{2} \sin ^{2} \omega t\right\rangle=\frac{1}{2} \hat{V}_{\mathrm{cy}}^{2}$. In accelerator design, we prefer sometimes to use

\footnotetext{
${ }^{1}$ The shunt impedance is defined in the literature sometimes by $P_{\mathrm{cy}}=V_{\mathrm{cy}}^{2} / R_{\mathrm{s}}$ in which case the numerical value of the shunt impedance is larger by a factor of two.
} 
the shunt impedance per unit length or the specific shunt impedance. The required length depends on the accelerating voltage needed and the rf-power available. With the cavity shunt impedance per unit length

$$
r_{\mathrm{s}}=\frac{R_{\mathrm{s}}}{d}
$$

the cavity losses are instead of (18.74)

$$
P_{\mathrm{cy}}=\frac{\hat{V}_{\mathrm{cy}}^{2}}{2 r_{\mathrm{s}} L_{\mathrm{cy}}},
$$

where $L_{\text {cy }}$ is the total length of all cavities producing the voltage $\hat{V}_{\text {cy }}$. Since the cavity shunt impedance scales like $R_{\mathrm{S}} \propto 1 / \sqrt{\omega}$ and the length for a resonant cavity like $d \propto 1 / \omega$, the specific shunt impedance is proportional to the square root of the rf-frequency $r_{\mathrm{s}} \propto \sqrt{\omega}$ favoring high frequencies. A practical limit is reached when the cavity apertures become too small for the particle beam to pass through or when the size of the cavities prevents an efficient cooling of wall losses.

As an example, we calculate from (18.75) the shunt impedance for a pill box cavity designed for a resonance frequency of $358 \mathrm{MHz}$. The wavelength is $\lambda=$ $85 \mathrm{~cm}$, the cavity length $d=42.5 \mathrm{~cm}$ and the cavity radius $a_{1}=32.535 \mathrm{~cm}$. This cavity was constructed with nose cones for the storage ring PEP [7] from aluminum. With a skin depth of $\delta_{\mathrm{s}}=4.44 \mu \mathrm{m}$ the specific shunt impedance becomes $r_{\mathrm{s}}=15.2$ $\mathrm{M} \Omega / \mathrm{m}$ while the measured value for this cavity is $18.0 \mathrm{M} \Omega / \mathrm{m}$.

The difference is due to two competing effects. The open aperture along the axis for the beam has the tendency to reduce the shunt impedance while the nose cones being a part of the actual cavity increase the transient time factor and thereby the effective shunt impedance (18.75). The simple example of a pill box cavity produces rather accurate results, however, for more precise estimates computer programs have been developed to calculate the mode frequencies and shunt impedances for all modes in arbitrary rotational symmetric cavities (for example, SUPERFISH [8] or URMEL [9]). More sophisticated three-dimensional programs are available (for example, MAFIA [9]) to simulate rf-properties of arbitrary forms of cavities.

The specific shunt impedance for a pill box cavity can be expressed in a simple form as a function of the rf-frequency only and is for realistic cavities approximately

$$
\begin{array}{ll}
r_{\mathrm{s}}(\mathrm{M} \Omega / \mathrm{m}) \approx 1.28 \sqrt{f_{\mathrm{rf}}(\mathrm{MHz})} & \text { for copper and } \\
r_{\mathrm{s}}(\mathrm{M} \Omega / \mathrm{m}) \approx 1.06 \sqrt{f_{\mathrm{rf}}(\mathrm{MHz})} & \text { for aluminum }
\end{array}
$$

The shunt impedance should be maximum in order to minimize cavity losses for a given acceleration. Since the interior of the cavity must be evacuated $\mu=\epsilon=1$ and $\mu_{\mathrm{w}}=1$ because we do not consider magnetic materials to construct a cavity. The only adjustable design parameters left are the skin depth and the transient time 
factor. The skin depth can be minimized by using well conducting materials like copper or aluminum.

To derive the quality factor of the cavity the energy $W$ stored in the electromagnetic field within the cavity must be calculated. The field energy is the volume integral of the square of the electrical or magnetic field and we have in case of a $\mathrm{TM}_{010}$-mode with $W=\frac{1}{2} \epsilon_{0} \epsilon \int_{V} E_{z}^{2} \mathrm{~d} V$ and (18.49) for the stored cavity energy

$$
W=\frac{1}{2} \epsilon_{0} \epsilon E_{010}^{2} d a_{1}^{2} J_{1}^{2}(2.405)
$$

The quality factor $Q$ of a resonator is defined as the ratio of the stored energy to the energy loss per radian

$$
Q=2 \pi \frac{\text { stored energy }}{\text { energy loss/cycle }}=\omega \frac{W}{P_{\text {cy }}},
$$

or with (18.73), (18.79)

$$
Q=\frac{d}{\delta_{\mathrm{s}}} \frac{\mu_{\mathrm{w}}}{\mu} \frac{a_{1}}{a_{1}+d} .
$$

The quality factor determines the cavity time constant since the fields decay exponentially like $\mathrm{e}^{-t / \tau_{\mathrm{cy}}}$ due to wall losses, where $\tau_{\text {cy }}$ is the cavity time constant and the decay rate of the stored energy in the cavity is

$$
\frac{\mathrm{d} W}{\mathrm{~d} t}=-\frac{2}{\tau_{\mathrm{cy}}} W
$$

The change in the stored energy is equal to the cavity losses $P_{\text {cy }}$ and the cavity time constant is with (18.80)

$$
\tau_{\mathrm{cy}}=\frac{2 W}{P_{\mathrm{cy}}}=\frac{2 Q}{\omega},
$$

which is equal to (18.62) and also called the cavity filling time because it describes the build up time of fields in a cavity following a sudden application of rf-power.

\subsection{Rf-Parameters}

A variety of rf-parameters has to be chosen for a circular accelerator. Some parameters relate directly to beam stability criteria and are therefore easy to determine. Other parameters have less of an impact on beam stability and are often determined by nonphysical criteria like availability and economics. Before rf-parameters can be determined a few accelerator and lattice parameters must be 
known. Specifically, we need to know the desired minimum and maximum beam energy, the beam current, the circumference of the ring, the momentum compaction factor, and the bending radius of the magnets. Further, we make a choice of the maximum desired rate of particle acceleration per turn or determine the energy loss per turn to synchrotron radiation which needs to be compensated. During the following discussion we assume that these parameters are known.

One of the most prominent parameters for rf-accelerating systems is the rffrequency of the electromagnetic fields. For highly relativistic beams there is no fundamental reason for a particular choice of the rf-frequency and it can therefore be selected on technical and economic grounds. The rf-frequency must, however, be an integer multiple, the harmonic number, of the particle revolution frequency. The harmonic number can be any integer from a beam stability point of view. In specific cases, the harmonic number need to be a multiple of a smaller number. Considering, for example, a colliding beam facility with $N_{\mathrm{IP}}$ collision points an optimum harmonic number is divisible by $N_{\mathrm{IP}} / 2$. In this case $N_{\mathrm{IP}} / 2$ bunches could be filled in each of the two counter rotating beams leading to a maximum collision rate. Other such considerations may require the harmonic number to contain additional factors. In general, most flexibility is obtained if the harmonic number is divisible by small prime numbers.

Within these considerations the harmonic number can be chosen from a large range of rf-frequencies without generally affecting beam stability. Given complete freedom of choice, however, a low frequency is preferable to a high frequency. For low rf-frequencies the bunch length is longer and electromagnetic interaction with the beam environment is reduced since high frequency modes are not excited significantly. A longer bunch length also reduces the particle density in the bunch and thereby potentially troublesome intra-beam scattering $[10,11]$. In proton and heavy ion beams a longer bunch length leads to a reduced space charge tune shift and therefore allows to accelerate a higher beam intensity. For these reasons lower frequency systems are used mostly in low energy circular accelerators. The downside of low rf-frequencies is the fact that the accelerating cavities become very large or less efficient and rf-sources are limited in power capability.

The size of circular accelerators imposes a lower limit on the rf-frequency since the synchronicity condition requires that the rf-frequency be at least equal to the revolution frequency in which case the harmonic number is equal to unity. A higher harmonic number to accommodate more than a single particle bunch further increases the required rf-frequency. Most electron and very high energy proton accelerators operate at rf-frequencies of a few hundred $\mathrm{MHz}$, while lower frequencies are preferred for ion or medium energy proton accelerators.

For some applications it is critical to obtain short particle bunches which is much easier to achieve with a high rf-frequency. The appropriate choice of the rffrequency therefore dependents much on the desired parameters for the particular application and is mostly chosen as a compromise between competing requirements including economic considerations like cost and availability. 


\subsubsection{Synchronous Phase and Rf-voltage}

The most common use of an rf-system is for acceleration while particles pass through a resonant cavity at the moment when the voltage reaches the crest of the rf-wave and particles gain a kinetic energy equivalent to the full cavity voltage. This is the general accelerating mode in linear accelerators. In circular accelerators, however, the principle of phase focusing requires that particles be accelerated off the crest at a synchronous phase $\psi_{\mathrm{s}}$, where the effective accelerating voltage is $V_{\mathrm{a}}=\hat{V}_{\text {cy }} \sin \psi_{\mathrm{s}}$. The peak rf-voltage $\hat{V}_{\text {cy }}$ and the synchronous phase are determined by the desired energy acceptance and acceleration per turn.

The energy acceptance of a circular accelerator has been derived in Chap. 9, is proportional to the square root of the cavity voltage and must be adjusted for the larger of several energy acceptance requirements. To successfully inject a beam into a circular accelerator the voltage must be sufficiently large to accept the finite energy spread in the injected beam. In addition, any phase spread or timing error of the incoming beam translates into energy errors due to synchrotron oscillations. For acceleration of a high intensity beam an additional allowance to the rf-voltage must be made to compensate beam loading, which will be discussed later in more detail.

After injection into a circular accelerator an electron beam may change considerably its energy spread due to quantum excitation as a result of emitting synchrotron radiation. This energy spread has a Gaussian distribution and to assure long beam lifetime the energy acceptance must be large enough to contain at least seven standard deviations. In proton and heavy ion accelerators some phase space manipulation may be required during the injection process which contributes another lower limit for the required rf-voltage. In general, there are a number of requirements that determine the ultimate energy acceptance of an accelerator and the most stringent requirement may very well be different for different accelerator designs and applications. Generally, circular accelerators are designed for an energy acceptance of a few percent.

\subsection{Linear Accelerator}

The phase velocity $v_{\mathrm{ph}}$ must be equal to the particle velocity $v_{\mathrm{p}}$ for efficient acceleration and we need therefore to modify or "load" the wave guide structure to reduce the phase velocity to become equal to the particle velocity. This can be done by inserting metallic structures into the aperture of the circular wave guide. Many different ways are possible, but we will consider only the disk loaded waveguide which is the most common accelerating structure for electron linear accelerators.

In a disk loaded waveguide metallic plates are inserted normal to the waveguide axis at periodic intervals with iris apertures to allow for the passage of the particle beam as shown in Fig. 18.6. 


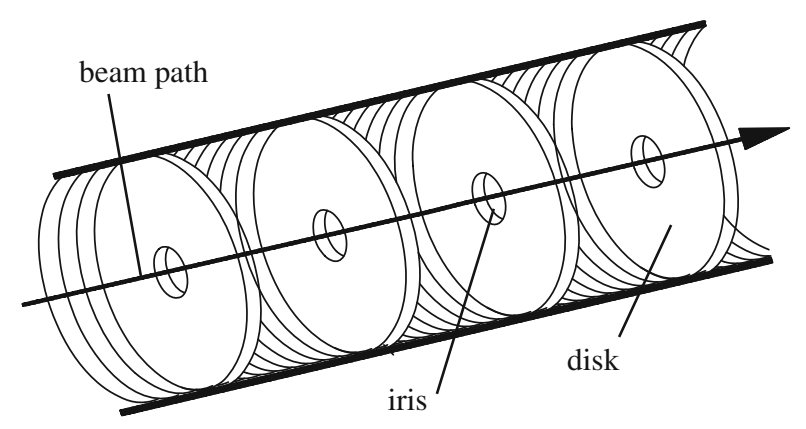

Fig. 18.6 Disk loaded accelerating structure for an electron linear accelerator (schematic)

The boundary conditions and therefore the electromagnetic fields in such a structure are significantly more complicated than those in a simple circular tube. It would exceed the goal of this text to derive the theory of disk loaded waveguides and the interested reader is referred to the review article by Slater [12].

Insertion of disks in periodic intervals into a uniform waveguide essentially creates a sequence of cavities with electromagnetic coupling through either the central hole, holes at some radius on the disks or external coupling cavities. The whole arrangement of cells acts like a band pass filter allowing electromagnetic fields of certain frequencies to propagate. By proper choice of the geometric dimensions the pass band can be adjusted to the desired frequency and the phase velocity can be designed to be equal to the velocity of the particles. For electron linear accelerators the phase velocity is commonly adjusted to the velocity of light since electrons quickly reach such a velocity.

\subsubsection{Basic Waveguide Parameters}

Without going into structure design and detailed determination of geometric parameters we can derive parameters relating to the acceleration capability of such structures. Conservation of energy requires that

$$
\frac{\partial W}{\partial t}+\frac{\partial P}{\partial z}+P_{\mathrm{w}}+n e v E_{z}=0,
$$

where $W$ is the stored energy per unit length, $P$ the energy flux along $z, P_{\mathrm{w}}$ wall losses per unit length and nev $E_{z}$ the energy transferred to $n$ particles with charge $e$ each moving with the velocity $v$ in the electric field $E_{z}$. The wall losses are related to the quality factor $Q$ of the structure defined by

$$
Q=\frac{\omega W}{P_{\mathrm{w}}},
$$


where $P_{\mathrm{w}} / \omega$ are wall losses per unit length and per radian of field oscillation. The energy flux $P$ is with the group velocity $v_{\mathrm{g}}$

$$
P=v_{\mathrm{g}} W
$$

In case of equilibrium, the stored energy in the accelerating structure does not change with time, $\partial W / \partial t=0$, and

$$
\frac{\partial P}{\partial z}=-P_{\mathrm{w}}-i_{\mathrm{b}} E_{z}=-\frac{\omega P}{v_{\mathrm{g}} Q}-i_{\mathrm{b}} E_{z},
$$

where $i_{\mathrm{b}}=n e v$ is the beam current. Considering the case of small beam loading $i_{\mathrm{b}} E_{z} \ll \omega P /\left(v_{\mathrm{g}} Q\right)$ we may integrate (18.87) to get

$$
P=P_{0} \exp \left(-\frac{\omega}{v_{\mathrm{g}} Q} z\right)=P_{0} \mathrm{e}^{-2 \alpha z},
$$

where we have defined the attenuation coefficient

$$
2 \alpha=\frac{\omega}{v_{\mathrm{g}} Q}
$$

Equation (18.88) shows an exponential decay of the energy flux along the accelerating structure with the attenuation coefficient $2 \alpha$. The wall losses are often expressed in terms of the total voltage or the electrical field defined by

$$
P_{\mathrm{w}}=\frac{\hat{V}_{0}^{2}}{Z_{\mathrm{s}} L}=\frac{\hat{E}^{2}}{r_{\mathrm{s}}},
$$

where $Z_{\mathrm{s}}=r_{\mathrm{s}} L$ is the shunt impedance for the whole section, $\hat{E}$ the maximum value of the accelerating field, $E_{z}=\hat{E} \cos \psi_{\mathrm{s}}, \psi_{\mathrm{s}}$ the synchronous phase at which the particle interacts with the wave, $r_{\mathrm{s}}$ the shunt impedance per unit length, and $L$ the length of the cavity. From (18.90) we get with (18.87) and (18.89) for negligible beam current the accelerating field

$$
\hat{E}^{2}=\frac{\omega}{v_{\mathrm{g}}} \frac{r_{\mathrm{s}}}{Q} P=2 \alpha r_{\mathrm{s}} P
$$

The total accelerating voltage along a structure of length $L$ is

$$
V_{0}=\int_{0}^{L} E_{z} \mathrm{~d} z=\hat{E} \cos \psi_{\mathrm{s}} \int_{0}^{L} \mathrm{e}^{-\alpha z} \mathrm{~d} z
$$


or after integration

$$
V_{0}=\frac{1-\mathrm{e}^{-\alpha L}}{\alpha} \hat{E} \cos \psi_{\mathrm{s}}
$$

Defining an attenuation factor $\tau$ by

$$
\tau=\alpha L
$$

we get with (18.91) for the total accelerating voltage per section of length $L$

$$
V_{0}=\sqrt{r_{\mathrm{s}} L P_{0}} \sqrt{2 \tau} \frac{1-\mathrm{e}^{-\tau}}{\tau} \cos \psi_{\mathrm{s}}
$$

The maximum energy is obtained if the particles are accelerated at the crest of the wave, where $\psi_{\mathrm{s}}=0$.

Tacitly it has been assumed that the shunt impedance $r_{\mathrm{s}}$ is constant resulting in a variation of the electrical field strength along the accelerating section. Such a structure is called a constant impedance structure and is characterized physically by equal geometric dimensions for all cells.

In a constant impedance structure the electric field is maximum at the beginning of the section and drops off toward the end of the section. A more efficient use of accelerating sections would keep the electric field at the maximum possible value just below field break down throughout the whole section. A structure with such characteristics is called a constant gradient structure because the field is now constant along the structure.

As an example for an electron linear accelerator, the SLAC constant gradient linac structure has the following parameters [13]

$$
\begin{array}{ll}
f_{\mathrm{rf}}=2856 \mathrm{MHz} & L=10 \mathrm{ft}=3.048 \mathrm{~m} \\
r_{\mathrm{s}}=53 \mathrm{M} \Omega / \mathrm{m} & a_{\mathrm{i}}=0.040 \mathrm{~m} \\
Q \approx 12000 & \tau=0.57
\end{array}
$$

A constant gradient structure can be realized by varying the iris holes in the disks to smaller and smaller apertures along the section. This kind of structure is actually used in the SLAC accelerator as well as in most modern linear electron accelerators. The field $\hat{E}=$ const and therefore from (18.88) with (18.94)

$$
\frac{\partial P}{\partial z}=\frac{P(L)-P_{0}}{L}=-\left(1-\mathrm{e}^{-2 \tau}\right) \frac{P_{0}}{L}
$$

On the other hand, we have from (18.87)

$$
\frac{\partial P}{\partial z}=-\frac{\omega P_{0}}{Q v_{\mathrm{g}}}=\mathrm{const}
$$


and to make $\partial P / \partial z$ constant the group velocity must vary linearly with the local rf-power like

$$
v_{\mathrm{g}} \sim P(z)=P_{0}+\frac{\partial P}{\partial z} z
$$

Furthermore, since $\partial P / \partial z<0$ the group velocity is made to decrease along the section by reducing gradually the iris radii. From (18.98)

$$
v_{\mathrm{g}}(z)=-\frac{\omega}{Q} \frac{P(z)}{\partial P / \partial z}
$$

or with (18.97)

$$
v_{\mathrm{g}}(z)=-\frac{\omega}{Q} \frac{P_{0}+\frac{\partial P}{\partial z} z}{\partial P / \partial z}=+\frac{\omega}{Q} \frac{L-\left(1-\mathrm{e}^{-2 \tau}\right) z}{1-\mathrm{e}^{-2 \tau}}
$$

and the filling time is after integration of (18.101)

$$
t_{\mathrm{F}}=\int_{0}^{L} \frac{\mathrm{d} z}{v_{\mathrm{g}}}=2 \tau \frac{Q}{\omega} .
$$

The electric field in the accelerating section is from (18.90) with (18.87)

$$
\hat{E}=\sqrt{r_{\mathrm{s}}\left|\frac{\partial P}{\partial z}\right|}
$$

and the total accelerating voltage $V_{0}$ or gain in kinetic energy per section is

$$
\Delta E_{\text {kin }}=e V_{0}=e \int_{0}^{L} E_{z} \mathrm{~d} z=e \sqrt{r_{\mathrm{s}} L P_{0}} \sqrt{1-\mathrm{e}^{-2 \tau}} \cos \psi_{\mathrm{s}},
$$

where $\psi_{\mathrm{s}}$ is the synchronous phase at which the particles travel with the electromagnetic wave. The energy gain scales with the square root of the accelerating section length and rf-power delivered.

As a numerical example, we find for the SLAC structure from (18.104) the gain of kinetic energy per $10 \mathrm{ft}$ section as

$$
\Delta E_{\text {kin }}(\mathrm{MeV})=10.48 \sqrt{P_{0}(\mathrm{MW})}
$$

where $P_{0}$ is the rf-power delivered to the section. The energy gain (18.105) is the maximum value possible ignoring beam loading or energy extraction from the fields by the beam. The total accelerating voltage is reduced when we include beam loading due to a pulse current $i_{\mathrm{b}}$. Referring the interested reader to reference [13] 
we only quote the result for the energy gain in a linear accelerator with constant gradient sections including beam loading

$$
V_{i}=\sqrt{r_{\mathrm{s}} L P_{0}} \sqrt{1-\mathrm{e}^{-2 \tau}}-\frac{1}{2} i_{\mathrm{b}} r_{\mathrm{s}} L\left(1-\frac{2 \tau \mathrm{e}^{-2 \tau}}{1-\mathrm{e}^{-2 \tau}}\right) .
$$

For the SLAC linac structure this equation becomes with $\tau=0.57$

$$
E_{\mathrm{kin}}(\mathrm{MeV})=10.48 \sqrt{P_{0}(\mathrm{MW})}-37.47 i_{\mathrm{b}}(\mathrm{A}) .
$$

The beam loading depends greatly on the choice of the attenuation factor $\tau$ as is shown in Figs. 18.7 and 18.8 where the coefficients $f_{\mathrm{v}}=\sqrt{1-\mathrm{e}^{2 \tau}}$ and $f_{\mathrm{i}}=\frac{1}{2}\left(1-\frac{2 \tau \mathrm{e}^{-2 \tau}}{1-\mathrm{e}^{-2 \tau}}\right)$ are plotted as functions of $\tau$. Both coefficients increase as the attenuation factor is increased and reach asymptotic limits. The ratio $f_{\mathrm{v}} / f_{\mathrm{i}}$, however, decreases from infinity to a factor two which means that beam loading occurs much stronger for large values of the attenuation factor compared to low values. During the

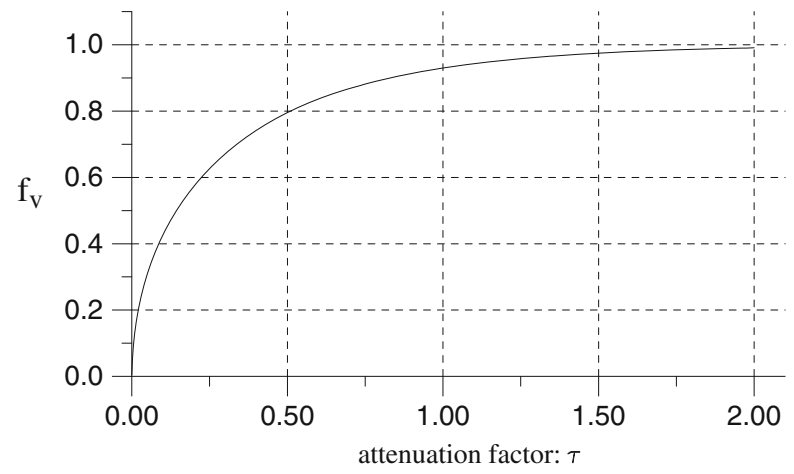

Fig. 18.7 Energy coefficient $f_{\mathrm{v}}$ as a function of $\tau$

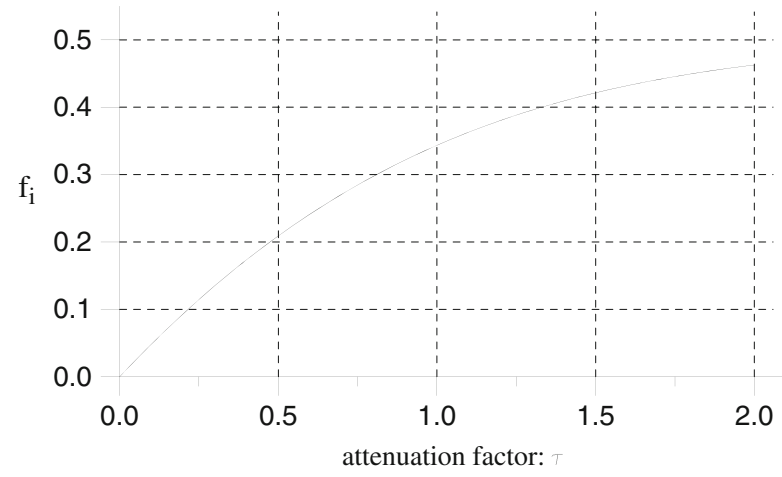

Fig. 18.8 Beam loading coefficient $f_{\mathrm{i}}$ as a function of $\tau$ 


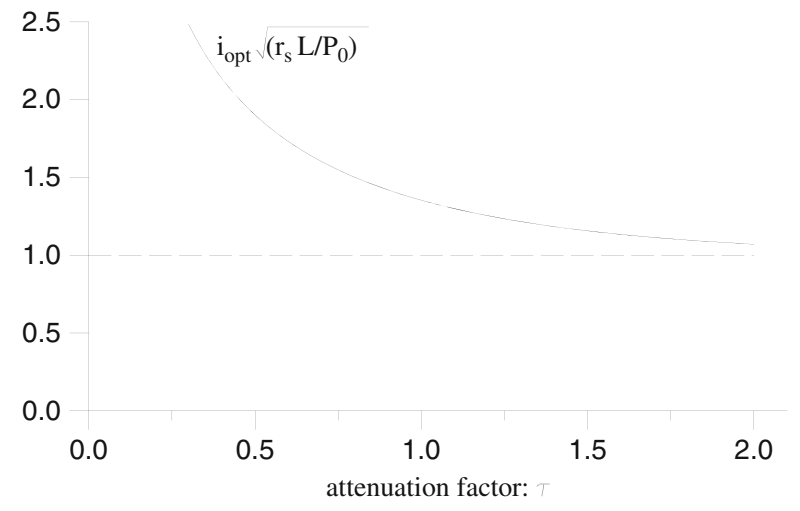

Fig. 18.9 Optimum beam current as a function of $\tau$

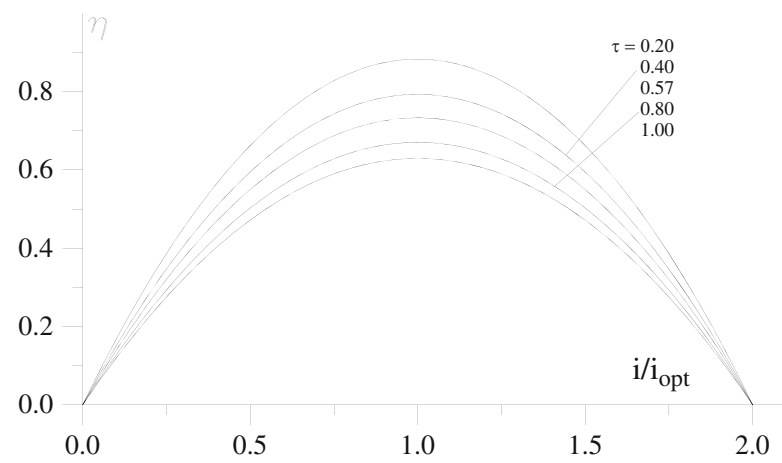

Fig. 18.10 Linac efficiency as a function of beam current

design of the linac structure it is therefore useful to know the intended use requiring different optimization for high-energy or high-current acceleration.

We may also ask for the efficiency of transferring rf-power into beam power which is defined by

$$
\eta=\frac{i_{\mathrm{b}} V_{\mathrm{i}}}{P_{0}}=i_{\mathrm{b}} \sqrt{\frac{r_{\mathrm{s}} L}{P_{0}}} \sqrt{1-\mathrm{e}^{-2 \tau}}-\frac{1}{2} i_{\mathrm{b}}^{2} r_{\mathrm{s}} \frac{L}{P_{0}}\left(1-\frac{2 \tau \mathrm{e}^{-2 \tau}}{1-\mathrm{e}^{-2 \tau}}\right) .
$$

The linac efficiency has clearly a maximum and the optimum beam current is

$$
i_{\mathrm{b}, \mathrm{opt}}=\sqrt{\frac{P_{0}}{r_{\mathrm{s}} L}} \frac{\left(1-\mathrm{e}^{-2 \tau}\right)^{3 / 2}}{1-(1+2 \tau) \mathrm{e}^{-2 \tau}} .
$$

The optimum beam current is plotted in Fig. 18.9 as a function of the attenuation coefficient $\tau$ and the linac efficiency is shown in Fig. 18.10 as a function of beam current in units of the optimum current with the attenuation factor as a parameter. 
The optimum beam current increases as the attenuation factor is reduced while the linac efficiency reaches a maximum for the optimum beam current.

\subsubsection{Particle Capture in a Linear Accelerator Field*}

The capture of particles and the resulting particle energy at the end of the accelerating section depends greatly on the relative synchronism of the particle and wave motion. If particles with velocity $v_{\mathrm{p}}$ are injected at low energy $\left(v_{\mathrm{p}} \ll c\right)$ into an accelerator section designed for a phase velocity $v_{\mathrm{ph}} \geq v_{\mathrm{p}}$ the electromagnetic wave would roll over the particles with reduced acceleration. The particle velocity and phase velocity must be equal or at least close to each other. Because small mismatches are quite common, we will discuss particle dynamics under those circumstances and note that there is no fundamental difference between electron and proton linear accelerators. The following discussion is therefore applicable to any particle type being accelerated by traveling electromagnetic fields in a linear accelerator.

We observe the relative motion of both the particle and the wave from the laboratory system. During the time $\Delta t$ particles move a distance $\Delta z_{\mathrm{p}}=v_{\mathrm{p}} \Delta t$ and the wave a distance $\Delta z_{\mathrm{ph}}=v_{\mathrm{ph}} \Delta t$. The difference in the distance traveled can be expressed in terms of a phase shift

$$
\Delta \psi=-k\left(\Delta z_{\mathrm{ph}}-\Delta z_{\mathrm{p}}\right)=-k\left(v_{\mathrm{ph}}-v_{\mathrm{p}}\right) \frac{\Delta z_{\mathrm{p}}}{v_{\mathrm{p}}} .
$$

The wave number $k$ is

$$
k=\frac{\omega}{v_{\mathrm{ph}}}=\frac{2 \pi c}{\lambda_{\mathrm{rf}} v_{\mathrm{ph}}}
$$

and inserted into (18.110) the relative phase shift over a distance $\Delta z_{\mathrm{p}}$ becomes

$$
\Delta \psi=-\frac{2 \pi c}{\lambda_{\mathrm{rf}}} \frac{v_{\mathrm{ph}}-v_{\mathrm{p}}}{v_{\mathrm{ph}} v_{\mathrm{p}}} \Delta z_{\mathrm{p}} .
$$

To complete the equation of motion we consider the energy gain of the particles along the same distance $\Delta z_{\mathrm{p}}$ which is

$$
\Delta E_{\text {kin }}=-e E_{z}(\psi) \Delta z_{\mathrm{p}}
$$

Equations (18.112) and (18.113) form the equations of motion for particles in phase space. Both equations are written as difference equations for numerical integration since no analytic solution exists. For the most trivial case $v_{\mathrm{ph}}=v_{\mathrm{p}}$ and $\psi=$ const allowing easy integration of (18.113). This trivial case becomes the 


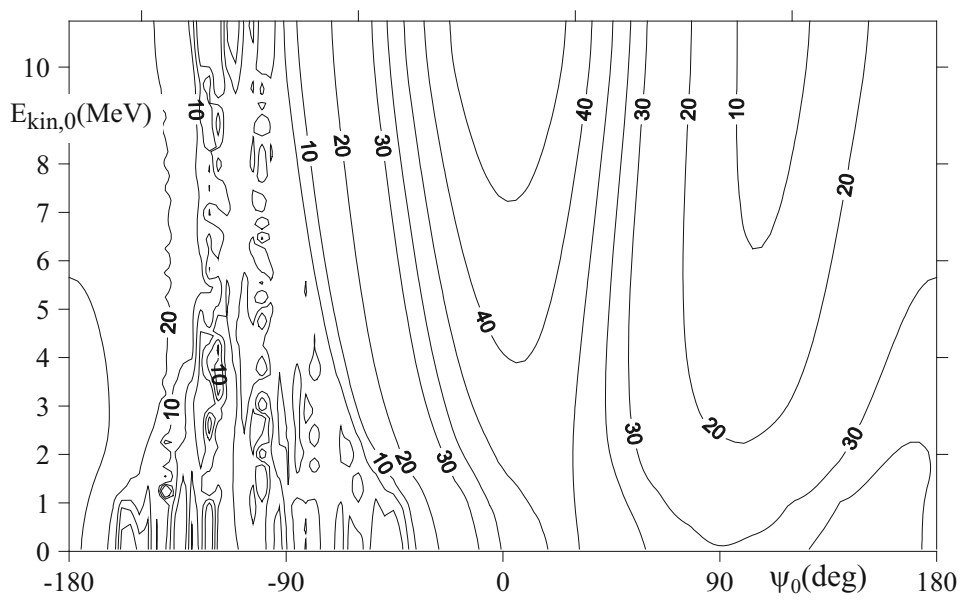

Fig. 18.11 Capture of electrons in a $3 \mathrm{~m}$ linac section for initial phase $\psi_{0}$ and initial kinetic energy $E_{\text {kin, }, .}$. Contour lines are lines of constant particle energy in $\mathrm{MeV}$ at the end of the section. The phase $\psi_{0}=0$ corresponds to the crest of the accelerating wave

overwhelming common case for electrons which reach a velocity very close to the speed of light. Consistent with this, most accelerating sections are dimensioned for a phase velocity equal to the speed of light.

As an illustrative example, we integrate (18.112) and (18.113) numerically to determine the beam parameters at the end of a single $3 \mathrm{~m}$ long accelerating section $\left(v_{\mathrm{ph}}=c\right)$ for an initial particle distribution in phase and momentum at the entrance to the accelerating section. This situation is demonstrated in Fig. 18.11 for a constant field gradient of $\hat{E}=12.0 \mathrm{MeV} / \mathrm{m}$. The momentum and phase at the end of the accelerating section are shown as functions of the initial momentum and phase. We note from Fig. 18.11 that particles can be captured in the accelerating field only in the vicinity of $\psi_{0} \approx 0$ to $+90^{\circ}$ at almost any initial phase and momentum. At phases from $\psi_{0} \approx-45$ to $-160^{\circ}$ slow particles at sub-relativistic energies loose whatever little energy they had to move randomly in the rf-wave rolling over them. On the other hand, particles which enter the accelerating section ahead of the crest $\left(\psi_{0} \gtrsim 0^{\circ}\right)$ gain maximum momentum while the wave's crest moves over them.

Such diagrams calculated for particular parameters under consideration provide valuable information needed to prepare the beam for optimum acceleration. The most forgiving operating parameters are, where the contour lines are far apart. In those areas a spread in initial phase or energy has little effect on the final phase or energy. If a beam with a small energy spread at the end of acceleration is desired, the initial phase should be chosen to be at small positive values or just ahead of the wave crest as shown in Fig. 18.11. Even for a long bunch the final energy spread is small while reaching the highest total energy.

On the other hand, if a short bunch length at the end of acceleration is of biggest importance, an initial phase of around $\psi_{0} \approx 100^{\circ}$ seems to be more appropriate. In 
this case, however, the final energy is lower than the maximum possible energy and the energy spread is large.

Once the particular particle distribution delivered to the linear accelerator and the desired beam quality at the end is known one can use Fig. 18.11 for optimization. Conversely such diagrams can be used to judge the feasibility of a particular design to reach the desired beam characteristics.

\subsection{Preinjector and Beam Preparation*}

Although the proper choice of the initial rf-phase with respect to the particle beam greatly determines the final beam quality, the flexibility of such adjustments is limited. Special attention must be given to the preparation of the beam before acceleration. In most cases, particles are generated in a continuous stream or from a microwave source of different frequency. Depending on the particle source, special devices are used for initial acceleration and bunching of the beam. We will discuss basic principles of beam preparation.

\subsubsection{Prebuncher}

Many particle sources, be it for electrons, protons or ions, produce a continuous stream of particles at modest energies limited by electrostatic acceleration between two electrodes. Not all particles of such a beam will be accelerated because of the oscillatory nature of the accelerating field. For this reason and also in case short bunches or a small energy spread at the end of the linac is desired, the particles should be concentrated at a particular phase. This concentration of particles in the vicinity of an optimum phase maximizes the particle intensity in the bunch in contrast to a mechanical chopping of a continuous beam. To bunch particles requires specific beam manipulation which we will discuss here in more detail.

A bunched beam can be obtained from a continuous stream of nonrelativistic particles by the use of a prebuncher. The basic components of a prebuncher is an rf-cavity followed by a drift space. As a continuous stream of particles passes through the prebuncher, some particles get accelerated and some are decelerated. The manipulation of the continuous beam into a bunched beam is best illustrated in the phase space diagrams of Fig. 18.12.

Figure 18.12a shows the continuous particle distribution in energy and phase at the entrance of the prebuncher. Depending on the phase of the electric field in the prebuncher at the time of passage, a particle becomes accelerated or decelerated and the particle distribution at the exit of the prebuncher is shown in Fig. 18.12b. The particle distribution has been distorted into a sinusoidal energy variation. Since the particles are nonrelativistic the energy modulation reflects also a velocity modulation. We concentrate on the origin of the distribution at $\varphi=0$ and 


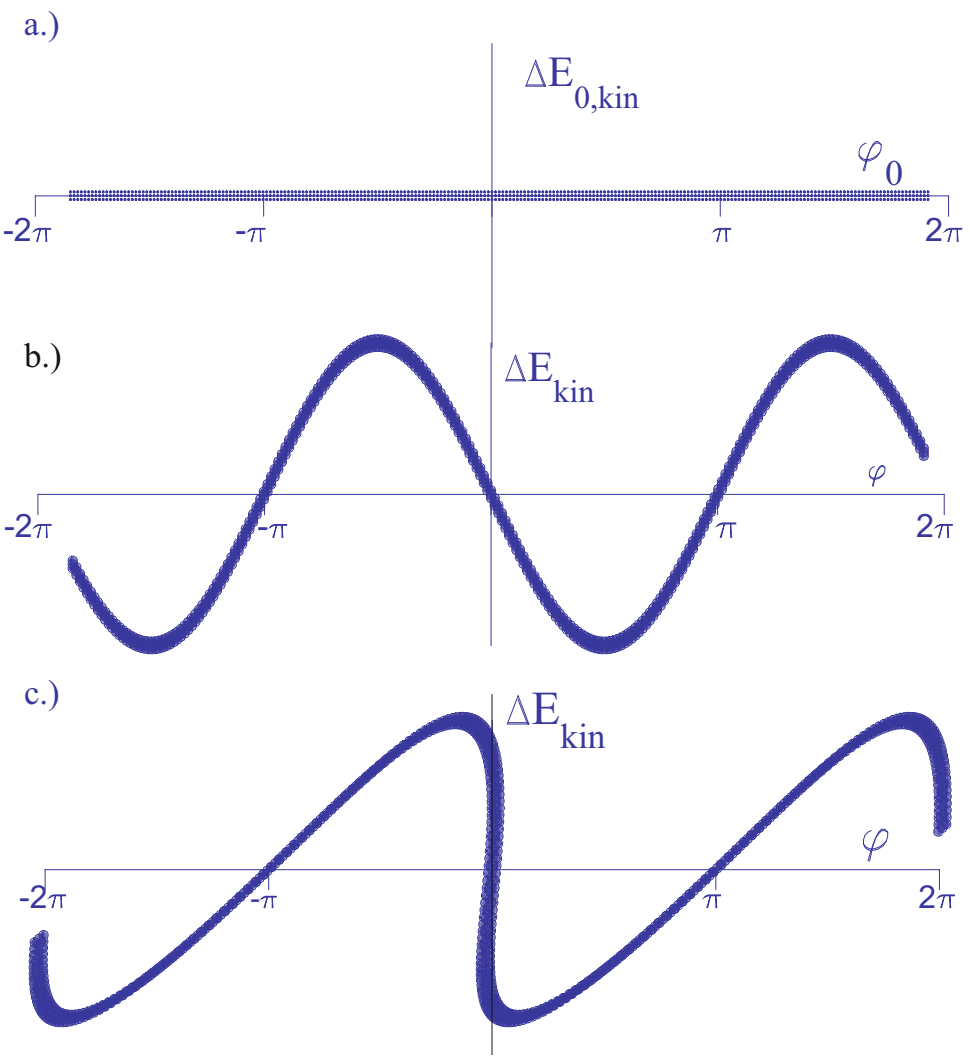

Fig. 18.12 Phase space diagrams for a continuous beam passing through a prebuncher. Before acceleration (a) and right after (b). A distance $L$ downstream of the buncher cavity the phase space distribution shows strong bunching (c). [Note: the beam moves from left to right]

$\Delta E_{\text {kin }}=0$ as the reference phase and note that particles ahead of this reference phase have been decelerated and particles behind the reference phase have been accelerated. Following this modulated beam through the drift space we observe due to the velocity modulation a bunching of the particle distribution which reaches a maximum at some distance as shown in Fig. 18.12c. A significant beam intensity has been concentrated close to the reference phase of the prebuncher.

The frequency used in the prebuncher depends on the desired bunch distribution. For straight acceleration in a linear accelerator one would choose the same frequency for both systems. Often, however, the linear accelerator is only an injector into a bigger circular accelerator which generally operates at a lower frequency. For optimum matching to the final circular accelerator the appropriate prebuncher frequency would be the same as the cavity frequency in the circular accelerator cavity. 
The effect of the prebuncher can be formulated analytically in the vicinity of the reference phase. At the exit of the prebuncher, operating at a voltage $V=V_{0} \sin \varphi$, the energy spread is

$$
\Delta E_{\mathrm{kin}}=e V_{0} \sin \varphi=m c^{2} \beta \gamma^{3} \Delta \beta,
$$

which is related to a velocity spread $\Delta \beta$. Perfect bunching occurs a time $\Delta t$ later when for $\sin \varphi \approx \varphi$

$$
c \Delta \beta \Delta t=\frac{\varphi}{2 \pi} \lambda_{\mathrm{rf}},
$$

where $\lambda_{\text {rf }}$ is the rf-wavelength in the prebuncher cavity. Solving for $\Delta t$ we get for nonrelativistic particles with $\gamma=1$ and $\beta \ll 1$

$$
\Delta t=\frac{\lambda_{\mathrm{rf}}}{2 \pi} \frac{m v}{e V_{0}}
$$

and optimum bunching occurs a distance $L$ downstream from the cavity

$$
L=v_{0} \Delta t=\frac{2 E_{\mathrm{kin}}}{k_{\mathrm{rf}} e V_{0}},
$$

where $v_{0}$ is the velocity of the reference particle and $k_{\mathrm{rf}}=2 \pi / \lambda_{\mathrm{rf}}$. The minimum bunch length in this case is then

$$
\delta L=\frac{\delta E_{\mathrm{kin}}}{k_{\mathrm{rf}} e V_{0}}
$$

where $\delta E_{\text {kin }}$ is the total energy spread in the beam before the prebuncher.

In this derivation, we have greatly idealized the field variation being linear instead of sinusoidal. The real bunching is therefore less efficient than the above result and shows some wings as is obvious from Fig. 18.12c. In a compromise between beam intensity and bunch length one might let the bunching go somewhat beyond the optimum and thereby pull in more of the particle intensity in the wings.

There are still particles between the bunches which could either be eliminated by an rf-chopper or let go to be lost in the linear accelerator because they are mainly distributed over the decelerating field period in the linac.

\subsubsection{Beam Chopper}

A conceptually simple way to produce a bunched beam is to pass a continuous beam from the source through a chopper system, where the beam is deflected across 


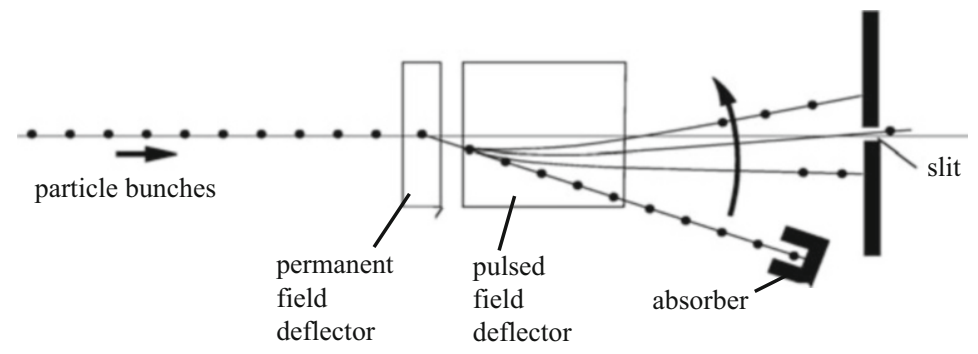

Fig. 18.13 Principal functioning of a chopper system

a narrow slit resulting in a pulsed beam behind the slit. The principle components of such a chopper system are shown in Fig. 18.13.

As was mentioned in the previous section this mode of bunching is rather wasteful and therefore an rf-prebuncher which concentrates particles from a large range of phases towards a particular phase is more efficient for particle bunching. However, we still might want to add a beam chopper.

One such reason could be to eliminate most of the remaining particles between the bunches from the prebuncher. Although these particles most likely get lost during the acceleration process a significant fraction will still reach the end of the linac with an energy spread between zero and maximum energy. Because of their large energy deviation from the energy of the main bunches, such particles will be lost in a subsequent beam transport system and therefore create unnecessary high radiation levels. It is therefore prudent to eliminate such particles at low energies. A suitable device for that is a chopper which consists of an rf-cavity excited similar to the prebuncher cavity but with the beam port offset by a distance $r$ from the cavity axis. In this case the same rf-source as for the prebuncher or main accelerator can be used and the deflection of particles is effected by the azimuthal magnetic field in the cavity.

The prebuncher produces a string of bunches at the prebuncher frequency. For many applications, however, a different bunch structure is desired. Specifically it often occurs that only one single bunch is desired. To produce a single pulse, the chopper system may consist of a permanent magnet and a fast pulsed magnet. The permanent magnet deflects the beam into an absorber while the pulsed magnet deflects the beam away from the absorber across a small slit. The distance between the center of the pulsed magnet and the slit be $D$ (Fig. 18.13), the slit aperture $\Delta$ and the rate of change of the magnetic field $\dot{B}$. For an infinitely thin beam the pulse length behind the slit is then

$$
\tau_{b}=\frac{\Delta}{D \dot{\varphi}}=\frac{\Delta}{D} \frac{c p}{e \dot{B} \ell},
$$

where $\varphi$ is the deflection angle, $\ell$ the effective magnetic length of the pulsed magnet and $c p$ the momentum of the particles. In order to clean the beam between bunches 
or to select a single bunch from a train of bunches the chopper parameters must be chosen such that only the desired part of the beam passes through.

\subsubsection{Buncher Section}

A buncher section is similar to an ordinary electron linac section but dimensioned for sub relativistic particle. Particles arriving from the source, prebuncher or chopper may not be at relativistic energies and therefore cannot follow the rf-wave in an ordinary linac section which have a phase velocity equal to the velocity of light. To optimize the whole acceleration system a buncher section is inserted as the first linac section. The length of each cell is shorter than in a normal linac structure and changes from a length appropriate for the velocity of the incoming particles to longer and longer cell lengths until the particle has reached the constant velocity of light for which the normal linac structure is dimensioned. In this case the particle would be accelerated from beginning at the desired phase. In case of an rf-gun the electrons emerge relativistic and no buncher is necessary. In many cases, especially at smaller facilities, the buncher section is omitted at some degradation of beam performance.

\section{Problems}

18.1 (S). Determine within a factor of two or less the longest TE or TM-mode wavelength that can propagate through a round tube of diameter $2 R$.

18.2 (S). Consider a pill box cavity made of copper and calculate the frequency shift per $1{ }^{\circ} \mathrm{C}$ temperature change. The linear expansion coefficient for copper is $\eta_{\mathrm{T}}=16.6 \cdot 10^{-6} \mathrm{~m} /\left(\mathrm{m}^{\circ} \mathrm{C}\right)$. What is the temperature tolerance if the rf frequency should not change by more than $\pm 10^{-6}$.

18.3 (S). Determine the frequency scaling of cavity dimensions, transit time factor, quality factor, shunt impedance, specific shunt impedance and cavity filling time.

18.4 (S). In electron linear accelerators operating at $3 \mathrm{GHz}$ accelerating fields of more than $50 \mathrm{MeV} / \mathrm{m}$ can be reached. Why can such high fields not be used in a storage ring? Discuss quantitatively, while scaling linac parameters to the frequency of your choice in the storage ring.

18.5 (S). Discuss the graph in Fig. 18.11. Specifically explain in words the particle dynamics within random features. How come particles get accelerated even though they enter the linac while the accelerating field is negative? (note: interpretation of the graph for initial energies $\ll 1 \mathrm{MeV}$ does not have enough resolution to be reliable.) 
18.6 (S). Design a $500 \mathrm{MHz}$ prebuncher system for a $3 \mathrm{GHz}$ linear accelerator. Particles in a continuous beam from the source have a kinetic energy of $E_{0}=$ $100 \mathrm{keV}$ with an energy spread of $\pm 0.02 \%$. Specify the optimum prebuncher voltage and drift length to compress the maximum intensity into a bunch with a phase distribution of less than $\pm 12^{\circ}$ at $3 \mathrm{GHz}$.

18.7 (S). Calculate for a SLAC type linac section the no-load energy gain and the energy gain for a pulse current of $i_{\mathrm{b}}=20 \mathrm{~mA}$. The rf-power is $P_{0}=15 \mathrm{MW}$ per section at a pulse length of $2.5 \mu \mathrm{s}$. Compare the efficiency to the situation when only one bunch of $n^{\mathrm{b}}=10^{10}$ electrons is accelerated. What is the linac efficiency for this current and what is the energy gain in this case?

18.8. Consider a rectangular box cavity with copper walls and dimensioned for an rf-wavelength of $\lambda=10.5 \mathrm{~cm}$. Calculate the wall losses due to the fundamental field only and determine the shunt impedance per unit length $r_{\mathrm{s}}$ and the quality factor $Q$ for this cavity. These losses are due to surface currents within a skin depth generated by the rf-fields on the cavity surface. Compare these parameters with those of (18.96). Is the shape of the cavity very important? Determine the resonance width and temperature tolerance for the cavity.

18.9. Plot the electrical and magnetic field distribution for the three lowest order modes in a rectangular and cylindrical cavity. Calculate the shunt impedance and compare the results. Which type of cavity is more efficient?

18.10. Derive a general expression of the shunt impedance for general TM-modes in a cylindrical cavity.

18.11. Derive expressions for the maximum electric field strength and the waveguide losses per unit length for the $\mathrm{TE}_{10}$ mode in a rectangular waveguide. Use this result to design a waveguide for $3 \mathrm{GHz}$. Calculate the cut-off frequency, the phase and group velocities and the waveguide wavelength. What criteria did you use to choose the dimensions $a$ and $b$ ? Sketch the electrical and magnetic fields.

18.12. Consider a $8 \mathrm{GeV}$ electron storage ring with a FODO lattice and a beam current of $200 \mathrm{~mA}$. Determine the equilibrium energy spread and specify rfparameters which will be sufficient to compensate for synchrotron radiation losses and provide an energy acceptance for all particles in a Gaussian energy distribution up to $7 \sigma_{\epsilon} / E$. What is the synchrotron tune and the bunch length in your storage ring?

18.13. Consider a pill box cavity with copper walls for a storage ring and choose a rf-frequency of $750 \mathrm{MHz}$. Derive an expression for the wall losses due to the fundamental field only and derive an expression for the shunt impedance of the cavity defined by $R_{\mathrm{cy}}=V_{\mathrm{rf}}^{2} / P_{\mathrm{rf}}$, where $V_{\mathrm{rf}}$ is the maximum rf-voltage and $P_{\mathrm{rf}}$ the cavity wall losses. What are the rf-losses if this cavity is used in the ring of Problem 18.12? Assume that you can cool only about $150 \mathrm{~kW} / \mathrm{m}$ of cavity length. How many cavities would you need for your ring example? 
18.14. The electromagnetic field for a cylindrical waveguide have been derived in Sect. 18.1.3. Derive in a similar way expressions for resonant field modes in a rectangular waveguide.

\section{References}

1. F.T. Cole, Nonlinear transformations in action-angle variables. Technical Report TM-179, FERMI Lab, Batavia (1969)

2. W.T. Weng, Fundamentals - longitudinal motion, in Physics of Particle Accelerators, vol. 184 (American Institute of Physics, New York, 1989), p. 4243

3. J.D. Jackson, Classical Electrodynamics, 2nd edn. (Wiley, New York, 1975)

4. S. Ramo, J.R. Whinnery, T. van Duzer, Fields and Waves in Communication Electronic (Wiley, New York, 1984)

5. M. Abramovitz, I. Stegun, Handbook of Mathematical Functions (Dover, New York, 1972)

6. I.S. Gradshteyn, I.M. Ryzhik, Table of Integrals, Series, and Products, 4th edn. (Academic, New York, 1965)

7. M.A. Allen, L.G. Karvonen, J.L. Pelegrin, P.B. Wilson, IEEE Trans. Nucl. Sci. 24, 1780 (1977)

8. K. Halbach, F. Holsinger, Part. Accel. 7, 213 (1976)

9. T. Weiland, Nucl. Instrum. Methods 212, 13 (1983)

10. A. Piwinski, CERN Accelerator School, CAS, CERN 85-19 (CERN, Geneva, 1986), p. 29

11. J.D. Bjorken, S.K. Mtingwa, Part. Accel. 13, 115 (1983)

12. J.C. Slater, Rev. Mod. Phys. 20, 473 (1948)

13. R. Neal (ed.), The 2 Mile Linear Accelerator (Benjamin, New York, 1968) 


\section{Chapter 19 \\ Beam-Cavity Interaction*}

The proper operation of the rf-system in a particle accelerator depends more than any other component on the detailed interaction with the particle beam. This results from the observation that a particle beam can induce fields in the accelerating cavities of significant magnitude compared to the generator produced voltages and we may therefore not neglect the presence of the particle beam. This phenomenon is called beam loading and can place severe restrictions on the beam current that can be accelerated. In this section, main features of such interaction and stability conditions for most efficient and stable particle acceleration will be discussed.

\subsection{Coupling Between rf-Field and Particles}

In our discussions about particle acceleration we have tacitly assumed that particles would gain energy from the fields in accelerating cavities merely by meeting the synchronicity conditions. This is true for a weak particle beam which has no significant effect on the fields within the cavity. As we try, however, to accelerate an intense beam, the actual accelerating fields become modified by the presence of considerable electrical particle beam currents. This beam loading can ultimately limit the maximum beam intensity.

The phenomenon of beam loading will be defined and characterized in this section leading to conditions and parameters to assure positive energy flow from the rf-power source to the beam. Fundamental consideration to this discussion are the principles of energy conservation and linear superposition of fields which allow us to study field components from one source independent of fields generated by other sources. Specifically, we may treat beam induced fields separately from fields generated by rf-power sources.

This chapter has been made Open Access under a CC BY 4.0 license. For details on rights and licenses please read the Correction https://doi.org/10.1007/978-3-319-18317-6_28 


\subsubsection{Network Modelling of an Accelerating Cavity}

The electrical excitation of a rf-cavity can be accurately described by an oscillator as discussed in Sect. 18.2.4 and we will use therefore characteristic parameters and terminology of externally driven, damped oscillators in our further discussions of rf-systems. Electrically, an accelerating cavity can be represented by a parallel resonant circuit (Fig. 19.1) which is driven by an external rf-current source $I_{\mathrm{g}}$ from a generator and the particle beam $I_{\mathrm{b}}$.

The amount of rf-power available from the generator in the accelerating cavity depends greatly on the relative impedance of cavity and generator. Both have to be matched to assure optimum power transfer. To derive conditions for that we define the internal impedance of the current source or rf-generator in terms of the cavity shunt impedance $R_{\mathrm{S}}$ of an empty cavity as defined in (18.74)

$$
R_{\mathrm{g}}=\frac{R_{\mathrm{s}}}{\beta},
$$

where $\beta$ is the coupling coefficient still to be defined. This coefficient depends on the actual hardware of the coupling arrangement for the rf-power from the generator at the entrance to the cavity and quantifies the generator impedance as seen from the cavity in units of the cavity shunt impedance $R_{\mathrm{S}}$ (Fig. 19.1). Since this coupling coefficient depends on the hardware, we need to specify the desired operating condition to determine the proper adjustment of the coupling during assembly. This adjustment is done by either rotating a loop coupler with respect to the cavity axis or adjustment of the aperture in case of capacitive coupling through a hole.

The inductance $L$ and capacitance $C$ form a parallel resonant circuit with the resonant frequency

$$
\omega_{\mathrm{r}}=\frac{1}{\sqrt{L C}} .
$$

The rf-power available at the cavity from the generator is

$$
P_{\mathrm{g}}=\frac{1}{2} Y_{\mathrm{L}} V_{\mathrm{g}}^{2},
$$

Fig. 19.1 Network model for an rf generator and an accelerating cavity

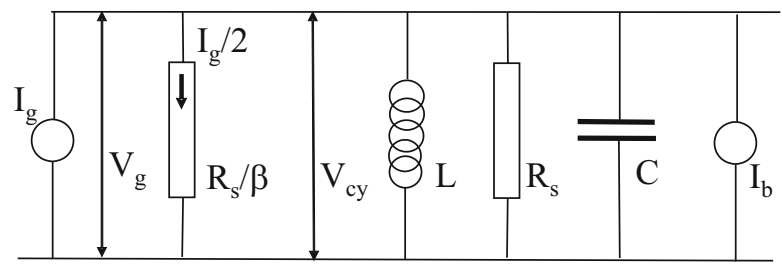


where $Y_{\mathrm{L}}$ is the loaded cavity admittance including energy transfer to the beam and $V_{\mathrm{g}}$ is the generator voltage. Unless otherwise noted, the voltages, currents and power used in this section are the amplitudes of otherwise oscillating quantities. At resonance where all reactive power vanishes we use the generator current $I_{\mathrm{g}}$ and network admittance $Y=Y_{\mathrm{g}}+Y_{\mathrm{L}}$ to replace the generator voltage

$$
V_{\mathrm{g}}=\frac{I_{\mathrm{g}}}{Y}=\frac{I_{\mathrm{g}}}{Y_{\mathrm{g}}+Y_{\mathrm{L}}}
$$

and get after insertion into (19.3) the generator power in the form

$$
P_{\mathrm{g}}=\frac{1}{2} \frac{Y_{\mathrm{L}}}{\left(Y_{\mathrm{g}}+Y_{\mathrm{L}}\right)^{2}} I_{\mathrm{g}}^{2} .
$$

Noting that the generator power has a maximum, which can be determined from $\partial P_{\mathrm{g}} / \partial Y_{\mathrm{L}}=0$, we obtain the well-known result that the rf-power transfer from the generator becomes a maximum if the load is matched to the internal impedance of the generator by adjusting

$$
Y_{\mathrm{g}}=Y_{\mathrm{L}} \quad \text { or } \quad R_{\mathrm{L}}=\frac{R_{\mathrm{s}}}{\beta},
$$

replacing the admittances by the respective impedances. The maximum available rf-power at the cavity is therefore with $Y_{\mathrm{g}}=\beta / R_{\mathrm{s}}$

$$
P_{\mathrm{g}}=\frac{1}{8} \frac{R_{\mathrm{s}}}{\beta} I_{\mathrm{g}}^{2} \text {. }
$$

To calculate the quality factor for a cavity, we note the stored energy is $W=$ $\frac{1}{2} C V^{2}$ and the energy loss rate $P_{\text {cy }}=\frac{1}{2} V^{2} / R$. Using the definition (18.80) the unloaded quality factor becomes with $R=R_{\mathrm{S}}$ at resonance

$$
Q_{0}=\omega_{\mathrm{r}} C R_{\mathrm{s}} .
$$

The admittance for the total circuit as seen by the beam is that of cavity plus generator or

$$
\frac{1}{R_{\mathrm{b}}}=\frac{\beta}{R_{\mathrm{s}}}+\frac{1}{R_{\mathrm{s}}}=\frac{1+\beta}{R_{\mathrm{s}}} .
$$

From this and (19.7) we get the loaded quality factor

$$
Q=\omega_{\mathrm{r}} C R_{\mathrm{b}}=\frac{Q_{0}}{1+\beta}
$$


Off resonance the generator voltage and current are no more in phase. The phase difference can be derived from the complex impedance of the network, which is the same seen from the generator as well as seen from the beam

$$
\frac{1}{Z}=\frac{1}{R_{\mathrm{b}}}+\mathrm{i} \omega C+\frac{1}{\mathrm{i} \omega L}
$$

The complex impedance becomes with (19.2), (19.9)

$$
\frac{1}{Z}=\frac{1}{R_{\mathrm{b}}}\left(1+\mathrm{i} Q \frac{\omega^{2}-\omega_{\mathrm{r}}^{2}}{\omega \omega_{\mathrm{r}}}\right)
$$

and with $I_{\mathrm{g}}=V_{\mathrm{g}} / Z$ the generator current is

$$
I_{\mathrm{g}}=\frac{V_{\mathrm{g}}}{R_{\mathrm{b}}}\left(1+\mathrm{i} Q \frac{\omega^{2}-\omega_{\mathrm{r}}^{2}}{\omega \omega_{\mathrm{r}}}\right)=\frac{V_{\mathrm{g}}}{R_{\mathrm{b}}}(1-\mathrm{i} \tan \Psi) .
$$

Close to resonance the tuning angle $\Psi$ becomes from (19.12) with $\omega \approx \omega_{\mathrm{r}}$

$$
\tan \Psi \approx-Q \frac{\omega^{2}-\omega_{\mathrm{r}}^{2}}{\omega \omega_{\mathrm{r}}} \approx-2 Q \frac{\omega-\omega_{\mathrm{r}}}{\omega_{\mathrm{r}}}
$$

in agreement with (18.64) except for a phase shift of $-90^{\circ}$, which was introduced here to be consistent with our definition of the synchronous phase $\psi_{\mathrm{s}}$. The variation of the tuning angle is shown in Fig. 19.2 as a function of the generator frequency. From (19.12), the generator voltage at the cavity is finally

$$
V_{\mathrm{g}}=\frac{I_{\mathrm{g}} R_{\mathrm{b}}}{1-\mathrm{i} \tan \Psi}=I_{\mathrm{g}} R_{\mathrm{b}} \cos \Psi \mathrm{e}^{\mathrm{i} \Psi}
$$

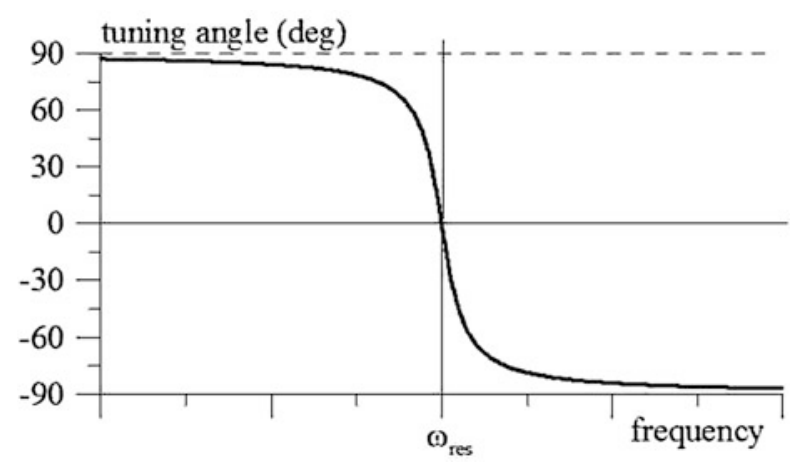

Fig. 19.2 Tuning angle $\psi$ as a function of the generator frequency 
At frequencies below the resonance frequency the tuning angle is positive and therefore the generator current lags the voltage by the phase $\Psi$. This case is also called inductive detuning since the impedance looks mainly inductive. Conversely, the detuning is called capacitive detuning because the impedance looks mostly capacitive for frequencies above resonance frequency.

A bunched particle beam passing through a cavity acts as a current just like the generator current and therefore the same relationships with respect to beam induced voltages exist. In case of capacitive detuning, for example, the beam induced voltage $V_{\mathrm{b}}$ lags in phase behind the beam current $I_{\mathrm{b}}$.

The effective accelerating voltage in the cavity is a composition of the generator voltage, the induced voltage, and the phase relationships between themselves and relative to the particle beam. To assure a stable beam, the resulting cavity voltage must meet the requirements of particle acceleration to compensate, for example, lost energy into synchrotron radiation. We determine the conditions for that by deriving first the generator voltage $V_{\mathrm{gr}}$ at resonance and without beam loading while voltage and current are in phase. From Fig. 19.1 we get

$$
V_{\mathrm{gr}}=\frac{I_{\mathrm{g}}}{Y_{\mathrm{g}}+Y_{\mathrm{L}}}=\frac{I_{\mathrm{g}}}{\frac{1}{R_{\mathrm{s}}}+\frac{\beta}{R_{\mathrm{s}}}}=\frac{R_{\mathrm{s}} I_{\mathrm{g}}}{1+\beta}
$$

and with (19.6) the generator voltage at resonance becomes

$$
V_{\mathrm{gr}}=\frac{2 \sqrt{2 \beta}}{1+\beta} \sqrt{R_{\mathrm{s}} P_{\mathrm{g}}} .
$$

The generator voltage at the cavity is therefore with (19.6)

$$
V_{\mathrm{g}}=V_{\mathrm{gr}} \cos \Psi \mathrm{e}^{\mathrm{i} \Psi}
$$

This is the cavity voltage seen by a negligibly small beam and can be adjusted to meet beam stability requirements by varying the tuning angle $\Psi$ and rf-power $P_{\mathrm{g}}$.

\subsection{Beam Loading and Rf-System}

For more substantial beam currents the effect of beam loading must be included to obtain the effective cavity voltage. Similar to the derivation of the generator voltage in a cavity, we may derive the induced voltage from the beam current passing through that cavity. Since there is no fundamental difference between generator and beam current, the induced voltage is in analogy to (19.17)

$$
V_{\mathrm{b}}=-V_{\mathrm{br}} \cos \Psi \mathrm{e}^{\mathrm{i} \Psi}
$$


where the negative sign indicates that the induced voltage is decelerating the beam. The particle distribution in the beam occurs in bunches and the beam current therefore can be expressed by a Fourier series. Here we are only interested in the harmonic $I_{\mathrm{h}}$ of the beam current and find for bunches short compared to the rfwavelength

$$
I_{\mathrm{h}}=2 I_{\mathrm{b}}
$$

where $I_{\mathrm{b}}$ is the average beam current and $h$ the harmonic number. The approximation

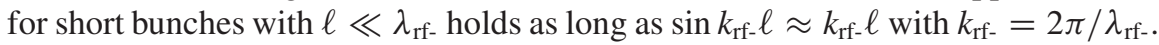
For longer bunches the factor 2 becomes a more complicated formfactor as can be derived from an appropriate Fourier expansion. At the resonance frequency $\omega_{\mathrm{r}}=$ $h \omega_{0}$, the beam induced voltage in the cavity is then with (19.8) from (19.15)

$$
V_{\mathrm{br}}=\frac{R_{\mathrm{s}} I_{\mathrm{h}}}{1+\beta}=\frac{2 R_{\mathrm{s}} I_{\mathrm{b}}}{1+\beta} .
$$

The resulting cavity voltage is the superposition of both voltages, the generator and the induced voltage. This superposition, including appropriate phase factors, is often represented in a phasor diagram. In such a diagram a complex quantity $\tilde{z}$ is represented by a vector of length $|\tilde{z}|$ with the horizontal and vertical components being the real and imaginary part of $\tilde{z}$, respectively. The phase of this vector increases counter clockwise and is given by $\tan \varphi=\operatorname{Im}(\tilde{z}) / \operatorname{Re}(\tilde{z})$. In an application to rf-parameters we represent voltages and currents by vectors with a length equal to the magnitude of voltage or current and a counter clockwise rotation of the vector by the phase angle $\varphi$.

The particle beam current can be chosen as the reference being parallel to the real axis and we obtain from the quantities derived so far the phasor diagram as shown in Fig. 19.3. First we determine the relationships between individual vectors and phases and then the correct adjustments of variable rf-parameters. In Fig. 19.3 the generator current is assumed to have the still to be determined phase $\vartheta$ with respect to the beam current while the generator voltage and beam induced voltage lag by the phase $\Psi$ behind the beam current. The resulting cavity voltage $\tilde{\boldsymbol{V}}_{\text {cy }}$ is the phasor addition of both voltages $\tilde{\boldsymbol{V}}_{\mathrm{g}}+\tilde{\boldsymbol{V}}_{\mathrm{b}}$ as shown in Fig. 19.3.

The adjustment of the rf-system must now be performed in such a way as to provide the desired gain in kinetic energy $U_{0}=e \hat{V}_{\text {cy }} \sin \psi_{\mathrm{s}}$ where $\hat{V}_{\text {cy }}$ is the maximum value of the cavity voltage and $\psi_{\mathrm{s}}$ the synchronous phase. To maximize the energy flow from the generator to the cavity the load must be matched such that it appears to the generator purely resistive. This is achieved by adjusting the phase $\psi_{\mathrm{g}}$ to get the cavity voltage $V_{\text {cy }}$ and generator current $I_{\mathrm{g}}$ in phase which occurs for

$$
\psi_{\mathrm{g}}=\frac{1}{2} \pi-\psi_{\mathrm{s}}
$$

as shown in Fig. 19.4. Obviously, this is only true for a specific value of the beam current. General operation will deviate from this value and therefore we often match 


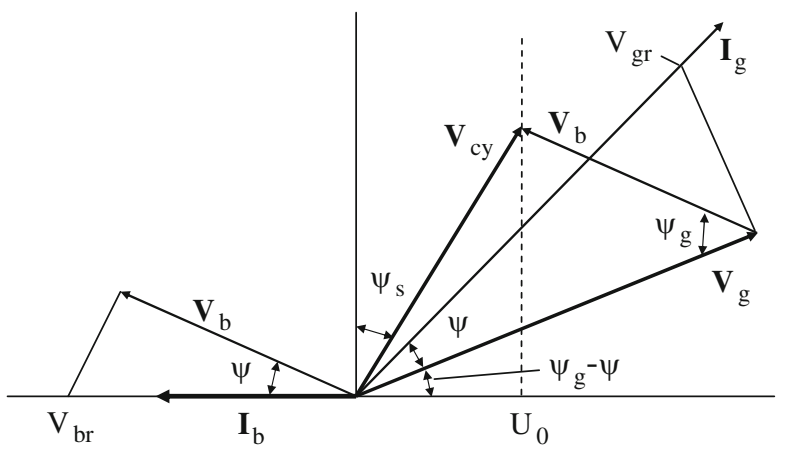

Fig. 19.3 Phasor diagram for an accelerating cavity and arbitrary tuning angle

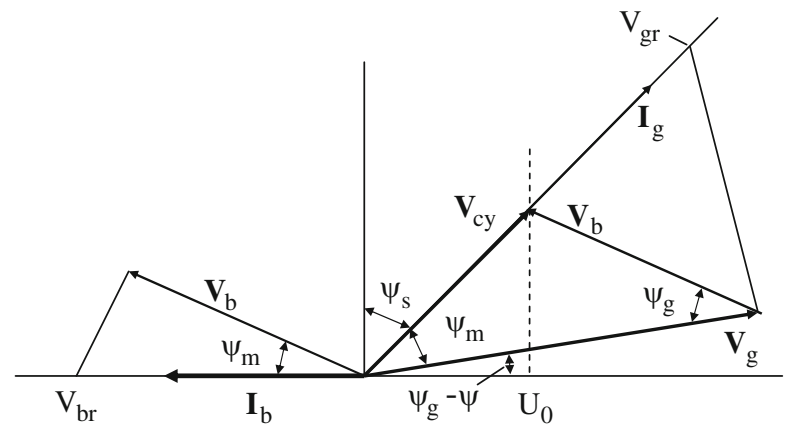

Fig. 19.4 Phasor diagram with optimum tuning angle

to the maximum desired beam current. For lower currents, where the energy transfer is not optimum anymore, some loss of efficiency is acceptable.

The tuning angle adjustment for optimum matching can be derived from Fig. 19.4 and applying the law of sines we have with (19.17)

$$
\frac{V_{\mathrm{b}}}{V_{\mathrm{cy}}}=\frac{V_{\mathrm{br}} \cos \Psi_{\mathrm{m}}}{V_{\mathrm{cy}}}=\frac{\sin \Psi_{\mathrm{m}}}{\sin \psi_{\mathrm{g}}}=\frac{\sin \Psi_{\mathrm{m}}}{\cos \psi_{\mathrm{s}}} .
$$

The optimum tuning angle is from (19.22)

$$
\tan \Psi_{\mathrm{m}}=\frac{V_{\mathrm{br}}}{V_{\mathrm{cy}}} \cos \psi_{\mathrm{s}}
$$

This tuning is effected by a shift in the resonant frequency of the cavity with respect to the generator frequency by, for example, moving a tuner in or out. From (19.13) we get with (19.9), (19.20), (19.23) for the frequency shift or 
frequency tuning

$$
\delta \omega=\omega-\omega_{\mathrm{r}}=-\frac{\omega_{\mathrm{r}}}{2 Q_{0}} \frac{P_{\mathrm{b}}}{P_{\mathrm{cy}}} \cot \psi_{\mathrm{s}}
$$

where the cavity power is defined by

$$
P_{\mathrm{cy}}=\frac{V_{\mathrm{cy}}^{2}}{2 R_{\mathrm{s}}}
$$

and the beam power by

$$
P_{\mathrm{b}}=I_{\mathrm{b}} V_{\mathrm{cy}} \sin \psi_{\mathrm{s}}
$$

To determine the required generator power the components of the cavity voltage vector can be expressed by other quantities and we get from Fig. 19.4

$$
V_{\mathrm{cy}} \sin \psi_{\mathrm{s}}=V_{\mathrm{gr}} \cos \Psi_{\mathrm{m}} \cos \left(\psi_{\mathrm{g}}-\Psi_{\mathrm{m}}\right)-V_{\mathrm{br}} \cos ^{2} \Psi_{\mathrm{m}}
$$

and

$$
V_{\mathrm{cy}} \cos \psi_{\mathrm{s}}=V_{\mathrm{gr}} \cos \Psi_{\mathrm{m}} \sin \left(\psi_{\mathrm{g}}-\Psi_{\mathrm{m}}\right)+V_{\mathrm{br}} \cos \Psi_{\mathrm{m}} \sin \Psi_{\mathrm{m}}
$$

Combining both equations to eliminate the phase $\left(\psi_{\mathrm{g}}-\Psi_{\mathrm{m}}\right)$, we get

$$
V_{\mathrm{gr}}^{2}=\left(V_{\mathrm{cy}} \frac{\sin \psi_{\mathrm{s}}}{\cos \Psi_{\mathrm{m}}}+V_{\mathrm{br}} \cos \Psi_{\mathrm{m}}\right)^{2}+\left(V_{\mathrm{cy}} \frac{\cos \psi_{\mathrm{s}}}{\cos \Psi_{\mathrm{m}}}-V_{\mathrm{br}} \sin \Psi_{\mathrm{m}}\right)^{2}
$$

and with (19.16), (19.20) the required generator power for the condition of optimum matching is

$$
\begin{gathered}
P_{\mathrm{g}}=\frac{V_{\mathrm{cy}}^{2}}{2 R_{\mathrm{s}}} \frac{(1+\beta)^{2}}{4 \beta}\left[\left(\frac{\sin \psi_{\mathrm{s}}}{\cos \Psi_{\mathrm{m}}}+\frac{2 R_{\mathrm{s}} I_{\mathrm{b}}}{V_{\mathrm{cy}}(1+\beta)} \cos \Psi_{\mathrm{m}}\right)^{2}\right. \\
\left.+\left(\frac{\cos \psi_{\mathrm{s}}}{\cos \Psi_{\mathrm{m}}}-\frac{2 R_{\mathrm{s}} I_{\mathrm{b}}}{V_{\mathrm{cy}}(1+\beta)} \sin \Psi_{\mathrm{m}}\right)^{2}\right]
\end{gathered}
$$

This expression can be greatly simplified with (19.23) to become

$$
P_{\mathrm{g}, \mathrm{opt}}=\frac{(1+\beta)^{2}}{8 \beta R_{\mathrm{s}}}\left(V_{\mathrm{cy}}+\frac{2 R_{\mathrm{s}} I_{\mathrm{b}}}{1+\beta} \sin \psi_{\mathrm{s}}\right)^{2} \text {. }
$$

Equation (19.31) represents a combination of beam current through $I_{\mathrm{b}}$, rfgenerator power $P_{\mathrm{g}}$, coupling coefficient $\beta$, and shunt impedance $R_{\mathrm{S}}$ to sustain 
a cavity voltage $V_{\text {cy }}$. Specifically, considering that the rf-power $P_{\mathrm{g}}$ and coupling coefficient $\beta$ is fixed by the hardware installed a maximum supportable beam current can be derived as a function of the desired or required cavity voltage. Solving for the cavity voltage, (19.31) becomes after some manipulation

$$
V_{\mathrm{cy}}=\frac{\sqrt{2 \beta R_{\mathrm{s}}}}{1+\beta}\left(\sqrt{P_{\mathrm{g}, \mathrm{opt}}}+\sqrt{P_{\mathrm{g}, \mathrm{opt}}-\frac{1+\beta}{\beta} P_{\mathrm{b}}}\right) .
$$

This expression exhibits a limit for the beam current above which the second square root becomes imaginary. The condition for real solutions requires that

$$
P_{\mathrm{b}} \leq \frac{\beta}{1+\beta} P_{\mathrm{g}, \mathrm{opt}}
$$

leading to a limit of the maximum sustainable beam current of

$$
I_{\mathrm{b}} \leq \frac{\beta}{1+\beta} \frac{P_{\mathrm{g}}}{V_{\mathrm{cy}} \sin \psi_{\mathrm{s}}}
$$

Inspection of (19.31) shows that the required generator power can be further minimized by adjusting for the optimum coupling coefficient $\beta$. Optimum coupling can be derived from $\partial P_{\mathrm{g}} / \partial \beta=0$ with the solution

$$
\beta_{\mathrm{opt}}=1+\frac{2 R_{\mathrm{s}} I_{\mathrm{b}}}{V_{\mathrm{cy}}} \sin \psi_{\mathrm{s}}=1+\frac{P_{\mathrm{b}}}{P_{\mathrm{cy}}} .
$$

The minimum generator power required to produce an accelerating voltage $V_{\text {cy }} \sin \psi_{\mathrm{s}}$ is therefore from (19.31) with (19.35)

$$
P_{\mathrm{g}, \min }=\frac{V_{\mathrm{cy}}^{2}}{2 R_{\mathrm{s}}} \beta_{\mathrm{opt}}=\beta_{\mathrm{opt}} P_{\mathrm{cy}}
$$

and the optimum tuning angle from (19.23)

$$
\tan \Psi_{\mathrm{opt}}=\frac{\beta_{\mathrm{opt}}-1}{\beta_{\mathrm{opt}}+1} \cot \psi_{\mathrm{s}} .
$$

In this operating condition all rf-power from the generator is absorbed by the beam loaded cavity and no power reflection occurs. The maximum beam power is therefore $P_{\mathrm{b}}=P_{\mathrm{g}}-P_{\text {cy }}$ and the maximum beam current

$$
I_{\mathrm{b}} \leq \frac{P_{\mathrm{g}}}{V_{\mathrm{cy}} \sin \psi_{\mathrm{s}}}-\frac{V_{\mathrm{cy}}}{2 R_{\mathrm{s}} \sin \psi_{\mathrm{s}}} .
$$


Conditions have been derived assuring most efficient power transfer to the beam by proper adjustment of the cavity power input coupler to obtain the optimum coupling coefficient. Of course this coupling coefficient is optimum only for a specific beam current which in most cases is chosen to be the maximum desired beam current.

We are now in a position to determine the total rf-power flow. From conservation of energy we have

$$
P_{\mathrm{g}}=P_{\mathrm{cy}}+P_{\mathrm{b}}+P_{\mathrm{r}}
$$

where $P_{\mathrm{r}}$ is the reflected power which vanishes for the case of optimum coupling.

\subsection{Higher-Order Mode Losses in an Rf-Cavity}

The importance of beam loading for accurate adjustments of the rf-system has been discussed qualitatively but not yet quantitatively. In this paragraph, quantitative expressions will be derived for beam loading. Accelerating cavities constitute an impedance to a particle current and a bunch of particles with charge $q$ passing through a cavity induces electromagnetic fields into a broad frequency spectrum limited at the high frequency end by the bunch length. The magnitude of the excited frequencies in the cavity depends on the frequency dependence of the cavity impedance, which is a function of the particular cavity design and need not be known for this discussion. Fields induced within a cavity are called modes, oscillating at different frequencies with the lowest mode being the fundamental resonant frequency of the cavity. Although cavities are designed primarily for one resonant frequency, many higher-order modes or HOM'scan be excited at higher frequencies. Such modes occur above the fundamental frequency first at distinct well-separated frequencies with increasing spectral densities at higher frequencies.

For a moment we consider here only the fundamental frequency and deal with higher-order modes later. Fields induced by the total bunch charge act back on individual particles modifying the overall accelerating voltage seen by the particle. To quantify this we use the fundamental theorem of beam loading formulated by Wilson [1] which states that each particle within a bunch sees one half of the induced field while passing through the cavity.

We prove this theorem by conducting a Gedanken experiment proposed by Wilson. Consider a bunch of particles with charge $q$ passing through a lossless cavity inducing a voltage $V_{\mathrm{i} 1}$ in the fundamental mode. This induced field is opposed to the accelerating field since it describes a loss of energy. While the bunch passes through the cavity this field increases from zero reaching a maximum value at the moment the particle bunch leaves the cavity. Each particle will have interacted with this field and the energy loss corresponds to a fraction $f$ of the induced voltage $V_{\mathrm{i}, h}$, where the index $h$ indicates that we consider only the fundamental mode. The total energy 
lost by the bunch of charge $q$ is

$$
\Delta E_{1}=-q_{1} f V_{\mathrm{i}, h} .
$$

This energy appears as field energy proportional to the square of the voltage

$$
W_{1}=c_{1} V_{\mathrm{i}, h}^{2},
$$

where $c_{1}$ is a constant.

Now consider another bunch with the same charge $q_{2}=q_{1}=q$ following behind the first bunch at a distance corresponding to half an oscillation period at the fundamental cavity frequency. In addition to its own induced voltage this second bunch will see the field from the first bunch, now being accelerating, and will therefore gain an energy

$$
\Delta E_{2}=q_{1} V_{\mathrm{i}, h}-q_{2} f V_{\mathrm{i}, h}=q V_{\mathrm{i}, h}(1-f) .
$$

After passage of the second charge, the cavity returns to the original state before the first charge arrived because the field from the first charge having changed sign exactly cancels the induced field from the second charge. The cavity has been assumed lossless and energy conservation requires therefore that $\Delta E_{1}+\Delta E_{2}=0$ or $-q f V_{\mathrm{i}, h}+q V_{\mathrm{i}, h}(1-f)=0$ from which we get

$$
f=\frac{1}{2}
$$

proving the statement of the fundamental theorem of beam loading. The energy loss of a bunch of charge $q$ due to its own induced field is therefore

$$
\Delta E_{1}=-\frac{1}{2} q V_{\mathrm{i}, h}
$$

This theorem will be used to determine the energy transfer from cavity fields to a particle beam. To calculate the induced voltages in rf-cavities, or in arbitrarily shaped vacuum chambers providing some impedance for the particle beam can become very complicated. For cylindrically symmetric cavities the induced voltages can be calculated numerically with programs like SUPERFISH [2], URMEL[3] or MAFIA [3].

For a more practical approach Wilson [1] introduced a loss parameter $k$ which can be determined either by electronic measurements or by numerical calculations. This loss parameter for the fundamental mode loss of a bunch with charge $q$ is defined by

$$
\Delta E_{h}=k_{h} q^{2}
$$

and together with (19.44) we get the induced voltage

$$
V_{\mathrm{i}, h}=-2 k_{h} q
$$


or after elimination of the charge

$$
\Delta E_{h}=\frac{V_{\mathrm{i}, h}^{2}}{4 k_{h}},
$$

where the index $h$ indicates that the parameter should be taken at the fundamental frequency. The loss parameter can be expressed in terms of cavity parameters. From the definition of the cavity quality factor (18.80) and cavity losses from (18.77) we get

$$
\frac{2 R_{\mathrm{cy}}}{Q}=\frac{V^{2}}{\omega W},
$$

where $\omega$ is the frequency and $W$ the stored field energy in the cavity. Applying this to the induced field, we note that $\Delta E_{h}$ is equal to the field energy $W_{h}$ and combining (19.47), (19.48) the loss parameter to the fundamental mode in a cavity with shunt impedance $R_{h}$ and quality factor $Q_{h}$ is

$$
k_{h}=\frac{\omega_{h}}{4} \frac{R_{h}}{Q_{h}} .
$$

The excitation of higher-order mode fields by the passing particle bunch leads to additional energy losses which are conveniently expressed in units of the energy loss to the fundamental mode

$$
\Delta E_{\mathrm{hom}}=\left(r_{\mathrm{hom}}-1\right) \Delta E_{h},
$$

where $r_{\text {hom }}$ is the ratio of the total energy losses into all cavity modes to the loss into the fundamental mode only. The induced higher order field energy in the cavity is therefore

$$
W_{\text {hom }}=\left(r_{\text {hom }}-1\right) W_{h} .
$$

Again we may define a loss parameter $k_{n}$ for an arbitrary $n$ th-mode and get analogous to (19.49)

$$
k_{n}=\frac{\omega_{n}}{4} \frac{2 R_{n}}{Q_{n}},
$$

where $R_{n}$ and $Q_{n}$ are the shunt impedance and quality factor for the $n$ th-mode or frequency $\omega_{n}$, respectively. The total loss parameter due to all modes is by linear superposition

$$
k=\sum_{n} k_{n} .
$$


The task to determine the induced voltages has been reduced to the determination of the loss parameters for individual modes or if this is not possible or desirable we may use just the overall loss parameter $k$ as may be determined experimentally. This is particularly convenient for cases where it is difficult to calculate the mode losses but much easier to measure the overall losses by electronic measurements.

The higher-order mode losses will become important for discussion of beam stability since these fields will act back on subsequent particles and bunches thus creating a coupling between different parts of one bunch or different bunches.

\subsubsection{Efficiency of Energy Transfer from Cavity to Beam}

Higher-order mode losses affect the efficiency by which energy is transferred to the particle beam. Specifically, since the higher-order mode losses depend on the beam current we must expect some limitation in the current capability of the accelerator.

With these preparations we have now all information to calculate the transfer of energy from the cavity to the particle beam. Just before the arrival of a particle bunch let the cavity voltage as generated by the rf-power source be

$$
V_{\text {cy }}=-V_{\mathrm{g}} \mathrm{e}^{\mathrm{i} \Psi_{\mathrm{g}}},
$$

where $V_{\mathrm{g}}$ is the generator voltage and $\Psi_{\mathrm{g}}$ the generator voltage phase with respect to the particle beam. To combine the generator voltage with the induced voltage we use phasor diagrams in the complex plane.

The generator voltage is shown in Fig. 19.5 as a vector rotated by the angle $\Psi_{\mathrm{g}}$ from the real axis representing the cavity state just before the beam passes. The beam induced voltage is parallel and opposite to the real axis. Both vectors add up to the voltage $V$ just after the beam has left the cavity.

The difference of the fundamental field energy before and after passage of the particle bunch is equal to the energy transferred to the passing particle bunch minus

Fig. 19.5 Phasor diagram for cavity voltages with beam loading

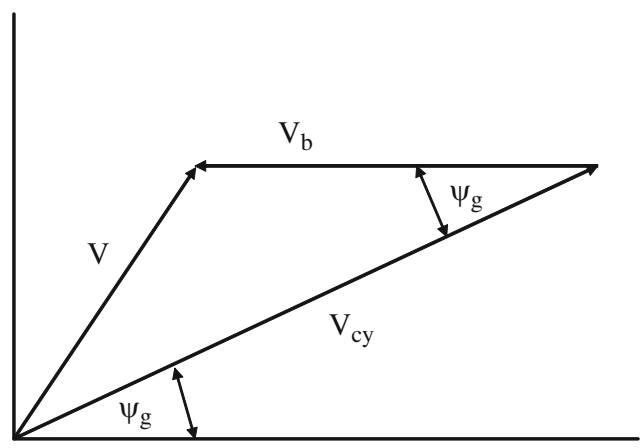


the higher-order field energy and is from the phasor diagram

$$
\Delta E_{\mathrm{hom}}=\alpha\left(V_{\mathrm{cy}}^{2}-V^{2}\right)-W_{\mathrm{hom}}=\alpha\left(2 V_{\mathrm{cy}} V_{\mathrm{b}} \cos \Psi_{\mathrm{g}}-V_{\mathrm{b}}^{2}\right)-W_{\mathrm{hom}},
$$

where $\alpha$ is the proportionality factor between the energy gain $\Delta E$ and the square of the voltage defined by $\alpha=\Delta E / V^{2}$. With (19.45), (19.46), we get from (19.55) the net energy transfer to a particle bunch [4]

$$
\Delta E_{\mathrm{hom}}=\alpha\left(2 V_{\mathrm{cy}} V_{\mathrm{b}} \cos \Psi_{\mathrm{g}}-r_{\mathrm{hom}} V_{\mathrm{b}}^{2}\right) .
$$

The energy stored in the cavity before arrival of the beam is $W_{\mathrm{cy}}=\alpha V_{\mathrm{cy}}^{2}$ and the energy transfer efficiency to the beam becomes

$$
\eta=\frac{\Delta E_{h}}{W_{\mathrm{cy}}}=2 \frac{V_{\mathrm{b}}}{V_{\mathrm{cy}}} \cos \Psi_{\mathrm{g}}-r_{\mathrm{hom}} \frac{V_{\mathrm{b}}^{2}}{V_{\mathrm{cy}}^{2}} .
$$

It is obvious from (19.57) that energy transfer is not guaranteed by the synchronicity condition or the power of the generator alone. Specifically, the second term in (19.57) becomes dominant for a large beam intensity and the efficiency may even become negative indicating reversed energy transfer from the beam to cavity fields. The energy transfer efficiency has a maximum for $V_{\mathrm{b}}=\frac{\cos \Psi_{\mathrm{g}}}{r_{\mathrm{hom}}} V_{\text {cy }}$ and is

$$
\eta_{\max }=\frac{\cos ^{2} \Psi_{\mathrm{g}}}{r_{\mathrm{hom}}},
$$

a result first derived by Keil et al. [5] and is therefore frequently called the KeilSchnell-Zotter criterion. The maximum energy transfer efficiency is limited by the phase of the generator voltage and the higher-order mode losses in the cavity.

\subsection{Beam Loading}

Only one passage of a bunch through a cavity has been considered in the previous section. In circular accelerators, however, particle bunches pass periodically through the accelerating cavities and we have to consider the cumulative build up of induced fields. Whenever a particle bunch is traversing a cavity the induced voltage from this passage is added to those still present from previous bunch traversals. For simplicity, we assume a number of equidistant bunches along the circumference of the ring, where adjacent bunches are separated by an integer number $m_{\mathrm{b}}$ of the fundamental rf-wavelength. The induced voltage decays exponentially by a factor $\mathrm{e}^{-\rho}$ between 
Fig. 19.6 Phasor diagram for the superposition of induced voltages in an accelerating cavity

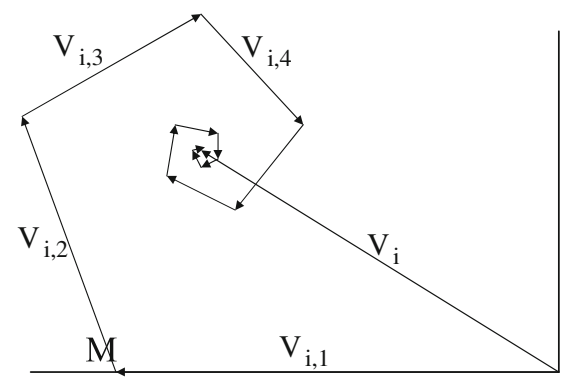

two consecutive bunches with

$$
\rho=\frac{t_{\mathrm{b}}}{t_{\mathrm{d}}},
$$

where $t_{\mathrm{b}}$ is the time between bunches and $t_{\mathrm{d}}$ the cavity voltage decay time for the fundamental mode. The phase of the induced voltage varies between the passage of two consecutive bunches by

$$
\varphi=\omega_{h} t_{\mathrm{b}}-2 \pi m_{\mathrm{b}}
$$

At the time a bunch passes through the cavity the total induced voltages are then the superposition of all fields induced by previous bunches

$$
V_{\mathrm{i}}=V_{\mathrm{i}, h}\left(1+\mathrm{e}^{-\rho} \mathrm{e}^{\mathrm{i} \varphi}+\mathrm{e}^{-2 \rho} \mathrm{e}^{\mathrm{i} 2 \varphi}+\cdots\right)
$$

shown in Fig. 19.6 as the superposition of all induced voltages in form of a phasor diagram together with the resultant induced voltage $V_{\mathrm{i}}$. The sum (19.61) can be evaluated to be

$$
V_{\mathrm{i}}=V_{\mathrm{i}, h} \frac{1}{1-\mathrm{e}^{-\rho} \mathrm{e}^{\mathrm{i} \varphi}},
$$

which is the total induced voltage in the cavity just after the last bunch passes; however, the voltage seen by this last bunch is only half of the induced voltage and the total voltage $V_{\mathrm{b}}$ acting back on the bunch is therefore

$$
V_{\mathrm{b}}=V_{\mathrm{i}, h}\left(\frac{1}{1-\mathrm{e}^{-\rho} \mathrm{e}^{\mathrm{i} \varphi}}-\frac{1}{2}\right) .
$$

The voltage $V_{\mathrm{i}, h}$ can be expressed in more practical units. Considering the damping time (18.62) for fields in a cavity we note that two damping times exist, one for the empty unloaded cavity $t_{\mathrm{d} 0}$ and a shorter damping time $t_{\mathrm{d}}$ when there is also a beam present. For the unloaded damping time we have from (18.62) 


$$
t_{\mathrm{d} 0}=\frac{2 Q_{0 h}}{\omega_{h}},
$$

where $Q_{0 h}$ is the unloaded quality factor. From (19.45), (19.47) we get with $q=I_{0} t_{\mathrm{b}}$, where $I_{0}$ is the average beam current,

$$
V_{\mathrm{i}, h}=\frac{\omega_{h}}{2 Q_{0 h}} R_{h} I_{0} t_{\mathrm{b}}
$$

and with (19.64)

$$
V_{\mathrm{i}, h}=R_{h} I_{0} \frac{t_{\mathrm{b}}}{t_{\mathrm{d} 0}} .
$$

Introducing the coupling coefficient $\beta$, we get from (19.9), (19.64)

$$
t_{\mathrm{d} 0}=(1+\beta) t_{\mathrm{b}} .
$$

In analogy to (19.59) we define

$$
\rho_{0}=\frac{\rho}{1+\beta}=\frac{t_{\mathrm{b}}}{t_{\mathrm{d} 0}}
$$

and (19.65) becomes

$$
V_{\mathrm{i}, h}=\rho_{0} R_{h} I_{0} .
$$

We are finally in a position to calculate from (19.63), (19.68) the total beam induced cavity voltage $V_{\mathrm{b}}$ in the fundamental mode for circular accelerators.

\subsection{Phase Oscillation and Stability}

In the course of discussing phase oscillations we found it necessary to select carefully the synchronous phase depending on the particle energy being below or above the transition energy. Particularly, we found that phase stability for particles above transition energy requires the rf-voltage to decrease with increasing phase. From the derivative of (19.27) with respect to $\psi_{\mathrm{s}}$ we find with (19.21) and since $V_{\mathrm{gr}} \cos \Psi>0$ the condition for phase stability $\sin \left(\psi_{\mathrm{g}}-\Psi_{\mathrm{m}}\right)<0$ or

$$
\frac{1}{2} \pi<\psi_{\mathrm{s}}+\Psi_{\mathrm{m}}<\frac{3}{2} \pi .
$$


From (19.28) and (19.69) we get

$$
-V_{\mathrm{cy}}\left|\cos \psi_{\mathrm{s}}\right|-V_{\mathrm{br}} \sin \Psi_{\mathrm{m}} \cos \Psi_{\mathrm{m}}<0
$$

or with (19.23)

$$
V_{\mathrm{br}} \sin \psi_{\mathrm{s}}<V_{\mathrm{cy}}
$$

which is Robinson's phase-stability criterion or the Robinson condition [6] for the tuning angle of the accelerator cavity. The maximum current that can be accelerated in a circular accelerator with stable phase oscillations is limited by the effective cavity voltage. In terms of rf-power, (19.70) is with (19.20) equivalent to

$$
P_{\mathrm{b}} \leq(1+\beta) P_{\mathrm{cy}}
$$

and the stability condition for the coupling coefficient is from (19.35)

$$
\beta>\beta_{\text {opt }}-2
$$

The stability condition is always met for rf-cavities with optimum coupling $\beta=$ $\beta_{\text {opt }}$.

\subsubsection{Robinson Damping}

Correct tuning of the rf-system is a necessary but not a sufficient condition for stable phase oscillations. In Chap. 12 we found the occurrence of damping or antidamping due to forces that depend on the energy of the particle. Such a case occurs in the interaction of bunched particle beams with accelerating cavities or vacuum chamber components which act like narrow band resonant cavities. The revolution time of a particle bunch depends on the average energy of particles within a bunch and the Fourier spectrum of the bunch current being made up of harmonics of the revolution frequency is therefore energy dependent. On the other hand by virtue of the frequency dependence of the cavity impedance, the energy loss of a bunch in the cavity due to beam loading depends on the revolution frequency. We have therefore an energy dependent loss mechanism which can lead to damping or worse anti-damping of coherent phase oscillation and we will therefore investigate this phenomenon in more detail. Robinson [6] studied first the dynamics of this effect generally referred to as Robinson damping or Robinson instability.

Above transition energy the revolution frequency is lower for higher bunch energies compared to the reference energy and vice versa. To obtain damping of coherent phase oscillations, we would therefore tune the cavity such that the bunch would loose more energy in the cavity while at higher energies (lower frequency) during the course of coherent synchrotron oscillation and loose less energy at 
a)

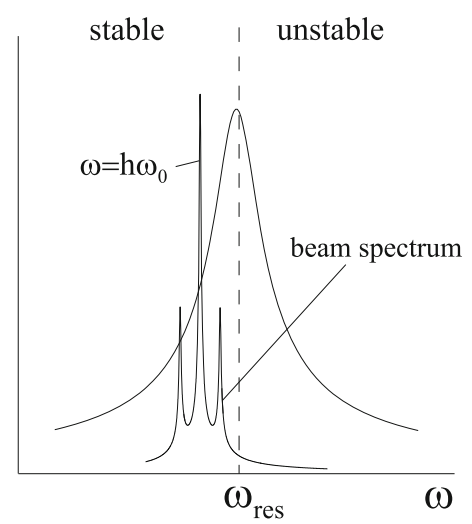

below transition b)

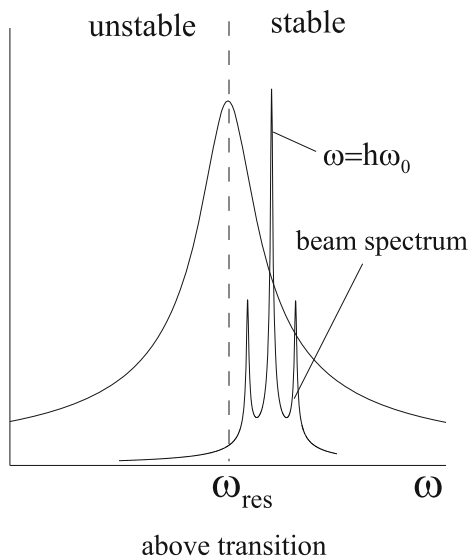

Fig. 19.7 Cavity tuning for positive Robinson damping below and above transition energy. (a) Below transition. (b) Above transition

lower energies (higher frequency). In this situation, the impedance of the cavity should decrease with increasing frequency for damping to occur as demonstrated in Fig. 19.7.

Here the resonance curve or impedance spectrum is shown for the case of a resonant frequency above the beam frequency $h \omega_{0}$ in Fig. 19.7a and below the beam frequency in Fig 19.7b. Consistent with the arguments made above we would expect damping in case of Fig. 19.7b for a beam above transition and anti-damping in case of Fig. 19.7a. Adjusting the resonance frequency of the cavity to a value below the beam frequency $h \omega_{0}$ where $\omega_{0}$ is the revolution frequency, is called capacitive detuning. Conversely, we would tune the cavity resonance frequency above the beam frequency $\left(\omega_{\mathrm{r}}>h \omega_{0}\right)$ or inductively detune the cavity for damping below transition energy (Fig. 19.7a).

In a more formal way we fold the beam-current spectrum with the impedance spectrum of the cavity and derive scaling laws for the damping as well as the shift in synchrotron frequency. During phase oscillations the revolution frequency is modulated and as a consequence the beam spectrum includes in addition to the fundamental frequency two side bands or satellites. The beam-current spectrum is composed of a series of harmonics of the revolution frequency up to frequencies with wavelength of the order of the bunchlength

$$
I(t)=I_{\mathrm{b}}+\sum_{n>0} I_{\mathrm{n}} \cos \left(n \omega_{0} t-\varphi\right)
$$

where $I_{\mathrm{b}}$ is the average circulating beam current and $\varphi$ a phase shift with respect to the reference particle. The Fourier coefficient for bunches short compared to the 
wavelength of the harmonic is given by

$$
I_{\mathrm{n}}=2 I_{\mathrm{b}}
$$

Here we restrict the discussion to the interaction between beam and cavity at the fundamental cavity frequency and the only harmonic of interest in the beam spectrum is therefore the $h$ th harmonic

$$
I_{h}(t)=2 I_{\mathrm{b}} \cos \left(h \omega_{0} t-\varphi\right)
$$

By virtue of coherent synchrotron oscillations the phase oscillates for each particle in a bunch like

$$
\varphi(t)=\varphi_{0} \sin \Omega_{\mathrm{s}} t
$$

where $\varphi_{0}$ is the maximum amplitude and $\Omega_{\mathrm{s}}$ the synchrotron oscillation frequency of the phase oscillation. We insert this into (19.75) and get after expanding the trigonometric functions for small oscillation amplitudes $\varphi_{0} \ll 1$

$$
I_{h}(t)=2 I_{\mathrm{b}} \cos \left(h \omega_{0} t\right)-I_{\mathrm{b}} \varphi_{0}\left[\cos \left(h \omega_{0} t+\Omega_{\mathrm{s}} t\right)-\cos \left(h \omega_{0} t-\Omega_{\mathrm{s}} t\right)\right] .
$$

This expression exhibits clearly sidebands or satellites in the beam spectrum at $h \omega_{0} \pm \Omega_{\mathrm{s}}$. Folding the expression for the beam current with the cavity impedance defines the energy loss of the particle bunch while passing through the cavity. The cavity impedance is a complex quantity which was derived in (19.11) and its real part is shown together with the beam spectrum in Fig. 19.8. The induced voltage in the cavity by a beam $I_{h}(t)=I_{h} \cos h \omega_{0} t$ is

$$
V_{h}=-Z I_{h}(t)=-Z_{\mathrm{r}} I_{h} \cos \left(h \omega_{0} t\right)-Z_{\mathrm{i}} I_{h} \sin \left(h \omega_{0} t\right),
$$

Fig. 19.8 Cavity impedance and beam spectrum in the vicinity of the fundamental $\mathrm{rf}$ frequency $\omega_{\mathrm{rf}}=h \omega_{0}$

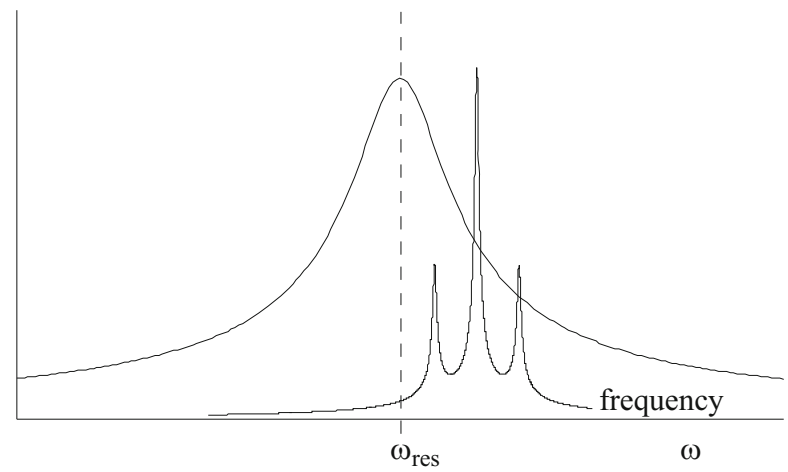


where we have split the impedance in its real and imaginary part and have expressed the imaginary part of the induced voltage by a $-\pi / 2$ phase shift. Applying (19.78) to all components of the beam current (19.77) we get the induced voltage in the cavity

$$
\begin{aligned}
V_{h}= & -Z_{\mathrm{r}}^{0} 2 I_{\mathrm{b}} \cos h \omega_{0} t-Z_{\mathrm{i}}^{0} 2 I_{\mathrm{b}} \sin h \omega_{0} t \\
& +Z_{\mathrm{r}}^{+} I_{\mathrm{b}} \varphi_{0} \cos h \omega_{0} t \cos \Omega_{\mathrm{s}} t-Z_{\mathrm{r}}^{+} I_{\mathrm{b}} \varphi_{0} \sin h \omega_{0} t \sin \Omega_{\mathrm{s}} t \\
& +Z_{\mathrm{i}}^{+} I_{\mathrm{b}} \varphi_{0} \sin h \omega_{0} t \cos \Omega_{\mathrm{s}} t+Z_{\mathrm{i}}^{+} I_{\mathrm{b}} \varphi_{0} \cos h \omega_{0} t \sin \Omega_{\mathrm{s}} t \\
& -Z_{\mathrm{r}}^{-} I_{\mathrm{b}} \varphi_{0} \cos h \omega_{0} t \cos \Omega_{\mathrm{s}} t-Z_{\mathrm{r}}^{-} I_{\mathrm{b}} \varphi_{0} \sin h \omega_{0} t \sin \Omega_{\mathrm{s}} t \\
& -Z_{\mathrm{i}}^{-} I_{\mathrm{b}} \varphi_{0} \sin h \omega_{0} t \cos \Omega_{\mathrm{s}} t+Z_{\mathrm{i}}^{-} I_{\mathrm{b}} \varphi_{0} \cos h \omega_{0} t \sin \Omega_{\mathrm{s}} t,
\end{aligned}
$$

where $Z^{0}, Z^{+}$and $Z^{-}$are the real $\mathrm{r}$ and imaginary $\mathrm{i}$ cavity impedances at the frequencies $h \omega_{0}, h \omega_{0}+\Omega_{\mathrm{s}}, h \omega_{0}-\Omega_{\mathrm{s}}$ respectively. We make use of the expression for the phase oscillation (19.76) and its derivative

$$
\dot{\varphi}(t)=\varphi_{0} \Omega_{\mathrm{s}} \cos \Omega_{\mathrm{s}} t,
$$

multiply the induced voltage spectrum (19.79) by the current spectrum (19.77) and get after averaging over fast oscillating terms at frequency $h \omega_{0}$

$$
\left\langle V_{h} I_{h}\right\rangle=-2 I_{\mathrm{b}}^{2}\left\{Z_{\mathrm{r}}^{0}-\left[Z_{\mathrm{i}}^{0}-\frac{1}{2}\left(Z_{\mathrm{i}}^{+}+Z_{\mathrm{i}}^{-}\right)\right] \varphi-\frac{Z_{\mathrm{r}}^{+}-Z_{\mathrm{r}}^{-}}{2 \Omega_{\mathrm{s}}} \dot{\varphi}\right\} .
$$

This is the rate of energy loss of the particle bunch into the impedance of the cavity. Dividing by the total circulating charge $T_{0} I_{\mathrm{b}}$ we get the rate of relative energy loss per unit charge

$$
\frac{\mathrm{d} \varepsilon}{\mathrm{d} t}=-\frac{\left\langle e V_{h} I_{h}\right\rangle}{T_{0} I_{\mathrm{b}} E_{0}}=+\frac{\ddot{\varphi}}{\beta c k_{h}\left|\eta_{\mathrm{c}}\right|},
$$

where $T_{0}$ is the revolution time and $I_{\mathrm{b}}$ the average beam current.

We made use of the relation between the energy deviation from the ideal energy and the rate of change of the phase (9.17) on the r.h.s. of the equation. From (19.81), (19.82) and making use of the definition of the synchrotron frequency in (9.32) $\Omega_{\mathrm{s} 0}^{2}=\frac{c k_{\mathrm{h}}\left|\eta_{\mathrm{c}}\right|}{E_{0} T_{0}} e V_{\mathrm{cy}}\left|\cos \psi_{\mathrm{s}}\right|$, we get a differential equation of the form

$$
\ddot{\varphi}+2 \alpha_{R} \dot{\varphi}+\Delta \Omega^{2} \varphi=0
$$

with a Robinson damping decrement

$$
\alpha_{R}=-\frac{\beta \Omega_{\mathrm{s} 0}}{2 V_{\mathrm{cy}}\left|\cos \psi_{\mathrm{s}}\right|}\left(Z_{\mathrm{r}}^{+}-Z_{\mathrm{r}}^{-}\right) I_{\mathrm{b}},
$$


and a shift in synchrotron oscillation frequency

$$
\Delta \Omega^{2}=-\frac{2 \beta \Omega_{\mathrm{s} 0}^{2}}{V_{\mathrm{cy}}\left|\cos \psi_{\mathrm{s}}\right|}\left[Z_{\mathrm{i}}^{0}-\frac{1}{2}\left(Z_{\mathrm{i}}^{+}+Z_{\mathrm{i}}^{-}\right)\right] I_{\mathrm{b}} .
$$

The unperturbed phase equation (9.26) is

$$
\ddot{\varphi}+2 \alpha_{\mathrm{s} 0} \dot{\varphi}+\Omega_{\mathrm{s} 0}^{2} \varphi=0
$$

and combining both, we derive a modification of both the damping and oscillation frequency. The combined damping decrement is

$$
\alpha_{\mathrm{s}}=\alpha_{\mathrm{s} 0}-\frac{\beta \Omega_{\mathrm{s} 0}}{V_{\mathrm{cy}}\left|\cos \psi_{\mathrm{s}}\right|}\left(Z_{\mathrm{r}}^{+}-Z_{\mathrm{r}}^{-}\right) I_{\mathrm{b}}>0
$$

where $\alpha_{\mathrm{s} 0}$ is the radiation damping in electron accelerators. The total damping decrement must be positive for beam stability. The interaction of the beam with the accelerating cavity above transition is stable for all values of the beam current if $Z_{\mathrm{r}}^{+}<Z_{\mathrm{r}}^{-}$or if the cavity resonant frequency is capacitively detuned. Due to the imaginary part of the impedance the interaction of beam and cavity leads to a synchrotron oscillation frequency shift given by

$$
\Omega_{\mathrm{s}}^{2}=\Omega_{\mathrm{s} 0}^{2}-\frac{2 \beta \Omega_{\mathrm{s} 0}^{2}}{V_{\mathrm{cy}}\left|\cos \psi_{\mathrm{s}}\right|}\left[Z_{\mathrm{i}}^{0}-\frac{1}{2}\left(Z_{\mathrm{i}}^{+}+Z_{\mathrm{i}}^{-}\right)\right] I_{\mathrm{b}} .
$$

This frequency shift has two components, the incoherent frequency shift due to the impedance $Z_{\mathrm{i}}^{0}$ at the fundamental beam frequency $h \omega_{0}$ and a frequency shift for coherent bunch-phase oscillations due to the imaginary part of the cavity impedances. For small frequency shifts $\Delta \Omega_{\mathrm{s}}=\Omega_{\mathrm{s}}-\Omega_{\mathrm{s} 0},(19.88)$ can be linearized for

$$
\frac{\Delta \Omega_{\mathrm{s}}}{\Omega_{\mathrm{s} 0}}=-\frac{I_{\mathrm{b}} \beta}{V_{\mathrm{cy}}\left|\cos \psi_{\mathrm{s}}\right|}\left[Z_{\mathrm{i}}^{0}-\frac{1}{2}\left(Z_{\mathrm{i}}^{+}+Z_{\mathrm{i}}^{-}\right)\right] .
$$

The cavity impedance is from (19.10)

$$
Z=R_{\mathrm{s}} \frac{1-\mathrm{i} Q_{0} \frac{\omega^{2}-\omega_{\mathrm{r}}^{2}}{\omega_{\mathrm{r}} \omega}}{1+Q_{0}^{2}\left(\frac{\omega^{2}-\omega_{\mathrm{r}}^{2}}{\omega_{\mathrm{r}} \omega}\right)^{2}} .
$$

From the imaginary part of the cavity impedance and capacitive detuning we conclude that above transition energy, the incoherent synchrotron tune shift is positive

$$
\Delta \Omega_{\text {s,incoh }}>0
$$


while the coherent synchrotron tune shift is negative

$$
\Delta \Omega_{\mathrm{s}, \mathrm{coh}}<0 .
$$

This conclusion may in special circumstances be significantly different due to other passive cavities in the accelerator. The shift in the synchrotron tune is proportional to the beam current and can be used as a diagnostic tool to determine the cavity impedance or its deviation from the ideal model (19.90).

In the preceding discussion it was assumed that only resonant cavities contribute to Robinson damping. This is correct to the extend that other cavity like structures of the vacuum enclosure in a circular accelerator have a low quality factor $Q$ for the whole spectrum or at least at multiples of the revolution frequency and therefore do not contribute significantly to this effect through a persistent energy loss over many turns. Later we will see that such low- $Q$ structures in the vacuum chamber may lead to other types of beam instability.

\subsubsection{Potential Well Distortion}

The synchrotron frequency is determined by the slope of the rf-voltage at the synchronous phase. In the last subsection the effect of beam loading at the cavity fundamental frequency was discussed demonstrating the need to include the induced voltages in the calculation of the synchrotron oscillation frequency. These induced voltages cause a perturbation of the potential well and as a consequence a change in the bunch length. In this subsection we will therefore also include higher-order interaction of the beam with its environment.

It is not possible to derive a general expression for the impedance of all components of a vacuum chamber in a circular accelerator. However, measurements [7] have shown that the impedance spectrum of circular accelerator vacuum chambers, while excluding accelerating cavities, has the form similar to that of the SPEAR storage ring shown in Fig. 19.9.

Fig. 19.9 SPEAR impedance spectrum [7]

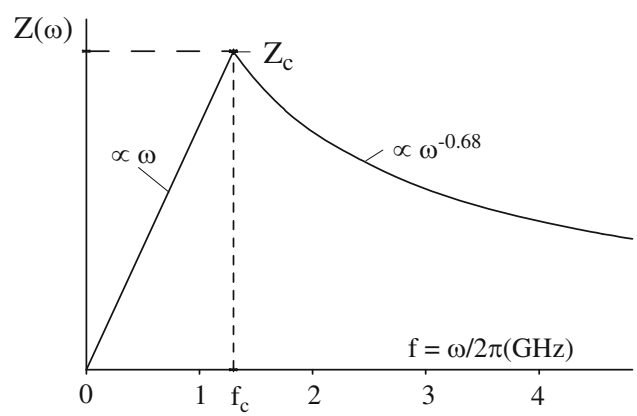


Up to the transition frequency $f_{\mathrm{t}}$, which is determined by vacuum chamber dimensions, the impedance is predominantly inductive and becomes capacitive above the transition frequency. We are looking here only for fields with wavelength longer than the bunch length which may distort the rf-voltage waveform such as to change the slope for the whole bunch. Later we will consider shorter wavelength which give rise to perturbations within the bunch. Because the bunch length is generally of the order of vacuum chamber dimensions we only need to consider the impedance spectrum below transition frequency which is predominantly inductive. To preserve generality, however, we assume a more general but still purely imaginary impedance defined by

$$
Z(\omega)_{\|}=\mathrm{i} \omega \mathcal{Z}_{\|}
$$

Studying the modification of a finite bunch length due to potential-well distortions we use for mathematical simplicity a parabolic particle distribution [8] in phase (Fig. 19.10) normalized to the bunch current $\int_{-\varphi_{\ell}}^{\varphi_{\ell}} I(\varphi) \mathrm{d} \varphi=I_{\mathrm{b}}$

$$
I(\varphi)=\frac{3 I_{\mathrm{b}}}{4 \varphi_{\ell}}\left(1-\frac{\varphi^{2}}{\varphi_{\ell}^{2}}\right)
$$

where $2 \varphi_{\ell}$ is the bunch length expressed in terms of a phase with respect to the fundamental rf-wavelength. The combined induced voltage in the whole vacuum chamber is

$$
V_{\mathcal{Z}}=\mathcal{Z}_{\|} \frac{\mathrm{d} I}{\mathrm{~d} t}=h \omega_{0} \mathcal{Z}_{\|} \frac{\mathrm{d} I}{\mathrm{~d} \varphi}=h \operatorname{Im}\left(Z_{\|} / n\right) \frac{\mathrm{d} I}{\mathrm{~d} \varphi}
$$

where we have introduced the normalized impedance

$$
\frac{Z_{\|}}{n}=\mathrm{i} \omega_{0} \mathcal{Z}_{\|}
$$

which is the longitudinal impedance divided by the frequency in units of the revolution frequency or by the mode number $n=\omega / \omega_{0}$. Inserting (19.94)

Fig. 19.10 Current distribution for potential-well distortion

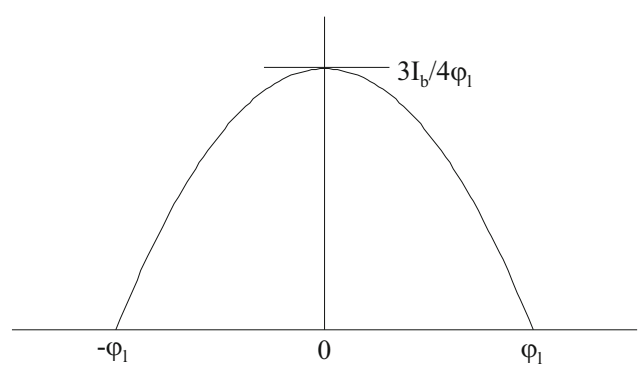


into (19.95) we get the induced voltage

$$
V_{\mathcal{Z}}=-\frac{3 h I_{\mathrm{b}} \operatorname{Im}\left(Z_{\|} / n\right)}{2 \varphi_{\ell}^{3}} \varphi,
$$

which must be added to the rf-voltage $V_{\text {rf- }}=V_{\text {cy }} \sin \left(\psi_{\mathrm{s}}+\varphi\right)$. Forming an effective voltage we get

$$
V_{\text {eff }}=V_{\text {cy }} \cos \psi_{\mathrm{s}}\left(1-\frac{3 h I_{\mathrm{b}} \operatorname{Im}\left(Z_{\|} / n\right)}{2 \varphi_{\ell}^{3} V_{\mathrm{cy}} \cos \psi_{\mathrm{s}}}\right) \varphi+V_{\mathrm{cy}} \sin \psi_{\mathrm{s}} .
$$

This modification of the effective cavity voltage leads to an incoherent shift of the synchrotron oscillation frequency

$$
\frac{\Omega_{\mathrm{s}}^{2}}{\Omega_{\mathrm{s} 0}^{2}}=1-\frac{3 \eta_{\mathrm{c}} e I_{\mathrm{b}}}{4 \pi \varphi_{\ell}^{3} E v_{\mathrm{s}}^{2}} \operatorname{Im}\left(Z_{\|} / n\right),
$$

where we used the definition of the synchrotron tune $v_{\mathrm{s}}^{2}=\frac{\eta_{\mathrm{c}} e V_{\mathrm{cy}} \cos \psi_{\mathrm{s}}}{2 \pi h E}$.

Above transition energy $\eta_{\mathrm{c}} \cos \psi_{\mathrm{s}}>0$ and therefore the frequency shift is positive for $\operatorname{Im}\left(Z_{\|} / n\right)<0$ and negative for $\operatorname{Im}\left\{Z_{\|} / n\right\}>0$. We note specifically that the shift depends strongly on the bunch length and increases with decreasing bunch length, a phenomenon we observe in all higher-order mode interactions.

Note that this shift of the synchrotron oscillation frequency does not appear for coherent oscillations since the induced voltage also moves with the bunch oscillation. The bunch center actually sees always the unaltered rf-field and oscillates according to the slope of the unperturbed rf-voltage. The coherent synchrotron oscillation frequency therefore need not be the same as the incoherent frequency. This has some ramification for the experimental determination of the synchrotron oscillation frequency.

The shift in incoherent synchrotron oscillation frequency reflects also a change in the equilibrium bunch length which is different for proton or ion beams compared to an electron bunch. The energy spread of radiating electron beams is determined only by quantum fluctuations due to the emission of synchrotron radiation and is independent of rf-fields. The electron bunch length scales therefore inversely proportional to the synchrotron oscillation frequency and we get with $\Omega_{\mathrm{s}} / \Omega_{\mathrm{s} 0}=$ $\sigma_{\ell 0} / \sigma_{\ell}$ from (19.99) after solving for $\sigma_{\ell} / \sigma_{\ell 0}$

$$
\frac{\sigma_{\ell}^{3}}{\sigma_{\ell 0}^{3}}-\frac{\sigma_{\ell}}{\sigma_{\ell 0}}-\frac{8 \eta_{\mathrm{c}} e I_{\mathrm{b}}}{9 \pi^{2} \sqrt{2 \pi} \sigma_{\ell 0}^{3} E v_{\mathrm{s}}^{2}} \operatorname{Im}\left(\frac{Z_{\|}}{n}\right)=0,
$$

where we replaced the parabolic current distribution by a Gaussian distribution with equal total bunch current and equal intensity in the bunch center by setting $\varphi_{\ell}=$ $3 \sqrt{2 \pi} / 4 h \sigma_{\ell} / \bar{R}$ and where $\sigma_{\ell 0}$ is the unperturbed bunch length. 
Non-radiating particles, in contrast, must obey Liouville's theorem and the longitudinal beam emittance $\ell \Delta p$ will not change due to potential-well distortions. For proton or ion bunches we employ the same derivation for the bunch lengthening but note that the bunch length scales with the energy spread in such a way that the product of bunch length $\ell$ and momentum spread $\Delta p$ remains constant. Therefore $\ell \propto 1 / \sqrt{\Omega_{\mathrm{s}}}$ and the perturbed bunch length is from (19.99) with $\ell=(\bar{R} / h) \varphi_{\ell}$

$$
\frac{\ell^{4}}{\ell_{0}^{4}}-\frac{3 \eta_{\mathrm{c}} e I_{\mathrm{b}}}{4 \pi E v_{\mathrm{s}}^{2}} \frac{\bar{R}^{3}}{\ell_{0}^{3}} \operatorname{Im}\left(\frac{Z_{\|}}{n}\right) \frac{\ell}{\ell_{0}}-1=0 .
$$

Of course, along with this perturbation of the proton or ion bunch length goes an opposite perturbation of the energy spread.

\section{Problems}

19.1 (S). Consider an electron storage ring to be used as a damping ring for a linear collider. The energy is $E=1.21 \mathrm{GeV}$, circumference $C=35.27 \mathrm{~m}$, bending radius $\rho=2.037 \mathrm{~m}$, momentum compaction factor $\alpha_{\mathrm{c}}=0.01841$, rf harmonic number $h=84$, cavity shunt impedance of $R_{\mathrm{cy}}=8.4 \mathrm{M} \Omega$. An intense bunch of $N_{\mathrm{e}}=$ $5 \times 10^{10}$ particles is injected in a single pulse and is stored for only a few msec to damp to a small beam emittance. Specify and optimize a suitable rf-system and calculate the required rf-cavity power, cavity voltage, coupling factor first while ignoring beam loading and then with beam loading. Assume a quantum lifetime of $1 \mathrm{~h}$.

19.2 (S). Show that for bunches short compared to the rf-wavelength the harmonic amplitudes are $I_{\mathrm{h}}=2 I_{\mathrm{b}}$.

\section{References}

1. P.B. Wilson, R. Servranckx, A.P. Sabersky, J. Gareyte, G.E. Fischer, A.W. Chao, IEEE Trans. Nucl. Sci. 24, 1211 (1977)

2. K. Halbach, F. Holsinger, Part. Accel. 7, 213 (1976)

3. T. Weiland, Nucl. Instrum. Methods 212, 13 (1983)

4. P.L. Morton, V.K. Neil, The interaction of a ring of charge passing through a cylindrical rf cavity. vol. UCRL-18103 (LBNL, Berkeley, 1968), p. 365

5. E. Keil, W. Schnell, B. Zotter, Technical Report, CERN-ISR-LTD/76-22, CERN, CERN, Geneva (1976)

6. K.W. Robinson, Stability of beam in radiofrequency system. Technical Report, CEA-11, CEAL1010, Harvard University, Cambridge (1956/1966)

7. A.W. Chao, J. Gareyte, Technical Report, Int Note-197, SLAC, Stanford, CA (1976)

8. A. Hofmann, in Lecture Notes of Physics, vol. 296 (Springer, Berlin/Heidelberg, 1986), p. 99 

Part VII

Coupled Motion 


\section{Chapter 20 \\ Dynamics of Coupled Motion*}

In linear beam dynamics transverse motion of particles can be treated separately in the horizontal and vertical plane. This can be achieved by proper selection, design and alignment of beam transport magnets. Fabrication and alignment tolerances, however, will introduce, for example, rotated quadrupole components where only upright quadrupole fields were intended. In other cases like colliding beams for high energy physics large solenoid detectors are installed at the collision points to analyse secondary particles. Such solenoids cause coupling which must be compensated. The perturbation caused creates a coupling of both the horizontal and vertical oscillation and independent treatment is no longer accurate. Such linear coupling can be compensated in principle by additional rotated or skew quadrupoles, but the beam dynamics for coupling effects must be known to perform a proper compensation.

Since coupling is caused by linear as well as nonlinear fields, we observe this effect in virtually any accelerator. In order to be able to manipulate coupling in a controlled and predictable way, we need to understand its dynamics in more detail. In this chapter, we will derive first the equations of motion for the two most general sources of coupling, the solenoid field and the field of a rotated quadrupole, solve the equations of motion and formulate modifications to beam dynamics parameters and functions of linear uncoupled motion.

\subsection{Equations of Motion in Coupled Systems}

The most generally used magnets that introduce coupling in beam transport systems are rotated quadrupoles and solenoid magnets and we will restrict our discussion of coupled beam dynamics to such magnets defining the realm of linear coupling. Equations (6.95), (6.96) include all linear and nonlinear coupling terms up to third order while longitudinal fields are treated in Sect. 6.6. The equations of motion in

This chapter has been made Open Access under a CC BY 4.0 license. For details on rights and licenses please read the Correction https://doi.org/10.1007/978-3-319-18317-6_28 
the presence of upright and rotated quadrupoles as well as solenoid fields are

$$
\begin{gathered}
x^{\prime \prime}+k x=-\underline{k} y+S y^{\prime}+\frac{1}{2} S^{\prime} y, \\
y^{\prime \prime}-k y=-\underline{k} x-S x^{\prime}-\frac{1}{2} S^{\prime} x
\end{gathered}
$$

where the solenoid field is expressed by

$$
S(z)=\frac{e}{p} B_{\mathrm{s}}(z)
$$

We use in this chapter the symbol $S$ for the solenoid field not to be confused with the sine-like solution. In the following subsections we will derive separately the transformation through both rotated quadrupoles and solenoid magnets.

\subsubsection{Coupled Beam Dynamics in Skew Quadrupoles}

The distribution of rotated or skew quadrupoles and solenoid magnets is arbitrary and therefore no analytic solution can be expected for the differential equations (20.1). Similar to other beam line elements, we discuss solutions for the equations of motion within individual magnets only and assume that strength parameters within hard-edge model magnets stay constant. We discuss first solutions of particle motion in skew quadrupoles alone and ignore solenoid fields. The equations of motion for skew quadrupoles are from (20.1)

$$
\begin{aligned}
& x^{\prime \prime}+\underline{k} y=0, \\
& y^{\prime \prime}+\underline{k} x=0 .
\end{aligned}
$$

These equations look very similar to the equations for ordinary upright quadrupoles except that the restoring forces now depend on the particle amplitude in the other plane. We know the solution of the equation of motion for an upright focusing and defocusing quadrupole and will try to apply these solutions to (20.3). Combining the observation that each quadrupole is focusing in one plane and defocusing in the other with the apparent mixture of both planes for a skew quadrupole, we will try an ansatz for (20.3) which is made up of four principal solutions

$$
\begin{aligned}
& x=a \cos \varphi+\frac{b}{\sqrt{k}} \sin \varphi+c \cosh \varphi+\frac{d}{\sqrt{k}} \sinh \varphi, \\
& y=A \cos \varphi+\frac{B}{\sqrt{\underline{k}}} \sin \varphi+C \cosh \varphi+\frac{D}{\sqrt{\underline{k}}} \sinh \varphi,
\end{aligned}
$$

where $\varphi=\sqrt{k} z$ and the variable $z$ varies between zero and the full length of the quadrupole, $0<z<\ell_{q}$. The coefficients $a, b, c, \ldots D$ must be determined to be consistent with the initial parameters of the trajectories $\left(x_{0}, x_{0}^{\prime}, y_{0}, y_{0}^{\prime}\right)$. For $z=0$ we 
get

$$
\begin{array}{ll}
x_{0}=a+c, & y_{0}=A+C, \\
x_{0}^{\prime}=b+d, & y_{0}^{\prime}=B+D .
\end{array}
$$

Solutions (20.4) must be consistent with (20.3) from which we find

$$
\begin{array}{ll}
a=A, & c=-C, \\
b=B, & d=-D .
\end{array}
$$

From (20.5), (20.6) we get finally the coefficients consistent with the initial conditions and the differential equations (20.3)

$$
\begin{array}{ll}
a=A=\frac{1}{2}\left(x_{0}+y_{0}\right), & b=B=\frac{1}{2}\left(x_{0}^{\prime}+y_{0}^{\prime}\right), \\
c=-C=\frac{1}{2}\left(x_{0}-y_{0}\right), & d=-D=\frac{1}{2}\left(x_{0}^{\prime}-y_{0}^{\prime}\right) .
\end{array}
$$

With these definitions the transformation through a skew quadrupole is

$$
\left(\begin{array}{c}
x \\
x^{\prime} \\
y \\
y^{\prime}
\end{array}\right)=\mathcal{M}_{\mathrm{sq}}\left(\begin{array}{c}
x_{0} \\
x_{0}^{\prime} \\
y_{0} \\
y_{0}^{\prime}
\end{array}\right)
$$

where $\mathcal{M}_{\mathrm{sq}}$ is the transformation matrix for a skew quadrupole,

$$
\mathcal{M}_{\mathrm{sq}}(s \mid 0)=\frac{1}{2}\left(\begin{array}{cccc}
\mathcal{C}^{+} & \frac{1}{\sqrt{k}} \mathcal{S}^{+} & \mathcal{C}^{-} & \frac{1}{\sqrt{k}} \mathcal{S}^{-} \\
-\sqrt{\underline{k}} \mathcal{S}^{-} & \mathcal{C}^{+} & -\sqrt{\underline{k}} \mathcal{S}^{+} & \mathcal{C}^{-} \\
\mathcal{C}^{-} & \frac{1}{\sqrt{k}} \mathcal{S}^{-} & \mathcal{C}^{+}(\varphi) & \frac{1}{\sqrt{k}} \mathcal{S}^{+} \\
-\sqrt{\underline{k}} \mathcal{S}^{+} & \mathcal{C}^{-} & -\sqrt{\underline{k}} \mathcal{S}^{-} & \mathcal{C}^{+}
\end{array}\right)
$$

with $\mathcal{C}^{ \pm}=\mathcal{C}^{ \pm}(\varphi)=\cos \varphi \pm \cosh \varphi$ and $\mathcal{S}^{ \pm}=\mathcal{S}^{ \pm}(\varphi)=\sin \varphi \pm \sinh \varphi$ and $\varphi=\sqrt{\underline{k}} z$.

This transformation matrix is quite elaborate and becomes useful only for numerical calculations on computers. We employ again thin lens approximation where the quadrupole length vanishes $\left(\ell_{\mathrm{sq}} \rightarrow 0\right)$ in such a way as to preserve the integrated magnet strength or the focal length $f$. The matrix (20.9) then reduces to the simple form

$$
\mathcal{M}_{\mathrm{sq}}\left(0 \mid \ell_{\mathrm{sq}}\right)=\left(\begin{array}{cccc}
1 & \ell_{\mathrm{sq}} & 0 & 0 \\
0 & 1 & -1 / f & 0 \\
0 & 0 & 1 & \ell_{\mathrm{sq}} \\
-1 / f & 0 & 0 & 1
\end{array}\right)
$$


where the focal length is defined as $f^{-1}=\underline{k} \ell_{\mathrm{sq}}$. Note, that we have not set $\ell_{\mathrm{sq}}=0$ but retained the linear terms in $\ell_{\mathrm{sq}}$, which is a more appropriate thin-lens approximation for weak skew quadrupoles of finite length. Along the diagonal, the transformation matrix looks like a drift space of length $\ell_{\mathrm{sq}}$ while the off-diagonal elements describe the coupling due to the thin skew quadrupole.

\subsubsection{Particle Motion in a Solenoidal Field}

The equations of motion in a solenoid can be derived from (6.92a), neglecting all transverse beam deflection and electric fields

$$
\begin{aligned}
& x^{\prime \prime}-\frac{1}{2} \frac{x^{\prime}}{z^{\prime 2}} \frac{\mathrm{d} z^{\prime 2}}{\mathrm{~d} z}=\frac{e}{p} z^{\prime}\left(y^{\prime} B_{\mathrm{s}}-B_{y}\right), \\
& y^{\prime \prime}-\frac{1}{2} \frac{y^{\prime}}{z^{\prime 2}} \frac{\mathrm{d} z^{\prime 2}}{\mathrm{~d} z}=\frac{e}{p} z^{\prime}\left(B_{x}-x^{\prime} B_{\mathrm{s}}\right),
\end{aligned}
$$

where the solenoid field component $B_{\mathrm{s}}$, assumed to be colinear with the $z$-direction, can be derived from (6.103)

$$
\boldsymbol{B}=\left(-\frac{1}{2} B_{\mathrm{s}}^{\prime} x,-\frac{1}{2} B_{\mathrm{s}}^{\prime} y, B_{\mathrm{s}}\right)
$$

Following the same derivation as in Sect. 6.5, the general equations of motion in a solenoid field including up to third-order terms are

$$
\begin{aligned}
x^{\prime \prime}= & +\frac{e}{p} B_{\mathrm{s}} y^{\prime}+\frac{1}{2} \frac{e}{p} B_{\mathrm{s}}^{\prime} y \\
& +\frac{1}{4} \frac{e}{p}\left(2 x^{\prime 2} y^{\prime} B_{\mathrm{s}}+x^{\prime 2} y B_{\mathrm{s}}^{\prime}+2 y^{\prime 3} B_{\mathrm{s}}+y y^{\prime 2} B_{\mathrm{s}}^{\prime}\right)+\mathcal{O}(4), \\
y^{\prime \prime}=- & \frac{e}{p} B_{\mathrm{s}} x^{\prime}-\frac{1}{2} \frac{e}{p} B_{\mathrm{s}}^{\prime} x \\
& -\frac{1}{4} \frac{e}{p}\left(2 x^{\prime} y^{\prime 2} B_{\mathrm{s}}+x y^{\prime 2} B_{\mathrm{s}}^{\prime}+2 x^{\prime 3} B_{\mathrm{s}}+x x^{\prime 2} B_{\mathrm{s}}^{\prime}\right)+\mathcal{O}(4) .
\end{aligned}
$$

Considering only linear terms, the equations of motion in a solenoidal field simplify to

$$
\begin{aligned}
& x^{\prime \prime}=+\frac{e}{p} B_{\mathrm{s}} y^{\prime}+\frac{1}{2} \frac{e}{p} B_{\mathrm{s}}^{\prime} y, \\
& y^{\prime \prime}=-\frac{e}{p} B_{\mathrm{s}} x^{\prime}-\frac{1}{2} \frac{e}{p} B_{\mathrm{s}}^{\prime} x,
\end{aligned}
$$

exhibiting clearly coupling terms. In a uniform field, where $B_{\mathrm{s}}^{\prime}=0$, the particle trajectory assumes the form of a helix parallel to the axis of the solenoid field.

The equations of motion (20.15) have been derived under the assumption of paraxial rays so that we can set $v \approx v_{\mathrm{z}}$. In a solenoid field this approximation is not generally acceptable since we may, for example, be interested in using a solenoid 
to focus particles emerging from a target at large angles. We therefore replace all derivatives with respect to $z$ by derivatives with respect to the time, use the particle velocity $v$, and replace $\frac{\mathrm{d}}{\mathrm{d} z} \rightarrow \frac{1}{v} \frac{\mathrm{d}}{\mathrm{d} t}$. In a uniform solenoid field the equations of motion are then

$$
\begin{aligned}
& \ddot{x}=+\left(\frac{e}{p} B_{\mathrm{s}} v\right) \dot{y}=\omega_{\mathrm{L}} \dot{y}, \\
& \ddot{y}=-\left(\frac{e}{p} B_{\mathrm{s}} v\right) \dot{x}=-\omega_{\mathrm{L}} \dot{x},
\end{aligned}
$$

where the Larmor frequency $\omega_{\mathrm{L}}$ is defined by

$$
\omega_{\mathrm{L}}=\frac{e B_{\mathrm{s}} v}{p}=\frac{e c^{2}}{E} B_{\mathrm{s}},
$$

and $E$ is the total particle energy. Multiplying (20.16) by $\dot{x}$ and $\dot{y}$, respectively, and adding both equations we get $\mathrm{d}\left(\dot{x}^{2}+\dot{y}^{2}\right) / \mathrm{d} t=0$ or

$$
\dot{x}^{2}+\dot{y}^{2}=v_{t}^{2}=\text { const } .
$$

The transverse particle velocity $v_{\mathrm{t}}$ or total transverse momentum of the particle $c p_{\mathrm{t}}$ stays constant during the motion in a uniform solenoid field. For $\dot{x}_{0}=0$ and $\dot{y}_{0}=v_{\mathrm{t}}$, for example, the transverse velocities can be expressed by

$$
\begin{aligned}
& \dot{x}=v_{\mathrm{t}} \sin \omega_{\mathrm{L}} t, \\
& \dot{y}=v_{\mathrm{t}} \cos \omega_{\mathrm{L}} t
\end{aligned}
$$

and the solutions of the equations of motion are

$$
\begin{aligned}
& x(t)=x_{0}-\frac{v_{\mathrm{t}}}{\omega_{\mathrm{L}}} \cos \omega_{\mathrm{L}} t, \\
& y(t)=y_{0}+\frac{v_{\mathrm{t}}}{\omega_{\mathrm{L}}} \sin \omega_{\mathrm{L}} t .
\end{aligned}
$$

The amplitude of the oscillating term in (20.20) is equal to the radius of the helical path

$$
\rho_{\mathrm{h}}=\frac{p_{\mathrm{t}}}{e B_{\mathrm{s}}}
$$

where we have used the Larmor frequency (20.17) and set the transverse momentum $p_{\mathrm{t}}=\gamma m v_{\mathrm{t}}$. The longitudinal motion is unaffected for not too strong solenoid fields and $\dot{v}_{\mathrm{z}}=0$ as can be derived from the Lorentz equation since all transverse field components vanish and

$$
z(t)=z_{0}+v_{z} t
$$


The time to complete one period of the helix is

$$
T=\frac{2 \pi}{\omega_{\mathrm{L}}}
$$

during which time the particle moves along the $z$-axis a distance

$$
\Delta z=2 \pi \frac{p_{z}}{e B_{\mathrm{s}}}
$$

where $p_{z}$ is the $z$-component of the particle momentum.

The solutions of the equations of motion for a solenoid magnet are more complex since we must now include terms that depend on the slope of the particle trajectories as well. Ignoring skew quadrupoles the differential equations of motion in a solenoid magnet becomes from (20.15)

$$
\begin{aligned}
& x^{\prime \prime}-S(z) y^{\prime}-\frac{1}{2} S^{\prime}(z) y=0 \\
& y^{\prime \prime}+S(z) x^{\prime}+\frac{1}{2} S^{\prime}(z) x=0 .
\end{aligned}
$$

Coupling between both planes is obvious and the variation of coordinates in one plane depends entirely on the coordinates in the other plane. We note a high degree of symmetry in the equations in the sense that both coordinates change similar as a function of the other coordinates. This suggests that a rotation of the coordinate system may help simplify the solution of the differential equations. We will therefore try such a coordinate rotation in complex notation by defining

$$
R=(x+\mathrm{i} y) \mathrm{e}^{-\mathrm{i} \phi(z)}
$$

where the rotation angle $\phi$ may be a function of the independent variable $z$. A single differential equation can be formed from (20.25) in complex notation

$$
(x+\mathrm{i} y)^{\prime \prime}+\mathrm{i} S(z)(x+\mathrm{i} y)^{\prime}+\mathrm{i} \frac{1}{2} S^{\prime}(z)(x+\mathrm{i} y)=0 .
$$

The rotation (20.26) can now be applied directly to (20.27) and with

$$
(x+\mathrm{i} y)^{\prime}=R^{\prime} \mathrm{e}^{\mathrm{i} \phi}+\mathrm{i} \phi^{\prime} R \mathrm{e}^{+\mathrm{i} \phi}
$$

and

$$
(x+\mathrm{i} y)^{\prime \prime}=R^{\prime \prime} \mathrm{e}^{\mathrm{i} \phi}+2 \mathrm{i} \phi^{\prime} R^{\prime} \mathrm{e}^{\mathrm{i} \phi}+\mathrm{i} \phi^{\prime \prime} R \mathrm{e}^{\mathrm{i} \phi}-\phi^{\prime 2} R \mathrm{e}^{\mathrm{i} \phi} .
$$

After insertion into (20.26) and sorting of terms

$$
R^{\prime \prime}-\left[S(z) \phi^{\prime}+\phi^{\prime 2}\right] R+\mathrm{i} 2\left[\phi^{\prime}+\frac{1}{2} S(z)\right] R^{\prime}+\mathrm{i}\left[\phi^{\prime \prime}+\frac{1}{2} S^{\prime}(z)\right] R=0
$$


At this point, the introduction of the coordinate rotation allows a great simplification (20.28) by assuming a continuous rotation along the beam axis with a rotation angle defined by

$$
\phi(z)=-\frac{1}{2} \int_{z_{0}}^{z} S(\zeta) \mathrm{d} \zeta
$$

where the solenoid field starts at $z_{0}$. We are able to eliminate two terms in the differential equation (20.28). Since a positive solenoid field generates Lorentz forces that deflect the particles onto counter clockwise spiraling trajectories, we have included the negative sign in (20.30) to remain consistent with our sign convention. From (20.30) it follows that $\phi^{\prime}=-\frac{1}{2} S(z)$ and $\phi^{\prime \prime}=-\frac{1}{2} S^{\prime}(z)$, which after insertion into (20.28) results in the simple equation of motion

$$
R^{\prime \prime}+\frac{1}{4} S^{2}(z) R=0 .
$$

With $R=v+\mathrm{i} w$, we finally get two uncoupled equations

$$
\begin{aligned}
v^{\prime \prime}+\frac{1}{4} S^{2}(z) v & =0, \\
w^{\prime \prime}+\frac{1}{4} S^{2}(z) w & =0 .
\end{aligned}
$$

Introducing a coordinate rotation allow us to reduce the coupled differential equations (20.25) to the form of uncoupled equations of motion exhibiting focusing in both planes. At the entrance to the solenoid field $\phi=0$ and therefore $v_{0}=x_{0}$ and $w_{0}=y_{0}$. To determine the particle motion through the solenoid field of length $L_{\mathrm{s}}$ we simply follow the particle coordinates $(v, w)$ through the solenoid as if it were a quadrupole of strength $k_{\mathrm{s}}=\frac{1}{4} S^{2}\left(L_{\mathrm{s}}\right)$ followed by a rotation of the coordinate system by the angle $-\phi\left(L_{\mathrm{s}}\right)$ thus reverting to Cartesian coordinates $(x, y)$.

\subsubsection{Transformation Matrix for a Solenoid Magnet}

Similar to the transformation through quadrupoles and other beam transport magnets, we may formulate a transformation matrix for a solenoid magnet. Instead of uncoupled $2 \times 2$ transformation matrices, however, we must use $4 \times 4$ matrices to include coupling effects. Each coordinate now depends on the initial values of all coordinates, $x(z)=\left(x_{0}, x_{0}^{\prime}, y_{0}, y_{0}^{\prime}\right)$, etc. The transformation through a solenoid is performed in two steps in which the first is the solution of (20.32) in the form of the matrix $\mathcal{M}_{\mathrm{s}}$, and the second is a coordinate rotation introduced through the matrix 
$\mathcal{M}_{\mathrm{r}}$. The total transformation is therefore

$$
\left(\begin{array}{c}
x \\
x^{\prime} \\
y \\
y^{\prime}
\end{array}\right)=\mathcal{M}_{\mathrm{r}} \mathcal{M}_{\mathrm{s}}\left(\begin{array}{c}
x_{0} \\
x_{0}^{\prime} \\
y_{0} \\
y_{0}^{\prime}
\end{array}\right)
$$

In analogy to the transformation through an upright quadrupole, we get from (20.32) the transformation matrix $\mathcal{M}_{\mathrm{s}}$ from the beginning of the solenoid field at $z_{0}$ to a point $z$ inside the solenoid magnet. The strength parameter in this case is $\frac{1}{4} S^{2}$ assumed to be constant along the length of the magnet and the transformation matrix is

$$
\mathcal{M}_{\mathrm{s}}\left(z_{0} \mid z\right)=\left(\begin{array}{cccc}
\cos \phi & \frac{2}{S} \sin \phi & 0 & 0 \\
-\frac{S}{2} \sin \phi & \cos \phi & 0 & 0 \\
0 & 0 & \cos \phi & \frac{2}{S} \sin \phi \\
0 & 0 & -\frac{S}{2} \sin \phi & \cos \phi
\end{array}\right)
$$

where $\phi=\frac{1}{2} S z$. The next step is to introduce the coordinate rotation $\mathcal{M}_{\mathrm{r}}$ which we derive from the vector equation

$$
(\boldsymbol{x}+\mathrm{i} \boldsymbol{y})=(\boldsymbol{v}+\mathrm{i} \boldsymbol{w}) \mathrm{e}^{-\mathrm{i} \phi(z)},
$$

where the vectors are defined like $\boldsymbol{x}=\left(x, x^{\prime}\right)$, etc. Note that the value of the rotation angle $\phi$ is proportional to the strength parameter and the sign of the solenoid field defines the orientation of the coordinate rotation. Fortunately, we need not keep track of the sign since the components of the focusing matrix $\mathcal{M}_{\mathrm{s}}$ are even functions of $z$ and do not depend on the direction of the solenoid field.

By separating (20.35) into its real and imaginary part and applying Euler's identity $\mathrm{e}^{\alpha}=\cos \alpha+\mathrm{i} \sin \alpha$, we get for the rotation matrix at the point $z$ within the solenoid magnet

$$
\mathcal{M}_{\mathrm{r}}=\left(\begin{array}{cccc}
\cos \phi & 0 & \sin \phi & 0 \\
-\frac{S}{2} \sin \phi & \cos \phi & \frac{S}{2} \cos \phi & \sin \phi \\
-\sin \phi & 0 & \cos \phi & 0 \\
\frac{S}{2} \cos \phi & -\sin \phi & -\frac{S}{2} \sin \phi & \cos \phi
\end{array}\right)
$$

The total transformation matrix for a solenoid magnet from $z_{0}=0$ to $z$ finally is the product of (20.34) and (20.36)

$$
\mathcal{M}_{\mathrm{sol}}(0 \mid z<L)=\left(\begin{array}{cccc}
\cos ^{2} \phi & \frac{1}{S} \sin 2 \phi & \frac{1}{2} \sin 2 \phi & \frac{2}{S} \sin ^{2} \phi \\
-\frac{S}{2} \sin 2 \phi & \cos 2 \phi & \frac{S}{2} \cos 2 \phi & \sin 2 \phi \\
-\frac{1}{2} \sin 2 \phi & -\frac{2}{S} \sin ^{2} \phi & \cos ^{2} \phi & \frac{1}{S} \sin 2 \phi \\
-\frac{S}{2} \cos 2 \phi & -\sin 2 \phi & -\frac{S}{2} \sin 2 \phi & \cos 2 \phi
\end{array}\right) .
$$


This transformation matrix is correct inside the solenoid magnet but caution must be taken applying this transformation matrix for the whole solenoid by setting $z=$ $L_{\mathrm{s}}$. The result would be inaccurate because of a discontinuity caused by the solenoid fringe field. Only the focusing matrix $\mathcal{M}_{\mathrm{s}}$ for the whole solenoid becomes a simple extension of (20.34) to the end of the solenoid by setting $\phi\left(L_{\mathrm{s}}\right)=\Phi=\frac{1}{2} S L_{\mathrm{s}}$.

Due to the solenoid fringe field, which in hard-edge approximation adopted here is a thin slice, the rotation matrix exhibits a discontinuity. For $z=L_{\mathrm{s}}+\epsilon$, where $\epsilon \rightarrow 0$ the phase is $\phi\left(L_{\mathrm{s}}\right)=\Phi$ but the solenoid strength is now zero, $S=0$. Therefore, the rotation matrix (20.36) assumes the form

$$
\mathcal{M}_{\mathrm{r}}=\left(\begin{array}{cccc}
\cos \Phi & 0 & \sin \Phi & 0 \\
0 & \cos \Phi & 0 & \sin \Phi \\
-\sin \Phi & 0 & \cos \Phi & 0 \\
0 & -\sin \Phi & 0 & \cos \Phi
\end{array}\right)
$$

Notice that this matrix at the solenoid entrance is just the unit matrix. This does not mean that we ignored the entrance fringe field, it only indicates that this effect is already included in (20.37). After multiplication of (20.34) with (20.38), the transformation matrix for a complete solenoid magnet is finally

$$
\mathcal{M}_{\mathrm{sol}}(0 \mid L)=\left(\begin{array}{cccc}
\cos ^{2} \Phi & \frac{1}{S} \sin 2 \Phi & \frac{1}{2} \sin 2 \Phi & \frac{2}{S} \sin ^{2} \Phi \\
-\frac{S}{4} \sin 2 \Phi & \cos ^{2} \Phi & -\frac{S}{2} \sin ^{2} \Phi & \frac{1}{2} \sin 2 \Phi \\
-\frac{1}{2} \sin 2 \Phi & -\frac{2}{S} \sin ^{2} \Phi & \cos ^{2} \Phi & \frac{1}{S} \sin 2 \Phi \\
\frac{S}{2} \sin ^{2} \Phi & -\frac{1}{2} \sin 2 \Phi & -\frac{S}{4} \sin 2 \Phi & \cos ^{2} \Phi
\end{array}\right) .
$$

Comparing matrices (20.37), (20.39), we find no continuous transition between both matrices since only one matrix includes the effect of the fringe field. In reality, the fringe field is not a thin-lens and therefore a continuous transition between both matrices could be derived. To stay consistent with the rest of this book, however, we assume for our discussions hard-edge magnet models.

From the matrix (20.34) some special properties of particle trajectories in a solenoid can be derived. For $\Phi=\frac{1}{2} \pi$ a parallel beam becomes focused to a point at the magnet axis. A trajectory entering a solenoid with the strength $\Phi=\frac{1}{2} S L=\pi / 2$ at say $y_{0}$ will follow one quarter of a spiraling trajectory with a radius $\rho=y_{0} / 2$ and exit the solenoid at $x=y=0$. Similarly, a beam emerging from a point source on axis and at the start of the solenoid field will have been focused to a parallel beam at the end of the solenoid. Such a solenoid is used to focus, for example, a divergent positron beam emerging from the target source and is called a $\lambda / 4$-lens or quarter-wavelength solenoid for obvious reasons.

The focusing properties of the whole solenoid are most notable when the field strength is weak and the focal length is long compared to the length of the solenoid. In this case, the focal length can be taken immediately from the $M_{21}$ and $M_{43}$ element of the transformation matrix as we did for quadrupoles and other focusing devices 
and is with $\phi=\frac{1}{2} S L_{\mathrm{s}}$

$$
\begin{aligned}
& \frac{1}{f_{x}}=M_{21}=-\frac{1}{2} S \sin \phi \cos \phi, \\
& \frac{1}{f_{y}}=M_{43}=-\frac{1}{2} S \sin \phi \cos \phi .
\end{aligned}
$$

In contrast to quadrupole magnets, the focal length of a solenoid magnet is the same in both planes and is in thin-lens approximation, $\phi=\frac{1}{2} S L_{\mathrm{s}} \rightarrow 0$ while $S^{2} L_{\mathrm{s}}=$ const.

$$
\frac{1}{f_{\mathrm{sol}}}=\frac{1}{4} S^{2} L_{\mathrm{s}}=\frac{1}{4}\left(\frac{e}{p}\right)^{2} B_{\mathrm{s}}^{2} L_{\mathrm{s}}
$$

The thin lens transformation matrix for a weak solenoid is thereby

$$
\mathcal{M}_{\mathrm{sol}}(0 \mid L)=\left(\begin{array}{cccc}
1 & 0 & 0 & 0 \\
-\frac{1}{f_{\mathrm{sol}}} & 1 & 0 & 0 \\
0 & 0 & 1 & 0 \\
0 & 0 & -\frac{1}{f_{\mathrm{sol}}} & 1
\end{array}\right) \text {. }
$$

The focal length is always positive and a solenoid will therefore always be focusing independent of the sign of the field or the sign of the particle charge.

Transformation matrices have been derived for the two most important coupling magnets in beam transport systems, the skew quadrupole and the solenoid magnet, which allows us now to employ linear beam dynamics in full generality including linear coupling. Using $(4 \times 4)$-transformation matrices any particle trajectory can be described whether coupling magnets are included or not. Specifically, we may use this formalism to incorporate compensating schemes when strongly coupling magnets must be included in a particular beam transport line.

\subsection{Betatron Functions for Coupled Motion}

For the linear uncoupled motion of particles in electromagnetic fields we have derived powerful mathematical methods to describe the dynamics of single particles as well as that of a beam composed of a large number of particles. Specifically, the concept of phase space to describe a beam at a particular location and the ability to transform this phase space from one point of the beam transport line to another allow us to design beam transport systems with predictable results. These theories derived for particle motion in one degree of freedom can be expanded to describe coupled motion in both the horizontal and vertical plane. 


\subsection{Conjugate Trajectories}

Lattice functions have been defined to express solutions to the equations of motion for individual trajectories. Conversely, there must be a way to express these lattice functions by the principal solutions of the equation of motion. This would enable us to determine lattice functions for coupled particle motion by integrating the equations of motion for two orthogonal trajectories. To do this, we start from the differential equation of motion in normalized coordinates for which two linearly independent principal solutions are given by

$$
\begin{aligned}
& w_{1}(\varphi)=\cos (\nu \varphi) \\
& w_{2}(\varphi)=\sin (\nu \varphi) .
\end{aligned}
$$

For simplicity, we set the initial amplitudes equal to unity and get in regular coordinates with $u(z)=w \sqrt{\beta(z)}$ the conjugate trajectories are

$$
\begin{aligned}
& u_{1}(z)=\sqrt{\beta(z)} \cos \psi(z), \\
& u_{2}(z)=\sqrt{\beta(z)} \sin \psi(z),
\end{aligned}
$$

where $u(z)$ stands for $x(z)$ or $y(z)$, and their derivatives

$$
\begin{aligned}
& u_{1}^{\prime}(z)=-\frac{\alpha(z)}{\sqrt{\beta(z)}} \cos \psi(z)-\frac{1}{\sqrt{\beta(z)}} \sin \psi(z), \\
& u_{2}^{\prime}(z)=-\frac{\alpha(z)}{\sqrt{\beta(z)}} \sin \psi(z)+\frac{1}{\sqrt{\beta(z)}} \cos \psi(z) .
\end{aligned}
$$

Using (20.45), (20.46) all lattice functions can be expressed in terms of conjugate trajectories like

$$
\begin{aligned}
& \beta(z)=u_{1}^{2}(z)+u_{2}^{2}(z), \\
& \alpha(z)=-u_{1}(z) u_{1}^{\prime}(z)-u_{2}(z) u_{2}^{\prime}(z), \\
& \gamma(z)=u_{1}^{\prime 2}(z)+u_{2}^{\prime 2}(z) .
\end{aligned}
$$

The betatron phase advance $\Delta \psi=\psi-\psi_{0}$ between the point $z=0$ and the point $z$ can be derived from

$$
\cos \left(\psi-\psi_{0}\right)=\cos \psi \cos \psi_{0}+\sin \psi \sin \psi_{0},
$$

where $\psi_{0}=\psi(0)$ and $\psi=\psi(z)$. With (20.45), (20.47) we get

$$
\cos \psi(z)=\frac{u_{1}(z)}{\sqrt{\beta(z)}}=\frac{u_{1}(z)}{\sqrt{u_{1}^{2}(z)+u_{2}^{2}(z)}}
$$


and similarly,

$$
\sin \psi(z)=\frac{u_{2}(z)}{\sqrt{\beta(z)}}=\frac{u_{2}(z)}{\sqrt{u_{1}^{2}(z)+u_{2}^{2}(z)}} .
$$

The betatron phase advance then is given by

$$
\cos \left(\psi-\psi_{0}\right)=\frac{u_{1} u_{10}+u_{2} u_{20}}{\sqrt{u_{1}^{2}+u_{2}^{2}} \sqrt{u_{10}^{2}+u_{20}^{2}}}
$$

where $u_{i}=u_{i}(z)$ and $u_{i 0}=u_{i}(0)$. Finally, we can express the elements of the transformation matrix from $z=0$ to $z$ by

$$
\mathcal{M}(z \mid 0)=\left(\begin{array}{ll}
M_{11} M_{12} \\
M_{21} M_{22}
\end{array}\right)=\left(\begin{array}{c}
u_{1} u_{20}^{\prime}-u_{2} u_{10}^{\prime} u_{10} u_{2}-u_{1} u_{20} \\
u_{1}^{\prime} u_{10}^{\prime}-u_{2}^{\prime} u_{20}^{\prime} u_{10} u_{2}^{\prime}-u_{20} u_{1}^{\prime}
\end{array}\right) .
$$

The two linearly independent solutions (20.45) also can be used to define and characterize the phase space ellipse. At the start of a beam line we set $z=0$ and $\psi(0)=0$ and define an ellipse by the parametric vector equation

$$
\mathbf{u}(0)=a\left[\mathbf{u}_{1}(0) \cos \phi-\mathbf{u}_{2}(0) \sin \phi\right],
$$

where

$$
\mathbf{u}(0)=\left(\begin{array}{l}
u_{0} \\
u_{0}^{\prime}
\end{array}\right) \quad \text { and } \quad \mathbf{u}_{i}(0)=\left(\begin{array}{c}
u_{i 0} \\
u_{i 0}^{\prime}
\end{array}\right)
$$

As $\phi$ varies over a period of $2 \pi$, the vector follows the outline of an ellipse. To parametrize this ellipse we calculate the area enclosed by the phase ellipse. The area element is $\mathrm{d} A=u^{\prime} \mathrm{d} u_{0}$, from (20.52) we get

$$
\mathrm{d} u_{0}=a\left[u_{10} \sin \phi-u_{20} \cos \phi\right] \mathrm{d} \phi
$$

and the area enclosed by the ellipse is

$$
\begin{aligned}
A & =2 a^{2} \int_{0}^{\pi}\left(u_{10}^{\prime} \cos \phi-u_{20}^{\prime} \sin \phi\right)\left(u_{10} \sin \phi-u_{20} \cos \phi\right) \mathrm{d} \phi \\
& =a^{2} \pi\left(u_{10} u_{20}^{\prime}-u_{10}^{\prime} u_{20}\right)=a^{2} \pi,
\end{aligned}
$$

since the expression in the brackets is the Wronskian, which we choose to normalize to unity. The Wronskian is an invariant of the motion and therefore the area of the phase ellipse along the beam transport line is preserved. The vector equation (20.52) describes the phase ellipse enclosing a beam with the emittance $a^{2}=\epsilon$. 
The formalism of conjugate trajectories has not produced any new insight into beam dynamics that we did not know before but it is an important tool for the discussion of coupled particle motion and provides a simple way to trace individual particles through complicated systems.

Ripken [1] developed a complete theory of coupled betatron oscillations and of particle motion in four-dimensional phase space. In our discussion of coupled betatron motion and phase space transformation we will closely follow his theory. The basic idea hinges on the fact that the differential equations of motion provide the required number of independent solutions, two for oscillations in one plane and four for coupled motion in two planes, to define a two- or four-dimensional ellipsoid which serves as the boundary in phase space for the beam enclosed by it. Since the transformations in beam dynamics are symplectic, we can rely on invariants of the motion which are the basis for the determination of beam characteristics at any point along the beam transport line if we only know such parameters at one particular point.

Before we discuss coupled motion in more detail it might be useful to recollect some salient features of linear beam dynamics. The concept of conjugate trajectories can be used to define a phase ellipse at $z=0$ in parametric form. Due to the symplecticity of the transformations we find the area of the phase ellipse to be a constant of motion and we may describe the phase ellipse at any point $z$ along the beam line is given by (20.52). The Wronskian is a constant of motion normalized to unity in which case the phase ellipse (20.52) has the area $A=\pi \epsilon$, where $\epsilon$ is the beam emittance for the beam enclosed by the ellipse. The solutions are of the form (20.45) and forming the Wronskian we find the normalization

$$
\beta \phi^{\prime}=1
$$

as we would expect.

To describe coupled motion we try analogous to (20.52) the ansatz

$$
\begin{aligned}
\mathbf{v}(z)= & \sqrt{\epsilon_{\mathrm{I}}}\left[\mathbf{v}_{1}(z) \cos \vartheta_{\mathrm{I}}-\mathbf{v}_{2}(z) \sin \vartheta_{\mathrm{I}}\right] \cos \chi \\
& +\sqrt{\epsilon_{\mathrm{II}}}\left[\mathbf{v}_{3}(z) \cos \vartheta_{\mathrm{II}}-\mathbf{v}_{4}(z) \sin \vartheta_{\mathrm{II}}\right] \sin \chi
\end{aligned}
$$

As the independent variables $\chi, \vartheta_{\text {I }}$ and $\vartheta_{\text {II }}$ vary from 0 to $2 \pi$ the vector $\mathbf{v}$ covers all points on the surface of a four-dimensional ellipsoid while the shape of the ellipse varies along the beam line consistent with the variation of the vector functions $\mathbf{v}_{i}$. In this ansatz we chose two modes of oscillations indicated by the index I and II. If the oscillations were uncoupled, we would identify mode-I with the horizontal oscillation and mode-II with the vertical motion and (20.57) would still hold with $\chi=0$ having only horizontal nonvanishing components while $\mathbf{v}_{3,4}$ contain nonzero components only in the vertical plane for $\chi=\pi / 2$. For independent solutions $\mathbf{v}_{i}$ of 
coupled motion, we try

$$
\begin{array}{ll}
x_{1}(z)=\sqrt{\beta_{x_{\mathrm{I}}}(z)} \cos \phi_{x_{\mathrm{I}}}(z), & y_{1}(z)=\sqrt{\beta_{y_{\mathrm{I}}}(z)} \cos \phi_{y_{\mathrm{I}}}(z), \\
x_{2}(z)=\sqrt{\beta_{x_{\mathrm{I}}}(z)} \sin \phi_{x_{\mathrm{I}}}(z), & y_{2}(z)=\sqrt{\beta_{y_{\mathrm{I}}}(z)} \sin \phi_{y_{\mathrm{I}}}(z), \\
x_{3}(z)=\sqrt{\beta_{x_{\mathrm{II}}}(z)} \cos \phi_{x_{\mathrm{II}}}(z), & y_{3}(z)=\sqrt{\beta_{y_{\mathrm{II}}}(z)} \cos \phi_{y_{\mathrm{II}}}(z), \\
x_{4}(z)=\sqrt{\beta_{x_{\mathrm{II}}}(z)} \sin \phi_{x_{\mathrm{II}}}(z), & y_{4}(z)=\sqrt{\beta_{y_{\mathrm{II}}}(z)} \sin \phi_{y_{\mathrm{II}}}(z),
\end{array}
$$

which is consistent with the earlier definitions of conjugate trajectories. Earlier in this section we defined conjugate trajectories to be independent solutions normalized to the same phase ellipse and developed relationships between these trajectories and betatron functions. These relationships can be expanded to coupled motion by defining betatron functions for both modes of oscillations similar to (20.47)

$$
\begin{array}{ll}
\beta_{x_{\mathrm{I}}}=x_{1}^{2}+x_{2}^{2}, & \beta_{x_{\mathrm{II}}}=x_{3}^{2}+x_{4}^{2}, \\
\beta_{y_{\mathrm{I}}}=y_{1}^{2}+y_{2}^{2}, & \beta_{y_{\mathrm{II}}}=y_{3}^{2}+y_{4}^{2} .
\end{array}
$$

The phase functions can be defined like (20.48) by

$$
\begin{array}{ll}
\cos \phi_{x_{\mathrm{I}}}=\frac{x_{1}}{\sqrt{x_{1}^{2}+x_{2}^{2}}}, & \cos \phi_{x_{\mathrm{II}}}=\frac{x_{3}}{\sqrt{x_{3}^{2}+x_{4}^{2}}}, \\
\cos \phi_{y_{\mathrm{I}}}=\frac{y_{1}}{\sqrt{y_{1}^{2}+y_{2}^{2}}}, & \cos \phi_{y_{\mathrm{II}}}=\frac{y_{3}}{\sqrt{y_{3}^{2}+y_{4}^{2}}} .
\end{array}
$$

All other lattice functions can be defined in a similar way. By following the conjugate trajectories and utilizing the $(4 \times 4)$-transformation matrices including coupling effects we are able to determine the betatron functions at any point along the coupled beam transport line. To correlate parameters of the four-dimensional phase ellipse with quantities that can be measured, we write the solutions in the form

$$
\begin{array}{ll}
x_{1}(z)=\sqrt{\beta_{x_{\mathrm{I}}}(z)} \cos \phi_{x_{\mathrm{I}}}(z), & x_{2}(z)=\sqrt{\beta_{x_{\mathrm{I}}}(z)} \sin \phi_{x_{\mathrm{I}}}(z), \\
x_{1}^{\prime}(z)=\sqrt{\gamma_{x_{\mathrm{I}}}(z)} \cos \psi_{x_{\mathrm{I}}}(z), & x_{2}^{\prime}(z)=\sqrt{\gamma_{x_{\mathrm{I}}}(z)} \sin \psi_{x_{\mathrm{I}}}(z),
\end{array}
$$

and similar for all other solutions. Comparing the second equations in (20.63) with the derivative of the first equations we find the definitions

$$
\gamma_{x_{\mathrm{I}}}=\frac{\beta_{x_{\mathrm{I}}}^{2} \phi_{x_{\mathrm{I}}}^{\prime 2}+\alpha_{x_{\mathrm{I}}}^{2}}{\beta_{x_{\mathrm{I}}}}
$$

and

$$
\psi_{x_{\mathrm{I}}}=\phi_{x_{\mathrm{I}}}-\arctan \frac{\beta_{x_{\mathrm{I}}} \phi_{x_{\mathrm{I}}}^{\prime}}{\alpha_{x_{\mathrm{I}}}} .
$$


The other parameters $\gamma_{x_{I}}$, etc. are defined similarly and the phase ellipse (20.57) can now be expressed by the four-dimensional vector

$$
\begin{gathered}
\mathbf{v}(z)=\sqrt{\epsilon_{I}}\left(\begin{array}{c}
\sqrt{\beta_{x I}} \cos \left(\phi_{x I}+\vartheta_{I}\right) \\
\sqrt{\gamma_{x I}} \cos \left(\psi_{x I}+\vartheta_{I}\right) \\
\sqrt{\beta_{y I}} \cos \left(\phi_{y I}+\vartheta_{I}\right) \\
\sqrt{\gamma_{y I}} \cos \left(\psi_{y I}+\vartheta_{I}\right)
\end{array}\right) \cos \chi \\
+\sqrt{\epsilon_{I I}}\left(\begin{array}{c}
\sqrt{\beta_{x I I}} \cos \left(\phi_{x I I}+\vartheta_{I I}\right) \\
\sqrt{\gamma_{x I I}} \cos \left(\psi_{x I I}+\vartheta_{I I}\right) \\
\sqrt{\beta_{y I I}} \cos \left(\phi_{y I I}+\vartheta_{I I}\right) \\
\sqrt{\gamma_{y I I}} \cos \left(\psi_{y I I}+\vartheta_{I I}\right)
\end{array}\right) \sin \chi
\end{gathered}
$$

This vector covers all points on the surface of the four-dimensional ellipsoid as $\chi, \vartheta_{\text {I }}$ and $\vartheta_{\text {II }}$ vary independently from 0 to $2 \pi$. For one-dimensional oscillations we know from the definition of the phase ellipse that the product $\sqrt{\epsilon_{u}} \sqrt{\beta_{u}}$ is equal to the beam size or beam envelope $E_{u}$ and $\sqrt{\epsilon_{u}} \sqrt{\gamma_{u}}$ equal to the angular beam envelope $A_{u}$, where $u=x$ or $y$. These definitions of beam envelopes can be generalized to coupled motion but we find from (20.66) that the envelopes have two contributions. Each point on the phase ellipse for an uncoupled beam appears now expanded into an ellipse with an area $\pi \epsilon_{\mathrm{II}}$ as shown in Fig. 20.1.

In a real beam transport line we are not able to observe experimentally the fourdimensional phase ellipse. By methods of emittance measurements, however, we may determine the area for the projection of the four-dimensional ellipsoid onto the $\left(x-x^{\prime}\right)$, the $\left(y-y^{\prime}\right)$ or the $(x-y)$-plane.

To do that we note in (20.66) that the maximum amplitude of a particle in the $u$ plane occurs for $\phi_{u_{\mathrm{I}, \mathrm{II}}}=-\vartheta_{u_{\mathrm{I}, \mathrm{II}}}$ and a projection angle $\chi$ given by $\sin ^{2} \chi=\frac{\epsilon_{u_{\mathrm{II}}} \beta_{u_{\mathrm{II}}}}{E_{u}}$, where the beam envelope for coupled motion is given by

$$
E_{u}=\sqrt{\epsilon_{u_{\mathrm{I}}} \beta_{u_{\mathrm{I}}}+\epsilon_{u_{\mathrm{II}}} \beta_{u_{\mathrm{II}}}} .
$$

Fig. 20.1 Phase space ellipse for coupled motion

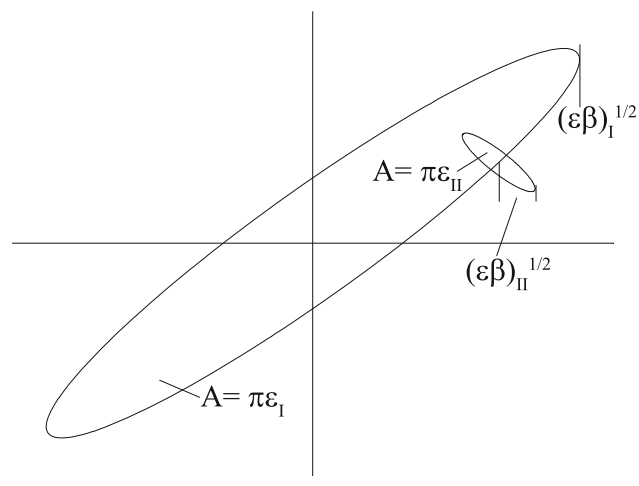


Similarly, we get from the second component of (20.66) the angular envelope

$$
A_{u}=\sqrt{\epsilon_{u_{\mathrm{I}}} \gamma_{u_{\mathrm{I}}}+\epsilon_{u_{\mathrm{I}}} \gamma_{u_{\mathrm{II}}}}
$$

for $\psi_{u_{I, I I}}=-\tilde{\vartheta}_{u_{\mathrm{III}}}$ and a projection angle given by

$$
\sin ^{2} \chi=\frac{\epsilon_{u_{\Pi}} \beta_{u_{\amalg}}}{A_{u}} .
$$

To completely determine the phase ellipse we calculate also the slope $x^{\prime}$ for the particle at $x=E_{x}$ which is the slope of the envelope $E^{\prime}$. Taking the derivative of (20.67) we get

$$
E_{u}^{\prime}=-\frac{\epsilon_{u_{\mathrm{I}}} \alpha_{u_{\mathrm{I}}}+\epsilon_{u_{\mathrm{II}}} \alpha_{u_{\mathrm{I}}}}{\sqrt{\epsilon_{u_{\mathrm{I}}} \beta_{u_{\mathrm{I}}}+\epsilon_{u_{\mathrm{I}}} \beta_{u_{\mathrm{I}}}}} .
$$

Expressing the equation of the phase ellipse in terms of these envelope definitions we get

$$
A_{u}^{2} u^{2}-2 E_{u}^{\prime} E_{u} u u^{\prime}+E_{u}^{2} u^{\prime 2}=\epsilon_{u}^{2}
$$

and inserting $u=E_{u}$ and $u^{\prime}=E_{u}^{\prime}$ into (20.71) we get for the emittance of the projection ellipse

$$
\epsilon_{u}=E_{u} \sqrt{A_{u}^{2}-E_{u}^{\prime 2}} .
$$

The envelope functions can be measured noting that $E^{2}=\sigma_{11}, A^{2}=\sigma_{22}$ and $E E^{\prime}=-\sigma_{12}$ where the $\sigma_{i j}$ are elements of the beam matrix. Because of the deformation of the four-dimensional phase ellipse through transformations, we cannot expect that the projection is a constant of motion and the projected emittance is therefore of limited use.

A more important and obvious projection is that onto the $(x, y)$-plane which shows the actual beam cross section under the influence of coupling. For this projection we use the first and third equation in (20.66) and find an elliptical beam cross section. The spatial envelopes $E_{x}$ and $E_{y}$ have been derived before in (20.67) and become here

$$
\begin{aligned}
& E_{x}=\sqrt{\epsilon_{x_{\mathrm{I}}} \beta_{x_{\mathrm{I}}}+\epsilon_{x_{\mathrm{II}}} \beta_{x_{\mathrm{II}}}}, \\
& E_{y}=\sqrt{\epsilon_{y_{\mathrm{I}}} \beta_{y_{\mathrm{I}}}+\epsilon_{y_{\mathrm{II}}} \beta_{y_{\mathrm{II}}}} .
\end{aligned}
$$


The $y$-coordinate for $E_{x}$, which we denote by $E_{x y}$, can be derived from the third equation in (20.66) noting that now $\vartheta_{y_{\mathrm{I}, \mathrm{II}}}=-\phi_{x_{\mathrm{I}, \mathrm{II}}}, \chi$ is given by (20.69) and

$$
E_{x y}=\frac{\epsilon_{\mathrm{I}} \sqrt{\beta_{x_{\mathrm{I}}} \beta_{y_{\mathrm{I}}}} \cos \Delta \phi_{\mathrm{I}}+\epsilon_{\mathrm{II}} \sqrt{\beta_{x_{\mathrm{II}}} \beta_{y_{\mathrm{II}}}} \cos \Delta \phi_{\mathrm{II}}}{\sqrt{\epsilon_{x_{\mathrm{I}}} \beta_{x_{\mathrm{I}}}+\epsilon_{x_{\mathrm{II}}} \beta_{x_{\mathrm{II}}}}},
$$

where $\Delta \phi_{\mathrm{I}, \mathrm{II}}=\phi_{x_{\mathrm{I}, \mathrm{II}}}-\phi_{y_{\mathrm{I}, \mathrm{II}}}$. The beam cross section is tilted due to coupling whenever $E_{x, y} \neq 0$. The tilt angle $\psi$ of the ellipse is determined by

$$
\tan 2 \psi=\frac{2 E_{x} E_{x y}}{E_{x}^{2}-E_{y}^{2}}
$$

or more explicitly

$$
\tan 2 \psi=2 \frac{\epsilon_{\mathrm{I}} \sqrt{\beta_{x_{\mathrm{I}}} \beta_{y_{\mathrm{I}}}} \cos \Delta \phi_{\mathrm{I}}+\epsilon_{\mathrm{II}} \sqrt{\beta_{x_{\mathrm{II}}} \beta_{y_{\mathrm{II}}}} \cos \Delta \phi_{\mathrm{II}}}{\epsilon_{x_{\mathrm{I}}} \Delta \beta_{\mathrm{I}}+\epsilon_{x_{\mathrm{II}}} \Delta \beta_{\mathrm{II}}}
$$

The beam cross section of a coupled beam is tilted as can be directly observed, for example, through a light monitor which images the beam cross section by the emission of synchrotron light. This rotation vanishes as we would expect for vanishing coupling when $\beta_{x_{\mathrm{II}}} \rightarrow 0$ and $\beta_{y_{\mathrm{I}}} \rightarrow 0$. The tilt angle is not a constant of motion and therefore different tilt angles can be observed at different points along a beam transport line.

We have discussed Ripken's theory [1] of coupled betatron motion which allows the formulation of beam dynamics for arbitrary strength of coupling. The concept of conjugate trajectories and transformation matrices through skew quadrupoles and solenoid magnets are the basic tools required to determine coupled betatron functions and the tilt of the beam cross section.

\subsection{Hamiltonian and Coupling}

In practical beam transport systems particle motion is not completely contained in one or the other plane although special care is being taken to avoid coupling effects as much as possible. Coupling of the motion from one plane into the other plane can be generated through the insertion of actual rotated magnets or in a more subtle way by rotational misalignments of upright magnets. Since such misalignments are unavoidable, it is customary to place weak rotated quadrupoles in a transport system to provide the ability to counter what is known as linear coupling caused by unintentional magnet misalignments. Whatever the source of coupling, we consider such fields as small perturbations to the particle motion.

The Hamiltonian treatment of coupled motion follows that for motion in a single plane in the sense that we try to find cyclic variables while transforming away 
those parts of the motion which are well known. For a single particle normalized coordinates can be defined which eliminate the $z$-dependence of the unperturbed part of the equations of motion. Such transformations cannot be applied in the case of coupled motion since they involve the oscillation frequency or betatron phase function which is different for both planes.

\subsubsection{Linearly Coupled Motion}

We will derive some properties of coupled motion for the case of linear coupling introduced, for example, by a rotated quadrupole. Equations of linearly coupled motion are with $\underline{k}=p(z)$ of the form

$$
\begin{aligned}
x^{\prime \prime}+k x & =-p(z) y, \\
y^{\prime \prime}-y x & =-p(z) x,
\end{aligned}
$$

which can be derived from the Hamiltonian for linearly coupled motion

$$
H=\frac{1}{2} x^{\prime 2}+\frac{1}{2} y^{\prime 2}+\frac{1}{2} k x^{2}-\frac{1}{2} k y^{2}+p(z) x y .
$$

This Hamiltonian is composed of an uncoupled Hamiltonian $H_{0}$ and the perturbation Hamiltonian for linear coupling

$$
H_{1}=p(z) x y \text {. }
$$

The solutions for the uncoupled equations with integration constants $c_{u}$ and $\phi$ are of the form

$$
\begin{aligned}
& u(z)=c_{u} \sqrt{\beta_{u}} \cos \left[\psi_{u}(z)+\phi\right], \\
& u^{\prime}(z)=-\frac{c_{u}}{\sqrt{\beta_{u}}}\left\{\alpha_{u}(z) \cos \left[\psi_{u}(z)+\phi\right]+\sin \left[\psi_{u}(z)+\phi\right]\right\} .
\end{aligned}
$$

Applying the method of variation of integration constants, we try the ansatz

$$
\begin{aligned}
& u(z)=\sqrt{2 a_{u}(z)} \sqrt{\beta_{u}} \cos \left[\psi_{u}(z)+\phi(z)\right], \\
& u^{\prime}(z)=-\sqrt{\frac{2 a_{u}(z)}{\beta_{u}}}\left\{\alpha_{u}(z) \cos \left[\psi_{u}(z)+\phi(z)\right]+\sin \left[\psi_{u}(z)+\phi(z)\right]\right\},
\end{aligned}
$$

for the coupled motion. Now we use the integration constants $(a, \phi)$ as new variables and to show that the new variables are canonical, we use the Hamiltonian equations $\partial H / \partial u^{\prime}=\mathrm{d} u / \mathrm{d} z$ and $\partial H / \partial u=-\mathrm{d} u^{\prime} / \mathrm{d} z$ and get

$$
\frac{\partial H}{\partial u^{\prime}}=\frac{\partial H_{0}}{\partial u^{\prime}}+\frac{\partial H_{1}}{\partial u^{\prime}}=\frac{\mathrm{d} u}{\mathrm{~d} z}=\frac{\partial u}{\partial z}+\frac{\partial u}{\partial a} \frac{\partial a}{\partial z}+\frac{\partial u}{\partial \phi} \frac{\partial \phi}{\partial z} .
$$


A similar expression exists for the second Hamiltonian equation of motion

$$
\frac{\partial H}{\partial u}=\frac{\partial H_{0}}{\partial u}+\frac{\partial H_{1}}{\partial u}=-\frac{\mathrm{d} u^{\prime}}{\mathrm{d} z}=-\frac{\partial u^{\prime}}{\partial z}-\frac{\partial u^{\prime}}{\partial a} \frac{\partial a}{\partial z}-\frac{\partial u^{\prime}}{\partial \phi} \frac{\partial \phi}{\partial z} .
$$

For uncoupled oscillators we know that $a=$ const. and $\phi=$ const. and therefore $\partial u / \partial z=\partial H_{0} / \partial u^{\prime}$ and $\partial u^{\prime} / \partial z=-\partial H_{0} / \partial u$. With this we derive from (20.81)(20.84) the equations

$$
\begin{aligned}
& \frac{\partial H_{1}}{\partial \phi}=\frac{\partial H_{1}}{\partial u} \frac{\partial u}{\partial \phi}+\frac{\partial H_{1}}{\partial u^{\prime}} \frac{\partial u^{\prime}}{\partial \phi}=-\frac{\mathrm{d} a}{\mathrm{~d} z}, \\
& \frac{\partial H_{1}}{\partial a}=+\frac{\partial H_{1}}{\partial u} \frac{\partial u}{\partial a}+\frac{\partial H_{1}}{\partial u^{\prime}} \frac{\partial u^{\prime}}{\partial a}=\frac{\mathrm{d} \phi}{\mathrm{d} z},
\end{aligned}
$$

demonstrating that the new variables $(\phi, a)$ are canonical variables and (20.82) are canonical transformations. Applying (20.82) to the perturbation Hamiltonian (20.80) with appropriate indices to distinguish between horizontal and vertical plane, the perturbation Hamiltonian becomes

$$
H_{1}=2 p(z) \sqrt{\beta_{x} \beta_{y}} \sqrt{a_{x} a_{y}} \cos \left(\psi_{x}+\phi_{x}\right) \cos \left(\psi_{y}+\phi_{y}\right),
$$

where $z$ is still the independent variable. The dynamics of linearly coupled motion becomes more evident after isolating the periodic terms in (20.86). For the trigonometric functions we set

$$
\cos \left(\psi_{u}+\phi_{u}\right)=\frac{1}{2}\left[\mathrm{e}^{\mathrm{i}\left(\psi_{u}+\phi_{u}\right)}+\mathrm{e}^{-\mathrm{i}\left(\psi_{u}+\phi_{u}\right)}\right]
$$

and the Hamiltonian takes the form

$$
H_{1}=\frac{1}{2} p(z) \sqrt{\beta_{x} \beta_{y}} \sqrt{a_{x} a_{y}} \sum_{l_{x}, l_{y}} \mathrm{e}^{\mathrm{i}\left[l_{x}\left(\psi_{x}+\phi_{x}\right)+l_{y}\left(\psi_{y}+\phi_{y}\right)\right]},
$$

where the non-zero integers $l_{x}$ and $l_{y}$ are integers defined by

$$
l_{x}, l_{y} \exists(-1,1) .
$$

Similar to the one-dimensional case we try to separate constant or slowly varying terms from the fast oscillating terms and expand the exponent in (20.88) like

$$
\begin{aligned}
l_{x} \psi_{x}+l_{y} \psi_{y}- & l_{x} v_{0 x} \varphi-l_{y} v_{0 y} \varphi \\
& +l_{x} v_{0 x} \varphi+l_{y} v_{0 y} \varphi+l_{x} \phi_{x}+l_{y} \phi_{y},
\end{aligned}
$$

where $\phi_{u}=v_{0 u} \varphi, v_{0 u}$ are the tunes for the periodic lattice, $\varphi=2 \pi z / L$ and $L$ is the length of the lattice period. The first four terms in (20.90) are periodic with the 
period $\varphi(L)=2 \pi+\varphi(0)$. Inserting (20.90) into (20.88) we get with $\psi_{u}(L)=$ $2 \pi v_{0 u}+\psi_{u}(0)$

$$
\begin{aligned}
\bar{H}_{1}=\frac{1}{2} \sum_{l_{x}, l_{y}} p(z) \sqrt{\beta_{x} \beta_{y}} \mathrm{e}^{\mathrm{i}\left[l_{x} \psi_{x}+l_{y} \psi_{y}-l_{x} \nu_{0 x} \varphi-l_{y} \nu_{0 y} \varphi\right]} \\
\times \sqrt{a_{x} a_{y}} \sum_{l_{x}, l_{y}} \mathrm{e}^{\mathrm{i}\left[l_{x} \nu_{0 x} \varphi+l_{y} \nu_{0 y} \varphi+l_{y} \psi_{y}+l_{y} \phi_{y}\right]} .
\end{aligned}
$$

In this form we recognize the periodic factor

$$
A(\varphi)=p(z) \sqrt{\beta_{x} \beta_{y}} \mathrm{e}^{\mathrm{i}\left[l_{x} \psi_{x}+l_{y} \psi_{y}-l_{x} \nu_{0 x} \varphi-l_{y} \nu_{0 y} \varphi\right]}
$$

since betatron functions and perturbations $p(z)=\underline{k}(z)$ are periodic. After expanding (20.92) into a Fourier series

$$
\frac{L}{2 \pi} A(\varphi)=\sum_{q} \kappa_{q, l_{x}, l_{y}} \mathrm{e}^{\mathrm{i} q N \varphi}
$$

coupling coefficients can be defined by

$$
\begin{aligned}
\kappa_{q, l_{x}, l_{y}} & =\frac{1}{2 \pi} \int_{0}^{2 \pi} \frac{L}{2 \pi} A(\varphi) \mathrm{e}^{-\mathrm{i} q N \varphi} \mathrm{d} \varphi \\
& =\frac{1}{2 \pi} \int_{0}^{L} \underline{k} \sqrt{\beta_{x} \beta_{y}} \mathrm{e}^{\mathrm{i}\left[l_{x} \psi_{x}+l_{y} \psi_{y}-\left(l_{x} \nu_{0 x}+l_{y} \nu_{0 y}-q N\right) \frac{2 \pi}{L} z\right]} \mathrm{d} z
\end{aligned}
$$

Since $\kappa_{q, 1,1}=\kappa_{q,-1,-1}$ and $\kappa_{q, 1,-1}=\kappa_{q,-1,1}$, we have with $-1 \leq l \leq+1$

$$
\kappa_{q, l}=\frac{1}{2 \pi} \int_{0}^{L} \underline{k} \sqrt{\beta_{x} \beta_{y}} \mathrm{e}^{\mathrm{i}\left[\psi_{x}+l \psi_{y}-\left(\nu_{0 x}+l \nu_{0 y}-q N\right) \frac{2 \pi}{L} z\right]} \mathrm{d} z .
$$

The coupling coefficient is a complex quantity indicating that there are two orthogonal and independent contributions which require also two orthogonally independent corrections. Now that the coupling coefficients are defined in a convenient form for numerical evaluation we replace the independent variable $z$ by the angle variable $\varphi=2 \pi z / L$ and obtain the new Hamiltonian $\tilde{H}_{1}=\frac{2 \pi}{L} H_{1}$ or

$$
\tilde{H}=\sum_{q} \kappa_{q, l} \sqrt{a_{x} a_{y}} \cos \left(\phi_{x}+l \phi_{y}+\Delta_{q} \varphi\right)
$$

where

$$
\Delta_{q}=v_{0 x}+l v_{0 y}-q N
$$


Most terms in (20.96) are fast oscillating and therefore cancel before any damage can be done to particle stability. One term, however, is slowly varying for $q=r$ defining the resonance condition for coupled motion $\left(\Delta_{q} \approx 0\right)$ or

$$
r N \approx v_{0 x}+l v_{0 y}
$$

In this resonant case, the quantity $\Delta_{\mathrm{r}}$ is the distance of the tunes from the coupling resonance as defined by (20.97) with $q=r$. Neglecting all fast oscillating terms we apply one more canonical transformation $\left(\phi_{u}, a_{u}\right) \rightarrow\left(\tilde{\phi}_{u}, \tilde{a}_{u}\right)$ to eliminate the independent variable $\varphi$ from the Hamiltonian. In essence, we thereby use a coordinate system that follows with the unperturbed particle and exhibits only the deviations from the ideal motion. From the generating function

$$
G=\tilde{a}_{x}\left(\phi_{x}+\frac{1}{2} \Delta_{\mathrm{r}} \varphi\right)+\tilde{a}_{y}\left(\phi_{y}+l \frac{1}{2} \Delta_{\mathrm{r}} \varphi\right)
$$

we get for the new variables

$$
\begin{aligned}
& \tilde{\phi}_{x}=\frac{\partial G}{\partial \tilde{a}_{x}}=\phi_{x}+\frac{1}{2} \Delta_{\mathrm{r}} \varphi, \quad \tilde{a}_{x}=\frac{\partial G}{\partial \phi_{x}}=a_{x}, \\
& \tilde{\phi}_{y}=\frac{\partial G}{\partial \tilde{a}_{y}}=\phi_{y}+l \frac{1}{2} \Delta_{\mathrm{r}} \varphi, \quad \tilde{a}_{y}=\frac{\partial G}{\partial \phi_{y}}=a_{y} \text {, }
\end{aligned}
$$

and the new Hamiltonian for the rotating coordinate system is

$$
\tilde{H}_{\mathrm{r}}=\tilde{H}+\frac{\partial G}{\partial \varphi}=\tilde{H}+\frac{1}{2} \Delta_{\mathrm{r}} a_{x}+l \frac{1}{2} \Delta_{\mathrm{r}} a_{y} .
$$

For simplicity we drop the tilde on the amplitudes and use $\left(a_{x}, a_{y}\right)$. The resonant Hamiltonian becomes after this transformation

$$
\tilde{H}_{\mathrm{r}}=\frac{1}{2} \Delta_{\mathrm{r}}\left(a_{x}+l a_{y}\right)+\kappa_{\mathrm{r}, l} \sqrt{a_{x} a_{y}} \cos \left(\tilde{\phi}_{x}+l \tilde{\phi}_{y}\right)
$$

and the equations of motion are

$$
\begin{aligned}
& \frac{\partial a_{x}}{\partial \varphi}=-\frac{\partial \tilde{H}_{\mathrm{r}}}{\partial \tilde{\phi}_{x}}=\kappa_{\mathrm{r}, l} \sqrt{a_{x} a_{y}} \sin \left(\tilde{\phi}_{x}+l \tilde{\phi}_{y}\right), \\
& \frac{\partial a_{y}}{\partial \varphi}=-\frac{\partial \tilde{H}_{\mathrm{r}}}{\partial \tilde{\phi}_{y}}=l \kappa_{\mathrm{r}, l} \sqrt{a_{x} a_{y}} \sin \left(\tilde{\phi}_{x}+l \tilde{\phi}_{y}\right),
\end{aligned}
$$

and

$$
\begin{aligned}
& \frac{\partial \tilde{\phi}_{x}}{\partial \varphi}=\frac{\partial \tilde{H}_{\mathrm{r}}}{\partial a_{x}}=\frac{1}{2} \Delta_{\mathrm{r}}+\frac{1}{2} \kappa_{\mathrm{r}, l} \sqrt{\frac{a_{y}}{a_{x}}} \cos \left(\tilde{\phi}_{x}+l \tilde{\phi}_{y}\right), \\
& \frac{\partial \tilde{\phi}_{y}}{\partial \varphi}=\frac{\partial \tilde{H}_{\mathrm{r}}}{\partial a_{y}}=l \frac{1}{2} \Delta_{\mathrm{r}}+\frac{1}{2} \kappa_{\mathrm{r}, l} \sqrt{\frac{a_{x}}{a_{y}}} \cos \left(\tilde{\phi}_{x}+l \tilde{\phi}_{y}\right)
\end{aligned}
$$

From these equations we can derive criteria for the stability or resonance condition of coupled systems. Depending on the value of $l$ we distinguish a sum resonance if $l=+1$ or a difference resonance if $l=-1$. 


\section{Linear Difference Resonance}

In case of a difference resonance $(l=-1)$ we add both Eqs. (20.103) and get

$$
\frac{\mathrm{d}}{\mathrm{d} \varphi}\left(a_{x}+a_{y}\right)=0 .
$$

The coupled motion is stable because the sum of both amplitudes does not change. Both amplitudes $a_{x}$ and $a_{y}$ will change such that one amplitude increases at the expense of the other but the sum of both will not change and therefore neither amplitude will grow indefinitely. Since $a_{x}$ and $a_{y}$ are proportional to the beam emittance, we note that the sum of the horizontal and vertical emittance stays constant as well,

$$
\epsilon_{x}+\epsilon_{y}=\text { const. }
$$

The resonance condition (20.98) for a difference resonance becomes [2]

$$
v_{x}-v_{y} m_{r}=N .
$$

Our discussion of linear coupling resonances reveals the feature that a difference resonances will cause an exchange of oscillation amplitudes between the horizontal and vertical plane but will not lead to beam instability. This result is important for lattice design. If emittance coupling is desired, one would choose tunes which closely meet the resonance condition. Conversely, when coupling is to be avoided or minimized, tunes are chosen at a save distance from the coupling resonance.

There exists a finite stop-band width also for the coupling resonance just as for any other resonance and we have all the mathematical tools to calculate that width. Since the beam is not lost at a difference coupling resonance, we are also able to measure experimentally the stop-band width by moving the tunes through the resonance. The procedure becomes obvious after linearizing the equations of motion (20.103), (20.104). Following a suggestion by Guignard [3], we define new variables similar in form to normalized coordinates

$$
\begin{aligned}
& w=\sqrt{a_{x}} \mathrm{e}^{\mathrm{i} \tilde{\phi}_{x}}, \\
& v=\sqrt{a_{y}} \mathrm{e}^{\mathrm{i} \tilde{\phi}_{y}} .
\end{aligned}
$$

Taking derivatives of (20.108) with respect to $\varphi$ and using (20.103), (20.104) we get after some manipulation the linear equations

$$
\begin{aligned}
& \frac{\mathrm{d} w}{\mathrm{~d} \varphi}=\mathrm{i} \frac{1}{2}\left(\kappa v+\Delta_{\mathrm{r}} w\right), \\
& \frac{\mathrm{d} v}{\mathrm{~d} \varphi}=\mathrm{i} \frac{1}{2}\left(\kappa w-\Delta_{\mathrm{r}} v\right),
\end{aligned}
$$

where we have set for simplicity $\kappa_{\mathrm{r},-1}=\kappa$. 
These equations can be solved analytically and will provide further insight into the dynamics of coupled oscillations. We will look for characteristics of coupled motion which do not depend on initial conditions but are general for all particles. Expecting the solutions $w$ and $v$ to describe oscillations, we assume that the motion in both planes depends on the initial conditions $w_{0}, v_{0}$ in both planes due to the effect of coupling. For simplicity, however, we study the dynamics of a particle which starts with a finite amplitudes $w_{0} \neq 0$ in the horizontal plane only and set $v_{0}=0$. The ansatz for the oscillations be

$$
\begin{aligned}
& w(\varphi)=w_{0}\left(a \mathrm{e}^{\mathrm{i} v \varphi}+b \mathrm{e}^{-\mathrm{i} \nu \varphi}\right), \\
& v(\varphi)=w_{0}\left(c \mathrm{e}^{\mathrm{i} \nu \varphi}+d \mathrm{e}^{-\mathrm{i} \nu \varphi}\right),
\end{aligned}
$$

where we define an as yet undefined frequency $v$. Inserting (20.110) into (20.109) the coefficients of the exponential functions vanish separately and we get from the coefficients of $\mathrm{e}^{\mathrm{i} \nu \varphi}$ the two equations

$$
\begin{aligned}
& 2 \nu a=\kappa c+\Delta_{\mathrm{r}} a, \\
& 2 \nu c=\kappa a+\Delta_{\mathrm{r}} c,
\end{aligned}
$$

from which we may eliminate the unknowns $a$ and $c$ to get the defining equation for the oscillation frequency

$$
v=\frac{1}{2} \sqrt{\Delta_{\mathrm{r}}^{2}+\kappa^{2}}
$$

While determining the coefficients $a, b, c, d$, we note that due to the initial conditions $a+b=1$ and $c+d=0$. Similar to (20.111) we derive another pair of equations from the coefficients of $\mathrm{e}^{-\mathrm{i} \nu \varphi}$

$$
\begin{aligned}
& 2 v b=\kappa d-\Delta_{\mathrm{r}} b, \\
& 2 v d=\kappa b+\Delta_{\mathrm{r}} d,
\end{aligned}
$$

which completes the set of four equations required to determine with (20.112) the four unknown coefficients

$$
\begin{array}{ll}
a=\frac{2 v+\Delta_{\mathrm{r}}}{4 v}, & b=\frac{2 v-\Delta_{\mathrm{r}}}{4 v}, \\
c=\frac{\kappa}{4 v}, & d=-\frac{\kappa}{4 v},
\end{array}
$$

With this, the solutions (20.110) become

$$
\begin{aligned}
& w(\varphi)=w_{0} \cos \nu \varphi+\mathrm{i} w_{0} \frac{\Delta_{\mathrm{r}}}{2 v} \sin \nu \varphi, \\
& v(\varphi)=\quad+\mathrm{i} w_{0} \frac{\kappa}{2 v} \sin \nu \varphi,
\end{aligned}
$$


and by multiplication with the complex conjugate and (20.108) we get expressions for the coupled beam emittances $\left(\epsilon_{u}=2 a_{u}\right)$

$$
\begin{aligned}
& a_{x}=a_{x 0} \frac{1}{4 v^{2}}\left(\Delta_{\mathrm{r}}^{2}+\kappa^{2} \cos ^{2} \nu \varphi\right), \\
& a_{y}=a_{x 0} \frac{\kappa^{2}}{4 v^{2}} \sin ^{2} \nu \varphi .
\end{aligned}
$$

The ratio of maximum values for beam emittances in both planes under the influence of linear coupling is from (20.116)

$$
\frac{\epsilon_{y}}{\epsilon_{x}}=\frac{\kappa^{2}}{\Delta_{\mathrm{r}}^{2}+\kappa^{2}} .
$$

The emittance coupling increases with the strength of the coupling coefficient and is equal to unity at the coupling resonance or for large values of $\kappa$. At the coupling resonance we observe complete exchange of emittances at the frequency $v$. If on the other hand, the tunes differ and $\Delta_{\mathrm{r}} \neq 0$, there will always be a finite oscillation amplitude left in the horizontal plane because we started with a finite amplitude in this plane. A completely symmetric result would be obtained only for a particle starting with a finite vertical amplitude as well.

We may now collect all results and derive the particle motion as a function of time or $\varphi$. For example, the horizontal particle position is determined from (20.82) where we set $\sqrt{a_{x}}=w \mathrm{e}^{-\mathrm{i} \tilde{\phi}_{x}}$ and further replace $w$ by (20.110). Here, we are only interested in the oscillation frequencies of the particle motion and note that the oscillatory factor in (20.82) is $\operatorname{Re}\left[\mathrm{e}^{\mathrm{i}\left(\psi_{x}+\phi_{x}\right)}\right]$. Together with other oscillatory quantities $\mathrm{e}^{-\mathrm{i} \tilde{\phi}_{x}}$ and $w$ we get both in the horizontal and vertical plane terms with oscillatory factors

$$
\operatorname{Re}\left[\mathrm{e}^{\mathrm{i}\left(\psi_{u}+\phi_{u}-\tilde{\phi}_{u} \pm \nu \varphi\right)}\right]
$$

where the index $u$ stands for either $x$ or $y$. The phase $\psi_{u}=v_{u} \varphi$ and from (20.100) and $l=-1$ for the difference resonance $\tilde{\phi}_{u}=\phi_{u} \pm \frac{1}{2} \Delta_{\mathrm{r}} \varphi$. These expressions used in (20.118) define two oscillation frequencies

$$
v_{I, I I}=v_{x, y} \mp \frac{1}{2} \Delta_{\mathrm{r}} \pm v
$$

or with (20.112)

$$
v_{I, I I}=v_{x, y} \mp \frac{1}{2} \Delta_{\mathrm{r}} \pm \frac{1}{2} \sqrt{\Delta_{\mathrm{r}}^{2}+\kappa^{2}}
$$

We have again found the result that under coupling conditions the betatron oscillations assume two modes. In a real accelerator only these mode frequencies can be measured while close to the coupling resonance. For very weak coupling $(\kappa \approx 0)$ the mode frequencies are approximately equal to the uncoupled frequencies 
$v_{x, y}$, respectively. Even for large coupling this equality is preserved as long as the tunes are far away from the coupling resonance or $\Delta_{\mathrm{r}} \gg \kappa$.

The mode frequencies can be measured while adjusting quadrupoles such that the beam is moved through the coupling resonance. During this adjustment the detuning parameter $\Delta_{\mathrm{r}}$ varies and changes sign as the coupling resonance is crossed. For example, if we vary the vertical tune across a coupling resonance from below, we note that the horizontal tune or $v_{I}$ does not change appreciably until the resonance is reached, because $-\Delta_{r}+\sqrt{\Delta_{\mathrm{r}}^{2}+\kappa^{2}} \approx 0$. Above the coupling resonance, however, $\Delta_{r}$ has changed sign and $v_{I}$ increase with $\Delta_{r}$. The opposite occurs with the vertical tune. Going through the coupling resonance the horizontal tune has been transformed into the vertical tune and vice versa without ever getting equal.

Actual tune measurements [4] are shown in Fig. 20.2 as a function of the excitation current of a vertically focusing quadrupole. The vertical tune change is proportional to the quadrupole current and so is the parameter $\Delta_{r}$. While increasing the quadrupole current, the vertical tune is increased and the horizontal tune stays practically constant. We note that the tunes actually do not cross the linear coupling resonance during that procedure, rather the tune of one plane is gradually transformed into the tune of the other plane and vice versa. Both tunes never become equal and the closest distance is determined by the magnitude of the coupling coefficient $\kappa$.

The coupling coefficient may be nonzero for various reasons. In some cases coupling may be caused because special beam characteristics are desired. In most cases, however, coupling is not desired or planned for and a finite linear coupling of the beam emittances is the result of rotational misalignments of upright quadrupoles. Where this coupling is not desired and must be minimized, we may introduce a pair or two sets of rotated quadrupoles into the lattice to cancel the coupling due to misalignments. The coupling coefficient (20.95) is defined in the form of a complex quantity. Both orthogonal components must therefore be compensated

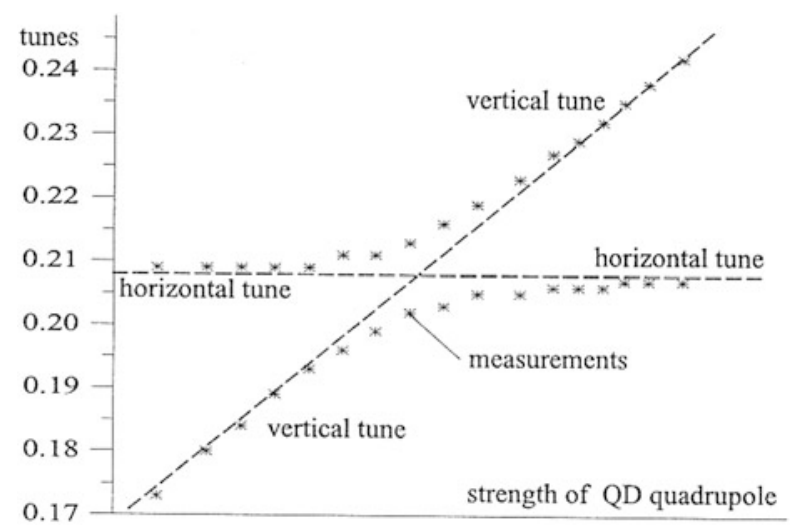

Fig. 20.2 Measurements of mode frequencies as a function of detuning for linearly coupled motion [4] 
by two orthogonally located skew quadrupoles and the proper adjustment of these quadrupoles can be determined by measuring the width of the linear coupling resonance.

\section{Linear Sum Resonance}

To complete the discussion, we will now set $l=+1$ and get from (20.98) the resonance condition for a sum resonance

$$
v_{x}+v_{y}=m_{r} N
$$

Taking the difference of both Eqs. (20.103), we get

$$
\frac{\mathrm{d}}{\mathrm{d} \varphi}\left(a_{x}-a_{y}\right)=0,
$$

which states only that the difference of the emittances remains constant. Coupled motion in the vicinity of a sum resonance is therefore unstable allowing both emittances to grow unlimited. To solve the equations of motion (20.103), (20.104), we try the ansatz

$$
u=\sqrt{a_{x}} \mathrm{e}^{\mathrm{i} \Phi_{x}}+\mathrm{i} \sqrt{a_{y}} \mathrm{e}^{\mathrm{i} \Phi_{y}} .
$$

From the derivative $\mathrm{d} u / \mathrm{d} \varphi$, we get with (20.103), (20.104)

$$
\frac{\mathrm{d} u}{\mathrm{~d} \varphi}=\mathrm{i} \frac{1}{2}\left(\Delta_{r} u-\kappa u^{*}\right),
$$

and for the complex conjugate

$$
\frac{\mathrm{d} u^{*}}{\mathrm{~d} \varphi}=-\mathrm{i} \frac{1}{2}\left(\Delta_{r} u^{*}+\kappa u\right) .
$$

Solving these differential equations with the ansatz

$$
u=a \mathrm{e}^{\mathrm{i} \nu \varphi}+b \mathrm{e}^{-\mathrm{i} \nu \varphi},
$$

and the complex conjugate

$$
u^{*}=a \mathrm{e}^{-\mathrm{i} \nu \varphi}+b \mathrm{e}^{\mathrm{i} \nu \varphi},
$$

we get after insertion into (20.124), (20.125) analogous to (20.111) the oscillation frequency

$$
v=\frac{1}{2} \sqrt{\Delta_{\mathrm{r}}^{2}-\kappa^{2}}
$$


This result shows that motion in the vicinity of a linear sum resonance becomes unstable as soon as the detuning is less than the coupling coefficient. The condition for stability is therefore

$$
\Delta_{\mathrm{r}}>\kappa
$$

By a careful choice of the tune difference to avoid a sum resonance and careful alignment of quadrupoles, it is possible in real circular accelerators to reduce the coupling coefficient to very small values. Perfect compensation of the linear coupling coefficient eliminates the linear emittance coupling altogether. However, nonlinear coupling effects become then dominant which we cannot compensate for.

\subsubsection{Higher-Order Coupling Resonances}

So far all discussions on coupled motions and resonances have been based on linear coupling effects caused by rotated quadrupole fields. For higher-order coupling the mathematical treatment of the beam dynamics is similar although more elaborate. The general form of the $n$ th-order resonance condition (20.98) is

$$
l_{x} v_{x}+l_{y} v_{y}=m_{\mathrm{r}} N \quad \text { with } \quad\left|l_{x}\right|+\left|l_{y}\right| \leq n
$$

The factors $l_{x}$ and $l_{y}$ are integers and the sum $\left|l_{x}\right|+\left|l_{y}\right|$ is called the order of the resonance. In most cases it is sufficient to choose a location in the resonance diagram which avoids such resonances since circular accelerators are generally designed for minimum coupling. In special cases, however, where strong sextupoles are used to correct chromaticities, coupling resonances can be excited in higher order. For example, the difference resonance $2 v_{x}-2 v_{y}$ has been observed at the $400 \mathrm{GeV}$ proton synchrotron at the Fermi National Laboratory [5].

\subsubsection{Multiple Resonances}

We have only discussed isolated resonances. In general, however, nonlinear fields of different orders do exist, each contributing to the stop-band of resonances. A particularly strong source of nonlinearities occurs due to the beam-beam effect in colliding-beam facilities where strong and highly nonlinear fields generated by one beam cause significant perturbations to particles in the other beam. The resonance patterns from different resonances are superimposed creating new features of particle instability which were not present in any of the resonances while treated as isolated resonances. Of course, if one of these resonances is unstable for any oscillation amplitude the addition of other weaker resonances will not change this situation. 
Combining the effects of several resonances should cause little change for small amplitude oscillations since the trajectory in phase space is close to a circle for resonances of any order provided there is stability at all. Most of the perturbations of resonance patterns will occur in the vicinity of the island structures. When island structures from different resonances start to overlap, chaotic motion can occur and may lead to stochastic instability. The onset of island overlap is often called the Chirikov criterion after Chirikov [6], who has studied extensively particle motion in such situations.

It is beyond the scope of this text to evaluate the mathematical criteria of multi resonance beam dynamics. For further insight and references the interested reader may consult articles in [7-10]. A general overview and extensive references can also be found in [11].

\section{Problems}

20.1 (S). Consider a lattice made of 61 FODO cells with $90^{\circ}$ per cell in both planes. The half cell length be $L=5 \mathrm{~m}$ and the full quadrupole length $\ell=0.2 \mathrm{~m}$. Introduce a Gaussian distribution of rotational quadrupole misalignments. Calculate and plot the coupling coefficient for the ring and the emittance ratio as a function of the rms misalignment. If the emittance coupling is to be held below $1 \%$ how must the lattice be retuned and how well must the quadrupoles be aligned? Insert two rotated quadrupoles into the lattice such that they can be used to compensate the coupling due to misalignments. Calculate the required quadrupole strength.

20.2 (S). Use the measurement in Fig. 20.2 and determine the coupling coefficient $\kappa$.

20.3. Can we rotate a horizontally flat $10 \mathrm{GeV}$ beam by $90^{\circ}$ with a solenoid? If yes, what is the strength of the solenoid and where along the $z$-axis do we have a flat vertical beam?

20.4. In circular accelerators rotated quadrupoles may be inserted to compensate for coupling due to misalignments. Assume a statistical distribution of rotational quadrupole errors which need to be compensated by special rotated quadrupoles. How many such quadrupoles are required and what criteria would you use for optimum placement in the ring?

20.5. Consider a point source of particles (e.g. a positron conversion target) on the axis of a solenoidal field. Determine the solenoid parameters for which the particles would exit the solenoid as a parallel beam. Such a solenoid is also called a $\lambda / 4$-lens, why? Let the positron momentum be $10 \mathrm{MeV} / \mathrm{c}$. What is the maximum solid angle accepted from the target that can be focused to a beam of radius $r=1 \mathrm{~cm}$ ? What is the exit angle of a particle which emerges from the target at a radius of $1 \mathrm{~mm}$ ? Express the transformation of this $\lambda / 4$-lens in matrix formulation. 
20.6. Choose a FODO lattice for a circular accelerator and insert at a symmetry point a thin rotated quadrupole. Calculate the tilt of the beam cross section at this point as a function of the strength of the rotated quadrupole. Place the same skew quadrupole in the middle of a FODO half cell and determine if the rotation of the beam aspect ratio at the symmetry point requires a stronger or a weaker field. Explain why.

20.7. Assume two cells of a symmetric FODO lattice and determine the betatron functions for a phase advance of $90^{\circ}$ per cell. Now introduce a rotational misalignment of the first quadrupole by an angle $\alpha$ which generates coupling of the horizontal and vertical betatron oscillations: a.) Calculate and plot the perturbed betatron functions $\beta_{\mathrm{I}}$ and $\beta_{\mathrm{II}}$ and compare with the unperturbed solution. b.) If the beam emittances are $\epsilon_{\mathrm{I}}=\epsilon_{\mathrm{II}} \mathrm{mm}-\mathrm{mrad}$, what is the beam aspect ratio and beam rotation at the end of cell one and two with and without the rotation of the first quadrupole?

20.8. Use the Fokker-Planck equation and derive an expression for the equilibrium beam emittance of a coupled beam

\section{References}

1. G. Ripken, Technical Report, R1-70/04, DESY, Hamburg (1970)

2. E.D. Courant, M.S. Livingston, H.S. Snyder, Phys. Rev. 88, 1190 (1952)

3. G. Guignard, The general theory of all sum and difference resonances in a three dimensional magnetic field in a synchrotron. Technical Report, CERN 76-06, CERN, Geneva (1976)

4. J. Safranek, SPEAR Lattice for high brightness synchrotron radiation. Ph.D. thesis, Stanford University, 1992

5. S.Ohnuma, Quarter integer resonance by sextupoles. Technical Report, TM-448, FERMI Lab, Batavia, IL (1973)

6. B. Chirikov, Phys. Rep. 52, 263 (1979)

7. in Nonlinear Dynamics Aspects in Particle Accelerators. Lecture Notes in Physics, vol. 247 (Springer, Berlin/Heidelberg, 1986)

8. M. Month, J.C. Herrera (eds.), in Nonlinear Dynamics and the Beam-Beam Interaction. AIP Conference Proceedings, vol. 57 (American Institute of Physics, New York, 1979)

9. M. Month, M. Dienes (eds.), Physics of Particle Accelerators. AIP Conference Proceedings, vol. 184 (American Institute of Physics, New York, 1989)

10. J.M. Greene, J. Math. Phys. 20, 1183 (1979)

11. in Space Charge Dynamics. Lecture Notes in Physics, vol. 296 (Springer, Berlin/Heidelberg, 1988) 


\section{Part VIII}

Intense Beams 


\section{Chapter 21 \\ Statistical and Collective Effects*}

Transverse and longitudinal beam dynamics as discussed in earlier chapters is governed by purely single-particle effects where the results do not depend on the presence of other particles or any interactive environment. Space-charge effects were specifically excluded. This restriction is sometimes too extreme and collective effects must be taken into account where significant beam intensities are desired. In most applications high beam intensities are desired and it is therefore prudent to test for the appearance of space charge and other intensity effects.

Collective effects can be divided into two distinct groups according to the physics involved. The compression of a large number of charged particles into a small volume increases the probability for collisions of particles within the same beam. Because particles perform synchrotron and betatron oscillations, statistical collisions occur in longitudinal, as well as transverse phase space often causing a mixing of phase space coordinates. The other group of collective effects includes effects which are associated with electromagnetic fields generated by the collection of all particles in a beam.

The study and detailed understanding of the cause and nature of collective effects or collective instabilities with corrective measures is important for a successful design of the accelerator. Most accelerator design and developments are conducted to eliminate collective effects as much as possible through self-imposed limitation on the performance or installation of feedback systems and other stabilizing control mechanisms. Beyond that, we also must accept limitations in beam performance imposed by nature or lack of understanding and technological limits. Pursuit of accelerator physics is the attempt to explore and push such limits as far as nature and general understanding of the subject allows.

This chapter has been made Open Access under a CC BY 4.0 license. For details on rights and licenses please read the Correction https://doi.org/10.1007/978-3-319-18317-6_28 


\subsection{Statistical Effects}

Coupling of individual particles to the presence of other particles may occur through very short range forces in collisions with each other. In this section, we will discuss statistical effects related to the finite number of particles and from collision processes within a particle bunch.

\subsubsection{Schottky Noise}

Electrical current is established by moving charged particles. The finite electrical charge and finite number of particles gives rise to statistical variations of the electrical current. This phenomenon has been observed and analyzed by Schottky [1] and we will discuss this Schottky noise in the realm of particle dynamics in circular accelerators. The information included in the Schottky noise is of great diagnostic importance for the nondestructive determination of particle beam parameters, a technique which has been developed at the CERN Intersecting Storage Ring (ISR) [2] and has become a standard tool of beam diagnostics.

We consider a particle $k$ with charge $q$ orbiting in an accelerator with the angular revolution frequency $\omega_{k}$ and define a particle line density by $2 \pi \bar{R} \lambda(t)=1$ where $2 \pi \bar{R}$ is the circumference of the ring. On the other hand, we may describe the orbiting particle by delta functions

$$
q=q \int_{0}^{2 \pi} \sum_{m=-\infty}^{+\infty} \delta\left(\omega_{k} t+\theta_{k}-2 \pi m\right) \mathrm{d} \theta,
$$

where $\omega_{k}$ is the angular revolution frequency of the particle $k$ and $\theta_{k}$ its phase at time $t=0$. The delta function can be expressed by a Fourier series and the line-charge density at time $t$ becomes

$$
q \lambda_{k}(t)=\frac{q}{2 \pi \bar{R}}\left[1+2 \sum_{n=0}^{\infty} \cos \left(n \omega_{k} t+n \theta_{k}\right)\right]
$$

From a pick up electrode close to the circulating particle, we would obtain a signal with a frequency line spectrum $\omega=n \omega_{k}$ where $n$ is an integer. In a real particle beam there are many particles with a finite spread of revolution frequencies $\omega_{k}$ and therefore the harmonic lines $n \omega_{k}$ spread out proportionally to $n$. For not too high harmonic numbers the frequency spreads do not yet overlap and we are able to measure the distribution of revolution frequencies. Tuning the spectrum analyzer to $\omega$, we observe a signal with an amplitude proportional to $N(\omega / n) \frac{\delta \omega}{n}$ where $N(\omega / n)$ is the particle distribution in frequency space and $\delta \omega$ the frequency resolution of the spectrum analyzer. The signal from the pick up electrode is proportional to the 
line-charge density which is at the frequency $\omega$ from (21.1)

$$
q \lambda_{\mathrm{rms}}(\omega)=\frac{\sqrt{2} q}{2 \pi \bar{R}} \sqrt{N(\omega / n) \frac{\delta \omega}{n}}
$$

and has been derived first by Schottky for a variety of current sources [1]. The spread in the revolution frequency originates from a momentum spread in the beam and measuring the Schottky spectrum allows its nondestructive determination.

Individual particles orbiting in an accelerator perform transverse betatron oscillations which we describe, for example, in the vertical plane by

$$
y_{k}(t)=a_{k} \cos \left(v_{k} \omega_{k} t+\psi_{k}\right),
$$

where $a_{k}$ is the amplitude and $\psi_{k}$ the phase of the betatron oscillation for the particle $k$ at time $t=0$. The difference signal from two pick up electrodes above and below the particle beam is, in linear approximation, proportional to the product of the betatron amplitude (21.3) and the line-charge density (21.1) and of the form

$$
\begin{aligned}
D_{k}(t)=A_{k} & \sum_{n=0}^{\infty} \cos \left[\left(n-v_{k}\right)\left(\omega_{k} t+\phi_{k}\right)\right] \\
& +A_{k} \sum_{n=0}^{\infty} \cos \left[\left(n+v_{k}\right)\left(\omega_{k} t+\varphi_{k}\right)\right],
\end{aligned}
$$

where we have ignored terms at frequencies $n \omega_{k}$. The transverse Schottky signal is composed of two side bands for each harmonic at frequencies

$$
\omega=\left(n \pm v_{k}\right) \omega_{k}
$$

which are also called the fast wave for $\omega=\left(n+v_{k}\right) \omega_{k}$ and the slow wave for $\omega=\left(n-v_{k}\right) \omega_{k}$.

The longitudinal Schottky noise depends on the rms contribution of all particles which are spread over a range of revolution frequencies due to a momentum spread and over betatron frequencies by virtue of the chromaticity. For $\Delta \omega_{\mathrm{rms}}=\eta_{\mathrm{c}} \omega_{0} \delta_{\mathrm{rms}}$ and $\Delta v_{\mathrm{rms}}=\xi_{y} \delta_{\mathrm{rms}}$ where $\omega_{0}$ is the revolution frequency of the bunch center, $\delta_{\mathrm{rms}}=$ $\Delta p_{\text {rms }} / p_{0}$ the rms relative momentum error, $\eta_{\mathrm{c}}$ the momentum compaction and $\xi_{y}$ the vertical chromaticity, the frequency distribution of the signal from the pick up is

$$
\begin{aligned}
\omega & =\left[n \pm\left(v_{y 0}+\xi_{y} \delta_{k}\right)\right]\left(\omega_{0}+\eta_{\mathrm{c}} \omega_{0} \delta_{k}\right) \\
& =\left(n \pm v_{y 0}\right) \omega_{0}+\left[\left(n \pm v_{y 0}\right) \eta_{\mathrm{c}} \pm \xi_{y}\right] \omega_{0} \delta_{k}+O\left(\delta^{2}\right) .
\end{aligned}
$$

The momentum spread $\delta_{k}$ causes a frequency spread which is different for the slow and fast wave. For example, for positive chromaticity above transition, $\eta_{\mathrm{c}}<0$ and the frequency spreads add up for the slow wave and cancel partially for the fast 
wave. This has been verified experimentally for a coasting proton beam in the ISR [2].

A transverse Schottky scan may exhibit the existence of weak resonances which may dilute the particle density, specifically in a coasting proton or ion beam. To control coasting beam instabilities, it is desirable to make use of Landau damping by introducing a large momentum and tune spread. This tune spread, however, can be sufficiently large to spread over higher order resonances and blow up that part of the beam which oscillates at those resonance frequencies. A Schottky scan can clearly identify such a situation as reported in [2].

In this text we are able to touch only the very basics of Schottky noise and the interested reader is referred to references [3-6] for more detailed discussions on the theory and experimental techniques to obtain Schottky scans and how to interpret the signals.

\subsubsection{Stochastic Cooling}

The "noise" signal from a circulating particle beam includes information which can be used to drive a feedback system in such a way as to reduce the beam emittance, longitudinal as well as transverse. Due to the finite number of particles in a realistic particle beam, the instantaneous center of a beam at the location of a pick up electrode exhibits statistical variations. This statistical displacement of a slice of beam converts to a statistical slope a quarter betatron wavelength downstream. The signal from the small statistical displacement of the beam at the pick up electrode can be amplified and fed back to the beam through a kicker magnet located an odd number of quarter wavelength downstream, assuming that the statistical variations do not smear out between pick up electrode and kicker. Van der Meer [7] proposed this approach to reduce the transverse proton beam emittance in ISR for increased luminosity and the process is now known as stochastic cooling.

This process of correction is not a statistical process and we must ask ourselves if this is an attempt to circumvent Liouville's theorem. It is not. Due to the finite number of particles in the beam, the phase space is not uniformly covered by particles but rather exhibits many holes. The method of stochastic cooling detects the moment one of these holes appears on one or the other side of the beam in phase space. At the same moment, the whole emittance is slightly shifted with respect to the center of the phase space and this shift can be both detected and corrected. The whole process of stochastic cooling therefore only squeezes the "air" out of the particle distribution in phase space. The most prominent application of this method occurs in the cooling of an antiproton beam to reach a manageable beam emittance for injection into high energy proton antiproton colliders. To discuss this process in more detail, theoretically as well as technically, would exceed the scope of this text and the interested reader is referred to a series of articles published in [8]. 


\subsubsection{Touschek Effect}

The concentration of many particles into small bunches increases the probability for elastic collisions between particles. This probability is further enhanced considering that particles perform transverse betatron as well as longitudinal synchrotron oscillations. In each degree of freedom, we have acceptance limits and if a particle's oscillation amplitude exceeds such limits due, for example, to a collision with another particle one or both particles can get lost. In this section, we discuss the process of single collisions where the momentum transfer is large enough to lead to the loss of both particles involved in the collision and postpone the discussion of multiple collisions with small momentum transfer to the next section.

We may consider two collision processes which could lead to beam loss. First, we observe two particles performing synchrotron oscillations and colliding headon in such a way that they transfer their longitudinal momentum into transverse momentum. This collision process is insignificant in particle accelerators because the longitudinal motion includes not enough momentum to increase the betatron oscillation amplitude enough for particle loss. On the other hand, transverse oscillations of particles represent large momenta and a transfer into longitudinal momenta can lead to the loss of both particles. This effect was discovered on the first electron storage ring ever constructed $[9,10]$ and we therefore call this the Touschek effect.

In this text, we will not pursue a detailed derivation of the collision process and refer the interested reader to references [11-13]. Of particular interest is the expression for the beam lifetime as a result of particle losses due to a momentum transfer into the longitudinal phase space exceeding the rf-bucket acceptance of $\Delta p /\left.p_{0}\right|_{\mathrm{rf}}$. Whenever such a transfer occurs both particles involved in the collision are lost. The beam decay rate is proportional to the number of particles in the bunch and the beam current therefore decays exponentially. Last, but not least, a loss occurs only if there is sufficient momentum in the transverse motion to exceed the rf-momentum acceptance. We assume the momentum acceptance to be limited by the rf-voltage and combining these parameters in a collision theory results in a beam lifetime for a Gaussian particle distribution given by

$$
\frac{1}{\tau}=-\frac{1}{N_{\mathrm{b}}} \frac{\mathrm{d} N_{\mathrm{b}}}{\mathrm{d} t}=\frac{r_{\mathrm{c}}^{2} c N_{\mathrm{b}}}{8 \pi \sigma_{x} \sigma_{y} \sigma_{\ell}} \frac{\lambda^{3}}{\gamma^{2}} D(\epsilon),
$$

where $r_{\mathrm{c}}$ is the classical particle radius, $\sigma_{x}, \sigma_{y}, \sigma_{\ell}$ are the standard values of the Gaussian bunch width, height and length, respectively, and $\lambda^{-1}=\Delta p /\left.p_{0}\right|_{\mathrm{rf}}$ the momentum acceptance parameter. The function $D(\epsilon)$ (Fig. 21.1) is defined by [13]

$$
\begin{aligned}
D(\epsilon)=\sqrt{\epsilon}\left[-\frac{3}{2} \mathrm{e}^{-\epsilon}+\frac{\epsilon}{2} \int_{\epsilon}^{\infty} \frac{\ln u}{u} \mathrm{e}^{-u} \mathrm{~d} u\right. \\
\left.\quad+\frac{1}{2}(3 \epsilon-\epsilon \ln \epsilon+2) \int_{\epsilon}^{\infty} \frac{\mathrm{e}^{-u}}{u} \mathrm{~d} u\right],
\end{aligned}
$$




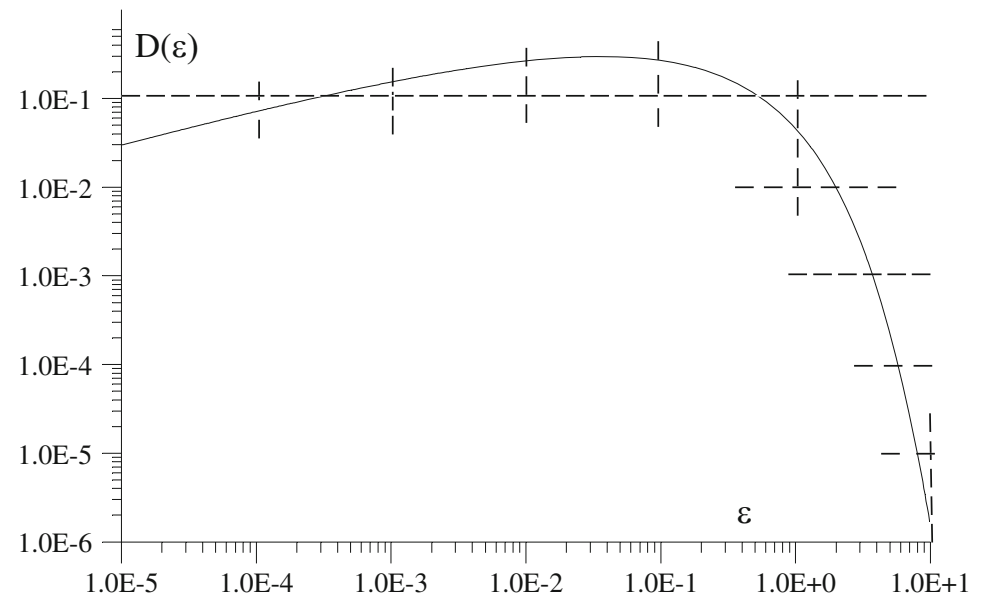

Fig. 21.1 Touschek lifetime function $D(\epsilon)$

where the argument is

$$
\epsilon=\left(\frac{\Delta p_{\mathrm{rf}}}{\gamma \sigma_{p}}\right)^{2} \quad \text { with } \quad \sigma_{\mathrm{p}}=\frac{m c \gamma \sigma_{x}}{\beta_{x}}
$$

Particle losses due to the Touschek effect is particularly effective at low energies and where the rf-acceptance is small. For high particle densities $N_{\mathrm{b}} /\left(\sigma_{x} \sigma_{y} \sigma_{\ell}\right)$ the rf-acceptance should therefore be maximized. This seems to be the wrong thing to do because the bunch length is reduced at the same time and the particle density becomes even higher but a closer look at (21.7) shows us that the Touschek lifetime increases faster with rf-acceptance than it decreases with bunch length.

\subsubsection{Intra-Beam Scattering}

The Touschek effect describes collision processes which lead to immediate loss of both colliding particles. In reality, however, there are many other collisions with only small exchanges of momentum. While these collisions do not lead to immediate particle loss, there might be sufficiently many during a damping time in electron storage rings or during the storage time for proton and ion beams to cause a significant increase in the bunch volume, or in the case of a coasting beam an increase in beam cross section. During the discussion of the Touschek effect we neglected the transfer from the longitudinal momentum space into transverse momentum space because the transverse momentum acceptance is larger than the longitudinal acceptance and particles are generally not lost during such an exchange. 
This is not appropriate any more for the multiple Touschek effect or intra-beam scattering where we are interested in all collisions.

The multiple Touschek effect was observed in the first ever constructed storage ring, AdA (Anello di Accumulatione) in Frascati, Italy. The Touschek effect had been expected and analyzed before but did give too pessimistic beam lifetimes compared to those observed in AdA. A longer beam lifetime had been obtained because of multiple elastic scattering between particles increasing the bunch volume and thereby reducing the Touschek effect [10].

During the exchange of momentum as a consequence of collisions between particles within the same bunch or beam, each degree of freedom can increase its energy or temperature because the beam is able to absorb any amount of energy from the rf- system. We are particularly interested in the growth times of transverse and longitudinal emittances to asses the long-term integrity of the particle beam. The multiple Touschek effect or intra-beam scattering has been studied extensively $[14,15]$ and we will not repeat here the derivations but merely recount the results.

The growth time of the beam emittances for Gaussian particle distributions are for the longitudinal phase space or momentum and bunch distribution $[14,15]$

$$
\tau_{p}^{-1}=\frac{1}{2 \sigma_{p}^{2}} \frac{\mathrm{d} \sigma_{p}^{2}}{\mathrm{~d} t}=A \frac{\sigma_{h}^{2}}{\sigma_{p}^{2}} f(a, b, c)
$$

where the particle bunch density is expressed by

$$
A=\frac{r_{\mathrm{c}}^{2} c N_{\mathrm{b}}}{64 \pi^{2} \sigma_{z} \sigma_{p} \sigma_{x} \sigma_{y} \sigma_{x^{\prime}} \sigma_{y^{\prime}} \beta^{3} \gamma^{4}}
$$

with the standard dimensions of a Gaussian distribution for the bunch length $\sigma_{z}$, the relative momentum spread $\sigma_{p}$, horizontal and vertical betatron amplitudes $\left(\sigma_{x}, \sigma_{y}\right)$ and divergences $\left(\sigma_{x^{\prime}}, \sigma_{y^{\prime}}\right)$ and number of particles per bunch $N_{\mathrm{b}}$. The constants $r_{\mathrm{c}}$ and $\beta=v / c$, finally, are the classical particle radius and velocity in units of the velocity of light.

The function

$$
f(a, b, c)=8 \pi \int_{0}^{1}\left\{\ln \left[\frac{c^{2}}{2}\left(\frac{1}{\sqrt{p}}+\frac{1}{\sqrt{q}}\right)\right]-0.577 . .\right\} \frac{1-3 x^{2}}{\sqrt{p q}} \mathrm{~d} x
$$

where

$$
\begin{array}{ll}
p=a^{2}+x^{2}\left(1-a^{2}\right), & q=b^{2}+x^{2}\left(1-b^{2}\right), \\
a=\frac{\sigma_{h}}{\gamma \sigma_{x^{\prime}}}, & b=\frac{\sigma_{h}}{\gamma \sigma_{y^{\prime}}}, \\
\sigma_{h}^{2}=\frac{\sigma_{p}^{2} \sigma_{x}^{2}}{\sigma_{x}^{2}+\eta^{2} \sigma_{p}^{2}}, & c^{2}=\beta^{2} \sigma_{h}^{2} \frac{\sqrt{2 \pi} \sigma_{y}}{r_{\mathrm{c}}} .
\end{array}
$$


The transverse emittance growth times are similarly given by

$$
\tau_{x}^{-1}=\frac{1}{2 \sigma_{x}^{2}} \frac{\mathrm{d} \sigma_{x}^{2}}{\mathrm{~d} t}=A\left[f\left(\frac{1}{a}, \frac{b}{a}, \frac{c}{a}\right)+\frac{\eta^{2} \sigma_{p}^{2}}{\sigma_{x}} f(a, b, c)\right],
$$

and

$$
\tau_{y}^{-1}=\frac{1}{2 \sigma_{y}^{2}} \frac{\mathrm{d} \sigma_{y}^{2}}{\mathrm{~d} t}=A f\left(\frac{1}{b}, \frac{a}{b}, \frac{c}{b}\right) .
$$

These expressions allow the calculations of the emittance growth rate, which for most electron accelerators is small compared to radiation damping but become significant in proton and ion storage rings where high particle densities and long storage times are desired. Progress in the design of modern synchrotron radiation facilities allow ever smaller emittances which have reached a level where intrabeam scattering is significant again. From the density factor $A$ it is apparent that high particle density in six-dimensional phase space increases the growth rates while this effect is greatly reduced at higher beam energies.

\subsection{Collective Self Fields}

The electric charges of a particle beam can become a major contribution to the forces encountered by individual particles while travelling along a beam transport line or orbiting in a circular accelerator. These forces may act directly from beam to particle or may originate from electromagnetic fields being excited by the beam interaction with its surrounding vacuum chamber. In this section, we will derive expressions for the fields from a collection of particles and determine the force due to these fields on an individual test particle. We use the particle charge $q$ rather than the elementary charge $e$ to cover particles with multiple charges like ions for which $q=e Z$. For all cases to be correct, we should distinguish between the electrical charge of particles in the beam and that of the individual test particle. This, however, would significantly complicate the expressions and we use therefore the same charge for both the beam and test particle. In a particular situation whenever particles of different charges are considered, the sign and value of the charge factors in the formulas must be reconsidered.

Individual particles in an intense beam are under the influence of strong repelling electrostatic forces creating the possibility of severe stability problems. Particle beam transport over long distances could be greatly restricted unless these spacecharge forces can be kept under control. First, it is interesting to calculate the magnitude of the problem.

If all particles would be at rest within a small volume, we would clearly expect the particles to quickly diverge from the center of charge under the influence of the 
repelling forces from the other particles. This situation may be significantly different in a particle beam where all particles propagate in the same direction.

In Sect. 1.5.10 we obtained the encouraging result that at least relativistic particle beams become stable under the influence of their own fields. For lower particle energies, however, significant diverging forces must be expected and adequate focusing measures must be applied. The physics of such space charge dominated beams is beyond the scope of this book and is treated elsewhere, for example in considerable detail in [16].

\subsubsection{Self Field for Elliptical Particle Beams}

The self fields of a beam depend on beam parameters like particle type, particle distribution, bunching, and energy of the particle. Here, we will derive the nature and effect of these self fields in a more restricted way for common particle beam cases in accelerators.

To determine self fields, we consider a continuous beam of particles with a line charge $\lambda$, or a volume charge $\rho(x, y)$. The electric fields within a beam are derived from a potential $V$ defined by

$$
\triangle V=-\frac{1}{\epsilon_{0}} \rho(x, y),
$$

where $\rho$, being the electric charge density in the beam, is finite within and zero outside the beam. Similarly, the magnetic vector potential is defined by

$$
\boldsymbol{\Delta A}=-\frac{1}{\epsilon_{0}} \boldsymbol{v} \rho(x, y) .
$$

For a particle beam, we may set $\boldsymbol{v} \approx(0,0, v)$ and the vector potential therefore contains only a longitudinal component $\boldsymbol{A}=\left(0,0, A_{z}\right)$.

In Sect. 1.5.10 we discussed the self fields of a round beam. Generally, however, particle beams have an elliptical cross section and the solution to (21.15) for such a beam with constant charge density ( $\rho=$ const) has been derived by Teng [17, 18]. Within the elliptical beam cross section, where $x \leq a$ and $y \leq b$, the electric potential is

$$
V(x, y)=-\frac{1}{2 \epsilon_{0}} \rho \frac{a b}{a+b}\left[\frac{x^{2}}{a}+\frac{y^{2}}{b}\right]
$$

and $a, b$ are the horizontal and vertical half axis respectively. The vector potential for the magnetic field is from the discussions above

$$
A_{z}(x, y)=-\frac{1}{2 \epsilon_{0}} \rho \frac{v}{c} \frac{a b}{a+b}\left[\frac{x^{2}}{a}+\frac{y^{2}}{b}\right]
$$


and both the electric and magnetic field can be derived by simple differentiations

$$
\mathbf{E}=-\nabla V \quad \text { and } \quad \mathbf{B}=\nabla \times \mathbf{A}
$$

for

$$
E_{x}=\frac{1}{4 \pi \epsilon_{0}} \frac{4 q \lambda}{a(a+b)} x, \quad E_{y}=\frac{1}{4 \pi \epsilon_{0}} \frac{4 q \lambda}{b(a+b)} y,
$$

and

$$
B_{x}=-\frac{c \mu_{0}}{4 \pi} \frac{4 q \lambda \beta}{b(a+b)} y, \quad B_{y}=\frac{c \mu_{0}}{4 \pi} \frac{4 q \lambda \beta}{a(a+b)} x,
$$

where $\beta=v / c$ and the linear charge density $\lambda$ is defined by

$$
\lambda=\pi a b \rho(x, y) .
$$

Comparing (21.20) and (21.21) reveals the relationship between electric and magnetic self fields of the beam to be (21.22)

$$
c B_{x}=-\beta E_{y}, \quad c B_{y}=+\beta E_{x} .
$$

The electric as well as the magnetic field scales linearly with distance from the beam center and therefore both cause focusing and a tune shift in a circular accelerator.

In many applications it is not acceptable to assume a uniform transverse charge distribution. Most particle beams either have a bell shaped particle distribution or a Gaussian distribution as is specially the case for electrons in circular accelerators. We therefore use in the transverse plane a Gaussian charge distribution given by

$$
\rho(x, y)=\frac{\lambda}{2 \pi \sigma_{x} \sigma_{y}} \exp \left[-\frac{x^{2}}{2 \sigma_{x}^{2}}-\frac{y^{2}}{2 \sigma_{y}^{2}}\right],
$$

which also well describes a beam with bell shaped distribution. Although many particle beams, but specifically electron beams, come in bunches with a Gaussian distribution in all degrees of freedom, we will only introduce a bunching factor for the longitudinal particle distribution and refer the interested reader for the study of a fully six dimensional Gaussian charge distribution to reference [19].

The potential for a transverse bi-Gaussian charge distribution (21.24) can be expressed by [18]

$$
V(x, y)=-\frac{e}{4 \pi \epsilon_{0}} \lambda \int_{0}^{\infty} \frac{1-\exp \left[-\frac{x^{2}}{2\left(\sigma_{x}^{2}+t\right)}-\frac{y^{2}}{2\left(\sigma_{y}^{2}+t\right)}\right]}{\sqrt{\left(\sigma_{x}^{2}+t\right)\left(\sigma_{y}^{2}+t\right)}} \mathrm{d} t
$$


Equation (21.25) can be verified by back insertion into (21.15). From this potential we obtain for example the vertical electric field component by differentiation

$$
E_{y}=-\frac{\partial V(x, y)}{\partial y}=\frac{e}{4 \pi \epsilon_{0}} \lambda y \int_{0}^{\infty} \frac{\exp \left[-\frac{x^{2}}{2\left(\sigma_{x}^{2}+t\right)}-\frac{y^{2}}{2\left(\sigma_{y}^{2}+t\right)}\right]}{\left(\sigma_{y}^{2}+t\right) \sqrt{\left(\sigma_{x}^{2}+t\right)\left(\sigma_{y}^{2}+t\right)}} \mathrm{d} t
$$

No closed analytical expression exists for these integrals unless we restrict ourselves to a symmetry plane with $x=0$ or $y=0$ and small amplitudes $y \ll \sigma_{y}$ or $x \ll \sigma_{x}$ respectively. These assumptions are appropriate for most space-charge effects and the potential in the vertical midplane becomes

$$
V\left(x=0, y \ll \sigma_{y}\right)=-\frac{1}{4 \pi \epsilon_{0}} \frac{\lambda}{\sigma_{y}\left(\sigma_{x}+\sigma_{y}\right)} y^{2} .
$$

For reasons of symmetry a similar expression can be derived for the horizontal mid plane by merely interchanging $x$ and $y$ in (21.27). The associated electric fields are for $x=0$ and $y \ll \sigma_{y}$

$$
E_{x}=\frac{1}{4 \pi \epsilon_{0}} \frac{2 \lambda}{\sigma_{x}\left(\sigma_{x}+\sigma_{y}\right)} x, \quad E_{y}=\frac{1}{4 \pi \epsilon_{0}} \frac{2 \lambda}{\sigma_{y}\left(\sigma_{x}+\sigma_{y}\right)} y,
$$

and the magnetic fields according to (21.23) are from (21.28)

$$
B_{x}=-\frac{c \mu_{0}}{4 \pi} \frac{2 \lambda \beta}{\sigma_{y}\left(\sigma_{x}+\sigma_{y}\right)} y, \quad B_{y}=+\frac{c \mu_{0}}{4 \pi} \frac{2 \lambda \beta}{\sigma_{x}\left(\sigma_{x}+\sigma_{y}\right)} x .
$$

All fields increase linearly with amplitude and we note that the field components in the horizontal midplane are generally much smaller compared to those in the vertical midplane because most particle beams in circular accelerators are flat and $\sigma_{y} \ll \sigma_{x}$.

\section{Forces from Space-Charge Fields}

The electromagnetic self fields generated by the collection of all particles within a beam exert forces on individual particles of the same beam or of another beam. The Lorentz force due to these fields can be expressed by

$$
\boldsymbol{F}=e \boldsymbol{E} f_{\mathrm{e}}+e[\boldsymbol{v} \times \boldsymbol{B}] f_{\mathrm{e}} f_{\mathrm{v}},
$$

where we have added to the usual expression for the Lorentz force the factors $f_{\mathrm{e}}$ and $f_{\mathrm{v}}$. Because the fields act differently depending on the relative directions and charge of beam and individual particle distinct combinations occur. We set $f_{\mathrm{e}}=1$ if both the beam particles and the test particle have the same sign of their charge and 
Table 21.1 Self field force factors

\begin{tabular}{c|c|c|c}
\hline$++\uparrow \uparrow$ & $+-\uparrow \uparrow$ & $++\uparrow \downarrow$ & $+-\uparrow \downarrow$ \\
\hline$--\uparrow \uparrow$ & $-+\uparrow \uparrow$ & $--\uparrow \downarrow$ & $-+\uparrow \downarrow$ \\
\hline$+\left(1-\beta^{2}\right)$ & $-\left(1-\beta^{2}\right)$ & $+\left(1+\beta^{2}\right)$ & $-\left(1+\beta^{2}\right)$ \\
\hline
\end{tabular}

$f_{\mathrm{e}}=-1$ if their charges are of opposite sign. Similarly we set $f_{\mathrm{v}}=1$ or $f_{\mathrm{v}}=-1$ depending on whether the beam and test particle have the same or opposite direction of movement with respect to each other.

The vertical force from the self field, for example, of a proton beam on an individual proton within the same beam moving with the same velocity is from (21.30)

$$
F_{y}(\uparrow \uparrow,++)=+e\left(1-\beta^{2}\right) E_{y} .
$$

An antiproton moving in the opposite direction through a proton beam would feel the vertical force

$$
F_{y}(\uparrow \downarrow,+-)=-e\left(1+\beta^{2}\right) E_{y} .
$$

Expansion to other combinations of particles and directions of velocities are straightforward. For ions the charge multiplicity $Z$ must be added to the fields or the individual particle or both depending on the case. The possible combinations of the force factors $\pm\left(1 \pm \beta^{2}\right)$ are summarized in Table 21.1.

The \pm -signs in Table 21.1 indicate the charge polarity of beam and test particle and the arrows the relative direction. We note a great difference between the case, where particles move in the same direction, and the case of beams colliding head on.

\subsubsection{Beam-Beam Effect}

In colliding beam facilities two counter rotating beams within one storage ring or counter rotating beams from two intersecting storage rings are brought into collision to create a high center of mass energy at the collision point which transforms into known or unknown particles to be studied by high energy experimentalists. The event rate is given by the product of the cross section for the particular event and the luminosity which is determined by storage ring operating conditions. By definition, the luminosity is the density of collision centers in the target multiplied by the number of particles colliding with this target per unit time. In the case of a colliding beam facility a bunch of one beam is the target for the other beam. For simplicity we assume here that both beams have the same cross section. We also assume that 
each beam consists of $n_{\mathrm{b}}$ bunches. In this case the luminosity is

$$
\mathcal{L}=\frac{N_{1}}{n_{\mathrm{b}} A} N_{2} v_{\text {rev }}
$$

where $N_{1}$ and $N_{2}$ are the total number of particles in each beam, $A$ the cross section of the beams, and $v_{\text {rev }}$ the revolution frequency in the storage ring. In most storage rings the transverse particle distribution is Gaussian or bell shaped and since only the core of the beam contributes significantly to the luminosity we may define standard beam sizes for all kinds of particles. For a Gaussian particle distribution the effective beam cross section is

$$
A_{\mathrm{g}}=4 \pi \sigma_{x} \sigma_{y}
$$

and the luminosity

$$
\mathcal{L}=\frac{N_{1}}{4 \pi \sigma_{x} \sigma_{y} B} N_{2} \nu_{\text {rev }} .
$$

The recipe for high luminosity is clearly to maximize the beam intensity and to minimize the beam cross section. This approach, however, fails because of the beam-beam effect which, due to electromagnetic fields created by the beams themselves, causes a tune shift and therefore limits the amount of beam that can be brought into collision in a storage ring. The beam-beam effect has first been recognized and analyzed by Amman and Ritson [20].

In case of counter rotating beams colliding at particular interaction points in a colliding-beam facility, we always have $f_{\mathrm{v}}=-1$ but the colliding particles still may be of equal or opposite charge. In addition, there is no contribution from magnetic image fields since collisions do not occur within magnets. Even image fields from vacuum chambers are neglected because the beam-beam interaction happens only over a very short distance. A particle in one beam will feel the field from the other beam only during the time it travels through the other beam which is equal to the time it takes the particle to travel half the effective length of the oncoming bunch. With these considerations in mind, we obtain for the beam-beam tune shift in the vertical plane from (21.63) with $f_{\text {corr }}=1$ and assuming head on collisions of particle-antiparticle beams $\left(f_{\mathrm{e}}=-1\right)$

$$
\Delta v_{y, \mathrm{bb}}=\frac{r_{\mathrm{c}} N_{\mathrm{tot}}}{2 \pi B \gamma} \frac{\beta_{y}^{*}}{\sigma_{y}^{*}\left(\sigma_{x}^{*}+\sigma_{y}^{*}\right)}
$$

and in the horizontal plane

$$
\Delta v_{x, \mathrm{bb}}=\frac{r_{\mathrm{c}} N_{\mathrm{tot}}}{2 \pi B \gamma} \frac{\beta_{x}^{*}}{\sigma_{x}^{*}\left(\sigma_{x}^{*}+\sigma_{y}^{*}\right)},
$$


where ${ }^{*}$ indicates that the quantities be taken at the interaction point. In cases where other particle combinations are brought into collision or when both beams cross under an angle these equations must be appropriately modified to accurately describe the actual situation.

From (21.32) and (21.28)we find for two counter rotating beams of particle and antiparticle a vertical beam-beam force of

$$
F_{y}=-\frac{1}{4 \pi \epsilon_{0}} \frac{e\left(1+\beta^{2}\right) 2 \lambda}{\sigma_{y}\left(\sigma_{x}+\sigma_{y}\right)} y .
$$

This force is attractive and therefore focusing, equivalent to that of a quadrupole of strength

$$
k=-\frac{F_{y} / y}{c^{2} \beta^{2} \gamma m}
$$

causing a vertical tune shift of

$$
\delta v_{y}=\frac{1}{4 \pi} \int_{\text {coll }} \beta_{y} k \mathrm{~d} z .
$$

Integrating over the collision length which is equal to half the bunch length $\ell$ because colliding beams move in opposite directions, we note that the linear charge density is $\lambda=e N /(B \ell)$, where $N$ is the total number of particles per beam and $B$ the number of bunches per beam. With these replacements the beam tune shift becomes finally

$$
\delta v_{y}=\frac{r_{\mathrm{c}} N \beta_{y}}{2 \pi B \gamma \sigma_{y}\left(\sigma_{x}+\sigma_{y}\right)},
$$

where $r_{\mathrm{c}}$ is the classical particle radius of the particle which is being disturbed. Obviously, the tune shift scales linear with particle intensity or particle beam current and inversely with the beam cross section. Upon discovery of this effect it was thought that the particle beam intensity is limited when the tune shift is of the order of $\approx 0.15-0.2$ which is the typical distance to the next resonance. Experimentally, however, it was found that the limit is much more restrictive with maximum tune shift values of $\approx 0.04-0.06$ for electrons [20-23] and less for proton beams [24].

A definitive quantitative description of the actual beam-beam effect has not been possible yet due to its highly nonlinear nature. Only particles with very small betatron oscillation amplitudes will experience the linear tune shift derived above. For betatron oscillations larger than one $\sigma$, however, the field becomes very nonlinear turning over to the well known $1 / r$-law at large distances from the beam center. 
In spite of the inability to quantitatively describe the beam-beam effect by the linear tune shift it is generally accepted practice to quantify the beam-beam limit by the value of the linear tune shift. This is justified since the nonlinear fields of a particle beam are strictly proportional to the linear field and therefore the linear tune shift is a good measure for the amount of nonlinear fields involved.

\subsubsection{Transverse Self Fields}

Expressions for space-charge fields originating from a beam of charged particles have been derived earlier and we obtained for a Gaussian transverse distribution of particles with charge $q$ the electric fields in (21.28) and the magnetic fields in (21.29).

The local linear particle density $\lambda$ is defined by

$$
\lambda(z)=\iint \rho(x, y, z) \mathrm{d} x \mathrm{~d} y,
$$

where $\rho(x, y, z)$ is the local particle density normalized to the total number of particles in the beam $\int_{-\infty}^{\infty} \lambda(z) \mathrm{d} z=N_{\mathrm{p}}$. With these fields and the Lorentz equation, we formulate the transverse force acting on a single particle within the same particle beam. Since both expressions for the electrical and magnetic field differ only by the factor $\beta$ we may, for example, derive from the Lorentz equation the vertical force on a particle with charge $q$

$$
F_{y}=q\left(1-\beta^{2}\right) E_{y}=\frac{1}{4 \pi \epsilon_{0}} \frac{2 q \lambda}{\gamma^{2} \sigma_{y}\left(\sigma_{x}+\sigma_{y}\right)} y .
$$

The space-charge force appears at its strongest for nonrelativistic particles and diminishes quickly like $1 / \gamma^{2}$ for relativistic particles. In accelerator physics, however, particle beams are carried from low to high energies and therefore spacecharge effects may become important during some or all phases of acceleration. This is specifically true for heavy particles like protons and ions for which the relativistic parameter $\gamma$ is rather low for most any practically achievable particle energies.

\subsubsection{Fields from Image Charges}

Discussing space charges, we ignored so far the effect of metallic and magnetic surfaces close to the beam. The electromagnetic self fields of the beam circulating in a metallic vacuum chamber and between ferromagnetic poles of magnets must meet certain boundary conditions on such surfaces. Laslett [25] derived appropriate 
corrections to free space electromagnetic fields by adding the electromagnetic fields from all image charges to the fields of the particle beam itself.

Following his reasoning, we consider a particle beam with metallic and ferromagnetic boundaries as shown in Fig. 21.2. For full generality, let the elliptical particle beam be displaced in the vertical plane by $\bar{y}$ from the midplane, the metallic vacuum chamber and magnet pole are simulated as pairs of infinitely wide parallel surfaces at $\pm b$ and $\pm g$, respectively, and the observation point of the fields be at $y$. The linear particle density is

$$
\lambda=\frac{N_{\text {tot }}}{n_{\mathrm{b}} \ell_{\mathrm{b}}}=\frac{N_{\mathrm{tot}}}{n_{\mathrm{b}} \sqrt{2 \pi} \sigma_{\ell}},
$$

where $N_{\text {tot }}$ is the total number of particle in the circulating beam, $n_{\mathrm{b}}$ the number of bunches, $\ell_{\mathrm{b}}=\sqrt{2 \pi} \sigma_{\ell}$ the effective bunch length and $\sigma_{\ell}$ the standard bunch length for a Gaussian distribution.

The locations and strength of the electrical images of a line current in the configuration of Fig. 21.2 are shown in Fig. 21.3. The boundary condition for electric fields is $E_{z}(b)=0$ on the surface of the metallic vacuum chamber and is satisfied if the image charges change sign from image to image. To calculate the

Fig. 21.2 Particle beam with metallic and ferromagnetic boundaries

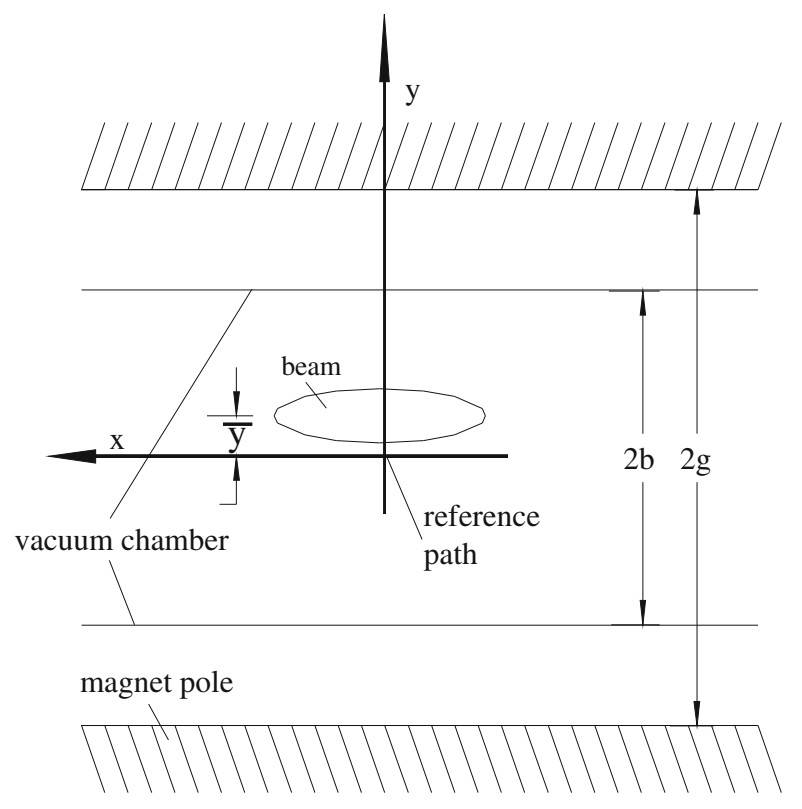


Fig. 21.3 Location and source of image fields

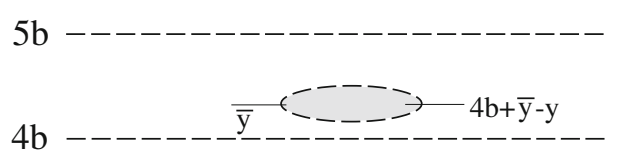

$3 b$
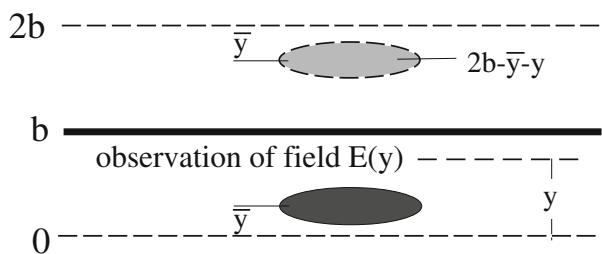

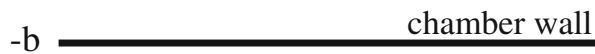
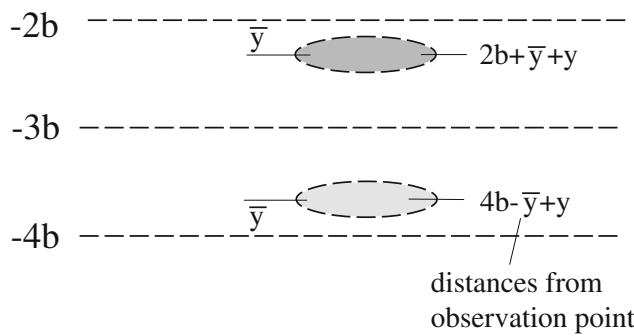

electrical field $E_{y}(y)$, with (21.28) we add the contributions from all image fields in the infinite series

$$
\begin{aligned}
E_{y, \text { image }}(y)= & \frac{1}{4 \pi} \epsilon_{0} 2 \lambda \\
& \times\left(\frac{1}{2 b-\bar{y}-y}-\frac{1}{2 b+\bar{y}+y}-\frac{1}{4 b+\bar{y}-y}+\frac{1}{4 b-\bar{y}+y}\right. \\
& +\frac{1}{6 b-\bar{y}-y}-\frac{1}{6 b+\bar{y}+y}-\frac{1}{8 b+\bar{y}-y}+\frac{1}{8 b-\bar{y}+y} \\
& \left.+\frac{1}{10 b-\bar{y}-y}-\frac{1}{10 b+\bar{y}+y}-\ldots\right)
\end{aligned}
$$

These image fields must be added to the direct field of the line charge to meet the boundary condition that the electric field enter metallic surfaces perpendicular. Equation (21.45) can be split into two series with factors $(\bar{y}+y)$ and $(\bar{y}-y)$ in the 
numerator. We get after some manipulations with $\bar{y}+y \ll b$ and $\bar{y}-y \ll b$

$$
\begin{aligned}
E_{y, \text { image }}(y) & =\frac{1}{4 \pi \epsilon_{0}} \frac{\lambda}{b^{2}}\left[\sum_{m=1}^{\infty} \frac{\bar{y}+y}{(2 m-1)^{2}}+\sum_{m=1}^{\infty} \frac{\bar{y}-y}{4 m^{2}}\right], \\
& =\frac{1}{4 \pi \epsilon_{0}} \frac{\lambda}{b^{2}}\left[(\bar{y}+y) \frac{\pi^{2}}{8}+(\bar{y}-y) \frac{\pi^{2}}{24}\right], \\
& =\frac{1}{4 \pi \epsilon_{0}} \frac{\lambda}{b^{2}} \frac{\pi^{2}}{12}(2 \bar{y}+y)=\frac{1}{4 \pi \epsilon_{0}} \frac{4 q \lambda}{b^{2}} \epsilon_{1}(2 \bar{y}+y) .
\end{aligned}
$$

The electric image fields depend linearly on the deviations $\bar{y}$ and $y$ from the axis of bunch center and test particle, respectively, and act therefore like a quadrupole causing a tune shift.

A similar derivation is used to get the magnetic image fields due to ferromagnetic surfaces at $\pm g$ above and below the midplane. The magnetic field lines must enter the magnetic pole faces perpendicular and the image currents therefore flow in the same direction as the line current causing a magnetic force on the test particle which is opposed to that by the magnetic field of the beam itself.

Bunched beams generate high frequency electromagnetic fields which do not reach ferromagnetic surfaces because of eddy current shielding by the metallic vacuum chamber. For magnetic image fields we distinguish therefore between dc and ac image fields. The dc Fourier component of a bunched beam current is equal to twice the average beam current $c \beta \lambda B$, where the Laslett bunching factor $B$ is the bunch occupation along the ring circumference defined by

$$
B=\frac{\bar{\lambda}}{\lambda}=\frac{n_{\mathrm{b}} \ell_{\mathrm{b}}}{2 \pi R} .
$$

The dc magnetic image fields are derived similar to electric image fields with $B_{\varphi}=-2 \lambda \beta / r$ from (21.29) and are with (21.47)

$$
\begin{aligned}
B_{x, \text { image, dc }}(y) & =\frac{c \mu_{0}}{4 \pi} \frac{2 \lambda \beta}{g^{2}} B\left[\sum_{m=1}^{\infty} \frac{\bar{y}+y}{(2 m-1)^{2}}+\sum_{m=1}^{\infty} \frac{\bar{y}-y}{4 m^{2}}\right] \\
& =\frac{c \mu_{0}}{4 \pi} \frac{\lambda \beta}{g^{2}} B\left[(\bar{y}+y) \frac{\pi^{2}}{8}+(\bar{y}-y) \frac{\pi^{2}}{24}\right] \\
& =\frac{c \mu_{0}}{4 \pi} \frac{4 \lambda \beta}{g^{2}} B \epsilon_{2}(2 \bar{y}+y) .
\end{aligned}
$$

The magnetic image fields must penetrate the metallic vacuum chamber to reach ferromagnetic poles. This is no problem for dc or low frequency field components but in case of bunched beams relevant frequencies are rather high and eddy current shielding of the vacuum chamber for ac magnetic fields must be taken into account. 
In most cases we may assume that they do not penetrate the thick metallic vacuum chamber. Consequently, we ignore here the effect of ferromagnetic poles and consider only the contribution of magnetic ac image fields due to eddy currents in vacuum chamber walls. Similar to electric image fields, the magnetic image fields are in analogy to (21.46)

$$
\begin{aligned}
B_{x, \text { image }, \mathrm{ac}}(y) & =-\frac{c \mu_{0}}{4 \pi} \frac{\lambda \beta}{b^{2}}(1-B) \frac{\pi^{2}}{12}(2 \bar{y}+y), \\
& =-\frac{c \mu_{0}}{4 \pi} \frac{4 \lambda \beta}{b^{2}}(1-B) \epsilon_{1}(2 \bar{y}+y),
\end{aligned}
$$

where the factor $(1-B)$ accounts for the subtraction of the dc component $\beta \lambda B$. Similar to the electric image fields, the magnetic image fields must be added to the direct magnet fields (21.29) from the beam current to meet the boundary condition of normal field components at ferromagnetic surfaces. The coefficients $\epsilon_{1}$ and $\epsilon_{2}$ are the Laslett form factors which are for infinite parallel plate vacuum chambers and magnetic poles

$$
\epsilon_{1}=\frac{\pi^{2}}{48} \quad \text { and } \quad \epsilon_{2}=\frac{\pi^{2}}{24} .
$$

The vacuum chamber and ferromagnetic poles are similar to infinitely wide surfaces. While this is a sufficiently accurate approximation for the magnet poles, corrections must be applied for circular or elliptical vacuum chambers. Laslett [25] has derived what we call now Laslett form factors for vacuum chambers with elliptical cross sections and variable aspect ratios which are compiled in Table 21.2.

All relevant field components have been identified and we collect these fields first for $\bar{y}=0$ and obtain from (21.28), (21.46) for the electric field in the vertical mid plane

$$
E_{y}(y)=\frac{c^{2} \mu_{0}}{4 \pi} \frac{2 \lambda}{\sigma_{y}\left(\sigma_{x}+\sigma_{y}\right)}\left[1+\frac{2 \sigma_{y}\left(\sigma_{x}+\sigma_{y}\right)}{b^{2}} \epsilon_{1}\right] y .
$$

From (21.29), (21.48) the dc magnetic field is

$$
B_{x, \mathrm{dc}}=-\frac{c \mu_{0}}{4 \pi} \frac{2 \lambda \beta B}{\sigma_{y}\left(\sigma_{x}+\sigma_{y}\right)}\left[1-\frac{2 \sigma_{y}\left(\sigma_{x}+\sigma_{y}\right)}{g^{2}} \epsilon_{2}\right] y
$$

Table 21.2 Laslett incoherent tune shift form factors for elliptical vacuum chambers

\begin{tabular}{l|l|l|l|l|l|l}
\hline$a / b:^{\mathrm{a}}$ & 1 & $5 / 4$ & $4 / 3$ & $3 / 2$ & $2 / 1$ & $\infty$ \\
\hline$\epsilon_{1}:$ & 0 & 0.090 & 0.107 & 0.134 & 0.172 & 0.206 \\
\hline
\end{tabular}
a $a$ is the horizontal and $b$ the vertical half-axis of an
elliptical vacuum chamber


and from (21.49) the ac magnetic field

$$
B_{x, \mathrm{ac}}=-\frac{c \mu_{0}}{4 \pi} \frac{2 \lambda \beta}{\sigma_{y}\left(\sigma_{x}+\sigma_{y}\right)}\left[1+\frac{2 \sigma_{y}\left(\sigma_{x}+\sigma_{y}\right.}{b^{2}} \epsilon_{1}\right](1-B) y .
$$

Tacitly, we have assumed that the transverse particle distribution is Gaussian which is a true representation of an electron beam but may not be correct for proton or ion beams. The standard deviations $\sigma$ of a Gaussian distribution are very well defined and can therefore be replaced by other quantities like the full-width half maximum or as the particle distribution may require.

The electromagnetic force due to space charge on individual particles in a beam has been derived and it became obvious that image field effects can play a significant role in the perturbation of the beam. The fields scale linear with amplitude for very small amplitudes and act therefore like focusing quadrupoles. At larger amplitudes, however, the fields reach a maximum and then evanesce like $1 / r$. Consequently, the field gradient is negative decaying quickly with amplitude.

A complete set of direct and image fields have been derived which must be considered to account for space-charge effects. Similar derivations lead to other field components necessary to determine horizontal space-charge forces. In most accelerators, however, the beam cross section is flat and so is the vacuum chamber and the magnet pole aperture. As a consequence, we expect the space-charge forces to be larger in the vertical plane than in the horizontal plane.

\subsubsection{Space-Charge Effects}

The Lorentz force on individual particles can be calculated from the space-charge fields and we get

$$
F_{y}=\frac{1}{4 \pi \epsilon_{0}} \frac{2 f_{\mathrm{p}} \lambda\left(1-\beta^{2} f_{\mathrm{v}}\right)}{\sigma_{y}\left(\sigma_{x}+\sigma_{y}\right)} f_{\text {corr }} y=q \mathcal{F} y,
$$

where the correction factor due to image fields is with $\beta^{2} \gamma^{2}=\gamma^{2}-1$,

$$
f_{\text {corr }}=1+\frac{2 \sigma_{y}\left(\sigma_{x}+\sigma_{y}\right)}{b^{2}} \epsilon_{1}\left[1+\left(\gamma^{2}-1\right) B\right]+\epsilon_{2}\left(\gamma^{2}-1\right) \frac{b^{2}}{g^{2}} B
$$

and

$$
\mathcal{F}=\frac{1}{4 \pi \epsilon_{0}} \frac{2 f_{\mathrm{p}} \lambda\left(1-\beta^{2} f_{\mathrm{v}}\right)}{\sigma_{y}\left(\sigma_{x}+\sigma_{y}\right)} f_{\text {corr }},
$$

The factors $f_{\mathrm{p}}$ and $f_{\mathrm{v}}$ determine signs depending on the kind of particles interacting and the direction of travel with respect to each other. Specifically, $f_{\mathrm{p}}=\operatorname{sign}\left(q q_{\mathrm{b}}\right)$ 
where $q$ is the charge of a test particle and $q_{\mathrm{b}}$ the charge of the field creating particles, e.g. the charge of a bunch. Similarly, $f_{\mathrm{v}}=\operatorname{sign}\left(\boldsymbol{v} \boldsymbol{v}_{\mathrm{b}}\right)$ where $\boldsymbol{v}$ is the direction of travel for the test particle and $\mathbf{v}_{\mathrm{b}}$ the direction of travel of the bunch. To calculate the space-charge force of head-on colliding proton and antiproton beams, for example, we would set $f_{\mathrm{p}}=-1$ and $f_{\mathrm{v}}=-1$.

There is a significant cancellation of two strong terms, the repulsive electrical field and the focusing magnetic field, expressed by the factor $1-\beta^{2}$ for spacecharge forces within a highly relativistic beam. This cancellation can be greatly upset if particle beams become partially neutralized by collecting other particles of opposite charge within the beams potential well. For example, proton beams can trap electrons in the positive potential well as can electron beams trap positive ions in the negative potential well. To avoid such partial neutralization and appearance of unnecessarily strong space-charge effects, clearing electrodes must be installed over much of the ring circumference to extract with electrostatic fields low energy electrons or ions from the particle beam.

The electromagnetic space-charge force on an individual particle within a particle beam increases linearly with its distance from the axis. A similar force occurs for the horizontal plane and both fields therefore act like a quadrupole causing a tune shift. This has been recognized and analyzed early by Kerst [26] and Blewett [27]. A complete treatment of space charge dominated beams can be found in [16]. The equation of motion under the influence of space charge forces can be written in the form

$$
m \gamma \ddot{u}+D u=\frac{\partial F_{u}}{\partial u} u \quad \text { with } \quad u=(x, y) .
$$

We get the regular form $u^{\prime \prime}+\left(k_{0}+\Delta k\right) u=0$ with $\ddot{u}=u^{\prime \prime}(c \beta)^{2}$ and $f_{v}=1$, where $k_{0}$ describes the quadrupole strength and the space-charge strength is expressed by

$$
\Delta k=\frac{1}{m c^{2} \gamma \beta^{2}} \frac{\partial F_{u}}{\partial u}=-\frac{2 r_{\mathrm{c}}}{\beta^{2} \gamma^{3}} \frac{\lambda}{\sigma_{y}\left(\sigma_{x}+\sigma_{y}\right)} f_{\mathrm{corr}}
$$

where $r_{\mathrm{c}}$ is the classical particle radius. For ions with charge multiplicity $Z$ and atomic number $A$ the classical particle radius is $r_{\text {ion }}=r_{\mathrm{p}} Z^{2} / A$.

\section{Space Charge Dominated Beams}

So far, space-charge effects or space-charge focusing has been consistently neglected in the discussions on transverse beam dynamics. In cases of low beam energy and high particle densities, it might become necessary to include spacecharge effects. They are defocusing in both planes and compensation therefore requires additional focusing in both planes. However, it should be noted that particles closer to the beam surface will not experience the same linear space-charge defocusing as those near the axis and therefore a compensation of space-charge 
focusing works only for part of the beam. Here, we will not get involved with the dynamics of heavily space charge dominated particle beams but try to derive a criterion by which we can decide whether or not space-charge forces are significant in transverse particle beam optics.

This distinction becomes obvious from the equation of motion including space charges. From (21.57), (21.58) we get the equation of motion

$$
u^{\prime \prime}+\left[k_{0}-\frac{2 r_{\mathrm{c}}}{\beta^{2} \gamma^{3}} \frac{\lambda}{\sigma_{y}\left(\sigma_{x}+\sigma_{y}\right)} f_{\text {corr }}\right] u=0,
$$

where we ignored the image current corrections. Space-charge forces can be neglected if the integral of the space-charge force over a length $L$ which is characteristic for the average distance between quadrupoles in the beam line is small compared to the typical integrated quadrupole length $k_{0} \ell_{\mathrm{q}}$ or if

$$
\frac{2 r_{\mathrm{c}}}{\beta^{2} \gamma^{3}} \int_{L} \frac{\lambda f_{\text {corr }}}{\sigma_{y}\left(\sigma_{x}+\sigma_{y}\right)} \mathrm{d} z \ll k_{0} \ell_{\mathrm{q}} .
$$

The effect of space-charge focusing is most severe where the beam cross section is smallest and (21.60) should therefore be applied specifically to such sections of the beam transport line. Obviously, the application of this formula requires some subjective judgement as to how much smaller space-charge effects should be. To aid this judgement, one might also calculate the average betatron phase shift caused by space-charge forces and compare it with the total phase advance along the beam line under investigation. In this case we look for

$$
\frac{2 r_{\mathrm{c}}}{\beta^{2} \gamma^{3}} \int_{L} \frac{\beta_{u} \lambda f_{\text {corr }}}{\sigma_{y}\left(\sigma_{x}+\sigma_{y}\right)} \mathrm{d} z \ll \psi_{0}(L)
$$

to determine the severity of space-charge effects. The nominal phase advance $\psi_{0, u}(L)$ is defined such that $\psi_{0, u}(0)=0$ at the beginning of the beam line.

\section{Space-Charge Tune Shift}

Space-charge focusing may not significantly perturb the lattice functions but may cause a big enough tune shift in a circular accelerator moving the beam onto a resonance. The beam current is therefore limited by the maximum allowable tune shift in the accelerator which is for a linear focusing force $F(z)$ given by

$$
\Delta v_{u}=-\frac{1}{4 \pi} \frac{r_{\mathrm{c}}}{\beta^{2} \gamma} \int_{0}^{L_{\mathrm{int}}} F(z) \beta_{u} \mathrm{~d} z .
$$

The integration in (21.62) is taken over that part of the path in each revolution where the force is effective. For the effect on particles within the same beam this is the 
circumference and for the beam-beam effect it is the total length of all head on collisions per turn.

The tune shifts are not the same for all particles due to the nonuniform charge distribution within a beam. Only particles close to the beam center suffer the maximum tune shift while particles with increasing betatron oscillation amplitudes are less affected. The effect of space charge therefore introduces a tune spread rather than a specific tune shift and we refer to this effect as the incoherent space-charge tune shift.

As a particular case, consider the space-charge tune shift of a particle within a beam of equal species particles. Applying the Lorentz force (21.54) with (21.56) the space-charge tune shift becomes from (21.62)

$$
\Delta v_{u, \mathrm{sc}}=-\frac{r_{\mathrm{c}} \lambda}{2 \pi} \frac{f_{\mathrm{p}}\left(1-\beta^{2} f_{\mathrm{v}}\right)}{\beta^{2} \gamma} \int \frac{\beta_{u}}{\sigma_{u}\left(\sigma_{x}+\sigma_{y}\right)} f_{\text {corr }} \mathrm{d} z,
$$

where the local linear particle density $\lambda$ is defined by (21.44).

The maximum incoherent space-charge tune shift is from (21.63) with $f_{\mathrm{p}}=1$, $f_{\mathrm{v}}=1,\left(1-\beta^{2}\right)=1 / \gamma^{2}$ and $(21.56)$

$$
\begin{aligned}
\Delta v_{u, \text { sc }, \text { incoh }} & =-\frac{r_{\mathrm{c}} \lambda}{2 \pi \beta^{2} \gamma^{3}}\left[\int_{0}^{2 \pi \bar{R}} \frac{\beta_{u}}{\sigma_{u}\left(\sigma_{x}+\sigma_{y}\right)} \mathrm{d} z\right. \\
& \left.+2\left(1+\beta^{2} \gamma^{2} B\right) \int_{0}^{L_{\mathrm{vac}}} \frac{\beta_{u} \epsilon_{1}}{b^{2}} \mathrm{~d} z+2 \beta^{2} \gamma^{2} B \int_{0}^{L_{\mathrm{mag}}} \frac{\beta_{u} \epsilon_{2}}{g^{2}} \mathrm{~d} z\right],
\end{aligned}
$$

where the integration length $L_{\mathrm{vac}}$ is equal to the total length of the vacuum chamber and $L_{\mathrm{mag}}$ is the total length of magnets along the ring circumference. Note, however, that this last term appears only at low frequencies because of eddy-current shielding in the vacuum chamber at high frequencies. Observing the tune on a betatron side band at a high harmonic of the revolution frequency may not exhibit a tune shift due to this term while one might have a contribution at low frequencies.

A coherent space-charge tune shift can be identified by setting $y=\bar{y}$ in the field expressions (21.46), (21.48), (21.49) to determine the fields at the bunch center. The calculation is similar to that for the incoherent space-charge tune shift except that we define new Laslett form factors for this case

$$
\xi_{2}=\frac{\pi^{2}}{16}
$$

for the image fields from the magnetic pole and form factors $\xi_{1}$ which depend on the aspect ratio of an elliptical vacuum chamber (Table 21.3). 
Table 21.3 Laslett coherent tune shift form factors for elliptical vacuum chambers

\begin{tabular}{l|l|l|l|l|l|l}
\hline$a / b:^{\mathrm{a}}$ & 1 & $5 / 4$ & $4 / 3$ & $3 / 2$ & $2 / 1$ & $\infty$ \\
\hline$\xi_{1}:$ & 0 & 0.090 & 0.107 & 0.134 & 0.172 & 0.206 \\
\hline
\end{tabular}

${ }^{\mathrm{a}} a$ is the horizontal and $b$ the vertical half-axis of an elliptical vacuum chamber

The coherent space-charge tune shift is analogous to (21.64)

$$
\begin{aligned}
\Delta v_{u, \mathrm{sc}, \mathrm{coh}}=- & \frac{r_{\mathrm{c}} \lambda}{2 \pi \beta^{2} \gamma^{3}}\left[\int_{0}^{2 \pi \bar{R}} \frac{\beta_{u}}{\sigma_{u}\left(\sigma_{x}+\sigma_{y}\right)} \mathrm{d} z\right. \\
& \left.+2\left(1+\beta^{2} \gamma^{2} B\right) \int_{0}^{L_{\mathrm{vac}}} \frac{\beta_{u} \xi_{1}}{b^{2}} \mathrm{~d} z+2 \beta^{2} \gamma^{2} B \int_{0}^{L_{\mathrm{mag}}} \frac{\beta_{u} b^{2} \xi_{2}}{g^{2}} \mathrm{~d} z\right] .
\end{aligned}
$$

In both cases, we may simplify the expressions significantly for an approximate calculation by applying smooth approximation $\overline{\beta_{u}} \approx \bar{R} / \nu_{0 u}$ and assuming a uniform vacuum chamber and magnet pole gaps. With these approximations, (21.63) becomes

$$
\Delta v_{u, \mathrm{sc}}=-\frac{r_{\mathrm{c}} N_{\mathrm{tot}} \bar{R}}{2 \pi v_{0 u} B} \frac{f_{\mathrm{p}}\left(1-\beta^{2} f_{\mathrm{v}}\right)}{\beta^{2} \gamma} \frac{\left\langle f_{\text {corr }}\right\rangle}{\bar{\sigma}_{u}\left(\bar{\sigma}_{x}+\bar{\sigma}_{y}\right)},
$$

where

$$
\left\langle f_{\text {corr }}\right\rangle=1+\frac{\bar{\sigma}_{u}\left(\bar{\sigma}_{x}+\bar{\sigma}_{y}\right)}{\bar{b}^{2}}\left[\epsilon_{1}\left(1+\beta^{2} \gamma^{2} B\right)+\epsilon_{2} \beta^{2} \gamma^{2} \frac{\bar{b}^{2}}{\bar{g}^{2}} B\right] .
$$

Symbols with an overbar are the values of quantities averaged over the circumference of the ring and $v_{0 u}$ is the unperturbed tune in the plane $(x, y)$. The incoherent tune shift (21.64) becomes then

$$
\begin{aligned}
\Delta v_{u, \mathrm{sc}, \mathrm{incoh}} \approx & -\frac{r_{\mathrm{c}} N_{\mathrm{tot}} \bar{R}}{2 \pi v_{0 u} B \beta^{2} \gamma^{3}}\left[\frac{1}{\bar{\sigma}_{u}\left(\bar{\sigma}_{x}+\bar{\sigma}_{y}\right)}\right. \\
& \left.+2\left(1+\beta^{2} \gamma^{2} B\right) \frac{\epsilon_{1}}{\bar{b}^{2}}+2 \beta^{2} \gamma^{2} B \frac{\epsilon_{2}}{\bar{g}^{2}} \eta_{\mathrm{b}}\right],
\end{aligned}
$$

where $\eta_{\mathrm{b}}=L_{\mathrm{mag}} /(2 \pi \bar{R})$ is the magnet fill factor and the coherent tune shift (21.67) becomes

$$
\begin{aligned}
\Delta v_{u, \mathrm{sc}, \mathrm{coh}} \approx & -\frac{r_{\mathrm{c}} N_{\mathrm{tot}} \bar{R}}{2 \pi v_{0 u} B \beta^{2} \gamma^{3}}\left[\frac{1}{\overline{\bar{\sigma}}_{u}\left(\bar{\sigma}_{x}+\bar{\sigma}_{y}\right)}\right. \\
& \left.+\frac{2\left(1+\beta^{2} \gamma^{2} B\right)}{\bar{b}^{2}} \xi_{1}+\frac{2 \beta^{2} \gamma^{2} B}{\bar{g}^{2}} \xi_{2} \eta_{\mathrm{b}}\right] .
\end{aligned}
$$


The tune shift diminishes proportional to the third power of the particle energy. As a matter of fact in electron machines of the order of $1 \mathrm{GeV}$ or more, space-charge tune shifts are generally negligible. For low energy protons and ions, however, this tune shift is of great importance and must be closely controlled to avoid beam loss due to nearby resonances. While a maximum allowable tune shift of $0.15-0.25$ seems reasonable to avoid crossing a strong third order or half-integer resonance, practically realized tune shifts can be significantly larger of the order $0.5-0.6$ [2830]. Independent of the maximum tune shift actually achieved in a particular ring, space charge forces ultimately lead to a limitation of the beam current.

\subsubsection{Longitudinal Space-Charge Field}

Within a continuous particle beam travelling along a uniform vacuum chamber we do not expect longitudinal fields to arise. We must, however, consider what happens if the longitudinal charge density is not uniform since this is a more realistic assumption. For the case of a round beam of radius $r_{0}$ in a circular vacuum tube of radius $r_{\mathrm{W}}$ (Fig. 21.4), the fields can be derived by integrating Maxwell's equation $\nabla \times \boldsymbol{E}=-\frac{\partial \boldsymbol{B}}{\partial t}$ and with Stoke's law

$$
\oint \boldsymbol{E} \mathrm{d} \boldsymbol{s}=-\frac{\partial}{\partial t} \int \boldsymbol{B} \mathrm{d} \boldsymbol{A},
$$

where $\mathrm{d} A$ is an element of the area enclosed by the integration path $s$. The integration path shown in Fig. 21.4 leads to the determination of the electrical field $E_{z 0}$ in the center of the beam.

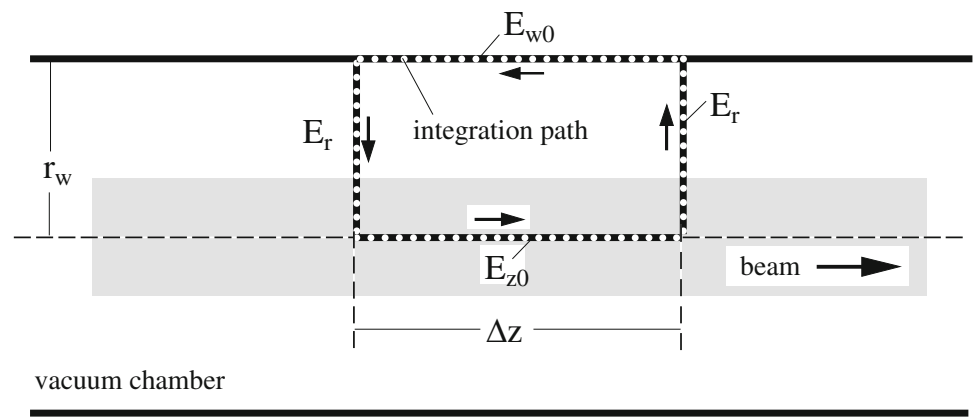

Fig. 21.4 Space-charge fields due to a particle beam travelling inside a circular metallic vacuum chamber 
Integrating the 1.h.s. of (21.72) along the integration path we get with (21.28) for a round beam $(r=\sigma)$

$$
\begin{aligned}
& E_{z 0} \Delta z+\int_{0}^{r_{\mathrm{w}}} E_{r}(z+\Delta z) \mathrm{d} r-E_{z \mathrm{w}} \Delta z-\int_{0}^{r_{\mathrm{w}}} E_{r}(z) \mathrm{d} r \\
& =\left(E_{z 0}-E_{z \mathrm{~W}}\right) \Delta z+\frac{q}{4 \pi \epsilon_{0}}\left(1+2 \ln \frac{r_{\mathrm{w}}}{r_{0}}\right) \frac{\partial \lambda}{\partial z} \Delta z,
\end{aligned}
$$

where a Taylor's expansion was applied to the linear particle density $\lambda(z+\Delta z)$ and only linear terms were retained. $E_{z \mathrm{w}}$ is the longitudinal electrical field on the vacuum chamber wall.

For the r.h.s. of (21.72) we use the expressions for the magnetic field (21.29) and get with $\int B_{\varphi} \mathrm{d} A=\Delta z \int B_{\varphi} \mathrm{d} r$

$$
-\frac{\beta}{c} q\left(1+2 \ln \frac{r_{\mathrm{w}}}{r_{0}}\right) \frac{\partial \lambda}{\partial t} \Delta z=\beta^{2} q\left(1+2 \ln \frac{r_{\mathrm{w}}}{r_{0}}\right) \frac{\partial \lambda}{\partial z} \Delta z
$$

while using the continuity equation

$$
\frac{\partial \lambda}{\partial t}+\beta c \frac{\partial \lambda}{\partial z}=0
$$

The longitudinal space-charge field is therefore

$$
E_{z 0}=E_{z \mathrm{w}}-\frac{q}{4 \pi \epsilon_{0}} \frac{1}{\gamma^{2}}\left(1+2 \ln \frac{r_{\mathrm{w}}}{r_{0}}\right) \frac{\partial \lambda}{\partial z}
$$

and vanishes indeed for a uniform charge distribution because $E_{z \mathrm{w}}=0$ for a dc current. However, variations in the charge distribution cause a longitudinal field which together with the associated ac field in the vacuum chamber wall, acts on individual particles.

The perturbation of a uniform particle distribution in a circular accelerator is periodic with the circumference of the ring and we may set for the longitudinal particle distribution keeping only the $n$th harmonic for simplicity

$$
\lambda=\lambda_{0}+\lambda_{n} \mathrm{e}^{\mathrm{i}\left(n \theta-\omega_{n} t\right)},
$$

where $\omega_{n}$ is the $n$th harmonic of the perturbation $\left(\omega_{n}=n \omega_{0}\right)$. Of course a real beam may have many modes and we need therefore to sum over all modes $n$. In case of instability, it is clear that the whole beam is unstable if one mode is unstable.

With the derivative $\mathrm{d} \lambda / \mathrm{d} z$, smooth approximation and $\theta=z / \bar{R}$ with $\bar{R}$ the average ring radius an integration of (21.76) around the circular accelerator gives 
the total induced voltage due to space-charge fields

$$
V_{z 0}=2 \pi \bar{R} E_{z \mathrm{w}}-\mathrm{i} \frac{I_{n}}{4 \pi \epsilon_{0}} \frac{2 \pi n}{\beta c \gamma^{2}}\left(1+2 \ln \frac{r_{\mathrm{w}}}{r_{0}}\right) \mathrm{e}^{\mathrm{i}\left(n \theta-\omega_{n} t\right)} .
$$

In this expression we have also introduced the $n$th harmonic of the beam-current perturbation $I_{n}=\beta c q \lambda_{n}$. Equation (21.78) exhibits a relation of the induced voltage to the beam current. Borrowing from the theory of electrical currents, it is customary to introduce here the concept of a frequency dependent impedance which will become a powerful tool to describe the otherwise complicated coupling between beam current and induced voltage. We will return to this point in Chap. 22 .

\subsection{Beam-Current Spectrum}

In the last section a beam stability issue appeared based on instantaneous current variations. This is particularly true in circular accelerators where the particle distribution is periodic with the circumference of the ring. On one hand, we have an orbiting particle beam which constitutes a harmonic oscillator with many eigenfrequencies and harmonics thereof and on the other hand, there is an environment with a frequency dependent response to electromagnetic excitation. Depending on the coupling of the beam to its environment at a particular frequency, periodic excitations occur which can create perturbations of particle and beam dynamics. This interaction is the subject of this discussion. In this text, we will concentrate in Chap. 22 on the discussion of basic phenomena of beam-environment interactions or beam instabilities. For a more detailed introduction into the field of beam instabilities, the interested reader is referred to the general references for this chapter. In this discussion, we will follow mainly the theories as formulated by Chao [31], Laclare [32], Sacherer [33] and Zotter [34].

Since the coupling of the beam to its environment depends greatly on the frequency involved, it seems appropriate to discuss first the frequency spectrum of a circulating particle beam.

\subsubsection{Longitudinal Beam Spectrum}

In case of a single circulating particle of charge $q$ in each of $n_{\mathrm{b}}$ equidistant bunches, a pick up electrode located at azimuth $\varphi$ would produce a signal proportional to the single-particle beam current which is composed of a series of delta function signals

$$
i_{\|}(t, \varphi)=\frac{q}{T_{0}} \sum_{k=-\infty}^{+\infty} \delta\left(t-\frac{\varphi}{2 \pi} T_{0}-k \frac{T_{0}}{n_{\mathrm{b}}}-\tau\right),
$$


where $\tau$ is the longitudinal offset of the particle from the reference point, $n_{\mathrm{b}}$ the number of equidistant bunches and $T_{0}$ the revolution time (Fig. 21.4). With the revolution frequency $\omega_{0}=2 \pi / T_{0}$, we use the mathematical relations $2 \pi \sum_{k=-\infty}^{+\infty} \delta(y-2 \pi k)=$ $\sum_{p=-\infty}^{+\infty} \mathrm{e}^{\mathrm{i} p y}$ and $|c| \delta(c y)=\delta(y)$ for

$$
\sum_{k=-\infty}^{+\infty} \delta\left(x-\frac{2 \pi k}{n_{\mathrm{b}} \omega_{0}}\right)=\frac{n_{\mathrm{b}} \omega_{0}}{2 \pi} \sum_{p=-\infty}^{+\infty} \mathrm{e}^{\mathrm{i} p n_{\mathrm{b}} \omega_{0} x}
$$

where $x=t-\frac{\varphi}{\omega_{0}}-\tau$, to replace the delta functions. We also replace the exponential function

$$
\mathrm{e}^{\mathrm{i} y \sin \psi}=\sum_{n=-\infty}^{+\infty} J_{n}(y) \mathrm{e}^{\mathrm{i} n \psi}
$$

and replace $\tau$ by the synchrotron oscillation $\tau=\hat{\tau} \cos \left[\left(m+v_{\mathrm{s}}\right) \omega_{0} t+\zeta_{i}\right]$ where $v_{\mathrm{s}}$ is the synchrotron oscillation tune. The term $m \omega_{0} t$ reflects the mode of the longitudinal particle distribution in all buckets. This distribution is periodic with the periodicity of the circumference and the modes are the harmonics of the distribution in terms of the revolution frequency (Fig. 21.5).

Inserting (21.80) on the r.h.s. of (21.79) and replacing the term $\mathrm{e}^{-\mathrm{i} p n_{\mathrm{b}} \omega_{0} \tau}$ with (21.81) one gets

$$
\begin{aligned}
i_{\|}(t, \varphi)=\frac{q n_{\mathrm{b}} \omega_{0}}{2 \pi} \sum_{p=-\infty}^{+\infty} \sum_{n=-\infty}^{+\infty} \mathrm{i}^{-n} J_{n}\left(q n_{\mathrm{b}} \omega_{0} \hat{\tau}\right) \\
\times \mathrm{e}^{\mathrm{i}\left[\left(p n_{\mathrm{b}}+n m+n v_{\mathrm{s}}\right) \omega_{0} t-p n_{\mathrm{b}} \varphi+n \zeta_{\mathrm{i}}\right]},
\end{aligned}
$$

Fig. 21.5 Particle distribution along the circumference of a circular accelerator and definition of parameters

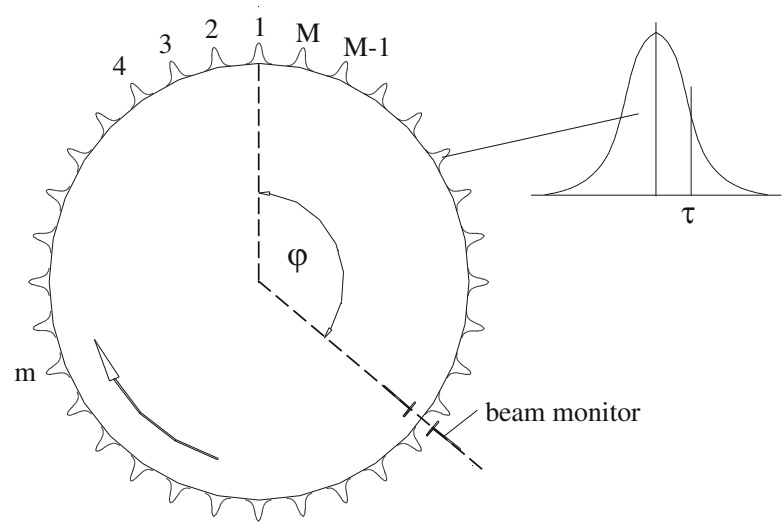




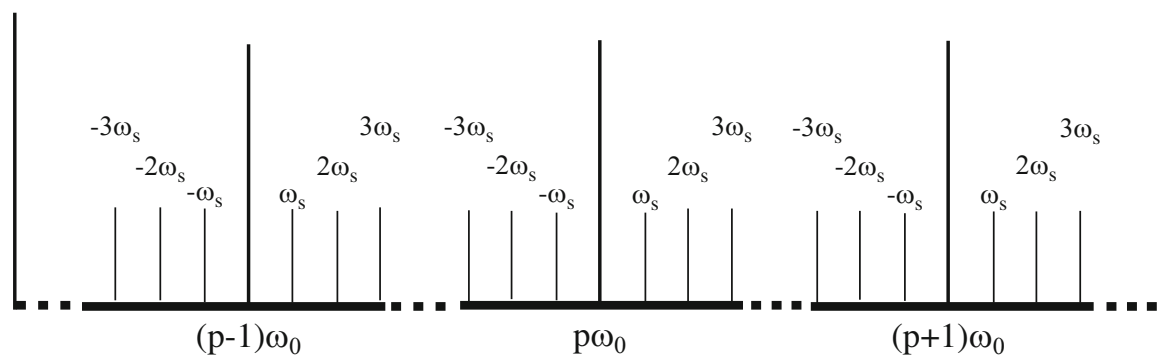

Fig. 21.6 Current spectrum of a single particle orbiting in a circular accelerator and executing synchrotron oscillations

Performing a Fourier transform

$$
i_{\|}(\omega, \varphi)=\frac{1}{2 \pi} \int_{-\infty}^{+\infty} i(t, \varphi) \mathrm{e}^{-\mathrm{i} \omega t} \mathrm{~d} t
$$

we get instead of (21.82) the single particle longitudinal current spectrum

$$
i_{\|}(\omega, \varphi)=\frac{q n_{\mathrm{b}} \omega_{0}}{2 \pi} \sum_{\substack{p=\infty \\=}}^{+\infty} \sum_{-\infty}^{+\infty} \mathrm{i}^{-n} J_{n}\left(p n_{\mathrm{b}} \omega_{0} \hat{\tau}\right) \mathrm{e}^{-\mathrm{i}\left(p n_{\mathrm{b}} \varphi-n \zeta_{i}\right)} \delta(\Omega),
$$

where $\Omega=\omega-\left(p n_{\mathrm{b}}+n m+n v_{\mathrm{s}}\right) \omega_{0}$ and making use of the identity $\int \mathrm{e}^{-\mathrm{i} \omega t} \mathrm{~d} t=$ $2 \pi \delta(\omega)$. This spectrum is a line spectrum with harmonics of the revolution frequency separated by $n_{\mathrm{b}} \omega_{0}$. Each of these main harmonics is accompanied on both sides with satellites separated by $\Omega_{\mathrm{s}}=v_{\mathrm{s}} \omega_{0}$. Schematically, some of the more important lines of this spectrum are shown in Fig. 21.6 for a single particle.

In the approximation of small synchrotron oscillation amplitudes, one may neglect all terms with $|n|>1$ and the particle beam includes only the frequencies $\omega=\left[p n_{\mathrm{b}} \pm\left(m+v_{\mathrm{s}}\right)\right] \omega_{0}$. In Sect. 19.5.1 the interaction of this spectrum for $p=h$ with the narrow-band impedance of a resonant cavity was discussed in connection with Robinson damping.

A real particle beam consists of many particles which are distributed in initial phase $\zeta_{i}$ as well as in oscillation amplitudes $\hat{\tau}$. Assuming the simple case of equal and equidistant bunches with uniform particle distributions in synchrotron phase $\zeta_{i}$ we may set $n=0$. The time independent particle distribution is then $\Phi_{0}(t, \hat{\tau})=$ $\phi_{0}(\hat{\tau})$ which is normalized to unity and the total beam-current spectrum is given by

$$
I_{\|}(\omega, \varphi)=I_{\mathrm{b}} \sum_{p=-\infty}^{+\infty} \delta\left(\omega-\Omega_{0}\right) \mathrm{e}^{-\mathrm{i} p \varphi} \int_{-\infty}^{+\infty} J_{0}\left(p n_{\mathrm{b}} \omega_{0} \hat{\tau}\right) \phi_{0}(\hat{\tau}) \mathrm{d} \hat{\tau}
$$


a.

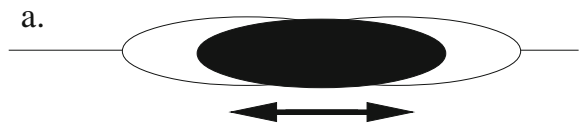

b.

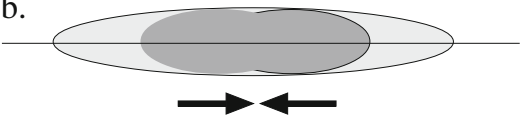

Fig. 21.7 Dipole mode oscillation (a) and quadrupole bunch shape oscillations (b)

where $I_{\mathrm{b}}=q / T_{0}$ is the bunch current and $\Omega_{0}=p n_{\mathrm{b}} \omega_{0}$. All synchrotron satellites vanished because of the uniform distribution of synchrotron phases and lack of coherent bunch oscillations. Observation of synchrotron satellites, therefore, indicates a perturbation from this condition either by coherent oscillations of one or more bunches $(n \neq 0, \hat{\tau} \neq 0)$ or coherent density oscillations within a bunch $\Phi_{0}(t, \hat{\tau})=f\left(\zeta_{i}\right)$.

The infinite sum over $p$ represents the periodic bunch distribution along the circumference over many revolutions whether it be single or multiple bunches. The beam-current spectrum is expected to interact with the impedance spectrum of the environment and this interaction may result in a significant alteration of the particle distribution $\Phi(t, \hat{\tau})$. As an example for what could happen, the two lowest order modes of bunch oscillations are shown in Fig. 21.7.

In lowest order a collection of particles contained in a bunch may perform dipole mode oscillations where all particles and the bunch center oscillate coherently (Fig. 21.7a). In the next higher mode, the bunch center does not move but particles at the head or tail of the bunch oscillate $180^{\circ}$ out of phase. This bunch shape oscillation is in its lowest order a quadrupole mode oscillation as shown in Fig. 21.7b. Similarly, higher order mode bunch shape oscillations can be defined.

\subsubsection{Transverse Beam Spectrum}

Single particles and a collection of particles in a bunch may also perform transverse betatron oscillations constituting a transverse beam current which can interact with its environment. Again, we observe first only a single particle performing betatron oscillations

$$
u=\hat{u} \cos \psi(t)
$$

where $u=x$ or $y, \psi(t)$ is the betatron phase, and the transverse current is

$$
i_{\perp}(t, \varphi)=i_{\|}(t, \varphi) \hat{u} \cos \psi(t)
$$

Note that the transverse current has the dimension of a current moment represented by the same spectrum as the longitudinal current plus additional spectral lines due to betatron oscillations. The betatron phase is a function of time and depends on the revolution frequency and the chromaticity, which both depend on the momentum 
of the particle. From the definition of the momentum compaction $\mathrm{d} \omega / \omega_{0}=\eta_{\mathrm{c}} \delta$, chromaticity $\xi_{u}=\mathrm{d} v / \delta$ and relative momentum deviation $\delta=\mathrm{d} p / p_{0}$, the variation of the betatron phase with time is

$$
\begin{aligned}
\dot{\psi}(t) & =\omega_{u}=v_{0}\left(1+\frac{\xi_{u}}{v_{0}} \delta\right) \omega_{0}\left(1+\eta_{\mathrm{c}} \delta\right), \\
& \approx v_{0} \omega_{0}+\left(v_{0}+\frac{\xi_{u}}{\eta_{\mathrm{c}}}\right) \omega_{0} \dot{\tau},
\end{aligned}
$$

where we have kept only linear terms in $\delta$ and used $\dot{\tau}=-\eta_{\mathrm{c}} \delta$. Equation (21.88) can be integrated for

$$
\psi(t)=v_{0} \omega_{0}(t-\tau)-\omega_{0} \frac{\xi_{u}}{\eta_{\mathrm{c}}} \tau+\psi_{0}
$$

and (21.87) becomes with (21.74), (21.81), (21.84)

$$
\begin{aligned}
i_{\perp}(t, \varphi) & =i_{\|}(t, \varphi) \hat{u} \cos \psi(t) \\
& =q \hat{u} \cos \psi(t) \sum_{m=-\infty}^{+\infty} \delta\left(t-\frac{\varphi}{2 \pi} T_{0}-m \frac{T_{0}}{n_{\mathrm{b}}}\right) \\
& =q \hat{u} \frac{\mathrm{e}^{\mathrm{i} \psi(t)}+\mathrm{e}^{-\mathrm{i} \psi(t)}}{2} \frac{n_{\mathrm{b}}}{T_{0}} \sum_{p=-\infty}^{+\infty} \mathrm{e}^{\mathrm{i}\left[p n_{\mathrm{b}} \omega_{0}(t-\tau)-p \varphi\right]} .
\end{aligned}
$$

Following the derivation for the longitudinal current and performing a Fourier transform we get the transverse beam spectrum

$$
\begin{gathered}
i_{\perp}(\omega, \varphi)=\frac{q}{2 T_{0}} \hat{u} \mathrm{e}^{\mathrm{i} \psi_{0}} \sum_{p=-\infty}^{+\infty} \sum_{n=-\infty}^{+\infty} \mathrm{i}^{-n} J_{n}\left\{\left[\left(p+v_{0}\right) n_{\mathrm{b}} \omega_{0}-\frac{\xi_{u}}{\eta_{\mathrm{c}}}\right] \hat{\tau}\right\} \\
\times e^{-\mathrm{i}\left(p \varphi-n \zeta_{i}\right)} \delta\left(\Omega_{u}\right),
\end{gathered}
$$

where $\Omega_{u}=\omega-\left(p+v_{0}\right) n_{\mathrm{b}} \omega_{0}+n \Omega_{\mathrm{s}}$ defines the line spectrum of the transverse single particle current (Fig. 21.8).

We note that the betatron harmonics $\left(p+v_{0}\right) n_{\mathrm{b}} \omega_{0}$ are surrounded by synchrotron oscillation satellites, however, in such a way that the maximum amplitude is shifted in frequency by $\omega_{0} \xi_{u} / \eta_{\mathrm{c}}$. It is interesting to note at this point that the integer part of the tune $v_{0}$ cannot be distinguished from the integer $p$ of the same value. This is the reason why a spectrum analyzer shows only the fractional tune $\Delta v \omega_{0}$.

The transverse current spectrum is now just the sum of all contributions from each individual particles. If we assume a uniform distribution $\Phi(t, \hat{\tau}, \hat{u})$ in betatron phase, we get no transverse coherent signal because $\left\langle\mathrm{e}^{\mathrm{i} \psi_{0}}\right\rangle=0$, although the incoherent space-charge tune shift is effective. Additional coherent signals appear as a result of perturbations of a uniform transverse particle distribution. 


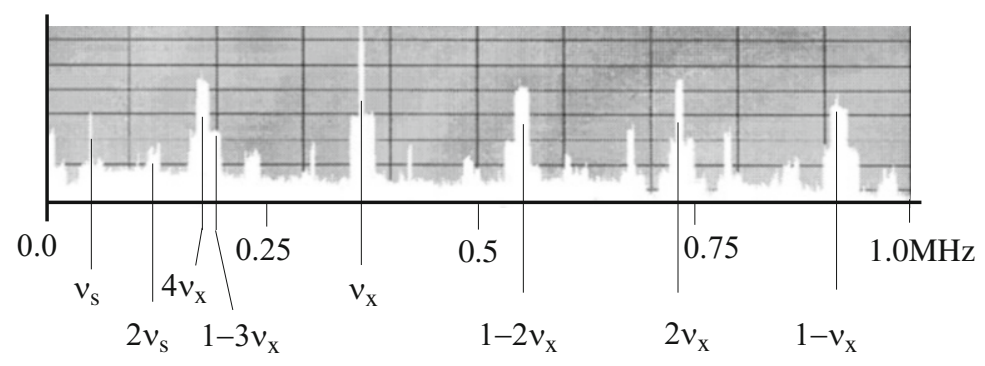

Fig. 21.8 Oscillation spectrum of a single particle orbiting in a circular accelerator and executing betatron and synchrotron oscillations

\section{Problems}

21.1 (S). The linear focusing of the beam-beam effect changes also the betatron function. Derive an expression that relates the change in the value of the betatron function $\beta_{y}^{*}$ at the collision point to the beam-beam tune shift $\delta v$.

21.2. Verify that (21.17) and (21.25) are indeed solutions of the respective Poisson equation.

21.3. Prove that (21.27) is indeed the potential for small vertical amplitudes and $x=0$.

21.4. Calculate the linear beam-beam tune shift for each beam under the following head on colliding beam conditions:

a) A $250 \mathrm{GeV}$ proton beam colliding with a fully ionized $30 \mathrm{GeV} / \mathrm{u}$ Au ion beam. (proton emittance $\epsilon_{x, y}=20 \mathrm{~mm}-\mathrm{mrad}$, gold ion emittance $\epsilon_{x, y}=33 \mathrm{~mm}-\mathrm{mrad}$, $\beta_{x, y}^{*}=2.0 \mathrm{~m}$, proton intensity $10^{11} \mathrm{p} / \mathrm{bunch}$, a total of 60 bunches per beam, gold ion intensity $10^{9} \mathrm{Au}$ ions/bunch).

b) A $250 \mathrm{GeV}$ proton beam colliding with a fully ionized $100 \mathrm{GeV} / \mathrm{u} \mathrm{Au}$ ion beam (parameters same as in a) but gold ion emittance $\epsilon_{x, y}=10 \mathrm{~mm}-\mathrm{mrad}$ ).

c) A $30 \mathrm{GeV}$ electron beam colliding with a $820 \mathrm{GeV}$ proton beam. The circumference of the rings is $6336 \mathrm{~m}$, there are $2.1 \times 10^{13}$ protons and $0.8 \times 10^{13}$ electrons in 210 bunches and the horizontal and vertical beam sizes at the collision point are $\sigma_{x / y}=0.29 / 0.07 \mathrm{~mm}$ for the proton beam and $0.26 / 0.02 \mathrm{~mm}$ for the electron beam, respectively.

d) A $1.5 \mathrm{GeV}$ electron beam colliding with a $1.5 \mathrm{GeV}$ positron beam at a collision point with $\epsilon_{x}=0.67 \mathrm{~mm}$-mrad, emittance coupling $27.7 \%, \beta_{x}^{*}=1.3 \mathrm{~m}, \beta_{y}^{*}=$ $0.1 \mathrm{~m}$ and a beam current of $66 \mathrm{~mA}$ [35].

21.5. Estimate the strength of the octupole field component of the proton beam in RHIC at the collision point. Would an octupole be technically feasible to compensate for the beam-beam octupole term? 
21.6. At the Stanford Linear Collider, SLC, an electron beam collides with a positron beam at up to $50 \mathrm{GeV}$ per beam. Each bunch contains $5 \times 10^{11}$ particles and is focused to a beam diameter of $2.0 \mu \mathrm{m}$ at the collision point where the betatron functions in both planes are $\beta^{*}=0.005 \mathrm{~m}$. Calculate the beam-beam tune shift and the focal length of the beam lens for a bunch length of $\ell=1 \mathrm{~mm}$. Compare with beam-beam limits in storage rings. Why can we tolerate a much greater beam-beam tune shift in a linear collider compared with a storage ring?

21.7. Show that the horizontal damping partition number is negative in a fully combined function FODO lattice as employed in older synchrotron accelerators. Why, if there is horizontal antidamping in such synchrotrons, is it possible to retain beam stability during acceleration? What happens if we accelerate a beam and keep it orbiting in the synchrotron at some higher energy?

21.8. Future colliding beam facilities for high-energy physics experimentation are based on two linear accelerators aimed at each other and producing beams of very high energy for collision. In this arrangement synchrotron radiation is avoided compared to a storage ring. We assume that such beams can be directed to different detectors. Design an $S$-shaped beam transport system based on a FODO lattice, which would allow the beams to be directed into a detector being displaced by the distance $D$ normal to the linac axis. The beams have an energy of $E_{0}=1,000 \mathrm{GeV}$ and a beam emittance of $\epsilon=1.0 \times 10^{-12} \mathrm{~m}$ which should not be diluted in this beam transport system by more than $10 \%$. Determine quadrupole and bending magnet parameters.

21.9. Strong focusing is required along a $500 \mathrm{GeV}$ linear accelerator. Misalignments and path correction introduce dipole fields which are the source of synchrotron radiation and quantum excitation. Assume a normalized emittance of $\gamma \epsilon=10^{-6} \mathrm{~m}$ and an initial beam energy of $1 \mathrm{GeV}$ at the entrance to the linac. The high-energy linac has a circular aperture of $3 \mathrm{~mm}$ diameter. Design a FODO cell with sufficient focusing to contain this beam within a radius of $0.5 \mathrm{~mm}$ leaving the rest for path distortions. The distance between quadrupoles increases linearly with energy. Determine with statistical methods the number and strength of the quadrupoles for an acceleration of $100 \mathrm{MeV} / \mathrm{m}$. Determine the alignment tolerances for these quadrupoles to keep the emittance increase due to quantum excitation in the dipole field from misaligned quadrupoles and due to correctors to $10 \%$.

21.10. Consider the FODO lattice along the linear accelerator in Problem 21.9 and estimate the increase in beam energy spread due to synchrotron radiation from the finite beam size in quadrupoles.

21.11. Consider an electron beam in a $6 \mathrm{GeV}$ storage ring with a bending radius of $\rho=20 \mathrm{~m}$ in the bending magnets. Calculate the rms energy spread $\sigma_{\epsilon} / E_{0}$ and the damping time $\tau$. What is the probability for a particle to emit a photon with an energy of $\sigma_{\epsilon}$ and $2 \sigma_{\epsilon}$. How likely is it that this particle emits another such photon within a damping time? In evaluating the particle distribution, do we need to consider multiple photon emissions? 
21.12. Consider one of the storage rings in Table 10.1 and calculate the equilibrium beam emittance and energy spread. To manipulate the beam emittance we vary the rf-frequency. Determine the maximum variation possible with this method.

21.13. A large hadron collider LHC operates in the LEP tunnel of $28 \mathrm{~km}$ circumference at CERN in Geneva. The maximum proton energy is $15 \mathrm{TeV}$. Determine the magnetic bending field required if $80 \%$ of the circumference can be used for bending magnets. Calculate the synchrotron radiation power for a circulating proton current of $200 \mathrm{~mA}$, damping times, equilibrium beam emittance and energy spread.

21.14. Determine basic FODO lattice parameters for a $2 \mathrm{GeV} \mathrm{e}^{+} / \mathrm{e}^{-}$-colliding beam storage ring with two collision points to reach a design luminosity of $\mathcal{L}_{\mathrm{e}}=10^{31} \mathrm{~cm}^{-2} \mathrm{~s}^{-1}$. The betatron functions at the collision point be $\beta_{y}^{*}=5$ $\mathrm{cm}$ and $\beta_{x}^{*}=1.3 \mathrm{~m}$ and the emittance coupling $10 \%$. Calculate beam sizes in the arc, aperture requirements, circumference and beam current. What is the total synchrotron radiation power? Adjust, if necessary, your design to keep the maximum synchrotron radiation power at the vacuum chamber wall below a practical limit of $5 \mathrm{~kW} / \mathrm{m}$.

\section{References}

1. W. Schottky, Ann. Phys. 57, 541 (1918)

2. J. Borer, P. Bramham, H.G. Hereward, K. Hübner, W. Schnell, L. Thorndahl, in 9th International Conference on High Energy Accelerators (Stanford Linear Accelerator Center, Stanford, 1974), p. 53

3. H.G. Hereward, Technical Report, CERN 77-13, CERN, Geneva (1977)

4. W. Schnell, Technical Report, CERN 57-23, CERN, Geneva (1977)

5. J. Gareyte, F. Sacherer, in 9th International Conference on High Energy Accelerators (Stanford Linear Accelerator Center, Stanford, 1974), p. 341

6. D. Boussard, Technical Report, CERN 87-03, CERN, Geneva (1987)

7. S. Van der Meer, Technical report, CERN (1972)

8. in Antiprotons for Colliding Beam Facilities, number CERN-84-15 in CERN Accelerator School (CERN, Geneva, 1984)

9. C. Bernardini, G.F. Caorazza, G. DiGiugno, G. Ghigo, J. Haissinski, P. Marin, R. Querzoli, B. Touschek, Phys. Rev. Lett. 10, 407 (1963)

10. B. Touschek, in Proceedings of 1963 Summer Study on Storage Rings, Acceleration and Experimentation at Super High Energies, BNL-Report vol. 7534 (Brookhaven, New York, 1956), p. 171

11. H. Bruck, Acceleratéurs Circulaires de Particules (Presses Universitaires de France, Paris, 1966)

12. U. Völkel, Particle loss by touschek effect in a storage ring. Technical Report, DESY 67-5, DESY, Hamburg (1967)

13. J. LeDuff, Technical Report, CERN 89-01, CERN, Geneva (1989)

14. A. Piwinski, CERN Accelerator School, CAS vol. 85-19 (CERN, Geneva, 1986), p. 29

15. J.D. Bjorken, S.K. Mtingwa, Part. Accel. 13, 115 (1983)

16. M. Reiser, Theory and Design of Charged Particle Beams (Wiley, New York, 1994)

17. L. Teng, Technical Report, ANLAD-59, Argonne Nat. Lab., Chicago (1960) 
18. L. Teng, Primer in beam dynamics in synchrotrons, in Physics of Particle Accelerators, vol. 184 (AIP, New York, 1989), p. 1878

19. J.E. Augustin, Technical Report, Rpt. Tech. 36-69 JEA-LN, Laboratoire de L'Accelerateur Lineaire, Orsay (1969)

20. F. Amman, D. Ritson, Space charge effects in electro-electron and positron-electron colliding or crossing beam rings, in Proceedings of International Conference on High Energy Accelerators (Brokhaven, New York, 1961), p. 471

21. R. Belbeoch, Technical Report, Rapp.Techn. 3-73, Laboratoire de L'Accelerateur Lineaire, Orsay (1973)

22. I.B. Vasserman, Stochastic effects under the interaction of colliding beams (in russian). in Proceedings of All-Union Conference on Charged Particle Accelerators (Dubna, 1978)

23. H. Wiedemann, Experiments on the beam-beam effect in $\mathrm{e}^{+}-\mathrm{e}^{-}$storage rings, in Nonlinear Dynamics and the Beam-Beam Interaction, vol. 57, ed. by M. Month, J.C. Herrera. (American Institute of Physics, New York, 1979), p. 84

24. G. Guignard, Review of the investigations on the beam-beam interaction at the isr, in Nonlinear Dynamics and the Beam-Beam Interaction, vol. 57 (American Institute of Physics, New York, 1979), p. 69

25. L.J. Laslett, On intensity limitations imposed by transverse space-charge effects. Technical Report, BNL-7534, BNL, Brookhaven, Upton (1963)

26. D.W. Kerst, R. Serber, Phys. Rev. 60, 47 (1941)

27. J.P. Blewett, Phys. Rev. 69, 87 (1946)

28. W.T. Weng, Space charge effects: tune shifts and resonances, in Physics of Particle Accelerators, vol. 153 (American Institute of Physics, New York, 1987), p. 4243

29. J.P. Delahaye, G. Gelato, L. Magnani, G. Nassibian, F. Pedersen, K.H. Reich, K. Schindl, H. Schœnauer, in 11th International Conference on High Energy Accelerators. Stanford Linear Accelerator Center (Birkæuser Verlag, Basel, 1980)

30. E. Raka, L. Ahrens, W. Frey, E. Gill, J.W. Glenn, R. Sanders, W.T. Weng, IEEE Trans. Nucl. Sci. 32, 3110 (1985)

31. A. Chao, Physics of Collective Beam Instabilities in High Energy Accelerators (Wiley, New York, 1993)

32. J.L. Laclare, Technical Report, CERN 87-03, CERN, Geneva (1987)

33. F. Sacherer, in 9th International Conference on High Energy Accelerators (Stanford Linear Accelerator Center, Stanford, 1974), p. 347

34. B. Zotter, F. Sacherer, Technical Report, CERN 77-13, CERN, Geneva (1977)

35. Summary of the preliminary design of beijing 2.2/2.8 gev electron positron collider (bepc). Technical Report, IHEP Academica Sinica, Beijing (1982) 


\section{Chapter 22 \\ Wake Fields and Instabilities*}

While discussing self fields of a charged particle bunch, we noticed a significant effect from nearby metallic surfaces. The dynamics of individual particles as well as collective dynamics of the whole bunch depends greatly on the electromagnetic interaction with the environment. Such interactions must be discussed in more detail to establish stability criteria for particle beams.

The electric field from a charge in its rest frame extends isotropic from the charge into all directions. In the laboratory frame, this field is Lorentz contracted and assumes for a charge in a uniform beam pipe the form shown in Fig. 22.1a. The contracted field lines spread out longitudinally only within an angle $\pm 1 / \gamma$. This angle is very small for most high energy electron beams and we may describe the single-particle current as well as its image current by a delta function. Some correction must be made to this assumption for lower energy protons and specifically ions for which the angle $1 / \gamma$ may still be significant. In the following discussions, however, we will assume that the particle energy is sufficiently large and $\gamma \gg 1$.

Electron storage rings are being planned, designed, constructed, and operated for a variety of applications. While in the past such storage rings were optimized mostly as colliding beam facilities for high energy physics, in the future most applications for storage rings seem to be connected with the production of synchrotron radiation. Some of these radiation sources will be designed for higher energy particle beams (few $\mathrm{GeV}$ ) to produce hard $\mathrm{X}$-rays while others have moderate to low beam energies $(\gtrsim 100 \mathrm{MeV}$ ) to, for example, produce VUV and soft X-rays or to drive free electron lasers.

The beam in an electron storage ring is composed of bunches which are typically a few centimeters long and are separated by a distance equal to one or more rfwavelengths. The total number of bunches in a storage ring can range from one bunch to a maximum of $h$ bunches, where $h$ is the harmonic number for the storage ring system. The particle beam covers therefore a wide frequency spectrum from the $\mathrm{kHz}$ regime of the order of the revolution frequency up to many $\mathrm{GHz}$ limited only by the bunch length or size of the vacuum chamber. On the other hand, the vacuum

This chapter has been made Open Access under a CC BY 4.0 license. For details on rights and licenses please read the Correction https://doi.org/10.1007/978-3-319-18317-6_28 
a.)

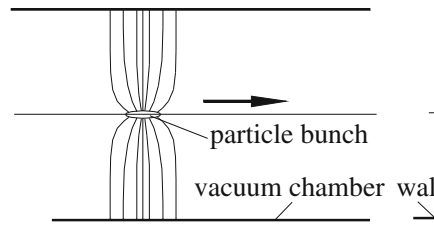

b.)

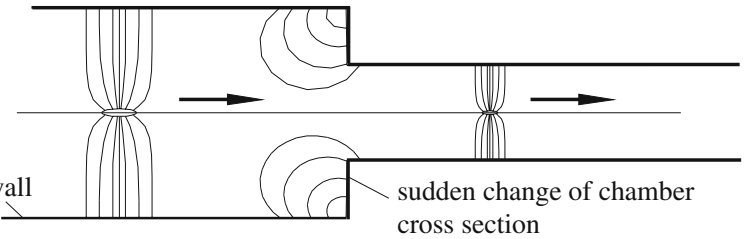

Fig. 22.1 Coupling of a charged particle beam to the environment; uniform chamber cross section (a), and obstacle on vacuum chamber surface (b)

chamber environment constitutes an impedance which can become significant in the same frequency regime and efficient coupling can occur leading to collective effects. The most important impedance in an accelerator is that of the accelerating cavity at the cavity fundamental frequency. Since the particle beam is bunched at the same frequency, we observe a very strong coupling which has been extensively discussed in Sect. 19.4 in connection with beam loading. In this section, we will therefore ignore beam loading effects in resonant cavities at the fundamental frequency and concentrate only on higher-order mode losses and interaction with the general vacuum chamber environment.

Depending on the particular application and experiment conducted, it may be desirable to store only a single bunch with the highest intensity possible. In other cases the maximum total achievable intensity is desired in as many bunches as possible and the particular bunch distribution around the ring does not matter. In either case the ultimate electron beam intensity will most probably be limited by instabilities caused by electromagnetic interaction of the beam current with the environment of the vacuum chamber. We ignore here technical limitations due to, for example, insufficient available rf-power or inability to cool the radiation heating of the vacuum chamber.

Since the radiation intensity produced is directly proportional to the stored electron beam current, it is obvious that the usefulness of such a radiation source depends among other parameters on the maximum electron beam current that can be stored in each bunch or in the storage ring.

\subsection{Definitions of Wake Field and Impedance}

The image currents of a charge $q$ travelling along the axis of a uniform and perfectly conducting tube move with the charge without losses and no forces are generated that would act back on the particle. This is different for a resistive wall where the image fields drag a significant distances behind the charge or in case of an obstacle extending into the tube or any other sudden variation of the tube cross section (Fig. 22.1b). 
In any of these cases, wake fields are created which have the ability to pull or push the charge $q$ or test particles following that charge. Because of causality, no such fields exist ahead of a relativistically moving charge.

Energy losses and gains of a single or collection of particles can cause significant modifications in the dynamics of particle motion. Specifically, we are concerned that such forces may lead to particle or beam instability which must be understood in detail to determine limitations or corrective measures in a particular accelerator design. The interaction of a charged particle beam with its environment can be described in time domain or frequency domain where both have their advantages and disadvantages when it comes to evaluate their effect on particle dynamics.

\subsubsection{Parasitic Mode Losses and Impedances}

In time domain, the interaction is described by wake fields which then act on charges. In frequency domain, vacuum chamber components can be represented as a frequency dependent impedance. We used this picture before while discussing properties of accelerating cavities. Many vacuum chamber components or sudden changes in cross section behave like cavities and represent therefore frequency dependent impedances. Together with the frequency spectrum of the beam, we find strong coupling to the vacuum chamber if the impedance and particle beam have a significant component at the same frequency. The induced voltage $V(\omega)$ from this interaction is proportional to the collective particle current $I(\omega)$ and the impedance $Z(\omega)$ acting as the proportionality factor, describes the actual coupling from the particle beam via the vacuum chamber environment to the test particle. Mathematically, we set

$$
V(\omega)=-Z(\omega) I(\omega)
$$

indicating by the minus sign that the induced voltage leads to an energy loss for beam particles. The impedance is in general complex and depends for each piece of vacuum chamber including accelerating cavities or accidental cavities, on its shape, material and on the frequency under consideration. The coupling impedance for a particular vacuum chamber component or system may be narrow band with a quality factor $Q \gg 1$ like that in an accelerating cavity or broad band with $Q \approx 1$ due to a sudden change in the vacuum chamber cross section.

Fields induced by the beam in a high $Q$ structure are restricted to a narrow frequency width and persist for a long time and can act back on subsequent particle bunches or even on the same particles after one or more revolutions. Such narrow-band impedances can be the cause for multi-bunch instabilities but rarely affect single bunch limits. The main source for a narrow-band impedance in a well-designed accelerator comes from accelerating cavities at the fundamental as well as higher-order mode frequencies. There is little we can or want do about the impedance at the fundamental frequency which is made large by design for 
efficiency. The design of modern accelerator cavities exhibit significantly reduced impedances for higher-order modesor HOMs.

The source for broad-band impedances are discontinuities in cross section or material along the vacuum chamber including accelerating cavities, flanges, kicker magnets with ferrite materials, exit chambers electrostatic plates, beam position monitors, etc. Many higher order modes over a wide frequency range can be excited in such discontinuities by a passing short particle bunch, but all modes decoher very fast. Only for a very short time are these mode fields in phase, adding up to a high field intensity but at the time of arrival of the next particle bunch or the same bunch after one or more revolutions these fields have essentially vanished. Broad-band wake fields are therefore mainly responsible for the appearance of single-bunch beam instabilities.

Due to tight particle bunching by the rf-system to about $5 \%$ of the rf-wavelength, we have large instantaneous currents with significant amplitudes of Fourier components at harmonics of the revolution frequency up to about 20 times the rf-frequency or down to wavelength of a few centimeters. Strong electromagnetic interaction between electron bunches and cavity like structures as part of the vacuum enclosure must therefore be expected. Any but the smallest steps in the cross section of the vacuum chamber constitute cavity like structures. A bunch passing by such a structure deposits electromagnetic energy which in turn causes heating of the structure and can act back on particles in a later segment of the same bunch or in a subsequent bunch. Schematically such fields, also called wake fields, are shown in Fig. 22.1 where the beam passes by a variation in the cross section of the vacuum chamber. We will discuss the nature and the frequency spectrum of these wake fields to determine the effect on the stability of the beam and to develop counter measures to minimize the strength and occurrence of these wake fields.

We distinguish broad band parasitic losses where the quality factor $Q$ is of the order of unity from narrow band losses with higher $Q$ values. Fields from broad band losses last only a very short time of the order of one period and are mainly responsible for single bunch instabilities, where the fields generated by electrical charges in the head of the bunch act back on the particles in the tail of the same bunch. Due to the low value of the quality factor $(Q \approx 1)$ these broad band wake fields decay before the next bunch arrives.

Wake fields can appear as longitudinal or transverse modes and cause correspondingly longitudinal or transverse instabilities. Obviously, a perfect vacuum chamber would have a superconducting surface and be completely uniform around the ring. This is not possible in real accelerators because we need rf-systems which by their nature are not smooth, injection/ejection components, synchrotron light ports, bellows, and beam position monitors. While we cannot avoid such lossy components we are able by proper design to minimize the detrimental effects of less than ideal components.

The loss characteristics of a particular piece of the vacuum chamber for the whole ring is generally expressed in terms of an impedance $Z$ or in terms of a loss factor $k$. To illustrate the different nature of wake fields we assume a cavity like change in the cross section of the vacuum chamber as shown in Fig. 22.2. 

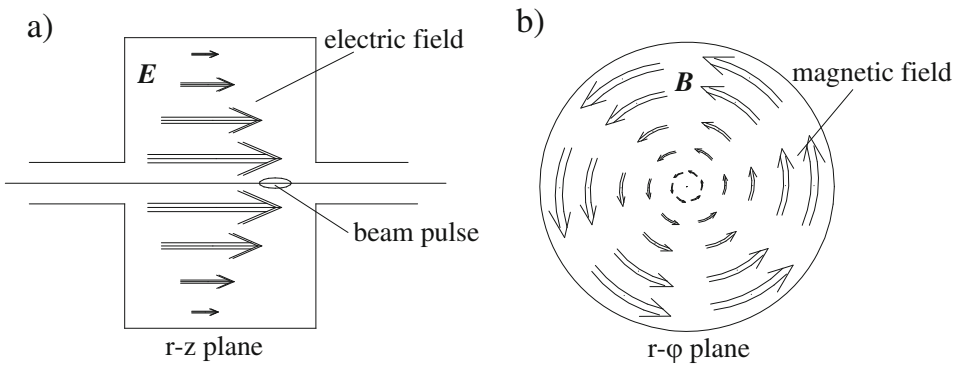

Fig. 22.2 Longitudinal parasitic mode
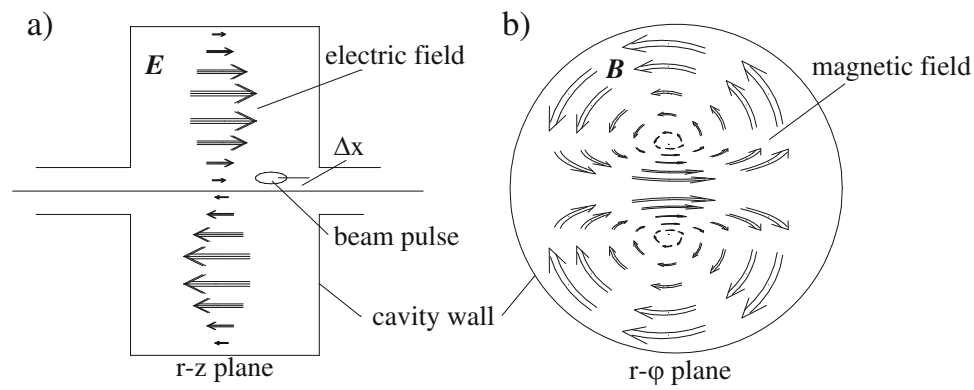

Fig. 22.3 Transverse parasitic mode

A bunch passing through such a structure on axis excites in lowest order a longitudinal electrical field and a transverse magnetic field as shown. Such a field pattern will not cause a transverse deflection of the whole beam since the electrical field is strictly longitudinal and the transverse magnetic field is zero on axis and out of phase. For this situation we define a longitudinal impedance $Z_{\|}$by

$$
Z_{\|}(\omega)=-\frac{\int \boldsymbol{E}(\omega) \mathrm{d} z}{I(\omega)},
$$

where $\mathbf{E}(\omega)$ is the electric field at the frequency $\omega$ and $I(\omega)$ the Fourier transform of the bunched beam current. The r.h.s. of (22.2) is the energy gained per unit charge and is equivalent to an accelerating voltage divided by the current, where the actual frequency dependence as determined by the specific physical shape of the "resonating" structure.

In a similar way we can define a transverse impedance. A beam passing off axis through a "cavity" excites asymmetric fields, as shown in Fig. 22.3, proportional to the moment of the beam current $I(\omega) \Delta x$, where $\Delta x$ is the displacement of the beam from the axis.

Such an electrical field is connected through Maxwell's equation with a finite transverse magnetic field on axis, as shown in Fig. 22.3, which causes a transverse 
deflection of the beam. Consistent with the definition of the longitudinal impedance we define a transverse impedance by

$$
Z_{\perp}(\omega)=\mathrm{i} \frac{\left.\int(\boldsymbol{E}(\omega)+[\boldsymbol{v} \times \boldsymbol{B}(\omega)])\right|_{\perp} \mathrm{d} \boldsymbol{z}}{I(\omega) \Delta x},
$$

where $\boldsymbol{v}$ is the velocity of the particle and $\boldsymbol{B}(\omega)$ the magnetic field component of the electromagnetic field at frequency $\omega$. In general the impedances are complex

$$
Z(\omega)=Z_{\operatorname{Re}}(\omega)+\mathrm{i} Z_{\operatorname{Im}}(\omega) .
$$

The resistive part of the impedance can lead to a shift in the betatron oscillation frequency of the particles while the reactive or imaginary part may cause damping or antidamping.

The impedance is a function of the frequency and its spectrum depends on the specific design of the vacuum chambers in a storage ring. The longitudinal impedance of vacuum chambers has been measured in SPEAR and in other existing storage rings and has been found to follow a general spectrum as a consequence of similar design concepts of storage ring components. SPEAR measurements, as shown in Fig. 19.9, demonstrate the general form of the frequency spectrum of the vacuum chamber impedance [1].

Characteristic for the spectrum is the cutoff frequency $f_{c}$ at which the linear impedance function reaches a maximum and above which the fields are able to propagate in the vacuum chamber. This cutoff frequency obviously is determined by the aperture of the vacuum chamber and therefore occurs at different frequencies for different rings with different vacuum chamber apertures. For the longitudinal broad band impedance at high frequencies above the cutoff frequency $f_{\mathrm{c}}$ we have the simple power law

$$
Z_{\|}(\omega)=Z_{\mathrm{c}} \omega^{-0.68}, \quad\left(\omega>\omega_{\mathrm{c}}\right)
$$

To simplify comparisons between different storage rings we define a normalized impedance $Z / n$ as the impedance at the cut off frequency divided by the mode number $n$ which is the ratio of the cutoff frequency $f_{\mathrm{c}}$ to the revolution frequency $f_{\text {rev }}$

$$
\left|\frac{Z}{n}\right|_{\mathrm{c}}=\left|\frac{Z_{\mathrm{c}}}{f_{\mathrm{c}} / f_{\mathrm{rev}}}\right|
$$

This definition of the normalized impedance can be generalized to all frequencies and together with (22.5) the impedance spectrum becomes

$$
\left|\frac{Z_{\|}}{n}\right|_{\mathrm{eff}}=\left|\frac{Z_{\|}}{n}\right|_{\mathrm{c}}\left(\frac{\omega}{\omega_{\mathrm{c}}}\right)^{-1.68} .
$$


Where only one is known, we can make an estimate of the other one through the approximate relation which is correct only for cylindrically symmetric structures $[2,3]$

$$
Z_{\perp}=\frac{2 R}{b^{2}} \frac{Z_{\|}}{n},
$$

where $2 \pi R$ is the ring circumference and $b$ the typical vacuum chamber radius. The longitudinal impedance of the whole storage ring vacuum system including rf-cavities can be determined by measuring the energy loss of particles in a high intensity bunch compared to the energy loss for particles in a low intensity bunch. Such loss measurements are performed by observing the shift in synchronous phase for the low and high intensity beam. The parasitic losses of rf-cavities can be calculated very accurately with computer programs or are known from laboratory measurements. From the separate knowledge of cavity and total ring losses we derive the vacuum chamber losses by simple subtraction.

A bunched particle beam of high intensity represents a source of electromagnetic fields, called wake fields [4] in a wide range of wavelengths down to the order of the bunch length. The same is true for a realistic coasting beam where fluctuations in beam current simulate short particle bunches on top of an otherwise uniform beam.

Introducing wake fields and higher-order mode losses, we distinguish two groups, the longitudinal and the transverse wake fields. The longitudinal wake fields being in phase with the beam current cause energy losses to the beam particles, while transverse wakes deflect particle trajectories. There is no field ahead of relativistically moving charge due to causality. From the knowledge of such wake fields in a particular environment we may determine the effect on a test charge moving behind a charge $q$.

The character of local wake fields depends greatly on the actual geometry and material of the vacuum chamber and we may expect a significant complication in the determination of wake field distributions along a vacuum enclosure of an actual accelerator. It is not practical to evaluate these fields in detail along the beam path and fortunately we do not need to. Since the effects of localized fields are small compared to the energy of the particles, we may integrate the wake fields over a full circumference. As we will see, this integral of the field can be experimentally determined.

\subsubsection{Longitudinal Wake Fields}

One may wonder how the existence of an obstacle in the vacuum chamber, like a disk which is relatively far away from the charge $q$, can influence a test particle following closely behind the charge $q$. To illustrate this, we consider the situation shown in Fig. 22.4. 
Fig. 22.4 Catch up of wake fields with test particle

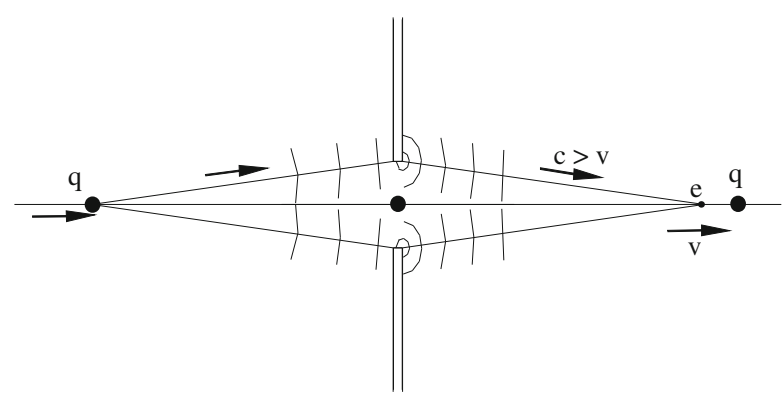

Long before the charge reaches the obstruction, fields start to diverge from the charge toward the obstruction to get scattered there. Some of the scattered fields move again toward the charge and catch up with it due to its slightly faster speed.

The details of this catch up process are, however, of little interest compared to the integrated effect of wake fields on the test particle. Each charge at the position $z$ creates a wake field for a particle at location $\tilde{z}<z$ and this wake field persists during the whole travel time along an accelerator segment $L$ assuming that the distance $\zeta=z-\tilde{z}$ does not change appreciably along $L$. We define now a longitudinal wake function by integrating the longitudinal wake fields $\boldsymbol{E}_{\|}$along the interaction length $L$, which might be the length of a vacuum chamber component, a linear accelerator or the circumference of a circular accelerator, and normalize it to a unit charge. By integrating, which is the same as averaging over the length $L$, we eliminate the need to calculate everywhere the complicated fields along the vacuum chambers. The wake field at the location of a test particle at $\tilde{z}$ from a charge $q$ at location $z$ is then (Fig. 22.4)

$$
W_{\|}(\zeta)=\frac{1}{q} \int_{L} \boldsymbol{E}_{\|}(z, t-\zeta / \beta c) \mathrm{d} z,
$$

where $\zeta=z-\tilde{z}>0$. The wake function is measured in $\mathrm{V} / \mathrm{Cb}$ using practical units and is independent of the sign of the charge. To get the full wake field for a test particle, one would integrate the wake function over all particles ahead of the test particle.

The longitudinal wake function allows us to calculate the total energy loss of the whole bunch by integrating over all particles. We consider a test particle with charge $e$ at position $\tilde{z}$ and calculate the energy loss of this particle due to wake fields from charges further ahead at $z \geq \tilde{z}$. The total induced voltage from a collection of particles with distribution $\lambda(z)$ on the test charge at $\tilde{z}$ is then determined by the wake potential $^{1}$

$$
V_{\text {ном }}(\tilde{z})=-e \int_{\tilde{z}}^{\infty} \lambda(z) W_{\|}(z-\tilde{z}) \mathrm{d} z
$$

\footnotetext{
${ }^{1}$ Expression (22.9) is sometimes called the wake potential. We do not follow this nomenclature because the expression (22.9) does not have the dimension of a potential but (22.10) does.
} 
where a negative sign was added to indicate that the wake fields are decelerating. Integrating over all slices $\mathrm{d} \tilde{z}$, the total energy loss of the bunch into HOM fields is

$$
\Delta U_{\text {ном }}=-\int_{-\infty}^{\infty} e \lambda(\tilde{z}) \mathrm{d} \tilde{z} \underbrace{\int_{\tilde{z}}^{\infty} e \lambda(z) W_{\|}(z-\tilde{z}) \mathrm{d} z}_{\text {wake potential at } \tilde{z}} .
$$

The linear distribution $\lambda(z)$ of particles with charge $e$ is normalized to the total number of particles $N_{\mathrm{b}}$ in the bunch $\int \lambda(z) \mathrm{d} z=N_{\mathrm{b}}$. It is interesting to perform the integrations in (22.11) for a very short bunch such that the wake function seen by particles in this bunch is approximately constant and equal to $W_{0}$. In this case, we define the function $w(\tilde{z})=\int_{\tilde{z}}^{\infty} e \lambda(z) \mathrm{d} z$ and the double integral assumes the form $-\int_{-\infty}^{\infty} w \mathrm{~d} w=\frac{1}{2}\left(e N_{\mathrm{b}}\right)^{2}$ where we have used the normalization $w(-\infty)=e N_{\mathrm{b}}$. Particles in a bunch see therefore only $50 \%$ of the wake fields produced by the same bunch consistent with our earlier formulation of the fundamental theorem of wake fields discussed in Sect. 19.3 in connection with wake fields in rf-cavities. By the same argument, each particle sees only half of its own wake field.

Wake functions describe higher-order mode losses in the time domain. For further discussions, we determine the relationship to the concept of impedance in the frequency domain and replace in (22.10)the charge distribution with the instantaneous current passing by $\tilde{z}$

$$
I(\tilde{z}, t)=\hat{I}_{0} \mathrm{e}^{\mathrm{i}(k \tilde{z}-\omega t)} .
$$

The beam current generally includes more than one mode $k$ but for simplicity we consider only one in this discussion. Integrating over all parts of the beam which have passed the location $\tilde{z}$ before, the wake potential (22.10) becomes

$$
V_{\text {ном }}(\tilde{z}, t)=-\frac{1}{c \beta} \int_{\tilde{z}}^{\infty} I\left(\tilde{z}, t+\frac{z-\tilde{z}}{c \beta}\right) W_{\|}(z-\tilde{z}) \mathrm{d} z .
$$

Consistent with a time dependent beam current, the induced voltage depends on location $\tilde{z}$ and time as well. The wake function vanishes due to causality for $z-\tilde{z}<0$ and the integration can therefore be extended over all values of $z$. With (22.12), $\zeta=z-\tilde{z}$ and applying a Fourier transform (22.13) becomes

$$
V_{\text {ном }}(t, \omega)=-I(t, \omega) \frac{1}{c \beta} \int_{-\infty}^{\infty} \mathrm{e}^{-\mathrm{i} \omega \zeta / c \beta} W_{\|}(\zeta) \mathrm{d} \zeta .
$$

From (22.14) we define the longitudinal coupling impedance in the frequency domain

$$
Z_{\|}(\omega)=\frac{1}{c \beta} \int_{-\infty}^{\infty} \mathrm{e}^{-\mathrm{i} \omega \zeta / c \beta} W_{\|}(\zeta) \mathrm{d} \zeta
$$


which has in practical units the dimension Ohm. The impedance of the environment is the Fourier transform of the wake fields left behind by the beam in this environment. Because the wake function has been defined in (22.9) for the length $L$ of the accelerator under consideration, the impedance is an integral parameter of the accelerator section $L$ as well. Conversely, we may express the wake function in terms of the impedance spectrum

$$
W_{\|}(z)=\frac{1}{2 \pi} \int_{-\infty}^{\infty} Z_{\|}(\omega) \mathrm{e}^{\mathrm{i} \omega z / c \beta} \mathrm{d} \omega .
$$

The interrelations between wake functions and impedances allows us to use the most appropriate quantity for the problem at hand. Generally, it depends on whether one wants to work in the frequency or the time domain. For theoretical discussions, the well defined impedance concept allows quantitative predictions for beam stability or instability to be made. In most practical applications, however, the impedance is not quite convenient to use because it is not well known for complicated shapes of the vacuum chamber. In a linear accelerator, for example, we need to observe the stability of particles in the time domain to determine the head-tail interaction. The most convenient quantity depends greatly on the problem to be solved, theoretically or experimentally.

\section{Loss Parameter}

In a real accelerator, the beam integrates over many different vacuum chamber pieces with widely varying impedances. The interaction of the beam with the vacuum chamber impedance leads to an energy loss which has to be compensated by the rf-system. We are therefore not able to experimentally determine the impedance or wake function of a particular vacuum chamber element. Only the integrated impedance for the whole accelerator can sometimes be probed at specific frequencies by observing specific instabilities as we will discuss later. The most accurate quantity to measure the total resistive impedance for the whole accelerator integrated over all frequencies is the loss factor or loss parameter.

We characterize this loss through the loss factor $k$ defined by

$$
k=\frac{\Delta U}{q^{2}},
$$

where $\Delta U$ is the total energy deposited by the passing bunch and $q$ is the total electrical charge in this bunch. This definition is a generalization of the energy loss of a single particle passing once through a resonator where $k=-(\omega / 4)\left(R_{\mathrm{S}} / Q\right)$ and $R_{\mathrm{S}}$ is the shunt impedance of this resonator. The loss factor is related to the real part of the impedance by

$$
k=\frac{2}{q^{2}} \int_{o}^{\infty} \operatorname{Re}[Z(\omega)] I^{2}(\omega) \mathrm{d} \omega
$$


Fig. 22.5 Dependence of the overall loss factor $k$ in the storage ring SPEAR on the bunch length [5]

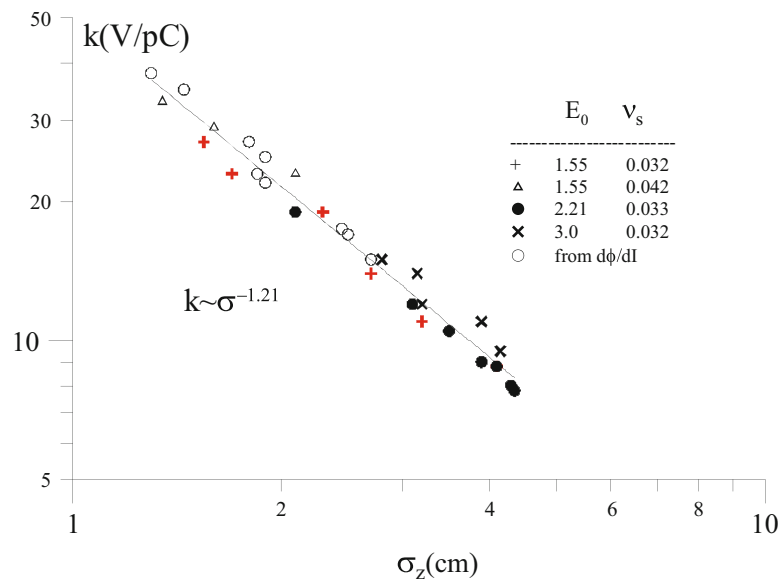

and depends strongly on the bunch length as can be seen from measurements of the loss factor in SPEAR [5] shown in Fig. 22.5. Specifically, we find the loss factor to scale with the bunch length like

$$
k\left(\sigma_{\ell}\right) \sim \sigma_{\ell}^{-1.21}
$$

Similar to the definitions of impedances, we also distinguish a longitudinal and a transverse loss factor. The loss factor can be related to the wake function and we get from comparison with (22.11) the relation

$$
k_{\|_{\mathrm{HOM}}}=\frac{1}{N_{\mathrm{b}}^{2}} \int_{-\infty}^{\infty} \lambda(\tilde{z}) \mathrm{d} \tilde{z} \int_{\tilde{z}}^{\infty} \lambda(z) W_{\|}(z-\tilde{z}) \mathrm{d} z .
$$

The loss parameter can be defined for the complete circular accelerator or for a specific vacuum chamber component installed in a beam line or accelerator. Knowledge of this loss factor is important to determine possible heating effects which can become significant since the total higher-order mode losses are deposited in the form of heat in the vacuum chamber component. In a circular accelerator, the energy loss rate or heating power of a beam circulating with revolution frequency $f_{0}$ is

$$
P_{\mathrm{HOM}}=k_{\mathrm{HOM}} \frac{I_{0}^{2}}{f_{0} n_{\mathrm{b}}},
$$

where $n_{\mathrm{b}}$ is the number of bunches in the beam and $I_{\mathrm{o}}=n_{\mathrm{b}} q N_{\mathrm{b}} f_{0}$ is the average circulating beam current in the accelerator. As an example, we consider a circulating beam of $1 \mathrm{~mA}$ in one bunch of the LEP storage ring where the revolution frequency is about $f_{0}=10 \mathrm{kHz}$. The heating losses in a component with loss factor $k_{\mathrm{Hом}}=0.1$ $\mathrm{V} / \mathrm{pCb}$ would be 10 Watts. This might not seem much if the component is large 
and an external cooling fan might be sufficient. On the other hand, if the vacuum component is small and not accessible like a bellows this heating power might be significant and must be prevented by design. The higher-order heating losses scale like the average current, the bunch current and inversely proportional with the revolution frequency. For a given circulating beam current, the losses depend therefore greatly on the number of bunches and the size of the circular accelerator. As the bunch length becomes smaller, higher and higher modes can be excited as demonstrated by the steep increase in loss parameter with decreasing bunch length (Fig. 22.5). Although we try to apply a careful design to all accelerator components to minimize the impedance it is prudent to be aware of this heating effect while developing accelerators that involve significantly reduced bunch length like those in quasi-isochronous storage rings or beams accelerated by laser beams.

The loss parameter can be measured by observing the shift in the synchronous phase. A bunch of particles circulating in an accelerator looses energy due to the resistive impedance of the vacuum chamber. This additional energy loss is compensated by an appropriate shift in the synchronous phase which is given by

$$
\Delta U_{\text {ном }}=e N_{\mathrm{b}} V_{\mathrm{rf}}\left|\sin \left(\phi_{\mathrm{s}}-\phi_{\mathrm{s} 0}\right)\right|,
$$

where $\phi_{\mathrm{s} 0}$ is the synchronous phase for a very small beam current and $V_{\mathrm{rf}}$ the peak rf-voltage. The loss factor is then with the number of particles per bunch $N_{\mathrm{b}}$

$$
k_{\text {ном }}=\frac{\Delta U_{\text {ном }}}{e^{2} N_{\mathrm{b}}^{2}} .
$$

Performing this measurement as a function of rf-voltage one can establish a curve similar to that shown in Fig. 22.5 for the storage ring SPEAR and the dependence of the loss parameter on the bunch length can be used to determine the total resistive impedance of the accelerator as a function of frequency. To do that, we write (22.19) in terms of Fourier transforms

$$
k_{\|_{\text {ном }}}=\frac{\pi}{e^{2} N_{\mathrm{b}}^{2}} \int_{-\infty}^{\infty} Z_{\text {res }}(\omega)|I(\omega)|^{2} \mathrm{~d} \omega
$$

and recall that the bunch or current distribution in a storage ring is Gaussian

$$
I(\tau)=\frac{I_{0}}{\sqrt{2 \pi} \sigma_{\tau}} \mathrm{e}^{-\tau^{2} / 2 \sigma_{\tau}^{2}} .
$$

The Fourier transform of a Gaussian distribution is

$$
I(\omega)=I_{0} \mathrm{e}^{-\frac{1}{2} \omega^{2} \sigma_{\tau}^{2}},
$$


where $I_{0}$ is the total bunch current and inserting (22.25) into (22.23), we get

$$
k_{\|_{\mathrm{HOM}}}=\frac{\pi I_{0}}{e^{2} N_{\mathrm{b}}^{2}} \int_{-\infty}^{\infty} Z_{\mathrm{res}}(\omega) \mathrm{e}^{-\omega^{2} \sigma_{\tau}^{2}} \mathrm{~d} \omega .
$$

With (22.26) and the measurement $k_{\|_{\text {Hом }}}\left(\sigma_{\ell}\right)$, where $\sigma_{\ell}=c \sigma_{\tau}$, one may solve for $Z_{\text {res }}(\omega)$ and determine the resistive-impedance spectrum of the ring.

Unfortunately, it is not possible to attach a resistance meter to an accelerator to determine its impedance and we will have to apply a variety of wake field effects on the particle beams to determine the complex impedance as a function of frequency. No single effect, however, will allow us to measure the whole frequency spectrum of the impedance.

\subsubsection{Transverse Wake Fields}

Similar to the longitudinal case we also observe transverse wake fields with associated impedances. Such fields exert a transverse force on particles generated by either transverse electrical or magnetic wake fields. Generally such fields appear when a charged particle beam passes off center through a nonuniform but cylindrical or through an asymmetric vacuum chamber. Transverse wake fields can be induced only on structures which also exhibit a longitudinal impedance. A beam travelling off center through a round pipe with perfectly conducting walls will not create longitudinal and therefore also no transverse wake fields.

We consider a charge $q$ passing through a vacuum chamber structure with an offset $\Delta u=(\Delta x, \Delta y)$ in the horizontal or vertical plane as shown in Fig. 22.3.

In analogy to the definition of the longitudinal wake function (22.9), we define a transverse wake function per unit transverse offset by

$$
W_{\perp}(\zeta, t)=+\frac{\int_{L}\{\boldsymbol{E}(t-\zeta / \beta c)+c[\boldsymbol{\beta} \times \boldsymbol{B}(t-\zeta / \beta c)]\}_{\perp} \mathrm{d} z}{q \Delta u}
$$

which is measured in units of $\mathrm{V} / \mathrm{Cb} / \mathrm{m}$. Consistent with the definition (22.15) of the longitudinal impedance, the transverse coupling impedance is the Fourier transform of the transverse wake functions defined by

$$
Z_{\perp}(\omega)=\mathrm{i} \frac{1}{c \beta} \int_{-\infty}^{\infty} \mathrm{e}^{-\mathrm{i} \omega \zeta / c \beta} W_{\perp}(\zeta) \mathrm{d} \zeta
$$

adding the factor $i$ to indicate that the action of the transverse force is a mere deflection while the particle energy stays constant. This transverse impedance is measured in $\mathrm{Ohm} / \mathrm{m}$. The inverse relation is similar to the longitudinal case

$$
W_{\perp}(z)=\mathrm{i} \frac{1}{2 \pi} \int_{-\infty}^{\infty} Z_{\perp}(\omega) \mathrm{e}^{\mathrm{i} \omega z / c \beta} \mathrm{d} \omega
$$




\subsubsection{Panofsky-Wenzel Theorem}

The general relationship between longitudinal and transverse wake fields is expressed by the Panofsky-Wenzel theorem [6]. Panofsky and Wenzel studied the effect of transverse electromagnetic fields on a particle trajectory and applied general relations of electromagnetic theory to derive a relationship between longitudinal and transverse electromagnetic forces. We will derive the same result in the realm of wake fields. The Lorentz force on a test particle at $\tilde{z}$ due to transverse wake fields from charges at location $z>\tilde{z}$ causes a deflection of the particle trajectory and the change in transverse momentum of the test particle is after integration over all charges at locations $z<\tilde{z}$

$$
c \boldsymbol{p}_{\perp}=\frac{e}{\beta} \int_{-\infty}^{\infty}[\boldsymbol{E}+(\boldsymbol{v} \times \boldsymbol{B})]_{\perp} \mathrm{d} z .
$$

Note that the wake fields vanish because of causality for $\zeta<0$. The fields can be expressed by the vector potential $\boldsymbol{E}_{\perp}=-\partial \boldsymbol{A}_{\perp} / \partial t$ and $\boldsymbol{B}_{\perp}=(\nabla \times \boldsymbol{A})_{\perp}$. The particle velocity has only one nonvanishing component $\boldsymbol{v}=(0,0, v)$ and (22.30) becomes with $\partial z / \partial t=v$

$$
c \boldsymbol{p}_{\perp}=-c e \underbrace{\int_{0}^{d} \overbrace{\left(\frac{\partial}{\partial t} \frac{\partial t}{\partial z}+\frac{\partial}{\partial z}\right)}^{=\mathrm{d} / \mathrm{d} z} A_{\perp} \mathrm{d} z}_{=0}+c e \nabla_{\perp} \int_{0}^{d} A_{\|} \mathrm{d} z,
$$

where we made use of the vector relation for $\boldsymbol{v} \times(\nabla \times A)+\underbrace{\boldsymbol{A} \times(\nabla \times v)}_{=0}$ which is equal to $\nabla_{\perp}(v A)-(v \nabla) A_{\perp}-\underbrace{(A \nabla) v}_{=0}$ noting that the particle velocity is a constant.

The integrand in the first integral of (22.31) is equal to the total derivative $\mathrm{d} A_{\perp} / \mathrm{d} z$ and the integral vanishes because the fields vanish for $\zeta= \pm \infty$. After differentiation with respect to the time $t$ (22.31) becomes

$$
\frac{\mathrm{d} \boldsymbol{p}_{\perp}}{\mathrm{d} t}=-e \nabla_{\perp} \int_{-\infty}^{\infty} E_{\|} \mathrm{d} z
$$

which is in terms of forces

$$
\frac{\partial}{\partial z} \boldsymbol{F}_{\perp}=-\nabla_{\perp} \boldsymbol{F}_{\|}
$$

The longitudinal gradient of the transverse force or electromagnetic field is proportional to the transverse gradient of the longitudinal force or electromagnetic field and knowledge of one allows us to calculate the other. 


\subsection{Impedances in an Accelerator Environment}

The vacuum chamber of an accelerator is too complicated in geometry to allow an analytical expression for its impedance. In principle each section of the chamber must be treated individually. By employing two or three-dimensional numerical codes it may be possible to determine the impedance for a particular component and during a careful design process for a new accelerator, this should be done to avoid later surprises. In [7] expressions for many geometries are compiled. Yet, every accelerator is somewhat different from another and will have its own particular overall impedance characteristics. For this reason, we focus in these discussions specifically on such instabilities which can be studied experimentally revealing impedance characteristics of the ring. However, depending on the frequency involved, there are a few classes of impedances which are common to all accelerators and may help understand the appearance and strength of certain instabilities. In this section, we will discuss such impedances to be used in later discussions on stability conditions and growth rate of instabilities.

Consistent with (21.88), (22.10) the longitudinal impedance for a circular accelerator is defined as the ratio of the induced voltage at frequency $\omega$ to the Fourier transform of the beam current at the same frequency

$$
\begin{aligned}
Z_{\|}(\omega) & =-\frac{\int \boldsymbol{E}_{\|}(\omega) \mathrm{d} z}{I(\omega)} \\
& =\frac{1}{4 \pi \epsilon_{0}} \frac{1}{e N_{\mathrm{b}}} \int_{L} \boldsymbol{E}_{\|}(z, t-\zeta / \beta c) \mathrm{e}^{-\mathrm{i} \omega \zeta / \beta c} \mathrm{~d} z
\end{aligned}
$$

Similarly the transverse impedance is from (22.27), (22.28) the ratio of induced transverse voltage to the transverse moment of the beam current

$$
Z_{\perp}(\omega)=-\mathrm{i} \frac{\left.\int\left(\boldsymbol{E}_{\perp}+[\boldsymbol{v} \times \boldsymbol{B}]_{\perp}\right)\right|_{(z, t-\zeta / \beta c)} \mathrm{e}^{-\mathrm{i} \omega \zeta / \beta c} \mathrm{~d} z}{I(\omega) \Delta u}
$$

where $\Delta u$ is the horizontal or vertical offset of the beam from the axis.

\subsubsection{Space-Charge Impedance}

In (21.78) we found an induced voltage leading to an energy gain or loss due to a collection of charged particles. It is customary to express (21.78) in a form exhibiting the impedance of the vacuum chamber. In case of a perfectly conducting vacuum chamber $E_{z \mathrm{w}}=0$ and (21.78) becomes

$$
V_{z}=-Z_{\| s c} I_{n} \mathrm{e}^{\mathrm{i}\left(n \theta-\omega_{n} t\right)}
$$


where the longitudinal space-charge impedance $Z_{\| \text {sc }}$ is defined by [8, Sect. 2.4.5]. ${ }^{2}$

$$
\frac{Z_{\| \mathrm{sc}}(\omega)}{n}=-\frac{\mathrm{i}}{\epsilon_{0} c} \frac{1}{2 \beta \gamma^{2}}\left(1+2 \ln \frac{r_{\mathrm{w}}}{r_{0}}\right),
$$

where $n=\omega / \omega_{0}$ and $\omega_{0}$ is the revolution frequency. This expression is correct for long wavelength below cut off of the vacuum chamber or for $\omega<c / r_{\mathrm{w}}$. The spacecharge impedance is purely reactive and, as we will see, capacitive. For a round beam of radius $r_{0}$ and offset from the axis of a round beam pipe with diameter $2 r_{\mathrm{w}}$ a transverse space-charge impedance can be derived [7]

$$
Z_{\perp \mathrm{sc}}(\omega)=-\frac{\mathrm{i}}{\epsilon_{0} c} \frac{\bar{R}}{\beta^{2} \gamma^{2}}\left(\frac{1}{r_{0}^{2}}-\frac{1}{r_{\mathrm{w}}^{2}}\right),
$$

where $\bar{R}$ is the average ring radius. The transverse space-charge impedance is inversely proportional to $\beta^{2}$ and is therefore especially strong for low energy particle beams.

\subsubsection{Resistive-Wall Impedance}

The particle beam induces an image current in the vacuum chamber wall in a thin layer with a depth equal to the skin depth. For less than perfect conductivity of the wall material, we observe resistive losses which exert a pull or decelerating field on the particles. This pull is proportional to the beam current and integrating the fields around a full circumference $2 \pi \bar{R}$ of the accelerator we get the longitudinal resistive wall impedance in a uniform tube of radius $r_{\mathrm{w}}$ at frequency $\omega_{n}$ for lowest order monopole oscillations [9]

$$
\left.Z_{\|}(\omega)\right|_{\text {res }}=(1-i) \frac{\bar{R}}{r_{\mathrm{w}} \sigma \delta_{\text {skin }}},
$$

where the skin depth is defined by [10] $\delta_{\text {skin }}\left(\omega_{n}\right)=\sqrt{\frac{2}{\mu_{0} \mu_{\mathrm{r}} \omega_{n} \sigma}}$. The longitudinal resistive wall impedance decays with increasing frequency and therefore plays an important role only for lower frequencies up to tens of $\mathrm{GHz}$ [9]. The transverse resistive wall impedance for a round beam pipe is from the Panofsky-Wenzel theorem [6]

$$
Z_{\perp}(\omega)_{\mathrm{res}}=\left.\frac{2 c}{\omega r_{\mathrm{w}}^{2}} Z_{\|}(\omega)\right|_{\mathrm{res}}
$$

\footnotetext{
${ }^{2}$ Note: the factor $1 /\left(\epsilon_{0} c\right)=\sqrt{\mu_{0} / \epsilon_{0}}=Z_{0}=376.73 \Omega$ is often called the free space impedance. We will not use it because it is not a physical quantity but only a convenient unit scaling factor. A current passing through vacuum will not loose energy into this impedance.
} 


\subsubsection{Cavity-Like Structure Impedance}

The impedance of accelerating cavities or cavity like objects of the vacuum chamber can be described by the equivalent of a parallel resonant circuit for which the impedance is from (19.11)

$$
\left.\frac{1}{Z_{\|}(\omega)}\right|_{\mathrm{cy}}=\frac{1}{R_{\mathrm{s}}}\left(1+\mathrm{i} Q \frac{\omega^{2}-\omega_{\mathrm{r}}^{2}}{\omega_{\mathrm{r}} \omega}\right),
$$

where $Q$ is the quality factor and $R_{\mathrm{s}}$ the cavity impedance at the resonance frequency $\omega_{\mathrm{r}}$ or cavity shunt impedance. Taking the inverse, we get for the normalized impedance

$$
\left.\frac{Z_{\|}(\omega)}{n}\right|_{\mathrm{cy}}=\left|\frac{Z_{\|}(\omega)}{n}\right|_{0} \frac{1-\mathrm{i} Q \frac{\omega^{2}-\omega_{\mathrm{r}}^{2}}{\omega_{\mathrm{r}} \omega}}{1+Q^{2} \frac{\left(\omega^{2}-\omega_{\mathrm{r}}^{2}\right)^{2}}{\omega_{\mathrm{r}}^{2} \omega^{2}}}
$$

where $\left|\frac{Z}{n}\right|_{0}=R_{\mathrm{s}}$ is purely resistive and $n=\omega / \omega_{0}$.

Vacuum chamber impedances occur, for example, due to sudden changes of cross section, flanges, beam position monitors, etc., and are collectively described by a cavity like impedance with a quality factor $Q \approx 1$. This is justified because fields are induced in these impedances at any frequency. From (22.42) the longitudinal broad-band impedance is therefore

$$
\left.\frac{Z_{\|}}{n}\right|_{\mathrm{bb}}(\omega)=\left|\frac{Z_{\|}}{n}\right|_{0} \frac{1-\mathrm{i} \frac{\omega^{2}-\omega_{\mathrm{r}}^{2}}{\omega_{\mathrm{r}} \omega}}{1+\frac{\left(\omega^{2}-\omega_{\mathrm{r}}^{2}\right)^{2}}{\omega_{\mathrm{r}}^{2} \omega^{2}}} .
$$

This broad-band impedance spectrum is shown in Fig. 22.6 and we note that the resistive and reactive part exhibit different spectra.

The resistive broad-band impedance has a symmetric spectrum and scales like $\omega^{2}$ for low frequencies decaying again for very high frequencies like $1 / \omega^{2}$. At low frequencies, the broad-band impedance (22.43) is almost purely inductive scaling linear with frequency

$$
\left.\frac{Z_{\|}(\omega)}{n}\right|_{\mathrm{bb}}=\mathrm{i}\left|\frac{Z_{\|}(\omega)}{n}\right|_{0} \frac{\omega}{\omega_{\mathrm{r}}} \quad \text { for } \quad \omega \ll \omega_{\mathrm{r}} .
$$

At high frequencies the impedance becomes capacitive

$$
\left.\frac{Z_{\|}(\omega)}{n}\right|_{\mathrm{bb}}=-\mathrm{i}\left|\frac{Z_{\|}(\omega)}{n}\right|_{0} \frac{\omega_{\mathrm{r}}}{\omega} \quad \text { for } \quad \omega \gg \omega_{\mathrm{r}}
$$


Fig. 22.6 Resistive and reactive broad-band impedance spectrum

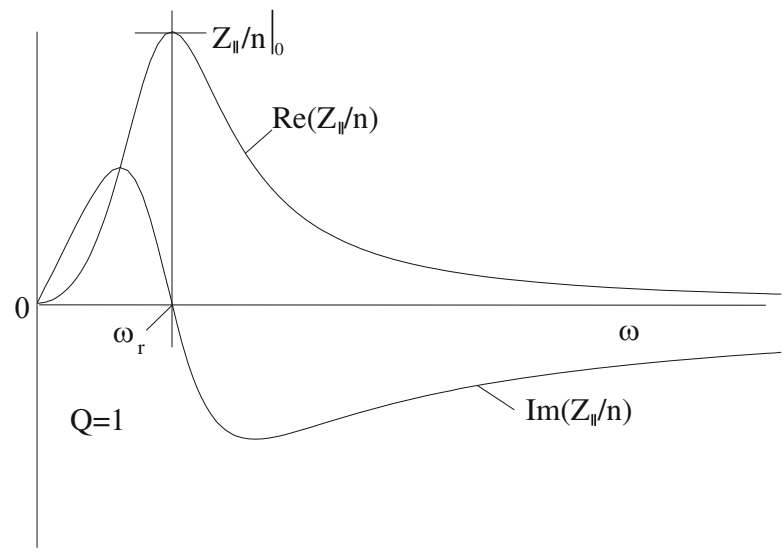

and decaying slower with frequency than the resistive impedance. We note, however, that the reactive broad-band impedance spectrum changes sign and beam stability or instability depend therefore greatly on the actual coupling frequency. At resonance, the broad-band impedance is purely resistive as would be expected.

Sometimes it is convenient to have a simple approximate correlation between longitudinal and transverse impedance in a circular accelerator as shown in (22.40). Although this correlation is valid only for the resistive wall impedance in a round beam pipe, it is often used for approximate estimates utilizing the broad-band impedance.

\subsubsection{Overall Accelerator Impedance}

At this point, we have identified all significant types of impedances we generally encounter in an accelerator which are the space charge, resistive wall, narrow-band impedances in high $Q$ cavities, and broad-band impedance. In Fig. 22.7 we show qualitatively these resistive as well as reactive impedance components as a function of frequency.

At low frequency the reactive as well as the resistive component of the resistive wall impedance dominates while the space charge impedance is independent of frequency. The narrow-band cavity spectrum includes the high impedances at the fundamental and higher mode frequencies.

Generally, it is not possible to use a uniform vacuum chamber in circular accelerators. Deviations from a uniform chamber occur at flanges, bellows, rfcavities, injection/ejection elements, electrostatic plates, etc. It is not convenient to consider the special impedance characteristics of every vacuum chamber piece and we may therefore look for an average impedance as seen by the beam. The broad-band impedance spectrum created by chamber components in a ring reaches a maximum at some frequency and then diminishes again like $1 / \omega$. This turn over of the broad-band impedance function depends on the general dimensions of all 

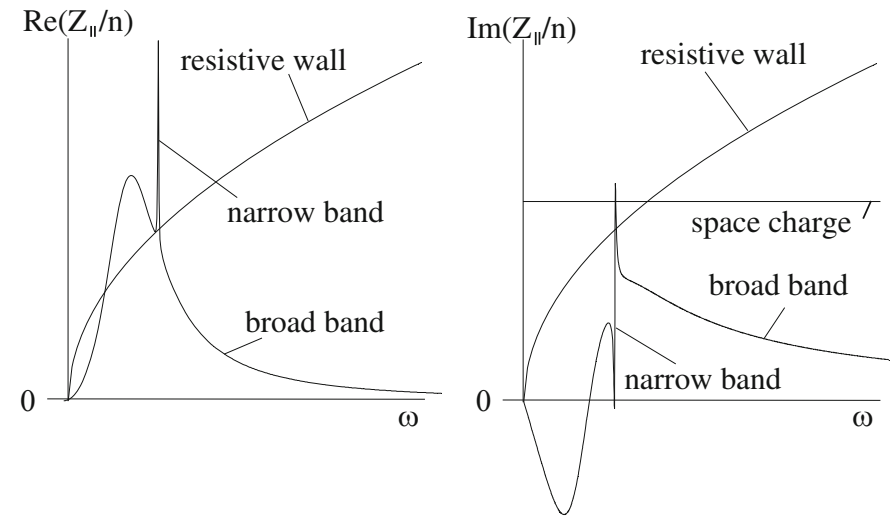

Fig. 22.7 Qualitative spectra of resistive and reactive coupling impedances in a circular accelerator

vacuum chamber components of a circular accelerator and has to do with the cut off frequency for travelling waves in tubes.

In Fig. 19.9 the measured impedance spectrum of a storage ring was shown and is typical for complex storage ring vacuum chambers which are generally composed of similar components exhibiting at low frequencies an inductive impedance increasing linearly with frequency and diminishing again at high frequencies. This is also the characteristics of broad-band cavity impedance and therefore expressions for broad-band impedance are useful tools in developing theories for beam instabilities and predicting conditions for beam stability. The induced voltage for the total ring circumference scales like $L_{\mathrm{w}} \dot{I}_{\mathrm{w}}$ where $L_{\mathrm{w}}$ is the wall inductance and $\dot{I}_{\mathrm{w}}$ the time derivative of the image current in the wall. The induced voltage is

$$
\Delta V_{z 0}=-L_{\mathrm{w}} \frac{\mathrm{d} I}{\mathrm{~d} t}=\mathrm{i} \omega L_{\mathrm{w}}(\omega) I_{n} \mathrm{e}^{\mathrm{i}\left(n \theta-\omega_{n} t\right)}
$$

where the inductive impedance is defined by

$$
Z_{\| \text {ind }}(\omega)=-\mathrm{i} \omega L_{\mathrm{w}}(\omega) \text {. }
$$

The total induced voltage due to space charge, resistive and inductive wall impedance is finally

$$
V_{z \mathrm{w}}=-Z_{\|} I_{n} \mathrm{e}^{\mathrm{i}\left(n \theta-\omega_{n} t\right)},
$$

where the total longitudinal normalized impedance at frequency $\omega_{n}$ is from (22.37), (22.39), (22.47)

$$
\frac{Z_{\|}\left(\omega_{n}\right)}{n}=\mathrm{i} \frac{1}{2 \epsilon_{0} \beta c \gamma^{2}}\left(1+2 \ln \frac{r_{\mathrm{w}}}{r_{0}}\right)+(1-\mathrm{i}) \frac{\bar{R}}{r_{\mathrm{w}} \sigma \delta_{\text {skin }}}-\mathrm{i} \frac{\omega_{0}}{4 \pi \epsilon_{0}} L_{\mathrm{w}}\left(\omega_{n}\right) .
$$


From the frequency dependence we note that space charge and inductive wall impedance becomes more important at high frequencies while the resistive wall impedance is dominant at low frequencies. The inductive wall impedance derives mostly from vacuum chamber discontinuities like sudden change in the vacuum chamber cross section, bellows, electrostatic plates, cavities, etc. In older accelerators, little effort was made to minimize the impedance and total ring impedances of $\left|Z_{\|} / n\right| \approx 20$ to $30 \Omega$ were not uncommon. Modern vacuum design have been developed to greatly reduce the impedance mostly by avoiding changes of vacuum chamber cross section or by introducing gentle transitions and impedances of the order of $\left|Z_{\|} / n\right| \lesssim 1 \Omega$ can be achieved whereby most of this remaining impedance comes from accelerating rf-cavities.

From (22.49), we note that the space-charge impedance has the opposite sign of the inductive impedance and is therefore capacitive in nature. In general, we encounter in a realistic vacuum chamber resistive as well as reactive impedances causing both real frequency shifts or imaginary shifts manifesting themselves in the form of damping or instability. In subsequent sections, we will discuss a variety of such phenomena and derive stability criteria, beam-current limits or rise times for instability. At this point, it is noteworthy to mention that we have not made any assumption as to the nature of the particles involved and we may therefore apply the results of this discussion to electron as well as proton and ion beams.

\subsubsection{Broad-Band Wake Fields in a Linear Accelerator}

The structure of a linear accelerator constitutes a large impedance for a charged particle beam, specifically, since particle bunches are very short compared to the periodicity of the accelerator lattice. Every single cell resembles a big sudden change of the vacuum chamber cross section and we expect therefore a large accumulation of wake fields or impedance along the accelerator. The wake fields can be calculated numerically [4] and results for both the longitudinal and transverse wakes from a point charge are shown in Fig. 22.8 as a function of the distance behind this point charge.

Broad-band wake fields for other structures look basically similar to those shown in Fig. 22.8. Specifically, we note the longitudinal wake to be strongest just behind the head of the bunch while the transverse wake builds up over some distance. For an arbitrary particle distribution, one would fold the particle distribution with these wake functions to obtain the wake potential at the location of the test particle.

\subsection{Coasting-Beam Instabilities}

The space-charge impedance as well as resistive and reactive wall impedances extract energy from a circulating particle beam. As long as the particle distribution 

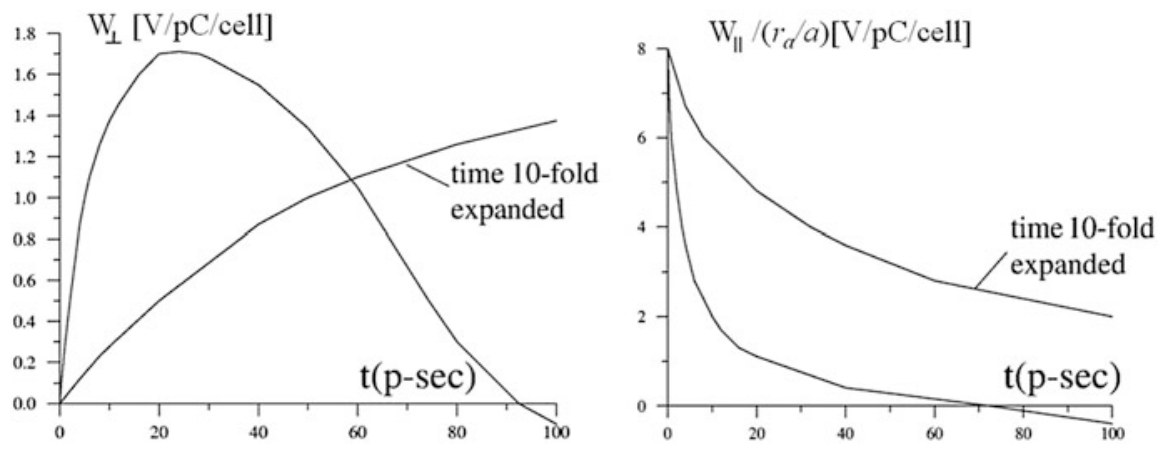

Fig. 22.8 Time dependence of transverse (left) and longitudinal (right) wake fields from a point charge moving through one $3.3 \mathrm{~cm}$ long cell of a SLAC type $3 \mathrm{GHz}$ linear accelerator structure [4]

is uniform, this energy loss is the same for all particles and requires simple replacement in acceleration cavities. In reality, however, some modulation of the longitudinal particle distribution cannot be avoided and we encounter therefore an uneven energy loss along the coasting particle beam. This can have serious consequences on beam stability and we therefore need to discuss stability criteria for coasting beams.

\subsubsection{Negative-Mass Instability}

Consider a beam in a ring below transition energy. The repulsive electrostatic field from a lump in the charge distribution causes particles ahead of the lump to be accelerated and particles behind the lump to be decelerated. Since accelerated particles will circulate faster and decelerated particles circulate slower, we observe a stabilizing situation and the lumpy particle density becomes smoothed out. Nature demonstrates this in the stability of Saturn's rings.which is equi8valent to this case below transition energy.

At energies above transition energy the situation changes drastically. Now the acceleration of a particle ahead of a lump leads to a slower revolution frequency and it will actually move closer to the lump with every turn. Similarly a particle behind the lump becomes decelerated and circulates therefore faster, again catching up with the lump. We observe an instability leading to a growing concentration of particles wherever a small perturbation started to occur. We call this instability the negativemass instability [11] because acceleration causes particles to circulate slower similar to the acceleration of a negative mass. The same mechanism can lead to stabilization of oscillations if the forces are attractive rather than repulsive.

We will derive conditions of stability for this effect in a more quantitative way. The stability condition depends on the variation of the revolution frequency for particles close to the small perturbation of an otherwise uniform longitudinal 
particle distribution and we therefore investigate the time derivative of the revolution frequency

$$
\frac{\mathrm{d} \omega}{\mathrm{d} t}=\frac{\partial \omega}{\partial t}+\frac{\partial \omega}{\partial \theta} \frac{\partial \theta}{\partial t}
$$

which can also be expressed in the form

$$
\frac{\mathrm{d} \omega}{\mathrm{d} t}=\frac{\mathrm{d} \omega}{\mathrm{d} E} \frac{\mathrm{d} E}{\mathrm{~d} t}=\frac{\eta_{\mathrm{c}} \omega_{0}}{\beta^{2} E_{0}} \frac{\mathrm{d} E}{\mathrm{~d} t},
$$

where $\eta_{\mathrm{c}}$ is the momentum compaction. The energy change per unit time is for a longitudinal impedance $Z_{z}$ and $n$th harmonic of the beam current

$$
\frac{\mathrm{d} E}{\mathrm{~d} t}=q V_{z 0} \frac{\omega_{0}}{2 \pi}=-q Z_{z} I_{n} \mathrm{e}^{\mathrm{i}(n \theta-\omega t)} \frac{\omega_{0}}{2 \pi},
$$

where $q=e Z>0$ is the electrical charge of the particle and $Z$ the charge multiplicity. Collecting all terms for (22.51) we get with

$$
\omega=\omega_{0}+\omega_{n} \mathrm{e}^{\mathrm{i}(n \theta-\Omega t)}
$$

the relation

$$
\omega_{n}\left(\Omega-n \omega_{0}\right)=-\mathrm{i} \frac{q \eta_{\mathrm{c}} \omega_{0}^{2}}{2 \pi \beta^{2}} \frac{I_{n} Z_{z}}{E_{0}} .
$$

This can be further simplified with the continuity equation

$$
\frac{\partial \lambda}{\partial t}+\frac{1}{\bar{R}} \frac{\partial}{\partial \theta}(\beta c \lambda)=\frac{\partial \lambda}{\partial t}+\frac{\partial \lambda}{\partial \theta} \omega_{0}+\frac{\partial \omega}{\partial \theta} \lambda_{0}=0
$$

and we get with (21.77), (22.53)

$$
\left(\Omega-n \omega_{0}\right) I_{n}=\omega_{n} n I_{0} .
$$

Replacing $\omega_{n}$ in (22.54) by the expression (22.55,) we finally get for the perturbation frequency $\Omega$ with $I_{0}=\beta c \lambda_{0}$

$$
\Delta \Omega^{2}=\left(\Omega-n \omega_{0}\right)^{2}=-\mathrm{i} \frac{n q \eta_{\mathrm{c}} \omega_{0}^{2} I_{0}}{2 \pi \beta^{2} E_{0}} Z_{\|} .
$$

Equation (22.56) determines the evolution of the charge or current perturbation $\lambda_{n}$ or $I_{n}$ respectively. With $\Delta \Omega=\Delta \Omega_{\mathrm{r}}+\mathrm{i} \Delta \Omega_{\mathrm{i}}$, the current perturbation is

$$
\left.I_{n} \mathrm{e}^{\mathrm{i}\left(n \theta-n \omega_{0} t-\Delta \Omega_{\mathrm{r}} t-\mathrm{i} \Delta \Omega_{\mathrm{i}} t\right.}\right)=I_{n} \mathrm{e}^{\Delta \Omega_{\mathrm{i}} t} \mathrm{e}^{\mathrm{i}\left(n \theta-n \omega_{0} t-\Delta \Omega_{\mathrm{r}} t\right)}
$$


exhibiting an exponential factor which can cause instability or damping since there is a positive as well as negative solution from (22.56) for the frequency shift $\Delta \Omega_{\mathrm{i}}$. The situation in a particular case will depend on initial conditions describing the actual perturbation of the density distribution, however, different initial perturbations must be expected to be present along a real particle distribution including at least one leading to instability.

Beam stability occurs only if the imaginary part of the frequency shift vanishes. This is never the case if the impedance has a resistive component causing a resistivewall instability [12]. From (22.56) and the resistive wall impedance (22.39) we may derive a growth rate for the instability

$$
\frac{1}{\tau_{\text {res.wall }}}=\operatorname{Im}\{\Delta \Omega\}=\frac{\sqrt{2}-1}{\sqrt{2}} \frac{n^{2} q \eta_{\mathrm{c}} \omega_{0}^{2} I_{0} \bar{R}}{2 \pi c \beta^{2} E_{0} r_{\mathrm{w}}} \sqrt{\frac{2 \pi \omega_{0} \mu}{n \sigma}} .
$$

This result requires some more discussion since we know that circular accelerators exist, work, and have metallic vacuum chambers with a resistive surface. The apparent discrepancy is due to the fact that we have assumed a monochromatic beam which indeed is unstable but also unrealistic. In the following sections, we include a finite momentum spread, find a stabilizing mechanism called Landau damping and derive new stability criteria.

Below transition energy, $\eta_{\mathrm{c}}>0$ will assure stability of a coasting beam as long as we consider only a purely capacitive impedance like the space-charge impedance (22.37) in which case $\Delta \Omega_{\mathrm{i}}=0$. Above transition energy $\eta_{\mathrm{c}}<0$ and the negative-mass instability appears as long as the impedance is capacitive or $Z_{\mathrm{i}}>0$. For an inductive impedance, the stability conditions are exchanged below and above transition energy. In summary, we have the following longitudinal coasting beam stability conditions:

$$
\begin{aligned}
& \text { if } \quad Z_{\mathrm{r}} \neq 0 \rightarrow \Delta \omega_{\mathrm{i}} \neq 0 \rightarrow\left\{\begin{array}{l}
\text { always stable } \\
\text { resistive-wall instability }
\end{array}\right.
\end{aligned}
$$

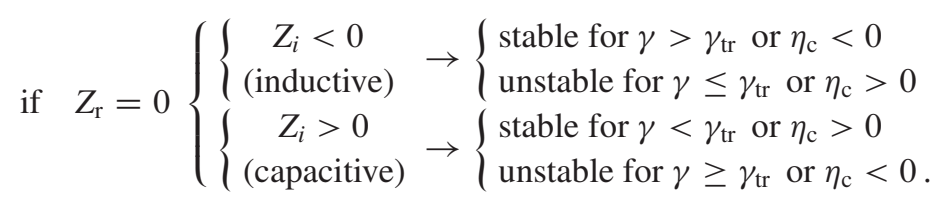

It is customary to plot the stability condition (22.56) in a $\left(Z_{\mathrm{r}}, Z_{\mathrm{i}}\right)$-diagram with $\Delta \Omega_{\mathrm{i}}$ as a parameter. We solve (22.56) for the imaginary impedance $Z_{\mathrm{i}}$ and get

$$
Z_{\mathrm{i}}=\operatorname{sgn}\left(\eta_{\mathrm{c}}\right) a\left[\left(\frac{Z_{\mathrm{r}}}{2 \Delta \Omega_{\mathrm{i}}}\right)^{2} \mp\left(\frac{\Delta \Omega_{\mathrm{i}}}{a}\right)^{2}\right]
$$


Fig. 22.9 Stability diagram for a coasting monochromatic particle beam

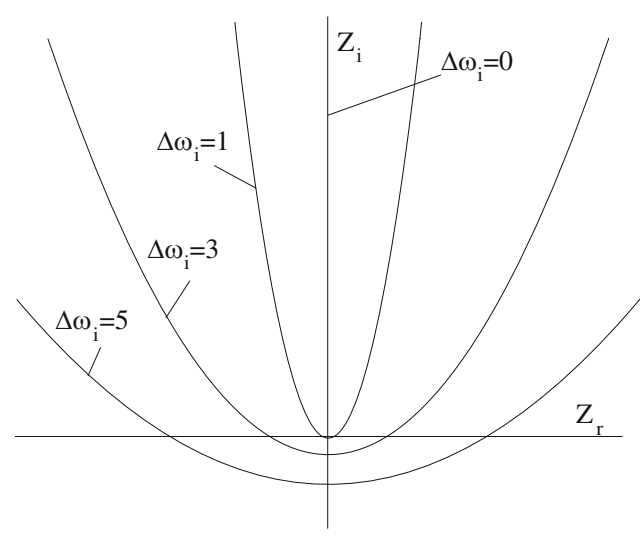

where

$$
a=\frac{n q\left|\eta_{\mathrm{c}}\right| \omega_{0}^{2} I_{0}}{2 \pi \beta^{2} E_{0}}
$$

and plot the result in Fig. 22.9. Only the case $\eta_{\mathrm{c}}>0$ is shown in Fig. 22.9 noting that the case $\eta_{\mathrm{c}}<0$ is obtained by a $180^{\circ}$ rotation of Fig. 22.9 about the $Z_{\mathrm{r}}$-axis. Figure 22.9 demonstrates that beam stability occurs only if $Z_{\mathrm{r}}=0$ and $Z_{\mathrm{i}}>0$. Knowing the complex impedance for a particular accelerator, Fig. 22.9 can be used to determine the rise time $1 / \tau=\Delta \Omega_{\mathrm{i}}$ of the instability.

The rise time or growth rate of the negative-mass instability above transition is for a beam circulating within a perfectly conducting vacuum chamber from (22.37) and (22.56)

$$
\frac{1}{\tau} \text { neg.mass }=\frac{n \omega_{0}}{\beta c \gamma} \sqrt{\frac{q\left|\eta_{\mathrm{c}}\right| c I_{0}\left(1+2 \ln \frac{r_{\mathrm{w}}}{r_{0}}\right)}{\beta E_{0}}} .
$$

In this section, it was implicitly assumed that all particles have the same momentum and therefore, the same revolution frequency $\omega_{0}$ allowing a change of the revolution frequency only for those particles close to a particle density perturbation. This restriction to a monochromatic beam is not realistic and provides little beam stability for particle beams in a circular accelerator. In the following section, we will discuss the more general case of a beam with a finite momentum spread and review beam stability conditions under more realistic beam parameters.

\subsubsection{Dispersion Relation}

In the previous section, conditions for beam stability were derived based on a monochromatic particle beam. The rise time of the instability depends critically 
on the revolution frequency and we may assume that the conditions for beam stability may change if we introduce the more realistic case of a beam with a finite momentum spread and therefore a finite spread of revolution frequencies. In Chap. 15, we discussed the mathematical tool of the Vlasov equation to describe collectively the dynamics of a distribution of particles in phase space. We will apply this tool to the collective interaction of a particle beam with its environment.

The canonical variables describing longitudinal motion of particles are the azimuth $\theta$ and relative momentum error $\delta=\Delta p / p_{0}$. Neglecting radiation damping, the Vlasov equation is

$$
\frac{\partial \Psi}{\partial t}+\dot{\theta} \frac{\partial \Psi}{\partial \theta}+\dot{\delta} \frac{\partial \Psi}{\partial \delta}=0
$$

where $\Psi(\delta, \theta, t)$ is the particle distribution. For a coasting beam with a small perturbation

$$
\Psi=\Psi_{0}+\Psi_{n} \mathrm{e}^{\mathrm{i}\left(n \theta-\omega_{n} t\right)}
$$

we get after insertion in (22.64) and sorting terms the relation

$$
\mathrm{i}\left(\omega_{n}-n \omega\right) \Psi_{n}=\frac{\dot{\delta}}{\mathrm{e}^{\mathrm{i}\left(n \theta-\omega_{n} t\right)}} \frac{\partial \Psi_{0}}{\partial \delta} .
$$

Making use of the correlation between particle momentum and revolution frequency, we get from (22.66) with $\frac{\partial \Psi_{0}}{\partial \delta}=\frac{\partial \Psi_{0}}{\partial \omega} \frac{\partial \omega}{\partial \delta}=\eta_{\mathrm{c}} \omega_{0} \frac{\partial \Psi_{0}}{\partial \omega}$

$$
\Psi_{n}=-\mathrm{i} \frac{\eta_{\mathrm{c}} \omega_{0} \dot{\delta}}{\mathrm{e}^{\mathrm{i}\left(n \theta-\omega_{n} t\right)}} \frac{\partial \Psi_{0}}{\partial \omega} \frac{1}{\omega_{n}-n \omega} .
$$

Integrating the 1.h.s. of (22.67) over all momenta, we get for the perturbation current

$$
q \frac{\beta c}{\bar{R}} \int_{-\infty}^{\infty} \Psi_{n}(\delta) \mathrm{d} \delta=I_{n} .
$$

At this point, it is convenient to transform from the variable $\delta$ to the frequency $\omega$ and obtain the particle distribution in these new variables

$$
\Psi(\delta, \theta)=\eta_{\mathrm{c}} \omega_{0} \Phi(\omega, \theta)
$$

Performing the same integration on the r.h.s. of (22.67), we get with (22.52) and $\dot{\delta}=(\mathrm{d} E / \mathrm{d} t) /\left(\beta^{2} E_{0}\right)$ the dispersion relation [13]

$$
1=-\mathrm{i} \frac{q^{2} \omega_{0}^{3} \eta_{\mathrm{c}} Z_{z}}{2 \pi \beta^{2} E_{0}} \int \frac{\partial \Phi_{0} / \partial \omega}{\omega_{n}-n \omega} \mathrm{d} \omega .
$$


The integration is to be taken over the upper or lower complex plane where we assume that the distribution function $\Phi$ vanishes sufficiently fast at infinity. Trying to establish beam stability for a particular particle distribution, we solve the dispersion relation for the frequency $\omega_{n}$ or frequency shift $\Delta \omega_{n}=\omega_{n}-n \omega$ which is in general complex. The real part causes a shift in the frequency while the imaginary part determines the state of stability or instability for the collective motion.

For example, it is interesting to apply this result to the case of a coasting beam of monochromatic particles as discussed in the previous section. Let the particle distribution be uniform in $\theta$ and a delta function in energy. In the dispersion relation, we need to take the derivative with respect to the revolution frequency and set therefore

$$
\frac{\partial \Phi_{0}}{\partial \omega}=\frac{N_{\mathrm{p}}}{2 \pi} \frac{\partial}{\partial \omega} \delta\left(\omega-\omega_{0}\right) .
$$

Insertion into (22.69) and integration by parts results in

$$
\int_{-\infty}^{\infty} \frac{\partial \Phi_{0} / \partial \omega}{\omega_{n}-n \omega} \mathrm{d} \omega=\frac{N_{\mathrm{b}}}{2 \pi} \frac{n}{\left(\omega_{n}-n \omega_{0}\right)^{2}}
$$

which is identical to the earlier result (22.56) in the previous section. Application of the Vlasov equation therefore gives the same result as the direct derivation of the negative-mass instability conditions as it should be.

We may now apply this formalism to a beam with finite momentum spread. In preparation to do that, we note that the integrand in (22.69) has a singularity at $\omega=\omega_{n} / n$ which we take care of by applying Cauchy's residue theorem for

$$
\int \frac{\partial \Phi_{0} / \partial \omega}{\omega_{n}-n \omega} \mathrm{d} \omega=\text { P.V. } \int_{n \omega \neq \omega_{n}} \frac{\partial \Phi_{0} / \partial \omega}{\omega_{n}-n \omega} \mathrm{d} \omega-\left.\mathrm{i} \pi \frac{\partial \Phi_{0}}{\partial \omega}\right|_{\omega_{n} / n} .
$$

The dispersion relation (22.69) then assumes the form

$$
1=\mathrm{i} \frac{q^{2} \omega_{0}^{3} \eta_{\mathrm{c}} Z_{z}}{2 \pi \beta^{2} E_{0}}\left[\left.\mathrm{i} \frac{\pi}{n} \frac{\partial \Phi_{0}}{\partial \omega}\right|_{\omega=\frac{\omega_{n}}{n}}-\mathrm{P} . \mathrm{V} \cdot \int \frac{\partial \Phi_{0} / \partial \omega}{\omega_{n}-n \omega} \mathrm{d} \omega\right],
$$

where P.V. indicates that only the principal value of the integral be taken.

The solutions of the dispersion function depend greatly on the particle distribution in momentum or revolution-frequency space. To simplify the expressions, we replace the revolution frequency by its deviation from the reference value [14]. With $2 S$ being the full-width half maximum of the particle momentum distribution (Fig. 22.10), we define the new variables

$$
x=\frac{\omega-\omega_{0}}{S}, \quad \text { and } \quad x_{1}=\frac{\Delta \omega_{n}}{n S}=\frac{\Omega-n \omega_{0}}{n S} .
$$


Fig. 22.10 Particle distribution $f(x)$

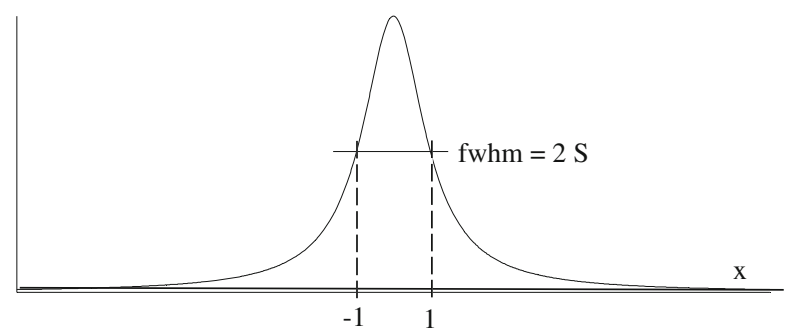

In these variables the particle distribution becomes

$$
f(x)=\frac{2 \pi S}{N_{\mathrm{b}}} \Phi(\omega)
$$

which is normalized to $f( \pm 1)=\frac{1}{2} f(0)$ and $\int f(x) \mathrm{d} x=1$. The full momentum spread at half maximum intensity is

$$
\frac{\Delta p}{p_{0}}=\frac{2 S}{\left|\eta_{\mathrm{c}}\right| \omega_{0}}
$$

and (22.73) becomes with this

$$
1=-\mathrm{i} \frac{2 q Z_{z} I_{0}}{\pi \beta^{2} E_{0} n \eta_{\mathrm{c}}\left(\frac{\Delta p}{p_{0}}\right)^{2}}\left[\mathrm{P} . \mathrm{V} . \int_{-\infty}^{\infty} \frac{\partial f_{0}(x) / \partial x}{x_{1}-x} \mathrm{~d} x-\left.\mathrm{i} \pi \frac{\partial f_{0}}{\partial x}\right|_{x_{1}}\right]
$$

It is customary to define parameters $U, V$ by

$$
V+\mathrm{i} U=\frac{2 q I_{0}}{\pi \beta^{2} E_{0} \eta_{\mathrm{c}}\left(\frac{\Delta p}{p_{0}}\right)^{2}} \frac{\left(Z_{\mathrm{r}}+\mathrm{i} Z_{\mathrm{i}}\right)_{z}}{n}
$$

and the dispersion relation becomes finally with this

$$
1=-(V+\mathrm{i} U) I
$$

where the integral

$$
I=\left[\operatorname{P.V} \cdot \int_{-\infty}^{\infty} \frac{\partial f_{0}(x) / \partial x}{x_{1}-x} \mathrm{~d} x-\left.\mathrm{i} \pi \frac{\partial f_{0}}{\partial x}\right|_{x_{1}}\right]
$$

For a particular accelerator all parameters in (22.79) are known, at least in principle, and we may determine the status of stability or instability for a desired beam current $I_{0}$ by solving for the generally complex frequency shift $\Delta \omega$. The specific 
Fig. 22.11 Particle distribution in momentum space

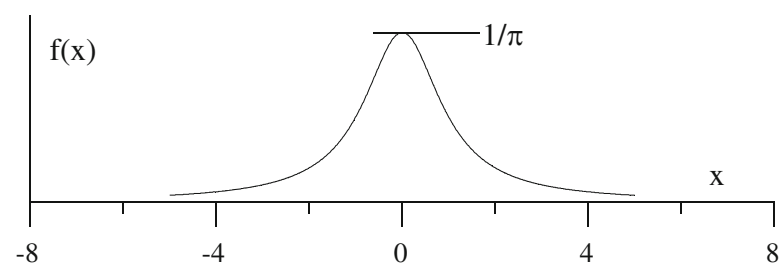

boundary of stability depends on the actual particle distribution in momentum. Unfortunately, (22.79) cannot be solved analytically for an arbitrary momentum distribution and we will have to either restrict our analytical discussion to simple solvable distributions or to numerical evaluation.

For reasonable representations of real particle distributions in an accelerator a central region of stability can be identified for small complex impedances and finite spread in momentum. Regions of stability have been determined for a number of particle distributions and the interested reader is referred for more detailed information on such calculations to references [15-19].

As an example, we use a simple particle distribution (Fig. 22.11)

$$
f(x)=\frac{1}{\pi} \frac{1}{1+x^{2}}
$$

and evaluate the dispersion relation (22.79). The integral in (22.80) becomes now after integration by parts

$$
I=\mathrm{P} . \mathrm{V} \cdot \int_{-\infty}^{\infty} \frac{1}{\left(1+x^{2}\right)\left(x_{1}-x\right)^{2}} \mathrm{~d} x
$$

exhibiting a new singularity at $x=i$ while the integration path still excludes the other singularity at $x=x_{1}$. Applying the residue theorem

$$
\int \frac{f(z) \mathrm{d} z}{z-z_{0}}=\mathrm{i} 2 \pi \operatorname{Res}\left[f(z), z_{0}\right]=\mathrm{i} 2 \pi \lim _{z \rightarrow z_{0}}\left(z-z_{0}\right) f(z)
$$

we get

$$
\text { P.V. } \int_{-\infty}^{\infty} \frac{1}{\left(1+x^{2}\right)\left(x_{1}-x\right)} \mathrm{d} x=\frac{1}{\left(x_{1}-\mathrm{i}\right)^{2}}
$$

The second term in (22.80) is

$$
-\left.\mathrm{i} \pi \frac{\partial f_{0}}{\partial x}\right|_{x_{1}}=\mathrm{i} \frac{2 x_{1}}{\left(1+x_{1}^{2}\right)^{2}}
$$


and the dispersion relation (22.79) becomes

$$
1=-\mathrm{i}(V+\mathrm{i} U)\left(\frac{1}{\left(x_{1}-\mathrm{i}\right)^{2}}+\mathrm{i} \frac{2 x_{1}}{\left(1+x_{1}^{2}\right)^{2}}\right) .
$$

We solve this for $\left(x_{1}-i\right)^{2}$ and get

$$
x_{1}=\mathrm{i} \pm \sqrt{-\mathrm{i}(V+\mathrm{i} U)\left(1+\mathrm{i} \frac{2 x_{1}}{\left(\mathrm{i}+x_{1}\right)^{2}}\right)} .
$$

For a small beam current $i_{0}$, we get $x_{1} \approx i$ and the second term in the square bracket becomes approximately $1 / 2$. Recalling the definition (22.74) for $x_{1}$, we get from (22.87)

$$
\Delta \Omega=\mathrm{i} n S \pm \sqrt{\frac{3}{2} n^{2} S^{2}(U-\mathrm{i} V)}
$$

where from (22.76) $S=\frac{1}{2}\left|\eta_{\mathrm{c}}\right| \omega_{0} \Delta p / p_{0}$. The significant result in (22.88) is the fact that the first term on the right-hand side has a definite positive sign and provides therefore damping which is called Landau damping [20].

Recalling the conditions for the negative-mass instability of a monochromatic beam, we did not obtain beam stability for any beam current if $Z_{\mathrm{r}} \propto V=0$ and the reactive impedance was inductive or $Z_{\mathrm{i}} \propto U<0$. Now with a finite momentum spread in the beam we get in the same case

$$
\Delta \Omega_{\text {neg.mass }}=\mathrm{i} n S \pm \mathrm{i} \sqrt{\frac{3}{2} n^{2} S^{2}|U|},
$$

where $S^{2}|U|$ is independent of the momentum spread. We note that it takes a finite beam current $\left(U \propto I_{0}\right)$ to overcome Landau damping and cause instability. Of course Landau damping is proportional to the momentum spread $S$ and does not occur for a monochromatic beam. Equation (22.88) serves as a stability criterion for longitudinal coasting-beam instabilities and we will try to derive a general expression by writing (22.88) in the form

$$
\Delta \Omega=\mathrm{i} n S \pm \sqrt{a-\mathrm{i} b}
$$

and get after evaluating the square root

$$
\Delta \Omega=\mathrm{i} n S \pm\left(\sqrt{\frac{r+a}{2}}-\mathrm{i} \sqrt{\frac{r-a}{2}}\right),
$$

where $r=\sqrt{a^{2}+b^{2}}$. Beam stability occurs for $\operatorname{Im}\{\Delta \Omega\}>0$ or

$$
n^{2} S^{2}=\frac{r-a}{2}
$$


Fig. 22.12 Stability diagram for the particle distribution (22.93)

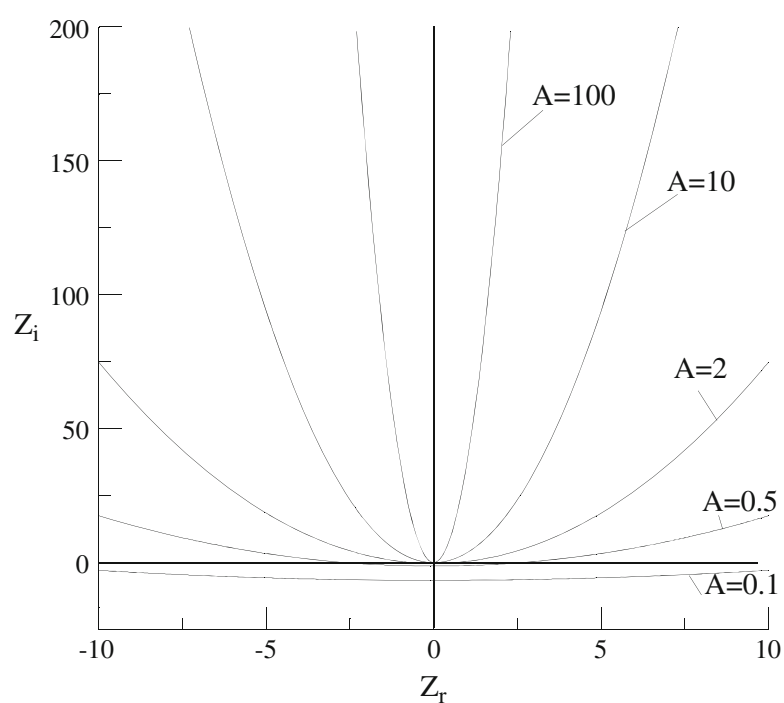

which is in more practical quantities recalling the definition (22.76) for $S$

$$
\left(\frac{\Delta p}{p_{0}}\right)^{2} \geq \frac{3}{2 \pi} \frac{q I_{0}}{\beta^{2} E_{0}\left|\eta_{\mathrm{c}}\right|}\left(\frac{\left|Z_{z}\right|}{n}-\frac{Z_{\mathrm{i}}}{n}\right) .
$$

We may solve (22.93) for the impedance and get an equation of the form

$$
Z_{\mathrm{i}}=A Z_{\mathrm{r}}^{2}-\frac{1}{4 A}
$$

which is shown in Fig. 22.12.

Any combination of actual resistive and reactive impedances below this curve cause beam instability for the particle distribution (22.81). We note the significant difference to Fig. 22.9 where the impedance had to be purely positive and reactive to obtain beam stability.

Other momentum distributions like $f(x) \propto\left(1-x^{2}\right)^{m}$ lead to similar results [15] although the stability curves allow less resistive impedance than the distribution (22.81). As a safe stability criterion which is true for many such distributions including a Gaussian distribution we define the area of stability by a circle with a radius $R=\left.Z_{\mathrm{i}}\right|_{Z_{\mathrm{r}}=0}=1 /(4 A)$. With this assumption, the stability criterion for the longitudinal microwave instability is

$$
\frac{\left|Z_{z}\right|}{n} \leq F \frac{\beta^{2} E_{0}\left|\eta_{\mathrm{c}}\right|}{q I_{0}}\left(\frac{\Delta p}{p_{0}}\right)^{2},
$$


where the form factor $F=\pi / 3$ for the distribution (22.81) and is generally of the order of unity for other bell shaped distributions. The criterion (22.95) has been derived by Keil and Schnell [21] and is known as the Keil-Schnell stability criterion. For a desired beam current and an allowable momentum spread an upper limit for the normalized impedance can be derived.

The impedance seen by the particle beam obviously should be minimized to achieve the highest beam-beam currents. A large value of the momentum compaction is desirable here to increase the mixing of the revolution frequencies as well as a large momentum spread to achieve high beam currents. A finite momentum spread increases beam stability where there was none for a monochromatic coasting beam as discussed earlier. This stabilization effect of a finite momentum spread is called Landau damping.

\subsubsection{Landau Damping}

In previous sections, we repeatedly encountered a damping phenomenon associated with the effect of collective fields on individual particle stability. Common to the situations encountered is the existence of a set of oscillators or particles, under the influence of an external driving force. Particularly, we are interested in the dynamics when the external excitation is caused by the beam itself. Landau [20] studied this effect first and later Hereward [22] formulated the theory for application to particle accelerators.

We consider a bunch of particles where each particle oscillates at a different frequency $\Omega$, albeit within a small range of frequencies. The equation of motion for each oscillator under the influence of the force $F e^{-\mathrm{i} \omega t}$ is

$$
\ddot{u}+\Omega^{2} u=F \mathrm{e}^{-\mathrm{i} \omega t}
$$

and the solution

$$
u=F \frac{\mathrm{e}^{-\mathrm{i} \omega t}}{2 \omega}\left(\frac{1}{\Omega-\omega}-\frac{1}{\Omega+\omega}\right) .
$$

Folding this solution with the distribution function of particles in frequency space

$$
\psi(\omega)=\frac{1}{N_{\mathrm{b}}} \frac{\mathrm{d} N_{\mathrm{b}}}{\mathrm{d} \Omega}
$$

one obtains the center of mass amplitude of the bunch

$$
\bar{u}=F \frac{\mathrm{e}^{-\mathrm{i} \omega t}}{2 \omega} \int_{-\infty}^{\infty}\left[\frac{\psi(\Omega)}{\Omega-\omega}-\frac{\psi(\Omega)}{\Omega+\omega}\right] \mathrm{d} \Omega
$$


or with $\psi(\Omega)=\psi(-\Omega)$ and $\int_{-\infty}^{\infty} \frac{\psi(\Omega)}{\Omega-\omega} \mathrm{d} \Omega=-\int_{-\infty}^{\infty} \frac{\psi(\Omega)}{\Omega+\omega} \mathrm{d} \Omega$

$$
\bar{u}=F \frac{\mathrm{e}^{-\mathrm{i} \omega t}}{\omega} \int_{-\infty}^{\infty} \frac{\psi(\Omega)}{\Omega-\omega} \mathrm{d} \Omega
$$

Here we apply again Cauchy's residue theorem and get

$$
\bar{u}=F \frac{\mathrm{e}^{-\mathrm{i} \omega t}}{\omega}\left[+\mathrm{i} \pi \psi(\omega)+\mathrm{P} . \mathrm{V} . \int_{-\infty}^{\infty} \frac{\psi(\Omega)}{\Omega-\omega} \mathrm{d} \Omega\right] .
$$

The derivation up to here appears quite abstract and we pause a moment to reflect on the physics involved here. We know that driving an oscillator at resonance leads to infinitely large amplitudes and that is what the mathematical formulation above expresses. However, we also know that infinite amplitudes take an infinite time to build up and the solutions gained above describe only the state after a long time. The same result can be obtained in a physical more realistic way if we apply the excitation at time $t=0$ and look for the solution at $t \rightarrow \infty$ as has been shown by Hofmann [23]. As an added result of this time evolution derivation, we obtain the correct sign for the residue which we have tacitly assumed to be negative, but mathematically could be of either sign.

To understand the damping effect, we calculate the velocity $\overline{\dot{u}}$ and get from $(22.101)$

$$
\begin{aligned}
\overline{\dot{u}} & =-\mathrm{i} \omega \bar{u} \\
& =F \mathrm{e}^{-\mathrm{i} \omega t}\left[+\pi \psi(\omega)-\mathrm{iP} . \mathrm{V} . \int_{-\infty}^{\infty} \frac{\psi(\Omega)}{\Omega-\omega} \mathrm{d} \Omega\right]
\end{aligned}
$$

The bunch velocity is in phase with the external force for the residue term allowing extraction of energy from the external force. The principal value term, on the other hand, is out of phase and no work is done. If, for example, the external force is due to a transverse wake field generated by a bunch performing coherent betatron oscillations, the described mechanism would extract energy from the wake field thus damping the coherent betatron oscillation. The question is where does the energy go?

For this, we study the time evolution of the solution for the inhomogeneous differential equation (22.96) in the form

$$
u=a \sin \Omega t+\frac{F}{\Omega^{2}-\omega^{2}} \sin \omega t .
$$

At time $t=0$ we require that the amplitude and velocity of the bunch motion be zero $u(t=0)=0$ and $\dot{u}(t=0)=0$. The oscillation amplitude

$$
a=-\frac{\omega}{\Omega} \frac{F}{\Omega^{2}-\omega^{2}}
$$


and the final expression for the solution to (22.96) is for $\Omega \neq \omega$

$$
u_{\Omega \neq \omega}(t)=\frac{F}{\Omega^{2}-\omega^{2}}\left(\sin \omega t-\frac{\omega}{\Omega} \sin \Omega t\right) .
$$

Close to or at resonance $\Omega=\omega+\Delta$ and (22.105) becomes

$$
u_{\Omega \approx \omega}(t)=-\frac{F}{2 \omega}\left(t \cos \omega t-\frac{\sin \omega t}{\omega}\right) .
$$

The oscillation amplitude of particles at resonance grows continuously with time while the width of the resonance shrinks like $1 / t$ thus absorbing energy linear in time. This Landau damping depends critically on the resistive interaction with the wake fields or external forces and is mathematically expressed by the residue term. This residue term, however, depends on the particle density at the excitation frequency $\omega$ and is zero if the particle distribution in frequency space does not overlap with the frequency $\omega$. For effective Landau damping to occur such an overlap is essential.

\subsubsection{Transverse Coasting-Beam Instability}

Particle beams travelling off center through vacuum chamber sections can induce transverse fields which act back on the beam. We express the strength of this interaction by the transverse coupling impedance. In terms of the transverse coupling impedance, the force is

$$
F_{\perp}=\mathrm{i} \frac{q Z_{\perp} I_{0} u}{2 \pi \bar{R}}
$$

where $I_{0}$ is the beam current, $u$ the transverse beam displacement, $Z_{\perp} /(2 \pi \bar{R})$ the average transverse coupling impedance and $2 \pi \bar{R}$ the ring circumference. The equation of motion is then

$$
\ddot{u}+v_{0}^{2} \omega_{0}^{2} u=-\mathrm{i} \frac{q Z_{\perp} I_{0}}{2 \pi \bar{R} m \gamma}(u+\bar{u})
$$

with $u$ the betatron oscillation amplitude of an individual particle and $\bar{u}$ the amplitude of the coherent bunch oscillation. Since the perturbation is linear in the amplitudes, we expect tune shifts from the perturbations. The incoherent tune shift due to individual particle motion will be incorporated on the 1.h.s. as a small tune shift

$$
\delta v_{0}=\mathrm{i} \frac{c q Z_{\perp} I_{0}}{4 \pi v_{0} \omega_{0} E_{0}}
$$


The transverse impedance is generally complex and we get therefore from the real part of the coupling impedance a real tune shift while the imaginary part leads to damping or antidamping depending on the sign of the impedance. The imaginary frequency shift is equal to the growth rate of the instability and is given by

$$
\frac{1}{\tau}=\operatorname{Im}\{\omega\}=\frac{q \operatorname{Re}\left\{Z_{\perp}\right\} I_{0}}{4 \pi \bar{R} m \gamma \omega_{\beta_{0}}} .
$$

For a resistive transverse impedance, we observe therefore always instability known as the transverse resistive-wall instability.

Similar to the case of a longitudinal coasting beam, we find instability for any finite beam current just due to the reactive space-charge impedance alone, and again we have to rely on Landau damping to obtain beam stability for a finite beam intensity. To derive transverse stability criteria including Landau damping, we consider the coherent tune shift caused by the coherent motion of the whole bunch for which the equation of motion is

$$
\ddot{u}+\omega_{\beta 0}^{2} u=2 v_{0} \omega_{0}[U+(1+\mathrm{i}) V] \bar{u},
$$

where

$$
U+(1+\mathrm{i}) V=-\mathrm{i} \frac{c q Z_{\perp} I_{0}}{4 \pi v_{0} E_{0}},
$$

The coherent beam oscillation must be periodic with the circumference of the ring and is of the form $\bar{u}=\hat{u} e^{\mathrm{i}(n \theta-\omega t)}$. As can be verified by back insertion the solution of (22.111) is

$$
y=[U+(1+i) V] \frac{2 v_{0} \omega_{0}}{v_{1}^{2} \omega_{0}^{2}-\left(n \omega_{0}-\omega\right)^{2}} \bar{u} .
$$

Now we must fold (22.113) with the distribution in the spread of the betatron oscillation frequency. This spread is mainly related to a momentum spread via the chromaticity and the momentum compaction. The distribution $\psi(\delta)$ where $\delta=\Delta p / p_{0}$, is normalized to unity $\int \psi(\delta) d \delta=1$ and the average particle position is $\bar{u}=\int u \psi(\delta) \mathrm{d} \delta$. The dispersion relation is then with this from (22.113)

$$
1=[U+(1+\mathrm{i}) V] \int_{-\infty}^{\infty} \frac{2 v_{0} \omega_{0} \psi(\delta) \mathrm{d} \delta}{v_{1}^{2} \omega_{0}^{2}-\left(n \omega_{0}-\omega\right)^{2}} .
$$

or simplified by setting $v_{1} \approx v_{0}$ and ignoring the fast wave $(n+v) \omega_{0}[24]$

$$
1=[U+(1+\mathrm{i}) V] \int_{-\infty}^{\infty} \frac{\psi(\delta) \mathrm{d} \delta}{\omega-\left(n-v_{0}\right) \omega_{0}} .
$$


This is the dispersion relation for transverse motion and can be evaluated for stability based on a particular particle distribution in momentum. As mentioned before, the momentum spread transforms to a betatron oscillation frequency spread by virtue of the momentum compaction

$$
\Delta v_{\beta}=v_{\beta 0} \Delta \omega_{0}=v_{\beta 0} \eta_{\mathrm{c}} \delta \omega_{0}
$$

and by virtue of the chromaticity

$$
\Delta v_{\beta}=\xi_{u} \delta
$$

Landau damping provides again beam stability for a finite beam current and finite coupling impedances, and the region of stability depends on the actual particle distribution in momentum.

\subsection{Longitudinal Single-Bunch Effects}

The dynamics in bunched particle beams is similar to that of a coasting beam with the addition of synchrotron oscillations. The frequency spectrum of the circulating beam current contains now many harmonics of the revolution frequency with sidebands due to betatron and synchrotron oscillations. The bunch length depends greatly on the interaction with the rf-field in the accelerating cavities but also with any other field encountered within the ring. It is therefore reasonable to expect that wake fields may have an influence on the bunch length which is know as potential well distortion.

\subsubsection{Potential-Well Distortion}

From the discussions on longitudinal phase space motion in circular accelerators, it is known that the particle distribution or bunch length depends on the variation in time of the rf-field interacting with the beam in the accelerating cavities. Analogous, we would expect that wake fields may have an impact on the longitudinal particle distribution. Pellegrini and Sessler [25] For a particular wake field, we have studied this effect in Chap. 15 recognizing that a bunch passing through an rf-cavity causes beam loading by exciting fields at the fundamental frequency in the cavity. These fields then cause a modification of the bunch length. In this section, we will expand on this thought and study the effect due to higher-order mode wake fields.

To study this in more detail, we ignore the transverse particle distribution. The rate of change in the particle momentum can be derived from the integral of all longitudinal forces encountered along the circumference and we set with $\delta=d p / p_{0}$

$$
\frac{\mathrm{d} \delta}{\mathrm{d} t}=\frac{q F(\tau)}{\beta^{2} E_{0} T_{0}},
$$


where $q F(\tau)$ is the sum total of all acceleration and energy losses of a particle at a position $z=\beta c \tau$ from the bunch center or reference point over the course of one revolution and $T_{0}$ is the revolution time. The change of $\tau$ per unit time depends on the momentum compaction of the lattice and the momentum deviation

$$
\frac{\mathrm{d} \tau}{\mathrm{d} t}=-\eta_{\mathrm{c}} \delta .
$$

Both equations can be derived from the Hamiltonian

$$
\mathcal{H}=-\frac{1}{2} \eta_{\mathrm{c}} \delta^{2}-\int_{0}^{\tau} \frac{q F(\bar{\tau})}{\beta^{2} E_{0} T_{0}} \mathrm{~d} \bar{\tau} .
$$

For an electron ring and small oscillation amplitudes, we have

$$
q F(\tau)=q V_{\mathrm{rf}}\left(\tau_{\mathrm{s}}+\tau\right)-U(E)+q V_{\mathrm{w}}(\tau)=\left.q \frac{\partial V_{\mathrm{rf}}}{\partial \tau}\right|_{\tau_{\mathrm{s}}} \tau+q V_{\mathrm{w}}(\tau),
$$

where we ignored radiation damping and where $V_{\mathrm{w}}(\tau)$ describes the wake field. In the last form, the equation is also true for protons and ions if we set the synchronous time $\tau_{\mathrm{s}}=0$. Inserting (22.121) into (22.120) and using the definition of the synchrotron oscillation frequency (9.35) we get the new Hamiltonian

$$
\mathcal{H}=-\frac{1}{2} \eta_{\mathrm{c}} \delta^{2}-\frac{1}{2} \frac{\Omega_{\mathrm{s} 0}^{2}}{\eta_{\mathrm{c}}} \tau^{2}-\int_{0}^{\tau} \frac{q V_{\mathrm{w}}(\bar{\tau})}{\beta^{2} E_{0} T_{0}} \mathrm{~d} \bar{\tau} .
$$

\section{Synchrotron Oscillation Tune Shift}

First we use the Hamiltonian to formulate the equation of motion and determine the effect of wake fields on the dynamics of the synchrotron motion. The equation of motion is from (22.122)

$$
\ddot{\tau}+\Omega_{\mathrm{s} 0}^{2} \tau=\operatorname{sign}\left(\eta_{\mathrm{c}}\right) \frac{2 \pi \Omega_{\mathrm{s} 0}^{2} V_{\mathrm{w}}}{\omega_{0} h V_{\mathrm{rf}} \mid \cos \psi_{\mathrm{s} 0}},
$$

where we have made use of the definition of the unperturbed synchrotron oscillation frequency $\Omega_{\mathrm{s} 0}$. We express the wake field in terms of impedance and beam spectrum

$$
V_{\mathrm{w}}(t)=-\int_{-\infty}^{\infty} Z_{\|}(\omega) I(t, \omega) \mathrm{e}^{\mathrm{i} \omega t} \mathrm{~d} \omega,
$$

and use (21.85) for

$$
V_{\mathrm{w}}(t)=-I_{\mathrm{b}} \sum_{p=-\infty}^{\infty} Z_{\|}(p) \Psi(p) \mathrm{e}^{-\mathrm{i} p \omega_{0} \tau},
$$


where $I_{\mathrm{b}}$ is the bunch current and

$$
\Psi(p)=\int_{-\infty}^{+\infty} J_{0}\left(p \omega_{0} \hat{\tau}\right) \Phi(t, \hat{\tau}) \mathrm{d} \hat{\tau}
$$

The maximum excursion $\hat{\tau}$ during phase oscillation is much smaller than the revolution time and the exponential factor

$$
\mathrm{e}^{\mathrm{i} p \omega_{0} \tau} \approx 1+\mathrm{i} p \omega_{0} \tau-\frac{1}{2} p^{2} \omega_{0}^{2} \tau^{2}+\mathcal{O}(3)
$$

can be expanded. After insertion of (22.120), (22.121) into (22.123) the equation of motion is

$$
\begin{aligned}
\ddot{\tau}+\Omega_{\mathrm{s} 0}^{2} \tau & \approx \\
& -\operatorname{sign}\left(\eta_{\mathrm{c}}\right) \frac{2 \pi I_{\mathrm{b}} \Omega_{\mathrm{s} 0}^{2}}{\omega_{0} h V_{\mathrm{rf}}\left|\cos \psi_{\mathrm{s} 0}\right|} \sum_{p=-\infty}^{\infty} Z_{\|}(p) \Psi(p)\left(1-\mathrm{i} p \omega_{0} \tau-\frac{1}{2} p^{2} \omega_{0}^{2} \tau^{2}\right) .
\end{aligned}
$$

The first term in the factor $\left(1-\mathrm{i} p \omega_{0} \tau-\frac{1}{2} p^{2} \omega_{0}^{2} \tau^{2}\right)$ is independent of $\tau$ and causes a synchronous phase shift due to resistive losses

$$
\Delta \psi_{\mathrm{s}}=\operatorname{sgn}\left(\eta_{\mathrm{c}}\right) \frac{2 \pi I_{\mathrm{b}}}{V_{\mathrm{rf}}\left|\cos \psi_{\mathrm{s} 0}\right|} \sum_{p=-\infty}^{\infty} \operatorname{Re}\left\{Z_{\|}(p)\right\} \Psi(p) .
$$

For a resistive positive impedance, for example, the phase shift is negative above transition indicating that the beam requires more energy from the rf-cavity. By measuring the shift in the synchronous phase of a circulating bunch as a function of bunch current, it is possible to determine the resistive part of the longitudinal impedance of the accelerator. To do this one may fill a small amount of beam in the bucket upstream from the high intensity bunch and use the signal from the small bunch as the time reference against which the big bunch will shift with increasing current.

The second term in (22.127) is proportional to $\tau$ and therefore acts like a focusing force shifting the incoherent synchrotron oscillation frequency by

$$
\Delta \Omega_{\mathrm{s}}=-\operatorname{sign}\left(\eta_{\mathrm{c}}\right) \frac{\pi I_{\mathrm{b}} \Omega_{\mathrm{s} 0}}{h V_{\mathrm{rf}}\left|\cos \psi_{\mathrm{s} 0}\right|} \sum_{p=-\infty}^{\infty} \operatorname{Im}\left\{Z_{\|}(p)\right\} p \Psi(p)
$$

The real part of the impedance is symmetric in $p$ and therefore cancels in the summation over $p$ which leaves only the imaginary part consistent with the expectation that the tune shift be real. At this point, it becomes necessary to introduce a particular particle distribution and an assumption for the impedance spectrum. For long bunches, the frequencies involved are low and one might use for 
the impedance the space charge and broad-band impedance which both are constant for low frequencies. In this case, the impedance can be extracted from the sum in (22.129) and the remaining arguments in the sum depend only on the particle distribution.

For a parabolic particle distribution, for example, (22.129) reduces to [26]

$$
\Delta \Omega_{\mathrm{s}}=-\operatorname{sgn}\left(\eta_{\mathrm{c}}\right) \frac{16 I_{\mathrm{b}}}{\pi^{3} B^{3} h V_{\mathrm{rf}}\left|\cos \psi_{0}\right|} \operatorname{Im}\left\{\frac{Z_{\|}(p)}{p}\right\},
$$

where $B$ is the bunching factor $B=\ell /(2 \pi \bar{R})$ with $\ell$ the effective bunch length.

A measurement of the incoherent synchrotron tune shift as a function of bunch current allows the determination of the reactive impedance of the accelerator for a given particle distribution. This tune shift is derived from a measurement of the unperturbed synchrotron frequency $\Omega_{\mathrm{s} 0}$ for a very small beam current combined with the observation of the quadrupole mode frequency $\Omega_{2 \mathrm{~s}}$ as a function of bunch current. The incoherent tune shift is then

$$
\Delta \Omega_{\mathrm{s}, \text { incoh }}=\mu\left(\Omega_{2 \mathrm{~s}}-2 \Omega_{\mathrm{s} 0}\right),
$$

where $\mu$ is a distribution dependent form factor of order 2 for a parabolic distribution [27].

The third and subsequent terms in (22.127) contribute nonlinear effects making the synchrotron oscillation frequency amplitude dependent similar to the effects of nonlinear fields in transverse beam dynamics.

\section{Bunch Lengthening}

A synchrotron frequency shift is the consequence of a change in the longitudinal focusing and one might expect therefore also a change in the bunch length. In first approximation, one could derive expressions for the new bunch length by scaling with the synchrotron tune shift. Keeping the phase space area constant in the proton and ion case or keeping only the energy spread constant in the electron case, a rough estimate for bunch lengthening can be obtained for a specific particle distribution. Since the electron bunch length scales inversely proportional to the synchrotron frequency, we have

$$
\frac{\sigma_{\ell}}{\sigma_{\ell 0}}=\frac{\Omega_{\mathrm{s}}}{\Omega_{\mathrm{s} 0}}=1+\frac{\Delta \Omega_{\mathrm{s}}}{\Omega_{\mathrm{s} 0}} .
$$

From (22.132), one can determine for an electron beam the potential-well bunch lengthening or shortening, depending on the sign of the reactive impedance. For a proton or ion beam, the scaling is somewhat different because of the preservation of phase space. 
This approach to understanding potential-well bunch lengthening assumes that the particle distribution does not change which is an approximate but not correct assumption. The deformation of the potential well is nonlinear and can create significant variations of the particle distribution specifically, for large amplitudes.

In this discussion, we determine the stationary particle distribution $\psi(\tau, \delta)$ under the influence of wake fields by solving the Vlasov equation

$$
\frac{\partial \psi}{\partial t}+\dot{\tau} \frac{\partial \psi}{\partial \tau}+\dot{\delta} \frac{\partial \psi}{\partial \delta}=0
$$

For a stationary solution, $\frac{\partial \psi}{\partial t}=0$ and therefore any function of the Hamiltonian is a solution of the Vlasov equation. Since the Hamiltonian does not exhibit explicitly the time variable, any such function could be the stationary solution which we are looking for and we set therefore $\psi(\tau, \delta)=\psi(H)$. The local particle density is then after integrating over all momenta

$$
\lambda(\tau)=N_{\mathrm{b}} \int_{-\infty}^{\infty} \psi(\mathcal{H}) \mathrm{d} \delta,
$$

where $N_{\mathrm{b}}$ is the number of particles per bunch or with (22.122)

$$
\lambda(\tau)=N_{\mathrm{b}} \int_{-\infty}^{\infty} \psi\left(-\frac{1}{2} \eta_{\mathrm{c}} \delta^{2}-\frac{1}{2} \frac{\Omega_{\mathrm{s} 0}^{2}}{\eta_{\mathrm{c}}} \tau^{2}-\int_{0}^{\tau} \frac{q V_{\mathrm{w}}(\bar{\tau})}{\beta^{2} E_{0} T_{0}} \mathrm{~d} \bar{\tau}\right) \mathrm{d} \bar{\delta} .
$$

Without wake fields, the distribution of an electron beam is Gaussian and the introduction of wake fields does not change that for the energy distribution. We make therefore the ansatz

$$
\psi(\tau, \delta)=A \exp \left(\frac{\mathcal{H}}{\eta_{\mathrm{c}} \sigma_{\delta}^{2}}\right)=A_{\delta} \exp \left(\frac{1}{2} \frac{\delta^{2}}{\sigma_{\delta}^{2}}\right) A_{\lambda} \lambda(\tau),
$$

where $A_{\delta}$ and $A_{\lambda}$ are normalization factors for the respective distributions. Integrating over all momenta, the longitudinal particle distribution is finally

$$
\lambda(\tau)=N_{\mathrm{b}} A_{\lambda} \exp \left(-\frac{1}{2} \frac{\tau^{2}}{\sigma_{\tau}^{2}}-\frac{q}{\eta_{\mathrm{c}} \beta^{2} E_{0} T_{0} \sigma_{\delta}^{2}} \int_{0}^{\tau} V_{\mathrm{w}}(\tilde{\tau}) \mathrm{d} \tilde{\tau}\right),
$$

where we used $\sigma_{\delta}=\Omega_{\mathrm{so}} \sigma_{\tau} /\left|\eta_{\mathrm{c}}\right|$ from (13.26). A self-consistent solution of this equation will determine the longitudinal particle distribution under the influence of wake fields. Obviously, this distribution is consistent with our earlier results for an electron beam in a storage ring, in the limit of no wake fields. The nature of the wake fields will then determine the distortion from the Gaussian distribution. 
As an example, we assume a combination of an inductive $(L)$ and a resistive $(R)$ wake field

$$
V_{\mathrm{w}}=-L \frac{\mathrm{d} I}{\mathrm{~d} t}-R I_{\mathrm{b}}
$$

Such a combination actually resembles rather well the real average impedance in circular accelerators at least at lower frequencies as evidenced in the impedance spectrum of the SPEAR storage ring shown in Fig. 19.9. Inserting (22.138) into (22.137) while setting for a moment the resistance to zero $(R=0)$ we get after integration the transcendental equation

$$
\lambda(\tau)=N_{\mathrm{b}} A_{\lambda} \exp \left[-\frac{1}{2} \frac{\tau^{2}}{\sigma_{\tau}^{2}}-\frac{q^{2} L N_{\mathrm{b}} \lambda(\tau)}{\eta_{\mathrm{c}} \beta^{2} E_{0} T_{0} \sigma_{\delta}^{2}}\right]
$$

which must be solved numerically to get the particle distribution $\lambda(\tau)$. We note that the inductive wake does not change the symmetry of the particle distribution in $\tau$. For large values of $\tau$, the particle distribution must approach zero to meet the normalization requirement $\left(\lim _{\tau \rightarrow \infty} \lambda(\tau)=0\right)$ and the particle distribution is always Gaussian for large amplitudes. The effect of the inductive wake field is mainly concentrated to the core of the particle bunch.

Evaluating numerically (22.139), we distinguish between an electron beam and a proton or ion beam. The momentum spread $\sigma_{\delta}$ in case of an electron beam is determined by quantum effects related to the emission of synchrotron radiation and is thereby for this discussion a constant. Not so for proton and ion beams which are subject to Liouville's theorem demanding a strong correlation between bunch length and momentum spread such that the longitudinal phase space of the beam remains constant. Equation (22.139) has the form

$$
f(t)=K \exp \left[-\frac{1}{2} t^{2}-f(t)\right]
$$

or after differentiation with respect to $t$

$$
\frac{\mathrm{d} f(t)}{\mathrm{d} t}=-\frac{t f(t)}{1+f(t)} .
$$

For strong wake fields $f(t) \gg 1$ and (22.141) can be integrated for

$$
f(t)=f_{0}-\frac{1}{2} t^{2}
$$

The particle distribution in the bunch center assumes more and more the shape of a parabolic distribution as the wake fields increase. Figure 22.13 shows the particle distribution for different strengths of the wake field.

Now we add the resistive wake field component. This field actually extracts energy from the bunch and therefore one expects that the whole bunch is shifted 


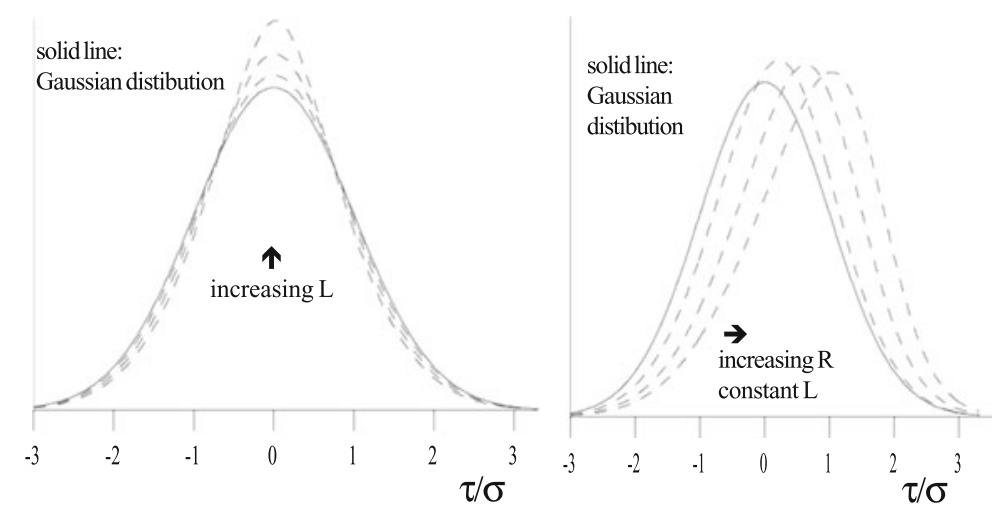

Fig. 22.13 Potential-well distortion of Gaussian particle distributions (a) for an inductive wake field, and (b) for a combination of an inductive and a resistive wake field

such as to compensate this extra loss by moving to a higher field in the accelerating cavities. Inserting the full wake field (22.138) into (22.137) results in the distribution

$$
\lambda(\tau)=N_{\mathrm{b}} A_{\lambda} \exp \left[-\frac{1}{2} \frac{\tau^{2}}{\sigma_{\tau}^{2}}-a L N_{\mathrm{b}} \lambda(\tau)-a R N_{\mathrm{b}} \int_{0}^{\tau} \lambda(\bar{\tau}) \mathrm{d} \bar{\tau}\right],
$$

where

$$
a=\frac{q^{2}}{\eta_{\mathrm{c}} \beta E_{0} T_{0} \sigma_{\delta}^{2}} .
$$

Looking for a shift of the tip of the particle distribution, we get from $\mathrm{d} \lambda / \mathrm{d} \tau=0$ the location of the distribution maximum

$$
\tau_{\max } \propto N_{\mathrm{b}} \lambda\left(\tau_{\max }\right) .
$$

The maximum of the particle distribution is therefore shifted proportional to the bunch intensity and the general distortion is shown in Fig. 22.13b for a resistive wake much larger than generally encountered in an accelerator. The distortion of the particle distribution leads to a deviation from a Gaussian distribution and a variation of the bunch length. In the limit of a strong and inductive wake field, for example, the full-width half maximum value of the bunch length increases like

$$
\tau_{\mathrm{fwhm}}=\sigma_{\tau} \sqrt{f_{0}}=\frac{q \sigma_{\tau}}{\beta \sigma_{\delta}} \sqrt{\frac{\beta L N_{\mathrm{b}} \lambda(\tau)}{\eta_{\mathrm{c}} E_{0} T_{0}}}
$$

The bunch length changes as the bunch intensity is increased while the sign and rate of change is dependent on the actual ring impedance spectrum on hand. We have 
used an induction as an example for the reactive impedance in a ring because it most appropriately represents the real impedance for lower frequencies or longer bunch length. In general, this potential-well bunch lengthening may be used to determine experimentally the nature and quantity of the ring impedance by measuring the bunch length as a function of bunch current.

\section{Turbulent Bunch Lengthening}

At higher bunch currents the bunch lengthening deviates significantly from the scaling of potential well distortion and actually proceeds in the direction of true lengthening. Associated with this lengthening is also an increase in the particle momentum spread. The nature of this instability is similar to the microwave instability for coasting beams.

Considering long bunches, a strong instability with a rise time shorter than the synchrotron oscillation period and high frequencies with wavelength short compared to the bunch length, we expect basically the same dynamics as was discussed earlier for a coasting beam. This was recognized by Boussard [28] who suggested a modification of the Keil-Schnell criterion by replacing the coastingbeam particle density by the bunch density. For a Gaussian particle distribution, the peak bunch current is

$$
\hat{I}=I_{0} \frac{2 \pi \bar{R}}{\sqrt{2 \pi} \sigma_{\ell}},
$$

where $I_{0}$ is the average circulating beam current per bunch, and the bunch length is related to the energy spread by

$$
\sigma_{\ell}=\frac{\beta c\left|\eta_{\mathrm{c}}\right|}{\Omega_{\mathrm{s} 0}} \frac{\sigma_{\epsilon}}{E_{0}} .
$$

With these modifications, the Boussard criterion is

$$
\left|\frac{Z_{z}}{n}\right| \leq F \frac{\beta^{3} E_{0}\left|\eta_{\mathrm{c}}\right|^{2}}{q I_{0} \sqrt{2 \pi} v_{\mathrm{s} 0}}\left(\frac{\sigma_{\epsilon}}{E_{0}}\right)^{3},
$$

where the form factor $F$ is still of the order unity.

As a consequence of this turbulent bunch lengthening we observe an increase of the energy spread as well as an increase of the bunch length. The instability does not necessarily lead to a beam loss but rather to an adjustment of energy spread and bunch length such that the Boussard criterion is met. For very low beam currents the stability criterion is always met up to a threshold where the r.h.s. of (22.149) becomes smaller than the 1.h.s. Upon further increase of the beam current beyond the threshold current the energy spread and consequently the bunch length increases to avoid the bunched beam microwave instability. 


\subsection{Transverse Single-Bunch Instabilities}

Transverse wake fields can also greatly modify the stability of a single bunch. Specifically at high frequencies, we note an effect of transverse wake fields generated by the head of a particle bunch on particles in the tail of the same bunch. Such interaction occurs for broad-band impedances where the bunch generates a short wake including a broad spectrum of frequencies. In the first moment all these fields add up being able to act back coherently on particles in the tail but they quickly decoher and vanish before the next bunch arrives. This effect is therefore a true single-bunch effect. In order to affect other bunches passing by later, the fields would have to persist a longer time which implies a higher $\mathrm{Q}$ value of the impedance structure which we ignore here.

\subsubsection{Beam Break-Up in Linear Accelerators}

A simple example of a transverse microwave instability is the phenomenon of beam break-up in linear accelerators. We noted repeatedly that the impedance of vacuum chambers originates mainly from sudden changes in cross section which therefore must be avoided to minimize impedance and microwave instabilities. This, however, is not possible in accelerating cavities of which there are particularly many in a linear accelerator. Whatever single-pass microwave instabilities exist they should become apparent in a linear accelerator. We have already discussed the effect of longitudinal wake fields whereby the fields from the head of a bunch act back as a decelerating field on particles in the tail. In the absence of corrective measures we therefore expect the particles in the tail to gain less energy than particles in the head of an intense bunch.

Transverse motion of particles is confined to the vicinity of the linac axis by quadrupole focusing in the form of betatron oscillations while travelling along the linear accelerator. However, coherent transverse betatron oscillations can create strong transverse wake fields at high bunch intensities. Such fields may act back on subsequent bunches causing bunch to bunch instabilities if the fields persist long enough. Here we are more interested in the effect on the same bunch. For example, the wake fields of the head of a bunch can act back on particles in the tail of the bunch. This interaction is effected by broad-band impedances like sudden discontinuities in the vacuum chamber which are abundant in a linear accelerator structure. The interaction between particles in the head of a bunch on particles in the tail of the same bunch can be described by a two macro particle model resembling the head and the tail.

Transverse wake fields are proportional to the transverse oscillation amplitude of the head and we describe the dynamics of the head and tail of a bunch in a two particle model where each particle represents half the charge of the whole bunch as shown in Fig. 22.14. 


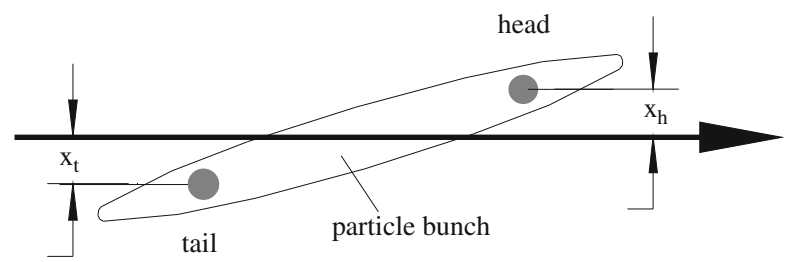

Fig. 22.14 Head-tail dynamics of a particle bunch represented by two macroparticles

The head particle with charge $\frac{1}{2} q N_{\mathrm{b}}$ performs free betatron oscillations while the tail particle responds like a driven oscillator. Since all particles travel practically at the speed of light, the longitudinal distribution of particles remains fixed along the whole length of the linear accelerator. The equations of motion in smooth approximation where $k_{\beta}=1 /\left(v_{0} \bar{\beta}_{u}\right)$ and $\bar{\beta}_{u}$ is the average value of the betatron function in the plane $u$, are for both macroparticles

$$
\begin{aligned}
& x_{\mathrm{h}}^{\prime \prime}+k_{\beta}^{2} x_{\mathrm{h}}=0, \\
& x_{\mathrm{t}}^{\prime \prime}+k_{\beta}^{2} x_{\mathrm{t}}=r_{\mathrm{c}} \frac{x_{\mathrm{h}}}{\gamma} \int_{\tilde{z}}^{\infty} \lambda(z) \tilde{W}_{\perp}(z-\tilde{z}) \mathrm{d} z=\frac{r_{\mathrm{c}} N_{\mathrm{b}} \tilde{W}_{\perp}}{2 \gamma} x_{\mathrm{h}},
\end{aligned}
$$

where we use the indices $\mathrm{h}$ and $\mathrm{t}$ for the head and tail particles respectively and introduce the average wake field per unit length

$$
\tilde{W}_{\perp}=\frac{W_{\perp}}{L_{\mathrm{acc}}} .
$$

For simplicity, it was assumed in (22.150) that the beam is just coasting along the linear accelerator to demonstrate the dynamics of the instability. If the beam is accelerated the adiabatic damping effect through the increase of the energy must be included.

Because of causality only the tail particle is under the influence of a wake field. The transverse wake field $W_{\perp}\left(2 \sigma_{z}\right)$, for example, which is shown in Fig. 22.8, is to be taken at a distance $2 \sigma_{z}$ behind the head particle. Inserting the solution $x_{\mathrm{h}}(z)=\hat{x}_{\mathrm{h}} \cos k_{\beta} z$ into the second equation, we obtain the solution for the betatron oscillation of the tail particle in the form

$$
x_{\mathrm{t}}(z)=\hat{x}_{\mathrm{h}} \cos k_{\beta} z+\hat{x}_{\mathrm{h}} \frac{r_{\mathrm{c}} N_{\mathrm{b}} \tilde{W}_{\perp}}{4 \gamma k_{\beta}} z \sin k_{\beta} z .
$$

The second term in this expression increases without bound leading to particle loss or beam break-up as soon as the amplitude reaches the edge of the aperture. If the bunch does reach the end of the linear accelerator of length $L_{\mathrm{acc}}$, the betatron oscillation amplitude of the tail has grown by a factor

$$
F_{\mathrm{bb}}=\frac{\hat{x}_{\mathrm{t}}}{\hat{x}_{\mathrm{h}}}=\frac{r_{\mathrm{c}} N_{\mathrm{b}} \tilde{W}_{\perp} L_{\mathrm{acc}}}{4 \gamma k_{\beta}} .
$$


One consequence of this instability is an apparent increase in beam emittance long before beam loss occurs. A straight bunch with a small cross section becomes bent first like a banana and later like a snake and the transverse distribution of all particles in the bunch occupies a larger cross-sectional area than before. This increase in apparent beam size has a big detrimental effect on the attainable luminosity in linear colliders and therefore must be minimized as much as possible. The two particle model adopted here is insufficient to determine a more detailed structure than that of a banana. However, setting up equations similar to (22.150) for more than two macroparticles will start to reveal the oscillatory nature of the transverse bunch perturbation.

One scheme to greatly reduce the beam break-up effect is called BNS damping in reference to its inventors Balakin et al. [29] and has been successfully implemented into the Stanford Linear Collider [30]. The technique utilizes the fact that the betatron oscillation frequency depends by virtue of the chromaticity on the energy of the particles. By accelerating the bunch behind the crest of the accelerating field the tail gains less energy than the head. Therefore the tail is focused more by the quadrupoles than the head. Since the transverse wake field introduces defocusing this additional chromatic focusing can be used for compensation.

Of course this method of damping the beam break-up by accelerating ahead of the crest is counter productive to compensating for the energy loss of tail particles due to longitudinal wake fields. In practice, BNS damping is applied only at lower energies where the instability is strongest and in that regime the energy reducing effect of the longitudinal wake field actually helps to maximize BNS damping. Toward the end of the linear accelerator at high beam energies, the beam break up effect becomes small $(\propto 1 / \gamma)$ and the bunch is now moved ahead of the crest to reduce the energy spread in the beam.

\subsubsection{Fast Head-Tail Effect}

Transverse bunch perturbations due to broad-band impedances are not restricted to linear accelerators but occur also in circular accelerators. In a circular proton accelerator, for example, the "length" is for all practical purposes infinite, there is no radiation damping and therefore even weak transverse wake fields can in principle lead to transverse bunch blow up and beam loss. This instability is known as the fast head-tail instability or strong head-tail instability and has been first discussed and analyzed by Kohaupt [31]. The dynamics in a circular accelerator is, however, different from that in a linear accelerator because particles in the head of a bunch will not stay there but rather oscillate between head and tail in the course of synchrotron oscillations. These synchrotron oscillations disturb the coherence between head and tail and the instability becomes much weaker.

On the other hand, particles in circular accelerators and especially in storage rings are expected to circulate for a long time and even a much reduced growth rate of the transverse bunch blow up may still be too strong. The dynamics of interaction 
is similar to that in a linear accelerator at least during about half a synchrotron oscillation period $\left(\frac{1}{2} t_{\mathrm{s}}\right)$, but during the next half period the roles are interchanged for individual particles. Particles travelling for one half period in the head of the bunch find themselves close to the tail for the next half period only to reach the head again and so forth. To understand the dynamics over many oscillations, we set up equations of motion for two macroparticles resembling the head and tail of a particle bunch similar to (22.150), but we now use the time as the independent variable. The distance $\zeta$ between head and tail particle varies between 0 and the maximum distance of the two macro particles $2 \ell$ during the course of a synchrotron oscillation period and since the transverse wake field increases linearly with $\zeta$, we set $W_{\perp}(\zeta)=W_{\perp}\left(2 \sigma_{\ell}\right) \sin \Omega_{\mathrm{s}} t$. With this the equations of motion are for $0 \leq t \leq$ $t_{\mathrm{s}} / 2$

$$
\begin{aligned}
& \ddot{x}_{1}+\omega_{\beta}^{2} x_{1}=0, \\
& \ddot{x}_{2}+\omega_{\beta}^{2} x_{2}=\frac{r_{\mathrm{c}} \beta^{2} c^{2} N_{\mathrm{b}} \tilde{W}_{\perp}\left(2 \sigma_{\ell}\right) \sin \Omega_{\mathrm{s}} t}{2 \gamma} x_{1},
\end{aligned}
$$

where $\tilde{W}_{\perp}=W_{\perp} /(2 \pi \bar{R})$ is the wake function per unit length. For the next half period $t_{\mathrm{s}} / 2 \leq t \leq t_{\mathrm{s}}$

$$
\begin{aligned}
& \ddot{x}_{1}+\omega_{\beta}^{2} x_{1}=\frac{r_{c} \beta^{2} c^{2} N_{\mathrm{b}} \tilde{W}_{\perp}(2 \ell) \sin \Omega_{\mathrm{s}} t}{2 \gamma} x_{2}, \\
& \ddot{x}_{2}+\omega_{\beta}^{2} x_{2}=0 .
\end{aligned}
$$

For further discussions we consider solutions to (22.154), (22.155) in the form of phasors defined by

$$
\boldsymbol{x}(t)=\boldsymbol{x}(0) \mathrm{e}^{\mathrm{i} \omega_{\beta} t}=x-\mathrm{i} \frac{\dot{x}}{\omega_{\beta}} .
$$

The first Eq. (22.154) can be solved immediately for

$$
\boldsymbol{x}_{1}(t)=\boldsymbol{x}_{1}(0) \mathrm{e}^{\mathrm{i} \omega_{\beta} t}
$$

and the second Eq. (22.154) becomes with (22.157)

$$
\ddot{\boldsymbol{x}}_{2}+\omega_{\beta}^{2} \boldsymbol{x}_{2}=A \sin \Omega_{\mathrm{s}} t \mathrm{e}^{\mathrm{i} \omega_{\beta} t} \boldsymbol{x}_{1}(0),
$$

where

$$
A=\frac{r_{\mathrm{c}} \beta^{2} c^{2} N_{\mathrm{b}} \tilde{W}_{\perp}(2 \ell)}{2 \gamma} .
$$


The synchrotron oscillation frequency is generally much smaller than the betatron oscillation frequency $\left(\Omega_{\mathrm{s}} \ll \omega_{\beta}\right)$ and the solution of (22.159) becomes with this approximation

$$
\boldsymbol{x}_{2}(t)=\boldsymbol{x}_{2}(0) \mathrm{e}^{\mathrm{i} \omega_{\beta} t}+\frac{1}{\omega_{\beta}} \int_{0}^{t}\left[A \boldsymbol{x}_{1}(0) \sin \Omega_{\mathrm{s}} t^{\prime} \mathrm{e}^{\mathrm{i} \omega_{\beta} t^{\prime}}\right] \sin \omega_{\beta}\left(t-t^{\prime}\right) \mathrm{d} t^{\prime}
$$

or after some manipulation

$$
\boldsymbol{x}_{2}(t)=\boldsymbol{x}_{2}(0) \mathrm{e}^{\mathrm{i} \omega_{\beta} t}-\mathrm{i} \boldsymbol{x}_{1}(0) \frac{1}{2} a\left(1-\cos \Omega_{\mathrm{s}} t\right) \mathrm{e}^{\mathrm{i} \omega_{\beta} t},
$$

where $a=A /\left(\omega_{\beta} \Omega_{\mathrm{s}}\right)$. During the second half synchrotron oscillation period, the roles of both macroparticles are exchanged. We may formulate the transformation through half a synchrotron oscillation period in matrix form and get with $1-$ $\cos \left(\Omega_{\mathrm{s}} \frac{1}{2} t_{\mathrm{s}}\right)=2$ since $\Omega_{\mathrm{s}} \frac{1}{2} t_{\mathrm{s}} \approx \pi$ for the first half period

$$
\left(\begin{array}{l}
x_{1}\left(t_{\mathrm{s}} / 2\right) \\
\boldsymbol{x}_{2}\left(t_{\mathrm{s}} / 2\right)
\end{array}\right)=\mathrm{e}^{\mathrm{i} \omega_{\beta} t_{\mathrm{s}} / 2}\left(\begin{array}{rr}
1 & 0 \\
-\mathrm{i} a & 1
\end{array}\right)\left(\begin{array}{l}
\boldsymbol{x}_{1}(0) \\
\boldsymbol{x}_{2}(0)
\end{array}\right)
$$

and for the second half period

$$
\left(\begin{array}{l}
x_{1}\left(t_{\mathrm{s}}\right) \\
\boldsymbol{x}_{2}\left(t_{\mathrm{s}}\right)
\end{array}\right)=\mathrm{e}^{\mathrm{i} \omega_{\beta} t_{\mathrm{s}} / 2}\left(\begin{array}{cc}
1 & -\mathrm{i} a \\
0 & 1
\end{array}\right)\left(\begin{array}{l}
\boldsymbol{x}_{1}\left(t_{\mathrm{s}} / 2\right) \\
\boldsymbol{x}_{2}\left(t_{\mathrm{s}} / 2\right)
\end{array}\right)
$$

Combining both half periods one gets finally for a full synchrotron oscillation period

$$
\left(\begin{array}{l}
x_{1}\left(t_{\mathrm{s}}\right) \\
\boldsymbol{x}_{2}\left(t_{\mathrm{s}}\right)
\end{array}\right)=\mathrm{e}^{\mathrm{i} \omega_{\beta} t_{\mathrm{s}}}\left(\begin{array}{cc}
1-a^{2} & -\mathrm{i} a \\
-\mathrm{i} a & 1
\end{array}\right)\left(\begin{array}{l}
\boldsymbol{x}_{1}(0) \\
\boldsymbol{x}_{2}(0)
\end{array}\right)
$$

The stability of the motion after many periods can be extracted from (22.163) by solving the eigenvalue equation

$$
\left(\begin{array}{cc}
1-a^{2} & -\mathrm{i} a \\
-\mathrm{i} a & 1
\end{array}\right)\left(\begin{array}{l}
\boldsymbol{x}_{1} \\
\boldsymbol{x}_{2}
\end{array}\right)=\lambda\left(\begin{array}{l}
\boldsymbol{x}_{1} \\
\boldsymbol{x}_{2}
\end{array}\right)
$$

The characteristic equation

$$
\lambda^{2}-\left(2-a^{2}\right) \lambda+1=0
$$

has the solution

$$
\lambda_{1,2}=\left(1-\frac{1}{2} a^{2}\right) \pm \sqrt{\left(1-\frac{1}{2} a^{2}\right)^{2}-1}
$$


and the eigenvalues can be expressed by

$$
\lambda=\mathrm{e}^{ \pm \mathrm{i} \Phi},
$$

where $\left(1-\frac{1}{2} a^{2}\right)=\cos \Phi$ for $|a| \leq 2$ or

$$
|a|=\frac{r_{\mathrm{c}} \beta^{2} c^{2} N_{\mathrm{b}} \tilde{W}_{\perp}(2 \ell)}{2 \gamma \omega_{\beta} \Omega_{\mathrm{s}}} \leq 2 .
$$

The motion remains stable since no element of the transformation matrix increases unbounded as the number of periods increases to infinity. In the form of a stability criterion, the single-bunch current $I_{\mathrm{b}}=q N_{\mathrm{b}} f_{\text {rev }}$ must not exceed the limit

$$
I_{\mathrm{b}} \leq \frac{4 q \gamma \omega_{0}^{2} v_{\beta} v_{\mathrm{s}}}{r_{\mathrm{c}} \beta c W_{\perp}(2 \ell)},
$$

where $q$ is the charge of the particles and $\left(v_{\beta}, v_{\mathrm{s}}\right)$ the betatron and synchrotron tune, respectively. In a storage ring, it is more convenient to use impedance rather than wake fields. Had we set up the equations of motion (22.150), (22.151) expressing the perturbing force in terms of impedance we would get the same results but replacing the wake field by

$$
W_{\perp}(2 \ell)=\frac{\omega_{0}}{\pi} \operatorname{Im}\left\{Z_{\perp}\right\}
$$

and the threshold beam current for the fast head-tail instability becomes

$$
I_{\mathrm{b}} \leq \frac{4 \pi q \gamma \omega_{0} v_{\beta} v_{\mathrm{s}}}{r_{\mathrm{c}} \beta c \operatorname{Im}\left\{\frac{Z_{\perp}}{n}\right\}} .
$$

The bunch current $I_{\mathrm{b}}$ is a threshold current which prevents us from filling more current into a single bunch. Exceeding this limit during the process of filling a bunch in a circular accelerator leads to an almost immediate loss of the excess current. This microwave instability is presently the most severe limitation on single-bunch currents in storage rings and special care must be employed during the design to minimize as much as possible the transverse impedance of the vacuum chamber system.

The strength of the instability becomes more evident when we calculate the growth time for a beam current just by an increment $\epsilon$ above the threshold. For $|a|>2$ we have $\left(1-\frac{1}{2} a^{2}\right)=-\cosh \mu$ and the eigenvalue is $\lambda=e^{ \pm \mu}$. The phase $\mu=0$ at threshold and $\cosh \mu \approx 1+\frac{1}{2} \mu^{2}$ for $a=2+\epsilon$ and we get

$$
\mu=2 \sqrt{\epsilon} .
$$


In each synchrotron oscillation period the eigenvalues increase by the factor $e^{\mu}$ or at a growth rate of $\frac{1}{\tau}=\frac{\mu}{t_{\mathrm{s}}}=\frac{2 \sqrt{\epsilon}}{t_{\mathrm{s}}}$. If, for example, the beam current exceeds the threshold by $10 \%$, we have $\epsilon=0.2$ and the rise time would be $\tau / t_{\mathrm{s}}=0.89$ or the oscillation amplitudes increase by more than a factor of two during a single synchrotron oscillation period. This is technically very difficult to counteract by a feedback system.

We have assumed that transverse wake fields are evenly distributed around the accelerator circumference. In a well designed accelerator vacuum chamber, however, most of the transverse wake field occur in the accelerating cavities and therefore only the transverse betatron oscillation amplitude in the cavities are relevant. In this case, one recalls the relation $v_{\beta} \approx \bar{R} / \beta_{u}$ and we replace in (22.171) the average value of the betatron function by the value in the cavities for

$$
I_{\mathrm{b}} \leq \frac{4 q \gamma \Omega_{\mathrm{s}}}{r_{\mathrm{c}} \beta_{u, \mathrm{cy}} W_{\perp, \mathrm{cy}}(2 \ell)} .
$$

This result suggest that the betatron function in the plane $u=x$ or $u=y$ at the location of cavities should be kept small and the synchrotron oscillation frequency should be large. The exchange of head and tail during synchrotron oscillation slows down considerably the growth rate of the instability. The result (22.173) is the same as the amplification factor (22.153) if we consider that in a linear accelerator the synchrotron oscillation period is infinite.

As we approach the threshold current, the beam signals the appearance of the head-tail instability on a spectrum analyzer with a satellite below the betatron frequency. The threshold for instability is reached when the satellite frequency reaches a value $\omega_{\text {sat }}=\omega_{\beta}-\frac{1}{2} \Omega_{\mathrm{s}}$. This becomes apparent when replacing the transformation matrix in (22.163) by the eigenvalue

$$
\left(\begin{array}{l}
\boldsymbol{x}_{1}\left(t_{\mathrm{s}}\right) \\
\boldsymbol{x}_{2}\left(t_{\mathrm{s}}\right)
\end{array}\right)=\mathrm{e}^{\mathrm{i} \omega_{\beta} t_{\mathrm{s}}} \mathrm{e}^{\mathrm{i} \Phi}\left(\begin{array}{l}
\boldsymbol{x}_{1}(0) \\
\boldsymbol{x}_{2}(0)
\end{array}\right) .
$$

The phase reaches a value of $\Phi=\pi$ at the stability limit and (22.174) becomes at this limit

$$
\left(\begin{array}{l}
\boldsymbol{x}_{1}\left(t_{\mathrm{s}}\right) \\
\boldsymbol{x}_{2}\left(t_{\mathrm{s}}\right)
\end{array}\right)=\mathrm{e}^{\mathrm{i}\left(\omega_{\beta}-\frac{1}{2} \Omega_{\mathrm{s}}\right) t_{\mathrm{s}}}\left(\begin{array}{l}
\boldsymbol{x}_{1}(0) \\
\boldsymbol{x}_{2}(0)
\end{array}\right) .
$$

At this point, it should be noted, however, that the shift of the betatron frequency to $\frac{1}{2} \Omega_{\mathrm{s}}$ is a feature of the two macro particle model. In reality there is a distribution of particles along the bunch and while increasing the beam current the betatron frequency decreases and the satellite $v_{\beta}-v_{\mathrm{s}}$ moves until both frequencies merge and become imaginary. This is the point of onset for the instability. It is this feature of merging frequencies which is sometimes called mode mixing or mode coupling. 


\section{Measurement of the Broad-Band Impedance}

As shown by Chao [9] the combined motion of head and tail represents a coherent transverse betatron motion which can be picked up by beam position monitors. The betatron frequency changes with increasing beam intensity and the initial slope can be used to determine the broad band impedance. From the lowest order instability mode we take the derivative of the betatron oscillation frequency and get

$$
\frac{\mathrm{d} \omega_{\beta}}{\mathrm{d} N_{\mathrm{b}}}=-\left.\frac{\omega_{\mathrm{s}}}{2 \pi}\left(\frac{\mathrm{d} \Phi}{\mathrm{d} N_{\mathrm{b}}}\right)\right|_{N_{\mathrm{b}}=0}=-\frac{r_{\mathrm{e}} c^{2} W_{0}}{16 \pi \gamma \bar{R} \omega_{\beta}}
$$

where $N_{\mathrm{b}}$ is the number of electrons per bunch. Measuring the slope $\mathrm{d} \omega_{\beta} / \mathrm{d} N_{\mathrm{b}}$ at low beam intensities allows to determine the wake function $W_{0}$. From this wake the transverse impedance is [9]

$$
Z_{\perp}=\frac{\bar{R}}{\beta_{z} v_{\beta}} \frac{b}{c} W_{0}
$$

and from (22.8) the relation to the longitudinal impedance is $Z_{\perp}=\frac{2 \bar{R}}{b^{2}} \frac{Z_{\|}}{n}$, where $\bar{R}$ is the average radius of the accelerator, $b$ the typical vacuum chamber radius, $v_{\beta}$ the betatron frequency and $\beta_{z}$ the average value of the betatron function around the ring. the mode number $n$ is the frequency in units of the revolution frequency $n=\omega / \omega_{0}$.

\subsubsection{Head-Tail Instability}

Discussing the fast head-tail instability we considered the effect of transverse wake fields generated by the head of a particle bunch on the transverse betatron motion of the tail. We assumed a constant betatron oscillation frequency which is only an approximation since the betatron frequency depends on the particle energy. On the other hand, there is a distinct relationship between particle energy and particle motion within the bunch, and it is therefore likely that the dynamics of the head-tail instability becomes modified by considering the energy dependence of the betatron oscillation frequency.

Like in the previous section, we represent the particle bunch by two macroparticles which perform synchrotron oscillations being $180^{\circ}$ apart in phase. The wake fields of the head particle act on the tail particle while the reverse is not true due to causality. However, during each half synchrotron oscillation period the roles become reversed.

In (22.160), we obtained an expression which includes the perturbation term and consider the variation of this term due to chromatic oscillations of the betatron 
frequency. The perturbation term is proportional to $\mathrm{e}^{\mathrm{i} \omega_{\beta} t}$ and we set therefore with $\delta=\Delta p / p_{0}$

$$
\omega_{\beta}=\omega_{\beta}(\delta)=\omega_{\beta 0}+\frac{\partial \omega_{\beta}}{\partial \delta} \delta+\mathcal{O}\left(\delta^{2}\right) .
$$

The chromaticity is defined by the betatron tune shift per unit relative momentum deviation

$$
\xi_{\beta}=\frac{\Delta v_{\beta}}{\delta}
$$

and (22.178) becomes with $\omega_{\beta}=v_{\beta} \omega_{0}$

$$
\omega_{\beta}=\omega_{\beta 0}+\xi_{\beta} \delta \omega_{0} .
$$

The momentum deviation is oscillating at the synchrotron frequency and is correlated with the longitudinal motion by

$$
\delta=-\frac{\Omega_{\mathrm{s}} \ell}{\beta c\left|\eta_{\mathrm{c}}\right|} \sin \Omega_{\mathrm{s}} t
$$

where $2 \ell$ is the maximum longitudinal distance between the two macroparticles. Combining (22.180), (22.181) we get

$$
\omega_{\beta}=\omega_{\beta 0}-\frac{\Omega_{\mathrm{s}} \ell \xi_{\beta}}{v_{\beta} \bar{R}\left|\eta_{\mathrm{c}}\right|} \sin \Omega_{\mathrm{s}} t,
$$

where the second term is much smaller than unity so that we may expand the exponential function of this term to get

$$
\mathrm{e}^{\mathrm{i} \omega_{\beta} t} \approx \mathrm{e}^{\mathrm{i} \omega_{\beta 0} t}\left[1-\mathrm{i} \frac{\Omega_{\mathrm{s}} \ell \xi_{\beta}}{v_{\beta} \bar{R}\left|\eta_{\mathrm{c}}\right|} t \sin \left(\Omega_{\mathrm{s}} t\right)\right] .
$$

The expression in the square bracket is the variation of the scaling factor $a$ in (22.160) and we note specifically, the appearance of the imaginary term which gives rise to an instability. The phase $\Phi$ in the eigenvalue equation (22.167) becomes for small beam currents $\Phi \approx a$ and with (22.183)

$$
\Phi=a\left[1-\mathrm{i} \frac{\Omega_{\mathrm{s}} \ell \xi_{\beta}}{\pi v_{\beta} \bar{R}\left|\eta_{\mathrm{c}}\right|} t_{\mathrm{s}}\right]
$$

where we have set $t=\frac{1}{2} t_{\mathrm{s}}$ and $\left\langle\sin \Omega_{\mathrm{s}} t\right\rangle \approx 2 / \pi$. The first term represents the fast head tail instability with its threshold characteristics discussed in the previous section. The second term is an outright damping or antidamping effect being 
effective at any beam current. This instability is called the head-tail effect discovered and analyzed by Pellegrini [32] and Sands [33] at the storage ring ADONE.

Considering only the imaginary term in (22.184), we note an exponential growth of the head-tail instability with a growth rate of

$$
\frac{1}{\tau}=\frac{\Omega_{\mathrm{s}} a \ell \xi_{\beta}}{\pi v_{\beta} \bar{R}\left|\eta_{\mathrm{c}}\right|}=\frac{\ell \xi_{\beta} r_{\mathrm{c}} \beta c N_{\mathrm{b}} \tilde{W}_{\perp}(2 \ell)}{2 \pi \gamma\left|\eta_{\mathrm{c}}\right| v_{\beta}^{2}} .
$$

Instability may occur either in the vertical or the horizontal plane depending on the magnitude of the transverse wake function in both planes. There are two modes, one stable and one unstable depending on the sign of the chromaticity and momentum compaction. Above transition $\eta_{\mathrm{c}}<0$ and the beam is unstable for negative chromaticity. This instability is the main reason for the insertion of sextupole magnets into circular accelerators to compensate for the naturally negative chromaticity. Below transition, the situation is reversed and no correction of chromaticity by sextupoles is required. From (22.185), we would conclude that we need to correct the chromaticity exactly to zero to avoid instability by one or the other mode. In reality, this is not the case because a two particle model overestimates the strength of the negative mode. Following a more detailed discussion including Vlasov's equation [9] it becomes apparent that the negative mode is much weaker to the point where, at least in electron accelerators, it can be ignored in the presence of radiation damping.

Observation of the head-tail damping for positive chromaticities or measuring the risetime as a function of chromaticity can be used to determine the transverse wake function or impedance of the accelerator [34, 35]. Measurements of head-tail damping rates have been performed in SPEAR [34] as a function of chromaticity and are reproduced in Figs. 22.15 and 22.16.
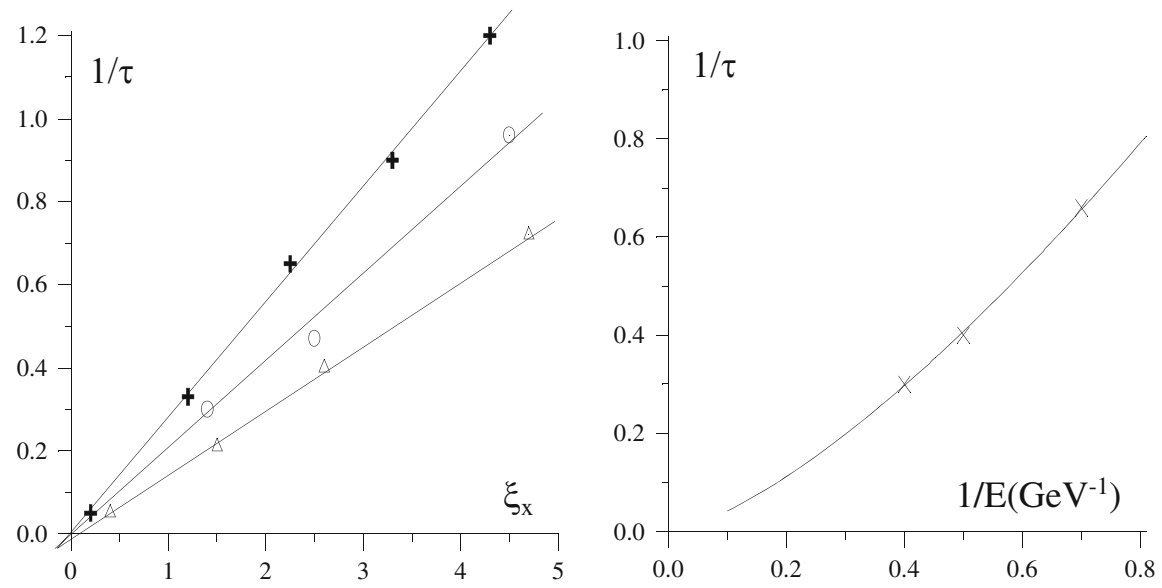

Fig. 22.15 Measurement of the head-tail damping rate in SPEAR as a function of chromaticity (a) and beam energy (b) [34] 
Fig. 22.16 Measurement of the head-tail damping rate in SPEAR as a function of beam current [34]

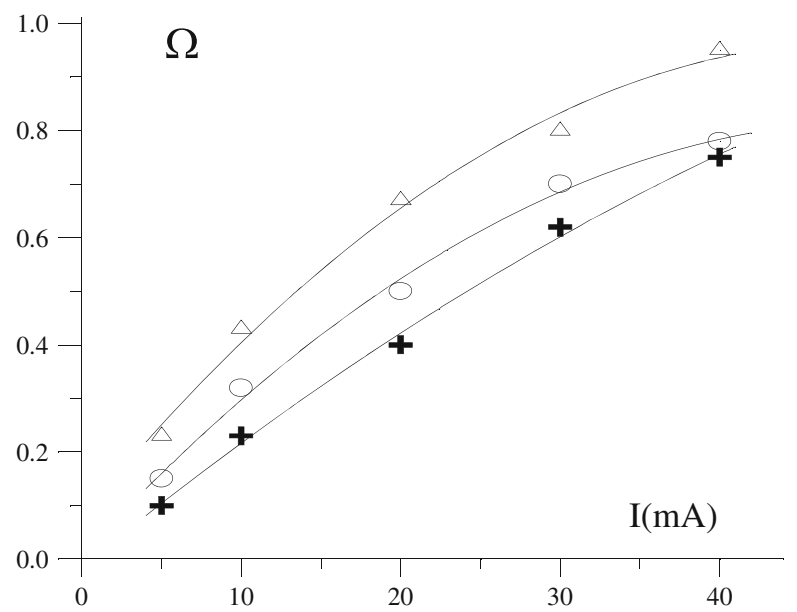

We clearly note the linear increase of the damping rate with chromaticity. The scaling with energy and beam current is less linear due to a simultaneous change in bunch length. Specifically the bunch length increases with beam intensity causing the wake fields to drop for a smaller damping rate.

\subsection{Multi-Bunch Instabilities}

Single-bunch dynamics is susceptible to all kinds of impedances or wake fields whether it be narrow or broad-band impedances. This is different for multi-bunch instabilities or coupled-bunch instabilities [27-37]. In order for wake fields to generate an effect on more than one bunch it must persist at least until the next bunch comes by the location of the impedance. We expect therefore multi-bunch instabilities only due to high $Q$ or narrow-band impedances like those encountered in accelerating cavities. Higher-order modes in such cavities persist some time after excitation and actually reach a finite amplitude in a circular accelerator where the orbiting beam may periodically excite one or the other mode. Because these modes have a high quality factor they are also confined to a narrow frequency spread. The impedance spectrum we need to be concerned with in the study of multi-bunch instabilities is therefore a line spectrum composed of many cavity modes.

To study the effect of these modes on the circulating beam, we must fold the beam current spectrum with the mode spectrum and derive from this interaction conditions for beam stability. We will do this for the case of the two lowest order mode oscillations only where all bunches oscillate in synchronism at the same phase or are $90^{\circ}$ out of phase from bunch to bunch respectively. Of course in a real accelerator higher-order modes can be present too and must be taken into account. Here we must limit ourself, however, to the discussion of the physical effect only and 
direct the interested reader to more detailed discussions on this subject in references $[3,9,19,26]$.

We consider the dynamics of rigid coupled bunches ignoring the internal motion of particles within a single bunch. The beam spectrum is then from (21.84) with $q$ the bunch charge and observing at $\varphi=0$ for simplicity

$$
I_{\|}(\omega, \varphi)=\frac{q n_{\mathrm{b}} \omega_{0}}{2 \pi} \sum_{p=-\infty}^{+\infty} \sum_{n=-\infty}^{+\infty} \mathrm{i}^{-n} J_{n}\left(p n_{\mathrm{b}} \omega_{0} \hat{\tau}\right) \delta\left(\omega-\Omega_{n}\right),
$$

where now $\Omega_{n}=\left(p n_{\mathrm{b}}+n m+n \nu_{\mathrm{s}}\right) \omega_{0}$ and where we have replaced the synchrotron frequency by the synchrotron tune and the phase $\zeta_{i}$ for individual particles by the mode of the bunch distribution setting $\zeta_{i}=m \omega_{0} t$ with $0 \leq m \leq n_{\mathrm{b}}$. A beam of $n_{\mathrm{b}}$ equidistant bunches can oscillate in $n_{\mathrm{b}}$ different modes. Two bunches, for example, can oscillate in phase or $180^{\circ}$ out of phase; four bunches can oscillate with a phase difference of $0^{\circ}, 90^{\circ}, 180^{\circ}$, and $270^{\circ}$ between consecutive bunches. In general the order of the mode $m$ defines the phase difference of consecutive bunches by

$$
\Delta \phi=m \frac{360^{\circ}}{n_{\mathrm{b}}} .
$$

To determine the multi-bunch dynamics we calculate first the induced voltage $V(t)$ by the beam current in the impedance $Z(\omega)$ and then fold the voltage function with the beam function to calculate the energy loss per turn by each particle. Knowing this, we will be able to formulate the equation of motion for the synchrotron oscillation. Specifically, we will be able to formulate frequency shifts and damping or antidamping due to the interaction of the multi-bunch beam with its environment to identify conditions for beam stability.

For simplicity we assume small phase oscillations $(\hat{\tau} \ll 1)$ and consider only the fundamental beam frequency and the first satellite $n=0,1$. With this (22.186) becomes

$$
I_{\|}(\omega)=\frac{q n_{\mathrm{b}} \omega_{0}}{2 \pi} \sum_{p=-\infty}^{+\infty} J_{0}\left(p n_{\mathrm{b}} \omega_{0} \hat{\tau}\right) \delta\left(\omega-\Omega_{0}\right)-\mathrm{i} J_{1}\left(p n_{\mathrm{b}} \omega_{0} \hat{\tau}\right) \delta\left(\omega-\Omega_{1}\right),
$$

where $\Omega_{0}=p n_{\mathrm{b}} \omega_{0}, \Omega_{1}=\left(p n_{\mathrm{b}}+m+v_{\mathrm{s}}\right) \omega_{0}$, and $J_{i}$ are Bessel's functions. The induced voltage spectrum is $V(\omega)=Z(\omega) I(\omega)$ and its Fourier transform $V(t)=$ $\int V(\omega) e^{i \omega t} \mathrm{~d} \omega$ or

$$
\begin{array}{r}
V_{\|}(t)=\frac{q n_{\mathrm{b}} \omega_{0}}{2 \pi} \sum_{p=-\infty}^{+\infty}\left[J_{0}\left(\hat{\tau} \Omega_{0}\right) Z\left(\Omega_{0}\right) \mathrm{e}^{\mathrm{i} \Omega_{0} t}\right. \\
\left.-\mathrm{i} J_{1}\left(\hat{\tau} \Omega_{0}\right) Z\left(\Omega_{1}\right) \mathrm{e}^{\mathrm{i} \Omega_{1} t}\right] .
\end{array}
$$


The energy loss per particle is then defined by integrating in time the product of voltage function and single-bunch current function

$$
U=\frac{1}{N_{\mathrm{b}}} \int V_{\|}(t) \frac{I_{\|}(t+\tau)}{n_{\mathrm{b}}} \mathrm{d} t,
$$

$N_{\mathrm{b}}$ is the number of particles per bunch and $T_{\mathrm{b}}=T_{0} / n_{\mathrm{b}}$ the time between passage of consecutive bunches. The bunch current can be expanded for $\tau \ll 1$

$$
I_{\|}(t+\tau) \approx I_{\|}(t)+\tau \frac{\mathrm{d}}{\mathrm{d} t} I_{\|}(t)
$$

The Fourier transforms of both current and its derivative with respect to time are correlated by

$$
\frac{\mathrm{d}}{\mathrm{d} t} I_{\|}(\omega)=\mathrm{i} \omega I_{\|}(\omega)
$$

and (22.191) becomes in frequency domain with (22.188)

$$
I_{\|}(t+\tau)=\frac{q n_{\mathrm{b}} \omega_{0}}{(2 \pi)^{2}} \int \sum_{p=-\infty}^{+\infty}(1+\mathrm{i} \omega \tau)\left(J_{0} \delta_{0}-\mathrm{i} J_{1} \delta_{1}\right) \mathrm{e}^{\mathrm{i} \omega t} \mathrm{~d} t
$$

where we have used some abbreviations which become obvious by comparison with (22.188). Inserting (22.193) and (22.189) into (22.190), we get

$$
\begin{aligned}
U=\frac{\left(q \omega_{0}\right)^{2} n_{\mathrm{b}}}{(2 \pi)^{2} N_{\mathrm{b}}} \int_{t} \int_{\omega} \sum_{p}\left(J_{0} Z_{0} \mathrm{e}^{\mathrm{i} \Omega_{0} t}-\mathrm{i} J_{1} Z_{1} \mathrm{e}^{\mathrm{i} \Omega_{1} t}\right) \\
\times(1+\mathrm{i} \omega \tau) \sum_{r}\left(J_{0} \delta_{0 r}-i J_{1} \delta_{1 r}\right) \mathrm{e}^{\mathrm{i} \omega t} \mathrm{~d} \omega \mathrm{d} t .
\end{aligned}
$$

For abbreviation we have set $\delta_{i}=\delta\left(\Omega_{i}\right), Z_{i}=Z\left(\Omega_{i}\right), J_{0}=J_{0}\left(\hat{\tau} \Omega_{0}\right)$, and $J_{1}=$ $J_{1}\left(\hat{\tau} \Omega_{0}\right)$. An additional index has been added to indicate whether the quantity is part of the summation over $p$ or $r$. Before we perform the time integration we reverse the first summation by replacing $p \rightarrow-p$ and get terms like $\int e^{-\mathrm{i}\left(\Omega_{0}-\omega\right) t} \mathrm{~d} t=2 \pi \delta_{0}$ etc. and (22.194) becomes

$$
\begin{aligned}
U=\frac{\left(q \omega_{0}\right)^{2} n_{\mathrm{b}}}{2 \pi N_{\mathrm{b}}} \int_{\omega} \sum_{p}\left(J_{0} Z_{0} \delta_{0 r}+\mathrm{i} J_{1} Z_{1} \delta_{1 r}\right) \\
\quad \times(1+\mathrm{i} \omega \tau) \sum_{r}\left(J_{0} \delta_{0}-\mathrm{i} J_{1} \delta_{1}\right) \mathrm{d} \omega .
\end{aligned}
$$


The integration over $\omega$ will eliminate many components. Specifically, we note that all cross terms $\delta_{0} \delta_{1}$ vanish after integration. We also note that the terms $\delta_{0 p} \delta_{0 r}$ vanish unless $r=p$. With this in mind we get from (22.195)

$$
U=\frac{\left(q \omega_{0}\right)^{2} n_{\mathrm{b}}}{2 \pi N_{\mathrm{b}}} \sum_{p}\left(J_{0}^{2} Z_{0}+\mathrm{i} \Omega_{0} \tau J_{0}^{2} Z_{0}+J_{1}^{2} Z_{1}+\mathrm{i} \Omega_{1} \tau J_{1}^{2} Z_{1}\right) .
$$

Finally the summation over $p$ leads to a number of cancellations considering that the resistive impedance is an even and the reactive impedance an odd function. With $Z_{0}=Z_{\mathrm{r} 0}+i Z_{\mathrm{i} 0}, Z_{\mathrm{r} 0}(\omega)=Z_{\mathrm{r} 0}(-\omega)$, and $Z_{\mathrm{i} 0}(\omega)=-Z_{\mathrm{i} 0}(-\omega)$ (22.196) becomes

$$
\begin{aligned}
U=\frac{\left(q \omega_{0}\right)^{2} n_{\mathrm{b}}}{2 \pi N_{\mathrm{b}}} \sum_{p=-\infty}^{+\infty}[ & J_{0}^{2}\left(\hat{\tau} \Omega_{0}\right) Z_{\mathrm{r}}\left(\Omega_{0}\right)+J_{1}^{2}\left(\hat{\tau} \Omega_{0}\right) Z_{\mathrm{r}}\left(\Omega_{1}\right) \\
& \left.+\mathrm{i} \tau \Omega_{1} J_{1}^{2}\left(\hat{\tau} \Omega_{1}\right) Z_{\mathrm{r}}\left(\Omega_{1}\right)-\tau \Omega_{1} J_{1}^{2}\left(\hat{\tau} \Omega_{1}\right) Z_{\mathrm{i}}\left(\Omega_{1}\right)\right] .
\end{aligned}
$$

The first and second term are the resistive energy losses of the circulating beam and synchrotron oscillations respectively while the third and fourth term are responsible for the stability of the multi bunch beam.

The equation of motion for synchrotron oscillations has been derived in Chap. 9 and we found that frequency and damping is determined by the accelerating rf-field and energy losses. We expect therefore that the energy loss derived for coupled bunch oscillations will also lead to a frequency shift and damping or anti damping. Specifically, we have for the equation of motion from (9.25)

$$
\ddot{\varphi}+\left.\omega_{0}^{2} \frac{h \eta_{\mathrm{c}}}{2 \pi \beta c c p_{0}} e \frac{\mathrm{d} V}{\mathrm{~d} \psi}\right|_{\psi_{\mathrm{s}}} \varphi-\left.\frac{1}{T_{0}} \frac{\mathrm{d} U}{\mathrm{~d} E}\right|_{E_{0}} \dot{\varphi}=0,
$$

where we notice the phase proportional term which determines the unperturbed synchrotron frequency

$$
\Omega_{\mathrm{s} 0}^{2}=\left.\omega_{0}^{2} \frac{h \eta_{\mathrm{c}}}{2 \pi \beta c p_{0}} e \frac{\mathrm{d} V}{\mathrm{~d} \psi}\right|_{\psi_{\mathrm{s}}}=\omega_{0}^{2} \frac{h \eta_{\mathrm{c}} e \hat{V}_{0} \cos \psi_{\mathrm{s}}}{2 \pi \beta c p_{0}} .
$$

The term proportional to $\dot{\varphi}$ gave rise to the damping decrement

$$
\alpha_{\mathrm{s} 0}=-\left.\frac{1}{2 T_{0}} \frac{\mathrm{d} U}{\mathrm{~d} E}\right|_{E_{0}} .
$$

The modification of the synchrotron frequency is with $=\tau=\varphi / h \omega_{0}$ from (22.93)-(22.95) similar to the derivation of the unperturbed frequency

$$
\Omega_{\mathrm{s}}^{2}=\Omega_{\mathrm{s} 0}^{2}+\omega_{0}^{2} \frac{h \eta_{\mathrm{c}} n_{\mathrm{b}}}{\beta c p_{0} N_{\mathrm{b}}} \sum_{p=-\infty}^{+\infty} \tau \Omega_{1}\left[q f_{0} J_{1}\left(\hat{\tau} \Omega_{1}\right)\right]^{2} Z_{\mathrm{i}}\left(\Omega_{1}\right),
$$


where $f_{0}=\omega_{0} / 2 \pi$ is the revolution frequency. Note that $\eta_{\mathrm{c}}<0$ above transition and the additional damping or energy loss due to narrow-band impedances reduces the frequency as one would expect.

Similarly we derive the modification of the damping decrement from the imaginary term in (22.197) noting that the solution of the synchrotron oscillation gives $\dot{\tau}=-\mathrm{i} \Omega_{\mathrm{s}} \tau$ with $\varphi=h \omega_{0} \tau$ and the damping decrement for a multi-bunch beam is

$$
\alpha_{\mathrm{s}}=\alpha_{\mathrm{s} 0}-\frac{\omega_{0} \eta_{\mathrm{c}} n_{\mathrm{b}}}{2 c p_{0} N_{\mathrm{b}}} \sum_{p=-\infty}^{+\infty} \frac{\Omega_{1}}{\Omega_{\mathrm{s}}}\left[q f_{0} J_{1}\left(\hat{\tau} \Omega_{1}\right)\right]^{2} Z_{\mathrm{r}}\left(\Omega_{1}\right) .
$$

For proton and ion beams we would set $\alpha_{\mathrm{s} 0}=0$ because there is no radiation damping and the interaction of a multi-bunch beam with narrow-band impedances would provide damping or antidamping depending on the sign of the damping decrement for each term. If, however, only one term is antidamped the beam would be unstable and get lost as was observed first at the storage ring DORIS [38]. It is therefore important to avoid the overlap of any line of the beam spectrum with a narrow-band impedance in the ring.

Since this is very difficult to achieve and to control, it is more convenient to minimize higher-order narrow-band impedances in the ring by design as much as possible to increase the rise time of the instabilities. In electron storage rings the situation is similar, but now the instability rise time must exceed the radiation damping time. Even though, modern storage rings are designed for high beam currents and great efforts are being undertaken to reduce the impedance of higher cavity modes by designing monochromatic cavities where the higher-order modes are greatly suppressed [39-42].

We have discussed here only the dipole mode of the longitudinal coupled-bunch instability. Of course, there are more modes and a similar set of instabilities in the transverse dimensions. A more detailed discussion of all aspects of multi-bunch instabilities would exceed the scope of this text and the interested reader is referred to the specific literature, specifically to $[3,9,19,26]$.

\section{Problems}

22.1 (S). Consider a storage ring with $250 \mathrm{~m}$ circumference and a stored beam current of $50 \mathrm{~mA}$ in 1 bunch. Assume that the bunch length is about $5 \%$ of the bunch spacing. A typical loss parameter for a BPM assembly is $k_{\|}=3.35 \cdot 10^{-2} \mathrm{~V} / \mathrm{pC}$ and for bellows its $k_{\|}=6.12 \cdot 10^{-2} \mathrm{~V} / \mathrm{pC}$. Calculate the induced power in both BPM with $50 \Omega$ termination and bellows. What is the power at the $50 \Omega$ termination?

22.2 (S). Show that (22.94) is the same as (22.93) and show that the constant $A$ in (22.94) is given by $A=\frac{3}{4 \pi} \frac{q I_{0}}{\beta^{2} E_{0}\left|\eta_{\mathrm{c}}\right| \delta^{2}}$, where $\delta=\Delta p / p_{0}$. 
22.3. Specify a damping ring at an energy of $1.5 \mathrm{GeV}$ and an emittance of $10^{-10}$ m-rad. The rf-frequency be $500 \mathrm{MHz}$ and $10^{11}$ electrons are to be stored into a single bunch at full coupling. Calculate the Touschek lifetime and the coherent and incoherent space-charge tune shift. Would the beam survive in case a tune shift of $\Delta Q_{y} \leq 0.05$ were permissible?

22.4. Use the wake field for the SLAC linear accelerator structure (Fig. 22.8) and calculate the energy loss of a particle in the tail of a $1 \mathrm{~mm}$ long bunch of $10^{11}$ electrons for the whole SLAC linear accelerator of $3 \mathrm{~km}$ length. This energy droop along a bunch is mostly compensated by accelerating the bunch ahead of the crest of the accelerating wave. This way the particles in the head of the bunch gain less energy than the particles in the tail of the bunch. The extra energy gain of the tail particles is then lost again due to wake field losses. How far off the crest must the bunch be accelerated for this compensation?

22.5. Consider the phenomenon of beam break-up in a linear accelerator and split the bunch into a head, center and tail part with a particle distribution $N_{\mathrm{b}} / 4$ to $N_{\mathrm{b}} / 2$ to $N_{\mathrm{b}} / 4$. Set up the equations of motion for all three particles including wake fields and solve the equations. Show the exponential build up of oscillation amplitudes of the tail particle. Perform the same derivation including BNS damping where each macroparticle has a different betatron oscillation frequency. Determine the condition for optimum BNS damping.

22.6. Determine the perturbation of a Gaussian particle distribution under the influence of a capacitive wake field. In particular, derive expressions for the perturbation of the distribution (if any) and the change in the fwhm bunch width as a function of $\sigma_{\tau}$ in the limit of small wakes. If there is a shift in the distribution what physical effects cause it? Hint: think of a loss mechanism for a purely capacitive wake field?

22.7. During the discussion of the dispersion relation we observed the stabilizing effect of Landau damping and found the stability criterion (22.95) stating that the threshold current can be increased proportional to the square of the momentum spread in the beam. How does this stability criterion in terms of a momentum spread relate to the conclusion in the section on Landau damping that the beam should have a frequency overlap with the excitation frequency? Why is a larger momentum spread better than a smaller spread?

22.8. Determine stability conditions for the fast head-tail instability in a storage ring of your choice assuming that all transverse wake fields come from accelerating cavities. Use realistic parameters for the rf-system and the number of cells appropriate for your ring. What is the maximum permissible transverse impedance for a bunch current of $100 \mathrm{~mA}$ ? Is this consistent with the transverse impedance of pill box cavities? If not how would you increase the current limit?

22.9. Calculate the real and imaginary impedance for the first longitudinal and transverse higher-order mode in a pill box cavity and apply these to determine the 
multi-bunch beam limit for a storage ring of your choice assuming that the beam spectrum includes the HOM frequency. Calculate also the frequency shift at the limit.

\section{References}

1. A.W. Chao, J. Gareyte, Technical Report, Int Note-197, SLAC, Stanford (1976)

2. W. Schnell, Technical Report, CERN ISR-RF/70-7, CERN, Geneva (1970)

3. F. Sacherer, in 9th International Conference on High Energy Accelerators (Stanford Linear Accelerator Center, Stanford, 1974), p. 347

4. P.B. Wilson, Introduction to wake fields and wake functions. Technical Report, SLAC PUB4547, SLAC (1989)

5. P.B. Wilson, R. Servranckx, A.P. Sabersky, J. Gareyte, G.E. Fischer, A.W. Chao, IEEE Trans. Nucl. Sci. 24, 1211 (1977)

6. W.K.H. Panofsky, W.A. Wenzel, Rev. Sci. Instrum. 27, 967 (1956)

7. A. Chao, K.H. Mess, M. Tigner, F. Zimmermann, (eds.), Handbook of Accelerator Physics and Engineering, 2nd edn. (World Scientific, Singapore, 2013)

8. A. Chao, K. Mess, M. Tigner, F. Zimmermann, (eds.), Handbook of Accelerator Physics and Engineering, 2nd edn. (World Scientific, Singapore, 2013)

9. A. Chao, Physics of Collective Beam Instabilities in High Energy Accelerators (Wiley, New York, 1993)

10. J.D. Jackson, Classical Electrodynamics, 2 edn. (Wiley, New York, 1975)

11. C.E. Nielsen, A.M. Sessler, K.R. Symon, in International Conference on High Energy Accelerators (CERN, Geneva, 1959), p. 239

12. V.K. Neil, A.M. Sessler, Rev. Sci. Instrum. 36, 429 (1965)

13. L.J. Laslett, K.V. Neil, A.M. Sessler, Rev. Sci. Instrum. 32, 279 (1961)

14. K. Hübner, A.G. Ruggiero, V.G. Vaccaro, in 8th International Conference on High Energy Accelerators (Yerevan Phusics Institute, Yerevan, 1969), p. 296

15. A.G. Ruggiero, V.G. Vaccaro, Technical Report, CERN isr-th/68-33, CERN, Geneva (1968)

16. K. Hübner, A.G. Ruggiero, V.G. Vaccaro, Technical Report, CERN ISR-TH/69-23, CERN, Geneva (1969)

17. K. Hübner, V.G. Vaccaro, Technical Report, CERN ISR-TH/70-44, CERN, Geneva (1970)

18. K. Hübner, P. Strolin, V.G. Vaccaro, B. Zotter, Technical Report, CERN ISR-TH/70-2, CERN, Geneva (1970)

19. B. Zotter, F. Sacherer, Technical Report, CERN 77-13, CERN, Geneva (1977)

20. J. Landau, J. Phys. 10, 25 (1946)

21. E. Keil, W. Schnell, Technical Report, CERN ISR-TH-RF/69-48, CERN, Geneva (1969)

22. H.G. Hereward, Technical Report, CERN 65-20, CERN, Geneva (1965)

23. A. Hofmann, Lecture Notes of Physics, vol. 296 (Springer, Berlin/Heidelberg, 1986), p. 112

24. L.R. Evans, Physics of Particle Accelerators, vol. 127 (American Institute of Physics, New York, 1985), p. 243

25. C. Pellegrini, A.M. Sessler, Nuovo Cimento 3A, 116 (1971)

26. J.L. Laclare, Technical Report, CERN 87-03, CERN, Geneva (1987)

27. F. Sacherer, Technical Report, CERN SI-BR/72-5, CERN, Geneva (1972)

28. D. Boussard, Technical Report, CERN Rept. Lab II/RF/Int 75-2, CERN, Geneva (1975)

29. V. Balakin, A. Novokhatsky, V. Smirnov, in 12th International Conference on High Energy Accelerators (Fermi Laboratory, FNAL, Chicago, 1983), p. 119

30. J.T. Seeman, Lecture Notes of Physics, vol. 400 (Springer, Berlin/Heidelberg, 1986), p. 255

31. R.D. Kohaupt, Excitation of a transverse instability by parasitic cavity modes. Technical Report, Int. Note DESY H1-74/2, DESY, Hamburg (1972) 
32. C. Pellegrini, Nuovo Cimento A64, 447 (1969)

33. M. Sands, Technical Report, TN-69-8, TN-69-10, SLAC, Stanford (1969)

34. J.M. Paterson, B. Richter, A.P. Sabersky, H. Wiedemann, P.B. Wilson, M.A. Allen, J.E. Augustin, G.E. Fischer, R.H. Helm, M.J. Lee, M. Matera, P.L. Morton, in 9th International Conference on High Energy Accelerators (Stanford Linear Accelerator Center, Stanford, 1974), p. 338

35. J. Gareyte, F. Sacherer, in 9th International Conference on High Energy Accelerators (Stanford Linear Accelerator Center, Stanford, 1974), p. 341

36. J. Haissinski, Nuovo Cimento B 18, 72 (1973)

37. D. Kohaupt, IEEE Trans. Nucl. Sci. 26, 3480 (1979)

38. J. LeDuff, J. Maidment, E. Dasskovski, D. Degele, H.D. Dehne, H. Gerke, D. Heins, K. Hoffmann, K. Holm, E. Jandt, R.D. Kohaupt, J. Kouptsidis, F. Krafft, N. Lehnart, G. Mülhaupt, H. Nesemann, S. Pæztold, H. Pingel, A. Piwinski, R. Rossmanith, H.D. Schulz, K.G. Steffen, H. Wiedemann, K. Wille, A. Wrulich, in 9th International Conference on High Energy Accelerators (Stanford Linear Accelerator Center, Stanford, 1974), p. 43

39. F. Voelker, G. Lambertson, R. Rimmer, in Proceedings of 1991 IEEE Particle Accelerator Conference, San Francisco, IEEE Cat. No. 91CH3038-7 (1991), p. 687

40. R. Rimmer, F. Voelker, G. Lambertson, M. Allen, J. Hodgeson, K. Ko, R. Pendelton, H. Schwartz, in Proceedings of 1991 IEEE Particle Accelerator Conference, San Francisco, IEEE Cat. No. 91CH3038-7 (1991), p. 2688

41. S. Bartalucci, R. Boni, A. Gallo, L. Palumbo, R. Parodi, M. Sergio, B. Spataro, G. Vignola, in Proceedings of 3th European Conference on Particle Accelerator, ed. by H. Henke, H. Homeyer, Ch. Petit-Jean-Genaz (Berlin, 1992)

42. M. Svandrlik, G. D'Auria, A. Fabris, A. masserotti, C. Passotti, C. Rossi, A pill-box resonator with very strong suppression of the hom spectrums. Technical Report, ST/M-92/14, Sincrotrone Trieste, Trieste (1992) 


\section{Part IX \\ Synchrotron Radiation}




\section{Chapter 23 \\ Fundamental Processes}

Ever since J.C. Maxwell formulated his unifying electromagnetic theory in 1873, the phenomenon of electromagnetic radiation has fascinated the minds of theorists as well as experimentalists. The idea of displacement currents was as radical as it was important to describe electromagnetic waves. It was only 14 years later when G. Hertz in 1887 succeeded to generate, emit and receive again electromagnetic waves, thus, proving experimentally the existence of such waves as predicted by Maxwell's equations. The sources of the radiation are oscillating electric charges and currents in a system of metallic wires. In this text, we discuss the generation of electromagnetic radiation emitted by free electrons from first principles involving energy and momentum conservation as well as Maxwell's equations.

\subsection{Radiation from Moving Charges}

Analytical formulation of the emission of electromagnetic radiation posed a considerable challenge. Due to the finite speed of light one cannot make a snapshot to correlate the radiation field at the observer with the position of radiating charges. Rather, the radiation field depends on the position of the radiating charges some time earlier, at the retarded time, when the radiation was emitted. Already $1867 \mathrm{~L}$. Lorenz included this situation into his formulation of the theory of electromagnetic fields and introduced the concept of retarded potentials. He did, however, not offer a solution to the retarded potentials of a point charge. Liénard [1] in 1898 and independently in 1900 Wiechert [2] derived for the first time expressions for retarded potentials of point charges like electrons. These potentials are now called the Liénard-Wiechert potentials relating the scalar and vector potential of electromagnetic fields at the observation point to the location of the emitting charges and currents at the time of emission. Using these potentials, Liénard was able to

This chapter has been made Open Access under a CC BY 4.0 license. For details on rights and licenses please read the Correction https://doi.org/10.1007/978-3-319-18317-6_28 
calculate the energy lost by electrons while circulating in a homogenous magnetic field.

In 1907 [3, 4] and 1912 [5] Schott formulated and published his classical theory of radiation from an orbiting electron. He was primarily interested in the spectral distribution of radiation and hoped to find an explanation for atomic radiation spectra. Verifying Liénard's conclusion on the energy loss, he derived the angular and spectral distribution and the polarization of radiation. Since this classical approach to explain atomic spectra was destined to fail, his paper was forgotten and only 40 years later were many of his findings rediscovered.

\subsubsection{Why Do Charged Particles Radiate?}

Before we dive into the theory of electromagnetic radiation in more detail we may first ask ourselves why do charged particles radiate at all? Emission of electromagnetic radiation from charged particle beams (microwaves or synchrotron radiation) is a direct consequence of the finite velocity of light. A charged particle in uniform motion through vacuum is the source of electric field lines emanating from the charge radially out to infinity. While the charged particle is at rest or moving uniformly these field lines also are at rest or in uniform motion together with the particle. Now, we consider a particle being suddenly accelerated for a short time. That means the field lines should also be accelerated. The fact that the particle has been accelerated is, however, still known only within the event horizon in a limited area close to the particle. The signal of acceleration travels away from the source (particle) only at the finite speed of light. Field lines close to the charged particle are directed radially toward the particle, but far away, the field lines still point to the location where the particle would be had it not been accelerated. Somewhere between those two regimes the field lines are distorted and it is this distortion travelling away from the particle at the speed of light what we call electromagnetic radiation. The magnitude of these field distortions is proportional to the acceleration.

In a linear accelerator, for example, electrons are accelerated along the linac axis and therefore radiate. The degree of actual acceleration, however, is very low because electrons in a linear accelerator travel close to the velocity of light. The closer the particle velocity is to the velocity of light the smaller is the actual acceleration gained from a given force, and the radiation intensity is very small. In a circular accelerator like a synchrotron, on the other hand, particles are deflected transversely to their direction of motion by magnetic fields. Orthogonal acceleration or the rate of change in transverse velocity is very large because the transverse particle velocity can increase from zero to very large values in a very short time while passing through the magnetic field. Consequently, the emitted radiation intensity is very large. Synchrotron radiation sources come therefore generally in form of circular synchrotrons. Linear accelerators can be the source of intense synchrotron radiation in conjunction with a transversely deflecting magnet. 


\subsubsection{Spontaneous Synchrotron Radiation}

Charged particles do not radiate while in uniform motion, but during acceleration a rearrangement of its electric fields is required and this field perturbation, traveling away from the charge at the velocity of light, is what we observe as electromagnetic radiation. Free accelerated electrons radiate similarly to those in a radio antenna, although now the source (antenna) is moving. Radiation from a fast moving particle source appears to the observer in the laboratory as being all emitted in the general direction of motion of the particle. This forward collimation is particularly effective for highly relativistic electrons where most of the radiation is concentrated into a small cone around the forward direction with an opening angle of $1 / \gamma$, typically 0.1 to $1 \mathrm{mrad}$, where $\gamma$ is the particle energy in units of its rest mass.

Radiation can be produced by magnetic deflection in a variety of ways. Whether it be a single kick-like deflection or a periodic right-left deflection, the radiation characteristics reflect the particular mode of deflection. Specific radiation characteristics can be gained through specific modes of deflections. Here, we will only shortly address the main processes of radiation generation and come back later for a much more detailed discussion of the physical dynamics.

In an undulator the electron beam is periodically deflected transversely to its direction of motion by weak sinusoidally varying magnetic fields, generating periodic perturbations of the electric field lines. A receiving electric field detector recognizes a periodic variation of the transverse electromagnetic field components and interprets this as quasi monochromatic radiation. In everyday life periodic acceleration of electrons occurs in radio and TV antennas and we may receive these periodic field perturbations with a radio or TV receiver tuned to the frequency of the periodic electron motion in the emitting antenna. The fact that we consider relativistic electrons is not fundamental, but we restrict ourselves in this text to high energy electrons only.

To the particle the wavelength of the emitted radiation is equal to the undulator period length $\left(\lambda_{\mathrm{p}}\right)$ divided by $\gamma$ due to relativistic Lorentz contraction. In a stationary laboratory system, this wavelength appears to the observer further reduced by another factor $2 \gamma$ due to the Doppler effect. The undulator period length of the order of centimeters is thus reduced by a factor $\gamma^{2}\left(10^{6}-10^{8}\right)$ to yield short wavelength radiation in the VUV and $\mathrm{x}$-ray regime. The spectral resolution of the radiation is proportional to the number of undulator periods $N_{\mathrm{p}}$ and its wavelength can be shifted by varying the magnetic field. Most radiation is emitted within the small angle of $\left(\gamma \sqrt{N_{\mathrm{p}}}\right)^{-1}$.

Increasing the magnetic field strength causes the pure sinusoidal transverse motion of electrons in an undulator to become distorted due to relativistic effects generating higher harmonic perturbations of the electron trajectory. Consequently, the monochromatic undulator spectrum exhibits higher harmonics and changes into a line spectrum. For very strong fields, many harmonics are generated which eventually merge into a continuous spectrum from IR to hard x-rays. In this extreme, we call the source magnet a wiggler magnet. The spectral intensity varies little over 
a broad wavelength range and drops off exponentially at photon energies higher than the critical photon energy, $\varepsilon_{\text {crit }} \propto B \gamma^{2}$. Changing the magnetic field, one may vary the critical photon energy to suit experimental requirements. Compared to bending magnet radiation, wiggler radiation is enhanced by the number of magnet poles $N_{\mathrm{pol}}$ and is well collimated within an angle of $1 / \gamma$ to say $10 / \gamma$, or a few mrad.

A bending magnet is technically the most simple radiation source. Radiation is emitted tangentially to the orbit similar to a search light while well collimated in the non-deflecting, or vertical plane. The observer at the experimental station sees radiation from only a small fraction of the circular path which can be described as a piece of a distorted sinusoidal motion. The radiation spectrum is therefore similar to that of a wiggler magnet while the intensity is due to only one pole. Because bending magnets define the geometry of the electron beam transport system or accelerator, it is not possible to freely choose the field strength and the critical photon energy is therefore fixed. Sometimes, specially in lower energy storage rings, it is desirable to extend the radiation spectrum to higher photon energies into the $\mathrm{x}$-ray regime. This can be accomplished by replacing one or more conventional bending magnet with a superconducting magnet or superbends at much higher field strength. To preserve the ring geometry the length of these superbends must be chosen such that the deflection angle is the same as it was for the conventional magnet that has been replaced. Again, superbends are part of the ring geometry and therefore the field cannot be changed.

A more flexible version of a radiation hardening magnet is the wavelength shifter. This is a magnet which consists of a high field central pole and two weaker outside poles to compensate the deflection by the central pole. The total deflection angle is zero and therefore the field strength can be chosen freely to adjust the critical photon energy. It's design is mostly based on superconducting magnet technology, particularly in low energy accelerators, to extend (shift) the critical photon energy available from bending magnets to higher values.

A variety of more complicated magnetic field arrangements have been developed to primarily generate circularly or elliptically polarized radiation. In such magnets horizontal as well as vertical magnetic fields are sequentially employed to deflect electrons into some sort of helical motion giving raise to the desired polarization effect.

\subsubsection{Stimulated Radiation}

The well defined time structure and frequency of undulator radiation can be used to stimulate the emission of even more radiation. In an optical klystron [6] coherent radiation with a wavelength equal to the fundamental undulator wavelength enters an undulator together with the electron beam. Since the electron bunch length is much longer than the radiation wavelength, some electrons loose energy to the radiation field and some electrons gain energy from the radiation field while interacting with the radiation field. This energy modulation can be transformed into 
a density modulation by passing the modulated electron beam through a dispersive section. This section consists of deflecting magnetic fields arranged in such a way that the total path length through the dispersive section depends on the electron energy. The periodic energy modulation of the electron bunch then converts into a periodic density modulation. Now we have microbunches at a distance of the undulator radiation wavelength. This microbunched beam travels through a second undulator where again particles can loose or gain energy from the radiation field. Due to the microbunching, however, most particles are concentrated at phases where there is only energy transfer from the particle to the radiation field, thus providing a high gain of radiation intensity.

In a more efficient variation of this principle, radiation emitted by electrons passing through an undulator is recycled by optical mirrors in such a way that it passes through the same undulator again together with another electron bunch. The external field stimulates more emission of radiation from the electrons, and is again recycled to stimulate a subsequent electron bunch until there are no more bunches in the electron pulse. Generating from a linear accelerator a train of thousands of electron bunches one can generate a large number of interactions, leading to an exponential growth of electromagnetic radiation. Such a devise is called a free electron laser or short FEL.

\subsubsection{Electron Beam}

In this text we consider radiation from relativistic electron beams only. Such beams can be generated efficiently by acceleration in microwave fields. The oscillatory nature of microwaves makes it impossible to produce a uniform stream of particles, and the electron beam is modulated into bunches at the distance of the microwave wavelength. The bunched nature of the electron beam and the fact that these bunches circulate in a storage ring determines the time structure and spectrum of the emitted radiation. Typically, the bunch length in storage rings is $30-100 \mathrm{ps}$ at a distance of 2-3 ns depending on the rf-frequency.

During the storage time of the particle beam, the electrons radiate and it is this radiation that is extracted and used in experiments of basic and applied research. Considering, for example, only one bunch rotating in the storage ring, the experimenter would observe a light flash at a frequency equal to the revolution frequency $f_{\text {rev }}$. Because of the extremely short duration of the light flash many harmonics of the revolution frequency appear in the light spectrum. At the low frequency end of this spectrum, however, no radiation can be emitted for wavelength longer than about the dimensions of the metallic vacuum chamber surrounding the electron beam. For long wavelengths the metallic boundary conditions for electromagnetic fields cannot be met prohibiting the emission of radiation. Practically, useful radiation is observed from storage rings only for wavelengths below the microwave regime, or for $\lambda \lesssim 1 \mathrm{~mm}$. 


\subsection{Conservation Laws and Radiation}

The emission of electromagnetic radiation from free electrons is a classical phenomenon. We may therefore use a visual approach to gain some insight into conditions and mechanisms of radiation emission. First, we will discuss necessary conditions that must be met to allow an electron to emit or absorb a photon. Once such conditions are met, we derive from energy conservation a quantity, the Poynting vector, relating energy transport or radiation to electromagnetic fields. This will give us the basis for further theoretical definitions and discussions of radiation phenomena.

The emission of electromagnetic radiation involves two components, the electron and the radiation field. For the combined system energy-momentum conservation must be fulfilled. These conservation laws impose very specific selection rules on the kind of emission processes possible. To demonstrate this, we plot the energy versus momentum for both electron and photon. In relativistic terms, we have the relation $\gamma=\sqrt{1+(\beta \gamma)^{2}}$ between energy $\gamma$ and momentum $\beta \gamma$. For consistency in quantities used we normalize the photon energy to the electron rest energy, $\gamma_{\mathrm{p}}=\varepsilon_{\mathrm{p}} / m c^{2}$, where $\varepsilon_{\mathrm{p}}=\hbar \omega$ is the photon energy and $m c^{2}$ the electron rest mass while the normalized photon momentum is $\beta_{\mathrm{p}} \gamma=\hbar k_{\mathrm{p}} / m c^{2}$. Similarly, we express the speed of light by $\beta_{\mathrm{p}}=c_{\mathrm{p}} / c=1 / n$ where $n>1$ is the refractive index of the medium surrounding the photon. With these definitions and assuming, for now, vacuum as the medium $(n=1)$ the location of a particle or photon in energy-momentum space is shown in Fig. 23.1(left).

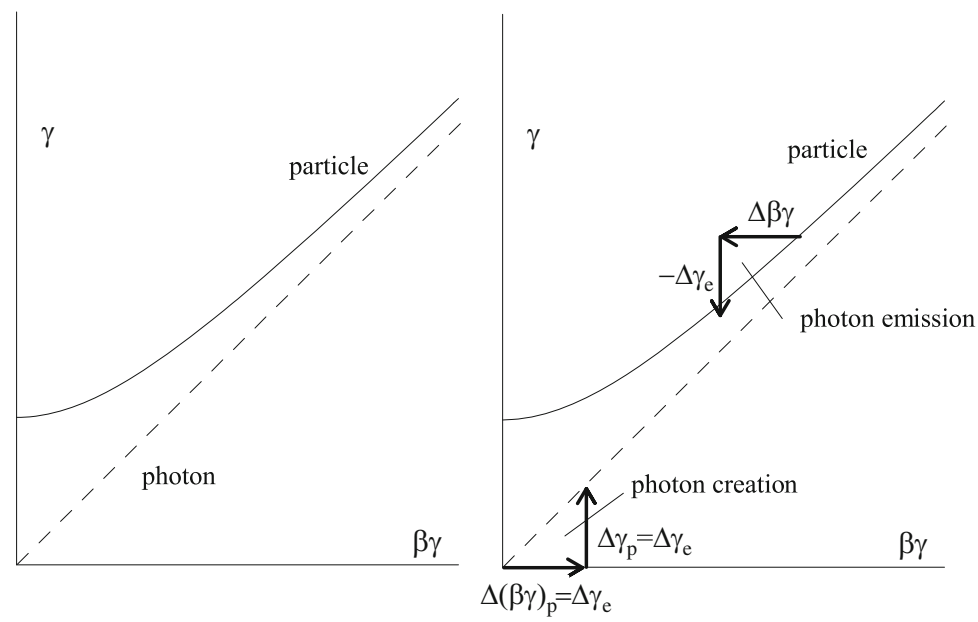

Fig. 23.1 Energy-momentum relationship for particles and photons (left). Violation of energy or momentum conservation during emission and absorption of electromagnetic radiation by a free electron travelling in perfect vacuum $\left(\beta_{\mathrm{p}}=1\right)($ right $)$ 
Energy and momentum of a particle are related such that it must be located on the "particle"-line in Fig. 23.1(left) while a photon is always located on the "photon"line. Transfer of energy between particle and photon must obey energy-momentum conservation. In Fig. 23.1(right) we apply this principle to a free electron in vacuum emitting (absorbing) a photon. To create a photon the electron would have to loose (gain) an amount of momentum which is numerically equal to the energy gained (lost) by the photon. Clearly, in this case the electron would end up at a location off the "particle"-line, thus violating momentum conservation. That cannot be, and such a process is therefore not permitted. A free electron in vacuum cannot emit or absorb a photon without violating energy-momentum conservation.

\subsubsection{Cherenkov Radiation}

We have been careful to assume an electron in perfect vacuum. What happens in a material environment is shown in Fig. 23.2. Because the refractive index $n>1$, the phase velocity of radiation is less than the velocity of light in vacuum and with $\beta=1 / n$, the "photon"-line is tilted towards the momentum axis.

Formally, we obtain this for a photon from the derivative $\mathrm{d} \gamma / \mathrm{d}(\beta \gamma)$ which we expand to $\frac{\mathrm{d} \gamma}{\mathrm{d}(\beta \gamma)}=\frac{\mathrm{d} \gamma}{\mathrm{d} \omega} \frac{\mathrm{d} \omega}{\mathrm{d} k} \frac{\mathrm{d} k}{\mathrm{~d}(\beta \gamma)}$ and get with $\gamma=\hbar \omega / m c^{2}, k=n \frac{\omega}{c}$, and the

Fig. 23.2 Energy and momentum conservation in a refractive environment with $n>1$

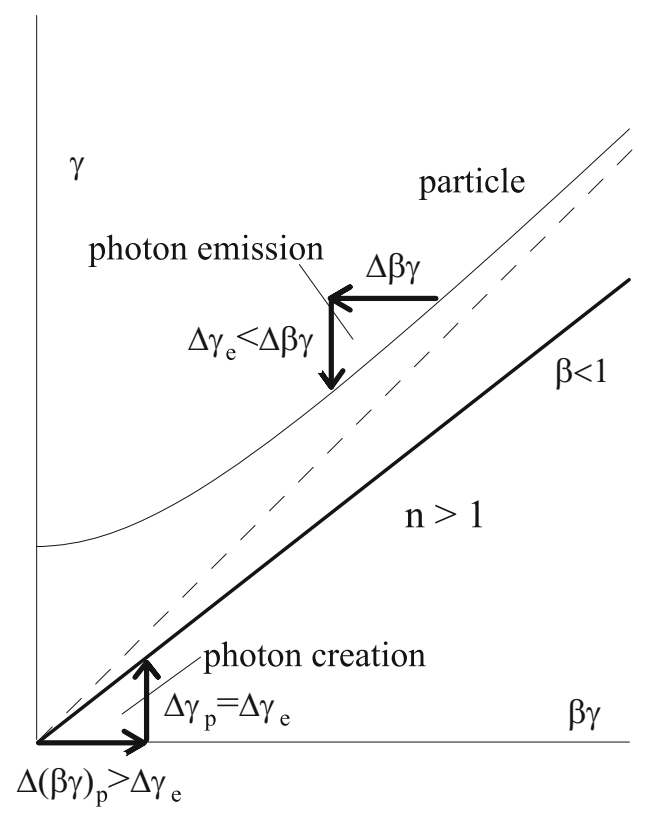


momentum $\beta \gamma=\frac{\hbar}{m c} k$, the derivative

$$
\frac{\mathrm{d} \gamma_{\mathrm{p}}}{\mathrm{d}(\beta \gamma)_{\mathrm{p}}}=\frac{1}{n}<1,
$$

where we have added the subscript ${ }_{p}$ to differentiate between photon and electron parameters.

The dispersion function for a photon in a material environment has a slope less than unity as shown in Fig. 23.2. In this case, the numerical value of the photon momentum is less than the photon energy, analogous to the particle case. To create a photon of energy $\gamma_{\mathrm{p}}$ we set $\gamma_{\mathrm{p}}=-\Delta \gamma=-\beta \Delta \beta \gamma$ from (1.30), where from (23.1) the photon energy $\gamma_{\mathrm{p}}=\frac{1}{n}(\beta \gamma)_{\mathrm{p}}$ and get from both relations $(\beta \gamma)_{\mathrm{p}}=-n \beta \Delta \beta \gamma$. Because of symmetry, no momentum transverse to the particle trajectory can be exchanged, which means radiation is emitted uniformly in azimuth. The change in longitudinal momentum along the trajectory is $-\Delta \beta \gamma=\left.(\beta \gamma)_{\mathrm{p}}\right|_{\|}=(\beta \gamma)_{\mathrm{p}} \cos \theta$. In a dielectric environment, free electrons can indeed emit or absorb a photon although, only in a direction given by the angle $\theta$ with respect to the electron trajectory. This radiation is called Cherenkov radiation, and the Cherenkov angle $\theta$ is given by the Cherenkov condition

$$
n \beta \cos \theta=1
$$

Note, that this condition is not the same as saying whenever an electron passes though a refractive medium with $n>1$ there is Cherenkov radiation. The Cherenkov condition requires that $n \beta>1$ which is, for example, not the case for an electron beam of less than $20 \mathrm{MeV}$ traveling through air.

\subsubsection{Compton Radiation}

To generate electromagnetic radiation from free electrons in vacuum without violating energy-momentum conservation, we may employ the Compton effect which is the scattering of an incoming photon by the electron. In energy-momentum space this process is shown in Fig. 23.3. The electron, colliding head-on with an incoming photon absorbs this photon and emits again a photon of different energy. In this process it gains energy but looses momentum bringing the electron in the energy-momentum space to an intermediate point, $P_{I}$, from where it can reach its final state on the "particle"-line by emitting a photon as shown in Fig. 23.3. This is the process involved in the generation of synchrotron radiation. Static magnetic fields in the laboratory system appear as electromagnetic fields like an incoming (virtual) photon in the electron system with which the electron can collide. Energymomentum conservation give us the fundamental and necessary conditions under 
Fig. 23.3 Energy and momentum conservation for Compton scattering process

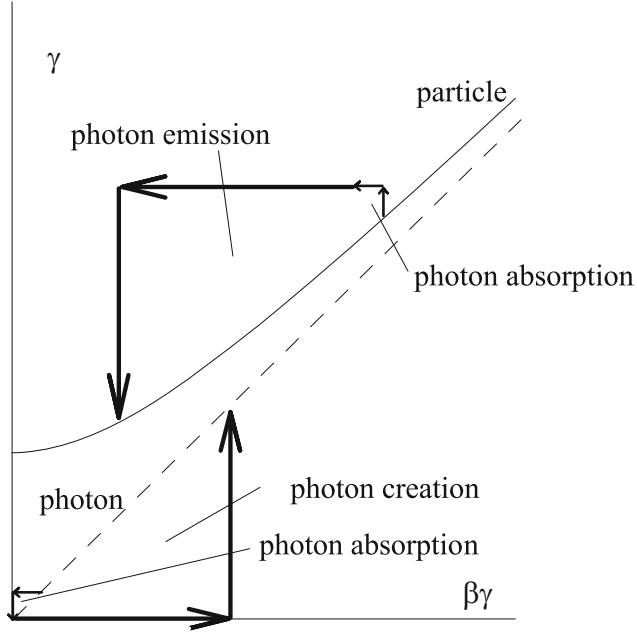

which a free charged particle can emit or absorb a photon. We turn our attention now to the actual interaction of charged particles with an electromagnetic field.

\subsection{Electromagnetic Radiation}

Phenomenologically, synchrotron radiation is the consequence of a finite value for the velocity of light. Electric fields extend infinitely into space from charged particles in uniform motion. When charged particles become accelerated, however, parts of these fields cannot catch up with the particle anymore and give rise to synchrotron radiation. This happens more so as the particle velocity approaches the velocity of light.

The emission of light can be described by applying Maxwell's equations to moving charged particles. The mathematical derivation of the theory of radiation from Maxwell's equations is straightforward although mathematically elaborate and we will postpone this to Chap. 25. Here we follow a more intuitive discussion ${ }^{1}$ which displays visually the physics of synchrotron radiation from basic physical principles.

Electromagnetic radiation occurs wherever electric and magnetic fields exist with components orthogonal to each other such that the Poynting vector

$$
S=\frac{1}{\mu_{0}}[\boldsymbol{E} \times \boldsymbol{B}] \neq 0 .
$$

\footnotetext{
${ }^{1}$ The author would like to thank Prof. M. Eriksson, Lund, Sweden for introducing him to this approach into the theory of synchrotron radiation.
} 
It is interesting to ask what happens if we have a static electric and magnetic field such that $[\boldsymbol{E} \times \boldsymbol{B}] \neq 0$. We know there is no radiation but the Poynting vector is nonzero. Applying energy conservation (1.87) we find the first two terms to be zero which renders the third term zero as well. For a static electric and magnetic field the integral defining the radiation loss or absorption is equal to zero and therefore no radiation or energy transport occurs.

Similarly, in case of a stationary electrostatic charge, we note that the electrostatic fields extend radially from the charge to infinity which violates the requirement that the field be orthogonal to the direction of observation or energy flow. Furthermore, the charge is stationary and therefore there is no magnetic field.

\subsubsection{Coulomb Regime}

Next, we consider a charge in uniform motion. In the rest frame of the moving charge we have no radiation since the charge is at rest as just discussed. In the laboratory system, however, the field components are different. Since the charge is moving, it constitutes an electric current which generates a magnetic field. Formulating the Poynting vector in the laboratory system we express the fields by the pure electric field in the particle rest frame $\mathcal{L}^{*}$. We accomplish that by an inverse Lorentz transformations to (1.9), where the laboratory system $\mathcal{L}$ now moves with the velocity $-\beta_{z}$ with respect to $\mathcal{L}^{*}$ and $\beta_{z}$ in (1.9) must be replaced by $-\beta_{z}$ for

$$
\left(\begin{array}{c}
E_{x} \\
E_{y} \\
E_{z} \\
c B_{x} \\
c B_{y} \\
c B_{z}
\end{array}\right)=\left(\begin{array}{cccccc}
\gamma & 0 & 0 & 0 & \beta_{z} \gamma & 0 \\
0 & \gamma & 0 & -\beta_{z} \gamma & 0 & 0 \\
0 & 0 & 1 & 0 & 0 & 0 \\
0 & -\beta_{z} \gamma & 0 & \gamma & 0 & 0 \\
\beta_{z} \gamma & 0 & 0 & 0 & \gamma & 0 \\
0 & 0 & 0 & 0 & 0 & 1
\end{array}\right)\left(\begin{array}{c}
E_{x}^{*} \\
E_{y}^{*} \\
E_{z}^{*} \\
c B_{x}^{*} \\
c B_{y}^{*} \\
c B_{z}^{*}
\end{array}\right) .
$$

In the laboratory system $\mathcal{L}$ the components of the Poynting vector (23.3) become then with $\boldsymbol{B}^{*}=0$

$$
\begin{aligned}
& c \mu_{0} S_{x}=-\gamma \beta_{z} E_{x}^{*} E_{z}^{*}, \\
& c \mu_{0} S_{y}=-\gamma \beta_{z} E_{y}^{*} E_{z}^{*}, \\
& c \mu_{0} S_{z}=+\gamma^{2} \beta_{z}\left(E_{x}^{* 2}+E_{y}^{* 2}\right),
\end{aligned}
$$

where ${ }^{*}$ indicates quantities in the moving system $\mathcal{L}^{*}$ and $\beta_{z}=v_{z} / c$. The Poynting vector is nonzero and describes the flow of field energy in the environment of a moving charged particle. The fields drop off rapidly with distance from the particle and the "radiation" is therefore confined close to the location of the particle. Specifically, the fields are attached to the charge and travel in the vicinity and with 
the charge. This part of electromagnetic radiation is called the Coulomb regime in contrast to the radiation regime and is, for example, responsible for the transport of electric energy along electrical wires and transmission lines.

We will ignore this regime in our further discussion of synchrotron radiation. It should be noted, however, that measurements of radiation parameters close to radiating charges may be affected by the presence of the Coulomb radiation regime. Such situations occur, for example, when radiation is observed close to the source point. Related theories deal with this mixing by specifying a formation length defining the minimum distance from the source required to sufficiently separate the Coulomb regime from the radiation regime.

\subsubsection{Radiation Regime}

In this text we are only interested in the radiation regime and therefore ignore from now on the Coulomb regime. To describe the physics of emission of radiation, we consider a coordinate system moving with a constant velocity equal to that of the charged particle and associated electric fields. The charge is at rest in the moving reference system, the electric field lines extend radially out to infinity, and there is no radiation as discussed before. Acceleration of the charge causes it to move with respect to this reference system generating a distortion of the purely radial electric fields of a uniformly moving charge (Fig. 23.4). This distortion, resulting in a rearrangement of field lines to the new charge position, travels outward at the velocity of light giving rise to what we call radiation.

Fig. 23.4 Distortion of fields due to longitudinal acceleration

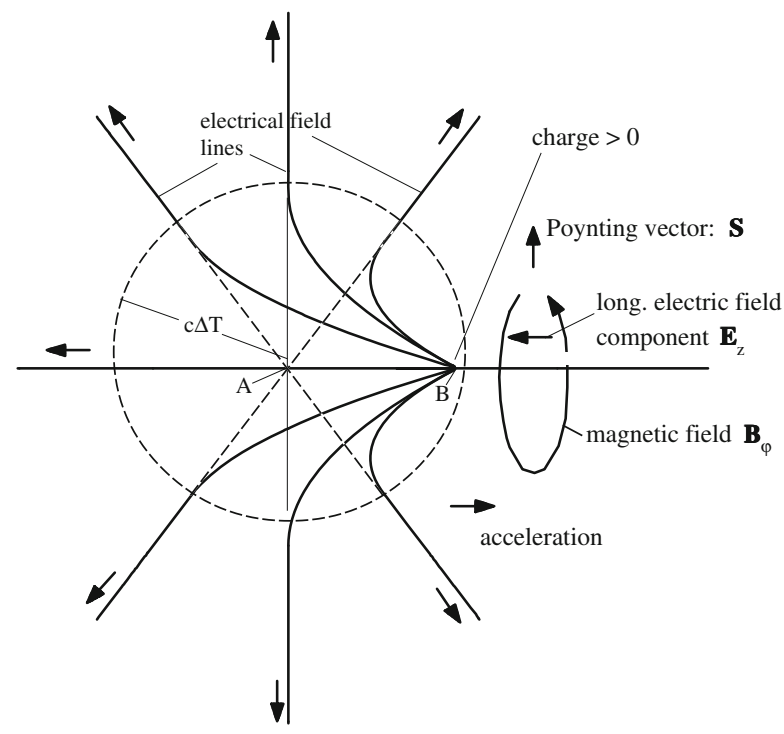


To be more specific, we consider a positive charge in uniform motion for $t \leqslant 0$, the we apply an accelerating force at time $t=0$ for a time $\Delta T$ and observe the charged particle and its fields in the uniformly moving frame of reference. Due to acceleration the charge moves in this reference system during the time $\Delta T$ from point $A$ to point $B$ and as a consequence the field lines become distorted within a radius $c \Delta T$ from the original location $A$ of the particle. It is this distortion, travelling away from the source at the speed of light, that we call radiation.

The effects on the fields are shown schematically in Fig. 23.4 for an acceleration of a positive charge along its direction of motion. At time $t=0$ all electric field lines extend radially from the charge located at point $A$ to infinity. During acceleration fieldlines emerge from the charge now at locations between $A$ and $B$. The new field lines must join the old field lines which, due to the finite velocity of light, are still unperturbed at distances larger than $c \Delta T$. As long as the acceleration lasts, a nonradial field component, parallel and opposite to the acceleration, is created. Furthermore, the moving charge creates an azimuthal magnetic field $B_{\varphi}^{*}(t)$ and the Poynting vector becomes nonzero causing the emission of radiation from an accelerated electrical charge.

Obviously, acceleration would not result in any radiation if the velocity of propagation for electromagnetic fields were infinite $(c \rightarrow \infty)$. In this case the radial fields at all distances from the charge would instantly move in synchrony with the movement of the charge. Only the Coulomb regime would exist.

The electrical field perturbation is proportional to the electrical charge $q$ and the acceleration $a^{*}$. Acceleration along the $z$-axis generates an electric field $\boldsymbol{E}_{z}^{*} \neq 0$ and its component normal to the direction of observation scales like $\sin \Theta^{*}$, where $\Theta^{*}$ is the angle between the line of observation and the direction of particle acceleration. During the acceleration a fixed amount of field energy is created which propagates radially outward from the source. Since the total radiation energy must stay constant and the volume of the expanding spherical sheath of field perturbation increases like $R^{2}$, the field strength decays linear with distance $R$. With this, we make the ansatz

$$
\boldsymbol{E}_{\|}^{*}=-\frac{1}{4 \pi \epsilon_{0}} \frac{e \boldsymbol{a}^{*}}{c^{2} R} \sin \Theta^{*}
$$

for the electric field, where we have added a factor $c^{2}$ in the denominator to be dimensionally correct. For an electron $(e<0)$ the field perturbation would be positive pointing in the direction of the acceleration. As expected from the definition of the Poynting vector, the radiation is emitted predominantly orthogonal to the direction of acceleration and is highly polarized in the direction of acceleration. From (1.89)

$$
S=\frac{1}{c \mu_{0}} E_{\|}^{* 2} \boldsymbol{n}^{*},
$$

where $\mathbf{n}^{*}$ is the unit vector in the direction of observation from the observer toward the radiation source. The result is consistent with our earlier finding that no free 
Fig. 23.5 Spatial radiation distribution in the rest frame of the radiating charge

Fig. 23.6 Distortion of field lines due to transverse acceleration
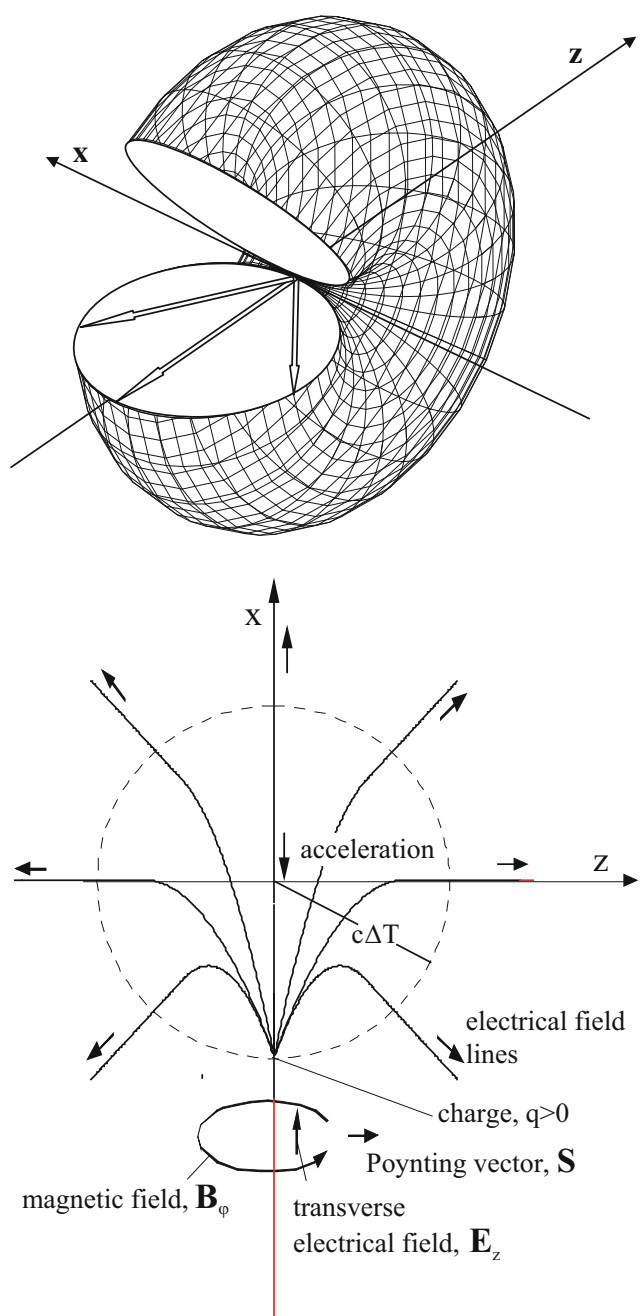

radiation is emitted from a charge at rest or uniform motion $\left(\boldsymbol{a}^{*} \rightarrow 0\right)$. The spatial radiation distribution is from (23.6) and (23.7) characterized by a $\sin ^{2} \Theta^{*}$ distribution resembling the shape of a doughnut as shown in Fig. 23.5, where the acceleration occurs along the $x$-axis.

Acceleration may not only occur in the longitudinal direction but also in the direction transverse to the velocity of the particle as shown in Fig. 23.6. The distortion of field lines in this case creates primarily transverse or radial field components. The radiation field component transverse to the direction of observation is

$$
\boldsymbol{E}_{\perp}^{*}=-\frac{\mu_{0}}{4 \pi} \frac{e \boldsymbol{a}^{*}}{R} \cos \Theta^{*}
$$


This case of transverse acceleration describes the appearance of synchrotron radiation created by charged particles being deflected in magnetic fields. Similar to (23.7) the Poynting vector for transverse acceleration is

$$
S=\frac{1}{c \mu_{0}} E_{\perp}^{* 2} n^{*}
$$

\section{Problems}

23.1 (S). Consider a relativistic electron traveling along the $z$-axis. In its own system, the electrical field lines extend radially from the charge. Considering only the $x z$-plane, derive an expressions for the electrical field lines in the laboratory frame of reference. Sketch the field pattern in the electron rest frame and in the laboratory system of reference.

23.2 (S). Use a $10 \mathrm{MeV}$ electron beam passing through atmospheric air. Can you observe Cherenkov radiation and if so at what angle? Answer the same questions also for a $50 \mathrm{MeV}$ electron beam. Describe and explain with Fig. 23.2 the fundamental difference of your results ( $n_{\text {air }}=1.0002769$ for $\left.\lambda=5,600 \AA\right)$.

23.3 (S). A $10 \mathrm{MeV}$ electron beam passes with normal incidence through a plate of polystyren scintilator $(n=1.58)$. Is there any Cherenkov radiation and if so at what angle? Where does this radiation escape the plate?

23.4 (S). An electron beam orbits in a circular accelerator with a circumference of $300 \mathrm{~m}$ at an average current of $250 \mathrm{~mA}$ and the beam consists of 500 equally spaced bunches each $1 \mathrm{~cm}$ long. How many particles are orbiting? How many particles are in each bunch? Assuming the time structure of synchrotron radiation is the same as the particle beam time-structure specify and plot the radiation time-structure in the photon beam line.

23.5 (S). From Heisenberg's uncertainty relation construct a "characteristic volume" of a photon with energy $\epsilon_{\mathrm{ph}}=\hbar \omega$. What is the average electric field in this volume for a $1 \mathrm{eV}$ photon and an X-ray photon of $10 \mathrm{keV}$ ?

23.6 (S). Derive from (1.38) the formula for the classical Doppler effect valid for sound waves emitted at a frequency $f_{\mathrm{s}}$ from a source moving with velocity $v$ and received at an angle $\vartheta$.

23.7 (S). Consider an electron storage ring at an energy of $800 \mathrm{MeV}$, a circulating current of $1 \mathrm{amp}$ and a bending radius of $\rho=1.784 \mathrm{~m}$. Calculate the energy loss per turn, and the total synchrotron radiation power from all bending magnets. What would the radiation power be if the particles were $800 \mathrm{MeV}$ muons.

23.8 (S). For the electron beam of exercise 23.7 calculate the critical energy and plot the radiation spectrum. What is the useful frequency range for experimentation 
assuming that the spectral intensity should be within $1 \%$ of the maximum value? Express the maximum useful photon energy in terms of the critical photon energy (only one significant digit!).

23.9 (S). What beam energy would be required to produce x-rays at a critical photon energy of $10 \mathrm{keV}$ from the storage ring of exercise 23.7? Is that energy feasible from a conventional magnet point of view or would the ring have to be larger? What would the new bending radius and energy have to be?

23.10. Verify that for a $10 \mathrm{MeV}$ electron colliding head-on with a Ti-Saphire laser $(\lambda=0.8 \mu \mathrm{m})$ the wavelength in it's own system is $\lambda^{*}=40.88 \mathrm{~nm}$. Also show that the wavelength of the backscattered photon in the laboratory system is $\lambda_{\gamma}=10.4 \AA$. What electron beam energy do you need to produce $1 \AA$ radiation? What is the maximum acceptance angle allowable to still get a photon beam with a band width of $10 \%$ or less? Show that the acceptance angle is $\pm 18.15 \mathrm{mrad}$.

23.11. Consider a ray of $123.8 \mathrm{meV}$ and $10 \mathrm{keV}$ photons, both at a power density of $100 \mathrm{Watt} / \mathrm{mm}^{2}$. How many photons occupy their respective "characteristic volumes"? Show that the photon flux density is $1.875 \times 10^{10}$ photons $(100 \mathrm{meV}) / \mathrm{mm}^{3}$ and $1.875 \times 10^{5}$ photons $(10 \mathrm{keV}) / \mathrm{mm}^{3}$. Verify that, 61.07 photons $(123.8 \mathrm{meV})$ and $1.44 \times 10^{-18}$ photons $(10 \mathrm{keV})$ occupy, on average, its own characteristic volume in a $100 \mathrm{~W} / \mathrm{mm}^{2}$ beam. The X-ray photon distribution is indeed sparse among it's characteristic volume. What are the respective characteristic volumes?

23.12. Show that Eqs. (1.88) and (1.89) are the same for electromagnetic waves.

\section{References}

1. A. Liènard, L'Eclaire Electrique 16(5) (1898)

2. E. Wiechert, Arch. Neerl. 546(5) (1900)

3. G.A. Schott, Ann. Physik 24, 635 (1907)

4. G.A. Schott, Philos. Mag. [6] 13, 194 (1907)

5. G.A. Schott, Electromagnetic Radiation (Cambridge University Press, Cambridge, 1912)

6. A.S. Skrinski, N.A. Vinokurov, Technical Report Preprint INP 77-59, Institute of Nuclear Physics, Novosibirsk, USSR (1977) 


\section{Chapter 24 \\ Overview of Synchrotron Radiation}

After Schott's [1] unsuccessful attempt to explain atomic radiation with his electromagnetic theory no further progress was made for some 40 years mainly because of lack of interest. Only in the mid 1940s did the theory of electromagnetic radiation from free electrons become interesting again with the successful development of circular high-energy electron accelerators. At this time powerful betatrons [2] have been put into operation and it was Ivanenko and Pomeranchouk [3], who first in 1944 pointed out a possible limit to the betatron principle and maximum energy due to energy loss from emission of electromagnetic radiation. This prediction was used by Blewett [4] to calculate the radiation energy loss per turn in a newly constructed $100 \mathrm{MeV}$ betatron at General Electric. In 1946 he measured the shrinkage of the orbit due to radiation losses and the results agreed with predictions. On April 24, 1947 visible radiation was observed for the first time at the $70 \mathrm{MeV}$ synchrotron built at General Electric [5-7] with a transparent glass vacuum chamber. Since then, this radiation is called synchrotron radiation.

The energy loss of particles to synchrotron radiation causes technical and economic limits for circular electron or positron accelerators. As the particle energy is driven higher and higher, more and more rf-power must be supplied to the beam not only to accelerate particles but also to overcome energy losses due to synchrotron radiation. The limit is reached when the radiation power grows to high enough levels exceeding technical cooling capabilities or exceeding the funds available to pay for the high cost of electrical power. To somewhat ameliorate this limit, high-energy electron accelerators have been constructed with ever increasing circumference to allow a more gentle bending of the particle beam. Since the synchrotron radiation power scales like the square of the particle energy (assuming constant magnetic fields) the circumference must scale similar for a constant amount of rf-power. Usually, a compromise is reached by increasing the circumference less and adding more rf-power in spaces along the ring lattice made available by the increased circumference. In general the maximum energy in large circular electron accelerators is limited by the available rf-power while the maximum energy

This chapter has been made Open Access under a CC BY 4.0 license. For details on rights and licenses please read the Correction https://doi.org/10.1007/978-3-319-18317-6_28 
of proton or ion accelerators is more likely limited by the maximum achievable magnetic fields in bending magnets.

What is a nuisance for researchers in one field can provide tremendous opportunities for others. Synchrotron radiation is emitted tangentially from the particle orbit and within a highly collimated angle of $\pm 1 / \gamma$. The spectrum reaches from the far infrared up to hard x-rays, the radiation is polarized and the intensities greatly exceed other sources specifically in the vacuum ultra violet to x-ray region. With these properties synchrotron radiation was soon recognized to be a powerful research tool for material sciences, crystallography, surface physics, chemistry, biophysics, and medicine to name only a few areas of research. While in the past most of this research was done parasitically on accelerators built and optimized for high-energy physics the usefulness of synchrotron radiation for research has become important in its own right to justify the construction and operation of dedicated synchrotron radiation sources all over the world.

\subsection{Radiation Sources}

Deflection of a relativistic particle beam causes the emission of electromagnetic radiation which can be observed in the laboratory system as broadband radiation, highly collimated in the forward direction. The emission is related to the deflection of a charged particle beam and therefore sweeps like a search light across the detection apparatus of the observer. It is this shortness of the observable radiation pulse which implies that the radiation is detected as synchrotron radiation with a broad spectrum. The width of the spectrum is characterized by the critical photon energy (24.49) and depends only on the particle energy and the bending radius of the magnet. Generally, the radiation is produced in bending magnets of a storage ring, where an electron beam is circulating for hours.

In order to adjust the radiation characteristics to special experimental needs, other magnetic devices are being used as synchrotron radiation sources. Such magnets are known as insertion devices since they do not contribute to the overall deflection of the particle beam in the circular accelerator. Their effect is localized and the total deflection in an insertion device is zero. In this chapter, we give a short overview of all radiation sources and their characteristics and postpone more detailed discussions of insertion device radiation to Chap. 26.

\subsubsection{Bending Magnet Radiation}

The radiation from bending magnets is emitted tangentially from any point along the curved path like that of a searchlight and appears therefore as a swath of radiation around the storage ring as shown in Fig. 24.1. In the vertical, nondeflecting plane, 
Fig. 24.1 Radiation swath from bending magnets in an electron storage ring

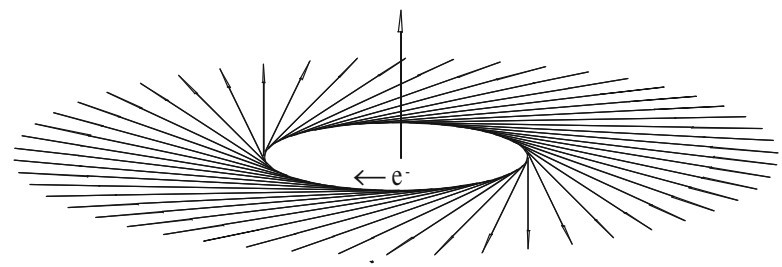

however, the radiation is very much collimated with a typical opening angle of $\pm 1 / \gamma$.

The temporal structure of synchrotron radiation reflects that of the electron beam. Electrons circulating in the storage ring are concentrated into equidistant bunches equal to an integer multiple (usually equal to unity) of the rf-wavelength $(60 \mathrm{~cm}$ for $500 \mathrm{MHz}$ ) while the bunch length itself is of the order of 1 to $3 \mathrm{~cm}$ or 30 to $100 \mathrm{ps}$ depending on beam energy and rf-voltage. As a consequence, the photon beam consists of a series of short 30-100 ps flashes every $2 \mathrm{~ns}(500 \mathrm{MHz})$ or integer multiples thereof.

Radiation is emitted in a broad spectrum reaching, in principal, from mircowaves up to the critically photon energy (24.49) and beyond with fast declining intensities. The long wavelength limit of the radiation spectrum is actually limited by the vacuum chamber, which causes the suppression of radiation at wavelength longer than its dimensions. The strength of bending magnets, being a part of the geometry of the storage ring cannot be freely varied to optimize for desired photon beam characteristics. This is specifically limiting in the choice of the critical photon energy. While the lower photon energy spectrum is well covered even for rather low energy storage rings, the x-ray region requires high beam energies and/or high magnetic fields. Often, the requirements for x-rays cannot be met with existing bending magnet and storage ring parameters.

\subsubsection{Superbends}

The critical photon energy from bending magnet radiation (24.51) is determined by the magnet field and the particle energy. The combination of both quantities may not be sufficient to extend the synchrotron radiation spectrum into the hard x-ray regime, especially in low energy storage rings. In this case, it is possible to replace some or all original bending magnets by much stronger but shorter magnets, called superbends. To be more specific, conventional bending magnets are replaced by high field, shorter superconducting magnets deflecting the electron beam by the same angle to preserve the storage ring geometry. Since conventional bending magnet fields rarely exceed $1.5 \mathrm{~T}$, but superconducting magnets can be operated at 5-6 T or higher, one can gain a factor of 3 to 4 in the critical photon energy and extend the photon spectrum towards or even into the hard x-ray regime and beyond. 


\subsubsection{Wavelength Shifter}

The installation of superbends is not always feasible or desirable. To still meet the need for harder $\mathrm{x}$-ray radiation in a low energy storage ring, it is customary to use a wavelength shifter. Such a device may consist of three or five superconducting dipole magnets with alternating magnetic field directions. For this latter reason, a wavelength shifter is a true insertion device. Figure 24.2 shows schematically a three-pole wavelength shifter.

The particle beam passing through this wavelength shifter is deflected up and down or left and right in such a way that no net deflection remains. To meet this condition, the longitudinal field distribution of a horizontally deflecting wavelength shifter must obey the condition

$$
\int_{-\infty}^{\infty} B_{y}(y=0, z) \mathrm{d} z=0 .
$$

A wavelength shifter with such field properties is neutral on the geometry of the particle beam path through a storage ring and therefore can be made in principle as strong as necessary or technically feasible.

Only the central high field pole is used as the radiation source, while the two side poles compensate the beam deflection from the central pole. In a five-pole wavelength shifter the three central poles would be used as radiators, while both end poles again act as compensators. Mostly, the end poles are longer than the central poles and operate at a lower field. As their name implies, the primary objective in wavelength shifters is to extend the photon spectrum while the enhancement of intensity through radiation accumulation from many poles, while desirable, is of secondary importance. To maximize the desired effect, wavelength shifters are often constructed as high field superconducting magnets to maximize the critical photon energy for the given particle beam energy. Some limitations apply for such devices as well as for any other insertion device. The end fields of magnets can introduce particle focusing and nonlinear field components may introduce aberrations and cause beam instability. Both effects must either be kept below a critical level or be compensated.

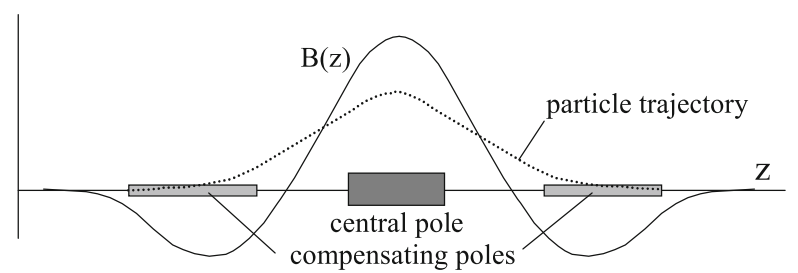

Fig. 24.2 Magnetic field distribution along the beam path for a wave length shifter 


\subsubsection{Wiggler Magnet Radiation}

The principle of a wavelength shifter is extended in the case of a wiggler-magnet. Such a magnet consists of a series of equal dipole magnets with alternating magnetic field direction. Again, the end poles must be configured to make the total device neutral to the geometry of the particle beam path such that the conditions $\int \boldsymbol{B} \mathrm{d} z=0$ are met in both planes.

The main advantage of using many magnet poles is to increase the photon flux. Like a single bending magnet, each of the $N_{\mathrm{pol}}$ magnet poles produces a fan of radiation in the forward direction and the total photon flux is $N_{\text {pol }}$-times larger than that from a single pole. Wiggler-magnets may be constructed as electromagnets with fields up to $2 \mathrm{~T}$ to function both as a flux enhancer and as a more modest wavelength shifter compared to the superconducting type. An example of an 8-pole, $1.8 \mathrm{~T}$ electromagnetic wiggler-magnet [8] is shown in Fig. 24.3.

In this picture, the magnet gap is wide open, to display the flat vacuum chamber running through the magnet between the poles. The pole pieces in the lower row are visible surrounded by water cooled excitation coils. During operation, both rows of wiggler poles are closed to almost touch the flat vacuum chamber. When the magnet is closed, a maximum magnetic field of $1.8 \mathrm{~T}$ can be obtained. Strong fields can be obtained from electromagnets, but the space requirement for the excitation coils limits the number of poles that can be installed within a given length.

Progress in the manufacturing of high field permanent magnet material permits the installation of many more poles into the same space compared to an electromagnet. An example of a modern 26 pole, $2.0 \mathrm{~T}$ permanent magnet wiggler magnet is shown in Fig. 24.4. ${ }^{1}$

Fig. 24.3 Electromagnetic wiggler magnet with eight $1.8 \mathrm{~T}$ poles

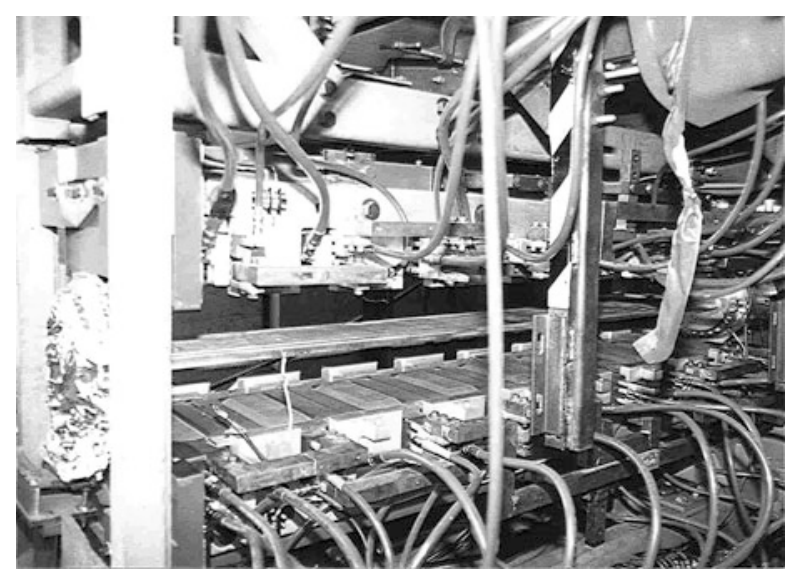

${ }^{1}$ The author would like to thank T. Rabedau, Stanford for this picture. 
BL11: 26 poles, $2.0 \mathrm{~T}, 175 \mathrm{~mm}$ period

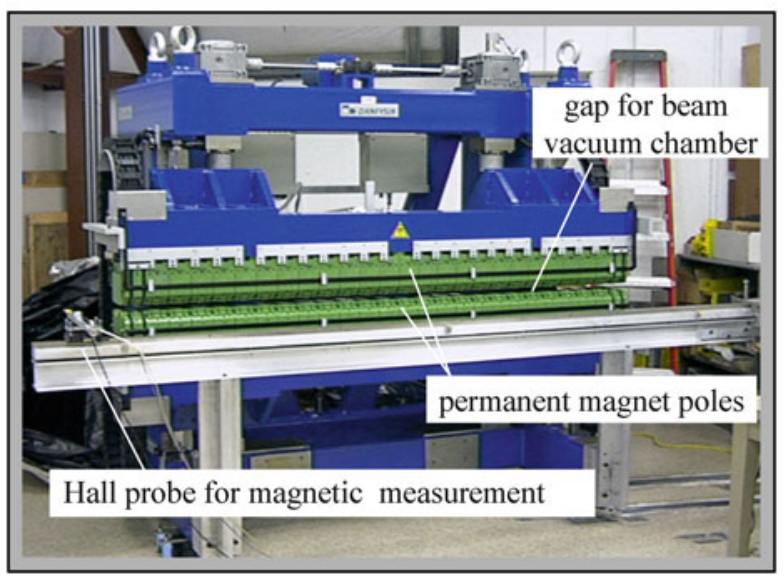

Fig. 24.4 Permanent magnet wiggler magnet with 26 poles, a $175 \mathrm{~mm}$ period length and a maximum field of $2.0 \mathrm{~T}$

Figure 24.4 shows the wiggler magnet during magnetic measurement with the rail in front of the magnet holding and guiding the Hall probe. The increased number of poles and simplified design compared to the electromagnetic wiggler in Fig. 24.3 are clearly visible.

For short wiggler poles, we express the magnetic field by

$$
B_{y}(x, y=0, z)=B_{0} \sin \frac{2 \pi z}{\lambda_{\mathrm{p}}}
$$

and the maximum beam deflection from the axis is equal to the deflection angle per half pole

$$
\vartheta=\frac{B_{0}}{B \rho} \int_{0}^{\lambda_{\mathrm{p}} / 4} \sin \frac{2 \pi z}{\lambda_{\mathrm{p}}} \mathrm{d} z=\frac{B_{0}}{B \rho} \frac{\lambda_{\mathrm{p}}}{2 \pi},
$$

where $B \rho$ is the beam rigidity. Multiplying this with the beam energy $\gamma$, we define the wiggler strength parameter

$$
K=\gamma \vartheta=\frac{e c B_{0}}{m c^{2}} \frac{\lambda_{\mathrm{p}}}{2 \pi}=0.934 B_{0}(\mathrm{~T}) \lambda_{\mathrm{p}}(\mathrm{cm}) .
$$

For longer magnet poles (24.2) must be replaced by a sum of harmonics. Most wiggler magnets, though, are designed for the lowest harmonic only. This wiggler strength parameter is generally much larger than unity. Conversely, a series of alternating magnet poles is called a wiggler magnet if the strength parameter $K \gg 1$ and condition (24.1) is met. As we will see later, a weak wiggler magnet with 
Fig. 24.5 On-axis field strength in a vanadium Permendur hybrid wiggler magnet as a function of gap aperture (24.5)

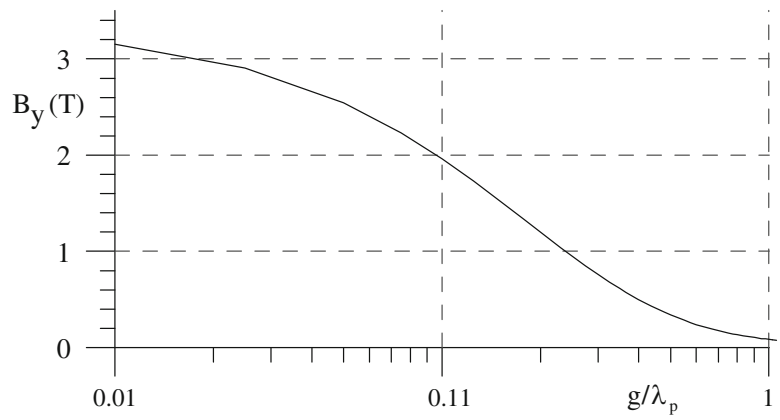

$K \ll 1$ is called an undulator and produces radiation with significant different characteristics. The magnetic field strength can be varied in both electromagnetic wigglers as well as in permanent magnet wigglers. While this is obvious for electromagnets, the magnetic field strength in permanent magnets depends on the distance between magnet poles or on the gap height $g$. By varying mechanically the gap height of a permanent magnet wiggler, the magnetic field strength can be varied as well. The field strength also depends on the period length and on the design and magnet materials used. For a wiggler magnet constructed as a hybrid magnet with Vanadium Permendur poles, the field strength along the midplane axis scales approximately like [9]

$$
B_{y}(\mathrm{~T}) \approx 3.33 \exp \left[-\frac{g}{\lambda_{\mathrm{p}}}\left(5.47-1.8 \frac{g}{\lambda_{\mathrm{p}}}\right)\right], \quad \text { for } 0.1 \lambda_{\mathrm{p}} \lesssim g \lesssim 10 \lambda_{\mathrm{p}},
$$

where $g$ is the gap aperture between magnet poles. This dependency is also shown in Fig. 24.5 and we note immediately that the field strength drops off dramatically for magnet gaps of the order of a period length or greater.

On the other hand, significant field strengths can be obtained for small gap apertures and it is therefore important to install the insertion device at a location, where the beam dimension normal to the deflection plane is small.

The total radiation power can be derived by integrating (24.32) through the wiggler magnet. The result of this integration is

$$
\left\langle P_{\gamma}\right\rangle=\frac{1}{3} r_{\mathrm{c}} m c^{2} c \gamma^{2} K^{2} \frac{4 \pi^{2}}{\lambda_{\mathrm{p}}^{2}}
$$

or in practical units

$$
\left\langle P_{\gamma}(\mathrm{W})\right\rangle=632.7 E^{2} B_{0}^{2} I L_{\mathrm{u}}
$$

where $I$ is the circulating beam current, and $L_{\mathrm{u}}=N_{\mathrm{p}} \lambda_{\mathrm{p}}$ the length of the wiggler magnet. 
For a sinusoidal field distribution $B_{0} \sin \frac{2 \pi}{\lambda_{\mathrm{p}}} z$, the desired wavelength shifting property of a strong wiggler magnet can be obtained only in the forward direction. Radiation emitted at a finite angle with respect to the wiggler axis is softer because it is generated at a source point where the field is lower. The hardest radiation is emitted in the forward direction from the crest of the magnetic field. For a distance $\Delta z$ away from the crest, the emission angle in the deflection plane is $\psi=\frac{1}{\rho_{0}} \frac{\lambda_{\mathrm{p}}}{2 \pi} \sin \left(\frac{2 \pi}{\lambda_{\mathrm{p}}} \Delta z\right)$ and the curvature at the source point is $\frac{1}{\rho}=\frac{1}{\rho_{0}} \sqrt{1-\left(\frac{\gamma \psi}{K}\right)^{2}}$, where we have made use of (24.4). Consequently, the critical photon energy for radiation in the direction $\psi$ with respect to the wiggler axis varies with the emission angle $\psi$ like

$$
\varepsilon_{\mathrm{c}}=\varepsilon_{\mathrm{c} 0} \sqrt{1-\left(\frac{\gamma \psi}{K}\right)^{2}} .
$$

At the maximum deflection angle $\psi_{\max }=K / \gamma$ the critical photon energy has dropped to zero, reflecting a zero magnetic field at the source point.

This property is undesirable if more than one experimental station is supposed to receive hard radiation from the same wiggler magnet. The strength of the wiggler magnet sweeps the electron beam over a considerable angle, a feature which can be exploited to direct radiation not only to one experimental station along the axis but also to two or more side-stations on either side of the wiggler axis. However, these side beam lines at an angle $\psi \neq 0$ receive softer radiation than the main beam line. This can be avoided if the poles of the wiggler magnet are lengthened thus flattening the sinusoidal field crest. As the flat part of the field crest is increased, hard radiation is emitted into an increasing angular cone.

\subsubsection{Undulator Radiation}

So far, we discussed insertion devices designed specifically to harden the radiation spectrum or to increase the radiation intensity. Equally common is the implementation of insertion devices to optimize photon beam quality by maximizing its brightness or to provide specific characteristics like elliptically polarized radiation. This is done with the use of undulator magnets, which are constructed similar to wiggler magnets, but are operated at a reduced field strength.

Fundamentally, an undulator magnet causes particles to be only very weakly deflected with an angle of less than $\pm 1 / \gamma$ and consequently the transverse motion of particles is nonrelativistic. In this picture, the electron motion viewed from far away along the beam axis appears as a purely sinusoidal transverse oscillation similar to the electron motion in a linear radio antenna driven by a transmitter and oscillating at the station's carrier frequency. The radiation emitted is therefore monochromatic with a period equal to the oscillation period. 
To be more precise, viewed from far away the particle appears to be at rest or uniform motion as long as the electron has not yet reached the undulator magnet. Upon entering the magnet the electron performs sinusoidal transverse oscillations and returns to its original motion again after it exits the undulator. As a consequence of this motion and in light of earlier discussions, we observe emission of radiation at the frequency of the transverse oscillating beam motion. If $N_{\text {per }}$ is the number of undulator periods, the electric field lines have been perturbed periodically $N_{\text {per }}{ }^{-}$ times and the radiation pulse is composed of $N_{\text {per }}$ oscillations. In the particle rest frame $\mathcal{L}^{*}$ the undulator period length is Lorentz contracted to $\lambda_{\gamma}^{*}=\lambda_{\mathrm{p}} / \gamma$ which is the wavelength of the emitted radiation. Because the radiation includes only a finite number of $N_{\text {per }}$ oscillations, the radiation is not quite monochromatic but rather quasi monochromatic with a band width of $1 / N_{\text {per }}$ as illustrated in Fig. 24.6 (top).

In Fig. 24.6 (bottom) the radiation lobe and spectrum is shown in the laboratory system. The monochromatic nature of the radiation is somewhat lost because radiation emitted at different angles experiences different Doppler shifts. Of course, the radiation is again quasi monochromatic even in the laboratory system when observed through a narrow pin hole along the axis. This monochromatic radiation is called the fundamental undulator radiation and has for $K \ll 1$ a wavelength of

$$
\lambda_{\gamma} \approx \frac{\lambda_{\mathrm{p}}}{2 \gamma^{2}} .
$$

a) electron rest system

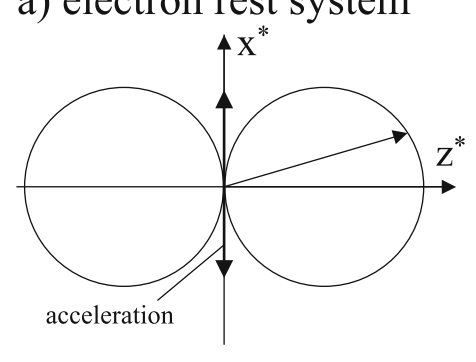

b) laboratory system

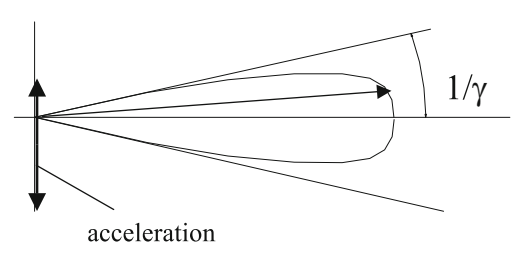

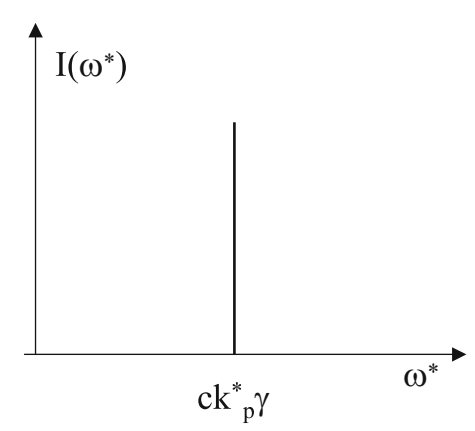

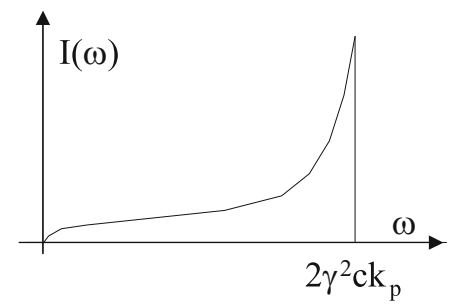

Fig. 24.6 Beam dynamics and radiation lobes in the particle rest system (a) and the laboratory system (b) for a weak undulator $(K \ll 1)$ 
Fig. 24.7 Distortion of sinusoidal motion due to relativistic perturbation of transverse motion

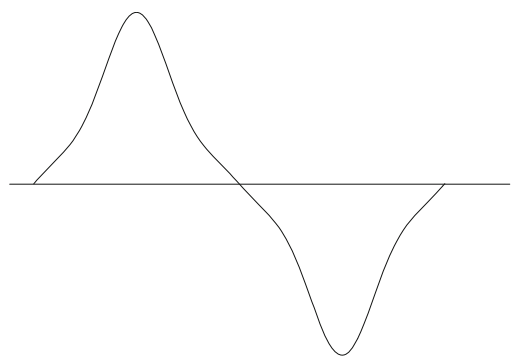

The situation becomes more complicated as the undulator strength is increased. Two new phenomena appear, an oscillatory forward motion and a transverse relativistic effect. The first phenomenon that we need to discuss is the fact that the transverse motion becomes relativistic. As a consequence of this, the pure sinusoidal transverse motion becomes distorted. There is a periodic Lorentz contraction of the longitudinal coordinate, which is larger when the particle travels almost parallel to the axis in the vicinity of the oscillation crests and is smaller when in between crests. The cusps and valleys of the sinusoidal motion become Lorentz-contracted in the particle system thus perturbing the sinusoidal motion as shown in Fig. 24.7. In addition with increasing undulator strength the transverse motion becomes relativistic and the transverse Lorentz contraction enhances the distortion of the sine-like motion.

This perturbation is symmetric about the cusps and valleys causing the appearance of odd and only odd $\left(3^{\text {rd }}, 5^{\text {th }}, 7^{\text {th }} \ldots\right)$ harmonics of the fundamental oscillation period. From an undulator of medium strength $(K \gtrsim 1)$ we observe therefore along the axis a line spectrum of odd harmonics in addition to the fundamental undulator radiation.

The second phenomenon to be discussed is the periodic modulation of the longitudinal motion. The longitudinal component of the particle velocity is maximum when the particle travels close to the crest of the oscillations and at a minimum when it is close to the axis crossings. In a reference system which moves uniformly with the average longitudinal particle velocity along the axis, the particle performs periodic longitudinal oscillations in addition to the transverse oscillations. For each transverse period, the particle performs two longitudinal oscillations and its path looks therefore like a figure of eight. This situation is shown in Fig. 24.8.

We have now two orthogonal accelerations, one transverse and one longitudinal, and two radiation lobes as indicated in Fig. 24.8. Since the longitudinal motion occurs at twice the frequency of the transverse motion, we observe now radiation also at twice the fundamental frequency. Of course, the relativistic perturbation applies here too and we have therefore a line spectrum which includes two series, one with all odd harmonics and one with only even harmonics. Even and odd harmonic radiation is emitted in the particle system in orthogonal directions and therefore we find both radiation lobes in the laboratory system spatially separated as well. The odd harmonics all have their highest intensities along the undulator 


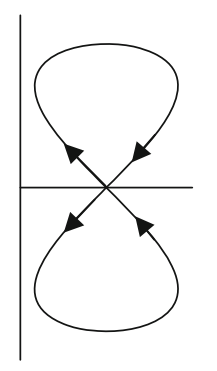

electron motion

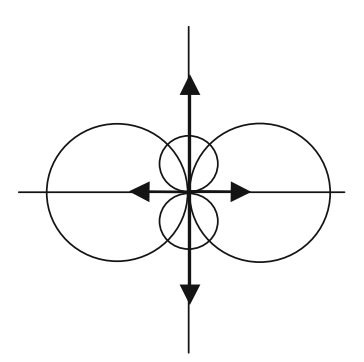

radiation lobes

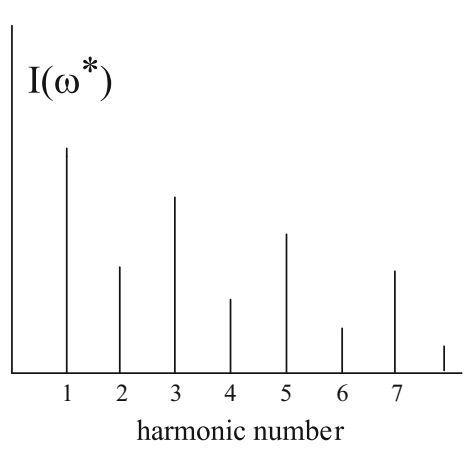

Fig. 24.8 Beam dynamics (left) and radiation lobes (middle) in the particle rest system together with the harmonics spectrum (right) for a stronger undulator $(K \gtrsim 1)$

axis, while the even harmonic radiation is emitted preferentially into an angle $1 / \gamma$ with respect to the axis and has zero intensity along the axis.

In another equally valid view of undulator radiation, the static and periodic magnetic undulator field appears in the rest frame of the electron as a Lorentz contracted electromagnetic field or as monochromatic photon of wavelength $\lambda^{*}=$ $\lambda_{\mathrm{p}} / \gamma$. The emission of photons can therefore be described as Thomson scattering of virtual photons by free electrons [10] resulting in monochromatic radiation in the direction of the particle path. Viewed from the laboratory system, the radiation is Doppler shifted and applying (1.38) the wavelength of the backscattered photons is

$$
\lambda_{\mathrm{ph}}=\frac{\lambda_{\mathrm{p}}}{\gamma^{2}\left(1+\beta n_{z}^{*}\right)} .
$$

Viewing the radiation parallel to the forward direction $(\vartheta \approx 0),(1.39)$ becomes with $n_{z}=\cos \vartheta^{*} \approx 1-\frac{1}{2} \vartheta^{* 2}$, and $\beta \approx 1$

$$
1+\beta n_{z}^{*}=\frac{\beta+n_{z}^{*}}{n_{z}} \approx 2-\frac{1}{2} \frac{\vartheta^{* 2}}{n_{z}} .
$$

Setting $n_{z} \approx 1$, the fundamental wavelength of the emitted radiation is

$$
\lambda_{1}=\frac{\lambda_{\mathrm{p}}}{\gamma^{2}} \frac{1}{2-\frac{1}{2} \frac{\vartheta^{* 2}}{n_{z}}} \approx \frac{\lambda_{\mathrm{p}}}{2 \gamma^{2}}\left(1+\frac{1}{4} \vartheta^{* 2}\right) .
$$

With (1.40) the angle $\vartheta^{*}$ of the particle trajectory with respect to the observation is transformed into the laboratory system like $\vartheta^{*}=2 \gamma \vartheta$. We distinguish two configurations. One where $\vartheta=K / \gamma=$ const. describing the particle motion in a helical undulator, where the magnetic field, being normal to the undulator axis, rotates about this axis. The other more common case is that of a flat undulator, where the particle motion follows a sinusoidal path in which case $\vartheta=\vartheta_{\text {und }}+\vartheta_{\text {obs }}$. 
Here $\vartheta_{\text {und }}=\frac{K}{\gamma} \sin k_{\mathrm{p}} z$ is the observation angle due to the periodic motion of the electrons in the undulator and $\vartheta_{\text {obs }}$ is the actual observation angle. With these definitions and taking the average $\left\langle\vartheta_{\text {und }}^{2}\right\rangle$ we get $\gamma^{2} \vartheta^{2}=\frac{1}{2} K^{2}+\gamma^{2} \vartheta_{\text {obs }}^{2}$. Depending on the type of undulator, the wavelength of radiation from an undulator with a strength parameter $K$ is

$$
\lambda_{1}= \begin{cases}\frac{\lambda_{\mathrm{p}}}{2 \gamma^{2}}\left(1+K^{2}+\gamma^{2} \vartheta_{\mathrm{obs}}^{2}\right) & \text { for a helical undulator } \\ \frac{\lambda_{\mathrm{p}}}{2 \gamma^{2}}\left(1+\frac{1}{2} K^{2}+\gamma^{2} \vartheta_{\mathrm{obs}}^{2}\right) & \text { for a flat undulator. }\end{cases}
$$

From now on only flat undulators will be considered in this text and readers interested in more detail of helical undulators are referred to [11]. No special assumptions have been made here which would prevent us to apply this derivation also to higher harmonic radiation and we get the general expression for the wavelength of the $k$ th harmonic

$$
\lambda_{k}=\frac{\lambda_{\mathrm{p}}}{2 \gamma^{2} k}\left(1+\frac{1}{2} K^{2}+\gamma^{2} \vartheta_{\mathrm{obs}}^{2}\right) .
$$

The additional terms $\frac{1}{2} K^{2}+\gamma^{2} \vartheta_{\text {obs }}^{2}$ compared to (24.9) comes from the correct application of the Doppler effect. Since the particles are deflected periodically in the undulator, we view even the on-axis radiation at a periodically varying angle which accounts for the $\frac{1}{2} K^{2}$-term. Of course, observation of the radiation at a finite angle $\vartheta_{\text {obs }}$ generates an additional red-shift expressed by the term $\gamma^{2} \vartheta_{\text {obs }}^{2}$.

In more practical units, the undulator wavelengths for the $k$ th harmonic are expressed from (24.14) by

$$
\lambda_{k}(\AA)=13.056 \frac{\lambda_{\mathrm{p}}(\mathrm{cm})}{k E^{2}\left(\mathrm{GeV}^{2}\right)}\left(1+\frac{1}{2} K^{2}+\gamma^{2} \vartheta_{\mathrm{obs}}^{2}\right)
$$

and the corresponding photon energies are

$$
\epsilon_{k}(\mathrm{eV})=950 \frac{k E^{2}\left(\mathrm{GeV}^{2}\right)}{\lambda_{\mathrm{p}}(\mathrm{cm})\left(1+\frac{1}{2} K^{2}+\gamma^{2} \vartheta_{\mathrm{obs}}^{2}\right)} .
$$

Recollecting the discussion of undulator radiation, we found that the first harmonic or fundamental radiation is the only radiation emitted for $K \ll 1$. As the undulator parameter increases, however, the oscillatory motion of the particle in the undulator deviates from a pure sinusoidal oscillation. For $K>1$ the transverse motion becomes relativistic, causing a deformation of the sinusoidal motion and the creation of higher harmonics. These harmonics appear at integral multiples of the fundamental radiation energy. Only odd harmonics are emitted along the axis $(\vartheta \approx 0)$ while even harmonics are emitted into a small angle from the axis. As 

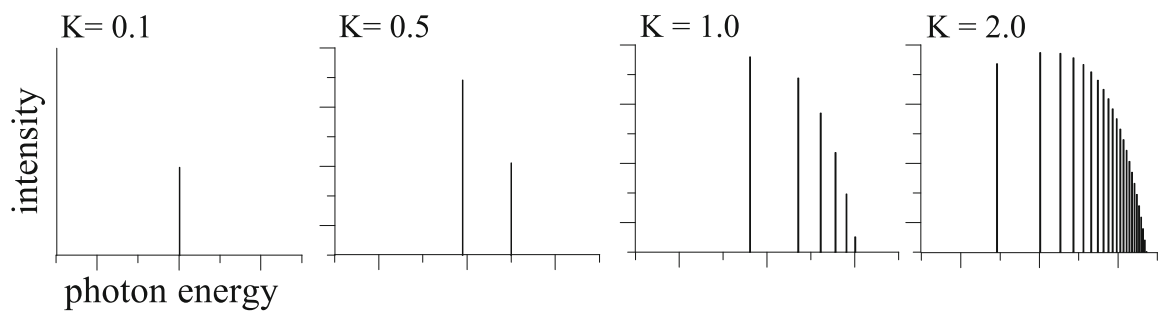

Fig. 24.9 Transition from quasi-monochromatic undulator radiation to broad band wiggler radiation

the undulator strength is further increased more and more harmonics appear, each of them having a finite width due to the finite number of undulator periods, and finally merging into the well-known broad spectrum of bending or wiggler magnet radiation (Fig. 24.9).

We find no fundamental difference between undulator and wiggler magnets, one being just a stronger/weaker version of the other. From a practical point of view, the radiation characteristics are very different and users of synchrotron radiation make use of this difference to optimize their experimental capabilities. In Chap. 26 we will discuss the features of undulator radiation in much more detail.

The electron motion through an undulator with $N_{\text {per }}$ periods includes that many oscillations and so does the radiation field. Applying a Fourier transformation to the field, we find the spectral width of the radiation to be

$$
\frac{\Delta \lambda}{\lambda}=\frac{1}{N_{\text {per }}} .
$$

In reality, this line width is increased due to the finite aperture of the radiation detection elements, and due to a finite energy spread and finite divergence of the electron beam. Typical experimental undulator spectra are shown in Fig. 24.10 for increasing undulator strength $K$ [12].

Although this radiation was measured through a pin hole and on-axis, we still recognize even harmonic radiation since the pin hole covers a finite solid angle and lets some even harmonic radiation through. Furthermore, the measured intensities of the line spectrum does not reflect the theoretical expectation for the lower harmonics at higher values of $K$. This is an artifact of the experimental circumstances, where the $\mathrm{x}$-rays have been extracted from the storage ring vacuum chamber through a Be-window. Such a window works very well for hard x-rays but absorbs heavily at photon energies below some $3 \mathrm{keV}$.

The concentration of all radiation into one or few spectral lines is very desirable for many experiments utilizing monochromatic photon beams since radiation is produced only in the vicinity of the desired wavelength at high brightness. Radiation at other wavelengths creating undesired heating effects on optical elements and samples is greatly eliminated. 


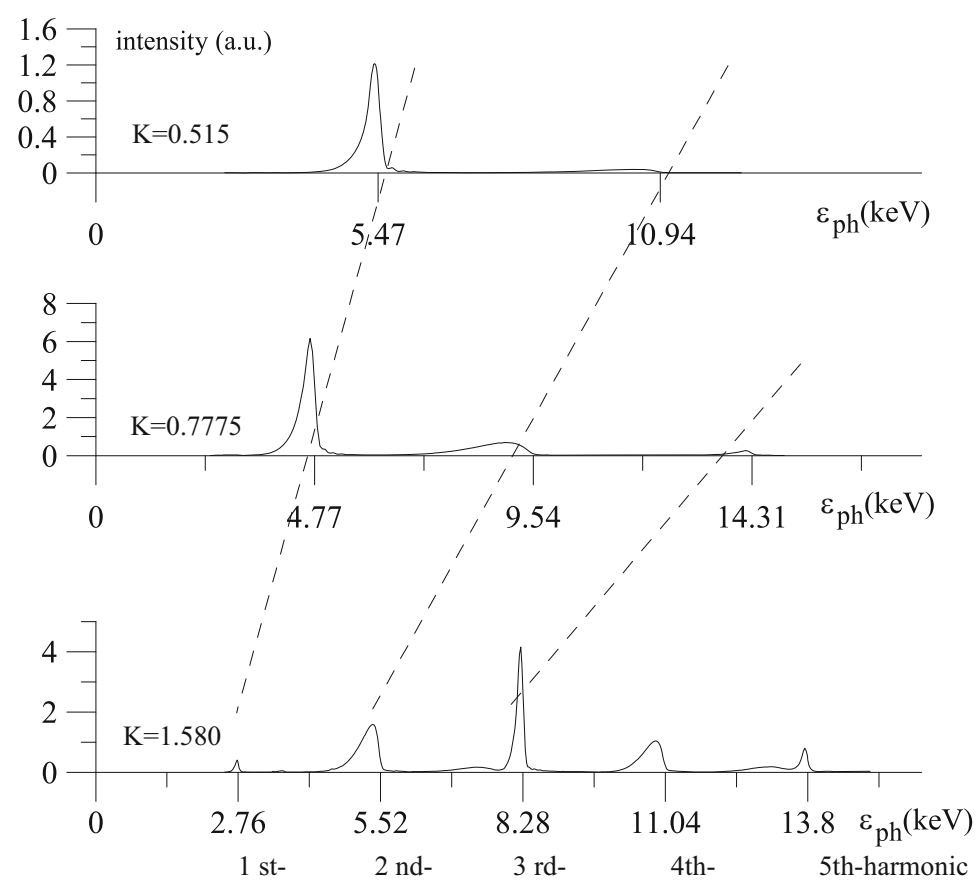

Fig. 24.10 Measured radiation spectrum from an undulator for different strength parameters $K$. The intensity at low photon energies are reduced by absorption in a Be-window [12]

\section{Back Scattered Photons}

The principle of Thomson backscattering or Compton scattering of the static undulator fields can be expanded to that of photon beams colliding head on with the particle beam. In the electron system of reference the electromagnetic field of this photon beam looks fundamentally no different than the electromagnetic field from the undulator magnet. We may therefore apply similar arguments to determine the wavelength of back scattered photons. The basic difference of both effects is that in the case of back scattered photons the photon beam moves with the velocity of light towards the electron beam and therefore the electron sees twice the Lorentz contracted photon frequency and we expect therefore a back scattered photon beam at twice the Doppler shifted frequency. That extra factor of two does not apply for undulator radiation since the undulator field is static and the relative velocity with respect to the electron beam is $c$. If $\lambda_{\mathrm{L}}$ is the wavelength of the incident radiation or incident laser, the wavelength of the backscattered photons is

$$
\lambda_{\gamma}=\frac{\lambda_{\mathrm{L}}}{4 \gamma^{2}}\left(1+\gamma^{2} \vartheta_{\mathrm{obs}}^{2}\right),
$$


where $\vartheta_{\text {obs }}$ is the angle between the direction of observation and the particle beam axis. Scattering, for example, a high intensity laser beam from high-energy electrons produces a monochromatic beam of hard x-rays which is highly collimated within an angle of $\pm 1 / \gamma$. If the laser wavelength is, for example, $\lambda_{\mathrm{L}}=10 \mu \mathrm{m}$ and the particle energy is $100 \mathrm{MeV}$ the wavelength of the backscattered $\mathrm{x}$-rays would be $1.3 \AA$ or the photon energy would be $9.5 \mathrm{keV}$ which is well within the hard $\mathrm{x}$-ray regime.

\section{Photon Flux}

The intensity of the backscattered photons can be calculated in a simple way utilizing the Thomson scattering cross section [10]

$$
\sigma_{\mathrm{Th}}=\frac{8 \pi}{3} r_{\mathrm{c}}^{2}=6.65 \times 10^{-25} \mathrm{~cm}^{2} \text {. }
$$

The total scattering event rate or the number of back scattered photons per unit time is then

$$
N_{\mathrm{sc}}=\sigma_{\mathrm{Th}} \mathcal{L},
$$

where $\mathcal{L}$ is called the luminosity. The value of the luminosity is independent of the nature of the physical reaction and depends only on the intensities and geometrical dimensions of the colliding beams. The definition of the luminosity is the product of the target density of one beam by the "particle"-flux of the other beam onto this target. Therefore the luminosity can be determined by folding the particle density in one beam with the incident "particles" per unit time of the other beam. Obviously, only those parts of the beam cross sections count which overlap with the cross section of the other beam. For simplicity, we assume a Gaussian distribution in both beams and assume that both beam cross sections are the same. In a real setup one would focus the electron beam and the photon beam to the same optimum cross section given by the Rayleigh length (27.59). We further consider the particle beam as the target for the photon beam.

With $N_{\mathrm{e}}$ electrons in each bunch of the particle beam within a cross section of $2 \pi \sigma_{x} \sigma_{y}$ the particle density is $N_{\mathrm{e}} / 2 \pi \sigma_{x} \sigma_{y}$. We consider now a photon beam with the same time structure as the electron beam. If this is not the case only that part of the photon beam which actually collides with the particle beam within the collision zone may be considered. For an effective photon flux $\dot{N}_{\text {ph }}$ the luminosity is

$$
\mathcal{L}=\frac{N_{\mathrm{e}} \dot{N}_{\mathrm{ph}}}{2 \pi \sigma_{x} \sigma_{y}} .
$$

Although the Thomson cross-section and therefore the photon yield is very small, this technique can be used to produce photon beams with very specific 
characteristics. By analyzing the scattering distribution this procedure can also be used to determine the degree of polarization of an electron beam in a storage ring.

So far, it was assumed that the incident and scattered photon energies are much smaller than the particle energy in which case it was appropriate to use the classical case of Thomson scattering. However, we note from (24.18) that the backscattered photon energy increases quadratically with the particle energy and therefore at some energy the photon energy becomes larger than the particle energy which is nonphysical. In case of large photon energies comparable with the particle energy, Compton corrections [13-15] must be included. The Compton cross-section for head-on collision is given by [16]

$$
\sigma_{\mathrm{C}}=\frac{3 \sigma_{\mathrm{Th}}}{4 x}\left[\left(1-\frac{4}{x}-\frac{8}{x^{2}}\right) \ln (1+x)+\frac{1}{2}+\frac{8}{x^{2}}-\frac{1}{2(1+x)^{2}}\right] \text {, }
$$

where $x=\frac{4 \gamma \hbar \omega_{0}}{m c^{2}}$, and $\hbar \omega_{0}$ the incident photon energy. The energy spectrum of the scattered photons is then [16]

$$
\frac{\mathrm{d} \sigma_{\mathrm{C}}}{\mathrm{d} y}=\frac{3 \sigma_{\mathrm{Th}}}{4 x}\left[1-y+\frac{1}{1-y}-\frac{4 y}{x(1+y)}+\frac{4 y^{2}}{x^{2}(1-y)^{2}}\right],
$$

where $y=\hbar \omega / E$ is the scattered photon energy in units of the particle energy.

\subsection{Radiation Power}

Synchrotron radiation properties can be described in more detail by integrating the Poynting vector (23.7) over a closed surface enclosing the radiating charge. With (23.9) and $\mathbf{n}^{*} \mathrm{~d} \mathbf{A}^{*}=R^{2} \sin \Theta^{*} \mathrm{~d} \Theta^{*} \mathrm{~d} \Phi^{*}$ we get the total radiation power from a single electron in its own rest frame

$$
P^{*}=\int S^{*} \mathrm{~d} A^{*}=\frac{2}{3} r_{\mathrm{c}} \frac{m c^{2}}{c^{3}} a^{* 2},
$$

where we have set $q^{2}=4 \pi \epsilon_{0} r_{\mathrm{c}} m c^{2}$. From the discussion of 4-vectors, we know that the square of the 4-acceleration is invariant to Lorentz transformations and get from (B.21) for the total radiation power in the laboratory system

$$
P=\frac{2}{3} r_{\mathrm{c}} m c \gamma^{6}\left[\dot{\boldsymbol{\beta}}^{2}-(\boldsymbol{\beta} \times \dot{\boldsymbol{\beta}})^{2}\right] \text {. }
$$

Equation (24.25) expresses the radiation power in a simple way and allows us to calculate other radiation characteristics based on beam parameters in the laboratory system. The radiation power is greatly determined by the geometric path of the 
particle trajectory through the quantities $\boldsymbol{\beta}$ and $\dot{\boldsymbol{\beta}}$. Specifically, if this path has strong oscillatory components we expect that motion to be reflected in the synchrotron radiation power spectrum. This aspect will be discussed later in more detail. Here we distinguish only between acceleration parallel $\dot{\boldsymbol{\beta}}_{\|}$or perpendicular $\dot{\boldsymbol{\beta}}_{\perp}$ to the propagation $\boldsymbol{\beta}$ of the charge and set therefore

$$
\dot{\boldsymbol{\beta}}=\dot{\boldsymbol{\beta}}_{\|}+\dot{\boldsymbol{\beta}}_{\perp}
$$

Insertion into (24.25) shows the total radiation power to be composed of separate contributions from parallel and orthogonal acceleration. Separating both contributions we get the synchrotron radiation power for both parallel and transverse acceleration respectively

$$
\begin{gathered}
P_{\|}=\frac{2}{3} r_{\mathrm{c}} m c \gamma^{6} \dot{\boldsymbol{\beta}}_{\|}^{2}, \\
P_{\perp}=\frac{2}{3} r_{\mathrm{c}} m c \gamma^{4} \dot{\boldsymbol{\beta}}_{\perp}^{2} .
\end{gathered}
$$

Expressions have been derived that define the radiation power for parallel acceleration like in a linear accelerator or orthogonal acceleration found in circular accelerators or deflecting systems. We note a similarity for both contributions except for the energy dependence. At highly relativistic energies the same acceleration force leads to much less radiation if the acceleration is parallel to the motion of the particle compared to orthogonal acceleration. Parallel acceleration is related to the accelerating force by $m \dot{\boldsymbol{v}}_{\|}=\frac{1}{\gamma^{3}} \mathrm{~d} \boldsymbol{p}_{\|} / \mathrm{d} t$ and after insertion into (24.27) the radiation power due to parallel acceleration becomes

$$
P_{\|}=\frac{2}{3} \frac{r_{\mathrm{c}}}{m c}\left(\frac{\mathrm{d} \boldsymbol{p}_{\|}}{\mathrm{d} t}\right)^{2}
$$

The radiation power for acceleration along the propagation of the charged particle is therefore independent of the energy of the particle and depends only on the accelerating force or with $\mathrm{d} \boldsymbol{p}_{\|} / \mathrm{d} t=\mathrm{d} \boldsymbol{E} / \mathrm{d} z$ on the energy increase per unit length of accelerator. Different from circular electron accelerators we encounter therefore no practical energy limit in a linear accelerator at very high energies. In contrast very different radiation characteristics exist for transverse acceleration as it happens, for example, during the transverse deflection of a charged particle in a magnetic field. The transverse acceleration $\dot{\boldsymbol{v}}_{\perp}$ is expressed by the Lorentz force

$$
\frac{\mathrm{d} \boldsymbol{p}_{\perp}}{\mathrm{d} t}=\gamma m \dot{\boldsymbol{v}}_{\perp}=e[\boldsymbol{v} \times \boldsymbol{B}]
$$


and after insertion into (24.28) the radiation power from transversely accelerated particles becomes

$$
P_{\perp}=\frac{2}{3} \frac{r_{\mathrm{c}}}{m c} \gamma^{2}\left(\frac{\mathrm{d} \boldsymbol{p}_{\perp}}{\mathrm{d} t}\right)^{2} .
$$

From (24.29), (24.31) we find that the same accelerating force leads to a much higher radiation power by a factor $\gamma^{2}$ for transverse acceleration compared to longitudinal acceleration. For all practical purposes, technical limitations prevent the occurrence of sufficient longitudinal acceleration to generate noticeable radiation. From here on we will stop considering longitudinal acceleration unless specifically mentioned and eliminate, therefore, the index $\perp$ setting for the radiation power $P_{\perp}=P_{\gamma}$. We also restrict from now on the discussion to singly charged particles and set $q=e$ ignoring extremely high energies, where multiple charged ions may start to radiate. Replacing the force in $(24.31)$ by the Lorentz force (24.30) we get

$$
P_{\gamma}=\frac{4 \pi}{\mu_{0}} \frac{2 r_{\mathrm{c}}^{2} c}{3\left(m c^{2}\right)^{2}} B^{2} E^{2}=C_{B} B^{2} E^{2},
$$

where $\beta \approx 1$ and

$$
C_{B}=\frac{4 \pi}{\mu_{0}} \frac{2 r_{\mathrm{c}}^{2} c}{3\left(m c^{2}\right)^{2}}=379.35 \frac{1}{\mathrm{~T}^{2} \mathrm{GeVs}} .
$$

The synchrotron radiation power scales like the square of the magnetic field and the square of the particle energy. Replacing the deflecting magnetic field $B$ by the bending radius $\rho$, the instantaneous synchrotron radiation power becomes

$$
P_{\gamma}=\frac{2}{3} r_{\mathrm{c}} m c^{3} \frac{\beta^{4} \gamma^{4}}{\rho^{2}}
$$

or in more practical units,

$$
P_{\gamma}=\frac{c C_{\gamma}}{2 \pi} \frac{E^{4}}{\rho^{2}}
$$

where

$$
C_{\gamma}=\frac{4 \pi}{3} \frac{r_{\mathrm{c}}}{\left(m c^{2}\right)^{3}}=8.8463 \times 10^{-5} \frac{\mathrm{m}}{\mathrm{GeV}^{3}}
$$

The electromagnetic radiation of charged particles in transverse magnetic fields is proportional to the fourth power of the particle momentum $\beta \gamma$ and inversely proportional to the square of the bending radius $\rho$. 
The synchrotron radiation power increases very fast for high-energy particles and provides the most severe limitation to the maximum energy achievable in circular accelerators. In storage rings with different magnets and including insertion devices it is important to formulate the average radiation power of an electron during the course of one turn. In this case we calculate the average

$$
\left\langle P_{\gamma}\right\rangle=\frac{c}{2 \pi} C_{\gamma} E^{4}\left\langle\frac{1}{\rho^{2}}\right\rangle=C_{\gamma} E^{4} \frac{f_{\mathrm{rev}}}{2 \pi} \oint \frac{\mathrm{d} z}{\rho^{2}} .
$$

We note, however, from also a strong dependence on the kind of particles involved in the process of radiation. Because of the much heavier mass of protons compared to the lighter electrons we find appreciable synchrotron radiation only in electron accelerators.

The radiation power of protons actually is smaller compared to that for electrons by the fourth power of the mass ratio or by the factor

$$
\frac{P_{\mathrm{e}}}{P_{\mathrm{p}}}=1836^{4}=1.1367 \times 10^{13} .
$$

In spite of this enormous difference measurable synchrotron radiation has been predicted by Coisson [17] and was indeed detected at the $400 \mathrm{GeV}$ proton synchrotron, SPS (Super Proton Synchrotron), at CERN in Geneva [18, 19]. Substantial synchrotron radiation is expected in multi-TeV proton colliders like the LHC (Large Hadron Collider) at CERN [20].

Knowledge of the synchrotron radiation power allows us now to calculate the energy loss per turn of a particle in a circular accelerator by integrating the radiation power along the circumference of the circular accelerator

$$
U_{0}=\oint P_{\gamma} \mathrm{d} t=\frac{2}{3} r_{\mathrm{c}} m c^{2} \beta^{3} \gamma^{4} \oint \frac{\mathrm{d} z}{\rho^{2}} .
$$

In an isomagnetic lattice, where the bending radius is the same for all bending magnets $\rho=$ const., the integration around a circular accelerator can be performed and the energy loss per turn due to synchrotron radiation is

$$
U_{0}=P_{\gamma} \frac{2 \pi \rho}{\beta c}=\frac{4 \pi}{3} r_{\mathrm{c}} m c^{2} \beta^{3} \frac{\gamma^{4}}{\rho} .
$$

In more practical units, the energy loss of relativistic electrons per revolution in a circular accelerator with an isomagnetic lattice and a bending radius $\rho$ is given by

$$
U_{0, \text { iso }}(\mathrm{GeV})=C_{\gamma} \frac{E^{4}\left(\mathrm{GeV}^{4}\right)}{\rho(m)} .
$$


For a beam of $N_{\mathrm{e}}$ particles or a circulating beam current $I=e f_{\mathrm{rev}} N_{\mathrm{e}}$ the total average radiation power is

$$
\left\langle P_{\mathrm{s}}\right\rangle=U_{0} \frac{I}{e}
$$

or in more practical units

$$
\left\langle P_{\mathrm{s}}(\mathrm{MW})\right\rangle_{\text {iso }}=0.088463 \frac{E^{4}(\mathrm{GeV})}{\rho(\mathrm{m})} I(\mathrm{~A}) .
$$

The total synchrotron radiation power scales like the fourth power of the particle energy and is inversely proportional to the bending radius. The strong dependence of the radiation on the particle energy causes severe practical limitations on the maximum achievable energy in a circular accelerator.

\subsection{Spectrum}

Synchrotron radiation from relativistic charged particles is emitted over a wide spectrum of photon energies. The basic characteristics of this spectrum can be derived from simple principles as suggested in [21]. For an observer synchrotron light has the appearance similar to the light coming from a lighthouse. Although the light is emitted continuously an observer sees only a periodic flash of light as the aperture mechanism rotates in the lighthouse. Similarly, synchrotron light emitted from relativistic particles will appear to an observer as a single flash if it comes from a bending magnet in a transport line passed through by a particle only once or as a series of equidistant light flashes as bunches of particles orbit in a circular accelerator.

Since the duration of the light flashes is very short the observer notes a broad spectrum of frequencies as his eyes or instruments Fourier analyze the pulse of electromagnetic energy. The spectrum of synchrotron light from a circular accelerator is composed of a large number of harmonics of the particle revolution frequency. These harmonics reach a cutoff, where the period of the radiation becomes comparable to the duration of the light pulse. Even though the aperture of the observers eyes or instruments are assumed to be infinitely narrow we still note a finite duration of the light flash. This is a consequence of the finite opening angle of the radiation as illustrated in Fig. 24.11. Synchrotron light emitted by a particle travelling along the orbit cannot reach the observer before it has reached the point $P_{0}$ when those photons emitted on one edge of the radiation cone at an angle $-1 / \gamma$ aim directly toward the observer. Similarly, the last photons to reach the observer are emitted from point $P_{1}$ at an angle of $+1 / \gamma$. Between point $P_{0}$ and point $P_{1}$ we have therefore a deflection angle of $2 / \gamma$. The duration of the light flash for the observer is not the time it takes the particle to travel from point $P_{0}$ to point 


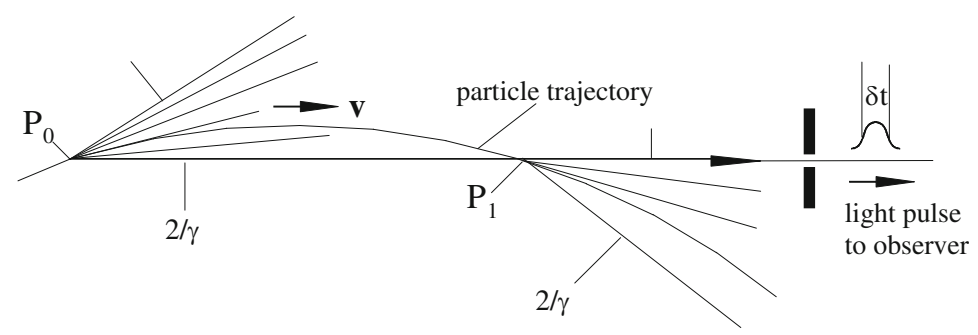

Fig. 24.11 Temporal pulse formation of synchrotron radiation

$P_{1}$ but must be corrected for the finite time of flight for the photon emitted at $P_{0}$. If particle and photon would travel toward the observer with exactly the same velocity the light pulse would be infinitely short. However, particles move slower following a slight detour and therefore the duration of the light pulse equals the time difference between the first photons from point $P_{0}$ arriving at the observer and the last photons being emitted by the particles at point $P_{1}$. Although the particle reaches point $P_{0}$ at time $t=0$ the first photon can be observed at point $P_{1}$ only after a time

$$
t_{\gamma}=\frac{2 \rho \sin \frac{1}{\gamma}}{c} .
$$

The last photon to reach the observer is emitted when the particle arrives at point $P_{1}$ at the time

$$
t_{\mathrm{e}}=\frac{2 \rho}{\beta c \gamma} .
$$

The duration of the light pulse $\delta t$ is therefore given by the difference of both travel times (24.44), (24.45)

$$
\delta t=t_{\mathrm{e}}-t_{\gamma}=\frac{2 \rho}{\beta c \gamma}-\frac{2 \rho \sin \frac{1}{\gamma}}{c} .
$$

The sine-function can be expanded for small angles keeping linear and third order terms only and the duration of the light pulse at the location of the observer is after some manipulation

$$
\delta t=\frac{4 \rho}{3 c \gamma^{3}} .
$$

The total duration of the electromagnetic pulse is very short scaling inversely proportional to the third power of $\gamma$. This short pulse translates into a broad spectrum. Using only half the pulse length for the effective pulse duration the 
spectrum reaches up to a maximum frequency of about

$$
\omega_{\mathrm{c}} \approx \frac{1}{\frac{1}{2} \delta t} \approx \frac{3}{2} c \frac{\gamma^{3}}{\rho}
$$

which is called the critical photon frequency of synchrotron radiation. The critical photon energy $\varepsilon_{\mathrm{c}}=\hbar \omega_{\mathrm{c}}$ is then given by

$$
\varepsilon_{\mathrm{c}}=C_{\mathrm{c}} \frac{E^{3}}{\rho},
$$

with

$$
C_{\mathrm{c}}=\frac{3 \hbar c}{2\left(m c^{2}\right)^{3}}
$$

For electrons numerical expressions are

$$
\varepsilon_{\mathrm{c}}(\mathrm{keV})=2.2183 \frac{E^{3}\left(\mathrm{GeV}^{3}\right)}{\rho(\mathrm{m})}=0.66503 E^{2}\left(\mathrm{GeV}^{2}\right) B(\mathrm{~T}) .
$$

The synchrotron radiation spectrum from relativistic particles in a circular accelerator is made up of harmonics of the particle revolution frequency $\omega_{0}$ with values up to and beyond the critical frequency (24.51). Generally, a real synchrotron radiation beam from say a storage ring will not display this harmonic structure. The distance between harmonics is extremely small compared to the extracted photon frequencies in the VUV and x-ray regime while the line width is finite due to the energy spread and beam emittance.

For a single pass of particles through a bending magnet in a beam transport line we observe the same spectrum. Specifically, the maximum frequency is the same assuming similar parameters. Synchrotron radiation is emitted in a particular spatial and spectral distribution, both of which will be derived in Chap. 25, and we will use here only some of these results. A useful parameter to characterize the photon intensity is the photon flux per unit solid angle into a frequency bin $\Delta \omega / \omega$ and from a circulating beam current $I$ defined by

$$
\frac{\mathrm{d}^{2} \dot{N}_{\mathrm{ph}}}{\mathrm{d} \theta \mathrm{d} \psi}=C_{\Omega} E^{2} I \frac{\Delta \omega}{\omega}\left(\frac{\omega}{\omega_{\mathrm{c}}}\right)^{2} K_{2 / 3}^{2}(\xi) F(\xi, \theta),
$$

where $\psi$ is the angle in the deflecting plane and $\theta$ the angle normal to the deflecting plane,

$$
C_{\Omega}=\frac{3 \alpha}{4 \pi^{2} e\left(m c^{2}\right)^{2}}=1.3255 \times 10^{16} \frac{\text { photons }}{\mathrm{s} \mathrm{mrad}^{2} \mathrm{GeV}^{2} \mathrm{~A} 100 \% \mathrm{BW}},
$$




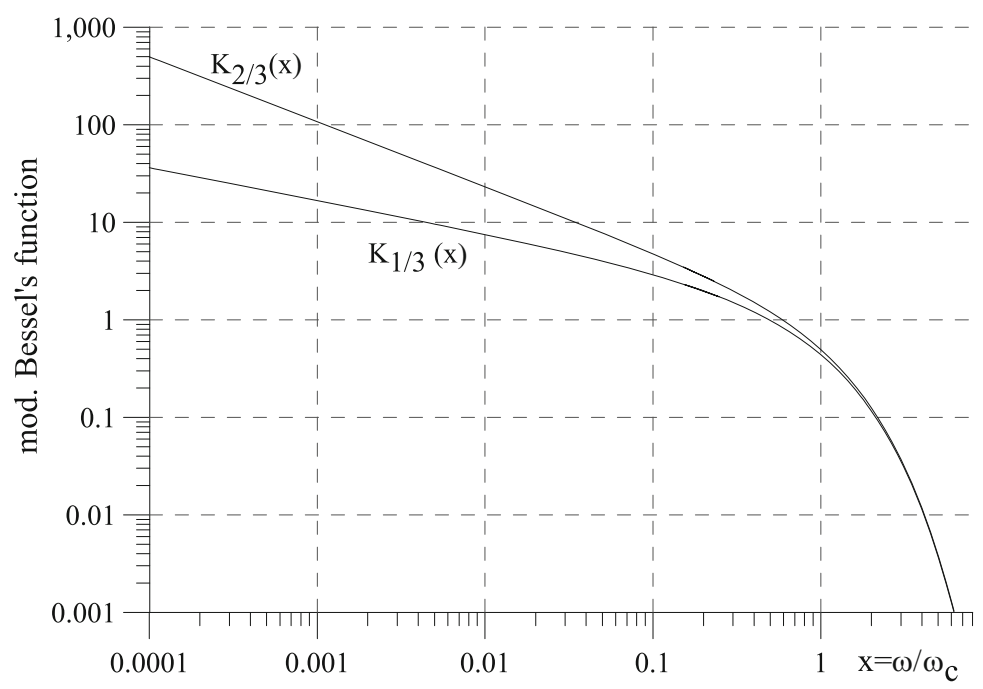

Fig. 24.12 Modified Bessel's functions $K_{1 / 3}(\xi)$ and $K_{2 / 3}(\xi)$

$\alpha$ the fine structure constant and

$$
F(\xi, \theta)=\left(1+\gamma^{2} \theta^{2}\right)^{2}\left(1+\frac{\gamma^{2} \theta^{2}}{1+\gamma^{2} \theta^{2}} \frac{K_{1 / 3}^{2}(\xi)}{K_{2 / 3}^{2}(\xi)}\right) .
$$

The functions $K_{1 / 3}(\xi)$ and $K_{2 / 3}(\xi)$, displayed in Fig. 24.12, are modified Bessel's functions with the argument

$$
\xi=\frac{1}{2} \frac{\omega}{\omega_{\mathrm{c}}}\left(1+\gamma^{2} \theta^{2}\right)^{3 / 2}
$$

Synchrotron radiation is highly polarized in the plane normal ( $\sigma$-mode), and parallel ( $\pi$-mode), to the deflecting magnetic field. The relative flux in both polarization directions is given by the two components in the second bracket of function $F(\xi, \theta)$ in (24.54). The first component is equal to unity and determines the photon flux for the polarization normal to the magnetic field or $\sigma$-mode, while the second term relates to the polarization parallel to the magnetic field which is also called the $\pi$ mode. Equation (24.52) expresses both the spectral and spatial photon flux for both the $\sigma$-mode radiation in the forward direction within an angle of about $\pm 1 / \gamma$ and for the $\pi$-mode off axis.

For highly relativistic particles the synchrotron radiation is collimated very much in the forward direction and we may assume that all radiation in the nondeflecting plane is accepted by the experimental beam line. In this case we are interested in the photon flux integrated over all angles $\theta$. This integration will be performed in 
Chap. 26 with the result (25.140)

$$
\frac{\mathrm{d} \dot{N}_{\mathrm{ph}}}{\mathrm{d} \psi}=\frac{4 \alpha}{9} \gamma \frac{I}{e} \frac{\Delta \omega}{\omega} S\left(\frac{\omega}{\omega_{\mathrm{c}}}\right),
$$

where $\psi$ is the deflection angle in the bending magnet, $\alpha$ the fine structure constant and the function $S(x)$ is defined by

$$
S\left(\frac{\omega}{\omega_{\mathrm{c}}}\right)=\frac{9 \sqrt{3}}{8 \pi} \frac{\omega}{\omega_{\mathrm{c}}} \int_{\omega / \omega_{\mathrm{c}}}^{\infty} K_{5 / 3}(\bar{x}) \mathrm{d} \bar{x}
$$

with $K_{5 / 3}(x)$ a modified Bessel's function. The function $S\left(\omega / \omega_{\mathrm{c}}\right)$ is known as the universal function of synchrotron radiation and is shown in Fig. 24.13. In practical units, the angle integrated photon flux is

$$
\frac{\mathrm{d} \dot{N}_{\mathrm{ph}}}{\mathrm{d} \psi}=C_{\psi} E I \frac{\Delta \omega}{\omega} S\left(\frac{\omega}{\omega_{\mathrm{c}}}\right)
$$

with $C_{\psi}$ defined by

$$
C_{\psi}=\frac{4 \alpha}{9 e m c^{2}}=3.9614 \times 10^{19} \frac{\text { photons }}{\mathrm{s} \mathrm{rad} \mathrm{A} \mathrm{GeV}} .
$$

The spectral distribution depends only on the particle energy, the critical frequency $\omega_{\mathrm{c}}$ and a purely mathematical function. This result has been derived originally by Ivanenko and Sokolov [22] and independently by Schwinger [23]. Specifically it should be noted that the spectral distribution, if normalized to the critical frequency, does not depend on the particle energy and can therefore be represented by a universal distribution shown in Fig. 24.13.

The energy dependence is contained in the cubic dependence of the critical frequency acting as a scaling factor for the actual spectral distribution. The synchrotron radiation spectrum in Fig. 24.13 is rather uniform up to the critical frequency beyond which the intensity falls off rapidly. This synchrotron radiation spectrum has been verified experimentally soon after such radiation sources became available [24, 25].

Equation (24.56) is not well suited for quick calculation of the radiation intensity at a particular frequency. We may, however, express (24.56) in much simpler form for very low and very large frequencies making use of limiting expressions of Bessel's functions for large and small arguments. For small arguments $x=\frac{\omega}{\omega_{\mathrm{c}}} \ll 1$ an asymptotic approximation [26] for the modified Bessel's function may be used to give instead of (24.58) with $\mathrm{AS}(9.6 .9)$ [26]

$$
\frac{\mathrm{d} \dot{N}_{\mathrm{ph}}}{\mathrm{d} \psi} \approx C_{\psi} E I \frac{\Delta \omega}{\omega} 1.333\left(\frac{\omega}{\omega_{\mathrm{c}}}\right)^{1 / 3} .
$$




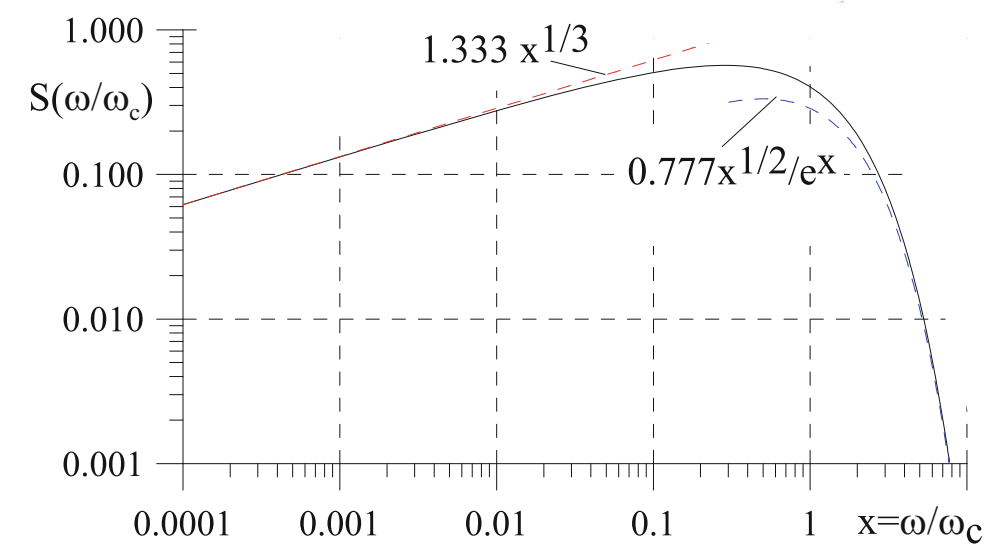

Fig. 24.13 Universal function of the synchrotron radiation spectrum, $S\left(\omega / \omega_{c}\right)$

Similarly, for high photon frequencies $x=\frac{\omega}{\omega_{\mathrm{c}}} \gg 1$ we get with $\operatorname{AS}(9.7 .2)$ [26]

$$
\frac{\mathrm{d} \dot{N}_{\mathrm{ph}}}{\mathrm{d} \psi} \approx C_{\psi} E I \frac{\Delta \omega}{\omega} 0.8460 \frac{\sqrt{x}}{e^{x}},
$$

where $x=\frac{\omega}{\omega_{\mathrm{c}}}$. Both approximations are included in Fig. 24.13 and display actually a rather good representation of the real spectral radiation distribution over all but the central portion of the spectrum where $S(x) \approx 0.4$. Specifically, we note the slow increase in the radiation intensity at low frequencies and the exponential drop off above the critical frequency.

\subsection{Spatial Photon Distribution}

The expressions for the photon fluxes (24.52), (24.56) provide the opportunity to calculate the spectral distribution of the photon beam divergence. Photons are emitted into a narrow angle and we may represent this narrow angular distribution by a Gaussian distribution. The effective width of a Gaussian distribution is $\sqrt{2 \pi} \sigma_{\theta}$ and we set

$$
\frac{\mathrm{d} \dot{N}_{\mathrm{ph}}}{\mathrm{d} \psi} \approx \frac{\mathrm{d}^{2} \dot{N}_{\mathrm{ph}}}{\mathrm{d} \theta \mathrm{d} \psi} \sqrt{2 \pi} \sigma_{\theta} .
$$




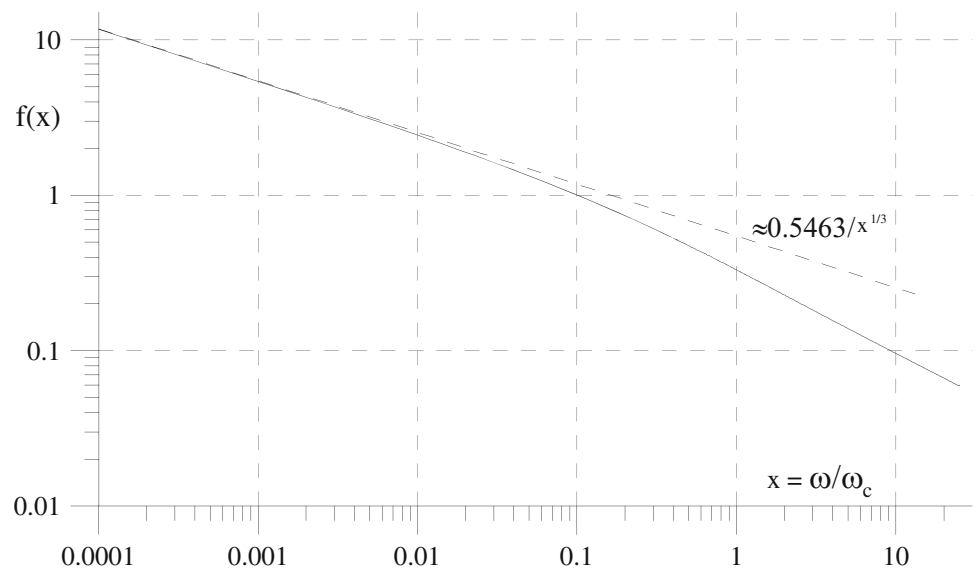

Fig. 24.14 Scaling function $f(x)=\sigma_{\theta}(\mathrm{mrad}) E(\mathrm{GeV})$ for the photon beam divergence in (24.63)

With (24.52), (24.58) the angular divergence of the forward lobe of the photon beam or for a beam polarized in the $\sigma$-mode is

$$
\sigma_{\theta}(\operatorname{mrad})=\frac{C_{\psi}}{\sqrt{2 \pi} C_{\Omega}} \frac{1}{E} \frac{S(x)}{x^{2} K_{2 / 3}^{2}\left(\frac{1}{2} x\right)}=\frac{f(x)}{E(\mathrm{GeV})},
$$

where $x=\omega / \omega_{\mathrm{c}}$. For the forward direction $\theta \approx 0$ the function $f(x)=$ $\sigma_{\theta}(\mathrm{mrad}) E(\mathrm{GeV})$ is shown in Fig. 24.14 for easy numerical calculations.

For wavelengths $\omega \ll \omega_{\mathrm{c}}$, (24.63) can be greatly simplified to become in more practical units

$$
\sigma_{\theta}(\mathrm{mrad}) \approx \frac{0.54626}{E(\mathrm{GeV})}\left(\frac{\omega}{\omega_{\mathrm{c}}}\right)^{1 / 3}=\frac{7.124}{\left[\rho(\mathrm{m}) \epsilon_{\mathrm{ph}}(\mathrm{eV})\right]^{1 / 3}}
$$

where $\rho$ is the bending radius and $\epsilon_{\mathrm{ph}}$ the photon energy. The photon beam divergence for low photon energies compared to the critical photon energy is independent of the particle energy and scales inversely proportional to the third root of the bending radius and photon energy.

\subsection{Fraunhofer Diffraction}

Synchrotron radiation is emitted from a rather small area equal to the cross section of the electron beam. In the extreme and depending on the photon wavelength the radiation may be spatially coherent because the beam cross section in phase space is smaller than the wavelength. This possibility to create spatially coherent radiation 


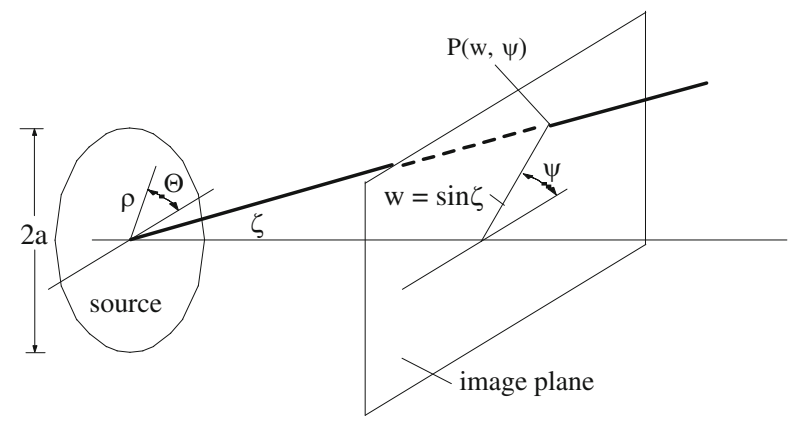

Fig. 24.15 Diffraction geometry

is important for many experiments specifically for holography and we will discuss in more detail the conditions for the particle beam to emit such radiation.

Reducing the particle beam cross section in phase space by diminishing the particle beam emittance reduces also the source size of the photon beam. This process of reducing the beam emittance is, however, effective only to some point. Further reduction of the particle beam emittance would have no effect on the photon beam emittance because of diffraction effects. A point like photon source appears in an optical instrument as a disk with concentric illuminated rings. For synchrotron radiation sources it is of great interest to maximize the photon beam brightness which is the photon density in phase space. On the other hand designing a lattice for a very small beam emittance can cause beam stability problems. It is therefore prudent not to push the particle beam emittance to values much less than the diffraction limited photon beam emittance. In the following we will therefore define diffraction limited photon beam emittance as a guide for low emittance lattice design.

For highly collimated synchrotron radiation it is appropriate to assume Fraunhofer diffraction. Radiation from an extended light source appears diffracted in the image plane with a radiation pattern which is characteristic for the particular source size and radiation distribution as well as for the geometry of the apertures involved. For simplicity, we will use the case of a round aperture being the boundaries of the beam itself although in most cases the beam cross section is more elliptical. In spite of this simplification, however, we will obtain all basic physical properties of diffraction which are of interest to us. We consider a circular light source with diameter $2 a$. The radiation field at point $P$ in the image plane is then determined by the Fraunhofer diffraction integral [27]

$$
U(P)=C \int_{0}^{a} \int_{0}^{2 \pi} \mathrm{e}^{-\mathrm{i} k \rho w \cos (\Theta-\psi)} \mathrm{d} \Theta \rho \mathrm{d} \rho,
$$

where $k$ is the wave number of the radiation and $w$ is the sine of the angle between the light ray and the optical axis as shown in Fig. 24.15. 


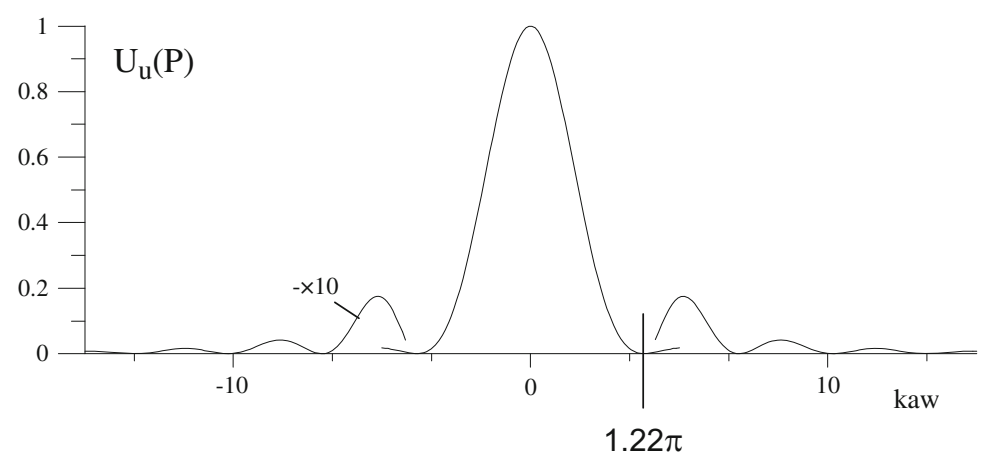

Fig. 24.16 Fraunhofer diffraction for a circular uniform light source

With $\alpha=\Theta-\psi$ and the definition of the lowest order Bessel's function $J_{0}(x)=$ $\frac{1}{2 \pi} \int_{0}^{2 \pi} \mathrm{e}^{-\mathrm{i} x \cos \alpha} \mathrm{d} \alpha,(24.65)$ can be expressed by the integral

$$
U(P)=2 \pi C \int_{0}^{a} J_{0}(k \rho w) \rho \mathrm{d} \rho .
$$

This integral can be solved analytically as well with the identity $\int_{0}^{x} J_{0}(y) y \mathrm{~d} y=$ $x J_{1}(x)$. The radiation intensity is proportional to the square of the radiation field and we get finally for the radiation intensity in the image plane at the point $P$

$$
I(P)=I_{0} \frac{4 J_{1}^{2}(k a w)}{(k a w)^{2}},
$$

where $I(P)=|U(P)|^{2}$ and $I_{0}=I(w \rightarrow 0)$ is the radiation intensity at the image center. This result has been derived first by Airy [28]. The radiation intensity from a light source of small circular cross section is distributed in the image plane due to diffraction into a central circle and concentric rings illuminated as shown in Fig. 24.16.

Tacitly, we have assumed that the distribution of emission at the source is uniform which is generally not correct for a particle beam. A Gaussian distribution is more realistic resembling the distribution of independently radiating particles. We must be careful in the choice of the scaling parameter. The relevant quantity for the Fraunhofer integral is not the actual particle beam size at the source point but rather the apparent beam size and distribution. By folding the particle density distribution with the argument of the Fraunhofer diffraction integral we get the radiation field from a round, Gaussian particle beam,

$$
U_{\mathrm{G}}(P) \propto \int_{0}^{\infty} \exp \left(-\frac{\rho^{2}}{2 \sigma_{r}^{2}}\right) J_{0}(k \rho w) \rho \mathrm{d} \rho,
$$


Fig. 24.17 Fraunhofer diffraction for a Gaussian luminescence at the light source

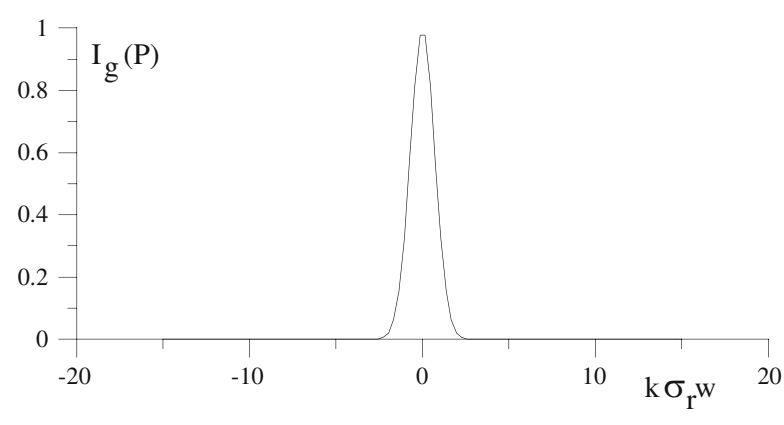

where $\sigma_{r}$ is the apparent standard source radius. Introducing the variable $x=$ $\rho / \sqrt{2} \sigma_{r}$ and replacing $k \rho w=\sqrt{2} x k \sigma_{r} w=2 x \sqrt{z}$ we get from (24.68)

$$
U_{\mathrm{G}}(P) \propto \int_{0}^{\infty} \mathrm{e}^{-x^{2}} x J_{0}(2 x \sqrt{z}) \mathrm{d} x
$$

and after integration

$$
U_{\mathrm{G}}(P) \propto \exp \left[-\frac{1}{2}\left(k \sigma_{r} w\right)^{2}\right]
$$

The diffraction pattern from a Gaussian light source (Fig. 24.17) does not exhibit the ring structure of a uniform source. The radiation field assumes rather the form of a Gaussian distribution in the emission angles $w$ with a standard width of $\sigma_{r^{\prime}}^{2}=\left\langle w^{2}\right\rangle$ or

$$
\sigma_{r^{\prime}}=\frac{1}{k \sigma_{r}}
$$

\subsection{Spatial Coherence}

Synchrotron radiation is emitted into a broad spectrum with the lowest frequency equal to the revolution frequency and the highest frequency not far above the critical photon energy. Detailed observation of the whole radiation spectrum, however, may reveal significant differences to these theoretical spectra at the low frequency end. At low photon frequencies we may observe an enhancement of the synchrotron radiation beyond intensities predicted by the theory of synchrotron radiation as discussed so far. We note from the definition of the Poynting vector that the radiation power is a quadratic effect with respect to the electric charge. For photon wavelengths equal and longer than the bunch length, we expect therefore all particles 
within a bunch to radiate coherently and the intensity to be proportional to the square of the number $N_{\mathrm{e}}$ of particles rather than linearly proportional as is the case for high frequencies. This quadratic effect can greatly enhance the radiation since the bunch population can be $10^{8}-10^{11}$ electrons.

Generally such radiation is not emitted from a storage ring beam because radiation with wavelengths longer than the vacuum chamber dimensions are shielded and will not propagate along a metallic beam pipe [29]. This radiation shielding is fortunate for storage ring operation since it eliminates an otherwise significant energy loss mechanism. Actually, since this shielding affects all radiation of sufficient wavelength both the ordinary synchrotron radiation and the coherent radiation is suppressed. New developments in storage ring physics, however, may make it possible to reduce the bunch length by as much as an order of magnitude below presently achieved short bunches of the order of 5-10 mm. Such bunches would then be much shorter than vacuum chamber dimensions and the emission of coherent radiation in some limited frequency range would be possible. Much shorter electron bunches down to a few fs can be produced in linear accelerators [30, 31], and specifically with bunch compression [32] a significant fraction of synchrotron radiation is emitted spontaneously as coherent radiation [33].

In this section we will discuss the physics of spontaneous coherent synchrotron radiation while distinguishing two kinds of coherence in synchrotron radiation, the temporal coherence and the spatial coherence. Temporal coherence occurs when all radiating electrons are located within a short bunch length of the order of the wavelength of the radiation. In this case the radiation from all electrons is emitted with about the same phase. For spatial coherence the electrons may be contained in a long bunch but the transverse beam emittance must be smaller than the radiation wavelength. In either case there is a smooth transition from incoherent radiation to coherent radiation as determined by a formfactor which depends on the bunch length or transverse emittance.

Similar to the particle beam characterization through its emittance we may do the same for the photon beam and doing so for the horizontal or vertical plane we have with $\sigma_{x, y}=\sigma_{r} / \sqrt{2}$ and $\sigma_{x^{\prime}, y^{\prime}}=\sigma_{r^{\prime}} / \sqrt{2}$ the photon beam emittance

$$
\epsilon_{\mathrm{ph}, x, y}=\frac{1}{2} \sigma_{r} \sigma_{r^{\prime}}=\frac{\lambda}{4 \pi}
$$

This is the diffraction limited photon emittance and reducing the electron beam emittance below this value would not lead to an additional reduction in the photon beam emittance. To produce a spatially coherent or diffraction limited radiation source the particle beam emittance must be less than the diffraction limited photon emittance

$$
\epsilon_{x, y} \leq \frac{\lambda}{4 \pi}
$$




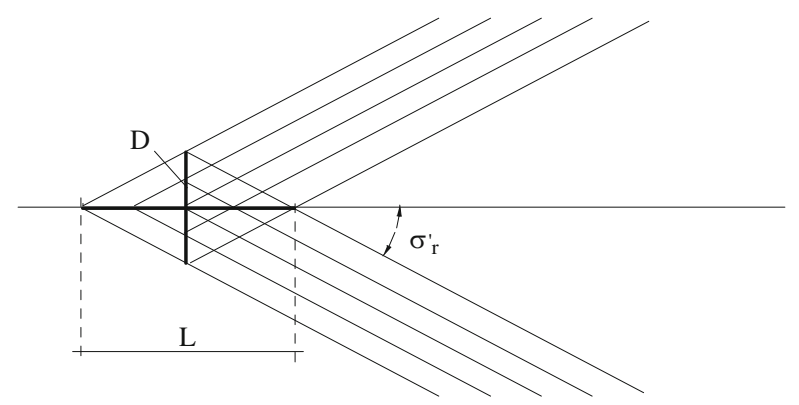

Fig. 24.18 Apparent photon source size

Obviously, this condition is easier to achieve for long wavelengths. For visible light, for example, the electron beam emittance must be smaller than about $5 \times$ $10^{-8}$ rad-m to be a spatially coherent radiation source. After having determined the diffraction limited photon emittance we may also determine the apparent photon beam size and divergence. The photon source extends over some finite length $L$ along the particle path which could be either the path length required for a deflection angle of $2 / \gamma$ or a much longer length in the case of an undulator to be discussed in the next chapter. With $\sigma_{r^{\prime}}$ the diffraction limited beam divergence the photons seem to come from a disc with diameter (Fig. 24.18)

$$
D=\sigma_{r^{\prime}} L
$$

On the other hand, we know from interference theory the correlation

$$
D \sin \sigma_{r^{\prime}} \approx D \sigma_{r^{\prime}}=\lambda
$$

and eliminating $D$ from both equations gives the diffraction limited photon beam divergence

$$
\sigma_{r^{\prime}}=\sqrt{\frac{\lambda}{L}}
$$

With this we get finally from (24.71) also the diffraction limited source size

$$
\sigma_{r}=\frac{1}{2 \pi} \sqrt{\lambda L}
$$

The apparent diffraction limited, radial photon beam size and divergence depend both on the photon wavelength of interest and the length of the source. 


\subsection{Temporal Coherence}

To discuss the appearance of temporal coherent synchrotron radiation, we consider the radiation emitted from each particle within a bunch. The radiation field at a frequency $\omega$ from a single electron is

$$
\mathcal{E}_{j} \propto \mathrm{e}^{\mathrm{i}\left(\omega t+\varphi_{j}\right)},
$$

where $\varphi_{j}$ describes the position of the jth electron with respect to the bunch center. With $z_{j}$ the distance from the bunch center, the phase is

$$
\varphi_{j}=\frac{2 \pi}{\lambda} z_{j} .
$$

Here we assume that the cross section of the particle beam is small compared to the distance to the observer such that the path length differences from any point of the beam cross section to observer are small compared to the shortest wavelength involved. The radiation power is proportional to the square of the radiation field and summing over all electrons we get

$$
\begin{aligned}
P(\omega) & \propto \sum_{j, l}^{N_{\mathrm{e}}} \mathcal{E}_{j} \mathcal{E}_{l}^{*} \propto \sum_{j, l}^{N_{\mathrm{e}}} \mathrm{e}^{\mathrm{i}\left(\omega t+\varphi_{j}\right)} \mathrm{e}^{-\mathrm{i}\left(\omega t+\varphi_{l}\right)} \\
& =\sum_{j, l}^{N_{\mathrm{e}}} \exp \mathrm{i}\left(\varphi_{j}-\varphi_{l}\right)=N_{\mathrm{e}}+\sum_{j \neq l}^{N_{\mathrm{e}}} \operatorname{expi}\left(\varphi_{j}-\varphi_{l}\right) .
\end{aligned}
$$

The first term $N_{\mathrm{e}}$ on the r.h.s. of (24.80) represents the ordinary incoherent synchrotron radiation with a power proportional to the number of radiating particles. The second term describes the coherent power averaging to zero for all but long wavelengths. The actual coherent radiation power spectrum depends on the particular particle distribution in the bunch. For a storage ring bunch it is safe to assume a Gaussian particle distribution and we use therefore the density distribution

$$
\Psi_{\mathrm{G}}(z)=\frac{N_{\mathrm{e}}}{\sqrt{2 \pi} \sigma} \exp \left(-\frac{z^{2}}{2 \sigma^{2}}\right),
$$

where $\sigma$ is the standard value of the Gaussian bunch length. Instead of summing over all electrons we integrate over all phases and folding the density distribution (24.81) with the radiation power (24.80) we get with (24.79)

$$
P(\omega) \propto N_{\mathrm{e}}+N_{\mathrm{e}} \frac{N_{\mathrm{e}}-1}{2 \pi \sigma^{2}} I_{1} I_{2},
$$


where the integrals $I_{1}$ and $I_{2}$ are defined by

$$
\begin{aligned}
& I_{1}=\int_{-\infty}^{+\infty} \exp \left(-\frac{z^{2}}{2 \sigma^{2}}+\mathrm{i} 2 \pi \frac{z}{\lambda}\right) \mathrm{d} z, \\
& I_{2}=\int_{-\infty}^{+\infty} \exp \left(-\frac{w^{2}}{2 \sigma^{2}}+\mathrm{i} 2 \pi \frac{w}{\lambda}\right) \mathrm{d} w,
\end{aligned}
$$

and $z=\frac{1}{2} \pi \lambda \varphi_{j}$ and $w=\frac{1}{2} \pi \lambda \varphi_{l}$. The factor $N_{\mathrm{e}}-1$ reflects the fact that we integrate only over different particles. Both integrals are equal to the Fourier transform for a Gaussian particle distribution. With

$$
\int_{-\infty}^{+\infty} \exp \left(-\frac{z^{2}}{2 \sigma^{2}}+\mathrm{i} 2 \pi \frac{z}{\lambda}\right) \mathrm{d} z=\sqrt{2 \pi} \sigma \exp \left[-2 \pi^{2}\left(\frac{\sigma}{\lambda}\right)^{2}\right]
$$

we get from (24.82) for the total radiation power at the frequency $\omega=2 \pi c / \lambda$

$$
P(\omega)=p(\omega) N_{\mathrm{e}}\left[1+\left(N_{\mathrm{e}}-1\right) g^{2}(\sigma, \lambda)\right],
$$

where $p(\omega)$ is the radiation power from one electron and the Fourier transform

$$
g^{2}(\sigma, \lambda)=\exp \left[-2 \pi^{2}\left(\frac{\sigma}{\lambda}\right)^{2}\right]
$$

is called the formfactor. With the effective bunch length

$$
\ell=\sqrt{2 \pi} \sigma
$$

this formfactor becomes finally

$$
g^{2}(\ell, \lambda)=\exp \left[-\pi \frac{\ell^{2}}{\lambda^{2}}\right]
$$

The coherent radiation power falls off rapidly for wavelengths as short or even shorter than the effective bunch length $\ell$. In Fig. 24.19 the relative coherent radiation power is shown as a function of the effective bunch length in units of the radiation wavelength. The fast drop off is evident and for an effective bunch length of about $\ell \approx 0.6 \lambda$ the radiation power is reduced to only about $10 \%$ of the maximum power for very short bunches. Particle beams from a linear accelerator have often a more compressed particle distribution of a form between a Gaussian and a rectangular distribution. If we take the extreme of a rectangular distribution

$$
\Psi_{\mathrm{r}}(z)= \begin{cases}1 & \text { for }-\frac{1}{2} \ell<z<\frac{1}{2} \ell \\ 0 & \text { otherwise }\end{cases}
$$




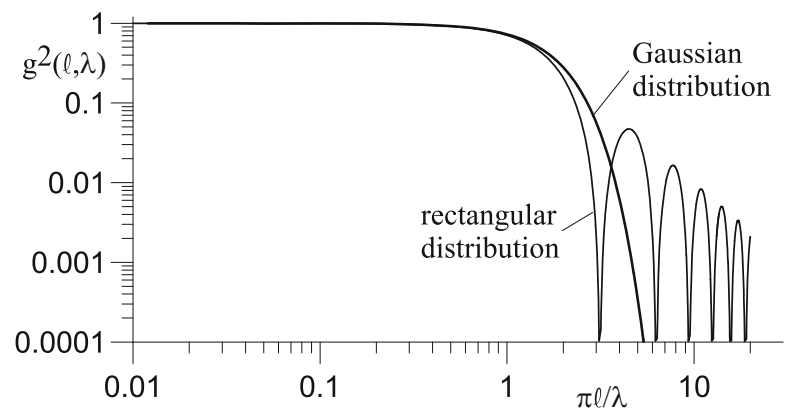

Fig. 24.19 Formfactor $g^{2}(\ell, \lambda)$ for a Gaussian and rectangular particle distribution

we expect to extend the radiation spectrum since the corners and sharp changes of the particle density require a broader spectrum in the Fourier transform. Following the procedure for the Gaussian beam we get for a rectangular particle distribution the Fourier transform

$$
g(\ell)=\frac{\sin x}{x}
$$

where $x=\pi \ell / \lambda$. Figure 24.19 also shows the relative coherent radiation power for this distribution and we note a significant but scalloping extension to higher radiation frequencies. Experiments have been performed with picosecond electron bunches from linear accelerators both at Tohoku University [30] and at Cornell University [31] which confirm the appearance of this coherent part of synchrotron radiation.

\subsection{Spectral Brightness}

The optical quality of a photon beam is characterized by the spectral brightness defined as the six-dimensional volume occupied by the photon beam in phase space

$$
\mathcal{B}=\frac{\dot{N}_{\mathrm{ph}}}{4 \pi^{2} \sigma_{x} \sigma_{x^{\prime}} \sigma_{y} \sigma_{y^{\prime}} \frac{\mathrm{d} \omega}{\omega}},
$$

where $\dot{N}_{\text {ph }}$ is the photon flux defined in (24.58). In the laser community, this quantity is called the radiance while the term spectral brightness is common in the synchrotron radiation community. ${ }^{2}$

\footnotetext{
${ }^{2}$ Sometimes the term brilliance is used. Since there is no common definition for brilliance and the dictionary does not connect brilliance with say luminescence of a source we do not use this term in this book.
} 
For bending magnet radiation there is a uniform angular distribution in the deflecting plane and we must therefore replace the Gaussian divergence $\sigma_{x^{\prime}}$ by the total acceptance angle $\Delta \psi$ of the photon beam line or experiment. The particle beam emittance must be minimized to achieve maximum spectral photon beam brightness. However, unlimited reduction of the particle beam emittance will, at some point, seize to further increase the brightness. Because of diffraction effects the electron beam emittance need not be reduced significantly below the limit (24.72) discussed in the previous section.

For a negligible particle beam emittance the maximum diffraction limited spectral brightness is from (24.72), (24.91)

$$
\mathcal{B}_{\max }=\frac{4}{\lambda^{2} \frac{\mathrm{d} \omega}{\omega}} \dot{N}_{\mathrm{ph}} .
$$

For a realistic synchrotron light source the finite beam emittance of the particle beam must be taken into account as well which is often even the dominant emittance being larger than the diffraction limited photon beam emittance. We may add both contributions in quadrature and have for the total source parameters

$$
\begin{aligned}
\sigma_{\mathrm{tot}, x} & =\sqrt{\sigma_{\mathrm{b}, x}^{2}+\frac{1}{2} \sigma_{r}^{2}}, & \sigma_{\mathrm{tot}, x^{\prime}} & =\sqrt{\sigma_{\mathrm{b}, x^{\prime}}^{2}+\frac{1}{2} \sigma_{r^{\prime}}^{2}}, \\
\sigma_{\mathrm{tot}, y} & =\sqrt{\sigma_{\mathrm{b}, y}^{2}+\frac{1}{2} \sigma_{r}^{2}}, & \sigma_{\mathrm{tot}, y^{\prime}} & =\sqrt{\sigma_{\mathrm{b}, y^{\prime}}^{2}+\frac{1}{2} \sigma_{r^{\prime}}^{2}},
\end{aligned}
$$

where $\sigma_{\mathrm{b}}$ refers to the respective particle beam parameters.

\subsubsection{Matching}

A finite particle beam emittance does reduce the photon beam brightness from it's ideal maximum. The amount of reduction, however, depends on the matching to the photon beam. The photon beam size and divergence are the result of folding the diffraction limited source emittance with the electron beam size and divergence. In cases where the electron beam emittance becomes comparable to the diffraction limited emittance the effective photon beam brightness can be greatly affected by the mutual orientation of both emittances. Matching both orientations will maximize the photon beam brightness. 


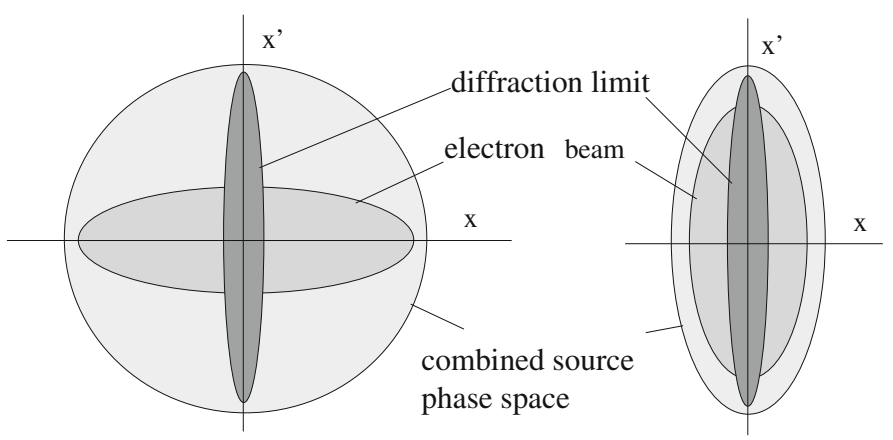

Fig. 24.20 Matching of the electron beam emittance to the diffraction limited emittance to gain maximum photon beam brightness

This matching process is demonstrated in Fig. 24.20. The left side shows a situation of poor matching in 2-dimensional $x-x^{\prime}$-phase space. In this case the electron beam width is much larger than the diffraction limited source size while its divergence is small compared to the diffraction limit. The effective photon beam distribution in phase space is the folding of both electron beam parameters and diffraction limit and is much larger than either one of its components. The photon beam width is dominated by the electron beam width and the photon beam divergence is dominated by the diffraction limit. Consequently, the effective photon density in phase space and photon beam brightness is reduced.

To improve the situation one would focus the electron beam to a smaller beam size at the source point at the expense of beam divergence. The reduction of the electron beam width increases directly the photon beam brightness while the related increase of the electron beam divergence is ineffective because the diffraction limit is the dominant term. Applying more focusing may give a situation shown on the right side of Fig. 24.20 where the folded photon phase space distribution is reduced and the brightness correspondingly increased. Of course, if the electron beam is focused too much we have the opposite situation as discussed. There is an optimum focusing for optimum matching. To find this optimum we use the particle beam parameters

$$
\sigma_{\mathrm{b}, x, y}^{2}=\epsilon_{x, y} \beta_{x, y} \quad \text { and } \quad \sigma_{\mathrm{b}, x^{\prime}, y^{\prime}}^{2}=\frac{\epsilon_{x, y}}{\beta_{x, y}}
$$

where $\beta_{x, y}$ are the betatron functions at the photon source location and $\epsilon_{x, y}$ the beam emittances, in the horizontal and vertical plane respectively. Including diffraction 
limits, the product

$$
\sigma_{\mathrm{tot}, x} \sigma_{\mathrm{tot}, x^{\prime}}=\sqrt{\epsilon_{x} \beta_{x}+\frac{1}{2} \sigma_{r}^{2}} \sqrt{\frac{\epsilon_{x}}{\beta_{x}}+\frac{1}{2} \sigma_{r^{\prime}}^{2}}
$$

has a minimum $\left(\frac{\mathrm{d}}{\mathrm{d} \beta_{x}} \sigma_{\mathrm{tot}, x} \sigma_{\mathrm{tot}, x^{\prime}}=0\right)$ for

$$
\beta_{x}=\frac{\sigma_{r}}{\sigma_{r^{\prime}}}=\frac{L}{2 \pi} .
$$

A similar optimum occurs for the vertical betatron function at the source point. The optimum value of the betatron functions at the source point depends only on the length of the undulator.

The values of the horizontal and vertical betatron functions should be adjusted according to (24.97) for optimum photon beam brightness. In case the particle beam emittance is much larger than the diffraction limited photon beam emittance, this minimum is very shallow and almost nonexistent in which case the importance of matching becomes irrelevant. As useful as matching may appear to be, it is not always possible to reach perfect matching because of limitations in the storage ring focusing system. Furthermore it is practically impossible to get a perfect matching for bending magnet radiation since the effective source length $L$ is very small, $L=$ $2 \rho / \gamma$.

\subsection{Photon Source Parameters}

In the previous paragraph, we have assumed that there is no dispersion at the source point. This is not always true and we have to modify our beam sizes to take the effect of energy spread and dispersion into account. Still simplifying, we use only the horizontal dispersion. Where this is not acceptable, the vertical dispersion effects have to be added in quadrature. The beam width or height is defined by the contribution of the betatron phase space $\sigma_{\beta, x, y}$ and the energy phase space $\sigma_{\eta, x, y}$ and is

$$
\sigma_{\mathrm{b}, x, y}=\sqrt{\sigma_{\beta, x, y}^{2}+\sigma_{\eta}^{2}}=\sqrt{\epsilon_{x, y} \beta, x, y+\left(\eta \frac{\sigma_{\varepsilon}}{E_{0}}\right)^{2}}
$$

with $\sigma_{\beta, x, y}^{2}=\epsilon_{x, y} \beta_{x, y}$ and $\sigma_{\eta}=\eta \frac{\sigma_{\varepsilon}}{E_{0}}, \gamma_{x, y}=\frac{1+\alpha_{x, y}^{2}}{\beta_{x, y}}$ and $\alpha_{x, y}=-\frac{1}{2} \beta_{x, y}^{\prime}$. Similarly, we get for the beam divergence

$$
\sigma_{\mathrm{b}, x^{\prime}, y^{\prime}}=\sqrt{\sigma_{\beta, x^{\prime}, y^{\prime}}^{2}+\sigma_{\eta^{\prime}}^{2}}=\sqrt{\epsilon_{x, y} \gamma_{x, y}+\left(\eta^{\prime} \frac{\sigma_{\varepsilon}}{E_{0}}\right)^{2}} .
$$


These beam parameters resemble in general the source parameters of the photon beam. Deviations occur when the beam emittance becomes very small, comparable to the photon wavelength of interest. First the matching conditions should be checked and modified if necessary. Second, the photon source parameters may be modified by diffraction effects which limit the apparent source size and divergence to some minimum values even if the electron beam cross section and divergence should be very small. For radiation at a wavelength $\lambda$, the diffraction limited radial photon source parameters are ${ }^{3}$

$$
\sigma_{r}=\frac{1}{2 \pi} \sqrt{\lambda L} \quad \text { and } \quad \sigma_{r^{\prime}}=\sqrt{\frac{\lambda}{L}} .
$$

Projection onto the horizontal or vertical plane gives $\sigma_{x, y}=\sigma_{r} / \sqrt{2}$ etc. Due to diffraction, it is not useful to push the electron beam emittance to values much smaller than

$$
\epsilon_{x, y}=\frac{\lambda}{4 \pi} .
$$

For an arbitrary electron beam cross section the photon source parameters are the quadratic sums of both contributions

$$
\begin{aligned}
\sigma_{\mathrm{ph}, x, y}^{2} & =\sigma_{\mathrm{b}, x, y}^{2}+\frac{1}{2} \sigma_{r}^{2}, \\
\sigma_{\mathrm{ph}, x^{\prime}, y^{\prime}}^{2} & =\sigma_{\mathrm{b}, x^{\prime}, y^{\prime}}^{2}+\frac{1}{2} \sigma_{r^{\prime}}^{2} .
\end{aligned}
$$

The contribution from diffraction can be ignored if

$$
\epsilon_{x, y} \gg \frac{\lambda}{4 \pi},
$$

which is generally true in the $x$-direction but not in the $y$-direction because of the small coupling in a storage ring.

\section{Problems}

24.1 (S). Bending magnet radiation $(\rho=2 \mathrm{~m})$ from a $800 \mathrm{MeV}, 500 \mathrm{~mA}$ storage ring includes a high intensity component of infrared radiation. Calculate the photon

\footnotetext{
${ }^{3}$ Many authors use a different definition $\sigma_{\mathrm{r}}=\sigma_{r} / \sqrt{2}$. The difference is mainly that the subscript $\mathrm{r}$ refers to radiation and the related beam parameters are already projected to the $x$ or $y$-plane. In this text, we use the subscript ${ }_{r}$ from the radial coordinate since we derive the diffraction effects from a round beam.
} 
beam brightness for $\lambda=10 \mu \mathrm{m}$ radiation at the experimental station which is $5 \mathrm{~m}$ away from the source. The electron beam cross section is $\sigma_{\mathrm{b}, x} \times \sigma_{\mathrm{b}, y}=1.1 \times 0.11 \mathrm{~mm}$ and its divergence $\sigma_{\mathrm{b}, x^{\prime}} \times \sigma_{\mathrm{b}, y^{\prime}}=0.11 \times 0.011 \mathrm{mrad}$. What is the corresponding brightness for infrared radiation from a black body radiator at 2,000 $\mathrm{K}$ with a source size of $x \times y=10 \times 2 \mathrm{~mm}$ ? (Hint: the source length $L=\rho 2 \theta_{\text {rad }}$ where $\pm \theta_{\text {rad }}$ is the vertical opening angle of the radiation.)

24.2 (S). What is the probability for a $6 \mathrm{GeV}$ electron to emit a photon with an energy of $\varepsilon=\sigma_{\varepsilon}$ per unit time travelling on a circle with radius $\rho=25 \mathrm{~m}$. How likely is it that this particle emits another such photon within a damping time? In evaluating quantum excitation and equilibrium emittances, do we need to consider multiple photon emissions? (use isomagnetic ring)

24.3 (S). Derive a formula for the average number of photons emitted by an electron of energy $E$ per turn. How many are these for $E=3 \mathrm{GeV}$ and $\rho=10 \mathrm{~m}$.

24.4 (S). In a $7 \mathrm{GeV}$ electron ring the circulating beam current is $200 \mathrm{~mA}$ and the bending radius $\rho=20 \mathrm{~m}$. Your experiment requires a photon flux of $10^{6}$ photons $/ \mathrm{sec}$ at a photon energy of $8 \mathrm{keV}$, within a band width of $10^{-4}$ onto a sample with a cross section of $10 \times 10 \mu \mathrm{m}^{2}$ and your experiment is $15 \mathrm{~m}$ away from the source point. Can you do your experiment on a bending magnet beam line of this ring?

24.5 (S). How well is the electron beam phase space of exercise 24.1 at the source matched to the photon beam? Show the phase space ellipses of both the electron and the photon beam in phase space and in $x$ and $y$.

24.6 (S). Derive an expression for the total synchrotron radiation power from a wiggler magnet.

24.7. Verify the numerical validity of Eqs. (24.4), (24.43), (24.51), (24.53), (24.59)

24.8 (S). In the SLAC linear accelerator operating at $100 \mathrm{~Hz}$ electrons can be accelerated to $50 \mathrm{GeV}$ at a rate of $17 \mathrm{MeV} / \mathrm{m}$. Calculate to total radiation power from $10^{9}$ electrons per pulse at $50 \mathrm{GeV}$ due to longitudinal acceleration. Compare with the radiation power if this bunch of $10^{9}$ electrons is deflected at the same energy by $1 \mathrm{mrad}$ in a $0.6 \mathrm{~T}$ bending magnet.

24.9 (S). Consider an electron storage ring at an energy of $1 \mathrm{GeV}$, a circulating current of $200 \mathrm{~mA}$ and a bending radius of $\rho=2.22 \mathrm{~m}$. Calculate the energy loss per turn, the critical energy and the total synchrotron radiation power. At what frequency in units of the critical frequency has the intensity dropped to $1 \%$ of the maximum? Plot the radiation spectrum and determine the frequency range available for experimentation.

24.10. What beam energy would be required to produce $x$-rays from the storage ring of problem 24.9 at a critical photon energy of $10 \mathrm{keV}$ ? Is that energy feasible from a conventional magnet point of view or would the ring have to be larger? What would the new beam energy and bending radius have to be? 
24.11. Consider a storage ring with an energy of $1 \mathrm{GeV}$ and a bending radius of $\rho=2.5 \mathrm{~m}$. Calculate the angular photon flux density $\mathrm{d} \dot{N} / \mathrm{d} \psi$ for a high photon energy $\hat{\varepsilon}$ where the intensity is still $1 \%$ of the maximum spectral intensity. What is this maximum photon energy? Installing a wavelength shifter with a field of $B=6 \mathrm{~T}$ allows the spectrum to be greatly extended. By how much does the spectral intensity increase at the photon energy $\hat{\varepsilon}$ and what is the new photon energy limit for the wavelength shifter?

24.12. Consider an electromagnetic wavelength shifter in a $1 \mathrm{GeV}$ storage ring with a central pole length of $30 \mathrm{~cm}$ and a maximum field of $6 \mathrm{~T}$. The side poles are $60 \mathrm{~cm}$ long and for simplicity assume that the field in all poles has a sinusoidal distribution along the axis. Determine the focal length due to edge focusing for the total wavelength shifter. To be negligible, the focal length should typically be longer than about $30 \mathrm{~m}$. Is this the case for this wavelength shifter?

24.13. Collide a $25 \mathrm{MeV}$ electron beam with a $1 \mathrm{~kW} \mathrm{CO}$-laser beam $(\lambda=10 \mu \mathrm{m})$. What is the energy of the backscattered photons? Assume a diffraction limited interaction length of twice the Rayleigh length and an electron beam cross section matching the photon beam. Calculate the x-ray photon flux for an electron beam from a $3 \mathrm{GHz}$ linear accelerator with a pulse length of $1 \mu \mathrm{s}$, a repetition rate of $10 \mathrm{~Hz}$ and a pulse current of $100 \mathrm{~mA}$.

\section{References}

1. G.A. Schott, Ann. Physik 24, 635 (1907)

2. D.W. Kerst, R. Serber, Phys. Rev. 60, 47 (1941)

3. D. Ivanenko, I.Ya. Pomeranchouk, Phys. Rev. 65, 343 (1944)

4. J.P. Blewett, Phys. Rev. 69, 87 (1946)

5. Describing work of C. Sutis, Sci. News Lett. 51, 339 (1947)

6. Describing work of F. Haber, Electronics 20, 136 (1947)

7. F. Elder, A. Gurewitsch, R. Langmuir, H. Pollock, Phys. Rev. 71, 829 (1947)

8. M. Berndt, W. Brunk, R. Cronin, D. Jensen, R. Johnson, A. King, J. Spencer, T. Taylor, H. Winick, IEEE Trans. Nucl. Sci. 26, 3812 (1979)

9. K. Halbach, F. Holsinger, Part. Accel. 7, 213 (1976)

10. W. Heitler, The Quantum Theory of Radiation (Clarendon, Oxford, 1954)

11. B.M. Kincaid, J. Appl. Phys. 48, 2684 (1977)

12. W.M. Lavender, Observation and analysis of $\mathrm{x}$-ray undulator radiation from PEP. PhD thesis, Stanford University (1988)

13. R.H. Milburn, Phys. Rev. Lett. 4, 75 (1963)

14. F.A. Arutyunian, V.A. Tumanian, Phys. Rev. Lett. 4, 176 (1963)

15. F.A. Arutyunian, I.I. Goldman, V.A. Tumanian, ZHETF(USSR) 45, 312 (1963)

16. I.F. Ginzburg, G.L. Kotyin, V.G. Serbo, V.I. Telnov, Colliding $\gamma$ e and $\gamma \gamma$ beams based on the single pass accelerators of vlepp type. Technical Report Preprint 81-102, Inst. of Nucl. phys., Novosibirsk (1981)

17. R. Coisson, Opt. Commun. 135, 135 (1977)

18. R. Bossart, J. Bosser, L. Burnod, E. D’Amico, G. Ferioli, J. Mann, F. Meot, Nucl. Instrum. Methods 184, 349 (1981) 
19. R. Bossart, J. Bosser, L. Burnod, R. Coisson, E. D’Amico, A. Hofmann, J. Mann, Nucl. Instrum. Methods 164, 275 (1979)

20. The large hadron collider in the lep tunnel. Technical Report CERN 87-05, CERN, Geneva (1985)

21. J.D. Jackson, Classical Electrodynamics, 2nd edn. (Wiley, New York, 1975)

22. D. Ivanenko, A.A. Sokolov, DAN(USSR) 59, 1551 (1972)

23. J.S. Schwinger, On the classical radiation of accelerated electrons. Phys. Rev. 75, 1912 (1949)

24. D.H. Tomboulian, P.L. Hartman, Phys. Rev. 102, 102 (1956)

25. G. Bathow, E. Freytag, R. Hänsel, J. Appl. Phys. 37, 3449 (1966)

26. M. Abramovitz, I. Stegun, Handbook of Mathematical Functions (Dover, New York, 1972)

27. M. Born, E. Wolf, Principles of Optics (Pergamon, Oxford, 1975)

28. G.B. Airy, Trans. Camb. Philos. Soc. 5, 283 (1835)

29. L.I. Schiff, Rev. Sci. Instrum. 17, 6 (1946)

30. T. Nakazato, M. Oyamada, N. Nimura, S. Urasawa, O. Konno, A. Kagaya, R. Kato, T. Kamiyama, Y. Torizuka, T. Nanba, Y. Kondo, Y. Shibata, K. Ishi, T. Oshaka, M. Ikezawa, Phys. Rev. Lett. 63, 1245 (1989)

31. E.B. Blum, U. Happek, A.J. Sievers, Nucl. Instrum. Methods A 307, 568 (1992)

32. H. Wiedemann, P. Kung, H.C. Lihn, Nucl. Instrum. Methods A 319, 1 (1992)

33. F.C. Michel, Phys. Rev. Lett. 48, 580 (1982) 


\section{Chapter 25 \\ Theory of Synchrotron Radiation}

The phenomenon of synchrotron radiation has been introduced in a conceptual way and a number of basic relations have been derived. In this chapter we will approach the physics of synchrotron radiation in a more formal way to exhibit detailed characteristics. Specifically, we will derive expressions for the spatial and spectral distribution of photon emission in a way which is applicable later for special insertion devices.

The theory of synchrotron radiation is intimately related to the electromagnetic fields generated by moving charged particles. Wave equations can be derived from Maxwell's equations and we will find that any charged particle under the influence of external forces can emit radiation. We will formulate the characteristics of this radiation and apply the results to highly relativistic particles.

\subsection{Radiation Field}

The electromagnetic fields for a single moving point charge will be derived first and then applied to a large number of particles. Fields are determined by Maxwell's equations (1.4) for moving charges in vacuum where $\epsilon_{\mathrm{r}}=\mu_{\mathrm{r}}=1$. The magnetic field can be derived from a vector potential $\boldsymbol{A}$ defined by

$$
\boldsymbol{B}=\nabla \times A .
$$

Inserting the vector potential into Faraday's law (1.4) we have $\boldsymbol{\nabla} \times\left(\boldsymbol{E}+\frac{\partial \boldsymbol{A}}{\partial t}\right)=0$, or after integration

$$
\boldsymbol{E}=-\frac{\partial \boldsymbol{A}}{\partial t}-\nabla \varphi
$$

This chapter has been made Open Access under a CC BY 4.0 license. For details on rights and licenses please read the Correction https://doi.org/10.1007/978-3-319-18317-6_28 
where $\varphi$ is the scalar potential. We choose the scalar potential such that $c \boldsymbol{\nabla} \boldsymbol{A}+$ $\frac{1}{c} \frac{\partial \varphi}{\partial t}=0$, a condition known as the Lorentz gauge. With (A.21) applied to $\boldsymbol{A}$ the expression for the electric field together with Ampere's law (1.4) results in the wave equation

$$
\nabla^{2} \boldsymbol{A}-\frac{1}{c^{2}} \frac{\partial^{2} \boldsymbol{A}}{\partial t^{2}}=\frac{1}{\epsilon_{0}} \rho \boldsymbol{\beta} .
$$

Similarly, we derive the wave equation for the scalar potential

$$
\nabla^{2} \varphi-\frac{1}{c^{2}} \frac{\partial^{2} \varphi}{\partial t^{2}}=-\frac{1}{\epsilon_{0}} \rho .
$$

These are the well-known wave equations with the solutions

$$
\boldsymbol{A}(t)=\left.\frac{\mu_{0}}{4 \pi} \int \frac{\boldsymbol{v} \rho(x, y, z)}{R}\right|_{t_{\mathrm{r}}} \mathrm{d} x \mathrm{~d} y \mathrm{~d} z
$$

and

$$
\varphi(t)=\left.\frac{1}{4 \pi \epsilon_{0}} \int \frac{\rho(x, y, z)}{R}\right|_{t_{\mathrm{r}}} \mathrm{d} x \mathrm{~d} y \mathrm{~d} z
$$

Because of the finite velocity of light, all quantities under the integrals must be evaluated at the retarded time

$$
t_{\mathrm{r}}=t-\frac{1}{c} R\left(t_{\mathrm{r}}\right)
$$

when the radiation was emitted by the moving charge, in contrast to the time $t$ when the radiation is observed at a distant point. The quantity $R$ is the distance between the observation point $P(x, y, z)$ and the location of the charge element $\rho\left(x_{\mathrm{r}}, y_{\mathrm{r}}, z_{\mathrm{r}}\right) \mathrm{d} x_{\mathrm{r}} \mathrm{d} y_{\mathrm{r}} \mathrm{d} z_{\mathrm{r}}$ at the retarded time $t_{\mathrm{r}}$. The vector

$$
\boldsymbol{R}=\left(x_{\mathrm{r}}-x, y_{\mathrm{r}}-y, z_{\mathrm{r}}-z\right)
$$

points away from the observation point to the charge element at the retarded time as shown in Fig. 25.1.

Special care must be exercised in performing the integrations. Although we consider only a point charge $q$, the integral in (25.6) cannot be replaced by $q / R$ but must be integrated over a finite volume followed by a transition to a point charge. As we will see this is a consequence of the fact that the velocity of light is finite and therefore the movement of charge elements must be taken into account.

To define the quantities involved in the integration we use Fig. 25.1. The combined field at the observation point $P$ at time $t$ comes from all charges located at 


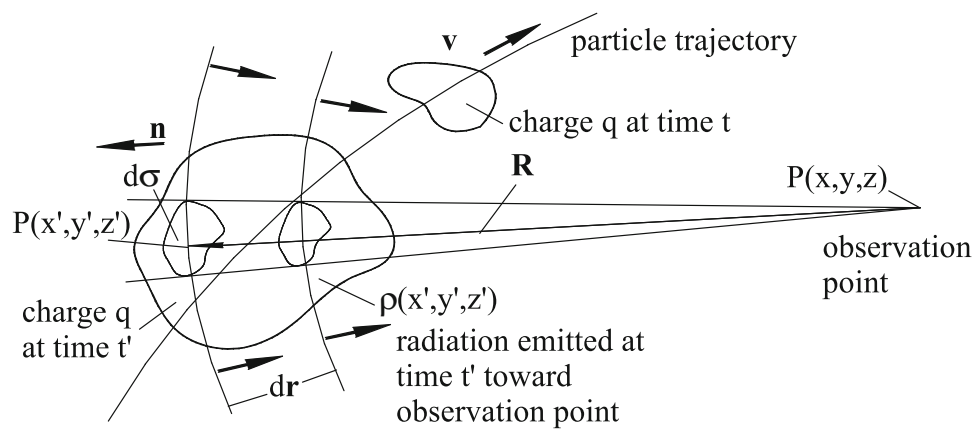

Fig. 25.1 Retarded position of a moving charge distribution

a distance $R$ away from $P$. We consider the contribution from all charges contained within a spherical shell centered at $P$ with a radius $R$ and thickness $\mathrm{d} r$ to the radiation field at $P$ and time $t$. Radiation emitted at time $t_{\mathrm{r}}$ will reach $P$ at the time $t$. If $\mathrm{d} \sigma$ is a surface element of the spherical shell, the volume element of charge is $\mathrm{d} x \mathrm{~d} y \mathrm{~d} z=\mathrm{d} \sigma \mathrm{d} r$. The retarded time for the radiation from the outer surface of the shell is $t_{\mathrm{r}}$ and the retarded time for the radiation from the charge element on the inner surface of the shell is $t_{\mathrm{r}}-\frac{\mathrm{d} r}{c}$. From Fig. 25.1 we find the electromagnetic field observed at $P$ at time $t$ to originate from the fractional charges within the volume element $\mathrm{d} \sigma \mathrm{d} r$ or from the charge element $\mathrm{d} q=\rho \mathrm{d} \sigma \mathrm{d} r$.

The radiation observed at point $P$ and time $t$ is the sum of all radiation arriving simultaneously at $P$. Elements of this radiation field may have been emitted by different charge elements and at different times and locations. In case of only one electrical charge moving with velocity $\boldsymbol{v}$, we have to include in the integration those charge elements that move across the inner shell surface into the volume $\mathrm{d} \sigma \mathrm{d} r$ during the time $\mathrm{d} r / c$. For a uniform charge distribution this additional charge is $\delta q=\rho \boldsymbol{v} \boldsymbol{n} \mathrm{d} t \mathrm{~d} \sigma$ where $\boldsymbol{n}$ is the vector normal to the surface of the shell and pointing away from the observer

$$
\boldsymbol{n}=\frac{\boldsymbol{R}}{R}
$$

With $\mathrm{d} t=\mathrm{d} r / c$ and $\boldsymbol{\beta}=\boldsymbol{v} / c$, we get then for both contributions to the charge element

$$
\mathrm{d} q=\rho(1+\boldsymbol{n} \boldsymbol{\beta}) \mathrm{d} r \mathrm{~d} \sigma .
$$

Depending on the direction of the velocity vector $\boldsymbol{\beta}$, we find an increase or a reduction in the radiation field from moving charges. We solve (25.10) for $\rho \mathrm{d} r \mathrm{~d} \sigma$ and insert into the integrals (25.5), (25.6). Now we may use the assumption that the electrical charge is a point charge and get for the retarded potentials of a moving 
point charge $q$ at time $t$ and observation point $P$

$$
\boldsymbol{A}(P, t)=\left.\frac{1}{4 \pi c \epsilon_{0}} \frac{q}{R} \frac{\boldsymbol{\beta}}{1+\boldsymbol{n} \boldsymbol{\beta}}\right|_{t_{\mathrm{r}}}
$$

and

$$
\varphi(P, t)=\left.\frac{1}{4 \pi \epsilon_{0}} \frac{q}{R} \frac{1}{1+\boldsymbol{n} \boldsymbol{\beta}}\right|_{t_{\mathrm{r}}} .
$$

These equations are known as the Liénard-Wiechert potentials and express the field potentials of a static or moving charge as functions of the charge parameters at the retarded time. To obtain the electric and magnetic fields we insert the retarded potentials into (25.1), (25.2) noting that the differentiation must be performed with respect to the time $t$ and location $P$ of the observer while the potentials are expressed at the retarded time $t_{\mathrm{r}}$.

In both equations for the vector and scalar potential we have the same denominator

$$
r=R(1+\boldsymbol{n} \boldsymbol{\beta}) .
$$

It will become necessary to calculate the derivative of the retarded time with respect to the time $t$ and since $t_{\mathrm{r}}=t-R / c$ the time derivative of $t_{\mathrm{r}}$ is

$$
\frac{\mathrm{d} t_{\mathrm{r}}}{\mathrm{d} t}=1-\frac{1}{c} \frac{\mathrm{d} R}{\mathrm{~d} t_{\mathrm{r}}} \frac{\mathrm{d} t_{\mathrm{r}}}{\mathrm{d} t}
$$

The variation of the distance $R$ with the retarded time depends on the velocity $v$ of the moving charge and is the projection of the vector $\boldsymbol{v} \mathrm{d} t_{\mathrm{r}}$ onto the unity vector $\boldsymbol{n}$. Therefore,

$$
\mathrm{d} R=\boldsymbol{v} \boldsymbol{n} \mathrm{d} t_{\mathrm{r}}
$$

and (25.14) becomes with (25.14) and (25.13)

$$
\frac{\mathrm{d} t_{\mathrm{r}}}{\mathrm{d} t}=\frac{1}{1+\boldsymbol{n} \boldsymbol{\beta}}=\frac{R}{r} .
$$

The electric field (25.2) is with (25.11), (25.12) and (25.16) after a few manipulations expressed by

$$
4 \pi \epsilon_{0} \frac{\boldsymbol{E}}{q}=-\frac{1}{c} \frac{R}{r^{2}} \frac{\partial \boldsymbol{\beta}}{\partial t_{\mathrm{r}}}+\frac{\beta \boldsymbol{R}}{c r^{3}} \frac{\partial r}{\partial t_{\mathrm{r}}}+\frac{1}{r^{2}} \nabla_{\mathrm{r}} r .
$$


In evaluating the nabla operator and other differentials we remember that all parameters on the r.h.s. must be taken at the retarded time (25.7) which itself depends on the location of the observation point $P$. To distinguish between the ordinary nabla operator and the case where the dependence of the retarded time on the position $P(x, y, z)$ must be considered, we add to the nabla symbol the index r like $\nabla_{\mathrm{r}}$. The components of this operator are then $\left.\frac{\partial}{\partial x}\right|_{\mathrm{r}}=\frac{\partial}{\partial x}+\frac{\partial t_{\mathrm{r}}}{\partial x} \frac{\partial}{\partial t_{\mathrm{r}}}$, and similar for the other components. We evaluate first

$$
\nabla_{\mathrm{r}} r=\nabla_{\mathrm{r}} R+\nabla_{\mathrm{r}}(\beta R)
$$

and with $\nabla R=-\boldsymbol{n}$ from $(25.8)$

$$
\nabla_{\mathrm{r}} R=-\boldsymbol{n}+\frac{\partial R}{\partial t_{\mathrm{r}}} \nabla t_{\mathrm{r}}
$$

For the gradient of the retarded time, we get

$$
\nabla t_{\mathrm{r}}=\nabla\left[t-\frac{1}{c} R\left(t_{\mathrm{r}}\right)\right]=-\frac{1}{c} \nabla_{\mathrm{r}} R=-\frac{1}{c}\left(-\boldsymbol{n}+\frac{\partial R}{\partial t_{\mathrm{r}}} \nabla t_{\mathrm{r}}\right)
$$

and performing the differentiation we get with $\frac{\partial x_{\mathrm{r}}}{\partial t_{\mathrm{r}}}=v_{x}, \ldots$

$$
\frac{\partial R}{\partial t_{\mathrm{r}}}=\frac{\partial R}{\partial x_{\mathrm{r}}} \frac{\partial x_{\mathrm{r}}}{\partial t_{\mathrm{r}}}+\frac{\partial R}{\partial y_{\mathrm{r}}} \frac{\partial y_{\mathrm{r}}}{\partial t_{\mathrm{r}}}+\frac{\partial R}{\partial z_{\mathrm{r}}} \frac{\partial z_{\mathrm{r}}}{\partial t_{\mathrm{r}}}=\boldsymbol{n} \boldsymbol{v}
$$

Solving (25.20) for $\nabla t_{\mathrm{r}}$ we get

$$
\nabla t_{\mathrm{r}}=\frac{\boldsymbol{R}}{c r}
$$

and (25.19) becomes finally

$$
\nabla_{\mathrm{r}} R=-\boldsymbol{n}+\frac{\boldsymbol{R}}{r}(\boldsymbol{\beta} \boldsymbol{n}) .
$$

For the second term in (25.18) we note that the velocity $v$ does not depend on the location of the observer and with $\nabla_{\mathrm{r}} \boldsymbol{R}-1,(25.22)$ and

$$
\frac{\mathrm{d} \boldsymbol{R}}{\mathrm{d} t_{\mathrm{r}}}=\boldsymbol{v}
$$

we get for the second term in (25.18)

$$
\nabla_{\mathrm{r}}(\boldsymbol{\beta} \boldsymbol{R})=-\boldsymbol{\beta}+\frac{\partial(\boldsymbol{\beta} \boldsymbol{R})}{\partial t_{\mathrm{r}}} \nabla t_{\mathrm{r}}=-\boldsymbol{\beta}+\left(\boldsymbol{R} \frac{\partial \boldsymbol{\beta}}{\partial t_{\mathrm{r}}}\right) \frac{\boldsymbol{R}}{c r}+\beta^{2} \frac{\boldsymbol{R}}{r} .
$$


To complete the evaluation of the electric field in (25.17), we express the derivative $\frac{\partial r}{\partial t_{\mathrm{r}}}$ with

$$
\frac{\partial r}{\partial t_{\mathrm{r}}}=\frac{\partial R}{\partial t_{\mathrm{r}}}+\frac{\partial(\boldsymbol{\beta} \boldsymbol{R})}{\partial t_{\mathrm{r}}}=\operatorname{cn} \beta+c \beta^{2}+\boldsymbol{R} \frac{\partial \boldsymbol{\beta}}{\partial t_{\mathrm{r}}},
$$

where we made use of (25.21). Collecting all differential expressions required in (25.17) we get with (25.18), (25.23), (25.25), (25.26)

$$
\begin{aligned}
4 \pi \epsilon_{0} \frac{\boldsymbol{E}}{q} & =\frac{1}{r^{2}}\left[-\boldsymbol{n}-\boldsymbol{\beta}+\frac{\boldsymbol{R}}{r}\left(\boldsymbol{n} \boldsymbol{\beta}+\beta^{2}+\frac{1}{c} \dot{\boldsymbol{\beta}} \boldsymbol{R}\right)\right]_{\mathrm{r}} \\
& -\frac{R}{c r^{2}} \dot{\boldsymbol{\beta}}+\boldsymbol{\beta} \frac{R}{r^{3}}\left(\boldsymbol{n} \boldsymbol{\beta}+\beta^{2}+\frac{1}{c} \dot{\boldsymbol{\beta}} \boldsymbol{R}\right)_{\mathrm{r}},
\end{aligned}
$$

where $\dot{\boldsymbol{\beta}}=\mathrm{d} \boldsymbol{\beta} / \mathrm{d} t_{\mathrm{r}}$. After some manipulation and using (A.10), the equation for the electrical field of a charge $q$ moving with velocity $\boldsymbol{v}$ becomes

$$
4 \pi \epsilon_{0} \frac{\boldsymbol{E}}{q}=\frac{1-\beta^{2}}{r^{3}}(\boldsymbol{R}+R \boldsymbol{\beta})_{\mathrm{r}}+\left.\frac{1}{c r^{3}}\left\{\boldsymbol{R} \times\left[(\boldsymbol{R}+R \boldsymbol{\beta})_{\mathrm{r}} \times \frac{\mathrm{d} \boldsymbol{\beta}}{\mathrm{d} t_{\mathrm{r}}}\right]\right\}\right|_{\mathrm{r}},
$$

where we have added the index $\mathrm{r}$ as a reminder that all quantities on the r.h.s. of (25.28) must be taken at the retarded time $t_{\mathrm{r}}$.

This equation for the electric field of a moving charge has two distinct parts. The first part is inversely proportional to the square of the distance between radiation source and observer and depends only on the velocity of the charge. For a charge at rest $\beta=0$ this term reduces to the Coulomb field of a point charge $q$. The area close to the radiating charge where this term is dominant is called the Coulomb regime. The field is directed toward the observer for a positive charge at rest and tilts into the direction of propagation as the velocity of the charge increases. For highly relativistic particles we note the Coulomb field becomes very small.

We will not further consider this regime since we are interested only in the radiation field far away from the moving charge. The second term in (25.28) depends on the velocity as well as on the acceleration of the charge. This term scales linear with the distance $r$ falling off much slower than the Coulomb term and therefore reaches out to large distances from the radiation source. We call this regime the radiation regime and the remainder of this chapter will focus on the discussion of the radiation from moving charges. The electrical field in the radiation regime is

$$
\left.4 \pi \epsilon_{0} \frac{\boldsymbol{E}(t)}{q}\right|_{\mathrm{rad}}=\left.\frac{1}{c r^{3}}\left\{\boldsymbol{R} \times\left[(\boldsymbol{R}+R \boldsymbol{\beta})_{\mathrm{r}} \times \frac{\mathrm{d} \boldsymbol{\beta}}{\mathrm{d} t_{\mathrm{r}}}\right]\right\}\right|_{\mathrm{r}} .
$$

The polarization of the electric field at the location of the observer is purely orthogonal to the direction of observation $\mathbf{R}$. Similar to the derivation of the electric field, we can derive the expression for the magnetic field and get from (25.1) 
with (25.11)

$$
\boldsymbol{B}=\nabla_{\mathrm{r}} \mathbf{x} \boldsymbol{A}=q\left[\nabla_{\mathrm{r}} \times \frac{\boldsymbol{\beta}}{r}\right]=\frac{q}{r}\left[\nabla_{\mathrm{r}} \times \boldsymbol{\beta}\right]-\frac{q}{r^{2}}\left[\nabla_{\mathrm{r}} r \times \boldsymbol{\beta}\right],
$$

where again all parameters on the r.h.s. must be evaluated at the retarded time. The evaluation of the "retarded" curl operation $\nabla_{\mathrm{r}} \times \boldsymbol{\beta}$ becomes obvious if we evaluate one component only, for example, the $x$ component

$$
\left(\frac{\partial}{\partial y}+\frac{\partial t_{\mathrm{r}}}{\partial y} \frac{\partial}{\partial t_{\mathrm{r}}}\right) \beta_{z}-\left(\frac{\partial}{\partial z}+\frac{\partial t_{\mathrm{r}}}{\partial z} \frac{\partial}{\partial t_{\mathrm{r}}}\right) \beta_{y}=[\nabla \times \boldsymbol{\beta}]_{x}+\left[\nabla t_{\mathrm{r}} \times \frac{\mathrm{d} \boldsymbol{\beta}}{\mathrm{d} t_{\mathrm{r}}}\right]_{x}
$$

In a similar way, we get the other components and find with (25.22) and the fact that the particle velocity $\boldsymbol{\beta}$ does not depend on the coordinates of the observation point $(\nabla \times \boldsymbol{\beta}=0)$,

$$
\left[\nabla_{\mathrm{r}} r \times \boldsymbol{\beta}\right]=[\nabla \times \boldsymbol{\beta}]+\left[\nabla t_{\mathrm{r}} \times \frac{\mathrm{d} \boldsymbol{\beta}}{\mathrm{d} t_{\mathrm{r}}}\right]=\frac{1}{c r}\left[\boldsymbol{R} \times \frac{\mathrm{d} \boldsymbol{\beta}}{\mathrm{d} t_{\mathrm{r}}}\right],
$$

The gradient $\nabla_{\mathrm{r}} r$ has been derived earlier in (25.18) and inserting this into (25.30) we find the magnetic field of an electrical charge moving with velocity $\mathbf{v}$

$$
\begin{aligned}
4 \pi c \epsilon_{0} \frac{\boldsymbol{B}}{q} & =-\frac{1}{r^{2}}(\boldsymbol{\beta} \times \boldsymbol{n})-\left.\frac{R}{c r^{2}}\left[\frac{\mathrm{d} \boldsymbol{\beta}}{\mathrm{d} t} \times \boldsymbol{n}\right]\right|_{\mathrm{r}} \\
& +\left.\frac{R}{r^{3}}\left(\boldsymbol{\beta} \boldsymbol{n}+\beta^{2}+\frac{1}{c} \frac{\mathrm{d} \boldsymbol{\beta}}{\mathrm{d} t} \boldsymbol{R}\right)[\boldsymbol{\beta} \times \boldsymbol{n}]\right|_{\mathrm{r}} .
\end{aligned}
$$

Again, there are two distinct groups of field terms. In case of the electrical field the terms that fall off like the square of the distance are the Coulomb fields. For magnetic fields such terms appear only if the charge is moving $\beta \neq 0$ and are identical to the Biot-Savart fields. Here we concentrate only on the far fields or radiation fields which decay inversely proportional to the distance from the source. The magnetic radiation field is then given by

$$
\left.4 \pi c \epsilon_{0} \frac{\boldsymbol{B}(t)}{q}\right|_{\mathrm{rad}}=-\frac{R}{c r^{2}}\left[\frac{\mathrm{d} \boldsymbol{\beta}}{\mathrm{d} t} \times \boldsymbol{n}\right]_{\mathrm{r}}+\frac{R}{c r^{3}}\left(\frac{\mathrm{d} \boldsymbol{\beta}}{\mathrm{d} t} \boldsymbol{R}\right)[\boldsymbol{\beta} \times \boldsymbol{n}]_{\mathrm{r}}
$$

Comparing the magnetic field (25.33) with the electrical field (25.28) reveals a very simple correlation between both fields. The magnetic field can be obtained from the electric field, and vice versa, by mere vector multiplication with the unit vector $\boldsymbol{n}$

$$
\boldsymbol{B}=\frac{1}{c}[\boldsymbol{E} \times \boldsymbol{n}]_{\mathrm{r}} .
$$


From this equation we can deduce special properties for the field directions by noting that the electric and magnetic fields are orthogonal to each other and both are orthogonal to the direction of observation $\boldsymbol{n}$. The existence of electric and magnetic fields can give rise to radiation for which the Poynting vector is

$$
\boldsymbol{S}=\frac{1}{c \mu_{0}}[\boldsymbol{E} \times \boldsymbol{B}]_{\mathrm{r}}=\epsilon_{0} c[\boldsymbol{E} \times(\boldsymbol{E} \times \boldsymbol{n})]_{\mathrm{r}} .
$$

Using again the vector relation (A.10) and noting that the electric field is normal to $\boldsymbol{n}$, we get for the Poynting vector or the radiation flux in the direction to the observer

$$
\boldsymbol{S}=-\left.\epsilon_{0} c \boldsymbol{E}_{\mathrm{r}}^{2} \boldsymbol{n}\right|_{\mathrm{r}} .
$$

Equation (25.36) defines the energy flux density measured at the observation point $P$ and time $t$ in form of synchrotron radiation per unit cross section and parallel to the direction of observation $\boldsymbol{n}$. All quantities expressing this energy flux are still to be taken at the retarded time. For practical reasons it becomes desirable to express the Poynting vector at the retarded time as well. The energy flux at the observation point in terms of the retarded time is then $\mathrm{d} W / \mathrm{d} t_{\mathrm{r}}=(\mathrm{d} W / \mathrm{d} t)\left(\mathrm{d} t / \mathrm{d} t_{\mathrm{r}}\right)$ and instead of (25.36) we express the Poynting vector with (25.16) like

$$
\boldsymbol{S}_{\mathrm{r}}=\boldsymbol{S} \frac{\mathrm{d} t}{\mathrm{~d} t_{\mathrm{r}}}=-\epsilon_{0} c \boldsymbol{E}^{2}[(1+\boldsymbol{\beta} \boldsymbol{n}) \boldsymbol{n}]_{\mathrm{r}} .
$$

The Poynting vector in this form can be readily used for calculations like those determining the spatial distribution of the radiation power.

\subsection{Total Radiation Power and Energy Loss}

So far, no particular choice of the reference system has been assumed, but a particularly simple reference frame $\mathcal{L}^{*}$ is the one which moves uniformly with the charge before acceleration. From now on, we use a single particle with a charge $e$. To an observer in this reference system, the charge moves due to acceleration and the electric field in the radiation regime is from (25.29)

$$
\boldsymbol{E}^{*}(t)=\left.\frac{1}{4 \pi \epsilon_{0}} \frac{e}{c R}\left[\boldsymbol{n} \times\left(n \times \frac{\mathrm{d} \boldsymbol{\beta}^{*}}{\mathrm{~d} t}\right)\right]\right|_{\mathrm{r}} .
$$


The synchrotron radiation power per unit solid angle and at distance $R$ from the source is from (25.37) with $v=0$

$$
\frac{\mathrm{d} P^{*}}{\mathrm{~d} \Omega}=-\boldsymbol{n} \boldsymbol{S}^{*} R_{\mathrm{r}}^{2}=\left.\epsilon_{0} c \boldsymbol{E}^{* 2} R^{2}\right|_{\mathrm{r}}
$$

Introducing the classical particle radius by $e^{2}=4 \pi \epsilon_{0} r_{\mathrm{c}} m c^{2}$ we obtain expressions which are independent of electromagnetic units and with (25.38)

$$
\frac{\mathrm{d} P^{*}}{\mathrm{~d} \Omega}=\frac{r_{\mathrm{c}} m c^{2}}{4 \pi c}\left|\boldsymbol{n} \times\left(\boldsymbol{n} \times \frac{\mathrm{d} \boldsymbol{\beta}^{*}}{\mathrm{~d} t}\right)\right|_{\mathrm{r}}^{2}=\left.\frac{r_{\mathrm{c}} m c^{2}}{4 \pi c} \frac{\mathrm{d} \boldsymbol{\beta}^{*}}{\mathrm{~d} t}\right|_{\mathrm{r}} ^{2} \sin ^{2} \vartheta_{\mathrm{r}},
$$

where $\vartheta_{\mathrm{r}}$ is the retarded angle between the direction of acceleration and the direction of observation $\boldsymbol{n}$. Integration over all solid angles gives the total radiated power. With $\mathrm{d} \Omega=\sin \vartheta_{\mathrm{r}} \mathrm{d} \vartheta_{\mathrm{r}} \mathrm{d} \phi$, where $\phi$ is the azimuthal angle with respect to the direction of acceleration, the total radiation power is in agreement with (24.24)

$$
P^{*}=\frac{2}{3} r_{\mathrm{c}} m c\left|\frac{\mathrm{d} \boldsymbol{\beta}^{*}}{\mathrm{~d} t}\right|_{\mathrm{r}}^{2}
$$

This equation has been derived first by Larmor [1] within the realm of classical electrodynamics. The emission of a quantized photon, however, exerts a recoil on the electron varying its energy slightly. Schwinger [2] investigated this effect and derived a correction to the radiation power like

$$
P^{*}=P_{\text {classical }}^{*}\left(1-\frac{55}{16 \sqrt{3}} \frac{\epsilon_{\mathrm{c}}}{E}\right),
$$

where $\epsilon_{\mathrm{c}}$ is the critical photon energy and $E$ the electron energy. The correction is generally very small and we ignore therefore this quantum mechanical effect in our discussions.

Equation (25.41) must be transformed from the particle system to the laboratory frame of reference. This has been done already in Sect. 24.2.

\subsubsection{Transition Radiation}

Digressing slightly from the discussion of synchrotron radiation we turn our attention to the solution of (25.39). Generally, we do not know the fields $\boldsymbol{E}^{*}$ and to solve (25.40) we need to know more about the particular trajectory of the particle motion. In the case of transition radiation, we have, however, all information to formulate a solution. Transition radiation is emitted when a charged particle passes through the boundary of two media with different dielectric constant. We will not go into the detailed general theory of transition radiation but concentrate on the 
case where a charged particle passes through a thin metallic foil in vacuum. As the particle passes through the foil backward transition radiation is emitted when the particle enters the foil and forward radiation is emitted when it appears on the other side. The emitted radiation energy can be derived directly from (25.39). First, we replace the electric radiation field by the magnetic field component and (25.39) becomes simply

$$
\frac{\mathrm{d} \varepsilon(t)}{\mathrm{d} t}=\left.\epsilon_{0} c \boldsymbol{B}^{* 2}(t) R^{2}\right|_{\mathrm{r}} \mathrm{d} \Omega
$$

From Parseval's theorem (A.42) we know that

$$
\int_{-\infty}^{\infty} B^{2}(t) \mathrm{d} t=\frac{1}{2 \pi} \int_{-\infty}^{\infty} B^{2}(\omega) \mathrm{d} \omega
$$

The emission of transition radiation occurs in a very short time $\tau \approx \omega_{\mathrm{p}}^{-1}$, where $\omega_{\mathrm{p}}$ is the plasma frequency. For this reason, the transition radiation frequency reaches into the X-ray regime. We limit ourselves here to frequencies $\omega$, which are much lower such that $\tau \ll \omega^{-1}$. The magnetic field is nonzero only during the emission process and we can therefore set

$$
B(\omega)=\int_{-\infty}^{\infty} B(t) e^{\mathrm{i} \omega t} \mathrm{~d} t \approx \int_{-\tau / 2}^{\tau / 2} B(t) \mathrm{d} t .
$$

To solve this integral we recall the definition of the vector potential $\boldsymbol{B}(t)=\nabla \times \boldsymbol{A}_{\mathrm{r}}$ and keep in mind that all quantities are to be taken at the retarded time. Expressing in component form $\nabla \times \boldsymbol{A}_{\mathrm{r}}=\left\{\frac{\partial A_{z}}{\partial y}-\frac{\partial A_{y}}{\partial z}, \frac{\partial A_{x}}{\partial z}-\frac{\partial A_{z}}{\partial x}, \frac{\partial A_{x}}{\partial z}-\frac{\partial A_{z}}{\partial x}\right\}_{t_{\mathrm{r}=t-\frac{1}{c} R(t)}}$ the derivatives are $\frac{\partial A_{z}}{\partial y}=\frac{\partial A_{z}}{\partial t_{\mathrm{r}}} \frac{\partial t_{\mathrm{r}}}{\partial y}$ etc. With $\frac{\partial t_{\mathrm{r}}}{\partial y}=\frac{1}{c} \frac{y_{\mathrm{r}}-y}{R}=\frac{n_{y}}{c}$ we get $\frac{\partial A_{z}}{\partial y}-\frac{\partial A_{y}}{\partial z}=\frac{1}{c} \frac{\partial A_{z}}{\partial t_{\mathrm{r}}} n_{y}-\frac{1}{c} \frac{\partial A_{y}}{\partial t_{\mathrm{r}}} n_{z}$ or finally

$$
\boldsymbol{B}(t)=\nabla \times \boldsymbol{A}_{\mathrm{r}}=\frac{1}{c} \boldsymbol{n}_{\mathrm{r}} \times \frac{\partial}{\partial t_{\mathrm{r}}} \boldsymbol{A}_{\mathrm{r}}=\frac{1}{c} \frac{\partial}{\partial t_{\mathrm{r}}}[\boldsymbol{n} \times \boldsymbol{A}]_{\mathrm{r}} .
$$

The magnetic field spectrum (25.45) becomes then simply

$$
B(\omega)=\int_{-\tau / 2}^{\tau / 2} B(t) \mathrm{d} t=\left.\frac{1}{c}[\boldsymbol{n} \times \boldsymbol{A}]_{\mathrm{r}}\right|_{\text {initial }} ^{\text {final }} .
$$

Initially, while the electron has not yet vanished into the metallic foil, the vector potential is made up of the Liènard-Wiechert potentials of a free electron and its image charge (a positron) moving in the opposite direction. The vector potential is 
therefore

$$
\boldsymbol{A}=\underbrace{4 \pi c \epsilon_{0} \frac{e \boldsymbol{\beta}}{R(1+\boldsymbol{\beta} \boldsymbol{n})}}_{\text {electron }}+\underbrace{4 \pi c \epsilon_{0} \frac{e \boldsymbol{\beta}}{R(1-\boldsymbol{\beta} \boldsymbol{n})}}_{\text {positron }} .
$$

Instead of (25.43) we use the spectral radiation energy $\mathrm{d} \varepsilon(\omega)=\frac{1}{\mu_{0}} R^{2} \mathrm{~d} \Omega \frac{1}{2 \pi} \boldsymbol{B}_{\mathrm{r}}^{* 2}$ $(t) \mathrm{d} \omega 2$, where the extra factor of two comes from using only positive frequencies $\omega>0$, and get with (25.48) and $e^{2}=r_{\mathrm{c}} m c^{2} 4 \pi \epsilon_{0}$

$$
\begin{aligned}
\frac{\mathrm{d}^{2} \varepsilon}{\mathrm{d} \omega \mathrm{d} \Omega} & =\frac{1}{4 \pi^{2}} \frac{r_{\mathrm{c}} m c^{2}}{c}\left\{\frac{\boldsymbol{n} \times \boldsymbol{\beta}}{1+\boldsymbol{\beta} \boldsymbol{n}}+\frac{\boldsymbol{n} \times \boldsymbol{\beta}}{1-\boldsymbol{\beta} \boldsymbol{n}}\right\}^{2} \\
& =\frac{r_{\mathrm{c}} m c^{2}}{\pi^{2} c}|\boldsymbol{n} \times \boldsymbol{z}|^{2}\left(\frac{\beta}{1-\beta^{2}(\boldsymbol{n z})^{2}}\right)^{2},
\end{aligned}
$$

where we used $\beta \approx \beta z$ and where $z$ is the unit vector along the $z$-axis. The emission angle $\vartheta$ is taken with respect to the $z$-axis. The spectral and spatial transition radiation distribution from a single electron is finally with $\boldsymbol{n z}=\cos \vartheta$ and $\boldsymbol{n} \times \boldsymbol{z}=\sin \vartheta$

$$
\frac{\mathrm{d}^{2} \varepsilon}{\mathrm{d} \omega \mathrm{d} \Omega}=\frac{r_{\mathrm{c}} m c^{2}}{\pi^{2} c} \frac{\beta^{2} \sin ^{2} \vartheta}{\left(1-\beta^{2} \cos ^{2} \vartheta\right)^{2}} .
$$

The spatial radiation distribution of transition radiation is shown in Fig. 25.2. No radiation is emitted along the axis $\vartheta=0$ while the radiation intensity reaches a maximum at an emission angle of $1 / \gamma$. Equation (25.49) does not exhibit any frequency dependence, which is due to the fact that the emission process occurs in a very short time generating a uniform spectrum. Very high frequencies in the x-ray regime, where the spectral intensity is expected to drop, have been excluded in this derivation.

Fig. 25.2 Intensity distribution $\frac{\mathrm{d}^{2} \varepsilon}{\mathrm{d} \omega \mathrm{d} \Omega} \frac{\pi^{2} c}{r_{\mathrm{c}} m c^{2}}$ of transition radiation

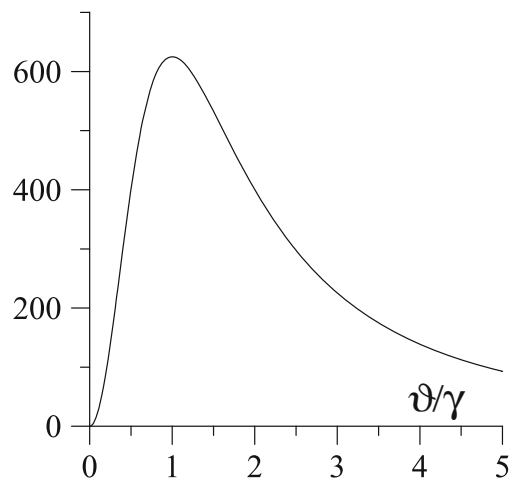


Integrating (25.49) over a half space, we get

$$
\begin{aligned}
\frac{\mathrm{d} \varepsilon}{\mathrm{d} \omega} & =\frac{2 r_{\mathrm{c}} m c^{2}}{\pi c} \int_{0}^{\pi / 2} \frac{\beta^{2} \sin ^{2} \vartheta}{\left(1-\beta^{2} \cos ^{2} \vartheta\right)^{2}} \sin \vartheta \mathrm{d} \vartheta \\
& =\frac{2 r_{\mathrm{c}} m c^{2}}{\pi c} \frac{1}{4 \beta}\left[\left(1+\beta^{2}\right) \ln \frac{1+\beta}{1-\beta}-2 \beta\right],
\end{aligned}
$$

which is for relativistic particles $\gamma \gg 1$

$$
\frac{\mathrm{d} \varepsilon(\omega)}{\mathrm{d} \omega} \approx \frac{2 r_{\mathrm{c}} m c^{2}}{\pi c} \ln \gamma .
$$

The spectral energy emitted into one half space by a single electron in form of transition radiation is uniform for all frequencies reaching up into the $\mathrm{x}$-ray regime and depends only logarithmically on the particle energy.

\subsection{Spatial Radiation Distribution}

Coming back to synchrotron radiation we must define the electron motion in great detail. It is this motion which determines many of the photon beam characteristics. The radiation power and spatial distribution of synchrotron radiation in the electron frame of reference is identical to that from a linear microwave antenna being emitted normal to the direction of acceleration with a $\sin ^{2}$-distribution.

Expressions for the radiation fields and Poynting vector exhibit strong vectorial dependencies on the directions of motion and acceleration of the charged particles and on the direction of observation. These vectorial dependencies indicate that the radiation may not be emitted isotropic but rather into specific directions forming characteristic radiation patterns. Similarly, we note a strong dependence on the photon frequency. In the following paragraphs, we will investigate theses dependencies closer.

\subsubsection{Radiation Lobes}

In this section we will derive these spatial radiation characteristics and determine the direction of preferred radiation emission.

In (25.40) the radiation power per unit solid angle is expressed in the reference frame of the particle

$$
\frac{\mathrm{d} P}{\mathrm{~d} \Omega}=\frac{r_{\mathrm{c}} m c}{4 \pi} \dot{\beta}_{\mathrm{r}}^{* 2} \sin ^{2} \Theta
$$


Fig. 25.3 Radiation pattern in the particle frame of reference or for nonrelativistic particles in the laboratory system

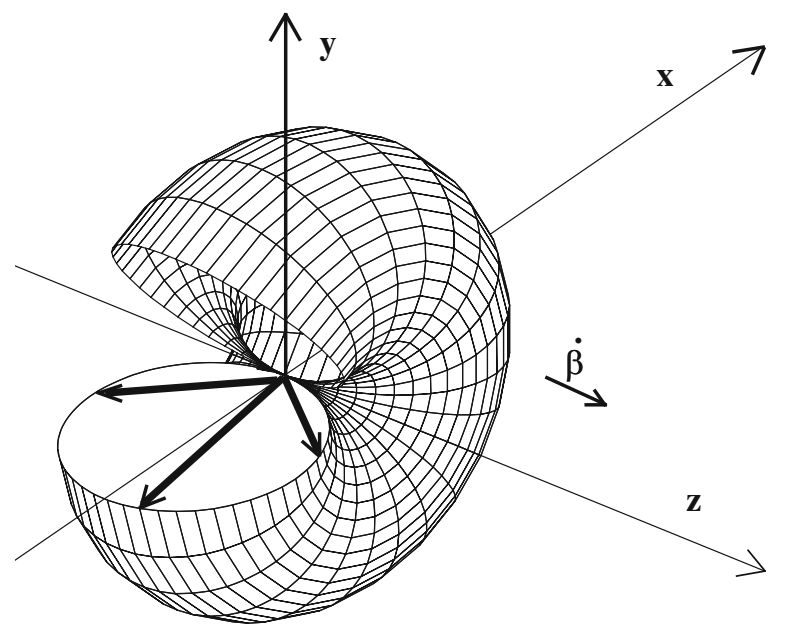

showing a particular directionality of the radiation as shown in Fig. 25.3. The radiation power is mainly concentrated in the $x, y$-plane and is proportional to $\sin ^{2} \Theta$ where $\Theta$ is the angle between the direction of acceleration, in this case the $z$-axis, and the direction of observation $\boldsymbol{n}$. The radiation pattern in Fig. 25.3 is formed by the end points of vectors with the length $\mathrm{d} P / \mathrm{d} \Omega$ and angles $\Theta$ with respect to the $z$ axis. Because of symmetry, the radiation is isotropic with respect to the polar angle $\varphi$ and therefore the radiation pattern is rotation symmetric about the direction of acceleration or in this case about the $z$-axis.

This pattern is the correct representation of the radiation for the reference frame of the radiating particle. We may, however, also consider this pattern as the radiation pattern from non relativistic particles like that from a linear radio antenna. For relativistic particles the radiation pattern differs significantly from the non relativistic case. The Poynting vector in the form of (25.37) can be used to calculate the radiation power per unit solid angle in the direction to the observer $-\boldsymbol{n}$

$$
\frac{\mathrm{d} P}{\mathrm{~d} \Omega}=-\left.\boldsymbol{n S} R^{2}\right|_{\mathrm{r}}=\left.\epsilon_{0} c \boldsymbol{E}^{2}(1+\boldsymbol{\beta} \boldsymbol{n}) R^{2}\right|_{\mathrm{r}} .
$$

We calculate the spatial distribution of the synchrotron radiation for the case of acceleration orthogonal to the propagation of the particle as it happens in beam transport systems where the particles are deflected by a transverse magnetic fields. The particle is assumed to be located at the origin of a right-handed coordinate system as shown in Fig. 25.4 propagating in the $z$-direction and the orthogonal acceleration in this coordinate system occurs along the $x$-axis.

With the expression (25.29) for the electric fields in the radiation regime the spatial radiation power distribution (25.53) becomes 


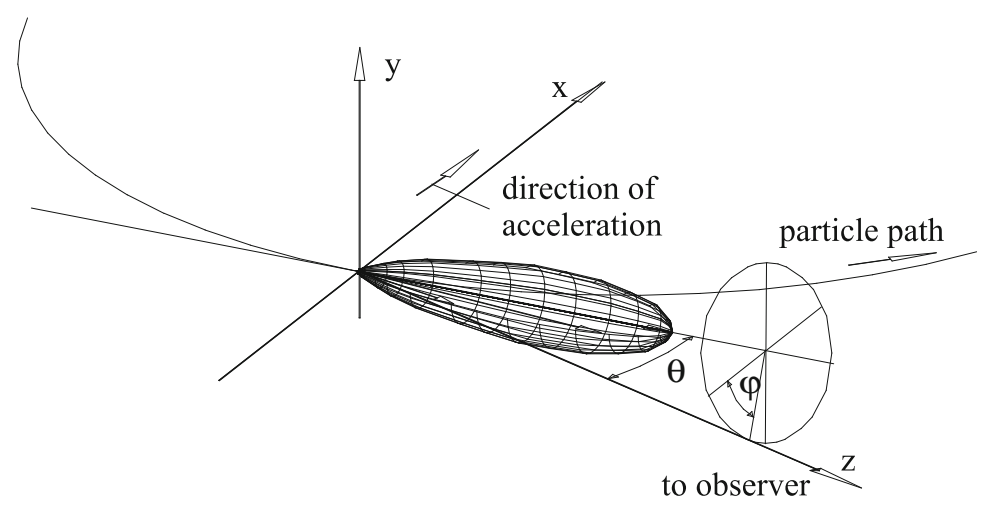

Fig. 25.4 Radiation geometry in the laboratory frame of reference for highly relativistic particles

$$
\frac{\mathrm{d} P}{\mathrm{~d} \Omega}=\frac{c}{4 \pi} r_{\mathrm{c}} m c^{2} \frac{R^{5}}{c^{3} r^{5}}\{\boldsymbol{n} \times[(\boldsymbol{n}+\boldsymbol{\beta}) \times \dot{\boldsymbol{\beta}}]\}^{2} .
$$

We will now replace all vectors by their components to obtain the directional dependency of the synchrotron radiation. The vector $\boldsymbol{n}$ pointing from the observation point to the source point of the radiation has from Fig. 25.4 the components

$$
\boldsymbol{n}=(-\sin \theta \cos \varphi,-\sin \theta \sin \varphi, \cos \theta),
$$

where the angle $\theta$ is the angle between the direction of particle propagation and the direction of emission of the synchrotron light $\boldsymbol{n}$. The $x$-component of the acceleration can be derived from the Lorentz equation

$$
\gamma m \dot{\boldsymbol{v}}_{x}=\frac{\mathrm{d} p_{x}}{\mathrm{~d} t}=c e \beta_{z} B_{y} .
$$

With $v_{z} \approx v$ we have $1 / \rho=c e B_{y} / c p=c e B_{y} /(\gamma m c v)$ and the acceleration vector is

$$
\dot{\boldsymbol{v}}_{\perp}=(\dot{v}, 0,0)=\left(\frac{v^{2}}{\rho}, 0,0\right)
$$

The velocity vector is

$$
\boldsymbol{v}=(0,0, v)
$$

and after replacing the double vector product in (25.54) by a single vector sum

$$
n \times[(n+\beta) \times \beta]=(n+\beta)(n \boldsymbol{\beta})-\boldsymbol{\beta}(\mathbf{1}+n \boldsymbol{\beta}),
$$


we may now square the r.h.s. of (25.54) and replace all vectors by their components. The denominator in (25.54) then becomes

$$
r^{5}=R^{5}(1+\boldsymbol{n} \boldsymbol{\beta})^{5}=R^{5}(1-\beta \cos \theta)^{5},
$$

and the full expression for the radiation power exhibiting the spatial distribution is finally

$$
\frac{\mathrm{d} P}{\mathrm{~d} \Omega}=\frac{r_{\mathrm{c}} m c^{2} c}{4 \pi} \frac{\beta^{4}}{\rho^{2}} \frac{(1-\beta \cos \theta)^{2}-\left(1-\beta^{2}\right) \sin ^{2} \theta \cos ^{2} \varphi}{(1-\beta \cos \theta)^{5}} .
$$

This equation describes the instantaneous synchrotron radiation power per unit solid angle from charged particles moving with velocity $v$ and being accelerated normal to the propagation by a magnetic field. Integration over all angles results again in the total synchrotron radiation power (24.34).

In Fig. 25.5 the radiation power distribution is shown in real space as derived from (25.61). We note that the radiation is highly collimated in the forward direction along the $z$-axis which is also the direction of particle propagation. Synchrotron radiation in particle accelerators or beam lines is emitted whenever there is a deflecting electromagnetic field and emerges mostly tangentially from the particle trajectory. An estimate of the typical opening angle can be derived from (25.61). We set $\varphi=0$ and expand the cosine function for small angles $\cos \theta \approx 1-\frac{1}{2} \theta^{2}$. With $\beta \approx 1-\frac{1}{2} \gamma^{-2}$ we find the radiation power to scale like $\left(\gamma^{-2}+\theta^{2}\right)^{-3}$. The radiation

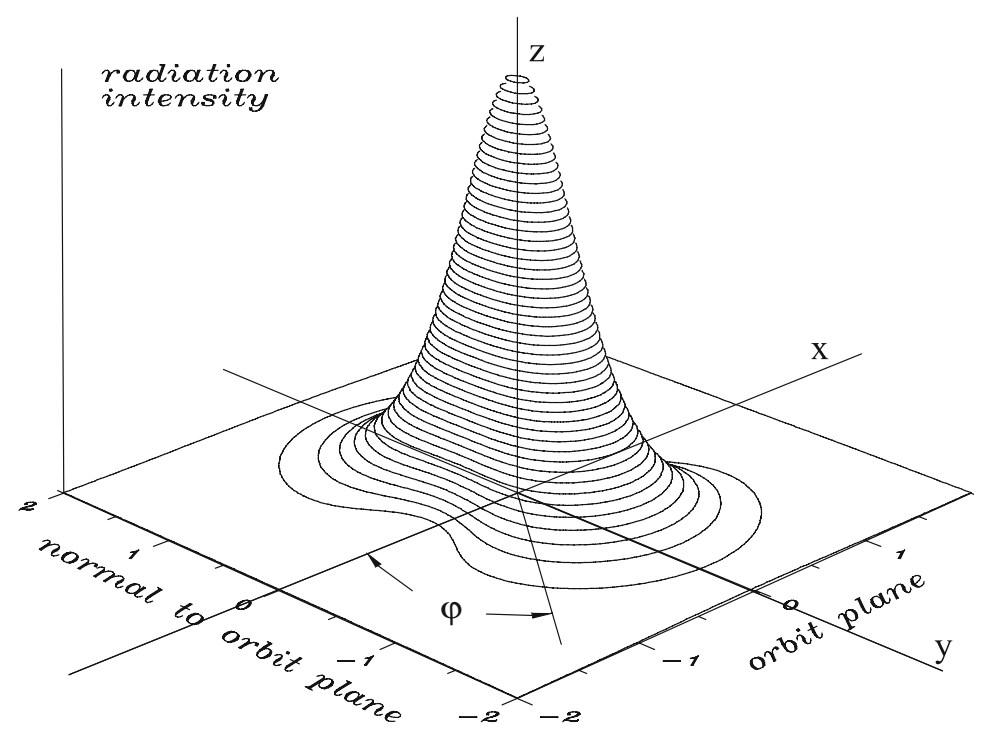

Fig. 25.5 Spatial synchrotron radiation distribution 
power therefore is reduced to about one eighth the peak intensity at an emission angle of $\theta_{\gamma}=1 / \gamma$ or virtually all synchrotron radiation is emitted within an angle of

$$
\theta_{\gamma}= \pm \frac{1}{\gamma}
$$

with respect to the direction of the particle propagation.

From Fig. 25.5 we observe a slightly faster fall off for an azimuthal angle of $\varphi=0$ which is in the plane of particle acceleration and propagation. Although the synchrotron radiation is emitted symmetrically within a small angle of the order of $\pm \frac{1}{\gamma}$ with respect to the direction of particle propagation, the radiation pattern from a relativistic particle as observed in the laboratory is very different in the deflecting plane from that in the nondeflecting plane. While the particle radiates from every point along its path, the direction of this path changes in the deflecting plane but does not in the nondeflecting plane. The synchrotron radiation pattern from a bending magnet therefore resembles the form of a swath where the radiation is emitted evenly and tangentially from every point of the particle trajectory as shown in Fig. 25.6.

The extreme collimation of the synchrotron radiation and its high intensity in high energy electron accelerators can cause significant heating problems as well as desorption of gas molecules from the surface of the vacuum chamber. In addition, the high density of thermal energy deposition on the vacuum chamber walls can cause significant mechanical stresses causing cracks in the material. A careful design of the radiation absorbing surfaces to avoid damage to the integrity of the material is required. On the other hand, this same radiation is a valuable source of photons for a wide variety of research applications where, specifically, the collimation of the radiation together with the small source dimensions are highly desired features of the radiation.

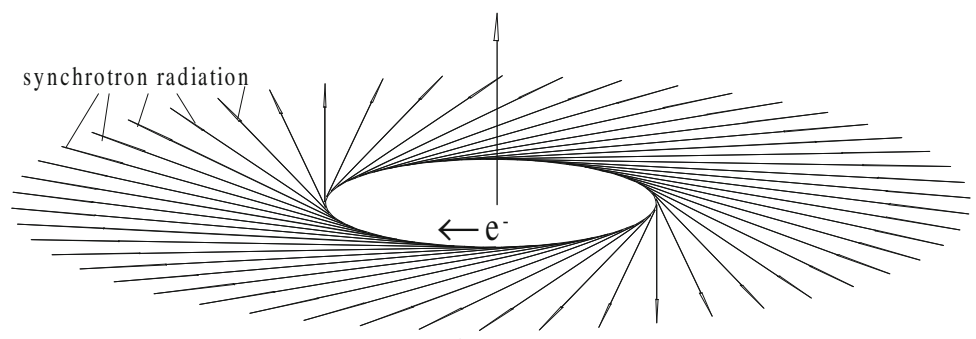

Fig. 25.6 Synchrotron radiation from a circular particle accelerator 


\subsection{Radiation Field in the Frequency Domain}

Synchrotron radiation is emitted within a wide range of frequencies. As we have seen in the previous paragraph, a particle orbiting in a circular accelerator emits light flashes at the revolution frequency. We expect therefore in the radiation frequency spectrum all harmonics of the revolution frequency up to very high frequencies limited only by the very short duration of the radiation pulse being sent into a particular direction toward the observer. The number of harmonics increases with beam energy and reaches at the critical frequency the order of $\gamma^{3}$.

The frequency spectrum of synchrotron radiation has been derived by many authors. In this text, we will stay closer to the derivation by Jackson [3] than others. The general method to derive the frequency spectrum is to transform the electric field from the time domain to the frequency domain by the use of Fourier transforms. Applying this method, we will determine the radiation characteristics of the light emitted by a single pass of a particle in a circular accelerator at the location of the observer. The electric field at the observation point has a strong time dependence and is given by (25.29) while the total radiation energy for one pass is from (25.38)

$$
\frac{\mathrm{d} W}{\mathrm{~d} \Omega}=-\int_{-\infty}^{\infty} \frac{\mathrm{d} P}{\mathrm{~d} \Omega} \mathrm{d} t=\int_{-\infty}^{\infty} \boldsymbol{S}_{\mathrm{r}} \boldsymbol{n} R^{2} \mathrm{~d} t=\epsilon_{0} c R^{2} \int_{-\infty}^{\infty} \boldsymbol{E}_{\mathrm{r}}^{2}(t) \mathrm{d} t .
$$

The transformation from the time domain to the frequency domain is performed by a Fourier transform or an expansion into Fourier harmonics. This is the point where the particular characteristics of the transverse acceleration depend on the magnetic field distribution and are, for example, different in a single bending magnet as compared to an oscillatory wiggler magnet. We use here the method of Fourier transforms to describe the electric field of a single particle passing only once through a homogeneous bending magnet. In case of a circular accelerator the particle will appear periodically with the period of the revolution time and we expect a correlation of the frequency spectrum with the revolution frequency. This is indeed the case and we will later discuss the nature of this correlation. Expressing the electrical field $\mathbf{E}_{\mathrm{r}}(t)$ by its Fourier transform, we set

$$
\boldsymbol{E}_{\mathrm{r}}(\omega)=\int_{-\infty}^{\infty} \boldsymbol{E}_{\mathrm{r}}(t) \mathrm{e}^{-\mathrm{i} \omega t} \mathrm{~d} t
$$

where $-\infty<\omega<\infty$. Applying Parseval's theorem (A.42) the total absorbed radiation energy from a single pass of a particle is therefore

$$
\frac{\mathrm{d} W}{\mathrm{~d} \Omega}=\epsilon_{0} c \frac{R^{2}}{2 \pi} \int_{-\infty}^{\infty}\left|\boldsymbol{E}_{\mathrm{r}}(\omega)\right|^{2} \mathrm{~d} \omega .
$$


Evaluating the electrical field by its Fourier components, we derive an expression for the spectral distribution of the radiation energy

$$
\frac{\mathrm{d}^{2} W}{\mathrm{~d} \Omega \mathrm{d} \omega}=\epsilon_{0} \frac{c}{\pi}\left|\boldsymbol{E}_{\mathrm{r}}(\omega)\right|^{2} R_{\mathrm{r}}^{2},
$$

where we have implicitly used the fact that $\boldsymbol{E}_{\mathrm{r}}(\omega)=\boldsymbol{E}_{\mathrm{r}}(-\omega)$ since $\boldsymbol{E}_{\mathrm{r}}(t)$ is real. To calculate the Fourier transform, we use (25.29) and note that the electrical field is expressed in terms of quantities at the retarded time. The calculation is simplified if we express the whole integrand in (25.64) at the retarded time and get with $t_{\mathrm{r}}=$ $t-\frac{1}{c} R\left(t_{\mathrm{r}}\right)$ and $\mathrm{d} t_{\mathrm{r}}=\frac{R\left(t_{\mathrm{r}}\right)}{r} \mathrm{~d} t$ instead of (25.64)

$$
\boldsymbol{E}_{\mathrm{r}}(\omega)=\left.\frac{1}{4 \pi \epsilon_{0}} \frac{e}{c} \int_{-\infty}^{\infty} \frac{\boldsymbol{R} \times[(\boldsymbol{R}+\boldsymbol{\beta} R) \times \dot{\boldsymbol{\beta}}]}{r^{2} R}\right|_{\mathrm{r}} \mathrm{e}^{-\mathrm{i} \omega\left(t_{\mathrm{r}}+\frac{R_{\mathrm{r}}}{c}\right)} \mathrm{d} t_{\mathrm{r}}
$$

We require now that the radiation be observed at a point sufficiently far away from the source that during the time of emission the vector $\boldsymbol{R}\left(t_{\mathrm{r}}\right)$ does not change appreciably in direction. This assumption is generally justified since the duration of the photon emission is of the order of $1 /\left(\omega_{\mathrm{L}} \gamma\right)$, where $\omega_{\mathrm{L}}=c / \rho$ is the Larmor frequency. The observer therefore should be at a distance from the source large compared to $\rho / \gamma$. Equation (25.67) together with (25.14) may then be written like

$$
\boldsymbol{E}_{\mathrm{r}}(\omega)=\left.\frac{1}{4 \pi \epsilon_{0}} \frac{e}{c R} \int_{-\infty}^{\infty} \frac{\boldsymbol{n} \times[(\boldsymbol{n}+\boldsymbol{\beta}) \times \dot{\boldsymbol{\beta}}]}{(1+\boldsymbol{n} \boldsymbol{\beta})^{2}}\right|_{\mathrm{r}} \mathrm{e}^{-\mathrm{i} \omega\left(t_{\mathrm{r}}+\frac{R_{\mathrm{r}}}{c}\right)} \mathrm{d} t_{\mathrm{r}}
$$

With

$$
\frac{\boldsymbol{n} \times[(\boldsymbol{n}+\boldsymbol{\beta}) \times \dot{\boldsymbol{\beta}}]}{(1+\boldsymbol{n} \boldsymbol{\beta})^{2}}=\frac{\mathrm{d}}{\mathrm{d} t_{\mathrm{r}}} \frac{\boldsymbol{n} \times(\boldsymbol{n} \times \boldsymbol{\beta})}{1+\boldsymbol{n} \boldsymbol{\beta}},
$$

we integrate (25.68) by parts while noting that the integrals vanish at the boundaries and get

$$
\boldsymbol{E}_{\mathrm{r}}(\omega)=\frac{1}{4 \pi \epsilon_{0}} \frac{-\mathrm{i} e \omega}{c R} \int_{-\infty}^{\infty}[\boldsymbol{n} \times(\boldsymbol{n} \times \boldsymbol{\beta})]_{\mathrm{r}} \mathrm{e}^{-\mathrm{i} \omega\left(t_{\mathrm{r}}+\frac{R_{\mathrm{r}}}{c}\right)} \mathrm{d} t_{\mathrm{r}} .
$$

After insertion into (25.66) the spectral and spatial intensity distribution is

$$
\frac{\mathrm{d}^{2} W}{\mathrm{~d} \Omega \mathrm{d} \omega}=\frac{r_{\mathrm{c}} m c^{2}}{4 \pi c} \omega^{2}\left|\int_{-\infty}^{\infty}[\boldsymbol{n} \times(\boldsymbol{n} \times \boldsymbol{\beta})] \mathrm{e}^{-\mathrm{i} \omega\left(t_{\mathrm{r}}+\frac{R_{\mathrm{r}}}{c}\right)} \mathrm{d} t_{\mathrm{r}}\right|_{\mathrm{r}}^{2} .
$$




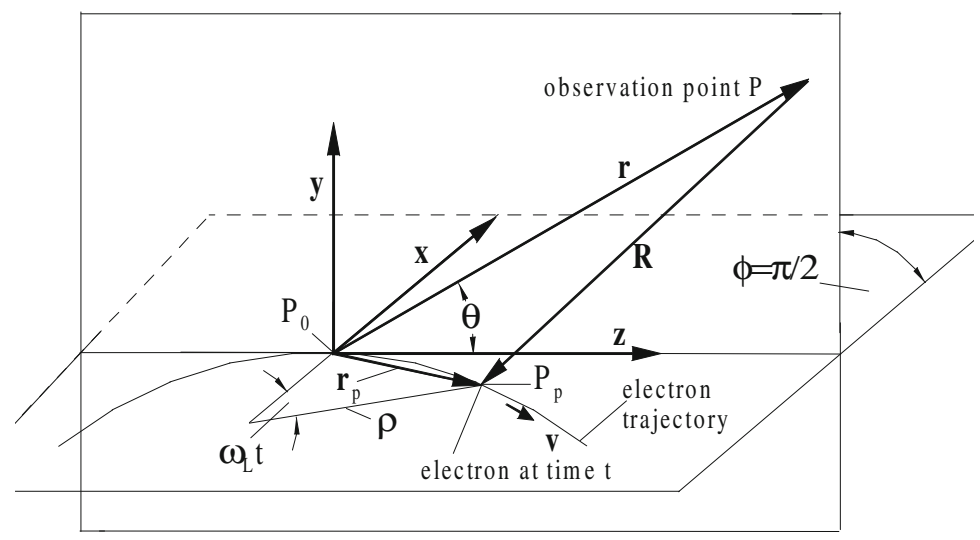

Fig. 25.7 Radiation geometry

The spectral and spatial radiation distribution depends on the Fourier transform of the particle trajectory which itself is a function of the magnetic field distribution. The trajectory in a uniform dipole field is different from say the step function of real lumped bending magnets or oscillating deflecting fields from wiggler magnets and the radiation characteristics may therefore be different. In this chapter, we will concentrate only on a uniform dipole field and postpone the discussion of specific radiation characteristics for insertion devices to Chap. 26.

The integrand in (25.71) can be expressed in component form to simplify integration. For that we consider a fixed coordinate system $(x, y, z)$ as shown in Fig. 25.7. The observation point is far away from the source point and we focus on the radiation that is centered about the tangent to the orbit at the source point. The observation point $P$ and the vectors $\boldsymbol{R}$ and $\boldsymbol{n}$ are therefore within the $(y, z)$-plane and radiation is emitted at angles $\theta$ with respect to the $z$-axis.

The vector from the origin of the coordinate system $P_{0}$ to the observation point $P$ is $\boldsymbol{r}$, the vector $\boldsymbol{R}$ is the vector from $P$ to the particle at $P_{\mathrm{p}}$ and $\boldsymbol{r}_{\mathrm{p}}$ is the vector from the origin to $P_{\mathrm{p}}$. With this we have

$$
\boldsymbol{r}=\boldsymbol{r}_{\mathrm{p}}-\boldsymbol{R}\left(t_{\mathrm{r}}\right)
$$

where $\boldsymbol{r}_{\mathrm{p}}$ and $\boldsymbol{R}_{\mathrm{r}}$ are taken at the retarded time. The exponent in (25.71) is then

$$
\omega\left(t_{\mathrm{r}}+R_{\mathrm{r}} / c\right)=\omega\left(t_{\mathrm{r}}+\boldsymbol{n} \boldsymbol{R}_{\mathrm{r}} / c\right)=\frac{\omega}{c}\left(c t_{\mathrm{r}}+\boldsymbol{n} \boldsymbol{r}_{\mathrm{p}}-\boldsymbol{n} \boldsymbol{r}\right)
$$

and the term $-\frac{\omega}{c} \boldsymbol{n} \boldsymbol{r}$ is independent of the time generating only a constant phase factor which is completely irrelevant for the spectral distribution and may therefore be ignored.

Following the above discussion the azimuthal angle is constant and set to $\varphi=\frac{1}{2} \pi$ because we are interested only in the vertical radiation distribution. The 
horizontal distribution is uniform by virtue of the tangential emission along the orbit. With these assumptions, we get the vector components for the vector $\boldsymbol{n}$ from (25.55)

$$
\boldsymbol{n}=(0,-\sin \theta,-\cos \theta) .
$$

The vector $\mathbf{r}_{\mathrm{p}}$ is defined by Fig. 25.7 and depends on the exact variation of the deflecting magnetic field along the path of the particles. Here we assume a constant bending radius $\rho$ and have

$$
\boldsymbol{r}_{\mathrm{p}}=\left[-\rho \cos \left(\omega_{\mathrm{L}} t_{\mathrm{r}}\right), 0, \rho \sin \left(\omega_{\mathrm{L}} t_{\mathrm{r}}\right)\right],
$$

where $\omega_{\mathrm{L}}=\beta c / \rho$ is the Larmor frequency. From these component representations the vector product

$$
\boldsymbol{n} \boldsymbol{r}_{\mathrm{p}}=-\rho \sin \left(\omega_{\mathrm{L}} t_{\mathrm{r}}\right) \cos \theta
$$

Noting that both arguments of the trigonometric functions in (25.76) are very small, we may expand the r.h.s. of (25.76) up to third order in $t_{\mathrm{r}}$ and the factor $t_{\mathrm{r}}+\boldsymbol{n} \boldsymbol{r}_{\mathrm{p}} / c$ in (25.73) becomes

$$
c t_{\mathrm{r}}+\boldsymbol{n} \boldsymbol{r}_{\mathrm{p}}=c t_{\mathrm{r}}-\rho\left[\omega_{\mathrm{L}} t_{\mathrm{r}}-\frac{1}{6}\left(\omega_{\mathrm{L}} t_{\mathrm{r}}\right)^{3}\left(1-\frac{1}{2} \theta^{2}\right)\right] .
$$

With $\omega_{\mathrm{L}}=\beta c / \rho$ we get $t_{\mathrm{r}}\left(1-\rho \omega_{\mathrm{L}} / c\right)=(1-\beta) t_{\mathrm{r}} \approx t_{\mathrm{r}} /\left(2 \gamma^{2}\right)$. Keeping only up to third order terms in $\omega_{\mathrm{L}} t_{\mathrm{r}}$ and $\theta$ we have finally for high energetic particles $\beta \approx 1$

$$
t_{\mathrm{r}}+\frac{\boldsymbol{n} \boldsymbol{r}_{\mathrm{p}}}{c}=\frac{1}{2}\left(\gamma^{-2}+\theta^{2}\right) t_{\mathrm{r}}+\frac{1}{6} \omega_{\mathrm{L}}^{2} t_{\mathrm{r}}^{3} .
$$

The triple vector product in (25.71) can be evaluated in a similar way. For the velocity vector we derive from Fig. 25.7

$$
\boldsymbol{\beta}=\beta\left[-\operatorname{sign}(1 / \rho) \sin \left(\omega_{\mathrm{L}} t_{\mathrm{r}}\right), 0, \cos \left(\omega_{\mathrm{L}} t_{\mathrm{r}}\right)\right] .
$$

Consistent with the definition of the curvature, the sign of the curvature sign $(1 / \rho)$ is positive for a positive charge and a positive magnetic field vector $B_{y}$. The vector relation (A.10) and (25.74), (25.79) can be used to express the triple vector product in terms of its components

$$
\boldsymbol{n} \times(\boldsymbol{n} \times \boldsymbol{\beta})=\beta\left[\operatorname{sign}(1 / \rho) \sin \left(\omega_{\mathrm{L}} t_{\mathrm{r}}\right), \frac{1}{2} \sin 2 \theta \cos \left(\omega_{\mathrm{L}} t_{\mathrm{r}}\right),-\sin ^{2} \theta \cos \left(\omega_{\mathrm{L}} t_{\mathrm{r}}\right)\right] .
$$

Splitting this three-dimensional vector into two parts will allow us to characterize the polarization states of the radiation. To do this, we take the unit vector $\boldsymbol{u}_{\perp}$ in the $x$-direction and $\boldsymbol{u}_{\|}$a unit vector normal to $\boldsymbol{u}_{\perp}$ and normal to $\boldsymbol{r}$. The $y$ and $z$ - components of (25.80) are then also the components of $\boldsymbol{u}_{\|}$and we may express 
the vector (25.80) by

$$
\boldsymbol{n} \times(\boldsymbol{n} \times \boldsymbol{\beta})=\beta \operatorname{sign}(1 / \rho) \sin \left(\omega_{\mathrm{L}} t_{\mathrm{r}}\right) \boldsymbol{u}_{\perp}+\beta \sin \theta \cos \left(\omega_{\mathrm{L}} t_{\mathrm{r}}\right) \boldsymbol{u}_{\|},
$$

Inserting (25.78) and (25.81) into the integrand (25.70) we get with $\beta \approx 1$

$$
\boldsymbol{E}_{\mathrm{r}}(\omega)=-\frac{1}{4 \pi \epsilon_{0}} \frac{e}{R} \frac{\omega}{c} \int_{-\infty}^{\infty}\left[\operatorname{sign}(1 / \rho) \sin \left(\omega_{\mathrm{L}} t_{\mathrm{r}}\right) \boldsymbol{u}_{\perp}+\sin \theta \cos \left(\omega_{\mathrm{L}} t_{\mathrm{r}}\right) \boldsymbol{u}_{\|}\right] \mathrm{e}^{X} \mathrm{~d} t_{\mathrm{r}},
$$

where

$$
X=-\mathrm{i} \frac{\omega}{2 \gamma^{2}}\left[\left(1+\gamma^{2} \theta^{2}\right) t_{\mathrm{r}}+\frac{1}{3} \gamma^{2} \omega_{\mathrm{L}}^{2} t_{\mathrm{r}}^{3}\right]
$$

Two polarization directions have been defined for the electric radiation field. One of which $\left(\boldsymbol{u}_{\perp}\right)$ is in the plane of the particle path being perpendicular to the particle velocity and to the deflecting magnetic field. Following Sokolov and Ternov [4] we call this the $\sigma$-mode $\left(\boldsymbol{u}_{\perp}=\boldsymbol{u}_{\sigma}\right)$. The other polarization direction in the plane containing the deflecting magnetic field and the observation point is perpendicular to $\boldsymbol{n}$ and is called the $\pi$-mode $\left(\boldsymbol{u}_{\|}=\boldsymbol{u}_{\pi}\right)$. Since the emission angle $\theta$ is very small, we find this polarization direction to be mostly parallel to the magnetic field. Noting that most accelerators or beam lines are constructed in the horizontal plane, the polarizations are also often referred to as the horizontal polarization for the $\sigma$-mode and as the vertical polarization for the $\pi$-mode.

\subsubsection{Spectral Distribution in Space and Polarization}

As was pointed out by Jackson [3], the mathematical need to extend the integration over infinite times does not invalidate our expansion of the trigonometric functions where we assumed the argument $\omega_{\mathrm{L}} t_{\mathrm{r}}$ to be small. Although the integral (25.82) extends over all past and future times, the integrand oscillates rapidly for all but the lowest frequencies and therefore only times of the order $c t_{\mathrm{r}}= \pm \rho / \gamma$ centered about $t_{\mathrm{r}}$ contribute to the integral. This is a direct consequence of the fact that the radiation is emitted in the forward direction and therefore only photons from a very small segment of the particle trajectory reach the observation point. For very low frequencies of the order of the Larmor frequency, however, we must expect considerable deviations from our results. In practical circumstances such low harmonics will, however, not propagate in the vacuum chamber [5] and the observed photon spectrum therefore is described accurately for all practical purposes.

The integral in (25.82) can be expressed by modified Bessel's functions in the form of Airy's integrals as has been pointed out by Schwinger [6]. Since the deflection angle $\omega_{\mathrm{L}} t_{\mathrm{r}}$ is very small, we may use linear expansions $\sin \left(\omega_{\mathrm{L}} t_{\mathrm{r}}\right) \approx$ 
$\omega_{\mathrm{L}} t_{\mathrm{r}}$ and $\cos \left(\omega_{\mathrm{L}} t_{\mathrm{r}}\right) \approx 1$. Inserting the expression for the electric field (25.82) into (25.65) we note that cross terms of both polarizations vanish $\boldsymbol{u}_{\perp} \boldsymbol{u}_{\|}=0$ and the radiation intensity can therefore be expressed by two separate orthogonal polarization components. Introducing in (25.82) the substitutions [6]

$$
\begin{aligned}
\omega_{\mathrm{L}} t_{\mathrm{r}} & =\sqrt{\frac{1}{\gamma^{2}}+\theta^{2}} x, \\
\xi & =\frac{1}{3} \frac{\omega}{\omega_{\mathrm{L}}} \frac{1}{\gamma^{3}}\left(1+\gamma^{2} \theta^{2}\right)^{3 / 2}=\frac{1}{2} \frac{\omega}{\omega_{\mathrm{c}}}\left(1+\gamma^{2} \theta^{2}\right)^{3 / 2},
\end{aligned}
$$

where $\hbar \omega_{\mathrm{c}}$ is the critical photon energy, the argument in the exponential factor of (25.82) becomes

$$
\frac{\omega}{2 \gamma^{2}}\left[\left(1+\gamma^{2} \theta^{2}\right) t_{\mathrm{r}}+\frac{1}{3} \gamma^{2} \omega_{\mathrm{L}}^{2} t_{\mathrm{r}}^{3}\right]=\frac{1}{2} \xi\left(3 x+x^{3}\right) .
$$

With these substitutions, (25.82) can be evaluated noting that only even terms contribute to the integral. With $\omega_{\mathrm{L}} t_{\mathrm{r}}$ and $\theta$ being small quantities we get integrals of the form [7]

$$
\begin{aligned}
& \int_{0}^{\infty} \cos \left[\frac{1}{2} \xi\left(3 x+x^{3}\right)\right] \mathrm{d} x=\frac{1}{\sqrt{3}} K_{1 / 3}(\xi), \\
& \int_{0}^{\infty} \sin \left[\frac{1}{2} \xi\left(3 x+x^{3}\right)\right] \mathrm{d} x=\frac{1}{\sqrt{3}} K_{2 / 3}(\xi),
\end{aligned}
$$

where the functions $K_{v}$ are modified Bessels's functions of the second kind. These functions assume finite values for small arguments but vanish exponentially for large arguments as shown in Fig. 24.12. Fast converging series for these modified Bessels's functions with fractional index have been derived by Kostroun [8]. The Fourier transform of the electrical field (25.82) finally becomes

$$
\boldsymbol{E}_{\mathrm{r}}(\omega)=\frac{-1}{4 \pi \epsilon_{0}} \frac{\sqrt{3} e}{c R} \frac{\omega}{\omega_{\mathrm{c}}} \gamma\left(1+\gamma^{2} \theta^{2}\right)\left[\operatorname{sign}\left(\frac{1}{\rho}\right) K_{2 / 3}(\xi) \boldsymbol{u}_{\sigma}-\mathrm{i} \frac{\gamma \theta K_{1 / 3}(\xi)}{\sqrt{1+\gamma^{2} \theta^{2}}} \boldsymbol{u}_{\pi}\right],
$$

describing the spectral radiation field far from the source for particles traveling through a uniform magnetic dipole field. Later, we will modify this expression to make it suitable for particle motion in undulators or other nonuniform fields.

The spectral synchrotron radiation energy emitted by one electron per pass is proportional to the square of the electrical field (25.87) and is from (25.66)

$$
\frac{\mathrm{d}^{2} W}{\mathrm{~d} \Omega \mathrm{d} \omega}=\frac{3 r_{\mathrm{c}} m c}{4 \pi^{2}} \gamma^{2}\left(\frac{\omega}{\omega_{\mathrm{c}}}\right)^{2}\left(1+\gamma^{2} \theta^{2}\right)^{2}\left[K_{2 / 3}^{2}(\xi) \boldsymbol{u}_{\sigma}^{2}+\frac{\gamma^{2} \theta^{2} K_{1 / 3}^{2}(\xi)}{1+\gamma^{2} \theta^{2}} \boldsymbol{u}_{\pi}^{2}\right] .
$$


Fig. 25.8 Radiation lobes for $\sigma$ - and $\pi$-mode polarization

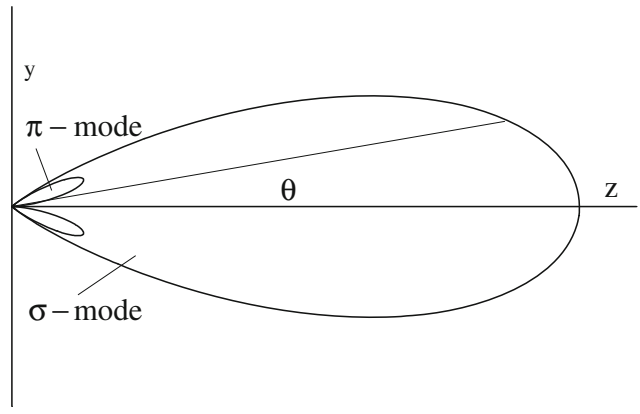

The radiation spectrum has two components of orthogonal polarization, one in the plane of the particle trajectory and the other almost parallel to the deflecting magnetic field. In (25.87) both polarizations appear explicitly through the orthogonal unit vectors. Forming the square of the electrical field to get the radiation intensity, cross terms disappear because of the orthogonality of the unit vectors $\boldsymbol{u}_{\sigma}$ and $\boldsymbol{u}_{\pi}$. The expression for the radiation intensity therefore preserves separately the two polarization modes in the square brackets of (25.88) representing the $\sigma$-mode and $\pi$-mode of polarization, respectively.

It is interesting to study the spatial distribution for the two polarization modes in more detail. Not only are the intensities very different but the spatial distribution is different too. The spatial distribution of the $\sigma$-mode is directed mainly in the forward direction while the $\pi$-mode radiation is emitted into two lobes at finite angles and zero intensity in the forward direction $\theta=0$. In Fig. 25.8 the instantaneous radiation lobes are shown for both the $\sigma$ - and the $\pi$-mode at the critical photon energy and being emitted tangentially from the orbit at the origin of the coordinate system.

\subsubsection{Spectral and Spatial Photon Flux}

The radiation intensity $W$ from a single electron and for a single pass may not always be the most useful parameter. A more useful parameter is the spectral photon flux per unit solid angle into a frequency bin $\Delta \omega / \omega$ and for a circulating beam current $I$

$$
\frac{\mathrm{d}^{2} \dot{N}_{\mathrm{ph}}(\omega)}{\mathrm{d} \theta \mathrm{d} \psi}=\frac{\mathrm{d}^{2} W(\omega)}{\mathrm{d} \omega \mathrm{d} \Omega} \frac{1}{\hbar} \frac{I}{e} \frac{\Delta \omega}{\omega} .
$$

Here we have replaced the solid angle by its components, the vertical angle $\theta$ and the bending angle $\psi$. In more practical units the differential photon flux is

$$
\frac{\mathrm{d}^{2} \dot{N}_{\mathrm{ph}}(\omega)}{\mathrm{d} \theta \mathrm{d} \psi}=C_{\Omega} E^{2} I \frac{\Delta \omega}{\omega}\left(\frac{\omega}{\omega_{\mathrm{c}}}\right)^{2} K_{2 / 3}^{2}(\xi) F(\xi, \theta),
$$



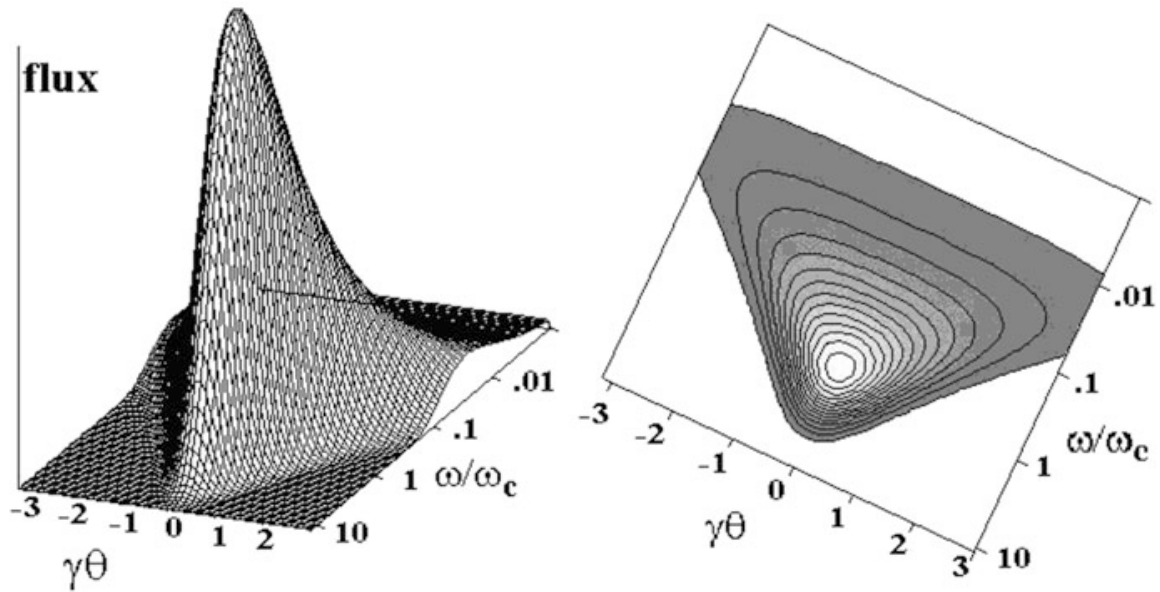

Fig. 25.9 Distribution in frequency and angular space for $\sigma$-mode radiation

where

$$
C_{\Omega}=\frac{3 \alpha}{4 \pi^{2} e\left(m c^{2}\right)^{2}}=1.3273 \times 10^{16} \frac{\text { photons }}{\mathrm{s} \mathrm{mrad}^{2} \mathrm{GeV}^{2} \mathrm{~A}},
$$

$\alpha$ the fine structure constant and

$$
F(\xi, \theta)=\left(1+\gamma^{2} \theta^{2}\right)^{2}\left[1+\frac{\gamma^{2} \theta^{2}}{1+\gamma^{2} \theta^{2}} \frac{K_{1 / 3}^{2}(\xi)}{K_{2 / 3}^{2}(\xi)}\right] .
$$

For approximate numerical calculations of photon fluxes, we may use the graphic representation in Fig. 24.12 for the modified Bessel's function.

The spatial radiation pattern varies with the frequency of the radiation. Specifically, the angular distribution concentrates more and more in the forward direction as the radiation frequency increases. The radiation distribution in frequency and angular space is shown for both the $\sigma$ - (Fig. 25.9) and the $\pi$-mode (Fig. 25.10) at the fundamental frequency. The high collimation of synchrotron radiation in the forward direction makes it a prime research tool to probe materials and its atomic and molecular properties.

\subsubsection{Harmonic Representation}

Expression (25.88) can be transformed into a different formulation emphasizing the harmonic structure of the radiation spectrum. The equivalence between both formulations has been shown by Sokolov and Ternov [4] expressing the modified 

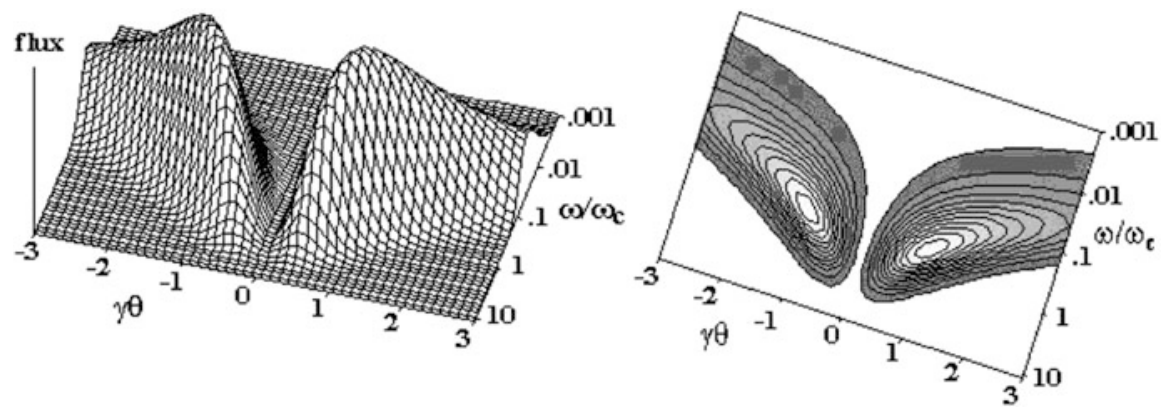

Fig. 25.10 Distribution in frequency and angular space for $\pi$-mode radiation

Bessel's functions $K_{1 / 3}$ and $K_{2 / 3}$ by regular Bessel's functions of high order. With $v=\frac{\omega}{\omega_{\mathrm{L}}}$ the asymptotic formulas for $v \gg 1$ are

$$
\begin{aligned}
& K_{1 / 3}(\xi)=\frac{\sqrt{3} \pi}{\sqrt{1-\beta^{2} \cos ^{2} \theta}} J_{\nu}(\nu \beta \cos \theta), \\
& K_{2 / 3}(\xi)=\frac{\sqrt{3} \pi}{1-\beta^{2} \cos ^{2} \theta} J_{\nu}^{\prime}(\nu \beta \cos \theta),
\end{aligned}
$$

where $\xi=\frac{v}{3}\left(1-\beta^{2} \cos ^{2} \theta\right)^{3 / 2} \approx \frac{v}{3}\left(\gamma^{-2}+\beta^{2} \theta^{2}\right)^{3 / 2}$ for small angles. These approximations are justified since we are only interested in very large harmonics of the revolution frequency. The harmonic number $v$ for the critical photon frequency, for example, is given by $v_{\mathrm{c}}=\omega_{\mathrm{c}} / \omega_{\mathrm{L}}=\frac{3}{2} \gamma^{3}$ which for practical cases is generally a very large number. Inserting these approximations into (25.88) gives the formulation that has been derived first by Schott [9-11] in 1907 long before synchrotron radiation was discovered in an attempt to calculate the radiation intensity of atomic spectral lines

$$
\frac{\mathrm{d}^{2} P}{\mathrm{~d} \Omega \mathrm{d} \nu}=\frac{r_{\mathrm{c}} m c^{3}}{2 \pi \rho^{2}} v^{2}\left[J_{v}^{\prime 2}(v \cos \theta)+\theta^{2} J_{v}^{2}(v \cos \theta)\right],
$$

where we have introduced the radiation power $P=W \frac{c}{2 \pi \rho}$. This form still exhibits the separation of the radiation into the two polarization modes.

\subsubsection{Spatial Radiation Power Distribution}

Integrating over all frequencies we obtain the angular distribution of the synchrotron radiation. From (25.88) we note the need to perform integrals of the form $\int_{-\infty}^{\infty} \omega^{2} K_{\mu}^{2}(a \omega) \mathrm{d} \omega$, where $a \omega=\xi$. The solution can be found in the integral tables 
of Gradshteyn and Ryzhik [12] as solution number GR(6.576.4) ${ }^{1}$

$$
\int_{0}^{\infty} \omega^{2} K_{\mu}^{2}(a \omega) d \omega=\frac{\pi^{2}}{32 a^{3}} \frac{1-4 \mu^{2}}{\cos \pi \mu},
$$

for $a>0$, and $-1.5<\mu<1.5$. Applying this solution to (25.88) and integrating over all frequencies, we get for the angular energy distribution of the synchrotron radiation per electron

$$
\frac{\mathrm{d} W}{\mathrm{~d} \Omega}=\frac{7}{16} \frac{r_{\mathrm{c}} m c^{2}}{\rho} \frac{\gamma^{5}}{\left(1+\gamma^{2} \theta^{2}\right)^{5 / 2}}\left(1+\frac{5}{7} \frac{\gamma^{2} \theta^{2}}{1+\gamma^{2} \theta^{2}}\right) .
$$

This result is consistent with the angular radiation power distribution (25.61) where we found that the radiation is collimated very much in the forward direction with most of the radiation energy being emitted within an angle of $\pm 1 / \gamma$. There are two contributions to the total radiation intensity, the $\sigma$-mode and the $\pi$-mode. The $\sigma$-mode has a maximum intensity in the forward direction, while the maximum intensity for the $\pi$-mode occurs at an angle of $\theta_{\pi}=1 /(\sqrt{5 / 2} \gamma)$. The quantity $\mathrm{d} W / \mathrm{d} \Omega$ is the radiation energy per unit solid angle from a single electron and a single pass and the average radiation power is therefore $P_{\gamma}=W / T_{\text {rev }}$ or (25.97) becomes

$$
\frac{\mathrm{d} P_{\gamma}}{\mathrm{d} \Omega}=\frac{7 r_{\mathrm{c}} m c^{3}}{32 \pi \rho^{2}} \frac{\gamma^{5}}{\left(1+\gamma^{2} \theta^{2}\right)^{5 / 2}}\left(1+\frac{5}{7} \frac{\gamma^{2} \theta^{2}}{1+\gamma^{2} \theta^{2}}\right) .
$$

Integrating (25.98) over all angles $\theta$, we find the synchrotron radiation power into both polarization modes. In doing so, we note first that (25.98) can be simplified with (24.34) and $\beta=1$

$$
\frac{\mathrm{d} P_{\gamma}}{\mathrm{d} \Omega}=\frac{21}{32} \frac{P_{\gamma}}{2 \pi} \frac{\gamma}{\left(1+\gamma^{2} \theta^{2}\right)^{5 / 2}}\left(1+\frac{5}{7} \frac{\gamma^{2} \theta^{2}}{1+\gamma^{2} \theta^{2}}\right) .
$$

This result is consistent with (25.61) although it should be noted that (25.99) gives the average radiation power from a circular accelerator with uniform intensity in $\psi$, while (25.61) is the instantaneous power into the forward lobe. Equation (25.99) exhibits the power into each polarization mode for which the total power can be obtained by integration over all angles. First, we integrate over all points along the circular orbit and get a factor $2 \pi$ since the observed radiation power does not depend on the location along the orbit. Continuing the integration over all angles of $\theta$, we find the contributions to the integral to become quickly negligible for angles larger than $1 / \gamma$. If it were not so, we could not have used (25.99) where the

\footnotetext{
${ }^{1}$ In this chapter we will need repeatedly results from mathematical tables. We abbreviate such solutions with the first letters of the authors names and the formula number.
} 
trigonometric functions have been replaced by their small arguments. Both terms in (25.99) can be integrated readily and the first term becomes with GR(2.271.6) [12]

$$
\int_{\theta_{\max } \gamma \ll 1}^{\theta_{\max } \gamma \gg 1} \frac{\gamma \mathrm{d} \theta}{\left(1+\gamma^{2} \theta^{2}\right)^{5 / 2}}=\frac{4}{3} .
$$

The second term is with GR[2.272.7] [12]

$$
\int_{\theta_{\max } \gamma \ll 1}^{\theta_{\max } \gamma \gg 1} \frac{\gamma^{3} \theta^{2} \mathrm{~d} \theta}{\left(1+\gamma^{2} \theta^{2}\right)^{7 / 2}}=\frac{4}{15} .
$$

With these integrals and (25.99) the radiation power into the $\sigma$ - and $\pi$-mode with $P_{\gamma}$ from (24.34) is

$$
\begin{aligned}
& P_{\sigma}=\frac{7}{8} P_{\gamma}, \\
& P_{\pi}=\frac{1}{8} P_{\gamma} .
\end{aligned}
$$

The horizontally polarized component of synchrotron radiation greatly dominates the photon beam characteristics and only $12.5 \%$ of the total intensity is polarized in the vertical plane. In the forward direction the $\sigma$-polarization even approaches $100 \%$. Obviously, the sum of both components is equal to the total radiation power. This high polarization of the radiation provides a valuable characteristic for experimentation with synchrotron radiation. In addition, the emission of polarized light generates a slow polarizing reaction on the particle beam orbiting in a circular accelerator like in a storage ring [13].

\subsection{Asymptotic Solutions}

Expressions for the radiation distribution can be greatly simplified if we restrict the discussion to very small or very large arguments of the modified Bessel's functions for which approximate expressions exist [14]. Knowledge of the radiation distribution at very low photon frequencies becomes important for experiments using such radiation or for beam diagnostics where the beam cross section is being imaged to a TV camera using the visible part of the radiation spectrum. To describe this visible part of the spectrum, we may in most cases assume that the photon frequency is much lower than the critical photon frequency. 


\subsubsection{Low Frequencies and Small Observation Angles}

For very small arguments or low frequencies and small angles, we find the following approximations AS(9.6.9) [14]

$$
\begin{aligned}
& K_{1 / 3}^{2}(\xi \longrightarrow 0) \approx \frac{\Gamma^{2}(1 / 3)}{2^{2 / 3}}\left(\frac{\omega}{\omega_{\mathrm{c}}}\right)^{-2 / 3} \frac{1}{1+\gamma^{2} \theta^{2}}, \\
& K_{2 / 3}^{2}(\xi \longrightarrow 0) \approx 2^{2 / 3} \Gamma^{2}(2 / 3)\left(\frac{\omega}{\omega_{\mathrm{c}}}\right)^{-4 / 3} \frac{1}{\left(1+\gamma^{2} \theta^{2}\right)^{2}},
\end{aligned}
$$

where the Gamma functions $\Gamma(1 / 3)=2.6789385$ and $\Gamma(2 / 3)=1.351179$ and from (25.85)

$$
\xi=\frac{1}{2} \frac{\omega}{\omega_{\mathrm{c}}}\left(1+\gamma^{2} \theta^{2}\right)^{3 / 2}
$$

Inserting this into (25.90) the photon flux spectrum in the forward direction becomes for $\theta=0$ and $\frac{\omega}{\omega_{\mathrm{c}}} \ll 1$

$$
\frac{\mathrm{d}^{2} \dot{N}_{\mathrm{ph}}}{\mathrm{d} \theta \mathrm{d} \psi} \approx C_{\Omega} E^{2} I \Gamma^{2}(2 / 3)\left(\frac{2 \omega}{\omega_{\mathrm{c}}}\right)^{2 / 3} \frac{\Delta \omega}{\omega} .
$$

The photon spectrum at very low frequencies is independent of the particle energy since $\omega_{\mathrm{c}} \propto E^{3}$. Clearly, in this approximation there is no angular dependence for the $\sigma$-mode radiation and the intensity increases with frequency. The $\pi$-mode radiation on the other hand is zero for $\theta=0$ and increases in intensity with the square of $\theta$ as long as the approximation is valid.

\subsubsection{High Frequencies or Large Observation Angles}

For large arguments of the modified Bessel's functions or for high frequencies and large emission angles different approximations hold. In this case, the approximate expressions are actually the same for both Bessel's functions indicating the same exponential drop off for high energetic photons $\operatorname{AS}(9.7 .2)$ [14]

$$
\begin{aligned}
& K_{1 / 3}^{2}(\xi \longrightarrow \infty) \approx \frac{\pi}{2} \frac{\mathrm{e}^{-2 \xi}}{\xi}, \\
& K_{2 / 3}^{2}(\xi \longrightarrow \infty) \approx \frac{\pi}{2} \frac{\mathrm{e}^{-2 \xi}}{\xi} .
\end{aligned}
$$


The photon flux distribution in this approximation becomes from (25.90)

$$
\frac{\mathrm{d}^{2} N_{\mathrm{ph}}}{\mathrm{d} \theta \mathrm{d} \psi} \approx \frac{3 r_{\mathrm{c}} m c^{2}}{4 \pi \hbar c} \gamma^{2} \frac{\omega}{\omega_{\mathrm{c}}} \mathrm{e}^{-2 \xi} \sqrt{1+\gamma^{2} \theta^{2}} \frac{\Delta \omega}{\omega} \frac{I}{e}\left(1+\frac{\gamma^{2} \theta^{2}}{1+\gamma^{2} \theta^{2}}\right),
$$

where $N_{\mathrm{ph}}$ is the number of photons emitted per pass. The spatial radiation distribution is greatly determined by the exponential factor and the relative amplitude with respect to the forward direction scales therefore like

$$
\exp \left\{-\frac{\omega}{\omega_{c}}\left[\left(1+\gamma^{2} \theta^{2}\right)^{3 / 2}-1\right]\right\} .
$$

We look now for the specific angle for which the intensity has fallen to $1 / e$. Since $\omega \gg \omega_{\mathrm{c}}$, this angle must be very small $\gamma \theta \ll 1$ and we can ignore other $\theta$-dependent factors. The exponential factor becomes equal to $1 / e$ for

$$
\frac{3}{2} \frac{\omega}{\omega_{\mathrm{c}}} \gamma^{2} \theta_{1 / e}^{2} \approx 1
$$

and solving for $\theta_{1 / e}$ we get finally

$$
\theta_{1 / e}=\sqrt{\frac{2}{3}} \frac{1}{\gamma} \frac{\omega_{\mathrm{c}}}{\omega} \quad \text { for } \omega \gg \omega_{\mathrm{c}}
$$

The high energy end of the synchrotron radiation spectrum is more and more collimated into the forward direction. The angular distribution is graphically illustrated for both polarization modes in Figs. 25.9 and 25.10.

\subsection{Angle-Integrated Spectrum}

Synchrotron radiation is emitted over a wide range of frequencies and it is of great interest to know the exact frequency distribution of the radiation. Since the radiation is very much collimated in the forward direction, it is useful to integrate over all angles of emission to obtain the total spectral photon flux that might be accepted by a beam line with proper aperture. To that goal, $(25.88)$ will be integrated with respect to the emission angles to obtain the frequency spectrum of the radiation. The emission angle $\theta$ appears in (25.88) in a rather complicated way which makes it difficult to perform the integration directly. We replace therefore the modified Bessel's functions by Airy's functions defined by AS(10.4.14) and AS(10.4.31) [14]

$$
\mathcal{A i}(z)=\frac{\sqrt{z}}{\sqrt{3} \pi} K_{1 / 3}(\xi)
$$




$$
\mathcal{A i}^{\prime}(z)=-\frac{z}{\sqrt{3} \pi} K_{2 / 3}(\xi)
$$

With the definition

$$
\eta=\frac{3}{4} \frac{\omega}{\omega_{\mathrm{c}}}
$$

we get from (25.85)

$$
z=\left(\frac{3}{2} \xi\right)^{2 / 3}=\eta^{2 / 3}\left(1+\gamma^{2} \theta^{2}\right)
$$

We apply this to the periodic motion of particles orbiting in a circular accelerator. In this case the spectral distribution of the radiation power can be obtained by noting that the differential radiation energy (25.88) is emitted every time the particle passes by the source point. A short pulse of radiation is sent towards the observation point at periodic time intervals equal to the revolution time. The spectral power distribution (25.88) expressed by Airy functions is

$$
\frac{\mathrm{d}^{2} P_{\gamma}}{\mathrm{d} \omega \mathrm{d} \Omega}=\frac{9 P_{\gamma}}{2 \pi} \frac{\gamma}{\omega_{\mathrm{c}}}\left[\eta^{2 / 3} \mathcal{A i}^{\prime 2}(z)+\eta^{4 / 3} \gamma^{2} \theta^{2} \mathcal{A} \mathrm{i}^{2}(z)\right] \text {. }
$$

To obtain the photon frequency spectrum, we integrate over all angles of emission which is accomplished by integrating along the orbit contributing a mere factor of $2 \pi$ and over the angle $\theta$. Although this latter integration is to be performed between $-\pi / 2$ and $+\pi / 2$, we choose the mathematically easier integration from $-\infty$ to $+\infty$ because the Airy functions fall off very fast for large arguments. In fact, we have seen already that most of the radiation is emitted within a very small angle of $\pm 1 / \gamma$. The integrals to be solved are of the form $\int_{0}^{\infty} \theta^{n} \mathcal{A} i^{2}\left[\eta^{2 / 3}\left(1+\gamma^{2} \theta^{2}\right] \mathrm{d} \theta\right.$ where $n=$ 0 or 2 . We concentrate first on the second term in (25.114) and form with (25.86) and (25.111a) the square of the Airy function

$$
\theta^{2} \mathcal{A i}^{2}(z)=\frac{1}{\pi^{2}} \int_{0}^{\infty} \theta^{2} \cos \left[\frac{1}{3} x^{3}+z x\right] \mathrm{d} x \int_{0}^{\infty} \theta^{2} \cos \left[\frac{1}{3} y^{3}+z y\right] \mathrm{d} y .
$$

We solve these integrals by making use of the trigonometric relation

$$
\cos \left(\alpha+\frac{1}{2} \beta\right) \cos \left(\alpha-\frac{1}{2} \beta\right)=\cos \alpha \cos \beta
$$

After introducing the substitutions $x+y=s$ and $x-y=t$, we obtain integrals over two terms which are symmetric in $s$ and $t$ and therefore can be set equal to get

$$
\theta^{2} \mathcal{A i}^{2}(z)=\frac{1}{2 \pi^{2}} \int_{0}^{\infty} \int_{0}^{\infty} \theta^{2} \cos \left[\frac{1}{12} s^{3}+3 s t^{2}+z s\right] \mathrm{d} s \mathrm{~d} y,
$$


where the factor $\frac{1}{2}$ comes from the transformation of the area element $\mathrm{d} s \mathrm{~d} y=\frac{\mathrm{d} s}{\sqrt{2}} \frac{\mathrm{d} t}{\sqrt{2}}$. In our problem we replace the argument $z$ by the expression $z=\eta^{2 / 3}\left(1+\gamma^{2} \theta^{2}\right)$ and integrate over the angle $\theta$

$$
\pi^{2} \int_{-\infty}^{\infty} \theta^{2} \mathcal{A i}^{2}(z) \mathrm{d} \theta=\iiint_{-\infty}^{\infty} \theta^{2} \cos \left[\frac{1}{12} s^{3}+3 s t^{2}+s \eta^{2 / 3}\left(1+\gamma^{2} \theta^{2}\right)\right] \mathrm{d} s \mathrm{~d} y \mathrm{~d} \theta .
$$

The integrand is symmetric with respect to $\theta$ and the integration therefore needs to be performed only from 0 to $\infty$ with the result being doubled. We also note that the integration is taken over only one quadrant of the $(s, t)$-space. Further simplifying the integration, the number of variables in the argument of the cosine function can be reduced in the following way. We note the coefficient $\frac{1}{4} t^{2}+\eta^{2 / 3} \gamma^{2} \theta^{2}$ which is the sum of squares. Setting $\frac{1}{2} t=r \cos \varphi$ and $\eta^{1 / 3} \gamma \theta=r \sin \varphi$ this term becomes simply $r^{2}$. The area element transforms like $\mathrm{d} t \mathrm{~d} \theta=2 /\left(\eta^{1 / 3} \gamma\right) r \mathrm{~d} r \mathrm{~d} \varphi$ and integrating over $\varphi$ from 0 to $\pi / 2$, since we need integrate only over one quarter plane, (25.118) becomes finally

$$
\int_{-\infty}^{\infty} \theta^{2} \mathcal{A} \mathrm{i}^{2}(z) \mathrm{d} \theta=\frac{1}{2 \pi \eta \gamma^{3}} \iint_{0}^{\infty} r^{2} \cos \left[\frac{1}{12} s^{3}+s \eta^{2 / 3}+r^{2}\right] r \mathrm{~d} r \mathrm{~d} s .
$$

The integrand of (25.119) has now a form close to that of an Airy integral and we will try to complete that similarity. With $q=(3 \xi / 2)^{1 / 3} x$ the definition of the Airy functions $\mathrm{AS}(10.4 .31)$ [14] are consistent with (25.111)

$$
\mathcal{A i}(z)=\frac{1}{\pi} \int_{0}^{\infty} \cos \left[\frac{1}{3} q^{3}+z q\right] \mathrm{d} q .
$$

Equation (25.119) can be modified into a similar form by setting

$$
w^{3}=\frac{1}{4} s^{3} \quad \text { and } \quad s\left(\eta^{2 / 3}+r^{2}\right)=y w .
$$

Solving for $w$ we get $w=s / 2^{2 / 3}$ and with $y=2^{2 / 3}\left(\eta^{2 / 3}+r^{2}\right), \mathrm{d} s=2^{2 / 3} \mathrm{~d} w$ and $\mathrm{d} y=2^{5 / 3} r \mathrm{~d} r$ Eq. (25.119) becomes

$$
\int_{-\infty}^{\infty} \theta^{2} \mathcal{A} \mathrm{i}^{2}(z) \mathrm{d} \theta=\frac{1}{4 \eta \gamma^{3}} \int_{y_{0}}^{\infty}\left(\frac{y}{2^{2 / 3}}-\eta^{2 / 3}\right) \mathcal{A} \mathrm{i}(y) \mathrm{d} y,
$$


where we have used the definition of Airy's function and where the integration starts at

$$
y_{0}=(2 \eta)^{2 / 3}=\left(\frac{3}{2} \frac{\omega}{\omega_{\mathrm{c}}}\right)^{2 / 3}
$$

corresponding to $r=0$.

We may separate this integral into two parts and get a term $y \mathcal{A i}(y)$ under one of the integrals. This term is by the definition of Airy's functions AS(10.4.1) [14] equal to $\mathcal{A i}{ }^{\prime \prime}$. Integration of this second derivative gives

$$
\int_{y_{0}}^{\infty} \mathcal{A} \mathrm{i}^{\prime \prime}(y) \mathrm{d} y=-\mathcal{A} \mathrm{i}^{\prime}\left(y_{0}\right)
$$

and collecting all terms in (25.122) we have finally

$$
\int_{-\infty}^{\infty} \theta^{2} \mathcal{A i}^{2}(z) \mathrm{d} \theta=-\frac{1}{4 \eta^{1 / 3} \gamma^{3}}\left[\frac{\mathcal{A i}\left(y_{0}\right)}{y_{0}}+\int_{y_{0}}^{\infty} \mathcal{A i}(y) \mathrm{d} y\right] .
$$

The derivation of the complete spectral radiation power distribution (25.114) requires also the evaluation of the integral $\int \mathcal{A i}^{\prime \prime}(z) \mathrm{d} \theta$. This can be done with the help of the integral $\int \mathcal{A i}{ }^{\prime \prime}(z) \mathrm{d} \theta$ and (25.125). We follow a similar derivation that led us just from (25.118) to (25.119) and get instead of (25.125)

$$
\int_{-\infty}^{\infty} \mathcal{A} \mathrm{i}^{2}(z) \mathrm{d} \theta=-\frac{1}{2 \eta^{1 / 3} \gamma} \int_{y_{0}}^{\infty} \mathcal{A i}(y) \mathrm{d} y .
$$

Recalling the definition of the argument $y=\eta^{2 / 3}\left(1+\gamma^{2} \theta^{2}\right)$, we differentiate (25.126) twice with respect to $\eta^{2 / 3}$ to get

$$
2 \int_{-\infty}^{\infty}\left[\mathcal{A i}{ }^{\prime \prime}(z)+\mathcal{A i}^{\prime 2}(z)\right] \mathrm{d} \theta=-\frac{2^{1 / 3}}{\eta^{1 / 3} \gamma} \mathcal{A} \mathrm{i}^{\prime}\left(y_{0}\right)
$$

Using the relation $\mathcal{A i}^{\prime \prime}(z)=z \mathcal{A i}(z)$ and the results (25.124), (25.125) in (25.127) we get

$$
\int_{-\infty}^{\infty} \mathcal{A} \mathrm{i}^{2}(z) \mathrm{d} \theta=-\frac{\eta^{1 / 3}}{4 \gamma}\left[\frac{3 \mathcal{A} \mathrm{i}^{\prime}\left(y_{0}\right)}{y_{0}}+\int_{y_{0}}^{\infty} \mathcal{A i}(y) \mathrm{d} y\right] .
$$

At this point, all integrals have been derived that are needed to describe the spectral radiation power separately in both polarization modes and the spectral 
radiation power from $(25.114)$ becomes

$$
\begin{aligned}
\frac{\mathrm{d} P_{\gamma}}{\mathrm{d} \omega}=\frac{27 P_{\gamma} \omega}{16 \omega_{\mathrm{c}}^{2}}\left[\left(-\frac{3 \mathcal{A i}{ }^{\prime}\left(y_{0}\right)}{y_{0}}\right.\right. & \left.-\int_{y_{0}}^{\infty} \mathcal{A i}(y) \mathrm{d} y\right) \\
& \left.-\left(\frac{\mathcal{A i}^{\prime}\left(y_{0}\right)}{y_{0}}+\int_{y_{0}}^{\infty} \mathcal{A i}(y) \mathrm{d} y\right)\right] .
\end{aligned}
$$

The first term describes the $\sigma$-mode of polarization and the second term the $\pi$ mode. Combining both polarization modes, we may derive a comparatively simple expression for the spectral radiation power. To this goal, we replace the Airy's functions by modified Bessel's functions

$$
\frac{\mathcal{A i}{ }^{\prime}\left(y_{0}\right)}{y_{0}}=-\frac{1}{\sqrt{3} \pi} K_{2 / 3}\left(x_{0}\right),
$$

where from (25.111), (25.112), and (25.122) $x_{0}=\omega / \omega_{\mathrm{c}}$. With $\sqrt{y} \mathrm{~d} y=\mathrm{d} x$, the recurrence formula $2 K_{2 / 3}^{\prime}=-K_{1 / 3}+K_{5 / 3}$ and (25.111) the Airy integral is

$$
\begin{aligned}
\int_{y_{0}}^{\infty} \mathcal{A i}(y) \mathrm{d} y & =-\frac{2}{\sqrt{3} \pi} \int_{x_{0}}^{\infty} K_{2 / 3}^{\prime}(x) \mathrm{d} x-\frac{1}{\sqrt{3} \pi} \int_{x_{0}}^{\infty} K_{5 / 3}(x) \mathrm{d} x \\
& =\frac{2}{\sqrt{3} \pi} K_{2 / 3}\left(x_{0}\right)-\frac{1}{\sqrt{3} \pi} \int_{x_{0}}^{\infty} K_{5 / 3}(x) \mathrm{d} x .
\end{aligned}
$$

We use (25.130) and (25.131) in (25.129) and get the simple expression for the synchrotron radiation spectrum

$$
\frac{\mathrm{d} P_{\gamma}}{\mathrm{d} \omega}=\frac{P_{\gamma}}{\omega_{\mathrm{c}}} \frac{9 \sqrt{3}}{8 \pi} \frac{\omega}{\omega_{\mathrm{c}}} \int_{x_{0}}^{\infty} K_{5 / 3}(x) \mathrm{d} x=\frac{P_{\gamma}}{\omega_{\mathrm{c}}} S\left(\frac{\omega}{\omega_{\mathrm{c}}}\right),
$$

where we defined the universal function

$$
S\left(\frac{\omega}{\omega_{\mathrm{c}}}\right)=\frac{9 \sqrt{3}}{8 \pi} \frac{\omega}{\omega_{\mathrm{c}}} \int_{\omega / \omega_{\mathrm{c}}}^{\infty} K_{5 / 3}(x) \mathrm{d} x .
$$

The spectral distribution depends only on the critical frequency $\omega_{\mathrm{c}}$, the total radiation power and a purely mathematical function. This result has been derived originally by Ivanenko and Sokolov [15] and independently by Schwinger [6]. Specifically, it should be noted that the synchrotron radiation spectrum, if normalized to the critical frequency, does not depend on the particle energy and is represented by the universal function shown in Fig. 25.11. The energy dependence is contained in the cubic dependence of the critical frequency acting as a scaling factor for the real spectral distribution. The mathematical function is properly normalized 
Fig. 25.11 Universal function:

$S(\xi)=\frac{9 \sqrt{3}}{8 \pi} \xi \int_{\xi}^{\infty} K_{5 / 3}(x) \mathrm{d} x$, with $\xi=\omega / \omega_{\mathrm{c}}$

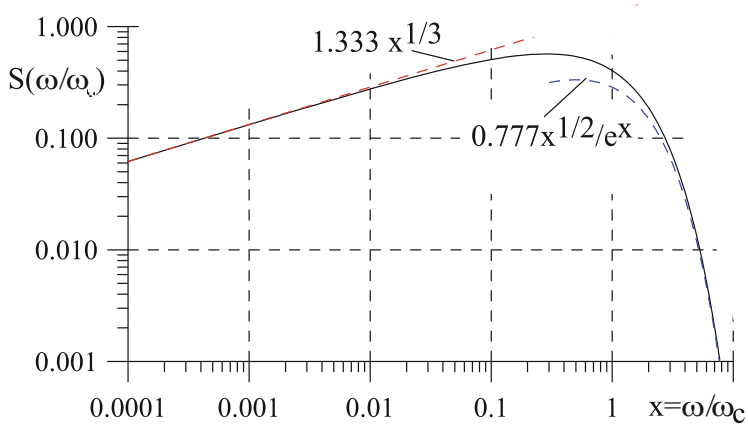

as we can see by integrating over all frequencies.

$$
\int_{0}^{\infty} \frac{\mathrm{d} P_{\gamma}}{\mathrm{d} \omega} \mathrm{d} \omega=\frac{9 \sqrt{3}}{8 \pi} P_{\gamma} \int_{0}^{\infty} x_{0} \int_{x_{0}}^{\infty} K_{5 / 3}(x) \mathrm{d} x \mathrm{~d} x_{0} .
$$

After integration by parts, the result can be derived from GR[6.561.16] [12]

$$
\int_{0}^{\infty} \frac{\mathrm{d} P_{\gamma}}{\mathrm{d} \omega} \mathrm{d} \omega=\frac{9 \sqrt{3}}{16 \pi} P_{\gamma} \int_{0}^{\infty} x_{0}^{2} K_{5 / 3}\left(x_{0}\right) \mathrm{d} x_{0}=\Gamma(4 / 3) \Gamma(2 / 3) .
$$

Using the triplication formula AS(6.1.19) [14] the product of the gamma functions becomes

$$
\Gamma(4 / 3) \Gamma(2 / 3)=\frac{4}{9} \frac{2 \pi}{\sqrt{3}} .
$$

With this equation the proper normalization of (25.134) is demonstrated

$$
\int_{0}^{\infty} \frac{\mathrm{d} P_{\gamma}}{\mathrm{d} \omega} \mathrm{d} \omega=P_{\gamma}
$$

Of more practical use is the spectral photon flux per unit angle of deflection in the bending magnet. With the photon flux $\mathrm{d} \dot{N}_{\mathrm{ph}}=\mathrm{d} P / \hbar \omega$ we get from (25.132)

$$
\frac{\mathrm{d} \dot{N}_{\mathrm{ph}}}{\mathrm{d} \psi}=\frac{P_{\gamma}}{2 \pi \hbar \omega_{\mathrm{c}}} \frac{\Delta \omega}{\omega} S\left(\frac{\omega}{\omega_{\mathrm{c}}}\right)
$$

and with (24.34) and (24.49)

$$
\frac{\mathrm{d} \dot{N}_{\mathrm{ph}}}{\mathrm{d} \psi}=\frac{4 \alpha}{9} \gamma \frac{I}{e} \frac{\Delta \omega}{\omega} S\left(\frac{\omega}{\omega_{\mathrm{c}}}\right),
$$


where $\psi$ is the deflection angle in the bending magnet and $\alpha$ the fine structure constant. In practical units, this becomes

$$
\frac{\mathrm{d} \dot{N}_{\mathrm{ph}}}{\mathrm{d} \psi}=C_{\psi} E I \frac{\Delta \omega}{\omega} S\left(\frac{\omega}{\omega_{\mathrm{c}}}\right)
$$

with

$$
C_{\psi}=\frac{4 \alpha}{9 e m c^{2}}=3.9614 \times 10^{16} \frac{\text { photons }}{\mathrm{s} \mathrm{mrad} \mathrm{A} \mathrm{GeV}} .
$$

The synchrotron radiation spectrum in Fig. 25.11 is rather uniform up to the critical frequency beyond which the intensity falls off rapidly. Equation (25.132) is not well suited for quick calculation of the radiation intensity at a particular frequency. We may, however, express (25.132) in much simpler form for very low and very large frequencies as discussed in Sect. 24.3.

\subsection{Statistical Radiation Parameters}

The emission of synchrotron radiation is a classical phenomenon. For some applications it is, however, useful to express some parameters in statistical form. Knowing the spectral radiation distribution, we may follow Sands [16] and express some quantities in the photon picture. We have used such to derive expressions for the equilibrium beam size and energy spread. Equilibrium beam parameters are determined by the statistical emission of photons and its recoil on the particle motion. For this purpose, we are mainly interested in an expression for $\varepsilon_{\mathrm{ph}}^{2}$ and the photon flux at energy $\varepsilon_{\mathrm{ph}}$. From these quantities, we may derive an expression for the average photon energy $\left\langle\varepsilon_{\mathrm{ph}}^{2}\right\rangle_{z}$ emitted along the circumference of the storage ring. With $\Pi\left(\varepsilon_{\mathrm{ph}}\right)$ being the probability to emit a photon with energy $\varepsilon_{\mathrm{ph}}$ we have

$$
\left\langle\varepsilon_{\mathrm{ph}}^{2}\right\rangle_{z}=\int_{0}^{\infty} \varepsilon_{\mathrm{ph}}^{2} \Pi\left(\varepsilon_{\mathrm{ph}}\right) \mathrm{d} \varepsilon_{\mathrm{ph}} .
$$

The probability $\Pi\left(\varepsilon_{\mathrm{ph}}\right)$ is defined by the ratio of the photon flux $\dot{n}\left(\varepsilon_{\mathrm{ph}}\right)$ emitted at energy $\varepsilon_{\text {ph }}$ to the total photon flux $\dot{N}_{\text {ph }}$

$$
\Pi\left(\varepsilon_{\mathrm{ph}}\right)=\frac{\dot{n}\left(\varepsilon_{\mathrm{ph}}\right)}{\dot{N}_{\mathrm{ph}}},
$$


where

$$
\dot{n}\left(\varepsilon_{\mathrm{ph}}\right)=\frac{P_{\gamma}}{\varepsilon_{\mathrm{c}}^{2}} \frac{S(x)}{x}, \quad \text { with } \quad x=\frac{\varepsilon_{\mathrm{ph}}}{\varepsilon_{\mathrm{c}}} .
$$

The photon flux at $\varepsilon_{\mathrm{ph}}$ is related to the spectral photon power by $\varepsilon_{\mathrm{ph}} \dot{n}\left(\varepsilon_{\mathrm{ph}}\right)=P\left(\varepsilon_{\mathrm{ph}}\right)$. Integrating (25.138) over all angles $\psi$ and multiplying by $\hbar \omega=\varepsilon_{\mathrm{ph}}$ we get for the spectral radiation power

$$
P\left(\varepsilon_{\mathrm{ph}}\right) \mathrm{d} \varepsilon_{\mathrm{ph}}=\varepsilon_{\mathrm{ph}} \frac{\mathrm{d} \dot{N}}{\mathrm{~d} \varepsilon_{\mathrm{ph}}} \mathrm{d} \varepsilon_{\mathrm{ph}}=\frac{P_{\gamma}}{\varepsilon_{\mathrm{c}}} S\left(\frac{\varepsilon_{\mathrm{ph}}}{\varepsilon_{\mathrm{c}}}\right) \mathrm{d} \varepsilon_{\mathrm{ph}} .
$$

The total number of emitted photons per unit time is just the integral

$$
\dot{N}_{\mathrm{ph}}=\int_{0}^{\infty} \dot{n}\left(\varepsilon_{\mathrm{ph}}\right) \mathrm{d} \varepsilon_{\mathrm{ph}}=\frac{P_{\gamma}}{\varepsilon_{\mathrm{c}}} \int_{0}^{\infty} \frac{S(x)}{x} \mathrm{~d} x=\frac{15 \sqrt{3}}{8} \frac{P_{\gamma}}{\varepsilon_{\mathrm{c}}} .
$$

With this, the probability to emit a photon of energy $\varepsilon_{\mathrm{ph}}$ is finally

$$
\Pi\left(\varepsilon_{\mathrm{ph}}\right)=\frac{8}{15 \sqrt{3}} \frac{1}{\varepsilon_{\mathrm{c}}} \frac{S(x)}{x},
$$

and

$$
\left\langle\varepsilon_{\mathrm{ph}}^{2}\right\rangle_{z}=\frac{8 \varepsilon_{\mathrm{c}}^{2}}{15 \sqrt{3}} \int_{0}^{\infty} x S(x) \mathrm{d} x=\frac{11}{27} \varepsilon_{\mathrm{c}}^{2} .
$$

To calculate equilibrium beam parameters in Chaps. 11.3 and 11.4, for example, we need to know the quantity $\left\langle\dot{N}_{\mathrm{ph}}\left\langle\varepsilon_{\mathrm{ph}}^{2}\right\rangle\right\rangle_{z}$ which is now from (25.146), (25.148)

$$
\left\langle\dot{N}_{\mathrm{ph}}\left\langle\varepsilon^{2}\right\rangle\right\rangle_{z}=\frac{55}{24 \sqrt{3}}\left\langle\varepsilon_{\mathrm{c}} P_{\gamma}\right\rangle_{z}
$$

where the average is to be taken along the orbit and around the storage ring through all magnets. Expressing the critical photon energy by (24.49) and the radiation power by (24.34) and we get finally

$$
\left\langle\dot{N}_{\mathrm{ph}}\left\langle\varepsilon^{2}\right\rangle\right\rangle_{z}=\frac{55}{24 \sqrt{3}} r_{\mathrm{c}} c m c^{2} \hbar c \gamma^{7}\left\langle\frac{1}{\rho^{3}}\right\rangle_{z} .
$$




\section{Problems}

25.1 (S). Integrate the radiation power distribution (25.61) over all solid angles and prove that the total radiation power is equal to (24.34).

25.2 (S). In the ESRF (European Synchrotron Radiation Facility) synchrotron radiation source in Grenoble (France) an electron beam of $200 \mathrm{~mA}$ circulates at an energy of $6 \mathrm{GeV}$. The bending magnet field is $1.0 \mathrm{~T}$. Derive and sketch the spectral photon flux into a band width of $1 \%$ and an acceptance angle of $10 \mathrm{mrad}$ as a function of photon energy.

25.3 (S). Derive an expression identifying the angle at which the spectral intensity has dropped to $p \%$ from the maximum intensity. Derive approximate expressions for very low or very large photon energies. Find the angle at which the total radiation intensity has dropped to $10 \%$.

25.4. Derive the wave equations (25.3) and (25.4).

25.5. Derive (25.17).

25.6. Derive (25.28) from (25.27). Show that the electrical field in the radiation regime is purely orthogonal to the direction of observation. Is the field also parallel to the acceleration?

25.7. Design a synchrotron radiation source for a critical photon energy of your choice. Use a simple FODO lattice and specify the minimum beam energy, beam current, and bending radius which will produce a bending magnet photon flux of $10^{14}$ photons $/ \mathrm{s} / \mathrm{mrad}$ at the desired photon energy and into a band width of $\Delta \omega / \omega=$ $1 \%$. What is the minimum and maximum photon energy for which the photon flux is at least $10^{11}$ photons $/ \mathrm{s} / \mathrm{mrad}$ ? How big is your ring assuming a $30 \%$ fill factor for bending magnets?

\section{References}

1. J. Larmor, Philos. Mag. 44, 503 (1897)

2. J.S. Schwinger, Proc. Natl. Acad. Sci. 40, 132 (1954)

3. J.D. Jackson, Classical Electrodynamics, 2nd edn. (Wiley, New York, 1975)

4. A.A. Sokolov, I.M. Ternov, Synchrotron Radiation (Pergamon, Oxford, 1968)

5. L.I. Schiff, Rev. Sci. Instrum. 17, 6 (1946)

6. J.S. Schwinger, On the classical radiation of accelerated electrons. Phys. Rev. 75, 1912 (1949)

7. G.N. Watson, Bessel Functions (Macmillan, New York, 1945)

8. V.O. Kostroun, Nucl. Instrum. Methods 172, 371 (1980)

9. G.A. Schott, Ann. Physik 24, 635 (1907)

10. G.A. Schott, Philos. Mag. [6] 13, 194 (1907)

11. G.A. Schott, Electromagnetic Radiation (Cambridge University Press, Cambridge, 1912)

12. I.S. Gradshteyn, I.M. Ryzhik, Table of Integrals, Series, and Products, 4th edn. (Academic, New York, 1965) 
13. V.N. Baier, Radiative polarization of electrons in storage rings, in Physics with Intersecting Storage Rings, ed. by B. Touschek (Academic, New York, 1971)

14. M. Abramovitz, I. Stegun, Handbook of Mathematical Functions (Dover, New York, 1972)

15. D. Ivanenko, A.A. Sokolov, DAN(USSR) 59, 1551 (1972)

16. M. Sands, The physics of electron storage rings, an introduction, in Physics with Intersecting Storage Rings, ed. by B. Touschek (Academic, New York, 1971) 


\section{Chapter 26 \\ Insertion Device Radiation}

Synchrotron radiation from bending magnets is characterized by a wide spectrum from microwaves up to soft or hard x-rays as determined by the critical photon energy. To optimally meet the needs of basic research with synchrotron radiation, it is desirable to provide radiation characteristics that cannot be obtained from ring bending magnets but require special magnets. The field strength of bending magnets and the maximum particle beam energy in circular accelerators like a storage ring is fixed leaving no adjustments to optimize the synchrotron radiation spectrum for particular experiments. To generate specific synchrotron radiation characteristics, radiation is often produced from insertion devices installed along the particle beam path. Such insertion devices introduce no net deflection of the beam and can therefore be incorporated in a beam line without changing its geometry. Motz [1] proposed first the use of wiggler magnets to optimize characteristics of synchrotron radiation. By now, such magnets have become the most common insertion devices consisting of a series of alternating magnet poles deflecting the beam periodically in opposite directions as shown in Fig. 26.1.

In Chap. 24 the properties of wiggler radiation were discussed shortly in an introductory way. Here we concentrate on more detailed and formal derivations of radiation characteristics from relativistic electrons passing through periodic magnets.

There is no fundamental difference between wiggler and undulator radiation. One is the stronger/weaker version of the other. The deflection in an undulator is weak and the transverse particle momentum remains nonrelativistic. The motion is purely sinusoidal in a sinusoidal field, and the emitted radiation is monochromatic at the particle oscillation frequency which is the Lorentz-contracted periodicity of the undulator period. Since the radiation is emitted from a moving source the observer in the laboratory frame of reference then sees a Doppler shifted frequency. We call this monochromatic radiation the fundamental radiation or radiation at the fundamental frequency of the undulator.

This chapter has been made Open Access under a CC BY 4.0 license. For details on rights and licenses please read the Correction https://doi.org/10.1007/978-3-319-18317-6_28 
Fig. 26.1 Trajectory of a particle beam in a flat wiggler magnet

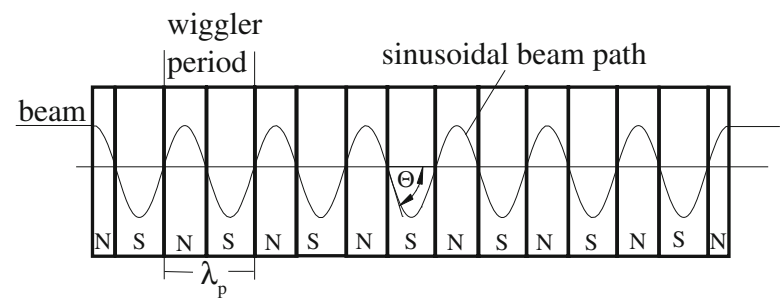

As the undulator field is increased, the transverse motion becomes stronger and the transverse momentum starts to become relativistic. As a consequence, the so far purely sinusoidal motion becomes periodically distorted causing the appearance of harmonics of the fundamental monochromatic radiation. These harmonics increase in number and density with further increase of the magnetic field and, at higher frequencies, eventually merge into one broad spectrum characteristic for wiggler or bending magnet radiation. At very low frequencies, the theoretical spectrum is still a line spectrum showing the harmonics of the revolution frequency. Of course, there is a low frequency cut-off at a wavelength comparable or longer than vacuum chamber dimensions which therefore do not show-up as radiation.

An insertion device does not introduce a net deflection of the beam and we may therefore choose any arbitrary field strength which is technically feasible to adjust the radiation spectrum to experimental needs. The radiation intensity from a wiggler magnet also can be made much higher compared to that from a single bending magnet. A wiggler magnet with say ten poles acts like a string of ten bending magnets or radiation sources aligned in a straight line along the photon beam direction. The effective photon source is therefore ten times more intense than the radiation from a single bending magnet with the same field strength.

Wiggler magnets come in a variety of types with the flat wiggler magnet being the most common. In this wiggler type only the component $B_{y}$ is nonzero deflecting the beam in the horizontal plane. To generate circularly or elliptically polarized radiation, a helical wiggler magnet [2] may be used or a combination of several flat wiggler magnets deflecting the beam in orthogonal planes which will be discussed in more detail in Sect. 26.3.2.

\subsection{Particle Dynamics in a Periodic Field Magnet}

Insertion devices are characterized by the requirement that

$$
\int B_{\perp} \mathrm{d} z=0 .
$$

As discussed in Chap. 15 this requirement demands that the first and second integral must be made zero with the use of steering magnets before and after the undulator. 
This correction is sufficient from the beam stability point of view. However, it does not address the effect of field tolerances on the intensity of radiation into harmonics. For example, the curved trajectory in Fig. 15.4 can reduce the radiation intensity because not all periods radiate in the same direction and constructive interference of light emitted by individual periods is not optimum. Therefore the overall trajectory curvature in Fig. 15.4 should be corrected as discussed in Chap. 15. Furthermore, variations in field strength and period length from period to period in the undulator can seriously diminish the radiation intensity especially in the higher harmonics. The effect of such errors on individual harmonic intensities have been studied [3]. A special shimming procedure has been proposed by Elleaume [4] to transform an undulator with construction tolerances to an almost ideal undulator giving close to perfect intensities for about a dozen harmonics. If the shimming is done correctly the long coil mentioned in Chap. 15 is not necessary anymore. In the following discussion we assume that the integrals have been corrected and that the undulator has been shimmed.

Particle dynamics and resulting radiation characteristics for an undulator have been derived first by Motz [1] and later in more detail by other authors [5, 6]. A sinusoidally varying vertical field causes a periodic deflection of particles in the $(x, z)$-plane shown in Fig. 26.1. To describe the particle trajectory, we use the equation of motion

$$
\frac{\mathbf{n}}{\rho}=\frac{e c}{m c^{2} \gamma \beta^{2}}[\boldsymbol{\beta} \times \boldsymbol{B}],
$$

where $\beta$ is the particle velocity and get with (6.110) the equations of motion in component form

$$
\begin{aligned}
& \frac{\mathrm{d}^{2} x}{\mathrm{~d} t^{2}}=-\frac{e B_{0}}{\gamma \beta m} \frac{\mathrm{d} z}{\mathrm{~d} t} \cos \left(k_{\mathrm{p}} z\right) \\
& \frac{\mathrm{d}^{2} z}{\mathrm{~d} t^{2}}=+\frac{e B_{0}}{\gamma \beta m} \frac{\mathrm{d} x}{\mathrm{~d} t} \cos \left(k_{\mathrm{p}} z\right),
\end{aligned}
$$

where we have set $k_{\mathrm{p}}=2 \pi / \lambda_{\mathrm{p}}$ and $\mathrm{d} z=\beta c \mathrm{~d} t$ with $\beta=v / c$.

Equations (26.2) describe the coupled motion of a particle in the sinusoidal field of a flat wiggler magnet. This coupling is common to the particle motion in any magnetic field but generally in beam dynamics we set $\mathrm{d} z / \mathrm{d} t \approx v$ and $\mathrm{d} x / \mathrm{d} t \approx 0$ because $\mathrm{d} x / \mathrm{d} t \ll \mathrm{d} z / \mathrm{d} t$. This approximation is justified in most beam transport applications for relativistic particles, but here we have to be cautious not to neglect effects that might be of relevance on a very short time or small geometric scale comparable to the oscillation period and wavelength of synchrotron radiation.

We will keep the $\mathrm{d} x / \mathrm{d} t$-term and get from (26.2) with $\mathrm{d} z / \mathrm{d} t \approx v$ and after integrating twice that the particle trajectory follows the magnetic field in the sense that the oscillatory motion reaches a maximum where the magnetic field reaches a maximum and crosses the beam axis where the field is zero. We start at the time $t=0$ in the middle of a magnet pole where the transverse velocity $\dot{x}_{0}=0$ while 
the longitudinal velocity $\dot{z}_{0}=\beta c$ and integrate both equations (26.2) utilizing the integral of the first equation in the second to get

$$
\begin{aligned}
& \frac{\mathrm{d} x}{\mathrm{~d} t}=-\beta c \frac{K}{\beta \gamma} \sin \left(k_{\mathrm{p}} z\right), \\
& \frac{\mathrm{d} z}{\mathrm{~d} t}=\beta c\left[1-\frac{K^{2}}{2 \beta^{2} \gamma^{2}} \sin ^{2}\left(k_{\mathrm{p}} z\right)\right] .
\end{aligned}
$$

The transverse motion describes the expected oscillatory motion and the longitudinal velocity $v$ exhibits a periodic modulation reflecting the varying projection of the velocity vector to the $z$-axis. Closer inspection of this velocity modulation shows that its frequency is twice that of the periodic motion. It is convenient to describe the longitudinal particle motion with respect to a Cartesian reference frame moving uniformly along the $z$-axis with the average longitudinal particle velocity $\bar{\beta} c=\langle\dot{z}\rangle$ which can be derived from (26.3b)

$$
\bar{\beta}=\beta\left(1-\frac{K^{2}}{4 \beta^{2} \gamma^{2}}\right) .
$$

In this reference frame the particle follows a figure-of-eight trajectory composed of the transverse oscillation and a longitudinal oscillation with twice the frequency. We will come back to this point since both oscillations contribute to the radiation spectrum. A second integration of (26.3b) results finally in the equation of motion in component representation

$$
\begin{aligned}
& x(t)=\frac{K}{\beta \gamma k_{\mathrm{p}}} \cos \left(k_{\mathrm{p}} \bar{\beta} c t\right), \\
& z(t)=\bar{\beta} c t+\frac{K^{2}}{8 \beta^{2} \gamma^{2} k_{\mathrm{p}}} \sin \left(2 k_{\mathrm{p}} \bar{\beta} c t\right),
\end{aligned}
$$

where we set $z=\bar{\beta}$ ct. The maximum amplitude $a$ of the transverse particle oscillation is finally

$$
a=\frac{K}{\beta \gamma k_{\mathrm{p}}}=\frac{\lambda_{\mathrm{p}} K}{2 \pi \beta \gamma} .
$$

This last expression gives another simple relationship between the wiggler strength parameter and the transverse displacement of the beam trajectory

$$
a(\mu \mathrm{m})=0.8133 \frac{\lambda_{\mathrm{p}}(\mathrm{cm}) K}{E(\mathrm{GeV})} .
$$

For most cases, this beam displacement is very small. 


\subsection{Undulator Radiation}

The physical process of undulator radiation is not different from the radiation produced from a single bending magnet. However, the radiation received at great distances from the undulator exhibits special features which we will discus in more detail. Basically, we observe an electron performing $N_{\mathrm{p}}$ oscillations while passing through an undulator with $N_{\mathrm{p}}$ undulator periods. The observed radiation spectrum is the Fourier transform of the electron motion and therefore quasimonochromatic with a finite line width inversely proportional to the number of oscillations performed.

\subsubsection{Fundamental Wavelength}

Undulator radiation can also be viewed as a superposition of radiation fields from $N_{\mathrm{p}}$ sources yielding quasi-monochromatic radiation as a consequence of interference. To see that, we observe the radiation at an angle $\vartheta$ with respect to the path of the electron as shown in Fig. 26.2.

The electron travels on its path at an average velocity given by (26.4) and it takes the time

$$
\tau=\frac{\lambda_{\mathrm{p}}}{c \bar{\beta}}=\frac{\lambda_{\mathrm{p}}}{c \beta\left[1-K^{2} /\left(4 \gamma^{2}\right)\right]}
$$

to move along one undulator period. During that same time, the radiation front proceeds a distance

$$
s_{\mathrm{ph}}=\tau c=\frac{\lambda_{\mathrm{p}}}{\beta\left[1-K^{2} /\left(4 \gamma^{2}\right)\right]}
$$

moving ahead of the particle since $s_{\mathrm{ph}}>\tau c \bar{\beta}$. For constructive superposition of radiation from all undulator periods, we require that the difference $s_{\mathrm{ph}}-\lambda_{\mathrm{p}} \cos \vartheta$ be equal to an integer multiple of the wavelength $\lambda_{k}$ or for small observation angles $\vartheta \ll 1$

$$
k \lambda_{k}=\frac{\lambda_{\mathrm{p}}}{\beta\left[1-K^{2} /\left(4 \gamma^{2}\right)\right]}-\lambda_{\mathrm{p}}\left(1-\frac{1}{2} \vartheta^{2}\right) .
$$

Fig. 26.2 Interference of undulator radiation

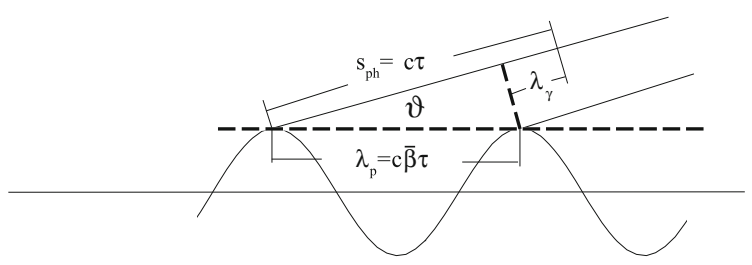


After some manipulations, we get with $K^{2} / \gamma^{2} \ll 1$ and $\beta \approx 1$ for $\lambda_{k}$

$$
\lambda_{k}=\frac{\lambda_{\mathrm{p}}}{2 \gamma^{2} k}\left(1+\frac{1}{2} K^{2}+\gamma^{2} \vartheta^{2}\right) .
$$

The lowest harmonics is defined by $k=1$ and is called the fundamental undulator wavelength.

From an infinitely long undulator, the radiation spectrum consists of spectral lines at a wavelength determined by (26.11). In particular, we note that the shortest wavelength is emitted into the forward direction while the radiation at a finite angle $\vartheta$ appears red shifted by the Doppler effect. For an undulator with a finite number of periods, the spectral lines are widened to a width of about $1 / N_{\mathrm{p}}$ or less as we will discuss in the next section.

\subsubsection{Radiation Power}

The radiation power is from $(25.41)$

$$
P=\frac{2}{3} r_{\mathrm{c}} m c\left|\dot{\beta}^{*}\right|_{\mathrm{r}}^{2}
$$

where $*$ indicates quantities to be evaluated in the particle reference system. We may use this expression in the particle system to calculate the total radiated energy from an electron passing through an undulator. The transverse particle acceleration is expressed by $m \dot{\mathbf{v}}^{*}=\mathrm{d} \mathbf{p}_{\perp} / \mathrm{d} t^{*}=\gamma \mathrm{d} \mathbf{p}_{\perp} / \mathrm{d} t$ where we used $t^{*}=t / \gamma$ and inserting into (26.12) we get

$$
P=\frac{2}{3} \frac{r_{\mathrm{c}} \gamma^{2}}{m c}\left(\frac{\mathrm{d} \mathbf{p}_{\perp}}{\mathrm{d} t}\right)^{2} .
$$

The transverse momentum is determined by the particle deflection in the undulator with a period length $\lambda_{\mathrm{p}}$ and is for a particle of momentum $c p_{0}$

$$
p_{\perp}=\hat{p} \sin \omega_{\mathrm{p}} t
$$

where $\hat{p}=p_{0} \theta$ and $\omega_{\mathrm{p}}=c k_{\mathrm{p}}=2 \pi c / \lambda_{\mathrm{p}}$. The angle $\theta=K / \gamma$ is the maximum deflection angle defined in (6.121). With these expressions and averaging over one period, we get from (26.13) for the instantaneous radiation power from a charge $e$ traveling through an undulator

$$
P_{\text {inst }}=\frac{1}{3} c r_{\mathrm{c}} m c^{2} \gamma^{2} K^{2} k_{\mathrm{p}}^{2},
$$

where $r_{\mathrm{c}}$ is the classical electron radius. The duration of the radiation pulse is equal to the travel time through an undulator of length $L_{\mathrm{u}}=\lambda_{\mathrm{p}} N_{\mathrm{p}}$ and the total radiated 
energy per electron is therefore

$$
\Delta E=\frac{1}{3} r_{\mathrm{c}} m c^{2} \gamma^{2} K^{2} k_{\mathrm{p}}^{2} L_{\mathrm{u}}
$$

In more practical units

$$
\Delta E(\mathrm{eV})=C_{\mathrm{u}} \frac{E^{2} K^{2}}{\lambda_{\mathrm{p}}^{2}} L_{\mathrm{u}}=725.69 \frac{E^{2} K^{2}}{\lambda_{\mathrm{p}}^{2}(\mathrm{~cm})} L_{\mathrm{u}}
$$

with

$$
C_{\mathrm{u}}=\frac{4 \pi^{2} r_{\mathrm{c}}}{3 m c^{2}}=7.2569 \times 10^{-20} \frac{\mathrm{m}}{\mathrm{eV}} .
$$

The average total undulator radiation power for an electron beam circulating in a storage ring is then just the radiated energy (26.16) multiplied by the number of particles $N_{\mathrm{b}}$ in the beam and the revolution frequency or

$$
P_{\mathrm{avg}}=\frac{1}{3} r_{\mathrm{c}} c m c^{2} \gamma^{2} K^{2} k_{\mathrm{p}}^{2} N_{\mathrm{b}} \frac{L_{\mathrm{u}}}{2 \pi \bar{R}}
$$

or

$$
P_{\mathrm{avg}}(\mathrm{W})=633.6 E^{2} B_{0}^{2} I L_{\mathrm{u}},
$$

where $I$ is the circulating electron beam current. The total angle integrated radiation power from an undulator in a storage ring is proportional to the square of the beam energy and maximum undulator field $B_{0}$ and proportional to the beam current and undulator length.

\subsubsection{Spatial and Spectral Distribution}

For bending magnet radiation, the particle dynamics is relatively simple being determined only by the particle velocity and the bending radius of the magnet. In a wiggler magnet, the magnetic field parameters are different from those in a constant field magnet and we will therefore derive again the synchrotron radiation spectrum for the beam dynamics in a general wiggler magnet. No special assumptions on magnetic field configurations have been made to derive the radiation spectrum (25.71) and we can therefore use this expression together with the appropriate beam dynamics to derive the radiation spectrum from a wiggler magnet

$$
\frac{\mathrm{d}^{2} W}{\mathrm{~d} \omega \mathrm{d} \Omega}=\frac{r_{\mathrm{c}} m c \omega^{2}}{4 \pi^{2}}\left|\int_{-\infty}^{\infty} \boldsymbol{n} \times[\boldsymbol{n} \times \boldsymbol{\beta}] \mathrm{e}^{-\mathrm{i} \omega\left(t_{\mathrm{r}}+\frac{R}{c}\right)} \mathrm{d} t_{\mathrm{r}}\right|^{2} .
$$


Fig. 26.3 Particle trajectory and radiation geometry in a wiggler magnet

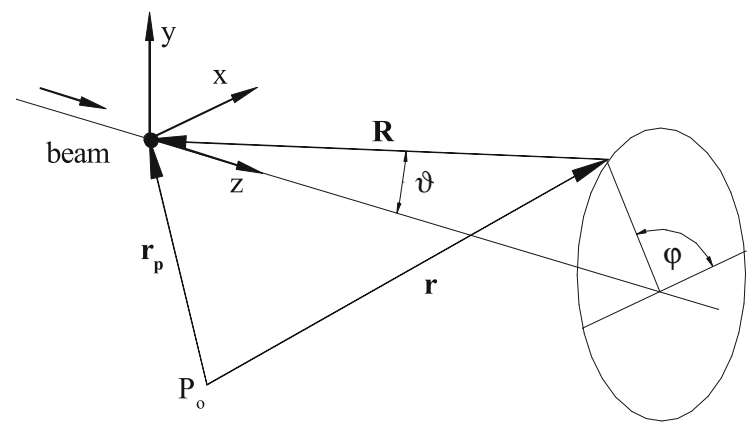

The integrand in (26.21) can be evaluated from known particle dynamics in a wiggler magnet noting that all quantities are to be taken at the retarded time $t_{\mathrm{r}}$. The unit vector from the observer to the radiating particle is from Fig. 26.3

$$
\boldsymbol{n}=-\cos \varphi \sin \vartheta \hat{\boldsymbol{x}}-\sin \varphi \sin \vartheta \hat{\boldsymbol{y}}-\cos \vartheta \hat{\boldsymbol{z}}
$$

where $(\hat{\boldsymbol{x}}, \hat{\boldsymbol{y}}, \hat{z})$ are coordinate unit vectors. The exponent in (26.21) includes the term $R / c=\boldsymbol{n} \boldsymbol{R} / c$. We express again the vector $\boldsymbol{R}$ from the observer to the particle by the constant vector $\boldsymbol{r}$ from the origin of the coordinate system to the observer and the vector $\boldsymbol{r}_{\mathrm{p}}$ from the coordinate origin to the particle for $\boldsymbol{R}=-\boldsymbol{r}+\boldsymbol{r}_{\mathrm{p}}$ as shown in Fig. 26.3.

The $\boldsymbol{r}$-term gives only a constant phase shift and can therefore be ignored. The location vector $\boldsymbol{r}_{\mathrm{p}}$ of the particle with respect to the origin of the coordinate system is

$$
\boldsymbol{r}_{\mathrm{p}}\left(t_{\mathrm{r}}\right)=x\left(t_{\mathrm{r}}\right) \hat{\boldsymbol{x}}+z\left(t_{\mathrm{r}}\right) \hat{z}
$$

and with the solutions (26.5) we have

$$
\boldsymbol{r}_{\mathrm{p}}\left(t_{\mathrm{r}}\right)=\frac{K}{k_{\mathrm{p}} \gamma} \cos \left(\omega_{\mathrm{p}} t_{\mathrm{r}}\right) \hat{\boldsymbol{x}}+\left[\bar{\beta} c t_{\mathrm{r}}+\frac{K^{2}}{8 \pi k_{\mathrm{p}}} \sin \left(2 \omega_{\mathrm{p}} t_{\mathrm{r}}\right)\right] \hat{\boldsymbol{z}}
$$

where

$$
\omega_{\mathrm{p}}=k_{\mathrm{p}} \bar{\beta} c
$$

The velocity vector finally is just the time derivative of (26.23)

$$
\boldsymbol{\beta}\left(t_{\mathrm{r}}\right)=-\frac{K}{\gamma} \bar{\beta} \sin \left(\omega_{\mathrm{p}} t_{\mathrm{r}}\right) \hat{\boldsymbol{x}}+\bar{\beta}\left[1+\frac{K^{2}}{4 \gamma^{2}} \cos \left(2 \omega_{\mathrm{p}} t_{\mathrm{r}}\right)\right] \hat{\boldsymbol{z}} .
$$


We use these vector relations to evaluate the integrand in (26.21). First, we express the triple vector product $\boldsymbol{n} \times[\boldsymbol{n} \times \boldsymbol{\beta}]$ by its components and get with (26.22), (26.25)

$$
\begin{aligned}
& \boldsymbol{n} \times[\boldsymbol{n} \times \boldsymbol{\beta}]=+\hat{\boldsymbol{x}}\left[-\frac{K}{\gamma} \bar{\beta} \sin ^{2} \vartheta \cos ^{2} \varphi \cos \omega_{\mathrm{p}} t_{\mathrm{r}}+\frac{K}{\gamma} \bar{\beta} \sin \omega_{\mathrm{p}} t_{\mathrm{r}}\right. \\
&\left.+\bar{\beta}\left(1+\frac{K^{2}}{4 \gamma^{2}} \cos 2 \omega_{\mathrm{p}} t_{\mathrm{r}}\right) \sin \vartheta \cos \vartheta \cos \varphi\right] \\
&+\hat{\boldsymbol{y}}\left[-\frac{K}{\gamma} \bar{\beta} \sin ^{2} \vartheta \sin \varphi \cos \varphi \sin \omega_{\mathrm{p}} t_{\mathrm{r}}\right. \\
&\left.+\bar{\beta}\left(1+\frac{K^{2}}{4 \gamma^{2}} \cos 2 \omega_{\mathrm{p}} t_{\mathrm{r}}\right) \sin \vartheta \cos \vartheta \sin \varphi\right] \\
&+\hat{\boldsymbol{z}}\left[-\frac{K}{\gamma} \bar{\beta} \sin \vartheta \cos \vartheta \cos \varphi \cos \omega_{\mathrm{p}} t_{\mathrm{r}}\right. \\
&+\left.\bar{\beta}\left(1+\frac{K^{2}}{4 \gamma^{2}} \cos 2 \omega_{\mathrm{p}} t_{\mathrm{r}}\right)\left(\cos ^{2} \vartheta-1\right)\right]
\end{aligned}
$$

This expression can be greatly simplified considering that the radiation is emitted into only a very small angle $\vartheta \ll 1$. Furthermore, we note that the deflection due to the wiggler field is in most practical cases very small and therefore $K \ll \gamma$ and $\bar{\beta}=\beta\left(1-\frac{K^{2}}{4 \gamma^{2}}\right) \approx \beta$. Finally, we carefully set $\beta \approx 1$ where this term does not appear as a difference to unity. With this and ignoring second order terms in $\vartheta$ and $K / \gamma$ we get from (26.26)

$$
\boldsymbol{n} \times[\boldsymbol{n} \times \boldsymbol{\beta}]=\left(\bar{\beta} \vartheta \cos \varphi+\bar{\beta} \frac{K}{\gamma} \sin \left(\omega_{\mathrm{p}} t_{\mathrm{r}}\right)\right) \hat{\boldsymbol{x}}+(\bar{\beta} \vartheta \sin \varphi) \hat{\boldsymbol{y}} .
$$

The vector product in the exponent of the exponential function is just the product of (26.22) and (26.23)

$$
\frac{1}{c} \boldsymbol{n} \boldsymbol{r}_{\mathrm{p}}\left(t_{\mathrm{r}}\right)=-\frac{K \bar{\beta}}{\gamma \omega_{\mathrm{p}}} \sin \vartheta \cos \varphi \cos \left(\omega_{\mathrm{p}} t_{\mathrm{r}}\right)-\left(\bar{\beta} t_{\mathrm{r}}+\frac{K_{\mathrm{c}}^{2-}}{8 \gamma^{2} \omega_{\mathrm{p}}} \sin 2 \omega_{\mathrm{p}} t_{\mathrm{r}}\right) \cos \vartheta
$$

Employing again the approximation $\vartheta \ll 1$ and keeping only linear terms we get from (26.28)

$$
t_{\mathrm{r}}+\frac{1}{c} \boldsymbol{n} \boldsymbol{r}_{\mathrm{p}}\left(t_{\mathrm{r}}\right)=t_{\mathrm{r}}(1-\bar{\beta} \cos \vartheta)-\frac{K_{\iota}^{-} \vartheta}{\gamma \omega_{\mathrm{p}}} \cos \varphi \cos \left(\omega_{\mathrm{p}} t_{\mathrm{r}}\right)-\frac{K_{\iota}^{2-}}{8 \gamma^{2} \omega_{\mathrm{p}}} \sin \left(2 \omega_{\mathrm{p}} t_{\mathrm{r}}\right) .
$$

With (26.4) and $\cos \vartheta \approx 1-\frac{1}{2} \vartheta^{2}$, the first term becomes 


$$
1-\bar{\beta} \cos \vartheta=\frac{1}{2 \gamma^{2}}\left(1+\frac{1}{2} K^{2}+\gamma^{2} \vartheta^{2}\right)=\frac{\omega_{\mathrm{p}}}{\omega_{1}},
$$

where we have defined the fundamental wiggler frequency $\omega_{1}$ by

$$
\omega_{1}=\omega_{\mathrm{p}} \frac{2 \gamma^{2}}{1+\frac{1}{2} K^{2}+\gamma^{2} \vartheta^{2}}
$$

or the fundamental wavelength of the radiation

$$
\lambda_{1}=\frac{\lambda_{\mathrm{p}}}{2 \gamma^{2}}\left(1+\frac{1}{2} K^{2}+\gamma^{2} \vartheta^{2}\right)
$$

in full agreement with (26.11). At this point, it is worth to remember that the term $\frac{1}{2} K^{2}$ becomes $K^{2}$ for a helical wiggler [2]. With (26.30), the complete exponential term $-\mathrm{i} \omega\left[t_{\mathrm{r}}+\frac{1}{c} \boldsymbol{n} \boldsymbol{r}_{\mathrm{p}}\left(t_{\mathrm{r}}\right)\right]$ in $(26.21)$ can be evaluated to be equal to

$$
-\mathrm{i} \frac{\omega}{\omega_{1}}\left[\omega_{\mathrm{p}} t_{\mathrm{r}}-\frac{K \bar{\beta} \vartheta}{\gamma} \frac{\omega_{1}}{\omega_{\mathrm{p}}} \cos \varphi \cos \left(\omega_{\mathrm{p}} t_{\mathrm{r}}\right)-\frac{K^{2} \bar{\beta}}{8 \gamma^{2}} \frac{\omega_{1}}{\omega_{\mathrm{p}}} \sin \left(2 \omega_{\mathrm{p}} t_{\mathrm{r}}\right)\right],
$$

and (26.21) can be modified with this expression into a form suitable for integration by inserting (26.27) and (26.30) into (26.21) for

$$
\begin{aligned}
\frac{\mathrm{d}^{2} W}{\mathrm{~d} \omega \mathrm{d} \Omega} & =\frac{r_{\mathrm{c}} m c \omega^{2}}{4 \pi^{2}} \bar{\beta} \\
& \times\left|\int_{-\infty}^{\infty}\left[\vartheta \cos \varphi+\frac{K}{\gamma} \sin \left(\omega_{\mathrm{p}} t_{\mathrm{r}}\right)\right] \boldsymbol{x}+(\vartheta \sin \varphi) \boldsymbol{y} \mathrm{e}^{X} \mathrm{~d} t_{\mathrm{r}}\right|^{2},
\end{aligned}
$$

where

$$
X=\left\{-\mathrm{i} \frac{\omega}{\omega_{1}}\left[\omega_{\mathrm{p}} t_{\mathrm{r}}-\frac{K \vartheta}{\gamma} \frac{\omega_{1}}{\omega_{\mathrm{p}}} \cos \varphi \cos \left(\omega_{\mathrm{p}} t_{\mathrm{r}}\right)-\frac{K^{2}}{8 \gamma^{2}} \frac{\omega_{1}}{\omega_{\mathrm{p}}} \sin \left(2 \omega_{\mathrm{p}} t_{\mathrm{r}}\right)\right]\right\} .
$$

We are now ready to perform the integration of (26.34) noticing that the integration over all times can be simplified by separation into an integral along the wiggler magnet alone and an integration over the rest of the time while the particle is traveling in a field free space. We write symbolically

$$
\int_{-\infty}^{\infty}=\int_{-\pi N_{\mathrm{p}} / \omega_{\mathrm{p}}}^{\pi N_{\mathrm{p}} / \omega_{\mathrm{p}}}(K \neq 0)+\int_{-\infty}^{\infty}(K=0)-\int_{-\pi N_{\mathrm{p}} / \omega_{\mathrm{p}}}^{\pi N_{\mathrm{p}} / \omega_{\mathrm{p}}}(K=0) .
$$

First, we evaluate the second integral for $K=0$ which is of the form

$$
\int_{-\infty}^{\infty} \mathrm{e}^{\mathrm{i} \kappa \omega t} \mathrm{~d} t=\frac{2 \pi}{|\kappa|} \delta(\omega)
$$


where $\delta(\omega)$ is the Dirac $\delta$-function. The value of the integral is nonzero only for $\omega=0$ in which case the factor $\omega^{2}$ in (26.34) causes the whole expression to vanish. The second integral is therefore zero.

The third integral has the same form as the second integral, but since the integration is conducted only over the length of the wiggler magnet we get

$$
\int_{-\pi N_{\mathrm{p}} / \omega_{\mathrm{p}}}^{\pi N_{\mathrm{p}} / \omega_{\mathrm{p}}} \mathrm{e}^{-\mathrm{i} \frac{\omega}{2 \gamma^{2}} t_{\mathrm{r}}} \mathrm{d} t_{\mathrm{r}}=\frac{2 \pi N_{\mathrm{p}}}{\omega_{\mathrm{p}}} \frac{\sin \frac{\pi N_{\mathrm{p}}}{2 \gamma^{2}} \frac{\omega}{\omega_{\mathrm{p}}}}{\frac{\pi N_{\mathrm{p}}}{2 \gamma^{2}} \frac{\omega}{\omega_{\mathrm{p}}}} .
$$

The value of this integral reaches a maximum of $2 \pi \frac{N_{\mathrm{p}}}{\omega_{\mathrm{p}}}$ for $\omega \rightarrow 0$. From (26.34) we note the coefficient of this integral to include the angle $\vartheta \gtrsim 1 / \gamma$ and the whole integral is therefore of the order or less than $L_{\mathrm{u}} /(c \gamma)$, where $L_{\mathrm{u}}=N_{\mathrm{p}} \lambda_{\mathrm{p}}$ is the total length of the wiggler magnet. This value is in general very small compared to the first integral and can therefore be neglected. Actually, this statement is only partially true since the first integral, as we will see, is a fast varying function of the radiation frequency with a distinct line spectrum. Being, however, primarily interested in the peak intensities of the spectrum we may indeed neglect the third integral. Only between the spectral lines does the radiation intensity from the first integral become so small that the third integral would be a relatively significant although absolutely a small contribution.

To evaluate the first integral in (26.35) with $K \neq 0$ we follow Alferov [5] and introduce with (26.31) the abbreviations

$$
\begin{aligned}
& C=\frac{2 K \bar{\beta} \gamma \vartheta \cos \varphi}{1+\frac{1}{2} K^{2}+\gamma^{2} \vartheta^{2}}, \\
& S=\frac{K^{2} \bar{\beta}}{4\left(1+\frac{1}{2} K^{2}+\gamma^{2} \vartheta^{2}\right)}
\end{aligned}
$$

to get from (26.34) the exponential functions in the form

$$
\mathrm{e}^{-\mathrm{i} \frac{\omega}{\omega_{1}} \omega_{\mathrm{p}} t_{\mathrm{r}}} \mathrm{e}^{\mathrm{i} \frac{\omega}{\omega_{1}} C \cos \omega_{\mathrm{p}} t_{\mathrm{r}}} \mathrm{e}^{\mathrm{i} \frac{\omega}{\omega_{1}} S \sin 2 \omega_{\mathrm{p}} t_{\mathrm{r}}} .
$$

The integral in the radiation power spectrum (26.34) has two distinct forms, one where the integrand is just the exponential function multiplied by a time independent factor while the other includes the sine function $\sin \omega_{\mathrm{p}} t_{\mathrm{r}}$ as a factor of the exponential function. To proceed further we replace the exponential functions by an infinite sum of Bessel's functions

$$
\mathrm{e}^{\mathrm{i} \kappa \sin \psi}=\sum_{p=-\infty}^{p=\infty} J_{p}(\kappa) \mathrm{e}^{\mathrm{i} p \psi}
$$


and apply this identity to the first integral type in (26.34). Applying the identity (26.39) also to the second and third exponential factors in (26.38), we get with $\mathrm{e}^{a \cos x}=\mathrm{e}^{a \sin (x+\pi / 2)}$ the product of the exponential functions

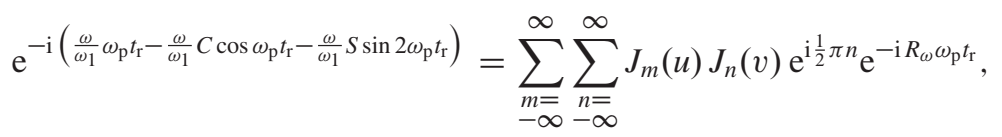

where

$$
R_{\omega}=\frac{\omega}{\omega_{1}}-n-2 m, \quad u=\frac{\omega}{\omega_{1}} S, \quad \text { and } \quad v=\frac{\omega}{\omega_{1}} C .
$$

The time integration along the length of the wiggler magnet is straight forward for this term since no other time dependent factors are involved and we get

$$
\int_{-\pi N_{\mathrm{p}} / \omega_{\mathrm{p}}}^{\pi N_{\mathrm{p}} / \omega_{\mathrm{p}}} \mathrm{e}^{-\mathrm{i}\left(\frac{\omega}{\omega_{1}}-n-2 m\right) \omega_{\mathrm{p}} t_{\mathrm{r}}} \mathrm{d} t_{\mathrm{r}}=\frac{2 \pi N_{\mathrm{p}}}{\omega_{\mathrm{p}}} \frac{\sin \left(\pi N_{\mathrm{p}} R_{\omega}\right)}{\pi N_{\mathrm{p}} R_{\omega}} .
$$

In the second form of the integrand, we replace the trigonometric factor, $\sin \omega_{\mathrm{p}} t_{\mathrm{r}}$, by exponential functions and get with (26.42) integrals of the form

$$
\begin{aligned}
& \int_{-\pi N_{\mathrm{p}} / \omega_{\mathrm{p}}}^{\pi N_{\mathrm{p}} / \omega_{\mathrm{p}}} \sin \omega_{\mathrm{p}} t_{\mathrm{r}} \mathrm{e}^{-\mathrm{i} R_{\omega} \omega_{\mathrm{p}} t_{\mathrm{r}}} \mathrm{d} t_{\mathrm{r}} \\
& =-\mathrm{i} \frac{1}{2} \int_{-\pi N_{\mathrm{p}} / \omega_{\mathrm{p}}}^{\pi N_{\mathrm{p}} / \omega_{\mathrm{p}}}\left(\mathrm{e}^{\mathrm{i} \omega_{\mathrm{p}} t_{\mathrm{r}}}-\mathrm{e}^{-\mathrm{i} \omega_{\mathrm{p}} t_{\mathrm{r}}}\right) \mathrm{e}^{-\mathrm{i} R_{\omega} \omega_{\mathrm{p}} t_{\mathrm{r}}} \mathrm{d} t_{\mathrm{r}} \\
& =\mathrm{i} \frac{\pi N_{\mathrm{p}}}{\omega_{\mathrm{p}}} \frac{\sin \left[\pi N_{\mathrm{p}}\left(R_{\omega}+1\right)\right]}{\pi N_{\mathrm{p}}\left(R_{\omega}+1\right)}-\mathrm{i} \frac{\pi N_{\mathrm{p}}}{\omega_{\mathrm{p}}} \frac{\sin \left[\pi N_{\mathrm{p}}\left(R_{\omega}-1\right)\right]}{\pi N_{\mathrm{p}}\left(R_{\omega}-1\right)} .
\end{aligned}
$$

Both integrals (26.42) and (26.43) exhibit the character of multibeam interference spectra well known from optical interference theory. The physical interpretation here is that the radiation from the $N_{\mathrm{p}}$ wiggler periods consists of $N_{\mathrm{p}}$ photon beamlets which have a specific phase relationship such that the intensities are strongly reduced for all frequencies but a few specific frequencies as determined by the $\frac{\sin x}{x}$ factors. The resulting line spectrum, characteristic for undulator radiation, is the more pronounced the more periods or beamlets are available for interference. To get a more complete picture of the interference pattern, we collect now all terms derived separately so far and use them in (26.34) which becomes with (26.38)

$$
\begin{aligned}
\frac{\mathrm{d}^{2} W}{\mathrm{~d} \omega \mathrm{d} \Omega} & =a \mid \int_{-\pi N_{\mathrm{p}} / \omega_{\mathrm{p}}}^{\pi N_{\mathrm{p}} / \omega_{\mathrm{p}}}\left[\left(A_{0}+A_{1} \sin \omega_{\mathrm{p}} t_{\mathrm{r}}\right) \hat{\boldsymbol{x}}+B_{0} \hat{\boldsymbol{y}}\right] \\
& \times\left.\mathrm{e}^{-\mathrm{i} \frac{\omega}{\omega_{1}} \omega_{\mathrm{p}} t_{\mathrm{r}}} \mathrm{e}^{\mathrm{i} v \cos \omega_{\mathrm{p}} t_{\mathrm{r}}} \mathrm{e}^{\mathrm{i} u \sin 2 \omega_{\mathrm{p}} t_{\mathrm{r}}} \mathrm{d} t_{\mathrm{r}}\right|^{2},
\end{aligned}
$$


where $a=\frac{r_{\mathrm{c}} m c \bar{\beta}^{2}}{4 \pi^{2}} \omega^{2}, A_{0}=\vartheta \cos \varphi, A_{1}=\frac{K}{\gamma}$, and $B_{0}=\vartheta \sin \varphi$. Introducing the identity (26.38), the photon energy spectrum becomes

$$
\begin{aligned}
\frac{\mathrm{d}^{2} W}{\mathrm{~d} \omega \mathrm{d} \Omega}=a & \mid \int_{-\pi N_{\mathrm{p}} / \omega_{\mathrm{p}}}^{\pi N_{\mathrm{p}} / \omega_{\mathrm{p}}}\left[\left(A_{0}+A_{1} \sin \omega_{\mathrm{p}} t_{\mathrm{r}}\right) \hat{\boldsymbol{x}}+B_{0} \hat{\boldsymbol{y}}\right] \\
& \times\left.\sum_{m=-\infty}^{\infty} \sum_{n=-\infty}^{\infty} J_{m}(u) J_{n}(v) \mathrm{e}^{\mathrm{i} \frac{1}{2} \pi n} \mathrm{e}^{-\mathrm{i} R_{\omega} \omega_{\mathrm{p}} t_{\mathrm{r}}} \mathrm{d} t_{\mathrm{r}}\right|^{2}
\end{aligned}
$$

and after integration with (26.42) and (26.43)

$$
\begin{aligned}
& \frac{\mathrm{d}^{2} W}{\mathrm{~d} \omega \mathrm{d} \Omega}= a \mid \boldsymbol{x} A_{0} \sum_{\substack{m=\infty \\
-\infty}}^{\infty} \sum_{n=\infty}^{\infty} J_{m}(u) J_{n}(v) \mathrm{e}^{\mathrm{i} \frac{1}{2} \pi n} \frac{2 \pi N_{\mathrm{p}}}{\omega_{\mathrm{p}}} \frac{\sin \left(\pi N_{\mathrm{p}} R_{\omega}\right)}{\pi N_{\mathrm{p}} R_{\omega}} \\
&+\hat{\boldsymbol{x}} A_{1} \sum_{\substack{m=\infty \\
-\infty}}^{\infty} \sum_{n=\infty}^{\infty} J_{m}(u) J_{n}(v) \mathrm{e}^{\mathrm{i} \frac{1}{2} \pi n} \\
& \\
& \quad \times \mathrm{i} \frac{\pi N_{\mathrm{p}}}{2 \omega_{\mathrm{p}}}\left[\frac{\sin \left[\pi N_{\mathrm{p}}\left(R_{\omega}+1\right)\right]}{\pi N_{\mathrm{p}}\left(R_{\omega}+1\right)}-\mathrm{i} \frac{\pi N_{\mathrm{p}}}{\omega_{\mathrm{p}}} \frac{\sin \left[\pi N_{\mathrm{p}}\left(R_{\omega}-1\right)\right]}{\pi N_{\mathrm{p}}\left(R_{\omega}-1\right)}\right] \\
&+\left.\hat{\boldsymbol{y}} B_{0} \sum_{m=}^{\infty} \sum_{-\infty}^{\infty} J_{m}(u) J_{n}(v) \mathrm{e}^{\mathrm{i} \frac{1}{2} \pi n} \frac{2 \pi N_{\mathrm{p}}}{\omega_{\mathrm{p}}} \frac{\sin \left(\pi N_{\mathrm{p}} R_{\omega}\right)}{\pi N_{\mathrm{p}} R_{\omega}}\right|^{2}
\end{aligned}
$$

To determine the frequency and radiation intensity of the line maxima, we simplify the double sum of Bessel's functions by selecting only the most dominant terms. The first and third sums in (26.46) show an intensity maximum for $R_{\omega}=0$ at frequencies

$$
\omega=(n+2 m) \omega_{1},
$$

and intensity maxima appear therefore at the frequency $\omega_{1}$ and harmonics thereof. The transformation of a lower frequency to very high values has two physical components. In the system of relativistic particles, the static magnetic field of the wiggler magnet appears Lorentz contracted by the factor $\gamma$, and particles passing through the wiggler magnet oscillate with the frequency $\gamma \omega_{\mathrm{p}}$ in its own system emitting radiation at that frequency. The observer in the laboratory system receives this radiation from a source moving with relativistic velocity and experiences therefore a Doppler shift by the factor $2 \gamma$. The wavelength of the radiation emitted in the forward direction, $\vartheta=0$, from a weak wiggler magnet, $K \ll 1$, with the period length $\lambda_{\mathrm{p}}$ is therefore reduced by the factor $2 \gamma^{2}$. In cases of a stronger wiggler 
magnet or when observing at a finite angle $\vartheta$, the wavelength is somewhat longer as one would expect from higher order terms of the Doppler effect.

From (26.46) we determine two more dominant terms originating from the second term for $R_{\omega} \pm 1=0$ at frequencies

$$
\begin{aligned}
& \omega=(n+2 m-1) \omega_{1} \\
& \omega=(n+2 m+1) \omega_{1},
\end{aligned}
$$

respectively. The summation indices $n$ and $m$ are arbitrary integers between $-\infty$ and $\infty$. Among all possible resonant terms we collect such terms which contribute to the same harmonic $k$ of the fundamental frequency $\omega_{1}$. To collect these dominant terms for the same harmonic we set $\omega=\omega_{k}=k \omega_{1}$ where $k$ is the harmonic number of the fundamental and express the index $n$ by $k$ and $m$ to get

$$
\begin{aligned}
\text { from (26.47): } & n=k-2 m, \\
\text { from (26.48a): } & n=k-2 m+1 \\
\text { and from (26.48b): } & n=k-2 m-1 .
\end{aligned}
$$

Introducing these conditions into (26.46) all trigonometric factors assume the form $\frac{\sin \left(\pi N_{\mathrm{p}} \Delta \omega_{k} / \omega_{1}\right)}{\pi N_{\mathrm{p}} \Delta \omega_{k} / \omega_{1}}$, where

$$
\frac{\Delta \omega_{k}}{\omega_{1}}=\frac{\omega}{\omega_{1}}-k
$$

and we get the photon energy spectrum of the $k$ th harmonic for radiation from a single electron passing through an undulator

$$
\begin{aligned}
\frac{\mathrm{d}^{2} W_{k}(\omega)}{\mathrm{d} \omega \mathrm{d} \Omega}= & \frac{r_{\mathrm{c}} m c \bar{\beta}^{2} N_{\mathrm{p}}^{2}}{\gamma^{2}} \frac{\omega^{2}}{\omega_{\mathrm{p}}^{2}}\left[\frac{\sin \left(\pi N_{\mathrm{p}} \Delta \omega_{k} / \omega_{1}\right)}{\pi N_{\mathrm{p}} \Delta \omega_{k} / \omega_{1}}\right]^{2} \\
\times \mid & +\hat{\boldsymbol{x}} A_{0} \sum_{m=-\infty}^{\infty} J_{m}(u) J_{k-2 m}(v) \mathrm{e}^{\mathrm{i} \frac{1}{2} \pi(k-2 m)} \\
& +\hat{\boldsymbol{y}} B_{0} \sum_{m=-\infty}^{\infty} J_{m}(u) J_{k-2 m}(v) \mathrm{e}^{\mathrm{i} \frac{1}{2} \pi(k-2 m)} \\
& +\mathrm{i} \hat{\boldsymbol{x}} \frac{1}{2} A_{1} \sum_{m=-\infty}^{\infty} J_{m}(u) J_{k-2 m+1}(v) \mathrm{e}^{\mathrm{i} \frac{1}{2} \pi(k-2 m+1)} \\
& -\left.\mathrm{i} \hat{\boldsymbol{x}} \frac{1}{2} A_{1} \sum_{m=-\infty}^{\infty} J_{m}(u) J_{k-2 m-1}(v) \mathrm{e}^{\mathrm{i} \frac{1}{2} \pi(k-2 m-1)}\right|^{2} .
\end{aligned}
$$




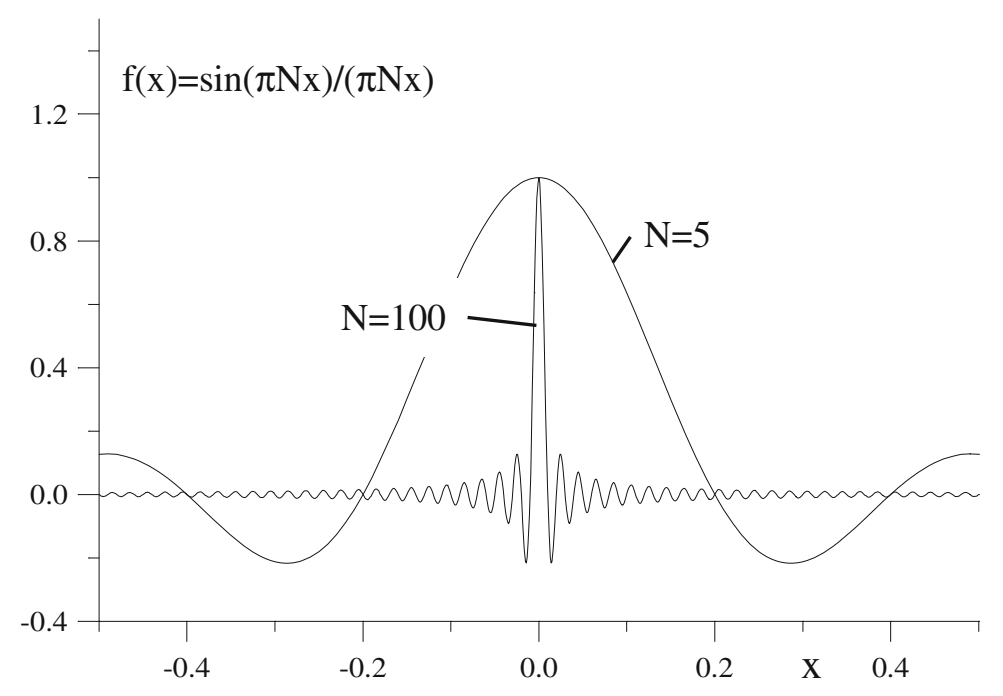

Fig. 26.4 $\frac{\sin \left(\pi N_{\mathrm{p}} x\right)}{\pi N_{\mathrm{p}} x}$ distribution for $N_{\mathrm{p}}=5$ and $N_{\mathrm{p}}=100$

All integrals exhibit the resonance character defining the locations of the spectral lines. The $(\sin x / x)$-terms represents the line spectrum of the radiation. Specifically, the number $N_{\mathrm{p}}$ of beamlets, here source points, determines the spectral purity of the radiation. In Fig. 26.4 the $(\sin x / x)$-function is shown for $N_{\mathrm{p}}=5$ and $N_{\mathrm{p}}=100$. It is clear that the spectral purity improves greatly as the number of undulator periods is increased. This is one of the key features of undulator magnets to gain spectral purity by maximizing the number of undulator periods.

The spectral purity or line width is determined by the shape of the $(\sin x / x)$ function. We define the line width by the frequency at which $\sin x / x=0$ or where $\pi N_{\mathrm{p}} \Delta \omega_{k} / \omega_{1}=\pi$ defining the line width for the $k$ th harmonic

$$
\frac{\Delta \omega_{k}}{\omega_{k}}= \pm \frac{1}{k N_{\mathrm{p}}} .
$$

The spectral width of the undulator radiation is reduced proportional to the number of undulator periods, but reduces also proportional to the harmonic number.

The Bessel functions $J_{m}(u)$ determine mainly the intensity of the line spectrum. For an undulator with $K \ll 1$, the argument $u \propto K^{2} \ll 1$ and the contributions of higher order Bessel's functions are very small. The radiation spectrum consists therefore only of the fundamental line. For stronger undulators with $K>1$, higher order Bessel's functions grow and higher harmonic radiation appears in the line spectrum of the radiation. 
Summing over all harmonics of interest, one gets the total power spectrum. In the third and fourth terms of (26.51) we use the identities i $\mathrm{e}^{ \pm \mathrm{i} \pi / 2}=\mp 1, J_{m}(u) \mathrm{e}^{\mathrm{i} \pi m}=$ $J_{-m}(u)$ and abbreviate the sums of Bessel's functions by the symbols

$$
\begin{aligned}
& \sum_{1}=\sum_{m=-\infty}^{\infty} J_{-m}(u) J_{k-2 m}(v) \\
& \sum_{2}=\sum_{m=-\infty}^{\infty} J_{-m}(u)\left[J_{k-2 m-1}(v)+J_{k-2 m+1}(v)\right] .
\end{aligned}
$$

The total number of photons $N_{\text {ph }}$ emitted into a spectral band width $\Delta \omega / \omega$ by a single electron moving through a wiggler magnet is finally with $N_{\mathrm{ph}}(\omega)=$ $W(\omega) /(\hbar \omega)$

$$
\begin{aligned}
\frac{\mathrm{d} N_{\mathrm{ph}}(\omega)}{\mathrm{d} \Omega}=\alpha & \gamma^{2} \bar{\beta}^{2} N_{\mathrm{p}}^{2} \frac{\Delta \omega}{\omega} \sum_{k=1}^{\infty} k^{2}\left[\frac{\sin \left(\pi N_{\mathrm{p}} \Delta \omega_{k} / \omega_{1}\right)}{\pi N_{\mathrm{p}} \Delta \omega_{k} / \omega_{1}}\right]^{2} \\
& \times \frac{\left(2 \gamma \vartheta \sum_{1} \cos \varphi-K \sum_{2}\right)^{2} \hat{\boldsymbol{x}}^{2}+\left(2 \gamma \vartheta \sum_{1} \sin \varphi\right)^{2} \hat{\boldsymbol{y}}^{2}}{\left(1+\frac{1}{2} K^{2}+\gamma^{2} \vartheta^{2}\right)^{2}}
\end{aligned}
$$

where $\alpha$ is the fine structure constant and where we have kept the coordinate unit vectors to keep track of the polarization modes. The vectors $\mathbf{x}$ and $\mathbf{y}$ are orthogonal unit vectors indicating the directions of the electric field or the polarization of the radiation. Performing the squares does therefore not produce cross terms and the two terms in (26.54) with the expressions (26.53) represent the amplitude factors for both polarization directions, the $\sigma$-mode and $\pi$-mode respectively.

We also made use of (26.50) and the resonance condition

$$
\frac{\omega}{\omega_{\mathrm{p}}}=\frac{k \omega_{1}+\Delta \omega_{k}}{\omega_{\mathrm{p}}} \approx k \frac{\omega_{1}}{\omega_{\mathrm{p}}}=\frac{2 \gamma^{2} k}{1+\frac{1}{2} K^{2}+\gamma^{2} \vartheta^{2}},
$$

realizing that the photon spectrum is determined by the $(\sin x / x)^{2}$-function. For not too few periods, this function is very small for frequencies away from the resonance conditions.

Storage rings optimized for very small beam emittance are being used as modern synchrotron radiation sources to reduce the line width of undulator radiation and concentrate all radiation to the frequency desired. The progress in this direction is demonstrated in the spectrum of Fig. 26.5 derived from the first electron storage ring operated at a beam emittance below $10 \mathrm{~nm}$ at $7.1 \mathrm{GeV}$ [7]. In Fig. 26.5 a measured undulator spectrum is shown as a function of the undulator strength $K$ [8]. For a 

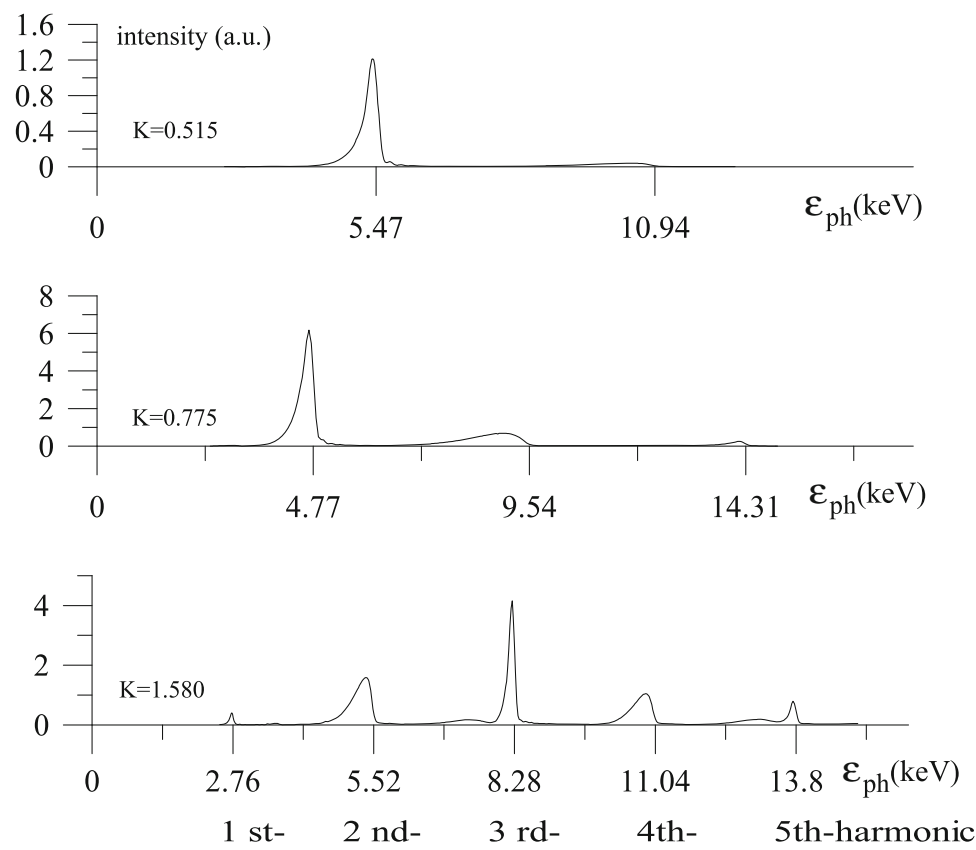

Fig. 26.5 Measured frequency spectrum from an undulator for different strength parameters $K$ [8]

strength parameter $K \ll 1$ there is only one line at the fundamental frequency. As the strength parameter increases, additional lines appear in addition to being shifted to lower frequencies. The spectral lines from a real synchrotron radiation source are not infinitely narrow as (26.66) would suggest. Because of the finite size of the pinhole opening, some light at small angles with respect to the axis passes through, and we observe therefore also some signal of the even order harmonic radiation.

Even for an extremely small pin hole, we would observe a similar spectrum as shown in Fig. 26.5 because of the finite beam divergence of the electron beam. The electrons follow oscillatory trajectories due not only to the undulator field but also due to betatron oscillations. We observe therefore always some radiation at a finite angle given by the particle trajectory with respect to the undulator axis. Figure 26.5 also demonstrates the fact that all experimental circumstances must be included to meet theoretical expectations. The amplitudes of the measured low energy spectrum is significantly suppressed compared to theoretical expectations which is due to a Be-window being used to extract the radiation from the ultra high vacuum chamber of the accelerator. This material absorbs radiation significantly below a photon energy of about $3 \mathrm{keV}$.

While we observe a line spectrum expressed by the $(\sin x / x)^{2}$-function, we also notice that this line spectrum is red shifted as we increase the observation angle $\vartheta$. Only, when we observe the radiation though a very small aperture (pin hole) 


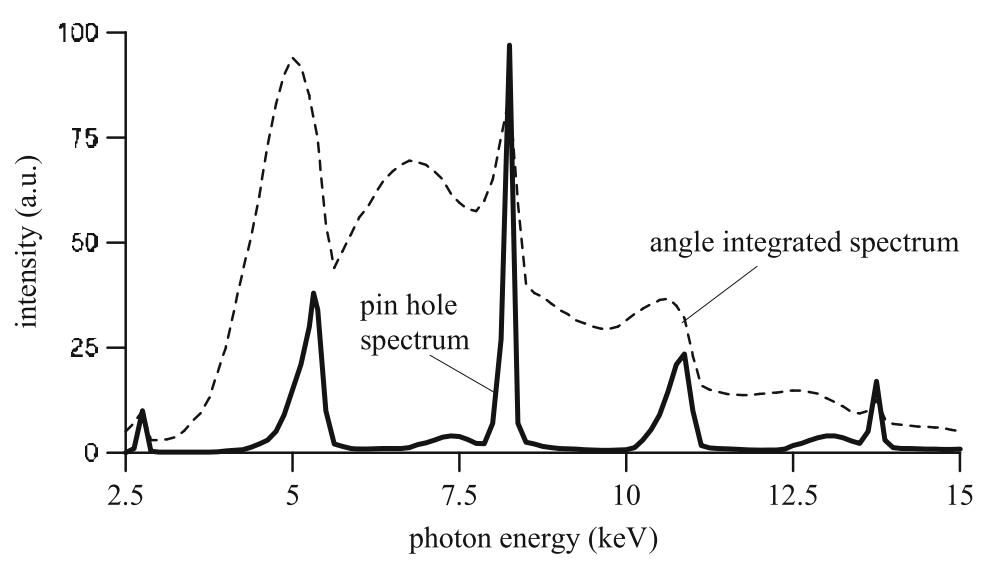

Fig. 26.6 Actual radiation spectra from an undulator with a maximum field of $0.2 \mathrm{~T}$ and a beam energy of $7.1 \mathrm{GeV}$ through a pin hole and angle-integrated after removal of the pin hole [7]

do we actually see this line spectrum. Viewing the undulator radiation through a large aperture integrates the linespectra over a finite range of angles $\vartheta$ producing an almost continuous spectrum with small spikes at the locations of the harmonic lines.

The difference between a pin hole undulator spectrum and an angle-integrated spectrum becomes apparent from the experimental spectra shown in Fig. 26.6 [7]. While the pin hole spectrum demonstrates well the line character of undulator radiation, much radiation appears between these spectral lines as the pin hole is removed and radiation over a large solid angle is collected by the detector. The pin hole undulator line spectrum shows up as mere spikes on top of a broad continuous spectrum.

The overall spatial intensity distribution includes a complex set of different radiation lobes depending on frequency, emission angle and polarization. In Fig. 26.7 the radiation intensity distributions described by the last factor in (26.54)

$$
I_{\sigma, k}=\frac{\left(2 \gamma \vartheta \Sigma_{1} \cos \varphi-K \Sigma_{2}\right)^{2}}{\left(1+\frac{1}{2} K^{2}+\gamma^{2} \vartheta^{2}\right)^{2}}
$$

for the $\sigma$-mode polarization and

$$
I_{\pi, k}=\frac{\left(2 \gamma \vartheta \Sigma_{1} \sin \varphi\right)^{2}}{\left(1+\frac{1}{2} K^{2}+\gamma^{2} \vartheta^{2}\right)^{2}}
$$

for the $\pi$-mode polarization are shown for the lowest order harmonics.

We note clearly the strong forward lobe at the fundamental frequency in $\sigma$-mode while there is no emission in $\pi$-mode along the path of the particle. The second 
$\sigma$-mode $\quad \pi$-mode
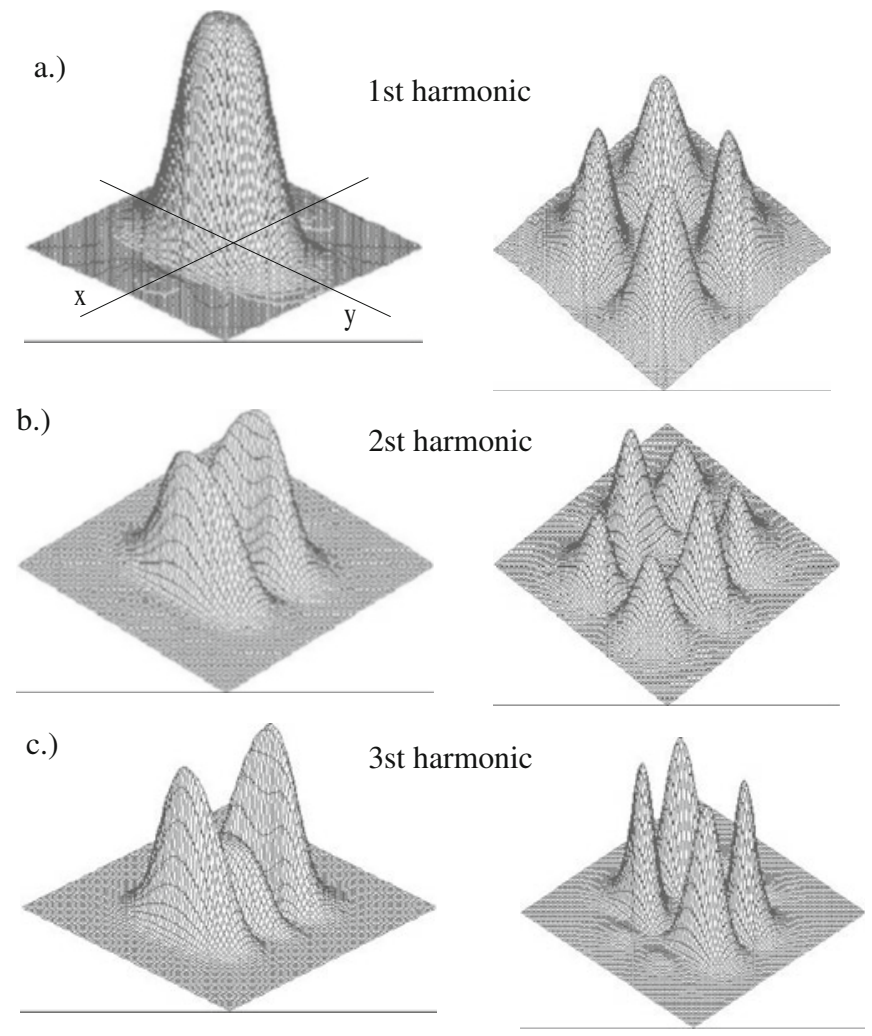

Fig. 26.7 Undulator radiation distribution in $\sigma$-and $\pi$-mode for the lowest order harmonics

harmonic radiation vanishes in the forward direction, an observation that is true for all even harmonics. By inspection of (26.54), we note that $v=0$ for $\vartheta=0$ and the square bracket in (26.53b) vanishes for all odd indices or for all even harmonics $k$. There is therefore no forward radiation for even harmonics of the fundamental undulator frequency.

A contour plot of the first harmonic $\sigma$ - and $\pi$-mode radiation is shown in Fig. 26.8. There is a slight asymmetry in the radiation distribution between the deflecting and nondeflecting plane as one might expect. It is obvious that the pin hole radiation is surrounded by many radiation lobes not only from the first harmonics but also from higher harmonics compromising the pure line spectrum for larger apertures. 
Fig. 26.8 Contour plot of the first harmonic $\sigma$-mode (solid) and $\pi$-mode (dashed) undulator radiation distribution

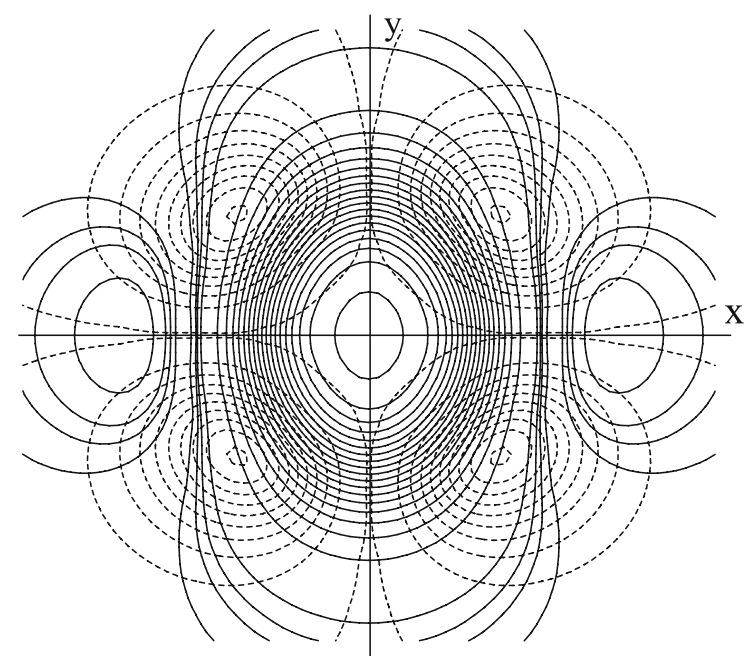

\subsubsection{Line Spectrum}

To exhibit other important and desirable features of the radiation spectrum (26.54), we ignore the actual frequency distribution in the vicinity of the harmonics and set $\Delta \omega_{k}=0$ because the spectral lines are narrow for large numbers of wiggler periods $N_{\mathrm{p}}$. Further, we are interested for now only in the forward radiation where $\vartheta=0$ keeping in mind that the radiation is mostly emitted into a small angle $\langle\vartheta\rangle=1 / \gamma$.

There is no radiation for the $\pi$-mode in the forward direction and the only contribution to the forward radiation comes from the second term in (26.54) of the $\sigma$-mode. From (26.41) we get for this case with $\omega / \omega_{1}=k$

$$
u_{0}=\frac{k K^{2}}{4+2 K^{2}} \quad \text { and } \quad v_{0}=0 .
$$

The sums of Bessel's functions simplify in this case greatly because only the lowest order Bessel's function has a nonvanishing value for $v_{0}=0$. In the expression for $\Sigma_{2}$ all summation terms vanish except for the two terms for which the index is zero or for which

$$
k-2 m-1=0, \quad \text { or } \quad k-2 m+1=0
$$

and

$$
\begin{aligned}
\sum_{2} & =\sum_{m=-\infty}^{\infty} J_{-m}(u)\left[J_{k-2 m-1}(0)+J_{k-2 m+1}(0)\right] \\
& =J_{-\frac{k-1}{2}}\left(u_{0}\right)+J_{-\frac{k+1}{2}}\left(u_{0}\right) .
\end{aligned}
$$


The harmonic condition (26.57) implies that $k$ is an odd integer. For even integers, the condition cannot be met as we would expect from earlier discussions on harmonic radiation in the forward direction. Using the identity $J_{-n}=(-1)^{n} J_{n}$ and (26.56), we get finally with $N_{\text {ph }}=W / \hbar \omega$ the photon flux per unit solid angle from a highly relativistic particle passing through an undulator

$$
\left.\frac{\mathrm{d} N_{\mathrm{ph}}(\omega)}{\mathrm{d} \Omega}\right|_{\theta=0}=\alpha \gamma^{2} N_{\mathrm{p}}^{2} \frac{\Delta \omega}{\omega} \frac{K^{2}}{\left(1+\frac{1}{2} K^{2}\right)^{2}} \sum_{k=1}^{\infty} k^{2}\left(\frac{\sin \pi N_{\mathrm{p}} \Delta \omega_{k} / \omega_{1}}{\pi N_{\mathrm{p}} \Delta \omega_{k} / \omega_{1}}\right)^{2} J J^{2},
$$

where the $J J$-function is defined by

$$
J J=\left[J_{\frac{1}{2}(k-1)}\left(\frac{k K^{2}}{4+2 K^{2}}\right)-J_{\frac{1}{2}(k+1)}\left(\frac{k K^{2}}{4+2 K^{2}}\right)\right] .
$$

The amplitudes of the harmonics are given by

$$
A_{k}(K)=\frac{k^{2} K^{2}}{\left(1+\frac{1}{2} K^{2}\right)^{2}} J J^{2} .
$$

The strength parameter greatly determines the radiation intensity as shown in Fig. 26.9 for the lowest order harmonics. For the convenience of numerical calculations the values $A_{k}(K)$ are tabulated for odd harmonics in Table 26.1. For weak magnets $(K \ll 1)$ the intensity increases with the square of the magnet field or undulator strength parameter. There is an optimum value for the strength parameter for maximum photon flux depending on the harmonic under consideration. In particular, radiation in the forward direction at the fundamental frequency reaches a maximum photon flux for strength parameters $K \approx 1.3$. The photon flux per unit solid angle increases like the square of the number of wiggler periods $N_{\mathrm{p}}$, which is a result of the interference effect of many beams concentrating the radiation more and more into one frequency and its harmonics as the number of interfering beams is increased.

Fig. 26.9 Undulator radiation intensity $A_{k}(K)$ in the forward direction as a function of the strength parameter $K$ for the six lowest order odd harmonics

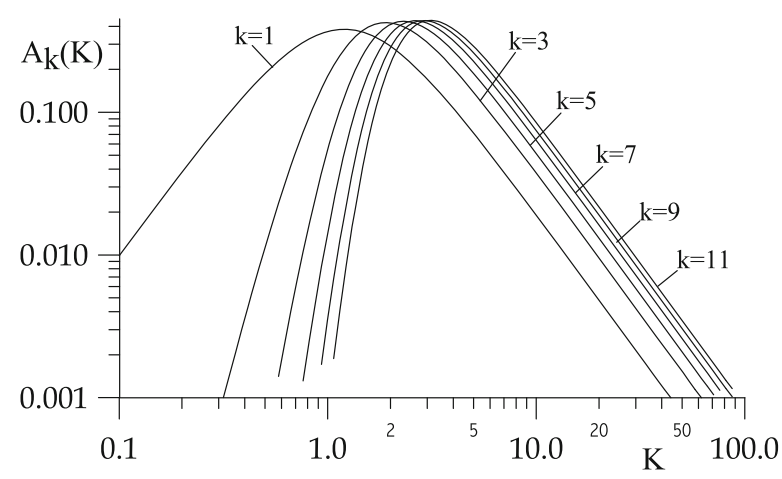


Table 26.1 Amplitudes $A_{k}(K)$ for $k=1,3,5,7,9,11$

\begin{tabular}{l|l|l|l|l|l|l}
\hline$K$ & $A_{1}$ & $A_{3}$ & $A_{5}$ & $A_{7}$ & $A_{9}$ & $A_{11}$ \\
\hline 0.1 & 0.010 & 0 & 0 & 0 & 0 & 0 \\
\hline 0.2 & 0.038 & 0 & 0 & 0 & 0 & 0 \\
\hline 0.4 & 0.132 & 0.004 & 0 & 0 & 0 & 0 \\
\hline 0.6 & 0.238 & 0.027 & 0.002 & 0 & 0 & 0 \\
\hline 0.8 & 0.322 & 0.087 & 0.015 & 0.002 & 0 & 0 \\
\hline 1.0 & 0.368 & 0.179 & 0.055 & 0.015 & 0.004 & 0.001 \\
\hline 1.2 & 0.381 & 0.276 & 0.128 & 0.051 & 0.019 & 0.007 \\
\hline 1.4 & 0.371 & 0.354 & 0.219 & 0.118 & 0.059 & 0.028 \\
\hline 1.8 & 0.320 & 0.423 & 0.371 & 0.286 & 0.206 & 0.142 \\
\hline 2.0 & 0.290 & 0.423 & 0.413 & 0.354 & 0.285 & 0.220 \\
\hline 5.0 & 0.071 & 0.139 & 0.188 & 0.228 & 0.261 & 0.290 \\
\hline 10.0 & 0.019 & 0.037 & 0.051 & 0.064 & 0.075 & 0.085 \\
\hline 20.0 & 0.005 & 0.010 & 0.013 & 0.016 & 0.019 & 0.022 \\
\hline
\end{tabular}

The radiation opening angle is primarily determined by the $(\sin x / x)^{2}$-term. We define the opening angle for the $k$ th harmonic radiation by $\vartheta_{k}$ being the angle for which $\sin x / x=0$ for the first time. In this case $x=\pi$ or $N_{\mathrm{p}} \Delta \omega_{k} / \omega_{1}=1$. With $\omega_{1}=\omega_{\mathrm{p}} \frac{2 \gamma^{2}}{1+\frac{1}{2} K^{2}}, \omega_{k}=k \omega_{\mathrm{p}} \frac{2 \gamma^{2}}{1+\frac{1}{2} K^{2}+\gamma^{2} \vartheta_{k}^{2}}$ and $\frac{\Delta \omega_{k}}{\omega_{1}}=\left|\frac{\omega_{k}}{\omega_{1}}-k\right|$, we get $\frac{N_{\mathrm{p}} k \gamma^{2} \vartheta_{k}^{2}}{1+\frac{1}{2} K^{2}+\gamma^{2} \vartheta_{k}^{2}}=$ 1 or after solving for $\vartheta_{k}$

$$
\vartheta_{k}^{2}=\frac{1+\frac{1}{2} K^{2}}{\gamma^{2}\left(k N_{\mathrm{p}}-1\right)}
$$

Assuming an undulator with many periods $\left(k N_{\mathrm{p}} \gg 1\right)$ the rms opening angle of undulator radiation is finally

$$
\sigma_{r} \approx \frac{1}{\sqrt{2}} \vartheta_{k}=\frac{1}{\gamma} \sqrt{\frac{1+\frac{1}{2} K^{2}}{2 k N_{\mathrm{p}}}} .
$$

Radiation emitted into a solid angle defined by this small opening angle

$$
\Delta \Omega=\pi \sigma_{r}^{2}
$$

is referred to as the forward radiation cone. The opening angle of undulator radiation becomes more collimated as the number of periods and the order of the harmonic increases. On the other hand, the radiation cone opens up as the undulator strength $K$ is increased. We may use this opening angle to calculate the total photon flux of the $k$ th harmonic within a bandwidth $\frac{\Delta \omega}{\omega}$ into the forward cone

$$
\left.N_{\mathrm{ph}}\left(\omega_{k}\right)\right|_{\vartheta=0}=\frac{1}{2} \pi \alpha N_{\mathrm{p}} \frac{\Delta \omega}{\omega_{k}} \frac{k K^{2}}{1+\frac{1}{2} K^{2}} J J^{2},
$$


where $\omega_{k}=k \omega_{1}$. The radiation spectrum from an undulator magnet into the forward direction has been reduced to a simple form exhibiting the most important characteristic parameters. Utilizing (26.61) the number of photons emitted into a band width $\frac{\Delta \omega}{\omega_{k}}$ from a single electron passing through an undulator in the $k$ th harmonic is

$$
\left.N_{\mathrm{ph}}\left(\omega_{k}\right)\right|_{\vartheta=0}=\frac{1}{2} \pi \alpha N_{\mathrm{p}} \frac{\Delta \omega}{\omega_{k}} \frac{1+\frac{1}{2} K^{2}}{k} A(K) .
$$

Equation (26.66) is to be multiplied by the number of particles in the electron beam to get the total photon intensity. In case of a storage ring, particles circulate with a high revolution frequency and we get from (26.66) by multiplication with $I / e$, where $I$ is the circulating beam current, the photon flux

$$
\left.\frac{\mathrm{d} N_{\mathrm{ph}}\left(\omega_{k}\right)}{\mathrm{d} t}\right|_{\vartheta=0}=\frac{1}{2} \pi \alpha N_{\mathrm{p}} \frac{I}{e} \frac{\Delta \omega}{\omega_{k}} \frac{1+\frac{1}{2} K^{2}}{k} A(K) .
$$

The spectrum includes only odd harmonic since all even harmonics are suppressed through the cancellation of Bessel's functions. This photon flux represents fully spatial coherent radiation as long as the beam divergence does not significantly contribute to the photon divergence (26.63).

\subsubsection{Spectral Undulator Brightness}

Similar to Chap. 27 we define the spectral brightness of undulator radiation as the photon density in six-dimensional phase space. The actual photon brightness is reduced from the diffraction limit due to betatron motion of the particles, transverse beam oscillation in the undulator, apparent source size on axis and under an oblique angle. All of these effects tend to increase the source size and reduce brightness.

The particle beam cross section varies in general along the undulator. We assume here for simplicity that the beam size varies symmetrically along the undulator with a waist in its center. From beam dynamics it is then known that, for example, the horizontal beam size varies like $\sigma_{\mathrm{b}}^{2}=\sigma_{\mathrm{b} 0}^{2}+\sigma_{\mathrm{b} 0}^{\prime 2} s^{2}$, where $\sigma_{\mathrm{b} 0}$ is the beam size at the waist, $\sigma_{\mathrm{b} 0}^{\prime}$ the divergence of the beam at the waist and $-\frac{1}{2} L \leqq s \leqq \frac{1}{2} L$ the distance from the waist. The average beam size along the undulator length $L$ is then

$$
\left\langle\sigma_{\mathrm{b}}^{2}\right\rangle=\sigma_{\mathrm{b} 0}^{2}+\frac{1}{12} \sigma_{\mathrm{b} 0}^{\prime 2} L^{2} .
$$

Similarly, due to an oblique observation angle $\vartheta$ with respect to the $(y, z)$-plane or $\psi$ with respect to the $(x, z)$-plane we get a further additive contribution $\frac{1}{6} \vartheta L$ to the apparent beam size. Finally, the apparent source size is widened by the transverse beam wiggle in the periodic undulator field. This oscillation amplitude is from (26.6) $a=\lambda_{\mathrm{p}} K /(2 \pi \gamma)$. 
Collecting all contributions and adding them in quadrature, the total effective beam-size parameters are given by

$$
\begin{aligned}
\sigma_{t, x}^{2} & =\frac{1}{2} \sigma_{r}^{2}+\sigma_{\mathrm{b} 0, x}^{2}+\left(\frac{\lambda_{\mathrm{p}} K}{2 \pi \gamma}\right)^{2}+\frac{1}{12} \sigma_{\mathrm{b} 0, x^{\prime}}^{2} L^{2}+\frac{1}{36} \vartheta^{2} L^{2}, \\
\sigma_{t, x^{\prime}}^{2} & =\frac{1}{2} \sigma_{r^{\prime}}^{2}+\sigma_{\mathrm{b} 0, x^{\prime}}^{2}, \\
\sigma_{t, y}^{2} & =\frac{1}{2} \sigma_{r}^{2}+\sigma_{\mathrm{b} 0, y}^{2}+\left(\frac{\lambda \mathrm{p} K}{2 \pi \gamma}\right)^{2}+\frac{1}{12} \sigma_{\mathrm{b} 0, y^{\prime}}^{2} L^{2}+\frac{1}{36} \psi^{2} L^{2}, \\
\sigma_{t, y^{\prime}}^{2} & =\frac{1}{2} \sigma_{r^{\prime}}^{2}+\sigma_{\mathrm{b} 0, y^{\prime}}^{2},
\end{aligned}
$$

where the particle beam sizes can be expressed by the beam emittance and betatron function as $\sigma_{\mathrm{b}}^{2}=\epsilon \beta, \sigma_{\mathrm{b}}^{\prime 2}=\epsilon / \beta$, and the diffraction limited beam parameters are $\sigma_{r^{\prime}}=\sqrt{\lambda / L}$, and $\sigma_{r}=\sqrt{\lambda L} /(2 \pi)$.

\subsection{Elliptical Polarization}

During the discussion of bending magnet radiation in Chap. 25 and insertion radiation in this chapter, we noticed the appearance of two orthogonal components of the radiation field which we identified with the $\sigma$-mode and $\pi$-mode polarization. The $\pi$-mode radiation is observable only at a finite angle with the plane defined by the particle trajectory and the acceleration force vector, which is in general the horizontal plane. As we will see, both polarization modes can, under certain circumstances, be out of phase giving rise to elliptical polarization. In this section, we will shortly discuss such conditions.

\subsubsection{Elliptical Polarization from Bending Magnet Radiation}

The direction of the electric component of the radiation field is parallel to the particle acceleration. Since radiation is the perturbation of electric field lines from the charge at the retarded time to the observer, we must take into account all apparent acceleration. To see this more clear, we assume an electron to travel counter clockwise on an orbit travelling from say a 12-o' clock position to 9-o' clock and then 6-o'clock. Watching the particle in the plane of deflection, the midplane, we notice only a horizontal acceleration which is maximum at 9-o'clock. Radiation observed in the midplane is therefore linearly polarized in the plane of deflection.

Now we observe the same electron at a small angle above the midplane. Apart from the horizontal motion, we notice now also an apparent vertical motion. Since the electron follows pieces of a circle this vertical motion is not uniform but exhibits 
acceleration. Specifically, at 12-o'clock the particle seems to be accelerated only in the vertical direction (downward), horizontally it is in uniform motion; at 9o'clock the acceleration is only horizontal (towards 3-o'clock) and the vertical motion is uniform; finally, at 6-o'clock the electron is accelerated only in the vertical plane again (upward). Because light travels faster than the electron, we observe radiation first coming from the 12-o'clock position, then from 9-o'clock and finally from 6-o'clock. The polarization of this radiation pulse changes from downward to horizontal (left-right) to upward which is what we call elliptical polarization where the polarization vector rotates with time. Of course, in reality we do not observe radiation from half the orbit, but only from a very short arc segment of angle $\pm 1 / \gamma$. However, if we consider Lorentz contraction the 9-o'clock trajectory in the particle system looks very close to a half circle radiation into \pm 180 degrees which appears in the laboratory system within $\pm 1 / \gamma$. Therefore the short piece of arc from which we observe the radiation has all the features just used to explain elliptical polarization in a bending magnet.

If we observe the radiation at a small angle from below the midplane, the sequence of accelerations is opposite, upward-horizontal (left-right)-downward. The helicity of the polarization is therefore opposite for an observer below or above the midplane. This qualitative discussion of elliptical polarization must become obvious also in the formal derivation of the radiation field. Closer inspection of the radiation field (25.87) from a bending magnet

$$
\boldsymbol{E}_{\mathrm{r}}(\omega)=-\frac{\sqrt{3}}{4 \pi \epsilon_{0}} \frac{e}{c R} \frac{\omega}{\omega_{\mathrm{c}}} \gamma\left(1+\gamma^{2} \vartheta^{2}\right)\left[\operatorname{sign}\left(\frac{1}{\rho}\right) K_{2 / 3}(\xi) \boldsymbol{u}_{\sigma}-\mathrm{i} \frac{\gamma \vartheta K_{1 / 3}(\xi)}{\sqrt{1+\gamma^{2} \vartheta^{2}}} \boldsymbol{u}_{\pi}\right]
$$

shows that both polarization terms are $90^{\circ}$ out of phase. As a consequence, the combination of both terms does not just introduce a rotation of the polarization direction but generates a time dependent rotation of the polarization vector which we identify with circular or elliptical polarization. In this particular case, the polarization is elliptical since the $\pi$-mode radiation is always weaker than the $\sigma$-mode radiation. The field rotates in time just as expected from the qualitative discussion above. The linear dependence of the second term in (26.70) also defines the helicity proportional to the sign of $\vartheta$.

We may quantify the polarization property considering that the electrical field is proportional to the acceleration vector $\dot{\boldsymbol{\beta}}$. Observing radiation at an angle with the horizontal plane, we note that the acceleration being normal to the trajectory and in the midplane can be decomposed into two components $\dot{\beta}_{x}$ and $\dot{\beta}_{z}$ as shown in Fig. 26.10a.

The longitudinal acceleration component together with a finite observation angle $\vartheta$ gives rise to an apparent vertical acceleration with respect to the observation direction and the associated vertical electric field component is

$$
\boldsymbol{E}_{y} \propto \dot{\beta}_{y}=n_{y} \dot{\beta}_{z}+n_{x} n_{y} \dot{\beta}_{x} .
$$


a)

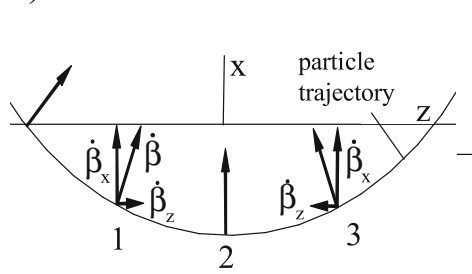

b)

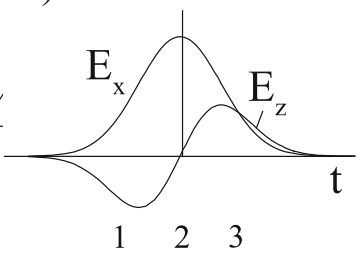

c)

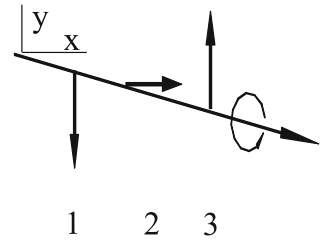

Fig. 26.10 Acceleration along an arc-segment of the particle trajectory in (a) a bending magnet, (b) polarization as a function of time, and (c) radiation field components as a function of time

An additional component appears, if we observe the radiation also at an angle with respect to the $(x, y)$-plane which we, however, ignore here for this discussion. The components $n_{x}, n_{y}$ are components of the observation unit vector from the observer to the source with $n_{y}=-\sin \vartheta$. We observe radiation first from an angle $\vartheta>0$. The horizontal and vertical radiation field components as a function of time are shown in Fig. 26.10b. Both being proportional to the acceleration (Fig. 26.10a), we observe a symmetric horizontal field $E_{x}$ and an antisymmetric vertical field $E_{y}$. The polarization vector (Fig. 26.10c) therefore rotates with time in a counter clockwise direction giving rise to elliptical polarization with lefthanded helicity. Observing the radiation from below with $\vartheta<0$, the antisymmetric field switches sign and the helicity becomes righthanded. The visual discussion of the origin of elliptical polarization of bending magnet radiation is in agreement with the mathematical result (26.70) displaying the sign dependence of the $\pi$-mode component with $\vartheta$.

The intensities for both polarization modes are shown in Fig. 26.11 as a function of the vertical observation angle $\vartheta$ for different photon energies. Both intensities are normalized to the forward intensity of the $\sigma$-mode radiation. From Fig. 26.11 it becomes obvious that circular polarization is approached for large observation angles. At high photon energies both radiation lobes are confined to very small angles but expand to larger angle distributions for photon energies much lower than the critical photon energy.

The elliptical polarization is left or right handed depending on whether we observe the radiation from above or below the horizontal mid plane. Furthermore, the helicity depends on the direction of deflection in the bending magnet or the sign of the curvature sign $(1 / \rho)$. By changing the sign of the bending magnet field the helicity of the elliptical polarization can be reversed. This is of no importance for radiation from a bending magnet since we cannot change the field without loss of the particle beam but is of specific importance for elliptical polarization state of radiation from wiggler and undulator magnets. 


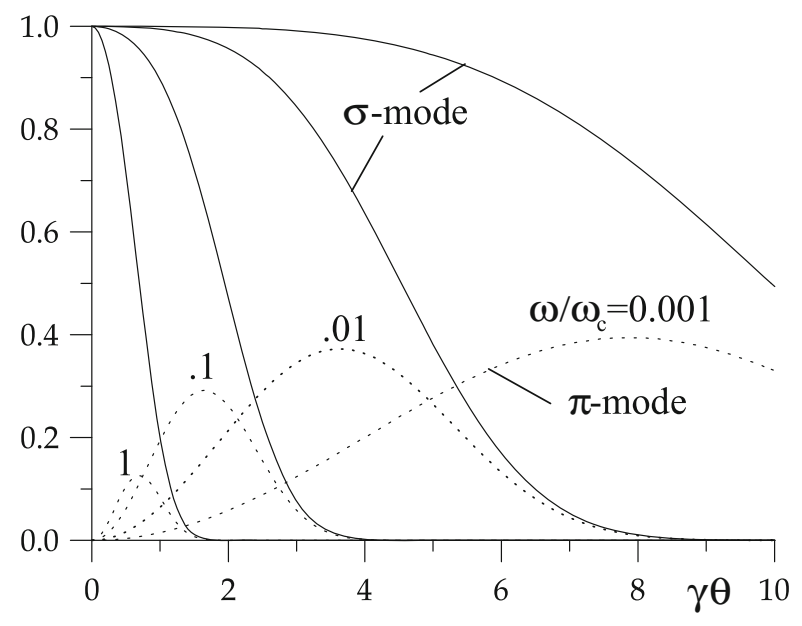

Fig. 26.11 Relative intensities of $\sigma$-mode and $\pi$-mode radiation as a function of vertical observation angle $\theta$ for different photon energies

\subsubsection{Elliptical Polarization from Periodic Insertion Devices}

We apply the visual picture for the formation of elliptically polarized radiation in a bending magnet to the periodic magnetic field of wiggler and undulator magnets. The acceleration vectors and associated field vectors are shown in Fig. 26.12a, b for one period and similar to the situation in bending magnets we do not expect any elliptical polarization in the mid plane where $\vartheta=0$. Off the mid-plane, we observe now the radiation from a positive and a negative pole. From each pole we get elliptical polarization but the combination of lefthanded polarization from one pole with righthanded polarization from the next pole leads to a cancellation of elliptical polarization from periodic magnets (Fig. 26.12c). In bending magnets, this cancellation did not occur for lack of alternating deflection. Since there are generally an equal number of positive and negative poles in a wiggler or undulator magnet the elliptical polarization is completely suppressed. Ordinary wiggler and undulator magnets do not produce elliptically polarized radiation.

\section{Asymmetric Wiggler Magnet}

The elimination of elliptical polarization in periodic magnets results from a compensation of left and righthanded helicity and we may therefore look for an insertion device in which this symmetry is broken. Such an insertion device is the asymmetric wiggler magnet which is designed similar to a wavelength shifter with one strong central pole and two weaker poles on either side such that the total integrated field vanishes or $\int B_{y} \mathrm{~d} s=0$. A series of such magnets may be 


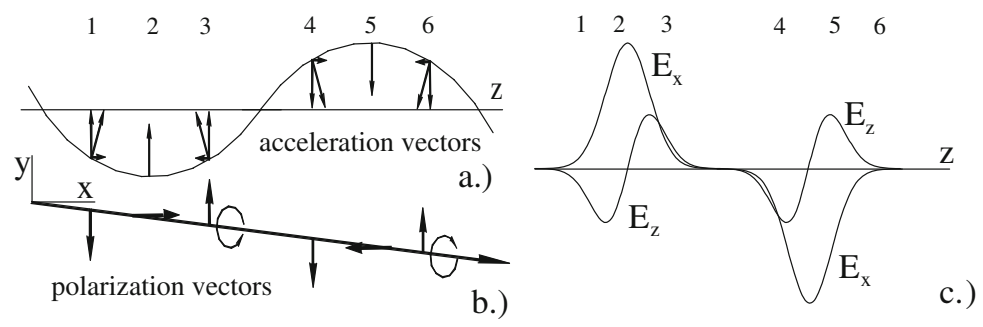

Fig. 26.12 Acceleration vectors along one period of (a) a wiggler magnet, (b) associated polarization vectors, and (c) corresponding radiation fields

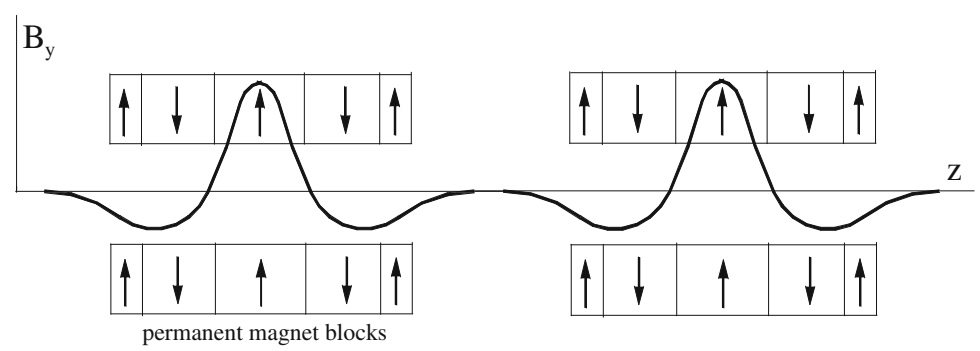

Fig. 26.13 Asymmetric wiggler magnet

aligned to produce an insertion device with many poles to enhance the intensity. The compensation of both helicities does not work anymore since the radiation depends on the magnetic field and not on the total deflection angle. A permanent magnet rendition of an asymmetric wiggler magnet is shown schematically in Fig. 26.13

The degree of polarization from an asymmetric wiggler depends on the desired photon energy. The critical photon energy is high for radiation from the high field pole $\left(\epsilon_{\mathrm{c}}^{+}\right)$and lower for radiation from the low field pole $\left(\epsilon_{\mathrm{c}}^{-}\right)$. For high photon energies $\left(\epsilon_{\mathrm{ph}} \approx \epsilon_{\mathrm{c}}^{+}\right)$the radiation from the low field poles is negligible and the radiation is essentially the same as from a series of bending magnets with its particular polarization characteristics. For lower photon energies $\left(\epsilon_{\mathrm{c}}^{-}<\epsilon_{\mathrm{ph}}<\epsilon_{\mathrm{c}}^{+}\right)$ the radiation intensity from high and low field pole become similar and cancellation of the elliptical polarization occurs. At low photon energies $\left(\epsilon_{\mathrm{ph}}<\epsilon_{\mathrm{c}}^{-}\right)$the intensity from the low field poles exceeds that from the high field poles and we observe again elliptical polarization although with reversed helicity.

\section{Elliptically Polarizing Undulator}

The creation of elliptically and circularly polarized radiation is important for a large class of experiments using synchrotron radiation and special insertion devices have therefore been developed to meet such needs in an optimal way. Different approaches have been suggested and realized as sources for elliptically 


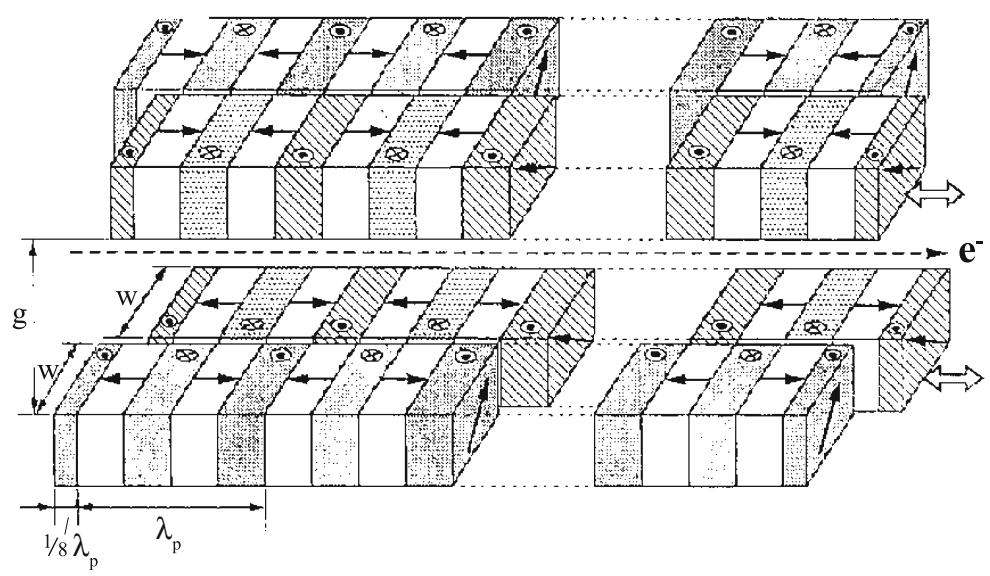

Fig. 26.14 Permanent magnet arrangement to produce elliptically polarized undulator radiation [11]

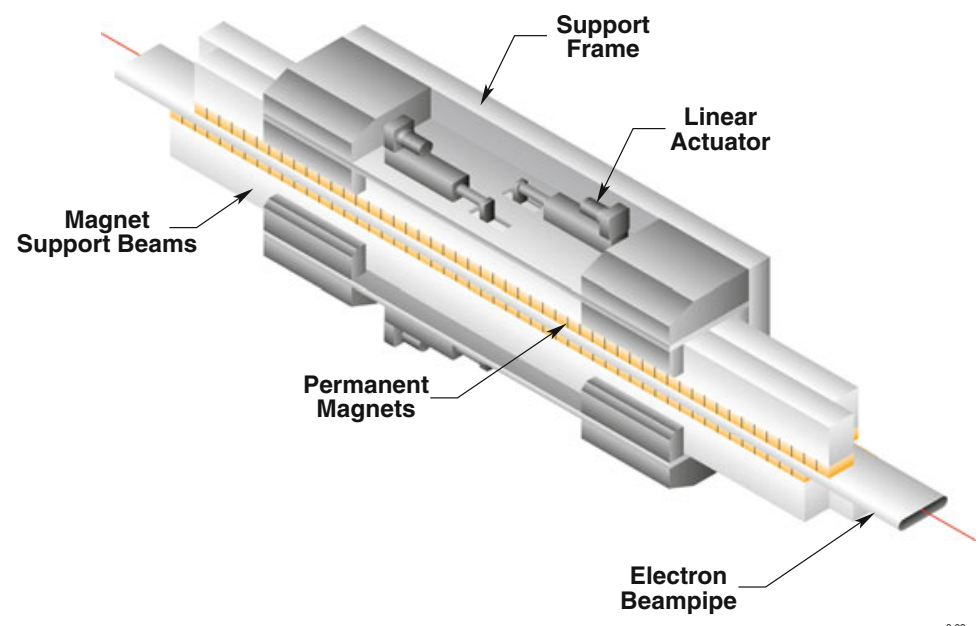

Fig. 26.15 3-D view of an elliptically polarizing undulator, EPU [11]

polarized radiation, among them for example, those described in $[9,10]$. All methods are based on permanent magnet technology, sometimes combined with electromagnets, to produce vertical and horizontal fields shifted in phase such that elliptically polarized radiation can be produced. Utilizing four rows of permanent magnets which are movable with respect to each other and magnetized as shown in Fig. 26.14, elliptically polarized radiation can be obtained.

Figure 26.15 shows the arrangement in a three dimensional rendition to visualize the relative movement of the magnet rows $[9,11]$. 


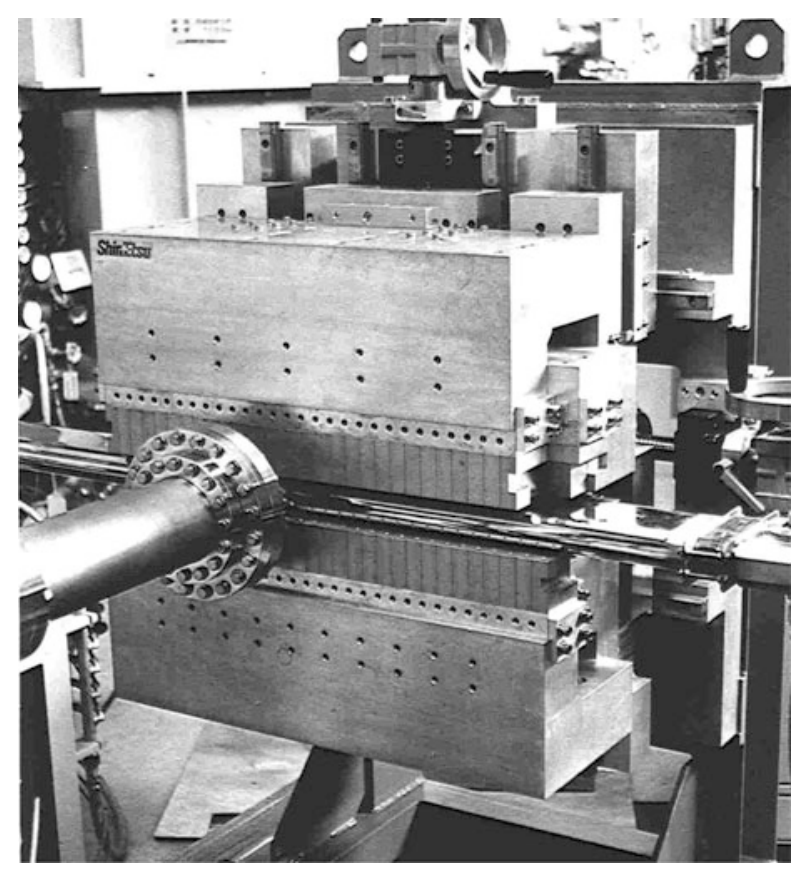

Fig. 26.16 Undulator for elliptically polarized radiation [10]

The top as well as the bottom row of magnet poles are split into two rows, each of which can be shifted with respect to each other. This way, a continuous variation of elliptical polarization from left to linear to right handed helicity can be obtained. By shifting the top magnet arrays with respect to the bottom magnets the fundamental frequency of the undulator radiation can be varied as well. Figure 26.16 shows a photo of such a magnet [10].

\section{Problems}

26.1 (S). Consider an undulator magnet with a period length of $\lambda_{\mathrm{p}}=5 \mathrm{~cm}$ in a $7 \mathrm{GeV}$ storage ring. The strength parameter be $K=1$. What is the maximum oscillation amplitude of an electron passing through this undulator? What is the maximum longitudinal oscillation amplitude with respect to the reference system moving with velocity $\bar{\beta}$ ?

26.2 (S). An undulator with 50 poles, a period length of $\lambda_{\mathrm{p}}=5 \mathrm{~cm}$ and a strength parameter of $K=1$ is to be installed into a $1 \mathrm{GeV}$ storage ring. Calculate the focal length of the undulator magnet. Does the installation of this undulator require compensation of its focusing properties? How about a wiggler magnet with $K=5$ ? 
26.3 (S). Consider the expression (26.67) for the photon flux into the forward cone. We also know that the band width of undulator radiation scales like $\Delta \omega / \omega \propto 1 / N_{\mathrm{p}}$. With this, the photon flux (26.67) becomes independent of the number of undulator periods!? Explain in words, why this expression for the photon flux is indeed a correct scaling law.

26.4 (S). A hybrid undulator is to be installed into a $7 \mathrm{GeV}$ storage ring to produce undulator radiation in a photon energy range of $4 \mathrm{keV}$ to $15 \mathrm{keV}$. The maximum undulator field shall not exceed a value of $B_{0} \leq 2 \mathrm{~T}$ at a gap aperture of $10 \mathrm{~mm}$. The available photon flux in the forward cone shall be at least $10 \%$ of the maximum flux within the whole spectral range. Specify the undulator parameters and show that the required photon energy range can be covered by changing the magnet gap only.

26.5 (S). Consider an electron colliding head-on with a laser beam. What is the wavelength of the laser as seen from the electron system. Derive from this the wavelength of the "undulator" radiation in the laboratory system.

26.6 (S). An electron of energy $2 \mathrm{GeV}$ performs transverse oscillations in a wiggler magnet of strength $K=1.5$ and period length $\lambda_{\mathrm{p}}=7.5 \mathrm{~cm}$. Calculate the maximum transverse oscillation amplitude. What is the maximum transverse velocity in units of $c$ during those oscillations. Define and calculate a transverse relativistic factor $\gamma_{\perp}$. Note, that for $K \gtrsim 1$ the transverse relativistic effect becomes significant in the generation of harmonic radiation.

26.7 (S). Calculate for a $3 \mathrm{GeV}$ electron beam the fundamental photon energy for a 100 period-undulator with $K=1$ and a period length of $\lambda_{\mathrm{p}}=5 \mathrm{~cm}$. What is the maximum angular acceptance angle $\vartheta$ (as determined by adjustable slits) of the beam line, if the radiation spectrum is to be restricted to a bandwidth of $10 \%$ ?

26.8 (S). Strong mechanical forces exist between the magnetic poles of an undulator when energized. Are these forces attracting or repelling the poles? Why? Consider a $\ell=1 \mathrm{~m}$ long undulator with a pole width $w=0.1 \mathrm{~m}, 15$ periods each $\lambda_{\mathrm{p}}=7 \mathrm{~cm}$ long and a maximum field of $B_{0}=1.5 \mathrm{~T}$. Estimate the total force between the two magnet poles?

26.9 (S). In Chap. 23 we mentioned undulator radiation as a result of Compton scattering of the undulator field by electrons. Derive the fundamental undulator wavelength from the process of Compton scattering.

26.10 (S). The undulator radiation intensity is a function of the strength parameter $K$. Find the strength parameter $K$ for which the fundamental radiation intensity is a maximum. Determine the range of $K$-values for which the intensity of the fundamental radiation is within $10 \%$ of the maximum.

26.11 (S). Show from $(26.54)$ that along the axis $(\vartheta=0)$ radiation is emitted only in odd harmonics.

26.12 (S). Show from (26.51) that undulator radiation does not produce elliptically polarized radiation in the forward direction $(\vartheta=0)$. 
26.13 (S). Try to design a hybrid undulator for a $3 \mathrm{GeV}$ storage ring to produce $4 \mathrm{keV}$ to $15 \mathrm{keV}$ photon radiation. Is it possible? Why not? Optimize the undulator parameters such that this photon energy range can be covered with the highest flux possible and utilizing lower order harmonics (order 7 or less). Plot the radiation spectrum that can be covered by changing the gap height of the undulator.

26.14 (S). An undulator is constructed from hybrid permanent magnet material with a period length of $\lambda_{, \mathrm{p}}=5.0 \mathrm{~cm}$. What is the fundamental wavelength range in a $800 \mathrm{MeV}$ storage ring and in a $7 \mathrm{GeV}$ storage ring if the undulator gap is to be at least $10 \mathrm{~mm}$ ?

26.15 (S). Determine the tuning range for a hybrid magnet undulator in a $2.5 \mathrm{GeV}$ storage ring with an adjustable gap $g \geq 10 \mathrm{~mm}$. Plot the fundamental wavelength as a function of magnet gap for two different period lengths, $\lambda_{, \mathrm{p}}=15 \mathrm{~mm}$ and $\lambda_{, \mathrm{p}}=75 \mathrm{~mm}$. Why are the tuning ranges so different?

26.16. Consider a 26-pole wiggler magnet with a field $B_{y}(\mathrm{~T})=1.5 \sin \left(\frac{2 \pi}{\lambda, \mathrm{p}} z\right)$ and a period length of $\lambda_{, p}=15 \mathrm{~cm}$ as the radiation source for a straight through photon beam line and two side stations at an angle $\vartheta=4 \mathrm{mr}$ and $\vartheta=8 \mathrm{mr}$ in a storage ring with a beam energy of $2.0 \mathrm{GeV}$. What is the critical photon energy of the photon beam in the straight ahead beam line and in the two side stations?

26.17. Verify the relative intensities of $\sigma$-mode and $\pi$-mode radiation in Fig.26.12 for two quantitatively different pairs of observation angles $\vartheta$ and photon energies $\varepsilon / \varepsilon_{\mathrm{c}}$.

26.18. Design an asymmetric wiggler magnet assuming hard edge fields and optimized for the production of elliptical polarized radiation at a photon energy of your choice. Calculate and plot the photon flux of polarized radiation in the vicinity of the optimum photon energy.

26.19. Calculate the total undulator $\left(N_{\mathrm{p}}=50, \lambda_{\mathrm{p}}=4.5 \mathrm{~cm}, K=1.0\right)$ radiation power from a $200 \mathrm{~mA}, 6 \mathrm{GeV}$ electron beam. Pessimistically, assume all radiation to come from a point source and be contained within the central cone. This is a safe assumption for the design of the vacuum chamber or mask absorbers. Determine the power density at a distance of $15 \mathrm{~m}$ from the source. Compare this power density with the maximum acceptable of $10 \mathrm{~W} / \mathrm{mm}^{2}$. How can you reduce the power density, on say a mask, to the acceptable value or below?

26.20. Use the beam and undulator from problem 26.19 and estimate the total radiation power into the forward cone alone. What percentage of all radiation falls within the forward cone? [hint: make reasonable approximations to simplify the math but keep the result reasonably close to the correct answer].

26.21. Derive an expression for the average velocity component $\bar{\beta}=\bar{v} / c$ of a particle traveling through an undulator magnet of strength $K$. 


\section{References}

1. H. Motz, J. Appl. Phys. 22, 527 (1951)

2. B.M. Kincaid, J. Appl. Phys. 48, 2684 (1977)

3. S. Chunjarean, Supercond. Sci. Technol. 24, 055013 (2011)

4. P. Elleaume, Synchrotron radiation and free electron laser, in The CERN Accelerator School (CAS), ed. by D. Brandt, number CERN-2005-012 (CERN, Geneva, 2003)

5. D.F. Alferov, Y.A. Bashmakov, E.G. Bessonov, Sov. Phys. Tech. Phys. 18, 1336 (1974)

6. S. Krinsky, IEEE Trans. Nucl. Sci. 30, 3078 (1983)

7. A. Bienenstock, G. Brown, H. Winick, H. Wiedemann, Rev. Sci. Instrum. 60, 1393 (1989)

8. W.M. Lavender, Observation and analysis of $\mathrm{x}$-ray undulator radiation from PEP. PhD thesis, Stanford University (1988)

9. R. Carr, Nucl. Instrum. Methods A 306, 391 (1991)

10. S. Sasaki, K. Kakuno, T. Takada, T. Shimada, K. Yanagida, Y. Miyahara, Nucl. Instrum. Methods A 331, 763 (1993)

11. R. Carr, S. Lidia, The adjustable phase planar helical undulator. SPIE Proc., vol. 2013 (1993) 


\section{Chapter 27 \\ Free Electron Lasers}

Synchrotron radiation is emitted when electromagnetic fields exert a force on a charged particle. This opens the possibility to apply external fields with specific properties for the stimulation of electrons to emit more radiation. Of course, not just any external electromagnetic field would be useful. Fields at some arbitrary frequency would not work because particles interacting with such fields would in general be periodically accelerated and decelerated without any net energy transfer. The external field must have a frequency and phase such that a particle may continuously lose energy into synchrotron radiation. Generally, it is most convenient to recycle and use spontaneous radiation emitted previously by the same emission process. In this part, we will discuss in some detail the process of stimulation as it applies to a free electron laser.

In a free electron laser (FEL) quasi-monochromatic, spontaneous radiation emitted from an undulator is recycled in an optical cavity to interact with the electron beam causing accelerations which are periodic with the frequency of the undulator radiation. In order to couple the particle motion to the strictly transverse electromagnetic radiation field, the path of the electrons is modulated by periodic deflections in a magnetic field to generate transverse velocity components. In a realistic setup, this magnetic field is provided in an undulator magnet serving both as the source of radiation and the means to couple to the electric field. The transverse motion of the particle results in a gain or loss of energy from/to the electromagnetic field depending on the location of the particle with respect to the phase of the external radiation field. The principle components of a FEL are shown in Fig. 27.1.

An electron beam is guided by a bending magnet unto the axis of an undulator. Upon exiting the undulator, the beam is again deflected away from the axis by a second bending magnet, both deflections to protect the mirrors of the optical cavity. Radiation that is emitted by the electron beam while travelling through the undulator is reflected by a mirror, travels to the mirror on the opposite side of the undulator and is reflected there again. Just as this radiation pulse enters the undulator again, another electron bunch joins to establish the emission of stimulated radiation. The

This chapter has been made Open Access under a CC BY 4.0 license. For details on rights and licenses please read the Correction https://doi.org/10.1007/978-3-319-18317-6_28 


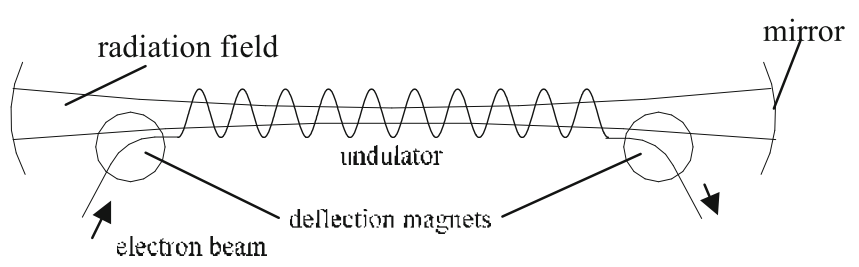

Fig. 27.1 Free electron laser setup (schematic)

electron beam pulse consists of a long train of many bunches, much longer than the length of the optical cavity such that many beam-radiation interactions can be established.

\subsection{Small Gain Regime}

We may follow this process in great detail observing an electron as it travels through say the positive half period of its oscillatory trajectory. During this phase, the electron experiences a negative acceleration from the undulator magnet field which is in phase with the oscillation amplitude. Acceleration causes a perturbation of the electric fields of the electron as was discussed in detail in Chap. 12. This perturbation travels away from the source at the speed of light, which is what we call electromagnetic radiation. For an electron, the electric radiation field points in the direction of the acceleration. As the electron travels through the positive half wave, it emits a radiation field made of half a wave. Simultaneously, this radiation field, being faster than the electron, travels ahead of the electron by precisely half a wavelength. This process tells us that the radiation wavelength is closely related to the electron motion and that it is quasi-monochromatic. Of course, for a strong undulator the sinusoidal motion becomes perturbed and higher harmonics appear, but the principle arguments made here are still true. Now, the electron starts performing the negative half of its oscillation and, experiencing a positive acceleration, emits the second halfwave of the radiation field matching perfectly the first halfwave. This happens in every period of the undulator and when the electron reaches the end of the last period a radiation wave composed of $N_{\mathrm{p}}$ oscillations exists ahead of the electron. This process describes the spontaneous radiation emission from an electron in an undulator magnet.

The radiation pulse just created is recycled in the optical cavity to reenter the undulator again at a later time. The length of the optical cavity must be adjusted very precisely to an integer multiple of both the radiation wavelength and the distance between electron bunches. Under these conditions, electron bunches and radiation pulses enter the undulator synchronously. A complication arises from the fact that the electrons are contained in a bunch which is much longer than the wavelength of the radiation. The electrons are distributed for all practical purposes uniformly over many wavelengths. For the moment, we ignore this complication and note that there is an electron available whenever needed. 
We pick now an electron starting to travel through a positive half wave of its oscillation exactly at the same time and location as the radiation wave starts its positive field halfperiod. The electron, experiences then a downward acceleration from the radiation field. During its motion the electron is continuously accelerated until it has completed its travel through the positive half oscillation. At the same time, the full positive have wave of the radiation field has moved over the electron. At this moment the electron and the radiation field are about to start their negative half periods. Continuing its motion now through the negative half period, the electron still keeps loosing energy because it now faces a negative radiation field. The fact that the radiation field "slides" over the electron just one wavelength per undulator period ensures a continuous energy transfer from electron to the radiation field. The electron emits radiation which is now exactly in synchronism with the existing radiation field and the new radiation intensity is proportional to the acceleration or the external radiation field. Multiple recycling and interaction of radiation field with electron bunches results therefore in an exponential increase in radiation intensity.

At this point, we must consider all electrons, not just the one for which the stimulation works as just described. This process does not work that perfect for all particles. An electron just half a wavelength behind the one discussed above would continuously gain energy from the radiation field and any other electron would loose or gain energy depending on its phase with respect to the radiation. It is not difficult to convince oneself that on average there may not be any net energy transfer one way or another and therefore no stimulation or acceleration. To get actual stimulation, some kind of asymmetry must be introduced.

To see this, we recollect the electron motion in a storage ring in the presence of the rf-field in the accelerating cavity. In Sect. 9.2.1 we discussed the phase space motion of particles under the influence of a radiation field. The radiation field of a FEL acts exactly the same although at a much shorter wavelength. The electron beam extends over many buckets as shown in Fig.27.2 and it is obvious that in its interaction with the field half of the electrons gain and the other half loose energy from/to the radiation field. The effect of the asymmetry required to make the FEL work is demonstrated in Fig. 27.3. Choosing an electron beam energy to be off-

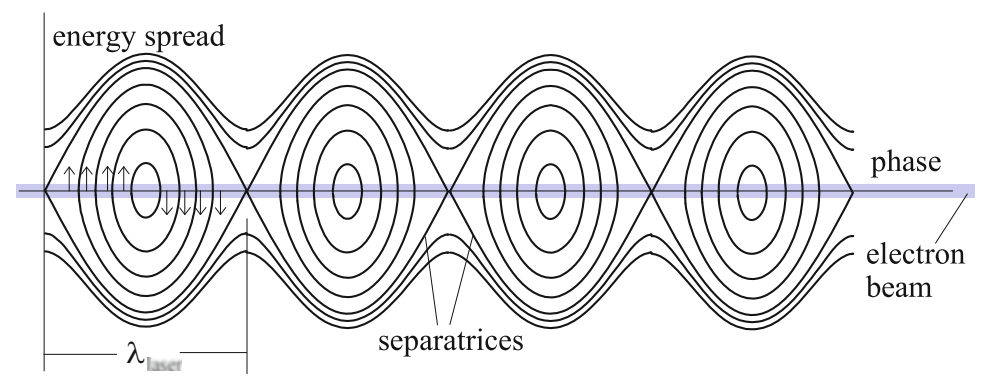

Fig. 27.2 Interaction of an electron beam (on-resonance energy) with the radiation field of a FEL. The arrows in the first bucket indicate the direction of particle motion in its interaction with the electromagnetic field 


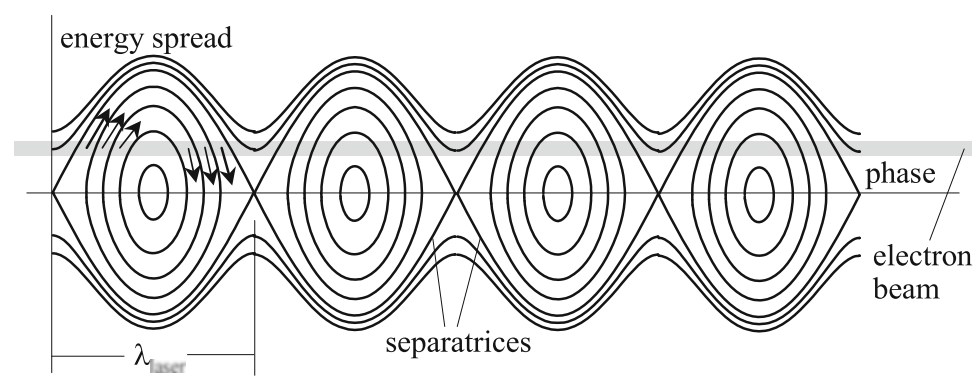

Fig. 27.3 Interaction of an electron beam (off-resonance energy) with the radiation field of a FEL

resonance by a small amount, the energy gain and losses for all electrons within a bucket becomes unbalanced and we can choose a case where all electrons on average loose energy into (FEL) or gain energy (particle acceleration by a radiation field) from the radiation field. The arrows in the first bucket of Fig. 27.3 show clearly the imbalance of energy gain or loss. What it means to choose an electron beam energy off-resonance will be discussed in more detail in the next section, where we formulate quantitatively the processes discussed so far only qualitatively.

We concentrate on the case where only a small fraction of the particle energy is extracted such that we can neglect effects on particle parameters. This regime is called the "small-gain" regime. Specifically, we ignore changes in the particle energy and redistribution in space as a consequence of the periodic energy modulation.

\subsubsection{Energy Transfer}

Transfer of energy between a charged particle and an electromagnetic wave is effected by the electric field term of the Lorentz force equation and the amount of transferred energy is

$$
\Delta W=e \int \boldsymbol{E}_{\mathrm{L}} \mathrm{d} z=e \int_{\mathrm{L}} \boldsymbol{E} \boldsymbol{v} \mathrm{d} t
$$

where $\boldsymbol{E}_{\mathrm{L}}$ is the external field or the Laser field in the optical cavity and $\boldsymbol{v}$ the particle velocity. In free space $\boldsymbol{v} \perp \boldsymbol{E}_{\mathrm{L}}$ and therefore there is no energy transfer possible $(\Delta W \equiv 0)$. Generating some transverse velocity $\boldsymbol{v}_{\perp}$ through periodic deflection in an undulator, we get from (26.3)

$$
v_{x}=+\beta c \frac{K}{\gamma} \sin \left(k_{\mathrm{p}} z\right),
$$

where $k_{\mathrm{p}}=2 \pi / \lambda_{\mathrm{p}}$. The external radiation field can be expressed by

$$
\boldsymbol{E}_{\mathrm{L}}=\boldsymbol{E}_{0 \mathrm{~L}} \cos \left(\omega_{\mathrm{L}} t-k_{\mathrm{L}} z+\varphi_{0}\right)
$$


and the energy transfer is

$$
\begin{aligned}
\Delta W & =e \int \boldsymbol{v} \boldsymbol{E}_{\mathrm{L}} \mathrm{d} t=e \int v_{x} E_{\mathrm{L}} \mathrm{d} t \\
& =e \beta c \frac{K}{\gamma} E_{0 \mathrm{~L}} \int \cos \left(\omega_{\mathrm{L}} t-k_{\mathrm{L}} z+\varphi_{0}\right) \sin \left(k_{\mathrm{p}} z\right) \mathrm{d} t \\
& =\frac{1}{2} e \beta c \frac{K}{\gamma} E_{0 \mathrm{~L}} \int\left(\sin \Psi^{+}-\sin \Psi^{-}\right) \mathrm{d} t
\end{aligned}
$$

where

$$
\Psi^{ \pm}=\omega_{\mathrm{L}} t-\left(k_{\mathrm{L}} \pm k_{\mathrm{p}}\right) z+\varphi_{0}
$$

The energy transfer appears to be oscillatory, but continuous energy transfer can be obtained if either $\Psi^{+}=$const. or $\Psi^{-}=$const. In this case

$$
\frac{\mathrm{d} \Psi^{ \pm}}{\mathrm{d} t}=\omega_{\mathrm{L}}-\left(k_{\mathrm{L}} \pm k_{\mathrm{p}}\right) \dot{z}=0
$$

and we must derive conditions for this to be true. The velocity $\dot{z}$ is from (26.3)

$$
\dot{z}=\bar{\beta} c+\beta c \frac{K^{2}}{4 \gamma^{2}} \cos \left(2 k_{\mathrm{p}} z\right)
$$

where the average drift velocity $\bar{\beta} c$ is defined by

$$
\frac{\mathrm{d} \bar{z}}{\mathrm{~d} t}=\bar{\beta} c=\beta c\left(1-\frac{K^{2}}{4 \gamma^{2}}\right) .
$$

We modify slightly the condition (27.6) and require that it be true only on average

$$
\frac{\mathrm{d} \Psi^{ \pm}}{\mathrm{d} t}=\omega_{\mathrm{L}}-\left(k_{\mathrm{L}} \pm k_{\mathrm{p}}\right) \frac{\mathrm{d} \bar{z}}{\mathrm{~d} t}=0,
$$

or

$$
\left(k_{\mathrm{L}} \pm k_{\mathrm{p}}\right) \beta\left(1-\frac{K^{2}}{4 \gamma^{2}}\right)-k_{\mathrm{L}}=0 .
$$

With $\beta \approx 1-1 / 2 \gamma^{2}$ and $k_{\mathrm{p}} \ll k_{\mathrm{L}},(27.10)$ becomes

$$
k_{\mathrm{L}}\left[\left(1-\frac{1}{2 \gamma^{2}}\right)\left(1-\frac{K^{2}}{4 \gamma^{2}}\right)-1\right] \pm k_{\mathrm{p}} \approx 0,
$$


or for $\gamma \gg 1$

$$
-\frac{k_{\mathrm{L}}}{2 \gamma^{2}}\left(1+\frac{1}{2} K^{2}\right) \pm k_{\mathrm{p}}=0 .
$$

Equation (27.12) can be met only for the + sign or for

$$
k_{\mathrm{p}}=\frac{k_{\mathrm{L}}}{2 \gamma^{2}}\left(1+\frac{1}{2} K^{2}\right),
$$

which is identical to the definition of the fundamental undulator radiation wavelength

$$
\lambda_{\mathrm{L}}=\frac{\lambda_{\mathrm{p}}}{2 \gamma^{2}}\left(1+\frac{1}{2} K^{2}\right) .
$$

Radiation at the fundamental wavelength of undulator radiation guarantees a continuous energy transfer from the particles to the electromagnetic wave or stimulation of radiation emission by an external field. For this reason, it is most convenient to use spontaneous undulator radiation as the external field to start the build-up of the free electron laser.

\subsubsection{Equation of Motion}

The energy gain $\mathrm{d} W$ of the electromagnetic field is related to the energy change $\mathrm{d} \gamma$ of the electron by

$$
\frac{\mathrm{d} \gamma}{\mathrm{d} z}=-\frac{1}{m c^{2}} \frac{\mathrm{d} W}{\beta c \mathrm{~d} t}
$$

or with (27.4)

$$
\frac{\mathrm{d} \gamma}{\mathrm{d} z}=-\frac{e K E_{0 \mathrm{~L}}}{2 \gamma m c^{2}}\left(\sin \Psi^{+}-\sin \Psi^{-}\right) .
$$

With the substitution $\sin x=-\operatorname{Re}\left(\mathrm{i} e^{\mathrm{i} x}\right)$

$$
\frac{\mathrm{d} \gamma}{\mathrm{d} z}=\frac{e K E_{0 \mathrm{~L}}}{2 \gamma m c^{2}} \operatorname{Re}\left(\mathrm{ie}^{\mathrm{i} \psi^{+}}-\mathrm{ie}^{\mathrm{i} \psi^{-}}\right) .
$$

In $\Psi^{ \pm}=\omega_{\mathrm{L}} t-\left(k_{\mathrm{L}} \pm k_{\mathrm{p}}\right) z(t)+\varphi_{0}$, we replace the location function $z(t)$ by its expression (26.5)

$$
z(t)=\underbrace{\bar{\beta} c t}_{=\bar{s}}+\underbrace{\frac{K^{2}}{8 \gamma^{2} k_{\mathrm{p}}} \sin \left(2 k_{\mathrm{p}} \bar{\beta} c t\right)}_{\ll \bar{\beta} c t},
$$


composed of an average position $\bar{z}$ and an oscillatory term. With $k_{\mathrm{p}} \ll k_{\mathrm{L}}$

$$
\frac{\mathrm{d} \gamma}{\mathrm{d} z}=\frac{e \beta K E_{0 \mathrm{~L}}}{2 \gamma m c^{2}} \operatorname{Re}\left\{\mathrm{i} \exp \left[\mathrm{i} \frac{k_{\mathrm{L}} K^{2}}{8 \gamma^{2} k_{\mathrm{p}}} \sin \left(2 k_{\mathrm{p}} \bar{z}\right)\right]\left[\mathrm{e}^{\mathrm{i} \bar{\Psi}^{+}}-\mathrm{e}^{\mathrm{i} \bar{\Psi}^{-}}\right]\right\}
$$

and the phase

$$
\bar{\Psi}^{ \pm}=\omega_{\mathrm{L}} t-\left(k_{\mathrm{L}} \pm k_{\mathrm{p}}\right) \bar{z}+\varphi_{0}
$$

With the definition $\exp (\mathrm{i} x \sin \phi)=\sum_{n=-\infty}^{n=+\infty} J_{n}(x) \mathrm{e}^{\mathrm{i} n \phi}$ we get finally

$$
\frac{\mathrm{d} \gamma}{\mathrm{d} z}=\frac{e \beta K E_{0 \mathrm{~L}}}{2 \gamma m c^{2}} \operatorname{Re}\left[\mathrm{i} \sum_{n=-\infty}^{n=+\infty} J_{n}\left(\frac{k_{\mathrm{L}} K^{2}}{8 \gamma^{2} k_{\mathrm{p}}}\right) \mathrm{e}^{\mathrm{i} 2 n k_{\mathrm{p}} \bar{z}}\left(\mathrm{e}^{\mathrm{i} \bar{\Psi}^{+}}-\mathrm{e}^{\mathrm{i} \bar{\Psi}^{-}}\right)\right] .
$$

The infinite sum reflects the fact that the condition for continuous energy transfer can be met not only at one wavenumber but also at all harmonics of that frequency. Combining the exponential terms and sorting for equal wavenumbers $h k_{\mathrm{p}}$, where $h$ is an integer, we redefine the summation index by setting

$$
\begin{aligned}
& 2 n k_{\mathrm{p}}+k_{\mathrm{p}}=h k_{\mathrm{p}} \quad \longrightarrow \quad n=\frac{h-1}{2} \\
& 2 n k_{\mathrm{p}}-k_{\mathrm{p}}=h k_{\mathrm{p}} \quad \longrightarrow \quad n=\frac{h+1}{2}
\end{aligned}
$$

and get

$$
\frac{\mathrm{d} \gamma}{\mathrm{d} z}=\frac{e \beta K E_{0 \mathrm{~L}}}{2 \gamma m c^{2}} \sum_{h=1}^{\infty}\left[J_{\frac{h-1}{2}}(x)-J_{\frac{h+1}{2}}(x)\right] \underbrace{\operatorname{Re}\left\{i \mathrm{e}^{\mathrm{i}\left[\left(k_{\mathrm{L}}+h k_{\mathrm{p}}\right) \bar{z}-\omega_{\mathrm{L}} t+\varphi_{0}\right]}\right\}}_{=-\sin \left[\left(k_{\mathrm{L}}+h k_{\mathrm{p}}\right) \bar{z}-\omega_{\mathrm{L}} t+\varphi_{0}\right]},
$$

where $x=\frac{K^{2}}{4+2 K^{2}}$. Using the $J J$-function (26.60) the energy transfer is

$$
\frac{\mathrm{d} \gamma}{\mathrm{d} z}=-\frac{e \beta K E_{0 \mathrm{~L}}}{2 \gamma m c^{2}} \sum_{h=1}^{\infty}[J J] \sin \Psi
$$

For maximum continuous energy transfer $\sin \Psi=$ const. or

$$
\begin{aligned}
\frac{\mathrm{d} \Psi}{\mathrm{d} t} & =\left(k_{\mathrm{L}}+h k_{\mathrm{p}}\right) \frac{\mathrm{d} \bar{z}}{\mathrm{~d} t}-\omega_{\mathrm{L}} \\
& =\left(k_{\mathrm{L}}+h k_{\mathrm{p}}\right) \beta c\left(1-\frac{K^{2}}{4 \gamma^{2}}\right)-\omega_{\mathrm{L}}
\end{aligned}
$$




$$
\begin{aligned}
& =\left(k_{\mathrm{L}}+h k_{\mathrm{p}}\right)\left(1-\frac{1}{2 \gamma^{2}}\right) c\left(1-\frac{K^{2}}{4 \gamma^{2}}\right)-c k_{\mathrm{L}} \\
\frac{\mathrm{d} \Psi}{\mathrm{d} t} & =-\frac{c k_{\mathrm{L}}}{2 \gamma_{\mathrm{r}}^{2}}\left(1+\frac{1}{2} K^{2}\right)+h k_{\mathrm{p}} c=0,
\end{aligned}
$$

where we assumed that $k_{\mathrm{L}} \gg h k_{\mathrm{p}}$, which is true since $\lambda_{\mathrm{p}} \gg \lambda_{\mathrm{L}}$ and the harmonic number of interest is generally unity or a single digit number. This condition confirms our earlier finding (27.14) and extends the synchronicity condition to multiples $h$ of the fundamental radiation frequency

$$
\lambda_{\mathrm{L}}=\frac{\lambda_{\mathrm{p}}}{2 \gamma^{2} h}\left(1+\frac{1}{2} K^{2}\right) .
$$

The integer $h$ therefore identifies the harmonic of the radiation frequency with respect to the fundamental radiation.

In a real particle beam with a finite energy spread we may not assume that all particles exactly meet the synchronicity condition. It is therefore useful to evaluate the tolerance for meeting this condition. To do this, we define a resonance energy

$$
\gamma_{\mathrm{r}}^{2}=\frac{k_{\mathrm{L}}}{2 h k_{\mathrm{p}}}\left(1+\frac{1}{2} K^{2}\right),
$$

which is the energy at which the synchronicity condition is met exactly. For any other particle energy $\gamma=\gamma_{\mathrm{r}}+\delta \gamma$ we get from (27.25) and (27.27)

$$
\frac{\mathrm{d} \Psi}{\mathrm{d} z}=2 h k_{\mathrm{p}} \frac{\delta \gamma}{\gamma_{\mathrm{r}}}
$$

With the variation of the energy deviation $\frac{\mathrm{d}}{\mathrm{d} z} \delta \gamma=\left.\frac{\mathrm{d} \gamma}{\mathrm{d} z}\right|_{\gamma_{\mathrm{r}}}-\frac{\mathrm{d} \gamma_{\mathrm{r}}}{\mathrm{d} z}=\left.\frac{\mathrm{d} \gamma}{\mathrm{d} z}\right|_{\gamma_{\mathrm{r}}}$ and (27.24) we get from (27.28) after differentiating with respect to $z$

$$
\frac{\mathrm{d}^{2} \Psi}{\mathrm{d} z^{2}}=2 h k_{\mathrm{p}} \frac{\mathrm{d}}{\mathrm{d} z} \frac{\delta \gamma}{\gamma_{\mathrm{r}}}=-\frac{e h k_{\mathrm{p}} K E_{0 \mathrm{~L}}}{\gamma_{\mathrm{r}}^{2} m c^{2}}[J J] \sin \Psi(z),
$$

where, for simplicity, we use only one harmonic $h$. This equation can be written in the form

$$
\frac{\mathrm{d}^{2} \Psi}{\mathrm{d} z^{2}}+\Omega_{\mathrm{L}}^{2} \sin \Psi=0
$$

exhibiting the dynamics of a harmonic oscillator. Equation (27.30) is known as the Pendulum equation [1] with the frequency

$$
\Omega_{\mathrm{L}}^{2}=\frac{e h k_{\mathrm{p}} K E_{0 \mathrm{~L}}}{\gamma_{\mathrm{r}}^{2} m c^{2}}|J J| .
$$


While interacting with the external radiation field, the particles perform harmonic oscillations in a potential generated by this field. This situation is very similar to the synchrotron oscillation of particles in a storage ring interacting with the field of the rf-cavities as was discussed in Sect. 9.2.1. In phase space, the electron perform synchrotron oscillations at the frequency $\Omega_{\mathrm{L}}$ while exchanging energy with the radiation field.

\subsubsection{FEL-Gain}

Having established the possibility of energy transfer from an electron to a radiation field, we may evaluate the magnitude of this energy transfer or the gain in field energy per interaction process or per pass. One pass is defined by the interaction of an electron bunch with the radiation field while passing through the entire length of the undulator. The gain in the laser field $\Delta W_{\mathrm{L}}=-m c^{2} n_{\mathrm{e}} \Delta \gamma$, where $\Delta \gamma$ is the energy loss per electron and pass to the radiation field and $n_{\mathrm{e}}$ the number of electrons per bunch. The energy in the laser field

$$
W_{\mathrm{L}}=\frac{1}{4} \epsilon_{0} E_{0 \mathrm{~L}}^{2} V
$$

where $V$ is the volume of the radiation field. With this, we may define the average FEL-gain for the $h$ th harmonic by

$$
G_{h}=\frac{\left\langle\Delta W_{\mathrm{L}}\right\rangle}{W_{\mathrm{L}}}=-\frac{m c^{2} n_{\mathrm{e}}\langle\Delta \gamma\rangle_{n_{\mathrm{e}}}}{\frac{\epsilon_{0}}{4} E_{0 \mathrm{~L}}^{2} V}=-\frac{2 m c^{2} \gamma_{\mathrm{r}} n_{\mathrm{e}}}{\epsilon_{0} h k_{\mathrm{p}} E_{0 \mathrm{~L}}^{2} V}\left\langle\Delta \Psi^{\prime}\right\rangle_{n_{\mathrm{e}}},
$$

making use of (27.28). $\left\langle\Delta \Psi^{\prime}\right\rangle_{n_{\mathrm{e}}}$ is the average value of $\Delta \Psi^{\prime}=\Psi_{\mathrm{f}}^{\prime}-\Psi_{0}^{\prime}$ for all electrons per bunch, where $\Psi_{0}^{\prime}$ is defined at the beginning of the undulator and $\Psi_{\mathrm{f}}^{\prime}$ at the end of the undulator. To further simplify this expression, we use (27.31), solve for the laser field

$$
E_{0 \mathrm{~L}}=\frac{m c^{2} \gamma_{\mathrm{r}}^{2} \Omega_{\mathrm{L}}^{2}}{e h K k_{\mathrm{p}}[J J]}
$$

and define the electron density $n_{\mathrm{b}}=n_{\mathrm{e}} / V$. Here we have tacitly assumed that the volume of the radiation field perfectly overlaps the volume of the electron beam. This is not automatically the case and must be achieved by carefully matching the electron beam to the diffraction dominated radiation field. If this cannot be done, the volume $V$ is the overlap volume, or the larger of both. With this the FEL-gain becomes

$$
G=-\frac{8 \pi e^{2} n_{\mathrm{b}} h K^{2} k_{\mathrm{p}}[J J]^{2}}{m c^{2} \gamma_{\mathrm{r}}^{3} \Omega_{\mathrm{L}}^{4}}\left\langle\Delta \Psi^{\prime}\right\rangle_{n_{\mathrm{e}}}
$$


Numerical evaluation of $\left\langle\Delta \Psi^{\prime}\right\rangle_{n_{\mathrm{e}}}$ can be performed with the pendulum equation. Multiplying the pendulum equation $2 \Psi^{\prime}$ and integrating we get

$$
\Psi^{\prime 2}-2 \Omega_{\mathrm{L}}^{2} \cos \Psi=\text { const. }
$$

Evaluating this at the beginning of the undulator

$$
\Psi^{\prime 2}-\Psi_{0}^{\prime 2}=2 \Omega_{\mathrm{L}}^{2}\left(\cos \Psi-\cos \Psi_{0}\right),
$$

which becomes with $\Psi_{0}^{\prime}=2 h k_{\mathrm{p}} \frac{\gamma_{0}-\gamma_{\mathrm{r}}}{\gamma_{\mathrm{r}}}$

$$
\Psi^{\prime 2}=\left(2 h k_{\mathrm{p}} \frac{\gamma_{0}-\gamma_{\mathrm{r}}}{\gamma_{\mathrm{r}}}\right)^{2}+2 \Omega_{\mathrm{L}}^{2}\left(\cos \Psi-\cos \Psi_{0}\right)
$$

Finally,

$$
\Psi^{\prime}(z)=2 h k_{\mathrm{p}} \frac{\gamma-\gamma_{\mathrm{r}}}{\gamma_{\mathrm{r}}} \sqrt{1+\frac{\Omega_{\mathrm{L}}^{2}}{2 k^{2} k_{\mathrm{p}}^{2}} \frac{\gamma_{\mathrm{r}}^{2}}{\left(\gamma-\gamma_{\mathrm{r}}\right)^{2}}\left[\cos \Psi(z)-\cos \Psi_{0}\right]},
$$

or with

$$
w=h k_{\mathrm{p}} L_{\mathrm{u}} \frac{\gamma-\gamma_{\mathrm{r}}}{\gamma_{\mathrm{r}}}
$$

where $L_{\mathrm{u}}=N_{\mathrm{p}} \lambda_{\mathrm{p}}$ is the undulator length,

$$
\Psi^{\prime}(z)=\frac{2 w}{L_{\mathrm{u}}} \sqrt{1+\frac{L_{\mathrm{u}}^{2} \Omega_{\mathrm{L}}^{2}}{2 w^{2}}\left[\cos \Psi(z)-\cos \Psi_{0}\right]} .
$$

We solve this by expansion and iteration. For a low gain FEL, the field $E_{0 \mathrm{~L}}$ is weak and does not influence the particle motion. Therefore $\Omega_{\mathrm{L}} \ll 1$ and (27.41) becomes

$$
\begin{aligned}
\Psi^{\prime} \approx \frac{2 w}{L}[ & 1+\frac{1}{2} \frac{L^{2} \Omega_{\mathrm{L}}^{2}}{2 w^{2}}\left(\cos \Psi-\cos \Psi_{0}\right) \\
& \left.-\frac{1}{8} \frac{L^{4} \Omega_{\mathrm{L}}^{4}}{4 w^{4}}\left(\cos \Psi-\cos \Psi_{0}\right)^{2}+\ldots\right] .
\end{aligned}
$$

In the lowest order of iteration $\Psi^{\prime}=\Psi_{0}^{\prime}=\frac{2 w}{L}$ and $\Delta \Psi_{(0)}^{\prime}=0$ for all particles, which means there is no energy transfer. For first order approximation, we integrate $\Psi_{0}^{\prime}(z)=\frac{2 w}{L_{\mathrm{u}}}$ to get $\Psi_{(1)}(z)=\frac{2 w}{L_{\mathrm{u}}} z+\Psi_{0}$ and

$$
\Delta \Psi_{(1)}^{\prime}=\Psi^{\prime}\left(L_{\mathrm{u}}\right)-\Psi_{1}^{\prime}(0)=\frac{L \Omega_{\mathrm{L}}^{2}}{2 w}\left[\cos \left(2 w+\Psi_{0}\right)-\cos \Psi_{0}\right]+\mathcal{O}(2)
$$


from (27.42). Averaging over all initial phases occupied by electrons $0 \leq \Psi_{0} \leq 2 \pi$

$$
\left\langle\Delta \Psi_{1}^{\prime}\right\rangle=\frac{L \Omega_{\mathrm{L}}^{2}}{2 w} \frac{1}{2 \pi} \int_{0}^{2 \pi}\left[\cos \left(2 w+\Psi_{0}\right)-\cos \Psi_{0}\right] \mathrm{d} \Psi_{0}=0 .
$$

No energy transfer to the laser field occurs in this approximation either. We need a still higher order approximation. The higher order correction to $\Psi_{1}^{\prime}(s)=\Psi_{0}^{\prime}(s)+$ $\delta \Psi_{1}^{\prime}(s)$ is from (27.42)

$$
\delta \Psi_{(1)}^{\prime}=\frac{L \Omega_{\mathrm{L}}^{2}}{2 w}\left[\cos \Psi-\cos \Psi_{0}\right]
$$

and the correction to $\Psi_{1}(s)$ is

$$
\begin{aligned}
\delta \Psi_{(1)} & =\frac{L \Omega_{\mathrm{L}}^{2}}{2 w} \int_{0}^{L}\left[\cos \left(\frac{2 w}{L} z+\Psi_{0}\right)-\cos \Psi_{0}\right] \mathrm{d} s \\
& =\frac{L^{2} \Omega_{\mathrm{L}}^{2}}{4 w^{2}}\left[\sin \left(2 w+\Psi_{0}\right)-\sin \Psi_{0}-2 w \cos \Psi_{0}\right]
\end{aligned}
$$

The second order approximation to the phase is then $\Psi_{1}(z)=\frac{2 w}{L_{u}} z+\Psi_{0}+$ $\delta \Psi_{(1)}$ and using (27.42) in second order as well we get

$$
\begin{aligned}
\Delta \Psi_{(2)}^{\prime} & =\frac{L \Omega_{\mathrm{L}}^{2}}{2 w}\left[\cos \left(2 w+\Psi_{0}+\delta \Psi_{(1)}\right)-\cos \Psi_{0}\right] \\
& -\frac{L^{3} \Omega_{\mathrm{L}}^{4}}{4 w^{2}}\left[\cos \left(2 w+\Psi_{0}\right)-\cos \Psi_{0}\right]^{2}+\ldots,
\end{aligned}
$$

where in the second order term only the first order phase $\Psi_{1}(z)=\frac{2 w}{L_{\mathrm{u}}} z+\Psi_{0}$ is used. The first term becomes with $\delta \Psi_{(1)} \ll \Psi_{0}+2 w$

$$
\begin{aligned}
& \cos \left(2 w+\Psi_{0}+\delta \Psi_{1}\right)-\cos \Psi_{0} \\
& \approx \cos \left(2 w+\Psi_{0}\right)-\delta \Psi_{1} \sin \left(2 w+\Psi_{0}\right)-\cos \Psi_{0}
\end{aligned}
$$

and

$$
\begin{aligned}
\Delta \Psi_{2}^{\prime}= & \frac{L_{\mathrm{u}}^{3} \Omega_{\mathrm{L}}^{4}}{16 w^{3}}\left\{\frac{8 w^{2}}{L_{\mathrm{u}}^{2} \Omega^{2}}\left[\cos \left(2 w+\Psi_{0}\right)-\cos \Psi_{0}\right]\right. \\
& -2 \sin \left(2 w+\Psi_{0}\right)\left[\sin \left(2 w+\Psi_{0}\right)-\sin \Psi_{0}-2 w \cos \Psi_{0}\right] \\
& \left.-\left[\cos \left(2 w+\Psi_{0}\right)-\cos \Psi_{0}\right]^{2}+\ldots\right\} .
\end{aligned}
$$


Now, we average over all initial phases assuming a uniform distribution of particles in $z$ or in phase. The individual terms become then

$$
\begin{aligned}
\left\langle\cos \left(2 w+\Psi_{0}\right)-\cos \Psi_{0}\right\rangle & =0 \\
\left\langle\sin ^{2}\left(2 w+\Psi_{0}\right)\right\rangle & =\frac{1}{2} \\
\left\langle\sin \left(2 w+\Psi_{0}\right) \sin \Psi_{0}\right\rangle & =\frac{1}{2} \cos (2 w) \\
\left\langle\sin \left(2 w+\Psi_{0}\right) \cos \Psi_{0}\right\rangle & =\frac{1}{2} \sin (2 w) \\
\left\langle\cos \left(2 w+\Psi_{0}\right) \cos \Psi_{0}\right\rangle & =\frac{1}{2} \cos (2 w) .
\end{aligned}
$$

With this

$$
\left\langle\Delta \Psi_{2}^{\prime}\right\rangle=-\frac{L_{\mathrm{u}}^{3} \Omega_{\mathrm{L}}^{4}}{16 w^{3}}[1-\cos (2 w)-w \sin (2 w)]
$$

and finally with $[1-\cos (2 w)-w \sin (2 w)] / w^{3}=-\frac{\mathrm{d}}{\mathrm{d} w}\left(\frac{\sin w}{w}\right)^{2}$

$$
\left\langle\Delta \Psi_{2}^{\prime}\right\rangle=\frac{L_{\mathrm{u}}^{3} \Omega_{\mathrm{L}}^{4}}{8} \frac{\mathrm{d}}{\mathrm{d} w}\left(\frac{\sin w}{w}\right)^{2} .
$$

The FEL-gain is finally from (27.35)

$$
G_{h}=-\frac{\pi r_{\mathrm{c}} n_{\mathrm{b}} h K^{2} L_{\mathrm{u}}^{3} k_{\mathrm{p}}}{\gamma_{\mathrm{r}}^{3}}[J J]^{2} \frac{\mathrm{d}}{\mathrm{d} w}\left(\frac{\sin w}{w}\right)^{2},
$$

where we may express the particle density $n_{\mathrm{b}}$ by beam parameters as obtained from the electron beam source

$$
n_{\mathrm{b}}=\frac{n_{\mathrm{e}}}{V}=\frac{n_{\mathrm{e}}}{2 \pi \sigma^{2} \ell},
$$

where $\sigma$ is the radius of a round beam. With these definitions, and $\hat{I}=c e n_{\mathrm{e}} / \ell$ the electron peak current, the gain per pass becomes

$$
G_{h}=-\frac{2^{2 / 3} \pi r_{\mathrm{c}} h \lambda^{3 / 2} L_{\mathrm{u}}^{3}}{c \sigma^{2} \lambda_{\mathrm{p}}^{5 / 2}} \frac{\hat{I}}{e} \frac{K^{2}[J J]^{2}}{\left(1+\frac{1}{2} K^{2}\right)^{3 / 2}} \frac{\mathrm{d}}{\mathrm{d} w}\left(\frac{\sin w}{w}\right)^{2} .
$$

The gain depends very much on the choice of the electron beam energy through the function (27.40), which is expressed by the gain curve as shown in Fig. 27.4.

There is no gain if the beam energy is equal to the resonance energy $\left(\gamma=\gamma_{\mathrm{r}}\right)$. As has been discussed in the introduction to this chapter, we must introduce an asymmetry to gain stimulation of radiation or gain and this asymmetry is generated by a shift in energy. For a monochromatic electron beam maximum gain can be reached for $w \approx 1.2$. A realistic beam, however, is not monochromatic and the 
Fig. 27.4 Free electron laser gain curve $G \propto-\frac{\mathrm{d}}{\mathrm{d} w}\left(\frac{\sin w}{w}\right)^{2}$

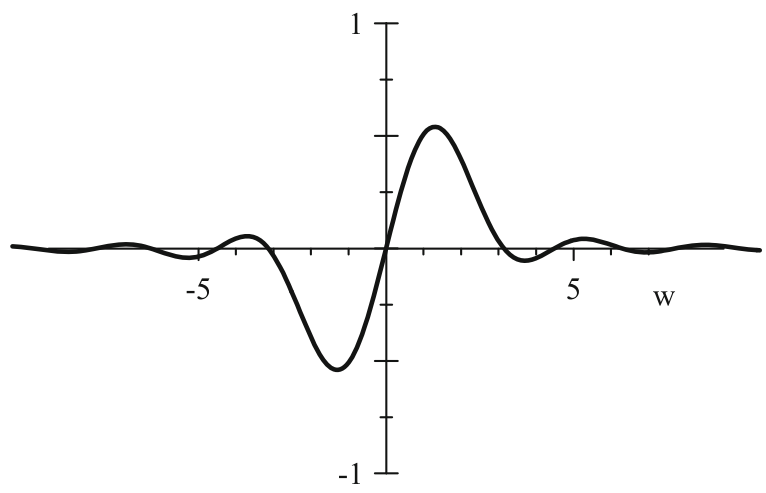

narrow gain curve indicates that a beam with too large an energy spread may not produce any overall gain. There is no precise upper limit for the allowable energy spread but from Fig. 27.4 we see that gain is all but gone when $|w| \gtrsim 5$. We use this with (27.40) and (27.27) to formulate a condition for the maximum allowable energy spread

$$
\left|\frac{\delta \gamma}{\gamma}\right| \ll \frac{2 \gamma_{\mathrm{r}}^{2} \lambda_{\mathrm{L}}}{1+\frac{1}{2} K^{2}} .
$$

For efficient gain the geometric size of the electron beam and the radiation field must be matched. In (27.55) we have introduced a volume for the electron bunch. Actually, this volume is the overlap volume of radiation field and electron bunch. Ideally, one would try to get a perfect overlap by forming both beams to be equal. This is in fact possible and we will discuss the conditions for this to happen. First, we assume that the electron beam size varies symmetrically about the center of the undulator. The beam size develops like

$$
\sigma^{2}(z)=\sigma_{0}^{2}+\left(\frac{\epsilon}{\sigma_{0}}\right)^{2} z^{2}
$$

with distance $z$ from the beam waist. To maximize gain we look for the minimum average beam size within an undulator. This minimum demands a symmetric solution about the undulator center. Furthermore, we may select the optimum beam size at the center by looking for the minimum value of the maximum beam size within the undulator. From $\mathrm{d} \sigma^{2} / \mathrm{d} \sigma_{0}^{2}=0$, the optimum solution is obtained for $z=\frac{1}{2} L_{\mathrm{u}}=\sigma_{0}^{2} / \epsilon=\beta_{0}$. For $\beta_{0}=\frac{1}{2} L_{\mathrm{u}}$ the beam cross section grows from a value of $\sigma_{0}^{2}$ in the middle of the undulator to a maximum value of $2 \sigma_{0}^{2}$ at either end.

The radiation field is governed by diffraction. Starting at a beam waist, the growth of the radiation field cross section due to diffraction is quantified by the Rayleigh length

$$
z_{\mathrm{R}}=\pi \frac{w_{0}^{2}}{\lambda},
$$


where $w_{0}$ is the beam size at the waist and $\lambda$ the wavelength. This length is defined as the distance from the radiation source (waist) to the point at which the cross section of the radiation beam has grown by a factor of two. For a Gaussian beam, we have for the beam size at a distance $z$ from the waist

$$
w^{2}(z)=w_{0}^{2}+\Theta^{2} z^{2}
$$

where $\Theta=\frac{\lambda}{\pi w_{0}}$ is the divergence angle of the radiation field. This is exactly the same condition as we have just discussed for the electron beam assuming the center of the undulator as the source of radiation.

\subsection{High Gain Free Electron Laser}

We have discussed the interaction of an electron beam with an external electromagnetic field and found that repeated recycling of the photon beam by reflecting mirrors this photon beam intensity can be made to grow until it is big enough to modulate the electron beam into microbunches at a distance equal to the radiation wavelength. This interaction works only at wavelength where good reflectors are available. This is, for example, not possible for UV and x-rays. The question arises what would happen if an electron beam would travel through a very long undulator instead of being reflected many times. This is the principle of self-amplified-spontaneousemission or SASE.

The goal is to look for electron dynamics which leads to micro bunching at the wavelength of interest. Any bunch radiates coherently at wavelengths equal or longer than the bunch length as was discussed in Sect. 24.7. This coherent radiation scales like the square of the number of particles per bunch $n_{\mathrm{b}}^{2}$ rather than linear with $n_{\mathrm{b}}$ as is the case of incoherent radiation emitted at wavelength shorter than the bunch length. Since the number of electrons per bunch can be very large we gain a large increase in the photon intensity. Actually this is the highest photon intensity one can extract from a bunch of electrons. Unfortunately, it is technically not possible to generate bunches at visible or shorter wavelength and preserve such bunches along a beam line. The way out is to possibly generate microbunches at the place of the radiation source. This was possible in the FEL and we will now discuss this possibility in the realm of SASE.

\subsubsection{Electron Dynamics in a SASE FEL}

In this section we aim at producing coherent radiation at any wavelength specifically at very short wavelength like x-rays without the support of reflecting mirrors. The electron beam appears in bunches which are long compared to the desired wavelength. Although the electron distribution is assumed to be uniform, there will 
be statistical fluctuations due to the finite number of electrons per bunch. This beam is travelling through an undulator of as yet undetermined length. An x-ray single pass FEL consists basically of a high brightness electron source, a linac followed by a long undulator, both with parameters to produce the desired photon radiation wavelength as the fundamental wavelength of the undulator.

In a perfect uniform beam every electron radiates in the undulator at an arbitrary phase resulting in in-coherent radiation. The radiation travels faster than the electrons and therefore the radiation field will interact with them. This interaction however is incoherent and will not lead to anything. Now we assume that along the bunch there is a density fluctuation or whisker which is very short of the order of the desired wavelength. That whisker radiates coherently at wavelengths equal to the temporal length of the whisker. The coherent radiation, although very small at first, interacts with the electron beam. However, only the fundamental wavelength as determined by electron beam energy and undulator properties will constructively grow from undulator period to period. As this fundamental radiation travels over the bunch ahead of the whisker it interacts coherently with the electrons and modulates their energy periodically at the fundamental wavelength. This energy modulation together with the deflection in the undulator leads to a density modulation at the desired wavelength. This process occurs because electrons which have been decelerated by the photon field get deflected more in the undulator field while electrons being accelerated by the photon field get deflected less. Both effects lead to a density modulation.

Such whiskers can and do occur at any place along the bunch. Therefore a number of coherent fields will be created and grow along the undulator. Eventually though there will be a strongest radiation field and all others, being spread over statistical phases, will decoher and vanish in the one largest spike. This spike keeps growing along the undulator and reaches a point from which on the photon field is strong enough to microbunch the electron beam at which point the intensity does not grow anymore. The SASE-FEL has reached its saturation. At the same time the energy change introduced by the photon field is big enough to spoil the SASE principle leading also to saturation. The theory of SASE-FEL has been first developed by [2] as a 1-D theory. Later this was extended to a 3-D theory [3, 4]. We will however restrict ourselves in this text to the 1-D approximation which is well met for a high quality electron beam. Where ideal parameters are not available some degradation of the photon beam parameters must be accepted and the actual characteristics are mostly determined by numerical simulations.

The increase of photon intensity along the undulator is exponential because the bunching depends on the photon intensity itself and is given by

$$
I_{\mathrm{ph}} \propto I_{0} \exp \left(\frac{z}{L_{\mathrm{G}}}\right),
$$


where $L_{\mathrm{G}}$ the power gain length and $I_{0}$ the initial spontaneous coherent intensity for an undulator of length $L_{\mathrm{G}}$ (26.66). The gain length is defined by

$$
L_{\mathrm{G}}=\frac{\lambda_{\mathrm{u}}}{4 \sqrt{3} \pi \rho}
$$

where $\rho$ is the FEL-parameter

$$
\rho=\left(\frac{K \cdot J J}{4 \sqrt{2}} \frac{\lambda_{\mathrm{u}} \Omega_{\mathrm{p}}}{2 \pi c \gamma}\right)^{2 / 3} .
$$

Here the $J J$-function is defined by (26.60) with the argument $x=\frac{K^{2}}{4+2 K^{2}}, \Omega_{\mathrm{p}}=$ $\sqrt{4 \pi c^{2} r_{\mathrm{e}} n_{\mathrm{e}} \frac{1}{\gamma}}$ is the plasma frequency and $n_{\mathrm{e}}$ the electron density. Tacitly we have assumed a planar undulator which could also generate third harmonic radiation albeit at a lower intensity $(\sim 1 \%)$ while a helical undulator would only produce the fundamental harmonic. Numerical simulations indicate that for presently achievable electron beam parameters about 20 gain lengths are required to reach saturation while the FEL-parameter is of the order of $10^{-3}$.

The peak photon pulse power at saturation is expected [2] to be

$$
P_{\text {peak }}=\frac{\rho_{\mathrm{eff}} N_{\mathrm{e}} E}{\sqrt{2 \pi} \tau_{\mathrm{b}, \mathrm{rms}}}=\rho_{\mathrm{eff}} I_{\text {peak }} \frac{E}{e},
$$

which is about $14 \mathrm{GW}$ for SLAC-LCLS parameters [5] $\left(\rho_{\mathrm{eff}}=2.9 \cdot 10^{-4}, \tau_{\mathrm{b}, \mathrm{rms}}=77\right.$ fs, $I_{\text {peak }}=3,400 \mathrm{~A}, E=14.35 \mathrm{GeV}$ ). Simulations give a somewhat lower power of about $8 \mathrm{GW}$ by taking all inefficiencies like increase of beam emittance along linac and undulator into account.

A high photon intensity (27.61) demand a short gain length for a given length of the facility while the gain length (27.62) itself is only related to the undulator period length and FEL-period. The period length is limited to a minimum of a few $\mathrm{cm}$ by technical considerations and the available linear accelerator energy and desired radiation wavelength. The FEL-parameter (27.63) is greatly determined by the electron density, e.g. by the electron beam emittance and bunch length. Therefore a small beam emittance and bunch length is of paramount importance. In addition the electron beam emittance must be close to the photon emittance for the desired wavelength to get maximum overlap of both beams. Theoretical considerations also require that the beam energy spread should be less than the FEL-parameter $\left(\frac{\sigma_{E}}{E}<\rho\right)$.

To make SASE work well, a very high quality electron beam must be produced and preserved along the linac and undulator. In the following sections we will shortly discuss the requirements and the solutions employed in the first few x-ray facilities. The development in this newest accelerator system is still flowing and experimental experience from the first facilities contribute to a vigorous development especially toward more compact solutions. 
Different from storage ring which can provide radiation to many users simultaneously an x-ray laser can do so only for one user at one wavelength at a time. This is acceptable because of the extraordinary properties of the radiation in terms of photon intensity, brightness, coherence and femto-second pulse length. A high desired pulse repetition rate obviously pushes the facility designs more and more to superconducting technology.

\subsubsection{Electron Source}

The electron source determines the ultimate performance of the x-ray laser. For maximum radiation intensity the number of electrons per microbunch should be as large as possible. This strongly points to a laser gun where it is possible to generate a large electron charge within a pulse of less than $100 \mathrm{fs}$. Not to loose spatial coherence the beam emittance must be very small of the order of less than $10^{-10} \mathrm{~m}$ at the end of the linac. At source energies of $\gamma=1$ the lowest possible normalized emittance is $1-210^{-6} \mathrm{~m}$ at high electron intensity of about $1 \mathrm{pC}$ per bunch. Lower emittances are possible for lower charges. The design requirements are determined by many detailed simulations with specially developed numerical programs to find solutions close to desired performance.

\subsubsection{Beam Dynamics}

Along the linac and undulator the beam should be focused as much as possible to maximize the electron density. However, if the beam size is too small diffraction effects will appear. Therefore there is an optimum beam size which can be realized by quadrupole focusing in a FODO channel. Numerical simulations are required to determine the optimum beam size for the parametrization of the FODO channel. The focusing requirements must also include the effect of beam steering which is stronger in strong focusing FODO channels.

To reach a realistic gain length a high peak current or short bunch length in the fs regime must be achieved. That is not possible with present day technology and bunch compression schemes must be included in the beam dynamics design.

In the low energy section of the linac (up to $200-300 \mathrm{MeV}$ ) the electron bunch is accelerated "off-crest" to obtain a mostly linear correlation of energy with phase along the electron bunch in preparation for the bunch compression system. There is a small non-linearity left from the sinusoidal variation of the acceleration field. Simulations show that part of this non-linearity can be eliminated by deceleration in a higher harmonic accelerating section. If the main linac operates at $3 \mathrm{GHz}$ then this linearizing section could operate in the X-band or about $12 \mathrm{GHz}$ where suitable power sources exist [6]. The decelerating in the X-band section is small and has no 
detrimental effect on the overall beam dynamics. After passing through the X-band section the beam travels through a four-bend bunch compressor.

Following Liouville's theorem the bunch compression is obtained in exchange with energy spread. In order not to increase the energy spread too much two bunch compression systems must be employed to reach the desired short bunch length. After the first bunch compression the beam is further accelerated (still "off-crest") to a higher energy and the energy spread is reduced due to adiabatic damping. At some intermediate energy a second four-bend bunch compressor is installed. The choice of the intermediate energy should be chosen such that the remaining acceleration is enough to reduce the beam energy spread by adiabatic damping to the final value of $\frac{\Delta E}{E} \sim \rho$ for optimum SASE. No beam heater is necessary here because the bunch length is already much shorter and the non-linearity is very small. Both bunch compressors must be designed such as not to perturb beam parameters like beam emittance too much. The $R_{56}$-term is therefore chosen to be about the same in both. Final distribution of compression is determined though by numerical simulations.

The resulting beam after the second bunch compressor includes now a very high peak current which can drive a micro-bunching instability $[7,8]$ thus possibly ruining emittance and energy spread. Therefore a "beam heater" is installed just before the second bunch compressor. This beam heater is a short and strong wiggler magnet which by emission of synchrotron radiation increases the incoherent energy spread. While this seems to be the wrong method only a very small insignificant increase of the energy spread is required to suppress the instability. After the second bunch compressor the beam is ready to be accelerated "on-crest" to the final energy.

A significant problem arises if the bunch length is reduced too much such that coherent radiation (CSR) can be emitted with detrimental effect on the beam emittance and energy spread. Other problems arise from the interaction of the beam with surface resistance of the vacuum chamber (resistive wall effect). As a consequence vacuum chamber materials with low wall resistance should be used, e.g. aluminum rather than steel or copper-plated steel. In addition the surface must be polished to reduce the roughness which can cause beam instabilities. Satisfactory polishing to a roughness of well below $100 \mathrm{~nm}$ must be followed.

The specific techniques described here are not the only way to solve problems. The interested reader is therefore encouraged to review the design reports of various $\mathrm{X}$-FEL facilities. The performance is determined by simulation of the electron beam propagating through linac and undulator as well as the simulation of the photon built-up in the undulator. While it is possible to calculate order of magnitude parameters theoretically, small effects from actual particle distribution from source to end can significantly affect the outcome. Therefore the whole process must be simulated and any undesirable effect be studied and possibly eliminated or corrected. 


\subsubsection{Undulator}

The undulator parameters determine together with the electron beam energy the wavelength of the photon beam. This is the fundamental wavelength of the undulator and at reduced intensity one can contemplate the third-harmonic. To reduce the undulator length for a desired wavelength a short period length is desired. There are, however, technical limits for period length below $2-3 \mathrm{~cm}$. When the gap aperture becomes close to the period length the field drops off rapidly. The period length in the SLAC-LCLS is $3 \mathrm{~cm}$ and the desired undulator strength $K=3.71$ which requires an electron beam energy of $14.35 \mathrm{GeV}$ to reach a fundamental wavelength of $1.5 \AA$. This high undulator strength requires a very small aperture of $6 \mathrm{~mm}$ which is acceptable for a linac beam because no Gaussian tails must be preserved for lifetime.

The built-up of photon intensity occurs exponentially from noise and therefore many gain length are needed to get the intensity into desired values. In other words, the undulator must be very long. In the SLAC-LCLS case the undulator length is $120 \mathrm{~m}$ long of which theoretically $91 \mathrm{~m}$ are required to reach saturation. Such a long undulator cannot be built in one piece and is therefore broken down into shorter pieces of, in this case, $3.42 \mathrm{~m}$. This breakup allows some space for beam monitoring and beam control.

\section{Problems}

27.1 (S). Consider an electron travelling through an undulator producing radiation. Show, that the radiation front moves ahead of the electron by one fundamental radiation wavelength per undulator period.

27.2 (S). Why does a helical undulator not produce higher harmonics?

27.3 (S). From the peak power at saturation derive the number of x-ray photons $\left(\varepsilon_{\mathrm{x}} \sim 10^{4} \mathrm{eV}\right)$ per electron. For the SLAC-LCLS $K=3.711, \lambda_{\mathrm{p}}=3 \mathrm{~cm}$ and $N_{\mathrm{p}}=$ 3,070 . Compare this with incoherent radiation. For the band width use $\frac{\Delta \omega}{\omega}=\frac{1}{N_{\mathrm{p}}}$.

\section{References}

1. W. Colson, Phys. Rev. 64A, 190 (1977)

2. R. Bonifacio, C. Pellegrini, L. Narducci, Opt. Commun. 50, 373 (1984)

3. K.-J. Kim, Three-dimensional analysis of coherent amplification and self-amplified spontaneous emission in free electron lasers. Phys. Rev. Lett. 57, 1871 (1986)

4. Y.H. Chin, K.-J. Kim, M. Xie, Three-dimensional free electron laser theory including betatron oscillations. Phys. Rev. A46, 6662 (1992) 
5. Linac Coherent Light Source (LCLS), Conceptual Design Report, Technical Report SLAC-R593, SLAC, Stanford (2002)

6. P. Emma, Technical Report LCLS TN-01-01, SLAC, Stanford (2001)

7. S. Heifets, S. Krinsky, G. Stupakov, Csr instability in a bunch compressor. Technical Report SLAC-PUB-9165, SLAC, Stanford (2002)

8. E.L. Saldin, E.A. Schneidmiller, M.V. Yurkov, Longitudinal phase space distortions in magnetic bunch compressors. Technical Report DESY 01-129, DESY, Hamburg (2001) 


\title{
Correction to: Particle Accelerator Physics
}

\author{
H. Wiedemann
}

\section{Correction to:}

\section{H. Wiedemann, Particle Accelerator Physics, https://doi.org/10.1007/978-3-319-18317-6}

The original version of the book was published in 2015 with exclusive rights reserved by the Publisher. As of November 2019 it has been changed to an open access publication: (c) The Editor(s) (if applicable) and The Author(s) 2015.

The copyright of the individual chapters has therefore changed to: (C) The Author(s) 2015 All chapters in the book are licensed under the terms of the Creative Commons Attribution 4.0 International License.

Any third party material is under the same Creative Commons license as the book unless specified otherwise below. 


\section{Solutions}

\section{Solutions for Chap. 1}

1.1 We start with the total energy and solve for the momentum $c p=$ $\sqrt{E^{2}-\left(m c^{2}\right)^{2}}=m c^{2} \sqrt{\gamma^{2}-1}=\gamma m c^{2} \sqrt{1-1 / \gamma^{2}}=\beta\left(E_{\mathrm{kin}}+m c^{2}\right)$. In terms of kinetic energy $c p=\sqrt{\left(E_{\mathrm{kin}}+m c^{2}\right)^{2}-\left(m c^{2}\right)^{2}}=\sqrt{E_{\mathrm{kin}}^{2}+2 m c^{2} E_{\mathrm{kin}}}$ and the kinetic energy is $E_{\mathrm{kin}}=E-m c^{2}=m c^{2}(\gamma-1)$. For very large energies we get with $\gamma \gg 1$ for the momentum $c p \approx E$ and for the kinetic energy $E_{\text {kin }} \approx E$. For $\beta \ll 1$ the kinetic energy is with $\gamma-1 \approx \frac{1}{2} \beta^{2}$ just $E_{\text {kin }}=\frac{1}{2} m v^{2}$. The non-relativistic momentum is from $p=\frac{1}{c} \beta\left(E_{\text {kin }}+m c^{2}\right) \approx \beta m c=m v$.

1.2 From Maxwell's equations we have $\nabla \boldsymbol{E}=\frac{1}{\epsilon_{0}} \rho$. We integrate this over a cylindrical volume including part or all of the charge and get with Gauss's Integral Theorem: $\int \nabla \boldsymbol{E} \mathrm{d} V=\oint \boldsymbol{E} \mathrm{d} a=E_{r} 2 \pi r L$, where $L$ is the length of the cylindrical beam considered. The electrical field has for symmetry reasons only a radial component. The r.h.s. of Maxwell's equation is then the integral over the volume contained within the surface used on the 1.h.s. $\frac{1}{\epsilon_{0}} \int \rho \mathrm{d} V=\frac{\rho}{\epsilon_{0}} \pi r^{2} L$ for $(r<R)$ and $\frac{\rho}{2 \epsilon_{0}} \pi R^{2} L$ for $(r>R)$, and the radial electrical field component is $E_{r}=\frac{\rho}{2 \epsilon_{0}} r$ for $(r<R)$ and $\frac{\rho}{2 \epsilon_{0}} \frac{R^{2}}{r}$ for $(r>R)$. Similarly we get for the magnetic field after integration $\int \nabla \times \boldsymbol{B} \mathrm{d} V=\frac{\rho}{\epsilon_{0}} \int \boldsymbol{v} \mathrm{d} V=\frac{\rho}{\epsilon_{0}} \beta \pi r^{2} L$ for $(r<R)$ and $\frac{\rho}{\epsilon_{0}} \beta \pi R^{2} L$ for $(r>R)$. Since $v=\left(0,0, v_{z}\right)$, symmetry restricts the r.h.s. to only a $z$-component and the field to only a $\varphi$-component. We get $\int \nabla \times \boldsymbol{B} \mathrm{d} V=\int \frac{\partial B_{\varphi}}{\partial r} \mathrm{~d} V=B_{\varphi} L 2 \pi r$. Solving for the field, we get $B_{\varphi}=\frac{1}{2} \mu_{0} \rho \beta r$ for $(r<R)$ and $\frac{1}{2} \mu_{0} \rho \beta \frac{R^{2}}{r}$ for $(r>R)$.

1.3 We integrate Maxwell's equation $\nabla \boldsymbol{E}=\frac{\rho(r)}{\epsilon_{0}}$ over a cylindrical volume concentric to the beam. The 1.h.s. becomes $\int \nabla \boldsymbol{E} \mathrm{d} V=\oint \boldsymbol{E} \mathrm{d} a=E_{r} 2 \pi r L$, where $\mathrm{d} a$ is an element of the co-centric cylindrical surface and $L$ an arbitrary length along the beam axis. Since an infinitely long beam is assumed, only a radial 
electric field component exists. The r.h.s. is integrated over the same cylinder $2 \pi \frac{\rho}{\epsilon_{0}} L \int_{0}^{r} \exp \left(-\frac{\bar{r}^{2}}{2 \sigma^{2}}\right) \bar{r} \mathrm{~d} \bar{r}=2 \pi \frac{\rho}{\epsilon_{0}} L \sigma^{2}\left[1-\exp \left(-\frac{r^{2}}{2 \sigma^{2}}\right)\right]$ and the radial electric field component is finally $E_{r}=\frac{\rho}{\epsilon_{0}} \frac{\sigma^{2}}{r}\left[1-\exp \left(-\frac{r^{2}}{2 \sigma^{2}}\right)\right]$. In a similar way the magnetic field can be obtained. Only the azimuthal component is non-zero given by $B_{\varphi}=\mu_{0} \rho \beta \frac{\sigma^{2}}{r}\left[1-\exp \left(-\frac{r^{2}}{2 \sigma^{2}}\right)\right]$. The fields vanish for $r \rightarrow 0$ and for $r=\sigma$ are $E_{r}(\sigma)=\frac{\rho}{\epsilon_{0}} \sigma\left[1-\exp \left(-\frac{1}{2}\right)\right]$ and $B_{\varphi}(\sigma)=\mu_{0} \rho \beta \sigma\left[1-\exp \left(-\frac{1}{2}\right)\right]$.

1.4 The circulating beam current is defined by $i=e n f_{\mathrm{rev}}=e n v / C$ where $n$ is the number of particles circulating, $f_{\text {rev }}$ is the revolution frequency, $v$ the particle velocity and $C$ the accelerator circumference. The number of particles representing a current of $1 \mathrm{~A}$ are: $n=i C /(E v)=6.2458 \cdot 10^{12}$. The ejected beam resembles a pulse with a pulse current of $1 \mathrm{~A}$ since particles are assumed to be distributed uniformly. The pulse length is given by the revolution time $\tau=\frac{C}{c \beta}=1.0007 \mu$ s assuming $\beta \approx 1$. The synchrotron produces ten pulses of $1.0007 \mu$ s duration and at a pulse current of $1 \mathrm{~A}$. The average beam current is therefore $i_{\text {avg }}=10 \cdot \tau \cdot 1 \mathrm{~A}=10 \mu \mathrm{A}$.

1.5 The bending radius $\rho$ of a particle's path due to a force $F$ is given by the equality of this force with the centrifugal force. $\frac{\gamma m v^{2}}{\rho}=F$ or $\rho=\beta^{2} \gamma m c^{2} / F$. The gravitational force is $F=f \frac{m M}{R^{2}}=1.6397 \cdot 10^{-26} \mathrm{~kg} \mathrm{~m} \mathrm{~s}^{-2}$, where the proton mass $m_{\mathrm{p}}=1.67262 \cdot 10^{-27} \mathrm{~kg}$, the mass of earth $M=5.98 \cdot 10^{24} \mathrm{~kg}$, and the earth's radius $R=6.380 \cdot 10^{6} \mathrm{~m}$. Numerically, the gravitational constant is $f=6.67259 \cdot 10^{-11} \mathrm{~m}^{3}$ $\mathrm{kg}^{-1} \mathrm{~s}^{-2}$ and for a $1 \mathrm{eV}$ proton $\left(\gamma=1+\frac{1}{0.938 \cdot 10^{9}}=1+1.0661 \cdot 10^{-9}\right)$ the velocity $\beta \approx \sqrt{\frac{2 E_{\mathrm{kin}}}{m c^{2}}}=4.6175 \cdot 10^{-5}$. With these parameters, the bending radius is $\rho=1.955 \cdot 10^{7} \mathrm{~m}$ and therefore negligible compared to any bending radius occurring in a realistic beam transport. Equivalent electromagnetic fields can be derived from the Lorentz equation. The electrical field equivalent to the gravitational force is $E_{\mathrm{el}}=F / e=1.023 \cdot 10^{-7} \mathrm{~V} / \mathrm{m}$ and the corresponding magnetic field is $B=F /(e c \beta)=7.393 \cdot 10^{-12} \mathrm{~T}$. The ratio of electrical to magnetic field is 13,837 . For an intergalactic $10 \mathrm{TeV}$ proton the gravitational force is the same and therefore the bending radius is increased by the increase in the factor $\beta^{2} \gamma=4.696 \cdot 10^{9}$ and the bending radius is $\rho=9.18 \cdot 10^{10} \mathrm{~km}$. The required electrical field to bend the same does not depend on the particle energy, while the required magnetic field scales inversely proportional to $\beta$ and is therefore reduced to $B=3.414 \cdot 10^{-16} \mathrm{~T}$. The field ratio finally has changed from 13,837 to $2.996 \cdot 10^{8}=c$ making the magnetic field the more efficient field to bend relativistic particles. This is indeed a small field, actually about a million times smaller than intergalactic magnetic fields of some $10^{-10} \mathrm{~T}$. Cosmic rays therefore are more affected by intergalactic magnetic fields than by gravitational field.

1.6 The fields at the surface of the beam are $E_{r}=\frac{\rho}{2 \epsilon_{0}} R$ and $B_{\varphi}=\frac{1}{2} \mu_{0} \rho \beta R$. The charge density for the cylindrical slug is $\rho=e \frac{n}{\pi R^{2} \ell}=1.275 \cdot 10^{7} \mathrm{C} / \mathrm{m}^{3}$. The electrical field on the surface of the beam is then $(\beta \approx 1) E_{r}=\frac{\rho}{2 \epsilon_{0}} R=$ $1.44 \cdot 10^{11} \mathrm{~V} / \mathrm{m}$ and the magnetic field is $B_{\varphi}=\frac{1}{2} \mu_{0} \rho \beta R=480 \mathrm{~T}$. The peak electrical 
current is defined by $i=Q / \tau$, where $Q=e \cdot 10^{10}$ is the total charge, and $\tau$ is the duration of the current pulse $\tau=\frac{1}{\beta c}=3.3356 \cdot 10^{-12} \mathrm{~s}$. The peak current is then $I=480.33$ A. Two particle beams either attract or repel each other depending on whether we use an $\mathrm{e}^{+}-\mathrm{e}^{-}$-system or an $\mathrm{e}^{-}-\mathrm{e}^{-}$or $\mathrm{e}^{+}-\mathrm{e}^{+}$-system. For $\gamma \gg 1$ the forces due to electrical and magnetic field are the same and act along a line including the particle in one beam and the center of charge of the other beam. The longitudinal forces cancel as both beams pass each other and the radial forces are $F_{r}=e \frac{\rho \beta}{2 \epsilon_{0}} \frac{R^{2}}{r}$, where now $r=10 \mu \mathrm{m}$. From problem 1.5 we have for the curvature $F /\left(\gamma m v^{2}\right)$ and the deflection angle is then $\theta=\frac{2 F \ell}{\gamma m v^{2}}=1.152 \cdot 10^{-5} \mathrm{rad}$. This deflection is significant and can be used as a diagnostic means to probe the closeness of both beams which eventually must be steered to collide head-on.

1.7 Plane waves can be expressed by $\boldsymbol{a}=\boldsymbol{a}_{0} \exp [\mathrm{i}(\omega-\boldsymbol{k r})]$, where the wave propagation vector $\boldsymbol{k}=\boldsymbol{k n}, k=\omega / c$ and $\boldsymbol{n}$ the unit vector in the direction of wave propagation. Applied to the fields $(\boldsymbol{E}, \boldsymbol{B})$ and using Amperes law $\boldsymbol{\nabla} \times \boldsymbol{E}=-\frac{\partial \boldsymbol{B}}{\partial t}$, we get on the 1.h.s. $\nabla \times \boldsymbol{E}=\nabla \exp [\mathrm{i}(\omega-\boldsymbol{k r})] \times \boldsymbol{E}_{0}=\mathrm{i} \nabla(\boldsymbol{k r}) \times \boldsymbol{E}=\mathrm{i} k \boldsymbol{\nabla}(\boldsymbol{n r}) \times \boldsymbol{E}=\mathrm{i} k(\boldsymbol{n} \times \boldsymbol{E})$ The r.h.s. is $-\frac{\partial \boldsymbol{B}}{\partial t}=\mathrm{i} \omega \boldsymbol{B}$. Equating both sides gives finally $\boldsymbol{n} \times \boldsymbol{E}=\boldsymbol{c} \boldsymbol{B}$.

1.8 We multiply two 4-vectors $\tilde{a}$ and $\tilde{b}$ and apply a Lorentz transformation:

$$
\begin{aligned}
\overline{\boldsymbol{a}}^{*} \overline{\boldsymbol{b}}^{*} & =a_{1}^{*} b_{1}^{*}+a_{2}^{*} b_{2}^{*}+a_{3}^{*} b_{3}^{*}-a_{4}^{*} b_{4}^{*} \\
& =a_{1} b_{1}+a_{2} b_{2}+\gamma^{2}\left(a_{3}-\beta a_{4}\right)\left(b_{3}-\beta b_{4}\right)-\gamma^{2}\left(\beta a_{3}-a_{4}\right)\left(\beta b_{3}-b_{4}\right) \\
& =a_{1} b_{1}+a_{2} b_{2}+\underbrace{\gamma^{2}\left(1-\beta^{2}\right)}_{=1} a_{3} b_{3}-\underbrace{\gamma^{2}\left(1-\beta^{2}\right)}_{=1} a_{4} b_{4} \\
& =a_{1} b_{1}+a_{2} b_{2}+a_{3} b_{3}-a_{4} b_{4}
\end{aligned}
$$

1.9 The 4-acceleration $\tilde{\boldsymbol{a}}=\gamma^{2} \frac{\mathrm{d}^{2} \tilde{\boldsymbol{s}}}{\mathrm{d} t^{2}}+\tilde{\boldsymbol{v}} \frac{\gamma^{4}}{c^{2}}(\boldsymbol{v} \boldsymbol{a})$ or in component form $\tilde{\boldsymbol{a}}=$ $\left(\tilde{a}_{x}, \tilde{a}_{y}, \tilde{a}_{z}, \mathrm{i} \tilde{a}_{t}\right)$ we get $\tilde{a}_{x}=\gamma^{2} a_{x}+\gamma^{4} \beta_{x}(\boldsymbol{\beta} \boldsymbol{a}), \tilde{a}_{y}=\ldots, \tilde{a}_{z}=\ldots, \tilde{a}_{t}=\gamma^{4}(\boldsymbol{\beta a})$, where $\boldsymbol{a}$ is the ordinary acceleration. The other components can be obtained in a similar way. Experimental verification through, for example, observation of synchrotron radiation parameters.

1.10 We formulate in laboratory frame the 4-vectors before and after scattering. To describe electron and photons we use the 4-velocity $\tilde{u}=(\gamma, \gamma \boldsymbol{u})$ with $\boldsymbol{u}=\boldsymbol{v} / c$ and energy-momentum 4-vector $c \tilde{k}=(\omega, c \boldsymbol{k})$. In the lab frame $\tilde{u}_{\mathrm{L}}=\left(\gamma, \gamma u_{\mathrm{L}} \hat{z}\right)$ and $c \tilde{k}_{\mathrm{L}}=\left(\omega_{\mathrm{L}},-\omega_{\mathrm{L}} \hat{z}\right)$ where $\hat{z}$ is a unit vector in the direction of photon motion. We assume both the electron and photons to travel along the $z$-axis. After scattering, $\tilde{u}_{\mathrm{L}}=\left(\gamma, \gamma u_{\mathrm{L}} \hat{z}\right)$ and $c \tilde{k}_{\mathrm{L}}=\left(\omega_{\chi}, \omega_{\chi} \hat{z}\right)$, where $\omega_{\chi}$ is the frequency of the outgoing photon. Here, we have assumed that the photon energy is much less than the electron energy. The product of both 4-vectors is Lorentz invariant and is therefore the same before and after scattering, or $\omega_{\mathrm{L}}\left(1+u_{\mathrm{L}}\right)=\omega_{\chi}\left(1-u_{\mathrm{L}}\right)$ and solving for the scattered frequency $\omega_{\chi}=\omega_{\mathrm{L}} \frac{1+u_{\mathrm{L}}}{1-u_{\mathrm{L}}} \approx 4 \omega_{\mathrm{L}} \gamma^{2}$ where we made use of $\gamma^{-2}=1-u_{\mathrm{L}}^{2}$ and $u_{\mathrm{L}} \approx 1$. 
1.11 Here, the only difference to problem 1.10 is that the undulator field does not move, or that $c \tilde{k}=\left(\omega_{u}, 0\right)$, where $\omega_{u}=2 \pi c / \lambda_{u}$ and $\tilde{u}_{\mathrm{L}}=\left(\gamma, \gamma u_{\mathrm{L}} z\right)$. After scattering $c \tilde{k}_{\mathrm{L}}=\left(\omega_{\chi}, \omega_{\chi} z\right)$ and $\tilde{u}_{\mathrm{L}}=\left(\gamma, \gamma u_{\mathrm{L}} z\right)$ as in problem 1.10. Equating again the Lorentz invariant products gives $\omega_{u}=\omega_{\chi}\left(1-u_{\mathrm{L}}\right)$ or $\omega_{\chi}=\omega_{\mathrm{u}} \frac{1}{1-u_{\mathrm{L}}} \approx 2 \omega_{\mathrm{L}} \gamma^{2}$. This is the fundamental radiation frequency emitted by electrons from an undulator.

1.12 The total proton energy at the end of the $200 \mathrm{MeV}$ linac is $E=E_{\mathrm{kin}}+m c^{2}=$ $1,138.27 \mathrm{MeV}$ and the momentum is $c p=\sqrt{E^{2}-\left(m c^{2}\right)^{2}}=644.44 \mathrm{MeV}$. Finally the velocity is $\beta=\frac{c p}{E}=0.56616$ or about half the velocity of light.

1.13 Due to Lorentz contraction the length of the linac is reduced by the factor $\gamma$ which along the linac is $\gamma(z)=\gamma_{0}+\frac{20}{0.5109990} z=\gamma_{0}+39.139 \cdot z$. The initial energy is $\gamma_{0}=\frac{1}{\sqrt{1-\beta_{0}^{2}}}=1.1547$ and the final energy $\gamma(L)=27,418$. Now, the integrated length of the linac is $\ell=\int_{0}^{L} \frac{\mathrm{d} z}{\gamma_{0}+39.139 \cdot z}=\left.\frac{1}{39.139} \ln \left(\gamma_{0}+39.139 \cdot z\right)\right|_{0} ^{L}=$ $0.29458 \mathrm{~m}$. In the electron system the $3 \mathrm{~km}$ SLAC linac has contracted to some $30 \mathrm{~cm}$. Now coasting, a tube of $3 \mathrm{~km}$ would contract even more to $\frac{3,000}{\gamma(L)}=$ $0.10942 \mathrm{~m} \approx 11 \mathrm{~cm}$.

1.14 The invariant center of mass energy of colliding particles is defined by $E_{\mathrm{cm}}^{2}=$ $\sum E_{i}^{2}-\sum\left(c p_{i}\right)^{2}$. For the collision of a positron with a target electron assumed to be at rest this evaluates to $E_{\mathrm{cm}}^{2}=\left(\gamma m c^{2}+m c^{2}\right)^{2}-\left(\gamma \beta m c^{2}\right)^{2}=2(\gamma+1) m^{2} c^{4}$ or $E_{\mathrm{cm}}=\sqrt{2(\gamma+1)} m c^{2}$. This is also the available energy to produce new particles since no particles must be conserved in a positron-electron collision. In an electronelectron collision the available energy would be only $E_{\text {avail }}=E_{\mathrm{cm}}-2 m c^{2}$ because the lepton number must be conserved in the collision. The same calculation for head-on collision of such particles would produce a center of mass energy of $E_{\mathrm{cm}}=$ $2 \gamma m c^{2}$. Obviously, head-on collisions provide more available energy to produce new particles.

1.15 From the invariant $E_{\mathrm{cm}}^{2}=\sum E_{i}^{2}-\sum\left(c p_{i}\right)^{2}$ we get for a particle colliding with a target particle at rest $E_{\mathrm{cm}}^{2}=\left(\gamma m c^{2}+m_{\mathrm{t}} c^{2}\right)^{2}-\left(\gamma \beta m c^{2}\right)^{2}=\left(\gamma m c^{2}\right)^{2}+$ $2 \gamma m c^{2} m_{\mathrm{t}} c^{2}+\left(m_{\mathrm{t}} c^{2}\right)^{2}-\left(\gamma \beta m c^{2}\right)^{2}$, where $m_{\mathrm{t}}$ is the mass of the target particle. The available energy to produce a $\psi / J$ particle must be $3.1 \mathrm{GeV}$. In case of a proton colliding with a target proton, the center of mass energy must be at least $E_{\mathrm{cm}}=$ $3.1 \mathrm{GeV}+m c^{2}$, because the proton number must be preserved. With $m=m_{\mathrm{t}}$ we get $E_{\mathrm{cm}}=\sqrt{2(\gamma+1)} m c^{2}=3.1+2 m c^{2}$ and solving for the particle energy we get $\gamma=\frac{1}{2}\left(\frac{3.1}{0.938}+2\right)-1=13.071$. The proton energy must therefore be at least $E_{\mathrm{p}} \geq 12.264 \mathrm{GeV}$ which was available at the Brookhaven AGS. In case of a positron colliding with a target electron the center of mass energy is also the available energy since the lepton number in this case is zero. Therefore, $\sqrt{2(\gamma+1)} m c^{2}=3.1 \mathrm{GeV}$ and the minimum positron energy $\gamma=\frac{1}{2}\left(\frac{3.1}{0.000511}\right)^{2}-1=1.84 \cdot 10^{7}$. This energy is not available at any existing particle accelerator. Only in an electron-positron colliding beam storage ring like SPEAR is it possible in head-on collisions to reach sufficient center of mass energy to produce a $\psi / J$ particle. 
1.16 The pion lifetime will be lengthened by the factor $\gamma$. Therefore, the pion lifetime at $20 \mathrm{MeV}$ is $\tau_{\pi, 20}=29.759 \mathrm{~ns}$ and at $100 \mathrm{MeV} \tau_{\pi, 100}=44.679 \mathrm{n}$. The velocities of the pions are $\beta_{20}=0.48473$ and $\beta_{100}=0.81277$ for $20 \mathrm{MeV}$ and $100 \mathrm{MeV}$ respectively. The distances traveled are $\ell_{20}=\tau_{20} c \beta_{20}=4.3245 \mathrm{~m}$ and $\ell_{100}=10.887 \mathrm{~m}$. This is a significant difference when trying to transport a pion beam from the target to the patient.

1.17 We look for the minimum kinetic energy necessary to perform the reaction, which means, the resulting particles have after collision no kinetic energy left. The length of the 4-vector $(c \boldsymbol{p}, \mathrm{i} E)=p_{\mu}$ after the collision is therefore $-16 M^{2}$ which must be equal to the length of the 4-vector before collision or $-16 M^{2}=$ $\left(p_{1, \mu}+p_{2, \mu}\right)^{2}=p_{1, \mu}^{2}+2 p_{1, \mu} p_{2, \mu}+p_{2, \mu}^{2}=-2 M^{2}+2 p_{1, \mu} p_{2, \mu}$ and $p_{1, \mu} p_{2, \mu}=-7 M^{2}$. For the two protons before collision $p_{1, \mu} p_{2, \mu}=\left(c p_{1}, \mathrm{i} E\right)(0, \mathrm{i} M)=E M$. With this, we get finally $E=7 M$ or the minimum total proton energy required to produce antiprotons is $7 M$ or subtracting the mass of the incoming proton the minimum kinetic energy must be $E_{\text {kin }}=5.6 \mathrm{GeV}$. The $6.2 \mathrm{GeV}$ Berkeley Bevatron was designed to do just this allowing the discovery of the antiproton.

\section{Solutions for Chap. 2}

2.1 A horizontal deflection can be accomplished by a horizontal electric field generated between two vertical plane electrodes ( $x=$ const) connected to a potential $V$. Since the aperture is $2 \mathrm{~cm}$ we place the electrodes at a position of $x= \pm 1 \mathrm{~cm}$. The deflection angle is given by $\varphi=e|\boldsymbol{E}| \ell /\left(\beta^{2} E\right)$, where $\ell=0.1 \mathrm{~m}$ and $E=10.511 \mathrm{MeV}$ and $\gamma=20.57$. With this, we get $\beta=0.99882$ and $\beta^{2} E=$ $10.486 \mathrm{MeV}$. The required electrical field is $|\boldsymbol{E}|=1.0486 \mathrm{MV} / \mathrm{m} \approx 10.5 \mathrm{kV} / \mathrm{cm}$. A potential difference of $21 \mathrm{kV}$ between the electrodes would be sufficient. This is across a gap of $2 \mathrm{~cm}$ which is possible in dry air (rule of thumb: breakdown field is about $30 \mathrm{kV} / \mathrm{cm}$ in dry air) or in vacuum.

2.2 Assuming no friction, the beam power is the product of the beam energy in Volt and the beam current. The minimum power for the charging belt motor is therefore $500 \mathrm{~kW}$.

2.3 The particles travel from the source to the first gap where they gain an energy of 1 $\mathrm{MeV}$. At the end of the $n$th gap they have an energy of $E_{\mathrm{kin}, n}=100 \mathrm{keV}+n \cdot 1 \mathrm{MeV}$ $(n=1,2,3 \ldots)$. After each gap the particles travels protected from microwave fields in tubes for most of an rf-period. At $7 \mathrm{MHz}$ the period is $\tau_{\mathrm{rf}}=0.14286 \mu \mathrm{s}$. The velocities of the particles after each gap is $\beta_{n}=\sqrt{1-\gamma_{n}^{-2}}=\frac{\sqrt{E_{\mathrm{kin}, n}^{2}+2 A m c^{2} E_{\mathrm{kin}, n}}}{E_{\mathrm{kin}, n}+A m c^{2}}$ which translates for potassium ions to $\beta_{\mathrm{p} 1}=0.002265, \beta_{\mathrm{p} 2}=0.007511, \beta_{\mathrm{p} 3}=$ $0.01038, \beta_{\mathrm{p} 4}=0.01261$. For electrons, the equivalent velocities are $\beta_{\mathrm{e} 1}=$ $0.9484, \beta_{\mathrm{e} 2}=0.9807, \beta_{\mathrm{e} 3}=0.9899, \beta_{\mathrm{e} 4}=0.9938$. The related lengths of the drift tubes are now $l_{n}=c \beta_{n} \tau_{\mathrm{rf}}$. For potassium ions this gives $L_{\mathrm{p} 1}=0.097 \mathrm{~m}$, 
$L_{\mathrm{p} 2}=0.322 \mathrm{~cm}, L_{\mathrm{p} 3}=0.445 \mathrm{~m}, L_{\mathrm{p} 4}=0.540 \mathrm{~m}$. These are realistic drift tube lengths. For electrons, the drift tube lengths would have to be much longer $L_{\mathrm{p} 1}=20.315 \mathrm{~m}, L_{\mathrm{p} 2}=21.007 \mathrm{~m}, L_{\mathrm{p} 3}=21.204 \mathrm{~m}, L_{\mathrm{p} 4}=21.287 \mathrm{~m}$ due to the higher velocities It is obvious that much higher frequency microwave sources had to be developed before this principle could be applied to electrons. This became possible with the invention of the klystron.

\section{Solutions for Chap. 3}

3.1 The total electron energy at injection is $E_{\text {tot }}=(50+511) 10^{3}=c p=$ $\gamma m c^{2} \frac{v}{c}=\gamma m c^{2} \beta$. From (1.76) and an orbit radius of $1.23 \mathrm{~m}$, the magnetic field on the orbit is $\frac{B_{\mathrm{i}}}{\beta}=\frac{E_{\mathrm{tot}} / e}{0.3 R}=1.520 \times 10^{-3}[\mathrm{~T}]$. The velocity can be derived from $\gamma=E_{\mathrm{tot}} / m c^{2}=1.0978$ and $\beta_{\mathrm{i}}=0.41260$. Therefore the magnetic field at injection is $B_{\mathrm{i}}=6.273 \times 10^{-4}[\mathrm{~T}]$. The field increases at a $60 \mathrm{~Hz}$ cycle and the particle needs a time of $\tau=2 \pi R / c \beta_{\mathrm{i}}=6.2436 \times 10^{-8} \mathrm{~s}$ for the first turn. During that time, the magnetic field increases by $\Delta B \approx B_{0} \omega \tau=1.907 \times 10^{-5}[\mathrm{~T}]$. At $20 \mathrm{MeV}$, the particles have gained virtually velocity of light with $\beta \approx 1$. The field change per turn at $20 \mathrm{MeV}$ is now less by the factor $\beta_{\mathrm{i}}$ for $\Delta B \approx 7.8666 \times 10^{-6}[\mathrm{~T}]$. The reason for this drop of acceleration is due to the higher velocity of the particle making the go-around time shorter. The acceleration is indeed slow and it takes the particles more than a million turns to reach the final energy.

3.2 From (1.4) we choose Ampère's law while ignoring the electric field and apply it to the betatron magnet. We integrate over an area which includes one coil. The left hand side of this integral can be expressed with Stoke's Theorem as a line integral of the magnetic field along the boundary of the area chosen. The right hand side is just the total current in the coil multiplied by $\mu_{0}$. To be able to evaluate the line integral, we choose a boundary or integration path starting in the middle of the magnet aperture (orbit) and integrate the field $\left(\mu_{\mathrm{r}}=1\right)$ along a vertical line to the magnet pole. This field is just the desired field $B_{y 0}$ The integration path through the magnet iron to the mid plane is zero because we assume $\left(\mu_{\mathrm{r}}=\infty\right)$. Integration from the magnet iron along the midplane to the origin of the integration path is also zero because all fields in the midplane of a symmetric magnet are in the vertical direction while the integration path is in the horizontal direction. That gives the end result of $B_{y 0} \frac{1}{2} g=\mu_{0} I_{\text {coil }}$. Note, for the integration path chosen, we have to use here only half the gap. The field for $42 \mathrm{MeV}$ electrons is $B_{y 0}=\frac{c p}{0.3 \cdot R}=0.35 \mathrm{~T}$ and the total current in one coil is $I_{\text {coil }}=13,926 \mathrm{~A}$. This seems to be a large current, but actually isn't. Magnet coils are wound of many turns and the current from the power supply is only $I_{\mathrm{ps}}=I_{\text {coil }} / n_{\text {turns }}$. if the coil in this example has say 20 turns then the power supply current is only $I_{\mathrm{ps}}=696.33 \mathrm{~A}$ which is perfectly acceptable.

3.3 The microwave frequency scales inversely to the relativistic factor $\gamma$ or $f_{\mathrm{rf}, \max } / f_{\mathrm{r}, 0}=\gamma_{0} / \gamma$. The relativistic factor is $\gamma=1+\frac{E_{\mathrm{kin}}}{m c^{2}}$ and the microwave 
frequency is then given by $\frac{f_{\mathrm{rf}}}{f_{\mathrm{rf}, 0}}=\frac{E_{0}}{E}$. For protons $m c^{2}=938.27 \mathrm{MeV}$ and the end frequency $f_{\mathrm{rf}}=0.610 f_{\mathrm{rf}, 0}$. Deuterons are twice as heavy $m_{\mathrm{D}} c^{2}=1,875.61 \mathrm{MeV}$ and $f_{\mathrm{rf}}=0.758 f_{\mathrm{rf}, 0}$. The situation for electrons is quite different. The rest mass is $m c^{2}=0.511 \mathrm{MeV}$ and $f_{\mathrm{rf}}=1,175 f_{\mathrm{rf}, 0}$. The frequency swing is way too large to be practical and this is the reason why synchro-cyclotrons work only for heavier particles and at energies where the relativistic factor is not too far from unity.

3.4 The energy loss to synchrotron radiation power per electron is given by (24.41). This energy loss should be multiplied with the circulating current to get the total radiation power. $P_{\gamma}=8.85 \times 10^{-5} \frac{E^{4}}{R} \frac{I}{e}=55.976 I$. For $1 \mathrm{~W}$ of synchrotron radiation power the current should be $17.865 \mathrm{~mA}$.

3.5 a.) The bending radius at $25 \mathrm{MeV}$ is $\frac{1}{R}=0.3 \frac{B}{E}=2.568 \mathrm{~m}^{-1}$ (assuming $\beta \approx 1$ ) or $2 R=0.7788 \mathrm{~m}$. b.) We choose a gap of $10 \mathrm{~cm}$. c.) The excitation current for the field is derived similar to problem 27.2.4 and is $I_{\text {coil }}=\frac{B}{\mu_{0}} \frac{1}{2} g=4,259.6$ A. The length of the coil is $L_{\text {coil }}=2 \pi R+10 \%=2.69 \mathrm{~m}$ and the resistance is $R_{\mathrm{el}}=$ $\rho_{\mathrm{Cu}} \frac{L_{\text {coil }}}{A}=1.205 \times 10^{-4} \Omega\left(\rho_{\mathrm{Cu}}=1.68 \cdot 10^{-8} \Omega \mathrm{m}\right.$ at $\left.20^{\circ} \mathrm{C}\right)$ The electrical power loss in each coil is then $P_{\text {coil }}=R_{\mathrm{el}} \cdot I_{\text {coil }}^{2}=2186 \mathrm{~W}$ and, yes, you need water cooling. d.) The electrical power requirement does not depend on the number of turns, only on the amount of copper in the coil. If the coil is made of $n$ turns, then the current will be reduced by the factor $n$.Yet, the length of the coil is increased by $n$ and the cross section decreases by the factor $n$. To summarize, the coil resistance is increased by $n^{2}$ while the current scales like $1 / n$. Therefore the power is independent of $n$.

3.6 The frequency swing depend on the velocity of the protons. The relativistic factor at injection is $\gamma_{0}=\frac{\sqrt{\left(m c^{2}\right)^{2}+\left(c p_{0}\right)^{2}}}{m c^{2}}=10.706$ and at the maximum energy $\gamma=$ 426.35. The corresponding velocities are at injection $\beta_{0}=0.995$ and at maximum energy $\beta \approx 1.000$. The frequency changes only by $0.44 \%$.

\section{Solutions for Chap. 4}

4.1 From the variational principle (4.1) and the definition of the Hamiltonian (4.26) we have $\delta \int_{t_{0}}^{t_{1}} L(\varphi) \frac{\mathrm{d} \varphi}{\mathrm{d} t} \mathrm{~d} t=-\delta \int_{t_{0}}^{t_{1}}\left[\sum \dot{q}_{i} P_{i}-\mathcal{H}_{t}(t)\right] \mathrm{d} t=0$ and after changing to $\varphi$ this is $\delta \int_{t_{0}}^{t_{1}}\left[\frac{\partial q_{i}}{\partial \varphi} \frac{\mathrm{d} \varphi}{\mathrm{d} t} P_{i}-\mathcal{H}_{\varphi}(\varphi) \frac{\mathrm{d} \varphi}{\mathrm{d} t}\right] \mathrm{d} t=0$. Therefore $\mathcal{H}_{t}=\frac{\mathrm{d} \varphi}{\mathrm{d} t} \mathcal{H}_{\varphi}$.

4.3 We apply $\frac{\mathrm{d}}{\mathrm{d} t} \frac{\partial L}{\partial \dot{x}}-\frac{\partial L}{\partial x}=0$ to the Lagrangian (4.25) in curvilinear coordinates. With $\beta_{h}^{2}=\left(\dot{x}^{2}+\dot{y}^{2}+\dot{z}^{2}+h^{2} \dot{z}^{2}\right) / c^{2}$ we get $\frac{\partial L}{\partial x}=m \frac{h \kappa_{x} \dot{z}^{2}}{\sqrt{1-\beta_{h}^{2}}}+$ $e\left(\dot{x} \frac{\partial A_{x}}{\partial x}+\dot{y} \frac{\partial A_{y}}{\partial x}+h \dot{z} \frac{\partial A_{z}}{\partial x}+\kappa_{x} \dot{z} A_{z}\right)-e \frac{\partial \phi}{\partial x}$ and $\frac{\mathrm{d}}{\mathrm{d} t} \frac{\partial L}{\partial \dot{x}}=\frac{\mathrm{d}}{\mathrm{d} t}\left(\frac{\dot{x}}{\sqrt{1-\beta_{h}^{2}}}\right)$ $+e\left(\dot{x} \frac{\partial A_{x}}{\partial x}+\dot{y} \frac{\partial A_{y}}{\partial y}+\dot{z} \frac{\partial A_{z}}{\partial z}\right)=\gamma m h \kappa_{x} \dot{z}^{2}+e\left(\dot{x} \frac{\partial A_{x}}{\partial x}+\dot{y} \frac{\partial A_{y}}{\partial x}+\dot{z} \frac{\partial A_{z}}{\partial x}+\kappa_{x} \dot{z} A_{z}\right)$ 
$-e \frac{\partial \phi}{\partial x}$. In curvilinear coordinates

$$
\begin{aligned}
\boldsymbol{B} & =\boldsymbol{\nabla} \times \boldsymbol{A} \\
& =\left\{\frac{1}{h}\left[\frac{\partial}{\partial y}\left(h A_{z}\right)-\frac{\partial A_{y}}{\partial z}\right], \frac{1}{h}\left[\frac{\partial A_{x}}{\partial z}-\frac{\partial}{\partial z}\left(h A_{z}\right)\right],\left[\frac{\partial A_{y}}{\partial x}-\frac{\partial A_{x}}{\partial y}\right]\right\}
\end{aligned}
$$

and we can replace $\frac{\partial A_{x}}{\partial y}=\frac{\partial A_{y}}{\partial x}-B_{z}$ and $\frac{\partial A_{x}}{\partial z}=\frac{\partial}{\partial y}\left(h A_{z}\right)+h B_{y}=h \frac{\partial A_{x}}{\partial z}+\kappa_{x} A_{z}+h B_{y}$ and thereby replace the terms involving the vector potential by magnetic fields to give the equation of motion $\frac{\mathrm{d}}{\mathrm{d} t}(\gamma m \dot{x})=\gamma m h \kappa_{x} \dot{z}^{2}+e\left(\dot{y} B_{z}-\dot{z} B_{y}\right)+e E_{x}$ which is the same as (4.16).

\section{Solutions for Chap. 5}

5.1 We use the Lagrangian (4.25) and evaluate (4.5) to get first $\frac{\partial L}{\partial x}=$ $\gamma m \dot{x}+e A_{x}$ and second $\frac{\mathrm{d}}{\mathrm{d} t} \frac{\partial L}{\partial \dot{x}}=\frac{\mathrm{d}}{\mathrm{dt}}(\gamma m \dot{x})+e\left(\dot{x} \frac{\partial A_{x}}{\partial x}+\dot{y} \frac{\partial A_{x}}{\partial y}+\dot{z} \frac{\partial A_{x}}{\partial z}\right)$. Then $\frac{\partial L}{\partial x}=\gamma m \dot{z}^{2} h \kappa_{x}+e\left(\dot{x} \frac{\partial A_{x}}{\partial x}+\dot{y} \frac{\partial A_{x}}{\partial y}+\dot{z} \frac{\partial A_{x}}{\partial z} \frac{\partial\left(h A_{z}\right)}{\partial x}\right)+e E_{x}$ and the Lagrange equation is $\frac{\mathrm{d}(\gamma m \dot{x})}{\mathrm{d} t}+e\left(\dot{x} \frac{\partial A_{x}}{\partial x}+\dot{y} \frac{\partial A_{x}}{\partial y}+\dot{z} \frac{\partial A_{x}}{\partial z}\right)=\gamma m \dot{z}^{2} h \kappa_{x}+e\left(\dot{x} \frac{\partial A_{x}}{\partial x}+\dot{y} \frac{\partial A_{y}}{\partial y}+\dot{z} \frac{\partial\left(h A_{z}\right)}{\partial x}\right)+e E_{x}$ or after reordering and replacing $\frac{\partial A_{y}}{\partial x}-\frac{\partial A_{x}}{\partial y}=B_{z}$ and $\frac{\partial\left(h A_{z}\right)}{\partial x}-\frac{\partial A_{x}}{\partial z}=h B_{y}$ the equation of motion is finally $\frac{\mathrm{d}}{\mathrm{dt}}(\gamma m \dot{x})=\gamma m \dot{z}^{2} h \kappa_{x}+e\left(B_{z} \dot{y}+h B_{y} \dot{z}\right)+e E_{x}$. Inserting into the Lagrange equations we get first $\frac{\partial L}{\partial z}=\gamma m \dot{z}^{2} h h^{\prime}+e\left(\dot{x} \frac{\partial A_{x}}{\partial z}+\dot{y} \frac{\partial A_{x}}{\partial z}+\dot{z} h \frac{\partial A_{z}}{\partial z}\right)+$ $e E_{z}=\gamma m \dot{z}^{2} h\left(\kappa_{x}^{\prime} x+\kappa_{y}^{\prime} y\right)+e\left(\dot{x} \frac{\partial A_{x}}{\partial z}+\dot{y} \frac{\partial A_{y}}{\partial z}+\dot{z} h \frac{\partial A_{z}}{\partial z}\right)+e E_{z}$ and second $\frac{\mathrm{d}}{\mathrm{d} t} \frac{\partial L}{\partial \dot{z}}=$ $\frac{\mathrm{d}}{\mathrm{d} t}\left(\gamma m h^{2} \dot{z}\right)+e \frac{\mathrm{d}\left(h A_{z}\right)}{\mathrm{d} t}$. With $\frac{\partial A_{x}}{\partial z}=h B_{y}+\frac{\partial\left(h A_{z}\right)}{\partial x}$ and $\frac{\partial A_{y}}{\partial z}=-h B_{x}+\frac{\partial\left(h A_{z}\right)}{\partial y}$ the equation of motion is $\frac{\mathrm{d}}{\mathrm{dt}}\left(\gamma m h^{2} \dot{z}\right)=\gamma m \dot{z}^{2} h \kappa_{x}+\gamma m \dot{z}^{2} h\left(\kappa_{x}^{\prime} x+\kappa_{y}^{\prime} y\right)+e\left(h B_{y} \dot{x}-h B_{x} \dot{y}\right)+e E_{z}$.

5.2 Application of (5.33)-(5.30) gives with $A x=\left(x^{\prime} A_{x}+y^{\prime} A_{y}+h A_{z}\right)$ the Lagrangian $L=-m c^{2} \sqrt{1-\beta^{2}}+e \frac{v}{s^{\prime}} A x-e \phi$ and from this $\frac{L+\gamma m c^{2}}{\gamma m v} \frac{s^{\prime}}{v}=$ $s^{\prime}+\frac{e}{\gamma m v} A x-e \phi$. Dividing by the momentum $p=\gamma m v$ and setting $p=\frac{p_{0}}{1-\delta}$, $\frac{L+\gamma m c^{2}}{\gamma m v} \frac{s^{\prime}}{v}=s^{\prime}+(1-\delta) \frac{e}{p_{0}} A x-(1-\delta) \frac{e}{p_{0}} \frac{s^{\prime}}{v} \phi$. The variational principle $\Delta \int \frac{L+\gamma m c^{2}}{p} \frac{s^{\prime}}{v} \mathrm{~d} t=\Delta \int \frac{L+\gamma m c^{2}}{p} \mathrm{~d} z=0$, because constant additions or factors to the Lagrangian do not change the variational principle.

5.3 Use the definition of the bending radius (5.3) and remember that we express the particle energy only in Volts, which is $E / e$. For $1 \mathrm{GeV}$ particles we have then $E / e$ $=10^{9} \mathrm{~V}$. With this the curvature is $\frac{1}{\rho} \approx 0.2998 \frac{B}{\beta E}$. In the last step of the equation we get a bit sloppy by using the energy in $\mathrm{GeV}$ instead of the voltage $E / e$ as is commonly done. Numerically, however, we would ignore $e$ insert only the voltage in GV. 
5.4 We evaluate the second derivative of the solution $P^{\prime \prime}(z)$ and insert into (5.74). From the first derivative $P^{\prime}(z)$ we obtain with the property of the Wronskian (5.73) for the principal solutions the second derivative $P^{\prime \prime}(z)=S^{\prime \prime}(z) \int_{0}^{z} p(\tilde{z}) C(\tilde{z}) \mathrm{d} \tilde{z}-$ $C^{\prime \prime}(z) \int_{0}^{z} p(\tilde{z}) S(\tilde{z}) \mathrm{d} \tilde{z}$. Recalling that $S^{\prime \prime}=-K S$ and $C^{\prime \prime}=-K C$, we insert the expression for $P^{\prime \prime}(z)$ and (5.75) into (5.74) and verify the validity of the ansatz (5.75). The function $P(z)$ is therefore indeed a particular solution of the inhomogeneous differential equation (5.74).

5.5 The Cartesian coordinates $\left(x, x^{\prime}\right)$ are identical to the normalized coordinates $(w, \dot{w})$ except for some scaling factors if we consider the independent variable to be $z$ in both cases. The transformations are then given from (5.54) by $x^{\prime}=-v x \tan \psi$ and $J=\frac{1}{2} \frac{v x^{2}}{\cos ^{2} \psi}$ or $x=\sqrt{2 J / v} \cos \psi$ and $x^{\prime}=2 \nu \sin \psi$. With this, the Hamiltonian is $\mathcal{K}=v J \sin ^{2} \psi+D \frac{J}{v} \cos ^{2} \psi$. The frequency of the oscillator is $\dot{\psi}$ or from the equation of motion $\frac{\partial \mathcal{K}}{\partial J}=\dot{\psi}=v \sin ^{2} \psi+\frac{D}{v} \cos ^{2} \psi$ which requires that $D=v^{2}$. Finally, $\mathcal{K}=v J$ and $\frac{\partial \mathcal{K}}{\partial J}=\dot{\psi}=v$ as we would expect.

\section{Solutions for Chap. 6}

6.1 The coil power, assuming only one turn, is $P=R I^{2}$. The resistance is $R=\rho \frac{L}{A}$, where $\rho$ is the specific resistance of the coil material (copper), $L$ the length of the coil and $A$ the coil cross section. With $n_{\mathrm{t}}$ turns, the resistance changes to $R=\rho \frac{L n_{\mathrm{t}}}{A / n_{\mathrm{t}}}=$ $\rho \frac{L}{A} n_{\mathrm{t}}^{2}$, the current is $\frac{I}{n_{\mathrm{t}}}$ and the total power $P=\rho \frac{L}{A} n_{\mathrm{t}}^{2}\left(\frac{I}{n_{\mathrm{t}}}\right)^{2}$ is independent of the number of turns. The total coil power is $P=R I^{2}$. Utilizing the current density $j$ the total power is now $P=\rho L A j^{2}$. The weight of copper is $W=\sigma L A$ and the total power is finally $P=\rho \frac{W}{\sigma} j^{2}$ depending only on specific resistance, weight and current density.

6.2 The focal length of an electric quadrupole is $\frac{1}{f}=k \ell=\frac{e}{\beta^{2} E} g \ell$. Solving for the gradient $g$ we get $g=\frac{\beta^{2} E}{e l} \frac{1}{f}=19.81 \mathrm{MV} / \mathrm{m}^{2}$.The profile of the electrodes is given by $V_{2}=-\frac{1}{2} g\left(x^{2}-y^{2}\right)$ and the profile of the electrodes is therefore given by $V_{2}=9.905 \cdot 10^{6} R_{\mathrm{a}}^{2}=24,763 \mathrm{~V}$ where $R_{\mathrm{a}}$ is the aperture radius of the electric quadrupole. The r.h.s. derives from the potential for $R_{\mathrm{a}}=0.05 \mathrm{~m}$ and $y=0$. The electrode potentials are $V_{2}= \pm 24,763 \mathrm{~V}$.

6.3 For a purely transverse field $\boldsymbol{B}=\left(B_{x}, B_{y}, 0\right)=\nabla \times \boldsymbol{A}$. The field components are $B_{x}=\frac{\partial A_{z}}{\partial y}-\frac{\partial A_{y}}{\partial z}=\frac{\partial A_{z}}{\partial y}$ and $B_{y}=\frac{\partial A_{x}}{\partial z}-\frac{\partial A_{z}}{\partial x}=-\frac{\partial A_{z}}{\partial x}$. Here we made use of the fact that the fields do not depend on the longitudinal coordinate $z$. Therefore $A_{x}, A_{y}$ are constant which we may as well choose to be zero $A_{x}=A_{y}=0$ and the magnetic field is $\boldsymbol{B}=\left(\frac{\partial A_{z}}{\partial y},-\frac{\partial A_{z}}{\partial x}, 0\right)$. If we derive the magnetic field from a scalar potential $\boldsymbol{B}=-\nabla V$, we would get $\frac{\partial A_{z}}{\partial y}=-\frac{\partial V}{\partial x}$ and $\frac{\partial A_{z}}{\partial x}=\frac{\partial V}{\partial y}$ or $A_{z}=-\int \frac{\partial V}{\partial x} \mathrm{~d} y$ and $A_{z}=$ 
$\int \frac{\partial V}{\partial y} \mathrm{~d} x$. For a dipole magnet $B_{x}=0$ and $B_{y}=B_{0}$ and the potential is $V_{1}=-B_{0} y+$ $f(x)$ and $\left(\frac{\partial A_{z}}{\partial y}=g y, \frac{\partial A_{z}}{\partial x}=-g x\right)$. For a quadrupole we have a potential $V=-g x y$ and $\frac{\partial A_{z}}{\partial y}=g y, \frac{\partial A_{z}}{\partial x}=-g x$. After integration $A_{z}=\frac{1}{2} g y^{2}$ and $A_{z}=-\frac{1}{2} g x^{2}$ to give a combined potential $A_{z}=-\frac{1}{2} g\left(x^{2}-y^{2}\right)$. Following from their definition both, $V$ and $A_{z}$, depend differently on the coordinates but are equivalent otherwise for purely transverse fields. While the scalar potentials $V$ define equipotential lines, the vector potential $A_{z}$ defines the field lines which are orthogonal to the equipotential lines.

6.4 Such a magnet is basically a quadrupole which is displaced to generate a dipole field component along the path of the beam. From $\frac{1}{\rho}=k \Delta x$ the displacement is $\Delta x=0.00741 \mathrm{~m}$ or $7.41 \mathrm{~mm}$. The sextupole term is a small perturbation of the quadrupole profile. From (6.86) and the definitions for $A_{i j}$,we get for this combined field magnet the potential $V=\frac{\beta E}{e c}\left[\frac{1}{\rho} y+k x y+\frac{1}{6} m\left(3 x^{2} y-y^{3}\right)\right]=$ $0.557 y+75.15 x y+640\left(3 x^{2} y-y^{3}\right)=$ const. The constant can be estimated as follows: At a displacement $\Delta x=7.41 \mathrm{~mm}$ we require an aperture of $r=1 \mathrm{~cm}$ and so we define one point $(x=7.41 \mathrm{~mm}, y=10 \mathrm{~mm})$ of the profile. Inserted into the profile equation, we get $V(0.00741,0.01)=0.01155285$ and the pole profile equation is $0.557 y+75.15 x y+640\left(3 x^{2} y-y^{3}\right)=0.01155285$.

6.5 Forces between magnet pole exist because a change of the field volume is associated with a change of field energy. Increasing the gap of a dipole magnet while keeping the field constant results in an increase of field energy due to work against the force trying to reduce the gap or the distance between poles. Therefore the force is attracting the pole. The field energy in the dipole magnet with a pole gap $G$ is $E_{\mathrm{f}}=\frac{1}{2 \mu_{0}} B^{2} w l G=89570 \cdot G$. The force is $F=-\mathrm{d} E / \mathrm{d} G=89570 \mathrm{~N}=9.13$ tons. The force does not depend on the pole gap.

26.8 In first approximation, we assume that all the fields are contained within the volume between the two rows of poles and no field leaks out. Separating the poles by $g$ requires to generate the additional field energy $\mathrm{d} e=F \mathrm{~d} g$ where $F$ is the force between poles. Since $\mathrm{d} e>0$ for $\mathrm{d} g>0$ the force is attractive meaning that the poles are attracted. The force is then $F=\frac{\mathrm{d} e}{\mathrm{~d} g}=\frac{w}{2 \mu_{0}} \int_{0}^{15 \lambda_{\mathrm{p}}} B_{0}^{2} \sin ^{2}\left(2 \pi \frac{z}{\lambda_{\mathrm{p}}}\right) \mathrm{d} z=$ $\frac{w}{2 \mu_{0}} B_{0}^{2} \frac{\lambda_{\mathrm{p}}}{2 \pi} \frac{1}{2} N_{\mathrm{p}}=7,484 \mathrm{~N}=0.76$ tons.

6.6 See Sect. 6.2.2 for solution. With respect to the numerical part of the question, we calculate the gradient to be $g=\frac{k \ell}{0.3} \beta E=33.33 \mathrm{~T} / \mathrm{m}$ and the coil excitation current must be $I_{\text {coil }}=\frac{g R^{2}}{2 \mu_{0}}=11937$ A.turns.

6.7 Since this is a cylindrical problem, we use the definition of the magnetic potential in (6.7) $P(r, z)=\sum_{n \geq 0} C_{n} r^{n} \mathrm{e}^{\mathrm{i} n \varphi}$ from which we get the magnetic fields.

From Maxwell's equation $\boldsymbol{\nabla} \times \boldsymbol{E}=-\frac{\partial \boldsymbol{B}}{\partial t}$ and integrating over the cross section of the rotating coil we get an emf of $m V=-\partial \phi / \partial t$, where $\phi=\phi_{0} \cos 2 \pi \nu t$ is the flux through the coil at time $t$ and $m$ the number of turns in the coil. At time $t=0$ 
we assume the coil to be parallel with the midplane enclosing the maximum flux $\phi_{0}=B_{\varphi} 2 R$. We ignore the length of the coil because the field is assumed to be uniform longitudinally. From the potential, we get $B_{\varphi} \propto \mathrm{e}^{\mathrm{i} n \varphi}, \varphi=2 \pi \nu t$ and the induced voltage in the coil is $m P=2 \pi v \sum_{n \geq 0} C_{n} r^{n} \mathrm{e}^{\mathrm{i} n 2 \pi v t}$. The signal voltage from the rotating coil includes therefore all harmonics of the magnetic field. Usually the harmonics are normalized to the ideal field of the magnet at $r=1 \mathrm{~cm}$. The signal of the ideal magnet of order $N$ is $m V_{N} \propto 2 \pi \nu N \mathrm{e}^{\mathrm{i} N 2 \pi \nu t}$ and the relative strengths $r_{n N}$ of the harmonics are $r_{n N}=R^{n-N} \frac{V_{n}}{V_{N}}$.

6.8 A finite width of a quadrupole is like superimposing poles of opposite polarity on both sides of a pole. This is a symmetric perturbation with two negative poles within a $90^{\circ}$ quadrant. We may complete this picture by assuming that there is also an additional pole superimposed on the main pole with the same polarity. We have now in each quadrant a main pole with a somewhat lower quadrupole field and three poles describing the perturbation by a multipole. In the case of a quadrupole this would be a 12-pole. Since the perturbation is nonlinear but symmetric about the main pole, we observe all odd harmonics of the quadrupole field, $3 \times 4=12$-pole, $5 \times 4=20$ pole, etc. These perturbations are due to the finite width of the quadrupole and have nothing to do with tolerances. We call therefore these harmonics, "allowed" harmonics or multipole components.

6.9 The fields scale differently depending on the multipole field order. The quadrupole scales linearly and therefore $B_{2}(1 \mathrm{~cm})=B_{2} \frac{1}{1.79}=0.20832 \mathrm{~T}$. The next higher order field is the sextupole field which scales quadratically and the normalized field is therefore $B_{3}(1 \mathrm{~cm})=B_{2} \frac{B_{3}}{1.79^{2}}=1.8727 \cdot 10^{-4}$. Similar renormalizations lead to $B_{4}(1 \mathrm{~cm})=1.925 \cdot 10^{-5}, B_{5}(1 \mathrm{~cm})=1.6833 \cdot 10^{-5}$, $B_{6}(1 \mathrm{~cm})=1.8964 \cdot 10^{-5}, B_{7}(1 \mathrm{~cm})=2.9186 \cdot 10^{-7}, B_{8}(1 \mathrm{~cm})=1.875 \cdot 10^{-7}$, $B_{9}(1 \mathrm{~cm})=2.322 \cdot 10^{-8}, B_{10}(1 \mathrm{~cm})=1.8066 \cdot 10^{-7}$. The 12-pole and 20-pole components do not follow the general downward trend and are larger, because they are "allowed" harmonics due to the finite pole width.

6.10 The upright octupole potential is from Table 6.6 given by $-\frac{e c}{c p} V_{4}=$ $r \frac{1}{6}\left(x^{3} y-x y^{3}\right)=$ const. From the geometry of an octupole the pole tip is a radius $R$ and angle $22.5^{\circ}$ or at $x=R \cos \pi / 8, y=R \sin \pi / 8$. With these values the constant or potential of the pole is $\frac{1}{6}\left(x^{3} y-x y^{3}\right)=1.50 R^{4}$. It is actually easier to do the calculation for a rotated octupole, which has a pole tip in the midplane at $x=R$ and $y=0$. From Table 6.6 the potential is $-V_{4}(x, y)=\underline{s}_{4} \frac{1}{24}\left(x^{4}-6 x^{2} y^{2}+y^{4}\right)=$ const and the equation for the pole profile is $\frac{1}{24}\left(x^{4}-6 x^{2} y^{2}+y^{4}\right)=R$. The field in the midplane is $B_{x}(x, 0)=\underline{s}_{4} \frac{1}{6} x^{3}$, the field at the pole tip is expected to be $0.2 \mathrm{~T}$ and the octupole strength parameter is $\underline{s}_{4}=s_{4}=\frac{6 \times 0.2}{0.03^{3}}=44,444 \mathrm{~T} / \mathrm{m}^{3}$. To calculate the coil excitation current, we integrate from the octupole center along the $x$-axis to the pole giving $\int_{0}^{R} \frac{1}{6} x^{3} \mathrm{~d} x=\frac{1}{24} R^{4}$. The integration through the iron is zero as is the integration back along the $45^{\circ}$ line from the return yoke to the magnet center, because field and integration path are orthogonal. Similar to the calculation of the excitation current for the quadrupole, we have $I_{\text {coil }}=\frac{s_{4}}{24 \mu_{0}} R^{4}=1,193.7 \mathrm{~A} \cdot$ turns. 
6.11 For a pure dipole field the current distribution scales like $\cos \varphi$, we assume the coil thickness to be $h(\theta)=h_{0} \cos \varphi$ and the current density at $\varphi=0$ is from $\mathrm{d} I(\varphi)=I_{1} \cos \varphi \mathrm{d} \varphi$ and $\Delta A=h(\varphi) R \Delta \varphi$ just $j=\frac{I_{1}}{R \Delta \theta h_{0}}$. To generate a field of $B_{0}=\mu_{0} H_{0}=5 \mathrm{~T}$ we need a peak current of $I_{1}=2 R H_{1}=2 R \frac{B_{0}}{\mu_{0}}=2.387 \times 10^{5} \mathrm{~A}$ and $\hat{\jmath}=10^{9}=\frac{2.387 \times 10^{5} \Delta \varphi}{R \Delta \varphi h_{0}}$ with $h_{0}=7.96 \mathrm{~mm}$. The maximum coil thickness is therefore about $8 \mathrm{~mm}$.

\section{Solutions for Chap. 7}

7.1 Study Fig. 7.16 in detail. Now, make a similar sketch with the cosine-like trajectory for the horizontal and vertical plane both ending at the focal point. Tracing back both cosine-like trajectories from the focal point straight to the intersection with the incoming cosine-like trajectories. The distance from the focal point to the intersections should be equal to the doublet focal lengths from (7.20). The two intersections are also known from light optics as the principal planes of the system.

7.2 Transform through doublet and $5 \mathrm{~m}$ drift space to focal point. We need only the $M_{11}$ element of the total transformation matrix, which must be zero to let a parallel trajectory entering the doublet go through the focal point such that $x=M_{11} x_{0}=0$. We have $M_{11, x}=1-\frac{d+D}{\left|f_{1}\right|}-\frac{D}{\left|f_{2}\right|}+\frac{d D}{\left|f_{1} f_{2}\right|}=0$. Since we want the focal point in both planes, we have a second equation $M_{11, y}=1+\frac{d+D}{\left|f_{1}\right|}+\frac{D}{\left|f_{2}\right|}+\frac{d D}{\left|f_{1} f_{2}\right|}=0$. From both equations we isolate $f_{1}$ and $f_{2}$ for $f_{1}=\sqrt{6} \mathrm{~m}$ and $f_{2}=5 / \sqrt{6} \mathrm{~m}$. The total focal lengths in both planes are different $f_{x}^{*}=\frac{5 \sqrt{6}}{\sqrt{6}-1}=8.4495 \mathrm{~m}$ and $f_{y}^{*}=\frac{5 \sqrt{6}}{\sqrt{6}+1}=$ $3.5505 \mathrm{~m}$. The definition of these focal lengths is the distance of two principal planes from the focal point. The principal planes, one for each plane, are located at the intersection of the parallel incoming trajectory and the extension of the trajectories reaching the focal point. The principal planes are the positions of virtual lenses resembling the doublet. These lenses are at different location for both planes.

7.3 The transformation matrix of a quadrupole doublet with a drift space of length $d$ between quadrupoles is $\left(\begin{array}{cc}1-d / f_{1} & d \\ -1 / f^{*} & 1-d / f_{2}\end{array}\right)$, where $\frac{1}{f^{*}}=\frac{1}{f_{1}}+\frac{1}{f_{2}}-\frac{d}{f_{1} f_{2}}$. We set $\frac{1}{f_{1}}=-\frac{1}{f_{2}}=0.2 \mathrm{~m}^{-1}$ and get for the on-energy focal lengths in both planes $\frac{1}{f^{*}}=\frac{1}{25}$ or $f^{*}=25 \mathrm{~m}$. Since the combined focal length depends quadratically on the energy, we get a $\pm 10 \%$ spread in focal length. The beam width at the focal point is with $f_{1}=5 \mathrm{~m}$ is $r^{2}=\epsilon \beta=\left(1-d / f_{1}\right)^{2} \epsilon \beta_{0}+d^{2} \epsilon \gamma_{0}=0.64 \epsilon \beta_{0}+\frac{\epsilon^{2}}{r_{0}^{2}}$. The beam emittance is $\epsilon=r_{0} r_{0}^{\prime}$ and therefore the spot size in the horizontal plane is $r_{x}^{2}=0.64 r_{0}^{2}+r$ and in the vertical plane with $f_{1}=-5 \mathrm{~m}$ is $r_{y}^{2}=1.44 \epsilon \beta_{0}+\frac{\epsilon^{2}}{r_{0}^{2}}$. For the last question on dispersive beam sizes set $\left|f_{1}\right|=5 \cdot\left(1+\frac{\Delta p}{p_{0}}\right)$ and calculate $r_{x, y}=\left(\Delta p / p_{0}\right)$. 
7.4 Equations (7.63) and (7.67) give the focal lengths for a wedge magnet in both planes: $\frac{1}{\kappa f_{x}}=\left(\tan \eta_{0}+\tan \eta_{\mathrm{e}}\right) \cos \theta-\tan \eta_{0} \tan \eta_{\mathrm{e}}+\sin \theta$ and $\frac{1}{\kappa f_{y}}=\tan \eta_{0}+$ $\tan \eta_{\mathrm{e}}+\frac{1}{3} \delta_{f \mathrm{e}}+\delta$. We choose both edge angles to be the same and require that both focal lengths be the same as well. This results in the condition $2 \tan \eta \cos \theta-\tan ^{2} \eta+$ $\sin \theta=2 \tan \eta+\frac{2}{3} \delta_{f}$, which can be solved for the wedge angle $\eta$.

7.5 The deflection angle $\vartheta=u_{0}^{\prime}$ and we assume $u_{0}=0$. Then the betatron amplitude downstream from point $z_{0}$ is just $u(z)=\sqrt{\beta \beta_{0}} \vartheta \sin \left(\psi-\psi_{0}\right)$. From this it is clear that the value of the betatron function should be as large as possible at both points to get a large kick amplitude.

7.6 a.) a trim coil in $\mathrm{QF}_{1}$ causes a beam bump which will reach zero again in the middle of $\mathrm{QF}_{3}$. This is too early and we need to activate another trim coil in $\mathrm{QD}_{2}$ to be symmetric. The beam bump arrives in the middle of $\mathrm{QD}_{2}$ with a slope of $x^{\prime}$. A kick with an angle of $\theta_{2}=2 x^{\prime}\left(\mathrm{QD}_{2}\right)$ will symmetrize the beam bump ending at zero in the middle of $\mathrm{QF}_{4}$ where a third trim coil $\theta_{3}=\theta_{1}$ ends the beam bump. Because of symmetry we need to consider only half the three FODO cells from $\mathrm{QF}_{1}$ to $\mathrm{QD}_{2}$ for which the transformation matrix be $\mathcal{M}=\left(\begin{array}{ll}m_{11} & m_{12} \\ m_{21} & m_{22}\end{array}\right)$. The beam bump at $\mathrm{QD}_{2}$ is then $x\left(\mathrm{QD}_{2}\right)=m_{12} \theta_{1}$ and $x^{\prime}\left(\mathrm{QD}_{2}\right)=m_{22} \theta_{1}$. Since $x\left(\mathrm{QD}_{2}\right)=0.02$ m we get $\theta_{1}=\frac{0.02}{m_{12}}$ and $\theta_{3}=-2 \cdot x^{\prime}\left(\mathrm{QD}_{2}\right)$. The bump amplitude $A_{\mathrm{M}}$ is not the maximum bump amplitude which occurs in the $\mathrm{QF}_{2}$ and $\mathrm{QF}_{3}$.

\section{Solutions for Chap. 8}

8.1 We start from $u=\sqrt{\epsilon \beta} \cos (\psi+\delta)$, calculate the derivative $u^{\prime}=$ $-\sqrt{\epsilon} \frac{\alpha}{\sqrt{\beta}} \cos (\psi+\delta)-\frac{\sqrt{\epsilon}}{\sqrt{\beta}} \sin (\psi+\delta)$, and eliminate from both equations the

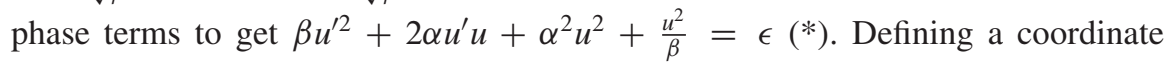
transformation by $w=u / \sqrt{\beta}$ and $\dot{w}=\sqrt{\beta} u^{\prime}+\alpha \frac{u}{\sqrt{\beta}}$ and inserting into (*) we get $w^{2}+\dot{w}^{2}=\epsilon$ which is the equation of a circle. The derivative $\dot{w}=\frac{\mathrm{d} w}{\mathrm{~d} \psi}$ and the new independent variable is the phase $\psi$.

8.2 First, we transform the phase ellipse as in problem 8.1. This transformation is scale preserving since its determinant is equal to unity. The phase ellipse is now a circle with radius $\sqrt{\epsilon}$ and therefore the area of the circle is $\pi \epsilon$.

8.3 Write (8.41) in component form and place beam matrix elements with their definitions (8.38)

8.4 The transformation matrix of such a transformer between symmetry points $P_{0}$ and $P_{1}$ is $\left(\begin{array}{cc}0 & \sqrt{\beta_{0} \beta} \\ -\frac{1}{\sqrt{\beta_{0} \beta}} & 0\end{array}\right)$. The transformation of $\beta_{0}$ with $\alpha_{0}=0$ through 
such a transformer is $\beta_{1}=C^{2} \beta_{0}+\frac{1}{\beta_{0}} S^{2}$ and $\gamma_{1}=C^{\prime 2} \beta_{0}+\frac{1}{\beta_{0}} S^{\prime 2}=\frac{1}{\beta_{1}}$. The dispersion function transforms through this matching section like a trajectory and we would look for $\eta_{1}=C \eta_{0}+S \eta_{0}^{\prime}=C \eta_{0}$. A $\pi / 2$-transformer does not work for the dispersion functions since $C=0$.

\section{Solutions for Chap. 9}

9.1 The proton energy after accelerating gap $i$ is $E_{\text {kin }}(\mathrm{MeV})=1+0.5 i$. At the energies under consideration the protons are nonrelativistic and their velocities are $v_{i}=c \sqrt{\frac{2 E_{\mathrm{kin}}}{m c^{2}}}=1.384 \times 10^{7} \sqrt{1+0.5 i} \mathrm{~m} / \mathrm{s}$. The period of the $500 \mathrm{MHz}$ rf-field is $2 \mathrm{~ns}$ and the tube lengths therefore must be $\ell_{i}+\ell_{\text {gap }}=n \cdot 2 \times 10^{-9} v_{i}$, where $n$ is an integer and $\ell_{i} \gg \ell_{\text {gap }}$. The first three sections are $(n=1) \ell_{1}+\ell_{\text {gap }}=3.39 \mathrm{~cm}$, $\ell_{2}+\ell_{\text {gap }}=3.92 \mathrm{~cm}$, and $\ell_{3}+\ell_{\text {gap }}=4.38 \mathrm{~cm}$. These are the minimum lengths of the tubes. To meet the requirement of a minimum tube length of $15 \mathrm{~cm}$ we need to choose $n_{1} \geq 5, n_{2} \geq 4, n_{3} \geq 4$. That means the first tube length is as long as 5 wavelengths and the second and third tube length is as long as 4 wavelengths.

9.2 Each proton travels at a slightly different velocity due to a finite energy spread. We assume nonrelativistic protons and get for the velocity spread $\frac{\Delta v}{v_{0}}= \pm \frac{1}{2} \frac{\Delta E}{E_{0}}$. The time $\tau$ it takes for debunching is equal to the time it take for the fastest particle to travel half the separation of bunches $\Delta=\frac{C}{1 n_{\mathrm{b}}} C$ where $C$ is the circumference. The other half of the bunch spacing is covered by particles with $\frac{\Delta E}{E_{0}}<0$. The debunching time is then $\tau=\frac{C}{2 n_{\mathrm{b}}} \frac{E_{0}}{v_{0} \Delta E}$.

9.3 The accelerating rate is given by $\frac{\mathrm{d} E_{\mathrm{kin}}}{\mathrm{d} t}=f_{\mathrm{rev}} e V_{\mathrm{rf}} \sin \psi_{\mathrm{s}}$. We solve for the synchronous phase and insert into (9.74) to get $\left(\frac{\Delta c p}{c p_{0}}\right)^{2}=\frac{e V_{0}}{\pi h\left|\eta_{\mathrm{c}}\right| c p_{0}}$ $\left[\cos \varphi+1+\left(2 \arcsin \frac{\mathrm{d} E_{\mathrm{kin}} / \mathrm{d} t}{f_{\mathrm{rev}} e V_{\mathrm{rf}}}+\varphi-\pi\right) \frac{\mathrm{d} E_{\mathrm{kin}} / \mathrm{d} t}{f_{\mathrm{rev}} e V_{\mathrm{rf}}}\right]$.

9.4 We start from (9.52) and write it in the form of a linear harmonic oscillator $\ddot{\varphi}+\Omega_{0}^{2} \frac{\sin \varphi \cos \psi_{\mathrm{s}}-\sin \psi_{\mathrm{s}}(1-\cos \varphi)}{\varphi \cos \psi_{\mathrm{s}}} \varphi=0$ where the synchrotron frequency is $\Omega^{2}=$ $\Omega_{0}^{2} \frac{\sin \varphi \cos \psi_{\mathrm{s}}-\sin \psi_{\mathrm{s}}(1-\cos \varphi)}{\varphi \cos \psi_{\mathrm{s}}}$. For small oscillation amplitudes the frequency $\Omega \approx \Omega_{0}$ and reaches zero at the separatrix. There is also a $\varphi$-dependence of the synchrotron frequency indicating a periodic variation as the particle travels along the phase space trajectory.

9.5 The longitudinal emittance is $\frac{\widehat{\Delta c p}}{c p_{0}} \hat{\varphi}_{0}=\frac{\Omega_{0}}{h \omega_{\text {rev }} \mid \eta_{\mathrm{c}}} \hat{\varphi}_{0}^{2}$ and does not change by increasing the rf-voltage. Since $\Omega_{0} \propto \sqrt{V_{\mathrm{rf}}}$, we get $\sqrt{V_{\mathrm{rf}, 0}} \hat{\varphi}_{0}^{2}=\sqrt{V_{\mathrm{rf}, 1}} \hat{\varphi}_{1}^{2}$ or $\hat{\varphi}_{1}=\hat{\varphi}_{1}\left(\frac{V_{\mathrm{rf}, 0}}{V_{\mathrm{rf}, 1}}\right)^{1 / 4}$. The bunch length scales like the fourth root of the rf-voltage. 


\section{Solutions for Chap. 10}

10.6 We use a FODO lattice with $\kappa=\sqrt{2}, \hat{\beta}=L(2+\sqrt{2})$ and $\hat{\eta}=\frac{L^{2}}{2 \rho}(4+\sqrt{2})$ to construct a ring. The beam width in such a lattice is $\hat{\sigma}_{x}^{2}=\epsilon \hat{\beta}+\hat{\eta}^{2}\left(\frac{\sigma_{E}}{E}\right)^{2}$ and the beam height $\check{\sigma}_{y}^{2}=\epsilon \check{\beta}$. Since $\check{\sigma}_{y}<\hat{\sigma}_{x}$ we get $\hat{\sigma}_{x}^{2}+\check{\sigma}_{y}^{2}=R^{2} \approx \epsilon(\hat{\beta}+\check{\beta})+$ $\hat{\eta}^{2}\left(\frac{\sigma_{E}}{E}\right)^{2}$. From the peak magnetic field $\frac{1}{\rho}=0.0036 \mathrm{~m}^{-1}$ and we can solve for $L$. The length of the bending magnet should be no more than $0.8 \mathrm{~L}$ and each bending magnet deflects the beam by $\psi_{\mathrm{b}}=\frac{0.8 L}{\rho}$. To complete a ring, we need $n=2 \pi / \psi_{\mathrm{b}}$ half cells. Numerically, we get $10^{4} R^{2} \approx 0.2 L+9.75 \times 10^{-5} L^{2}=4$ or solving for half the cell length $L=19.818 \mathrm{~m}$.

10.2 For a Gaussian beam the largest beam size is along one axis or in case of an optimum FODO lattice $\hat{\sigma}_{x}^{2}=\epsilon \hat{\beta}+\hat{\eta}^{2}\left(\frac{\sigma_{E}}{E}\right)^{2}$ from which we get $L$ since $\check{\sigma}_{y}^{2}=\epsilon \check{\beta} \ll \hat{\sigma}_{x}^{2}$. For a maximum field of $1.8 \mathrm{~T}$ the bending radius and angle $\frac{1}{\rho}=0.0108 \mathrm{~m}^{-1}$ and $\psi_{\mathrm{b}}=\frac{0.8 L}{\rho}$, respectively with the total number of cells $n_{\mathrm{c}}=2 \pi /\left(2 \psi_{\mathrm{b}}\right)$. The focal length of a half quadrupole is $f=\kappa L=\sqrt{2} L$ and $k^{-1}=\sqrt{2} L \ell_{\mathrm{q}}=0.05 \sqrt{2} L^{2}$ assuming that the total quadrupole length is $10 \%$ of $L$. Numerically, we get $10^{4} R^{2}=0.2 L+0.0292 L^{2}=9$ and solving for half the cell length $L=14.463 \mathrm{~m}$. The bending field is $1.8 \mathrm{~T}$ and therefore within practical limits, the quadrupole gradient is $g=\frac{k E}{0.3}=11.268 \mathrm{~T} / \mathrm{m}$ and the pole tip field is $B_{\mathrm{t}}=g R=11.268 \cdot 0.03=0.338 \mathrm{~T}$, which is well within practical limits of about $1 \mathrm{~T}$. The number of FODO cells is $n_{\mathrm{c}}=\pi / \psi_{\mathrm{b}}=25.141$ and to make it an even number of cell, say 26, we may decrease the bending magnet field by a factor $25.141 / 26$ to $1.740 \mathrm{~T}$. Now the tunes are $Q_{x, y}=6.25$ or right on a destructive half integer resonance. By raising the quadrupole strengths, we increase the tunes to say $Q_{x, y}=6.75$. The new quadrupole strengths are from $\sin \psi=\frac{1}{\kappa}=0.72815$ from which we get the new quadrupole strength $k=0.06962 \mathrm{~m}^{-2}$ and gradient $g=11.603 \mathrm{~T} / \mathrm{m}$ which is still within practical limits.

10.3 The transformation matrix of an unperturbed FOFO cell is $\mathcal{M}_{0}=$ $\left(\begin{array}{cc}\cos \psi_{0} & \beta_{0} \sin \psi_{0} \\ -\frac{1}{\beta_{0}} \sin \psi_{0} & \cos \psi_{0}\end{array}\right)$ and for $n_{\mathrm{c}}$ cells forming a half ring with $\Psi_{0}=n_{\mathrm{c}} \psi_{0}$ the transformation matrix is $\mathcal{M}_{0 n}=\mathcal{M}_{0}^{n_{\mathrm{c}}}=\left(\begin{array}{cc}\cos \Psi_{0} & \beta_{0} \sin \Psi_{0} \\ -\frac{1}{\beta_{0}} \sin \Psi_{0} & \cos \Psi_{0}\end{array}\right)$. Inserting a drift space of length $\ell$ at the beginning and end of the half ring results in a new 
transformation matrix with

$$
\begin{aligned}
\mathcal{M}_{n} & =\left(\begin{array}{ll}
1 & \ell \\
0 & 1
\end{array}\right)\left(\begin{array}{cc}
\cos \Psi_{0} & \beta_{0} \sin \Psi_{0} \\
-\frac{1}{\beta_{0}} \sin \Psi_{0} & \cos \Psi_{0}
\end{array}\right)\left(\begin{array}{ll}
1 & \ell \\
0 & 1
\end{array}\right) \\
& =\left(\begin{array}{cc}
\cos \Psi_{0}-\frac{\ell}{\beta_{0}} \sin \Psi_{0} & \beta_{0} \sin \Psi_{0}+2 \ell \cos \Psi_{0}-\frac{\ell^{2}}{\beta_{0}} \sin \Psi_{0} \\
-\frac{1}{\beta_{0}} \sin \Psi_{0} & \cos \Psi_{0}-\frac{\ell}{\beta_{0}} \sin \Psi_{0}
\end{array}\right) .
\end{aligned}
$$

This must be equal to a new symmetric matrix $\mathcal{M}_{n}=\left(\begin{array}{cc}\cos \Psi & \beta \sin \Psi \\ -\frac{1}{\beta} \sin \Psi & \cos \Psi\end{array}\right)$ and equating matrix elements on both sides we can solve for the new phase $\Psi$ and betatron function $\beta$. The tune change is $\Delta Q=\left(\Psi-\Psi_{0}\right) / \pi$ which we get from the equality $\cos \Psi=\cos \Psi_{0}-\frac{\ell}{\beta_{0}} \sin \Psi_{0}$ and the change of the betatron function is $\left(\beta-\beta_{0}\right) \approx \beta_{0} \frac{\sin \Psi_{0}-\sin \Psi}{\sin \Psi}$. Since $\eta^{\prime}=0$ at the insertion point, we don't find a change in the $\eta$-function by inserting a drift space.

10.4 For $\alpha_{1}=\alpha_{2}=0$ the transformation matrix of an arbitrary matching section is $\mathcal{M}_{\mathrm{m}}=\left(\begin{array}{cc}\sqrt{\frac{\beta_{2}}{\beta_{1}}} \cos \psi & \sqrt{\beta_{1} \beta_{2}} \sin \psi \\ -\frac{1}{\sqrt{\beta_{1} \beta_{2}}} \sin \psi & \sqrt{\frac{\beta_{1}}{\beta_{2}}} \cos \psi\end{array}\right)=\left(\begin{array}{cc}C & S \\ C^{\prime} & S^{\prime}\end{array}\right)$ and the transformation of the betatron function between two symmetry points is $\beta_{2}=C^{2} \beta_{1}+\frac{1}{\beta_{1}} S^{2}$. Especially simple solutions exist if the phases $\Delta \psi_{x}=\Delta \psi_{y}=\pi / 2$ in which case $C_{x}=C_{y}=0$ and the sine-like matrix element is adjusted such that $S_{x, y}^{2}=\beta_{1, x, y} \beta_{2, x, y}$. We have made no use of any FODO parameter and therefore the matching works between any two symmetry points where $\alpha_{1}=\alpha_{2}=0$.

10.5 The vertical betatron function is periodic since the vertical focusing is periodic from the quadrupoles. This should be true also for the horizontal betatron function but it is not because of the use of sector bending magnets which contribute to horizontal but not to vertical focusing. The dispersion function is even more perturbed because of the missing bending magnets.

\section{Solutions for Chap. 11}

11.1 The oscillation period for $500 \mathrm{MHz}$ is $T_{\mathrm{rf}}=\frac{1}{5 \cdot 10^{8}}=2.0 \times 10^{-9} \mathrm{~s}$. Therefore the bunches are separated by $2 \mathrm{~ns}$.

11.2 First we note that $J_{\mathrm{s}}=2$ for rectangular magnets and the synchrotron oscillation damping time is from (11.30) $\tau_{\mathrm{s}}^{-1}=\frac{2}{3} r_{\mathrm{e}} c \gamma^{3}\left\langle\frac{1}{\rho^{2}}\right\rangle=1139.6 \mathrm{~s}^{-1}$ or $\tau_{\mathrm{s}}=0.877 \mathrm{~ms}$. On the other hand, the energy loss per turn is $U(\mathrm{GeV})=$ $7.1685 \times 10^{-4} \mathrm{GeV}$ or $0.023895 \%$ of the particles energy. At this rate the particle radiates all its energy away in 4,185 turns. To orbit one turn it takes the particle $0.2096 \mu$ s or to radiate away all its energy $0.877 \mathrm{~ms}$. In other words, the synchrotron 
damping time is just as long as it takes the particle to radiate away all its energy at the initial energy rate.

\section{Solutions for Chap. 12}

12.1 We use the coordinates $\left(\psi, \Delta c p / c p_{0}\right)$ for which the changes per unit time are $\dot{\psi}=-h \omega_{0} \eta_{\mathrm{c}}\left(\frac{\Delta c p}{c p_{0}}\right)$ ( $h$ harmonic number, $\omega_{0}$ revolution frequency) and $\frac{\mathrm{d}}{\mathrm{d} t}\left(\frac{\Delta c p}{c p_{0}}\right)=\frac{e V_{\mathrm{rf}}(\psi)-U(E)}{c p_{0} T_{0}}$ with $V_{\mathrm{rf}}(\psi)$ the rf-voltage at the phase $\psi$ and $U(E)$ the energy loss per turn at energy $E$. Expanding we get $e V_{\mathrm{rf}}(\psi)-U(E) \approx$ $\left.e \frac{\mathrm{d} V_{\mathrm{rf}}}{\mathrm{d} \psi}\right|_{\psi_{\mathrm{s}}} \varphi-4 U\left(E_{0}\right) \beta^{2} \frac{\mathrm{d} c p}{c p_{0}}$ where $\varphi$ is the phase deviation from the synchronous phase $\psi_{\mathrm{s}}$. The last term gives rise to damping which we ignore here and $\frac{\mathrm{d}}{\mathrm{d} t}\left(\frac{\Delta c p}{c p_{0}}\right)=\left.\frac{1}{c p_{0} T_{0}} e \frac{\mathrm{d} V_{\mathrm{rf}}}{\mathrm{d} \psi}\right|_{\psi_{\mathrm{s}}} \varphi$. Following the discussion leading to the Vlasov equation we scale the coordinates $\varphi \longrightarrow \sqrt{\frac{c p_{0} T_{0}}{e \mathrm{~d} V_{\mathrm{rf}} /\left.\mathrm{d} \psi\right|_{\psi_{\mathrm{s}}}}} \varphi$ and $\frac{\Delta c p}{c p_{0}} \longrightarrow \sqrt{\frac{1}{-h \omega_{0} \eta_{\mathrm{c}}}} \frac{\Delta c p}{c p_{0}}$ to get $\sqrt{\frac{c p_{0} T_{0}}{e \mathrm{~d} V_{\mathrm{rf}} /\left.\mathrm{d} \psi\right|_{\psi_{\mathrm{s}}}}} \dot{\varphi}=-h \omega_{0} \eta_{\mathrm{c}} \sqrt{\frac{1}{-h \omega_{0} \eta_{\mathrm{c}}}} \frac{\Delta c p}{c p_{0}}$ or $\dot{\varphi}=\omega_{0} \sqrt{-\left.\frac{h \eta_{\mathrm{c}}}{2 \pi c p_{0}} e \frac{\mathrm{d} V_{\mathrm{rf}}}{\mathrm{d} \psi}\right|_{\psi_{\mathrm{s}}} \frac{\Delta c p}{c p_{0}}}$ and $\frac{\mathrm{d}}{\mathrm{d} t}\left(\frac{\Delta c p}{c p_{0}}\right)=\omega_{0} \sqrt{-\left.\frac{h \eta_{\mathrm{c}}}{2 \pi c p_{0}} e \frac{\mathrm{d} V_{\mathrm{rf}}}{\mathrm{d} \psi}\right|_{\psi_{\mathrm{s}}}} \varphi$. The synchrotron oscillation frequency is then just the coefficient to the coordinates $\Omega_{\mathrm{s}}=\omega_{0} \sqrt{-\left.\frac{h \eta_{\mathrm{c}}}{2 \pi c p_{0}} e \frac{\mathrm{d} V_{\mathrm{rf}}}{\mathrm{d} \psi}\right|_{\psi_{\mathrm{s}}}}$. To make the synchrotron oscillation frequency zero we would need a second rf-system adjusted such that $\left.\frac{\mathrm{d} V_{\mathrm{rf}}}{\mathrm{d} \psi}\right|_{\psi_{\mathrm{s}}}=0$. For simplicity of discussion we assume sinusoidal rf-voltages and an additional rf-system at twice the frequency of the first. For maximum efficiency, we phase the second rf-system such that $\psi_{2}=0$ at the synchronous phase of the first system. The conditions are then $V_{\mathrm{rf}} \sin \psi_{\mathrm{s}}=V_{1} \sin \psi_{\mathrm{s}}$ and $V_{\mathrm{rf}} \cos \psi_{\mathrm{s}}=V_{1} \cos \psi_{\mathrm{s}}+V_{2}=0$ from which we can isolate the voltages to be $V_{1}=V_{\mathrm{rf}}$ and $V_{2}=V_{\mathrm{rf}} \cos \psi_{\mathrm{s}}$.

12.2 The equilibrium bunch length is given by (11.48) and is with $\left.\frac{\mathrm{d} V_{\mathrm{rf}}}{\mathrm{d} \psi}\right|_{\psi_{\mathrm{s}}}=$ $\hat{V} \cos \psi_{\mathrm{s}}$ for this problem $\sigma_{\ell}=\frac{\sqrt{2 \pi} c}{\omega_{0}} \sqrt{\frac{\eta_{\mathrm{c}} E_{0}}{h e \mathrm{~d} V_{\mathrm{rf}} /\left.\mathrm{d} \psi\right|_{\psi_{\mathrm{s}}}}} \frac{\sigma_{E}}{E_{0}}$. The bunch length can be manipulated by adjusting the relative phase and voltages of both rf-systems. In general, for a two frequency rf-system the combined voltage is $V_{\mathrm{rf}}=V_{1} \sin \omega_{1} t+$ $V_{2} \sin \left(\omega_{2} t+\delta\right)$, where $\delta$ is the phase shift between both systems and $\left.\frac{\mathrm{d} V_{\mathrm{rf}}}{\mathrm{d} \psi}\right|_{\psi_{\mathrm{s}}}=$ $V_{1} \cos \omega_{1} t+V_{2} \cos \left(\omega_{2} t+\delta\right)$ with the synchronous time $t_{\mathrm{s}}=\psi_{\mathrm{s}} / \omega_{1}$.

12.3 An external field acts the same on all particles in a beam. If the beam as a whole performs coherent transverse or longitudinal oscillations, we may consider the situation being just one macroparticle. One could consider an external field which does depend on the amplitude of the macroparticle and this field would then damp the coherent oscillations because $\partial f / \partial w \neq 0$. This is the case for a feedback 
system to damp coherent oscillations within a beam. The beam position is measured at one point and the signal acts back on the beam after some amplification. If the feedback system is fast enough it works for slices of a bunch where the slice charge is offset from the equilibrium. This is exploited in stochastic cooling to reduce the beam emittance. However, damping of incoherent oscillations, as we get from the emission of synchrotron radiation, is not possible by external fields. Here each individual particle has a different coordinate but the external field is the same for all particles.

12.4 We use (12.106) and note that for a stationary solution $\alpha_{n}=0$ for all $n$. The remaining coefficients depend only on $\alpha_{w} / D$ or parameters that are determined by the storage ring containing the beam. This makes the Gaussian distribution of the injected beam irrelevant.

12.5 The beam lifetime without quantum effects is $21.4 \mathrm{~h}$ and we allow this lifetime to be reduced by $10 \%$. The quantum lifetime for all three degrees of freedom therefore should be longer than $\tau_{\mathrm{q}}=577.8 \mathrm{~h}$. Quantum effect lifetime is given by (12.132) where $2 x$ is the aperture in Gaussian standard units. The vertical damping time is $\frac{1}{\tau_{y}}=\frac{\left\langle P_{\gamma}\right\rangle}{2 E}$. The synchrotron radiation power is $\left\langle P_{\gamma}\right\rangle=1,155 \mathrm{GeV} / \mathrm{s}$ and the vertical damping time $\tau_{y}=2.60 \mathrm{~ms}$. The aperture factor is then $\frac{\mathrm{e}^{x}}{x}=2 \frac{\tau_{\mathrm{q}}}{\tau_{y}}=$ $4.4 \times 10^{5}$ which can be solved for $x \approx 15.75$ or for $\frac{A}{\sigma}=5.6$. The vertical aperture should therefore be at least 5-6 times the Gaussian standard height. The same is true for the other two degrees of freedom. The damping times may be slightly different which makes little difference on the number of "sigma's" because of the fast variation of the exponential function. To cover all three degrees of freedom it is common to use 7-10 Gaussian standard units.

\section{Solutions for Chap. 13}

13.1 The damping characteristics are determined by the partition numbers and the $\vartheta$ parameter. Horizontal motion becomes antidamped when $\vartheta=\frac{\oint \kappa^{2} \eta\left(1+2 \rho^{2} k\right) \mathrm{d} z}{\oint \kappa^{2} \mathrm{~d} z} \geq 1$. We evaluate this integral in thin lens approximation for only one half cell, since all others are the same and assume that $k_{\mathrm{QF}}=-k_{\mathrm{QD}}=k$ and $\ell_{\mathrm{QF}}=\ell_{\mathrm{QD}}=\ell$. Then $\vartheta=\kappa(\hat{\eta}+\check{\eta})+2 \rho(\hat{\eta}-\check{\eta}) k$. Furthermore $\hat{\eta}+\check{\eta}=\kappa L \frac{2}{k \ell}$ and $(\hat{\eta}-\check{\eta}) k=\kappa \frac{L}{\ell}$. With this the $\vartheta$-parameter is finally $\vartheta=2 \frac{L}{\ell}\left(\frac{\kappa^{2}}{k}+1\right)$. It follows from $\ell \ll L$ that $\vartheta>1$ and that the horizontal betatron function is antidamped. It is only because of adiabatic damping during acceleration that the beam does not blow up. If the acceleration is too slow the beam emittance will grow. 


\section{Solutions for Chap. 14}

12.2 For $\left\langle\frac{\mathcal{H}}{\left|\rho^{3}\right|}\right\rangle=\frac{1}{C} \int_{0}^{C} \frac{\mathcal{H}(z)}{\left|\rho^{3}\right|} \mathrm{d} z$ where $\langle\mathcal{H}(z)\rangle=\left\langle\beta \eta^{\prime 2}\right\rangle+\left\langle 2 \alpha \eta \eta^{\prime}\right\rangle+\left\langle\gamma \eta^{2}\right\rangle$ we need to formulate an analytical expression. Since all bending magnets contribute similarly, only one has to be evaluated. We estimate the average values from known solutions in the middle of FODO-cell quadrupoles (10.3), (10.74), and (10.5). Transforming through the $\mathrm{QF} \eta_{\mathrm{QF}}^{\prime}=-\hat{\eta} / f, \eta_{\mathrm{QD}}^{\prime}=-\check{\eta} / f$ and $\left\langle\beta \eta^{\prime 2}\right\rangle \approx \frac{1}{2}\left(\hat{\beta} \eta_{\mathrm{QF}}^{\prime 2}+\check{\beta} \eta_{\mathrm{QD}}^{\prime 2}\right)=$ $\frac{L^{3}}{\rho^{2}} \frac{4 \kappa^{2}+5}{4 \sqrt{\kappa^{2}-1}}$. For $\left\langle 2 \alpha \eta \eta^{\prime}\right\rangle$ we approximate $\langle\eta\rangle \approx \frac{\hat{\eta}+\check{\eta}}{2}=\frac{L^{2} \kappa^{2}}{\rho},\left\langle\eta^{\prime}\right\rangle \approx \frac{\hat{\eta}-\check{\eta}}{L}=\frac{L \kappa}{\rho}$ and with $\alpha=-\frac{1}{2} \beta^{\prime} \approx-\frac{1}{2} \frac{\hat{\beta}-\check{\beta}}{L}$ we get for the second term $\left\langle 2 \alpha \eta \eta^{\prime}\right\rangle=-\frac{L^{3}}{\rho^{2}} \frac{\kappa^{4}\left(4 \kappa^{2}+5\right)}{2 \sqrt{\kappa^{2}-1}}$. Finally, we note that $\gamma(z)$ is constant in a drift space (bending magnet) and is from matrix transformation at the exit of $\mathrm{QF} \gamma_{\mathrm{QF}}=\frac{1}{\kappa^{2} L^{2}} \hat{\beta}=$ const. With this $\left\langle\gamma \eta^{2}\right\rangle \approx$ $\left(\frac{1}{\kappa^{2} L^{2}} \hat{\beta}+\frac{1}{\bar{\beta}}\right) \frac{\hat{\eta}^{2}+\check{\eta}^{2}}{2}=\frac{L^{3}}{\rho^{2}} \frac{\kappa^{2}\left(4 \kappa^{2}+5\right)}{2 \sqrt{\kappa^{2}-1}}$. Collecting all terms $\langle\mathcal{H}(z)\rangle \approx \frac{L^{3}}{\rho^{2}} \frac{\kappa^{2}\left(4 \kappa^{2}-3\right)}{4 \sqrt{\kappa^{2}-1}}$ and the minimum is reached from $\partial \mathcal{H} / \partial \kappa=0$ for $\kappa \approx 1.071$. The associated FODO phase advance per cell is $\psi \approx 138^{\circ}$.

12.6 We use an energy of $E=2 \mathrm{GeV}$ and an optimized $90^{\circ}$ lattice for which from Fig. $14.5\langle\mathcal{H}\rangle /\left(\rho \Theta^{3}\right) \approx 3$. We also assume that only $75 \%$ of the ring is occupied by bending magnets and therefore $\ell_{\mathrm{b}} / \ell_{\mathrm{b}, 0}=0.75$. Solving $(14.25)$ for the minimum bending angle $\Theta$ per bending magnet we get $\Theta=\frac{\epsilon_{x}}{C_{\mathrm{q}} \gamma^{2}} \frac{1}{\langle\mathcal{H}\rangle /\left(\rho \Theta^{3}\right)} \frac{\ell_{\mathrm{b}}}{\ell_{\mathrm{b}, 0}} \frac{180}{\pi}=3.845^{\circ}$. To compose a ring, we need at least 94 bending magnets or quadrupoles to reach a minimum beam emittance of $\epsilon_{x}=5 \times 10^{-9} \mathrm{~m}$.

\section{Solutions for Chap. 15}

15.1 We use the perturbation $P_{22}(z)=\frac{1}{2} v_{0}^{2} \beta^{5 / 2} m w_{\beta}^{2}$ in the equation of motion $\ddot{w}_{\beta}+v_{0}^{2} w_{\beta}=\frac{1}{2} v_{0}^{2} \beta^{5 / 2} m w_{\beta}^{2}$ and get after reordering the equation $\ddot{w}_{\beta}+v_{0}^{2}\left(1-\frac{1}{2} \beta^{5 / 2} m w_{\beta}\right) w_{\beta}=0$. On average, the sextupoles do not contribute in linear approximation to a tune shift because $\left\langle\frac{1}{2} \beta^{5 / 2} m w_{\beta}\right\rangle=0$. In higher order, however there is some tune shift which we can expect from the fact that the contribution to the tune shift is greater while $w_{\beta}>0$ compared to the case when $w_{\beta}<0$. The $P_{21}(z)$-term contributes to a tune shift because a beam passing on a distorted orbit $w_{\mathrm{c}}$ through a sextupole feels a quadrupole field component and therefore a tune shift. The equation of motion shows this directly $\ddot{w}_{\beta}+v_{0}^{2}\left(1-\frac{1}{2} \beta^{5 / 2} m w_{\mathrm{c}}\right) w_{\beta}=0$ and the tune shift is $\delta v \approx \frac{1}{4} v_{0}\left(\beta^{5 / 2} m w_{\mathrm{c}}\right)$.

15.2 At the bending magnet exit the dispersion is $D=\rho\left(1-\cos \frac{\ell_{\mathrm{b}}}{\rho}\right)$ and the slope $D^{\prime}=\sin \frac{\ell_{\mathrm{b}}}{\rho}$. Extrapolating linearly back, we expect the dispersion to start with a slope of $D^{\prime}$ at a distance $s$ before the bending magnet exit. Therefore, $s \sin \frac{\ell_{\mathrm{b}}}{\rho}=$ 
$\rho\left(1-\cos \frac{\ell_{\mathrm{b}}}{\rho}\right)$ and using $\frac{\ell_{\mathrm{b}}}{\rho} \ll 1$ we get $s \approx \frac{1}{2} \ell_{\mathrm{b}}$ demonstrating that the dispersion function seems to start in the middle of the bending magnet.

15.3 With errors $\Delta \frac{1}{\rho} \ell=\theta$ and $A=\frac{\sqrt{\beta(z)}}{2 \sin \pi \nu}$ the vertical $\eta$-function is $\eta(z)=$ $A \oint \Delta \frac{1}{\rho} \sqrt{\beta(\bar{z})} \cos v[\varphi(z)-\varphi(\bar{z})+\pi] \mathrm{d} \bar{z}=A \sum_{i} \sqrt{\beta_{i}} \theta_{i} \cos v\left[\varphi(z)-\varphi_{i}+\pi\right]$ where we have omitted the index $y$. The expectation value for the dispersion function is for $N$ statistically distributed errors $\left\langle\eta^{2}\right\rangle=A^{2} \sum_{i} \beta_{i} \theta_{i}^{2} \cos ^{2} \nu \pi=$ $\frac{1}{4} \beta(z) N \bar{\beta} \sigma_{\theta}^{2} \cot \nu \pi$ where $\sigma_{\theta}=\sqrt{\left\langle\theta^{2}\right\rangle}$ is the rms deflection angle due to misalignment errors and $\bar{\beta}$ the average value of the betatron function at the location of errors. For random quadrupole misalignment errors $\sigma_{x}$ and $\ell$ the length of the quadrupole $\sigma_{\theta}=k \ell \sigma_{x}$ and for bending magnet rotational errors $\sigma_{\alpha}$ the rms errors are $\sigma_{\theta}=\frac{\ell}{\rho} \sigma_{\alpha}$. Use numerical values from lattice \#3 in Table 10.1 to estimate the actual expectation value of the vertical dispersion function.

15.4 The transformation $\mathcal{M}$ through both bending magnets and the drift space $L$ between them is with $D=\rho(1-\cos \theta)$

$$
\begin{aligned}
\mathcal{M} & =\left(\begin{array}{ccc}
1 & L+2 \ell-(L+\ell) \sin \theta \\
0 & 1 & \sin \theta \\
0 & 0 & 1
\end{array}\right) \\
& =\left(\begin{array}{ccc}
1 & \ell & D \\
0 & 1 & \sin \theta \\
0 & 0 & 1
\end{array}\right)\left(\begin{array}{lll}
1 & L & 0 \\
0 & 1 & 0 \\
0 & 0 & 1
\end{array}\right)\left(\begin{array}{ccc}
1 & \ell & -D \\
0 & 1 & -\sin \theta \\
0 & 0 & 1
\end{array}\right) .
\end{aligned}
$$

From this, we get the dispersion at the end of the second bending magnet $D=$ $-(L+\ell) \sin \theta=-d$ and $D^{\prime}=0$.

15.5 The tune change due to quadrupole field errors is $\Delta v=\frac{1}{4 \pi} \sum_{i} \beta_{i}\left(\Delta k_{i} \ell\right)$ and with $\sigma_{k}=\sqrt{\left\langle\left(\Delta k_{i} \ell\right)^{2}\right\rangle}$ the expectation value is $\sigma_{v}=\frac{1}{4 \pi} N_{\mathrm{q}} \bar{\beta} \sigma_{k}$. To have a $96 \%$ probability to avoid an integer or half integer resonance $2 \sigma_{v}$ should be less than 0.25 or $\sigma_{v, \mathrm{q}}<0.125$. With numerical values $N_{\mathrm{q}}$ and $\bar{\beta}$ one can solve for the rms quadrupole field tolerance $\sigma_{k}$. Manufacturing tolerances resulting in nonparallelism of bending magnet poles cause gradient field errors as well. If the angle between pole surfaces is $\alpha$ then the field will be $B(x) \approx \frac{B_{0} G_{0}}{G_{0}+\alpha x} \approx B_{0} G_{0}\left(1+\frac{\alpha}{G_{0}} x\right)$. From this, we extract the gradient field error $\delta k=-\frac{1}{\rho} \frac{\alpha}{G_{0}}$ and get the rms tune shift $\sigma_{v, \mathrm{~b}}=\frac{1}{4 \pi} N_{\mathrm{b}} \bar{\beta}_{\mathrm{b}} \frac{\ell}{\rho G_{0}} \sigma_{\alpha}$, where $G_{0}$ is the nominal gap between magnet poles, $\ell / \rho$ is the bending angle, $N_{\mathrm{b}}$ the number of magnets and $\bar{\beta}_{\mathrm{b}}$ the average value of the betatron function in the bending magnets. The total allowable tune shift is then $\sigma_{v}=\sqrt{\sigma_{v, \mathrm{q}}^{2}+\sigma_{v, \mathrm{~b}}^{2}}<0.125$ determining the gradient field and parallelism tolerances. 


\section{Solutions for Chap. 16}

16.1 We use an energy $E$, a FODO half cell length $L$ and a bending fill factor of $50 \%$ or $\ell_{\mathrm{b}}=L / 2$. We assume that there are $N=21$ FODO cells. The natural chromaticities are in both planes from (15.105) $\Delta \xi_{x, y}=-\frac{1}{2 \pi}$ per FODO half-cell and the sextupole strengths are $m_{\mathrm{SF}} \ell_{\mathrm{S}}=\frac{\Delta \xi}{\hat{\eta}(\hat{\beta}-\check{\beta})}$ and $m_{\mathrm{SD}} \ell_{\mathrm{S}}=\frac{\Delta \xi}{\check{\eta}(\hat{\beta}-\check{\beta})}$. The phase differences between similar sextupoles (SFs or SDs) are $\Delta \varphi=$ $2 \pi / N$. The stop-band width $\Delta v_{\text {stop }}^{(3)}=-\frac{v_{x, 0}}{4 \pi} \frac{x_{0}}{\sqrt{\beta_{0}}}\left|\int_{0}^{2 \pi} \beta_{x}^{5 / 2} m \mathrm{e}^{\mathrm{i} 3 v_{x 0} \varphi} \mathrm{d} \varphi\right|$ becomes with $v_{x, 0} \beta_{x} \mathrm{~d} \varphi=\mathrm{d} z$ and replacing the integral with sums over all SFs and SDs

$\Delta v_{\text {stop }}^{(3)}=\frac{-x_{0}}{4 \pi \sqrt{\beta_{0}}}\left|\sum_{k=0}^{N-1}\left(\left.\beta_{x}^{5 / 2} m \ell_{\mathrm{s}}\right|_{\mathrm{SF}} \mathrm{e}^{\mathrm{i} 3 v_{x 0} k \Delta \varphi}+\left.\beta_{x}^{5 / 2} m \ell_{\mathrm{s}}\right|_{\mathrm{SD}} \mathrm{e}^{\mathrm{i} 3 v_{x 0}(k+1 / 2) \Delta \varphi}\right)\right|$. The sums become $\sum_{k=0}^{N-1} e^{\mathrm{i} 3 v_{x 0} k \Delta \varphi}=\frac{\exp \left(\mathrm{i} 6 \pi v_{x 0}\right)}{\exp \left(\mathrm{i} 6 \pi v_{x 0} / N\right)-1}$ and $\exp \left(\mathrm{i} \frac{3 v_{x 0}}{2 N} \Delta \varphi\right) \sum_{k=0}^{N-1} \exp \left(\mathrm{i} 3 v_{x 0} k \Delta \varphi\right)=$ $\exp \left(\mathrm{i} \frac{3 \pi v_{x 0}}{N^{2}}\right) \frac{\exp \left(\mathrm{i} 6 \pi v_{x 0}\right)-1}{\exp \left(\mathrm{i} 6 \pi v_{x 0} / N\right)-1}$. Finally, the stop band width is $\Delta v_{\text {stop }}^{(3)}=\frac{-x_{0} \Delta \xi}{4 \pi \sqrt{\beta_{0}}} \mid\left[\frac{\hat{\beta}^{3 / 2}}{\hat{\eta}(\hat{\beta}-\check{\beta})}-\right.$ $\left.\frac{\check{\beta}^{3 / 2}}{\check{\eta}(\hat{\beta}-\check{\beta})} \exp \left(\mathrm{i} \frac{3 \pi v_{x 0}}{N^{2}}\right)\right] \frac{\exp \left(\mathrm{i} 6 \pi v_{x 0}\right)-1}{\exp \left(\mathrm{i} 6 \pi v_{x 0} / N\right)-1} \mid$. Close to the third order resonance $\left(v_{x 0} \approx 3+\delta \nu\right)$ we set $\exp \left(\mathrm{i} \frac{3 \pi v_{x 0}}{N^{2}}\right) \approx \exp \left(\mathrm{i} \frac{9 \pi}{N^{2}}\right)$ and $\frac{\exp \left(\mathrm{i} 6 \pi v_{x 0}\right)-1}{\exp \left(\mathrm{i} 6 \pi v_{x 0} / N\right)-1} \approx \frac{16 \pi \delta \nu}{\exp (\mathrm{i} 18 \pi / N)-1}$ and get $\Delta v_{\text {stop }}^{(3)} \propto x_{0} \delta v$. For finite betatron amplitudes $x_{0}$ the stop band width scales like the tune distance $\delta v$ from the third order resonance.

16.2 In the definition (16.33) we notice that $|m| \leq n$ and therefore there are odd values for $m$ if $m$ is even and vice versa. That means for $n$ even there can be a value $m=0$ while for an odd $n$ we have $m \neq 0$. Only terms $c_{n m}=c_{n 0} \neq 0$ give rise to a tune shift in this approximation which is the case for even values of $n$ only.

16.3 We write (16.33) like $\cos ^{n} \psi=c_{n 0}+c_{n 1} \mathrm{e}^{\mathrm{i} \psi}+c_{n 2} \mathrm{e}^{\mathrm{i} 2 \psi}+\ldots+c_{n n} \mathrm{e}^{\mathrm{i} n \psi}$. on the other hand $\cos ^{n} \psi=\frac{1}{2^{n}}\left(\mathrm{e}^{\mathrm{i} \psi}+\mathrm{e}^{-\mathrm{i} \psi}\right)^{n}=\frac{1}{2^{n}}\left(\mathrm{e}^{\mathrm{i} n \psi}+a \mathrm{e}^{\mathrm{i}(n-1) \psi}+\ldots\right)$. In both equations there is only one term $\mathrm{e}^{\mathrm{i} n \psi}$. Therefore $c_{n n} \mathrm{e}^{\mathrm{i} n \psi}=\frac{1}{2^{n}} \mathrm{e}^{\mathrm{i} n \psi}$ and $c_{n n}=\frac{1}{2^{n}}$.

\section{Solutions for Chap. 17}

17.1 First, we find from (6.95) all second order terms in $\delta$. The term $-\kappa \delta^{2}$ together with $C(\zeta)=\cos (\zeta / \rho)$ and $S(\zeta)=\rho \cos (\zeta / \rho)$ the perturbation $P(z, \delta)=$ $-\delta^{2} \int \kappa_{x}\left|S(z) C^{\prime}(\zeta)-C(z) S^{\prime}(\zeta)\right|=-\delta^{2} \rho(1-\cos z / \rho)$. Since no slopes are involved, $T_{166}=c_{166}=-\rho(1-\cos z / \rho)$. We could have guessed this result, since the perturbation term is the same as for the dispersion except for a factor $-\delta$. This second order term is therefore just the second order chromatic perturbation. 
17.2 From (6.95) we collect quadratic terms and with $x=C x_{0}$ and $x^{\prime}=C^{\prime} x_{0}$ we get the desired perturbation $p\left(\zeta \mid x_{0}^{2}\right)=\left[\left(-\frac{m}{2}-\kappa_{x}^{3}-2 \kappa_{x} k\right) C^{2}+\frac{\kappa_{x}}{2} C^{\prime 2}\right] x_{0}^{2}$. Note, that we ignore the $\kappa^{\prime}$-term since we exclude non-constant strength parameters. With this perturbation, the integral (5.75)

$$
\begin{aligned}
T_{111} & =\int_{0}^{z} p\left(\zeta \mid x_{0}^{2}\right)\left[S(z) C^{\prime}(\zeta)-C(z) S^{\prime}(\zeta)\right] d \zeta \\
& =\left(-\frac{1}{2} m-\kappa_{x}^{3}-2 \kappa_{x} k\right) \frac{1}{3 k}\left[k S^{2}+(1-C)\right]+\frac{5}{6}\left[2(1-C)-k S^{2}\right]
\end{aligned}
$$

where $C, S$ are the principal solutions of linear beam dynamics.

17.3 We consider a ring made of a total of $N_{c}=61$ FODO-cells, each with a phase advance of $90^{\circ}$ and the tune is then $v=15.25$. This ring has 61 QFs and 61 QDs. The betatron functions in the middle of the quadrupoles are $\beta=L(2 \pm \sqrt{2})$ and the $\eta$-function $\eta=\frac{L^{2}}{2 \rho}(4 \pm \sqrt{2})$. The natural chromaticity $\xi_{0, x, y}=-\frac{N_{\mathrm{c}}}{\pi}$ and the change in chromaticity due to sextupoles

$$
\begin{aligned}
\Delta \xi_{x, y} & =\frac{N_{\mathrm{c}}}{4 \pi}\left[\left(\beta \eta m \ell_{\mathrm{s}}\right)_{\mathrm{QF}} \pm\left(\beta \eta m \ell_{\mathrm{s}}\right)_{\mathrm{QD}}\right] \\
& =\frac{N_{\mathrm{c}}}{4 \pi} \frac{L^{3}}{2 \rho}\left[ \pm(10+6 \sqrt{2}) m_{\mathrm{QF}} \ell_{\mathrm{s}} \pm(10-6 \sqrt{2}) m_{\mathrm{QD}} \ell_{\mathrm{s}}\right]=\mp \xi_{x, y} .
\end{aligned}
$$

After evaluation $\Delta \xi_{x}=18.485 m_{\mathrm{QF}} \ell_{\mathrm{s}}+1.515 m_{\mathrm{QD}} \ell_{\mathrm{s}}=\frac{8 \rho}{L^{3}}$ and $\Delta \xi_{y}=$ $-1.515 m_{\mathrm{QF}} \ell_{\mathrm{s}}-18.4851 m_{\mathrm{QD}} \ell_{\mathrm{s}}=\frac{8 \rho}{L^{3}}$, which can be solved for the two sextupole families $m_{\mathrm{QF}} \ell_{\mathrm{s}}=-m_{\mathrm{QD}} \ell_{\mathrm{s}}=\frac{2 \rho}{3 \sqrt{2} L^{3}}$. We use this result for (17.45) and get $\sum_{i} m_{i} \ell_{i} \beta^{3 / 2} \exp \left(\mathrm{i} \psi_{x i}\right)=\left|m \ell_{s} \beta^{3 / 2}\right|_{\mathrm{QF}}-\left|m \ell_{s} \beta^{3 / 2}\right|_{\mathrm{QD}} \exp (\mathrm{i} \pi / 4) \neq 0$ because of cancellations within every four cells. Only the contribution of the last cell is uncompensated. If we place non-interleaved sextupoles in pairs $180^{\circ}$ apart there is total cancellation and the driving term is zero.

17.4 The tunes of the ring are in both planes $v=15.25$. Equations (17.45)-(17.49) exhibit mainly integer and third order resonances. A proper choice on the tunes can minimize these aberration terms. A tune of 15.25 is close to a multiple of 3 and therefore efficient at driving a third order resonance. A better tune would be say 16.25.16.75 or 17.25. One could argue that 16.75 or 17.25 might give the lowest driving terms, although this ignores the variation of sextupole strengths and betatron functions. To find the minimum driving terms (17.45)-(17.49) must be evaluated. 


\section{Solutions for Chap. 18}

18.1 The longest wavelength belong to the lowest TM and TE-mode. The lowest TM-mode is $\mathrm{TM}_{01}$. We use (18.44) with $B_{z}=0$ and note that $E_{r}$ meets the boundary condition by definition, $E_{\varphi}=0$ at the walls because $m=0$ and $E_{z}=0$ at the wall for $J_{0}\left(k_{\mathrm{c}} R\right)=0$ or $\lambda_{\mathrm{c}}=\frac{2 \pi R}{2.405}$. The longest TM-mode wavelength that could propagate in this tube is about $30 \%$ bigger than the diameter of the tube. The TEmode fields are given by (18.44) with $E_{z}=0$. The magnetic field $B_{z} \propto J_{m}\left(k_{\mathrm{c}} R\right)$ just like $E_{z}$. The radial electric field end normally at the wall, $E_{\varphi}=0$ for $J_{0}^{\prime}\left(k_{\mathrm{c}} R\right)=$ 0 or $k_{\mathrm{c}} R=1.84$. Solving for the cut-off wavelength $\lambda_{\mathrm{c}}=\frac{2 \pi R}{1.84}$ which is about $30 \%$ smaller than the tube diameter. Therefore, we may conclude that wavelength longer than the tube diameter do not propagate. One big consequence of this is that electron bunches with a bunch length of $1-2 \mathrm{~cm}$ or longer do not emit coherent synchrotron radiation because the wavelength would be of the order of the bunch length or vacuum chamber dimensions or longer.

18.2 A change of temperature would change all linear dimensions of a cavity as determined by the temperature coefficient of the cavity material, in this case copper. The fundamental cavity frequency is inversely proportional to the radial dimension $a_{1}$ of the cavity (18.48). From $\frac{\Delta a_{1}}{a_{1}}=-\frac{\Delta f_{\mathrm{rf}}}{f_{\mathrm{rf}}}=\eta_{\mathrm{T}} \Delta T$ and $\Delta f_{\mathrm{rf}}=-16.6 \cdot f_{\mathrm{rf}} \Delta T\left({ }^{\circ} \mathrm{C}\right)$. Assuming that the cavity is dimensioned for $500 \mathrm{MHz}\left|\frac{\Delta f_{\mathrm{rf}}}{f_{\mathrm{rf}}}\right|= \pm 10^{-6}=\mp 16.6 \times$ $10^{-6} \cdot \Delta T$ and the temperature tolerance is $\pm 0.06^{\circ} \mathrm{C}$.

18.3 The cavity length scales like $d \propto \frac{1}{f_{\mathrm{rf}}}$, the diameter like $a_{1} \propto \frac{1}{f_{\mathrm{rf}}}$, for a pill box cavity the transit time factor is independent of the frequency, the quality factor $Q \propto f_{\text {rf }}^{0}$, the shunt impedance $R_{\mathrm{s}} \propto \frac{1}{\sqrt{f_{\mathrm{rf}}}}$, the specific shunt impedance $r_{\mathrm{s}} \propto \sqrt{f_{\mathrm{rf}}}$ and cavity fill time $t_{\mathrm{F}} \propto \frac{1}{f_{\mathrm{rf}}}$.

18.4 Such high fields can be sustained only in pulsed linac systems. The wall losses per meter of accelerating structure are from (18.90) $P_{\mathrm{W}}=5.6 \mathrm{MW} / \mathrm{m}$. This is too large for financial and technical reasons because it would not be possible to cool that much power from one meter of structure. An electron linac is pulsed with a pulse length of say $2.5 \mu \mathrm{s}$ and a rep rate of say $100 \mathrm{~s}^{-1}$. The duty cycle is the $250 \mu \mathrm{s}$ per second or $2.510^{-4}$. The average wall losses are therefore $1.4 \mathrm{~kW}$ which is manageable.

18.5 The capture dynamics in linear accelerators depends on the particle velocity and initial phase at the time it enters the accelerating section. Particles can be accelerated even if the initial field is negative for example in the area $90^{\circ}$ to $180^{\circ} /-180^{\circ}$ to $-90^{\circ}$. Between 90 and $180^{\circ}$ initially low energy particles travel slower that the rf-wave and quickly fall back into the accelerating phase before they loos all energy. In the accelerating phase they get accelerated very fast and reach high energies. Higher energy particles do not reach equally high final energies because they are faster and will not fall back from the negative acceleration so fast and lose much of their initial energy before they get accelerated again in the 
accelerating phase. Particles starting at $<-90^{\circ}$ will stay longer in the decelerating phase and loose all their energy. After that they get accelerated/decelerated at random. The optimum phase should be $\psi_{0}=0^{\circ}$ and particles with higher initial relativistic energies gain the highest energy and stay at this phase. Lower energy particles at $1-2 \mathrm{MeV}$ are not relativistic yet and therefore fall back from the crest of the wave gaining less energy. This is the regime of a buncher section where the phase velocity of the rf-wave is reduced to match that of the particles.

18.6 We excite the prebuncher to a total effective voltage of $\hat{V}_{\text {rf }}$ and the linear part scales with $3 \mathrm{GHz}$ phase like $V_{\mathrm{rf}}=\hat{V}_{\mathrm{rf}} \varphi_{500}$. For simplicity, we assume the electrons to be nonrelativistic and the velocity deviation $\delta v$ from the $E_{0}=100 \mathrm{keV}$ reference particle $\sqrt{\frac{m}{2 E_{0}}} \delta v=\sqrt{\frac{E_{\text {kin }}}{E_{0}}}-1=\sqrt{1+\frac{e \hat{e}_{\mathrm{r}} \varphi_{500}}{E_{0}}}-1 \approx \frac{e \hat{V}_{\mathrm{rf}}}{2 E_{0}} \varphi_{500}$. A distance $\ell$ downstream from the prebuncher the reference particle arrives at a time $\tau=\ell / v_{0}=$ $\ell / \sqrt{2 E / m}$. During this same time a particle at phase $\varphi_{500}$ must advance or fall back with respect to the reference particle by the distance $\frac{\lambda_{\text {rf }}}{2 \pi} \varphi_{500}$ to arrive at the same time as the reference particle. Therefore, we require $\tau \delta v=\frac{\lambda_{\mathrm{rf}}}{2 \pi} \varphi_{500}$ and the shortest bunch length is obtained at a distance $\ell=\frac{\lambda_{\mathrm{rf}}}{2 \pi} \frac{2 E_{0}}{e \hat{V}_{\mathrm{ff}}}$. In this ideal (linear) case the resulting bunch length is zero independent of the original bunch length. In reality, the sinusoidal variation of the prebuncher voltage results in a finite S-like bunch distribution in phase space. We may over-compress the bunch by going slightly beyond the distance $\ell$ thus getting a slightly higher intensity within a finite phase distance of, for example, $\pm 12^{\circ}$ at $3 \mathrm{GHz}$.

18.7 The no-load energy gain is $E_{\text {kin }}(\mathrm{MeV})=10.48 \sqrt{P_{0}(\mathrm{MW})}=40.6 \mathrm{MeV}$ and with beam loading this energy gain is reduced by $\Delta E=-37.47 i_{\mathrm{b}}=-0.75 \mathrm{MeV}$. The fill time of one linac section is $t_{\mathrm{F}}=0.73 \mu \mathrm{s}$ and the rf-pulse remaining for beam acceleration is $1.77 \mu \mathrm{s}$. The beam pulse is therefore $\tau=1.77 \mu \mathrm{s}$ long with an energy gain of $E_{\text {load }}=39.85 \mathrm{MeV}$. The total beam energy is then $e_{\mathrm{b}}=$ $\left(E_{\text {load }} / e\right) i_{\mathrm{b}} \tau=2.0 \mathrm{~J}$. On the other hand the total rf-pulse energy is $e_{\mathrm{rf}}=37.5 \mathrm{~J}$ and the linac efficiency is then $\eta=5.3 \%$. If only one pulse is accelerated as is the case for a linear collider or a x-ray laser the total beam energy is $e_{\mathrm{b}}=n_{\mathrm{b}} E_{\mathrm{kin}}=0.065 \mathrm{~J}$ and the efficiency is $\eta=0.17 \%$.

\section{Solutions for Chap. 19}

19.1 The synchrotron radiation energy loss per turn is $U=93.0 \mathrm{keV}$, the beam current is $I=68.0 \mathrm{~mA}$ and the radiation power $P_{\text {syn }}=6.32 \mathrm{~kW}$. The energy spread is $\frac{\sigma_{E}}{E}=0.0727 \%$ and the required acceptance $6 \frac{\sigma_{E}}{E}=0.436 \%$ for a beam lifetime of at least one hour. From (9.64) we solve for the function $F(q)=1.202$ and $q=1.902$. Ignoring beam loading the required minimum rf-voltage is $V_{\mathrm{rf}}=q U=$ $176.9 \mathrm{kV}$. Including the cavity power $P_{\text {cy }}=1.86 \mathrm{~kW}$, the total minimum rf-power 
needed is $P_{\text {tot }}=8.18 \mathrm{~kW}$. Beam loading will change these parameters. From (19.35) the optimum coupling $\beta_{\text {opt }}=4.40$.

19.2 We express the current in an even Fourier series $I(t)=\frac{1}{2} a_{0}+\sum_{n=1}^{\infty} a_{n} \cos (n \omega t)$ and assume only one circulating bunch of charge $Q$ between $-\frac{1}{2} \tau \leq t \leq \frac{1}{2} \tau$ where $\tau \ll T_{0}$ is the revolution time and the average current $I_{0}=Q / \tau$. The current is $I(t)=\sum_{n=-\infty}^{\infty} c_{n} \mathrm{e}^{\mathrm{i} k \omega t}=c_{0}+2 \sum_{n>0} c_{n} \cos (n \omega t)$. Here $c_{n}=\frac{1}{T_{0}} \int_{-\frac{1}{2} T_{0}}^{\frac{1}{2} T_{0}} I(t) \mathrm{e}^{-\mathrm{i} k \omega t} \mathrm{~d} t$. After integration $c_{n}=I_{0} \frac{1}{T_{0}} \frac{2}{-\mathrm{i} \omega k} \int_{0}^{-\mathrm{i} \omega k \frac{1}{2} \tau} \mathrm{e}^{x} \mathrm{~d} x=I_{0} \frac{1}{T_{0}} \tau=I_{\mathrm{b}}$. The coefficient $a_{n}=$ $2 c_{n}=2 I_{\mathrm{b}}$ for all values of $n=0,1,2,3 \ldots$

\section{Solutions for Chap. 20}

20.1 From (20.95) we calculate the coupling from statistical errors to be $\kappa_{\mathrm{rms}}=$ $\sqrt{\left\langle\kappa^{2}\right\rangle}=\frac{1}{2 \pi}\left(\delta \underline{k} \ell_{\mathrm{q}}\right) \sqrt{\overline{\beta_{x} \beta_{y}}} N_{\mathrm{q}}$ where $\delta \underline{k}$ is the strength of the error, the length of the error is $\ell$ and $N_{\mathrm{q}}=122$ the number of errors. For $90^{\circ}$ cells, the quadrupole strength has to be $k=2 /\left(\sqrt{2} L \ell_{\mathrm{q}}\right)=\sqrt{2}$ and $\delta \underline{k}=k \delta \alpha$. In the lattice we have $N_{\mathrm{q}}=122$ quadrupoles, for $90^{\circ}$ cells $\overline{\beta_{x} \beta_{y}}=2 L^{2}$ and the rms coupling is $\kappa_{\mathrm{rms}}=38.8 \delta \alpha$. In the sample lattice we have equal tunes $v_{x}=v_{y}$ and therefore the emittance coupling is always $100 \%$. To get a finite emittance coupling we must separate the tunes by fine adjustment of the quadrupoles and choose, for example, tunes like $v_{x}=15.20$ and $v_{y}=15.15$. The emittance coupling is from (20.117) $\frac{\epsilon_{y}}{\epsilon_{x}}=\frac{\kappa^{2}}{\Delta v^{2}+\kappa^{2}}=0.01$. Solving for the coupling, we get $\kappa \approx 0.005$ which determines the rotational alignment tolerance of the quadrupoles to $\delta \alpha_{\mathrm{rms}} \approx 0.13 \mathrm{mrad}$.

20.2 From the graph we obtain $v_{\mathrm{I}}-v_{x}=\left|v_{\mathrm{II}}-v_{y}\right| \approx 0.05$. With this and $\Delta_{\mathrm{r}}=0$ at resonance (20.120) can be solved for the coupling resonance to be $\kappa \approx 0.1$. The coupling coefficient is just the narrowest distance between the tune measurements.

\section{Solutions for Chap. 21}

21.1 The beam-beam tune shift is $\delta v=\frac{1}{4 \pi} \beta_{0 y}^{*} \Delta(k \ell)$. We split the ring at the collision point and insert half of the beam-beam focusing on either side of the symmetry point. With the transformation matrix of the unperturbed ring being $\mathcal{M}_{0}$ we get for the perturbed ring $\mathcal{M}=\mathcal{P} \mathcal{M}_{0} P$, where $\mathcal{P}=\left(\begin{array}{cc}1 & 0 \\ -\frac{1}{2} \Delta(k \ell) & 1\end{array}\right)$, $\mathcal{M}_{0}=\left(\begin{array}{cc}\cos 2 \pi v_{0} & \beta_{0 y}^{*} \sin 2 \pi v_{0} \\ -\frac{1}{\beta_{0 y}^{*}} \sin 2 \pi v_{0} & \cos 2 \pi v_{0}\end{array}\right)$, and $\mathcal{M}=\left(\begin{array}{cc}\cos 2 \pi \nu & \beta_{y}^{*} \sin 2 \pi v \\ -\frac{1}{\beta_{y}^{*}} \sin 2 \pi v & \cos 2 \pi v\end{array}\right)$. 
Performing the matrix multiplications, we look for the determination of the perturbed betatron function $\beta_{y}^{*}$ at the collision point only for the terms $C=$ $\cos 2 \pi v_{0}-\frac{1}{2} \Delta(k \ell) \beta_{0 y}^{*} \sin 2 \pi v_{0}$ and $S=\beta_{0 y}^{*} \sin 2 \pi v_{0}$. The perturbed betatron function is

$\beta_{y}^{*}=\left(\cos 2 \pi v_{0}-\frac{1}{2} \Delta(k \ell) \beta_{0 y}^{*} \sin 2 \pi v_{0}\right)^{2} \beta_{0 y}^{*}+\left(\beta_{0 y}^{*} \sin 2 \pi v_{0}\right)^{2} \frac{1}{\beta_{0 y}^{*}}$ or the linear change of the betatron functions is $\frac{\Delta \beta_{y}^{*}}{\beta_{0 y}^{*}} \approx-2 \pi \delta \nu \sin 2 \pi \nu_{0}$.

\section{Solutions for Chap. 22}

22.1 To calculate the bunch charge we need to calculate the revolution frequency $f_{0}=1.199 \times 10^{6} \mathrm{~s}^{-1}$. A bunch current of $I_{\mathrm{b}}=50 \mathrm{~mA}$ resembles therefore a charge of $Q=41.70 \mathrm{pC}$. The induced voltage is then for the BPM $V_{\text {ind }}=2.55 \mathrm{~V}$ and the power into the $50 \mathrm{Ohm}$ terminator is $P=\frac{V^{2}}{R}=0.130 \mathrm{~W}$. This is very small but so are the BPMs which are very well insulated and continuously heat up. For the bellows these values are $V_{\text {ind }}=2.552 \mathrm{~V}$ and the power is from (22.20) $P_{\text {ном }}=$ $0.128 \mathrm{~W}$. Again this power is small enough without special cooling. However, this small impedance could only be achieved by shielding the inside of the bellows by smooth strips of flexible metal. An unshielded bellows has a loss factor of about $k_{\|}=50 \mathrm{~V} / \mathrm{pC}$. In this case the power would be $P_{\text {ном }} \approx 105 \mathrm{~W}$ which is too much to tolerate in a bellows. With shielding the bellows looks for the beam smooth while pumping is still possible through the small gaps between the metal strips. Similar techniques are now common practice to keep the impedance small in all components of a modern storage rings.

22.2 From (22.93) we get $a-\mathrm{i} b=\frac{r+a}{2}-\frac{r-a}{2}-\mathrm{i} 2 \sqrt{\frac{r^{2}-a^{2}}{4}}=a-\mathrm{i} b$. Furthermore $\delta^{2}=a \sqrt{\frac{Z_{\mathrm{r}}^{2}+Z_{\mathrm{i}}^{2}}{n^{2}}}-a \frac{Z_{\mathrm{i}}}{n}$ and $\left(\delta^{2}+a \frac{Z_{\mathrm{i}}}{n}\right)^{2}=a^{2} \frac{Z_{\mathrm{r}}^{2}+Z_{\mathrm{i}}^{2}}{n^{2}}=\delta^{4}+2 \delta^{2} a \frac{Z_{\mathrm{i}}}{n}+\left(a \frac{Z_{\mathrm{i}}}{n}\right)^{2}$. From this $a^{2} \frac{Z_{\mathrm{r}}^{2}}{n^{2}}=\delta^{4}+2 \delta^{2} a \frac{Z_{\mathrm{i}}}{n}$ and $\frac{Z_{\mathrm{i}}}{n}=\frac{a}{2 \delta^{2}} \frac{Z_{\mathrm{r}}^{2}}{n^{2}}-\frac{\delta^{2}}{2 a}$ or $\frac{Z_{\mathrm{i}}}{n}=A \frac{Z_{\mathrm{r}}^{2}}{n^{2}}-\frac{1}{4 A}$ with $A=\frac{a}{2 \delta^{2}}$.

\section{Solutions for Chap. 23}

23.1 The geometry of the field lines in the particle system may be expressed by $x=\tan \alpha \cdot z$ were $\alpha$ is the angle between the field line and the $z$-axis. In the laboratory system the $z$-coordinates are Lorentz contracted and the equation of the field lines becomes $x^{*}=\frac{\tan \alpha}{\gamma} \cdot z^{*}$.

23.2 The Cherenkov condition is $\beta n_{\text {air }} \cos \theta=1$. For electrons $\beta(10 \mathrm{MeV})=$ 0.99869 and $\beta(50 \mathrm{MeV})=0.99995$. The Cherenkov angle for $10 \mathrm{MeV}$ electrons is imaginary $(\cos \theta=1.001)$. In order to preserve energy and momentum, the electron energy must have a minimum energy such that $n \beta>1 \$$. For $50 \mathrm{MeV}$ electrons 
this condition is met and the Cherenkov angle is $\theta_{\mathrm{Ch}}=\arccos \left(\frac{1}{0.99995 \cdot 1.000277}\right)=$ $1.214^{\circ}$.

23.3 The Cherenkov angle is $\theta_{\mathrm{Ch}}=\frac{180}{\pi} \arccos \left(\frac{1}{0.99869 \cdot 1.58}\right)=50.673^{\circ}$. This radiation will meet the other side of the plate at this angle with respect to the surface normal and will be totally reflected, because according to Snell's law $\left(\frac{\sin \theta_{1}}{\sin \theta_{2}}=\frac{n_{2}}{n_{1}}\right)$ transmission is limited to a maximum angle of $n_{2} \sin \theta_{2}$ or $\theta_{2} \leq 36.0^{\circ}$. The radiation continues to be totally reflected until it reaches the small side of the plate in which case the incident angle is now $39.327^{\circ}$ which is still larger than the angle allowed by Snell's law. This is an ideal material for a scintillator in high energy physics experiments to detect secondary particles. In this case the radiation will eventually reach the surface of a photomultiplier and escape the plastic.

23.4 The revolution frequency is $f_{\mathrm{rev}}=c / C=1 \mathrm{MHz}$ and the total number of particles orbiting is $n_{\mathrm{e}}=1.5604 \times 10^{12}$ electrons or $3.1208 \times 10^{9}$ electrons per bunch. The photon pulses reflect exactly those of the electron bunches. We assume a uniform electron distribution over the bunch length. Therefore, there is a $1 \mathrm{~cm}$ long photon pulse every $0.6 \mathrm{~m}$ or one 30 ps photon pulse every $2 \mathrm{~ns}$.

23.5 We use the uncertainty relation $\Delta x \Delta p=\Delta x \cdot \hbar k \geq \hbar$ or $\Delta x \geq 1 / k$ and the characteristic volume of a photon may be set to $V_{\mathrm{ph}} \approx(\Delta x)^{3}=\left(\frac{\lambda}{2 \pi}\right)^{3}$. The average electric field within this volume is from the photon energy $\hbar \omega=\frac{1}{2 \epsilon_{0}} E^{2} V_{\mathrm{ph}}$ or $E=k^{2} \sqrt{2 \epsilon_{0} \hbar c}$. For a $0.1238 \mathrm{eV}$ photon $\left(\mathrm{CO}_{2}\right.$ Laser) the wavelength is $10 \mu \mathrm{m}$ and the average electric field is $E=2.96 \times 10^{-7} \mathrm{~V} / \mathrm{m}$. In the case of a $10 \mathrm{keV}$ photon the field is $E=1.93 \mathrm{kV} / \mathrm{m}$.

23.6 The relativistic Doppler effect is $\omega^{*} \gamma\left(1+\beta_{z} n_{z}^{*}\right)=\omega$ and for the classical case we set $\gamma=1, n_{z}^{*}=-\cos \vartheta$ and $\beta=v / v_{0}$, where $v_{0}$ is the velocity of the wave (light or acoustic). The relative Doppler shift is then $\frac{\Delta f}{f_{0}}=\frac{v}{v_{0}} \cos \vartheta$.

23.7 The energy loss per turn is from (24.41) $U_{0}=20.32 \mathrm{keV}$ and the total radiation power $P=20.32 \mathrm{~kW}$. In case of muons, we have the mass ratio $m_{\mu} / m_{\mathrm{e}}=206.8$ and the energy loss is reduced by the fourth-power of this ratio to become $U_{\mu}=$ $11.1 \mu \mathrm{eV}$, which is completely negligible. Besides electrons and positrons there are no other particles that would produce competitive synchrotron radiation.

23.8 The maximum photon flux occurs at a photon energy of about $\varepsilon=0.286 \varepsilon_{\mathrm{c}}$ and $S(0.286) \approx 0.569$. To find the $1 \%$ photon energy we use $(24.61)$ to scale the photon flux and have $0.777 \sqrt{x} / \mathrm{e}^{x}=0.00569$, which is solved by $x=5.795$. Appreciable radiation exists up to almost six times the critical photon energy.

23.9 From (24.51) the beam energy is $E=\left[0.4508 \varepsilon_{\mathrm{c}}(\mathrm{keV}) \rho(\mathrm{m})\right]^{1 / 3}=$ $2.0035 \mathrm{GeV}$. The magnetic field necessary for a bending radius of $\rho=1.784 \mathrm{~m}$ would be $B=3.75 \mathrm{~T}$, which is beyond conventional magnet saturation. Either superconducting magnets must be used to preserve the ring geometry or a new ring must be constructed with bending magnets which must be longer by about a factor 
of 2.5 for realistic magnetic fields, but then the beam energy must be increased to keep the critical photon energy.

\section{Solutions for Chap. 24}

24.1 In the horizontal plane the radiation distribution is uniform and an angle of $\Delta \psi=0.2 \mathrm{mr}$ will produce a photon beam width of $1 \mathrm{~mm}$ at a distance of $5 \mathrm{~m}$. The critical photon energy is $\varepsilon_{\mathrm{c}}=563 \mathrm{eV}$ and $\varepsilon / \varepsilon_{\mathrm{c}}=0.00022$. For the IR radiation the vertical opening angle $\theta_{\text {rad }}=11.3 \mathrm{mr}\left(\theta_{\mathrm{rad}} \gg 1 / \gamma\right)$ and the source length $L=0.045 \mathrm{~m}$. The total source height is $\sigma_{\text {tot }, y}=\sqrt{0.11^{2}+0.107^{2}}=0.153 \mathrm{~mm}$ and the vertical divergence $\sigma_{\text {tot }, y^{\prime}}=14.9 \mathrm{mr}$. The photon flux for $\lambda=10 \mu \mathrm{m}$ and $S(0.00022)=0.0805$ is $\frac{\mathrm{d} \dot{N}_{\mathrm{ph}}}{\mathrm{d} \psi}=1.275 \times 10^{15}$ photons $/ \mathrm{s} / \mathrm{mr} / 100 \% \mathrm{BW}$. The photon brightness is then $\mathcal{B}=\frac{\left(\mathrm{d} \dot{\hat{N}}_{\mathrm{ph}} / \mathrm{d} \psi\right) \Delta \psi}{2 \pi \sigma_{\text {tot }, y}^{2} \sigma_{\text {tot, } y^{\prime}}^{2}}=1.162 \times 10^{14} \mathrm{ph} / \mathrm{s} / \mathrm{mm}^{2} / \mathrm{mr}^{2} / 100 \% \mathrm{BW}$.

24.2 The probability to emit a photon of energy $\varepsilon$ in a unit time is $\dot{n}\left(\varepsilon_{\mathrm{ph}}\right)=\frac{P_{\gamma}}{\varepsilon_{\mathrm{c}}^{2}} \frac{S(x)}{x}$. We are looking for the case $\varepsilon=\sigma_{\varepsilon}=\frac{E^{2}}{m c^{2}} \sqrt{\frac{55 \hbar c}{64 \sqrt{3} m c^{2} J_{\mathrm{s}} \rho}}=10.9 \mathrm{MeV}$. For $\varepsilon_{\mathrm{c}}=$ $\frac{3}{2} \hbar c \frac{\gamma^{3}}{\rho}=19,166 \mathrm{eV}$, the ratio $x=\frac{\varepsilon}{\varepsilon_{\mathrm{c}}}=\frac{1}{\gamma} \sqrt{\frac{55 m c^{2} \rho}{144 \sqrt{3} J_{\mathrm{s}} \hbar c}}=277.54 \gg 1$ and $\frac{P_{\gamma}}{\varepsilon_{\mathrm{c}}^{2}}=$ $23,826 \frac{1}{\mathrm{eVs}}$. The probability becomes with this $\dot{n}\left(\varepsilon_{\mathrm{ph}}\right) \approx 2 \times 10^{-96}$ ! We may, safely assume that no second photon of this energy will be emitted within a damping time. Energy is emitted in very small fractions of the electron energy.

24.3 From (25.146) we get the number of photons emitted per unit time to be $\dot{N}_{\mathrm{ph}}=\frac{15 \sqrt{3}}{8} \frac{P_{\gamma}}{\varepsilon_{\mathrm{c}}}=3.158 \times 10^{6} \frac{\gamma}{\rho}$ and per radian $\dot{n}_{\mathrm{ph}}=0.01063 \gamma \approx \frac{\gamma}{100}$.

24.4 The critical photon energy is $\varepsilon_{\mathrm{c}}=38.04 \mathrm{keV}$ and $\varepsilon / \varepsilon_{\mathrm{c}}=0.21$. The universal function is $S(0.21)=0.5625$ and the photon flux $\frac{\mathrm{d} \dot{N}_{\mathrm{ph}}}{\mathrm{d} \psi}=3.1185 \times$ $10^{12}$ photons $/ \mathrm{s} / \mathrm{mrad}$. The vertical opening angle $\sqrt{2 \pi} \sigma_{\theta}=0.251 \mathrm{mrad}$ resulting in an effective beam height at the experiment of $Y=3.77 \mathrm{~mm}$. A beam size of 10 $\mu \mathrm{m}$ at $15 \mathrm{~m}$ corresponds to an angle of $0.667 \mu \mathrm{rad}$ at the source. The total photon flux into the required sample cross section is then $\dot{N}_{\text {ph }}=5.53 \times 10^{6} \mathrm{ph} / \mathrm{s}$, which is more than required. For a still higher photon flux one might apply some photon focusing.

24.5 From problem 24.1 $L=0.045 \mathrm{~m}$ and the diffraction limited source size and divergence are $\sigma_{r}=0.107 \mathrm{~mm}$ and $\sigma_{r^{\prime}}=14.9 \mathrm{mr}$, respectively. This is to be compared with the electron beam parameters $\left(\sigma_{\mathrm{b}, x}, \sigma_{\mathrm{b}, y}\right)=(1.1,0.11) \mathrm{mm}$ and $\left(\sigma_{\mathrm{b}, x^{\prime}}, \sigma_{\mathrm{b}, y^{\prime}}\right)=(0.11,0.011) \mathrm{mr}$. There is a considerable mismatch in the $x$-plane with $\sigma_{r} / \sqrt{2}=0.076 \mathrm{~mm}$ and $\sigma_{r^{\prime}} / \sqrt{2}=10.5 \mathrm{mr}$ while in the vertical plane the mismatch is small. In both planes the diffraction limited photon emittance is $\epsilon_{\mathrm{ph}, x, x}=797 \mathrm{~nm}$, which is much larger than the electron beam emittances in both planes. The $10 \mu \mathrm{m}$ IR radiation is therefore spatially coherent. 
24.6 The instantaneous radiation power is given by (24.32) as $P_{\gamma}(\mathrm{GeV} / \mathrm{s})=$ $379.35 B^{2} E^{2}$. The total energy loss of an electron due to wiggler radiation power can be obtained by integrating through the wiggler field for $\Delta E(\mathrm{GeV})=$ $189.67 B^{2} E^{2} \frac{L_{u}}{c \beta}$ and the total radiation power for a beam current $I$ is $P_{u}(\mathrm{~W})=$ $632.67 B^{2} E^{2} L_{\mathrm{u}} I$.

\section{Solutions for Chap. 25}

25.1 Integration of (25.61) over $\varphi$ results in factors $2 \pi$ and $\pi$ for the two terms in the nominator, respectively and we have the integrals $2 \pi \int_{0}^{\pi} \frac{\sin \theta}{(1-\beta \cos \theta)^{5}} \mathrm{~d} \theta-$ $\pi\left(1-\beta^{2}\right) \int_{0}^{\pi} \frac{\sin ^{3} \theta}{(1-\beta \cos \theta)^{5}} \mathrm{~d} \theta=4 \pi \gamma^{4} \frac{2}{3}$. With this the radiation power is $P_{\text {tot }}=$ $\frac{2}{3} r_{\mathrm{c}} m c^{2} \frac{c \beta^{4} \gamma^{4}}{\rho^{2}}$ which is (24.34).

25.2 The vertical opening angle is $1 / \gamma=0.085 \mathrm{mr}$ and therefore all radiation will be accepted. The spectral photon flux into an opening angle of $\Delta \psi=10 \mathrm{mr}$ is $\dot{N}_{\mathrm{ph}}=C_{\psi} E I \frac{\Delta \omega}{\omega} S\left(\omega / \omega_{\mathrm{c}}\right) \Delta \psi$ and with a critical photon energy of $\varepsilon_{\mathrm{c}}=23.94 \mathrm{keV}$ the spectral photon flux from an ESRF bending magnet is $\dot{N}_{\mathrm{ph}}=4.75 \times 10^{14}$. $S\left(\frac{\varepsilon_{\mathrm{ph}}(\mathrm{keV})}{23.94}\right)$.

25.3 We use (25.88) and get with $\xi=\frac{1}{2} \frac{\omega}{\omega_{\mathrm{c}}}\left(1+\gamma^{2} \theta^{2}\right)^{3 / 2}$ for the photon flux at $p \%$ the expression $\frac{\mathrm{d}^{2} W(10 \%)}{\mathrm{d} \Omega \mathrm{d} \omega} / \frac{\mathrm{d}^{2} W}{\mathrm{~d} \Omega \mathrm{d} \omega}=\left(1+\gamma^{2} \theta^{2}\right)^{2}\left[\frac{K_{2 / 3}^{2}(\xi)}{K_{2 / 3}^{2}(0)}+\frac{\gamma^{2} \theta^{2}}{1+\gamma^{2} \theta^{2}} \frac{K_{1 / 3}^{2}(\xi)}{K_{2 / 3}^{2}(0)}\right]=$ 0.1. Solving for $\theta$ gives the angle at which the intensity has dropped to $10 \%$. For low frequencies $\frac{\mathrm{d}^{2} W(10 \%)}{\mathrm{d} \Omega \mathrm{d} \omega} / \frac{\mathrm{d}^{2} W}{\mathrm{~d} \Omega \mathrm{d} \omega} \underset{\xi \rightarrow 0}{\longrightarrow} 1+\frac{\gamma^{2} \theta^{2}}{1+\gamma^{2} \theta^{2}} \frac{\Gamma^{4}(1 / 3)}{2^{8 / 3} \Gamma^{4}(2 / 3)}\left(\frac{\omega}{\omega_{\mathrm{c}}}\right)^{4 / 3}=p$ and for large arguments $\frac{\mathrm{d}^{2} W(10 \%)}{\mathrm{d} \Omega \mathrm{d} \omega} / \frac{\mathrm{d}^{2} W}{\mathrm{~d} \Omega \mathrm{d} \omega} \underset{\xi \rightarrow \infty}{\longrightarrow} \frac{1+2 \gamma^{2} \theta^{2}}{\sqrt{1+\gamma^{2} \theta^{2}}} \frac{\exp \left(\omega / \omega_{\mathrm{c}}\right)}{\exp \left[\frac{\omega}{\omega_{\mathrm{c}}}\left(1+\gamma^{2} \theta^{2}\right)^{3 / 2}\right]}=p$. All expressions have to be evaluated numerically. The angle at which the total radiation intensity has dropped to $10 \%$ is from (25.97) given by $\frac{\mathrm{d}^{2} W(10 \%)}{\mathrm{d} \Omega \mathrm{d} \omega} / \frac{\mathrm{d}^{2} W}{\mathrm{~d} \Omega \mathrm{d} \omega}=$ $\frac{1}{\left(1+\gamma^{2} \theta^{2}\right)^{5 / 2}}\left(1+\frac{5}{7} \frac{\gamma^{2} \theta^{2}}{1+\gamma^{2} \theta^{2}}\right)=p$, which can be solved by $\gamma \theta=1.390$ for $p=10 \%$.

\section{Solutions for Chap. 26}

26.1 The amplitude of the oscillatory motion in an undulator is from (26.6) $a_{\perp}=\frac{\lambda_{\mathrm{p}} K}{2 \pi \gamma}=0.581 \mu \mathrm{m}$. The longitudinal oscillation amplitude is from (26.5) $a_{\|}=\frac{K}{8 \gamma^{2} k_{\mathrm{p}}}=0.053 \AA$. Both amplitudes are very small, yet are responsible for the high intensities of radiation. 
26.2 The focal length of a single pole end is given by (7.75) $\frac{1}{f_{1}}=\frac{\pi^{2}}{2 \gamma^{2}} \frac{K^{2}}{\lambda_{\mathrm{p}}}=$ $2.58 \times 10^{-5} \mathrm{~m}^{-1}$ and for the whole undulator $\frac{1}{f_{y,}}=\frac{\pi^{2}}{2 \gamma^{2}} \frac{K^{2}}{\lambda_{\mathrm{p}}} 2 N=0.00258 \mathrm{~m}^{-1}$ or $f_{y},=387.60 \mathrm{~m}$. This focal length is very long compared to the focal lengths of the ring quadrupole, which are of the order of the distance between quadrupoles. Typically, the focal length of any insertion device should be more than about $50 \mathrm{~m}$ to be negligible. The wiggler magnet with $K=5$, on the other hand, produces a focal length of $f_{y,}=15.50 \mathrm{~m}$ which is too strong to be ignored and must be compensated. The difference comes from the fact that its the deflection angle which is responsible for focusing and $\frac{1}{f_{y}} \propto \theta^{2}$. Focusing occurs only in the nondeflecting plane and $\frac{1}{f_{x,}}=0$.

26.3 This result appears nonphysical, yet it is correct, but requires some interpretation. If the experimenter uses all radiation into the forward cone the photon flux is independent of $N_{\mathrm{p}}$, but as $N_{\mathrm{p}}$ becomes smaller the bandwidth increases. That is not as experimenters generally use synchrotron radiation. They use spectrometers to cut out a very small bandwidth $\frac{\delta \omega}{\omega} \ll \frac{1}{N_{\mathrm{p}}}$ and in this case the useful band width is independent of $N_{\mathrm{p}}$ and the photon flux is proportional to $N_{\mathrm{p}}$.

26.4 To solve this problem, we do not rely on exact calculations, but are satisfied with the precision of reading the graph (Fig. 24.5). We also use iterations to get the solution we want. The fundamental flux drops below $10 \%$ for $K<0.25$ and we use this value to get $15 \mathrm{keV}$ radiation. From the definition of the fundamental photon energy the periodlength is $\lambda_{\mathrm{p}}=3.0 \mathrm{~cm}$. To generate $4 \mathrm{keV}$ radiation we need to change $K$ enough to raise the factor $\left(1+\frac{1}{2} K^{2}\right)$ from a low value of 1.031 by a factor of $15 / 4$ to a value of 3.87 or to a value of $K=2.4$, which corresponds to a field of $B=0.857 \mathrm{~T}$. Unfortunately that field requires a gap of $8.1 \mathrm{~mm}$ which is less than allowed. We have to increase the period length to say $\lambda_{\mathrm{p}}=3.0 \mathrm{~cm}$, which gives a maximum photon energy for $K=0.25$ of $\varepsilon_{\mathrm{ph}}=12.9 \mathrm{keV}$. We plan to use the $3^{\text {rd }}$ harmonic to reach $15 \mathrm{keV}$. To reach $\varepsilon_{\mathrm{ph}}=4 \mathrm{keV}$ we need $K=2.16$ and a field of $B=0.661 \mathrm{~T}$ which requires an allowable gap of $g=11.7 \mathrm{~mm}$. We use the $3^{\text {rd }}$ harmonic to reach $\varepsilon_{\mathrm{ph}}=15 \mathrm{keV}$ at $K=1.82$. With this result we may even extend the spectral range on both ends.

26.5 In the electron system the wavelength of the laser beam is Lorentz contracted by a factor of $1 /(2 \gamma)$, where the factor of two is due to the fact that the relative velocity between both beams is $2 c$. The wavelength in the laboratory system is therefore $\lambda=\frac{\lambda_{\mathrm{L}}}{4 \gamma^{2}}$ since $K \ll 1$ for the laser field.

26.6 The maximum transverse oscillation amplitude is $4.57 \mu \mathrm{m}$ and the transverse velocity in units of $c$ is just equal to the maximum deflection angle $\beta \perp=\theta=$ $K / \gamma=0.38 \mathrm{mr}$. The transverse relativistic factor $\gamma \perp \approx 1+7.22 \cdot 10^{-8}$, indeed very small, yet enough to start generating relativistic perturbations in the transverse particle motion. 
26.7 The fundamental wavelength is $\lambda=\frac{\lambda \mathrm{p}}{2 \gamma^{2}}\left(1+\frac{1}{2} K^{2}+\gamma^{2} \vartheta^{2}\right)$ and for $\vartheta=0$ we have the fundamental wavelength $\lambda=10.88 \AA$. The natural bandwidth is $\frac{\Delta \omega}{\omega}=\frac{1}{N_{\mathrm{p}}}$ and we look therefore for an angle $\hat{\vartheta}$ such that the wavelength has increased by no more than $9 \%$, or $\frac{\gamma^{2} \hat{\vartheta}^{2}}{1+\frac{1}{2} K^{2}}=0.09$ and solving for $\hat{\vartheta}$, we get $\hat{\vartheta}= \pm 62.6 \mu \mathrm{rad}$.

26.8 In first approximation, we assume that all the fields are contained within the volume between the two rows of poles and no field leaks out. Separating the poles by $g$ requires to generate the additional field energy d $e=F \mathrm{~d} g$ where $F$ is the force between poles. Since $\mathrm{d} e>0$ for $\mathrm{d} g>0$ the force is attractive meaning that the poles are attracted. The force is then $F=\frac{\mathrm{d} e}{\mathrm{~d} g}=\frac{w}{2 \mu_{0}} \int_{0}^{15 \lambda_{\mathrm{p}}} B^{2}(z) \mathrm{d} z=47,024 \mathrm{~N}$ $=4.70$ tons.

26.9 In the electron rest frame energy conservation requires $\hbar \omega+m c^{2}=\hbar \omega^{\prime}+$ $\sqrt{c^{2} p^{2}+m c^{2}}$ where $\hbar \omega$ and $\hbar \omega^{\prime}$ are the incoming and outgoing photon energies, respectively and $c p$ the electron momentum after the scattering process. Solving for $c p$ we get $c^{2} p^{2}=\hbar^{2}\left(\omega-\omega^{\prime}\right)^{2}+2 \hbar m c^{2}\left(\omega-\omega^{\prime}\right)$. For momentum conservation, we require that $\hbar \boldsymbol{k}=\hbar \boldsymbol{k}^{\prime}+\boldsymbol{p}$ with the angle $\vartheta$ between $\boldsymbol{k}$ and $\boldsymbol{p}$. From this we get $c^{2} p^{2}=\left(\hbar \omega^{\prime}\right)^{2}+(\hbar \omega)^{2}-2 \hbar^{2} \omega \omega^{\prime} \cos \vartheta$. Comparing booth expressions for $c p$ we get $-2 \hbar \omega \omega^{\prime}+2 \hbar m c^{2}\left(\omega-\omega^{\prime}\right)=-2 \hbar^{2} \omega \omega^{\prime} \cos \vartheta$ or $\frac{\hbar}{m c^{2}}(1-\cos \vartheta)=\frac{1}{\omega^{\prime}}-\frac{1}{\omega}=$ $\frac{\lambda^{\prime}-\lambda}{2 \pi c}$. We look for radiation emitted in the forward direction or for $\vartheta=180^{\circ}$ and get for the scattered wavelength $\lambda^{\prime}=\lambda$ because $\frac{4 \pi \hbar c}{m c^{2}} \approx 4.8 \times 10^{12} \ll \lambda$. Note, that all quantities are still defined in the electron rest frame. The wavelength of the undulator field in the electron system is $\lambda=\lambda_{\mathrm{p}}^{*} / \gamma$, where now $\mathcal{L}^{*}$ the laboratory system of reference and the scattered radiation in the laboratory system due to the Doppler effect is $\lambda^{*}=\frac{\lambda_{\mathrm{p}}^{*}}{2 \gamma^{2}}\left(1+\frac{1}{2} K^{2}\right)$, which is the expression for the fundamental wavelength of undulator radiation.

26.10 The photon flux density in the forward cone from a given undulator and storage ring is proportional to (26.61). For the fundamental the maximum value is reached for $K=1.1985$ with an amplitude of $A_{1}(1.1985)=0.381$. An intensity of $90 \%$ has an amplitude of 0.343 which corresponds to $K$-values of $K=0.873$ and $K=1.642$.

26.11 Equation (26.54) is for $\vartheta=0$ proportional to $\left(-K \sum_{2}\right)^{2}$ and we look at the factor $\sum_{2}=\sum_{m=-\infty}^{\infty} J_{-m}(u)\left[J_{k-2 m-1}(v)+J_{k-2 m+1}(v)\right]$ to select harmonics. Here $u=\frac{\omega}{\omega_{1}} S$ and $v=\frac{\omega}{\omega_{1}} C$. Furthermore $C \propto \vartheta=0$ and $S \propto K^{2} \bar{\beta}$. Therefore $v=0$ and $\left[J_{k-2 m-1}(0)+J_{k-2 m+1}(0)\right]=0$ for all indices which are non-zero. Only for $k=2 m \pm 1$ are the indices equal to zero and $\left[J_{0}(0)+J_{0}(0)\right] \neq 0$. Finally $\sum_{2}=0$ for all even values of $k$ and $\sum_{2} \neq 0$ only for odd values of $k$.

26.12 To get elliptically polarized radiation we look at (26.51) and try to find one real term together with an imaginary term in the other plane to be non-zero at the same time. We also remember that undulator radiation is emitted only at odd harmonics $k=2 m \pm 1$. From (26.51) we note that the $\mathrm{i} \hat{\boldsymbol{x}}$-terms are both non-zero but the $\hat{\boldsymbol{y}} B_{0}$-term has a factor $J_{k-2 m}(v)$ for which $v=0$ in the forward direction and 
$J_{k-2 m}(0)=0$ for all non-zero indices. Since $k$ is odd $J_{k-2 m}(0)=0$ for all odd values of $k$ and with it the $\hat{\boldsymbol{y}} B_{0}$-term is zero too. Therefore there is no elliptical polarization. Of course the radiation from each pole is elliptically polarized, say, clock-wise but that polarization is compensated by the counter-clock-wise radiation from the next pole.

26.13 The best approach to solve this design problem is to use a computer program like EXCEL. There are many parameters and the photon wavelength must be calculated many times. Specify beam energy, period length and $\mathrm{min} / \mathrm{max}$ desired wavelengths. Calculate fundamental wavelength for $K_{\min } / K_{\max }$. For reasonable intensities $K_{\min } \gtrsim 0.5$. $K_{\max }$ determines the maximum field. At this point we can only guess, say $K_{\max }=6$. Calculate $\mathrm{min} / \mathrm{max}$ wavelength or photon energies for up to seventh harmonic. Try to find period length which covers the desired spectrum. From the undulator strength parameter calculate the maximum field strength. The maximum field in a permanent magnet undulator depends on the gap and period length. For a closed undulator assume a minimum gap of $\pm 10 \mathrm{~mm}$. That means to reach, say $1.5 \mathrm{~T}$, the period length should be longer than about $5-6 \mathrm{~cm}$ (see Fig. 24.5). Note, do not assume that you must find a perfect solution that covers the whole desired spectrum. If, for example, you desire low energy photons from an unduator in a high energy storage ring you will not find a solution with a reasonable value of $K$. Sometimes the harmonic spectra just will not close. In this case several undulators with different period length and automatically exchangeable can be used. Such undulators are also known as "revolver"-type undulators.

26.14 The fundamental wave length for a weak undulator $(K<1)$ are $\lambda(800 \mathrm{MeV})=102 \AA$ and $\lambda(7 \mathrm{GeV})=1.33 \AA$ which are the shortest achievable wavelength. For a $10 \mathrm{~mm}$ gap the field is from $(24.5) B=1.198 \mathrm{~T}$ and the maximum value of the strength parameter is $K=5.595$. With this, the longest fundamental wavelength is $\lambda=1698.5 \AA$ for the $800 \mathrm{MeV}$ ring and $\lambda=22.14 \AA$ for the $7 \mathrm{GeV}$ ring.

26.15 The short wavelength limits are given for a weak undulator $(K<1)$ and are $\lambda=3.13 \AA$ for $\lambda_{\mathrm{p}}=15 \mathrm{~mm}$ and $\lambda=15.7 \AA$ for $\lambda_{\mathrm{p}}=75 \mathrm{~mm}$. The long wavelength limits are determined by the magnetic fields when the undulator gaps are closed to $10 \mathrm{~mm}$. The fields are from $(24.5) B\left(\lambda_{\mathrm{p}}=15 \mathrm{~mm}\right)=$ $0.19 \mathrm{~T}$ and $B\left(\lambda_{\mathrm{p}}=75 \mathrm{~mm}\right)=1.66 \mathrm{~T}$, respectively. The undulator strengths are $K\left(\lambda_{\mathrm{p}}=15 \mathrm{~mm}\right)=0.270$ and $K\left(\lambda_{\mathrm{p}}=75 \mathrm{~mm}\right)=1.35$ and the wavelengths $\lambda\left(\lambda_{\mathrm{p}}=15 \mathrm{~mm}\right)=3.24 \AA$ and $\lambda\left(\lambda_{\mathrm{p}}=15 \mathrm{~mm}\right)=30.0 \AA$. The tuning range is very small for the $15 \mathrm{~mm}$ undulator and about a factor of two for the long period undulator. The tuning ranges are so different because the $K$-value can be varied much more for longer period undulators. 


\section{Solutions for Chap. 27}

27.1 We may solve this problem two ways. First, we use the average drift velocity $\bar{\beta}=\beta\left(1-\frac{K^{2}}{4 \gamma^{2}}\right)$ and calculate the time $t_{\mathrm{e}}=\frac{\lambda_{\mathrm{p}}}{c \bar{\beta}} \approx \frac{\lambda_{\mathrm{p}}}{c \beta}\left(1+\frac{K^{2}}{4 \gamma^{2}}\right)$ it takes the electron to travel along one period. During that same time the photon travels a distance $s_{\gamma}=c t_{\mathrm{e}}=\frac{\lambda_{\mathrm{p}}}{\beta}\left(1+\frac{K^{2}}{4 \gamma^{2}}\right)$ and the difference is $\delta s=s_{\gamma}-\lambda_{\mathrm{p}}=$ $\lambda_{\mathrm{p}}\left(\frac{1}{\beta}-1\right)+\frac{\lambda_{\mathrm{p}}}{\beta} \frac{K^{2}}{4 \gamma^{2}} \approx \frac{\lambda_{\mathrm{p}}}{2 \gamma^{2}}\left(1+\frac{1}{2} K^{2}\right)$, which is just equal to the fundamental radiation wave length. We may also integrate the path length along the sinusoidal trajectory and get for one quarter period $s_{\mathrm{e}}=\frac{\lambda_{\mathrm{p}}}{2 \gamma^{2}} \int_{0}^{\pi / 2} \sqrt{1+\theta^{2} \cos ^{2} x} \mathrm{~d} x=$ $\frac{\lambda_{\mathrm{p}}}{2 \gamma^{2}}$ EllipticE $\left(-\theta^{2}\right)$ which is the Legendre elliptical integral of the second kind. Since the argument will always be very small, we may expand $\operatorname{EllipticE}\left(-\theta^{2}\right) \approx$ $\frac{\pi}{2}+0.393 \theta^{2}$ for small arguments and get the electron travel time for one period $t_{\mathrm{e}}=4 \frac{\lambda_{\mathrm{p}}}{2 \pi} \frac{1}{c \beta}\left(\frac{\pi}{2}+0.393 \theta^{2}\right)$. The path length difference between the photon, $\lambda_{\mathrm{p}}$ and electron $c t_{\mathrm{e}}$ is $\delta s=c t_{\mathrm{e}}-\lambda_{\mathrm{p}}=\lambda_{\mathrm{p}} \frac{1}{\beta}\left(1+\frac{0.393 \theta^{2}}{\pi / 2}\right)-\lambda_{\mathrm{p}} \approx \frac{\lambda_{\mathrm{p}}}{2 \gamma^{2}}(1+\underbrace{\frac{8 \cdot 0.393}{\pi}}_{\approx 1} \frac{1}{2} K^{2})$ which is again the fundamental wavelength of radiation.

27.2 In a helical undulator the transverse as well as the longitudinal motion are uniform and therefore there are no varying relativistic effects perturbing the uniform motion. A Fourier transform of the motion will therefore only exhibit one, the fundamental harmonic .

27.3 The total energy of the photon flux is $E_{\gamma}=\rho N_{\mathrm{e}} E$ into a band width of $\frac{\Delta \omega}{\omega}=\frac{1}{N_{\mathrm{p}}}$ and the number of coherent x-ray photons of energy $\varepsilon_{\mathrm{x}}$ per electron is $n_{\text {ph,coh }}=\frac{E_{\gamma}}{\varepsilon_{\mathrm{x}} N_{\mathrm{e}}}=717.5$. On the other hand the radiation pulse energy from an undulator into the forward cone per electron and band width $\frac{\Delta \omega}{\omega}=\frac{1}{N_{\mathrm{p}}}$ is $n_{\text {ph,incoh }}=\frac{1}{2} \pi \alpha\left(1+\frac{1}{2} K^{2}\right) A_{1}(K)=0.015$. The SASE process accounts for about five orders of magnitude in photon flux per electron. 


\section{Appendix A \\ Useful Mathematical Formulae}

\section{A.1 Vector Algebra}

Electric and magnetic fields are vectors which are defined by direction and magnitude in space $\boldsymbol{E}(x, y, z)$ and $\boldsymbol{B}(x, y, z)$, where we use a Cartesian coordinate system $(x, y, z)$.The distribution of such vectors is called a vector field in contrast to a scalar field such as the distribution of temperature $T(x, y, z)$. In component form such vectors can be written as

$$
\begin{gathered}
\boldsymbol{E}=E_{x} \boldsymbol{x}+E_{y} \boldsymbol{y}+E_{z} z . \\
\boldsymbol{E}+\boldsymbol{B}=\left(E_{x}+B_{x}\right) \boldsymbol{x}+\left(E_{y}+B_{y}\right) \boldsymbol{y}+\left(E_{z}+B_{z}\right) z \\
\boldsymbol{E} \boldsymbol{B}=E_{x} B_{x}+E_{y} B_{y}+E_{z} B_{z}=|E||B| \cos \theta
\end{gathered}
$$

where $\theta$ is the angle between the vectors, and the

$$
\begin{gathered}
\boldsymbol{E} \times \boldsymbol{B}=\left(E_{y} B_{z}-E_{z} B_{y}, E_{z} B_{x}-E_{x} B_{z}, E_{x} B_{y}-E_{y} B_{x}\right), \\
|\boldsymbol{E} \times \boldsymbol{B}|=|E||B| \sin \theta .
\end{gathered}
$$

The resulting vector is orthogonal to both vectors $\boldsymbol{E}$ and $\boldsymbol{B}$ and the vectors $[\boldsymbol{E}, \boldsymbol{B}$, $\boldsymbol{E} \times \boldsymbol{B}$ ] form a right handed orthogonal coordinate system. 


\section{A.1.1 Differential Vector Expressions}

To describe the variation of scalar and vector fields a gradient for scalars if defined by

$$
\nabla T=\operatorname{grad} T=\left(\frac{\partial T}{\partial x}, \frac{\partial T}{\partial y}, \frac{\partial T}{\partial z}\right)
$$

which is a vector.

For vectors we define two differential expressions. The first is the divergence of the vector field:

$$
\boldsymbol{\nabla} \boldsymbol{E}=\operatorname{div} \boldsymbol{E}=\frac{\partial E_{x}}{\partial x}+\frac{\partial E_{y}}{\partial y}+\frac{\partial E_{z}}{\partial z},
$$

which is a scalar. Geometrically, the divergence of a vector is the outward flux of this vector per unit volume. As an example consider a small cube with dimensions $\mathrm{d} x, \mathrm{~d} y . \mathrm{d} z$. Put this cube in a uniform vector field and you get zero divergence, because the flux into the cube is equal to the flux out. Now, put the cube into a field free area and place a positive charge into the cube. The flux of fields is all outwards and the divergence is nonzero.

The divergence can be evaluated by integrating over all volume and we get with Gauss's integral theorem (A.25)

$$
\int_{V} \nabla \boldsymbol{E} \mathrm{d} V=\oint \boldsymbol{E} \boldsymbol{n} \mathrm{d} a
$$

where $\boldsymbol{n}$ is a unit vector normal to the surface and $\mathrm{d} a$ a surface element. The volume integral becomes an integral over the outer surface.

The second differential expression is the "curl" of a vector:

$$
\nabla \times \boldsymbol{B}=\left(\frac{\partial B_{z}}{\partial y}-\frac{\partial B_{y}}{\partial z}, \frac{\partial B_{x}}{\partial z}-\frac{\partial B_{z}}{\partial x}, \frac{\partial B_{y}}{\partial x}-\frac{\partial B_{x}}{\partial y}\right) .
$$

The "curl" of a vector per unit area is the circulation about the direction of the vector.

\section{A.1.2 Algebraic Relations}

$$
\begin{aligned}
a(b \times c) & =b(c \times a)=c(a \times b) \\
a \times(b \times c) & =b(a c)-c(a b) \\
(a \times b)(c \times d) & =(a c)(b d)-(b c)(a d)
\end{aligned}
$$




$$
\begin{aligned}
a \times(b \times c)+b \times(c \times a)+c \times(a \times b) & =0 \\
(a \times b) \times(c \times d) & =c[(a \times b) d]-d[(a \times b) c]
\end{aligned}
$$

\section{A.1.3 Differential Relations}

$$
\begin{aligned}
\nabla(\boldsymbol{a} \varphi) & =\varphi \nabla \boldsymbol{a}+\boldsymbol{a} \nabla \varphi \\
\nabla \times(\boldsymbol{a} \varphi) & =\varphi(\nabla \times \boldsymbol{a})-\boldsymbol{a} \times \nabla \varphi \\
\nabla(\boldsymbol{a} \times \boldsymbol{b}) & =\boldsymbol{b}(\nabla \times \boldsymbol{a})-\boldsymbol{a}(\nabla \times \boldsymbol{b}) \\
\nabla \times(\boldsymbol{a} \times \boldsymbol{b}) & =(\boldsymbol{b} \nabla) \boldsymbol{a}-(\boldsymbol{a} \nabla) \boldsymbol{b}+\boldsymbol{a}(\nabla \boldsymbol{b})-\boldsymbol{b}(\nabla \boldsymbol{a}) \\
\nabla(\boldsymbol{a} \boldsymbol{b}) & =(\boldsymbol{b} \nabla) \boldsymbol{a}+(\boldsymbol{a} \nabla) \boldsymbol{b}+\boldsymbol{a} \times(\nabla \times \boldsymbol{b})+\boldsymbol{b} \times(\nabla \times \mathbf{a}) \\
\nabla \times(\nabla \varphi) & =0 \\
\nabla(\nabla \times \boldsymbol{a}) & =0 \\
\nabla \times(\nabla \times \boldsymbol{a}) & =\nabla(\nabla \boldsymbol{a})-\Delta \boldsymbol{a}
\end{aligned}
$$

\section{A.1.4 Partial Integration}

Partial integration is defined by

$$
\begin{gathered}
\int_{a}^{b} u v^{\prime} d x=\left.u v\right|_{a} ^{b}-\int_{a}^{b} v u^{\prime} d x, \text { or } \\
\int_{a}^{b} u d v=\left.u v\right|_{a} ^{b}-\int_{a}^{b} v d u
\end{gathered}
$$

\section{A.1.5 Trigonometric and Exponential Functions}

$$
\begin{array}{cc}
\mathrm{e}^{\mathrm{i} x}=\cos x+\mathrm{i} \sin x & \\
\cos x=\frac{1}{2}\left(\mathrm{e}^{\mathrm{i} x}+\mathrm{e}^{-\mathrm{i} x}\right) & \sin x=\frac{1}{\mathrm{i} 2}\left(\mathrm{e}^{\mathrm{i} x}-\mathrm{e}^{-\mathrm{i} x}\right) \\
\cos (a \pm b)=\cos a \cos b \mp \sin a \sin b & \sin (a \pm b)=\sin a \cos b \pm \sin b \cos a \\
\tan (a \pm b)=\frac{\tan a \pm \tan b}{1 \mp \tan a \tan b} & \cot (a \pm b)=\frac{\cot a \cot b \mp 1}{\cot a \pm \cot b} \\
\frac{\mathrm{d}}{\mathrm{d} a} \tan a=\frac{1}{\cos ^{2} a}=1+\tan ^{2} a & \frac{\mathrm{d}}{\mathrm{d} a} \arctan a=\frac{1}{1+a^{2}}
\end{array}
$$




\section{A.1.6 Integral Relations}

$$
\begin{aligned}
\int_{V} \nabla \varphi \mathrm{d} \boldsymbol{r} & =\oint_{S} \varphi \hat{\boldsymbol{u}} \mathrm{d} \sigma \\
\int_{V} \nabla \boldsymbol{a} \mathrm{d} \boldsymbol{r} & =\oint_{S} \boldsymbol{a} \hat{\boldsymbol{u}} \mathrm{d} \sigma \quad \text { Gauss' theorem } \\
\int_{S}(\nabla \times \boldsymbol{a}) \hat{\boldsymbol{u}} \mathrm{d} \sigma & =\oint_{a \mathrm{~d} \boldsymbol{s}} \quad \text { Stokes' theorem }
\end{aligned}
$$

\section{A.1.7 Dirac's Delta Function}

$$
\begin{array}{cc}
\delta(x)=\left\{\begin{array}{lc}
\infty \text { for } x=0 & \int_{-\infty}^{\infty} \delta(x) \mathrm{d} x=1 \\
0 \text { for } x \neq 0 & \\
|\alpha| \int_{-\infty}^{\infty} \delta(\alpha x) \mathrm{d} x=\int_{-\infty}^{\infty} \delta(y) \mathrm{d} y=1 & \delta(\omega)=\int_{-\infty}^{\infty} \delta(t) \mathrm{e}^{-\mathrm{i} 2 \pi \omega t} \mathrm{~d} t=1
\end{array}\right. \\
\delta(x)=\frac{1}{2 \pi} \sum_{n=-\infty}^{\infty} \mathrm{e}^{\mathrm{i} n x} & \frac{1}{2 \pi} \sum_{n=-\infty}^{\infty} \mathrm{e}^{\mathrm{i} n x}=\sum_{m=-\infty}^{\infty} \delta(x-2 \pi m)
\end{array}
$$

\section{A.1.8 Bessel's Functions}

Order $n$ and first kind:

$$
\begin{gathered}
J_{n}(x)=\sum_{p=0}^{\infty} \frac{(-1)^{p}(x / 2)^{n+2 p}}{p !(n+p) !} \\
n=0 \quad J_{0}(0)=1 \\
n=1,2,3 \ldots \\
J_{n}(0)=0
\end{gathered}
$$

derivatives and recursion formulas with $J_{n}=J_{n}(x)$ :

$$
\begin{aligned}
J_{n+1} & =\frac{2 n}{x} J_{n}-J_{n-1} \\
J_{n}^{\prime} & =J_{n-1}-\frac{n}{x} J_{n}=\frac{n}{x} J_{n}-J_{n+1}=\frac{1}{2}\left(J_{n-1}-J_{n+1}\right)
\end{aligned}
$$


first four roots of Bessel's functions of the first kind: $J_{n}\left(x_{i}\right)=0$

$\begin{array}{ccccc}n & J_{n}\left(x_{1}\right) & J_{n}\left(x_{2}\right) & J_{n}\left(x_{3}\right) & J_{n}\left(x_{4}\right) \\ 0 & 2.4048 & 5.5200 & 8.6537 & 11.7954 \\ 1 & 3.8317 & 7.0155 & 10.1743 & \\ 2 & 5.1356 & 8.4172 & 11.6198 & \\ 3 & 6.3801 & 9.7610 & & \end{array}$

\section{A.1.9 Series Expansions}

For $\delta \ll 1$

$$
\begin{aligned}
\mathrm{e}^{x} & =!+x+\frac{1}{2 !} x^{2}+\frac{1}{3 !} x^{3}+\ldots \\
\ln (1-x) & =-x-\frac{1}{2} x^{2}-\frac{1}{3} x^{3}-\ldots \text { for }-1 \leq x<1 \\
\sin x & =x-\frac{1}{3 !} x^{3}+\frac{1}{5 !} x^{5}-\ldots \\
\cos x & =1-\frac{1}{2 !} x^{2}+\frac{1}{4 !} x^{4}-\ldots \\
\sqrt{1+\delta} & =1+\frac{1}{2} \delta-\frac{1}{2^{3}} \delta^{2}+\frac{1}{2^{5}} \delta^{3} \ldots \\
\frac{1}{1+\delta} & =1-\delta+\delta^{2}-\delta^{3}+\ldots
\end{aligned}
$$

\section{A.1.10 Fourier Series}

A function $f(t)$ is periodic if $f(t)=f(t+T)=f(t+n T)$ where $n$ is an integer and $T$ the lowest value for which this statement is true. Such a function can be expressed with $\tau=\frac{t}{T}$ by

$$
f(\tau)=\frac{1}{2} a_{0}+\sum_{n=1}^{\infty}\left[a_{n} \cos \left(2 \pi n \tau+\vartheta_{n}\right)+b_{n} \sin \left(2 \pi n \tau+\vartheta_{n}\right)\right]
$$


or using exponentials

$$
f(\tau)=\sum_{n=-\infty}^{\infty} c_{n} \mathrm{e}^{\mathrm{i} n \tau}
$$

where $c_{n}=c_{-n}$ are complex with $c_{n}=\overline{c_{-n}}$ and $c_{0}=\langle f(\tau)\rangle$. The coefficients are

$$
c_{n}=\int_{0}^{1} f(\tau) \mathrm{e}^{-\mathrm{i} n \tau} \mathrm{d} \tau \quad \text { where } \quad \tau=t / T
$$

\section{Parseval's Theorem}

$$
\int_{-\infty}^{\infty} F^{2}(t) \mathrm{d} t=\frac{1}{2 \pi} \int_{-\infty}^{\infty} F^{2}(\omega) \mathrm{d} \omega,
$$

where $F(t)=\frac{1}{2 \pi} \int F(\omega) \mathrm{e}^{-\mathrm{i} \omega t} \mathrm{~d} \omega$ and $F(\omega)=\int F(t) \mathrm{e}^{\mathrm{i} \omega t} \mathrm{~d} t$.

\section{Fourier Transform}

For non-periodic functions $f(t)$ with $(T \rightarrow \infty)$ the Fourier transform is

$$
F(\omega)=\int_{-\infty}^{\infty} f(t) e^{-\mathrm{i} \omega t} \mathrm{~d} t
$$

and

$$
f(t)=\frac{1}{2 \pi} \int_{-\infty}^{\infty} F(\omega) \mathrm{e}^{-\mathrm{i} n \omega t} \mathrm{~d} \omega
$$

\section{A.1.11 Coordinate Transformations}

\section{Cartesian coordinates}

$$
\begin{aligned}
\mathrm{d} s^{2} & =\mathrm{d} x^{2}+\mathrm{d} y^{2}+\mathrm{d} z^{2} \\
\mathrm{~d} V & =\mathrm{d} x \mathrm{~d} y \mathrm{~d} z \\
\nabla \psi & =\left(\frac{\partial \psi}{\partial x}, \frac{\partial \psi}{\partial y}, \frac{\partial \psi}{\partial z}\right) \\
\nabla \boldsymbol{a} & =\frac{\partial a_{x}}{\partial x}+\frac{\partial a_{y}}{\partial y}+\frac{\partial a_{z}}{\partial z}
\end{aligned}
$$




$$
\begin{aligned}
\boldsymbol{\nabla} \times \boldsymbol{a} & =\left(\frac{\partial a_{z}}{\partial y}-\frac{\partial a_{y}}{\partial z}, \frac{\partial a_{x}}{\partial z}-\frac{\partial a_{z}}{\partial x}, \frac{\partial a_{y}}{\partial x}-\frac{\partial a_{x}}{\partial y}\right) \\
\Delta \psi & =\frac{\partial^{2} \psi}{\partial x^{2}}+\frac{\partial^{2} \psi}{\partial y^{2}}+\frac{\partial^{2} \psi}{\partial z^{2}}
\end{aligned}
$$

\section{General Coordinate Transformation}

Transformation into new coordinates $(u, v, w)$, where $x=x(u, v, w)$, $y=y(u, v, w)$ and $z=z(u, v, w)$

$$
\begin{aligned}
& \mathrm{d} s^{2}=\frac{\mathrm{d} u^{2}}{U^{2}}+\frac{\mathrm{d} v^{2}}{V^{2}}+\frac{\mathrm{d} w^{2}}{W^{2}} \\
& \mathrm{~d} V=\frac{\mathrm{d} u}{U} \frac{\mathrm{d} v}{V} \frac{\mathrm{d} w}{W} \\
& \nabla \psi=\left(U \frac{\partial \psi}{\partial u}, V \frac{\partial \psi}{\partial v}, W \frac{\partial \psi}{\partial w}\right) \\
& \nabla \boldsymbol{a}=U V W\left[\frac{\partial}{\partial u} \frac{a_{u}}{V W}+\frac{\partial}{\partial v} \frac{a_{v}}{U W}+\frac{\partial}{\partial w} \frac{a_{w}}{U V}\right] \\
& \nabla \times \boldsymbol{a}=\left\{\begin{array}{c}
V W\left[\frac{\partial}{\partial v} \frac{a_{w}}{W}-\frac{\partial}{\partial w} \frac{a_{v}}{V}\right], U W\left[\frac{\partial}{\partial w} \frac{a_{u}}{U}-\frac{\partial}{\partial u} \frac{a_{w}}{W}\right], \\
U V\left[\frac{\partial}{\partial u} \frac{a_{v}}{V}-\frac{\partial}{\partial v} \frac{a_{u}}{U}\right]
\end{array}\right\} \\
& \Delta \psi=U V W\left[\frac{\partial}{\partial u}\left(\frac{U}{V W} \frac{\partial \psi}{\partial u}\right)+\frac{\partial}{\partial v}\left(\frac{V}{U W} \frac{\partial \psi}{\partial v}\right)+\frac{\partial}{\partial w}\left(\frac{W}{U V} \frac{\partial \psi}{\partial w}\right)\right]
\end{aligned}
$$

where

$$
\begin{aligned}
U^{-1} & =\sqrt{\left(\frac{\partial x}{\partial u}\right)^{2}+\left(\frac{\partial y}{\partial u}\right)^{2}+\left(\frac{\partial z}{\partial u}\right)^{2}}, \\
V^{-1} & =\sqrt{\left(\frac{\partial x}{\partial v}\right)^{2}+\left(\frac{\partial y}{\partial v}\right)^{2}+\left(\frac{\partial z}{\partial v}\right)^{2}} \\
W^{-1} & =\sqrt{\left(\frac{\partial x}{\partial w}\right)^{2}+\left(\frac{\partial y}{\partial w}\right)^{2}+\left(\frac{\partial z}{\partial w}\right)^{2}},
\end{aligned}
$$


and

$$
\begin{aligned}
& a_{u}=a_{x} U \frac{\partial x}{\partial u}+a_{y} U \frac{\partial y}{\partial u}+a_{z} U \frac{\partial z}{\partial u}, \\
& a_{v}=a_{x} V \frac{\partial x}{\partial v}+a_{y} V \frac{\partial y}{\partial v}+a_{z} V \frac{\partial z}{\partial v} \\
& a_{w}=a_{x} W \frac{\partial x}{\partial w}+a_{y} W \frac{\partial y}{\partial w}+a_{z} W \frac{\partial z}{\partial w}
\end{aligned}
$$

\section{Cylindrical Coordinates}

Transformation to cylindrical coordinates $(r, \varphi, \zeta)$

$$
\begin{aligned}
(x, y, z) & =(r \cos \varphi, r \sin \varphi, \zeta) \\
\mathrm{d} s^{2} & =\mathrm{d} r^{2}+r^{2} \mathrm{~d} \varphi^{2}+\mathrm{d} \zeta^{2} \\
\mathrm{~d} V & =r \mathrm{~d} r \mathrm{~d} \varphi \mathrm{d} \zeta \\
\nabla \psi & =\left[\frac{\partial \psi}{\partial r}, \frac{1}{r} \frac{\partial \psi}{\partial \varphi}, \frac{\partial \psi}{\partial \zeta}\right] \\
\nabla \boldsymbol{a} & =\frac{1}{r} \frac{\partial}{\partial r}\left(r a_{r}\right)+\frac{1}{r} \frac{\partial a_{\varphi}}{\partial \varphi}+\frac{\partial a_{\zeta}}{\partial \zeta} \\
\nabla \times \boldsymbol{a} & =\left[\frac{1}{r} \frac{\partial a_{\zeta}}{\partial \varphi}-\frac{\partial a_{\varphi}}{\partial \zeta}, \frac{\partial a_{r}}{\partial \zeta}-\frac{\partial a_{\zeta}}{\partial r}, \frac{1}{r} \frac{\partial}{\partial r}\left(r a_{\varphi}\right)-\frac{1}{r} \frac{\partial a_{r}}{\partial \varphi}\right] \\
\Delta \psi & =\frac{\partial^{2} \psi}{\partial r^{2}}+\frac{1}{r} \frac{\partial \psi}{\partial r}+\frac{1}{r^{2}} \frac{\partial^{2} \psi}{\partial \varphi^{2}}+\frac{\partial^{2} \psi}{\partial \zeta^{2}}
\end{aligned}
$$

\section{Polar Coordinates}

Transformation to polar coordinates $(r, \varphi, \theta)$

$$
\begin{aligned}
(x, y, z) & =(r \cos \varphi \sin \theta, r \sin \varphi \sin \theta, r \cos \theta) \\
\mathrm{d} s^{2} & =\mathrm{d} r^{2}+r^{2} \sin ^{2} \theta \mathrm{d} \varphi^{2}+r^{2} \mathrm{~d} \theta^{2} \\
\mathrm{~d} V & =r^{2} \sin \theta \mathrm{d} r \mathrm{~d} \varphi \mathrm{d} \theta \\
\nabla \psi & =\left[\frac{\partial \psi}{\partial r}, \frac{1}{r} \frac{\partial \psi}{\partial \varphi}, \frac{1}{r \sin \theta} \frac{\partial \psi}{\partial \theta},\right] \\
\nabla \boldsymbol{a} & =\frac{1}{r^{2}} \frac{\partial}{\partial r}\left(r^{2} a_{r}\right)+\frac{1}{r \sin \theta} \frac{\partial}{\partial \varphi}\left(\sin \varphi a_{\varphi}\right)+\frac{1}{r \sin \theta} \frac{\partial a_{\theta}}{\partial \theta}
\end{aligned}
$$




$$
\begin{aligned}
\nabla \times \boldsymbol{a} & =\left[\begin{array}{c}
\frac{1}{r \sin \theta}\left(\frac{\partial\left(\sin \theta a_{\zeta}\right)}{\partial \varphi}-\frac{\partial a_{\varphi}}{\partial \theta}\right), \frac{1}{r \sin \theta}\left(\frac{\partial a_{r}}{\partial \theta}-\sin \theta \frac{\partial\left(r a_{\theta}\right)}{\partial r}\right) \\
\frac{1}{r}\left(\frac{\partial}{\partial r}\left(r a_{\varphi}\right)-\frac{\partial a_{r}}{\partial \varphi}\right) \cdot
\end{array}\right] \\
\Delta \psi & =\frac{1}{r^{2}} \frac{\partial}{\partial r}\left(r^{2} \frac{\partial \psi}{\partial r}\right)+\frac{1}{r^{2} \sin ^{2} \theta} \frac{\partial^{2} \psi}{\partial \varphi^{2}}+\frac{1}{r^{2} \sin \theta} \frac{\partial}{\partial \theta}\left(\sin \theta \frac{\partial \psi}{\partial \theta}\right)
\end{aligned}
$$

\section{Curvilinear Coordinates}

Transformation to curvilinear coordinates of beam dynamics

$$
\begin{aligned}
\mathrm{d} s^{2} & =\mathrm{d} x^{2}+\mathrm{d} y^{2}+\left(1+\kappa_{x} x+\kappa_{y} y\right)^{2} \mathrm{~d} z^{2}=\mathrm{d} x^{2}+\mathrm{d} y^{2}+h^{2} \mathrm{~d} z^{2} \\
\mathrm{~d} V & =\mathrm{d} x \mathrm{~d} y h \mathrm{~d} z \\
\nabla \psi & =\frac{\partial \psi}{\partial x} \boldsymbol{x}+\frac{\partial \psi}{\partial y} \boldsymbol{y}+\frac{1}{h} \frac{\partial \psi}{\partial z} \boldsymbol{z}, \\
\nabla \boldsymbol{a} & =\frac{1}{h}\left[\frac{\partial\left(h a_{x}\right)}{\partial x}+\frac{\partial\left(h a_{y}\right)}{\partial y}+\frac{\partial a_{z}}{\partial z}\right], \\
\nabla \times \boldsymbol{a} & =\frac{1}{h}\left[\frac{\partial\left(h a_{z}\right)}{\partial y}-\frac{\partial a_{y}}{\partial z}\right] \boldsymbol{x}+\frac{1}{h}\left[\frac{\partial a_{x}}{\partial z}-\frac{\partial\left(h a_{z}\right)}{\partial x}\right] \boldsymbol{y}+\left[\frac{\partial a_{y}}{\partial x}-\frac{\partial a_{x}}{\partial y}\right] \boldsymbol{z} \\
\Delta \psi & =\frac{1}{h}\left[\frac{\partial}{\partial x}\left(h \frac{\partial \psi}{\partial x}\right)+\frac{\partial}{\partial y}\left(h \frac{\partial \psi}{\partial y}\right)+\frac{\partial}{\partial z}\left(\frac{1}{h} \frac{\partial \psi}{\partial z}\right)\right]
\end{aligned}
$$




\section{Appendix B \\ Physical Formulae and Parameters}

\section{B.1 Physical Constants}

$\begin{array}{llll}\text { Speed of light in vacuum } & c & =2.99792458 \times 10^{8} & \mathrm{~m} / \mathrm{s} \\ \text { Electric charge unit } & e & =1.602176462 \times 10^{-19} \mathrm{C} \\ \text { Electron rest energy } & m_{\mathrm{e}} c^{2} & =0.510998902 & \mathrm{MeV} \\ \text { Fine structure constant } & \alpha & =1 / 137.04 & \\ \text { Avogadro's number } & A & =6.0221367 \times 10^{23} & 1 / \mathrm{mol} \\ \text { Molar volume at STP } & & 22.41410 \times 10^{-3} & \mathrm{~m}^{3} / \mathrm{mol} \\ \text { Atomic mass unit } & \text { amu } & =931.49432 & \mathrm{MeV} \\ \text { Classical electron radius } & r_{\mathrm{e}} & =2.8179403 \times 10^{-15} & \mathrm{~m} \\ \text { Classical proton radius } & r_{\mathrm{p}} & =1.534698 \times 10^{-18} & \mathrm{~m} \\ \text { p/e mass ratio } & m_{\mathrm{p}} / m_{\mathrm{e}} & =1836.2 & \\ \text { Planck's constant } & h & =4.1356692 \times 10^{-15} & \mathrm{eVs} \\ \text { Planck's constant } & \hbar & =6.5821220 \times 10^{-16} & \mathrm{eVs} \\ & \hbar c & =197.327053 & \mathrm{MeV} \mathrm{s} \\ \text { Compton wavelength } & \lambda_{\mathrm{C}} & =2.42631058 \times 10^{-12} & \mathrm{~m} \\ \lambda \text { of a 1eV photon } & \hbar c / e & =12398.424 & \AA \\ \text { Thomson cross-section } & \sigma_{\mathrm{T}} & =0.66524616 \times 10^{-28} & \mathrm{~m}^{2} \\ \text { Boltzmann constant } & k & =1.3806568 \times 10^{-23} & \mathrm{~J} / \mathrm{K} \\ \text { Stephan-Boltzmann } & \sigma & =5.67051 \times 10^{-8} & \mathrm{Wm}{ }^{-2} \mathrm{~K}-4\end{array}$




\section{B.2 Relations of Fundamental Parameters}

$$
\text { fine structure constant } \quad \begin{aligned}
\alpha & =\frac{e^{2}}{4 \pi \epsilon_{0} \hbar c} \\
\text { classical electron radius } r_{\mathrm{c}} & =\frac{e^{2}}{4 \pi \epsilon_{0} m_{\mathrm{e}} c^{2}} \\
e^{2} & =14.399652 \mathrm{eVA}
\end{aligned}
$$

\section{B.3 Unit Conversions}

$$
\begin{aligned}
& \text { permittivity of vacuum } \epsilon_{0}=8.85418782 \times 10^{-12} \mathrm{C} /(\mathrm{Vm}) \\
& \text { permeability of vacuum } \mu_{0}=1.25663706 \times 10^{-6} \quad \mathrm{Vs} /(\mathrm{Am})
\end{aligned}
$$

See Tables B.1, B.2, and B.3.

Table B.1 Energy conversion table

\begin{tabular}{l|l|l|l|l|l}
\hline & $\begin{array}{l}\text { Calories } \\
(\mathrm{cal})\end{array}$ & $\begin{array}{l}\text { Joule } \\
(\mathrm{J})\end{array}$ & $\begin{array}{l}\text { eVolt } \\
(\mathrm{eV})\end{array}$ & $\begin{array}{l}\text { Wavenumber } \\
(1 / \mathrm{cm})\end{array}$ & $\begin{array}{l}\text { degKelvin } \\
\left({ }^{\circ} \mathrm{K}\right)\end{array}$ \\
\hline $1 \mathrm{cal}$ & 1 & 4.186 & $2.612710^{19}$ & $2.107310^{23}$ & $3.031910^{23}$ \\
\hline $1 \mathrm{~J}$ & 0.23889 & 1 & $6.241510^{18}$ & $5.034210^{22}$ & $7.242910^{22}$ \\
\hline $1 \mathrm{eV}$ & $3.827410^{-20}$ & $1.602210^{-19}$ & 1 & 8065.8 & 11604 \\
\hline $1 \mathrm{~cm}$ & $4.745310^{-24}$ & $1.986410^{-23}$ & $1.239810^{-4}$ & 1 & 1.4387 \\
\hline $1^{\circ} \mathrm{K}$ & $3.298410^{-24}$ & $1.380710^{-23}$ & $8.617610^{-5}$ & 0.69507 & 1 \\
\hline
\end{tabular}

Table B.2 Equation conversion factors

\begin{tabular}{l|l|l}
\hline Variable & Replace cgs variable & By SI variable \\
\hline Potential, voltage & $V_{\mathrm{cgs}}$ & $\sqrt{4 \pi \epsilon_{0}} V_{\mathrm{MKS}}$ \\
\hline Electric field & $E_{\mathrm{cgs}}$ & $\sqrt{4 \pi \epsilon_{0}} E_{\mathrm{MKS}}$ \\
\hline Current, current density & $I_{\mathrm{cgs}}, j_{\mathrm{cgs}}$ & $1 / \sqrt{4 \pi \epsilon_{0}} I_{\mathrm{MKS}}, j_{\mathrm{MKS}}$ \\
\hline Charge, charge density & $q, \rho$ & $1 / \sqrt{4 \pi \epsilon_{0}} q_{\mathrm{MKS}}, \rho_{\mathrm{MKS}}$ \\
\hline Resistance & $R_{\mathrm{cgs}}$ & $\sqrt{4 \pi \epsilon_{0}} R_{\mathrm{MKS}}$ \\
\hline Capacitance & $C_{\mathrm{cgs}}$ & $1 / \sqrt{4 \pi \epsilon_{0}} C_{\mathrm{MKS}}$ \\
\hline Inductance & $L_{\mathrm{cgs}}$ & $\sqrt{4 \pi \epsilon_{0}} L_{\mathrm{MKS}}$ \\
\hline Magnetic induction & $B_{\mathrm{cgs}}$ & $\sqrt{4 \pi / \mu_{0}} B_{\mathrm{MKS}}$ \\
\hline & &
\end{tabular}


Table B.3 Numerical conversion factors

\begin{tabular}{l|l|l|l}
\hline Quantity & Label & Replace cgs units & By SI units \\
\hline Voltage & $U$ & $1 \mathrm{esu}$ & $300 \mathrm{~V}$ \\
\hline Electric field & $E$ & $1 \mathrm{esu}$ & $310^{4} \mathrm{~V} / \mathrm{cm}$ \\
\hline Current & $I$ & $1 \mathrm{esu}$ & $10 c=2.997910^{9} \mathrm{~A}$ \\
\hline Charge & $q$ & $1 \mathrm{esu}$ & $(10 c)^{-1}=3.335610^{-10} \mathrm{C}$ \\
\hline Resistance & $R$ & $1 \mathrm{~s} / \mathrm{cm}$ & $8.987610^{11} \Omega$ \\
\hline Capacitance & $C$ & $1 \mathrm{~cm}$ & $(1 / 8.9876) 10^{-11} \mathrm{~F}$ \\
\hline Inductance & $L$ & $1 \mathrm{~cm}$ & $110^{9} \mathrm{Hy}$ \\
\hline Magnetic induction & $B$ & $1 \mathrm{G}$ & $310^{-4} \mathrm{~T}$ \\
\hline Magnetic field & $H$ & $1 \mathrm{Oersted}$ & $1000 / 4 \pi=79.577 \mathrm{~A} / \mathrm{m}$ \\
\hline Force & $f$ & $1 \mathrm{dyn}$ & $10^{-5} \mathrm{~N}$ \\
\hline Energy & $E$ & $1 \mathrm{erg}$ & $10^{-7} \mathrm{~J}$ \\
\hline
\end{tabular}

\section{B.4 Maxwell's Equations}

$$
\begin{aligned}
& \nabla \boldsymbol{E}=\quad \frac{1}{\epsilon_{0} \epsilon} \rho, \quad \text { Coulomb's law } \\
& \nabla \boldsymbol{B}=\quad 0 \\
& \boldsymbol{\nabla} \times \boldsymbol{E}=\quad-\frac{\partial \boldsymbol{B}}{\partial t}, \quad \text { Faraday's law } \\
& \boldsymbol{\nabla} \times \boldsymbol{B}=\mu_{0} \mu \rho \boldsymbol{v}+\frac{\epsilon \mu}{c^{2}} \frac{\partial \boldsymbol{E}}{\partial t} . \quad \text { Ampère's law } \\
& \boldsymbol{F}=q \boldsymbol{E}+q[\boldsymbol{v} \times \boldsymbol{B}] \quad \text { Lorentz force }
\end{aligned}
$$

where $\epsilon, \mu$ are the relative dielectricity and permeability, respectively.

\section{B.5 Wave and Field Equations}

\section{Definition of potentials}

$$
\begin{array}{ll}
\text { vector potential } \boldsymbol{A}: & \boldsymbol{B}=\boldsymbol{\nabla} \times \boldsymbol{A} \\
\text { scalar potential } \varphi: & \boldsymbol{E}=-\frac{\partial \boldsymbol{A}}{\partial t}-\nabla \varphi
\end{array}
$$

\section{Wave equations in vacuum}

$$
\boldsymbol{\Delta} \boldsymbol{A}-\frac{1}{c^{2}} \frac{\partial^{2} \boldsymbol{A}}{\partial t^{2}}=-\frac{\rho \boldsymbol{\beta}}{\epsilon_{0} c}
$$




$$
\Delta \varphi-\frac{1}{c^{2}} \frac{\partial^{2} \varphi}{\partial t^{2}}=-\frac{\rho}{\epsilon_{0}}
$$

Vector and scalar potential in vacuum

$$
\begin{aligned}
& \boldsymbol{A}(t)=\left.\frac{1}{4 \pi c^{2} \epsilon_{0}} \int \frac{\boldsymbol{v} \rho(x, y, z)}{R}\right|_{t_{\mathrm{ret}}} \mathrm{d} x \mathrm{~d} y \mathrm{~d} z \\
& \varphi(t)=\left.\frac{1}{4 \pi \epsilon_{0}} \int \frac{\rho(x, y, z)}{R}\right|_{t_{\mathrm{ret}}} \mathrm{d} x \mathrm{~d} y \mathrm{~d} z
\end{aligned}
$$

Vector and scalar retarded potentials for a point charge $q$ in vacuum

$$
\begin{aligned}
& \boldsymbol{A}(P, t)=\left.\frac{1}{4 \pi c \epsilon_{0}} \frac{q}{R} \frac{\boldsymbol{\beta}}{1+\boldsymbol{n} \boldsymbol{\beta}}\right|_{t_{\mathrm{ret}}} \\
& \varphi(P, t)=\left.\frac{1}{4 \pi \epsilon_{0}} \frac{q}{R} \frac{1}{1+\boldsymbol{n} \boldsymbol{\beta}}\right|_{t_{\mathrm{ret}}}
\end{aligned}
$$

\section{Radiation field in vacuum}

$$
\begin{aligned}
\boldsymbol{E}(t) & =\left.\frac{1}{4 \pi c \epsilon_{0}} \frac{q}{r^{3}}\{\boldsymbol{R} \times[(\boldsymbol{R}+\beta \boldsymbol{R}) \times \dot{\boldsymbol{\beta}}]\}\right|_{t_{\mathrm{ret}}} \\
c \boldsymbol{B}(t) & =[\boldsymbol{E} \times \boldsymbol{n}]_{t_{\mathrm{ret}}}
\end{aligned}
$$

\section{B.6 Relativistic Relations}

\section{B.6.1 Lorentz Transformation}

Quantities $x^{*}$ etc. are taken in the particle system $\mathcal{L}^{*}$, while quantities $x$ etc. refer to the laboratory system $\mathcal{L}$. The particle system $\mathcal{L}^{*}$ is assumed to move at the velocity $\beta$ along the $z$-axis with respect to the laboratory system $\mathcal{L}$.

\section{Lorentz transformation of coordinates}

$$
\left(\begin{array}{c}
x^{*} \\
y^{*} \\
z^{*} \\
c t^{*}
\end{array}\right)=\left(\begin{array}{cccc}
1 & 0 & 0 & 0 \\
0 & 1 & 0 & 0 \\
0 & 0 & \gamma & -\beta \gamma \\
0 & 0 & -\beta \gamma & \gamma
\end{array}\right)\left(\begin{array}{l}
x \\
y \\
z \\
c t
\end{array}\right) .
$$

Lorentz transformation of frequencies (relativistic Doppler effect)

$$
\omega=\omega^{*} \gamma\left(1+\beta n_{z}^{*}\right)
$$


Lorentz transformation of angles (collimation)

$$
\theta \approx \frac{\sin \theta^{*}}{\gamma\left(1+\beta \cos \theta^{*}\right)} .
$$

\section{B.6.2 Four-Vectors}

Properties of 4-vectors are used in this text to transform physical phenomena from one inertial system to another.

Space-time 4-vector

$$
\tilde{s}=(x, y, z, \mathrm{i} c t)
$$

\section{World time}

$$
c \tau=\sqrt{-\tilde{s}^{2}} .
$$

\section{Properties of 4-vectors}

Lorentz transformation of time. From (B.16)

$$
\begin{aligned}
c \mathrm{~d} \tau & =\sqrt{c^{2}(\mathrm{~d} t)^{2}-(\mathrm{d} x)^{2}-(\mathrm{d} y)^{2}-(\mathrm{d} z)^{2}} \\
& =\sqrt{c^{2}-\left(v_{x}^{2}+v_{y}^{2}+v_{z}^{2}\right)} \mathrm{d} t \\
& =\sqrt{c^{2}-v^{2}} \mathrm{~d} t=\sqrt{1-\beta^{2}} c \mathrm{~d} t
\end{aligned}
$$

or

$$
\mathrm{d} \tau=\frac{1}{\gamma} \mathrm{d} t
$$

Velocity 4-vector

$$
\tilde{v}=\frac{\mathrm{d} \tilde{s}}{\mathrm{~d} \tau}=\gamma \frac{\mathrm{d} \tilde{s}}{\mathrm{~d} t}=\gamma(\dot{x}, \dot{y}, \dot{z}, \mathrm{i} c)
$$

\section{4-acceleration}

$$
\tilde{a}=\frac{\mathrm{d} \tilde{v}}{\mathrm{~d} \tau}=\gamma \frac{\mathrm{d}}{\mathrm{d} t}\left(\gamma \frac{\mathrm{d} \tilde{s}}{\mathrm{~d} t}\right)
$$


4-acceleration $\tilde{a}=\left(\tilde{a}_{x}, \tilde{a}_{y}, \tilde{a}_{z}, \mathrm{i} \tilde{a}_{t}\right)$ in component form

$$
\tilde{a}_{x}=\gamma^{2} a_{x}+\gamma^{4} \beta_{x}(\boldsymbol{\beta} \boldsymbol{a}),
$$

where $\boldsymbol{a}$ is the ordinary acceleration.

\section{B.6.3 Square of the 4-Acceleration}

$$
\tilde{a}^{2}=\gamma^{6}\left\{\boldsymbol{a}^{2}-[\boldsymbol{\beta} \times \boldsymbol{a}]^{2}\right\}=\tilde{a}^{* 2} .
$$

in particle system $(\beta=0, \gamma=1)$

$$
\tilde{a}^{* 2}=a^{* 2} \text {. }
$$

\section{B.6.4 Miscellaneous 4-Vectors and Lorentz Invariant Properties}

\section{B.7 Transformation Matrices in Beam Dynamics}

In this section, we will collect transformation matrices for elements discussed in various parts of this book. Generally, we assume the following designations:

$$
\begin{aligned}
& C(z), S(z), D(z) \quad\left\{\begin{array}{l}
\text { cosine and sine like solution and dispersion } \\
\text { function, respectively }
\end{array}\right. \\
& C^{\prime}(z), S^{\prime}(z), . \text { etc. derivatives are taken with respect to } z \\
& u(z) \quad u(z) \text { can be either } x(z) \text { or } y(z) \\
& \ell \quad \text { path (arc) length of element } \\
& \delta=\Delta p / p_{0} \quad \text { relative momentum error } \\
& \varphi=\sqrt{\left|k_{0}\right| \ell} \quad \text { quadrupole phase } \\
& f \quad \text { quadrupole focal length } \\
& \Theta=\sqrt{\left|k+\kappa_{x}^{2}\right|} \ell \quad \text { deflection angle of synchrotron magnet } \\
& \theta=\kappa_{x} \ell=\ell / \rho_{0} \quad \text { deflection angle of bending magnet } \\
& \eta_{0} \text { and } \eta_{\mathrm{e}} \\
& L \\
& 2 G \quad \text { full magnet gap aperture } \\
& \left\{\begin{array}{l}
\text { magnet entrance and exit angles with respect to } \\
\text { sector magnet, } \eta_{0}=\eta_{\mathrm{e}}=-\theta / 2<0 \text { for rect. magnet }
\end{array}\right. \\
& \text { straight length of bending magnet }
\end{aligned}
$$




\begin{tabular}{ll}
\hline 4-vector: & invariance of \\
\hline space-time & world time \\
$\tilde{\boldsymbol{s}}=(\boldsymbol{r}, \mathrm{i} c t)$ & $\tilde{\boldsymbol{s}}^{2}=-c^{2} \tau^{2}$ \\
\hline momentum-energy & mass \\
$c \tilde{\boldsymbol{p}}=(c \boldsymbol{p}, \mathrm{i} E)$ & $c^{2} \tilde{\boldsymbol{p}}^{2}=-A^{2} m^{2} c^{4}$ \\
\hline wave number & isotropy of space \\
$c \tilde{\boldsymbol{k}}=(c \boldsymbol{k}, \mathrm{i} \omega)$ & $c^{2} \tilde{\boldsymbol{k}}^{2}=0$ \\
\hline velocity & speed of light \\
$\tilde{\boldsymbol{v}}=\gamma(\dot{\boldsymbol{r}}, \mathrm{i} c)$ & $\tilde{\boldsymbol{v}}^{2}=-c^{2}$ \\
\hline acceleration & radiation intensity or Poynting vector \\
$\tilde{\boldsymbol{a}}=\gamma^{2} \boldsymbol{a}+\gamma^{4} \boldsymbol{\beta}(\boldsymbol{\beta} \boldsymbol{a})$ & $\tilde{\boldsymbol{a}}^{2}=\left[\gamma^{2} \boldsymbol{a}+\gamma^{4} \boldsymbol{\beta}(\boldsymbol{\beta} \boldsymbol{a})\right]^{2}$ \\
\hline current divergence & charge conservation \\
$\widetilde{\nabla \boldsymbol{j}}=\left(-\nabla \boldsymbol{j}, \mathrm{i} \frac{\partial \rho}{\partial t}\right)$ & $(\boldsymbol{\nabla} \boldsymbol{j})^{2}-\left(\frac{\partial \rho}{\partial t}\right)^{2}=-\left(\frac{\partial \rho_{0}}{\partial t}\right)^{2}=0$ \\
\hline current density & charge density \\
$\tilde{\boldsymbol{j}}=(\boldsymbol{j}, \mathrm{i} \rho c)$ & $\widetilde{\boldsymbol{j}}^{2}=-\rho_{0}^{2} c^{2}$ \\
\hline force, & inertial system \\
$\widetilde{\tilde{\boldsymbol{p}}}=(c \dot{\boldsymbol{p}}, \mathrm{i} \dot{E})$ & $c^{2} \widetilde{\boldsymbol{p}}^{2}=0$ \\
\hline field potential & Maxwell's equations \\
$\widetilde{\boldsymbol{A}}=(\boldsymbol{A}, \mathrm{i} \phi)$ & \\
$4-$ divergence & $\mathrm{d}^{\prime}$ Alambertian \\
$\widetilde{\boldsymbol{\nabla}}=\left(-\boldsymbol{\nabla}, \mathrm{i} \frac{\partial}{\partial t}\right)$ & $\square=\nabla^{2}-\frac{\partial^{2}}{\partial t^{2}}$ \\
\hline$\tau:$ world time & \\
\hline
\end{tabular}

\section{B.8 General Transformation Matrix}

$$
\left(\begin{array}{c}
u(z) \\
u^{\prime}(z) \\
\delta
\end{array}\right)=\left(\begin{array}{ccc}
C(z) & S(z) & D(z) \\
C^{\prime}(z) & S^{\prime}(z) & D^{\prime}(z) \\
0 & 0 & 1
\end{array}\right)\left(\begin{array}{c}
u_{0}(z) \\
u_{0}^{\prime}(z) \\
\delta
\end{array}\right)=\mathcal{M}\left(\begin{array}{c}
u_{0}(z) \\
u_{0}^{\prime}(z) \\
\delta
\end{array}\right)
$$

\section{B.8.1 Symmetric Magnet Arrangement}

$$
\mathcal{M}_{\mathrm{tot}}=\mathcal{M}_{\mathrm{r}} \mathcal{M}=\left(\begin{array}{cc}
C S^{\prime}+S C^{\prime} & 2 S C S^{\prime} \\
2 C C^{\prime} & C S^{\prime}+S C^{\prime}
\end{array}\right)
$$

In a symmetric magnet or lattice segment, the diagonal elements of the transformation matrix are equal. 


\section{B.8.2 Inverse Transformation Matrix}

$$
\text { If } \mathcal{M}=\left(\begin{array}{cc}
C & S \\
C^{\prime} & S^{\prime}
\end{array}\right) \Longrightarrow \text { inverse matrix is } \mathcal{M}_{\mathrm{i}}=\left(\begin{array}{cc}
S^{\prime} & -S \\
-C^{\prime} & C
\end{array}\right) \text {, }
$$

and the total transformation matrix is $\mathcal{M}_{\text {tot }}=\mathcal{M}_{\mathrm{i}} \mathcal{M}=\left(\begin{array}{ll}1 & 0 \\ 0 & 1\end{array}\right)$

\section{B.9 Specific Transformation Matrices}

\section{B.9.1 Drift Space}

Length of drift space is $\ell$

$$
\mathcal{M}_{\mathrm{d}}=\left(\begin{array}{lll}
1 & \ell & 0 \\
0 & 1 & 0 \\
0 & 0 & 1
\end{array}\right)
$$

\section{B.9.2 Bending Magnets}

\section{Sector Magnet}

With $\ell$ being the arc length and $\rho_{0}$ the bending radius, the transformation matrices are in deflecting plane

$$
\mathcal{M}_{\mathrm{s}, \rho}=\left(\begin{array}{ccc}
\cos \theta & \rho \sin \theta & \rho(1-\cos \theta) \\
-\frac{1}{\rho} \sin \theta & \cos \theta & \sin \theta \\
0 & 0 & 1
\end{array}\right)
$$

and in the non-deflecting plane (including edge focusing)

$$
\mathcal{M}_{\mathrm{s}, 0}=\left(\begin{array}{ccc}
1+\frac{1}{3} \theta \delta_{\mathrm{f}} & \ell & 0 \\
\frac{2}{3} \frac{\delta_{\mathrm{f}}}{\rho}+\frac{1}{9} \theta \frac{\delta_{\mathrm{f}}^{2}}{\rho} & 1+\frac{1}{3} \theta \delta_{\mathrm{f}} & 0 \\
0 & 0 & 1
\end{array}\right),
$$

where $\delta_{\mathrm{f}}=G / \rho$ and $2 G$ the gap size between magnet poles. 


\section{Wedge Magnet}

In deflecting plane

$$
\mathcal{M}_{\mathrm{w}, \rho}=\left(\begin{array}{ccc}
\cos \theta-\sin \theta \tan \eta_{0} & \rho \sin \theta & \rho(1-\cos \theta) \\
-\frac{\tan \eta_{\mathrm{e}}+\tan \eta_{0}}{\rho} \cos \theta & \sin \theta \\
-\frac{1-\tan \eta_{\mathrm{e}} \tan \eta_{0}}{\rho} \sin \theta & \cos \theta-\sin \theta \tan \eta_{\mathrm{e}} & -\tan \eta_{\mathrm{e}}(1-\cos \theta) \\
0 & 0 & 1
\end{array}\right)
$$

and in the non-deflecting plane

$$
\mathcal{M}_{\mathrm{w}, 0}(\ell \mid 0)=\left(\begin{array}{ccc}
1-\frac{\ell}{\rho} t_{0} & \ell & 0 \\
-\frac{1}{\rho}\left(t_{\mathrm{e}}+t_{0}\right)+\frac{\ell}{\rho^{2}} t_{\mathrm{e}} t_{0} & 1-\frac{\ell}{\rho} t_{\mathrm{e}} & 0 \\
0 & 0 & 1
\end{array}\right),
$$

where $\mathrm{t}_{0, \mathrm{e}}=-\tan \eta_{0, \mathrm{e}}-\frac{1}{3} \delta_{0, \mathrm{e}}$ and $\delta_{0, \mathrm{e}}=\frac{G}{\rho \cos ^{2} \eta_{0, \mathrm{e}}}$.

\section{Rectangular Magnet}

In the deflecting plane with $\eta_{0}=\eta_{\mathrm{e}}=-\theta / 2$

$$
\mathcal{M}_{\mathrm{r}, \rho}(\ell \mid 0)=\left(\begin{array}{ccc}
1 & \rho \sin \theta & \rho(1-\cos \theta) \\
0 & 1 & 2 \tan (\theta / 2) \\
0 & 0 & 1
\end{array}\right),
$$

and in the non-deflecting plane

$$
\mathcal{M}_{\mathrm{r}, 0}(\ell \mid 0)=\left(\begin{array}{ccc}
1-\frac{\ell}{f_{y}} & \ell & 0 \\
-\frac{2}{f_{y}}+\frac{\ell}{f_{y}^{2}} & 1-\frac{\ell}{f_{y}} & 0 \\
0 & 0 & 1
\end{array}\right)
$$

where $\frac{1}{f_{y}}=\frac{1}{\rho} \tan \left(\frac{\theta}{2}\right)\left(1-\frac{2 G}{3 L}\right), L=2 \rho \sin \frac{\ell}{2 \rho_{0}}$ the straight length and $\ell$ the arc length of the magnet. 


\section{Synchrotron Magnet (Sector Type)}

For a focusing synchrotron magnet with $K=k+\kappa_{x}^{2}>0$ the transformation matrix in the deflecting plane is with $\left(\Theta=\sqrt{K} \ell, \kappa_{x}=1 / \rho\right)$

$$
\mathcal{M}_{\mathrm{sy}, \mathrm{f}}(\ell, 0)=\left(\begin{array}{ccc}
\cos \Theta & \frac{1}{\sqrt{K}} \sin \Theta & \frac{1-\cos \Theta}{\rho K} \\
-\sqrt{K} \sin \Theta & \cos \Theta & \frac{\sin \Theta}{\rho \sqrt{K}} \\
0 & 0 & 1
\end{array}\right)
$$

and when $K=k+\kappa_{x}^{2}<0$ the transformation matrix for a defocusing synchrotron magnet is

$$
\mathcal{M}_{\text {sy,d }}(\ell \mid 0)=\left(\begin{array}{ccc}
\cosh \Theta & \frac{\sinh \Theta}{\sqrt{|K|}} & -\frac{1-\cosh \Theta}{\rho|K|} \\
\sqrt{|K|} \sinh \Theta & \cosh \Theta & \frac{\sinh \Theta}{\rho \sqrt{|K|}} \\
0 & 0 & 1
\end{array}\right)
$$

In the non-deflecting plane the transformation matrices are with $(\psi=\sqrt{k} \ell>0)$

$$
\mathcal{M}_{s y, s, 0}=\left(\begin{array}{ccc}
\cos \psi & \frac{1}{\sqrt{k}} \sin \psi & 0 \\
-\sqrt{k} \sin \psi & \cos \psi & 0 \\
0 & 0 & 1
\end{array}\right)
$$

and for $k<0$ and $\psi=\sqrt{|k|} \ell$

$$
\mathcal{M}_{\mathrm{sy}, \mathrm{s}, \mathrm{d}}=\left(\begin{array}{ccc}
\cosh \psi & \frac{1}{\sqrt{|k|}} \sinh \psi & 0 \\
\sqrt{|k|} \sinh \psi & \cosh \psi & 0 \\
0 & 0 & 1
\end{array}\right)
$$

\section{Synchrotron Magnet (Rectangular Type)}

For a focusing synchrotron magnet $K=k_{0}+\kappa_{x}^{2}>0$ and with $\Theta=\sqrt{K} \ell$, $\theta=\ell / \rho, \kappa_{x}=1 / \rho, \frac{1}{f}=\sqrt{K} \tan \theta / 2$

$$
\mathcal{M}_{\mathrm{sy}, \mathrm{r}, \mathrm{f}}=\left(\begin{array}{ccc}
\cos \Theta+\frac{1}{f \sqrt{K}} \sin \Theta & \frac{\sin \Theta}{\sqrt{K}} & \frac{(1-\cos \Theta)}{\rho K} \\
\frac{1}{f}\left(\cos \Theta+\frac{1}{f \sqrt{K}} \sin \Theta\right) & \cos \Theta & \sin \Theta \\
+\frac{1}{f} \cos \Theta-\sqrt{K} \sin \Theta & +\frac{1}{f \sqrt{K}} \sin \Theta & \frac{\cos }{\rho \sqrt{K}} \\
0 & 0 & 1
\end{array}\right)
$$


In a defocusing synchrotron magnet $K=k_{0}+\kappa_{x}^{2}<0$ and with $\Theta=\sqrt{|K|} \ell, \theta=\ell / \rho, \kappa_{x}=1 / \rho, \frac{1}{f}=\sqrt{|K|} \tan \theta / 2$

$$
\mathcal{M}_{\mathrm{sy}, \mathrm{r}, \mathrm{d}}=\left(\begin{array}{ccc}
\cosh \Theta+\frac{\sinh \Theta}{f \sqrt{K}} & \frac{\sinh \Theta}{\sqrt{K}} & -\frac{(1-\cosh \Theta)}{\rho \sqrt{K}} \\
\frac{1}{f}\left(\cosh \Theta+\frac{\sinh \Theta}{f \sqrt{K}}\right) & \cosh \Theta & \frac{\sinh \Theta}{\rho \sqrt{K}} \\
+\frac{1}{f} \cosh \Theta+\sqrt{K} \sinh \Theta & +\frac{1}{f \sqrt{K}} \sinh \Theta & +\frac{1-\cosh \Theta}{f \rho|K|} \\
0 & 0 & 1
\end{array}\right)
$$

\section{B.9.3 Quadrupole}

Focusing Quadrupole $\left(k_{0}>0, \varphi=\sqrt{k} \ell\right)$

$$
\mathcal{M}_{\mathrm{QF}}=\left(\begin{array}{ccc}
\cos \varphi & \frac{1}{\sqrt{k}} \sin \varphi & 0 \\
-\sqrt{k} \sin \varphi & \cos \varphi & 0 \\
0 & 0 & 1
\end{array}\right)
$$

Defocusing Quadrupole $(k<0, \varphi=\sqrt{|k| \ell})$

$$
M_{\mathrm{QD}}=\left(\begin{array}{ccc}
\cosh \varphi & \frac{1}{\sqrt{|k|}} \sinh \varphi & 0 \\
\sqrt{|k|} \sinh \varphi & \cosh \varphi & 0 \\
0 & 0 & 1
\end{array}\right)
$$

\section{Quadrupole Doublet}

A quadrupole doublet formed by two quadrupoles of focal length $f_{1}$ and $f_{2}$ and separated by the distance $d$ has the transformation matrix

$$
\mathcal{M}_{\mathrm{db}}=\left(\begin{array}{ccc}
1-d / f_{1} & d & 0 \\
-1 / f^{*} & 1-d / f_{2} & 0 \\
0 & 0 & 1
\end{array}\right) \text {, }
$$

where $\frac{1}{f^{*}}=\frac{1}{f_{1}}+\frac{1}{f_{2}}-\frac{d}{f_{1} f_{2}}$. 


\section{Quadrupole Triplet}

Symmetric quadrupole triplet made of two equal doublets

$$
\mathcal{M}_{\mathrm{tr}}=\mathcal{M}_{\mathrm{r}} \mathcal{M}=\left(\begin{array}{ccc}
1-2 d^{2} / f^{2} & 2 d(1+d / f) & 0 \\
-1 / f^{*} & 1-2 d^{2} / f^{2} & 0 \\
0 & 0 & 1
\end{array}\right)
$$

where $\frac{1}{f^{*}}=\frac{1}{f_{1}}+\frac{1}{f_{2}}-\frac{d}{f_{1} f_{2}}$. 


\section{Index}

Aberration, 546, 573

chromatic, 108, 164, 338, 509, 514, 565. 584

geometric, 108, 164, 338, 513, 565, 575

Accelerating

cavities, 68

cavity fields, 253, 257

rf wave, 256

section, 44, 254, 255, 258, 272, 477, 629

voltage, 262, 263, 627

Acceleration, 256

charged particles, 270

electrostatic field, 253

longitudinal, 831

transverse, 831

Accelerator

Alvarez structure, 56

application, 10

circular, 43, 368

orbit, 315

quasi-isochronous, 279

Cockcroft-Walton, 46

ion, 21

laser, 278

linear, 43

focusing, 339

proton, 21

Van de Graaff, 47

Wideroe structure, 258

Acceptance, 281

efficiency, 283

longitudinal, 281

physical ring, 392

Accumulator ring, 8
Achromat, 207, 208, 240

double bend, 469

first order, 208

linear, 240

Achromatic lattice, 207

Action, 235

Action-angle variables, 115, 548, 581, 594

Adiabatic capture, 283

Adiabatic damping, 66, 67, 345, 356, 365, 404, 414, 437

ADONE, 336

Airy's functions, 885

Algebraic relations, 984

Alignment, 565

error, 477,565

tolerances, 490-492

Alpha magnet, 449

Alvarez structure, 258

Ampere turns, 140

Ampère's law, 14, 27, 35, 995

Amplification factor, 491

Antiprotons, 7

Aperture, 104, 133

Aperture limitation, 385

Approximations made

$$
c t_{\mathrm{r}}= \pm \rho / \gamma, 877
$$

$\sin \left(\omega_{\mathrm{L}} t_{\mathrm{r}}\right) \approx \omega_{\mathrm{L}} t_{\mathrm{r}}, 877$

Arc length, 192

AS(x.y.z), 882

Astigmatism, 164

Asymmetric wiggler, 921

Attenuation coefficient, 627, 628, 630

Avogadro's number, 388

Ampère's law, 30 
Backscattered photons, 828

Beam-beam effect, 337, 477, 713

Beam break-up, 779

Beam-cavity interaction, 641

Beam center displacement, 481

Beam current, 12, 354

average current, 354

bunch current, 354

Fourier spectrum, 657, 658

maximum, 649

optimum linac, 631

peak current, 354

pulsed beam, 354

Beam dynamics, 26, 30, 99, 106

with acceleration, 341

action, 116

basic elements, 205

beam size, 222

beam waist, 220

with betatron functions, 231

circular accelerator, 315

collective motion, 213

coupling, 213

dispersion function, 122

dispersive systems, 236

divergence, 375

equation of motion, 108, 110

Hamiltonian, 113, 234

non-linear, 565

higher order, 565

Lagrangian, 110

linear, 99, 131, 177

linear achromat, 240

linear approximation, 178

longidudinal focusing, 256

longitudinal synchronous phase, 257

matrix formalism, 118, 180

in normalized coordinates, 233

paraxial, 107

perturbation, 477, 480, 487

chromatic, linear, 207

methods, 524

phase space, 213

single particle, 177

skew quadrupole, 670

strong focusing, 116

thin lens approximation, 184

Beam emittance, 214, 223, 235

in achromat lattice minimum value, 462

colliding beams, 472

with damping wiggler, 381

DBA lattice, 469 definition, 357

equilibrium, 441, 442

transverse, 370, 371, 441, 442

geometric, 218, 219

lattice design, 459

longitudinal, 281

measurement, 224

minimum, 462

normalized, 218

in periodic lattice, 468

quantum excitation, 371, 440

scaling, 462

and scattering, 386

time dependance of, 433

in transport line, 371

transverse equilibrium, 368

variation of, 377

and wiggler magnets, 377

Beam energy, 353

Beam envelope, 230, 374

angular, 375

Beam focusing, 63

Beam heater, 946

Beam intensity, 12

Beam lifetime, 385

Bremsstrahlung, 394

Coulomb scattering, 386, 388

elastic scattering, 387

and vacuum, 386

Beam line, 94

Beam loading, 629, 641, 645, 654 fundamental theorem, 650

Beam matrix, 222, 223 transformation, 224

Beam momentum, 353

Beam monitoring, 492

Beam optics, 30, 99

linear, 177

paraxial, 97

Beam position monitors, 494

Beam pulse, 354

Beam rigidity, 101, 129

Beam size, 230, 373

Beam transformer, 60

Beam translation, 242

Beam transport line, 100, 178, 303

acceptance, 332

building blocks, 205

chromatic properties, 206

control of beam size, 531

general focusing properties, 205

isochronous, 208, 210

matching, 332

perturbations, 482 
Beam waist, 220 location, 220

Beat-beat, 508

Bending field, 62 magnet, 139,140 radiation, 802 radius, 33,101

Bessel's functions modified, 877

Beta beating, 585

Beta-beat, 506, 507, 585

Betatron, 5, 6, 60 Donald Kerst, 63 oscillation, 229, 485 phase, 116, 228 tune shift, 500

Betatron function, 227, 229, 356, 850 average, 320 beam dynamics, 231 beam waist, 232 optimum value, 232 beta beating, 507 coupled motion, 678 differential equation, 228, 229 independent variable, 228 measurement, 227, 409, 489 modes, 682 periodic, 305,318 perturbation, 506 smooth approximation, 320 symmetric, 305 symmetric solution, 319 transformation, 220, 318 transformation in drift space, 232 transformation through drift space, 221

variation of, 585

Betatron oscillation, 65, 110 amplitude, 567 damping, 416, 422 frequency, 65, 116 perturbation, 408

Betatron tunes coupled motion, 692

Bevatron, 6

Biot-Savart fields, 863

BNS damping, 781

Booster, 10

Bore radius, 143

BPM offset, 496

Bremsstrahlung, 393

Brho, 129
Brightness, 841, 917

diffraction limited, 849

spectral, 848

Broad band parasitic losses, 740

Bunch, 8, 12, 279

compression, 446 accelerating section, 447

current, 12

Buncher section, 972

Bunches, 354

Bunching, 634, 636

Bunch length, 275, 279, 368, 429, 444, 664, 803

equilibrium, 444

manipulation, 286

Byending, radius, 33

Canonical

momentum, 89

transformation, 90, 268, 548, 588, 589

variables, 92, 268

Capacitive detuning, 658

Carbon content, 144

Cascade generator, 5, 6, 46

Cathode rays, 4, 5

Cauchy-Riemann conditions, 126

Cauchy's residue theorem, 762

Cavity losses, 621 modes, 609 voltage, 649

$C_{B}, 832$

$C_{\mathrm{c}}, 836$

Central limit theorem, 367, 491

Centrifugal force, 33,48

$C_{\gamma}, 367,832$

Characteristic length, 135

Characteristic matrix equation, 310

Charged particles, in an electromagnetic field, 33

Charge multiplicity, 31

Chasman-Green lattice, 241, 469

Cherenkov angle, 806

condition, 806

radiation, 805,806

Chopper, 8, 634, 636 rf, 636

Chromatic aberration, 164, 573 effects, 161, 509 error, 122 
Chromaticity, 138, 509, 510

compensation, 575

correction, 513, 514

FODO cell, 512

function, 522

harmonic correction, 584

higher order, 514

horizontal, 516

measurement, 512

natural, 511

non linear, 517

vertical, 516

Circular accelerator, 9, 43, 59

characteristic parameters, 77

chromatic effects, 509

operating point, 320

periodic dispersion function, 327

periodic perturbation, 528

resonance, 327

superperiod, 330

tune, 320

$C_{\mathrm{K}}, 171$

Closed orbit, 324, 480, 484-487, 489

correction, 494

distortion, 486

equilibrium, 484

Cockcroft-Walton, 7, 46

Coherence

spatial, 844

temporal, 846

Coherent

radiation, 843

radiation power, 847

Coil slot, 144

Collective

effects, 701

instabilities, 701

Colliding beam facility, 9, 331, 337

luminosity, 331

Colliding beams

facility, 25

Collimation, 801 angle, 872, 997

Collins insertion, 336

Collision

head on, 25

particle, 24

point, 338

Combined function lattice, 159, 365

$C_{\Omega}, 836,880$

Complex potential, 126

Composite focusing system, 205

Compton effect, 806, 828
Conjugate

coordinates, 92

momenta, 92

trajectories, 679

Constant gradient structure, 628

Constant impedance structure, 628

Coordinates

Cartesian, 104, 115

curvilinear, 93

Floquet's, 233

Frenet-Serret, 87, 88, 94

normalized, 115, 229, 233

transformation, 988, 989

Coordinate system

Cartesian, 125, 988

curvilinear, 152, 160, 991

cylindrical, 27, 61, 990

Frenet-Serret, 61, 160

general, 989

polar, 26, 990

Cosine like solutions, 118

Cosmotron, 6

Coulomb, 3

egime, 809

field, 862

gauge, 29

multiple scattering, 386

regime, 808,862

scattering lifetime, 389

Coulomb scattering, 387

Coulomb's law, 14, 995

Coupled motion, 164, 669, 686

Coupling coefficient, 642, 648, 649, 657, 688

Coupling resonance, 544, 545

Courant-Snyder invariant, 228, 235, 407

$C_{p}, 77$

$C_{\mathrm{q}}, 367,442$

$C_{\psi}, 838,891$

Critical photon energy, 367, 836

$C_{\mathrm{u}}, 901$

Current, beam, 12

Current density, maximum, 144

Curvature, 33, 87, 100, 107, 129

Curvilinear coordinates, 991

Cutoff, wave number, 606

Cyclic, 115

Cyclic variables, 90, 115, 588

Cyclotron, 5, 70, 101

Cyenter of mass energy, 24

Cylindrical coordinates, 990

Cyolliding beams, 25

Cyollimation, 24

Cyompton effect, 39 
d'Alembert's principle, 48, 83

Damping, 263, 358, 404, 410

adiabatic, 66, 345, 437

distribution, 375

due synchrotron radiation, 412

in electron accelerator, 411

and rf-frequency, 375

ring, 8,447

synchrotron oscillations, 412

time, 67, 364, 485

wiggler, 167, 377, 379, 380

Damping criterion

Robinson, 358, 362

Damping decrement, 67, 263, 362, 364, 405, $410,412,419,422$

Robinson, 660

Damping partition, 364, 375

and synchrotron oscillations, 382

variation of, 375

DBA lattice, 241

Decapole magnet, 128, 154

Deflection angle, 101

Defocusing quadrupole, 183, 344

with acceleration, 344

Design orbit, 61

Deuteron, 6

Dielectric constant, 13

absolute, 14

relative, 14

Difference resonance, 689

Differential relations, 985

Diffraction, 841, 941

Fraunhofer, 841

intergral, Fraunhofer, 841

Diffraction limit, 852

emittance, 844

source divergence, 845

source size, 845

Diffusion coefficient, 425

due synchrotron radiation, 438

total, 439

Dipole, fringe fields, 194

Dipole field, 132

error, 480, 481, 484

statistical errors, 490

Dipole kick, single, 489

Dipole magnet, 132, 138, 139

cross section, 139

focusing in, 190

fringe field, 193, 195

rectangular type, 198

sector, 108, 192

sector type, 191

synchrotron, 192
Dispersion function, 122, 164, 237, 279, 325, 487

dipole field errors, 482

higher order, 287

matrix formalism, 237

measurement, 238

nonlinear terms, 250

periodic, 321, 322, 327

periodic lattice, general solution, 324

spurious, 443

Dispersion suppressor, 334

Dispersive effects, 108

Dispersive systems, 236

Dissipating forces, 120, 406

Divergence, photon beam, 839

Doppler effect, 801, 996

Double bend achromat, 241

Doublet, 205

6-D phase space motion in, 402

Drift space, 182

phase ellipse, 220

transformation betatron function, 221, 232

matrix, 1000

D's of a cyclotron, 71

Duty factor, 356

Dynamic aperture, 152, 385, 392, 513, 587, 588

Dyoppler effect, relativistic, 23

Effective magnet length, 187

Ehrenfest's theorem, 261

Eigenvalue equation, 310, 319, 326

Eigenvalues, 310, 326

Einzellens, 52

focusing properties, 53

Electric and magnetic field equivalence, 99

Electric charge of electron, 5

Electric field

point charge, 26

static, 7

uniform charged particle beam, 27

Electromagnetic

radiation, 4

waves, 4

Electromagnetic fields, 26

for a Gaussian beam, 38

orthogonality, 39

for uniform beam, 38

Electromagnetic radiation, 799, 800 
Electromagnetic waves

standing, 255

traveling, 255

Electromotive force, 30

Electron, 3, 4

beam, 803

gun, 8

source

cathode, 8

photo cathode, 8

Volt, 11

Electrostatic

deflector, 48

dipole, 56

einzellens, 52

focusing device, 49

generator, 47

iris electrode, 49

transformation matrix, 51

quadrupole, 172

Electrostatic field, 48, 159

multipole, 159

patterns, 159

Emittance

adiabatic damping, 345

coupling, 692

diffraction limited, 844, 852

longitudinal, 268

minimum, 462

normalized, 345, 356

Energy, 12, 21

center of mass, 24

conservation, 35, 36, 804

errors, 509

gain, total, 262

kinetic, 89

potential, 89

spectrum, measurement, 245

total, 20

Energy loss, 67, 833

due synchrotron radiation, 415

Energy spread, 366, 429, 625

elimination of, 384

equilibrium, 367, 444

variation of, 378

Envelope, coupled motion, 683

Equation of motion, 31, 32, 34, 44, 106

chromatic effects, 161, 164

cosine-like solution, 205

dispersion, 164, 207

general, 161-163

horizontal, 108, 113

inhomogeneous, 121

from Lagrangian, 98 linear, 117

with perturbations, 120

unperturbed, 117

perturbation terms, 163

in phase space, longitudinal, 259

sine-like solution, 206

in solenoid, 672

solutions, 116

third order, 160, 522

vertical, 109, 114

Equations

Euler-Lagrange, 85

Hamilton, 88

Equations of motion

coupled systems, 669

from Hamiltonian, 97

homogeneous, 117

Equilibrium orbit, 324, 325, 484, 486, 487

existence of, 484

Equipotential surface, 48, 105, 132, 133, 141

$\eta$-function, 322, 325

average, 329

Euler - Lagrange equations, 84, 85

Euler's formula, 311

Euler's identity, 676

Excitation, 140

Faraday's law, 14, 27, 30, 995

Fast head-tail instability, 781, 787 fast, 781

Feed-forward, 493

FEL, 261, 279, 803, 929

high gain, 942

parameter, 944

peak pulse power, 944

small gain, 932

Field errors, 142, 484, 566

sources, 478

statistical dipole, 490

Field gradient, 103, 104 maximum, 145

Field index, 64, 66, 74

Fields, 128

electrostatic, 43

high frequency electromagnetic, 254

linear, 99

linear magnetic, 178

longitudinal, 255

microwave, 43, 254

radio frequency, 254

transverse, 101

trapezoidal approximation, 187

Figure of eight trajectory, 898 
Filamentation, 282, 581

in phase space, 581

Filling time, 629

First integral, 493

First order achromat, 208

Fixed point

in phase space, 274

stable, 557

unstable, 557

Flat undulator, 825

Floquet's coordinates, 233

Floquet's theorem, 316

Focal length, 102, 105, 184, 205, 322

Focal point, 102

Focusing, 63

in bending magnets, 190

in electrostatic accelerator, 51

principle of, 102

in sector magnets, 190

strong, 6

transverse, 66

Focusing devices, 102, 133

Focusing quadrupole, 182

with acceleration, 344

Focusing strength, 105

FODO, 311

acceptance, 308

cell, 304

channel, 186, 304

general lattice, 311

lattice, 304, 461

necktie diagram, 312

optimum phase advance, 307

parameter, 305, 306

symmetric lattice, 305

FODO cell, 329

betatron phase, 306

maximum acceptance, 307

optimum, 307

stability criterion, 312

FODO channel

acceptance, 308

region of stability, 312

stability criterion, 313

FODO lattice, 304

and acceleration, 339

dispersion function, 321

emittance, 461, 470

Fokker-Planck equation, 236, 401, 422, 425, 430, 438

solutions, 425,427

Force, centrifugal, 100

Formation length, 809

Form factor, 847
Forward cone, 916

Forward radiation, 916

Fourier series, 525, 529, 987

Fourier transform, 988

Fraunhofer

diffraction, 840

diffraction integral, 841

Free electron laser, 261, 273, 279, 803, 929

Frenet-Serret

coordinates, 87, 94

coordinate system, 106

Frequency

Larmor, 101

tuning, 647

Fringe field, 145, 181

Fringe field effect, 193

Fundamental frequency, 825, 826, 895, 904

Fundamental theorem of beam loading, 650

Gain curve, 940

Gain length, 944

Gapheight magnetpole shimming, 142

Gas desorption

coeficient, 397

photo electron current, 396

synchrotron radiation, 396

thermal, 395

Gaussian distribution, 27, 367, 427

Gaussian tails, 430

Gauss' integral theorem, 26, 36, 984, 986

Generating function, 91, 92, 115, 589

Generator voltage, 645

Geometric aberrations, 164, 573, 574

Glow discharge, 7, 45

Good field region, 142

GR(x.y.z), 882

Gradient, 128

field error, 142, 408, 541

field quality, 142

magnet, 132

Green's function, 121

Green's function method, 122

Group velocity, 609, 613, 629

Gyromagnetic constant, 454

Hall probe, 147

Hamilton's equations, 88

Hamilton's integral principle, 83

Hamiltonian, 89, 112, 234, 406

beam dynamics, 97, 109, 113

coupling, 685, 686

equations, 89 
extended, 95, 96

non linear, 547

non-linear, 565, 589

perturbation theory, 565,588

resonant terms, 550

synchrotron oscillations, 268, 270

Hard edge model, 107, 181

Harmonic correction of chromaticity, 584

Harmonic number, 73, 76, 258, 264, 624, 737

Harmonic oscillator, 114, 116, 123, 229, 406, 619

damped, 66, 404, 618

damping decrement, 405

driven, 617

Q-value, 618

resonace curve, 618

Harmonics, 824

Head-tail instability fast, 787

Heavy ion storage rings, 277

Heavy ions, 12

Helical undulator, 825

Helicity, 920

Higher-order modes (HOMs), 650, 740 loss parameter, 651

Hill's equation, 315

Hybrid magnet, 821

Hydrogen atom, 4

Ideal orbit, 61

Ideal path, 94

Impedance, 663, 738

broad band, 740, 753

internal, 642

resistive wall, 752

space charge, 751

Independent variable change of, 96

Induction, 30

Induction accelerator, 9

Insertion, 319, 335

device, 168,816

low beta, 335,337

quadrupoles, 338

Instability, 385

single bunch, 740

Integer resonance, 327, 503

Interaction region, 9

Intra beam scattering, 357, 385

Inverse matrix, 186

Ion beam, 7, 31

Ionization, 7

Iris doublet, 51
Iris electrode, 49

Iron dominated magnets, 131

Isochronous

beam transport line, 210, 279

ring, 368 quasi, 368

storage ring, 286

system, 208

Isomagnetic

lattice, 833

$-\mathcal{I}$ transformation, 584

$J J$-function, 915, 935

Kanalstrahlen, 4

Keil-Schnell stability criterion, 767

Keil-Schnell-Zotter criterion, 654

Kinematic perturbations, 478

Kinetic energy, 629

Klystron, 6, 55, 954

Lagrange function, 86

Lagrangian, 85, 112

beam dynamics, 110

charged particle electro-magnetic field, 85

formulation of beam dynamics, 109

Lamor frequency, 874

Langragian, 88

Laplace equation, 125

Large Hadron Collider (LHC), 833

Larmor frequency, 101, 673

Larmor precession, 5

Laser beams, 7

Laslett form factors, 723

Lattice, 303

achromatic, 207

cell, 303

combined function, 159,178

DBA, 469

double bend achromat, 241

FODO, 186, 304

function, 229

periodic, 303

superperiod, 303

resonances, 539

separated function, 159, 178

symmetric, 303

triple bend achromat, 241

unit, 304

Lawrence Berkeley Laboratory, 73 
Legendre transformation, 92

Libration, 268

Liénard-Wiechert potentials, 29, 799, 860

Lifetime, 385

Coulomb scattering, 389

inelastic scattering, 393

Linac efficiency, 631, 632

Line spectrum, 909 undulator, 915

Linear accelerator, 5, 8, 43, 54, 59, 413, 800 Alvarez, 6 electron, 5, 625

Linear beam dynamics, 99, 179

Linear collider, 10, 60, 432, 459

Linear fields, 99

Linear superposition, 489 of perturbations, 487

Linear systems, 177

Liouville's theorem, 120, 215, 229, 282, 356, 401, 406, 414

Lithium lens, 103

Longitudinal

emittance, 268, 281

fields, 253

oscillations, 263

phase space, 265

phase space stability, 73

stability limit, 273

tune, 321

Lorentz

contraction, 5, 16, 801

equation, 99

force, 5, 30, 31, 33, 44, 61, 86, 99, 100, $160,450,711,995$

force equation, 66

gauge, 28,858

transformation, 15, 18, 996

electromagnetic fields, 16

space-time coordinates, 16

Loss parameter, 651, 746

Low beta insertion, 337

Luminosity, 331, 472, 712, 829

MAD, 332

MAGNET, 142

Magnet

arc length, 192

bending, 139

bore radius, 143

coils, 62

combined function, 365

decapole, 128,154 deflecting, 132

dipole, 132, 138, 139

effective length, 187

excitation, 140

free spaces, 335

fringe field, 193

gap, 140

hard edge, 107

hard edge model, 181, 187, 193

iron dominated, 138

lattice, 100, 178, 303

longitudinal field, 165

mechanical forces, 173,925

misalignment, 137, 478

multipole, 135

octupole, 128, 154

periodic, 167

permanent, 131

pole profile, 133

quadrupole, 133

return yoke, 139

rotated, 136

separated function, 365

sextupole, 128, 154

strength parameter, 128

superconducting, 131, 138

synchrotron, 133, 134, 178

trapezoidal model, 187

upright, 136

Magnetic field

charged particles in a, 33

equation, 131

expansion, 107, 158

measurement, 145

potential, 158

uniform charged particle beam, 27

Magnetic permeability, 138

Magnetization curve, 144

Markoff process, 423

Matching, 332

$\frac{1}{4}$ wave length transformer, 347

codes, 332

conditions, 331,332

dispersion, 332

phase space, 282

photon beam, 849

Twiss parameters, 331

Matrix formalism, 180

non-linear, 569

Matrix formulation, 118

betatron function, 220

Maxwell's equations, 14, 26, 44, 125, 799, 857,995 
Measurement

beam emittance, 224

betatron function, 227, 409, 489

broad-band impedance, 786

coupling coefficient, 692

dispersion function, 238

energy spectrum, 245

momentum and spectrum, 245

momentum resolution, 245

natural chromaticity, 512

tune, 321

Micro bunches, 354

Microwave instability, stability criterion, 766

Mid-plane symmetry, 127, 136

Modes, parasitic, 739

Moivre's formula, 311

Momentum, 21

canonical, 84, 86, 89, 112

compaction, 248, 250, 263, 266, 279, 286, 368,429

conjugate, 86,95

conservation, 804

error, 108

longitudinal, 97

ordinary, 86

resolution, optimum, 247

spread, 633

Momentum acceptance, 273, 275

higher order effects, 298

Momentum compaction factor, 249, 260, 329

approximate, 329

higher order, 291, 292

Moving rf-buckets, 272, 277

Multipole

magnets, 135,154

strength, 149

terms, 154

upright, 130

Multipole field errors, 565

allowed, 143

higher order, 141

random, 143

Nabla operator, 405

Necktie diagram, 312

Negative mass instability, 757

Network model, rf-cavity, 642

Neutrino horn, 104

Neutron, 6

Normalized

coordinates, 229

emittance, 345, 356
Octupole magnet, 128, 135, 154

Operating point, 320, 546

Optical klystron, 802

Orbit, 64

correction magnets, 494

correction schemes, 496

design- or ideal-, 61

equilibrium, 325

kick, 494

Orbit distortion, 138, 164, 494, 567

dominant harmonic, 488

expectation value, 526

harmonic content, 495

normalized coordinates, 488

Oscillation amplitude, 266

Oscillator, harmonic, 114

Over voltage factor, 276

Panofsky-Wenzel theorem, 384

Parabolic current sheet lens, 103

Parasitic losses, broad band, 740

Parasitic modes, 739

Paraxial approximation, 34

Paraxial beam optics, 97, 478

Paraxial beams, 107

Parseval's theorem, 873, 988

Particle

capture, 632

density distribution, 402

energy, 12

tracking, 587

trajectories, with betatron functions, 231

Particle beam

bunch length, 368

bunches, 354

continuous, 354

current, 354

damping, 358

dimension, 356

dynamics, 26, 106

emittance, 356

energy, 356

energy spread, 366

equilibrium transverse emittance, 368

focusing, 102

Gaussian distribution, 367

guidance, 102

halo, 385

intensity, 356

micro bunches, 354

parameters, 353 
polarization, 453

pulsed, 354

size, 356

stability, 37

standard sizes, 374

time structure, 354

Particle bunch, 279

bunch length, 279

Particle distribution, 223

aperture limited, 430

equilibrium, 437

Gaussian, 223, 846

longitudinal, 365, 429

no damping, 432

in phase space, 437

Vlasov equation, 405

Path length, 69, 103, 107, 208, 260

higher order, 289

momentum dependence, 248

Pendulum equation, 936

Periodic lattice

betatron functions, 316

betatron oscillation, 309

characteristic coefficient, 316

in circular accelerator, 329

determinant of matrix, 316

lattice functions, 318

perturbation in circular accelerators, 528

stability criterion, $310,311,319$

trace, 316

transformation matrix, 310 trace, 310

Period length, 168

Permanent magnet, wiggler, 821

Permeability, 144, 604

absolute, 14

magnetic, 13

relative, 14

vacuum chamber, 137

Perturbation(s), 121

alignment error, 477

in beam dynamics, 477

betatron function, 506

coupling terms, 544

detectors for high energy physics, 477

dipole field, 481

field error, 477

insertion devices, 477

kinematic, 478, 522

magnetic field, 522

periodic distribution, 525

quadrupole field, 499

self compensation, 479 statistical methods, 530

terms, 177, 408, 480, 566

Phase

diagram, 274

function, 228, 229, 320

oscillations, stability limits, 299

plane, 213

stability, 266

stable region, 272, 274

velocity, 256, 607, 612

Phase advance, drift space, 233

Phase ellipse, 218, 222, 229, 235, 332

parameters, 220

Phase equation, 264

longitudinal, 262

Phase focusing, 6, 73, 267

higher order, 286

Phase oscillation(s), 272, 656

coherent, 657

damping decrement, 263

focusing, 272

large amplitudes, 268

Robinson damping, 657

small amplitudes, 262

Phase space, 213, 356

constant area of, 216

beam matrix, 222

continuity equation, 215

current, 215

density, 283

density distribution, 215

diagram, 271, 272

ellipse, 277, 683

ellipse transformation, 220

exchange, 446

filamentation, 283, 581

focusing, 256

geometric beam emittance, 219

higher order dynamics, 292 secondary buckets, 294

longitudinal, 265, 428, 634

dynamics, 256

stability criteria, 296

stable fixed point, 274

unstable fixed point, 274

manipulation, 446

matching, 282, 331

motion, 235

parameters, longitudinal, 274

particle motion, 563

phase ellipse, 218

rotation, 446

stable, 280

transformation, 218, 219 
Phasor diagram, 646, 647, 655

Photo cathode, 8

Photo electron current, 396 emission coeficient, 397

Photo-electric effect, 4

Photon beam divergence, 839 matching, 849 temporal structure, 817

Photon energy critical, 802, 836

rms, 439

undulator, 826

Photon flux

angular, 838

differential, 879

per unit solid angle, 836 spectral, 439, 890 total, 439

Photon source parameters, 849,851

Photons, backscattered, 828

Photon spectrum, 367

Physical constants, 993

Pill box cavity, 616

Pin hole, 912

Plane wave, 44

Plasma frequency, 944

Plasma lens, 103

Poincaré integral, 268

Point charge, 26

Point to point imaging, 206

POISSON, 142

Poisson bracket, 93

Poisson's equation, 709

Polar coordinates, 990

Polarization, 879, 918

elliptical, 802, 918

of particle beam, 453

$\pi$-mode, 837, 918

$\sigma-$ mode, 837, 918

states, 876

time, 453

Pole face, 208

angles, 209

entrance, 208

exit, 208

Pole profile, 141

Pole root, 145 saturation, 145

Positron, 5, 6

Potential(s) retarded, 859, 996 scalar, 27-29, 858 vector, $27,28,857$

well distortion, 662, 771

wells, 269

Poynting vector, $36,804,807,808,864$

Prebuncher, 8, 634, 637

Preinjector, 634

Principal solutions, 117

Principle of phase focusing, 270, 282

Propagating waves, 606

Proton

beam, 5

radiation power, 833

source, 7

Proton accelerator, Alvarez-type, 7

Quadrupole, 104, 105, 133, 141

bore radius, 143

defocusing, 183, 344

design concepts, 140

doublet, 184, 205

focal length, 106

transformation matrix, 1003

electrostatic, 172

end field effects, 187

excitation, 143

field gradient, 187

focal length, 105

focusing, 182, 344

fringe field correction, 187

misalignments, 491

pole tip field, 145

rotated, 133, 669

strength, 35, 105

transformation matrix, 1003

upright, 133

Quadrupole triplet

transformation matrix, symmetric, 1004

Quad scan, 226

Quality factor, 626

loaded, 643

unloaded, 643

Quantum excitation, 6, 366, 371, 430

Quantum lifetime, 431

Q-value, 618, 623

$R_{56}, 449$

Race track microtron, 70

Radiance, 848

Radiation

bending magnet, 816

cleaning, 398

coherent, 843 
cone, 872

forward, 916

length, 394

lobes, 868

longitudinal acceleration, 811

regime, 809,862

shielding, 844

sources, 460

spatial and spectral distribution, 22, 23

spectrum, 834, 879

spontaneous, 930

stimulated, 929

synchrotron, 807

transverse acceleration, 831

undulator, 6

Radiation field, 862, 996

longitudinal acceleration, 810

spectral, 878

Radiation power, 830,831

instantaneous, 832

spatial distribution, 871,882

total, 834,865

undulator, 901

wiggler, 821

Radio antenna, 801

Radio frequency

antenna, 801

fields, 7

Rayleigh length, 941

Rectangular magnet, 198

focusing, 198

gap effect, 200

straight magnet length, 199

transformation matrix, 1001

Recursion formula, 153

Reference

orbit, 487

particle, 88

path, 324, 487

phase, 260

trajectory, 106

Relativistic

factor, 15,20

relations, 996

Residual gas atoms, 385

Resistive wall, 738

Resistive-wall instability, transverse, 770

Resonance, 236, 327, 503, 539

conditions, 540-543, 545, 695

coupling, 544, 545

curve, 618

diagram, 545

difference, 689, 690

forbidden, 544 frequency, 612

half integer, 502, 504, 541, 556, 558

Hamiltonian theory, 539

higher order, 546

higher-order coupling, 695

integer, 503, 541

lattice, 539,543

linear, 539

multiple, 695

nonlinear, 540, 542, 543

order of, 543,546

pattern, 553, 556

perturbation terms, 540

stop band, 503, 543

structural, 539, 543

sum, 689, 694

third order, 542, 560, 563

Resonant frequency, rf-cavity, 642

Response matrix, 494, 496

Restoring force, 64

Retarded

potentials, $5,799,859$

time, 29, 799, 858

Reversed matrix, 185

Revolution frequency, 12, 250, 264, 321, 803, 834

Revolution time, 68

rf-bucket, 268, 273

moving, 272, 277, 281

stationary, 269, 277, 281

rf cavity(ies), 254, 603, 614

cylindrical, 614

energy gain, 616

energy transfer, 653

field, 615

filling time, 619

impedance, 644, 659, 661

losses, 619

network modelling, 642

pill box, 616

resonant, 54

resonant frequency, 615

transit time, 255, 616

wall losses, 621

rf fields, 254

rf-fields and particles, 641

rf frequency, 264, 624

fundamental, 258

rf klystron, 59

rf-parameter, 623

rf phase, 258

rf-voltage, 625

rf-waveguide, 603

Ring acceptance, 388 


\author{
Robinson \\ criterion, 422 \\ damping, 660 \\ damping criterion, 362, 375, 419 \\ wiggler, 382 \\ Rotated magnet, 136 \\ Rotating coil, 148 \\ "bucked" signals, 150 \\ two coils, 150 \\ Rutherford scattering, 387
}

\section{SASE-FEL, 942}

Satellite frequencies, 321

Saturation, 143

Scalar potential, 104, 126, 133, 152, 858

Scattering angle, 386

Second integral, 493

Sector magnet, 190, 191, 208

fringe field, focal length, 195

transformation matrix, 1000

Self fields

electric, 710

magnetic, 710

space-charge, 711

Sensitivity, 151

Separated-function lattice, 159, 365

Separatrix, 268, 275, 556

Sextupole

magnet, 128, 135, 154

tune shift, 536

Shimming, 141 magnet pole, 142

Shunt impedance, 619, 621, 627, 642 specific, 622

Sine like solutions, 118

Single value decomposition (SVD), 497

Skew magnet, 127, 130

Skew quadrupole, 133, 669

Skin depth, 620

SLAC linac structure, 628

Small gain FEL, 932

Smooth approximation, 358

Solenoid, 165

field strength, 166, 670

focal length, 678

transformation matrix, 675, 677, 678

Space charge

fields, 715

forces, 37

self fields, 708, 711

tune shift, 624

Spatial coherence, 844
Spatial distribution, synchrotron radiation, 869

SPEAR, 336

Special relativity, 5, 15

Spectral brightness, 848, 917

Spectral line width, 909

Spectral photon flux, 439, 890

Spectral purity, 909

Spectrometer, 244

180 degree, 244

Spectrum, 834

Spill-down, 137, 138, 477

Spin, 453

rotator, 453

Spontaneous radiation, 930

Stability criterion, 64, 65, 310, 556

Steenbeck's, 65

Stability limit, longitudinal, 273

Stable phase space, 280

Standard size, 374

Standing wave, 614

Stationary buckets, 277

Steenbeck's stability criterion, 65

Stimulated radiation, 929

Stochastic cooling, 704

Stokes' theorem, 27, 30, 139, 986

Stop band, 503, 505, 555

half integer, 555

n-th order, 559

width, 544, 546, 553, 556, 558

Storage ring, $8,9,77,803$

beam emittance, 461

heavy ions, 277

isochronous, 286, 296

principle, 6

Strength parameter, 820

Strong focusing, 6, 75, 77, 116, 178

Structural resonance, 539

Sum resonance, 689

Superbend, 802, 817

Superconducting

dipole magnet, current distribution, 174

magnets, 138

Superperiodicity, 544

Superperiods, 330, 331, 543

Super Proton Synchrotron (SPS), 833

Surface resistance, 621

Symmetric quadrupole triplet, 186

Synchro cyclotron, 73

Synchronicity condition, 54, 68, 70, 75, 77, $256,257,272$

Synchronous particle, 271

Synchronous phase, 257, 260, 270, 277, 625 
Synchronous rf phase, 266

Synchrotron, 6, 800

booster, 10

electron, 6

lattice, 330

radiation, 807

Synchrotron magnet, 108, 132, 134, 192

defocusing, 239, 1002

focusing, 239

transformation matrix rectangular magnet, 1002

sector magnet, 1002

Synchrotron oscillation, 110, 263, 366

damping decrement, 415

frequency, 263, 264, 266, 276, 321, 429

frequency shift, 661,662

incoherent tune shift, 664

stability, 656

tune, 264,321

Synchrotron radiation, 5, 6, 9, 59, 67, 168, 261, $356,358,366,378,484,812$

angular distribution, 881

coherent, 843

diffusion coefficient, 438

energy loss, 277

energy loss per turn, 833

gas desorption, 396

harmonics, 880

polarization, 877

$\pi-$ mode, 877

$\sigma-$ mode, 877

power per unit solid angle, 865

spatial distribution, $869,871,874,878$

spectral distribution, 874,878

spectrum, 889

total power, 865

Synchrotron tune, 264

Target, 7

stationary, 25

TBA lattice, 241

TE-modes, 609

Temporal coherence, 844

Thales of Milet, 3

Thermal gas desorption, 395

Thermionic emission, 4

$\vartheta$-parameter, 416

Thin lens approximation, 184, 305

Thomson scattering, 828

cross section, 829

Time dilatation, 17

TM-modes, 609
Tolerance

alignment, 490

assembly, 477, 490

gradient, 142

quadrupole field, 499

requirements, 492

statistical, 490

Touschek effect, 705

Transformation

betatron function, 220

canonical, 90

nonlinear coefficient, 570

of phase space, 219

Transformation matrix, 118, 181

with acceleration, 343

in defocusing quadrupole, 344

drift space, 344

in drift space, 343

in focusing quadrupole, 344

in quadrupole, 343

chromatic, 322

composite beam line, 183

defocusing quadrupole, 183

drift space, 182

focusing quadrupole, 182

FODO cell, 305

inverse lattice, 186

in normalized coordinates, 234

quadrupole doublet, 184

rectangular magnet, 199, 239

reversed lattice, 185

sector magnet, 192

fringe field, 196

skew quadrupole, 671

solenoid, 675, 677, 678

symmetric quadrupole triplet, 186

synchrotron magnet, 192, 239, 1002

in terms of betatron functions, 231

triplet, 186

wedge magnet, 197, 198

$3 \times 3$ Transformation matrix, 123, 238

Transformer principle, 30

Transient time, 621

factor, 621

Transit time factor, 255,616

Transition energy, 250, 266

approximate, 329

Transition radiation, 865

spatial distribution, 867

spectral distribution, 868

total energy, 868

TRANSPORT, 332

nomenclature, 569

Transport line, non-isochronous, 447 
Transverse focusing, 66

Travel time, 249

Trim magnets, 494

Triple bend achromat, 243

Tune, 116, 320

amplitude dependent, 550

approximate, 320

measurement, 321, 501

spread, 409

synchrotron oscillation, 264

Tune shift, 409, 502, 527

amplitude dependence, 543, 597

chromatic, 512

coherent, 770

due general perturbations, 409

field errors, 500

higher order, 595

in sextupoles, 536

space charge, 624

synchrotron oscillation, 772, 773

Tuning angle, 644, 645, 657

optimum, 647, 649

Twin paradox, 17

Twiss function, 220, 227, 229

in terms of conjugate trajectories, 679

Two rf-system, 434

Ultra high vacuum (UHV), 395

Undulator, 801

flat, 825

fundamental wavelength, 823, 826, 900

helical, 825

line spectrum, 801, 915

magnet, 167, 821, 822, 895

period, 801

radiation, period length, 801

strength parameter, 820

Unit conversion, 994

Units, 11

cgs, 13

SI or mks, 11

Universal function, 838, 889

Upright magnets, 136

Upright multipoles, 127

\footnotetext{
Vacuum

chamber environment, 261

ultra high, 395

Van de Graaff

accelerator, 5, 7, 47

Tandem, 47

Vanadium Permendur, 821
}

\author{
Variables \\ canonical, 89, 92 \\ cyclic, 90, 115 \\ Variational principle, 83, 91 \\ Vector \\ algebra, 983 \\ curl, 984 \\ divergence, 984 \\ potential, 126, 857 \\ 4-vector, 18, 997 \\ acceleration, 19, 830, 997 \\ EM potential, 22 \\ energy-momentum, 20 \\ force, 22 \\ Lorentz invariance, 18, 39 \\ Lorentz invariants, 998 \\ photon, 22 \\ space-time, 18, 997 \\ velocity, 19, 997 \\ Velocity of light, 18 \\ Virtual photon, 16 \\ Vlasov equation, 236, 401, 402, 405, 419, \\ 775 \\ damping, 410 \\ Voltage breakdown, 253
}

Wake fields, 737, 738, 740

Wave equation(s), 28, 604, 858, 995

Wave number, 259

Waveguide

cutoff wave number, 612

cylindrical, 610

disk loaded, 6, 625

fields, 608, 613

modes, 607

parameter, 626

rectangular, 605

TE/TM-modes, 609

wall losses, 626

wavelength, 609

Wavelength

fundamental, 823, 900

shifter, 802,818

undulator, 826

Weak focusing, 63, 66, 74, 178

Wedge magnet, 196, 209

fringe field, focal length, 197

gap effect, 198

pole face rotation, 196

transformation matrix, 197, 198, 1001

Wideroe

condition, 60, 62, 63

structure, 55 
Wiggler magnet, 6, 167, 801, 819, 895 achromat, 243

asymmetric, 921

beam deflection, 171

critical photon energy, 822

dispersion function, 243

electromagnetic, 821

field, 170

flat, 167

focusing, 200

hard edge model, 203

effective length and rho, 204

helical, 167 period, 168

permanent magnet, 821

strength parameter, 171,820

transformation matrix, 203

Wire lens, 103

Wronskian, 119, 217, 404, 681

Wyorld time, 19

\author{
X-ray(s), 5 \\ beams, 63 \\ tube, 45
}

